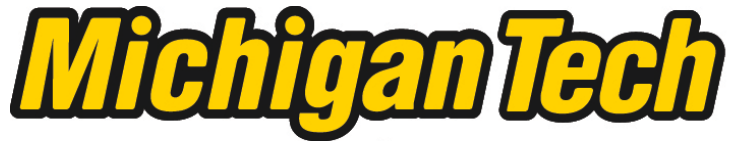 \\ Michigan Technological University Create the Future Digital Commons @ Michigan Tech
}

Dissertations, Master's Theses and Master's Reports - Open

Dissertations, Master's Theses and Master's

Reports

2012

\section{Investigation into the enhancement of polycarbonate with conductive nanomaterials}

Michael D. Via Jr.

Michigan Technological University

Follow this and additional works at: https://digitalcommons.mtu.edu/etds

Part of the Chemical Engineering Commons

Copyright 2012 Michael D. Via Jr.

\section{Recommended Citation}

Via Jr., Michael D., "Investigation into the enhancement of polycarbonate with conductive nanomaterials", Dissertation, Michigan Technological University, 2012.

https://doi.org/10.37099/mtu.dc.etds/10

Follow this and additional works at: https://digitalcommons.mtu.edu/etds

Part of the Chemical Engineering Commons 


\title{
INVESTIGATION INTO THE ENHANCEMENT OF POLYCARBONATE WITH CONDUCTIVE NANOMATERIALS
}

\author{
By \\ Michael D. Via Jr. \\ A DISSERTATION \\ Submitted in partial fulfillment of the requirements for the degree of \\ DOCTOR OF PHILOSOPHY \\ (Chemical Engineering) \\ MICHIGAN TECHNOLOGICAL UNIVERSITY
}

2012

(C) 2012 Michael D. Via Jr. 
This dissertation, "Investigation into the Enhancement of Polycarbonate with Conductive Nanomaterials," is hereby approved in partial fulfillment of the requirements for the Degree of DOCTOR OF PHILOSOPHY IN CHEMICAL ENGINEERING.

Department of Chemical Engineering

Signatures:

Dissertation Advisor

Department Chair
Dr. Julia A. King

Dr. Tony N. Rogers

Date 


\section{Table of Contents}

TABLE OF CONTENTS ...................................................................................................... i

LIST OF FIGURES................................................................................................................... vii

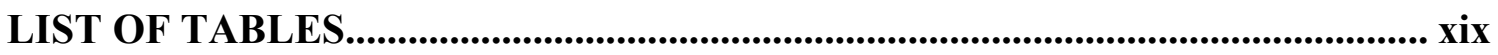

ACKNOWLEDGEMENTS ............................................................................................ xliii

NOMENCLATURE .............................................................................................................. xliv

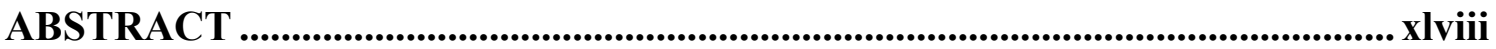

CHAPTER 1: INTRODUCTION ...............................................................................1

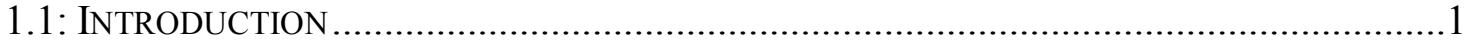

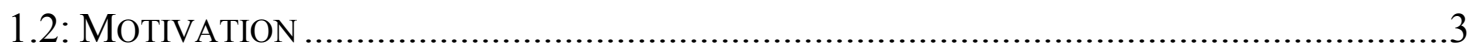

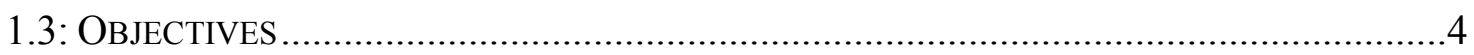

CHAPTER 2: BACKGROUND .....................................................................................5

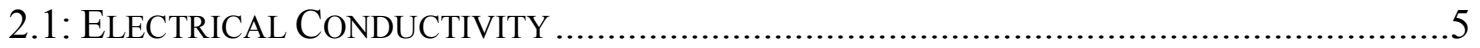

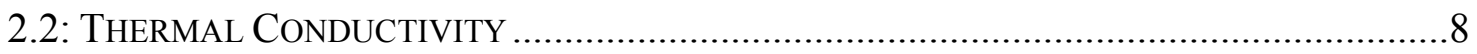

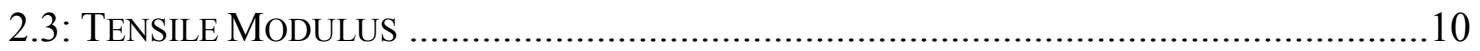

CHAPTER 3: MATERIALS ...............................................................................................14

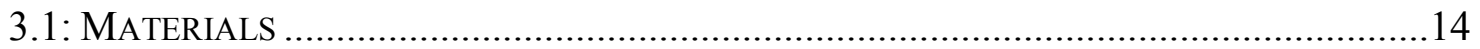

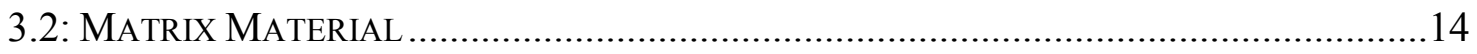

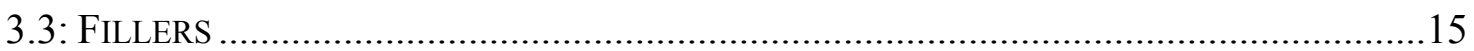

3.3.1: Hyperion Catalysis International's FIBRIL Multiwalled Carbon

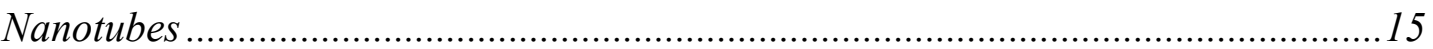

3.3.2: Ketjenblack EC-600 JD Carbon Black …………....................................... 17

3.3.3: XG Sciences $x G n P^{\mathrm{TM}}$ Graphene Nanoplatelets.............................................19

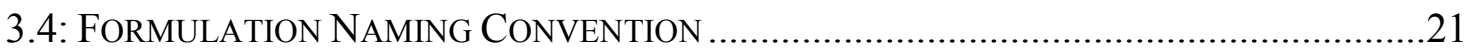

CHAPTER 4: FABRICATION AND EXPERIMENTAL METHODS.....................23

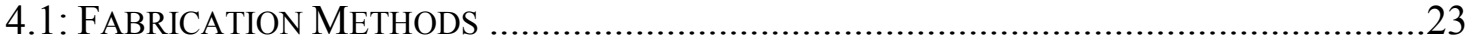

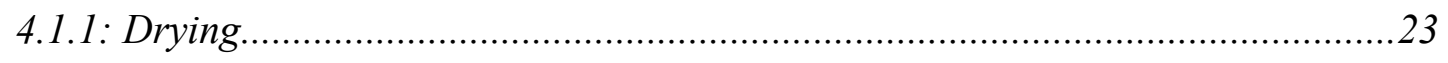

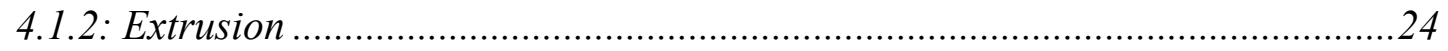

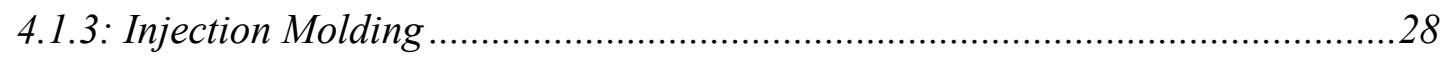

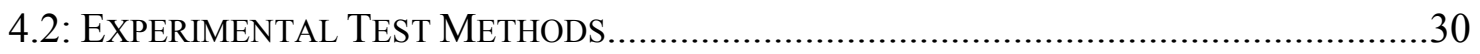

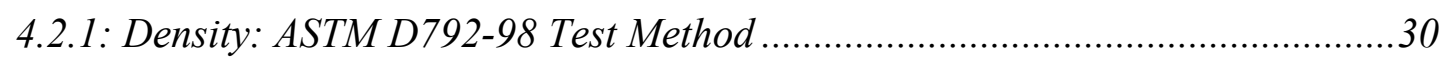

4.2.2: Melt-Flow Indexing (MFI) Test Method ………….........................................

4.2.3: Differential Scanning Calorimetry (DSC) Test Method.....................................32

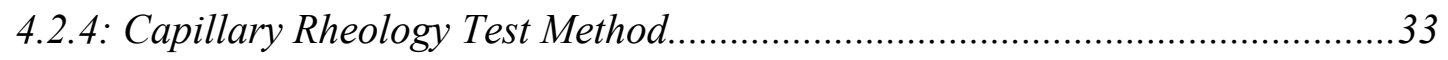


4.2.5: Small Amplitude Oscillatory Shear Rheology Test Method ...............................37

4.2.6: Through-Plane Electrical Resistivity Test Method ...........................................39

4.2.7: In-Plane Electrical Resistivity Test Method....................................................40

4.2.8: Thermal Conductivity: Guarded Heat Flow Meter Test Method ......................42

4.2.9: Mechanical Tensile Property Test Method ....................................................4

4.2.10: Mechanical Flexural Property Test Method ………………….......................4

4.2.11: Optical Polishing Method ........................................................................... 45

4.2.12: Field Emission Scanning Electron Microscope (FESEM) Test Method.........49

4.2.13: Transmission Electron Microscope (TEM) Test Method ...............................51

4.2.14: Environmental Scanning Electron Microscope (ESEM) Test Method............53

CHAPTER 5: MISCELLANEOUS RESULTS.............................................................55

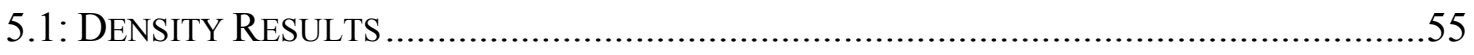

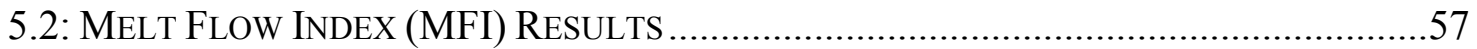

5.3: DifFERENTIAL SCANNING CALORIMETRY (DSC) RESULTS ......................................60

5.4: IN-PlANE ELECTRICAL RESISTIVITY OF EXTRUDED RodS .......................................64

CHAPTER 6: ELECTRICAL RESISTIVITY, THERMAL CONDUCTIVITY AND TENSILE AND FLEXURAL PROPERTIES OF CARBON NANOTUBE/POLYCARBONATE COMPOSITES................67

6.1: FieLd EMISSION SCANNING ELECTRON MicrosCope (FESEM) AND

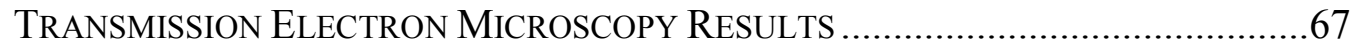

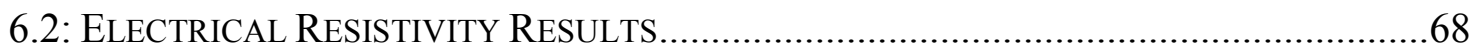

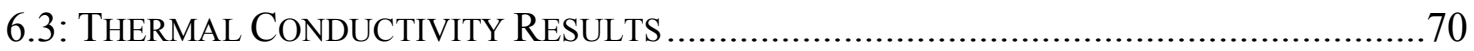

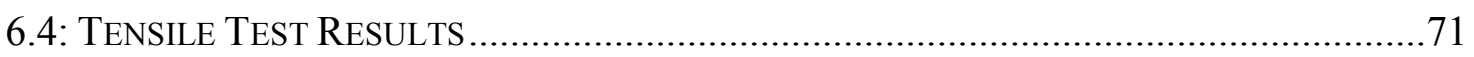

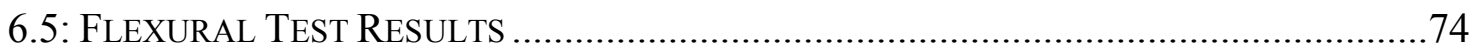

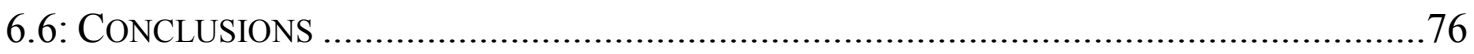

CHAPTER 7: ELECTRICAL RESISITIVITY, THERMAL CONDUCTIVITY AND TENSILE AND FLEXURAL PROPERTIES OF CARBON BLACK/POLYCARBONATE COMPOSITES AND COMPARISON WITH CARBON NANOTUBE/POLYCARBONATE COMPOSITES .............................................................................................77

7.1: Field EMisSion SCANNING ELECTRON Microscope (FESEM) ReSUltS.................77

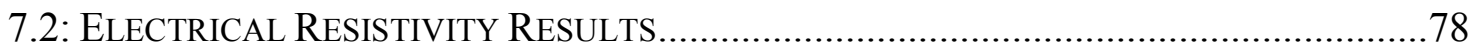

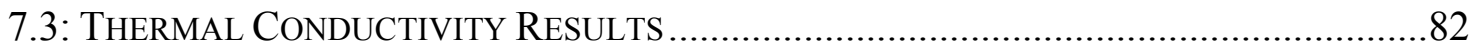

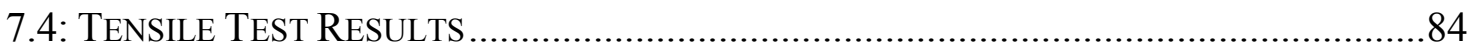

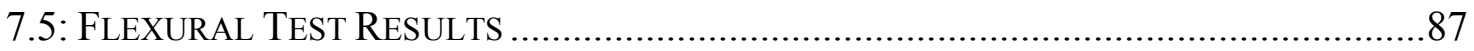

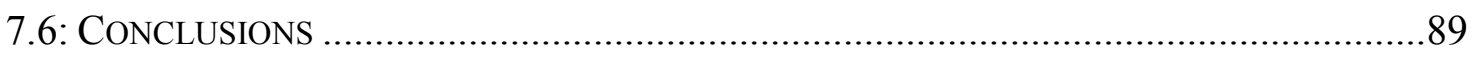




\section{CHAPTER 8: RHEOLOGICAL PROPERTIES OF CARBON}

NANOTUBE/POLYCARBONATE COMPOSITES AND CARBON

BLACK/POLYCARBONATE COMPOSITES.

8.1: TRANSMISSION ELECTRON MiCROSCOPE (TEM) RESUlTS.

8.2: SMALL-AMPLITUdE OSCILLATOR SHEAR (SAOS) RESUlTS ..................................91

8.3: CAPILLARY RHEOMETER RESUlTS: CNT/PC ......................................................95

8.4: CAPILLARY RHEOMETER RESUlTS AND DisCUSSION: CB/PC ...............................102

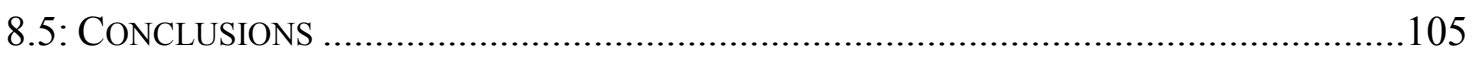

CHAPTER 9: ELECTRICAL RESISTIVITY, THERMAL

CONDUCTIVITY, AND TENSILE, FLEXURAL, AND

RHEOLOGICAL PROPERTIES OF EXFOLIATED GRAPHITE

NANOPLATELET/POLYCARBONATE COMPOSITES

9.1: ENVIRONMENTAL SCANNING ElECtRon Microscope (ESEM) RESUltS. 107

9.2: ELECTRICAL RESISTIVITY RESULTS.. 108

9.3: THERMAL CONDUCTIVITY RESULTS. 110

9.4: TENSILE TEST RESUlTS. 112

9.5: FLEXURAL TEST RESUlTS 114

9.6: CAPILLARY RHEOMETER RESULTS ... 117

9.7: CONCLUSIONS 120

CHAPTER 10: MODELING THE ELECTRICAL CONDUCTIVITY OF

CARBON BLACK/POLYCARBONATE, CARBON

NANOTUBE/POLYCARBONATE, AND EXFOLIATED

GRAPHITE NANOPLATELET/POLYCARBONATE COMPOSITES.

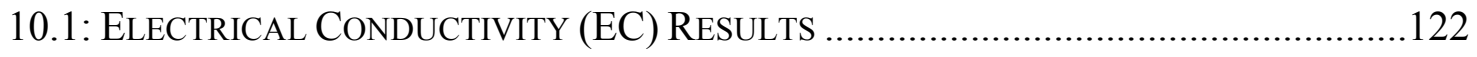

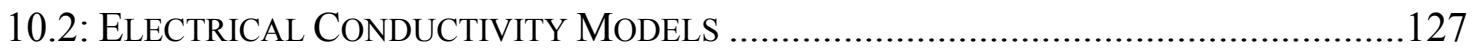

10.3: CONCLUSIONS .132

CHAPTER 11: TENSILE MODULUS MODELING OF CARBON

BLACK/POLYCARBONATE, CARBON

NANOTUBE/POLYCARBONATE, AND EXFOLIATED

GRAPHITE NANOPLATELET/POLYCARBONATE

COMPOSITES.

11.1: EleCtrical Conductivity AND PERCOLATION Models ................................134

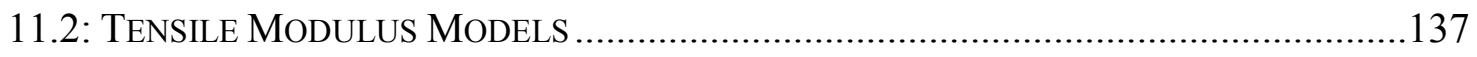

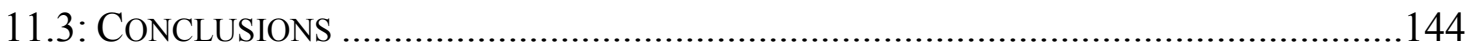

CHAPTER 12: EFFECTS OF MULTIPLE FILLERS ON THE ELECTRICAL RESISTIVITY, THERMAL CONDUCTIVITY AND 
TENSILE AND FLEXURAL PROPERTIES OF

POLYCARBONATE BASED COMPOSITES................................................146

12.1: TRANSMISSION ELECTRON MiCROSCOPE (TEM) RESULTS......................................146

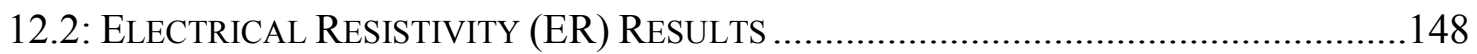

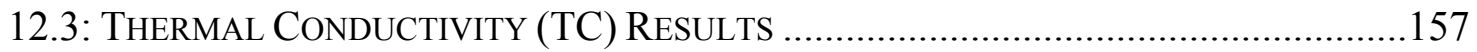

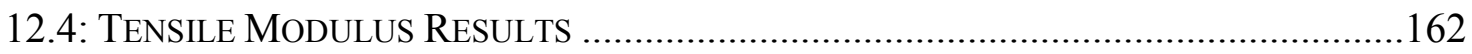

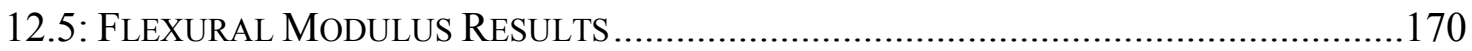

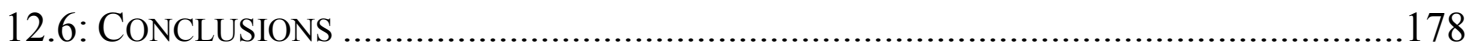

CHAPTER 13: EFFECTS OF MULTIPLE FILLERS ON THE RHEOLOGY OF POLYCARBONATE BASED RESINS ..............................180

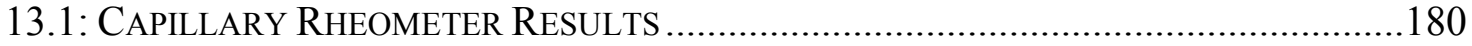

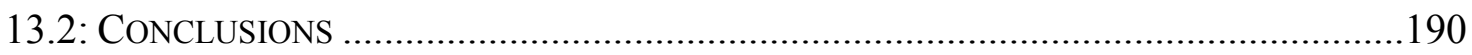

CHAPTER 14: CONCLUSIONS AND FUTURE WORK.........................................192

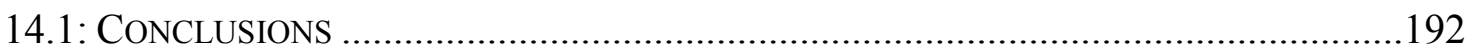

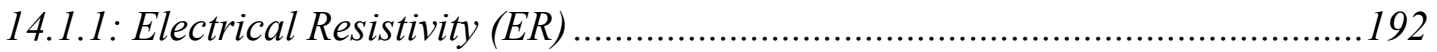

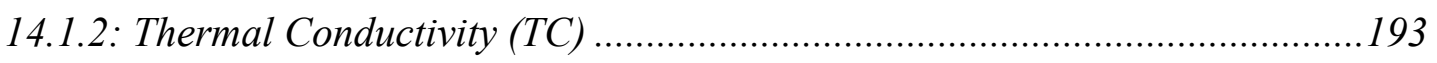

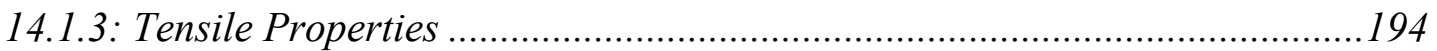

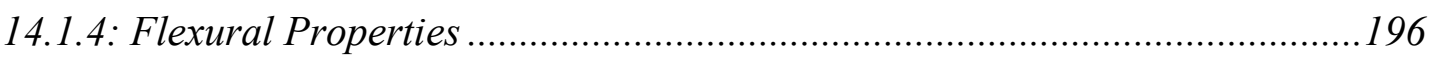

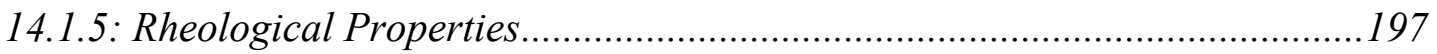

14.1.6: Electrical Conductivity and Tensile Modulus Modeling............................199

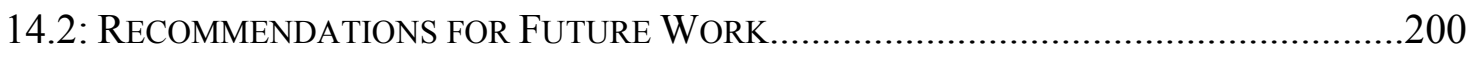

CHAPTER 15: REFERENCES ................................................................................205

\section{APPENDIX A: SCREW DESIGN AND EXTRUSION RUN CONDITIONS .......216}

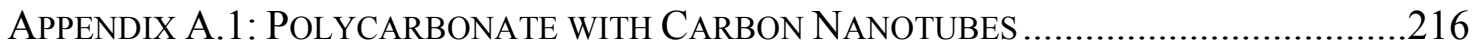

APPENDIX A.2: PolyCARBONATE WITH CARBON BLACK ………................................22

APPENDIX A.3: PolyCARBONATE WITH GRAPHITE NANOPLATELETS .............................226

APPENDIX A.4: PoLYCARBONATE WITH MULTIPLE FILLERS .......................................231

APPENDIX B: INJECTION MOLDING RUN CONDITIONS................................241

APPENDIX B.1: PolyCARBONATE WITH CARBON NANOTUBES .....................................241

APPENDIX B.2: PolyCARBONATE WITH CARBON BLACK ..........................................243

APPENDIX B.3: PolyCARBONATE WITH GRAPHITE NANOPLATELETS ............................247

APPENDIX B.4: PolyCARbONATE WITH MultiPLE FiLlerS ........................................251

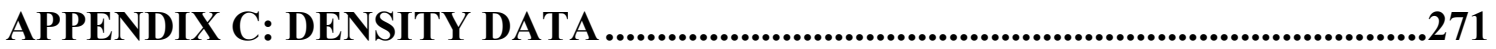

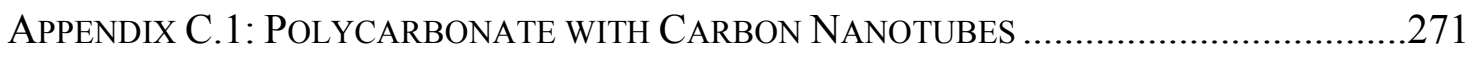


APPENDIX C.2: POLYCARBONATE WITH CARBON BLACK ..........................................275

APPENDIX C.3: PolyCARBONATE WITH GRAPHENE NANOPLATELETS ..........................2.

ApPENDiX C.4: PolyCARbONATE WITH Multiple Fillers .......................................285

APPENDIX D: MELT FLOW INDEXING RESULTS .......................................294

APPENDiX D.1: Polycarbonate With CARBon NANOTUBES ...................................294

APPENDIX D.2: POLYCARBONATE WITH CARBON BLACK ……...................................2.

APPENDIX D.3: PolYCARBONATE WITH GRAPHENE NANOPLATELETS..........................300

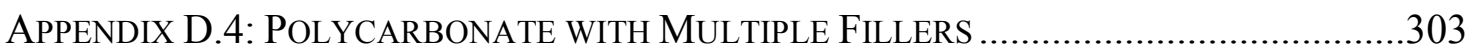

APPENDIX E: SAMPLE DIFFERENTIAL SCANNING CALORIMETRY (DSC) RESULTS......................................................................................309

ApPendix E.1: PolyCarbonate with CARbon NANOTUBeS ....................................309

APPENDIX E.2: PolyCARBONATE WITH CARBOn BlaCK..............................................316

APPENDIX E.3: PolyCARBONATE WITH GRAPHENE NANOPLATELETS ...........................321

APPENDiX E.4: PolyCARBONATE WITH Multiple Fillers .......................................326

APPENDIX F: IN-PLANE ELECTRICAL RESISTIVITY (TWO-PROBE) RESULTS ON EXTRUDED RODS ..........................................................356

APPENDIX F.1: PolyCARBONATE WITH CARBON BLACK .............................................356

APPENDIX F.2: PolyCARBONATE WITH GRAPHENE NANOPLATELETS ..........................363

APPENDIX F.3: PolyCARBONATE WITH MultiPLE FiLLERS..........................................366

APPENDIX G: THROUGH PLANE ELECTRICAL RESISTIVITY

(ASTM D257) RESULTS ............................................................................380

ApPendix G.1: Polycarbonate with Carbon NANOTUBeS ....................................380

APPENDIX G.2: PolyCARBONATE WITH CARBON BLACK ..............................................382

APPENDIX G.3: PolyCARBONATE WITH GRAPHITE NANOPARTICLES ..........................385

ApPENDiX G.4: PolyCARBONATE WITH Multiple FilLERS ........................................389

APPENDIX H: IN-PLANE ELECTRICAL RESISTIVITY (TWO-PROBE) RESULTS ....................................................................................................394

APPEndix H.1: PolyCARBonate with CARBOn NANOTUBES .....................................394

ApPENDIX H.2: PolyCARBONATE WITH CARBON BlACK .............................................398

APPENDIX H.3: PolyCARBONATE WITH GRAPHITE NANOPARTICLES ..........................405

APPENDiX H.4: POLYCARBONATE WITH Multiple FiLLERS .....................................407

APPENDIX I: THROUGH-PLANE THERMAL CONDUCTIVITY (ASTM F433) TEST RESULTS ......................................................................424

APPENDIX I.1: PolyCARBONATE WITH CARBON NANOTUBES .....................................424

APPENDiX I.2: PolyCARBONATE WITH CARBOn BLACK ..........................................428 
APPENDIX I.3: POLYCARBONATE WITH GRAPHITE NANOPARTICLES 432

APPENDIX I.4: POLYCARBONATE WITH MULTIPLE FILLERS. 437

APPENDIX J: MECHANICAL TENSILE PROPERTY RESULTS .448

APPENDIX J.1: PolyCARBONATE WITH CARBON NANOTUBES ..........................................448

APPENDIX J.2: POLYCARBONATE WITH CARBON BLACK...............................................464

APPENDIX J.3: POLYCARBONATE WITH GRAPHITE NANOPARTICLES ...............................482

APPENDIX J.4: PoLYCARBONATE WITH MULTIPLE FILLERS ………………………......500

APPENDIX K: MECHANICAL FLEXURAL PROPERTY RESULTS ................576

APPENDIX K.1: PolyCARBONATE With CARBON NANOTUBES ....................................576

APPENDIX K.2: POLYCARBONATE WITH CARBON BLACK ……………………….......592

APPENDIX K.3: PolyCARBONATE WITH GRAPHITE NANOPARTICLES ...........................610

APPENDIX K.4: POLYCARBONATE WITH MULTIPLE FILLERS ............................................628

APPENDIX L: SMALL AMPLITUDE OSCILLATORY SHEAR

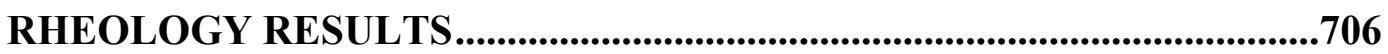

APPENDIX L.1: PolyCARBonate With CARBOn NANOTUBES .....................................706

APPENDIX F.2: PolYCARBONATE WITH CARBON BLACK ………...................................719

APPENDIX L.3: PolyCARBONATE WITH GRAPHENE NANOPLATELETS ............................731

APPENDIX M: CAPILLARY RHEOLOGY RESULTS..........................................749

APPENDIX M.1: PolyCARBONATE WITH CARBON NANOTUBES …………………..........749

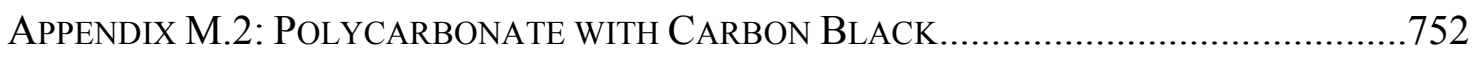

APPENDIX M.3: PoLYCARBONATE WITH GRAPHENE NANOPLATELETS ..........................754

APPENDIX M.4: PolyCARBONATE WITH MULTIPLE FILLERS …………………….........756 


\section{List of Figures}

Figure 2.1: Effect of increasing filler loading on electrical conductivity ............................6

Figure 2.2: Bond percolation theory with channels ......................................................

Figure 2.3: Two dimensional ball and spring model crystal lattice of sodium

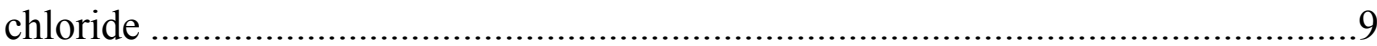

Figure 2.4: Generic ductile material tensile stress vs. tensile strain graph .......................11

Figure 3.1: Lexan HF1130-111 Polycarbonate Structure _............................................15

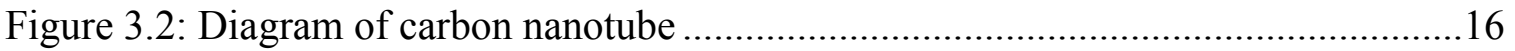

Figure 3.3: Diagram of Ketjenblack EC-600 JD carbon black structure .............................18

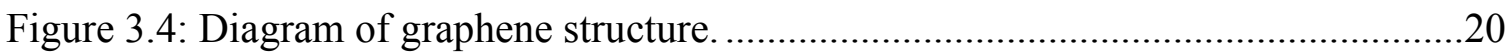

Figure 3.5: ASTM Type I Tensile Bar, 2.5" diameter thermal conductivity disk,

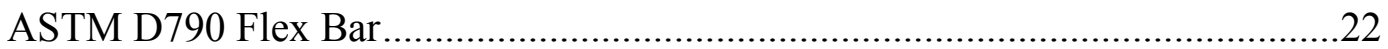

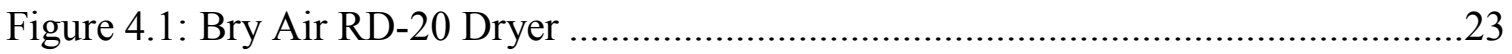

Figure 4.2: American Leistritz Extruder Corporation Model ZSE 27 twin screw

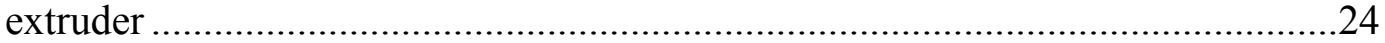

Figure 4.3: Side Stuffers seen disengaged from extruder .............................................25

Figure 4.4: Zone 1 Feeder (Feeder 3) used to feed PC and Masterbatch pellets ................25

Figure 4.5: Zone 5 Feeder (Feeder 4) used to add CB to extruder ...................................26

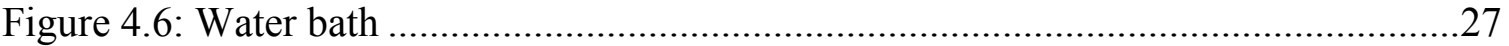

Figure 4.7: Conair Pelletizer and pre-pelletizer air-dryer .............................................27

Figure 4.8: Niigata model NE85UA4 injection molding machine....................................29

Figure 4.9: 4-cavity mold showing ASTM type I Tensile, 2.5" disk, ASTM type V

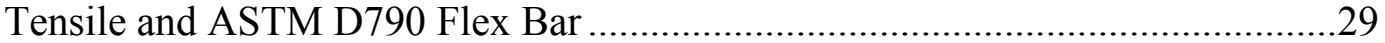

Figure 4.10: Olsen Plastometer MP 987/WL 987 Melt Indexer.........................................32

Figure 4.11: Metter Toledo 823E DSC .......................................................................33

Figure 4.12: Goettfert Rheo-Tester 1000 capillary rheometer............................................34

Figure 4.13: 30/1 capillary die and die nut, drive piston, 1400 bar pressure

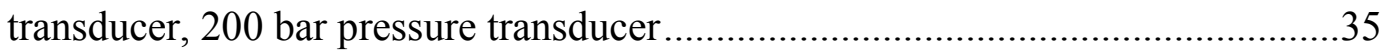

Figure 4.14: Bohlin C-VOR rotational rheometer with installed $25 \mathrm{~mm}$ parallel plates .38

Figure 4.15: ASTM D257 apparatus comprised of Keithley 6517A

Electrometer/High Resistance Meter and Keithley 8009 Resistivity Test fixture

Figure 4.16: Holometrix TCA-300 Thermal Conductivity Analyzer with constant temperature ethylene glycol/water circulator.

Figure 4.17: Instru-Met Sintech mechanical testing machine with tensile apparatus installed 
Figure 4.18: Instru-Met Sintech mechanical testing machine with flex apparatus installed

Figure 4.19: Diamond Pacific rotating lap with water drip apparatus .............................47

Figure 4.21: Buehler Vibromet I vibratory polisher .48

Figure 4.22: Olympus BX-60 System microscope with Optixcam Summit 1.3 camera on top

Figure 4.23: Hitachi S-4700 FESEM with energy dispersive x-ray spectrometer

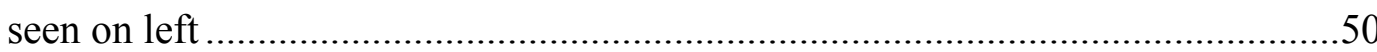

Figure 4.24: Leica EM UC6 ultramicrotome shown without cryo chamber ...................52

Figure 4.25: JEOL JEM-4000FX transmission electron microscope ............................52

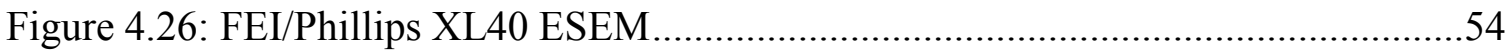

Figure 6.1: FESEM image of $6 \mathrm{wt} \%$ CNT in PC fracture surface..................................67

Figure 6.2: TEM images of $3 \mathrm{wt} \% \mathrm{CNT}$ in PC (a) and $6 \mathrm{wt} \% \mathrm{CNT}$ in PC (b) .................68

Figure 6.3: $\log ($ electrical resistivity) versus filler volume for $\mathrm{CNT} / \mathrm{PC}$ composites........69

Figure 6.4: Thermal conductivity versus filler volume for CNT/PC composites..............71

Figure 6.5: Tensile modulus of CNT/PC composites ................................................72

Figure 6.6: Ultimate tensile strength and strain at ultimate tensile strength for

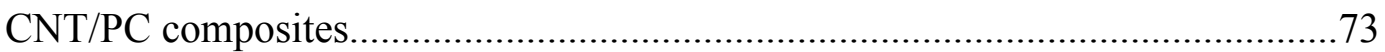

Figure 6.7: Typical stress-strain curves for CNT/PC composites ................................73

Figure 6.8: Flexural modulus of CNT/PC composites................................................ 75

Figure 6.9: Ultimate flexural strength and strain at ultimate flexural strength for

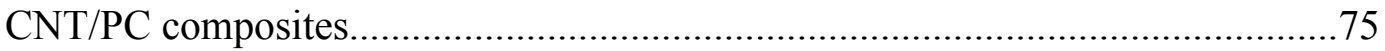

Figure 7.1: FESEM image of $6 \mathrm{wt} \% \mathrm{CB}$ in PC ........................................................... 77

Figure 7.2: Electrical resistivity (ER) versus filler volume for $\mathrm{CB} / \mathrm{PC}$ and

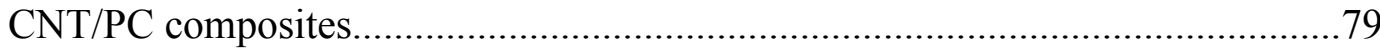

Figure 7.3: Thermal conductivity (TC) versus filler volume for $\mathrm{CB} / \mathrm{PC}$ and

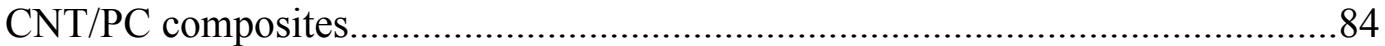

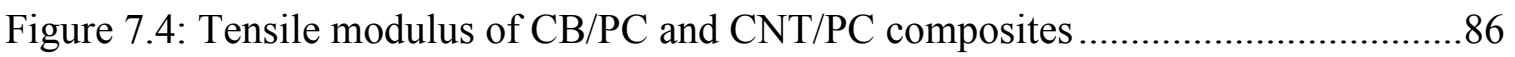

Figure 7.5: Ultimate tensile strength and strain at ultimate tensile strength for

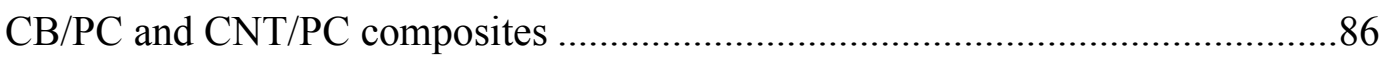

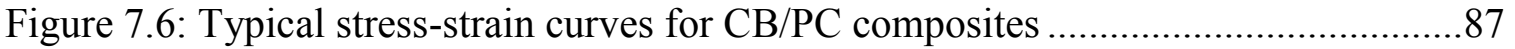

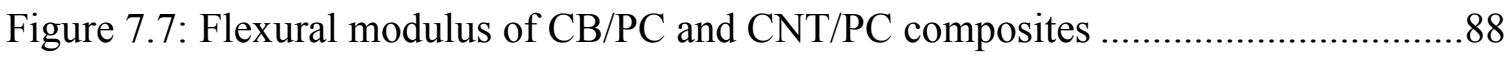

Figure 7.8: Ultimate flexural strength and strain at ultimate flexural strength for

$\mathrm{CB} / \mathrm{PC}$ and $\mathrm{CNT} / \mathrm{PC}$ composites

Figure 8.1: a.TEM image of $2 \mathrm{wt} \% \mathrm{CNT}$ in PC b. TEM image of $8 \mathrm{wt} \% \mathrm{CNT}$ in $\mathrm{PC}$. 
Figure 8.2: Time-temperature shifted complex viscosity as a function of shifted frequency for $\mathrm{CNT} / \mathrm{PC}$ studied at $270^{\circ} \mathrm{C}$

Figure 8.3: Time-temperature shift factors $a_{T}$ for both steady and oscillatory rheological data of $\mathrm{CNT} / \mathrm{PC}$ as a function of inverse temperature

Figure 8.4: Time-temperature shifted complex viscosity as a function of shifted frequency $\mathrm{CB} / \mathrm{PC}$ studied at $270^{\circ} \mathrm{C}$

Figure 8.5: Time-temperature shift factors $a_{T}$ for both steady and oscillatory rheological data of $\mathrm{CB} / \mathrm{PC}$ as a function of inverse temperature.

Figure 8.6: Time-temperature shifted steady-shear viscosity as a function of shifted shear rate for $\mathrm{CNT} / \mathrm{PC}$ studied at $270^{\circ} \mathrm{C}$. Compositions range from pure polycarbonate ( $0 \mathrm{wt} \% \mathrm{CNT}$ ) to $4 \mathrm{wt} \% \mathrm{CNT}$.

Figure 8.7: Time-temperature shifted steady-shear viscosity as a function of shifted shear rate for $\mathrm{CNT} / \mathrm{PC}$ studied at $270^{\circ} \mathrm{C}$. Compositions range from $4 \mathrm{wt} \% \mathrm{CNT}$ to $8 \mathrm{wt} \% \mathrm{CNT}$

Figure 8.8: Schematic of a possible mechanism for viscosity reduction effect observed at high shear rates.

Figure 8.9: Time-temperature shifted steady-shear viscosity as a function of shifted shear rate for $\mathrm{CB} / \mathrm{PC}$ studied at $270^{\circ} \mathrm{C}$. Compositions range from pure polycarbonate $(0 \mathrm{wt} \% \mathrm{CB})$ to $4 \mathrm{wt} \% \mathrm{CB}$.

Figure 8.10: Time-temperature shifted steady-shear viscosity as a function of shifted shear rate for $\mathrm{CB} / \mathrm{PC}$ studied at $270^{\circ} \mathrm{C}$. Compositions range from 4 wt $\%$ CB to 8 wt $\%$

Figure 9.1: ESEM micrograph of $5 \mathrm{wt} \% \mathrm{GNP}$ in $\mathrm{PC}$ composite

Figure 9.2: Electrical resistivity of $\mathrm{GNP} / \mathrm{PC}, \mathrm{CB} / \mathrm{PC}$, and $\mathrm{CNT} / \mathrm{PC}$ composites

Figure 9.3: Thermal conductivity of $\mathrm{GNP} / \mathrm{PC}, \mathrm{CB} / \mathrm{PC}$, and $\mathrm{CNT} / \mathrm{PC}$ composites 111

Figure 9.4: Tensile modulus of GNP/PC, CB/PC, and CNT/PC composites

Figure 9.5: Ultimate tensile strength and strain at ultimate tensile strength for

GNP/PC composites.

Figure 9.6: Tensile stress-strain curves for GNP/PC composites 114

Figure 9.7: Flexural modulus for GNP/PC, CB/PC and CNT/PC composites 115

Figure 9.8: Ultimate flexural strength and strain at ultimate flexural strength for

GNP/PC composites.

Figure 9.9: Time-temperature shifted steady-shear viscosity as a function of steady rate for $\mathrm{GNP} / \mathrm{PC}$ composites studied at $270^{\circ} \mathrm{C}$

Figure 9.10: Reduced viscosity versus volume fraction, $\phi$, GNP at a shear stress of $10^{5} \mathrm{~Pa}$ for GNP/PC composites: experimental data along with MaronPierce models for various filler shapes and a Kitano-modified MaronPierce model $(\mathrm{A}=0.3)$. 120 
Figure 10.1: $\mathrm{CB} / \mathrm{PC}$ electrical resistivity results along with Mamunya, additive and GEM models.

Figure 10.2: CNT/PC electrical resistivity results along with Mamunya, additive and GEM models.

Figure 10.3: GNP/PC electrical resistivity results along with Mamunya, additive and GEM models.

Figure 11.1: Tensile Modulus Results Along With Halpin-Tsai 2D and 3D Randomly Oriented Fiber and Nielsen Models for CB/PC Composites. 141

Figure 11.2: Tensile Modulus Results Along With Halpin-Tsai 2D and 3D Randomly Oriented Fiber and Nielsen Models for CNT/PC Composites

Figure 11.3: Tensile Modulus Results Along With Halpin-Tsai 2D and 3D Randomly Oriented Fiber and Nielsen Models for GNP/PC Composites

Figure 12.1: TEM Micrograph of Composite Containing $5 \mathrm{wt} \% \mathrm{CB}$ and $5 \mathrm{wt} \%$

$\mathrm{CNT}$ in $\mathrm{PC}$

Figure 12.2: TEM Micrograph of Composite Containing $5 \mathrm{wt} \% \mathrm{CB}$ and $5 \mathrm{wt} \%$

GNP in PC a) Bright Field Image b) Dark Field Image

Figure 12.3: TEM Micrograph of Composite Containing $5 \mathrm{wt} \% \mathrm{CNT}$ and $5 \mathrm{wt} \%$

GNP in PC a) Bright Field Image b) Dark Field Image

Figure 12.4. Contour Plot of Log (Log Electrical Resistivity, ohm-cm) for

$\mathrm{CB} / \mathrm{CNT}$ Composites

Figure 12.5. Contour Plot of Log (Log Electrical Resistivity, ohm-cm) for

CNT/GNP Composites. 156

Figure 12.6: Contour Plot of Tensile Modulus (MPa) for CB/CNT Composites ............168

Figure 12.7: Contour Plot of Tensile Modulus (MPa) for CB/GNP Composites 169

Figure 12.8: Contour Plot of Flexural Modulus (MPa) for CB/CNT Composites ..........176

Figure 12.9: Contour Plot of Flexural Modulus (MPa) for CB/GNP Composites ..... .177

Figure 13.1: Viscosity versus Shear Rate for $\mathrm{CB} / \mathrm{PC}$ Composites at $270^{\circ} \mathrm{C}$ 180

Figure 13.2: Viscosity versus Shear Rate for GNP/PC Composites at $270^{\circ} \mathrm{C}$ 181

Figure 13.3: Viscosity versus Shear Rate for CNT/PC Composites at $270^{\circ} \mathrm{C}$. .181

Figure 13.4: Viscosity versus Shear Rate for PC Composites with $\mathrm{CB}$ and GNP at $270^{\circ} \mathrm{C}$ 186

Figure 13.5: Viscosity versus Shear Rate PC Composites with CB and CNT at $270^{\circ} \mathrm{C}$

Figure 13.6: Viscosity versus Shear Rate for PC Composites with CNT and GNP at $270^{\circ} \mathrm{C}$ 188

Figure 13.7: Viscosity versus Shear-Rate PC Composites with $\mathrm{CB}$ and CNT at $270^{\circ} \mathrm{C}$ 190 


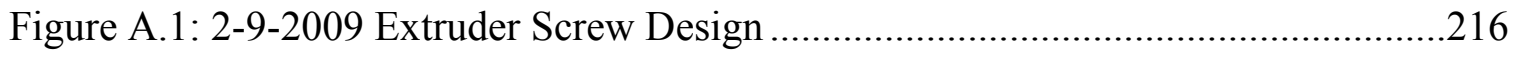

Figure A.2: Extruder Screw Design 2-9-09 used on 5-6-09 and 5-7-09 .......................217

Figure A.3: Extruder Screw Design 10-30-09 used on 11-5-09 and 11-11-09..............221

Figure A.4: Twin Screw Extruder Screw named 10-30-09 ..........................................222

Figure A.5: Extruder Screw Design 2-9-09 used on 5-26-10 and 5-27-10 .....................226

Figure A.6: Twin Screw Extruder Screw named 2-9-09 ............................................227

Figure A.7: Extruder Screw Design 2-9-09 ............................................................231

Figure A.8: Twin Screw Extruder Screw named 2-9-09 .........................................232

Figure E.1: DSC for Lexan HF1130-111 (BL) Run 1 vs. Time ......................................310

Figure E.2: DSC for Lexan HF1130-111 (BL) Run 2 vs. Time ...........................................

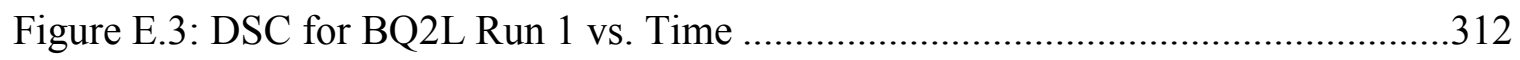

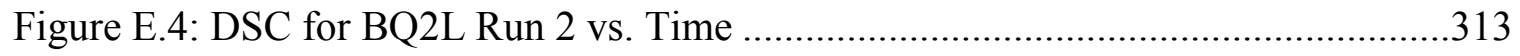

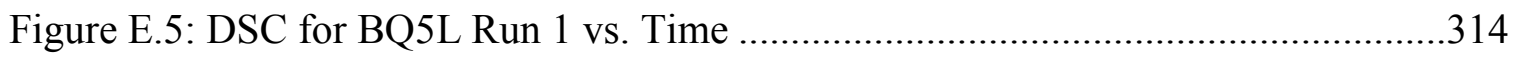

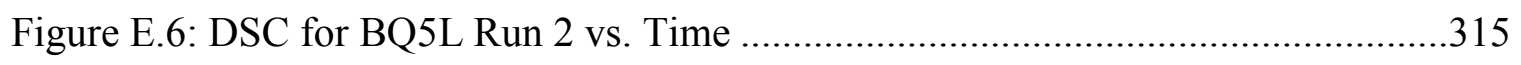

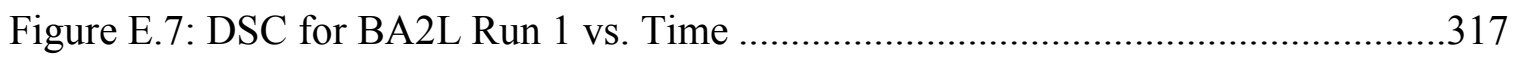

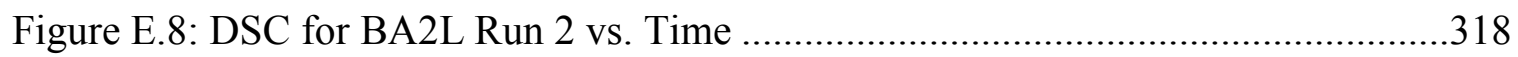

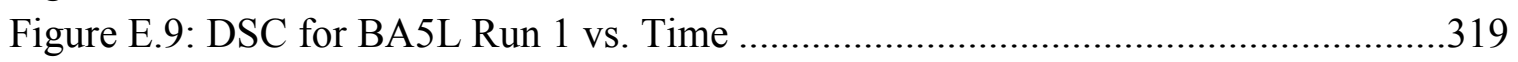

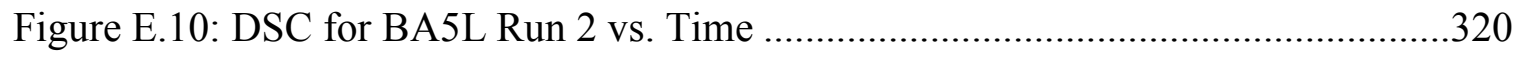

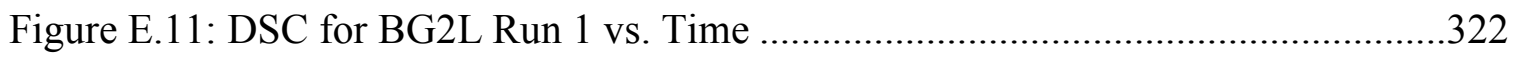

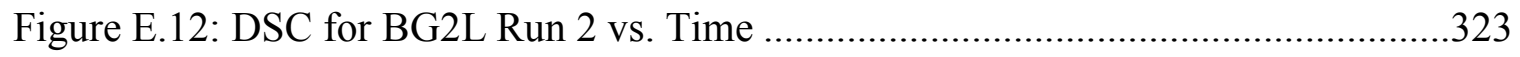

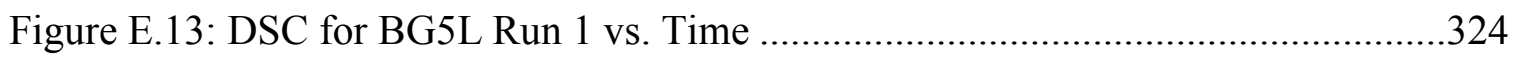

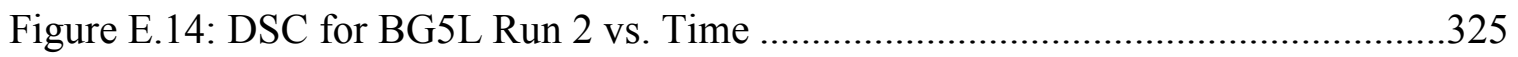

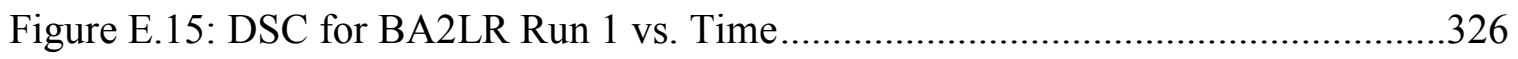

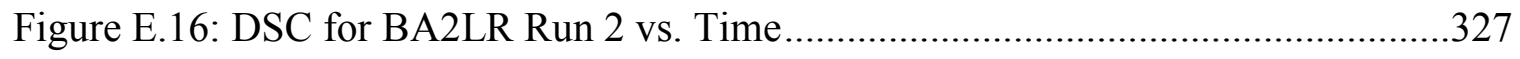

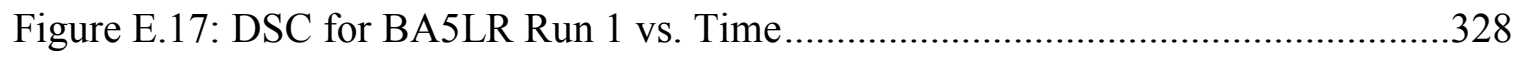

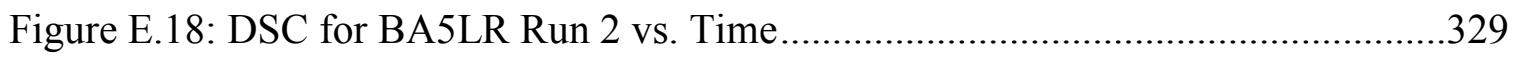

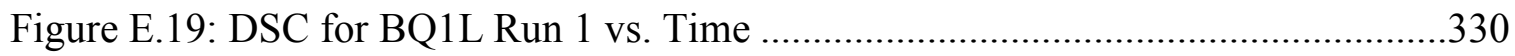

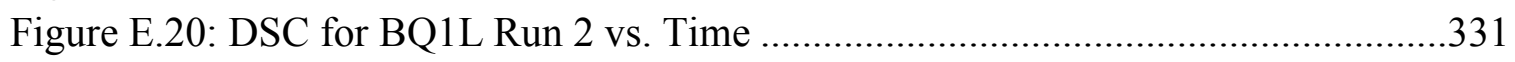

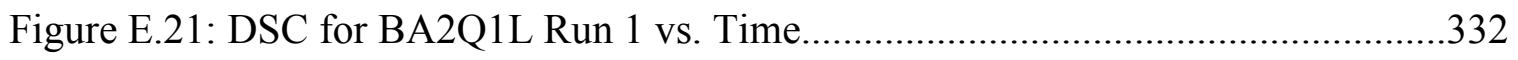

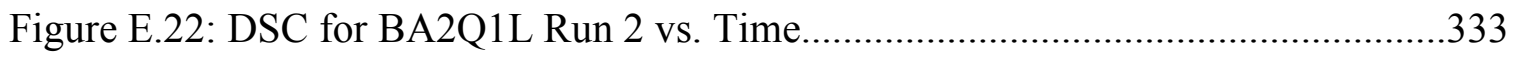

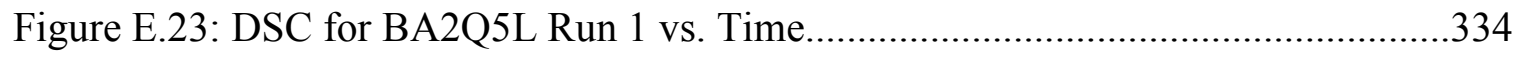

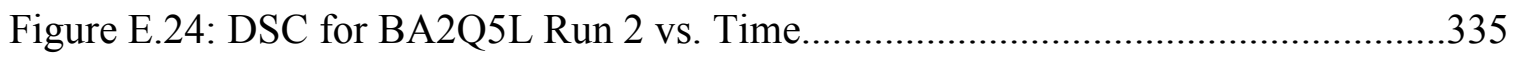

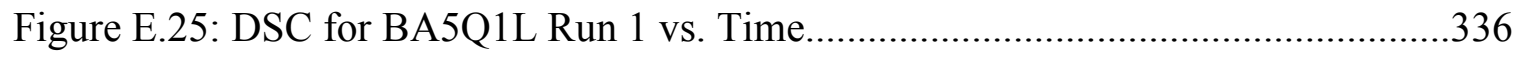

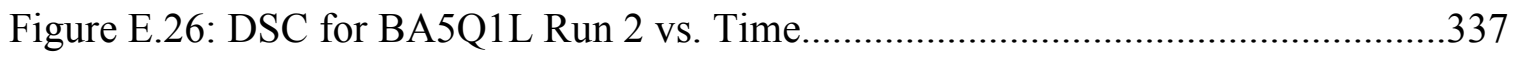

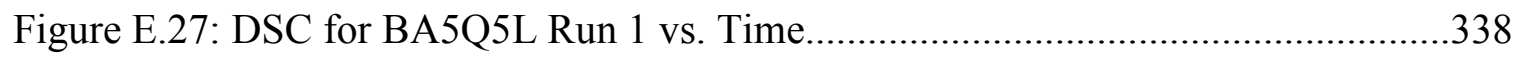

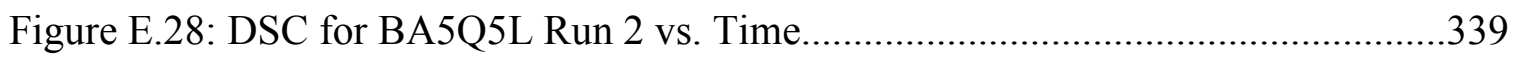

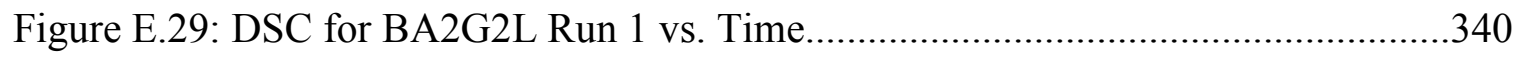

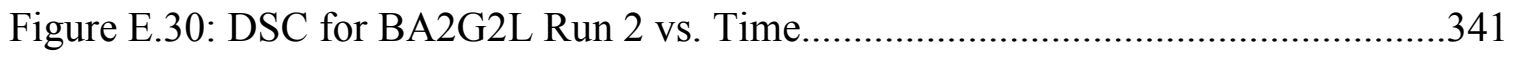




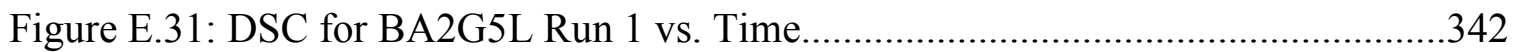

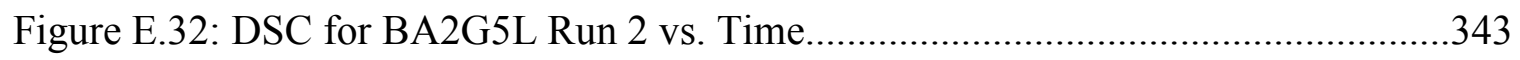

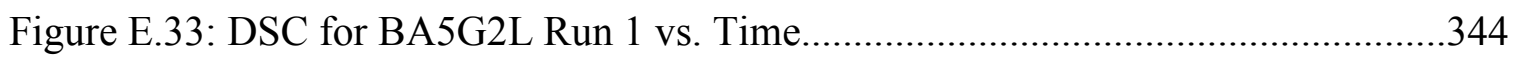

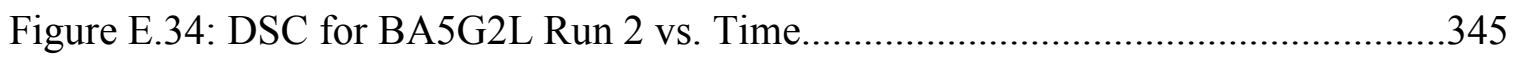

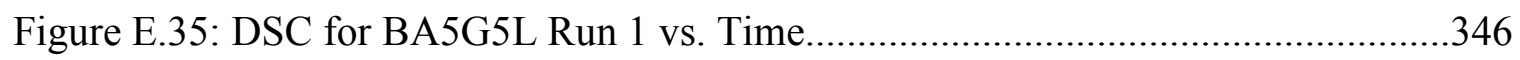

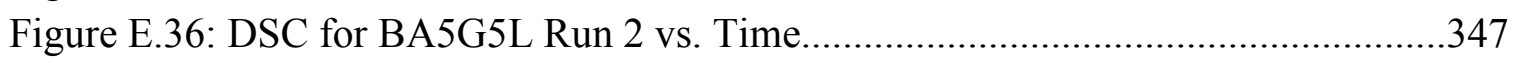

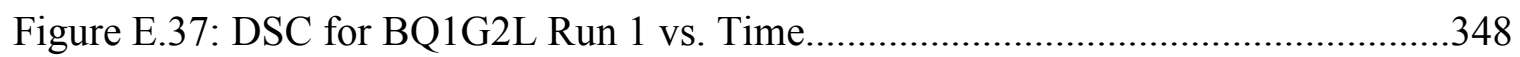

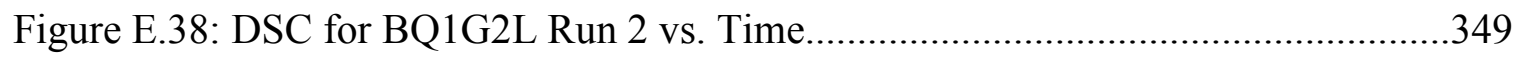

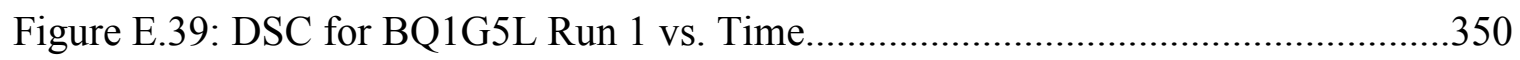

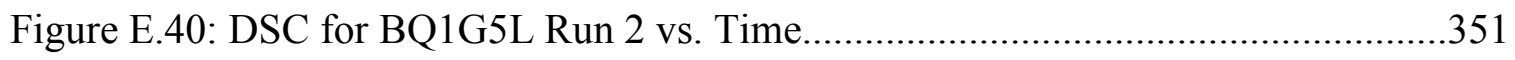

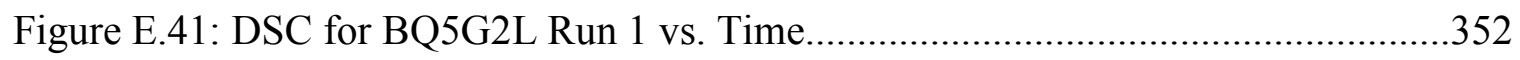

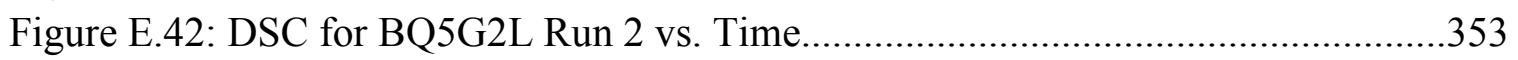

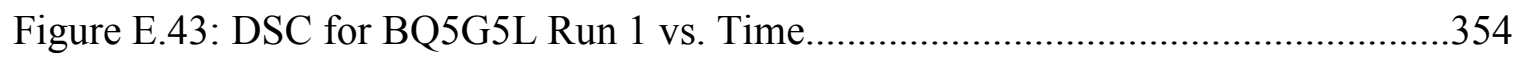

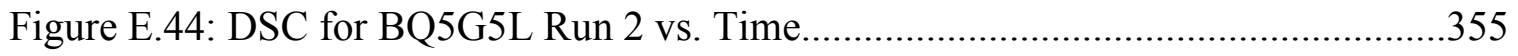

Figure J.1: Tensile Results for BL: Lexan HF1130-111: Injection Molded 5-26-09 ......448

Figure J.2: Tensile Results for BLE: Extruded Lexan HF1130-111: Injection

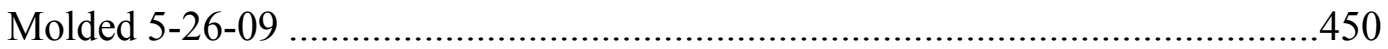

Figure J.3: Tensile Results for BQ2L: $2 \mathrm{wt} \%$ fibrils (carbon nanotubes) in Lexan

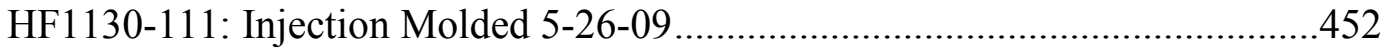

Figure J.4: Tensile Results for BQ3L: $3 \mathrm{wt} \%$ fibrils (carbon nanotubes) in Lexan

HF1130-111: Injection Molded 5-26-09....................................................454

Figure J.5: Tensile Results for BQ4L: $4 \mathrm{wt} \%$ fibrils (carbon nanotubes) in Lexan HF1130-111: Injection Molded 5-26-09..........................................................456

Figure J.6: Tensile Results for BQ5L: $5 \mathrm{wt} \%$ fibrils (carbon nanotubes) in Lexan HF1130-111: Injection Molded 5-26-09 ...................................................458

Figure J.7: Tensile Results for BQ6L: $6 \mathrm{wt} \%$ fibrils (carbon nanotubes) in Lexan HF1130-111: Injection Molded 5-26-09 ....................................................460

Figure J.8: Tensile Results for BQ8L: $8 \mathrm{wt} \%$ fibrils (carbon nanotubes) in Lexan HF1130-111: Injection Molded 5-26-09 ........................................................462

Figure J.9: Tensile Results for BL2: Lexan HF1130-111: Injection Molded 12-409. .464

Figure J.10: Tensile Results for BLE2: Extruded Lexan HF1130-111: Extruded 11-5-09, Injection Molded 12-4-09.................................................................466

Figure J.11: Tensile Results for BA2L: 2 wt\% Ketjenblack EC-600 JD (carbon black) in Lexan HF1130-111: Injection Molded 12-4-09.................................468

Figure J.12: Tensile Results for BA3L: 3 wt\% Ketjenblack EC-600 JD (carbon black) in Lexan HF1130-111: Injection Molded 12-4-09. .470

Figure J.13: Tensile Results for BA4L: 4 wt\% Ketjenblack EC-600 JD (carbon black) in Lexan HF1130-111: Injection Molded 12-4-09. xii 
Figure J.14: Tensile Results for BA5L: 5 wt\% Ketjenblack EC-600 JD (carbon black) in Lexan HF1130-111: Injection Molded 12-4-09

Figure J.15: Tensile Results for BA6L: $6 \mathrm{wt} \%$ Ketjenblack EC-600 JD (carbon black) in Lexan HF1130-111: Injection Molded 12-4-09 476

Figure J.16: Tensile Results for BA8L: 8 wt\% Ketjenblack EC-600 JD (carbon black) in Lexan HF1130-111: Injection Molded 12-4-09. 478

Figure J.17: Tensile Results for BA10L: $10 \mathrm{wt} \%$ Ketjenblack EC-600 JD (carbon black) in Lexan HF1130-111: Injection Molded 12-4-09. 480

Figure J.18: Tensile Results for BL3: Lexan HF1130-111: Injection Molded 6-310

Figure J.19: Tensile Results for BLE3: Extruded Lexan HF1130-111: Extruded 526-10, Injection Molded 6-3-10.

Figure J.20: Tensile Results for BG2L: $2 \mathrm{wt} \% \mathrm{xGnP}$ in Lexan HF1130-111:

Injection Molded 6-3-10, Extruded 5-26-10 486

Figure J.21: Tensile Results for BG3L: 3 wt $\%$ xGnP in Lexan HF1130-111:

Injection Molded 6-3-10, Extruded 5-26-10 488

Figure J.22: Tensile Results for BG4L: $4 \mathrm{wt} \% \mathrm{xGnP}$ in Lexan HF1130-111:

Injection Molded 6-3-10, Extruded 5-26-10

Figure J.23: Tensile Results for BG5L: $5 \mathrm{wt} \%$ xGnP in Lexan HF1130-111:

Injection Molded 6-3-10, Extruded 5-26-10

Figure J.24: Tensile Results for BG6L: $6 \mathrm{wt} \% \mathrm{xGnP}$ in Lexan HF1130-111:

Injection Molded 6-8-10, Extruded 5-27-10.

Figure J.25: Tensile Results for BG8L: $8 \mathrm{wt} \%$ xGnP in Lexan HF1130-111:

Injection Molded 6-8-10, Extruded 5-27-10

Figure J.26: Tensile Results for BG10L: $10 \mathrm{wt} \% \mathrm{xGnP}$ in Lexan HF1130-111:

Injection Molded 6-8-10, Extruded 5-27-10

Figure J.27: Tensile Results for BA2LR Set 1: Extruded 7-8-10, Injection Molded

7-26-10 .500

Figure J.28: Tensile Results for BA2LR Set 2: Extruded 7-8-10, Injection Molded

7-26-10

Figure J.29: Tensile Results for BA5LR Set 1: Extruded 7-8-10, Injection Molded

7-26-10

Figure J.30: Tensile Results for BA5LR Set 2: Extruded 7-8-10, Injection Molded

7-26-10 .506

Figure J.31: Tensile Results for BQ1L Set 1: Extruded 6-30-10, Injection Molded

7-26-10 .508

Figure J.32: Tensile Results for BQ1L Set 2: Extruded 6-30-10, Injection Molded

7-26-10

Figure J.33: Tensile Results for BQ5L Set 1: Extruded May 2009, Injection

Molded 5-26-09

xiii 
Figure J.34: Tensile Results for BQ5L Set 2: Extruded May 2009, Injection Molded 5-26-09

Figure J.35: Tensile Results for BG2L set 1: $2 \mathrm{wt} \% \mathrm{xGnP}$ in Lexan HF1130-111:

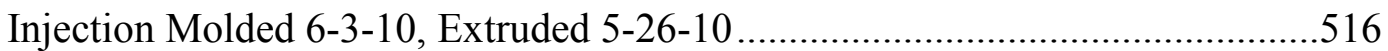

Figure J.36: Tensile Results for BG2L set 2: $2 \mathrm{wt} \% \mathrm{xGnP}$ in Lexan HF1130-111:

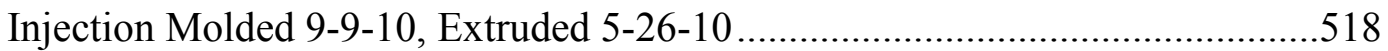

Figure J.37: Tensile Results for BG5L Set 1: $5 \mathrm{wt} \% \mathrm{xGnP}$ in Lexan HF1130-111: Injection Molded 6-3-10, Extruded 5-26-10 …………................................520

Figure J.38: Tensile Results for BG5L set 2: $2 \mathrm{wt} \% \mathrm{xGnP}$ in Lexan HF1130-111:

Extruded 5-26-10 .522

Figure J.39: Tensile Results for BG12L: $12 \mathrm{wt} \% \mathrm{xGnP}$ in Lexan HF1130-111:

Injection Molded 8-18-10, Extruded 7-8-10 .524

Figure J.40: Tensile Results for BG15L: $15 \mathrm{wt} \% \mathrm{xGnP}$ in Lexan HF1130-111:

Injection Molded 8-18-10, Extruded 7-8-10 .526

Figure J.41: Tensile Results for BA2Q1L set 1: Injection Molded 7-26-10, Extruded 7-7-10 .528

Figure J.42: Tensile Results for BA2Q1L set 2: Injection Molded 7-26-10, Extruded 7-7-10 .530

Figure J.43: Tensile Results for BA2Q5L set 1: Injection Molded 8-25-10, Extruded 7-7-10 .532

Figure J.44: Tensile Results for BA2Q5L set 2: Injection Molded 8-25-10, Extruded 7-7-10 .534

Figure J.45: Tensile Results for BA5Q1L set 1: Injection Molded 8-6-10, Extruded 7-7-10 .536

Figure J.46: Tensile Results for BA5Q1L set 2: Injection Molded 8-25-10, Extruded 7-7-10 .538

Figure J.47: Tensile Results for BA5Q5L set 1: Injection Molded 8-25-10, Extruded 7-7-10 .540

Figure J.48: Tensile Results for BA5Q5L set 2: Injection Molded 8-25-10, Extruded 7-7-10 542

Figure J.49: Tensile Results for BA2G2L set 1: Injection Molded 8-18-10,

Extruded 7-6-10 .544

Figure J.50: Tensile Results for BA2G2L set 2: Injection Molded 8-18-10,

Extruded 7-6-10 .546

Figure J.51: Tensile Results for BA2G5L set1: Injection Molded 8-18-10,

Extruded 7-6-10 .548

Figure J.52: Tensile Results for BA2G5L set 2: Injection Molded 8-18-10,

Extruded 7-6-10 .550

Figure J.53: Tensile Results for BA5G2L set 1: Injection Molded 8-18-10,

Extruded 7-6-10 .552 xiv 
Figure J.54: Tensile Results for BA5G2L set 2: Injection Molded 8-18-10, Extruded 7-6-10

Figure J.55: Tensile Results for BA5G5L set 1: Injection Molded 8-6-10,

Extruded 7-6-10 .556

Figure J.56: Tensile Results for BA5G5L set 2: Injection Molded 8-6-10,

Extruded 7-6-10 .558

Figure J.57: Tensile Results for BQ1G2L set 1: Injection Molded 8-18-10,

Extruded 7-1-10 .560

Figure J.58: Tensile Results for BQ1G2L set 2: Injection Molded 8-18-10,

Extruded 7-1-10

Figure J.59: Tensile Results for BQ1G5L set 1: Injection Molded 8-18-10,

Extruded 7-1-10 .564

Figure J.60: Tensile Results for BQ1G5L set 2: Injection Molded 8-6-10,

Extruded 7-1-10 .566

Figure J.61: Tensile Results for BQ5G2L set 1: Injection Molded 9-9-10,

Extruded 7-1-10 .568

Figure J.62: Tensile Results for BQ5G2L set 2: Injection Molded 9-9-10,

Extruded 7-1-10 .570

Figure J.63: Tensile Results for BQ5G5L set 1: Injection Molded 9-9-10,

Extruded 7-1-10 .572

Figure J.64: Tensile Results for BQ5G5L set 2: Injection Molded 9-9-10,

Extruded 7-1-10 .574

Figure K.1: Flexural Results for BL Lexan HF1130-111: Injection molded 5-2609. .576

Figure K.2: Flexural Results for BL E Extruded Lexan HF1130-111: Injection molded 5-26-09. .578

Figure K.3: Flexural Results for BQ2L: $2 \mathrm{wt} \%$ fibrils (carbon nanotubes) in

Lexan HF1130-111: Injection Molded 5-26-09 .580

Figure K.4: Flexural Results for BQ3L: $3 \mathrm{wt} \%$ fibrils (carbon nanotubes) in

Lexan HF1130-111: Injection Molded 5-26-09 .582

Figure K.5: Flexural Results for BQ4L: $4 \mathrm{wt} \%$ fibrils (carbon nanotubes) in

Lexan HF1130-111: Injection Molded 5-26-09 .584

Figure K.6: Flexural Results for BQ5L: $5 \mathrm{wt} \%$ fibrils (carbon nanotubes) in Lexan HF1130-111: Injection Molded 5-26-09 .586

Figure K.7: Flexural Results for BQ6L: $6 \mathrm{wt} \%$ fibrils (carbon nanotubes) in Lexan HF1130-111: Injection Molded 5-26-09 .588

Figure K.8: Flexural Results for BQ8L: $8 \mathrm{wt} \%$ fibrils (carbon nanotubes) in Lexan HF1130-111: Injection Molded 5-26-09 .590 
Figure K.9: Flexural Results for BL2 Lexan HF1130-111: Injection molded 12-409.

Figure K.10: Flexural Results for BLE2 Extruded Lexan HF1130-111: Extruded 11-5-09, Injection molded 12-4-09

Figure K.11: Flexural Results for BA2L: 2 wt\% Ketjenblack EC-600 JD (carbon black) in Lexan HF1130-111: Injection Molded 12-4-09.

Figure K.12: Flexural Results for BA3L: 3 wt\% Ketjenblack EC-600 JD (carbon black) in Lexan HF1130-111: Injection Molded 12-4-09.

Figure K.13: Flexural Results for BA4L: 4 wt\% Ketjenblack EC-600 JD (carbon black) in Lexan HF1130-111: Injection Molded 12-4-09.

Figure K.14: Flexural Results for BA5L: 5 wt\% Ketjenblack EC-600 JD (carbon black) in Lexan HF1130-111: Injection Molded 12-4-09

Figure K.15: Flexural Results for BA6L: 6 wt\% Ketjenblack EC-600 JD (carbon black) in Lexan HF1130-111: Injection Molded 12-4-09

Figure K.16: Flexural Results for BA8L: 8 wt\% Ketjenblack EC-600 JD (carbon black) in Lexan HF1130-111: Injection Molded 12-4-09.

Figure K.17: Flexural Results for BA10L: $10 \mathrm{wt} \%$ Ketjenblack EC-600 JD (carbon black) in Lexan HF1130-111: Injection Molded 12-4-09 .608

Figure K.18: Flexural Results for BL3 Lexan HF1130-111: Injection molded 6-310

Figure K.19: Flexural Results for BLE3 Extruded Lexan HF1130-111: Extruded 5-26-10, Injection molded 6-3-10

Figure K.20: Flexural Results for BG2L: $2 \mathrm{wt} \%$ xGnP in Lexan HF1130-111: Injection Molded 6-3-10

Figure K.21: Flexural Results for BG3L: $3 \mathrm{wt} \%$ xGnP in Lexan HF1130-111: Injection Molded 6-3-10

Figure K.22: Flexural Results for BG4L: $4 \mathrm{wt} \% \mathrm{xGnP}$ (carbon black) in Lexan HF1130-111: Injection Molded 6-3-10.

Figure K.23: Flexural Results for BG5L: $5 \mathrm{wt} \%$ xGnP in Lexan HF1130-111: Injection Molded 6-3-10

Figure K.24: Flexural Results for BG6L: $6 \mathrm{wt} \%$ xGnP in Lexan HF1130-111: Injection Molded 6-8-10

Figure K.25: Flexural Results for BG8L: 8 wt $\%$ xGnP in Lexan HF1130-111: Injection Molded 6-8-10

Figure K.26: Flexural Results for BG10L: $10 \mathrm{wt} \% \mathrm{xGnP}$ in Lexan HF1130-111: Injection Molded 6-8-10

Figure K.27: Flexural Results for BL4 Lexan HF1130-111: Injection molded 726-10 .628

Figure K.28: Flexural Results for BA2LR Set 1: Extruded 7-8-10, Injection molded 7-26-10 
Figure K.29: Flexural Results for BA2LR Set 2: Extruded 7-8-10, Injection molded 7-26-10

Figure K.30: Flexural Results for BA5LR Set 1: Extruded 7-8-10, Injection molded 7-26-10

Figure K.31: Flexural Results for BA5LR Set 2: Extruded 7-8-10, Injection molded 7-26-10.

Figure K.32: Flexural Results for BQ1L Set 1: Extruded 6-30-10, Injection molded 7-26-10

Figure K.33: Flexural Results for BQ1L Set 2: Extruded 6-30-10, Injection molded 7-26-10

Figure K.34: Flexural Results for BQ5LR Set 1: Extruded 6-30-10, Injection molded 8-25-10.

Figure K.35: Flexural Results for BQ5LR Set 2: Extruded 6-30-10, Injection molded 8-25-10

Figure K.36: Flexural Results for BG2L Set 1: Extruded May 2010, Injection molded 9-9-10

Figure K.37: Flexural Results for BG2L Set 2: Extruded May 2010, Injection molded 9-9-10

Figure K.38: Flexural Results for BG5L Set 1: Extruded May 2010, Injection molded 9-9-10

Figure K.39: Flexural Results for BG5L Set 2: Extruded May 2010, Injection molded 9-9-10

Figure K.40: Flexural Results for BG12L: Extruded 7-8-10, Injection molded 818-10

Figure K.41: Flexural Results for BG15L: Extruded 7-8-10, Injection molded 8$18-10$

Figure K.42: Flexural Results for BA2Q1L Set 1: Extruded 7-7-10, Injection molded 7-26-10

Figure K.43: Flexural Results for BA2Q1L Set 2: Extruded 7-7-10, Injection molded 7-26-10

Figure K.44: Flexural Results for BA2Q5L Set 1: Extruded 7-7-10, Injection molded 8-25-10

Figure K.45: Flexural Results for BA2Q5L Set 2: Extruded 7-7-10, Injection molded 8-25-10 .664

Figure K.46: Flexural Results for BA5Q1L Set 1: Extruded 7-7-10, Injection molded 7-26-10

Figure K.47: Flexural Results for BA5Q1L Set 2: Extruded 7-7-10, Injection molded 7-26-10 .668

Figure K.48: Flexural Results for BA5Q5L Set 1: Extruded 7-7-10, Injection molded 8-25-10 
Figure K.49: Flexural Results for BA5Q5L Set 2: Extruded 7-7-10, Injection molded 8-25-10

Figure K.50: Flexural Results for BA2G2L Set 1: Extruded 7-6-10, Injection molded 8-18-10

Figure K.51: Flexural Results for BA2G2L Set 2: Extruded 7-6-10, Injection molded 8-18-10

Figure K.52: Flexural Results for BA2G5L Set 1: Extruded 7-6-10, Injection molded 8-18-10

Figure K.53: Flexural Results for BA2G5L Set 2: Extruded 7-6-10, Injection molded 8-18-10

Figure K.54: Flexural Results for BA5G2L Set 1: Extruded 7-6-10, Injection molded 8-18-10.

Figure K.55: Flexural Results for BA5G2L Set 2: Extruded 7-6-10, Injection molded 8-18-10

Figure K.56: Flexural Results for BA5G5L Set 1: Extruded 7-6-10, Injection molded 8-18-10

Figure K.57: Flexural Results for BA5G5L Set 2: Extruded 7-6-10, Injection molded 8-18-10

Figure K.58: Flexural Results for BQ1G2L Set 1: Extruded 7-1-10, Injection molded 8-18-10

Figure K.59: Flexural Results for BQ1G2L Set 2: Extruded 7-1-10, Injection molded 8-18-10

Figure K.60: Flexural Results for BQ1G5L Set 1: Extruded 7-1-10, Injection molded 8-18-10

Figure K.61: Flexural Results for BQ1G5L Set 2: Extruded 7-1-10, Injection molded 8-18-10

Figure K.62: Flexural Results for BQ5G2L Set 1: Extruded 7-1-10, Injection molded 9-9-10

Figure K.63: Flexural Results for BQ5G2L Set 2: Extruded 7-1-10, Injection molded 9-9-10

Figure K.64: Flexural Results for BQ5G5L Set 1: Extruded 7-1-10, Injection molded 9-9-10

Figure K.65: Flexural Results for BQ5G5L Set 2: Extruded 7-1-10, Injection molded 9-9-10 


\section{List of Tables}

Table 1.1: Electrical conductivity of polymer, metals and carbon .....................................2

Table 1.2: Thermal conductivity of polymer, metals, ceramics and carbon .......................2

Table 1.3: Tensile modulus of polycarbonate and common reinforcing materials..............3

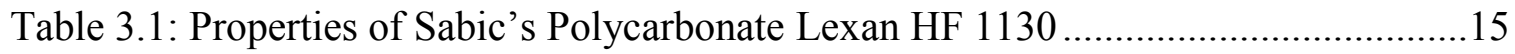

Table 3.2: Properties of FIBRIL ${ }^{\mathrm{TM}}$ Carbon Nanotubes ................................................ 17

Table 3.3: Properties of Akzo Nobel Ketjenblack EC-600 JD Carbon Black ...................19

Table 4.1: Niigata model NE85UA4 injection molding machine specifications ..............28

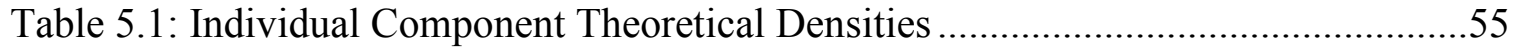

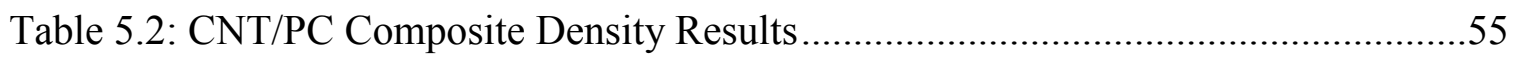

Table 5.3: CB/PC Composite Density Results ..............................................................56

Table 5.4: GNP/PC Composite Density Results..............................................................56

Table 5.5: CB/CNT, CB/GNP and CNT/GNP Density Results .....................................56

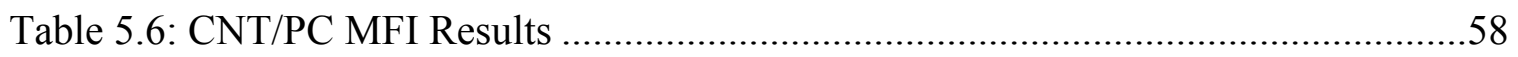

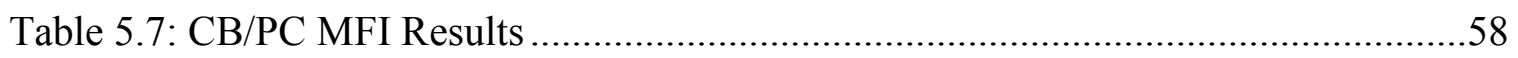

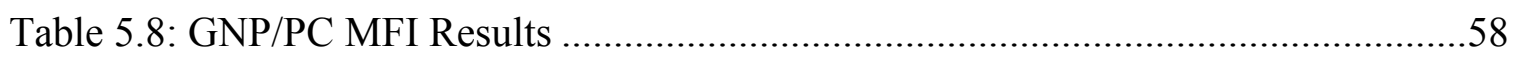

Table 5.9: CB/CNT, CB/GNP and CNT/GNP Composites MFI Results.........................59

Table 5.10: PC and CNT/PC DSC Midpoint $\mathrm{T}_{\mathrm{g}}$ Values .................................................60

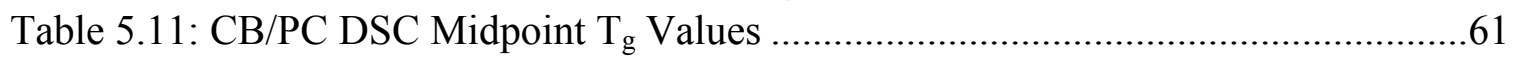

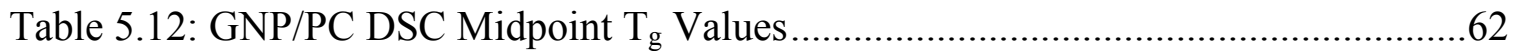

Table 5.13: CB/CNT, CB/GNP and CNT/GNP DSC Midpoint $\mathrm{T}_{\mathrm{g}}$ Values ......................63

Table 5.14: Average In-Plane Volume Electrical Resistivity of CB/PC Extruded

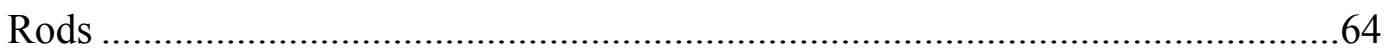

Table 5.15: Average In-Plane Volume Electrical Resistivity of GNP/PC Extruded Rods .....................................................................................................65

Table 5.16: Average In-Plane Volume Electrical Resistivity of CB/CNT, CB/GNP and GNP/CNT Extruded Rods...................................................................6

Table 6.1: Electrical resistivity results for CNT/PC composites ...................................69

Table 6.2: Thermal conductivity results for CNT/PC composites................................. 70

Table 7.1: Electrical resistivity results for $\mathrm{CB} / \mathrm{PC}$ and $\mathrm{CNT} / \mathrm{PC}$ composites ....................78

Table 7.2: Thermal conductivity results for $\mathrm{CB} / \mathrm{PC}$ and $\mathrm{CNT} / \mathrm{PC}$ composites.................83

Table 8.1: CNT/PC properties: ER, yield stress, matrix-zero-shear-viscosity and infinite-shear viscosity parameters 
Table 8.2: Composite properties: ER, yield stress, matrix-zero-shear-viscosity and infinite-shear viscosity parameters

Table 9.1: GNP/PC Electrical Resistivity Results 108

Table 9.2: GNP/PC Thermal Conductivity Results 111

Table 10.1: CB/PC, CNT/PC and GNP/PC Electrical Resistivity Results

Table 12.1: ER Results for Factorial Design Formulations Containing CB and CNT.

Table 12.2: ER Results for Factorial Design Formulations Containing CB and GNP 150

Table 12.3: ER Results for Factorial Design Formulations Containing CNT and GNP.

Table 12.4: $3^{2}$ Regression Results for Log (Log Electrical Resistivity, ohm-cm)

Table 12.5: TC Results for Factorial Design Formulations Containing CB and CNT. 158

Table 12.6: TC Results for Factorial Design Formulations Containing CB and GNP.

Table 12.7: TC Results for Factorial Design Formulations Containing CNT and GNP.

Table 12.8: $3^{2}$ Regression Results for Thermal Conductivity $(\mathrm{W} / \mathrm{m} \cdot \mathrm{K})$ 161

Table 12.10: Tensile Modulus Results for Factorial Design Formulations Containing CB and GNP. 164

Table 12.11: Tensile Modulus Results for Factorial Design Formulations Containing CNT and GNP .

Table 12.12. $3^{2}$ Regression Results for $3^{2}$ Regression Results for Tensile Modulus (MPa)

Table 12.13: Flexural Modulus Results for Factorial Design Formulations Containing CB and CNT.

Table 12.14: Flexural Modulus Results for Factorial Design Formulations Containing CB and GNP.

Table 12.16: $3^{2}$ Regression Results for $3^{2}$ Regression Results for Flexural Modulus (MPa)

Table 13.1: Newtonian Zero-Shear Plateau Results for Factorial Design Formulations Containing CB and GNP 182

Table 13.2: Electrical Resistivity and Zero-Shear Viscosity Results for Factorial Design Formulations Containing $\mathrm{CB}$ and $\mathrm{CNT}$ 183 
Table 13.3: Electrical Resistivity and Zero-Shear Viscosity Results for Factorial Design Formulations Containing CNT and GNP

Table A.1: Extrusion Conditions: Lexan HF1130-111 with Hyperion Fibrils MB6015-00 .218

Table A.2: Extrusion Conditions: Lexan HF1130-111 with Ketjenblack EC-600 JD .223

Table A.3: Extrusion Conditions: Lexan HF1130-111 with GNP. .228

Table A.4: Extrusion Conditions: Lexan HF1130-111 with 8 wt\% Ketjenblack EC-600 JD in Lexan HF1130-111 Masterbatch (extruder screw used = 10-30-09).

Table A.5: Extrusion Conditions: Lexan HF1130-111 Carbon Fillers for Factorial Design (extruder screw used $=2-9-09$ ). .235

Table B.1: Injection Molding Conditions for CNT/PC Composites 241

Table B.2: Injection Molding Conditions for $\mathrm{CB} / \mathrm{PC}$ Composites 243

Table B.3: Injection Molding conditions for GNP/PC composites .247

Table B.4: Injection Molding Conditions for Combinations in PC .251

Table C.1: Density Results for Lexan HF1130-111:BL: Injection Molded May 26, 2009.

Table C.2: Density Results for Extruded Lexan HF1130-111:BLE: Injection Molded May 26, 2009.

Table C.3: Density Results for $2 \mathrm{wt} \%$ fibrils in Lexan HF1130-111: BQ2L: Injection Molded May 26, 2009. .272

Table C.4: Density Results for 3 wt\% fibrils in Lexan HF1130-111: BQ3L: Injection Molded May 26, 2009.

Table C.5: Density Results for 4 wt\% fibrils in Lexan HF1130-111: BQ4L: Injection Molded May 26, 2009.

Table C.6: Density Results for 5 wt\% fibrils in Lexan HF1130-111: BQ5L: Injection Molded May 26, 2009.

Table C.7: Density Results for 6 wt\% fibrils in Lexan HF1130-111: BQ6L: Injection Molded May 26, 2009.

Table C.8: Density Results for 8 wt\% fibrils in Lexan HF1130-111: BQ8L: Injection Molded May 26, 2009. .274

Table C.9: Density Results for Lexan HF1130-111:BL2: Injection Molded December 4, 2009 .275

Table C.10: Density Results for Extruded Lexan HF1130-111:BLE2: Extruded Nov 5, 2009; Injection Molded December 4, 2009 .275 
Table C.11: Density Results for 2 wt\% Ketjenblack EC-600 JD in Lexan HF1130111: BA2L: Extruded November 5, 2009; Injection Molded December 4, 2009. .276

Table C.12: Density Results for 3 wt\% Ketjenblack EC-600 JD in Lexan HF1130111: BA3L: Extruded November 5, 2009; Injection Molded December 4, 2009

Table C.13: Density Results for 4 wt\% Ketjenblack EC-600 JD in Lexan HF1130-

111: BA4L: Extruded November 5, 2009; Injection Molded December 4, 2009

Table C.15: Density Results for 5 wt\% Ketjenblack EC-600 JD in Lexan HF1130-

111: BA5L: Extruded November 11, 2009; Injection Molded December 4, 2009

Table C.14: Density Results for 6 wt\% Ketjenblack EC-600 JD in Lexan HF1130-

111: BA6L: Extruded November 11, 2009; Injection Molded December 4, 2009

Table C.16: Density Results for 8 wt\% Ketjenblack EC-600 JD in Lexan HF1130-

111: BA8L: Extruded November 11, 2009; Injection Molded December 4, 2009

Table C.17: Density Results for 10 wt\% Ketjenblack EC-600 JD in Lexan

HF1130-111: BA10L: Extruded November 11, 2009; Injection Molded

December 4, 2009

Table C.18: Density Results for Lexan HF1130-111:BL3: Injection Molded June

3,2010 .

Table C.19: Density Results for Extruded Lexan HF1130-111:BLE3: Extruded

May 26, 2010; Injection Molded June 3, 2010 .280

Table C.20: Density Results for 2 wt\% xGnP in Lexan HF1130-111: BG2L:

Extruded May 26, 2010; Injection Molded June 3, 2010 .281

Table C.21: Density Results for $3 \mathrm{wt} \%$ xGnP in Lexan HF1130-111: BG3L:

Extruded May 26, 2010; Injection Molded June 3, 2010 .281

Table C.22: Density Results for $4 \mathrm{wt} \% \mathrm{xGnP}$ in Lexan HF1130-111: BG4L:

Extruded May 26, 2010; Injection Molded June 3, 2010 .282

Table C.23: Density Results for $5 \mathrm{wt} \% \mathrm{xGnP}$ in Lexan HF1130-111: BG5L:

Extruded May 26, 2010; Injection Molded June 3, 2010 .282

Table C.24: Density Results for 6 wt\% xGnP in Lexan HF1130-111: BG5L:

Extruded May 27, 2010; Injection Molded June 8, 2010 .283

Table C.25: Density Results for 8 wt\% xGnP in Lexan HF1130-111: BG5L:

Extruded May 27, 2010; Injection Molded June 8, 2010 .283

Table C.26: Density Results for $10 \mathrm{wt} \%$ xGnP in Lexan HF1130-111: BG5L:

Extruded May 27, 2010; Injection Molded June 8, 2010 .284

xxii 
Table C.27: Density Results for Lexan HF1130-111:BL4: Injection Molded July 26,2010 .

Table C.28: Density Results for BA2LR: Extruded 7-8-10; Injection Molded 7-

26-10 .285

Table C29: Density Results for BA5LR: Extruded 7-8-10; Injection Molded 7-2610. .286

Table C.30: Density Results for BG12L: Extruded 7-8-10; Injection Molded 8$18-10$ .286

Table C.31: Density Results for BG15L: Extruded 7-8-10; Injection Molded 8$18-10$

Table C.32: Density Results for BA2Q1L: Extruded 7-7-10; Injection Molded June 26, 2010 .287

Table C.33: Density Results for BA2Q5L: Extruded 7-7-10; Injection Molded 825-10 .288

Table C.34: Density Results for BA5Q1L: Extruded 7-7-10; Injection Molded 726-10 .288

Table C.35: Density Results for BA5Q5L: Extruded 7-7-10; Injection Molded 825-10 .289

Table C.36: Density Results for BA2G2L: Extruded 7-6-10; Injection Molded 818-10

Table C.37: Density Results for BA2G5L: Extruded 7-6-10; Injection Molded 818-10

Table C.38: Density Results for BA5G2L: Extruded 7-6-10; Injection Molded 818-10

Table C.39: Density Results for BA5G5L: Extruded 7-6-10; Injection Molded 818-10

Table C.40: Density Results for BQ1G2L: Extruded 7-1-10; Injection Molded 818-10

Table C.41: Density Results for BQ1G5L: Extruded 7-1-10; Injection Molded 818-10

Table C.42: Density Results for BQ5G2L: Extruded 7-1-10; Injection Molded 8$18-10$

Table C.43: Density Results for BQ5G5L: Extruded 7-1-10; Injection Molded 8$18-10$ .293

Table D.1: MFI Results for BL .294

Table D.2: MFI Results for BLE .294

Table D.3: MFI Results for BQ2L .295

Table D.4: MFI Results for BQ3L .295

Table D.5: MFI Results for BQ4L .296 xxiii 


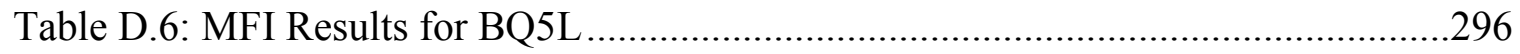

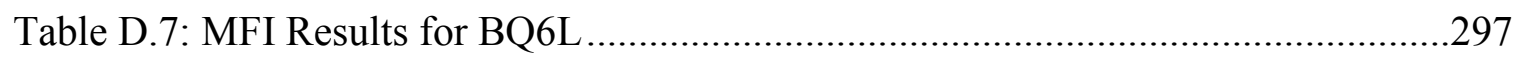

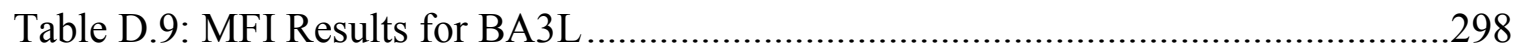

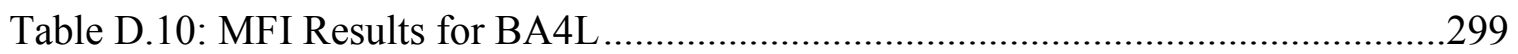

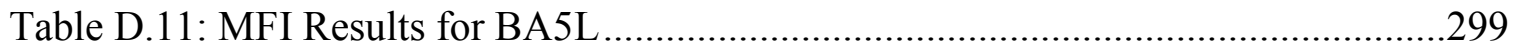

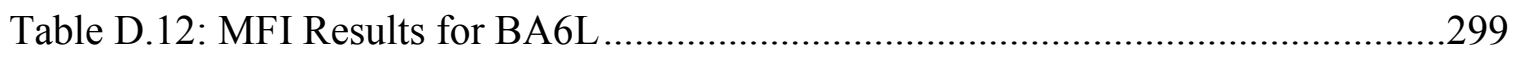

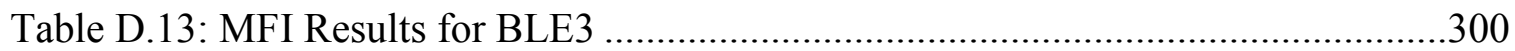

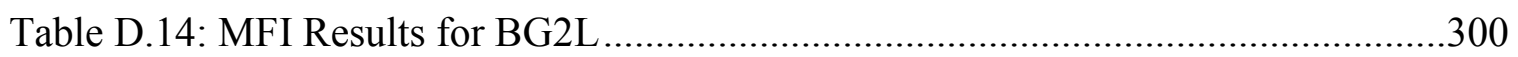

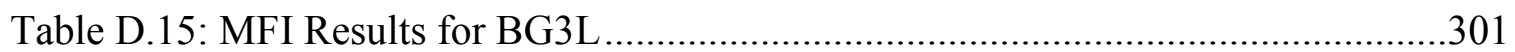

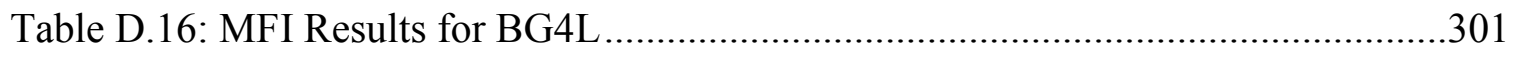

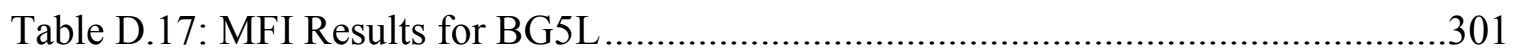

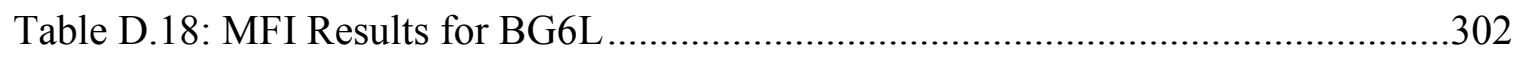

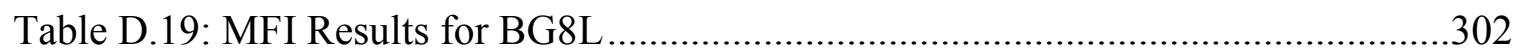

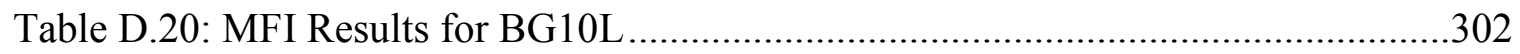

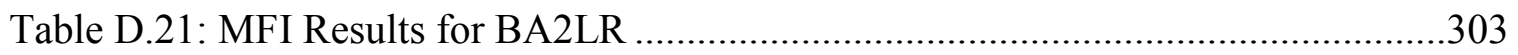

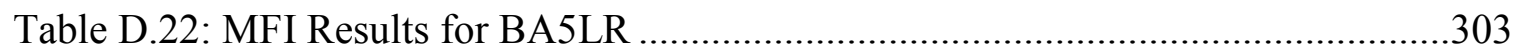

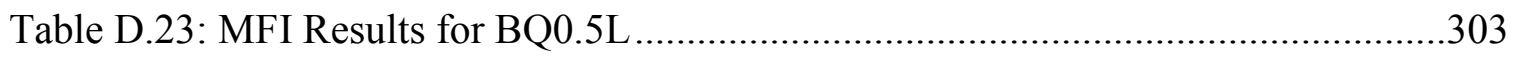

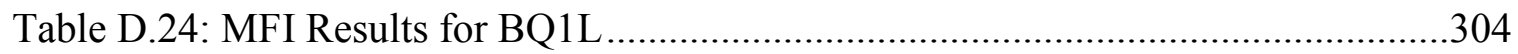

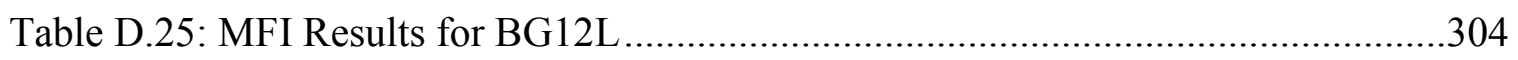

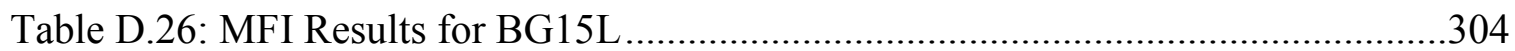

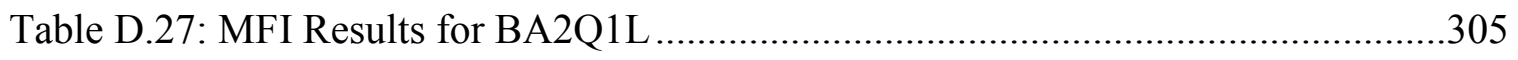

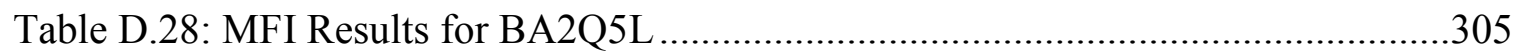

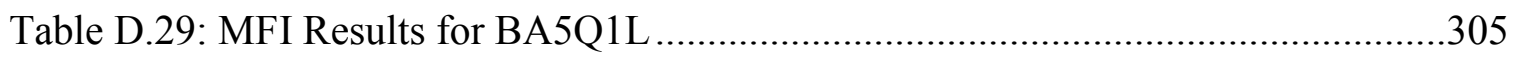

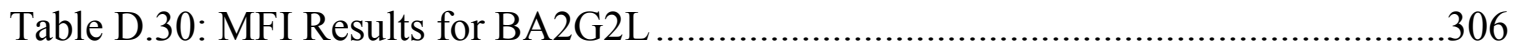

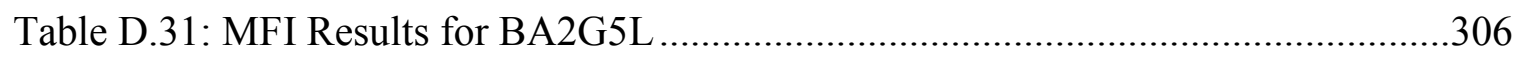

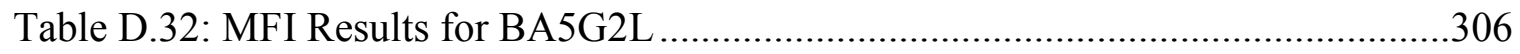

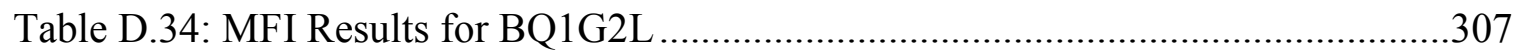

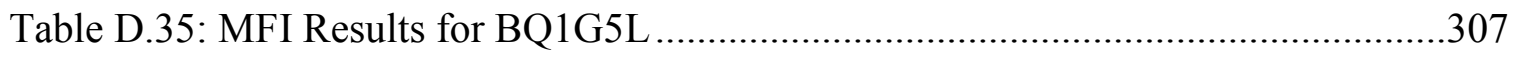

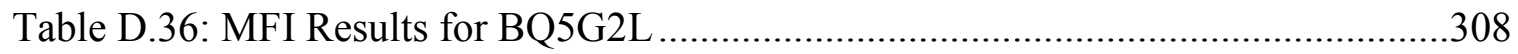

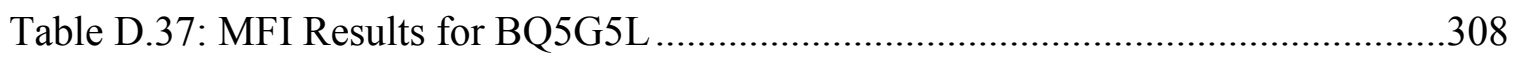

Table F.1: Two Point Two Probe In-Plane Electrical Resistivity Results for BA2L: 2 wt\% Ketjenblack EC-600 JD in Lexan HF1130-111: Extruded

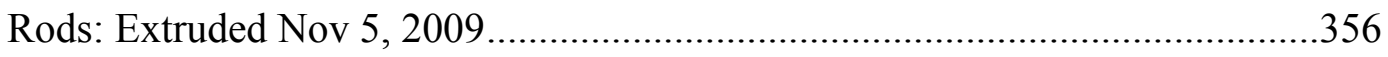

Table F.2: Two Point Two Probe In-Plane Electrical Resistivity Results for BA3L: 3 wt\% Ketjenblack EC-600 JD in Lexan HF1130-111: Extruded Rods: Extruded Nov 5, 2009

Table F.3: Two Point Two Probe In-Plane Electrical Resistivity Results for

BA4L: 4 wt\% Ketjenblack EC-600 JD in Lexan HF1130-111: Extruded

Rods: Extruded Nov 5, 2009 
Table F.4: Two Point Two Probe In-Plane Electrical Resistivity Results for BA5L: 5 wt\% Ketjenblack EC-600 JD in Lexan HF1130-111: Extruded

Rods: Extruded Nov 11, 2009

Table F.5: Two Point Two Probe In-Plane Electrical Resistivity Results for

BA6L: 6 wt\% Ketjenblack EC-600 JD in Lexan HF1130-111: Extruded

Rods: Extruded Nov 11, 2009

Table F.6: Two Point Two Probe In-Plane Electrical Resistivity Results for

BA8L: 8 wt\% Ketjenblack EC-600 JD in Lexan HF1130-111: Extruded

Rods: Extruded Nov 11, 2009

Table F.7: Two Point Two Probe In-Plane Electrical Resistivity Results for

BA10L: $10 \mathrm{wt} \%$ Ketjenblack EC-600 JD in Lexan HF1130-111: Extruded

Rods: Extruded Nov 11, 2009

Table F.8: Two Point Two Probe In-Plane Electrical Resistivity Results for

Ovation Polymers Extima GP MB PC 1515B As- Received Pellets: 15

wt $\%$ xGnP in Lexan HF1130-111.

Table F.9: Two Point Two Probe In-Plane Electrical Resistivity Results for

BG8L: 8 wt\% xGnP in Lexan HF1130-111: Extruded Rods: Extruded

May 27, 2010

Table F.10: Two Point Two Probe In-Plane Electrical Resistivity Results for

BG10L: 10 wt\% xGnP in Lexan HF1130-111: Extruded Rods: Extruded

May 27, 2010

Table F.11: Two Point Two Probe In-Plane Electrical Resistivity Results for

BA5LR Extruded Rods: Extruded on 7-8-10. .366

Table F.12: Two Point Two Probe In-Plane Electrical Resistivity Results for

BA8LR Extruded Rods: Extruded on 4-14-10 Masterbatch.

Table F.13: Two Point Two Probe In-Plane Electrical Resistivity Results for

BG12L Extruded Rods: Extruded on 7-8-10

Table F.14: Two Point Two Probe In-Plane Electrical Resistivity Results for

BG15L Extruded Rods: Extruded on 7-8-10

Table F.15: Two Point Two Probe In-Plane Electrical Resistivity Results for

Ovation Polymers Extima GP MB PC 1515B As- Received Pellets: 15

wt $\%$ xGnP in Lexan HF1130-111.

Table F.16: Two Point Two Probe In-Plane Electrical Resistivity Results for

BA2Q1L: Extruded Rods: Extruded 7-7-10

Table F.17: Two Point Two Probe In-Plane Electrical Resistivity Results for

BA2Q5L: Extruded Rods: Extruded 7-7-10

Table F.18: Two Point Two Probe In-Plane Electrical Resistivity Results for

BA5Q1L: Extruded Rods: Extruded 7-7-10

Table F.19: Two Point Two Probe In-Plane Electrical Resistivity Results for

BA5Q5L: Extruded Rods: Extruded 7-7-10 
Table F.20: Two Point Two Probe In-Plane Electrical Resistivity Results for

BA2G5L: Extruded Rods: Extruded 7-6-10

Table F.21: Two Point Two Probe In-Plane Electrical Resistivity Results for

BA5G2L: Extruded Rods: Extruded 7-6-10 .375

Table F.22: Two Point Two Probe In-Plane Electrical Resistivity Results for

BA5G5L: Extruded Rods: Extruded 7-6-10 .376

Table F.23: Two Point Two Probe In-Plane Electrical Resistivity Results for

BQ1G5L: Extruded Rods: Extruded 7-1-10

Table F.24: Two Point Two Probe In-Plane Electrical Resistivity Results for

BQ5G2L: Extruded Rods: Extruded 7-1-10

Table F.25: Two Point Two Probe In-Plane Electrical Resistivity Results for

BQ5G5L: Extruded Rods: Extruded 7-1-10

Table G.1: ASTM D257 Through Plane Electrical Resistivity Results for BL:

Lexan HF1130-111: Injection Molded May 26, 2009

Table G.2: ASTM D257 Through Plane Electrical Resistivity Results for BLE:

Extruded Lexan HF1130-111: Injection Molded May 26, 2009

Table G.3: ASTM D257 Through Plane Electrical Resistivity Results for BL2:

Lexan HF1130-111: Injection Molded December 4, 2009

Table G.4: ASTM D257 Through Plane Electrical Resistivity Results for BLE2:

Extruded Lexan HF1130-111: Extruded Nov 5, 2009; Injection Molded

December 4, 2009

Table G.5: ASTM D257 Through Plane Electrical Resistivity Results for BA2L: 2

wt\% Ketjenblack EC-600 JD in Lexan HF1130-111: Injection Molded

December 4, 2009

Table G.6: ASTM D257 Through Plane Electrical Resistivity Results for BA3L: 3 wt\% Ketjenblack EC-600 JD in Lexan HF1130-111: Injection Molded December 4, 2009 .384

Table G.7: ASTM D257 Through Plane Electrical Resistivity Results for BL3:

Lexan HF1130-111: Injection Molded 6-3-10.

Table G.8: ASTM D257 Through Plane Electrical Resistivity Results for BLE3:

Extruded Lexan HF1130-111: Extruded 5-26-10; Injection Molded 6-3-10.......386

Table G.9: ASTM D257 Through Plane Electrical Resistivity Results for BG2L: 2 wt $\%$ xGnP in Lexan HF1130-111: Injection Molded 6-3-10 .386

Table G.10: ASTM D257 Through Plane Electrical Resistivity Results for BG3L: $3 \mathrm{wt} \% \mathrm{xGnP}$ in Lexan HF1130-111: Injection Molded 6-3-10 .387

Table G.11: ASTM D257 Through Plane Electrical Resistivity Results for BG4L: $4 \mathrm{wt} \%$ xGnP in Lexan HF1130-111: Injection Molded 6-3-10

Table G.12: ASTM D257 Through Plane Electrical Resistivity Results for BG5L: $5 \mathrm{wt} \%$ xGnP in Lexan HF1130-111: Injection Molded 6-3-10 .388 xxvi 
Table G.13: ASTM D257 Through Plane Electrical Resistivity Results for BG6L:

$6 \mathrm{wt} \%$ xGnP in Lexan HF1130-111: Injection Molded 6-8-10 . .388

Table G.14: ASTM D257 Through Plane Electrical Resistivity Results for

BA2LR: Extruded 7-8-10, Injection Molded 7-26-10...

Table G.15: ASTM D257 Through Plane Electrical Resistivity Results for BQ0.5L: Extruded 6-30-10, Injection Molded 7-26-10.

Table G.16: ASTM D257 Through Plane Electrical Resistivity Results for BQ1L

Set 1: Extruded 6-30-10, Injection Molded 7-26-10.

Table G.17: ASTM D257 Through Plane Electrical Resistivity Results for BQ1L

Set 2: Extruded 6-30-10, Injection Molded 7-26-10.

Table G.18: ASTM D257 Through Plane Electrical Resistivity Results for BG2L

Set 2: Extruded 5-26-10, Injection Molded 6-3-10.

Table G.19: ASTM D257 Through Plane Electrical Resistivity Results for BG5L

Set 2: Extruded 5-26-10, Injection Molded 6-3-10.

Table G.20: ASTM D257 Through Plane Electrical Resistivity Results for

BA2G2L set 1: Extruded 7-6-10, Injection Molded 8-18-10

Table G.21: ASTM D257 Through Plane Electrical Resistivity Results for

BA2G2L set 2: Extruded 7-6-10, Injection Molded 8-18-10

Table G.22: ASTM D257 Through Plane Electrical Resistivity Results for

BQ1G2L set 1: Extruded 7-1-10, Injection Molded 8-18-10

Table G.23: ASTM D257 Through Plane Electrical Resistivity Results for BQ1G2L set 2: Extruded 7-1-10, Injection Molded 8-18-10

Table H.1: Two Point Two Probe In-Plane Electrical Resistivity Results for BQ2L: 2 wt\% fibrils in Lexan HF1130-111: Injection Molded May 26, 2009 .

Table H.2: Two Point Two Probe In-Plane Electrical Resistivity Results for BQ3L: 3 wt\% fibrils in Lexan HF1130-111: Injection Molded May 26, 2009

Table H.3: Two Point Two Probe In-Plane Electrical Resistivity Results for BQ4L: 4 wt\% fibrils in Lexan HF1130-111: Injection Molded May 26, 2009

Table H.4: Two Point Two Probe In-Plane Electrical Resistivity Results for BQ5L: 5 wt\% fibrils in Lexan HF1130-111: Injection Molded May 26, 2009. .396

Table H.5: Two Point Two Probe In-Plane Electrical Resistivity Results for BQ6L: 6 wt\% fibrils in Lexan HF1130-111: Injection Molded May 26, 2009 .396 
Table H.6: Two Point Two Probe In-Plane Electrical Resistivity Results for BQ8L: 8 wt\% fibrils in Lexan HF1130-111: Injection Molded May 26, 2009.

Table H.7: Two Point Two Probe In-Plane Electrical Resistivity (ER) Results for BA2L: 2 wt\% Ketjenblack EC-600 JD in Lexan HF1130-111: Tensile Bars: Injection Molded Dec 4, 2009 .398

Table H.8: Two Point Two Probe In-Plane Electrical Resistivity Results for BA3L: 3 wt \% Ketjenblack EC-600 JD in Lexan HF1130-111: Tensile Bars: Injection Molded Dec 4, 2009.

Table H.9: Two Point Two Probe In-Plane Electrical Resistivity Results for BA4L: 4 wt\% Ketjenblack EC-600 JD in Lexan HF1130-111: Tensile Bars: Injection Molded Dec 4, 2009 .400

Table H.10: Two Point Two Probe In-Plane Electrical Resistivity Results for BA5L: 5 wt\% Ketjenblack EC-600 JD in Lexan HF1130-111: Tensile Bars: Injection Molded Dec 4, 2009

Table H.11: Two Point Two Probe In-Plane Electrical Resistivity Results for BA6L: 6 wt\% Ketjenblack EC-600 JD in Lexan HF1130-111: Tensile Bars: Injection Molded Dec 4, 2009

Table H.12: Two Point Two Probe In-Plane Electrical Resistivity Results for BA8L: 8 wt\% Ketjenblack EC-600 JD in Lexan HF1130-111: Tensile Bars: Injection Molded Dec 4, 2009

Table H.13: Two Point Two Probe In-Plane Electrical Resistivity Results for BA10L: 10 wt\% Ketjenblack EC-600 JD in Lexan HF1130-111: Tensile Bars: Injection Molded Dec 4, 2009 .404

Table H.14: Two Point Two Probe In-Plane Electrical Resistivity (ER) Results for BG8L: $8 \mathrm{wt} \%$ xGnP in Lexan HF1130-111: Tensile Bars: Injection Molded June 8, 2010.

Table H.15: Two Point Two Probe In-Plane Electrical Resistivity (ER) Results for BG10L: $10 \mathrm{wt} \%$ xGnP in Lexan HF1130-111: Tensile Bars: Injection Molded June 8, 2010.

Table H.16: Two Point Two Probe In-Plane Electrical Resistivity (ER) Results for BA5LR set 1: 5 wt\% Ketjenblack EC-600 JD in Lexan HF1130-111:

Tensile Bars: Injection Molded July 26, 2010; Extruded 7-8-10; 2-9-09 extruder screw.

Table H.17: Two Point Two Probe In-Plane Electrical Resistivity (ER) Results for BA5LR set 2: 5 wt\% Ketjenblack EC-600 JD in Lexan HF1130-111: Tensile Bars: Injection Molded July 26, 2010; Extruded 7-8-10; 2-9-09 extruder screw ..... 
Table H.18: Two Point Two Probe In-Plane Electrical Resistivity (ER) Results for BQ5LR set 1: Tensile Bars: Injection Molded 8-25-10; Extruded 7-8-10;

2-9-09 extruder screw

Table H.19: Two Point Two Probe In-Plane Electrical Resistivity (ER) Results for BQ5LR set 2: Tensile Bars: Injection Molded 8-25-10; Extruded 7-8-10; 2-9-09 extruder screw

Table H.20: Two Point Two Probe In-Plane Electrical Resistivity (ER) Results for BG12L: 12 wt\% xGnP in Lexan HF1130-111: Tensile Bars: Injection Molded 8-18-10; Extruded 7-8-10; 2-9-09 extruder screw .410

Table H.21: Two Point Two Probe In-Plane Electrical Resistivity (ER) Results for BG15L: $15 \mathrm{wt} \%$ xGnP in Lexan HF1130-111: Tensile Bars: Injection Molded 8-18-10; Extruded 7-8-10; 2-9-09 extruder screw 411

Table H.22: Two Point Two Probe In-Plane Electrical Resistivity (ER) Results for BA2Q1L Set 1: Tensile Bars: Injection Molded 7-26-10

Table H.23: Two Point Two Probe In-Plane Electrical Resistivity (ER) Results for

BA2Q1L Set 2: Tensile Bars: Injection Molded 7-26-10

Table H.24: Two Point Two Probe In-Plane Electrical Resistivity (ER) Results for

BA2Q5L Set 1: Tensile Bars: Injection Molded 8-6-10

Table H.25: Two Point Two Probe In-Plane Electrical Resistivity (ER) Results for

BA2Q5L Set 2: Tensile Bars: Injection Molded 8-6-10

Table H.26: Two Point Two Probe In-Plane Electrical Resistivity (ER) Results for

BA5Q1L Set 1: Tensile Bars: Injection Molded 8-25-10

Table H.27: Two Point Two Probe In-Plane Electrical Resistivity (ER) Results for

BA5Q1L Set 2: Tensile Bars: Injection Molded 8-25-10

Table H.28: Two Point Two Probe In-Plane Electrical Resistivity (ER) Results for

BA5Q5L Set 1: Tensile Bars: Injection Molded 8-6-10

Table H.29: Two Point Two Probe In-Plane Electrical Resistivity (ER) Results for

BA5Q5L Set 2: Tensile Bars: Injection Molded 8-26-10

Table H.30 Two Point Two Probe In-Plane Electrical Resistivity (ER) Results for

BA2G5L Set 1: Tensile Bars: Injection Molded 8-18-10

Table H.31: Two Point Two Probe In-Plane Electrical Resistivity (ER) Results for

BA2G5L Set 2: Tensile Bars: Injection Molded 8-18-10 .418

Table H.32: Two Point Two Probe In-Plane Electrical Resistivity (ER) Results for

BA5G2L Set 1: Tensile Bars: Injection Molded 8-18-10 .418

Table H.33: Two Point Two Probe In-Plane Electrical Resistivity (ER) Results for

BA5G2L Set 2: Tensile Bars: Injection Molded 8-18-10

Table H.34: Two Point Two Probe In-Plane Electrical Resistivity (ER) Results for

BA5G5L Set 1: Tensile Bars: Injection Molded 8-18-10

Table H.35: Two Point Two Probe In-Plane Electrical Resistivity (ER) Results for

BA5G5L Set 2: Tensile Bars: Injection Molded 8-18-10

xxix 
Table H.36: Two Point Two Probe In-Plane Electrical Resistivity (ER) Results for BQ1G5L Set 1: Tensile Bars: Injection Molded 8-18-10 ....................................420

Table H.37: Two Point Two Probe In-Plane Electrical Resistivity (ER) Results for BQ1G5L Set 2: Tensile Bars: Injection Molded 8-18-10

Table H.38: Two Point Two Probe In-Plane Electrical Resistivity (ER) Results for BQ5G2L Set 1: Tensile Bars: Injection Molded 8-18-10

Table H.39: Two Point Two Probe In-Plane Electrical Resistivity (ER) Results for BQ5G2L Set 2: Tensile Bars: Injection Molded 8-18-10

Table H.40: Two Point Two Probe In-Plane Electrical Resistivity (ER) Results for BQ5G5L Set 1: Tensile Bars: Injection Molded 9-9-10.

Table H.41: Two Point Two Probe In-Plane Electrical Resistivity (ER) Results for BQ5G5L Set 2: Tensile Bars: Injection Molded 9-9-10

Table I.1: Through-Plane Thermal Conductivity at $55^{\circ} \mathrm{C}$ using Holometrix TCA300 Guarded Heat Flow Meter Method for Lexan HF1130-111 (BL): Injection Molded May 26, 2009.

Table I.2: Through-Plane Thermal Conductivity at $55^{\circ} \mathrm{C}$ using Holometrix TCA300 Guarded Heat Flow Meter Method for Extruded Lexan HF1130-111 (BLE): Injection Molded May 26, 2009

Table I.3: Through-Plane Thermal Conductivity at $55^{\circ} \mathrm{C}$ using Holometrix TCA300 Guarded Heat Flow Meter Method for $2 \mathrm{wt} \%$ Hyperion Fibrils in Lexan HF1130-111 (BQ2L): Injection Molded May 26, 2009 425

Table I.4: Through-Plane Thermal Conductivity at $55^{\circ} \mathrm{C}$ using Holometrix TCA300 Guarded Heat Flow Meter Method for $3 \mathrm{wt} \%$ Hyperion Fibrils in Lexan HF1130-111 (BQ3L): Injection Molded May 26, 2009

Table I.5: Through-Plane Thermal Conductivity at $55^{\circ} \mathrm{C}$ using Holometrix TCA300 Guarded Heat Flow Meter Method for $4 \mathrm{wt} \%$ Hyperion Fibrils in Lexan HF1130-111 (BQ4L): Injection Molded May 26, 2009

Table I.6: Through-Plane Thermal Conductivity at $55^{\circ} \mathrm{C}$ using Holometrix TCA300 Guarded Heat Flow Meter Method for $5 \mathrm{wt} \%$ Hyperion Fibrils in Lexan HF1130-111 (BQ5L): Injection Molded May 26, 2009 .426

Table I.7: Through-Plane Thermal Conductivity at $55^{\circ} \mathrm{C}$ using Holometrix TCA300 Guarded Heat Flow Meter Method for $6 \mathrm{wt} \%$ Hyperion Fibrils in Lexan HF1130-111 (BQ6L): Injection Molded May 26, 2009

Table I.8: Through-Plane Thermal Conductivity at $55^{\circ} \mathrm{C}$ using Holometrix TCA300 Guarded Heat Flow Meter Method for $8 \mathrm{wt} \%$ Hyperion Fibrils in Lexan HF1130-111 (BQ8L): Injection Molded May 26, 2009

Table I.9: Through-Plane Thermal Conductivity at $55^{\circ} \mathrm{C}$ using Holometrix TCA300 Guarded Heat Flow Meter Method for Lexan HF1130-111 (BL2): Injection Molded December 4, 2009 
Table I.10: Through-Plane Thermal Conductivity at $55^{\circ} \mathrm{C}$ using Holometrix TCA300 Guarded Heat Flow Meter Method for Extruded Lexan HF1130-111

(BLE2): Injection Molded Dec 4, 2009 428

Table I.11: Through-Plane Thermal Conductivity at $55^{\circ} \mathrm{C}$ using Holometrix TCA300 Guarded Heat Flow Meter Method for $2 \mathrm{wt} \%$ Ketjenblack EC-600 JD in Lexan HF1130-111 (BA2L): Injection Molded Dec 4, 2009

Table I.12: Through-Plane Thermal Conductivity at $55^{\circ} \mathrm{C}$ using Holometrix TCA300 Guarded Heat Flow Meter Method for $3 \mathrm{wt} \%$ Ketjenblack EC-600 JD in Lexan HF1130-111 (BA3L): Injection Molded Dec 4, 2009

Table I.13: Through-Plane Thermal Conductivity at $55^{\circ} \mathrm{C}$ using Holometrix TCA300 Guarded Heat Flow Meter Method for $4 \mathrm{wt} \%$ Ketjenblack EC-600 JD in Lexan HF1130-111 (BA4L): Injection Molded Dec 4, 2009

Table I.14: Through-Plane Thermal Conductivity at $55^{\circ} \mathrm{C}$ using Holometrix TCA300 Guarded Heat Flow Meter Method for $5 \mathrm{wt} \%$ Ketjenblack EC-600 JD in Lexan HF1130-111 (BA5L): Injection Molded Dec 4, 2009

Table I.15: Through-Plane Thermal Conductivity at $55^{\circ} \mathrm{C}$ using Holometrix TCA300 Guarded Heat Flow Meter Method for 6 wt \% Ketjenblack EC-600 JD in Lexan HF1130-111 (BA6L): Injection Molded Dec 4, 2009

Table I.16: Through-Plane Thermal Conductivity at $55^{\circ} \mathrm{C}$ using Holometrix TCA300 Guarded Heat Flow Meter Method for $8 \mathrm{wt} \%$ Ketjenblack EC-600 JD in Lexan HF1130-111 (BA8L): Injection Molded Dec 4, 2009

Table I.17: Through-Plane Thermal Conductivity at $55^{\circ} \mathrm{C}$ using Holometrix TCA300 Guarded Heat Flow Meter Method for $10 \mathrm{wt} \%$ Ketjenblack EC-600 JD in Lexan HF1130-111 (BA10L): Injection Molded Dec 4, 2009. 431

Table I.18: Through-Plane Thermal Conductivity at $55^{\circ} \mathrm{C}$ using Holometrix TCA300 Guarded Heat Flow Meter Method for Lexan HF1130-111 (BL3): Injection Molded June 3, 2010.

Table I.19: Through-Plane Thermal Conductivity at $55^{\circ} \mathrm{C}$ using Holometrix TCA300 Guarded Heat Flow Meter Method for Extruded Lexan HF1130-111 (BLE3): Injection Molded June 3, 2010

Table I.20: Through-Plane Thermal Conductivity at $55^{\circ} \mathrm{C}$ using Holometrix TCA300 Guarded Heat Flow Meter Method for $2 \mathrm{wt} \% \mathrm{xGnP}$ in Lexan HF1130-111 (BG2L): Injection Molded June 3, 2010

Table I.21: Through-Plane Thermal Conductivity at $55^{\circ} \mathrm{C}$ using Holometrix TCA300 Guarded Heat Flow Meter Method for $3 \mathrm{wt} \% \mathrm{xGnP}$ in Lexan HF1130-111 (BG3L): Injection Molded June 3, 2010

Table I.22: Through-Plane Thermal Conductivity at $55^{\circ} \mathrm{C}$ using Holometrix TCA300 Guarded Heat Flow Meter Method for $4 \mathrm{wt} \%$ xGnP in Lexan HF1130-111 (BG4L): Injection Molded June 3, 2010 434 
Table I.23: Through-Plane Thermal Conductivity at $55^{\circ} \mathrm{C}$ using Holometrix TCA300 Guarded Heat Flow Meter Method 5 wt\% xGnP in Lexan HF1130-

111 (BG5L): Injection Molded June 3, 2010

Table I.24: Through-Plane Thermal Conductivity at $55^{\circ} \mathrm{C}$ using Holometrix TCA300 Guarded Heat Flow Meter Method for $6 \mathrm{wt} \% \mathrm{xGnP}$ in Lexan HF1130-111 (BG6L): Injection Molded June 8, 2010 .435

Table I.25: Through-Plane Thermal Conductivity at $55^{\circ} \mathrm{C}$ using Holometrix TCA300 Guarded Heat Flow Meter Method for for $8 \mathrm{wt} \% \mathrm{xGnP}$ in Lexan HF1130-111 (BG8L): Injection Molded June 8, 2010 .435

Table I.26: Through-Plane Thermal Conductivity at $55^{\circ} \mathrm{C}$ using Holometrix TCA300 Guarded Heat Flow Meter Method for $10 \mathrm{wt} \%$ xGnP in Lexan HF1130-111 (BG10L): Injection Molded June 8, 2010 436

Table I.27: Through-Plane Thermal Conductivity at $55^{\circ} \mathrm{C}$ using Holometrix TCA300 Guarded Heat Flow Meter Method for BQ0.5L: Injection Molded 726-10

Table I.28: Through-Plane Thermal Conductivity at $55^{\circ} \mathrm{C}$ using Holometrix TCA300 Guarded Heat Flow Meter Method for BQ1L Set 1: Injection Molded 7-26-10

Table I.29: Through-Plane Thermal Conductivity at $55^{\circ} \mathrm{C}$ using Holometrix TCA300 Guarded Heat Flow Meter Method for BQ1L Set 2: Injection Molded 7-26-10

Table I.30: Through-Plane Thermal Conductivity at $55^{\circ} \mathrm{C}$ using Holometrix TCA300 Guarded Heat Flow Meter Method for BQ5L Set 2 .438

Table I.31: Through-Plane Thermal Conductivity at $55^{\circ} \mathrm{C}$ using Holometrix TCA300 Guarded Heat Flow Meter Method for BG2L Set 2: Injection Molded 6-3-10 .438

Table I.32: Through-Plane Thermal Conductivity at $55^{\circ} \mathrm{C}$ using Holometrix TCA300 Guarded Heat Flow Meter Method for BG5L Set 2: Injection Molded 6-3-10 .438

Table I.33: Through-Plane Thermal Conductivity at $55^{\circ} \mathrm{C}$ using Holometrix TCA300 Guarded Heat Flow Meter Method for BG12L: Injection Molded 8$18-10$

Table I.34: Through-Plane Thermal Conductivity at $55^{\circ} \mathrm{C}$ using Holometrix TCA300 Guarded Heat Flow Meter Method for BG15L: Injection Molded 8$18-10$

Table I.35: Through-Plane Thermal Conductivity at $55^{\circ} \mathrm{C}$ using Holometrix TCA300 Guarded Heat Flow Meter Method for BA2Q1L set 1: Injection Molded 7-26-10 
Table I.36: Through-Plane Thermal Conductivity at $55^{\circ} \mathrm{C}$ using Holometrix TCA300 Guarded Heat Flow Meter Method for BA2Q1L set 2: Injection

Molded 7-26-10

Table I.37: Through-Plane Thermal Conductivity at $55^{\circ} \mathrm{C}$ using Holometrix TCA-

300 Guarded Heat Flow Meter Method for BA2Q5L set 1: Injection

Molded 8-25-10

Table I.38: Through-Plane Thermal Conductivity at $55^{\circ} \mathrm{C}$ using Holometrix TCA-

300 Guarded Heat Flow Meter Method for BA2Q5L set 2: Injection

Molded 8-25-10

Table I.4-39: Through-Plane Thermal Conductivity at $55^{\circ} \mathrm{C}$ using Holometrix

TCA-300 Guarded Heat Flow Meter Method for BA5Q1L set 1: Injection

Molded 7-26-10

Table I.4-40: Through-Plane Thermal Conductivity at $55^{\circ} \mathrm{C}$ using Holometrix

TCA-300 Guarded Heat Flow Meter Method for BA5Q1L set 2: Injection

Molded 7-26-10

Table I.41: Through-Plane Thermal Conductivity at $55^{\circ} \mathrm{C}$ using Holometrix TCA-

300 Guarded Heat Flow Meter Method for BA5Q5L set 1: Injection

Molded 8-25-10

Table I.42: Through-Plane Thermal Conductivity at $55^{\circ} \mathrm{C}$ using Holometrix TCA-

300 Guarded Heat Flow Meter Method for BA5Q5L set 2: Injection

Molded 8-25-10

Table I.43: Through-Plane Thermal Conductivity at $55^{\circ} \mathrm{C}$ using Holometrix TCA-

300 Guarded Heat Flow Meter Method for BA2G2L set 1: Injection

Molded 8-18-10

Table I.44: Through-Plane Thermal Conductivity at $55^{\circ} \mathrm{C}$ using Holometrix TCA-

300 Guarded Heat Flow Meter Method for BA2G2L set 2: Injection

Molded 8-18-10

Table I.45: Through-Plane Thermal Conductivity at $55^{\circ} \mathrm{C}$ using Holometrix TCA-

300 Guarded Heat Flow Meter Method for BA2G5L set 1: Injection

Molded 8-18-10

Table I.46: Through-Plane Thermal Conductivity at $55^{\circ} \mathrm{C}$ using Holometrix TCA-

300 Guarded Heat Flow Meter Method for BA2G5L set 2: Injection

Molded 8-18-10

Table I.47: Through-Plane Thermal Conductivity at $55^{\circ} \mathrm{C}$ using Holometrix TCA-

300 Guarded Heat Flow Meter Method for BA5G2L set 1: Injection

Molded 8-18-10

Table I.48: Through-Plane Thermal Conductivity at $55^{\circ} \mathrm{C}$ using Holometrix TCA-

300 Guarded Heat Flow Meter Method for BA5G2L set 2: Injection

Molded 8-18-10

Xxxiii 
Table I.49: Through-Plane Thermal Conductivity at $55^{\circ} \mathrm{C}$ using Holometrix TCA300 Guarded Heat Flow Meter Method for BA5G5L set 1: Injection

Molded 8-18-10

Table I.50: Through-Plane Thermal Conductivity at $55^{\circ} \mathrm{C}$ using Holometrix TCA-

300 Guarded Heat Flow Meter Method for BA5G5L set 2: Injection

Molded 8-18-10

Table I.51: Through-Plane Thermal Conductivity at $55^{\circ} \mathrm{C}$ using Holometrix TCA-

300 Guarded Heat Flow Meter Method for BQ1G2L set 1: Injection

Molded 8-18-10

Table I.52: Through-Plane Thermal Conductivity at $55^{\circ} \mathrm{C}$ using Holometrix TCA-

300 Guarded Heat Flow Meter Method for BQ1G2L set 2: Injection

Molded 8-18-10

Table I-53: Through-Plane Thermal Conductivity at $55^{\circ} \mathrm{C}$ using Holometrix TCA-

300 Guarded Heat Flow Meter Method for BQ1G5L set 1: Injection

Molded 8-18-10

Table I.54: Through-Plane Thermal Conductivity at $55^{\circ} \mathrm{C}$ using Holometrix TCA-

300 Guarded Heat Flow Meter Method for BQ1G5L set 2: Injection

Molded 8-18-10

Table I.55: Through-Plane Thermal Conductivity at $55^{\circ} \mathrm{C}$ using Holometrix TCA-

300 Guarded Heat Flow Meter Method for BQ5G2L set 1: Injection

Molded 8-18-10

Table I.56: Through-Plane Thermal Conductivity at $55^{\circ} \mathrm{C}$ using Holometrix TCA300 Guarded Heat Flow Meter Method for BQ5G2L set 2: Injection Molded 8-18-10 .446

Table I.57: Through-Plane Thermal Conductivity at $55^{\circ} \mathrm{C}$ using Holometrix TCA-

300 Guarded Heat Flow Meter Method for BQ5G5L set 1: Injection

Molded 8-18-10

Table I.58: Through-Plane Thermal Conductivity at $55^{\circ} \mathrm{C}$ using Holometrix TCA300 Guarded Heat Flow Meter Method for BQ5G5L set 2: Injection Molded 8-18-10

Table J.1: Tensile Results for BL: Lexan HF1130-111: Injection Molded 5-26-09

Table J.2: Tensile Results for BLE: Extruded Lexan HF1130-111: Injection Molded 5-26-09

Table J.3: Tensile Results for BQ2L: $2 \mathrm{wt} \%$ fibrils (carbon nanotubes) in Lexan HF1130-111: Injection Molded 5-26-09

Table J.4: Tensile Results for BQ3L: 3 wt\% fibrils (carbon nanotubes) in Lexan

HF1130-111: Injection Molded 5-26-09

Table J.5: Tensile Results for BQ4L: 4 wt\% fibrils (carbon nanotubes) in Lexan

HF1130-111: Injection Molded 5-26-09 
Table J.6: Tensile Results for BQ5L: $5 \mathrm{wt} \%$ fibrils (carbon nanotubes) in Lexan HF1130-111: Injection Molded 5-26-09.

Table J.7: Tensile Results for BQ6L: $6 \mathrm{wt} \%$ fibrils (carbon nanotubes) in Lexan

HF1130-111: Injection Molded 5-26-09.

Table J.8: Tensile Results for BQ8L: $8 \mathrm{wt} \%$ fibrils (carbon nanotubes) in Lexan

HF1130-111: Injection Molded 5-26-09 (all these samples broke in the grips)

Table J.9: Tensile Results for BL2: Lexan HF1130-111: Injection Molded 12-4-

09. .465

Table J.10: Tensile Results for BLE2: Extruded Lexan HF1130-111: Extruded 11-

5-09, Injection Molded 12-4-09.

Table J.11: Tensile Results for BA2L: 2 wt\% Ketjenblack EC-600 JD (carbon

black) in Lexan HF1130-111: Injection Molded 12-4-09.

Table J.12: Tensile Results for BA3L: 3 wt\% Ketjenblack EC-600 JD (carbon

black) in Lexan HF1130-111: Injection Molded 12-4-09.

Table J.13: Tensile Results for BA4L: 4 wt\% Ketjenblack EC-600 JD (carbon

black) in Lexan HF1130-111: Injection Molded 12-4-09

Table J.14: Tensile Results for BA5L: 5 wt\% Ketjenblack EC-600 JD (carbon

black) in Lexan HF1130-111: Injection Molded 12-4-09..... .475

Table J.15: Tensile Results for BA6L: 6 wt\% Ketjenblack EC-600 JD (carbon

black) in Lexan HF1130-111: Injection Molded 12-4-09.

Table J.16: Tensile Results for BA8L: 8 wt\% Ketjenblack EC-600 JD (carbon

black) in Lexan HF1130-111: Injection Molded 12-4-09.

Table J.17: Tensile Results for BA10L: 10 wt\% Ketjenblack EC-600 JD (carbon

black) in Lexan HF1130-111: Injection Molded 12-4-09.

Table J.18: Tensile Results for BL3: Lexan HF1130-111: Injection Molded 6-3-

10

Table J.19: Tensile Results for BLE3: Extruded Lexan HF1130-111: Extruded 5-

26-10, Injection Molded 6-3-10

Table J.20: Tensile Results for BG2L: 2 wt\% xGnP in Lexan HF1130-111:

Injection Molded 6-3-10, Extruded 5-26-10

Table J.21: Tensile Results for BG3L: 3 wt\% xGnP in Lexan HF1130-111:

Injection Molded 6-3-10, Extruded 5-26-10

Table J.22: Tensile Results for BG4L: 4 wt\% xGnP in Lexan HF1130-111:

Injection Molded 6-3-10, Extruded 5-26-10

Table J.23: Tensile Results for BG5L: $5 \mathrm{wt} \%$ xGnP in Lexan HF1130-111:

Injection Molded 6-3-10, Extruded 5-26-10

Table J.24: Tensile Results for BG6L: 6 wt\% xGnP in Lexan HF1130-111:

Injection Molded 6-8-10, Extruded 5-27-10 
Table J.25: Tensile Results for BG8L: 8 wt\% xGnP in Lexan HF1130-111:

Injection Molded 6-8-10, Extruded 5-27-10

Table J.26: Tensile Results for BG10L: $10 \mathrm{wt} \%$ xGnP in Lexan HF1130-111:

Injection Molded 6-8-10, Extruded 5-27-10

Table J.27: Tensile Results for BA2LR Set 1: Extruded 7-8-10, Injection Molded

7-26-10.

Table J.28: Tensile Results for BA2LR Set 2: Extruded 7-8-10, Injection Molded

7-26-10

Table J.29: Tensile Results for BA5LR Set 1: Extruded 7-8-10, Injection Molded

7-26-10.

Table J.30: Tensile Results for BA5LR Set 2: Extruded 7-8-10, Injection Molded

7-26-10

Table J.31: Tensile Results for BQ1L Set 1: Extruded 6-30-10, Injection Molded

7-26-10

Table J.32: Tensile Results for BQ1L Set 2: Extruded 6-30-10, Injection Molded

7-26-10

Table J.33: Tensile Results for BQ5L Set 1: Extruded May 2009, Injection

Molded 5-26-09

Table J.34: Tensile Results for BQ5L Set 2: Extruded May 2009, Injection

Molded 5-26-09

Table J.35: Tensile Results for BG2L set 1: 2 wt\% xGnP in Lexan HF1130-111:

Injection Molded 6-3-10, Extruded 5-26-10.

Table J.36: Tensile Results for BG2L set 2: 2 wt\% xGnP in Lexan HF1130-111:

Injection Molded 9-9-10, Extruded 5-26-10

Table J.37: Tensile Results for BG5L set 1: $5 \mathrm{wt} \%$ xGnP in Lexan HF1130-111:

Injection Molded 6-3-10, Extruded 5-26-10

Table J.38: Tensile Results for BG5L set 2: 2 wt\% xGnP in Lexan HF1130-111:

Extruded 5-26-10

Table J.39: Tensile Results for BG12L: 12 wt\% xGnP in Lexan HF1130-111:

Injection Molded 8-18-10, Extruded 7-8-10

Table J.40: Tensile Results for BG15L: 15 wt\% xGnP in Lexan HF1130-111:

Injection Molded 8-18-10, Extruded 7-8-10.

Table J.41: Tensile Results for BA2Q1L set 1: Injection Molded 7-26-10,

Extruded 7-7-10

Table J.42: Tensile Results for BA2Q1L set 2: Injection Molded 7-26-10,

Extruded 7-7-10

Table J.43: Tensile Results for BA2Q5L set 1: Injection Molded 8-25-10,

Extruded 7-7-10

Table J.44: Tensile Results for BA2Q5L set 2: Injection Molded 8-25-10,

Extruded 7-7-10 .535

xxxvi 
Table J.45: Tensile Results for BA5Q1L set 1: Injection Molded 8-6-10, Extruded 7-7-10

Table J.46: Tensile Results for BA5Q1L set 2: Injection Molded 8-25-10, Extruded 7-7-10 .539

Table J.47: Tensile Results for BA5Q5L set 1: Injection Molded 8-25-10, Extruded 7-7-10 .541

Table J.48: Tensile Results for BA5Q5L set 2: Injection Molded 8-25-10, Extruded 7-7-10 .543

Table J.49: Tensile Results for BA2G2L set 1: Injection Molded 8-18-10, Extruded 7-6-10 .545

Table J.50: Tensile Results for BA2G2L set 2: Injection Molded 8-18-10, Extruded 7-6-10

Table J.51: Tensile Results for BA2G5L set 1: Injection Molded 8-18-10,

Extruded 7-6-10

Table J.52: Tensile Results for BA2G5L set 2: Injection Molded 8-18-10,

Extruded 7-6-10

Table J.53: Tensile Results for BA5G2L set 1: Injection Molded 8-18-10,

Extruded 7-6-10

Table J.54: Tensile Results for BA5G2L set 2: Injection Molded 8-18-10, Extruded 7-6-10 .555

Table J.55: Tensile Results for BA5G5L set 1: Injection Molded 8-6-10, Extruded 7-6-10 .557

Table J.56: Tensile Results for BA5G5L set 2: Injection Molded 8-6-10, Extruded 7-6-10 .559

Table J.57: Tensile Results for BQ1G2L set 1: Injection Molded 8-18-10, Extruded 7-1-10 .561

Table J.58: Tensile Results for BQ1G2L set 2: Injection Molded 8-18-10, Extruded 7-1-10 .563

Table J.59: Tensile Results for BQ1G5L set 1: Injection Molded 8-18-10, Extruded 7-1-10 .565

Table J.60: Tensile Results for BQ1G5L set 2: Injection Molded 8-6-10, Extruded 7-1-10. .567

Table J.61: Tensile Results for BQ5G2L set 1: Injection Molded 9-9-10, Extruded 7-1-10. .569

Table J.62: Tensile Results for BQ5G2L set 2: Injection Molded 9-9-10, Extruded 7-1-10 .571

Table J.63: Tensile Results for BQ5G5L set 1: Injection Molded 9-9-10, Extruded 7-1-10 .573

Table J.64: Tensile Results for BQ5G5L set 2: Injection Molded 9-9-10, Extruded 7-1-10 .575 xxxvii 
Table K.1: Flexural Results for BL Lexan HF1130-111: Injection molded 5-26-09 ......577

Table K.2: Flexural Results for BLE Extruded Lexan HF1130-111: Injection molded 5-26-09.

Table K.3: Flexural Results for BQ2L: $2 \mathrm{wt} \%$ fibrils (carbon nanotubes) in Lexan HF1130-111: Injection Molded 5-26-09.

Table K.4: Flexural Results for BQ3L: $3 \mathrm{wt} \%$ fibrils (carbon nanotubes) in Lexan HF1130-111: Injection Molded 5-26-09.

Table K.5: Flexural Results for BQ4L: 4 wt\% fibrils (carbon nanotubes) in Lexan HF1130-111: Injection Molded 5-26-09.

Table K.6: Flexural Results for BQ5L: $5 \mathrm{wt} \%$ fibrils (carbon nanotubes) in Lexan HF1130-111: Injection Molded 5-26-09.

Table K.7: Flexural Results for BQ6L: $6 \mathrm{wt} \%$ fibrils (carbon nanotubes) in Lexan HF1130-111: Injection Molded 5-26-09

Table K.8: Flexural Results for BQ8L: $8 \mathrm{wt} \%$ fibrils (carbon nanotubes) in Lexan HF1130-111: Injection Molded 5-26-09.

Table K.9: Flexural Results for BL2 Lexan HF1130-111: Injection molded 12-409.

Table K.10: Flexural Results for BLE2 Extruded Lexan HF1130-111: Extruded 11-5-09, Injection molded 12-4-09

Table K.11: Flexural Results for BA2L: 2 wt\% Ketjenblack EC-600 JD (carbon black) in Lexan HF1130-111: Injection Molded 12-4-09.

Table K.12: Flexural Results for BA3L: 3 wt\% Ketjenblack EC-600 JD (carbon black) in Lexan HF1130-111: Injection Molded 12-4-09.

Table K.13: Flexural Results for BA4L: 4 wt\% Ketjenblack EC-600 JD (carbon black) in Lexan HF1130-111: Injection Molded 12-4-09.

Table K.14: Flexural Results for BA5L: 5 wt\% Ketjenblack EC-600 JD (carbon black) in Lexan HF1130-111: Injection Molded 12-4-09.

Table K.15: Flexural Results for BA6L: 6 wt\% Ketjenblack EC-600 JD (carbon black) in Lexan HF1130-111: Injection Molded 12-4-09.

Table K.16: Flexural Results for BA8L: 8 wt\% Ketjenblack EC-600 JD (carbon black) in Lexan HF1130-111: Injection Molded 12-4-09.

Table K.17: Flexural Results for BA10L: $10 \mathrm{wt} \%$ Ketjenblack EC-600 JD (carbon black) in Lexan HF1130-111: Injection Molded 12-4-09: All samples fractured

Table K.18: Flexural Results for BL3 Lexan HF1130-111: Injection molded 6-310

Table K.19: Flexural Results for BLE3 Extruded Lexan HF1130-111: Extruded 526-10, Injection molded 6-3-10

Table K.20: Flexural Results for BG2L: 2 wt\% xGnP in Lexan HF1130-111: Injection Molded 6-3-10 
Table K.21: Flexural Results for BG3L: 3 wt\% xGnP in Lexan HF1130-111:

Injection Molded 6-3-10

Table K.22: Flexural Results for BG4L: 4 wt\% xGnP (carbon black) in Lexan HF1130-111: Injection Molded 6-3-10.

Table K.23: Flexural Results for BG5L: 5 wt\% xGnP in Lexan HF1130-111: Injection Molded 6-3-10

Table K.24: Flexural Results for BG6L: 6 wt\% xGnP in Lexan HF1130-111: Injection Molded 6-8-10: All samples fractured.

Table K.25: Flexural Results for BG8L: 8 wt\% xGnP in Lexan HF1130-111: Injection Molded 6-8-10 : All samples fractured. Flexural Maximum and Fracture Stress and Strain are identical.

Table K.26: Flexural Results for BG10L: $10 \mathrm{wt} \%$ xGnP in Lexan HF1130-111: Injection Molded 6-8-10: All samples fractured. Flexural Maximum and Fracture Stress and Strain are identical.

Table K.27: Flexural Results for BL4 Lexan HF1130-111: Injection molded 7-2610

Table K.28: Flexural Results for BA2LR Set 1: Extruded 7-8-10, Injection molded 7-26-10.

Table K.29: Flexural Results for BA2LR Set 2: Extruded 7-8-10, Injection molded 7-26-10.

Table K.30: Flexural Results for BA5LR Set 1: Extruded 7-8-10, Injection molded 7-26-10.

Table K.31: Flexural Results for BA5LR Set 2: Extruded 7-8-10, Injection molded 7-26-10

Table K.32: Flexural Results for BQ1L Set 1: Extruded 6-30-10, Injection molded 7-26-10

Table K.33: Flexural Results for BQ1L Set 2: Extruded 6-30-10, Injection molded 7-26-10.....

Table K.34: Flexural Results for BQ5LR Set 1: Extruded 6-30-10, Injection molded 8-25-10.

Table K.35: Flexural Results for BQ5LR Set 2: Extruded 6-30-10, Injection molded 8-25-10

Table K.36: Flexural Results for BG2L Set 1: Extruded May 2010, Injection molded 9-9-10

Table K.37: Flexural Results for BG2L Set 2: Extruded May 2010, Injection molded 9-9-10

Table K.38: Flexural Results for BG5L Set 1: Extruded May 2010, Injection molded 9-9-10

Table K.39: Flexural Results for BG5L Set 2: Extruded May 2010, Injection molded 9-9-10 
Table K.40: Flexural Results for BG12L: Extruded 7-8-10, Injection molded 8$18-10$

Table K.41: Flexural Results for BG15L: Extruded 7-8-10, Injection molded 8$18-10$

Table K.42: Flexural Results for BA2Q1L Set 1: Extruded 7-7-10, Injection molded 7-26-10.

Table K.43: Flexural Results for BA2Q1L Set 2: Extruded 7-7-10, Injection molded 7-26-10

Table K.44: Flexural Results for BA2Q5L Set 1: Extruded 7-7-10, Injection molded 8-25-10

Table K.45: Flexural Results for BA2Q5L Set 2: Extruded 7-7-10, Injection molded 8-25-10.....

Table K.46: Flexural Results for BA5Q1L Set 1: Extruded 7-7-10, Injection molded 7-26-10

Table K.47: Flexural Results for BA5Q1L Set 2: Extruded 7-7-10, Injection molded 7-26-10.

Table K.48: Flexural Results for BA5Q5L Set 1: Extruded 7-7-10, Injection molded 8-25-10.

Table K.49: Flexural Results for BA5Q5L Set 2: Extruded 7-7-10, Injection molded 8-25-10.

Table K.50: Flexural Results for BA2G2L Set 1: Extruded 7-6-10, Injection molded 8-18-10....

Table K.51: Flexural Results for BA2G2L Set 2: Extruded 7-6-10, Injection molded 8-18-10.

Table K.52: Flexural Results for BA2G5L Set 1: Extruded 7-6-10, Injection molded 8-18-10.

Table K.53: Flexural Results for BA2G5L Set 2: Extruded 7-6-10, Injection molded 8-18-10.....

Table K.54: Flexural Results for BA5G2L Set 1: Extruded 7-6-10, Injection molded 8-18-10.....

Table K.55: Flexural Results for BA5G2L Set 2: Extruded 7-6-10, Injection molded 8-18-10.

Table K.56: Flexural Results for BA5G5L Set 1: Extruded 7-6-10, Injection molded 8-18-10

Table K.57: Flexural Results for BA5G5L Set 2: Extruded 7-6-10, Injection molded 8-18-10

Table K.58: Flexural Results for BQ1G2L Set 1: Extruded 7-1-10, Injection molded 8-18-10.

Table K.59: Flexural Results for BQ1G2L Set 2: Extruded 7-1-10, Injection molded 8-18-10 
Table K.60: Flexural Results for BQ1G5L Set 1: Extruded 7-1-10, Injection molded 8-18-10.

Table K.61: Flexural Results for BQ1G5L Set 2: Extruded 7-1-10, Injection molded 8-18-10

Table K.62: Flexural Results for BQ5G2L Set 1: Extruded 7-1-10, Injection molded 9-9-10

Table K.63: Flexural Results for BQ5G2L Set 2: Extruded 7-1-10, Injection molded 9-9-10

Table K.64: Flexural Results for BQ5G5L Set 1: Extruded 7-1-10, Injection molded 9-9-10

Table K.65: Flexural Results for BQ5G5L Set 2: Extruded 7-1-10, Injection molded 9-9-10 .705

Table L.1: SAOS Results for BL: Lexan HF1130-111 at various temperatures .706

Table L.2: SAOS Results for BQ2L: $2 \mathrm{wt} \%$ Hyperion Fibrils (CNT) in Lexan HF1130-111 at various temperatures

Table L.3: SAOS Results for BQ3L: 3 wt \% Hyperion Fibrils (CNT) in Lexan HF1130-111 at various temperatures

Table L.4: SAOS Results for BQ4L: 4 wt\% Hyperion Fibrils (CNT) in Lexan HF1130-111 at various temperatures

Table L.5: SAOS Results for BQ5L: 5 wt \% Hyperion Fibrils (CNT) in Lexan HF1130-111 at various temperatures

Table L.6: SAOS Results for BQ6L: 6 wt\% Hyperion Fibrils (CNT) in Lexan HF1130-111 at various temperatures

Table L.7: SAOS Results for BQ8L: 8 wt\% Hyperion Fibrils (CNT) in Lexan HF1130-111 at various temperatures

Table L.8: SAOS Results for BA2L: 2 wt\% Carbon Black (CB) in Lexan HF1130-111 at various temperatures

Table L.9: SAOS Results for BA3L: 3 wt\% Carbon Black (CB) in Lexan

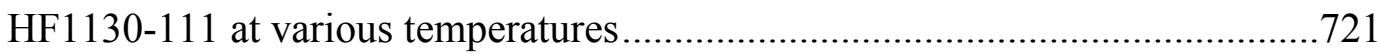

Formulation BA3L

Table L.10: SAOS Results for BA4L: $4 \mathrm{wt} \%$ Carbon Black (CB) in Lexan HF1130-111 at various temperatures

Table L.10: SAOS Results for BA4L: $4 \mathrm{wt} \%$ Carbon Black (CB) in Lexan HF1130-111 at various temperatures continued

Table L.11: SAOS Results for BA5L: 5 wt $\%$ Carbon Black (CB) in Lexan HF1130-111 at various temperatures .725

Table L.12: SAOS Results for BA6L: 6 wt $\%$ Carbon Black (CB) in Lexan HF1130-111 at various temperatures 
Table L.13: SAOS Results for BA8L: 8 wt\% Carbon Black (CB) in Lexan

HF1130-111 at various temperatures

Table L.14: SAOS Results for BG2L: 2 wt\% Graphene Nanoplatelets (GNP) in

Lexan HF1130-111 at various temperatures

Table L.15: SAOS Results for BG3L: 3 wt\% Graphene Nanoplatelets (GNP) in

Lexan HF1130-111 at various temperatures

Table L.16: SAOS Results for BG4L: 4 wt\% Graphene Nanoplatelets (GNP) in

Lexan HF1130-111 at various temperatures

Table L.17: SAOS Results for BG5L: 5 wt\% Graphene Nanoplatelets (GNP) in

Lexan HF1130-111 at various temperatures

Table L.18: SAOS Results for BG6L: $6 \mathrm{wt} \%$ Graphene Nanoplatelets (GNP) in

Lexan HF1130-111 at various temperatures

Table L.19: SAOS Results for BG8L: 8 wt\% Graphene Nanoplatelets (GNP) in

Lexan HF1130-111 at various temperatures

Table L.20: SAOS Results for BG10L: $10 \mathrm{wt} \%$ Graphene Nanoplatelets (GNP) in

Lexan HF1130-111 at various temperatures

Table L.21: SAOS Results for BG12L: $12 \mathrm{wt} \%$ Graphene Nanoplatelets (GNP) in

Lexan HF1130-111 at various temperatures

Table L.22: SAOS Results for BG15L: $15 \mathrm{wt} \%$ Graphene Nanoplatelets (GNP) in

Lexan HF1130-111 at various temperatures

Table M.1: Capillary Rheometer Results at $270^{\circ} \mathrm{C}$ 749

Table M.2: Capillary Rheometer Results at $300^{\circ} \mathrm{C}$ .750

Table M.3: Capillary Rheometer Results at $320^{\circ} \mathrm{C}$ .751

Table M.4: Capillary Rheometer Results at $270^{\circ} \mathrm{C}$ .752

Table M.5: Capillary Rheometer Results at $300^{\circ} \mathrm{C}$ .753

Table M.6: Capillary Rheometer Results at $270^{\circ} \mathrm{C}$ .754

Table M.7: Capillary Rheometer Results at $300^{\circ} \mathrm{C}$ .755

Table M.8: Capillary Rheometer Results at $270^{\circ} \mathrm{C}$ .756 


\section{Acknowledgements}

I would like to thank Dr. Julia King for being my Ph.D. advisor. She has been an invaluable asset in the pursuit of my degree. She has assisted in the procedural aspects of obtaining my degree as well as assisting in the experimental and theoretical work. Without her it would have been much more difficult for me to obtain this degree and it certainly would have taken much longer.

I would also like to thank my committee members Drs. Ibrahim Miskioglu, Tamara Olson, Warren Perger and Tony Rogers for taking the time out of their busy schedules to be on my committee.

My undergraduate research assistants have greatly assisted me over these past 4 years. There have been well over 2 dozen undergrads that have helped at various points in this project. I would particularly like to thank Jeffery Caspary, Daniel Alpers, Edsel Beach, Mark Cieslinski and Kyle Weise. I could always count on these 5 students to return for multiple semesters and perform tedious tasks or experiments without complaint and I cannot thank them enough for their time and effort.

Possibly most importantly, I would like to thank my family. Their support was critical to my entire education. They were always there to talk when things were tough and assist when it was needed.

Lastly, I would like to thank Beth Johnson, the previous graduate student working for Dr. King. She was a great mentor and has been very supportive and helpful ever since. 


\section{Nomenclature}

\begin{tabular}{|c|c|}
\hline A & Adjustable parameter in Kitano-modified Maron-Pierce model \\
\hline$a$ & Exponential parameter \\
\hline $\mathrm{a}_{\mathrm{T}}$ & Shift factor \\
\hline ASTM & American Society for Testing and Materials \\
\hline $\mathrm{c}$ & Volumetric heat capacity $\left(\mathrm{j} / \mathrm{m}^{3} \cdot \mathrm{K}\right)$ \\
\hline $\mathrm{CB}$ & Carbon black \\
\hline CNT & Carbon nanotubes \\
\hline $\mathrm{CNF}$ & Carbon nanofibers \\
\hline CVD & Chemical vapor deposition \\
\hline $\mathrm{CY}$ & Carreau-Yasuda model \\
\hline $\mathrm{d}$ & Diameter $(\mathrm{cm}$ or $\mathrm{nm})$ \\
\hline $\mathrm{D}$ & Diameter of capillary (mm) \\
\hline DSC & Differential scanning calorimetry \\
\hline $\mathrm{E}$ & Tensile modulus (MPa) \\
\hline E & Adjustable parameter for additive model \\
\hline $\mathrm{E}_{\mathrm{C}}$ & Composite tensile modulus (MPa) \\
\hline$E_{f}$ & Tensile modulus of filler (MPa) \\
\hline$E_{L}$ & Longitudinal composite tensile modulus (MPa) \\
\hline $\mathrm{E}_{\mathrm{m}}$ & Tensile modulus of matrix (MPa) \\
\hline $\mathrm{E}_{\mathrm{T}}$ & Transverse composite tensile modulus (MPa) \\
\hline $\mathrm{EC}$ & Electrical Conductivity (S/cm) \\
\hline ER & Electrical Resistivity (ohm-cm) \\
\hline ESEM & Environmental scanning electron microscopy \\
\hline $\mathrm{F}$ & Maximum filler volume fraction \\
\hline FESEM & Field-emission scanning electron microscopy \\
\hline G & Adjustable parameter for additive model \\
\hline$G^{\prime}(\omega)$ & Linear-viscoelastic elastic modulus $(\mathrm{Pa})$ \\
\hline $\mathrm{G}^{\prime}(\mathrm{T})$ & Linear-viscoelastic elastic modulus at the test temperature $(\mathrm{Pa})$ \\
\hline$G^{\prime \prime}(\omega)$ & Loss modulus $(\mathrm{Pa})$ \\
\hline$G^{\prime \prime}(\mathrm{T})$ & Loss modulus at the test temperature $(\mathrm{Pa})$ \\
\hline GEM & General effective media model \\
\hline GNP & Graphene nanoplatelets \\
\hline $\mathrm{H}$ & Adjustable parameter for additive model \\
\hline $\mathrm{i}$ & Electrical current (A) \\
\hline IPD & Electron hopping distance $(\mathrm{nm})$ \\
\hline K & Adjustable parameter for Mamunya model \\
\hline $\mathrm{k}$ & Thermal conductivity $(\mathrm{W} / \mathrm{m} \cdot \mathrm{K})$ \\
\hline $\mathrm{K}_{\mathrm{E}}$ & Einstein coefficient \\
\hline
\end{tabular}




\begin{tabular}{|c|c|}
\hline $\mathrm{L}$ & Length ( $\mathrm{cm}$ or $\mathrm{nm})$ \\
\hline $\mathrm{L}$ & Demagnification coefficient of dispersion \\
\hline $\mathrm{L} / \mathrm{d}$ & Aspect ratio of filler \\
\hline LVDT & Linear variable displacement transformer \\
\hline$m_{d r y}$ & Dry mass $(\mathrm{g})$ \\
\hline$m_{\text {wet }}$ & Submerged mass (g) \\
\hline MFI & Melt flow index \\
\hline MTU & Michigan Technological University \\
\hline$N$ & Proportionality constant \\
\hline$n$ & Power-law index \\
\hline$n$ & Adjustable parameter for Mamunya model \\
\hline$n$ & Adjustable parameter for additive model \\
\hline$\Delta \mathrm{P}$ & Pressure drop over the capillary (bar) \\
\hline $\mathrm{p}$ & Mean free path of phonons (m) \\
\hline $\mathrm{PC}$ & Polycarbonate \\
\hline PP & Polypropylene \\
\hline Q & Volumetric flow rate $\left(\mathrm{m}^{3} / \mathrm{s}\right)$ \\
\hline $\mathrm{q}$ & Heat flux $\left(\mathrm{W} / \mathrm{m}^{2}\right)$ \\
\hline $\mathrm{R}$ & Radius of the capillary (mm) \\
\hline$R_{0}$ & Sum of all contact thermal resistances $\left(\mathrm{m}^{2} \cdot \mathrm{K} / \mathrm{W}\right)$ \\
\hline$R_{S}$ & Specimen thermal resistance $\left(\mathrm{m}^{2} \cdot \mathrm{K} / \mathrm{W}\right)$ \\
\hline SAOS & Small-amplitude oscillatory shear \\
\hline $\mathrm{SiC}$ & Silicon carbide \\
\hline $\mathrm{T}$ & Temperature $(\mathrm{K})$ \\
\hline $\mathrm{t}$ & Thickness (cm or nm) \\
\hline$T_{g}$ & Glass-transition temperature $(\mathrm{K})$ \\
\hline$T_{L}$ & Lower surface temperature \\
\hline $\mathrm{T}_{\text {ref }}$ & Reference temperature $(\mathrm{K})$ \\
\hline$T_{U}$ & Upper surface temperature $(\mathrm{K})$ \\
\hline $\mathrm{TC}$ & Thermal conductivity $(\mathrm{W} / \mathrm{m} \cdot \mathrm{K})$ \\
\hline TEM & Transmission electron microscopy \\
\hline $\mathrm{u}$ & Velocity of phonons (m/s) \\
\hline UFS & Ultimate flexural strength (MPa) \\
\hline UTS & Ultimate tensile strength (MPa) \\
\hline$\Delta \mathrm{V}$ & Voltage drop over sample (V) \\
\hline$V_{f}$ & Volume fraction of filler \\
\hline vol\% & Volume percent of filler \\
\hline $\mathrm{w}$ & Sample width (cm) \\
\hline $\mathrm{w}_{\mathrm{CB}}$ & Weight percentage carbon black \\
\hline
\end{tabular}




\begin{tabular}{|c|c|}
\hline $\mathrm{w}_{\mathrm{CNT}}$ & Weight percentage carbon nanotubes \\
\hline $\mathrm{w}_{\mathrm{GNP}}$ & Weight percentage graphene nanoplatelets \\
\hline$w t \%$ & Weight percent of filler \\
\hline$\dot{\gamma}$ & Shear rate $(1 / \mathrm{s})$ \\
\hline$\dot{\gamma}_{a}$ & Apparent shear rate in the capillary $(1 / \mathrm{s})$ \\
\hline$\dot{\gamma}_{R}$ & True shear rate at the wall $(1 / \mathrm{s})$ \\
\hline$\frac{\partial T}{\partial x}$ & Temperature gradient in the $\mathrm{x}$-direction $(\mathrm{K} / \mathrm{m})$ \\
\hline$\varepsilon$ & Tensile strain $(\%)$ \\
\hline$\varepsilon$ & Total error \\
\hline$\eta$ & Steady-shear viscosity $(\mathrm{Pa} \cdot \mathrm{s})$ \\
\hline$\eta(T)$ & Viscosity at the test temperature $(\mathrm{Pa} \cdot \mathrm{s})$ \\
\hline$\eta^{*}(\omega)$ & Complex viscosity $(\mathrm{Pa} \cdot \mathrm{s})$ \\
\hline$\eta_{0}$ & Newtonian zero-shear viscosity $(\mathrm{Pa} \cdot \mathrm{s})$ \\
\hline$\left\langle\cos ^{2} \theta\right\rangle$ & Average of the squared cosine of orientation angle of filler \\
\hline$\lambda$ & Relaxation time (s) \\
\hline$\mu_{0}$ & Viscosity parameter \\
\hline$\rho$ & Specimen density $\left(\mathrm{g} / \mathrm{cm}^{3}\right)$ \\
\hline$\rho_{i}$ & Density of each individual component $\left(\mathrm{g} / \mathrm{cm}^{3}\right)$ \\
\hline$\rho_{\text {ref }}$ & Sample density at the reference temperature $\left(\mathrm{g} / \mathrm{cm}^{3}\right)$ \\
\hline$\rho_{\text {water }}$ & Density of test water $\left(\mathrm{g} / \mathrm{cm}^{3}\right)$ \\
\hline$\sigma$ & Tensile stress (MPa) \\
\hline$\sigma$ & Electical conductivity $(\mathrm{S} / \mathrm{cm})$ \\
\hline$\sigma_{\text {calc }}$ & Electrical conductivity predicted by model $(\mathrm{S} / \mathrm{cm})$ \\
\hline$\sigma_{\mathrm{f}}$ & Electrical conductivity of composite at $\mathrm{F}(\mathrm{S} / \mathrm{cm})$ \\
\hline$\sigma_{\mathrm{h}}$ & Electrical conductivity of conductive filler $(\mathrm{S} / \mathrm{cm})$ \\
\hline$\sigma_{\mathrm{m}}$ & Electrical conductivity of composite $(\mathrm{S} / \mathrm{cm})$ \\
\hline$\sigma_{\text {measured }}$ & Electrical conductivity measured from sample $(\mathrm{S} / \mathrm{cm})$ \\
\hline$\sigma_{\mathrm{p}}$ & Electrical conductivity of polymer $(\mathrm{S} / \mathrm{cm})$ \\
\hline$\tau_{R}$ & Shear stress at the wall $(\mathrm{Pa})$ \\
\hline$\tau_{0}$ & Yield stress $(\mathrm{Pa})$ \\
\hline$\tau_{21}$ & Shear stress $(\mathrm{Pa})$ \\
\hline$\phi$ & Filler volume fraction \\
\hline$\phi_{c}$ & Percolation threshold \\
\hline$\phi_{i}$ & Weight faction of each individual component \\
\hline
\end{tabular}


$\phi_{m} \quad$ Maximum packing fraction

$\omega \quad$ Shear rate $(\mathrm{rad} / \mathrm{s})$

xlvii 


\section{Abstract \\ "Investigation into the Enhancement of Polycarbonate with Conductive Nanomaterials"}

Polymers are typically electrically and thermally insulating materials. The electrical and thermal conductivities of polymers can be increased by the addition conductive fillers such as carbons. Once the polymer composites have been made electrically and thermally conductive, they can be used in applications where these conductivities are desired such as electromagnetic shielding and static dissipation.

In this project, three carbon nanomaterials are added to polycarbonate to enhance the electrical and thermal conductivity of the resulting composite. Hyperion Catalysis FIBRILs carbon nanotubes were added to a maximum loading of $8 \mathrm{wt} \%$. Ketjenblack EC-600 JD carbon black was added to a maximum loading of $10 \mathrm{wt} \%$. XG Sciences $\mathrm{xGnP} \mathrm{P}^{\mathrm{TM}}$ graphene nanoplatelets were added to a maximum loading of $15 \mathrm{wt} \%$. These three materials have drastically different morphologies and will have varying effects on the various properties of polycarbonate composites.

It was determined that carbon nanotubes have the largest effect on electrical conductivity with an $8 \mathrm{wt} \%$ carbon nanotube in polycarbonate composite having an electrical conductivity of $0.128 \mathrm{~S} / \mathrm{cm}$ (from a pure polycarbonate value of $10^{-17} \mathrm{~S} / \mathrm{cm}$ ). Carbon black has the next largest effect with an $8 \mathrm{wt} \%$ carbon black in polycarbonate composite having an electrical conductivity of $0.008 \mathrm{~S} / \mathrm{cm}$. Graphene nanoplatelets have the least effect with an $8 \mathrm{wt} \%$ graphene nanoplatelet in polycarbonate having an electrical conductivity of $2.53 \times 10^{-8} \mathrm{~S} / \mathrm{cm}$.

Graphene nanoplatelets show a significantly higher effect on increasing thermal conductivity than either carbon nanotubes or carbon black. Mechanically, all three materials have similar effects with graphene nanoplatelets being somewhat more effective at increasing the tensile modulus of the composite than the other fillers.

Carbon black and graphene nanoplatelets show standard carbon-filler rheology where the addition of filler increases the viscosity of the resulting composite. Carbon nanotubes, on the other hand, show an unexpected rheology. As carbon nanotubes are added to polycarbonate the viscosity of the composite is reduced below that of the original polycarbonate. It was seen that the addition of carbon nanotubes offsets the increased viscosity from a second filler, such as carbon black or graphene nanoplatelets. 


\section{Chapter 1: Introduction}

\section{1: Introduction}

Most polymers are electrical and thermal insulators. Traditionally applications that required some electrical or thermal conductivity were restricted to metals if both properties are required, or ceramics for thermal conductivity only. Increasing the electrical and thermal conductivity of polymers allows them to be used in these other applications. Thermoplastic polymers are resistant to corrosion, have high impact resistance and are lighter than metals (1). These properties have led to polymers being used to replace metal components in a variety of applications (2). Thermoplastic components can have properties tailored to the application by changing the type of polymer used as well as the additives that are added to the polymer.

It can be seen from Table 1.1 that the electrical conductivity of typical insulating polymers is dramatically lower than that of metals and carbon (upwards of 22 orders of magnitude). Table 1.2 shows some typical values for the thermal conductivity of polymer compared to metals, ceramics and carbon. Again polymers are seen to be much less conductive than metals. One of the ways to increase the conductivities of polymers is to add conductive fillers. Ceramics (i.e. aluminum nitride and boron nitride), metals (i.e. copper, steel and aluminum) and carbons (i.e. carbon black, carbon fibers, carbon nanotubes and graphite) are added to polymers to increase both thermal and electrical conductivity (1-16). When the electrical conductivity of the polymer composite has been increased to the $10^{-10}$ to $10^{-3} \mathrm{~S} / \mathrm{cm}$ range, they can be used in electrostatic dissipative applications such as electronic component handling trays and grounding straps for 
workers. Polymer composites that have electrical conductivities in the range of $10^{-2}$ or $10^{-1} \mathrm{~S} / \mathrm{cm}$ can be used for semiconductive applications such as fuel gages.

Table 1.1: Electrical conductivity of polymer, metals and carbon $(1,10,17)$

\begin{tabular}{|c|c|}
\hline Material & $\begin{array}{c}\text { Electrical Conductivity } \\
\text { (S/cm) }\end{array}$ \\
\hline Polymers & $10^{-7}$ to $10^{-17}$ \\
\hline Copper & $5.9 \times 10^{5}$ \\
\hline Silver & $6.3 \times 10^{5}$ \\
\hline Aluminum & $3.6 \times 10^{5}$ \\
\hline Nickel & $1.5 \times 10^{5}$ \\
\hline Carbon (amorphous) & 300 \\
\hline
\end{tabular}

Table 1.2: Thermal conductivity of polymer, metals, ceramics and carbon $(2,10,18)$

\begin{tabular}{|c|c|}
\hline Material & $\begin{array}{c}\text { Thermal Conductivity } \\
\text { (W/m.K) }\end{array}$ \\
\hline Polymer & 0.2 to 0.3 \\
\hline Copper & 400 \\
\hline Aluminum (extruded / cast) & $225 / 60$ \\
\hline Steel & 45 \\
\hline Aluminum Nitride & 140 to 180 \\
\hline Boron Nitride (cubic / hexagonal) & $1700 / 33$ \\
\hline Carbon (graphitic / amorphous) & $600 / 1.7$ \\
\hline
\end{tabular}

While the conductivities of the polymers are increased, it is also often important to increase the tensile modulus of the polymer to better match the properties of the materials they are replacing. Table 1.3 shows the tensile modulus of polycarbonate as well as metals, glasses and graphites that are typically used as reinforcements. The addition of these reinforcements to polymers helps to increase the modulus of polymers to levels that are required to replace metal components. 
Table 1.3: Tensile modulus of polycarbonate and common reinforcing materials $(19,20)$

\begin{tabular}{|c|c|}
\hline Material & Tensile Modulus (GPa) \\
\hline Polycarbonate & 2.2 \\
\hline Boron & 386 \\
\hline Steel & 407 \\
\hline E-glass & 72.4 \\
\hline S-glass & 85.5 \\
\hline $\begin{array}{c}\text { High-modulus graphite } \\
\text { fiber }\end{array}$ & 483 to 517 \\
\hline $\begin{array}{c}\text { High-strength graphite } \\
\text { fiber }\end{array}$ & 234 to 255 \\
\hline
\end{tabular}

\section{2: Motivation}

One emerging market for electrically conductive resins is aerospace components. With the introduction of the Boeing 787 Dreamliner $^{\mathrm{TM}}$ (a new highly efficient twin aisle commercial aircraft that will carry 230 passengers on routes of 8,000 nautical miles), new demands are being placed on the performance of polymer based composite materials (21). The 787 uses a significant amount (50\% by weight) of composites on its primary structure. Composite materials are chosen because of their increased fatigue life and resistance to corrosion, which reduces maintenance costs (21). Composites are also lighter weight versus metals, which increases aircraft fuel efficiency; and therefore, reduces the amount of greenhouse gases (primarily water and carbon dioxide) produced from burning fossil fuels. Fuel consumption is an important issue in today's society. Greenhouse gases could contribute to global climate change. With this increase in composite primary structure, there is an increase in the use of polymer materials for internal hardware and components such as brackets, shelves, ducts, interior support structure, and enclosures. Traditionally, these components would have used aluminum, but with the possibility of galvanic corrosion when aluminum is in contact with carbon fiber reinforced polymer composites, these components are now being designed with 
polymer composites, which raises a new issue. Aluminum is inherently electrically conductive and provides a level of electromagnetic interference (EMI) shielding and radio frequency interference (RFI) shielding that the polymer based composite does not provide. Therefore, it is necessary to use polymer compounds that have been modified to provide the necessary electrical conductivity. These conductive materials must also have moderate mechanical properties. The addition of conductive fillers such as carbon black and synthetic graphite particles to polymers often degrades tensile and impact strength. Hence, the challenge is to produce a cost effective composite that meets the electrical conductivity and mechanical property needs.

\section{3: Objectives}

The goals of this Ph.D. project are listed below:

- The electrical resistivity, thermal conductivity, as well as tensile, flexural and rheological properties of polycarbonate-based composites containing various amounts of different carbon-based conductive fillers will be determined.

- The synergistic effects of combinations of different carbon-based conductive fillers on the electrical resistivity, thermal conductivity and mechanical and rheological properties of polycarbonate-based composites will be determined.

- Electrical conductivity, tensile modulus, and rheological models will be applied to the composite systems tested herein and modified if required to suitably fit the behavior noted. 


\section{Chapter 2: Background}

\section{1: Electrical Conductivity}

Electrical conductivity of a composite describes the composite's ability to transmit electrons along its surface (surface electrical conductivity) or through its volume (volume electrical resistivity). Electrical conductivity is given in units of $\mathrm{S} / \mathrm{cm}$ and it the reciprocal of electrical resistivity (given in units of ohm-cm). Electrical resistance is the directly measureable property of the material that is then converted to electrical resistivity by multiplying by the cross-sectional area of the conducting volume and dividing by the length over which the measurement is being made. Electrical conductivity is then found by simply taking the reciprocal of this electrical resistivity.

When discussing electrical conductivity in relation to conductive-filler filled polymer composites, the composite can be in one of 3 stages based upon the type and amount of filler added as seen in Figure 2.1. At low filler concentrations, the electrical conductivity of the composite will be similar to that of the original polymer. Many polymers are highly electrically resistive with electrical conductivities on the order of $10^{-14}$ to $10^{-17}$ $\mathrm{S} / \mathrm{cm}$ (polycarbonate has a resistivity of about $10^{-17} \mathrm{~S} / \mathrm{cm}$ ). As more filler is added, the composite will reach a filler loading level at which the electrical conductivity rapidly increases by several orders of magnitude, known as the percolation threshold. After passing the percolation threshold, the electrical conductivity will flatten out and approach the electrical conductivity of the conductive filler as more filler is added. This percolation threshold is the point at which networks of conductive fillers begin to form 
creating continuous pathways for electricity to flow through. This is known as percolation theory $(22,23)$.

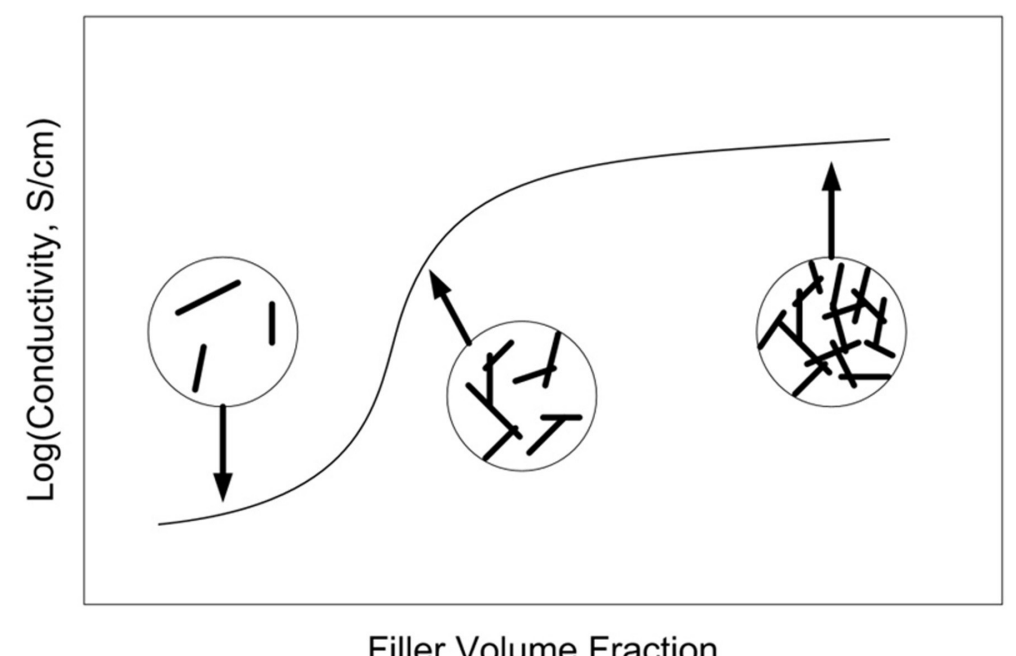

Figure 2.1: Effect of increasing filler loading on electrical conductivity

Percolation theory was introduced by Hammersley and Broadbent in 1957 (24). The theory was developed to describe how fluid flow would be affected by the random properties of the medium the fluid was flowing through. In this description, fluid and medium are used only in the broadest sense. The theory can describe liquid situations like solute flowing through a solvent, or it can describe other situations such as electrons flowing through the atomic lattice of a metal or, as suggested by Stauffer in 1985 (25), the spread of a fire through a forest. Hammersly and Broadbent suggested an example where water was flowing through a system of channels as illustrated in Figure 2.2. Each of the channels splits into two new channels. Each channel has a certain probability of being dammed. The flow of the water through the channels is then dependent upon the random set of dams. This system is known as bond percolation as each channel can be considered a bond through which the fluid flows. When enough of the channels are left 
not dammed (as illustrated in Figure 2.2) the system is percolated and water is able to flow (24). This system is analogous to electrical percolation in polymers. Conductive fillers, such as nanotubes, act as the channels that electrons can flow through. The polymer matrix acts as a dam through which electrons essentially cannot pass. If enough conductive filler is present, probability dictates that a continuous network will be formed across the sample and the electrons will be able to flow continuously from filler particle to filler particle drastically increasing the electrical conductivity of the sample.

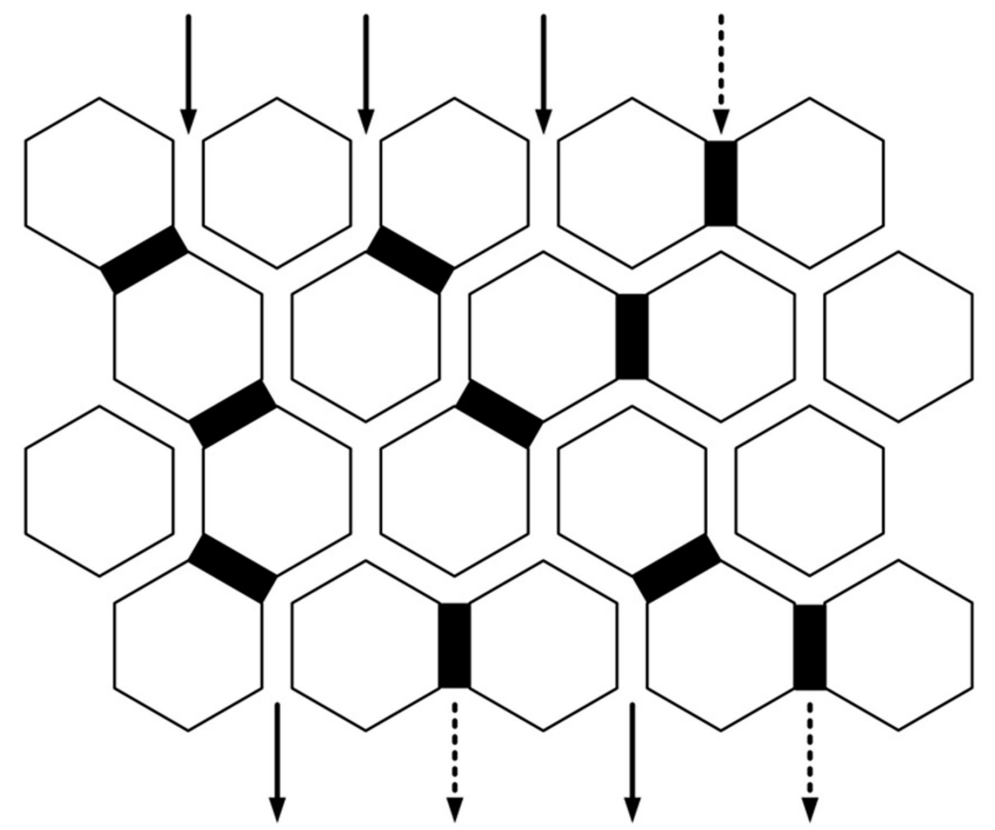

Figure 2.2: Bond percolation theory with channels

Conductive fillers that are added to polymers can vary greatly in properties. For example, multiwalled carbon nanotubes are hollow tubes that are nominally $10,000 \mathrm{~nm}$ long and $10 \mathrm{~nm}$ wide. Carbon black is a roughly spherical particle about $50 \mathrm{~nm}$ wide. Graphite nanoplatelets are flakes that are about $5000 \mathrm{~nm}$ wide and about $7 \mathrm{~nm}$ thick. These three fillers have very different geometries and will percolate differently. Fillers with high aspect ratios will align themselves with the direction of flow when melt 
processing. Samples made in this manner will exhibit increased electrical conductivity in the direction of the flow (26). Additionally, filler/matrix adhesion can have a significant effect on the percolation threshold. If there is poor bonding between the polymer matrix and the filler, the filler particles will not distribute evenly throughout the polymer instead forming clusters of filler (27).

\section{2: Thermal Conductivity}

Heat is the energy that is transferred between two bodies, or between regions of a single body, that are different temperatures. This energy can be transferred in three ways; convection, radiation and conduction. Convection is the transfer of heat caused by the flow of molecules in a fluid. Radiation is the transfer of heat as electromagnetic radiation (primarily through infrared radiation). Conduction is the primary form of heat transfer in solids, including polymer composites. Conduction in solids is described by Fourier's Law shown in Equation 2.1 below:

$$
q=-k \frac{\partial T}{\partial x}
$$

where $q$ is the heat flux (typically given in $\mathrm{W} / \mathrm{m}^{2}$ ), $k$ is the thermal conductivity (typically given in $\mathrm{W} / \mathrm{m} \cdot \mathrm{K})$ and $\frac{\partial T}{\partial x}$ is the temperature differential in the direction of heat transfer (typically in $\mathrm{K} / \mathrm{m}$ ) (28). It should be noted that heat always flows from areas of high temperature to areas of low temperature, thus the negative sign in Equation 2.1 (29). This heat is transferred through solids through phonons or, when available, free electrons. Free electrons are the primary energy carrier in metallic lattice solids. In non-metallic 
materials, including polymers, heat is transferred using phonons. Phonon transport theory characterizes collective waves of vibrational energy within a solid such as thermal energy. A phonon itself is described as a quanta of vibrational energy. These phonons transport themselves by interacting with the subatomic particles within the solid (30). This can be simply illustrated in two dimensional representation of a crystal lattice where atoms are shown as balls and springs represent the bonds between the atoms as shown in Figure 2.3. As can be seen in this representation, when a vibration, such as a thermal vibration, is applied to one side of the lattice, the heat will transmit through the springs across the lattice to the other side (31).

The efficiency of phonon transport is dependent upon the material through which it is moving. When irregularities in the lattice structure occur, the collective wave of vibrational energy is scattered in the directions of the irregular bonds. When the phonons are scattered, the heat transfer is impeded and thus the thermal conductivity of the material is lower than a material where the phonons are not scattered (30). The Debye model, below as Equation 2.2, describes how phonons transmit heat through a solid.

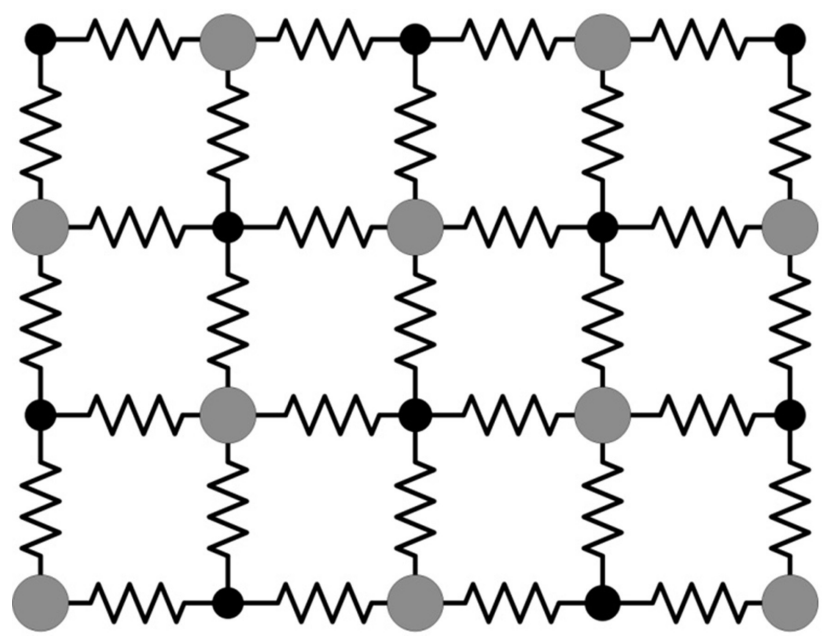

Figure 2.3: Two dimensional ball and spring model crystal lattice of sodium chloride 
$k=\frac{c \cdot u \cdot p}{3}$

In Equation 2.2, $k$ is thermal conductivity (typically in $\mathrm{W} / \mathrm{m}-\mathrm{K}$ ), $c$ is the volumetric heat capacity (typically in $\mathrm{J} / \mathrm{m}^{3} \cdot \mathrm{K}$ ), $u$ is the velocity of the phonons (approximately the speed

of sound through the material, typically in $\mathrm{m} / \mathrm{s}$ ), and $p$ is the mean free path (typically given in $\mathrm{m}$ ) or the average distance the vibrational wave can travel before scattering attenuates the wave intensity to $1 / \mathrm{e}$ (about 0.3679 ) of its original value (32). The heat conduction within polymers and carbon fillers, even ones with crystalline lattices with disassociated electrons like carbon nanotubes, is dominated by phonon transport $(32,33)$.

Thermal conductivity does not have a percolation threshold where it changes several orders of magnitudes with a small filler loading as electrical conductivity does (30). Previous work with carbon filled polymer systems has shown that thermal conductivity continually increases with increased filler loading as opposed to asymptotically tapering off at some value $(34,35)$. This observation indicates that thermal conductivity is not dependent upon the presence or absence of filler networks within the polymer $(4,36)$.

\section{3: Tensile Modulus}

Tensile modulus is a modulus of elasticity also known as Young's modulus. In general, modulus of elasticity is defined using Hooke's Law for an axially loaded member shown in Equation 2.3 below:

$$
E=\frac{\sigma}{\varepsilon}
$$


where $E$ is the tensile modulus, $\sigma$ is tensile stress, and $\varepsilon$ is tensile strain (20). This relationship allows the tensile modulus of a material to be found as the initial linear slope of a tensile stress vs. tensile strain curve. The initial linear portion of the curve must be used as Hooke's Law assumes Hookean behavior and by definition, any ductile material is non-Hookean and exhibits non-linear behavior as stress is applied (20). As seen in Figure 2.4 a generalized ductile material can exhibit up to 4 different behaviors as tensile stress is applied prior to failure.

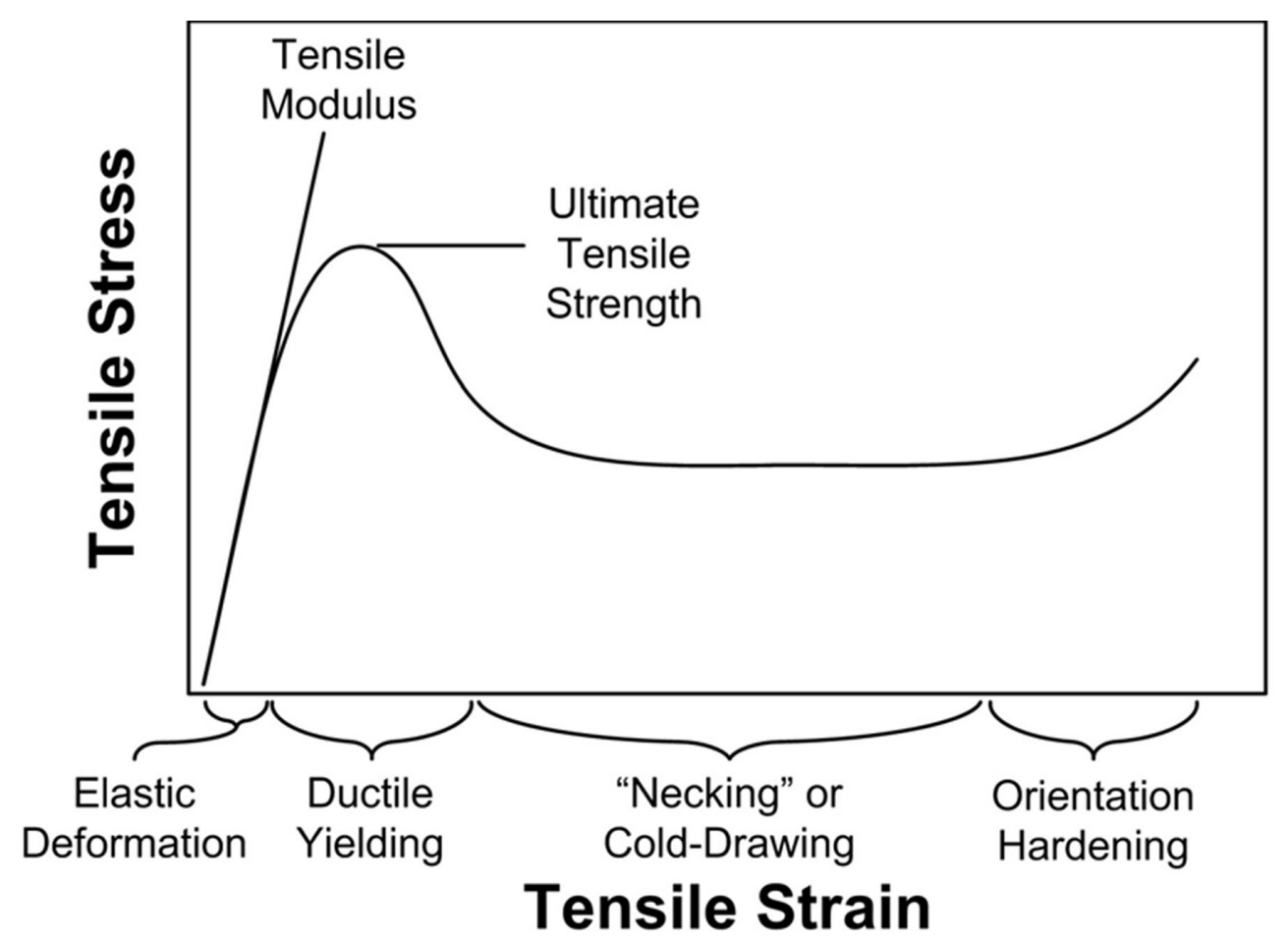

Figure 2.4: Generic ductile material tensile stress vs. tensile strain graph

Elastic deformation is the initial linear portion of the stress-strain curve where the material follows Hookean behavior and tensile modulus can be determined. Ductile yielding is the part following the initial linear portion of the stress strain curve. Ductile yielding is when plastic deformation begins to occur within the sample. Stress in the polymer will continue to increase to the ultimate tensile strength, then the composite will 
undergo strain softening where the stress will decrease as strain increases and "necking" forms (20). Following ductile yielding materials may begin "necking" or cold-drawing where they decrease in cross-sectional area while greatly expanding in the tensile direction. This necking behavior can propagate throughout the constant width area of the tensile bar and often appears as a line of constant stress over a large strain. After necking, it is possible that materials may orientation harden. Orientation hardening occurs when polymer chains within the "neck" become oriented in the tensile direction by the strain and then the individual bonds within the chains begin to be stretched by the strain. This often appears as a second increase in stress with increasing strain (20).

The tensile modulus of a filled polymer is dependent upon the properties of the polymer and the filler being added. The tensile modulus of the polymer itself (typically 0.5 to 4 GPa) acts as a baseline value. The carbon fillers then modify the tensile modulus from that point. Carbon fillers often have very high tensile moduli compared to the polymer. For example, carbon black has a tensile modulus of about $827 \mathrm{GPa}$, graphite and carbon nanotubes can have tensile moduli of over $1000 \mathrm{GPa}$ in the direction of their graphitic bonds, even in the c-axis, graphite has a tensile modulus of $37 \mathrm{GPa}(37,38)$.

By virtue of their high tensile moduli, carbon fillers will increase the tensile modulus of the resulting composite to a value between the original polymer tensile modulus and the filler tensile modulus. When tensile stress is applied to a composite, the stress begins to deform the composite according to Hooke's Law. When the polymer and higher tensile modulus fillers have good adhesion, the stress is transferred to those fillers. By having a higher tensile modulus, the fillers resist deformation more strongly than the polymer. In 
order for the composite to continue to deform, the fillers must deform thus bringing the higher tensile modulus of the filler to bear. As was mentioned, the level of adhesion between the polymer and the filler is another important factor. In order to transfer the tensile stress to the high modulus filler, the polymer must bond well to the filler surface. If poor adhesion is evident between the filler and the polymer, the filler will simply pull or separate from the polymer as the polymer is stretched away.

Filler orientation and aspect ratio are also important factors for tensile modulus. Increased filler orientation has been shown to increase the tensile modulus of the resultant composite $(39,40)$. Higher aspect ratios have also been shown to have a complex relationship with tensile modulus (as discussed in Chapter 11). Generally speaking, it has been shown that an increase in filler aspect ratio increases the tensile modulus of the resultant composite (41). A filler with a higher aspect ratio has the added benefit of increasing the orientation of the fillers in the direction of flow during sample creation. Many models of the tensile modulus, such as the Halpin-Tsai models, take the aspect ratio and orientation into account (37). 


\section{Chapter 3: Materials}

\section{1: Materials}

For this study, three different carbon fillers and one polymer matrix were used. The polymer matrix studied was SABIC Innovative Plastic Lexan HF1130-111 polycarbonate. The carbon fillers studied were Hyperion Catalysis International Fibril multi-walled carbon nanotubes, Ketjenblack EC-600JD carbon black, and XG Sciences graphene nanoplatelets. The following sections will discuss the materials in more detail.

\section{2: Matrix Material}

The polymer matrix used in this study was SABIC Innovative Plastics Lexan HF1130111 polycarbonate (PC). The chemical structure of polycarbonate is shown in Figure 3.1. Polycarbonate is a thermoplastic resin that can be re-melted and reshaped multiple times. Polycarbonate is primarily created through condensation polymerization of bisphenol-A and phosgene with a byproduct of hydrogen chloride or through condensation polymerization of the sodium salt of bisphenol-A and phosgene with sodium chloride as a byproduct (20). Polycarbonates are used in a variety of fields where durability or optical transparency is desired. Polycarbonate's very high impact strength and high optical transmittance along with high use temperature, tensile properties and flexural properties make it a very popular commercial and research plastic. It has been the focus of several other studies involving reinforcement with carbon fillers (42). This particular polycarbonate was chosen because it has high flow properties that are helpful for injection molding and allow for fillers to be added to the matrix. Table 3.1 shows properties of this material. 


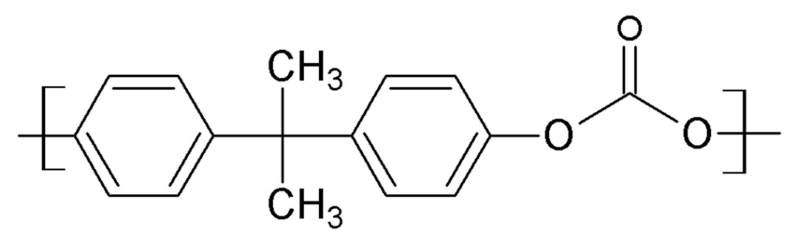

Figure 3.1: Lexan HF1130-111 Polycarbonate Structure

Table 3.1: Properties of Sabic's Polycarbonate Lexan HF 1130 (42).

\begin{tabular}{|l|l|}
\hline Melt Flow Rate $(300 \mathrm{C} / 1.2 \mathrm{~kg})$ & $25 \mathrm{~g} / 10 \mathrm{~min}$ \\
\hline Density & $1.2 \mathrm{~g} / \mathrm{cc}$ \\
\hline Electrical Resistivity & $1 \times 10^{17} \mathrm{ohm}-\mathrm{cm}$ \\
\hline Thermal Conductivity & $0.19 \mathrm{~W} / \mathrm{m} \cdot \mathrm{K}$ \\
\hline
\end{tabular}

\section{3: Fillers}

\subsection{1: Hyperion Catalysis International's FIBRIL Multiwalled Carbon Nanotubes}

Carbon nanotubes are hollow cylinders formed from sheets of graphene. They can be single walled, being formed from a single sheet of graphene, or multiwalled, having several sheets of graphene forming coaxial cylinders. For this project Hyperion Catalysis International FIBRIL multiwalled carbon nanotubes (CNT) was used. A diagram of these nanotubes is shown in Figure 3.2. These particular nanotubes are ideally 8-walled tubes wrapped around a hollow $5 \mathrm{~nm}$ core forming a tube $10 \mathrm{~nm}$ in diameter and 1000 microns in length creating a high aspect ratio of 1000 (43). This aspect ratio allows low loading of nanotubes to have significant effects on electrical conductivity as well as mechanical properties.

FIBRIL multiwalled nanotubes are produced using a chemical vapor deposition (CVD) process (43). The CVD process produces very high yields of carbon nanotubes, but the purity is lower than other methods (e.g. arc discharge or laser ablation). CVD has been used to produce carbon fibers since 1959. By passing a hydrocarbon vapor, such as 
benzene, over a catalyst at elevated temperatures (e.g. $1100^{\circ} \mathrm{C}$ ) fibers of carbon can be formed. By changing the vapor, catalyst type, catalyst particle size and temperature different fibers, including nanotubes, can be formed. Typical industrial production CVD methods occur in fluidized bed reactors. When the hydrocarbon vapor comes in contact with the catalyst, it is decomposed into carbon and hydrogen. The carbon adheres to the catalyst and further carbon attaches to this carbon forming the nanotubes. As the nanotubes grow, the catalyst is disintegrated and distributes with the carbon nanotube agglomerate that leaves the reactor as a product. Typical carbon nanotube agglomerates will have over $95 \%$ carbon content and high bulk density making material handling easier.

For this project, $\mathrm{CNT}$ was provided by Hyperion Catalysis International as a $15 \mathrm{wt} \%$ masterbatch in polycarbonate, MB6015-00. Table 3.2 shows the properties of these nanotubes.

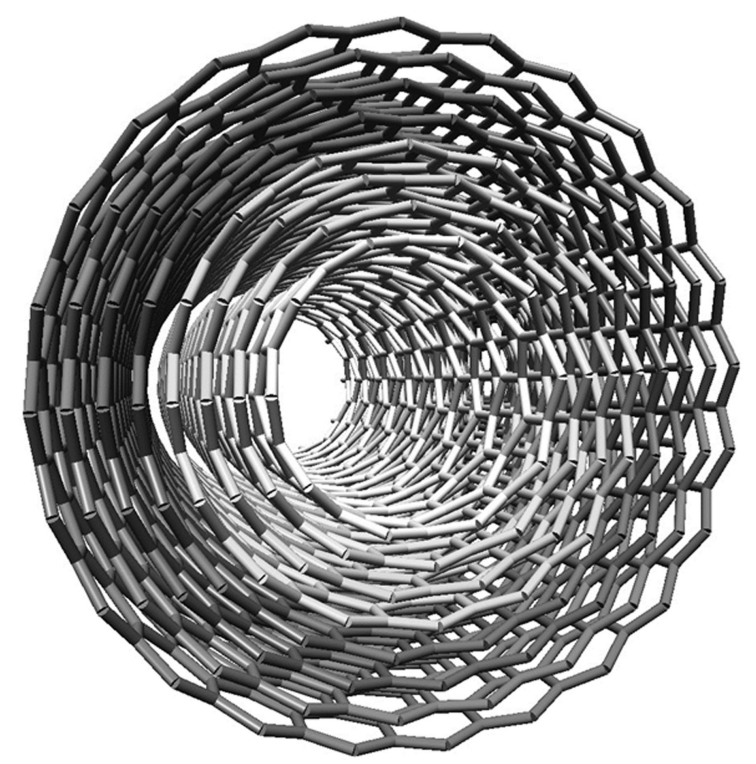

Figure 3.2: Diagram of carbon nanotube 
Table 3.2: Properties of FIBRIL ${ }^{\mathrm{TM}}$ Carbon Nanotubes (43).

\begin{tabular}{|l|l|}
\hline Composition & $\begin{array}{l}\text { carbon with trace residual of metal oxide } \\
\text { catalyst }\end{array}$ \\
\hline Diameter & $0.01 \mu \mathrm{m}$ \\
\hline Length & $10 \mu \mathrm{m}$ \\
\hline Morphology & 8 graphitic sheets wrapped around a hollow \\
& $0.005 \mu \mathrm{m}$ core \\
\hline Density & $2.0 \mathrm{~g} / \mathrm{cc}$ for the nanotube wall \\
& $1.75 \mathrm{~g} / \mathrm{cc}$ for the hollow nanotube \\
\hline
\end{tabular}

\subsection{2: Ketjenblack EC-600 JD Carbon Black}

Carbon black is a powdered form of amorphous carbon that is a created from the incomplete combustion of heavy hydrocarbons and as a side-product in thermal cracking (14). It is used as a UV stabilizer, pigment, and sometimes thermal and electrical property enhancer in plastics and rubbers, as well as a colorant in inks and printer toner.

The primary production method of carbon black is the furnace process. The furnace process begins by taking a feedstock, typically aromatic hydrocarbons, and preheats them to $200^{\circ} \mathrm{C}$ to $250^{\circ} \mathrm{C}$ (44). The feedstock is then injected into the hot-flame zone of a gasfired furnace (14). In the hot-flame zone, the feedstock is thermally decomposed into carbon and water vapor. Additional water is added to the furnace to quench the decomposition reaction. The reacted gas stream is then sent to filters where the carbon is separated from the unreacted feedstock and un-burnt furnace fuel. To increase the bulkdensity of the carbon to easily handled and shipped levels, the carbon is typically mixed in a pin machine or pelletized with additional water in pelletizing drums and then dried in a rotary kiln drier $(14,45)$. Carbon blacks can have different sizes and structures that are a function of the synthesis conditions of the carbon black. The type and concentration of 
the feedstock chemicals, reactor temperature, and the time spent in the hot-flame zone of the reactor can have a significant effect on the properties of the carbon black (44). Carbon black with small aggregate size, high structure (high surface area), and low concentrations of un-reacted feedstock are optimal for increasing the electrical conductivity of polymer composites (44).

Ketjenblack EC-600 JD carbon black (CB) is produced using a proprietary process and is designed to have high electrical conductivity when added to polymers, even in small amounts. This was the reason it was selected for this project. Ketjenblack EC-600 JD has a large specific surface area and highly-branched morphology that allows it to interact with a large amount of the polymer matrix, as seen in Figure 3.3. As received, Ketjenblack EC-600 JD is in the form of pellets ranging from 100 microns to 2 millimeters in diameter. When subjected to high shear forces, like those found in a twinscrew extruder, the pellets separate out into primary aggregates of 30 to 100 nanometers. Table 3.3 lists the properties of this carbon black.

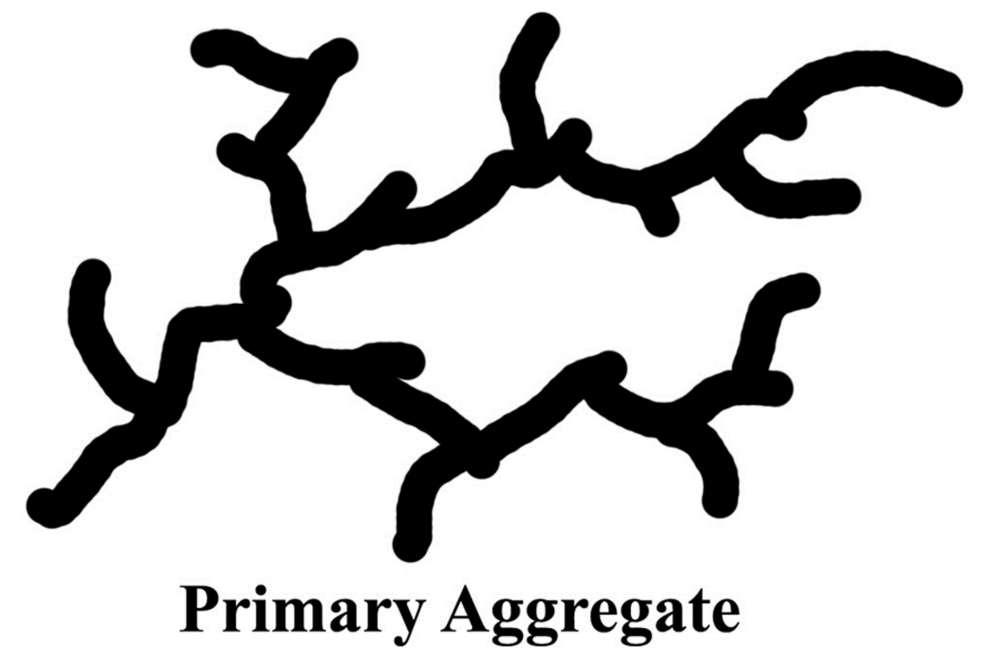

Figure 3.3: Diagram of Ketjenblack EC-600 JD carbon black structure 
Table 3.3: Properties of Akzo Nobel Ketjenblack EC-600 JD Carbon Black (46)

\begin{tabular}{|l|l|}
\hline Electrical Resistivity of Carbon Black & 0.01 to $0.1 \mathrm{ohm}-\mathrm{cm}$ \\
\hline Product Form & Pellet in size from 100 microns to a few mm \\
\hline Size of Carbon Black Aggregates & $30-100 \mathrm{~nm}$ \\
\hline Specific Gravity & $1.8 \mathrm{~g} / \mathrm{cc}$ \\
\hline Bulk Density & $100-120 \mathrm{~kg} / \mathrm{m}^{3}$ \\
\hline Ash, max & $0.1 \mathrm{wt} \%$ \\
\hline Moisture, max & $0.5 \mathrm{wt} \%$ \\
\hline BET $\left(\mathrm{N}_{2}\right)$ Surface Area & $1250 \mathrm{~m}^{2} / \mathrm{g}$ \\
\hline Pore Volume (DBP-absorption) & $480-510 \mathrm{~cm}^{3} / 100 \mathrm{~g}$ \\
\hline
\end{tabular}

\subsection{3: XG Sciences xGnPTM Graphene Nanoplatelets}

Graphite is made up of many layers of hexagonal carbon sheets. Individual hexagonal carbon sheets are known as graphene as seen in Figure 3.4. Several of these sheets $(\sim 20)$ stacked together form what is called a graphene nanoplatelet. Exfoliated graphene nanoplatelets are primarily produced through high-yield, low purity, exfoliation of graphite particles. Exfoliated graphene particles will have properties dependent upon their production method and the graphite from which they are produced. Other methods produce graphene without the graphite intermediary. Epitaxial growth on silicon carbide involves heating silicon carbide substrates above $1100^{\circ} \mathrm{C}$ reducing the silicon carbide to graphene. Graphene is also created through chemical vapor deposition on to metal substrates. Different metals used as substrates impart different electrical properties to the resulting graphene sheet.

Often graphite particles are intercalated using sulfuric acid. This intercalation process introduces guest molecules into the van der Waals gap between the individual sheets. The graphite is then exposed to a proprietary microwave treatment that rapidly raises the 
temperature of the graphite plates. This increase in temperature in the graphite causes the intercalated molecules to vaporize. The vaporization of the intercalated materials results in a separation of the individual sheets of graphite and expansion of around 500 times of the original graphite particle. The resultant particle is an accordion shaped expanded structure that can be broken down into individual nanoplatelets using ultrasonication. Following the ultrasonication the nanoplatelets are approximately 15 microns in diameter and nominally 5 nanometers in thickness. Further processing in a vibratory mill reduces the diameter of the nanoplatelets to 5 microns and maintains the nominal thickness (47). The graphene nanoparticles used in this project were made by XG Sciences. These particles are compounded into a 15 weight percent masterbatch with polycarbonate by Ovation Polymers Inc. as Extima GP MB PC 1515A which was then used for this project (48).

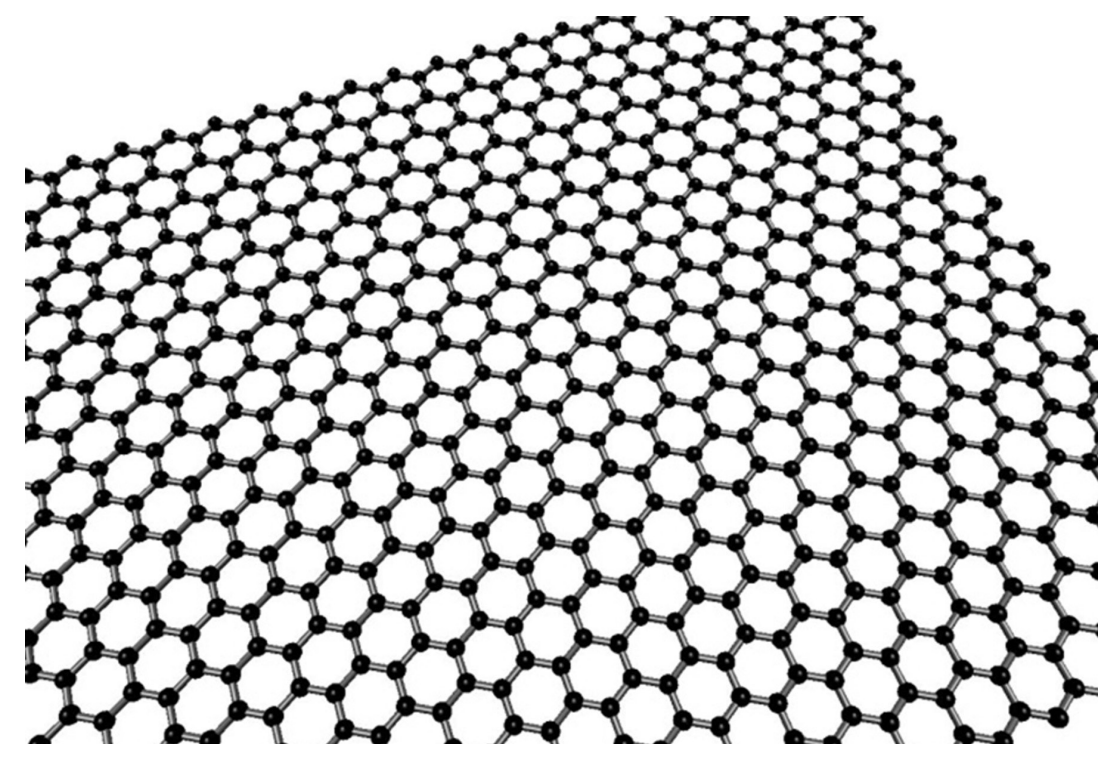

Figure 3.4: Diagram of graphene structure. 


\section{4: Formulation Naming Convention}

In an effort to make labeling and subsequent identification of samples simple and efficient, each sample will have a unique name and number. Many of the samples in this project will look similar, thus it is important to have a consistent and meaningful naming system. The labels will be applied to the samples immediately following injection molding and will describe the filler or fillers present in the sample, and to what weight percent they are present. The naming system will be as follows:

B w x L y $-\mathrm{z}-\#$

Where:

$\mathrm{B}=$ project description (Boeing project)

$\mathrm{W}=$ filler type $(\mathrm{A}=$ carbon black, $\mathrm{G}=$ graphene, $\mathrm{Q}=$ carbon nanotubes $)$

$\mathrm{X}=$ weight percent of conductive filler in composite

$\mathrm{L}=$ polymer matrix $(\mathrm{L}=\mathrm{Lexan} \mathrm{HF} 1130-111$ polycarbonate $)$

$\mathrm{y}=$ replicate number (none for original, $\mathrm{R}$ for first replicate)

$\mathrm{z}=$ specimen type $(\mathrm{F}=$ flex bar, $\mathrm{T}=$ tensile bar, $\mathrm{TC}=$ thermal conductivity disks (examples can be seen in Figure 3.5)

\# = specimen number (same as order it was injection molded)

An example of the naming system would be BQ4L-T-15. This would represent the $15^{\text {th }}$ injection molded tensile bar containing 4 weight percent carbon nanotubes in polycarbonate. When samples contain multiple fillers a second filler type and weight percent pair will follow the first pair. For example, BA2G5L-F-5 would represent the $5^{\text {th }}$ injection molded flex bar containing 2 weight percent carbon black, and 5 weight percent graphite nanoplatelets in polycarbonate. 


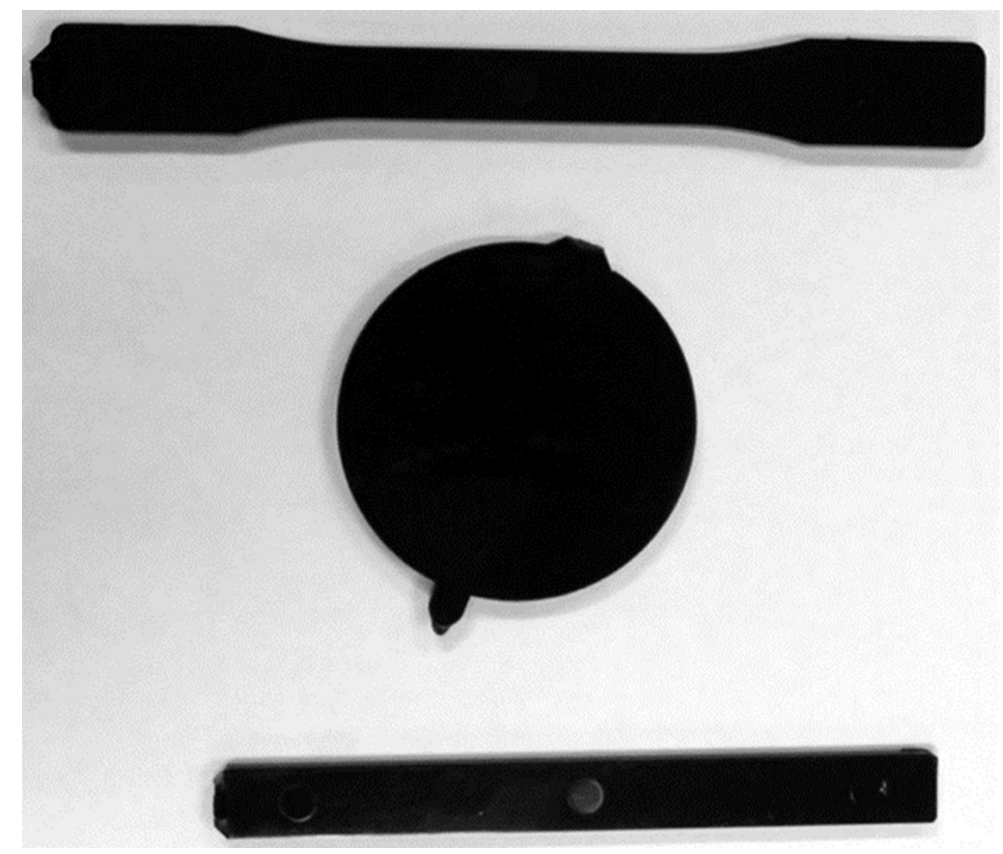

Figure 3.5: (Top to bottom) ASTM Type I Tensile Bar (T), 2.5" diameter thermal conductivity disk (TC), ASTM D790 Flex Bar (F) 


\section{Chapter 4: Fabrication and Experimental Methods}

\section{1: Fabrication Methods}

This section will describe the details of fabrication for the test specimens. Specific information on the screw designs, extruding conditions, and the injection molding conditions can be found in the Appendices.

\subsection{1: Drying}

Prior to extrusion the Lexan HF1130-111, Hyperion Catalysis MB6015-00 CNT masterbatch, and Ovation Extima GP MB PC 1515B GNP masterbatch composites were dried in a Bry Air RD-20 indirect-heated dehumidifying drying oven at $121^{\circ} \mathrm{C}$ for 12 hours as seen in Figure 4.1. Following drying, the materials are stored in moisture barrier bags until extrusion. Following extrusion and pelletization, the $\mathrm{PC}$ and filled $\mathrm{PC}$ composites are dried in the Bry Air RD-20 indirect-heated dehumidifying drying oven at $121^{\circ} \mathrm{C}$ for 12 hours. Following drying, the materials are again stored in moisture barrier bags until injection molding.

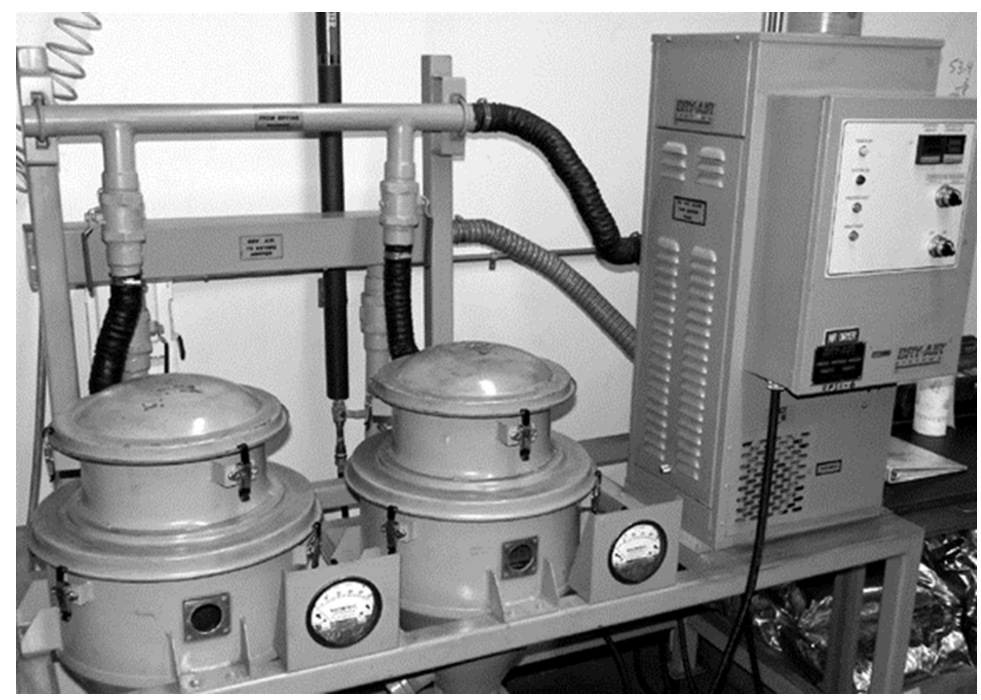

Figure 4.1: Bry Air RD-20 Dryer 


\subsection{2: Extrusion}

An American Leistritz Extruder Corporation Model ZSE 27 twin screw co-rotating extruder was used for this project and is shown in Figure 4.2. The extruder has a $27 \mathrm{~mm}$ co-rotating intermeshing twin screw, 10 independent heating zones, two side stuffers and a length to diameter ratio of 40 . The screw designs, shown in Appendix A, were selected to minimize filler degradation while providing good dispersion of the fillers throughout the polymer.

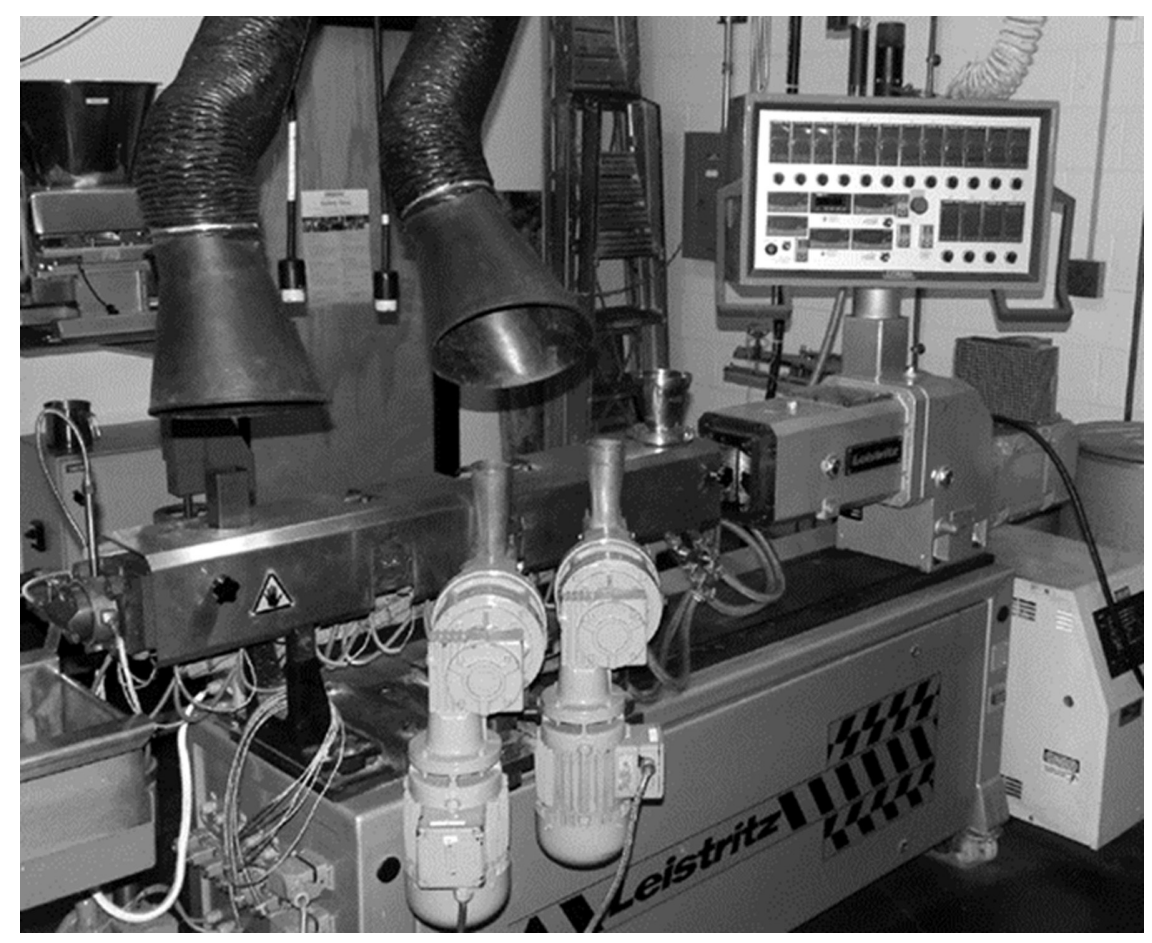

Figure 4.2: American Leistritz Extruder Corporation Model ZSE 27 twin screw extruder

The dried, pure polycarbonate pellets, dried CNT masterbatch and dried GNP masterbatch were introduced as received in the water-cooled Zone 1. For single filler systems, as received CB was introduced as received in Zone 5 by use of a side stuffer seen in Figure 4.2 and emphasized in Figure 4.3. Both Zones 1 and 5 were fed using 
Schenck AccuRate Flexwall gravimetric feeders seen in Figures 4.4 and 4.5. Detailed parameters for the extrusion conditions for all formulations is given in Appendix A.

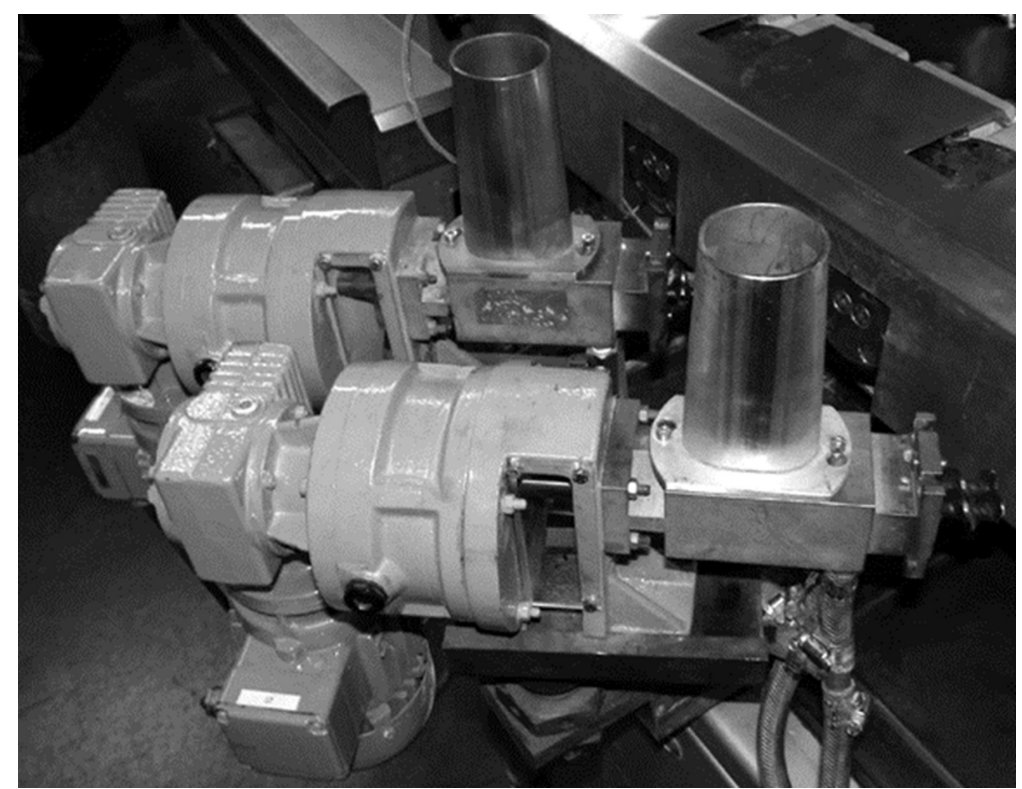

Figure 4.3: Side Stuffers seen disengaged from extruder

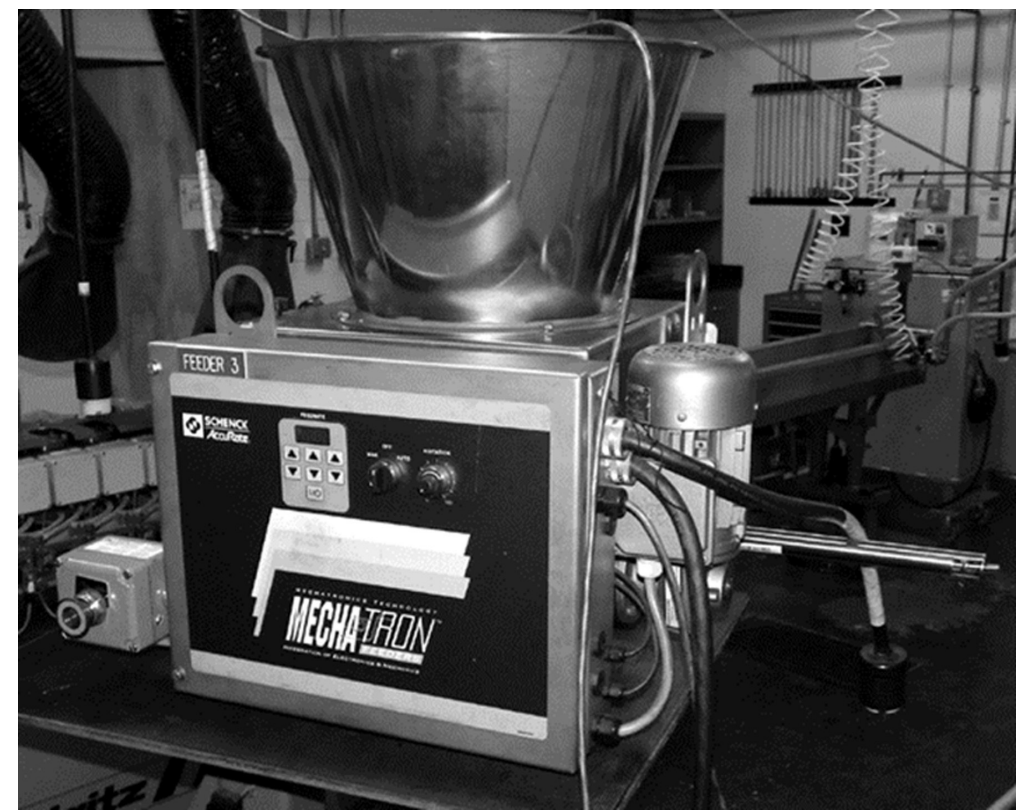

Figure 4.4: Zone 1 Feeder (Feeder 3) used to feed PC and Masterbatch pellets 


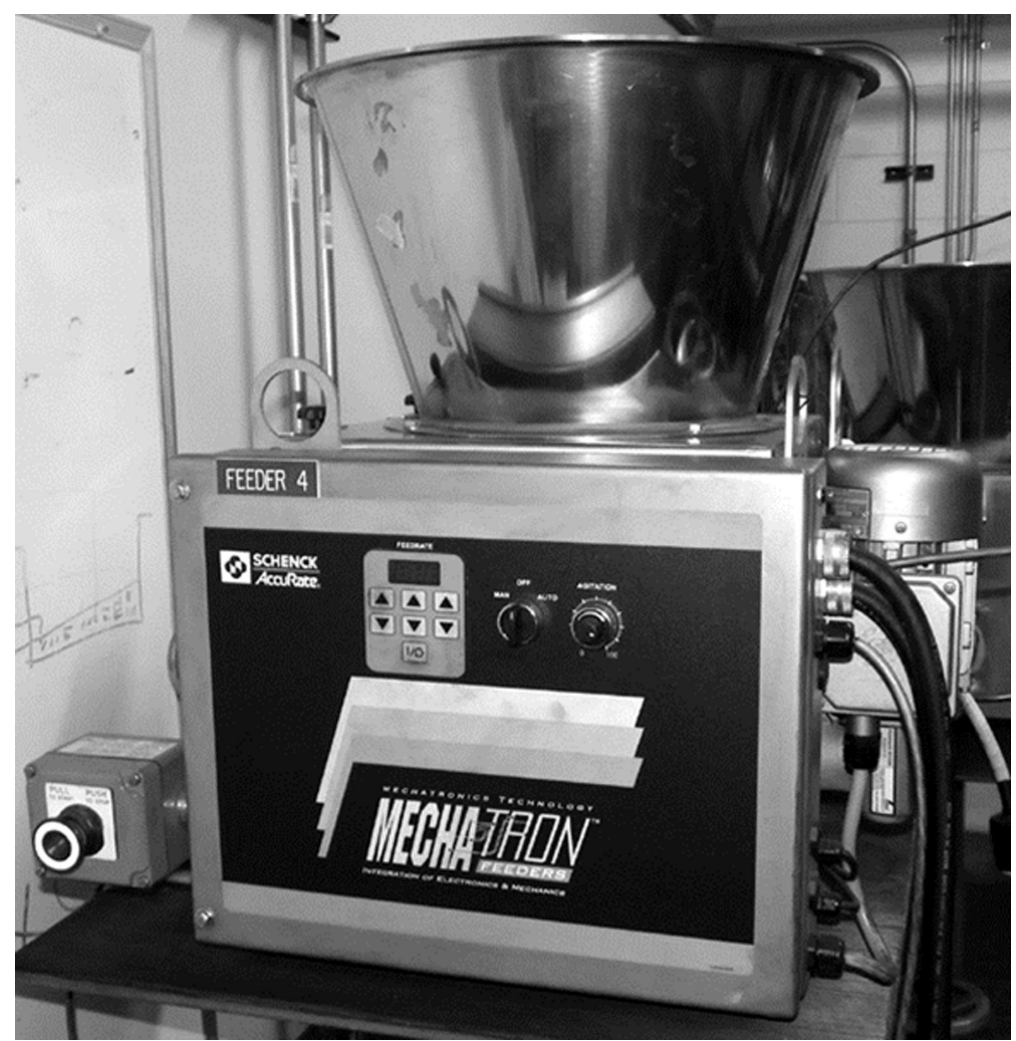

Figure 4.5: Zone 5 Feeder (Feeder 4) used to add CB to extruder (shown without feed pipe)

After being extruded the $3 \mathrm{~mm}$ polymer strands are cooled in a water bath, seen in Figure 4.6, then passed to a Conair Model 20402HP-14A pelletizer (Figure 4.7) that produces 3 mm long pellets. 


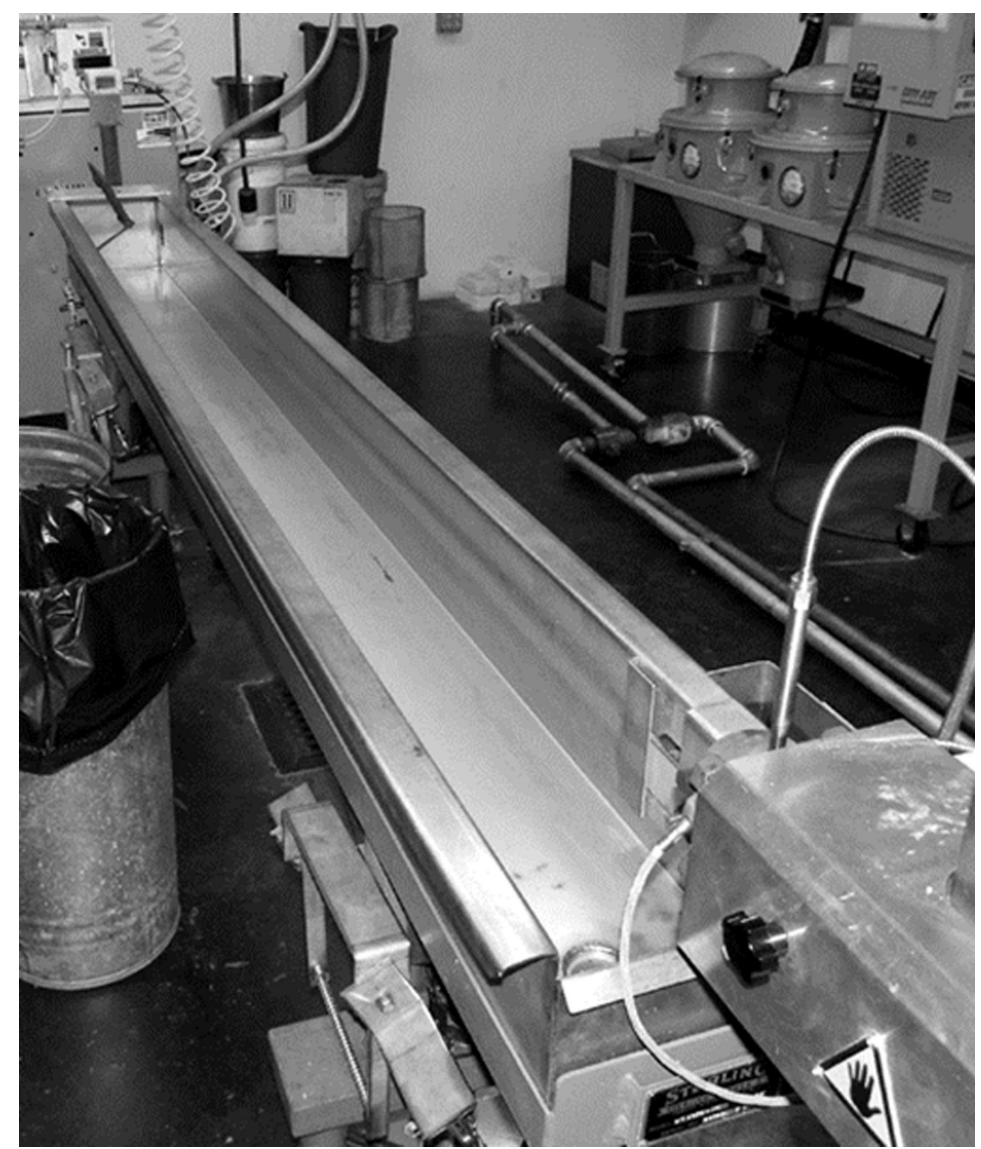

Figure 4.6: Water bath (here shown empty)

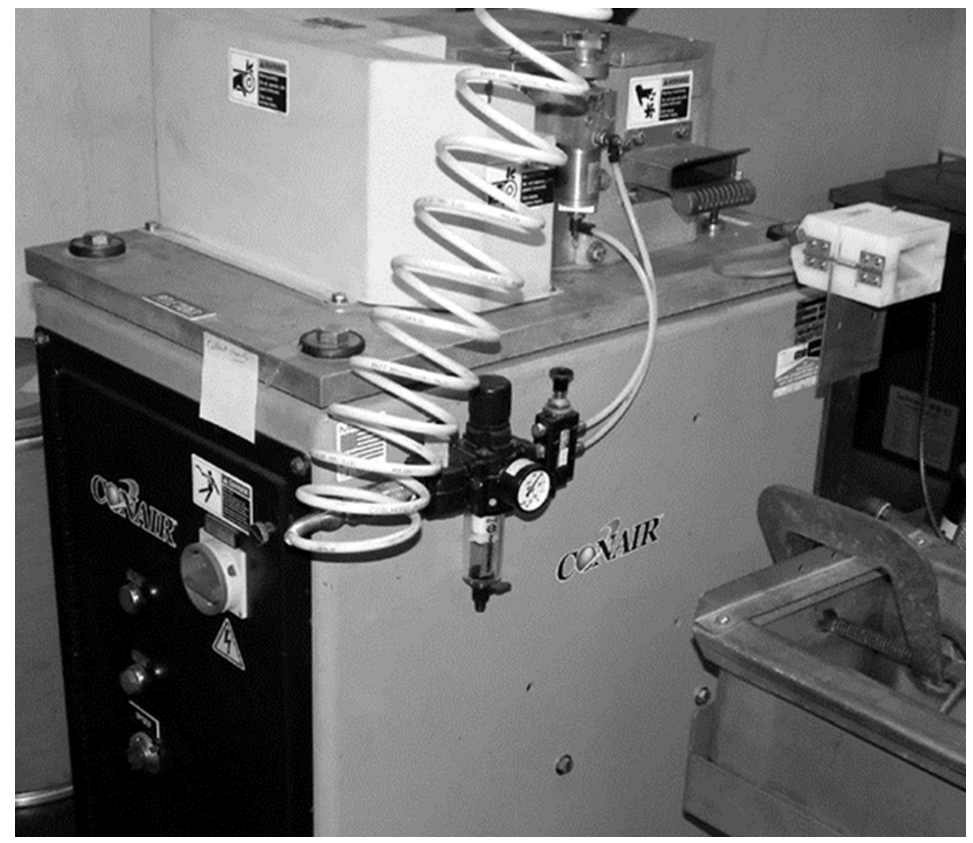

Figure 4.7: Conair Pelletizer and pre-pelletizer air-dryer 
For the multiple filler systems containing $\mathrm{CB}$, a masterbatch of $8 \mathrm{wt} \% \mathrm{CB}$ in $\mathrm{PC}$ was created using the extruder. This masterbatch was then cooled in the water bath and pelletized. After drying, this masterbatch was introduced in Zone 1 along with the pure PC and other fillers.

\subsection{3: Injection Molding}

A Niigata model NE85UA 4 injection molding machine (shown in Figure 4.8) was used to produce test specimens. The injection molder has a $40 \mathrm{~mm}$ single screw with a length to diameter ratio of 18. The specifications of the machine are shown in Table 4.1. A fourcavity mold (Figure 4.9) was used to produce the test specimens. Detailed parameters for injection molding of all formulations are given in Appendix B.

Table 4.1: Niigata model NE85UA 4 injection molding machine specifications

\begin{tabular}{|c|c|c|}
\hline \multicolumn{3}{|c|}{${\mathrm{NE} 85 \mathrm{UA}_{4} \text { Specifications }}$} \\
\hline \multicolumn{2}{|c|}{ Maximum Injection Pressure } & $22,610 \mathrm{psi}$ \\
\hline \multicolumn{2}{|c|}{ Maximum Screw Speed } & $320 \mathrm{rpm}$ \\
\hline \multicolumn{2}{|c|}{ Maximum Clamping Force } & 82.50 US tons \\
\hline \multirow{3}{*}{$\begin{array}{l}\text { Length of } \\
\text { Screw } \\
\text { Section }\end{array}$} & Feed & $396 \mathrm{~mm}$ \\
\hline & Compression & $180 \mathrm{~mm}$ \\
\hline & Metering & $144 \mathrm{~mm}$ \\
\hline
\end{tabular}




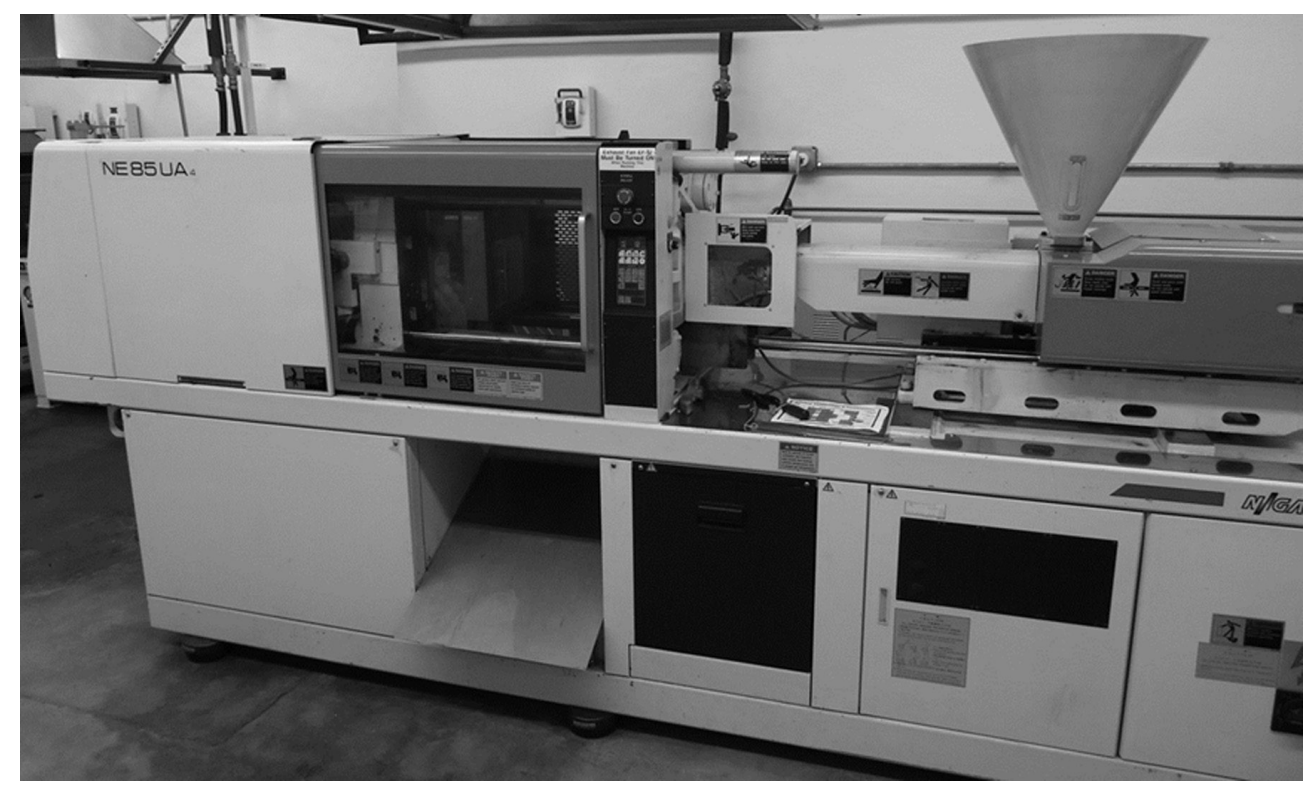

Figure 4.8: Niigata model $\mathrm{NE} 8 \mathrm{UA}_{4}$ injection molding machine

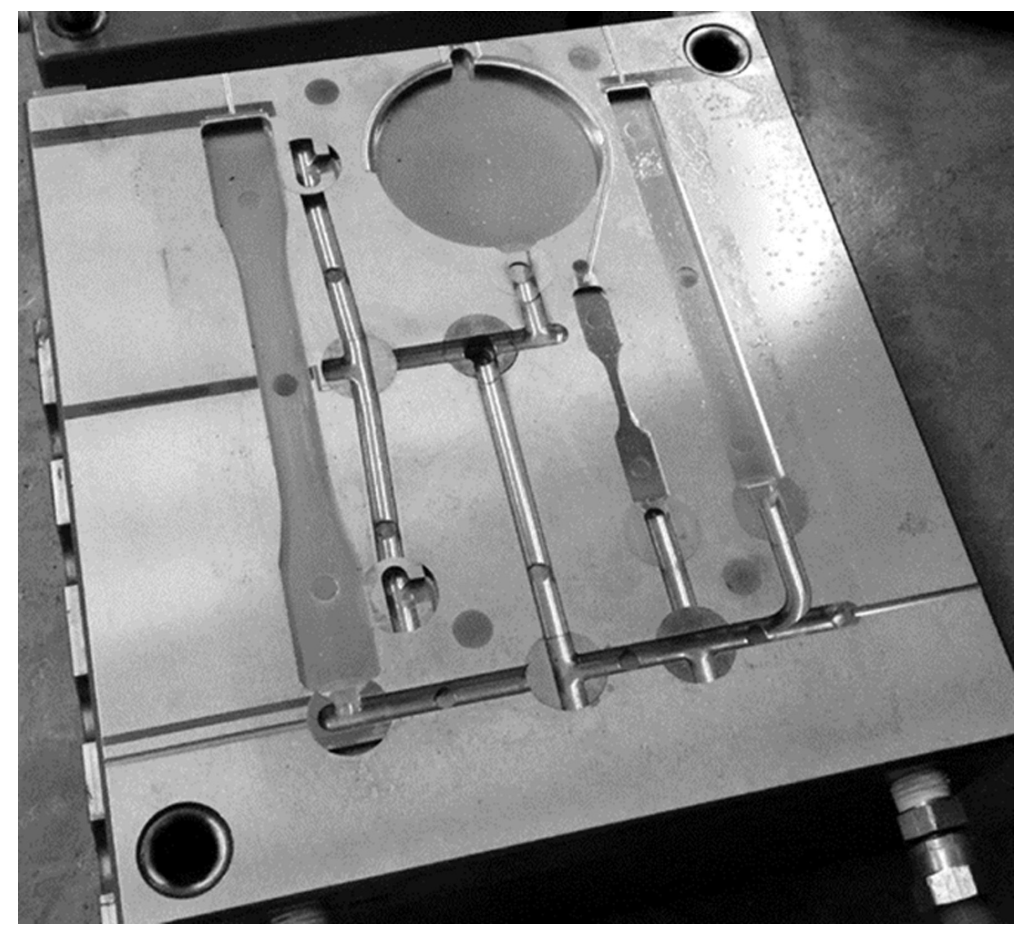

Figure 4.9: 4-cavity mold showing (left to right) ASTM type I Tensile, 2.5" disk, ASTM type V Tensile (not used) and ASTM D790 Flex Bar 


\section{2: Experimental Test Methods}

This section will describe the details of test methods for the test specimens. Results of these tests will be discussed in Chapters 5-12 and detailed results are available in the appendices.

\subsection{1: Density: ASTM D792-98 Test Method}

The density of the composite materials was found using the ASTM D792-98 test method, Specific Gravity and Density of Plastics by Displacement (49). For this test, $6.4 \mathrm{~cm}$ diameter injection molded disks are used. The dry mass of the samples is measured first. The samples are then submerged in water that has been allowed to sit overnight exposed to the atmosphere to equilibrate dissolved gases and temperature and the mass is measured in this state. Composite density can then be calculated using Equation 4.1,

$$
\rho=\frac{m_{d r y}}{m_{d r y}+m_{\text {wet }}} \cdot \rho_{\text {water }}
$$

Where $\rho$ is the specimen density, $m_{d r y}$ is the dry mass, $m_{w e t}$ is the submerged mass and $\rho_{\text {water }}$ is the density of the test water. The density can then be compared to a theoretical density for the composite given by Equation 4.2,

$$
\rho_{\text {theo }}=\frac{1}{\sum_{i} \frac{\phi_{i}}{\rho_{i}}}
$$

Where $\rho_{\text {theo }}$ is the theoretical specimen density, $\phi_{i}$ is the weight faction of each individual component and $\rho_{i}$ is the density of the individual component. 


\subsection{2: Melt-Flow Indexing (MFI) Test Method}

The Melt Flow Index (MFI) was determined using a Tinius Olsen Plastometer Melt Indexer (MP 987/WL 987) shown in Figure 4.10. For the as received Lexan HF1130-111 and the extruded Lexan HF1130-111 a weight of $1.25 \mathrm{~kg}$, temperature of $300^{\circ} \mathrm{C}$, and a dwell time of $360 \mathrm{~s}$, and a collection time of $15 \mathrm{~s}$ was used. For the filled composite samples the test was run at $320^{\circ} \mathrm{C}$ with a $5 \mathrm{~kg}$ weight and a dwell time of $360 \mathrm{~s}$. A higher temperature and weight was needed to allow the extrudate to exit the apparatus with the filled polymer as compared to the neat polymer. The amount of time needed to empty the barrel was measured since the test happened very 'quickly'. A test could not be conducted on the pure Lexan as the material emptied the barrel too quickly to be reliably timed. The $8 \mathrm{wt} \%$ carbon nanotubes in Lexan, $8 \mathrm{wt} \%$ carbon black in Lexan and $10 \mathrm{wt} \%$ carbon black in Lexan would not exit the barrel and were also not tested. 


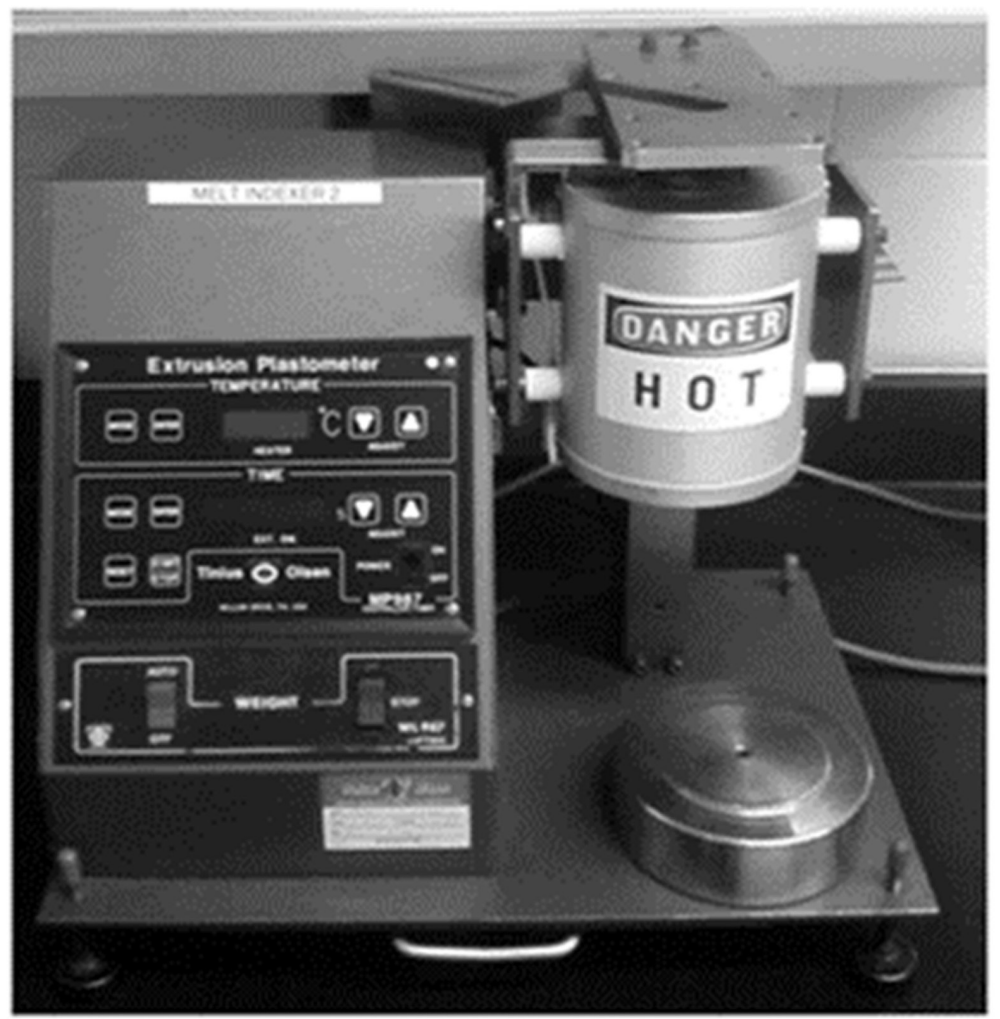

Figure 4.10: Olsen Plastometer MP 987/WL 987 Melt Indexer

\subsection{3: Differential Scanning Calorimetry (DSC) Test Method}

A Metter Toledo 823E DSC (shown in Figure 4.11) was used to measure the glass transition temperature $\left(\mathrm{T}_{\mathrm{g}}\right)$ of every composite. Approximately $10 \mathrm{mg}$ of each extruded pellet was placed in a sealed aluminum pan with a 'vent hole' in the lid. Nitrogen purge gas was used. The following heating cycle was used and $T_{g}$ was determined on the second heating cycle. $\mathrm{T}_{\mathrm{g}}$ was determined by a change in heat flow (i.e. heat capacity). The heating method is shown below. 
1) Heat $40^{\circ} \mathrm{C}$ to $350^{\circ} \mathrm{C}$ at $10^{\circ} \mathrm{C} / \mathrm{min}$

2) $\mathrm{Cool} 350^{\circ} \mathrm{C}$ to $40^{\circ} \mathrm{C}$ at $10^{\circ} \mathrm{C} / \mathrm{min}$

3) Hold $40^{\circ} \mathrm{C}$ and hold for $5 \mathrm{~min}$

4) Heat $40^{\circ} \mathrm{C}$ to $350^{\circ} \mathrm{C}$ at $10^{\circ} \mathrm{C} / \mathrm{min}$

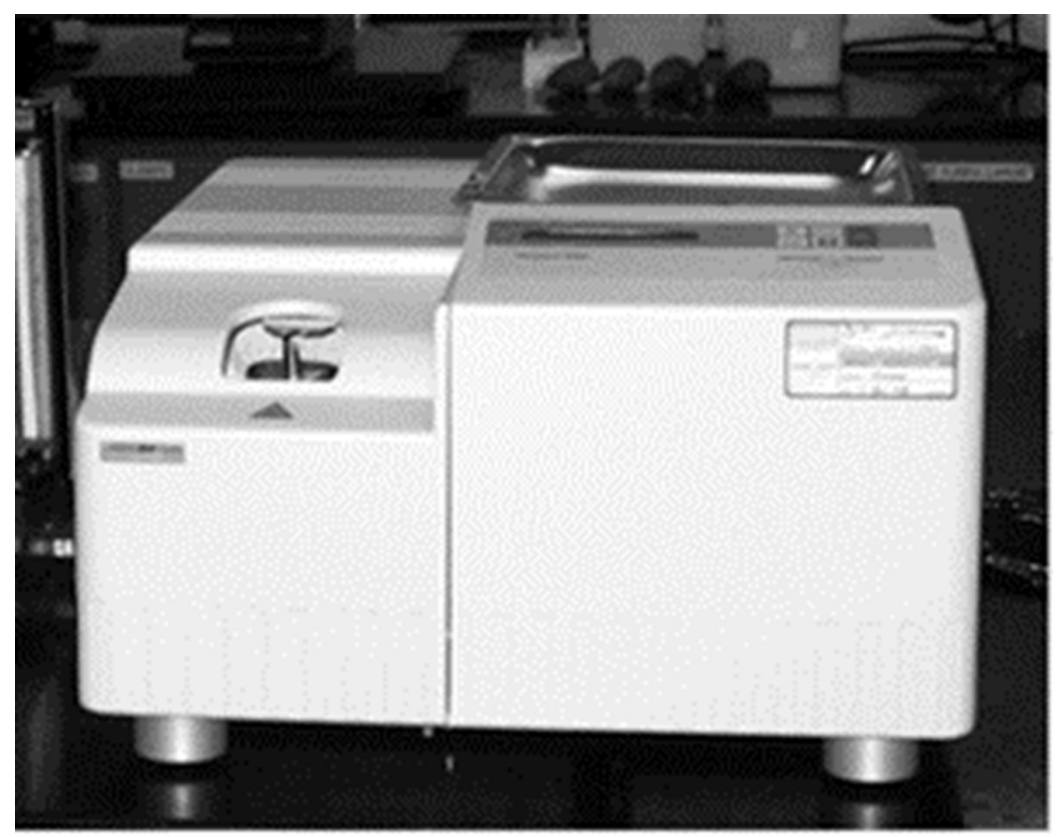

Figure 4.11: Metter Toledo 823E DSC

\subsection{4: Capillary Rheology Test Method}

Capillary rheology testing is performed according to ASTM D3835-96 using a Goettfert Rheo-Tester 1000 capillary rheometer seen in Figure 4.12 (50). The capillary rheometer is heated to a test temperature of $270^{\circ} \mathrm{C}, 300^{\circ} \mathrm{C}$ or $320^{\circ} \mathrm{C}$. A $1 \mathrm{~mm}$ diameter, $30 \mathrm{~mm}$ long capillary die was used for this project. A 200 bar pressure transducer was used for low viscosity formulations. A 1400 bar pressure transducer was used for high viscosity formulations. Figure 4.13 shows the various components of the rheometer. At least 4 
measurements were taken at 50,100, 200, 500, 1000 and 2000 1/s shear rates. Prior to testing samples were dried in a vacuum oven at $30^{\prime} \mathrm{Hg}$ vacuum and $100^{\circ} \mathrm{C}$ for 6 hours. $\mathrm{CNT} / \mathrm{PC}$ composites were tested at $270^{\circ} \mathrm{C}, 300^{\circ} \mathrm{C}$ and $320^{\circ} \mathrm{C} .320^{\circ} \mathrm{C}$ began to degrade the pure $\mathrm{PC}$ so $\mathrm{CB} / \mathrm{PC}$ samples were tested at $270^{\circ} \mathrm{C}$ and $300^{\circ} \mathrm{C}$. The $300^{\circ} \mathrm{C}$ data was found to be unnecessary thus GNP/PC samples and multiple filler samples were only tested at $270^{\circ} \mathrm{C}$.

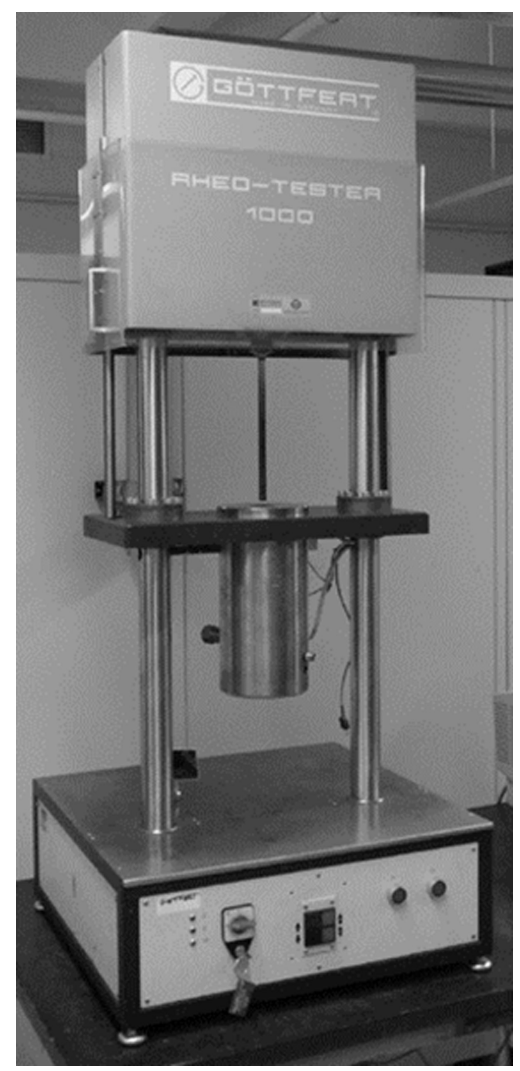

Figure 4.12: Goettfert Rheo-Tester 1000 capillary rheometer 


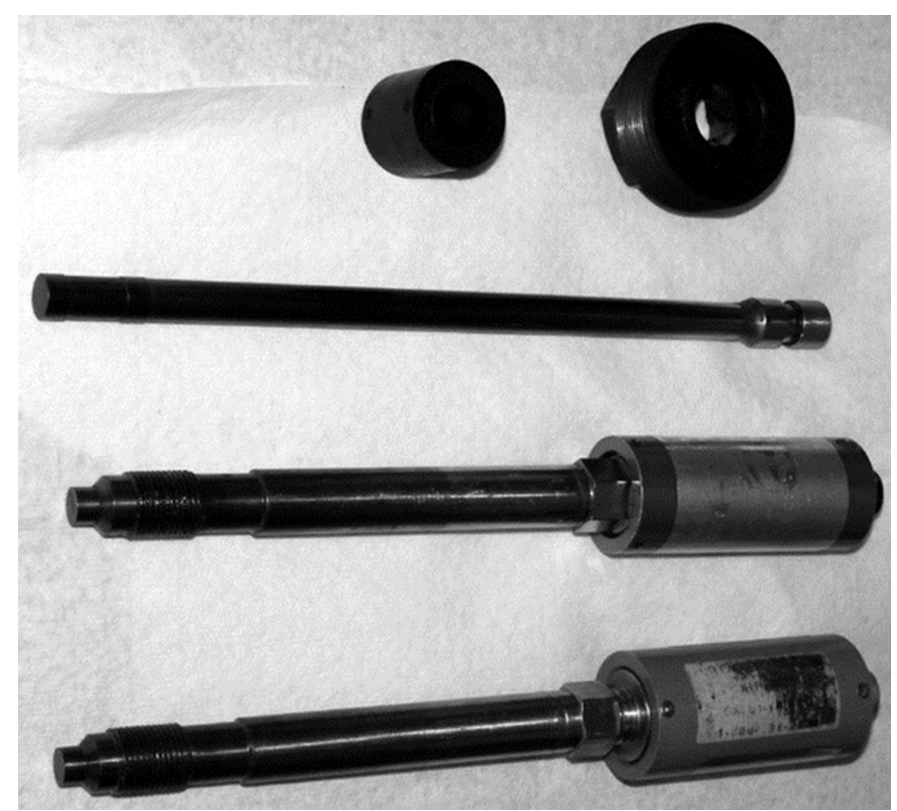

Figure 4.13: (Top to bottom) 30/1 capillary die and die nut, drive piston, 1400 bar pressure transducer, 200 bar pressure transducer

The apparent shear rate in the capillary, $\dot{\gamma}_{a}$, was calculated Equation 4.3 below

$\dot{\gamma}_{a}=\frac{4 Q}{\pi R^{3}}$

where $\mathrm{Q}$ is the volumetric flow rate, and $\mathrm{R}$ is the radius of the capillary (51). Shear stress at the wall, $\tau_{\mathrm{R}}$, in a capillary is given by:

$\tau_{R}=\frac{R \Delta P}{2 L}$

where $\Delta \mathrm{P}$ is the pressure drop over the capillary, $\mathrm{R}$ is the radius of the capillary and $\mathrm{L}$ is the length of the capillary. The relationship between the apparent shear rate and the true shear rate at the wall for any fluid, $\dot{\gamma}_{R}$, is given by Equation 4.5 (51). 
$\dot{\gamma}_{R}=\dot{\gamma}_{a}\left[\frac{1}{4}\left(3+\frac{d \ln \dot{\gamma}_{a}}{d \ln \tau_{R}}\right)\right]$

The Weissenberg-Rabinowitsch correction, seen in Equation 4.5 as the quantity within the square brackets, corrects for the effects of a non-Newtonian flow. When the fluid is Newtonian, the wall stress changes linearly with the shear rate. This causes the Weissenberg-Rabinositsch correction to be 1 (51).

To determine the value of the derivative in the Weissenberg-Rabinowitch correction, a quadratic equation was fit to the $\ln \left(\dot{\gamma}_{a}\right)$ vs. $\ln \left(\tau_{\mathrm{R}}\right)$ data. A quadratic fit provides good agreement with all data collected $\left(\mathrm{R}^{2}>0.995\right)$. The derivative of the quadratic equation can be simply determined. This allows calculation of a true shear rate that then allows the calculation of a steady-shear viscosity using Equation 4.6 (51).

$\eta=\frac{\tau_{R}}{\dot{\gamma}_{R}}$

Where steady-shear viscosity data is available at multiple test temperatures (CNT/PC and $\mathrm{CB} / \mathrm{PC}$ ) the data is time-temperature superpositioned to create a single master curve of shifted viscosity, $\eta_{\mathrm{r}}$, versus shear rates at $270^{\circ} \mathrm{C}$ according to Equation 4.7 below,

$\eta_{r}\left(a_{T} \dot{\gamma}\right) \equiv \frac{\eta(T) T_{r e f} \rho_{r e f}}{a_{T} T \rho}$

where $\mathrm{a}_{\mathrm{T}}$ is the shift factor, $\dot{\gamma}$ is the shear rate, $\eta(\mathrm{T})$ is the viscosity at the test temperature, $T_{\text {ref }}$ is the reference temperature $\left(270^{\circ} \mathrm{C}\right), \rho_{\text {ref }}$ is the sample density at the 
reference temperature, $T$ is the test temperature and $\rho$ is the sample density at the test temperature (51).

\subsection{5: Small Amplitude Oscillatory Shear Rheology Test Method}

Small-amplitude oscillatory shear (SAOS) complex viscosity was determined using a Bohlin C-VOR rotational rheometer (shown in Figure 4.14). For these materials $25 \mathrm{~mm}$ diameter parallel plates (seen installed in Figure 4.14) with a $3 \mathrm{~mm}$ nominal gap was used and testing was performed according to ISO $6721-99$ (52). Two $25 \mathrm{~mm}$ diameter disks were cut from an injection-molded $6.4 \mathrm{~cm}$ diameter disk. Five $25 \mathrm{~mm}$ disks were tested from each formulation. The $25 \mathrm{~mm}$ disks were vacuum dried for 6 hours at 30 inches of mercury vacuum. $\mathrm{CNT} / \mathrm{PC}$ samples were tested at $210^{\circ} \mathrm{C}, 230^{\circ} \mathrm{C}, 250^{\circ} \mathrm{C}, 270^{\circ} \mathrm{C}, 290^{\circ} \mathrm{C}$, $310^{\circ} \mathrm{C}, 330^{\circ} \mathrm{C}$ and $350^{\circ} \mathrm{C}$. Following this testing it was determined that $210^{\circ} \mathrm{C}, 230^{\circ} \mathrm{C}$ and $350^{\circ} \mathrm{C}$ testing did not provide helpful data thus $\mathrm{CB} / \mathrm{PC}$ samples were tested at $250^{\circ} \mathrm{C}$, $270^{\circ} \mathrm{C}, 290^{\circ} \mathrm{C}, 310^{\circ} \mathrm{C}$ and $330^{\circ} \mathrm{C}$. Testing started with an amplitude sweep varying from $0.1 \%$ to $10 \%$ strain to determine the limits of the linear-viscoelastic regime in which SAOS analysis is valid. Frequency sweeps varying logarithmically from 0.1 to $100 \mathrm{rad} / \mathrm{s}$ at an amplitude of $1 \%$ strain were taken in a "ramp-up then ramp-down" fashion to determine the rheological properties of the samples. 


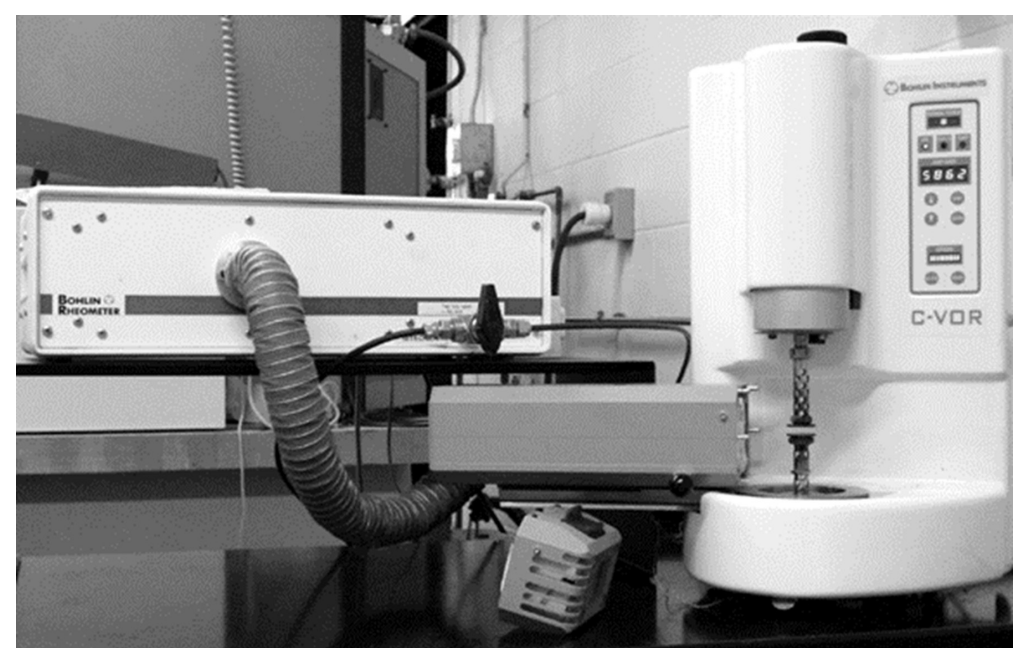

Figure 4.14: Bohlin C-VOR rotational rheometer with installed $25 \mathrm{~mm}$ parallel plates

Time-temperature superpositioning was performed using the linear-viscoelastic storage modulus, $G^{\prime}(\omega)$, and the loss modulus, $G^{\prime \prime}(\omega)$. The data was shifted by a shift factor $\left(a_{T}\right)$, according to Equations 4.8 and 4.9 below, to create master curves of $G^{\prime}(\omega)$ and $G^{\prime \prime}(\omega)$ with a reference temperature of $270^{\circ} \mathrm{C}(51)$.

$G_{r}^{\prime}\left(a_{T} \omega\right) \equiv \frac{G^{\prime}(T) T_{r e f} \rho_{r e f}}{T \rho}$

$G_{r}^{\prime \prime}\left(a_{T} \omega\right) \equiv \frac{G^{\prime \prime}(T) T_{r e f} \rho_{r e f}}{T \rho}$

where $\omega$ is the oscillation frequency in radians/s, $a_{T}$ is the shift factor, $G^{\prime}(T)$ is the storage modulus at the test temperature, G”(T) is the loss modulus at the test temperature, $\mathrm{T}_{\text {ref }}$ is the reference temperature for the superpositioning $\left(270^{\circ} \mathrm{C}\right), \rho_{\text {ref }}$ is the density of the material at the reference temperature, $\mathrm{T}$ is the test temperature, and $\rho$ is the sample density at the test temperature. The shift factors were chosen to optimize the fit of the 
shifted data to a single master curve. Complex viscosity, $\eta^{*}(\omega)$ can then be calculated from the elastic and viscous moduli using Equation 4.10 (51).

$$
\eta^{*}(\omega) \equiv \frac{\sqrt{G^{\prime 2}(\omega)+G^{\prime 2}(\omega)}}{\omega}
$$

When the steady-shear viscosity and SAOS complex viscosity are compared at $\omega=\dot{\gamma}$, many linear polymers show the same values. This is known as the Cox-Merz rule $(51,53)$. The Cox-Merz rule will be tested for $\mathrm{CNT} / \mathrm{PC}$ and $\mathrm{CB} / \mathrm{PC}$ in this project.

While the Bohlin C-VOR is capable of steady-shear testing, the high stiffness of these filled systems prevents the rheometer from attaining the shear rates that are of interest to this project, thus only capillary steady-shear testing will be performed.

\subsection{6: Through-Plane Electrical Resistivity Test Method}

Injection molded specimens that had high electrical resistivity, greater than $10^{4} \mathrm{ohm}-\mathrm{cm}$, were tested for electrical resistivity using the through-plane, volumetric electrical resistivity test method. Following ASTM D257, this test uses a Keithley 6517A Electrometer/High Resistance Meter equipped with an 8009 Resistivity Test Fixture to apply a constant voltage (typically 100V) to the as-molded $6.4 \mathrm{~cm}$ diameter, $3.2 \mathrm{~mm}$ thick disk (54). The testing is automated using Keithley 6524 High Resistance Measurement software. A minimum of six specimens were tested for each formulation. The testing apparatus is shown in Figure 4.15. 


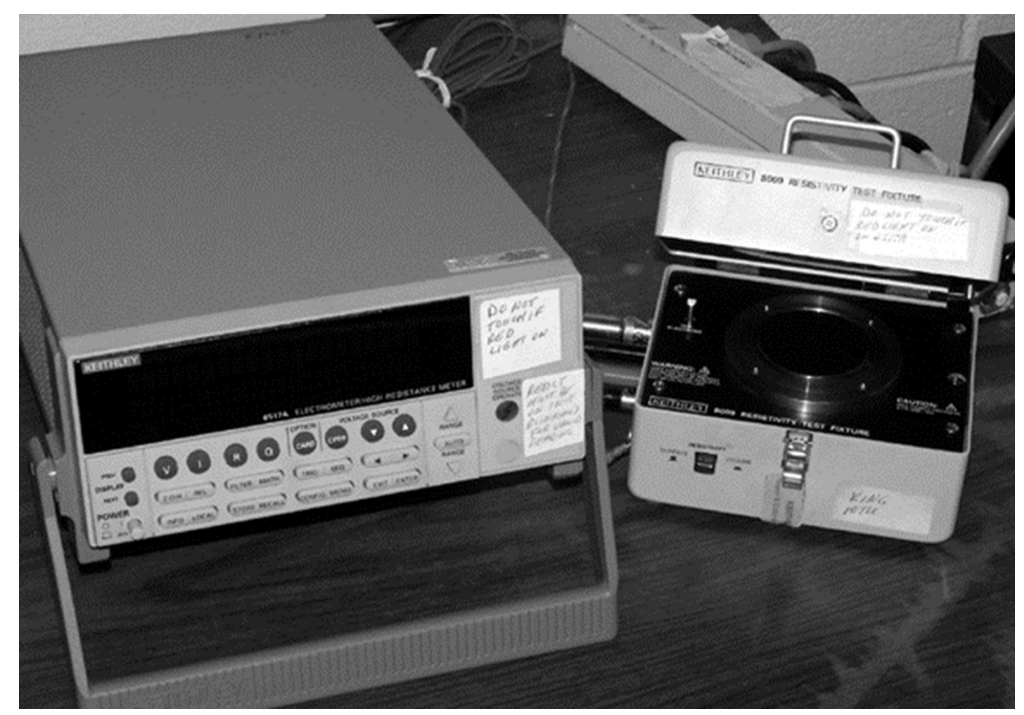

Figure 4.15: ASTM D257 apparatus comprised of Keithley 6517A Electrometer/High Resistance Meter (left) and Keithley 8009 Resistivity Test fixture (right)

\subsection{7: In-Plane Electrical Resistivity Test Method}

Injection molded samples that had low electrical resistivity, less than $104 \mathrm{ohm}-\mathrm{cm}$, were tested for electrical resistivity using the in-plane, volumetric electrical resistivity test method. The in-plane volumetric electrical resistivity of the center $60 \mathrm{~mm}$ long, $3.3 \mathrm{~mm}$ thick, $12.6 \mathrm{~mm}$ wide tensile bars (rectangular necked area) injection molded tensile bars was determined according to ASTM D 4496 at $23{ }^{\circ} \mathrm{C}$ (55). This test was conducted with 2 probes. In the two probe method, the tensile bar is scratched (1mm depth if possible) with a razor blade, placed in liquid nitrogen, and then broken by hand (using pliers) at the desired location. Hence, a fracture surface is created on both ends of the in plane sample. Then the $3.3 \mathrm{~mm}$ thick by $12.6 \mathrm{~mm}$ wide ends are coated with conductive silver paint (one coat) and allowed to dry for $1 \mathrm{hr}$. One probe is placed on each silver painted fracture surface and a constant voltage is placed across the sample using a Keithley 2400 Source Meter. The resulting current is also measured on this same Keithley 2400. The volume electrical resistivity is calculated from Equation 4.11. 
$E R=\frac{(\Delta V)(w)(t)}{(i)(L)}$

Where ER is the volume electrical resistivity, $\Delta \mathrm{V}$ is the voltage drop over length of sample, $w$ is the sample width, $t$ is the sample thickness, $i$ is the current, and $L$ is the length over which $\Delta \mathrm{V}$ is measured.

The in-plane volumetric electrical resistivity of $60 \mathrm{~mm}$ long, typically $3 \mathrm{~mm}$ diameter extruded rods were also tested according to ASTM D 4496 at $23^{\circ} \mathrm{C}$ (55). In the two probe method, fracture surface is created on both ends of the rod by scoring lines $60 \mathrm{~mm}$ apart on the rod and then, if necessary submersing the sample in liquid nitrogen for 2 minutes. The sample is retrieved from the liquid nitrogen, if used, and broken along the score lines. The ends of the $3 \mathrm{~mm}$ diameter rod are then coated with conductive silver paint (one coat) and allowed to dry for $1 \mathrm{hr}$. One probe is placed on each silver painted fracture surface and a constant voltage is placed across the sample using a Keithley 2400 Source Meter. The resulting current is also measured on this same Keithley 2400. The volume electrical resistivity (ER) is calculated from Equation 4.12 where $\mathrm{R}$ is radius of the rod and the other symbols retain their meaning from Equation 4.11.

$E R=\frac{(\Delta V)\left(\pi R^{2}\right)}{(i)(L)}$ 


\subsection{8: Thermal Conductivity: Guarded Heat Flow Meter Test Method}

Thermal conductivity testing of the specimens followed the ASTM F433 Guarded Heat Flow Meter method as seen in Figure 4.16 (56). To comply with this ASTM, a Holometrix (now Netzsch) Model TCA-300 Thermal Conductivity Analyzer was used to analyze $5 \mathrm{~cm}$ diameter $3.2 \mathrm{~mm}$ thick disks at $55^{\circ} \mathrm{C}$. The $5 \mathrm{~cm}$ diameter disks were cut from injection molded $6.4 \mathrm{~cm}$ diameter disks with a hole saw. The $55^{\circ} \mathrm{C}$ test temperature was selected to allow the machine to maintain a temperature gradient across the sample. For each formulation at least 5 specimens were tested with TCA-300 calibration "locon13".

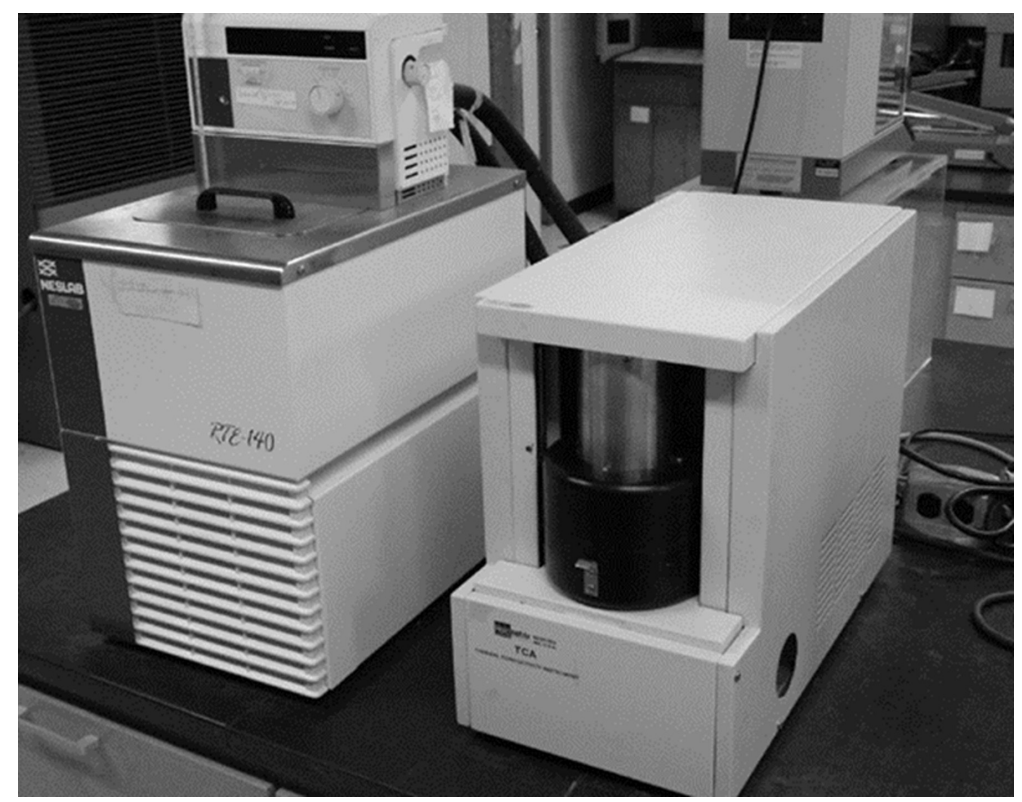

Figure 4.16: Holometrix TCA-300 Thermal Conductivity Analyzer (right) with constant temperature ethylene glycol/water circulator (left)

The TCA-300 controls the energy to the upper heater in an effort to maintain average temperature of the sample at the test temperature of $55^{\circ} \mathrm{C}$. Heat flows from the upper heater to the specimen through a layer of heat-sink compound (applied to ensure good thermal contact). Heat passes through the sample and into the lower surface (again using 
heat-sink compound to ensure thermal contact). In the lower surface the heat passes through a heat-flow meter. The lower surface also has a heater that helps maintain the average sample temperature at $55^{\circ} \mathrm{C}$. There are thermocouples that monitor the temperature at the upper and lower surface of the specimen. Using these temperatures and the heat-flow the machine calculates thermal conductivity using Equation 4.13 below:

$R_{S}=N\left(\frac{T_{U}-T_{L}}{q}\right)-R_{0}$

where $R_{S}$ is the specimen thermal resistance, $T_{U}$ is the upper surface temperature, $T_{L}$ is the lower surface temperature, $q$ is the heat flow, $N$ is a proportionality constant and $R_{0}$ is the sum of all contact thermal resistances. $N$ and $R_{0}$ are calibrated for the machine using reference specimens of known thermal conductivity (57). The specimen thermal conductivity is then calculated using Equation 4.14 below:

$$
R_{S}=\frac{t}{k}
$$

where $t$ is the sample thickness and $k$ is the thermal conductivity (57).

\subsection{9: Mechanical Tensile Property Test Method}

Tensile properties of the composites were obtained at ambient conditions using $16.5 \mathrm{~cm}$ long $3.3 \mathrm{~mm}$ thick injection molded ASTM Type 1 tensile bars. These samples are tested according to ASTM D638 with a crosshead rate of $5 \mathrm{~mm} / \mathrm{min}$ for reinforced plastics (58). Testing was performed on an Instru-Met Sintech screw driven mechanical testing machine as shown in Figure 4.17. Tensile modulus was calculated from the slope of the 
initial linear portion of the tensile stress versus tensile strain curve. At least 5 samples of each composite were tested. These samples were preconditioned at $23^{\circ} \mathrm{C}$ and $50 \%$ relative humidity for 2 days.

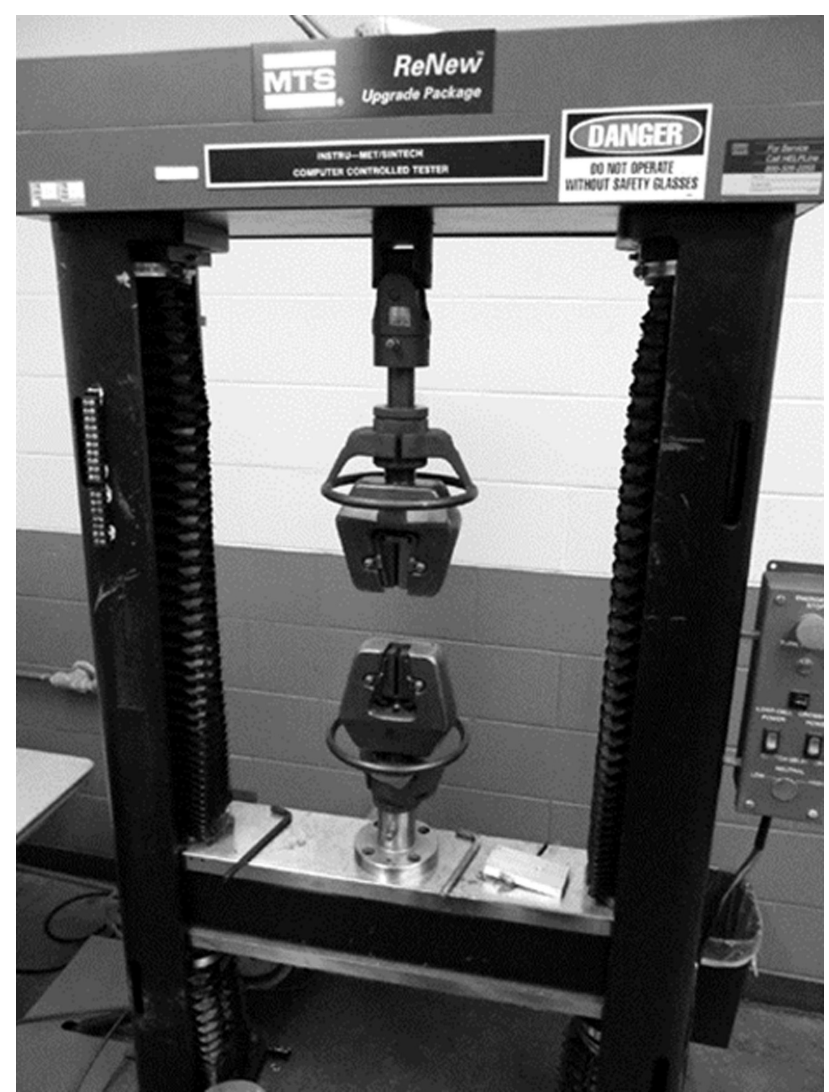

Figure 4.17: Instru-Met Sintech mechanical testing machine with tensile apparatus installed

\subsubsection{0: Mechanical Flexural Property Test Method}

Flexural properties of the composites were obtained at ambient conditions using $3.1 \mathrm{~mm}$ thick, $12.7 \mathrm{~mm}$ wide and $127 \mathrm{~mm}$ long flexural bars. The samples are tested using a three-point loading method in accordance with ASTM D790 with a crosshead rate of 5.3 $\mathrm{mm} / \mathrm{min}$ (59). At least 8 samples from each specimen is tested with a span to thickness ratio of 16:1 on a Instru-Met Sintech screw driven mechanical testing machine as shown in Figure 4.18. Deflection of the flex bar was measured using a linear variable 
displacement transformer (LVDT). The flexural moduli of the samples were calculated from the slope of the initial linear portion of the flexural stress versus flexural strain curve. These samples were preconditioned at $23^{\circ} \mathrm{C}$ and $50 \%$ relative humidity for 2 days.

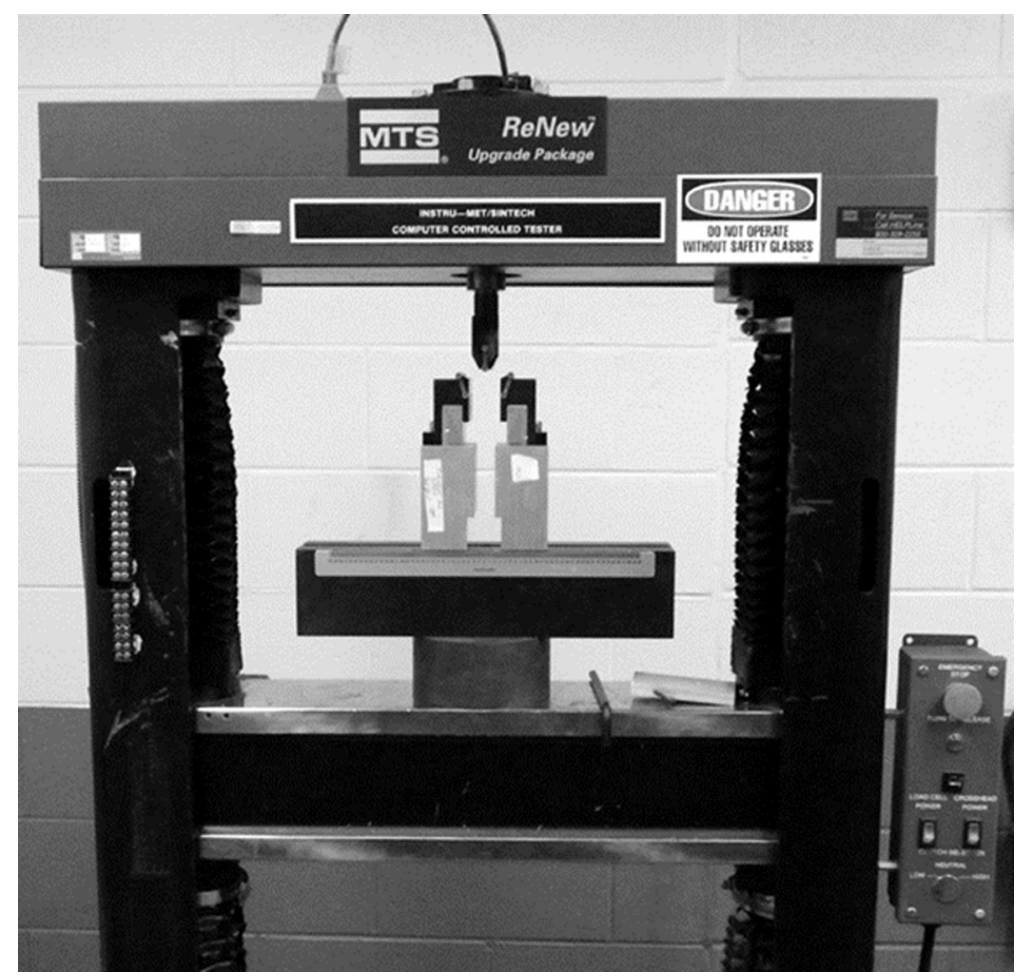

Figure 4.18: Instru-Met Sintech mechanical testing machine with flex apparatus installed

\subsubsection{1: Optical Polishing Method}

Optical imaging samples are created from flex bars. Approximately $1 \mathrm{~cm}$ squares were cut from the center of the flex bars using a scroll saw. Labeled 1.25 inch diameter reusable epoxy molds were prepared by first spraying the cylinders with mold release. Aluminum foil was placed over the removable bottoms. The bottoms were pressed onto the cylinder and the excess aluminum foil was trimmed off. The bottoms were again removed and double-sided tape was applied to the aluminum foil. The bottoms were pressed into the cylinder again and the excess tape was trimmed off. Disposable plastic 
sample clips were then applied to the $1 \mathrm{~cm}$ square specimens. The specimens were then pressed, cut side down, onto the tape. Two-part optical epoxy was then poured over the sample to a depth of about 2-cm, any air trapped under the tape was pressed out and the epoxy was allowed to cure creating pucks.

Following curing of the epoxy, the sample pucks are removed from the mold and labeled on the anti-sample side with an indelible marker. The aluminum foil and tape were removed as much as possible. The sample pucks were then hand ground on a Diamond Pacific rotating lap (Figure 4.19) with 320 grit $\mathrm{SiC}$ paper with water lubrication until the sample is exposed, the surface is plane and the remaining tape has been removed. The anti-sample side of the sample pucks was also ground until plane and parallel to the sample side to facilitate optical imaging. The sample pucks were re-labeled on the antisample side after grinding. At this time the edge on the anti-sample side was ground down to better facilitate handheld grinding. The sample pucks were subsequently placed in an ultrasonic bath for 5-minutes to remove lose grit then hand ground on the rotating lap with 600 grit $\mathrm{SiC}$ paper with water lubrication until the grind marks from the 320 grit paper were removed. The sample pucks were then placed in an ultrasonic bath for 5 minutes to remove loose grit then hand ground on the rotating lap with 1200 grit $\mathrm{SiC}$ paper with water lubrication until the grind marks from the 600 grit paper were removed. The sample pucks were again placed in an ultrasonic bath for 5 minutes to remove loose grit. 


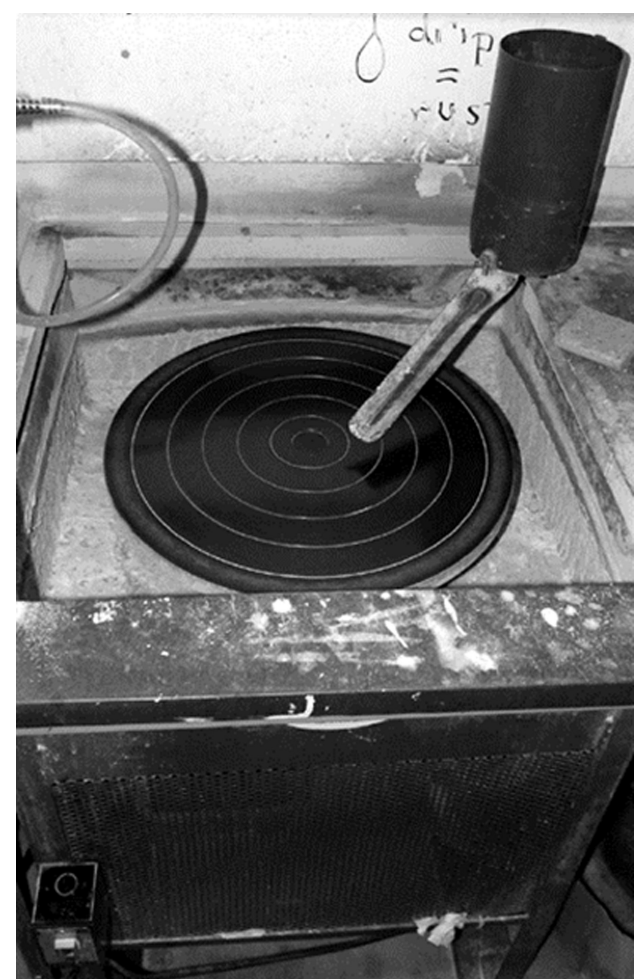

Figure 4.19: Diamond Pacific rotating lap with water drip apparatus

The cleaned sample pucks were then hand polished on a Buehler Ecomet (Figure 4.20) rotary polisher using a 9 micron diamond suspension until the grind marks from the 1200 grit $\mathrm{SiC}$ paper are removed. The sample pucks were cleaned after this and all following steps using an ultrasonicator with a $1 / 2$ " tip for 2 minutes after polishing except after the final step when they were cleaned for 4 minutes. The sample pucks were then hand polished on the rotary polished with a 3 micron diamond suspension until the grind marks from the 9 micron diamond suspension are removed as seen through a simple optical microscope. The sample pucks were then hand polished with a 1 micron deagglomerated alumina suspension until the grind marks from the 3 micron diamond suspension are removed as seen through a simple optical microscope. The sample pucks were then placed in a weighted vibratory polishing puck and placed on a Buehler Vibromet I vibratory polisher (seen in Figure 4.21) with 0.05 micron alumina suspension and 
polished for 2 hours to obtain a satisfactory surface. Optical imaging is done on an Olympus BX-60 System Microscope shown in Figure 4.22. Images are captured using an Optixcam Summit 1.3 camera seen in the top of Figure 4.22.
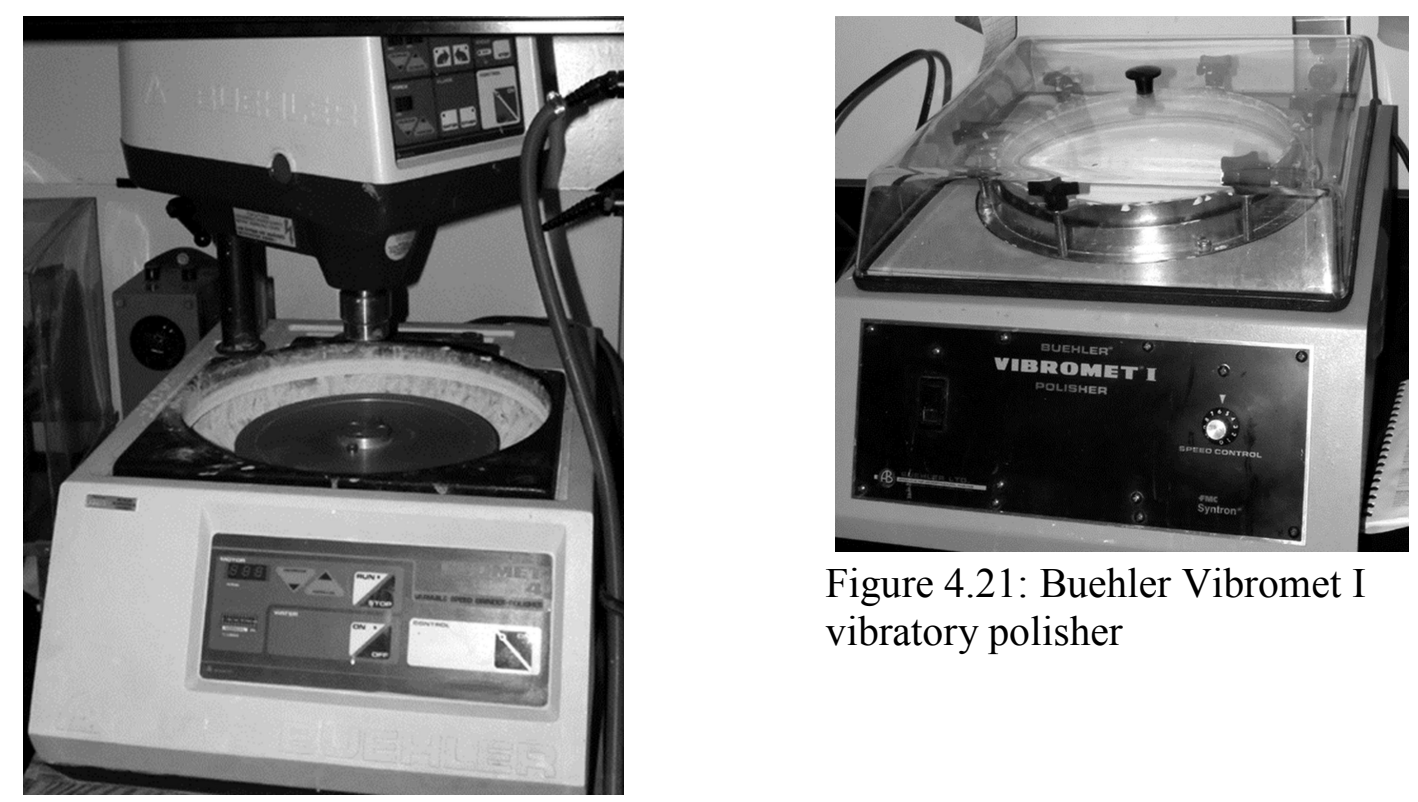

Figure 4.21: Buehler Vibromet I vibratory polisher

Figure 4.20: Buehler Ecomet rotary polisher with Automet 2 automated polishing head 


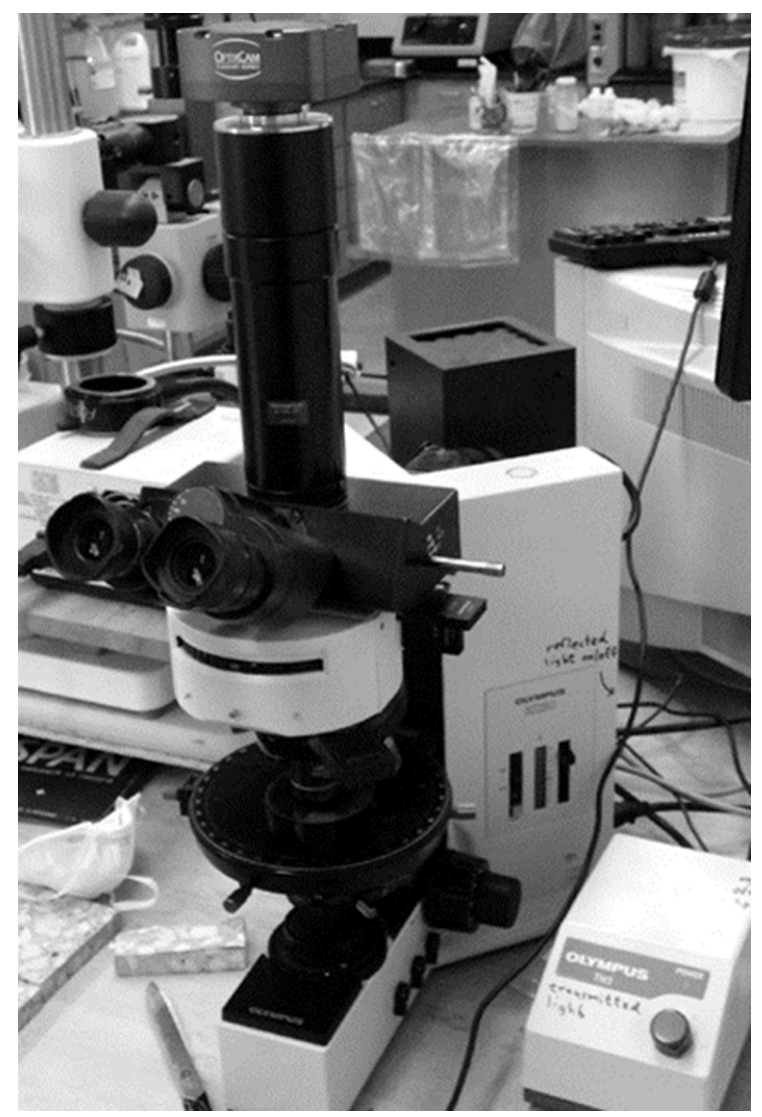

Figure 4.22: Olympus BX-60 System microscope with Optixcam Summit 1.3 camera on top

\subsubsection{2: Field Emission Scanning Electron Microscope (FESEM) Test Method}

CNT containing samples used for FESEM analysis were $2 \mathrm{~mm}$ by $2 \mathrm{~mm}$ sticks about 25 $\mathrm{mm}$ long cut from the center $6 \mathrm{~cm}$ section of tensile bars using a scroll saw. The sticks were placed in liquid nitrogen and then broken using two pairs of pliers. This created a cryogenic fracture surface that was the target of the imaging. The samples were mounted, with the cryogenic fracture surface facing upwards, to FESEM sample holders with hot-melt glue. Conductive carbon paint was applied from to sample over the hotmelt glue to the sample holder to ensure conductivity from the sample to the sample holder. The samples were then placed in the Hitachi S-4700 FESEM shown in Figure 
4.23. Imaging was done at $2 \mathrm{kV}$ accelerating voltage with a working distance of $1.9 \mathrm{~mm}$. Images were collected using the upper secondary electron detector.

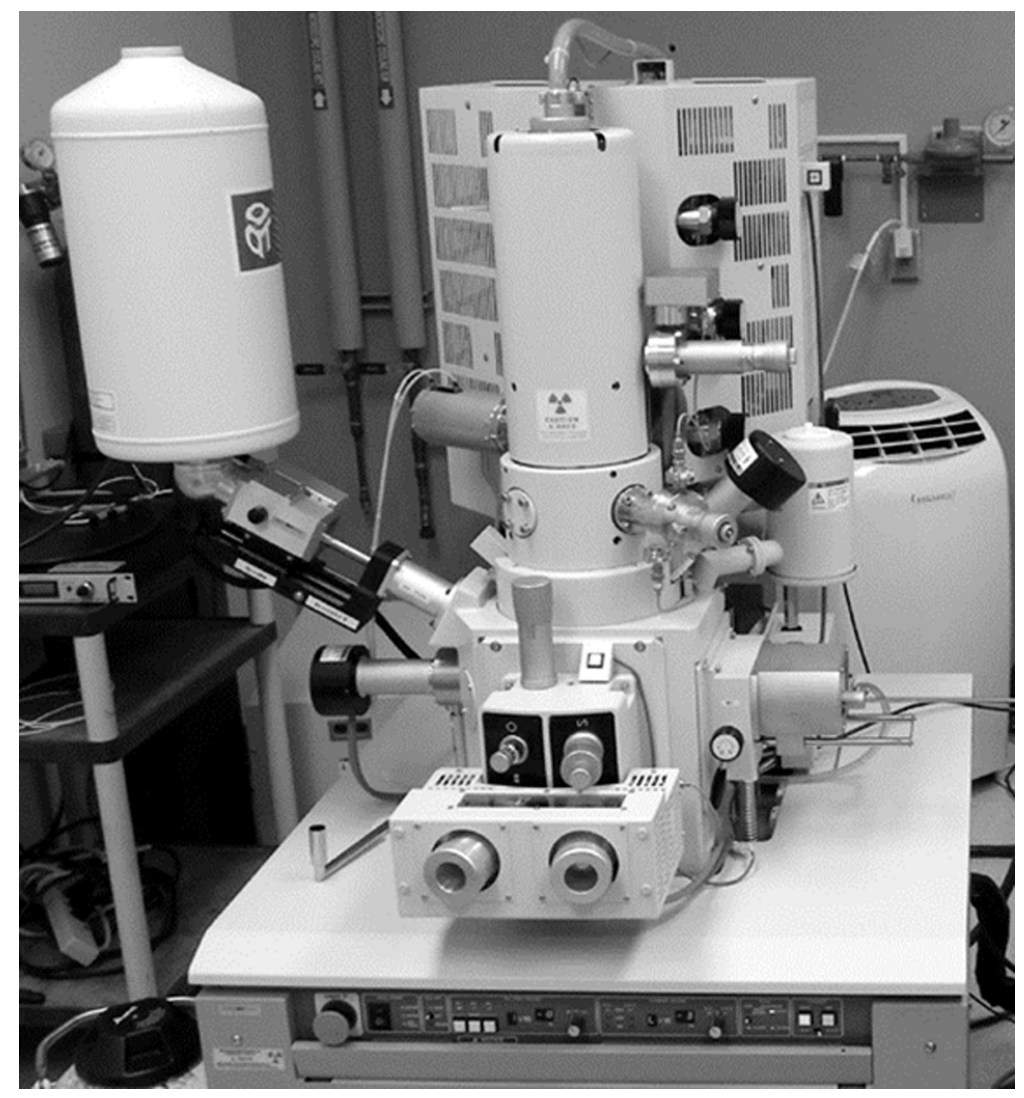

Figure 4.23: Hitachi S-4700 FESEM with energy dispersive $\mathrm{x}$-ray spectrometer seen on left

CB containing samples used for FESEM analysis were sections taken from $3.2 \mathrm{~mm}$ thick by $12.7 \mathrm{~mm}$ wide flex bars. The sample sections were then cast into epoxy pucks similarly to the optical samples. The surface of the resultant pucks were then polished on a rotary lap with $\# 4000 \mathrm{SiC}$ grit. They were then polished on the same lap with a 1micron alumina/water slurry. Following the polishing on the lap, the sample pucks were polished on a Buhler Vibromet vibratory polisher with 0.05 micron alumina/water slurry for 2 hours. The composite surface was then etched with $\mathrm{O}_{2}$ plasma at $23^{\circ} \mathrm{C}$ and 0.28 torr for 1 hour. The etched surface was then sputter coated with gold to a thickness of 
about $10 \mathrm{~nm}$. The coated samples were then imaged in a JEOL Ltd. JSM-7500F FESEM at $15 \mathrm{kV}$ accelerating voltage, $6 \mathrm{~mm}$ working distance with images captured with the upper secondary electron detector.

\subsubsection{3: Transmission Electron Microscope (TEM) Test Method}

TEM samples were taken from $2 \mathrm{~mm}$ by $2 \mathrm{~mm}$ by about $15 \mathrm{~mm}$ long sections the center 6 $\mathrm{cm}$ of a tensile bar with a scroll saw. The samples were then clamped and trimmed using a wire saw to get them down to less than $0.5 \mathrm{~mm}$ by $0.5 \mathrm{~mm}$ by about $5 \mathrm{~mm}$. These sections are then placed in a parallel jaw sample holder. The holder is placed into a Leica EM UC6 ultramicrotome (shown in Figure 4.24) equipped with an EM FC7 cryo chamber. The ultramicrotome is cooled to $-140^{\circ} \mathrm{C}$ and then the samples are cut until parallel with the diamond knives using a Diatome cryo-sectioning knife. Then the edges of the samples are sectioned about 30 microns deep leaving a mesa, approximately 50 microns wide by 50 microns tall, on the samples. The samples are then cut to approximately $70 \mathrm{~nm}$ thick slices. These slices are picked up and placed onto carbon coated, nickel TEM grids. The samples are then imaged in a JEOL Ltd. JEM-4000FX TEM shown in Figure 4.25. Composites containing GNP were imaged using bright field and dark field techniques. Bright field imaging allowed for the identification of $\mathrm{CB}$ or CNT if present and allowed the GNP particles to be seen in a true representation. Dark field imaging allowed for the positive identification of GNP particles (as opposed to torn TEM grid coating) by isolating the electrons that are diffracted by the crystalline structure of the GNP, following Bragg's Law, causing the GNP particles to brightly light up in the image. Dark field imaging was accomplished by positioning the objective 
aperture slightly off-center. Images were obtained from the TEM using a Gatan Orius camera. Imaging was performed at $15000 \mathrm{X}$.

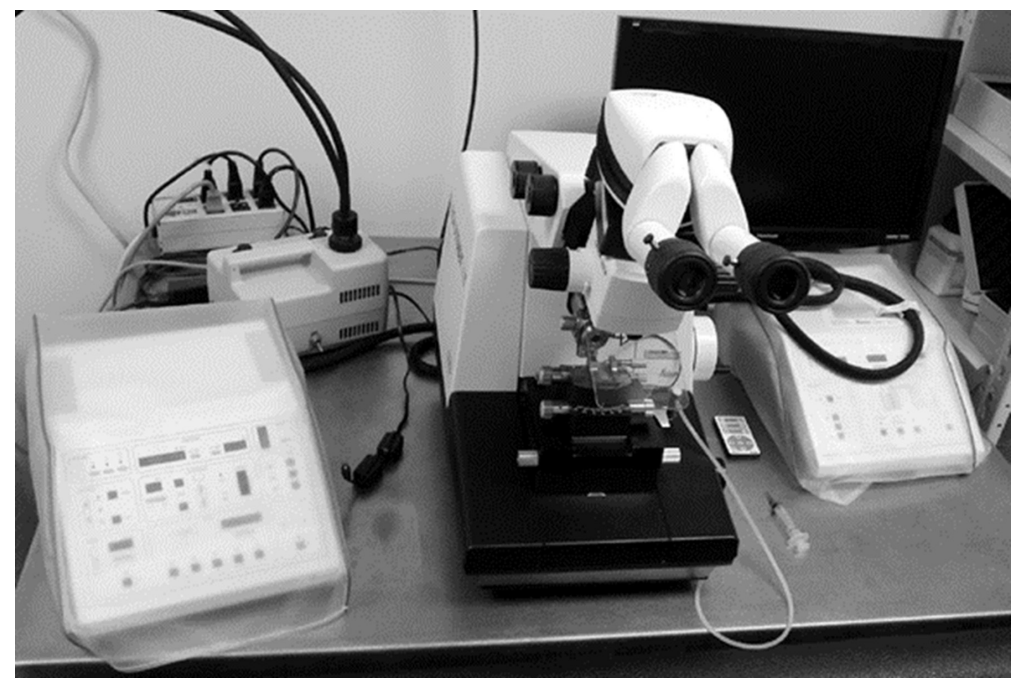

Figure 4.24: Leica EM UC6 ultramicrotome shown without cryo chamber

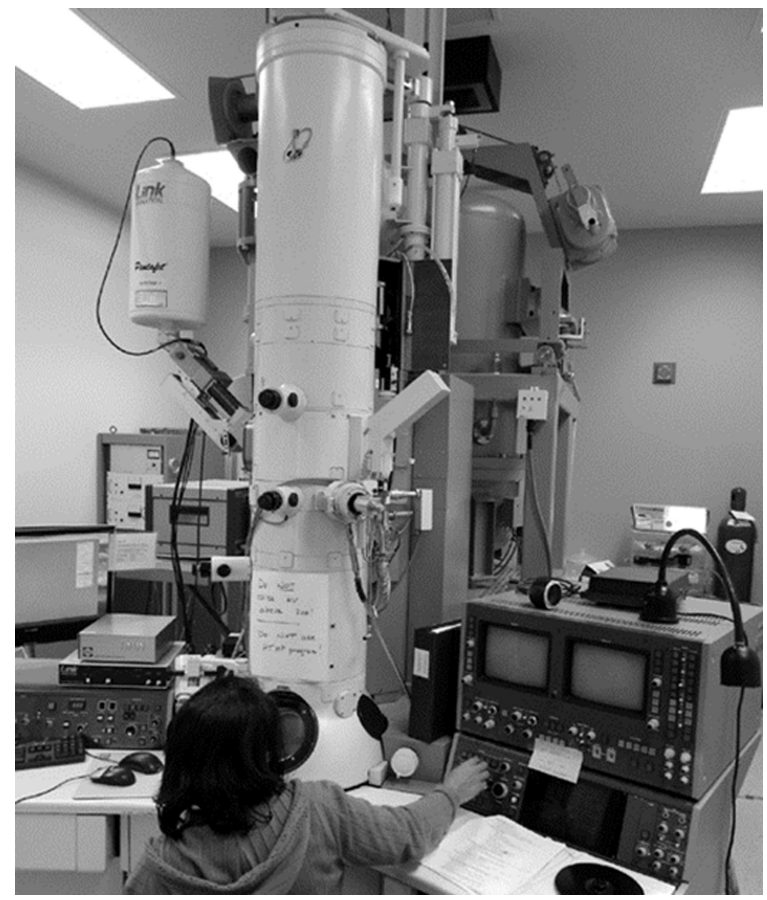

Figure 4.25: JEOL JEM-4000FX transmission electron microscope 


\subsubsection{4: Environmental Scanning Electron Microscope (ESEM) Test Method}

The samples for ESEM imaging were the previously polished optical imaging samples. The polished sample pucks were stored using a plastic end cap to maintain a clean, scratch-free surface following optical imaging. The sample pucks were then sputtercoated with gold/palladium alloy to a thickness of $5 \mathrm{~nm}$ on the sample side. Following coating, conductive carbon tape was placed on the edge of the sample surface of the pucks, not obscuring the sample, run down the side of the pucks, across the anti-sample side, up the other side of the pucks and back onto the sample side, again not obscuring the sample. This tape was then used to adhere the sample pucks to sample holders to be used in the ESEM. The sample was then placed into the ESEM chamber for imaging.

ESEM imaging was done on a FEI/Phillips XL40 Environmental Scanning Electron Microscope (shown in Figure 4.26) with a $15 \mathrm{kV}$ accelerating voltage at high vacuum and a working distance around $15 \mathrm{~mm}$. The images were captured using the secondary electron detector. 


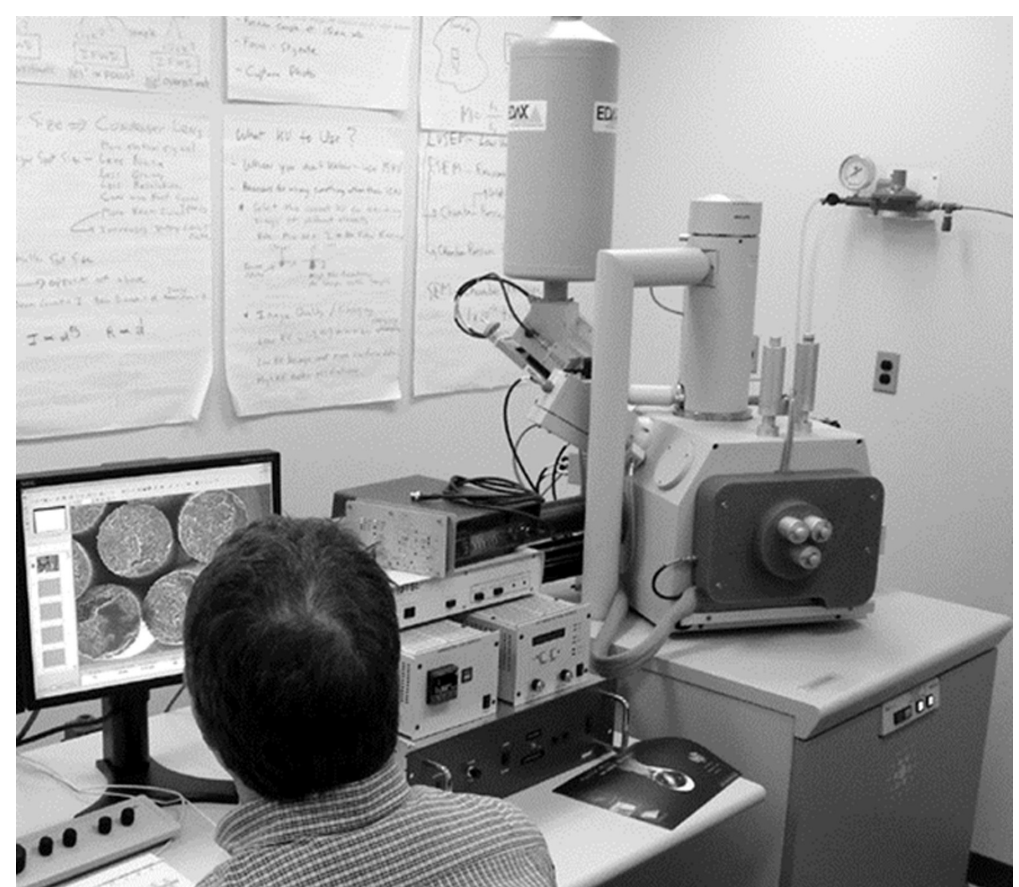

Figure 4.26: FEI/Phillips XL40 ESEM 


\section{Chapter 5: Miscellaneous Results}

\section{1: Density Results}

The density of each formulation was measured according to the procedure described in section 4.2.1. These measured values were then compared to theoretical density values. Theoretical density values, $\rho_{\text {Theo }}$, are determined using Equation 5.1 below:

$\rho_{\text {Theo }}=\frac{1}{\sum_{i} \frac{\Phi_{i}}{\rho_{i}}}$

where $\rho_{\mathrm{i}}$ is the density of the individual components (PC, CNT, CB or GNP) and $\Phi_{\mathrm{i}}$ is the weight fraction of individual component. This comparison verifies that each formulation has the target value of filler present. For this analysis it is assumed that the theoretical densities of the individual components are as given in Table 5.1.

Table 5.1: Individual Component Theoretical Densities

\begin{tabular}{|l|l|}
\hline Component & $\begin{array}{l}\text { Theoretical } \\
\text { Density }\left(\mathrm{g} / \mathrm{cm}^{3}\right)\end{array}$ \\
\hline Lexan HF1130-111 (PC) & 1.20 \\
\hline Ketjenblack EC-600JD (CB) & 1.80 \\
\hline FIBRILs MWCNT (CNT) & 1.75 \\
\hline xGnPM Graphite Nanoplatelets (GNP) & 2.00 \\
\hline
\end{tabular}

Tables 5.2to 5.4 show the theoretical and average experimental densities (along with the standard deviation) for $\mathrm{CNT} / \mathrm{PC}, \mathrm{CB} / \mathrm{PC}, \mathrm{GNP} / \mathrm{PC}$ composites respectively. Results of the individual specimens for each formulation can be found in Appendix C. These tables indicate that the samples were created with the target amount of filler.

Table 5.2: CNT/PC Composite Density Results

\begin{tabular}{|l|l|l|}
\hline Formulation & Theoretical Density & Average Experimental \\
\hline
\end{tabular}




\begin{tabular}{|l|l|ll|}
\hline & $\left(\mathrm{g} / \mathrm{cm}^{3}\right)$ & Density $\left(\mathrm{g} / \mathrm{cm}^{3}\right)$ & \\
\hline BL & 1.200 & $1.9123 \pm 0.0016$ & $\mathrm{n}=5$ \\
\hline BQ2L & 1.210 & $1.2015 \pm 0.0005$ & $\mathrm{n}=8$ \\
\hline BQ3L & 1.215 & $1.2052 \pm 0.0010$ & $\mathrm{n}=6$ \\
\hline BQ4L & 1.220 & $1.2123 \pm 0.0013$ & $\mathrm{n}=5$ \\
\hline BQ5L & 1.224 & $1.2168 \pm 0.0004$ & $\mathrm{n}=5$ \\
\hline BQ6L & 1.230 & $1.2214 \pm 0.0004$ & $\mathrm{n}=5$ \\
\hline BQ8L & 1.240 & $1.2313 \pm 0.0005$ & $\mathrm{n}=5$ \\
\hline
\end{tabular}

Table 5.3: CB/PC Composite Density Results

\begin{tabular}{|l|l|ll|}
\hline Formulation & $\begin{array}{l}\text { Theoretical Density } \\
\left(\mathrm{g} / \mathrm{cm}^{3}\right)\end{array}$ & $\begin{array}{l}\text { Average Experimental } \\
\text { Density }\left(\mathrm{g} / \mathrm{cm}^{3}\right)\end{array}$ \\
\hline BA2L & 1.208 & $1.2032 \pm 0.0004 \quad \mathrm{n}=7$ \\
\hline BA3L & 1.212 & $1.2080 \pm 0.0001 \quad \mathrm{n}=6$ \\
\hline BA4L & 1.216 & $1.2119 \pm 0.0007$ & $\mathrm{n}=6$ \\
\hline BA5L & 1.220 & $1.2153 \pm 0.0001$ & $\mathrm{n}=6$ \\
\hline BA6L & 1.224 & $1.2189 \pm 0.0004$ & $\mathrm{n}=5$ \\
\hline BA8L & 1.233 & $1.2268 \pm 0.0002$ & $\mathrm{n}=5$ \\
\hline BA10L & 1.241 & $1.2373 \pm 0.0003 \quad \mathrm{n}=5$ \\
\hline
\end{tabular}

Table 5.4: GNP/PC Composite Density Results

\begin{tabular}{|l|l|ll|}
\hline Formulation & $\begin{array}{l}\text { Theoretical Density } \\
\left(\mathrm{g} / \mathrm{cm}^{3}\right)\end{array}$ & $\begin{array}{l}\text { Average Experimental } \\
\text { Density }\left(\mathrm{g} / \mathrm{cm}^{3}\right)\end{array}$ \\
\hline BG2L & 1.2097 & $1.2027 \pm 0.0009$ & $\mathrm{n}=5$ \\
\hline BG3L & 1.2146 & $1.2062 \pm 0.0007$ & $\mathrm{n}=6$ \\
\hline BG4L & 1.2195 & $1.2104 \pm 0.0019$ & $\mathrm{n}=6$ \\
\hline BG5L & 1.2245 & $1.2154 \pm 0.0005$ & $\mathrm{n}=5$ \\
\hline BG6L & 1.2295 & $1.2229 \pm 0.0006$ & $\mathrm{n}=5$ \\
\hline BG8L & 1.2397 & $1.2306 \pm 0.0005$ & $\mathrm{n}=5$ \\
\hline BG10L & 1.2500 & $1.2402 \pm 0.0008$ & $\mathrm{n}=5$ \\
\hline BG12L & 1.2537 & $1.2521 \pm 0.0002$ & $\mathrm{n}=5$ \\
\hline BG15L & 1.2699 & $1.2683 \pm 0.0003$ & $\mathrm{n}=6$ \\
\hline
\end{tabular}

Table 5.5 shows the theoretical and average experimental densities for $\mathrm{CB} / \mathrm{CNT}$, CB/GNP, and CNT/GNP two filler composites. Results of the individual specimens for each formulation can be found in Appendix C. This table indicates that the samples were created with the target amount of fillers and the low standard deviations indicate the samples are made with significant reproducibility.

Table 5.5: CB/CNT, CB/GNP and CNT/GNP Density Results 


\begin{tabular}{|l|l|ll|}
\hline Formulation & $\begin{array}{l}\text { Theoretical } \\
\text { Density }\left(\mathrm{g} / \mathrm{cm}^{3}\right)\end{array}$ & $\begin{array}{l}\text { Average Experimental } \\
\text { Density }\left(\mathrm{g} / \mathrm{cm}^{3}\right)\end{array}$ \\
\hline BA2Q1L & 1.2050 & $1.2074 \pm 0.0004$ & $\mathrm{n}=7$ \\
\hline BA2Q5L & 1.2207 & $1.2252 \pm 0.0020$ & $\mathrm{n}=6$ \\
\hline BA5Q1L & 1.2174 & $1.2192 \pm 0.0002$ & $\mathrm{n}=6$ \\
\hline BA5Q5L & 1.2334 & $1.2379 \pm 0.0015$ & $\mathrm{n}=5$ \\
\hline BA2G2L & 1.2109 & $1.2096 \pm 0.0004$ & $\mathrm{n}=6$ \\
\hline BA2G5L & 1.2260 & $1.2252 \pm 0.0008$ & $\mathrm{n}=5$ \\
\hline BA5G2L & 1.2235 & $1.2241 \pm 0.0010$ & $\mathrm{n}=6$ \\
\hline BA5G5L & 1.2389 & $1.2382 \pm 0.0005$ & $\mathrm{n}=4$ \\
\hline BQ1G2L & 1.2066 & $1.2053 \pm 0.0004$ & $\mathrm{n}=4$ \\
\hline BQ1G5L & 1.2215 & $1.2194 \pm 0.0002$ & $\mathrm{n}=4$ \\
\hline BQ5G2L & 1.2223 & $1.2229 \pm 0.0006$ & $\mathrm{n}=4$ \\
\hline BQ5G5L & 1.2377 & $1.2376 \pm 0.0005$ & $\mathrm{n}=4$ \\
\hline
\end{tabular}

\section{2: Melt Flow Index (MFI) Results}

Melt flow indexing was performed according to the procedure listed in section 4.2.2. Typical filled system behavior indicates that as filler loading to a polymer is increased, MFI decreases. Tables 5.6 to 5.8 show the average MFIs of $\mathrm{CB} / \mathrm{PC}, \mathrm{CNT} / \mathrm{PC}$ and GNP/PC composites, respectively. Results of the individual specimens for each formulation can be found in Appendix D. As a general note, results in the appendices for factorial design formulations and supplemental formulations (BQ0.5L, BQ1L, BQ5LR, etc.) are given in the "Polycarbonate with Multiple Fillers" sections of the respective appendices. 
Table 5.6: CNT/PC MFI Results

\begin{tabular}{|c|c|c|}
\hline Formulation & \multicolumn{2}{|c|}{ MFI (g/10 min) } \\
\hline BQ0.5L & $326 \pm 23$ & $n=5$ \\
\hline BQ1L & $320 \pm 29$ & $\mathrm{n}=5$ \\
\hline BQ2L & $292 \pm 31$ & $\mathrm{n}=5$ \\
\hline BQ3L & $246 \pm 14$ & $\mathrm{n}=7$ \\
\hline BQ4L & $173 \pm 13$ & $\mathrm{n}=7$ \\
\hline BQ5L & $114 \pm 8$ & $\mathrm{n}=9$ \\
\hline BQ6L & $55 \pm 7$ & $\mathrm{n}=7$ \\
\hline
\end{tabular}

Table 5.7: CB/PC MFI Results

\begin{tabular}{|c|c|}
\hline Formulation & MFI (g/10 min) \\
\hline BA2L & $148.1 \pm 4.6 \mathrm{n}=5$ \\
\hline BA3L & $111.9 \pm 5.9 \mathrm{n}=6$ \\
\hline BA4L & $87.6 \pm 2.3 \mathrm{n}=6$ \\
\hline BA5L & $63.6 \pm 2.0 \mathrm{n}=6$ \\
\hline BA6L & $37.1 \pm 2.0 \mathrm{n}=6$ \\
\hline
\end{tabular}

Table 5.8: GNP/PC MFI Results

\begin{tabular}{|c|c|}
\hline Formulation & MFI (g/10 min) \\
\hline BG2L & $155.1 \pm 7.9 \mathrm{n}=5$ \\
\hline BG3L & $142.9 \pm 2.9 \mathrm{n}=4$ \\
\hline BG4L & $127.7 \pm 3.0 \mathrm{n}=5$ \\
\hline BG5L & $116.6 \pm 3.1 \mathrm{n}=5$ \\
\hline BG6L & $106.8 \pm 2.9 \mathrm{n}=5$ \\
\hline BG8L & $83.7 \pm 3.8 \mathrm{n}=5$ \\
\hline BG10L & $70.5 \pm 1.3 \mathrm{n}=5$ \\
\hline BG12L & $55.9 \pm 0.8 \mathrm{n}=5$ \\
\hline BG15L & $31.8 \pm 2.0 \mathrm{n}=5$ \\
\hline
\end{tabular}

It can be seen from these results that all 3 systems act in a typical fashion, increased filler loading leads to decreased MFI. We can also see from these results that $\mathrm{CB}$ has the 
largest effect on decreasing the MFI at equal $\mathrm{wt} \%$ filler loadings and CNT has the smallest effect on decreasing the MFI at equal wt $\%$ filler loadings. GNP has similar effect to $\mathrm{CB}$ at low filler loadings, but has much less effect at high filler loadings.

Table 5.9 shows the average MFIs of $\mathrm{CB} / \mathrm{CNT}, \mathrm{CB} / \mathrm{GNP}$ and $\mathrm{CNT} / \mathrm{GNP}$ composites. Results of the individual specimens for each formulation can be found in Appendix C.

Table 5.9: CB/CNT, CB/GNP and CNT/GNP Composites MFI Results

\begin{tabular}{|c|c|}
\hline Formulation & MFI (g/10 min) \\
\hline BA2Q1L & $292.8 \pm 6.1 \mathrm{n}=5$ \\
\hline BA2Q5L & $69.6 \pm 6.0 \mathrm{n}=5$ \\
\hline BA5Q1L & $119.0 \pm 5.1 \mathrm{n}=5$ \\
\hline BA5Q5L & Will not run- too viscous \\
\hline BA2G2L & $109.7 \pm 4.6 \mathrm{n}=5$ \\
\hline BA2G5L & $80.7 \pm 1.9 \mathrm{n}=5$ \\
\hline BA5G2L & $49.6 \pm 2.2 \mathrm{n}=5$ \\
\hline BA5G5L & $22.0 \pm 2.5 \mathrm{n}=5$ \\
\hline BQ1G2L & $218.9 \pm 14.4 \mathrm{n}=5$ \\
\hline BQ1G5L & $160.5 \pm 3.1 \mathrm{n}=5$ \\
\hline BQ5G2L & $143.9 \pm 7.8 \mathrm{n}=5$ \\
\hline BQ5G5L & $59.4 \pm 3.2 \mathrm{n}=5$ \\
\hline
\end{tabular}

These results show that all binary filler systems behave in a typical fashion with MFI decreasing with increased filler loading. We again see that $\mathrm{CB}$ has a greater effect on decreasing the MFI than GNP when we compare the BA2G5L and the BA5G2L. These two composites both contain $7 \mathrm{wt} \%$ carbon filler, but when the majority is $\mathrm{CB}$ (BA5G2L), the MFI is $39 \%$ lower than when the majority is GNP (BA2G5L). 


\section{3: Differential Scanning Calorimetry (DSC) Results}

Differential scanning calorimetry was performed according to the procedure given in section 4.2.3. The midpoint value of the glass transition temperature was chosen as the point to compare the different formulations. Table 5.10 shows the midpoint glass transition temperature $\left(\mathrm{T}_{\mathrm{g}}\right)$ of each run of pure $\mathrm{PC}$ and $\mathrm{CNT} / \mathrm{PC}$ composites.

Table 5.10: $\mathrm{PC}$ and CNT/PC DSC Midpoint $\mathrm{T}_{\mathrm{g}}$ Values

\begin{tabular}{|l|l|}
\hline Formulation: & $\mathbf{T}_{\mathbf{g}}$ Midpont $\left.\mathbf{(}^{\mathbf{}} \mathbf{C}\right)$ \\
\hline Lexan HF1130-111 $(\mathrm{BL})$ run 1 & 143.40 \\
\hline Lexan HF1130-111 (BL) run 2 & 141.96 \\
\hline BQ0.5L run 1 & 141.06 \\
\hline BQ0.5L run 2 & 140.87 \\
\hline BQ1L run 1 & 140.85 \\
\hline BQ1L run 2 & 140.2 \\
\hline BQ2L run 1 & 140.52 \\
\hline BQ2L run 2 & 140.93 \\
\hline BQ3L run 1 & 140.78 \\
\hline BQ3L run 2 & 141.40 \\
\hline BQ4L run 1 & 141.45 \\
\hline BQ4L run 2 & 141.20 \\
\hline BQ5L run 1 & 141.02 \\
\hline BQ5L run 2 & 140.88 \\
\hline BQ6L run 1 & 139.15 \\
\hline BQ6L run 2 & 138.84 \\
\hline BQ8L run 1 & 139.14 \\
\hline BQ8L run 2 & 137.80 \\
\hline
\end{tabular}

These results show very little change of the glass transition temperature with increased filler loading indicating that CNT does not restrict polymer chain movement unlike many filled systems. 
Tables 5.11 and 5.12 show the midpoint glass transition temperature of the $\mathrm{CB} / \mathrm{PC}$ and GNP/PC composites respectively.

Table 5.11: CB/PC DSC Midpoint $\mathrm{T}_{\mathrm{g}}$ Values

\begin{tabular}{|l|l|}
\hline Formulation: & $\mathbf{T}_{\mathbf{g}}$ Midpont $\left.\mathbf{~}^{\mathbf{0}} \mathbf{C}\right)$ \\
\hline BA2L run 1 & 143.36 \\
\hline BA2L run 2 & 143.26 \\
\hline BA2LR run 1 & 142.84 \\
\hline BA2LR run 2 & 142.26 \\
\hline BA3L run 1 & 143.79 \\
\hline BA3L run 2 & 144.48 \\
\hline BA4L run 1 & 143.29 \\
\hline BA4L run 2 & 143.87 \\
\hline BA5L run 1 & 144.63 \\
\hline BA5L run 2 & 143.84 \\
\hline BA5LR run 1 & 144.30 \\
\hline BA5LR run 2 & 144.35 \\
\hline BA6L run 1 & 143.88 \\
\hline BA6L run 2 & 143.91 \\
\hline BA8L run 1 & 144.96 \\
\hline BA8L run 2 & 143.04 \\
\hline BA10L run 1 & 145.92 \\
\hline BA10L run 2 & 145.68 \\
\hline
\end{tabular}


Table 5.12: GNP/PC DSC Midpoint $\mathrm{T}_{\mathrm{g}}$ Values

\begin{tabular}{|l|l|}
\hline Formulation: & $\mathbf{T}_{\mathbf{g}}$ Midpont $\left(^{\mathbf{0}} \mathbf{C}\right)$ \\
\hline BG2L run 1 & 144.40 \\
\hline BG2L run 2 & 141.89 \\
\hline BG3L run 1 & 142.53 \\
\hline BG3L run 2 & 143.46 \\
\hline BG4L run 1 & 143.95 \\
\hline BG4L run 2 & 142.89 \\
\hline BG5L run 1 & 143.91 \\
\hline BG5L run 2 & 143.24 \\
\hline BG6L run 1 & 143.55 \\
\hline BG6L run 2 & 142.44 \\
\hline BG8L run 1 & 144.31 \\
\hline BG8L run 2 & 143.96 \\
\hline BG10L run 1 & 144.49 \\
\hline BG10L run 2 & 144.60 \\
\hline BG12L run 1 & 144.18 \\
\hline BG12L run 2 & 144.38 \\
\hline BG15L run 1 & 145.00 \\
\hline BG15L run 2 & 145.42 \\
\hline
\end{tabular}

This data indicates that neither CB nor GNP have any significant effect on the $\mathrm{T}_{\mathrm{g}}$ of the composites. This indicates that none of the fillers restrict polymer chain motion. The midpoint glass transition temperature for the multiple filler formulations is shown in Table 5.13. 
Table 5.13: CB/CNT, CB/GNP and CNT/GNP DSC Midpoint $\mathrm{T}_{\mathrm{g}}$ Values

\begin{tabular}{|l|l|}
\hline Formulation: & Tg midpont $\left.{ }^{\circ} \mathbf{C}\right)$ \\
\hline BA2Q1L run 1 & 141.79 \\
\hline BA2Q1L run 2 & 141.05 \\
\hline BA2Q5L run 1 & 140.42 \\
\hline BA2Q5L run 2 & 139.25 \\
\hline BA5Q1L run 1 & 141.86 \\
\hline BA5Q1L run 2 & 142.15 \\
\hline BA5Q5L run 1 & 141.36 \\
\hline BA5Q5L run 2 & 140.63 \\
\hline BA2G2L run 1 & 143.11 \\
\hline BA2G2L run 2 & 143.52 \\
\hline BA2G5L run 1 & 144.37 \\
\hline BA2G5L run 2 & 143.72 \\
\hline BA5G2L run 1 & 144.61 \\
\hline BA5G2L run 2 & 143.97 \\
\hline BA5G5L run 1 & 144.19 \\
\hline BA5G5L run 2 & 144.51 \\
\hline BQ1G2L run 1 & 141.23 \\
\hline BQ1G2L run 2 & 141.60 \\
\hline BQ1G5L run 1 & 142.70 \\
\hline BQ1G5L run 2 & 142.52 \\
\hline BQ5G2L run 1 & 139.96 \\
\hline BQ5G2L run 2 & 139.55 \\
\hline BQ5G5L run 1 & 140.52 \\
\hline BQ5G5L run 2 & 140.29 \\
\hline
\end{tabular}

This data again indicates that none of the fillers have a significant effect on polymer chain mobility. It is interesting to note that for $10 \mathrm{wt} \%$ carbon filler formulations, the CNT containing composites (BA5Q5L and BQ5G5L) have $\mathrm{T}_{\mathrm{g}}$ 's that are about $4^{\circ} \mathrm{C}$ lower than the $\mathrm{CB} / \mathrm{GNP}$ composite (BA5G5L). This may indicate (as discussed later in Chapters 8 and 13) that CNT increases polymer chain mobility. The graphs of the heatflow versus time for all formulations are shown in Appendix E. 


\section{4: In-Plane Electrical Resistivity of Extruded Rods}

Extruded rods of each formulation were measured according to the procedure given in section 4.2.7. The average volume electrical resistivity of the extruded rods for the $\mathrm{CB} / \mathrm{PC}$ composites are given in Table 5.14. This table indicates the difference between the 2-9-09 screw design used for the majority of the formulations and the 10-30-09 screw design that was optimized for dispersion of the $\mathrm{CB}$ into PC. It also provides a baseline for the CB masterbatch material created at MTU for multiple filler formulations. Results for the individual samples are given in Appendix F.

Table 5.14: Average In-Plane Volume Electrical Resistivity of CB/PC Extruded Rods

\begin{tabular}{|l|l|}
\hline Formulation & $\begin{array}{l}\text { Average Volume Electrical } \\
\text { Resistivity (ohm-cm) }\end{array}$ \\
\hline BA2L (10-30-09 screw) & $>4 \mathrm{E} 7$ \\
\hline BA2LR (2-9-09 screw) & $>4 \mathrm{E} 7$ \\
\hline BA3L (10-30-09 screw) & $608 \pm 291$ \\
\hline BA4L(10-30-09 screw) & $99.3 \pm 18.7$ \\
\hline BA5L(10-30-09 screw) & $26.8 \pm 3.3$ \\
\hline BA5LR (2-9-09 screw) & $63.0 \pm 7.1$ \\
\hline BA6L (10-30-09 screw) & $14.1 \pm 1.7$ \\
\hline BA8L (10-30-09 screw) & $6.0 \pm 0.2$ \\
\hline $\begin{array}{l}\text { BA8LR (April 14, 2010 } \\
\text { masterbatch made at MTU, } \\
\text { 10-30-09 screw) }\end{array}$ & $6.1 \pm 0.2$ \\
\hline BA10L (10-30-09 screw) & $3.3 \pm 0.1$ \\
\hline
\end{tabular}

It can be seen that for the $5 \mathrm{wt} \%$ CB filled samples, the 10-30-09 screw design offered a $57 \%$ decrease in volume ER versus the formulation made with the 2-9-09 screw design. The further decrease in volume ER is due to better dispersion of the CB agglomerates provided by the optimized 10-30-09 screw design. This good dispersion allows the 8 
wt $\%$ CB msterbatch to be mixed with the pure PC and other fillers using the 2-9-09 screw design while maintaining sufficient $\mathrm{CB}$ dispersion. The data also shows that the $\mathrm{CB} / \mathrm{PC}$ composites percolate between 2 and $3 \mathrm{wt} \%$ filler.

Table 5.15 shows the average volume electrical resistivity of the extruded rods for the $\mathrm{GNP} / \mathrm{PC}$ formulations as well as a baseline for the as-received GNP masterbatch to determine the effect of further extrusion on the volume electrical resistivity of the material. Results for the individual samples are again given in Appendix F.

Table 5.15: Average In-Plane Volume Electrical Resistivity of GNP/PC Extruded Rods

\begin{tabular}{|l|l|}
\hline Formulation & $\begin{array}{l}\text { Average Volume Electrical } \\
\text { Resistivity }(\text { ohm-cm })\end{array}$ \\
\hline BG2L & $>8 \mathrm{E} 7$ \\
\hline BG3L & $>8 \mathrm{E} 7$ \\
\hline BG4L & $>8 \mathrm{E} 7$ \\
\hline BG5L & $>8 \mathrm{E} 7$ \\
\hline BG6L & $>8 \mathrm{E} 7$ \\
\hline BG8L & $5.7 \mathrm{E} 4 \pm 6.5 \mathrm{E} 3$ \\
\hline BG10L & $3.1 \mathrm{E} 3 \pm 2.2 \mathrm{E} 2$ \\
\hline BG12L & $610.0 \pm 56.9$ \\
\hline BG15L & $194.5 \pm 15.3$ \\
\hline $\begin{array}{l}\text { PC1515B Ovation } \\
\text { as received pellets }\end{array}$ & $149.5 \pm 47.8$ \\
\hline
\end{tabular}

This data shows that the further extrusion of the GNP masterbatch increases the volume electrical resistivity by $30 \%$. This is likely due to the degradation of the flake-like GNP particles. It can also be seen that the GNP/PC composites percolate between 6 and 8 wt $\%$, significantly higher than the $\mathrm{CB} / \mathrm{PC}$ composites.

The average in-plane volume electrical resistivity of the multiple filler formulations is given in Table 5.16. Results for the individual samples are given in Appendix F. 
Table 5.16: Average In-Plane Volume Electrical Resistivity of CB/CNT, CB/GNP and GNP/CNT Extruded Rods

\begin{tabular}{|l|l|}
\hline Formulation & $\begin{array}{l}\text { Volume Electrical } \\
\text { Resistivity }(\mathrm{ohm}-\mathrm{cm})\end{array}$ \\
\hline BA2Q1L & $300 \pm 60 \mathrm{n}=5$ \\
\hline BA2Q5L & $4.79 \pm 0.13 \mathrm{n}=10$ \\
\hline BA5Q1L & $18.23 \pm 0.93 \mathrm{n}=10$ \\
\hline BA5Q5L & $2.91 \pm 0.11 \mathrm{n}=10$ \\
\hline BA2G2L & $>$ E7 $\mathrm{n}=6$ \\
\hline BA2G5L & $692 \pm 114 \mathrm{n}=8$ \\
\hline BA5G2L & $22.1 \pm 2.0 \mathrm{n}=8$ \\
\hline BA5G5L & $11.2 \pm 0.4 \mathrm{n}=10$ \\
\hline BQ1G2L & $>\mathrm{E} 7 \mathrm{n}=6$ \\
\hline BQ1G5L & $1009 \pm 235.6 \mathrm{n}=6$ \\
\hline BQ5G2L & $4.91 \pm 0.18 \mathrm{n}=10$ \\
\hline BQ5G5L & $2.57 \pm 0.86 \mathrm{n}=9$ \\
\hline
\end{tabular}

It can be seen from this data that CNT has the largest effect on decreasing the volume electrical resistivity. When comparing $5 \mathrm{wt} \% \mathrm{CNT}$ with $2 \mathrm{wt} \%$ GNP (BQ5G2L) and 5 wt $\%$ CB and $2 \mathrm{wt} \%$ GNP (BA5G2L) it can be seen that the formulation containing CNT as opposed to $\mathrm{CB}$ has a $78 \%$ lower volume ER. When comparing the BQ5G5L and BA5G5L, we see that the CNT containing formulation has a 77\% lower volume ER than the $\mathrm{CB}$ containing formulation. It can also be seen that GNP has the least effect on lowering the composite volume ER. When comparing two formulations with $7 \mathrm{wt} \%$ carbon filler (BA5G2L and BA2G5L), we see when the majority filler is CB (BA5G2L), the volume ER is $97 \%$ lower than when the majority filler is GNP (BA2G5L). 


\section{Chapter 6: Electrical Resistivity, Thermal Conductivity and Tensile and Flexural Properties of Carbon Nanotube/Polycarbonate Composites}

\section{1: Field Emission Scanning Electron Microscope (FESEM) and Transmission Electron Microscopy Results}

An FESEM image of a BQ6L fracture surface is shown as Figure 6.1. CNT can be seen as white fibers. The distributed, interconnecting structure of CNT indicates that conductive networks are being formed, enhancing electrical conductivity. TEM images of BQ3L (a) and BQ6L (b) are shown in Figure 6.2. This figure shows the dispersion of the CNT within the PC matrix. It can be seen that the $6 \mathrm{wt} \%$ composite has significant conductive networking present.

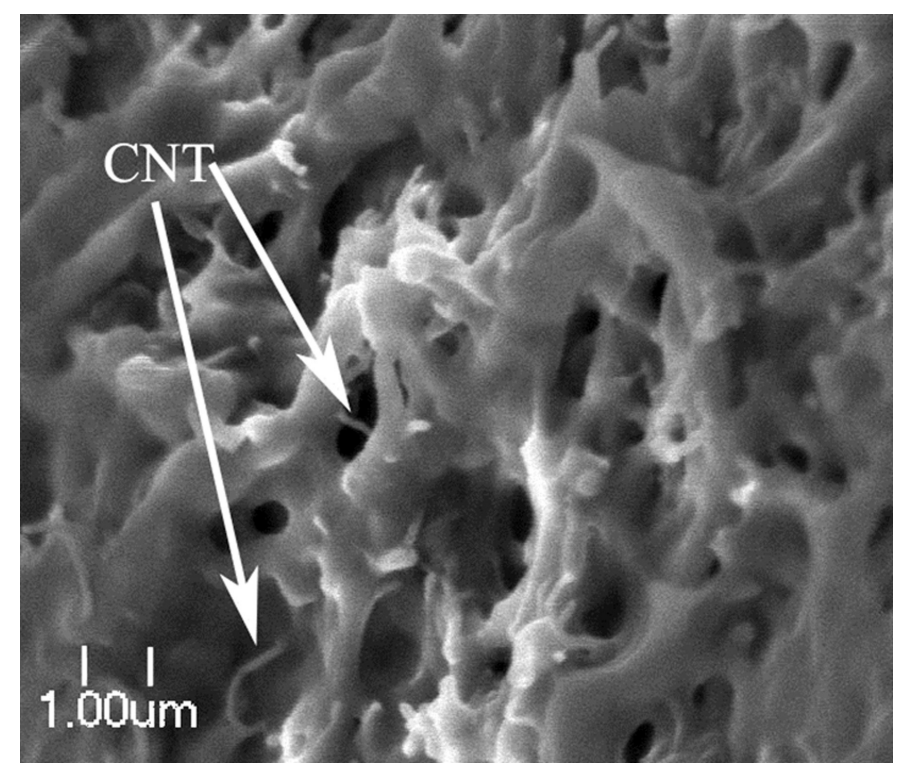

Figure 6.1: FESEM image of $6 \mathrm{wt} \% \mathrm{CNT}$ in PC fracture surface 

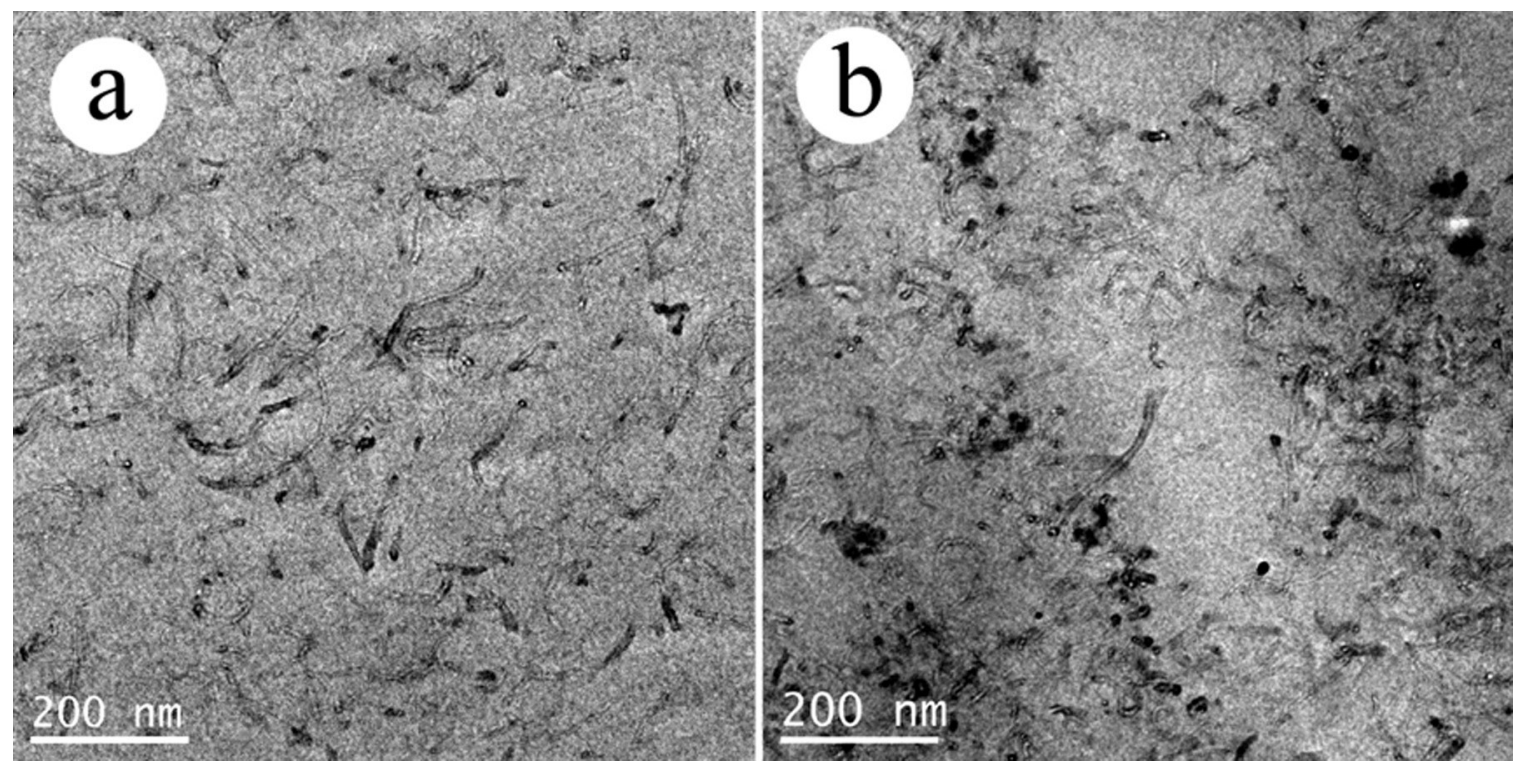

Figure 6.2: TEM images of $3 \mathrm{wt} \% \mathrm{CNT}$ in PC (a) and $6 \mathrm{wt} \% \mathrm{CNT}$ in PC (b)

\section{2: Electrical Resistivity Results}

Table 6.1 shows the mean electrical resistivity, standard deviation of the electrical resistivity and number of samples that were tested for each formulation. This data is plotted in Figure 6.3. This data is also given in Appendices G and H. We see a typical electrical resistivity curve in this figure. At low conductive filler loadings, the electrical resistivity of the composite will be similar to that of the pure polymer. As the loading of the conductive filler increases, there is a point at which conductive networks begin to form and the resistivity decreases dramatically over a very small range of filler loading. This point is known as the percolation threshold. After percolation occurs, the electrical resistivity of the composite levels out as additional conductive filler is added $(7,60)$. 
Table 6.1: Electrical resistivity results for CNT/PC composites

\begin{tabular}{|l|l|l|ll|}
\hline Formulation & Filler Wt \% & Filler Vol \% & Electrical Resistivity (ohm-cm) \\
\hline PC & 0 & 0.0 & $1.06 \times 10^{17} \pm 7.96 \times 10^{16}$ & $\mathrm{n}=7$ \\
\hline $2 \mathrm{CNT}$ & 2 & 1.38 & $4610 \pm 1120$ & $\mathrm{n}=6$ \\
\hline $3 \mathrm{CNT}$ & 3 & 2.08 & $216 \pm 44$ & $\mathrm{n}=6$ \\
\hline $4 \mathrm{CNT}$ & 4 & 2.78 & $73 \pm 10$ & $\mathrm{n}=6$ \\
\hline $5 \mathrm{CNT}$ & 5 & 3.48 & $43 \pm 7$ & $\mathrm{n}=6$ \\
\hline $6 \mathrm{CNT}$ & 6 & 4.19 & $18 \pm 2$ & $\mathrm{n}=6$ \\
\hline $8 \mathrm{CNT}$ & 8 & 5.63 & $7.8 \pm 0.4$ & $\mathrm{n}=6$ \\
\hline
\end{tabular}

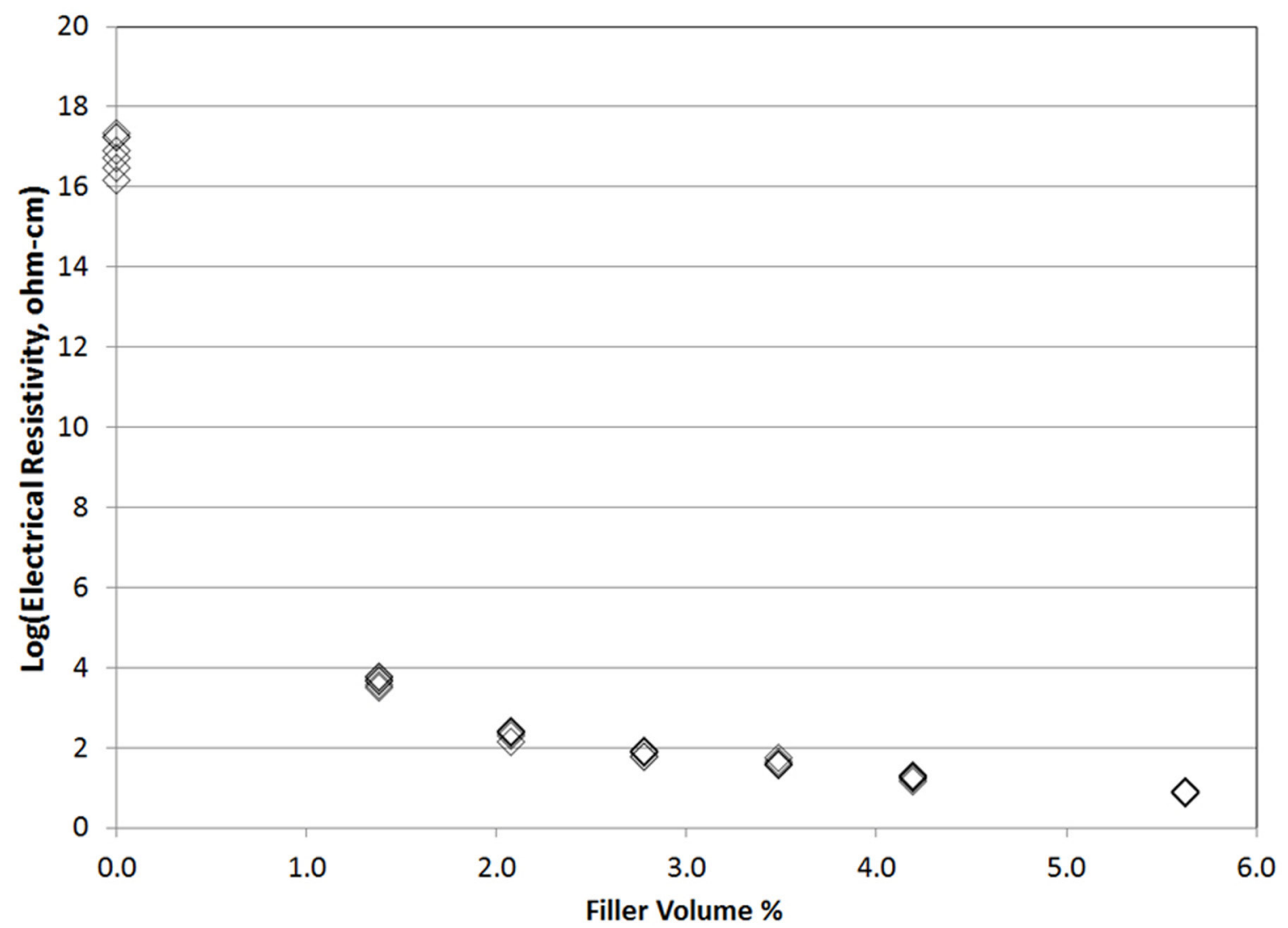

Figure 6.3: $\log ($ electrical resistivity) versus filler volume for CNT/PC composites

It can be seen from Figure 6.3 that CNT is effective at decreasing the electrical resistivity of composites, even at low filler loadings. The pure polymer begins at a electrical resistivity of $1.1 \times 10^{17} \mathrm{ohm}-\mathrm{cm}$, a value that coincides with data provided by the vendor. The percolation threshold occurs somewhere below 1.4 vol\% CNT for this system as the 
$2 \mathrm{wt} \%$ (1.4 vol\%) formulation is already percolated. At the highest filler loading studied, $8 \mathrm{wt} \%$ (5.6 vol\%) CNT, the electrical resistivity had dropped all the way down to $8 \mathrm{ohm}-$ cm. A low percolation threshold is found in this system likely due to the extreme geometry of the CNT. Low percolation thresholds have often been found for CNT-based polycarbonate systems, both single-walled and multi-walled (61-64).

\section{3: Thermal Conductivity Results}

The results of through-plane thermal conductivity testing on the CNT composites are shown in Figure 6.4 and listed in Table 6.2. More detailed results are contained in Appendix I. We see that CNT does increase the through-plane thermal conductivity of the composite from $0.22 \mathrm{~W} / \mathrm{m} \cdot \mathrm{K}$ for pure PC to $0.31 \mathrm{~W} / \mathrm{m} \cdot \mathrm{K}$ for $8 \mathrm{wt} \%$ CNT composites. This increase is similar to that reported by King et al. for CNT in polypropylene (17) but are lower than those reported by Lee et al. for a vulcanized silicone filled with CNT (65).

Table 6.2: Thermal conductivity results for CNT/PC composites

\begin{tabular}{|c|c|c|}
\hline Formulation & Filler Wt \% & $\begin{array}{c}\text { Thermal Conductivity } \\
\text { (W/m/K) }\end{array}$ \\
\hline PC & 0 & $0.218 \pm 0.002 \mathrm{n}=5$ \\
\hline $2 \mathrm{CNT}$ & 2 & $0.232 \pm 0.002 \mathrm{n}=5$ \\
\hline $3 \mathrm{CNT}$ & 3 & $0.241 \pm 0.003 \mathrm{n}=5$ \\
\hline $4 \mathrm{CNT}$ & 4 & $0.255 \pm 0.001 \quad \mathrm{n}=5$ \\
\hline $5 \mathrm{CNT}$ & 5 & $0.266 \pm 0.003 \quad \mathrm{n}=5$ \\
\hline $6 \mathrm{CNT}$ & 6 & $0.275 \pm 0.003 \quad \mathrm{n}=5$ \\
\hline $8 \mathrm{CNT}$ & 8 & $0.306 \pm 0.003 \quad \mathrm{n}=5$ \\
\hline
\end{tabular}




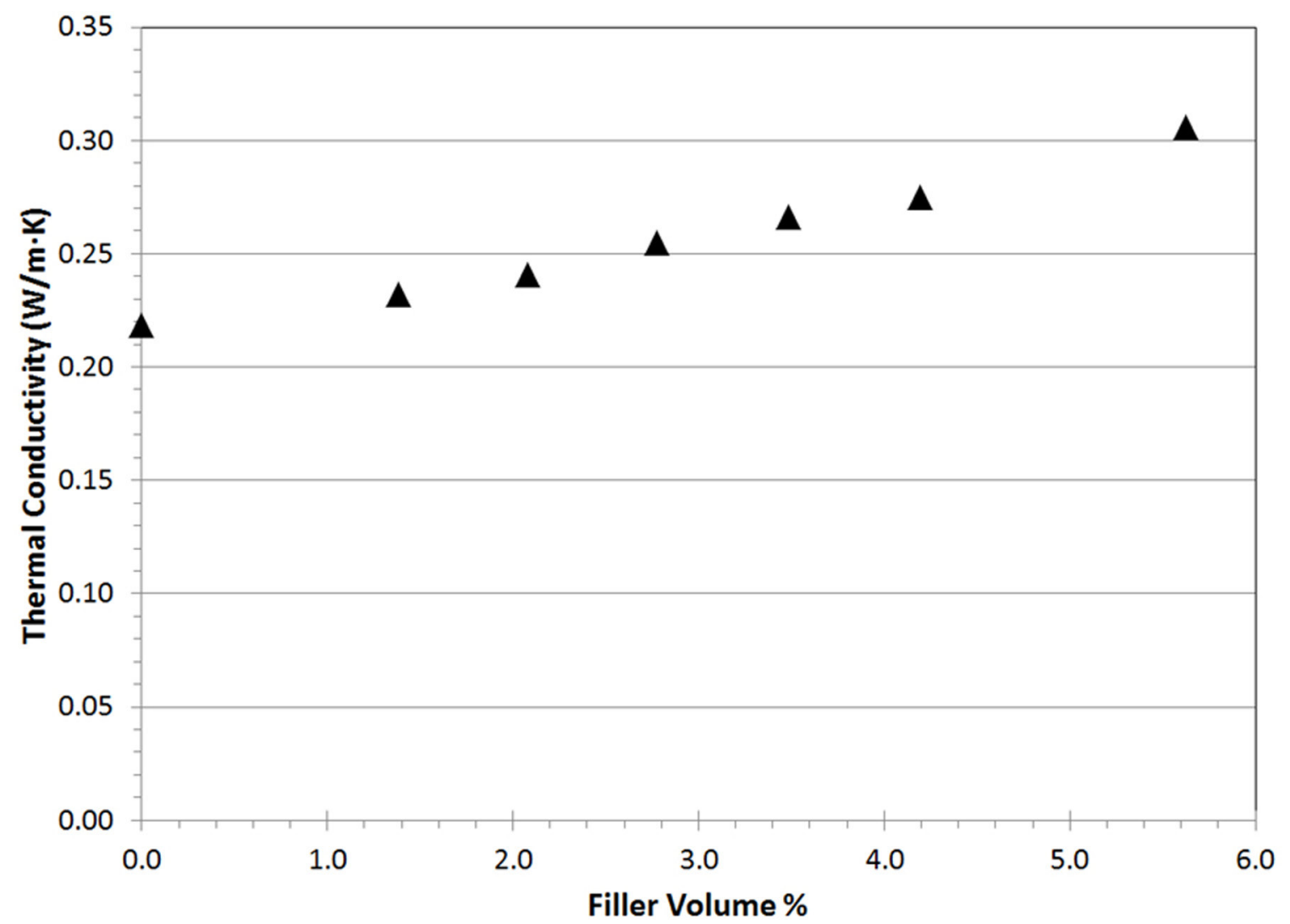

Figure 6.4: Thermal conductivity versus filler volume for CNT/PC composites

\section{4: Tensile Test Results}

The tensile modulus of the CNT based composites is shown in Figure 6.5. The associated ultimate tensile strengths (UTS) and the strains at the ultimate tensile strength are shown in Figure 6.6. Both of these figures display the mean value along with error bars representing one standard deviation. In cases where the error bars are smaller than the marker size, they have been omitted. It is shown in Figure 6.5 that the addition of 8 wt $\%$ (5.6 vol\%) CNT increased the tensile modulus of the composite from $2.2 \mathrm{GPa}$ for neat PC to $2.9 \mathrm{GPa}$. Figure 6.6 has omitted the results for the $8 \mathrm{wt} \% \mathrm{CNT}$ composite as this composite broke prematurely. It is likely that the $8 \mathrm{wt} \% \mathrm{CNT}$ composites were not injection molded optimally as the resin is very viscous and difficult to melt-process at this 
filler loading. For the samples with $\leq 6 \mathrm{wt} \% \mathrm{CNT}$, we see that the UTS tends to remain constant at around $60 \mathrm{MPa}$, meanwhile strain at UTS generally decreases with increased CNT loading. This trend agrees with previously reported trends for single-walled and multi-walled CNT in various polymer systems $(61,66,67)$. Figure 6.7 shows typical tensile stress-strain curves for the formulations created in this study with up to $6 \mathrm{wt} \%$ CNT including neat PC. As the stress increases, we initially see a linear Hookean response that can be used to calculate a tensile modulus. Following this initial linear response, all of the composites illustrated demonstrate a deviation below this line that indicates ductile yielding behavior. Detailed results for this data are listed in Appendix J.

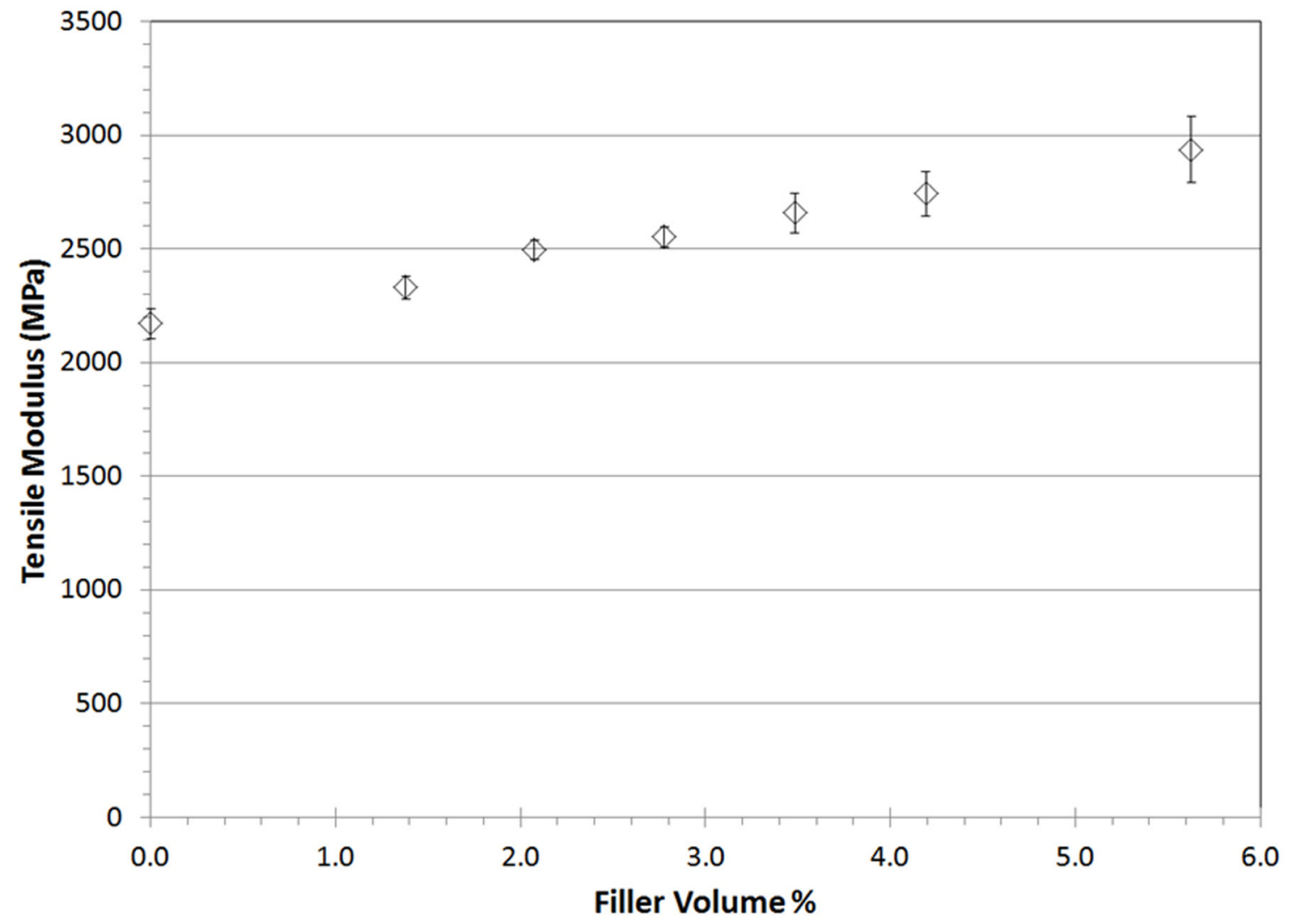

Figure 6.5: Tensile modulus of CNT/PC composites 


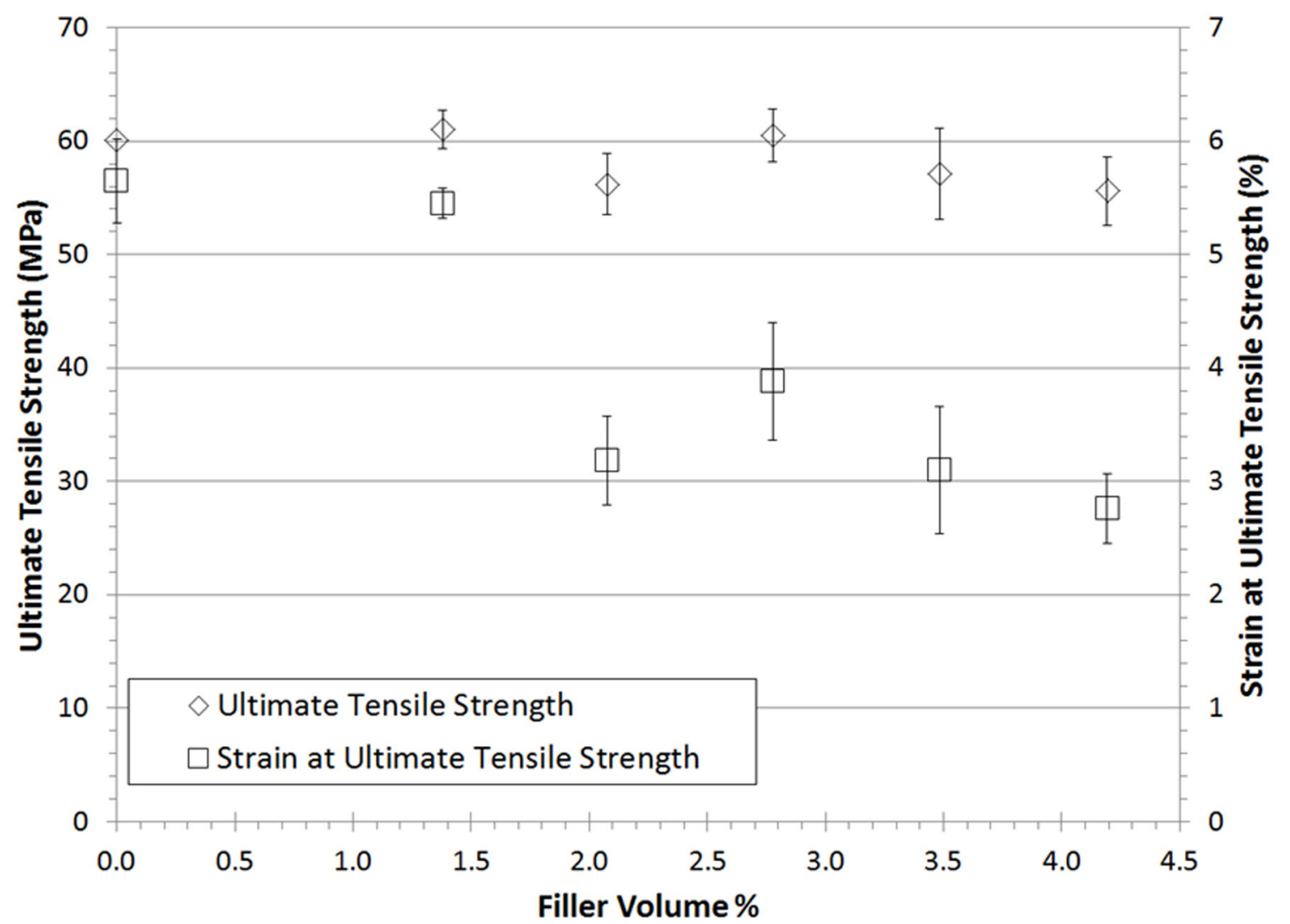

Figure 6.6: Ultimate tensile strength and strain at ultimate tensile strength for CNT/PC composites

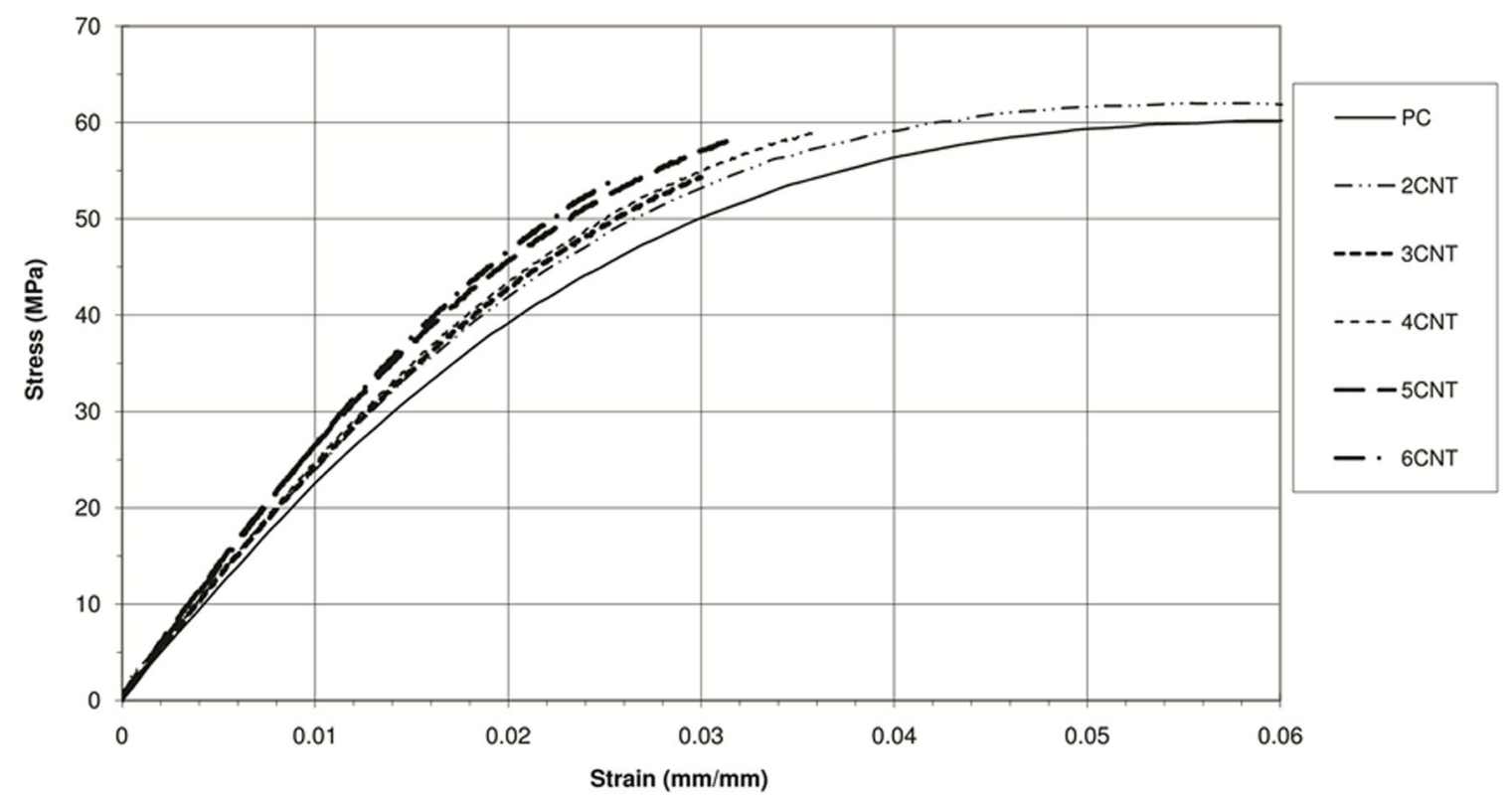

Figure 6.7: Typical stress-strain curves for CNT/PC composites 


\section{5: Flexural Test Results}

The flexural modulus of the CNT based composites is shown in Figure 6.8. The associated ultimate flexural strengths (UFS) and the strains at the ultimate flexural strength are shown in Figure 6.9. Both of these figures display the mean value along with error bars representing one standard deviation. In cases where the error bars are smaller than the marker size, they have been omitted. Similarly to the tensile modulus, the flexural modulus increases with increased CNT loading. From a pure PC value of 3.0 $\mathrm{GPa}$, the flexural modulus is increased to $3.6 \mathrm{GPa}$ for the $8 \mathrm{wt} \%$ (5.6 vol\%) $\mathrm{CNT}$ composite. In Figure 6.9 that we have again omitted the values for $8 \mathrm{wt} \% \mathrm{CNT}$ composites for the same reasons as discussed for tensile results. In this figure we do see a difference from the tensile results however. The addition of CNT increased the UFS from a value of $115 \mathrm{MPa}$ for the pure PC to $125 \mathrm{MPa}$ for a $6 \mathrm{wt} \%$ (4.2 vol\%) CNT composite. The strain at which this UFS was reached was approximately the same for all loading levels. Detailed results for this data are listed in Appendix K. 


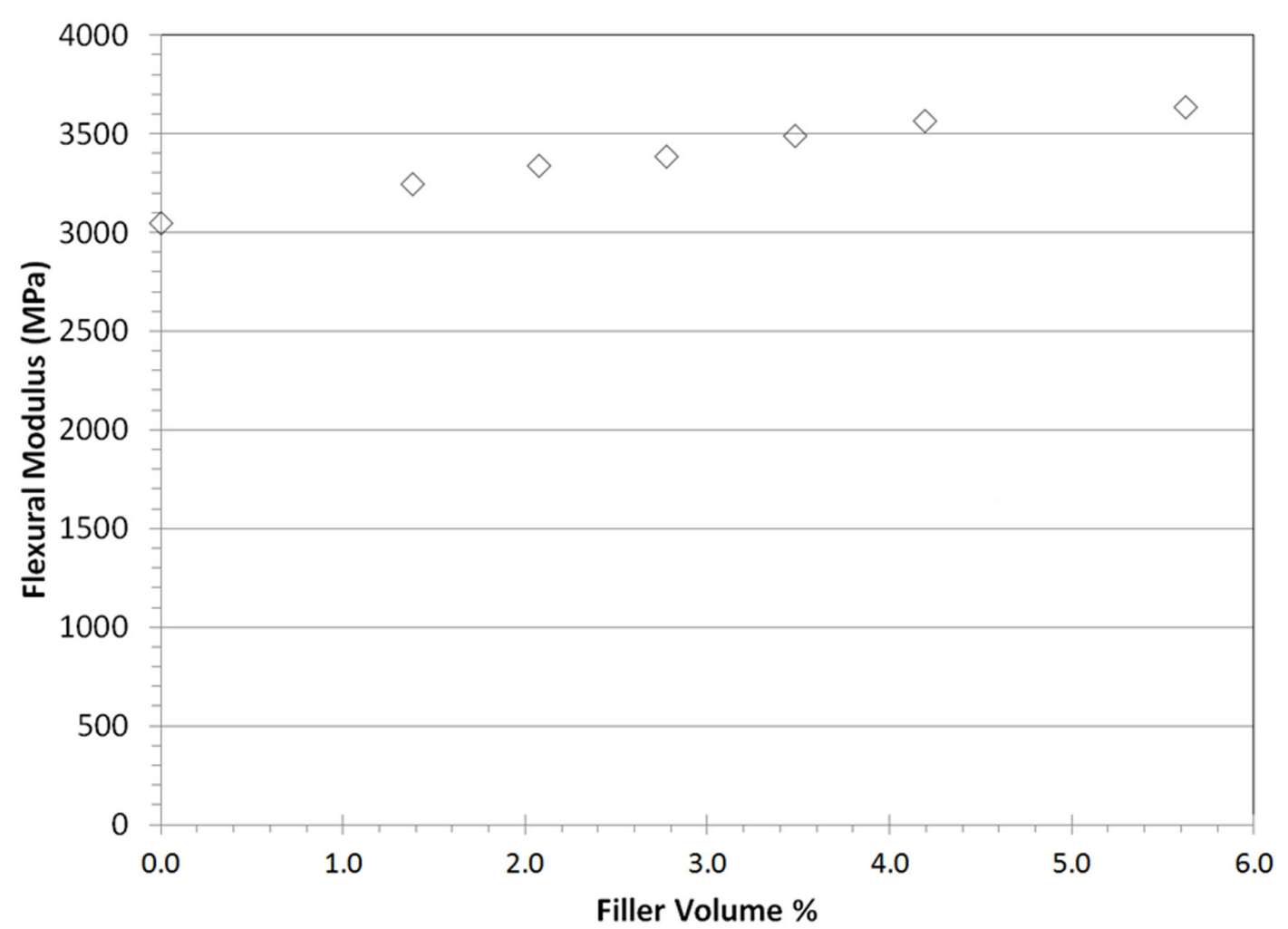

Figure 6.8: Flexural modulus of CNT/PC composites

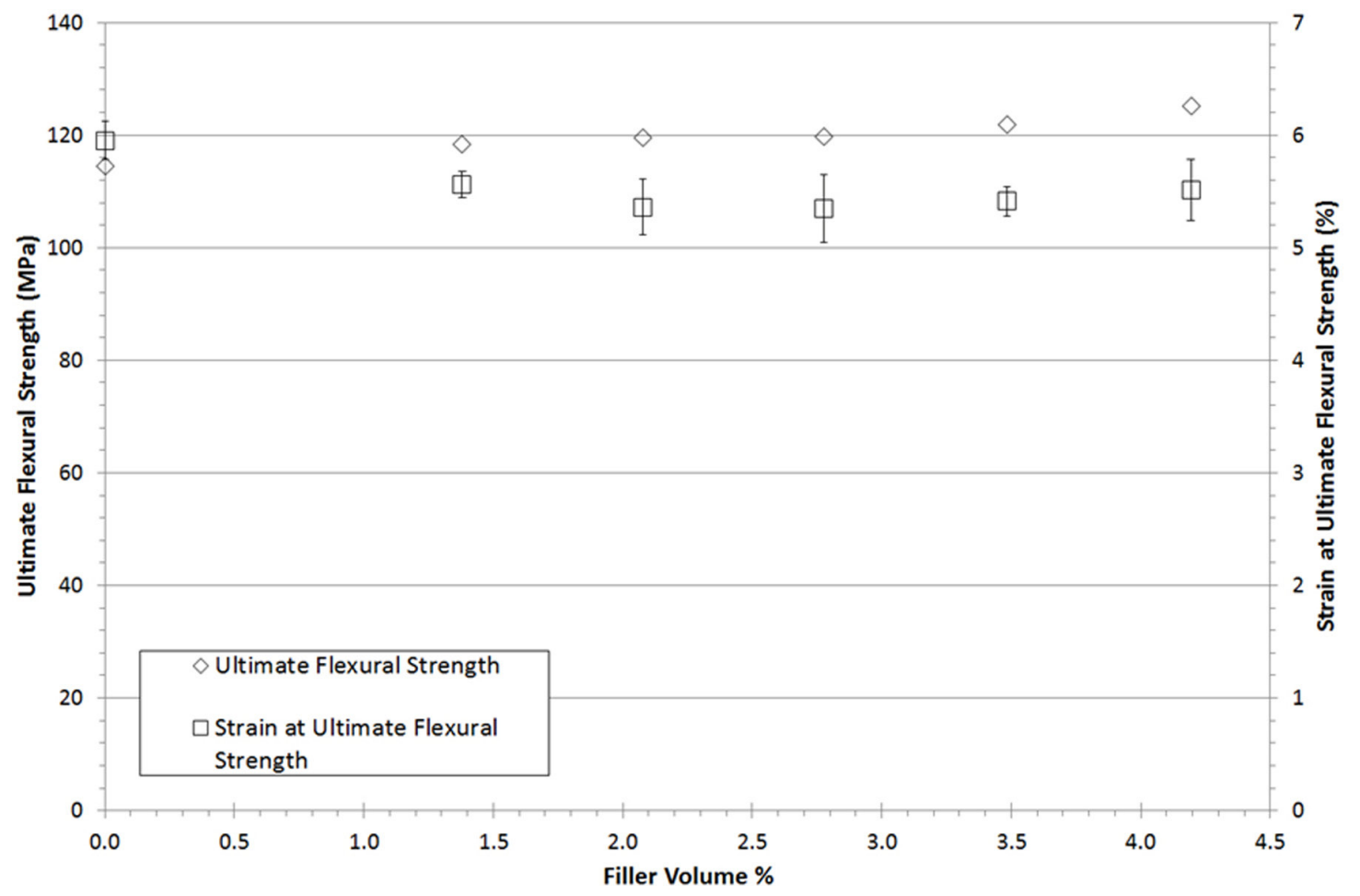

Figure 6.9: Ultimate flexural strength and strain at ultimate flexural strength for CNT/PC composites 


\section{6: Conclusions}

The purpose of this section of the project was to determine the effects of CNT on the electrical, thermal and mechanical properties of PC. Electrically, CNT exhibits a percolation threshold in PC somewhere below 1.4 vol\% CNT. This low percolation threshold is due to the high aspect ratio of the CNT. The addition of $5.6 \mathrm{vol} \%(8 \mathrm{wt} \%)$ CNT to PC reduced the ER of the composite from $1.1 \times 10^{17} \mathrm{ohm}-\mathrm{cm}$ (ER of neat PC) to 8 ohm-cm. Thermally, the addition of $5.6 \mathrm{vol} \% \mathrm{CNT}$ increased the TC from $0.22 \mathrm{~W} / \mathrm{m} \cdot \mathrm{K}$ for neat $\mathrm{PC}$ to $0.31 \mathrm{~W} / \mathrm{m} \cdot \mathrm{K}$.

Mechanically, the addition of $5.6 \mathrm{vol} \%(8 \mathrm{wt} \%) \mathrm{CNT}$ to PC increased the tensile modulus from 2.2 $\mathrm{GPa}$ for neat $\mathrm{PC}$ to $2.9 \mathrm{GPa}$. The ultimate tensile strength (UTS) was largely unchanged ranging from 56 to $62 \mathrm{MPa}$ for all filler loadings. The strain at UTS decreased with increased CNT loadings. The CNT/PC composites retained ductile behavior for all formulations with up to $6 \mathrm{wt} \% \mathrm{CNT}$. The addition of $5.6 \mathrm{vol} \%(8 \mathrm{wt} \%)$ CNT to PC increased the flexural modulus from 3.0 GPa for neat PC to 3.6 GPa. The ultimate flexural strength (UFS) increased with increased CNT loading changing from $115 \mathrm{MPa}$ for neat PC to $125 \mathrm{MPa}$ for $6 \mathrm{wt} \%(4.2 \mathrm{vol} \%) \mathrm{CNT}$. The strain at UTS stayed roughly the same for all CNT loadings around $5.7 \%$. 


\section{Chapter 7: Electrical Resisitivity, Thermal Conductivity and Tensile and Flexural Properties of Carbon Black/Polycarbonate Composites and Comparison with Carbon Nanotube/Polycarbonate Composites}

\section{1: Field Emission Scanning Electron Microscope (FESEM) Results}

An FESEM image of a $6 \mathrm{wt} \% \mathrm{CB}$ composite is shown in Figure 7.1. In this figure $\mathrm{CB}$ can be seen as white spheres that form an extended network, this is an expected result due to the high structure of $\mathrm{CB}$ and the proximity of the $\mathrm{CB}$ particles to each other (14). The FESEM image for CNT has been discussed in Chapter 6.

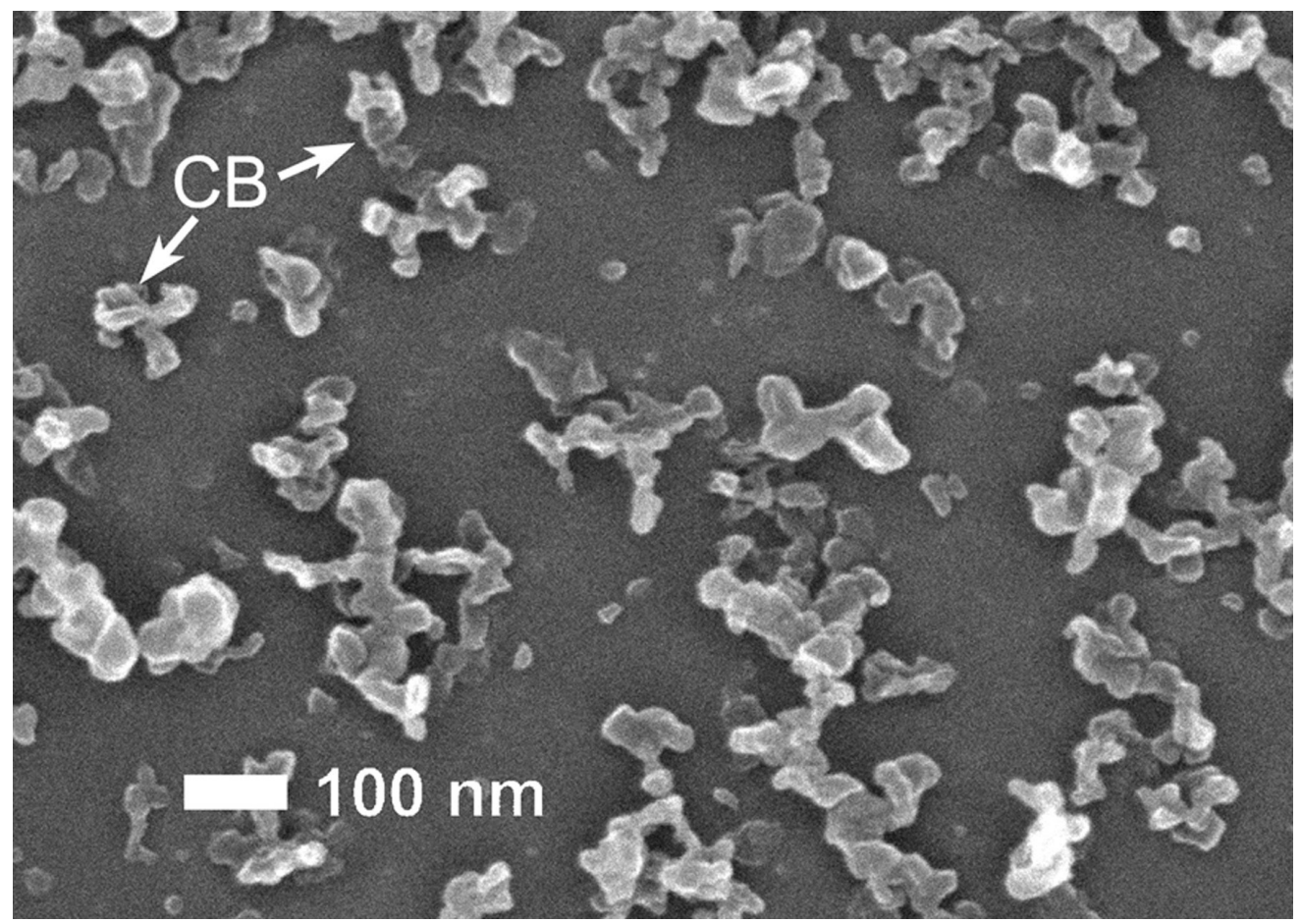

Figure 7.1: FESEM image of $6 \mathrm{wt} \% \mathrm{CB}$ in $\mathrm{PC}$ 


\section{2: Electrical Resistivity Results}

Table 7.1 shows the mean electrical resistivity, standard deviation of the electrical resistivity and number of samples that were tested for each CB-based formulation. Results for the individual values are found in Appendices G and H. This data is plotted in Figure 7.2 along with the CNT data from Chapter 6 for comparison. The CNT data shown has additional $0.5 \mathrm{wt} \%$ and $1 \mathrm{wt} \%$ CNT loadings. These loadings were added to better locate the percolation threshold of CNT (about 1.0 vol\%). In Figure 7.2 we again see a typical electrical resistivity curve similar to that of CNT.

Table 7.1: Electrical resistivity results for $\mathrm{CB} / \mathrm{PC}$ and $\mathrm{CNT} / \mathrm{PC}$ composites

\begin{tabular}{|c|c|c|c|}
\hline Formulation & $\begin{array}{l}\text { Filler } \\
\mathrm{Wt} \%\end{array}$ & $\begin{array}{l}\text { Filler } \\
\text { Vol \% }\end{array}$ & $\begin{array}{l}\text { Electrical Resistivity } \\
\text { (ohm-cm) }\end{array}$ \\
\hline $\mathrm{PC}$ & 0 & 0.0 & $1.26 \times 10^{17} \pm 3.35 \times 10^{16} \mathrm{n}=6$ \\
\hline $2 \mathrm{CB}$ & 2 & 1.34 & $4.05 \times 10^{16} \pm 2.66 \times 10^{16} \mathrm{n}=6$ \\
\hline $3 \mathrm{CB}$ & 3 & 2.01 & $2.85 \times 10^{15} \pm 4.58 \times 10^{14} \mathrm{n}=6$ \\
\hline $4 \mathrm{CB}$ & 4 & 2.69 & $1.17 \times 10^{5} \pm 7.77 \times 10^{4} \quad \mathrm{n}=8$ \\
\hline $5 \mathrm{CB}$ & 5 & 3.38 & $2474 \pm 646$ \\
\hline $6 \mathrm{CB}$ & 6 & 4.07 & $649 \pm 18$ \\
\hline $8 \mathrm{CB}$ & 8 & 5.46 & $122 \pm 4$ \\
\hline $10 \mathrm{CB}$ & 10 & 6.88 & $19.5 \pm 0.5$ \\
\hline $0.5 \mathrm{CNT}$ & 0.5 & 0.34 & $6.19 \times 10^{16} \pm 1.21 \times 10^{16} \mathrm{n}=6$ \\
\hline $1 \mathrm{CNT}$ & 1 & 0.69 & $2.02 \times 10^{16} \pm 6.62 \times 10^{15} \mathrm{n}=6$ \\
\hline $2 \mathrm{CNT}$ & 2 & 1.38 & $4610 \pm 1120$ \\
\hline $3 \mathrm{CNT}$ & 3 & 2.08 & $216 \pm 44$ \\
\hline $4 \mathrm{CNT}$ & 4 & 2.78 & $73 \pm 10$ \\
\hline $5 \mathrm{CNT}$ & 5 & 3.48 & $43 \pm 7$ \\
\hline $6 \mathrm{CNT}$ & 6 & 4.19 & $18 \pm 2$ \\
\hline $8 \mathrm{CNT}$ & 8 & 5.63 & $7.8 \pm 0.4$ \\
\hline
\end{tabular}




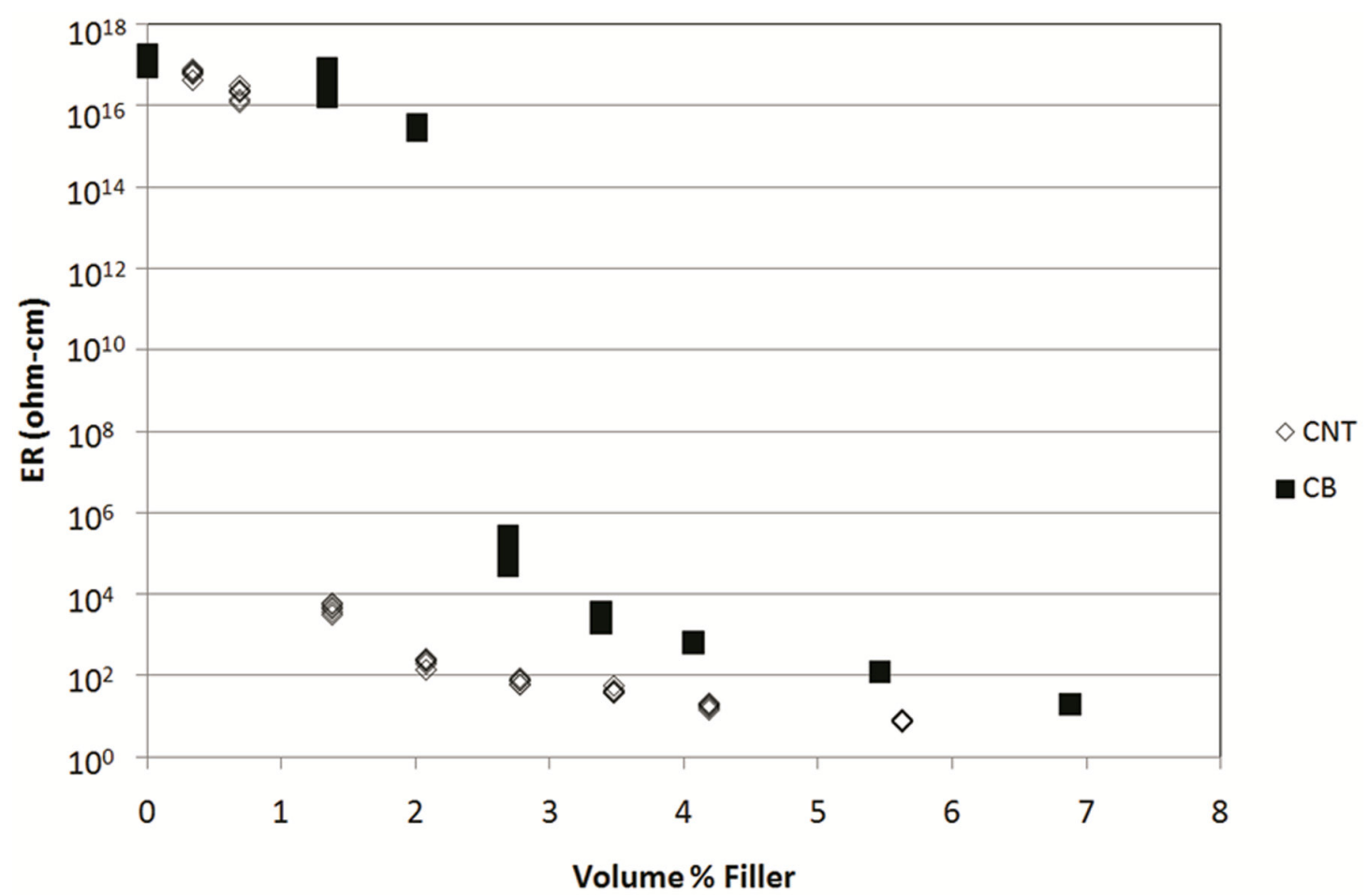

Figure 7.2: Electrical resistivity (ER) versus filler volume for $\mathrm{CB} / \mathrm{PC}$ and $\mathrm{CNT} / \mathrm{PC}$ composites

We note that $\mathrm{CB}$ is also effective at decreasing electrical resistivity at low filler loadings. The percolation threshold lies at approximately $2.3 \mathrm{vol} \%$. At the highest concentration made, $10 \mathrm{wt} \%$ (6.9 vol\%), the composite has an electrical resistivity of $20 \mathrm{ohm}-\mathrm{cm}$. If we compare these results with the CNT results from Chapter 6, we see that the percolation threshold for CB is above that of CNT ( 2.3 vol\% versus 1.0 vol\%). We also see that CNT typically shows a lower electrical resistivity than $\mathrm{CB}$ at equal volume loadings of filler. For example, at 5.6 vol $\% \mathrm{CNT}$, the electrical resistivity is $8 \mathrm{ohm}-\mathrm{cm}$. At $5.5 \mathrm{vol} \%$ $\mathrm{CB}$, the electrical resistivity is $122 \mathrm{ohm}-\mathrm{cm}$. The lower percolation threshold for CNT likely is a result of its high aspect ratio of 1000 compared to the spherical CB particles. This higher aspect ratio is also the likely cause of CNT's lower electrical resistivity at equivalent volume filler loadings. 
CB is a commonly studied filler for PC systems (7,68-72). At similar volume filler loadings, the $\mathrm{CB} / \mathrm{PC}$ composite we made are more conductive than Tchoudakov et al., Ezquerra et al, Narkis et al., Lee et al., and Potschke et al. for CB in PC (7,68-71). One primary difference that may account for the difference in electrical conductivity is the processing method. This project used a small commercial scale twin-screw extruder and commercial scale injection molder. The use of these devices allow for an easy scale-up to a large-scale production facility as twin-screw extruders and injection molders are more cost effective and commercially viable than their alternatives. The use of injection molding also encourages orientation of any agglomerates of $\mathrm{CB}$ in the flow direction, decreasing ER in that direction. These other studies will be described here in more detail.

Potschke et al. studied a Mitsubishi Engineering Plastics polycarbonate filled with Cabot Corporation Vulcan XC-72 electrically conductive carbon black. The project used a small scale conical co-rotating twin-screw extruder with a $4.5 \mathrm{~cm}^{3}$ capacity and used a compression molder to create $0.35 \mathrm{~mm}$ thick test samples (69). For $10 \mathrm{wt} \% \mathrm{CB}$ composites, Potschke's research group obtained a value of $10^{8} \mathrm{ohm}-\mathrm{cm}$. In our project, we obtained $20 \mathrm{ohm}-\mathrm{cm}$ for a $10 \mathrm{wt} \% \mathrm{CB}$ in PC composite.

Lee et al. studied SAMYANG Co. TRIREX 3022 polycarbonate filled with Korea Carbon Black co. Hiblack 30 electrically conductive carbon black. This project created $25 \mathrm{~mm}$ diameter disks with $1.5 \mathrm{~mm}$ thickness in a compression molding process (68). At $10 \mathrm{wt} \% \mathrm{CB}$, Lee's team found an ER of $10^{15} \mathrm{ohm}-\mathrm{cm}$. Again at $10 \mathrm{wt} \% \mathrm{CB}$, our project found an ER value of $20 \mathrm{ohm}-\mathrm{cm}$. 
Ezquerra et al. studied a Bayer polycarbonate filled with Philps Petroleum XE2 electrically conductive carbon black. This project compounded the composite in a small scale mechanical mixer at $50 \mathrm{rpm}$ for 10 minutes (71). This composite was then compression molded into test specimens. Ezquerra's group found an ER of $10^{4} \mathrm{ohm}-\mathrm{cm}$ for $6.4 \mathrm{vol} \% \mathrm{CB}$. Our project found an ER of $20 \mathrm{ohm}-\mathrm{cm}$ for $6.9 \mathrm{vol} \% \mathrm{CB}$ in PC.

Tchoudakov et al. studied GE Plastics (now SABIC Innovative Plastics) Lexan 103 polycarbonate filled with Akzo Ketjenblack EC carbon black. This project used a small scale mechanical mixer and compression molder to create test samples (70). Tchodakov's group obtained an ER of $10^{7} \mathrm{ohm}-\mathrm{cm}$ for an $8 \mathrm{wt} \% \mathrm{CB}$ composite compared to our value of $122 \mathrm{ohm}-\mathrm{cm}$ for the same loading.

Narkis et al. studied a SABIC Innovative Plastics Lexan filled with Kejenblack EC-600 JD carbon black, the same CB studied in our project. Narkis et al. used a twin-screw extruder and injection molder to obtain test specimens (7). Narkis's team obtained an ER value of $10^{8} \mathrm{ohm}-\mathrm{cm}$ for $6 \mathrm{wt} \% \mathrm{CB}$ compared to our result of $650 \mathrm{ohm}-\mathrm{cm}$ for the same CB loading.

Electrical resistivity of composites is very sensitive to processing conditions and filler/matrix interactions. Based upon comparison with similar systems, our combination of Lexan HF1130-111 and Ketjenblack EC-600 JD along with larger scale twin-screw extruding and injection molding provides very good electrical resistivity results and indicate that commercial applications may be more viable than what is suggested by small scale fabrication. 


\section{3: Thermal Conductivity Results}

Figure 7.2 shows mean through-plane thermal conductivity results for CNT/PC and $\mathrm{CB} / \mathrm{PC}$. These results correspond to the values listed in Tables 6.2 and 7.2. Detailed results for this test are given in Appendix I.

We see in Figure 7.3 that $\mathrm{CB}$ increases the thermal conductivity of the PC composite from $0.21 \mathrm{~W} / \mathrm{m} \cdot \mathrm{K}$ for pure $\mathrm{PC}$ to $0.29 \mathrm{~W} / \mathrm{m} \cdot \mathrm{K}$ for $10 \mathrm{wt} \%(6.9 \mathrm{vol} \%) \mathrm{CB}$ in $\mathrm{PC}$. This result compares well to previous studies of this $\mathrm{CB}$ in polypropylene and Vectra A950RX liquid crystal polymer performed by this research group $(73,74)$. When compared to CNT values, we see that on an equal vol\% filler basis that CNT increases thermal conductivity slightly more than $\mathrm{CB}$ for this system. 
Table 7.2: Thermal conductivity results for $\mathrm{CB} / \mathrm{PC}$ and $\mathrm{CNT} / \mathrm{PC}$ composites

\begin{tabular}{|c|c|c|}
\hline Formulation & $\begin{array}{c}\text { Filler } \\
\text { Wt \% }\end{array}$ & $\begin{array}{c}\text { Thermal Conductivity } \\
(\mathbf{W} / \mathbf{m} \cdot \mathbf{K})\end{array}$ \\
\hline PC & 0 & $0.214 \pm 0.001 \mathrm{n}=5$ \\
\hline $2 \mathrm{CB}$ & 2 & $0.228 \pm 0.001 \mathrm{n}=5$ \\
\hline $3 \mathrm{CB}$ & 3 & $0.234 \pm 0.002 \quad \mathrm{n}=4$ \\
\hline $4 \mathrm{CB}$ & 4 & $0.245 \pm 0.001 \quad \mathrm{n}=4$ \\
\hline $5 \mathrm{CB}$ & 5 & $0.254 \pm 0.001 \quad \mathrm{n}=4$ \\
\hline $6 \mathrm{CB}$ & 6 & $0.260 \pm 0.001 \quad \mathrm{n}=4$ \\
\hline $8 \mathrm{CB}$ & 8 & $0.275 \pm 0.003 \quad \mathrm{n}=4$ \\
\hline $10 \mathrm{CB}$ & 10 & $0.291 \pm 0.003 \quad \mathrm{n}=4$ \\
\hline & & \\
\hline $0.5 \mathrm{CNT}$ & 0.5 & $0.218 \pm 0.002 \mathrm{n}=4$ \\
\hline $1 \mathrm{CNT}$ & 1 & $0.225 \pm 0.005 \mathrm{n}=4$ \\
\hline $2 \mathrm{CNT}$ & 2 & $0.232 \pm 0.002 \quad \mathrm{n}=5$ \\
\hline $3 \mathrm{CNT}$ & 3 & $0.241 \pm 0.003 \quad \mathrm{n}=5$ \\
\hline $4 \mathrm{CNT}$ & 4 & $0.255 \pm 0.001 \quad \mathrm{n}=5$ \\
\hline $5 \mathrm{CNT}$ & 5 & $0.266 \pm 0.003 \quad \mathrm{n}=5$ \\
\hline $6 \mathrm{CNT}$ & 6 & $0.275 \pm 0.003 \quad \mathrm{n}=5$ \\
\hline $8 \mathrm{CNT}$ & 8 & $0.306 \pm 0.003 \mathrm{n}=5$ \\
\hline
\end{tabular}




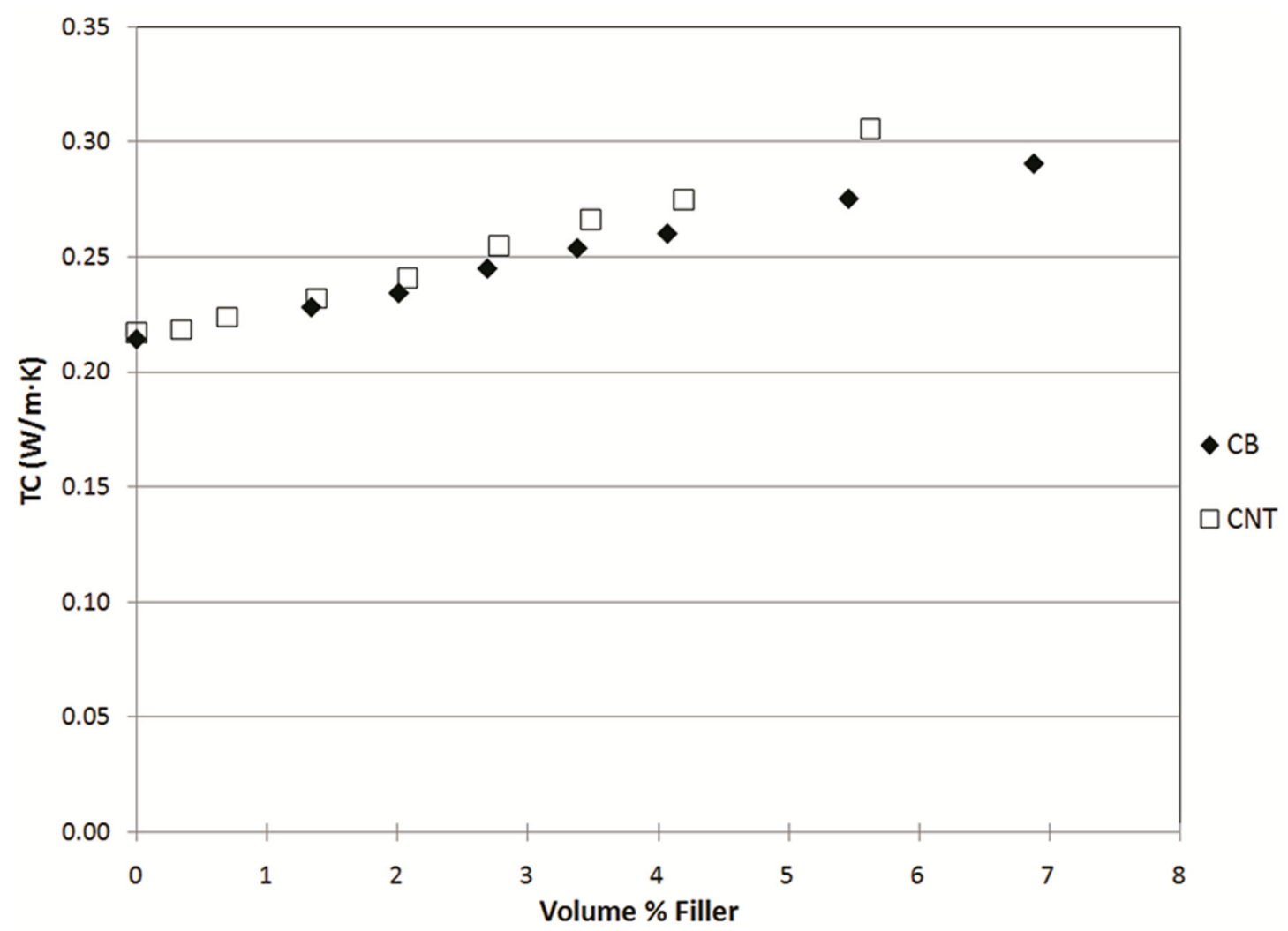

Figure 7.3: Thermal conductivity (TC) versus filler volume for $\mathrm{CB} / \mathrm{PC}$ and $\mathrm{CNT} / \mathrm{PC}$ composites

\section{4: Tensile Test Results}

The tensile moduli of the CB based and CNT based composites are shown in Figure 7.4. The associated ultimate tensile strengths (UTS) and the strains at the ultimate tensile strength are shown in Figure 7.4-2. Both of these figures display the mean value along with error bars representing one standard deviation. In cases where the error bars are smaller than the marker size, they have been omitted. It is shown in Figure 7.4-1 that the addition of $8 \mathrm{wt} \%(5.5 \mathrm{vol} \%) \mathrm{CB}$ increased the tensile modulus of the composite from 2.2 $\mathrm{GPa}$ for neat $\mathrm{PC}$ to $2.8 \mathrm{GPa}$. The increasing the filler loading to $10 \mathrm{wt} \%(6.9 \mathrm{vol} \%)$ increased the tensile modulus to $3.0 \mathrm{GPa}$. By comparing with the results from Section 
6.4 we see that $\mathrm{CB}$ and $\mathrm{CNT}$ have similar effects on the tensile modulus for similar volume loadings.

For Figure 7.5 we have omitted the 6.9 vol\% CB composite as the high viscosity of the composite made it very difficult to successfully injection mold. We can see from the figure that the UTS for CB composites stays roughly constant at 60 to $63 \mathrm{MPa}$ for all composites up to $8 \mathrm{wt} \% \mathrm{CB}$ while, similarly to CNT, the strain at UTS tends to decrease with increased CB loading. It is worth noting that in this study, CB provided a higher UTS than CNT for similar filler loadings. Detailed results for this data are listed in Appendix J.

Huang et al. found similar results while studying Miles Inc. Makrolon 2608 polycarbonate filled with Vulcan XR 72R carbon back (75). They utilized a $19 \mathrm{~mm}$ single screw extruder and 20 ton injection molding machine to fabricate specimens. Huang's group reported tensile strength increase from 59.3 MPa for a neat PC to 63.4 for a $5 \mathrm{wt} \% \mathrm{CB}$ composite. They also reported a $10 \%$ increase in tensile modulus over pure $\mathrm{PC}$ at $5 \mathrm{wt} \% \mathrm{CB}$.

Figure 7.6 illustrates a typical tensile stress-strain curve for $\mathrm{CB}$ systems up to $10 \mathrm{wt} \%$ CB. Similarly to the CNT system, CB shows ductile behavior for all the formulations shown as illustrated by the deviation of the data below the initial linear fit that represents the tensile modulus. 


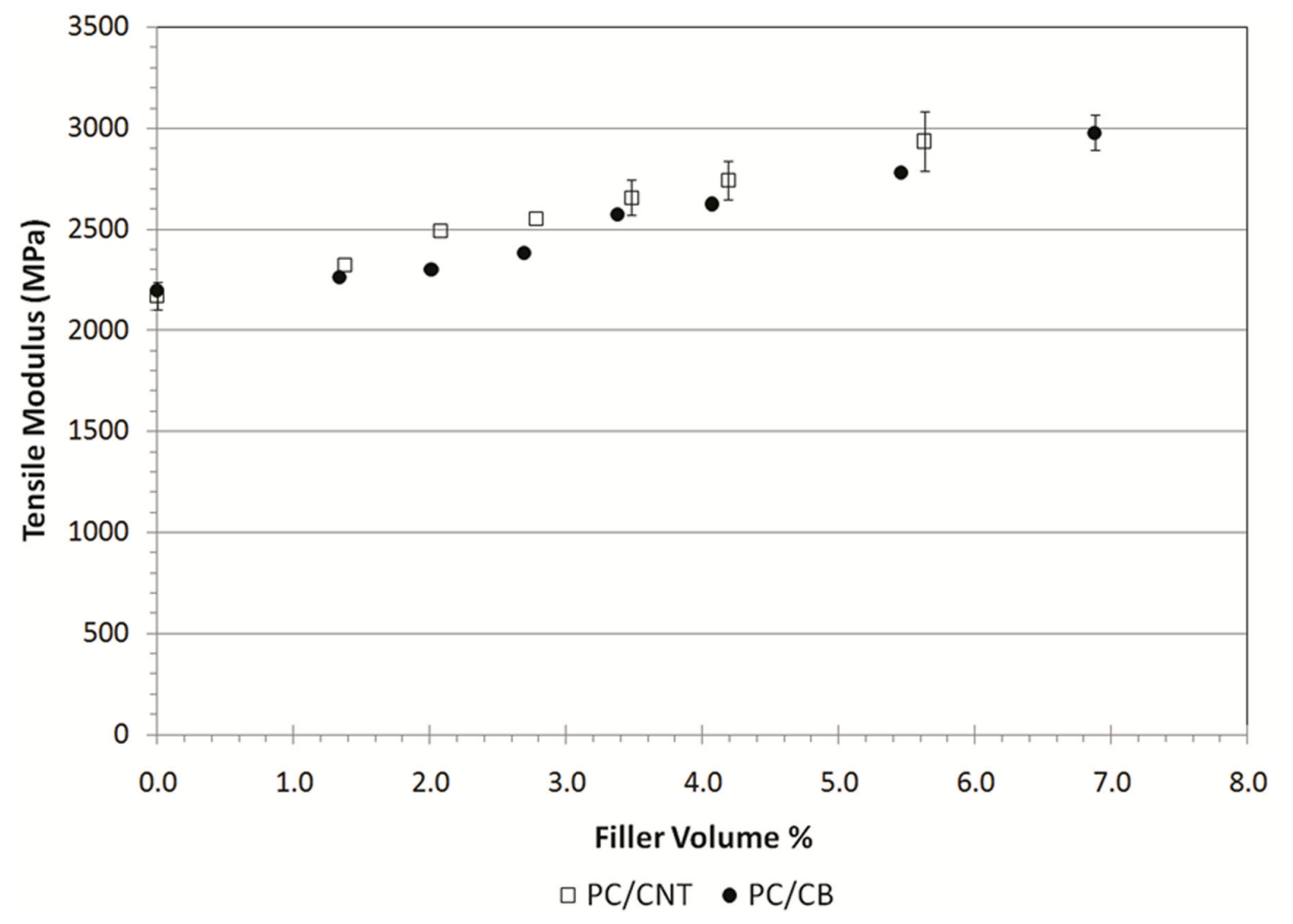

Figure 7.4: Tensile modulus of $\mathrm{CB} / \mathrm{PC}$ and $\mathrm{CNT} / \mathrm{PC}$ composites

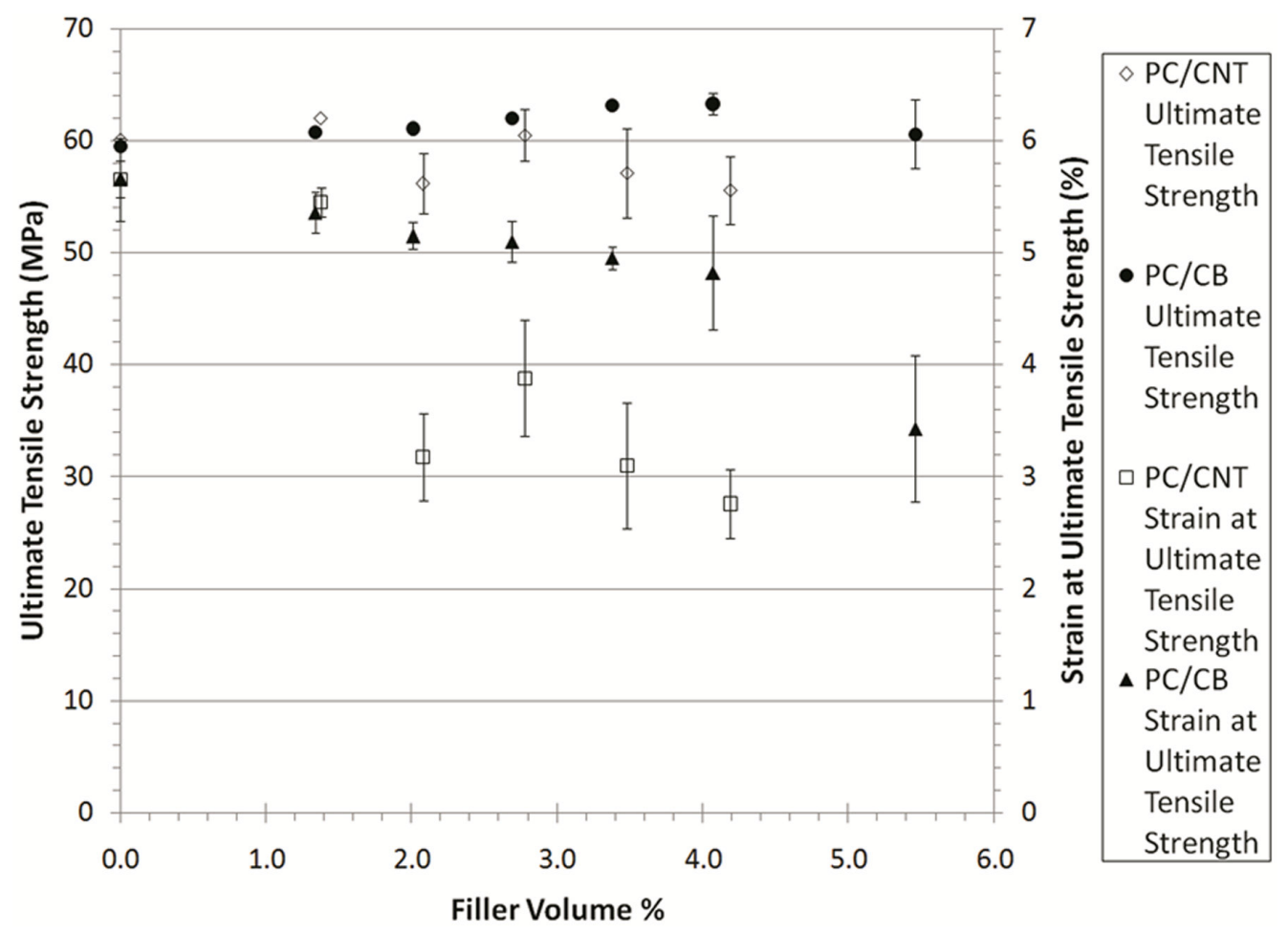

Figure 7.5: Ultimate tensile strength and strain at ultimate tensile strength for $\mathrm{CB} / \mathrm{PC}$ and $\mathrm{CNT} / \mathrm{PC}$ composites 


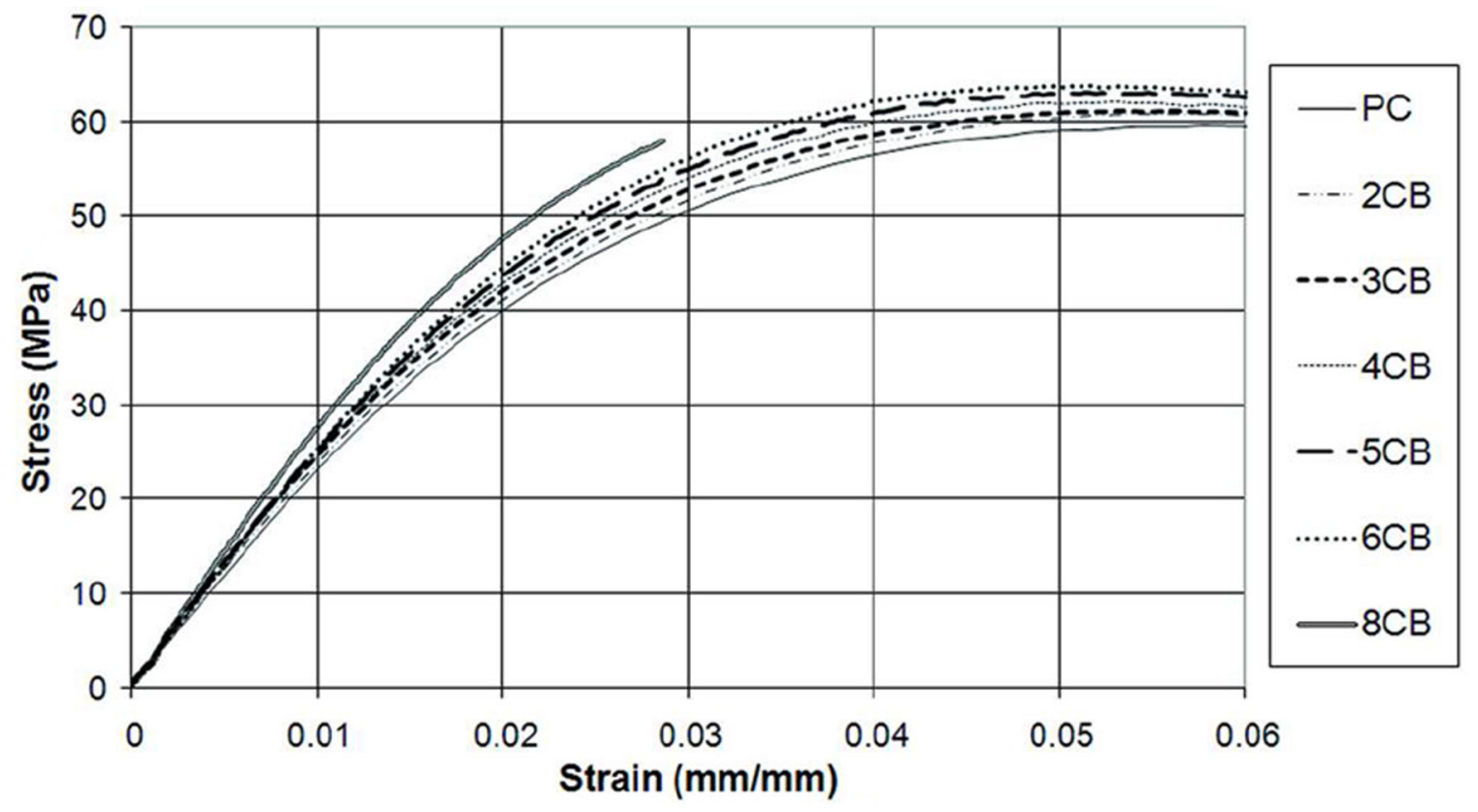

Figure 7.6: Typical stress-strain curves for $\mathrm{CB} / \mathrm{PC}$ composites

\section{5: Flexural Test Results}

The flexural modulus of the CB based composites is shown in Figure 7.7. The associated ultimate flexural strengths (UFS) and the strains at the ultimate flexural strength are shown in Figure 7.5-2. Both of these figures display the mean value along with error bars representing one standard deviation. In cases where the error bars are smaller than the marker size, they have been omitted. Similarly to the tensile modulus, the flexural modulus increases with increased CB loading. The flexural modulus increases from 2.6 $\mathrm{GPa}$ for the pure PC to $3.4 \mathrm{GPa}$ for the $8 \mathrm{wt} \% \mathrm{CB}$. These values are very similar to the results obtained for CNT as can be seen in Figure 7.7.

Figure 7.8 omits the results for $10 \mathrm{wt} \% \mathrm{CB}$ as the sample broke prematurely. Looking at the other data in the figure, similarly to CNT, the addition on CB increases the UFS of the composites. The UFS increases from $108 \mathrm{MPa}$ for pure PC to $116 \mathrm{MPa}$ for $8 \mathrm{wt} \%$ (5.5 vol\%) CB. The strain at UFS was fairly constant for all loading levels of CB around 
6.2\%. Detailed results for this data are given in Appendix K. It appears that the high structure and surface area of the $\mathrm{CB}$ allows it to form significant networks that allow for electrical conductivity and increase mechanical strengths. This trend has been seen before for $\mathrm{CB}$ in $\mathrm{PC}(75)$.

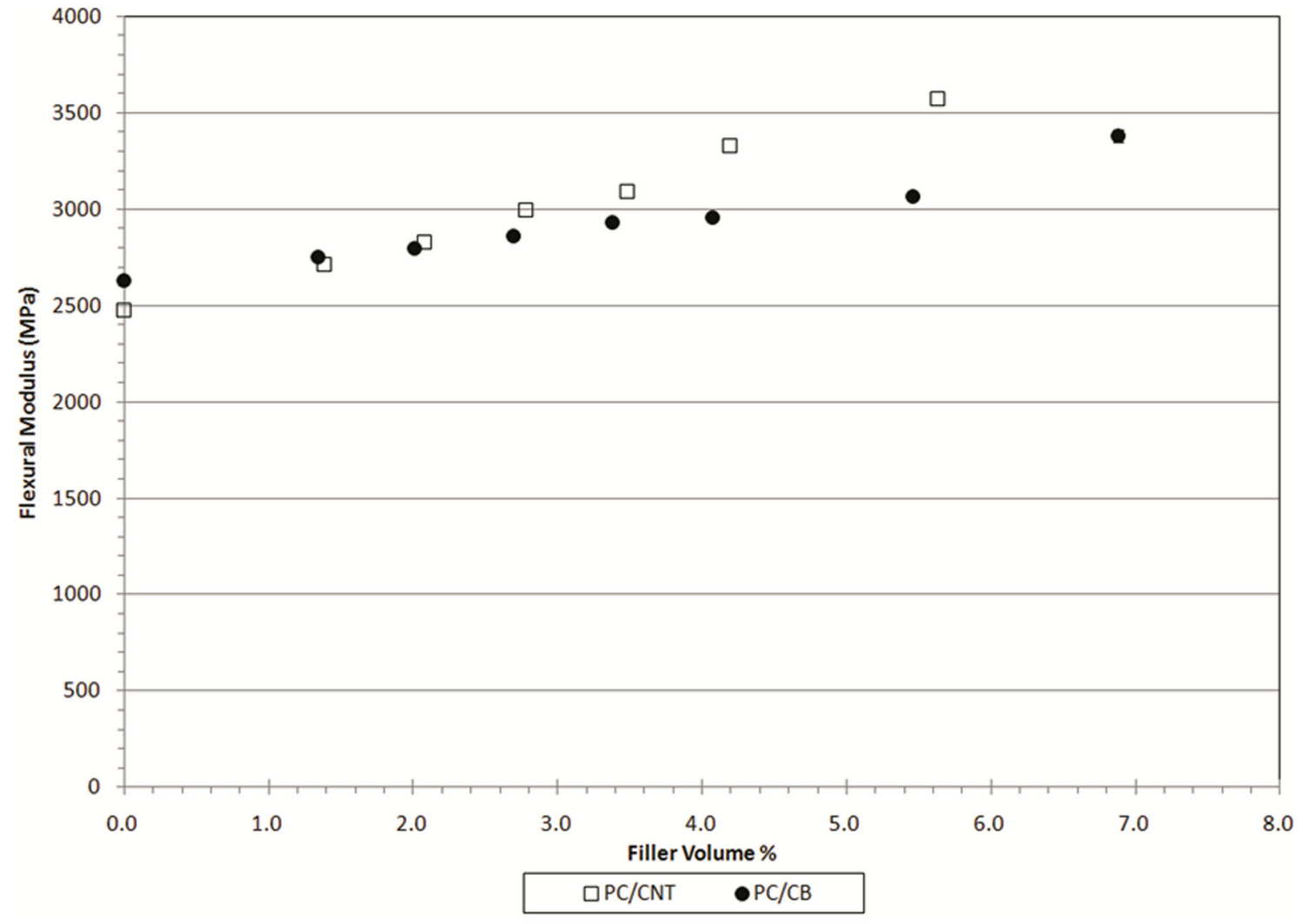

Figure 7.7: Flexural modulus of $\mathrm{CB} / \mathrm{PC}$ and $\mathrm{CNT} / \mathrm{PC}$ composites 


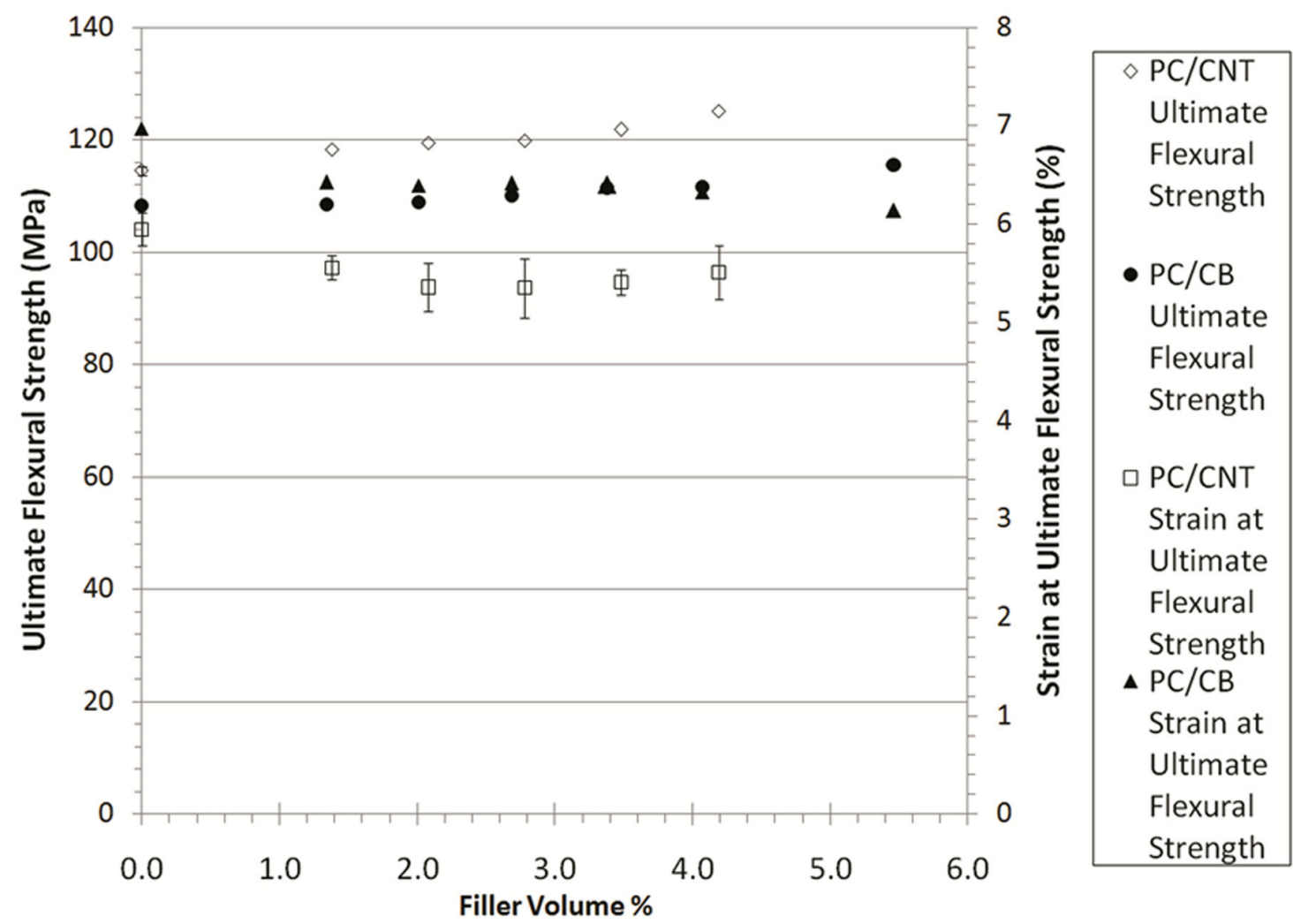

Figure 7.8: Ultimate flexural strength and strain at ultimate flexural strength for $\mathrm{CB} / \mathrm{PC}$ and $\mathrm{CNT} / \mathrm{PC}$ composites

\section{6: Conclusions}

The object of this section of the project was to measure the effects of carbon black on polycarbonate and compare these effects with those of carbon nanotubes as studied in Chapter 6. Electrically, we found the percolation threshold of CB in PC to be about 2.3 vol\% compared to a percolation threshold of about 1 vol \% for CNT in PC. CB decreased the electrical resistivity of PC from $1.3 \times 10^{17} \mathrm{ohm}-\mathrm{cm}$ for the neat PC to 20 ohm-cm for $10 \mathrm{wt} \%$ (6.9 vol\%) CB in PC. We see that CNT has a stronger effect on decreasing electrical resistivity in PC, CNT percolates at a lower filler volume (1.0 vol\% versus $2.3 \mathrm{vol} \%$ ) and has a lower resistivity when compared on an equal volume filler basis with CB (18 ohm-cm versus $649 \mathrm{ohm}-\mathrm{cm}$ for $\sim 4$ vol\%). For similar loading levels, 
these composites displayed a lower electrical conductivity than reported by others for $\mathrm{CB} / \mathrm{PC}$ composites $(7,19,34,37,42,75)$. The addition of $10 \mathrm{wt} \% \mathrm{CB}$ increased the thermal conductivity of PC from $0.21 \mathrm{~W} / \mathrm{m} \cdot \mathrm{K}$ for pure PC to $0.29 \mathrm{~W} / \mathrm{m} \cdot \mathrm{K}$, this is similar to the results for CNT/PC composites.

Mechanically, the addition of $10 \mathrm{wt} \%$ (6.9 vol\%) CB increased the tensile modulus of PC from 2.2 GPa for neat $\mathrm{PC}$ to $3.0 \mathrm{GPa}$. The ultimate tensile strength for all $\mathrm{CB}$ composites up to $8 \mathrm{wt} \%$ was approximately the same, 60 to $63 \mathrm{MPa}$. The strain at ultimate tensile strength decreased with increasing CB loading. Up to $8 \mathrm{wt} \% \mathrm{CB}$, all composites showed ductile behavior. The addition of $10 \mathrm{wt} \%$ ( $6.9 \mathrm{vol} \%) \mathrm{CB}$ increased the flexural modulus from 2.6 GPa for pure PC to $3.4 \mathrm{GPa}$. The ultimate flexural strength increased from $108 \mathrm{MPa}$ for pure PC to $116 \mathrm{MPa}$ for $8 \mathrm{wt} \%$ (5.5 vol\%) CB. The strain at ultimate flexural strength remained fairly constant (6.1 to 7\%) for all composites. All of these results are similar to those found for CNT. It is noteworthy that strain at ultimate tensile strength and ultimate flexural strength was higher in $\mathrm{CB} / \mathrm{PC}$ versus $\mathrm{CNT} / \mathrm{PC}$. 


\section{Chapter 8: Rheological Properties of Carbon Nanotube/Polycarbonate Composites and Carbon Black/Polycarbonate Composites}

\section{1: Transmission Electron Microscope (TEM) Results}

TEM images of CNT/PC composites were performed to get a better understanding of the morphology of the CNTs themselves and the conductive networks of CNTs as seen in the polymer matrix. Figure 8.1 shows the morphology of the CNTs within the PC matrix, it illustrates good dispersion of the CNT in $2 \mathrm{wt} \%$ and $8 \mathrm{wt} \% \mathrm{CNT}$ composites. Conductive networks of CNT are seen in the $8 \mathrm{wt} \%$ CNT sample.

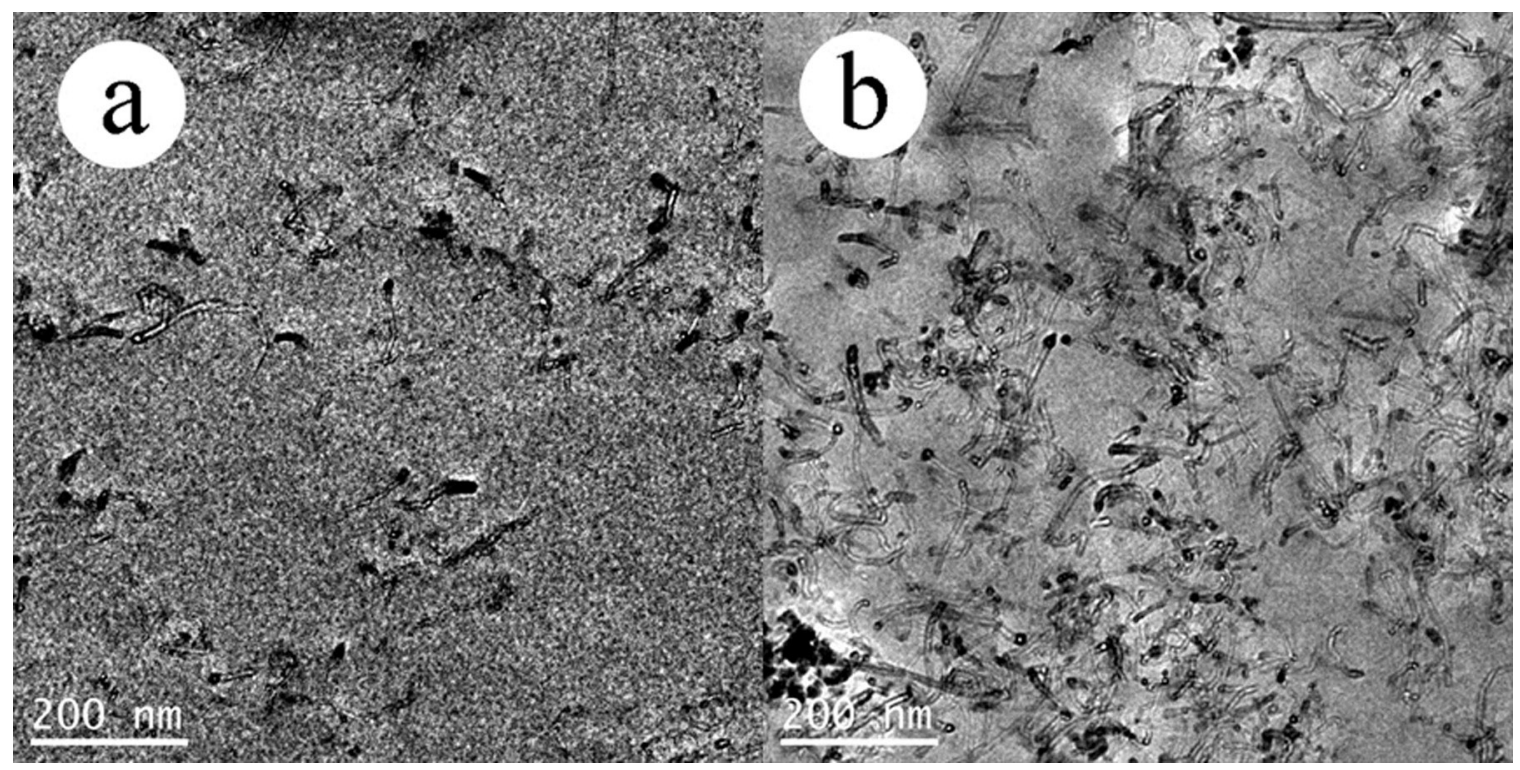

Figure 8.1: a.TEM image of $2 \mathrm{wt} \% \mathrm{CNT}$ in PC $\quad$ b. TEM image of $8 \mathrm{wt} \% \mathrm{CNT}$ in PC

\section{2: Small-Amplitude Oscillator Shear (SAOS) Results}

Figure 8.2 shows the SAOS master curves for the CNT/PC composites with the corresponding shift factors shown in Figure 8.3. The SAOS master curves and associated shift factors for the $\mathrm{CB} / \mathrm{PC}$ composites are shown in Figure 8.4 and Figure 8.5 
respectively. It can quickly be seen that in Figures 8.2 and 8.4 that the plots of the $\log \eta^{*}$ versus the $\log$ frequency show a slope of -1 for the filled systems. This behavior indicates the presence of a rheological yield stress (51). Detailed results for this data are given in Appendix L.

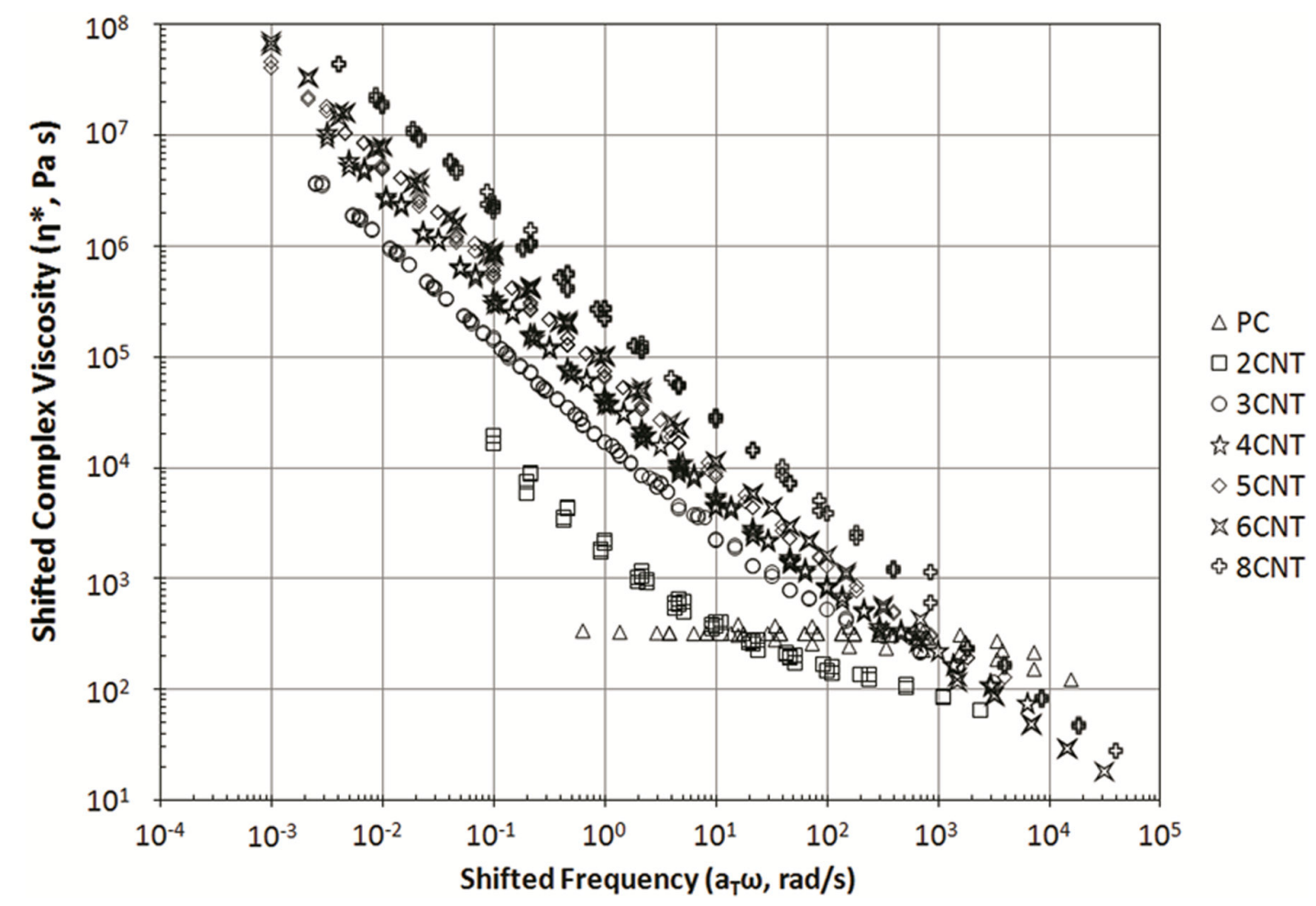

Figure 8.2: Time-temperature shifted complex viscosity as a function of shifted frequency for $\mathrm{CNT} / \mathrm{PC}$ studied at $270^{\circ} \mathrm{C}$ 


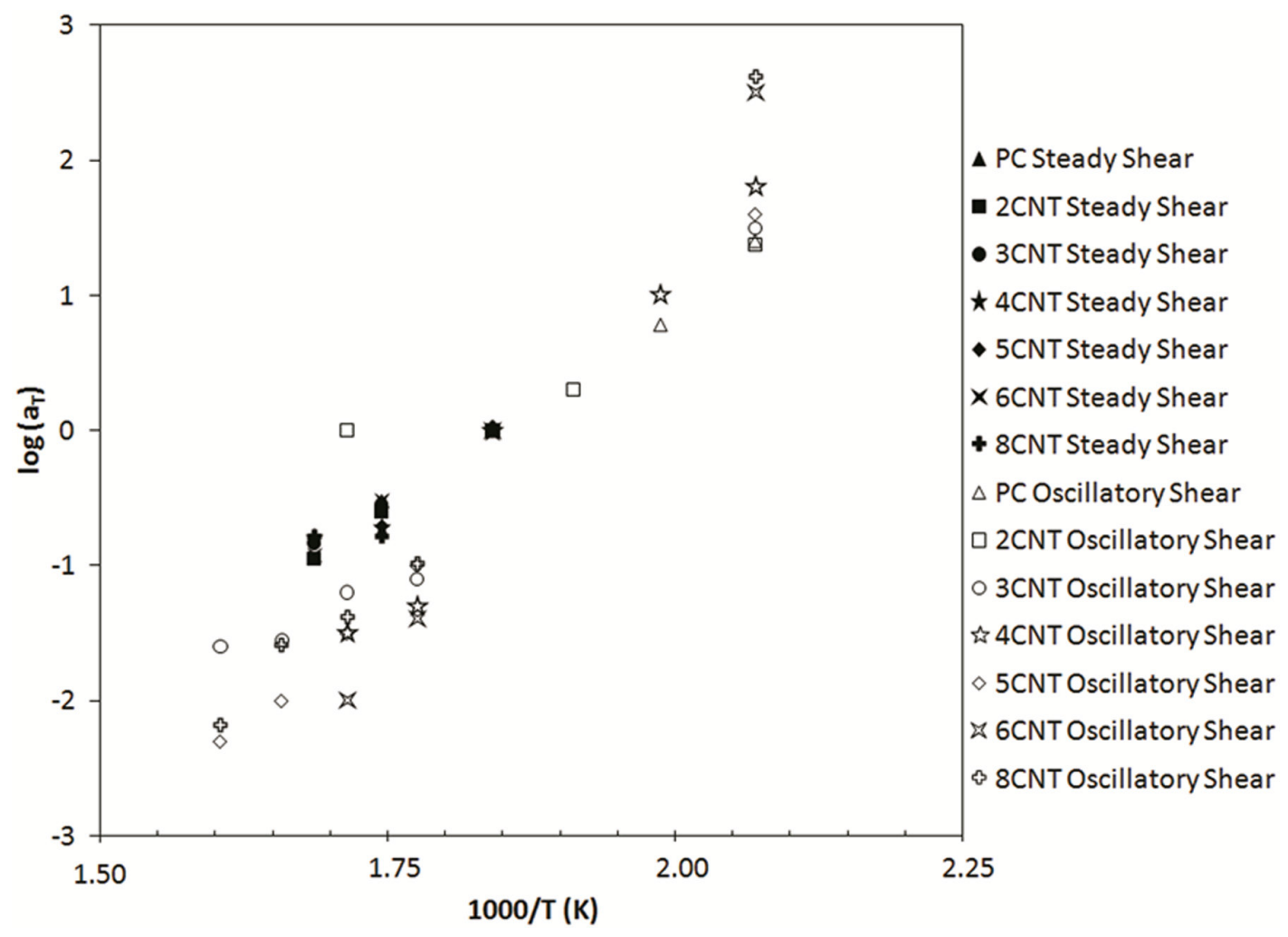

Figure 8.3: Time-temperature shift factors $a_{T}$ for both steady and oscillatory rheological data of CNT/PC as a function of inverse temperature

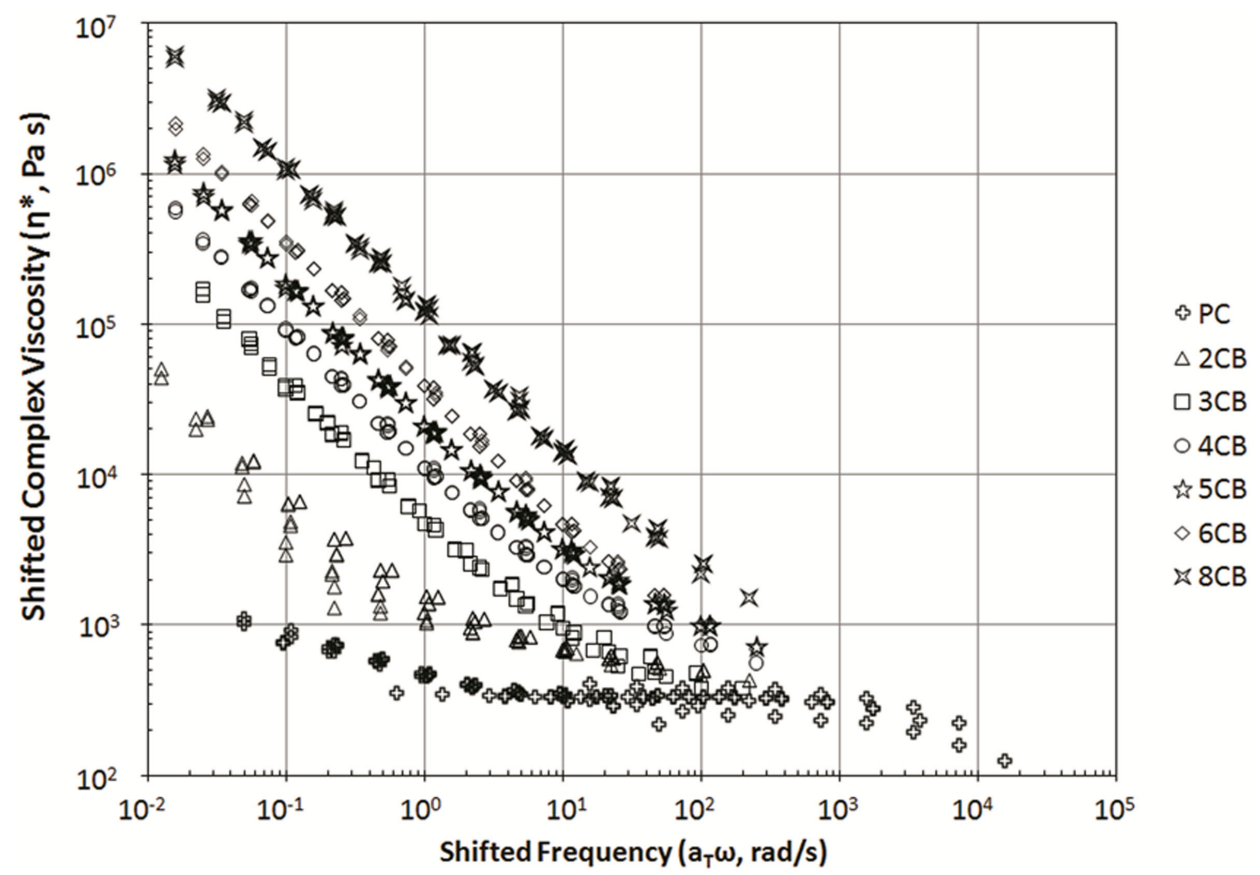

Figure 8.4: Time-temperature shifted complex viscosity as a function of shifted frequency $\mathrm{CB} / \mathrm{PC}$ studied at $270^{\circ} \mathrm{C}$ 


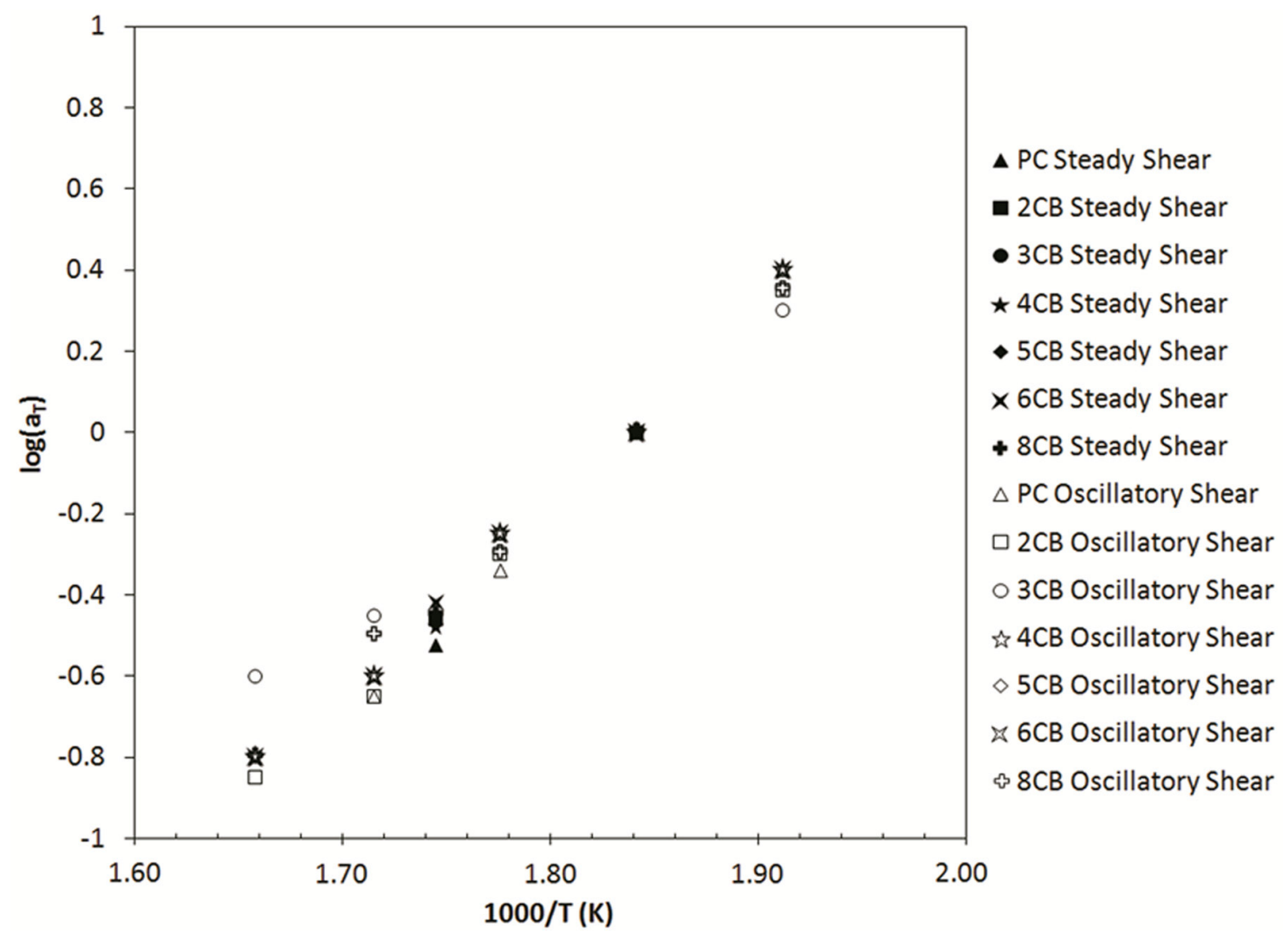

Figure 8.5: Time-temperature shift factors $a_{T}$ for both steady and oscillatory rheological data of $\mathrm{CB} / \mathrm{PC}$ as a function of inverse temperature

Bingham plastics are constant-viscosity fluids that have a yield-stress. The shear stress of these materials is given by Equation 8.1.

$\tau_{21}=\mu_{0} \dot{\gamma}+\tau_{0}$

In this equation, $\tau_{21}$ is the shear stress, $\dot{\gamma}$ is the shear rate, $\mu_{0}$ is the viscosity parameter of the model and $\tau_{0}$ is the yield stress (51). We can put this in terms of viscosity, $\eta$, by using the definition of viscosity, $\eta \equiv \tau_{21} / \dot{\gamma}$. When this is applied to Equation 8.1 we obtain Equation 8.2 known as the Bingham model.

$\eta=\mu_{0}+\frac{\tau_{0}}{\dot{\gamma}}$ 
By taking the logarithm of both sides of the equation then applying the limit as shear rate goes to zero, we can see the slope of -1 develop as shown in Equations 8.3 and 8.4.

$$
\begin{aligned}
& \log \eta=\log \left(\mu_{0}+\frac{\tau_{0}}{\dot{\gamma}}\right) \\
& \lim _{\dot{\gamma} \rightarrow 0}\left[\log \left(\mu_{0}+\frac{\tau_{0}}{\dot{\gamma}}\right)\right]=\log \tau_{0}-\log \dot{\gamma}
\end{aligned}
$$

This derivation allows us to estimate the yield stress of a system by measuring the viscosity at a shear rate of $1 \mathrm{rad}^{-1}$ as long as the systems exhibits a -1 slope on a plot of the $\log \eta^{*}$ versus the $\log$ frequency. The yield stresses for $\mathrm{CNT} / \mathrm{PC}$ and $\mathrm{CB} / \mathrm{PC}$ as measured using this method are given in Table 8.1 and Table 8.2 respectively.

\section{3: Capillary Rheometer Results: CNT/PC}

Figure 8.6 and Figure 8.7 shows the steady-shear master curves for the CNT/PC composites. Detailed results for this data are listed in Appendix M. The corresponding shift factors are shown in Figure 8.3. Figures 8.6 and 8.7 also include model fits (CY) that are discussed later in this section. 


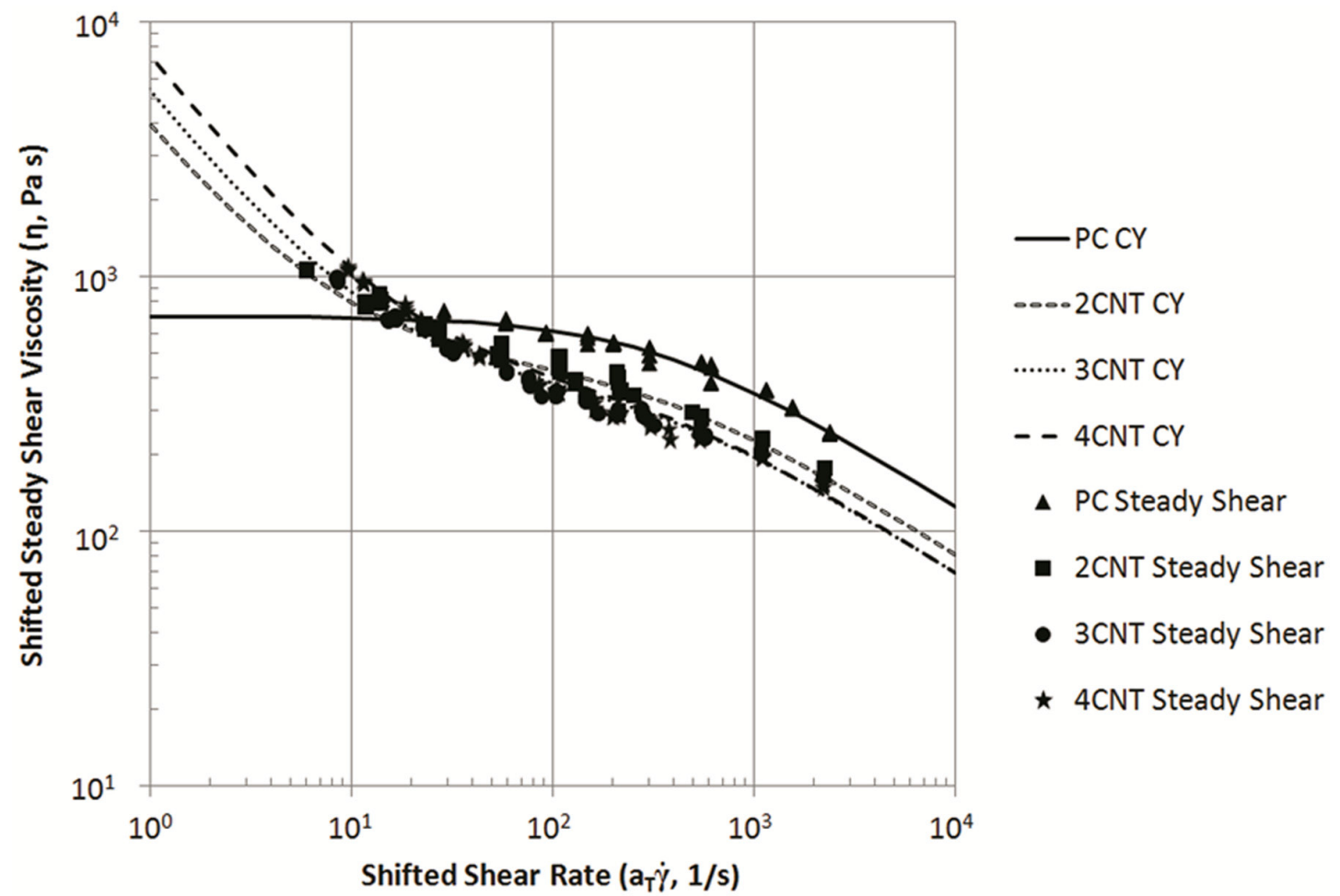

Figure 8.6: Time-temperature shifted steady-shear viscosity as a function of shifted shear rate for $\mathrm{CNT} / \mathrm{PC}$ studied at $270^{\circ} \mathrm{C}$. Compositions range from pure polycarbonate $(0 \mathrm{wt} \%$ $\mathrm{CNT}$ ) to $4 \mathrm{wt} \% \mathrm{CNT}$. Lines in the figure represent the modified Carreau-Yassuda (CY) model discussed in the text. 


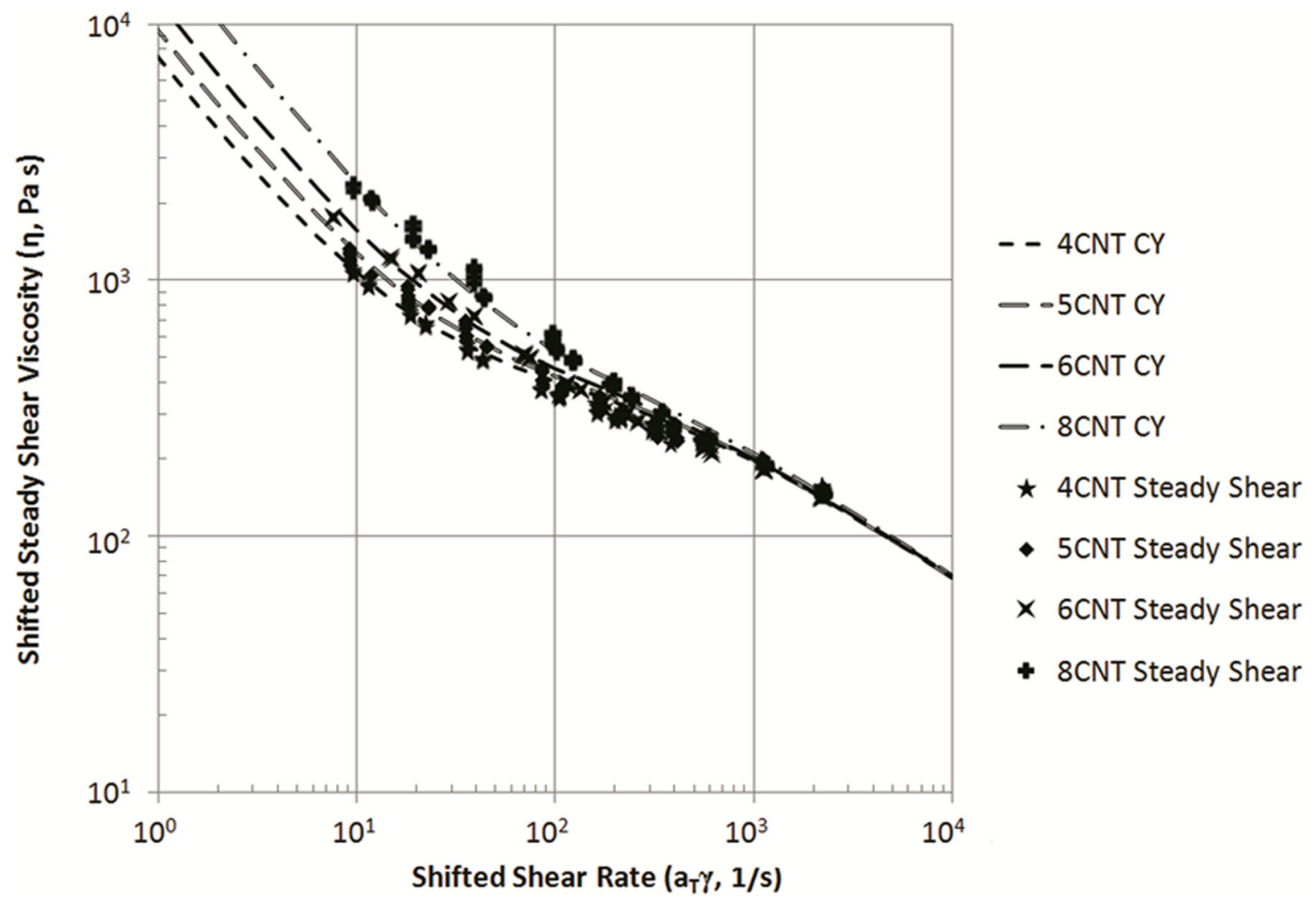

Figure 8.7: Time-temperature shifted steady-shear viscosity as a function of shifted shear rate for $\mathrm{CNT} / \mathrm{PC}$ studied at $270^{\circ} \mathrm{C}$. Compositions range from $4 \mathrm{wt} \% \mathrm{CNT}$ to $8 \mathrm{wt} \% \mathrm{CNT}$. Lines in the figure represent the modified Carreau-Yassuda (CY) model discussed in the text.

The pure PC curve in Figure 8.6 shows a typical polymeric flow curve. It displays a Newtonian zero-shear plateau, $\eta_{0}$, at about $700 \mathrm{~Pa}-\mathrm{s}$ which is present at low shear rates.

As the shear rate increases, the viscosity decreases, a condition known as shear-thinning. The addition of up to $4 \mathrm{wt} \% \mathrm{CNT}$ results in a decrease in the viscosity by up to a factor two. This is unusual behavior as the addition of most carbons to most thermoplastic systems results in an increase in viscosity. Addition of CNT past $4 \mathrm{wt} \%$ is seen to increase the viscosity of the system at low shear rates while maintaining the reduction in viscosity shown with $4 \mathrm{wt} \% \mathrm{CNT}$ loading at high shear rate. 
This reduction in viscosity, while unusual, has also been seen by other researchers in CNT/PC systems (68). In trying to determining the cause for this behavior, it was found that the Cox-Merz rule does not hold for this system $(51,53)$. The Cox-Merz rule states that, for many systems, when $\dot{\gamma}=\omega$, complex viscosity as obtained by SAOS, $\eta^{*}$, can be compared to steady shear viscosity, $\eta$, on a one-to-one basis. Even for pure PC, it was found that this rule did not hold, even at low shear rates $\left(\eta_{0}^{*}=320 \mathrm{~Pa}-s\right.$, $\eta_{0}=700 \mathrm{~Pa}-s$ ). It was still inferred from the SAOS data (Tables 8.1 and 8.2) that a yield stress was present, and should be included in any attempt to model steady-shear viscosity.

Using this information, it was postulated that the yield stress increases with increasing CNT loading and the increased CNT loading caused a decrease in the Newtonian zeroshear plateau, $\eta_{0}$, of the composite. This postulation was tested using a modified Carreau-Yasuda model ( (51), (76)) to fit our steady shear viscosity data. This model is shown as Equation 8.5. The model parameters used to fit the data are given in Table 8.1.

$\eta(\dot{\gamma})=\frac{\tau_{0}}{\dot{\gamma}}+\eta_{0}\left(1+(\dot{\gamma} \lambda)^{a}\right)^{\frac{n-1}{a}}$

In this equation, $\tau_{0}$ is the yield stress, $\dot{\gamma}$ is the shear rate, $\eta_{0}$ is the Newtonian zero-shear viscosity, $\lambda$ is the relaxation time, $n$ is the power-law index, and $a$ is an exponential parameter. This fits given by this model using the parameters listed in Table 8.1 are shown in Figures 8.6 and 8.7. 
Table 8.1: CNT/PC properties: ER, yield stress from the SAOS data; yield stress, matrixzero-shear-viscosity and infinite-shear viscosity parameters used in modeling discussed in text. For all fits $\lambda=0.003, a=1$, and $\mathrm{n}=0.5$.

\begin{tabular}{|c|c|c|c|c|c|}
\hline Formulation & $\begin{array}{c}\text { Filler } \\
\mathbf{W t} \%\end{array}$ & $\begin{array}{c}\text { Electrical } \\
\text { Resistivity } \\
(\boldsymbol{\Omega}-\mathbf{c m})\end{array}$ & $\begin{array}{c}\text { Yield stress } \\
\text { from SAOS } \\
\mathbf{( P a )}\end{array}$ & $\begin{array}{c}\text { Yield stress } \\
\text { in CY model } \\
\mathbf{( P a})\end{array}$ & $\eta_{\mathbf{o}}$ (Pa s) \\
\hline $\mathrm{PC}$ & 0.0 & $\begin{array}{c}1.06 \times 10^{17} \pm 7.96 \\
\times 10^{16} \mathrm{n}=7\end{array}$ & 0 & 0 & 700 \\
\hline $2 \mathrm{CNT}$ & 2.0 & $4605 \pm 1115 \mathrm{n}=6$ & 2500 & 3500 & 450 \\
\hline $3 \mathrm{CNT}$ & 3.0 & $216 \pm 44 \mathrm{n}=6$ & 17,000 & 5000 & 380 \\
\hline $4 \mathrm{CNT}$ & 4.0 & $73 \pm 10 \mathrm{n}=6$ & 43,000 & 7000 & 380 \\
\hline $5 \mathrm{CNT}$ & 5.0 & $43 \pm 7 \mathrm{n}=6$ & 75,000 & 9000 & 380 \\
\hline $6 \mathrm{CNT}$ & 6.0 & $18 \pm 2 \mathrm{n}=6$ & 87,000 & 12,000 & 380 \\
\hline $8 \mathrm{CNT}$ & 8.0 & $7.8 \pm 0.4 \mathrm{n}=6$ & 220,000 & 20,000 & 380 \\
\hline
\end{tabular}

This modified model has two primary parts, a Newtonian zero-shear viscosity with shearthinning and a yield stress. The yield stress causes the model to have a slope of -1 at low shear rates. The Newtonian zero-shear viscosity with shear-thinning portion of the model dominates the model at high shear rates. We see that using the parameters listed in Table 8.1 , our model generates crossover behavior of the viscosity, much as is seen in the experimental data.

An inspection of the parameters used in the fits can give us insight into the behavior of the CNT/PC composites. SAOS experimentation indicated that there was a yield stress and it increased with increasing filler loading. The steady-shear modeling shows the same behavior, the values, however, are not the same, this is expected as the Cox-Merz rule does not hold for this system. We also see from this data that the Newtonian zeroshear plateau decreases from $700 \mathrm{~Pa}-\mathrm{s}$ for pure PC to $380 \mathrm{~Pa}-\mathrm{s}$ as CNT loading is 
increased to $3 \mathrm{wt} \%$. The decrease in the zero-shear plateau saturates at this point and additional CNT loading, up to $8 \mathrm{wt} \%$ total CNT maintains the same level (380 Pa-s). The relaxation time, $\lambda$, is kept constant at 0.003 and the power-law index, $n$, is kept constant at 0.5 for all samples.

The increase in the yield strength as filler loading is increased (3.5 $\mathrm{kPa}$ for $2 \mathrm{wt} \% \mathrm{CNT}$ to $20 \mathrm{kPa}$ for $8 \mathrm{wt} \% \mathrm{CNT}$ ) is consistent with the idea of a conductive network being formed amongst the carbon nanotubes within the PC. The electrical percolation threshold for $\mathrm{CNT} / \mathrm{PC}$ is below the lowest loaded filler tested ( $2 \mathrm{wt} \%)$ thus it makes sense that all of the filled systems tested exhibit a yield stress as an electrically conductive network is likely a stress-bearing network (19).

The most notable parameter to look at when it comes to the unusual behavior seen in the $\mathrm{CNT} / \mathrm{PC}$ composites is the Newtonian zero-shear plateau. The decrease in the value for the plateau indicates a lubrication effect that is happening at high shear-rate. The high shear-rate data in the pure PC represents the entangled nature of the PC molecules. At high shear-rates, the individual PC molecules are disentangling and thus shear-thinning. Our model suggests that the presence of CNT in PC acts to laminarize, or layer, the flow of the PC molecules, enhancing the disentanglement. It is postulated that the cause of this laminarization is the high aspect-ratio CNT particles getting forced (by the flow of the PC) to align either in the flow direction or perpendicular to the flow direction where it would take on a log-rolling behavior as illustrated in Figure 8.8. This alignment thus allows for the PC layers to have a greater degree of relative motion, creating an overall decrease in friction, thus lower viscosity. This kind of behavior is similar to that believed 
to occur in drag reduction of turbulent flow (76). In turbulent flow drag reduction, polymers in a dilute solution are stretched out into the direction of the flow and to a degree straighten out the flow, this decreases overall friction.

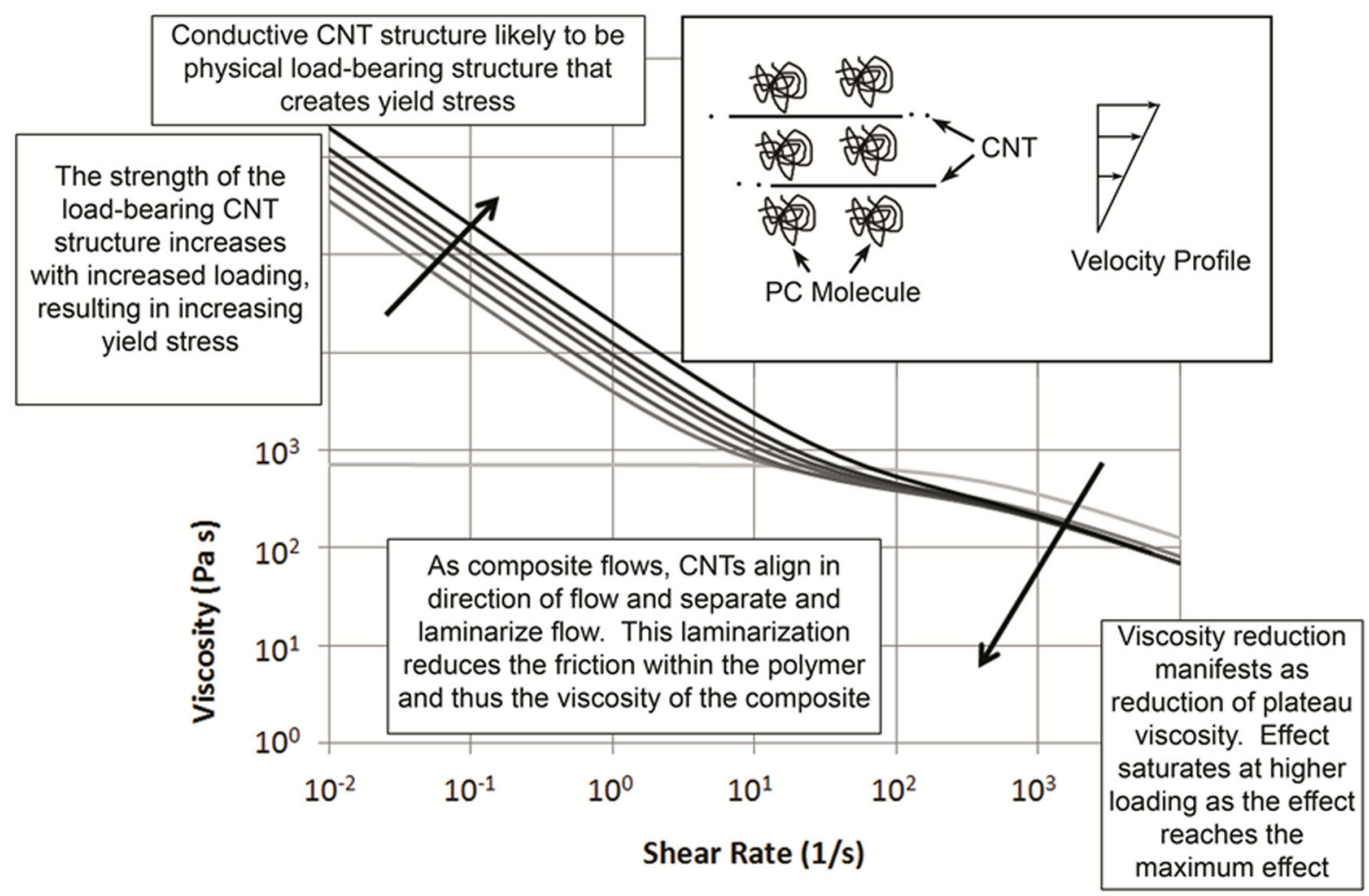

Figure 8.8: Schematic of a possible mechanism for viscosity reduction effect observed at high shear rates. 


\section{4: Capillary Rheometer Results and Discussion: $\mathrm{CB} / \mathrm{PC}$}

Figure 8.9 and Figure 8.10 shows the steady-shear master curves for the $\mathrm{CB} / \mathrm{PC}$ composites. The corresponding shift factors are shown in Figure 8.5. Figures 8.9 and 8.10 also include model fits $(\mathrm{CY})$ that were discussed in section 8.3. The parameters used for this model, is included in Table 8.2.

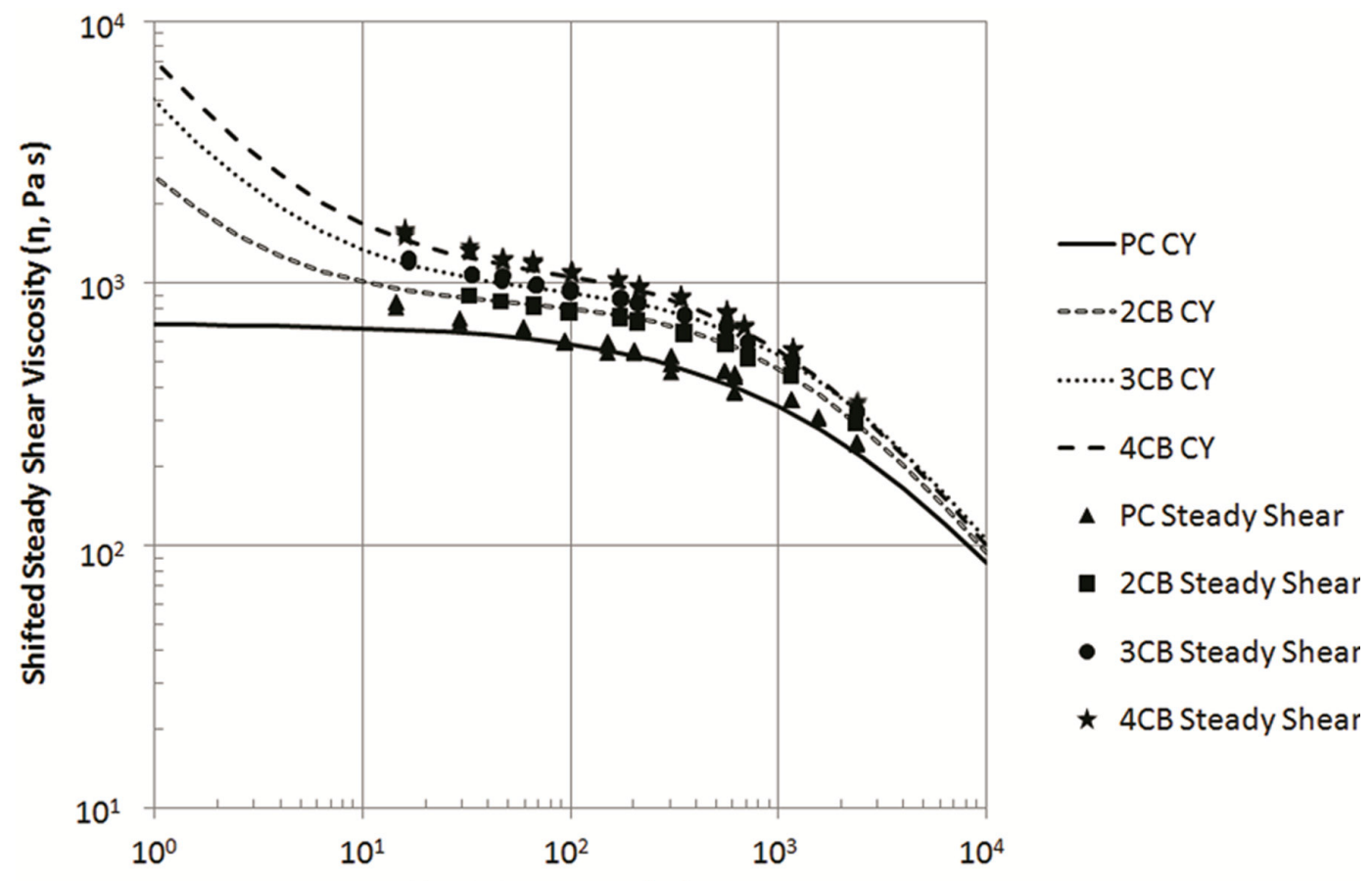

Figure 8.9: Time-temperature shifted steady-shear viscosity as a function of shifted shear rate for $\mathrm{CB} / \mathrm{PC}$ studied at $270^{\circ} \mathrm{C}$. Compositions range from pure polycarbonate $(0 \mathrm{wt} \%$ $\mathrm{CB})$ to $4 \mathrm{wt} \% \mathrm{CB}$. Lines in the figure represent the modified Carreau-Yassuda (CY) model discussed in the text. 


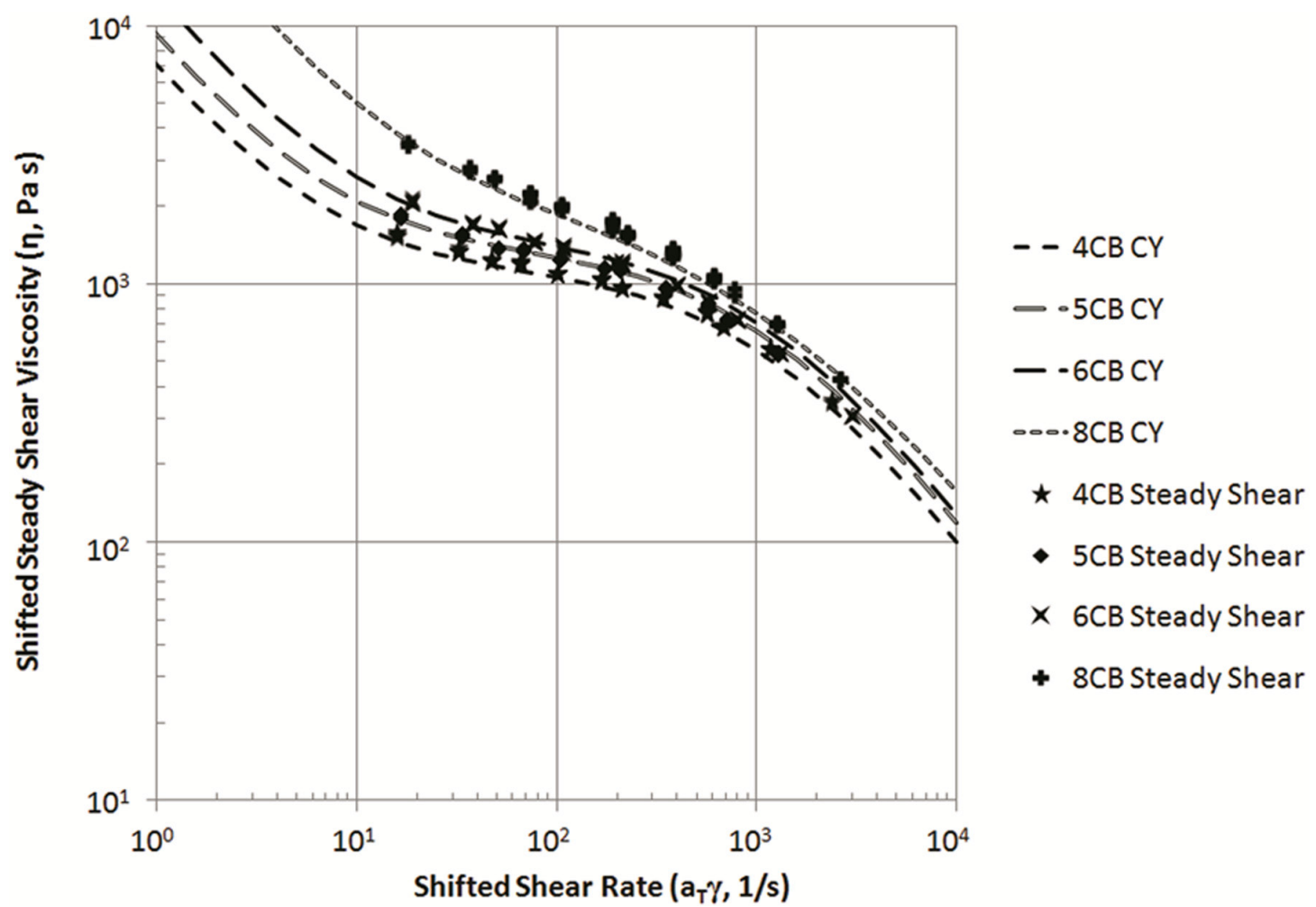

Figure 8.10: Time-temperature shifted steady-shear viscosity as a function of shifted shear rate for $\mathrm{CB} / \mathrm{PC}$ studied at $270^{\circ} \mathrm{C}$. Compositions range from $4 \mathrm{wt} \% \mathrm{CB}$ to $8 \mathrm{wt} \%$ $\mathrm{CB}$ Lines in the figure represent the modified Carreau-Yassuda (CY) model discussed in the text. 
Table 8.2: Composite properties: ER showing percolation between 3 and $4 \mathrm{wt} \%$; yield stress from the SAOS data; yield stress, matrix-zero-shear-viscosity and infinite-shear viscosity parameters used in modeling discussed in text. The parameters in the last three columns were used to fit steady shear data to the function given in Equation 12. For all fits $n=0$. Different yield stresses were obtained in SAOS and steady shear since the CoxMerz rule was not observed to hold.

\begin{tabular}{|c|c|c|c|c|c|c|c|}
\hline Formulation & $\begin{array}{c}\text { Filler } \\
\text { Wt } \\
\%\end{array}$ & $\begin{array}{l}\text { Electrical } \\
\text { Resistivity } \\
(\text { ohm-cm) }\end{array}$ & $\begin{array}{l}\text { Yield } \\
\text { stress } \\
\text { from } \\
\text { SAOS } \\
\text { (Pa) }\end{array}$ & $\begin{array}{c}\text { Yield } \\
\text { stress in } \\
\text { CY } \\
\text { model } \\
\text { (Pa) }\end{array}$ & $\begin{array}{c}\eta_{0} \\
(\text { Pa s) }\end{array}$ & $\mathbf{a}$ & $\lambda$ \\
\hline$\overline{\mathrm{PC}}$ & 0 & $\begin{array}{c}1.26 \times 10^{17} \pm \\
3.35 \times 10^{16} \\
n=6\end{array}$ & 0 & 0 & 700 & 0.7 & 0.0006 \\
\hline $2 \mathrm{CB}$ & 2 & $\begin{array}{c}4.05 \times 10^{16} \pm \\
2.66 \times 10^{16} \\
n=6\end{array}$ & 1200 & 1200 & 850 & 1 & 0.0008 \\
\hline $3 \mathrm{CB}$ & 3 & $\begin{array}{c}2.85 \times 10^{15} \pm \\
4.58 \times 10^{14} \\
n=6\end{array}$ & 5000 & 4000 & 950 & 1 & 0.0008 \\
\hline $4 \mathrm{CB}$ & 4 & $\begin{array}{c}1.17 \times 10^{5} \pm \\
7.77 \times 10^{4} \\
n=8\end{array}$ & 11,000 & 6000 & 1100 & 1 & 0.001 \\
\hline $5 \mathrm{CB}$ & 5 & $\begin{array}{c}2474 \pm \\
646.2 \mathrm{n}=8\end{array}$ & 21,000 & 9000 & 1300 & 1 & 0.001 \\
\hline $6 \mathrm{CB}$ & 6 & $\begin{array}{c}649.2 \pm 17.6 \\
n=8\end{array}$ & 37,000 & 12,000 & 1400 & 1 & 0.001 \\
\hline $8 \mathrm{CB}$ & 8 & $\begin{array}{c}122.2 \pm 4.0 \\
n=8\end{array}$ & 120,000 & 29,000 & 2000 & 0.7 & 0.001 \\
\hline
\end{tabular}

In Figures 8.9 and 8.10 along with the parameters used to obtain the models from Table 8.2, we see that the addition of $\mathrm{CB}$ to $\mathrm{PC}$ has the effect of increasing the Newtonian zeroshear plateau from $700 \mathrm{~Pa}-\mathrm{s}$ for pure PC to $2000 \mathrm{~Pa}-\mathrm{s}$ for an $8 \mathrm{wt} \% \mathrm{CB}$ composite. Again we see that the Cox-Merz rule does not hold for PC, thus we do not expect it to hold for any of the $\mathrm{CB} / \mathrm{PC}$ composite. For all shear rates, increasing the $\mathrm{CB}$ loading increases the 
viscosity. This increase in viscosity is substantial at low shear rates and high filler

loadings. This is the common behavior for carbon filled thermoplastic systems (77-82). No cross-over or lubrication effects are seen in these composites. The filled $\mathrm{CB} / \mathrm{PC}$ composites also show a yield stress which increases with increased filler loading, this is expected for $\mathrm{CB} / \mathrm{PC}$ for the same reasons that it was expected for $\mathrm{CNT} / \mathrm{PC}$ as discussed in section 8.3. The growth in yield stress from $1.2 \mathrm{kPa}$ for neat $\mathrm{PC}$ to $29 \mathrm{kPa}$ for $8 \mathrm{wt} \%$ $\mathrm{CB}$ is consistent with the building of a network of $\mathrm{CB}$ agglomerates within the $\mathrm{PC}$ matrix. For the $\mathrm{CB} / \mathrm{PC}$ composites, the power-law parameter, $n$, was maintained at 0 .

\section{5: Conclusions}

The object of this section of the project was to study the effect of CNT and CB on the viscosity of PC based composites. SAOS studies of $\mathrm{CNT} / \mathrm{PC}$ and $\mathrm{CB} / \mathrm{PC}$ composites showed that both CNT and CB created a yield-stress when added to PC. These yieldstresses were seen to increase monotonically with increasing filler loading for both systems. This yield-stress growth is postulated to be due to the creation of a stressbearing system that is consistent with electrical percolation for each system as seen in Chapters 6 and 7. Steady-shear rheometry testing on the CB/PC system showed a classic increase in viscosity at all shear-rates with increased filler loading. Steady-shear rheometry testing on the CNT/PC system showed a decrease in the Newtonian zero-shear plateau with increase CNT loading to about $3 \mathrm{wt} \% \mathrm{CNT}$. Further addition of CNT past 3 wt $\%$ increased the yield stress of the composite thus increasing the low shear-rate viscosity. This is not the common behavior seen in carbon-filled thermoplastics. The reduction in viscosity is consistent with a lubrication effect that would be caused by CNT 
within the matrix. The CNT are believed to laminarize the flow of PC molecules by either aligning in the flow direction, or aligning perpendicular to the flow direction and log-rolling. It is believed that this process saturates at $3 \mathrm{wt} \% \mathrm{CNT}$ thus the addition of further CNT past this amount does not further decrease viscosity. The unusual behavior seen in the CNT/PC system is captured in a viscosity model based on a modification of the Carreau-Yasuda equation. The Cox-Merz rule was not found to hold for PC or either of the filled systems. 


\section{Chapter 9: Electrical Resistivity, Thermal Conductivity, and Tensile, Flexural, and Rheological Properties of Exfoliated Graphite Nanoplatelet/Polycarbonate Composites}

\section{1: Environmental Scanning Electron Microscope (ESEM) Results}

An ESEM image of $5 \mathrm{wt} \%$ GNP in PC is shown in Figure 9.1. In this figure, the nanoplatelets appear as white elongated elements on the grey PC background. It is evident in this image that the GNP has a high aspect ratio.

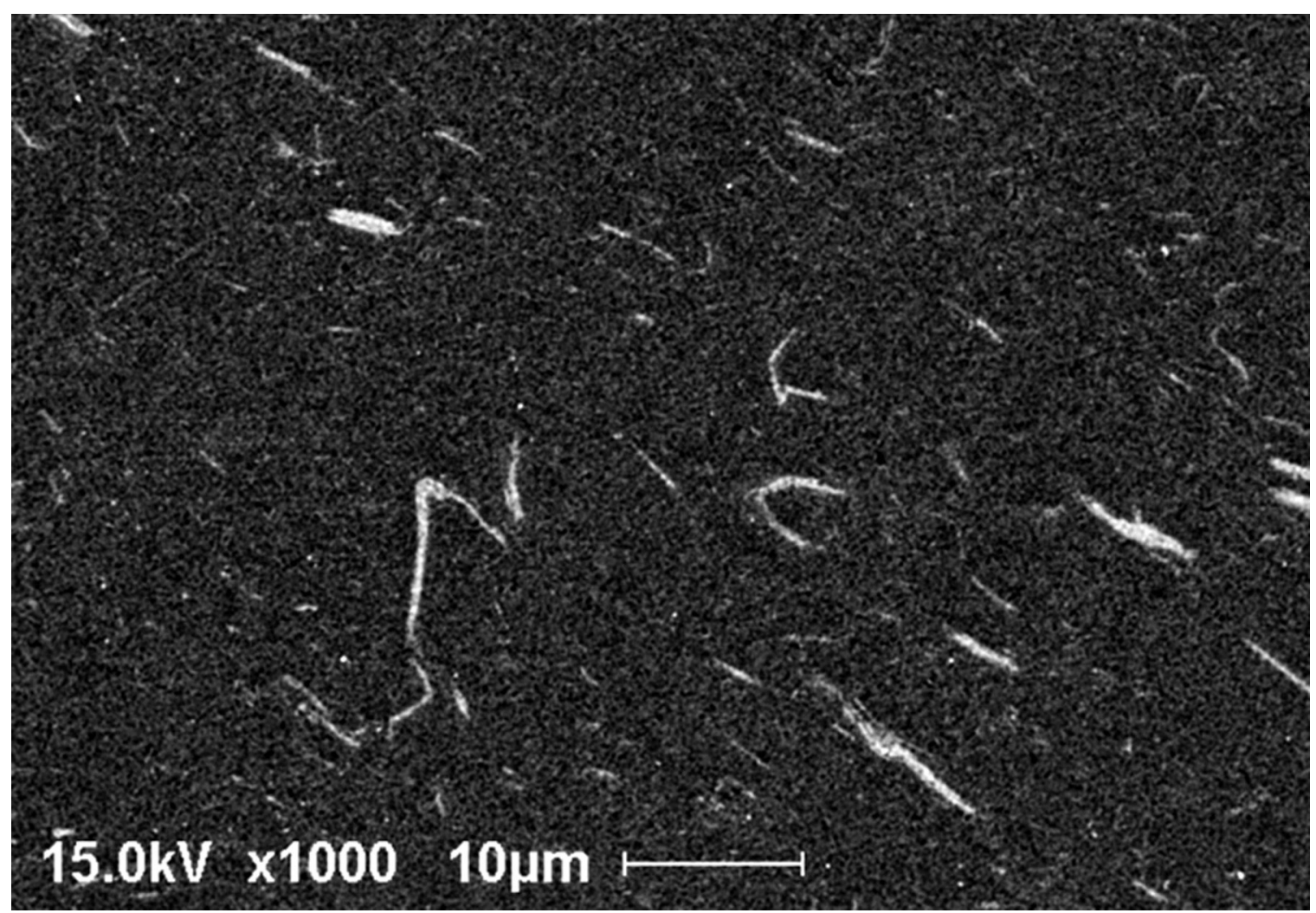

Figure 9.1: ESEM micrograph of $5 \mathrm{wt} \%$ GNP in PC composite 


\section{2: Electrical Resistivity Results}

Table 9.1 shows the mean electrical resistivity, standard deviation of the electrical resistivity and number of samples that were tested for each formulation. This data is plotted in Figure 9.2. Results for the individual samples are given in Appendices G and H. We see a typical electrical resistivity curve in this figure. At low conductive filler loadings, the electrical resistivity of the composite will be similar to that of the pure polymer. As the loading of the conductive filler increases, there is a point at which conductive networks begin to form and the resistivity decreases dramatically over a very small range of filler loading. This point is known as the percolation threshold. After percolation occurs, the electrical resistivity of the composite levels out as additional conductive filler is added $(7,60)$.

Table 9.1: GNP/PC Electrical Resistivity Results

\begin{tabular}{|l|l|l|l|}
\hline Formulation & $\begin{array}{l}\text { Filler } \\
\text { Wt } \%\end{array}$ & $\begin{array}{l}\text { Filler } \\
\text { Vol } \%\end{array}$ & $\begin{array}{l}\text { Electrical Resistivity } \\
\text { (ohm-cm })\end{array}$ \\
\hline PC & 0 & 0.0 & $9.37 \times 10^{16} \pm 2.00 \times 10^{16} \mathrm{n}=6$ \\
\hline $2 \mathrm{GNP}$ & 2 & 1.21 & $5.46 \times 10^{16} \pm 4.89 \times 10^{15} \mathrm{n}=6$ \\
\hline $3 \mathrm{GNP}$ & 3 & 1.82 & $3.23 \times 10^{16} \pm 7.22 \times 10^{15} \mathrm{n}=8$ \\
\hline $4 \mathrm{GNP}$ & 4 & 2.44 & $1.20 \times 10^{16} \pm 3.54 \times 10^{14} \mathrm{n}=6$ \\
\hline $5 \mathrm{GNP}$ & 5 & 3.06 & $3.76 \times 10^{15} \pm 2.83 \times 10^{14} \mathrm{n}=6$ \\
\hline $6 \mathrm{GNP}$ & 6 & 3.69 & $2.01 \times 10^{14} \pm 4.99 \times 10^{12} \mathrm{n}=6$ \\
\hline $8 \mathrm{GNP}$ & 8 & 4.96 & $3.95 \times 10^{7} \pm 1.53 \times 10^{7} \mathrm{n}=8$ \\
\hline $10 \mathrm{GNP}$ & 10 & 6.25 & $1.74 \times 10^{6} \pm 2.65 \times 10^{5} \mathrm{n}=8$ \\
\hline $12 \mathrm{GNP}$ & 12 & 7.56 & $3.07 \times 10^{5} \pm 9.50 \times 10^{3} \mathrm{n}=5$ \\
\hline $15 \mathrm{GNP}$ & 15 & 9.57 & $2.79 \times 10^{4} \pm 8.31 \times 10^{3} \mathrm{n}=8$ \\
\hline
\end{tabular}




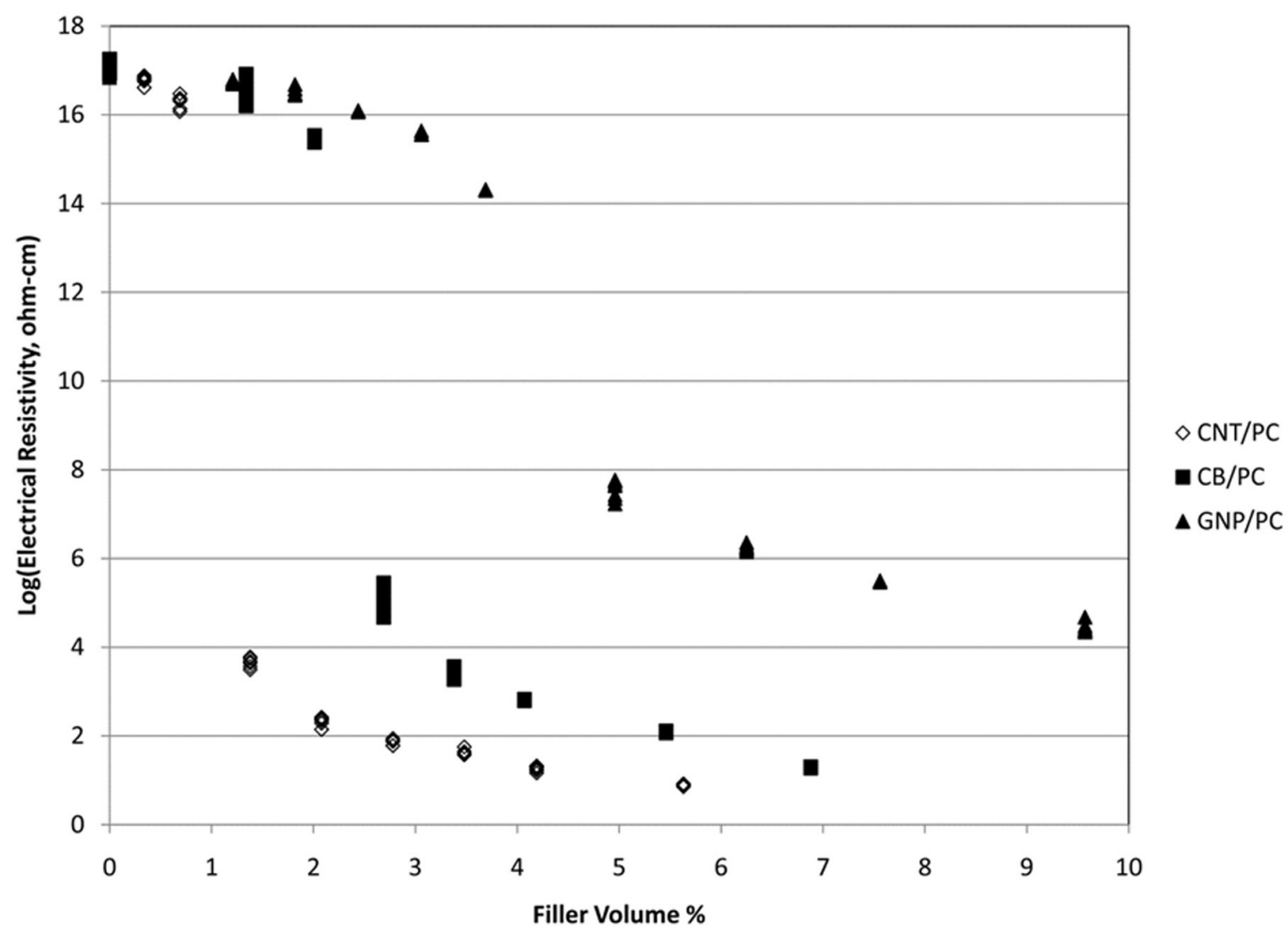

Figure 9.2: Electrical resistivity of $\mathrm{GNP} / \mathrm{PC}, \mathrm{CB} / \mathrm{PC}$, and $\mathrm{CNT} / \mathrm{PC}$ composites

Figure 9.2 shows that GNP/PC does not percolate until over 6 wt $\%$ GNP. The ER of the composite drops from about $10^{17} \mathrm{ohm}-\mathrm{cm}$ for neat PC down to $2.8 \times 10^{4} \mathrm{ohm}-\mathrm{cm}$ for 15 wt $\%$ GNP (9.6 vol\%). The percolation threshold for GNP/PC occurs at approximately 4.0 vol\% (about $6.5 \mathrm{wt} \%$ ) GNP. This is significantly higher than either the CNT or CB previously studied for this project ( 1 vol $\%$ and 2.3 vol $\%$ respectively). Additionally, the high end filler loadings of GNP had a much higher resistivity than the high end loadings of either the CNT or $\mathrm{CB}$, despite the vol\% of either of the other fillers being much lower. $15 \mathrm{wt} \%$ GNP (9.6 vol\%) in PC had a resistivity of $2.8 \times 10^{4} \mathrm{ohm}-\mathrm{cm}$ compared to $8 \mathrm{ohm}-$ $\mathrm{cm}$ for $8 \mathrm{wt} \%$ CNT (5.6 vol\%) and $20 \mathrm{ohm}-\mathrm{cm}$ for $10 \mathrm{wt} \% \mathrm{CB}(6.9 \mathrm{vol} \%)$. 
The relatively flat shape of the ER curve indicates that GNP/PC composites would be suitable for electrostatic dissipative applications $\left(10^{10}\right.$ to $\left.10^{3} \mathrm{ohm}-\mathrm{cm}\right)$ in formulations of 8 to $15 \mathrm{wt} \%$ GNP in PC. The high value for the percolation threshold and electrical resistivity for GNP in PC is consistent with studies done by Kalaitzidou et al. (83). In his research, Kalaitzidou found that the percolation threshold of GNP in polypropylene was around 6 vol\%. Additionally Kalaitzidou found that at 10 vol\% the ER of GNP in polypropylene was about $10^{5} \mathrm{ohm}-\mathrm{cm}$. Both of these findings fit well with our results for GNP in PC.

\section{3: Thermal Conductivity Results}

The thermal conductivity test results for GNP/PC composites have been shown in Figure 9.3. The results from the previous systems, $\mathrm{CNT} / \mathrm{PC}$ and $\mathrm{CB} / \mathrm{PC}$, have also been included for comparison. The results for the GNP/PC system have also been listed in Table 9.2. Detailed results for this data are given in Appendix I. 


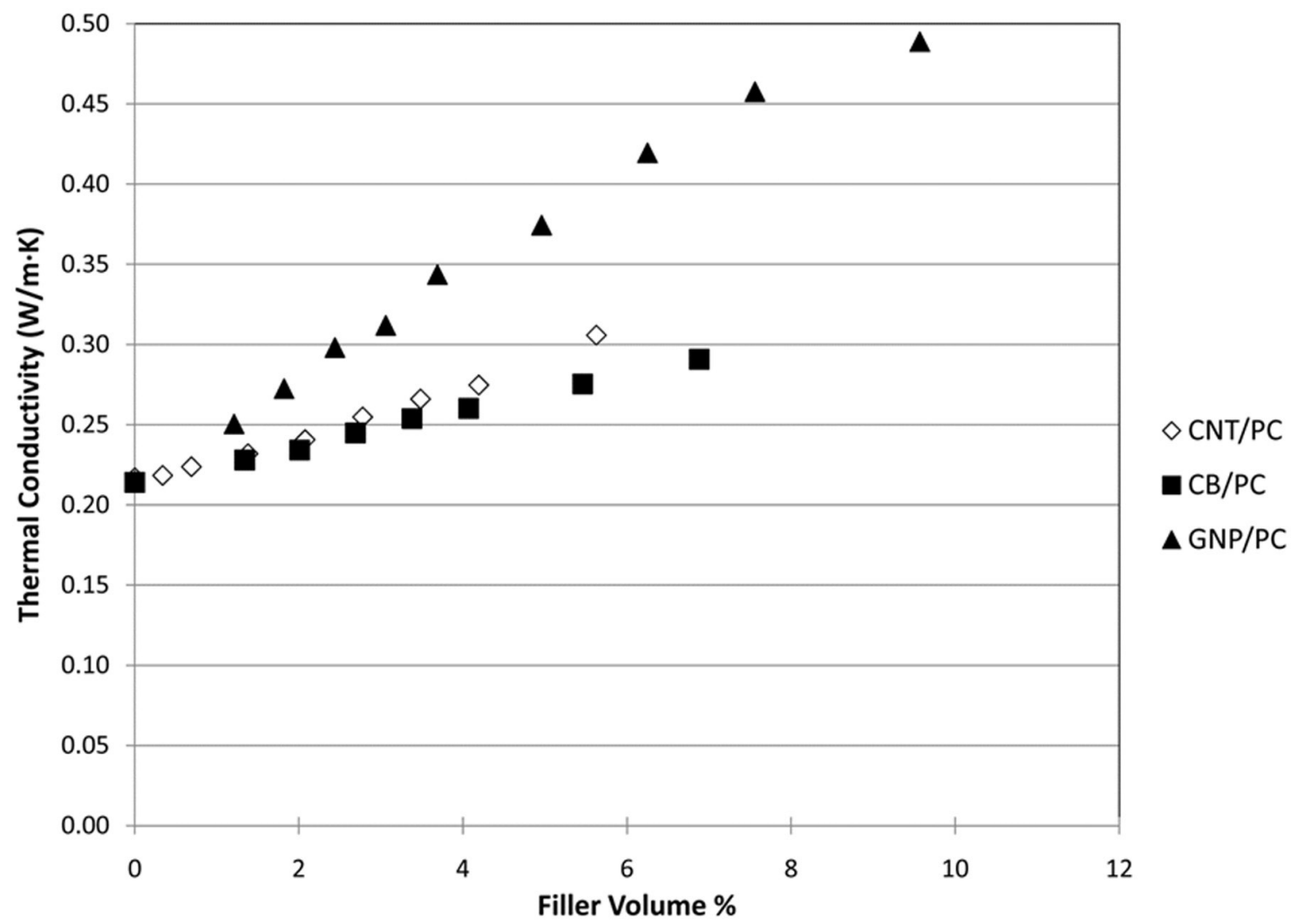

Figure 9.3: Thermal conductivity of GNP/PC, CB/PC, and CNT/PC composites

Table 9.2: GNP/PC Thermal Conductivity Results

\begin{tabular}{|l|l|l|l|}
\hline Formulation & Filler Wt $\%$ & Thermal Conductivity $(\mathrm{W} / \mathrm{m} \cdot \mathrm{K})$ \\
\hline PC & 0 & $0.215 \pm 0.002 \quad \mathrm{n}=4$ \\
\hline $2 \mathrm{GNP}$ & 2 & $0.251 \pm 0.003 \quad \mathrm{n}=5$ \\
\hline $3 \mathrm{GNP}$ & 3 & $0.273 \pm 0.005 \quad \mathrm{n}=5$ \\
\hline $4 \mathrm{GNP}$ & 4 & $0.298 \pm 0.003 \quad \mathrm{n}=4$ \\
\hline $5 \mathrm{GNP}$ & 5 & $0.319 \pm 0.001 \quad \mathrm{n}=5$ \\
\hline $6 \mathrm{GNP}$ & 6 & $0.344 \pm 0.002 \quad \mathrm{n}=4$ \\
\hline $8 \mathrm{GNP}$ & 8 & $0.374 \pm 0.007 \quad \mathrm{n}=5$ \\
\hline $10 \mathrm{GNP}$ & 10 & $0.420 \pm 0.002 \quad \mathrm{n}=5$ \\
\hline $12 \mathrm{GNP}$ & 12 & $0.458 \pm 0.016 \quad \mathrm{n}=4$ \\
\hline $15 \mathrm{GNP}$ & 15 & $0.489 \pm 0.016 \quad \mathrm{n}=4$ \\
\hline
\end{tabular}

We can see in this figure that the addition of $15 \mathrm{wt} \%$ GNP (9.6 vol\%) increases the thermal conductivity of the composite from $0.21 \mathrm{~W} / \mathrm{m} \cdot \mathrm{K}$ for neat PC to $0.49 \mathrm{~W} / \mathrm{m} \cdot \mathrm{K}$. We 
can also see from the graph that GNP has a significantly higher thermal conductivity than CNT or CB for similar filler loadings. Our results for GNP/PC conform well to research done before by Fukushima et al. who studied GNP in high-density polyethylene (84).

\section{4: Tensile Test Results}

The tensile modulus of the GNP based composites is shown in Figure 9.4 along with the results for the previously tested systems for comparison. The ultimate tensile strengths (UTS) and the strains at the ultimate tensile strength for GNP/PC are shown in Figure 9.5. Both of these figures display the mean value along with error bars representing one standard deviation. In cases where the error bars are smaller than the marker size, they have been omitted. Detailed results for this data are given in Appendix J.

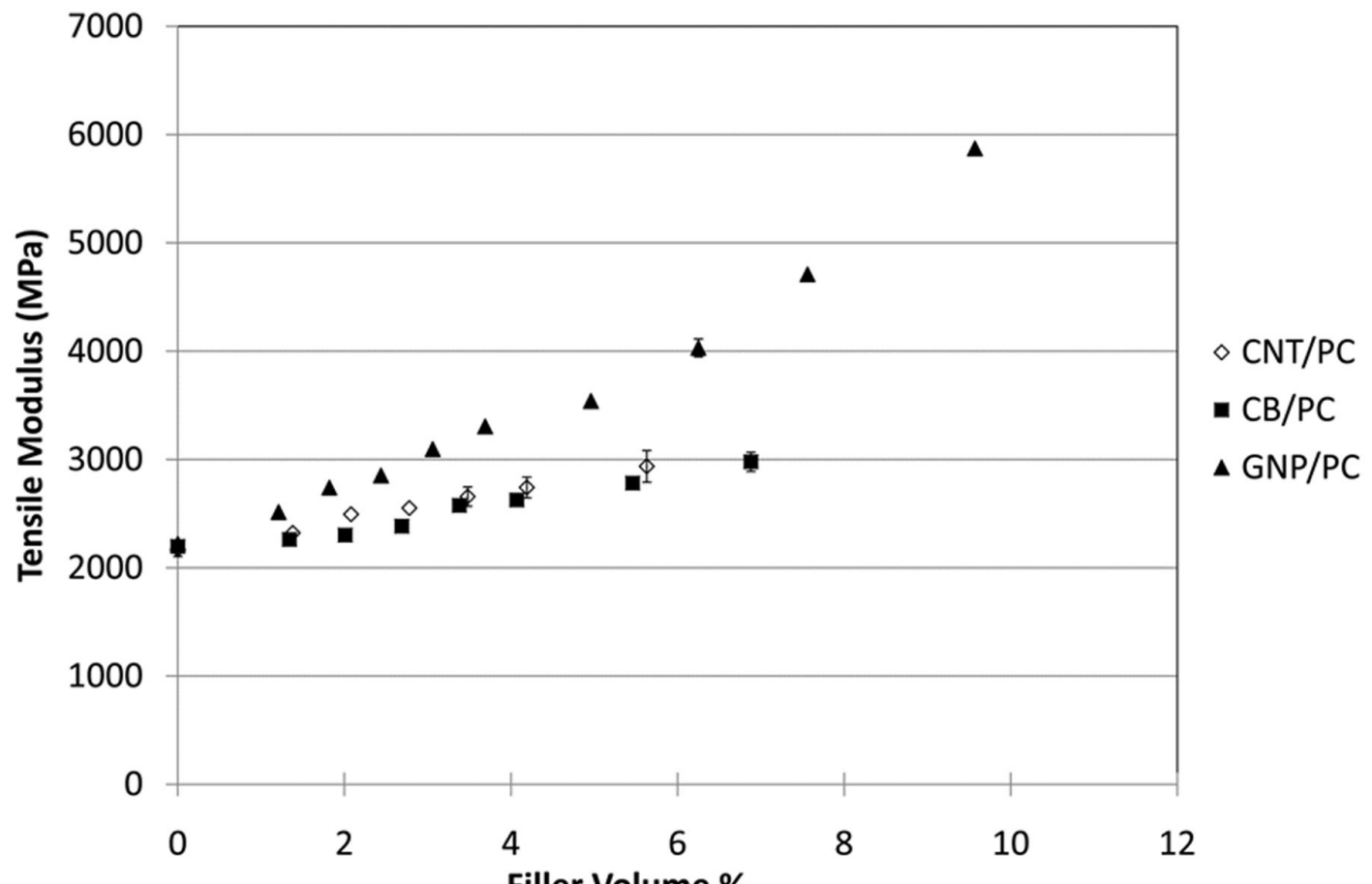

Figure 9.4: Tensile modulus of GNP/PC, $\mathrm{CB} / \mathrm{PC}$, and $\mathrm{CNT} / \mathrm{PC}$ composites 


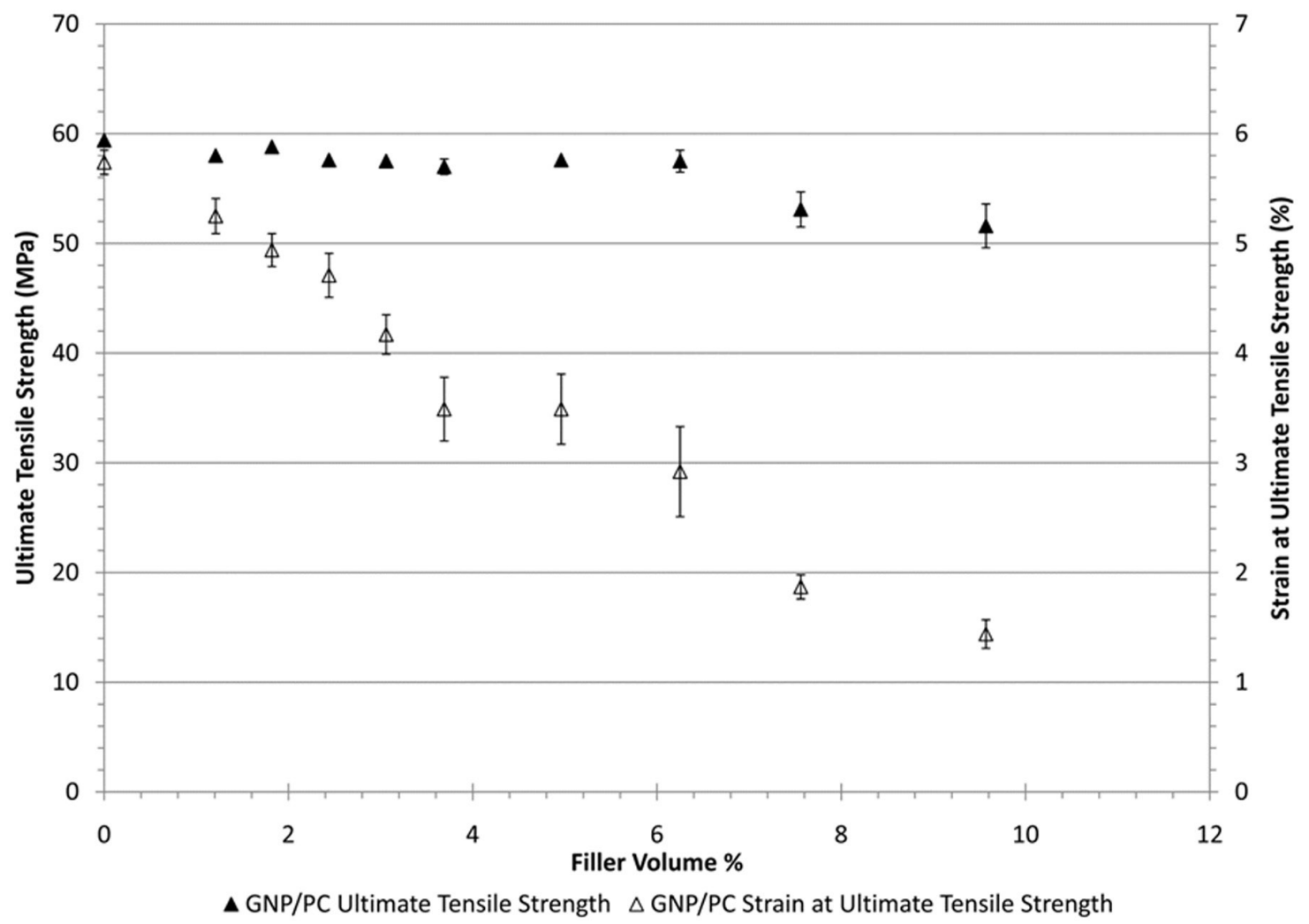

Figure 9.5: Ultimate tensile strength and strain at ultimate tensile strength for GNP/PC composites

The addition of GNP to PC increased the tensile modulus from 2.2 GPa for pure PC to 5.9 GPa for $15 \mathrm{wt} \%$ (9.6 vol\%) GNP. When compared to CNT and CB, GNP has a larger effect on increasing the tensile modulus, at $5 \mathrm{vol} \%(8 \mathrm{wt} \%)$ GNP the composite has a tensile modulus of $3.5 \mathrm{GPa}$. At $5.6 \mathrm{vol} \% \mathrm{CNT}(8 \mathrm{wt} \%)$ in PC, the tensile modulus is 2.9 GPa. At 5.5 vol\% CB (8 wt $\%)$ in PC the tensile modulus is $2.8 \mathrm{GPa}$. A previous study of GNP in polypropylene by Kalaitzidou et al. found similar results as those seen in our GNP/PC composites (85).

As seen in Figure 9.5, the UTS of the GNP/PC composite stayed fairly constant at about $59 \mathrm{MPa}$ for all formulations up to $10 \mathrm{wt} \% \mathrm{GNP}$. This compares similarly to the previous systems as seen in Figure 7.5. Above $10 \mathrm{wt} \%$ GNP, the composites became increasingly 
brittle and the UTS and strain at UTS decreased. Figure 9.6 shows typical stress-strain curves for some of the GNP/PC composites. In this figure we can see that the ductile behavior of PC is maintained in GNP filled composites that contain less than $10 \mathrm{wt} \%$ GNP.

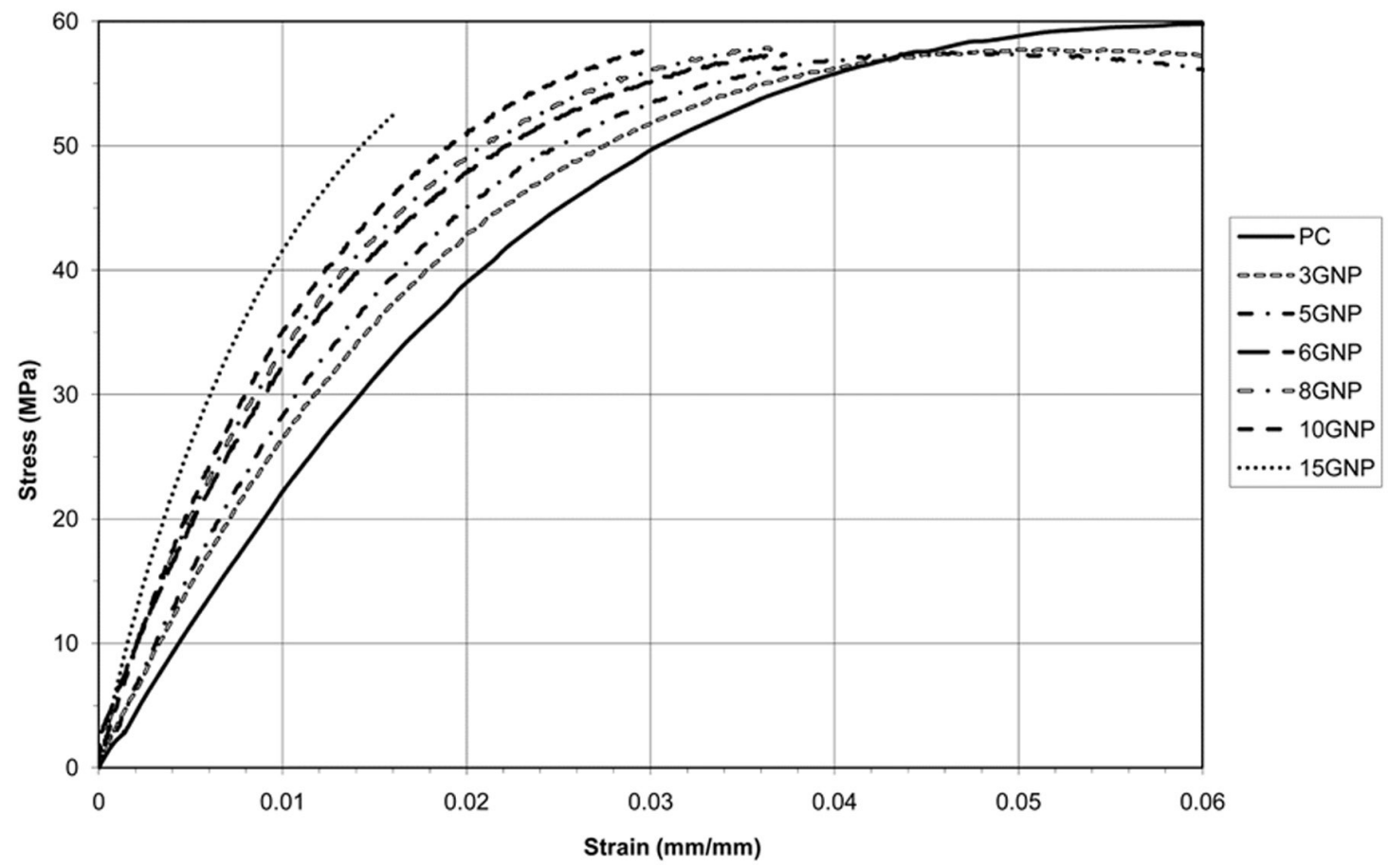

Figure 9.6: Tensile stress-strain curves for GNP/PC composites

\section{5: Flexural Test Results}

The flexural modulus of the GNP based composites is shown in Figure 9.7 along with the results for $\mathrm{CNT}$ and $\mathrm{CB}$ based composites for comparison. The ultimate flexural strengths (UFS) and the strains at the ultimate flexural strength for GNP/PC composites are shown in Figure 9.8. Both of these figures display the mean value along with error bars representing one standard deviation. In cases where the error bars are smaller than the marker size, they have been omitted. Detailed results for this data are given in Appendix K. 


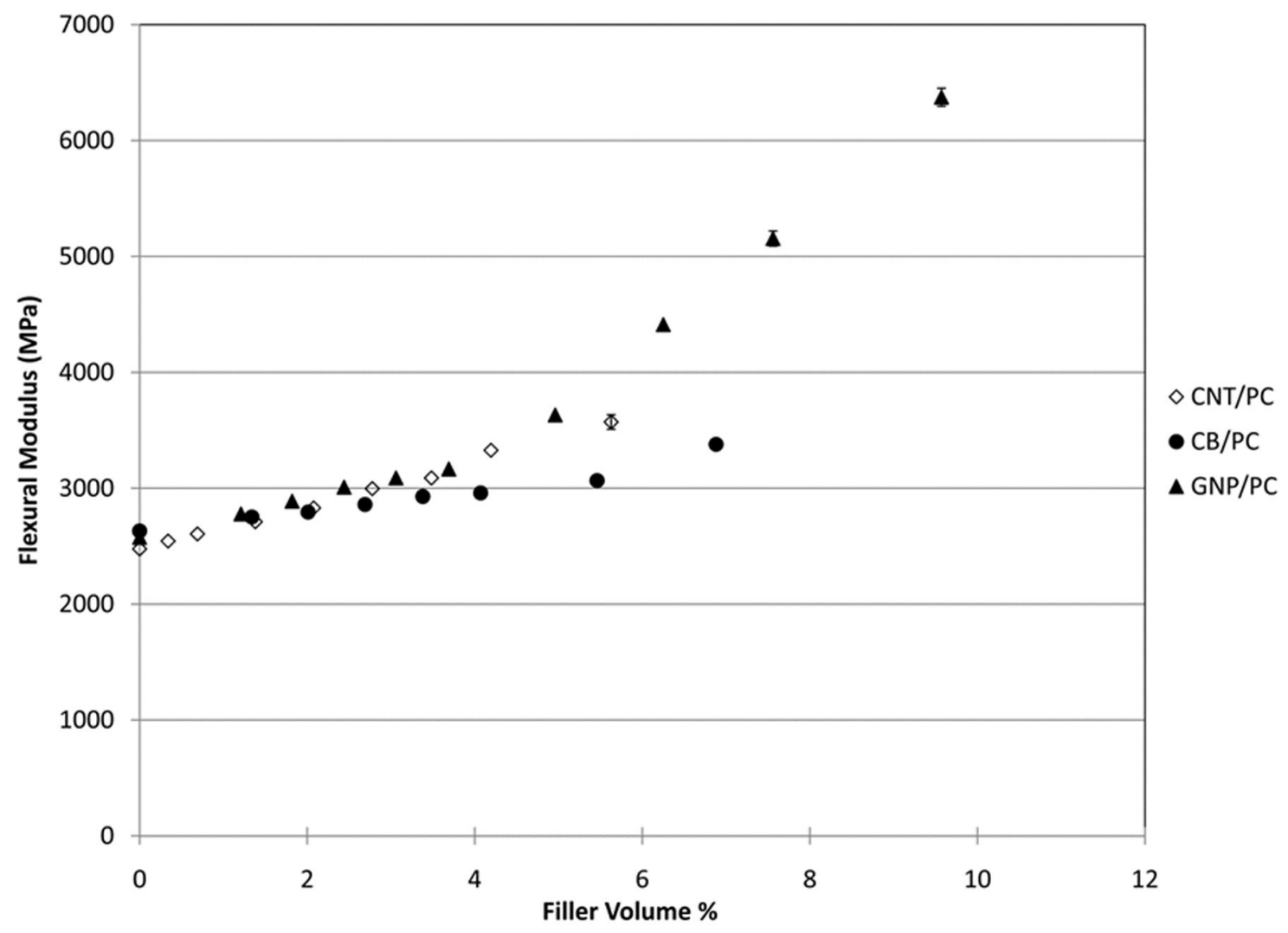

Figure 9.7: Flexural modulus for GNP/PC, CB/PC and CNT/PC composites 


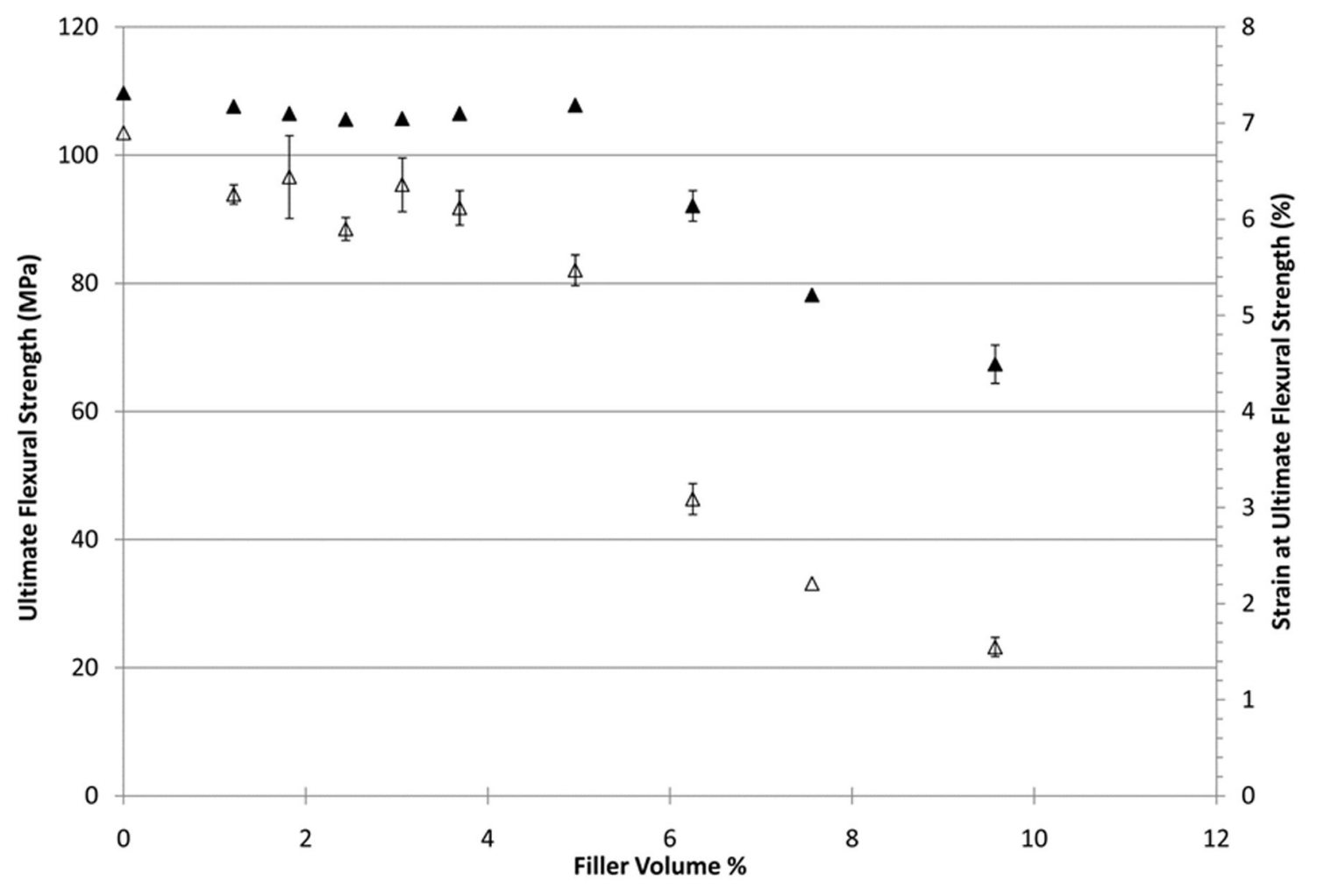

$\Delta$ GNP/PC Ultimate Flexural Strength $\triangle$ GNP/PC Strain at Ultimate Flexural Strength

Figure 9.8: Ultimate flexural strength and strain at ultimate flexural strength for GNP/PC composites

In Figure 9.7 it is again seen that the trends in flexural modulus are similar to those in tensile modulus. The addition of GNP to PC increases the flexural modulus from 2.6 GPa for pure PC to $6.4 \mathrm{GPa}$ for $15 \mathrm{wt} \% \mathrm{GNP}$ (9.6 vol\%). We see at high filler loadings that GNP has a larger effect on increasing the flexural modulus than either CNT or CB. At moderate filler loadings, it is similar to the other fillers. At $5 \mathrm{vol} \%$ GNP $(8 \mathrm{wt} \%)$ in $\mathrm{PC}$, the flexural modulus is $3.6 \mathrm{GPa}$. At $5.6 \mathrm{vol} \% \mathrm{CNT}$ in PC the flexural modulus is also $3.6 \mathrm{GPa}$. $\mathrm{CB}$ is somewhat lower requiring $6.9 \mathrm{vol} \%$ to reach $3.4 \mathrm{GPa}$. Again the reseach of Kalitzidou et al. of GNP in polypropylene shows similar results as those seen here for GNP/PC (85). 
As seen in Figure 9.8, the UFS for GNP/PC stayed fairly constant between 106 and 110 MPa for samples containing less than $10 \mathrm{wt} \%$ GNP. Samples with $10 \mathrm{wt} \%$ GNP of more again became increasingly brittle with decreasing UFS and strain at UFS. It is worth noting that the samples with 12 and $15 \mathrm{wt} \%$ GNP were quite viscous and difficult to injection mold.

\section{6: Capillary Rheometer Results}

Figure 9.9 shows the steady-shear viscosity master curve versus shear rate for GNP/PC composites. This figure shows all of the collected data points. This data is also listed in Appendix M. In the figure we see classical carbon filler in thermoplastic behavior where increased filler loading increases viscosity at all shear rates. We see common shear thinning behavior where the viscosity decreases at high shear rates. Also noticed is the common result in filled systems where the change in viscosity due to increased filler loading is more pronounced as shear rate decreases (note Figures 8.9 and 8.10 with $\mathrm{CB}$ in PC). The unusual viscosity reduction effects of CNT are not present in the GNP system. 


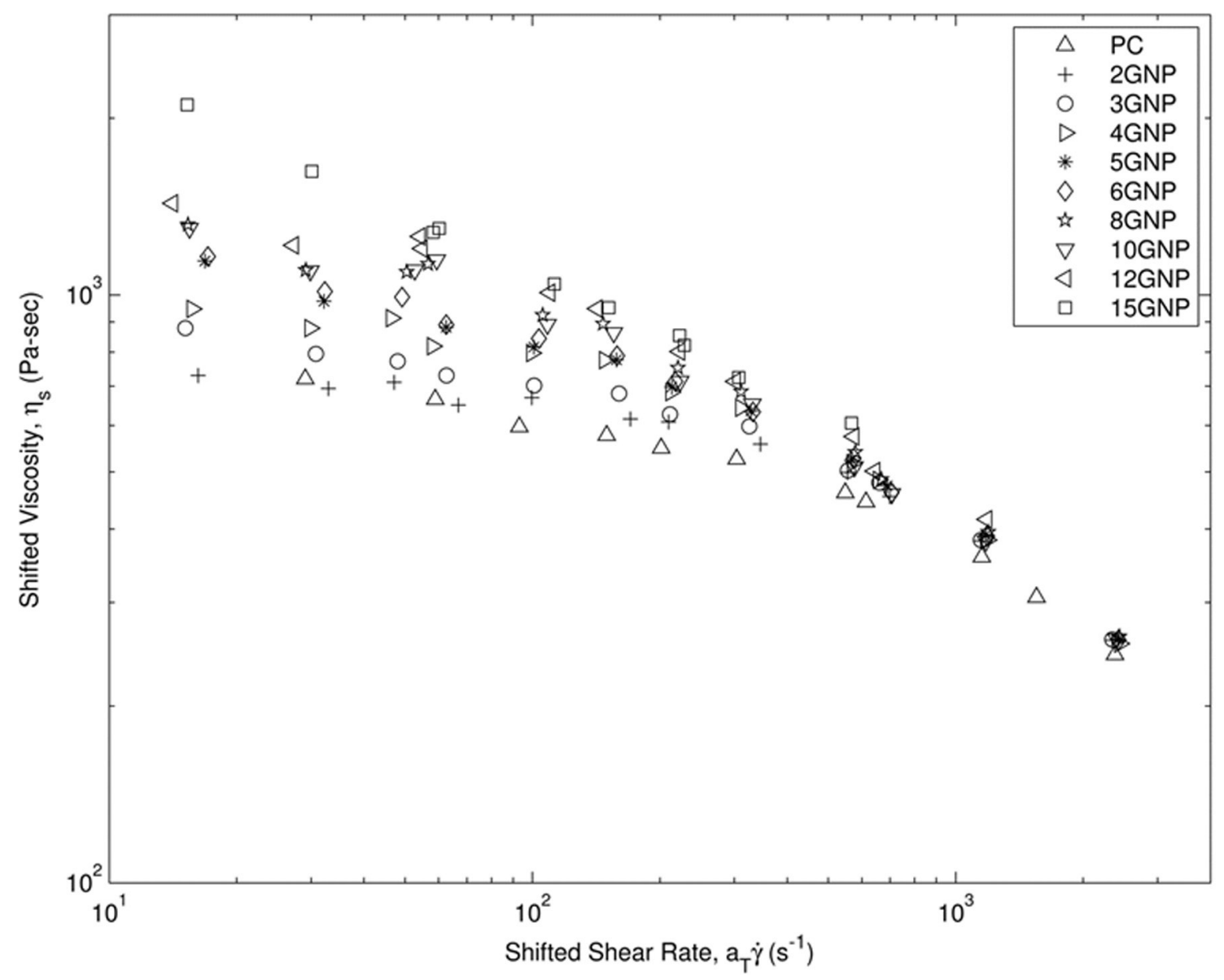

Figure 9.9: Time-temperature shifted steady-shear viscosity as a function of steady rate for GNP/PC composites studied at $270^{\circ} \mathrm{C}$

To model the GNP/PC system, the Maron-Pierce model for relative viscosity versus filler fraction in a concentrated suspension is employed (86). This system is effective for shear-thinning systems as long as the comparison is done at a constant shear stress. The Maron-Pierce model is shown in Equation 9.1 below:

$$
\eta_{r}=\frac{\eta(\phi)}{\eta(\phi=0)}=\frac{1}{\left(1-\frac{\phi}{\phi_{m}}\right)^{2}}
$$


Where $\eta_{r}$ is the reduced viscosity, $\eta(\phi)$ is the viscosity at filler volume, $\phi$, and $\phi_{m}$ is the maximum packing fraction of the filler. This model was based off of Einstein's experiments with glass spheres in water flows thus it assumes volume filling effects as the sole cause for viscosity increase. A value of 0.637 for the maximum packing fraction corresponds to random close packing of spheres, a value of 0.44 corresponds to random packing of rough irregular particles. Flaky mica particles have been found to have a value of 0.047 for the maximum packing fraction (87). As GNP is a flake-like particle with a high aspect ratio, it is expected to exhibit behavior somewhere between mica and rough irregular particles. To study the behavior of GNP/PC we follow the work of Kitano et al. and employ a modification of the Maron-Pierce model where the maximum packing fraction, $\phi_{m}$, is replaced with an adjustable parameter A as shown in Equation $9.2(88)$.

$\eta_{r}=\frac{\eta(\phi)}{\eta(\phi=0)}=\frac{1}{\left(1-\frac{\phi}{A}\right)^{2}}$

This model was found to fit the GNP/PC data well with a value for A of 0.30 . This model as well as the GNP/PC data is shown in Figure 9.10. The GNP/PC data shown in the figure corresponds to the viscosity of GNP/PC at a shear stress of $10^{5} \mathrm{~Pa}$. The value of 0.30 for GNP falls between the values for mica and rough irregular particles $(0.047$ and 0.44 respectively) as expected. The behavior of the GNP particles is more similar to that of rough irregular particles than flaky mica particles. 


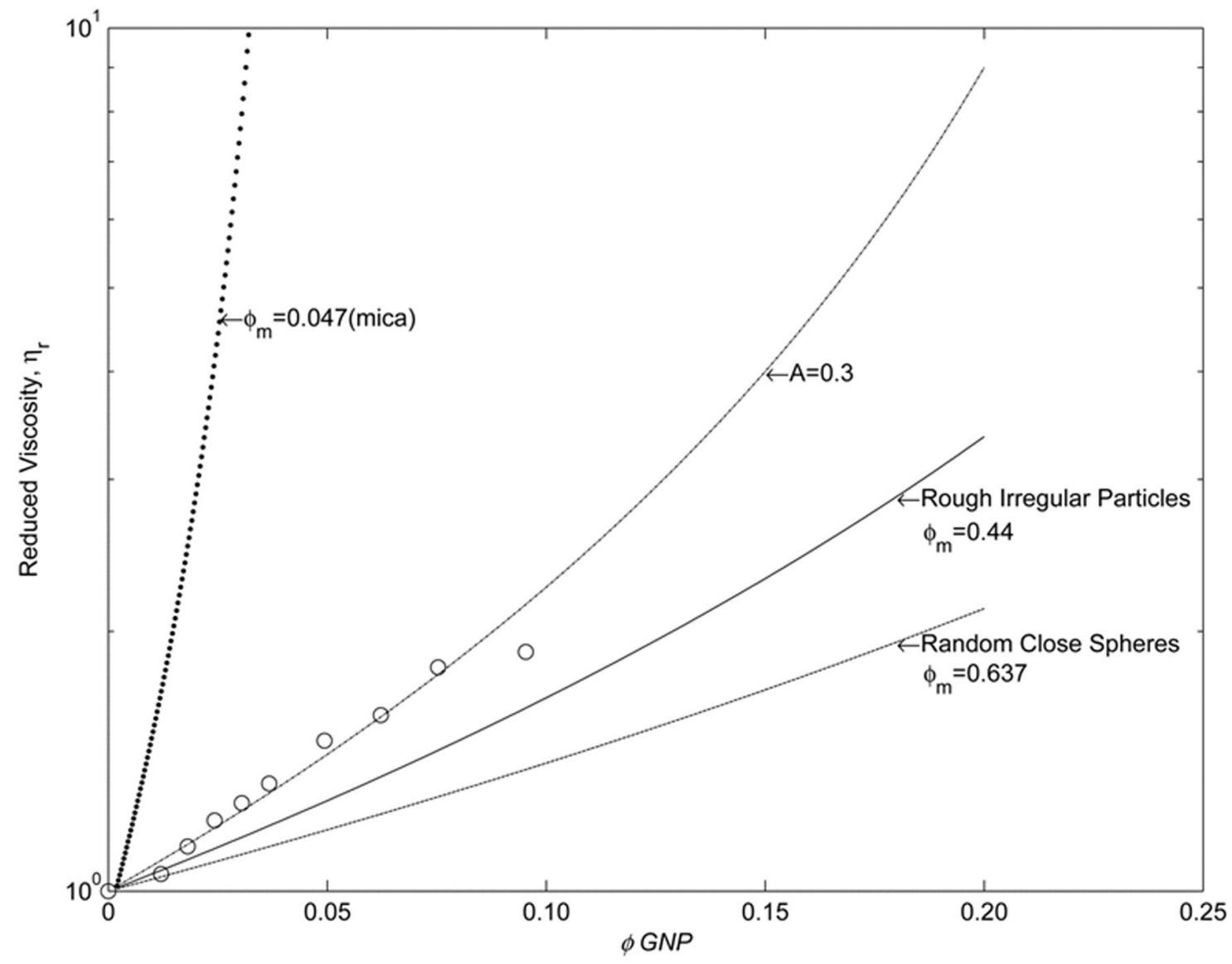

Figure 9.10: Reduced viscosity versus volume fraction, $\phi$, GNP at a shear stress of $10^{5}$ Pa for GNP/PC composites: experimental data along with Maron-Pierce models for various filler shapes and a Kitano-modified Maron-Pierce model $(\mathrm{A}=0.3)$

\section{7: Conclusions}

The object of this portion of the project was to study the effects of GNP on PC and compare them to CNT and CB. Electrically, GNP has a substantially higher percolation threshold than either CNT or CB $(4.0$ vol\% for GNP versus 1 vol\% for CNT and 2.3 vol\% for $\mathrm{CB}$ ). GNP also had less of an effect on reducing the electrical resistivity of the composite only reducing the ER from $\sim 10^{17} \mathrm{ohm}-\mathrm{cm}$ for pure PC to $2.8 \times 10^{4} \mathrm{ohm}-\mathrm{cm}$ for 9.6 vol\% GNP. GNP did have a relatively flat ER versus filler vol\% curve and has highend (8 to $15 \mathrm{wt} \% \mathrm{GNP}$ ) ER values that would place it in the electrostatic discharge range 
$\left(10^{10}\right.$ to $\left.10^{3} \mathrm{ohm}-\mathrm{cm}\right)$. Adding GNP to PC also increased the thermal conductivity of the neat PC from $0.21 \mathrm{~W} / \mathrm{m} \cdot \mathrm{K}$ to $0.49 \mathrm{~W} / \mathrm{m} \cdot \mathrm{K}$ for $15 \mathrm{wt} \% \mathrm{GNP}$ in PC.

Mechanically, GNP increased the tensile modulus of the resulting PC composite more than either CNT or $\mathrm{CB}$ reaching a value of $5.9 \mathrm{GPa}$ for $15 \mathrm{wt} \%$ (9.6 vol\%) GNP in PC up from 2.2 GPa for neat PC. The UTS for GNP remained constant at roughly $59 \mathrm{MPa}$ for all GNP/PC composites with up to $10 \mathrm{wt} \%$ GNP. Every composite containing less than $10 \mathrm{wt} \%$ GNP retained ductile behavior. The GNP also increased the flexural modulus of the resulting PC composite from 2.6 GPa for neat PC to $6.4 \mathrm{GPa}$ for $15 \mathrm{wt} \% \mathrm{GNP}$ in PC. The UFS stayed fairly constant at about $110 \mathrm{MPa}$ for all composites containing up to 8 $w t \%$ GNP.

GNP/PC composites were found to exhibit classical shear-thinning carbon-filled thermoplastic behavior (similar to $\mathrm{CB} / \mathrm{PC}$ ). The increase in viscosity in GNP/PC composites is due to the volume filling effects of the GNP platelet morphology. The viscosity of GNP/PC composites was found to be well described by the Kitano-modified Maron-Pierce model with value of A of 0.3. 


\section{Chapter 10: Modeling the Electrical Conductivity of Carbon Black/Polycarbonate, Carbon Nanotube/Polycarbonate, and Exfoliated Graphite Nanoplatelet/Polycarbonate Composites}

\section{1: Electrical Conductivity (EC) Results}

Table 10.1 shows the mean electrical conductivity, standard deviation of the electrical resistivity and number of samples that were tested for $\mathrm{CB} / \mathrm{PC}, \mathrm{CNT} / \mathrm{PC}$ and GNP/PC. This data is plotted in Figures 10.1 through 10.3 respectively along with models that will be discussed later. The results for the individual samples are given in Appendices $G$ and H. We see a typical electrical conductivity curves in these figures. At low conductive filler loadings, the electrical conductivity of the composite will be similar to that of the pure polymer. As the loading of the conductive filler increases, there is a point at which conductive networks begin to form and the conductivity increases dramatically over a very small range of filler loading. This point is known as the percolation threshold. After percolation occurs, the electrical conductivity of the composite levels out as additional conductive filler is added $(7,60)$. 
Table 10.1: CB/PC, CNT/PC and GNP/PC Electrical Resistivity Results

\begin{tabular}{|c|c|c|c|}
\hline Formulation & $\begin{array}{l}\text { Filler } \\
\text { Wt } \%\end{array}$ & $\begin{array}{l}\text { Filler } \\
\text { Vol \% }\end{array}$ & $\begin{array}{l}\text { Electrical Resistivity } \\
(\mathrm{ohm}-\mathrm{cm})\end{array}$ \\
\hline $\mathrm{PC}$ & 0 & 0.0 & $9.37 \times 10^{16} \pm 2.00 \times 10^{16} \mathrm{n}=6$ \\
\hline $2 \mathrm{CB}$ & 2 & 1.34 & $4.05 \times 10^{16} \pm 2.66 \times 10^{16} \mathrm{n}=6$ \\
\hline $3 \mathrm{CB}$ & 3 & 2.01 & $2.85 \times 10^{15} \pm 4.58 \times 10^{14} \mathrm{n}=6$ \\
\hline $4 \mathrm{CB}$ & 4 & 2.69 & $1.17 \times 10^{5} \pm 7.77 \times 10^{4} \mathrm{n}=8$ \\
\hline $5 \mathrm{CB}$ & 5 & 3.38 & $2474 \pm 646$ \\
\hline $6 \mathrm{CB}$ & 6 & 4.07 & $649 \pm 18$ \\
\hline $8 \mathrm{CB}$ & 8 & 5.46 & $122 \pm 4$ \\
\hline $10 \mathrm{CB}$ & 10 & 6.88 & $19.5 \pm 0.5$ \\
\hline $0.5 \mathrm{CNT}$ & 0.5 & 0.34 & $6.19 \times 10^{16} \pm 1.21 \times 10^{16} \mathrm{n}=6$ \\
\hline $1 \mathrm{CNT}$ & 1 & 0.69 & $2.02 \times 10^{16} \pm 6.62 \times 10^{15} \mathrm{n}=6$ \\
\hline $2 \mathrm{CNT}$ & 2 & 1.38 & $4610 \pm 1120$ \\
\hline $3 \mathrm{CNT}$ & 3 & 2.08 & $216 \pm 44$ \\
\hline $4 \mathrm{CNT}$ & 4 & 2.78 & $73 \pm 10$ \\
\hline $5 \mathrm{CNT}$ & 5 & 3.48 & $43 \pm 7$ \\
\hline $6 \mathrm{CNT}$ & 6 & 4.19 & $18 \pm 2$ \\
\hline $8 \mathrm{CNT}$ & 8 & 5.63 & $7.8 \pm 0.4$ \\
\hline $2 \mathrm{GNP}$ & 2 & 1.21 & $5.46 \times 10^{16} \pm 4.89 \times 10^{15} \mathrm{n}=6$ \\
\hline $3 \mathrm{GNP}$ & 3 & 1.82 & $3.23 \times 10^{16} \pm 7.22 \times 10^{15} \mathrm{n}=8$ \\
\hline $4 \mathrm{GNP}$ & 4 & 2.44 & $1.20 \times 10^{16} \pm 3.54 \times 10^{14} \mathrm{n}=6$ \\
\hline 5GNP & 5 & 3.06 & $3.76 \times 10^{15} \pm 2.83 \times 10^{14} \mathrm{n}=6$ \\
\hline $6 \mathrm{GNP}$ & 6 & 3.69 & $2.01 \times 10^{14} \pm 4.99 \times 10^{12} \mathrm{n}=6$ \\
\hline $8 \mathrm{GNP}$ & 8 & 4.96 & $3.95 \times 10^{7} \pm 1.53 \times 10^{7} \mathrm{n}=8$ \\
\hline $10 \mathrm{GNP}$ & 10 & 6.25 & $1.74 \times 10^{6} \pm 2.65 \times 10^{5} \mathrm{n}=8$ \\
\hline $12 \mathrm{GNP}$ & 12 & 7.56 & $3.07 \times 10^{5} \pm 9.50 \times 10^{3} \mathrm{n}=5$ \\
\hline 15GNP & 15 & 9.57 & $2.79 \times 10^{4} \pm 8.31 \times 10^{3} \mathrm{n}=8$ \\
\hline
\end{tabular}




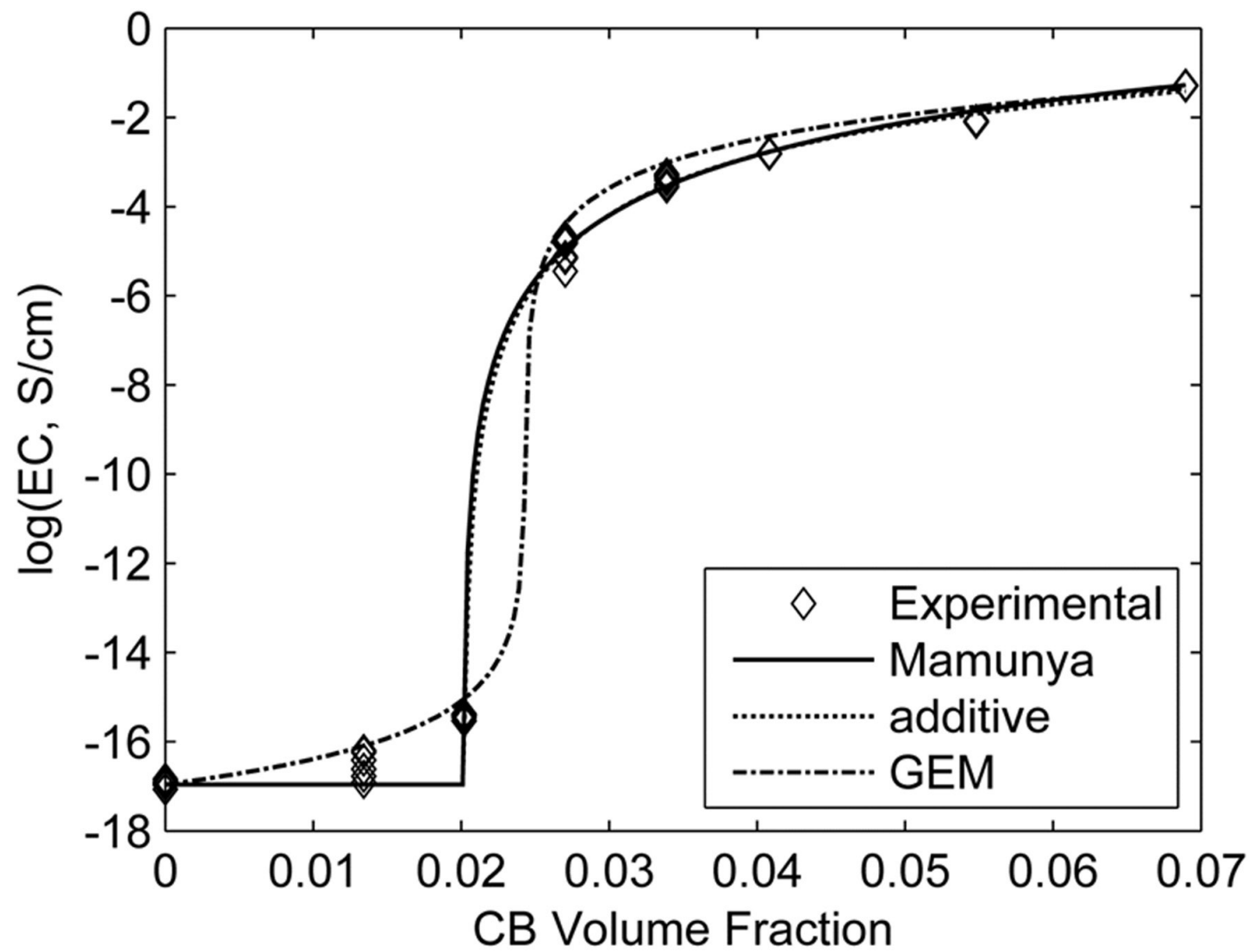

Figure 10.1: $\mathrm{CB} / \mathrm{PC}$ electrical resistivity results along with Mamunya, additive and GEM models. 


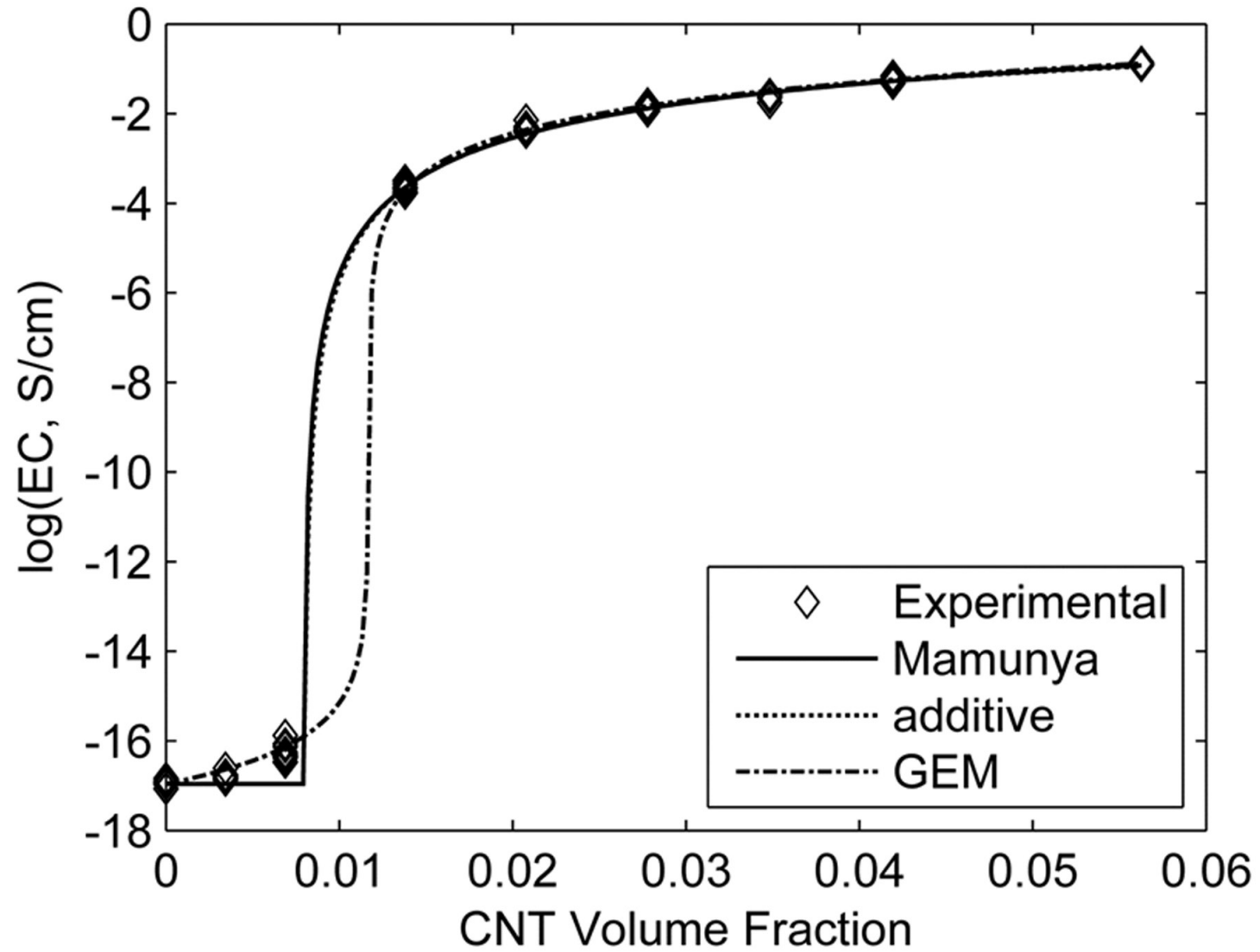

Figure 10.2: CNT/PC electrical resistivity results along with Mamunya, additive and GEM models. 


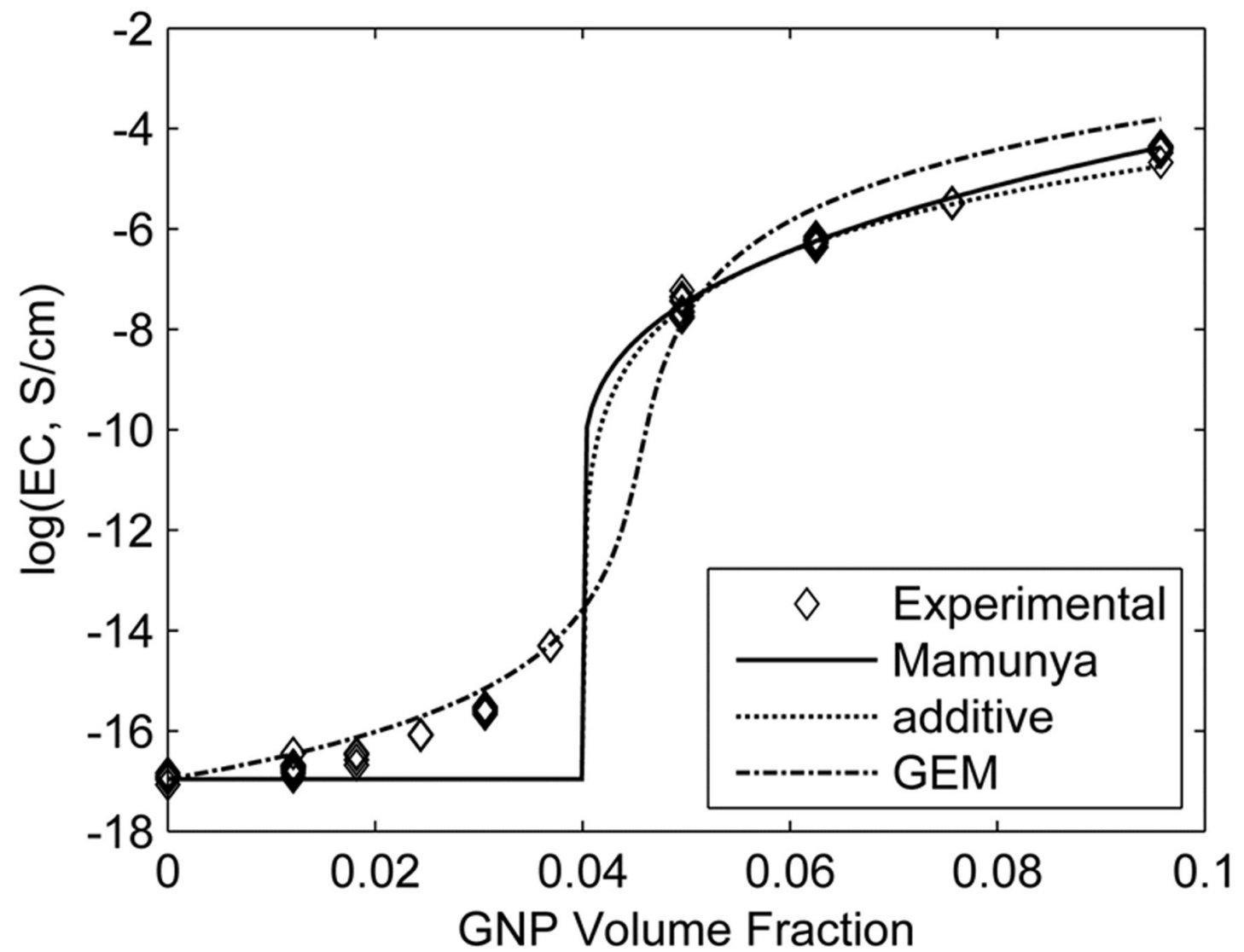

Figure 10.3: GNP/PC electrical resistivity results along with Mamunya, additive and GEM models.

In Figure 10.1 it is seen that $\mathrm{CB}$ decreases the ER of the composite at low loadings (percolation threshold 2.4 vol\%) as discussed in Chapter 7. Figure 10.2 illustrates that CNT has a very low percolation threshold (1 vol\%) and large effect on decreasing electrical conductivity $(7.8 \mathrm{ohm}-\mathrm{cm}$ at $5.6 \mathrm{vol} \%)$ as discussed in Chapter 6 . In Figure 10.3 we see that GNP has a higher percolation threshold than the other two fillers (4.6 vol\%) as discussed in Chapter 9. 


\section{2: Electrical Conductivity Models}

To model the electrical conductivity of these systems, we focused on a modified version of Mamunya et al.'s thermodynamic model $(89,90)$, Clingerman et al.'s additive model $(23,90)$ and McLachlan et al.'s General Effective Media (GEM) model (91-95). For all of these models, the adjustable parameters were chosen to minimize the total error as calculated by Equation 10.1 below:

$\varepsilon=\sum_{i=1}^{n}\left(\log \left(\sigma_{\text {calc }}\right)-\log \left(\sigma_{\text {measured }}\right)\right)^{2}$

where $\varepsilon$ is the total error, $\sigma_{\text {calc }}$ is the electrical conductivity for a formulation as predicted by the model and $\sigma_{\text {measured }}$ is the electrical conductivity of the same formulation as measured by experimentation. The total error as calculated by this method can also be used to compare the quality of fit of each model to each filler system. A lower total error would be indicative of a better quality of fit.

The modified Mamunya model is shown in Equation 10.2 below:

$$
\begin{array}{lr}
\log (\sigma)=\log \left(\sigma_{p}\right)+\left[\log \left(\sigma_{f}\right)-\log \left(\sigma_{p}\right)\left(\frac{\phi-\phi_{c}}{F-\phi_{c}}\right)^{k}\right. & \text { if } \phi>\phi_{C} \\
\log (\sigma)=\log \left(\sigma_{p}\right) & \text { if } \phi \leq \phi_{C}
\end{array}
$$

where $\sigma$ is the composite electrical conductivity, $\sigma_{p}$ is the electrical conductivity of the polymer, $\phi$ is the filler volume fraction, $\phi_{c}$ is the percolation threshold, $F$ is the maximum filler volume fraction and $\sigma_{f}$ is the electrical conductivity of the composite at $F(89)$. For this model, $k$ is given by Equation 10.3 below: 
$k=\frac{K \phi_{c}}{\left(\phi-\phi_{c}\right)^{n}}$

where $K$ and $n$ are adjustable parameters (89). It is important to note that for this model, when the filler volume fraction is below the percolation threshold, the composite electrical conductivity is assumed to be equal to the polymer electrical conductivity. This indicates that the percolation threshold for this model is defined as the filler volume fraction at which the electrical conductivity of the composite begins to dramatically increase.

For all of the formulations, the electrical conductivity of the polymer was measured to be $1.07 \times 10^{-17} \mathrm{~S} / \mathrm{cm}$. Figure 10.1 shows the fit of the Mamunya model to the CB/PC experimental data. For this fit, the percolation threshold, $\phi_{c}$, is estimated to be 0.020 , the maximum filler volume fraction, $F$, was 0.069 , the electrical conductivity at $F, \sigma_{f}$, was $0.052 \mathrm{~S} / \mathrm{cm}, K$ was set to 3.47 and $n$ was set to 0.13 . The Mamunya model resulted in a total error, $\varepsilon$, of $2.56(\log \mathrm{S} / \mathrm{cm})^{2}$ for the $\mathrm{CB} / \mathrm{PC}$ system. Figure 10.2 shows the fit of the Mamunya model to the CNT/PC experimental data. For this fit, the percolation threshold, $\phi_{c}$, is estimated to be 0.008 , the maximum filler volume fraction, $F$, was 0.056 , the electrical conductivity at $F, \sigma_{f}$, was $0.128 \mathrm{~S} / \mathrm{cm}, K$ was set to 4.11 and $n$ was set to 0.19. The Mamunya model resulted in a total error, $\varepsilon$, of $0.53(\log \mathrm{S} / \mathrm{cm})^{2}$ for the CNT/PC system. Figure 10.3 shows the fit of the Mamunya model to the GNP/PC experimental data. For this fit, the percolation threshold, $\phi_{c}$, is estimated to be 0.040 , the maximum filler volume fraction, $F$, was 0.096 , the electrical conductivity at $F, \sigma_{f}$, was 
$3.59 \times 10^{-5} \mathrm{~S} / \mathrm{cm}, K$ was set to 6.42 and $n$ was set to -0.099 . The Mamunya model resulted in a total error, $\varepsilon$, of $10.1(\log \mathrm{S} / \mathrm{cm})^{2}$ for the GNP/PC system. This model shows good agreement with the bulk of data from all three systems. The majority of the error comes from the data where the filler volume fraction is less than the percolation threshold thus the electrical conductivity is assumed to be that of the pure polymer. This assumption has the largest effect on the GNP/PC system as the EC curve decreases more gradually than the other two systems.

Clingerman et al.'s additive model has been shown in Equation 10.4 below:

$$
\begin{array}{lr}
\log (\sigma)=\log \left(\sigma_{p}\right)+H\left(\phi-\phi_{c}\right) \frac{G}{\left(\phi-\phi_{c}\right)^{n}}+E & \text { if } \phi \leq \phi_{C} \\
\log (\sigma)=\log \left(\sigma_{p}\right) & \text { if } \phi \leq \phi_{C}
\end{array}
$$

where $\sigma$ is the composite electrical conductivity, $\sigma_{p}$ is the electrical conductivity of the polymer, $\phi$ is the filler volume fraction and $\phi_{c}$ is the percolation threshold (90). In this model, $H, G, E$ and $n$ are adjustable model parameters (90). Again it should be noted that, similarly to the Mamunya model, this model assumes that the electrical conductivity of any specimen with a filler volume fraction less than the percolation threshold is equal to the pure polymer electrical conductivity. This means again that the percolation threshold used in the model is defined as the filler volume fraction at which the electrical conductivity of the composite begins to dramatically increase.

For all of the formulations, the electrical conductivity of the polymer was measured to be $1.07 \times 10^{-17} \mathrm{~S} / \mathrm{cm}$. Figure 10.1 shows the fit of the additive model to the $\mathrm{CB} / \mathrm{PC}$ experimental data. For this fit, the percolation threshold, $\phi_{c}$, is estimated to be $0.020, H$ 
is $18.3, G$ is $2.72 \times 10^{-2}, E$ is 0.0 and $n$ is 0.23 . The additive model resulted in a total error, $\varepsilon$, of $2.55(\log \mathrm{S} / \mathrm{cm})^{2}$ for the $\mathrm{CB} / \mathrm{PC}$ system. Figure 10.2 shows the fit of the additive model to the CNT/PC experimental data. For this fit, the percolation threshold, $\phi_{c}$, is estimated to be $0.008, H$ is $17.5, G$ is $1.28 \times 10^{-2}, E$ is 0.0 and $n$ is 0.28 . The additive model resulted in a total error, $\varepsilon$, of $0.52(\log \mathrm{S} / \mathrm{cm})^{2}$ for the CNT/PC system. Figure 10.3 shows the fit of the additive model to the GNP/PC experimental data. For this fit, the percolation threshold, $\phi_{c}$, is estimated to be $0.040, H$ is $17.5, G$ is $0.112, E$ is 0.0 and $n$ is 0.040 . The additive model resulted in a total error, $\varepsilon$, of $9.79(\log \mathrm{S} / \mathrm{cm})^{2}$ for the GNP/PC system. The additive model has a similar fit to the data as the Mamunya model did, fitting the bulk of the data for each system. Similarly to the Mamunya model, the majority of the total error comes from the assumption that the electrical conductivity of formulations with filler volume fractions less than the percolation threshold is equal to the electrical conductivity of the pure PC.

The GEM model is shown in Equation 10.5 below:

$$
\frac{(1-\phi)\left(\sigma_{p}^{1 / t}-\sigma_{m}^{1 / t}\right)}{\sigma_{p}^{1 / t}-A \sigma_{m}^{1 / t}}+\frac{\phi\left(\sigma_{h}^{1 / t}-\sigma_{m}^{1 / t}\right)}{\sigma_{h}^{1 / t}-A \sigma_{m}^{1 / t}}=0
$$

where $\sigma_{m}$ is the electrical conductivity of the composite, $\sigma_{p}$ is the electrical conductivity of the polymer, $\sigma_{h}$ is the electrical conductivity of the filler and $\phi$ is the filler volume fraction (91-93). $A$ is given by Equation 10.6 below:

$$
A=\frac{1-\phi_{c}}{\phi_{c}}
$$


where $\phi_{c}$ is the percolation threshold (91-93). $t$ is a dimensionless critical component that can be used to determine the effects of the filler morphology and is given in Equation 10.7 below:

$t=\frac{1-\phi_{c}}{1-L}$

where $\mathrm{L}$ is the demagnification coefficient of the dispersion (91-93).

The GEM model requires the electrical conductivity of the polymer $\left(1.07 \times 10^{-17} \mathrm{~S} / \mathrm{cm}\right)$ as well as the electrical conductivity of the conductive filler. CB has a conductivity of 100 $\mathrm{S} / \mathrm{cm}$ as given in Table 3.3. CNTs have a wide range of reported values for EC. For this modeling, we used $100 \mathrm{~S} / \mathrm{cm}$ as the EC (96-98). GNP has had measurements of the electrical conductivity range from 1 to $100 \mathrm{~S} / \mathrm{cm}(99)$. For this project, we take the logarithmic average of these values, $10 \mathrm{~S} / \mathrm{cm}$ for the EC of GNP.

It should be noted that due to the nature of the GEM model, the percolation threshold is assumed to be the inflection point of the large increase in electrical conductivity. This means that the values used for the percolation threshold for this model do not coincide with the values used for the Manmunya or additive models, but instead resembling the values we have been reporting as the percolation threshold in prior chapters. Figure 10.1 shows the fit of the GEM model to the $\mathrm{CB} / \mathrm{PC}$ experimental data. For this fit, the percolation threshold, $\phi_{c}$, is 0.024 and $t$ is 2.5 . The GEM model resulted in a total error, $\varepsilon$, of $0.55(\log \mathrm{S} / \mathrm{cm})^{2}$ for the $\mathrm{CB} / \mathrm{PC}$ system. Figure 10.2 shows the fit of the GEM model to the CNT/PC experimental data. For this fit, the percolation threshold, $\phi_{c}$, is 
0.012 and $t$ is 2.1. The GEM model resulted in a total error, $\varepsilon$, of $0.07(\log \mathrm{S} / \mathrm{cm})^{2}$ for the CNT/PC system. Figure 10.3 shows the fit of the GEM model to the GNP/PC experimental data. For this fit, the percolation threshold, $\phi_{c}$, is 0.046 and $t$ is 3.8. The GEM model resulted in a total error, $\varepsilon$, of $1.04(\log \mathrm{S} / \mathrm{cm})^{2}$ for the GNP/PC system. We can see from these parameters that $t$ has a large influence on the slope of the EC curve near percolation with a larger value for $t$ giving a more gradual slope. The GEM model showed the best quality of fit for all 3 systems as measured by the total errors. This was especially true for the GNP/PC system. These models have been studied before by this research group for other systems such as $\mathrm{CB}$ /polypropylene and $\mathrm{CNT}$ /polypropylene systems with similar results (100). These models were also used to study $\mathrm{CB} /$ liquid crystal polymer systems and again, the GEM model was found to fit the data well (94).

\section{3: Conclusions}

The purpose of this section of the project was to model the electrical conductivity of $\mathrm{CB} / \mathrm{PC}, \mathrm{CNT} / \mathrm{PC}$ and GNP/PC systems with a modified Mamunya thermodynamic model, Clingerman et al.'s additive model, and the Generalized Effective Media (GEM) model. The Mamunya and additive models produced similar results and showed very good agreement with formulations that had filler volume fractions above the percolation threshold. The assumption present in each model that, with filler volume fractions below the percolation threshold, the electrical conductivity of the composite equals that of the pure polymer does not conform to experimental results. The GEM model does not include this assumption and thus models this area of the data quite well. While the GEM model does not model the region of the curves above the percolation threshold as well as 
either the Mamunya or additive models, the ability to fit the data before the percolation threshold means it has a lower total error for all three filler systems. It is important to note that the percolation threshold for the GEM model coincides with the inflection point of the large increase of electrical conductivity versus filler volume fraction. This contrasts with the Mamunya and additive models which use the beginning of the large increase in electrical conductivity versus filler volume fraction as the percolation threshold. The GEM model has the benefit of not relying on the electrical conductivity of the highest filler loading composite in a system and is capable of being applied to multiple filler systems. The GEM model provides good estimations for $\mathrm{CB} / \mathrm{PC}, \mathrm{CNT} / \mathrm{PC}$ and GNP/PC over a wide range of filler loadings (above and below the percolation threshold) and thus would be a good model for the formulation of materials for semiconductive and electrostatic discharge applications. 


\section{Chapter 11: Tensile Modulus Modeling of Carbon Black/Polycarbonate, Carbon Nanotube/Polycarbonate, and Exfoliated Graphite Nanoplatelet/Polycarbonate Composites}

\section{1: Electrical Conductivity and Percolation Models}

Many of the most commonly used models to estimate the tensile modulus of filled composites require knowledge of the aspect ratio (typically length/diameter) of the filler. This is due to the filler aspect ratio having a significant effect on the tensile modulus of the composite. Typically, aspect ratios are known only for as-received materials. It is common knowledge that the high energy processing techniques used in composite manufacturing (i.e. extrusion, injection molding) causes some amount of filler degradation. It can be particularly difficult to determine the aspect ratio of nanomaterial fillers in polymer composites after processing. Nanomaterials are primarily too small to be optically imaged, and often have extreme geometries that are difficult to successfully measure using electron microscopy.

We hypothesize that the percolation threshold of the manufactured composite samples as calculated using electrical conductivity modeling can be used to calculate an effective aspect ratio for the fillers using modern percolation threshold modeling. For this calculation, we will assume that the degradation of the filler happens primarily in the extended dimensions of the filler. The extended dimensions of the fillers act as a long moment arm allowing for the moment felt by the particle during processing to be extremely high in these directions compared to the non-extended directions. Thus we 
will use the GEM modeling from Chapter 10 to determine a percolation threshold for these systems that can then be used with percolation threshold modeling techniques.

Recently, CNT percolation thresholds have been modeled by incorporating a conductive interparticale distance (IPD) into an analytical method (101). This paper suggests that there is a particular distance that conductive particles can be separated from each other without a loss of electrical conductivity. This distance is related to the quantum mechanical electron tunneling distance through the non-conductive matrix. For this model, a simple cylindrical geometry was assumed for CNT. The model then creates cubic elements containing single CNTs and uses these elements to determine a percolation threshold. The resulting formula for the percolation threshold of CNT/PC composites is shown below in Equation 11.1 (101).

$$
\phi_{C}=\frac{\pi d^{2} L}{4\left[\left\langle\cos ^{2} \theta\right\rangle \cdot(L+I P D)\right]^{\beta}}
$$

For this project, the percolation threshold as calculated in the GEM model in Chapter 10 will inserted into Equation 11.1 which will then be used to calculate an effective length and diameter (thus aspect ratio) for CNT in the CNT/PC system. Thus, $\phi_{c}$ is the percolation threshold as found in the GEM model (0.012), $d$ is the diameter of the CNT (10 $\mathrm{nm}$ as given by the vendor), $\left\langle\cos ^{2} \theta\right\rangle$ is the average of the squared cosine of the orientation angle of the CNT (for a randomly distributed 3D system this is equal to $1 / 3$ ), IPD is the electron tunneling distance (10 nm for many polymer systems) (101), and L is 
the effective length of the CNT which is calculated to be $405 \mathrm{~nm}$. The resulting aspect ratio that can be used in tensile modulus modeling of CNT/PC composites is thus 40.5 .

GNP percolation threshold has also recently been modeled using a similar method involving the IPD in an analytical approach (102). Again it is suggested that the electron tunneling distance through the non-conductive polymer allows the conductive particles to be separated by a certain distance without losing conductivity. This model assumes a high aspect ratio disc-like geometry that is suitable for GNP. This geometry is then used to create cubic elements that contain a single GNP particle. These elements are then used to estimate the percolation threshold. The resulting formula for the percolation threshold of GNP/PC systems is shown in Equation 11.2 below.

$$
\phi_{C}=\frac{27 \pi d^{2} t}{4(d+I P D)^{3}}
$$

For this project, the percolation threshold as calculated in the GEM model in Chapter 10 will inserted into Equation 11.2 which will then be used to calculate an effective thickness and diameter (thus aspect ratio) for GNP in the GNP/PC system. Thus, $\phi_{c}$ is the percolation threshold as found in the GEM model (0.046), $t$ is the thickness of the GNP (7 nm), IPD is the electron tunneling distance (10 $\mathrm{nm}$ for many polymer systems) (102), and $\mathrm{d}$ is the effective diameter of the GNP which is calculated to be $3200 \mathrm{~nm}$. The resulting aspect ratio that can be used in tensile modulus modeling of GNP/PC composites is thus 457 . 


\section{2: Tensile Modulus Models}

The Halpin-Tsai and Nielsen models will be investigated for this project. The HalpinTsai is a very common model used to estimate the tensile modulus of CNT based composites (103-105). It was originally developed as a modification of the Nielsen model as will be seen in the discussion.

As the Halpin-Tsai was based upon the Nielsen model, the Nielsen model will be discussed first. The Nielsen model requires the tensile modulus of the matrix, the tensile modulus of the filler, the volume fraction of the filler, the maximum volume fraction of the filler and the Einstein coefficient of the filler as inputs. The maximum packing fraction of $\mathrm{CB}$ and $\mathrm{CNT}$ have been determined in prior work to be 0.2 (37). The maximum packing fraction of GNP was determined using rheological analysis in prior work in Chapter 9 to be 0.3 .

This Einstein coefficient, $\mathrm{K}_{\mathrm{E}}$, is related to the aspect ratio of the filler. For spherical particles such as $\mathrm{CB}$, the Einstein coefficient is 2.5 (106). For rod-like fillers such as $\mathrm{CNT}$, the Einstein coefficient is $2 \mathrm{~L} / \mathrm{d}$, where $\mathrm{L} / \mathrm{d}$ is length/diameter (aspect ratio), or 81 for this system. For GNP the Einstein coefficient is calculated using Equation 11.3 (107) below.

$$
K_{E}=2.5\left(\frac{d}{t}\right)^{0.645}
$$


Where $d$ is the diameter of the platelet (3200 nm from percolation threshold modeling), $t$ is the thickness of the platelet $(7 \mathrm{~nm})$, and $K_{E}$ is the Einstein coefficient which is found to be 130 for this system.

The Nielsen model uses these parameters to predict the tensile modulus of composites according to Equations 11.4 through 11.7 below.

$$
\begin{aligned}
& \frac{E_{C}}{E_{m}}=\frac{1+A B V_{f}}{1-B \psi V_{f}} \\
& A=K_{E}-1 \\
& B=\frac{\left(E_{f} / E_{m}\right)-1}{\left(E_{f} / E_{m}\right)+A} \\
& \psi=1+\frac{1-\phi_{m}}{\phi_{m}^{2}} V_{f}
\end{aligned}
$$

In these equations, $E_{C}$ is the composite tensile modulus, $E_{m}$ is the tensile modulus of the matrix, $E_{f}$ is the tensile modulus of the filler, $K_{E}$ is the Einstein coefficient of the filler, $\phi_{m}$ is the maximum packing fraction of the filler and $V_{f}$ is the volume fraction of the filler $(30,108-110)$.

For this project, $E_{m}$ was the same value for all three systems, $2.20 \mathrm{GPa}$. Using experimental data from prior literature, $\mathrm{CB}$ composites used $E_{f}=827 \mathrm{GPa}, K_{E}=2.5$, and $\phi_{m}=0.2$ with the results of this modeling seen in Figure $11.1(30,37,108)$. CNT composites used $E_{f}=59.4 \mathrm{GPa}$ as determined from the Halpin-Tsai modeling discussed 
later, $K_{E}=81.0$, and $\phi_{m}=0.2$ with the results being shown in Figure 11.2. For GNP composites the value for the tensile modulus of the filler, $E_{f}$, was set at the modulus of exfoliation of graphite, $36.5 \mathrm{GPa}(38), K_{E}$ was set to 130 , and $\phi_{m}$ was set to 0.3 with the results shown in Figure 11.3. Graphene sheets are often reported to have tensile moduli in the range of $1000 \mathrm{GPa}$ in the plane of the graphene sheet (99). GNP is made of 10-15 graphene sheets stacked atop each other forming a graphite particle. When a tensile load is applied to the composite and the load is subsequently transferred to the GNP, it is likely that the weaker Van der Waals bonds between the graphene sheets will fail before the graphitic carbon-carbon bonds. This leads the effective modulus of the filler to be that of exfoliation of the plates, not the modulus of tearing of the individual plates. We see in Figures 11.1 through 11.3 that the Nielsen model shows good agreement for $\mathrm{CB} / \mathrm{PC}$ and GNP/PC systems but consistently over-estimates the modulus for CNT/PC composites.

The Halpin-Tsai model takes the Nielsen model then assumes fiber geometry for all fillers and removes the volume filler parameter, $\Psi$, to create an equation describing the tensile modulus in the direction of fiber orientation and adds a second equation that determines the tensile modulus of the composite transverse to the fiber orientation. Scaling factors are then used to combine the two equations into a final composite modulus. This focus on fiber geometries makes the Halpin-Tsai model particularly effective at modeling CNT systems and has thus often been used for these systems (103105). Equations 11.8 through 11.11 are used in the Halpin-Tsai model to determine the moduli of the composite in both the longitudinal and transverse directions of the fillers. 


$$
\begin{aligned}
& \frac{E_{L}}{E_{M}}=\frac{1+2(L / d) \eta_{L} V_{f}}{1-\eta_{L} V_{f}} \\
& \frac{E_{T}}{E_{M}}=\frac{1+2 \eta_{T} V_{f}}{1-\eta_{T} V_{f}} \\
& \eta_{L}=\frac{\left(E_{f} / E_{m}\right)-1}{\left(E_{f} / E_{m}\right)+2(L / d)} \\
& \eta_{T}=\frac{\left(E_{f} / E_{m}\right)-1}{\left(E_{f} / E_{m}\right)+2}
\end{aligned}
$$

In the preceding equations, $E_{L}$ is the longitudinal composite tensile modulus, $E_{T}$ is the transverse composite tensile modulus, $E_{M}$ is the tensile modulus of the matrix, $E_{f}$ is the tensile modulus of the filler, $L / d$ is the aspect ratio of the filler, and $V_{f}$ is the volume fraction of the filler (111-114). It is apparent that Equation 11.8when Equation 11.10 is incorporated is equivalent to Equation 11.4 after the incorporation of Equations 11.5 and 11.6 and the removal of $\Psi$ when $K_{E}$ is equal to $2(L / d)$. In order to better simulate the randomness of filler orientation within a composite system, the Halpin-Tsai model introduces two equations to determine the composite tensile modulus for primarily 2dimensional systems (i.e. films) and for 3-dimensional systems (i.e. bulk materials and structures). These equations are shown as Equations 11.12 and 11.13 below with $E_{C}$ being the composite tensile modulus. 
$E_{C}=\frac{3}{8} E_{L}+\frac{5}{8} E_{T} \quad$ 2D Randomly oriented fiber

$E_{C}=\frac{1}{5} E_{L}+\frac{4}{5} E_{T} \quad$ 3D Randomly oriented fiber

For all the formulations in this project, $E_{m}$ was set to be $2.20 \mathrm{GPa}$. For CB systems, $E_{f}=$ $827 \mathrm{GPa}$ and by following prior literature, $2(L / d)$ is replaced with the value 1.5 $(30,37,108)$. The results for the $\mathrm{CB}$ system are shown in Figure 11.1 where we see a consistent underestimation of the tensile modulus.

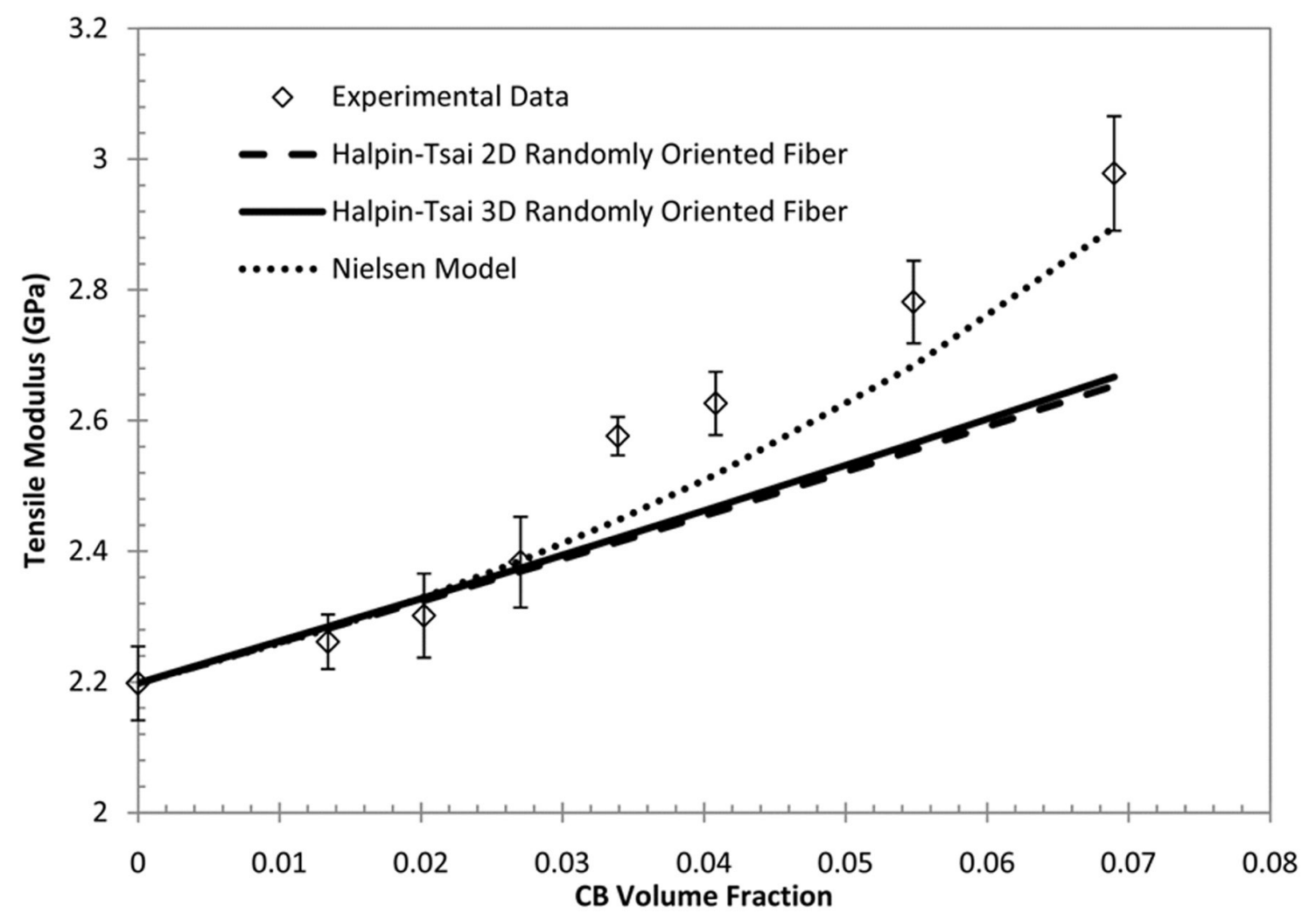

Figure 11.1: Tensile Modulus Results Along With Halpin-Tsai 2D and 3D Randomly Oriented Fiber and Nielsen Models for CB/PC Composites 
For CNT systems, $E_{f}$ has been reported to be anywhere from $12 \mathrm{GPa}$ to $1000 \mathrm{GPa}$ $(115,116)$. For this reason, $E_{f}$ was fit to minimize the error generated in the 3dimensional Halpin-Tsai model. Error was defined as the sum of the absolute values of the differences between the experimental data and model values for each formulation. 3dimensional random orientation was chosen for optimization due to the size difference between the filler and the tested sample. The smallest dimension of the sample, 3300 $\mu \mathrm{m}$, is 330 times larger than the length of the as-received CNT $(10 \mu \mathrm{m})$. Model fitting placed the value of $E_{f}$ for the CNT system at $59.4 \mathrm{GPa}$, similar to the 12 to $50 \mathrm{GPa}$ range given by Salvetat et al. (115). $L / d$ was set to 40.5 as determined by the percolation threshold modeling shown earlier. The results for the CNT system are shown in Figure 11.2 where it can be seen that the 3-dimensional Halpin-Tsai model shows good agreement with the experimental data.

For GNP based systems, $E_{f}$ is set to $36.5 \mathrm{GPa}$ for the same reasons as it was in the Nielsen model. L/d was equated to $\mathrm{d} / \mathrm{t}$ (diameter divided by thickness) and thus a value of 457 was used. The results for the GNP system are shown in Figure 11.3 where, similarly to CB systems, we see a consistent underestimation of the tensile modulus. 


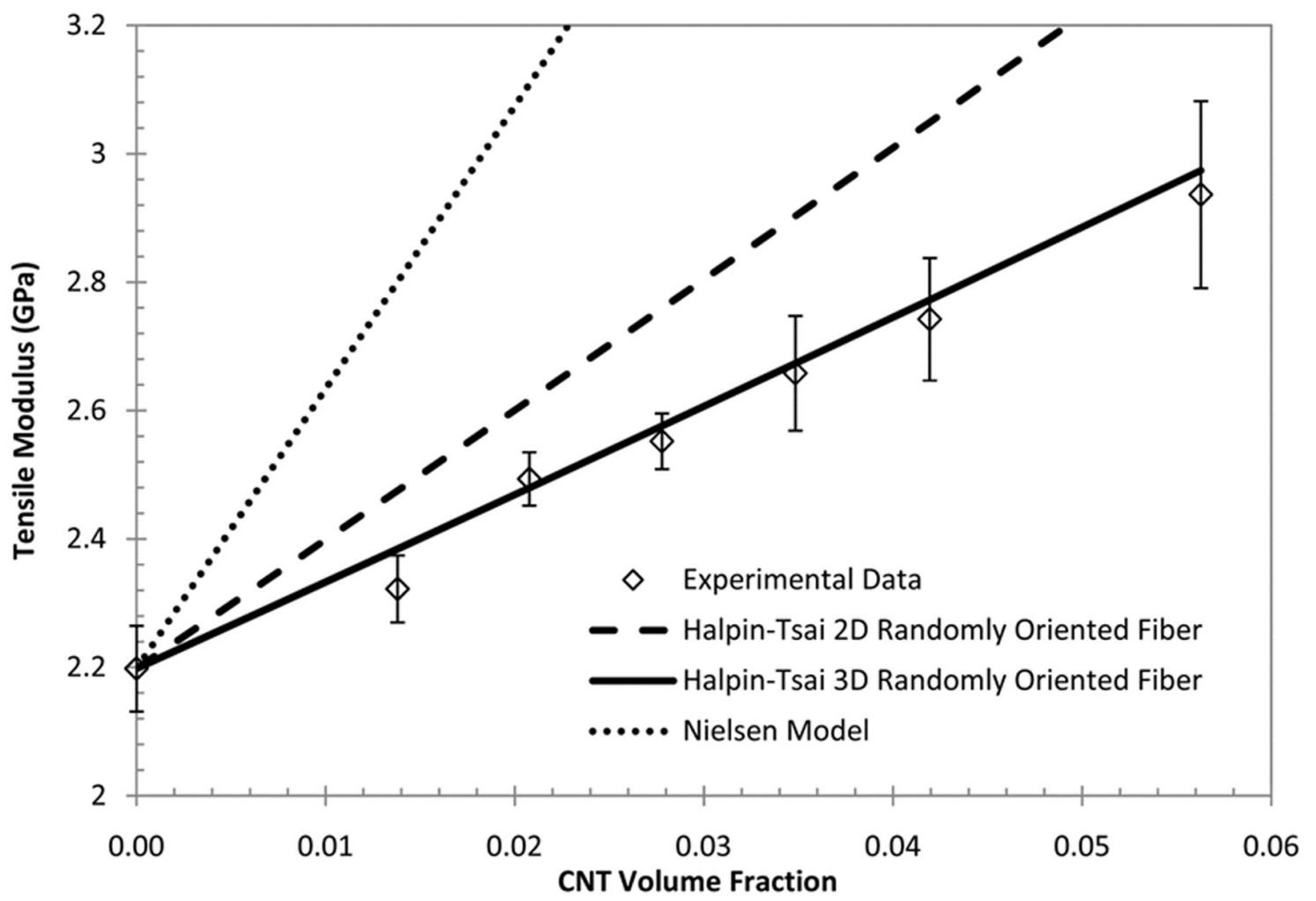

Figure 11.2: Tensile Modulus Results Along With Halpin-Tsai 2D and 3D Randomly Oriented Fiber and Nielsen Models for CNT/PC Composites 


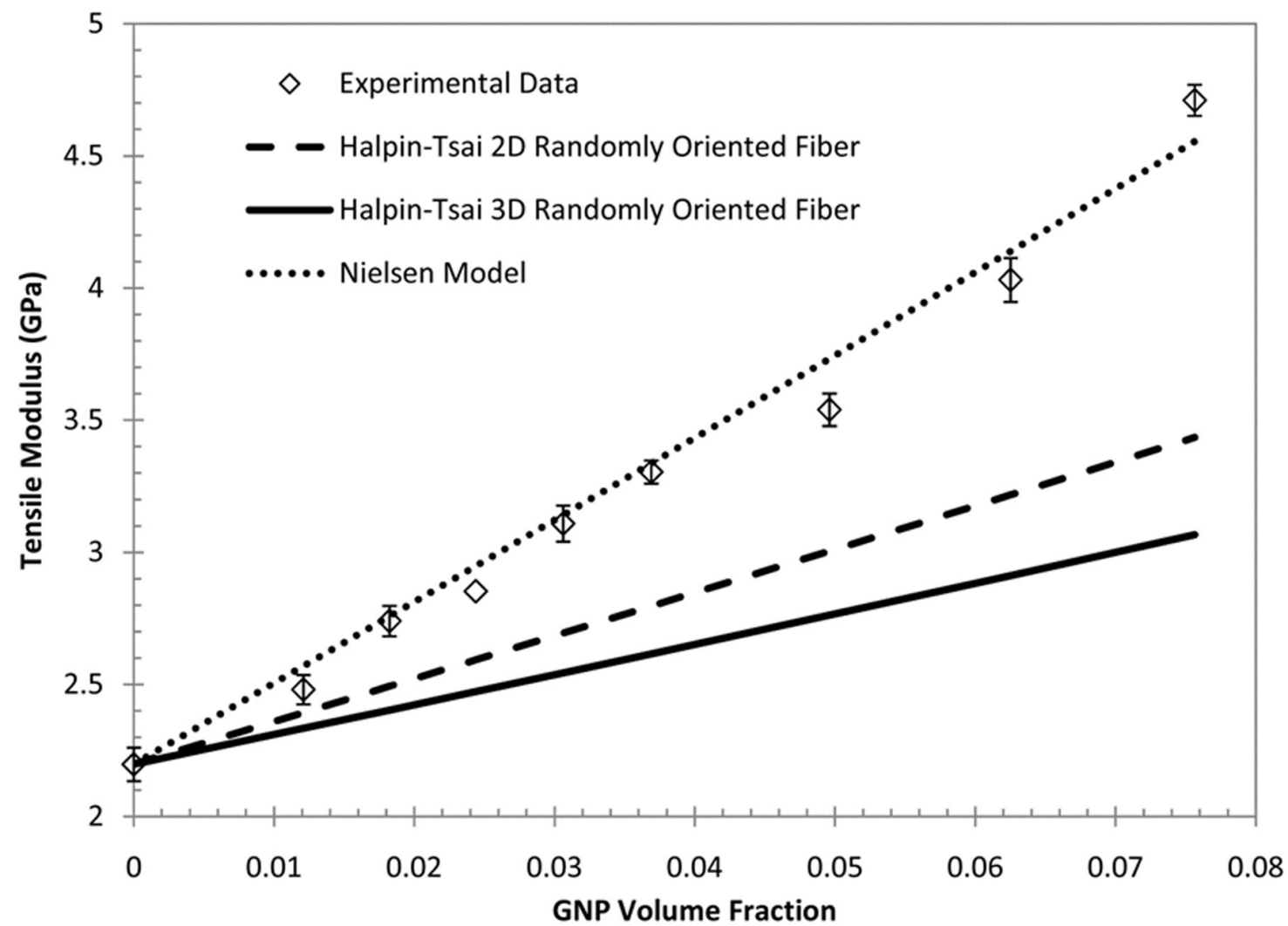

Figure 11.3: Tensile Modulus Results Along With Halpin-Tsai 2D and 3D Randomly Oriented Fiber and Nielsen Models for GNP/PC Composites

\section{3: Conclusions}

The purpose of this chapter is to measure the tensile moduli of single-filler PC composites ( $\mathrm{CB} / \mathrm{PC}, \mathrm{CNT} / \mathrm{PC}$ and $\mathrm{GNP} / \mathrm{PC})$ and apply the results to existing models (Nielsen and Halpin-Tsai) to determine which models are most applicable. Of these systems, GNP produced the largest increase in tensile modulus, followed by CNT, with CB having the least increase in tensile modulus.

The particular variation of the Halpin-Tsai model studied here assumes fiber-shaped filler geometry and is very commonly used to model the behavior of CNT in polymer systems. For the CNT/PC system studied here, the Halpin-Tsai model shows good agreement for all filler loadings. For the $\mathrm{CB} / \mathrm{PC}$ (spherical filler) and GNP/PC (platelet filler) systems, 
the Halpin-Tsai model was found to consistently underestimate the tensile modulus of the composites.

The Nielsen model is a precursor of the Halpin-Tsai model that does not assume the filler geometry. Instead of relying on assumptions for fiber geometry, the Nielsen model uses the Einstein coefficient of the filler and the maximum packing fraction to account for filler geometry and model the tensile modulus. Spherical particles like CB have a defined Einstein coefficient of 2.5. The Einstein coefficients of CNT and GNP are based upon the geometry of the filler particle, particularly the aspect ratio. The aspect ratio of the CNT and GNP in the PC was determined through modeling of the electrical percolation threshold as was determined previously using the GEM model as discussed in Chapter 10. Obtaining the model parameters using this method resulted in improved tensile models for the studied systems. The Einstein coefficient of the CNT in PC was calculated to be 81 . GNP was calculated to have an Einstein coefficient of 130 in PC. The maximum packing fractions of the fillers were obtained in prior work. The Nielsen model was found to show good agreement with the $\mathrm{CB} / \mathrm{PC}$ and GNP/PC composites, while consistently overestimating the tensile modulus of the CNT/PC composites. 


\section{Chapter 12: Effects of Multiple Fillers on the Electrical Resistivity, Thermal Conductivity and Tensile and Flexural Properties of Polycarbonate Based Composites}

\section{1: Transmission Electron Microscope (TEM) Results}

Figure 12.1 shows a TEM bright field micrograph of a composite containing $5 \mathrm{wt} \% \mathrm{CB}$ and $5 \mathrm{wt} \% \mathrm{CNT}$ in PC. Figure 12.2 shows TEM bright field (a) and dark field (b) micrographs of a composite containing $5 \mathrm{wt} \% \mathrm{CB}$ and $5 \mathrm{wt} \%$ GNP in PC. The dark field image is of the same region of the sample as the bright field. It was obtained by positioning the objective aperture off-center to isolate diffracted electrons from crystalline materials (GNP). This technique highlights only those crystalline particles that satisfy the Bragg condition, in this case, the GNP particle in the center of the image. Figure 12.3 similarly shows TEM bright field (a) and dark field (b) micrographs of a composite containing $5 \mathrm{wt} \% \mathrm{CNT}$ and $5 \mathrm{wt} \% \mathrm{GNP}$ in PC. 


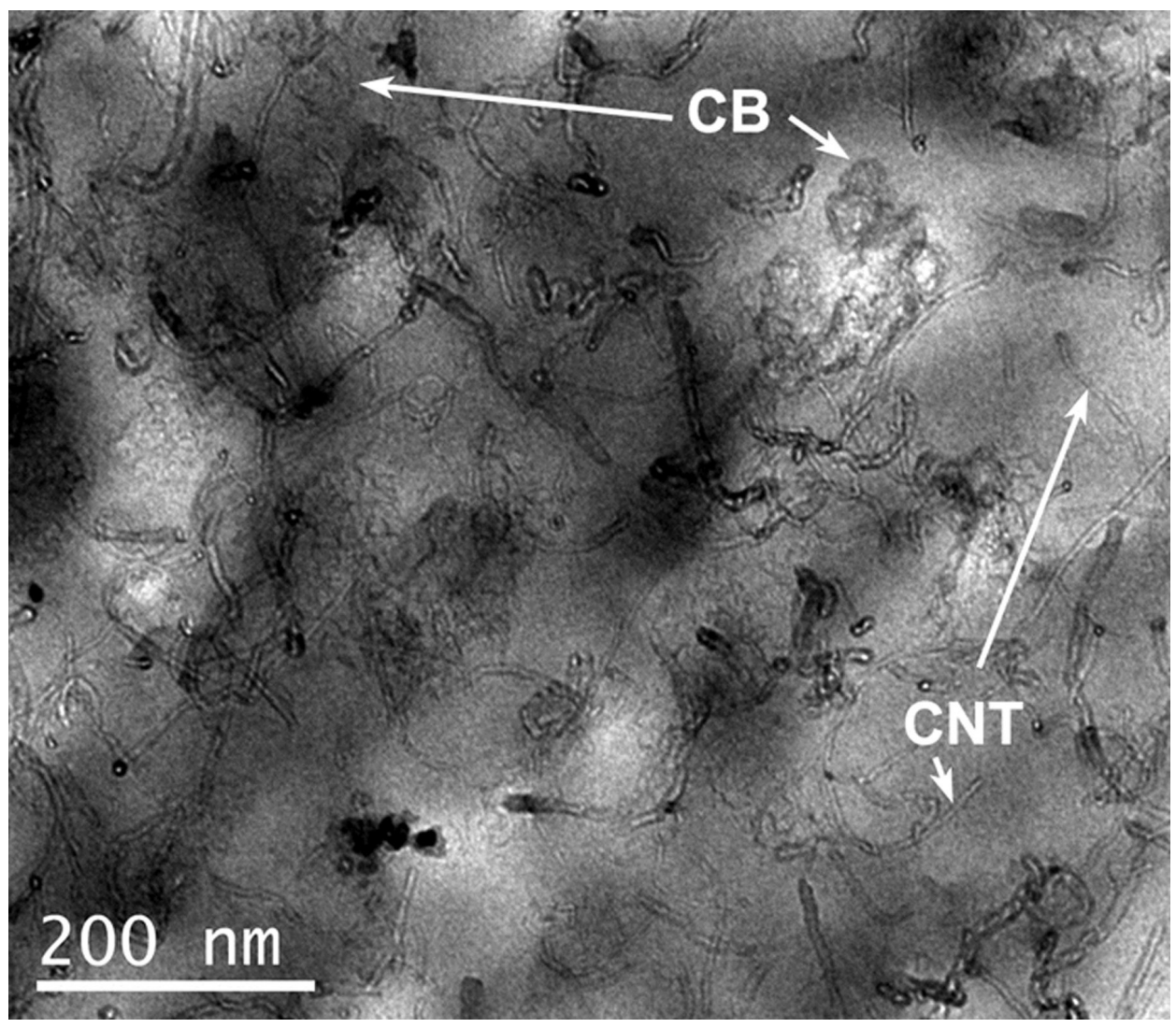

Figure 12.1: TEM Micrograph of Composite Containing $5 \mathrm{wt} \% \mathrm{CB}$ and $5 \mathrm{wt} \% \mathrm{CNT}$ in PC

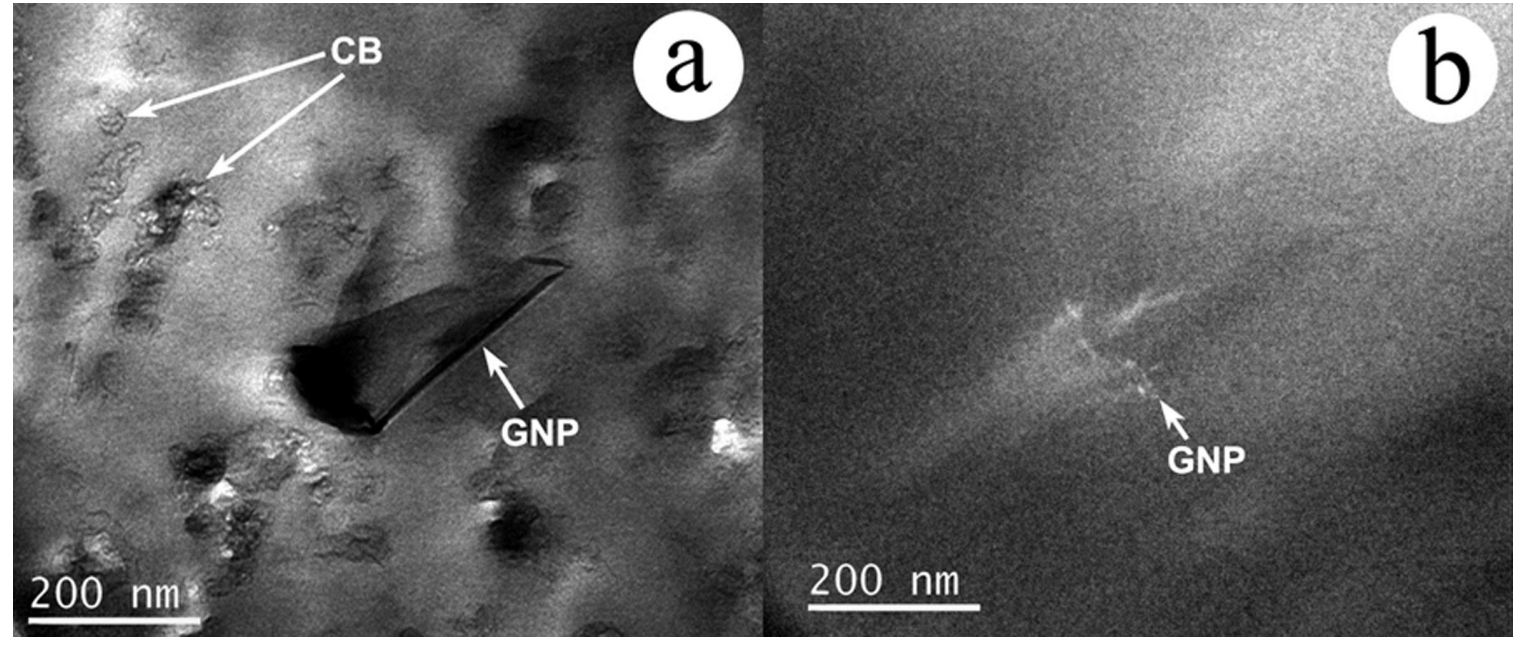

Figure 12.2: TEM Micrograph of Composite Containing $5 \mathrm{wt} \% \mathrm{CB}$ and $5 \mathrm{wt} \% \mathrm{GNP}$ in PC a) Bright Field Image b) Dark Field Image 


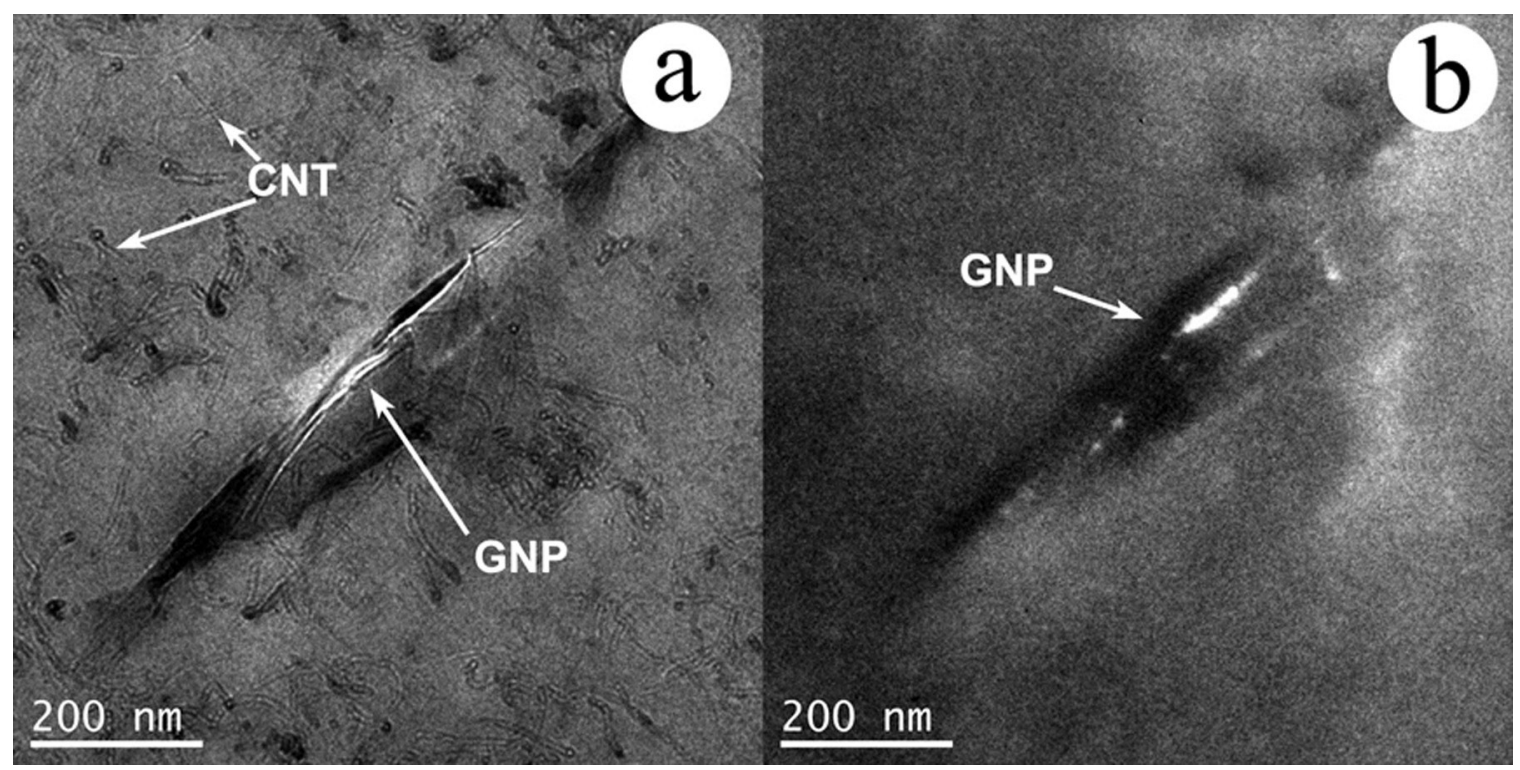

Figure 12.3: TEM Micrograph of Composite Containing $5 \mathrm{wt} \%$ CNT and $5 \mathrm{wt} \%$ GNP in PC a) Bright Field Image b) Dark Field Image

\section{2: Electrical Resistivity (ER) Results}

Table 12.1 shows the mean electrical resistivity, standard deviation of the electrical resistivity and number of samples that were tested for the $3^{2}$ factorial design ( 2 filler, 3 level) composites containing both $\mathrm{CB}$ and $\mathrm{CNT}(\mathrm{CB} / \mathrm{CNT})$. Table 12.2 shows these same data for factorial design composites containing both $\mathrm{CB}$ and GNP (CB/GNP). Table 12.3 shows the same data for the factorial design composites containing both CNT and GNP (CNT/GNP). Results for the individual samples are given in Appendices $\mathrm{G}$ and $\mathrm{H}$. Comparing the data in these tables shows that a composite with $5 \mathrm{wt} \% \mathrm{CB}$ and $5 \mathrm{wt} \%$ CNT has the lowest electrical resistivity with $15 \mathrm{ohm}-\mathrm{cm}$. As a general rule, we note that composites containing GNP have a higher electrical resistivity than composites that contain $\mathrm{CB}$ and $\mathrm{CNT}$. 
Table 12.1: ER Results for Factorial Design Formulations Containing CB and CNT

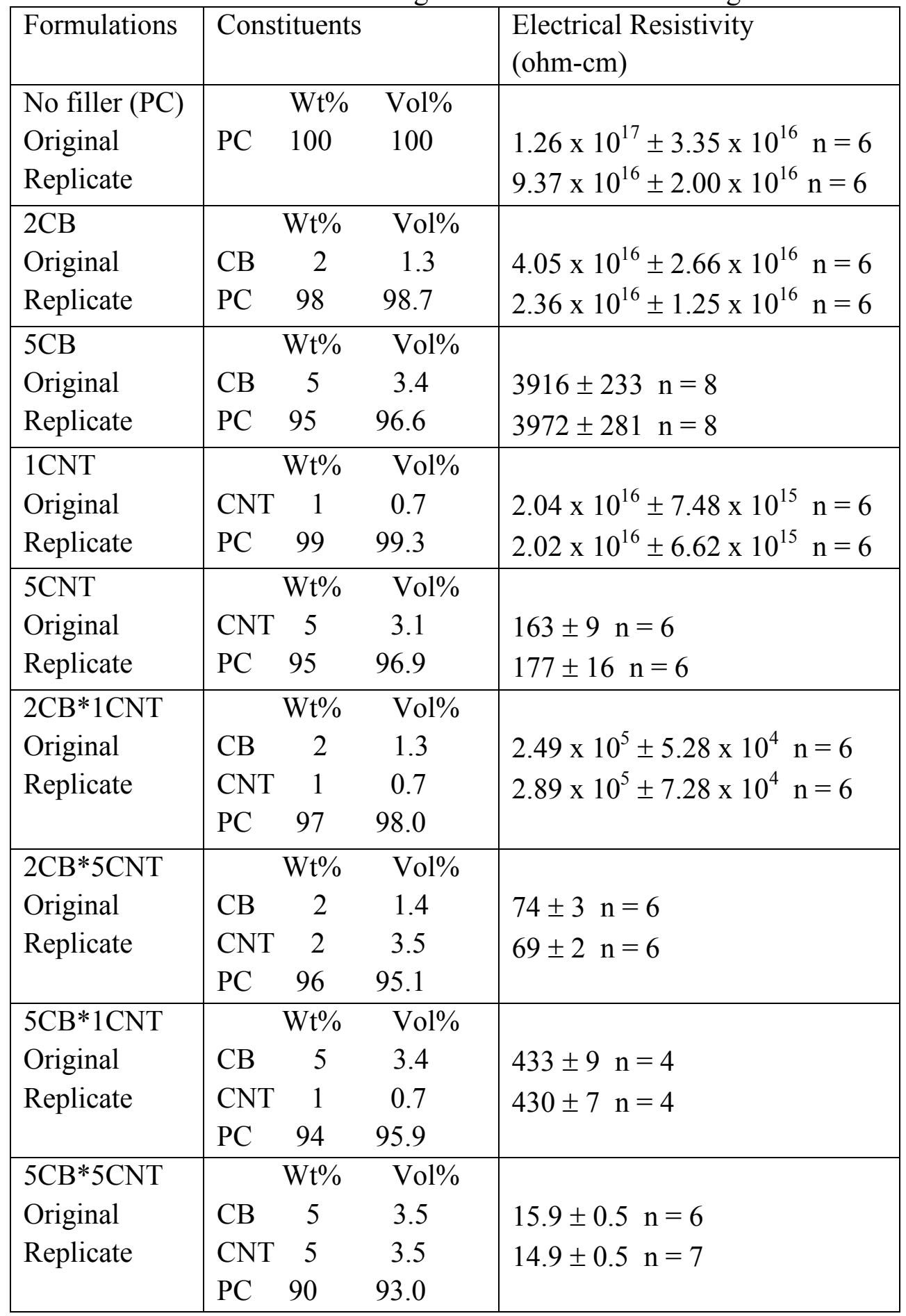


Table 12.2: ER Results for Factorial Design Formulations Containing CB and GNP

\begin{tabular}{|c|c|c|c|}
\hline Formulations & Constituent & & $\begin{array}{l}\text { Electrical Resistivity } \\
\text { (ohm-cm) }\end{array}$ \\
\hline $\begin{array}{l}\text { No filler (PC) } \\
\text { Original } \\
\text { Replicate }\end{array}$ & $\begin{array}{ll}\mathrm{Wt} \% \\
\mathrm{PC} & 100\end{array}$ & $\begin{array}{l}\text { Vol\% } \\
100\end{array}$ & $\begin{array}{l}1.26 \times 10^{17} \pm 3.35 \times 10^{16} \mathrm{n}=6 \\
9.37 \times 10^{16} \pm 2.00 \times 10^{16} \mathrm{n}=6\end{array}$ \\
\hline $\begin{array}{l}2 \mathrm{CB} \\
\text { Original } \\
\text { Replicate }\end{array}$ & $\begin{array}{lr} & \mathrm{Wt}^{\%} \% \\
\mathrm{CB} & 2 \\
\mathrm{PC} & 98\end{array}$ & $\begin{array}{r}\text { Vol\% } \\
1.3 \\
98.7\end{array}$ & $\begin{array}{l}4.05 \times 10^{16} \pm 2.66 \times 10^{16} \mathrm{n}=6 \\
2.36 \times 10^{16} \pm 1.25 \times 10^{16} \mathrm{n}=6\end{array}$ \\
\hline $\begin{array}{l}5 \mathrm{CB} \\
\text { Original } \\
\text { Replicate }\end{array}$ & $\begin{array}{cc} & \mathrm{Wt} \% \\
\mathrm{CB} & 5 \\
\mathrm{PC} & 95\end{array}$ & $\begin{array}{c}\text { Vol\% } \% \\
3.4 \\
96.6\end{array}$ & $\begin{array}{l}3916 \pm 233 n=8 \\
3972 \pm 281 \mathrm{n}=8\end{array}$ \\
\hline $\begin{array}{l}2 \text { GNP } \\
\text { Original } \\
\text { Replicate }\end{array}$ & $\begin{array}{lc} & \mathrm{Wt} \% \\
\text { GNP } & 2 \\
\text { PC } & 98\end{array}$ & $\begin{array}{c}\text { Vol\% } \\
1.2 \\
98.8\end{array}$ & $\begin{array}{l}5.46 \times 10^{16} \pm 4.89 \times 10^{15} \mathrm{n}=6 \\
5.20 \times 10^{16} \pm 2.25 \times 10^{16} \mathrm{n}=6\end{array}$ \\
\hline $\begin{array}{l}5 \mathrm{GNP} \\
\text { Original } \\
\text { Replicate }\end{array}$ & $\begin{array}{lc} & \mathrm{Wt} \% \\
\mathrm{GNP} & 5 \\
\mathrm{PC} & 95\end{array}$ & $\begin{array}{c}\mathrm{Vol} \% \\
3.1 \\
96.9\end{array}$ & $\begin{array}{l}3.78 \times 10^{15} \pm 5.59 \times 10^{14} \mathrm{n}=6 \\
3.76 \times 10^{15} \pm 2.83 \times 10^{14} \mathrm{n}=6\end{array}$ \\
\hline $\begin{array}{l}2 \mathrm{CB}^{*} 2 \mathrm{GNP} \\
\text { Original } \\
\text { Replicate }\end{array}$ & $\begin{array}{lr} & \mathrm{Wt} \% \\
\mathrm{CB} & 2 \\
\mathrm{GNP} & 2 \\
\mathrm{PC} & 96\end{array}$ & $\begin{array}{c}\text { Vol\% } \\
1.4 \\
1.2 \\
97.4\end{array}$ & $\begin{array}{l}3.99 \times 10^{15} \pm 9.22 \times 10^{14} \mathrm{n}=6 \\
3.77 \times 10^{15} \pm 1.31 \times 10^{15} \mathrm{n}=6\end{array}$ \\
\hline $\begin{array}{l}2 \mathrm{CB}^{*} 5 \mathrm{GNP} \\
\text { Original } \\
\text { Replicate }\end{array}$ & $\begin{array}{lc} & \mathrm{Wt}^{\circ} \% \\
\mathrm{CB} & 2 \\
\mathrm{GNP} & 5 \\
\mathrm{PC} & 93\end{array}$ & $\begin{array}{c}\text { Vol\% } \\
1.4 \\
3.1 \\
95.5\end{array}$ & $\begin{array}{l}2.42 \times 10^{7} \pm 3.14 \times 10^{6} \mathrm{n}=5 \\
1.85 \times 10^{7} \pm 4.31 \times 10^{6} \mathrm{n}=4\end{array}$ \\
\hline $\begin{array}{l}5 \mathrm{CB}^{*} 2 \mathrm{GNP} \\
\text { Original } \\
\text { Replicate }\end{array}$ & $\begin{array}{lr} & \mathrm{Wt} \% \\
\mathrm{CB} & 5 \\
\mathrm{GNP} & 2 \\
\mathrm{PC} & 93\end{array}$ & $\begin{array}{r}\mathrm{Vol} \% \\
3.4 \\
1.2 \\
95.4\end{array}$ & $\begin{array}{l}1337 \pm 16 n=6 \\
1387 \pm 32 n=5\end{array}$ \\
\hline $\begin{array}{l}5 \mathrm{CB}^{*} 5 \mathrm{GNP} \\
\text { Original } \\
\text { Replicate }\end{array}$ & $\begin{array}{lr} & \mathrm{Wt} \% \\
\text { CB } & 5 \\
\text { GNP } & 5 \\
\text { PC } & 90\end{array}$ & $\begin{array}{r}\text { Vol\% } \\
3.5 \\
3.1 \\
93.4\end{array}$ & $\begin{array}{l}729 \pm 42 n=5 \\
735 \pm 40 \mathrm{n}=5\end{array}$ \\
\hline
\end{tabular}


Table 12.3: ER Results for Factorial Design Formulations Containing CNT and GNP

\begin{tabular}{|c|c|c|c|}
\hline Formulations & \multicolumn{2}{|c|}{ Constituents } & $\begin{array}{l}\text { Electrical Resistivity } \\
\text { (ohm-cm) }\end{array}$ \\
\hline $\begin{array}{l}\text { No filler }(\mathrm{PC}) \\
\text { Original } \\
\text { Replicate }\end{array}$ & $\begin{array}{rl} & \mathrm{Wt} \% \\
\mathrm{PC} & 100\end{array}$ & $\begin{array}{l}\mathrm{Vol} \% \\
100\end{array}$ & $\begin{array}{l}1.26 \times 10^{17} \pm 3.35 \times 10^{16} \mathrm{n}=6 \\
9.37 \times 10^{16} \pm 2.00 \times 10^{16} \mathrm{n}=6\end{array}$ \\
\hline $\begin{array}{l}1 \mathrm{CNT} \\
\text { Original } \\
\text { Replicate }\end{array}$ & \begin{tabular}{|lr}
\multicolumn{2}{|c|}{$\mathrm{Wt}_{0}$} \\
$\mathrm{CNT}$ & 1 \\
$\mathrm{PC}$ & 99
\end{tabular} & $\begin{array}{r}\mathrm{Vol} \% \\
0.7 \\
99.3\end{array}$ & $\begin{array}{l}2.04 \times 10^{16} \pm 7.48 \times 10^{15} \mathrm{n}=6 \\
2.02 \times 10^{16} \pm 6.62 \times 10^{15} \mathrm{n}=6\end{array}$ \\
\hline $\begin{array}{l}\mathrm{CNT} \\
\text { Original } \\
\text { Replicate }\end{array}$ & \begin{tabular}{|lr}
\multicolumn{2}{|c}{$\mathrm{Wt}^{2} \%$} \\
CNT & 5 \\
PC & 95
\end{tabular} & $\begin{array}{r}\text { Vol } \% \\
3.1 \\
96.9\end{array}$ & $\begin{array}{l}163 \pm 9 n=6 \\
177 \pm 16 n=6\end{array}$ \\
\hline $\begin{array}{l}\text { GNP } \\
\text { Original } \\
\text { Replicate }\end{array}$ & \begin{tabular}{|lr}
\multicolumn{2}{|c|}{$\mathrm{Wt}_{0} \mathrm{o}$} \\
$\mathrm{GNP}$ & 2 \\
$\mathrm{PC}$ & 98 \\
\end{tabular} & $\begin{array}{r}\mathrm{Vol} \% \\
1.2 \\
98.8\end{array}$ & $\begin{array}{l}5.46 \times 10^{16} \pm 4.89 \times 10^{15} \mathrm{n}=6 \\
5.20 \times 10^{16} \pm 2.25 \times 10^{16} \mathrm{n}=6\end{array}$ \\
\hline $\begin{array}{l}\text { GNP } \\
\text { Original } \\
\text { Replicate }\end{array}$ & 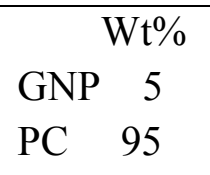 & $\begin{array}{r}\text { Vol\% } \\
3.1 \\
96.9\end{array}$ & $\begin{array}{l}3.78 \times 10^{15} \pm 5.59 \times 10^{14} \mathrm{n}=6 \\
3.76 \times 10^{15} \pm 2.83 \times 10^{14} \mathrm{n}=6\end{array}$ \\
\hline $\begin{array}{l}1 \mathrm{CNT} * 2 \mathrm{GNP} \\
\text { Original } \\
\text { Replicate }\end{array}$ & \begin{tabular}{|lc}
\multicolumn{2}{r}{$\mathrm{Wt} \%$} \\
$\mathrm{CNT}$ & 1 \\
GNP & 2 \\
PC & 97
\end{tabular} & $\begin{array}{c}\text { Vol\% } \\
0.7 \\
1.2 \\
98.1\end{array}$ & $\begin{array}{l}4.13 \times 10^{15} \pm 1.63 \times 10^{15} \mathrm{n}=7 \\
5.03 \times 10^{15} \pm 1.82 \times 10^{15} \mathrm{n}=6\end{array}$ \\
\hline $\begin{array}{l}1 \mathrm{CNT} * 5 \mathrm{GNP} \\
\text { Original } \\
\text { Replicate }\end{array}$ & \begin{tabular}{|cc} 
& $\mathrm{Wt} \%$ \\
$\mathrm{CNT}$ & 1 \\
$\mathrm{GNP}$ & 5 \\
$\mathrm{PC}$ & 94
\end{tabular} & $\begin{array}{c}\text { Vol\% } \\
0.7 \\
3.1 \\
96.2\end{array}$ & $\begin{array}{l}6.18 \times 10^{6} \pm 1.34 \times 10^{6} \mathrm{n}=4 \\
6.43 \times 10^{6} \pm 9.02 \times 10^{5} \mathrm{n}=4\end{array}$ \\
\hline $\begin{array}{l}5 \mathrm{CNT} * 2 \mathrm{GNP} \\
\text { Original } \\
\text { Replicate }\end{array}$ & \begin{tabular}{|cc} 
& $\mathrm{Wt} \%$ \\
$\mathrm{CNT}$ & 5 \\
$\mathrm{GNP}$ & 2 \\
$\mathrm{PC}$ & 93
\end{tabular} & $\begin{array}{c}\text { Vol\% } \\
3.5 \\
1.2 \\
95.3\end{array}$ & $\begin{array}{l}210 \pm 5 n=7 \\
198 \pm 6 n=6\end{array}$ \\
\hline $\begin{array}{l}5 \mathrm{CNT} * 5 \mathrm{GNP} \\
\text { Original } \\
\text { Replicate }\end{array}$ & $\begin{array}{lc} & \mathrm{Wt} \% \\
\mathrm{CNT} & 5 \\
\mathrm{GNP} & 5 \\
\mathrm{PC} & 90\end{array}$ & $\begin{array}{c}\text { Vol\% } \\
3.6 \\
3.1 \\
93.3\end{array}$ & $\begin{array}{l}126 \pm 11 n=6 \\
128 \pm 9 n=6\end{array}$ \\
\hline
\end{tabular}


Table 12.4 gives the results of regression analysis of $\log (\log E R$ in ohm-cm) for the $\mathrm{CB} / \mathrm{CNT}, \mathrm{CB} / \mathrm{GNP}$ and $\mathrm{CNT} / \mathrm{GNP}$ systems. For this analysis the t-statistic and associated p-value for each case has been calculated and reported. The t-statistic is calculated by dividing the estimated value of a property (in this case ER) by the estimated standard error of the property. When the absolute value of the t-statistic is high, and the associated p-value is low, the conclusion is that the associated factor (a filler in this case) may have a significant effect on the property. Typically, a larger t-statistic value indicates greater significance of the factor. For this project, the significance level, $\alpha$, is 0.05 .

Table 12.4: $3^{2}$ Regression Results for Log (Log Electrical Resistivity, ohm-cm)

\begin{tabular}{|c|c|c|c|c|}
\hline Response & Correlation Term & Coefficient & $\mathbf{t}$ & $\mathbf{p}$ \\
\hline \multirow{13}{*}{ 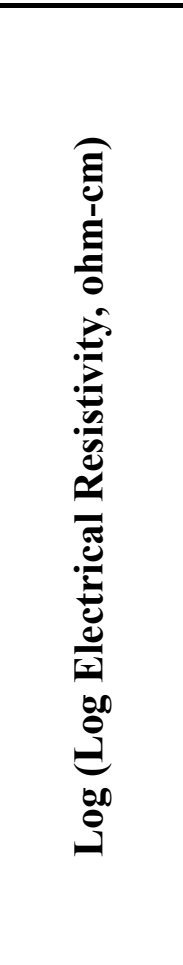 } & $\begin{array}{l}\text { Correlation for } \\
\text { CB/CNT Composites }\end{array}$ & & & \\
\hline & Constant & 1.34 & 26.9 & 0.000 \\
\hline & CB & -0.156 & -9.7 & 0.000 \\
\hline & CNT & -0.195 & -11.6 & 0.000 \\
\hline & $\mathrm{CB}^{*} \mathrm{CNT}$ & 0.0194 & 3.6 & 0.003 \\
\hline & $\begin{array}{l}\text { Correlation for } \\
\text { CB/GNP Composites }\end{array}$ & & & \\
\hline & Constant & 1.27 & 47.3 & 0.000 \\
\hline & $\mathrm{CB}^{2}$ & -0.0284 & -18.5 & 0.000 \\
\hline & $\mathrm{GNP}^{2}$ & -0.00646 & -4.2 & 0.001 \\
\hline & $\begin{array}{l}\text { Correlation for } \\
\text { CNT/GNP Composites }\end{array}$ & & & \\
\hline & Constant & 1.29 & 39.8 & 0.000 \\
\hline & CNT & -0.177 & -19.1 & 0.000 \\
\hline & $\mathrm{GNP}^{2}$ & -0.00624 & -3.4 & 0.004 \\
\hline
\end{tabular}


By studying the first part of Table 12.4 (ER of the CB/CNT system) more closely, we can see that both fillers individually ( $\mathrm{CB}$ and $\mathrm{CNT}$ ), as well as the interaction between the two fillers $\left(\mathrm{CB}^{*} \mathrm{CNT}\right)$, had a statistically significant interaction on the composite ER. As expected, the addition of either filler individually caused a statistically significant decrease (negative coefficient for both) in the composite ER. Of the two fillers, CNT caused a larger decrease in composite ER (as evidenced by the larger absolute coefficient). When $\mathrm{CB}$ and $\mathrm{CNT}$ are combined into a $\mathrm{CB} / \mathrm{CNT}$ composite, the composite ER is higher (positive coefficient) than one would expect from the additive effect of each filler individually (117). The $\mathrm{CB}^{*} \mathrm{CNT}$ interaction is much smaller than the other two effects as seen by the lower absolute value of the coefficient. For the CB/CNT system, the $\log (\log$ ER in ohm-cm) was found to vary with the filler percentage as shown in Equation 12.1 below:

$$
\begin{gathered}
\log (\log [E R, o h m-c m])=1.34-0.156 w_{C B}-0.195 w_{C N T}+0.0194 w_{C B} w_{C N T} \\
R^{2}=0.954
\end{gathered}
$$

where $w_{C B}$ and $w_{C N T}$ are the weight percentage of $\mathrm{CB}$ and CNT respectively. This corellation produced well behaved residuals and an $\mathrm{R}^{2}$ value close to 1 . Figure 12.4 shows a contour plot of Equation 12.1. It can be seen in the figure that CNT has a larger effect on decreasing the ER than CB. Additionally it is possible to see the small interaction effect of the fillers. 


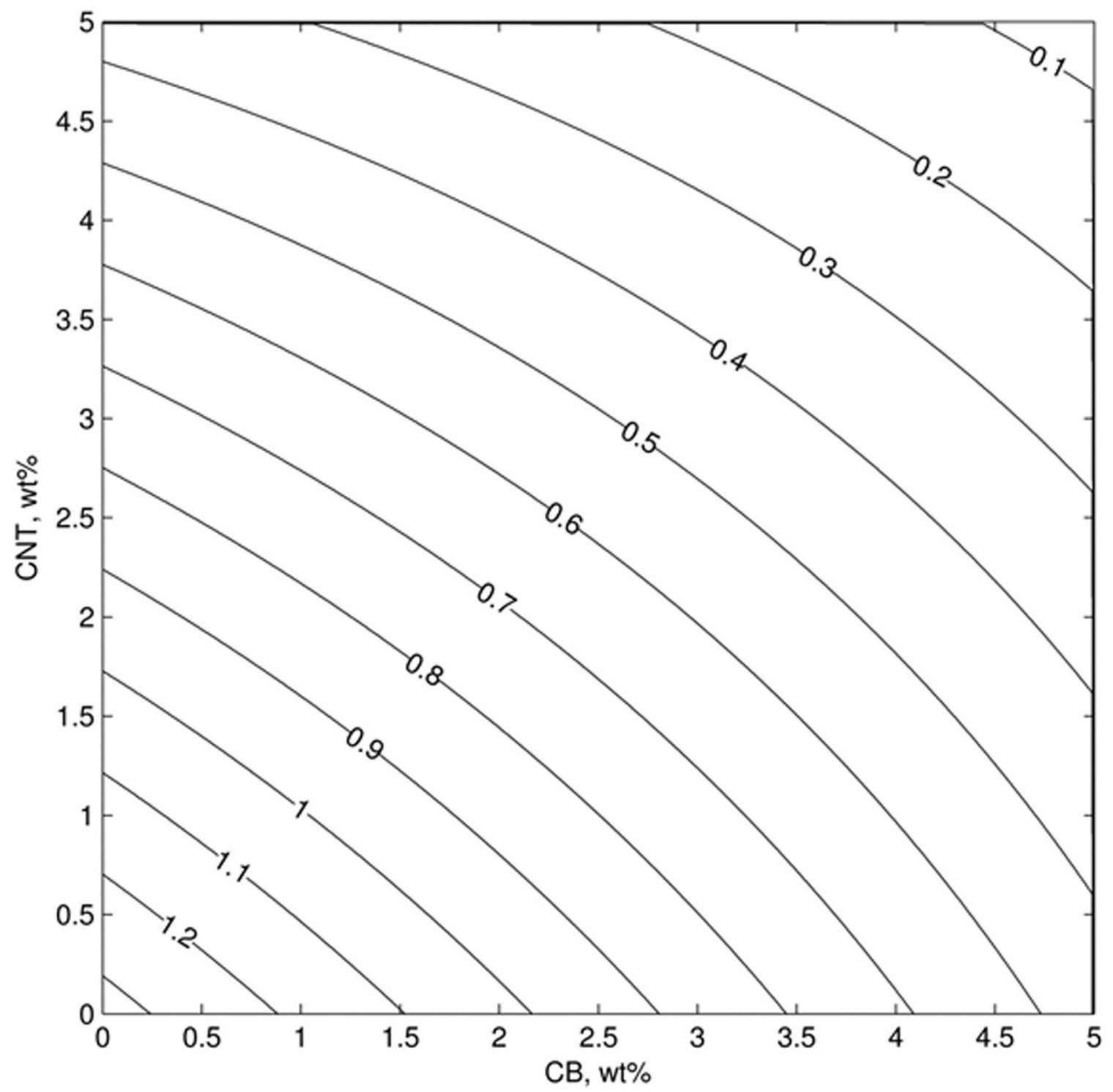

Figure 12.4. Contour Plot of Log (Log Electrical Resistivity, ohm-cm) for CB/CNT Composites

The second section of Table 12.4 (ER of the $\mathrm{CB} / \mathrm{GNP}$ system) gives the results of regression analysis of $\log (\log E R$ in ohm-cm $)$ for the CB and GNP system. In this system, we see that both individual fillers, CB and GNP, had statistically significant effects on the ER of the composite as expected. The effect of each filler was best correlated using the square of the filler $w \mathrm{t} \%$ versus the composite ER. CB had a larger effect on decreasing the composite ER than GNP as evidenced by the larger absolute 
value of the coefficient. The $\mathrm{CB} / \mathrm{GNP}$ system was found to be well correlated by the following Equation 12.2.

$$
\begin{gathered}
\log (\log [E R, o h m-c m])=1.27-0.0284 w_{C B}^{2}-0.00646 w_{G N P}^{2} \\
R^{2}=0.960
\end{gathered}
$$

In the previous equation, $w_{C B}$ and $w_{G N P}$ are the weight percentage of $\mathrm{CB}$ and GNP respectively.

The last section of Table 12.4 (ER of the CNT/GNP system) gives the results of regression analysis of $\log (\log$ ER in ohm-cm) for the CNT and GNP system. Again both individual fillers had a statistically significant decrease on the composite ER. The CNT has a larger effect on decreasing the composite ER in a similar fashion as is discussed for the CNT/CB system. The CNT/GNP system was found to be correlated best by Equation 12.3 below:

$$
\begin{gathered}
\log (\log [E R, o h m-c m])=1.29-0.177 w_{C N T}-0.00624 w_{G N P}^{2} \\
R^{2}=0.962
\end{gathered}
$$

where $w_{C N T}$ and $w_{G N P}$ are the weight percentage of CNT and GNP respectively. This equation is plotted in Figure 12.5. In this figure it can be seen again that CNT has a larger effect on decreasing the composite ER than the GNP. A related plot of Equation 12.2 has been omitted for brevity. When comparing Figures 12.4 and 12.5, we see that GNP has a smaller effect on reducing the composite ER than CNT or CB. Equations 12.1 through 12.3 could be used to estimate the ER of a theoretical formulation for material development purposes. 


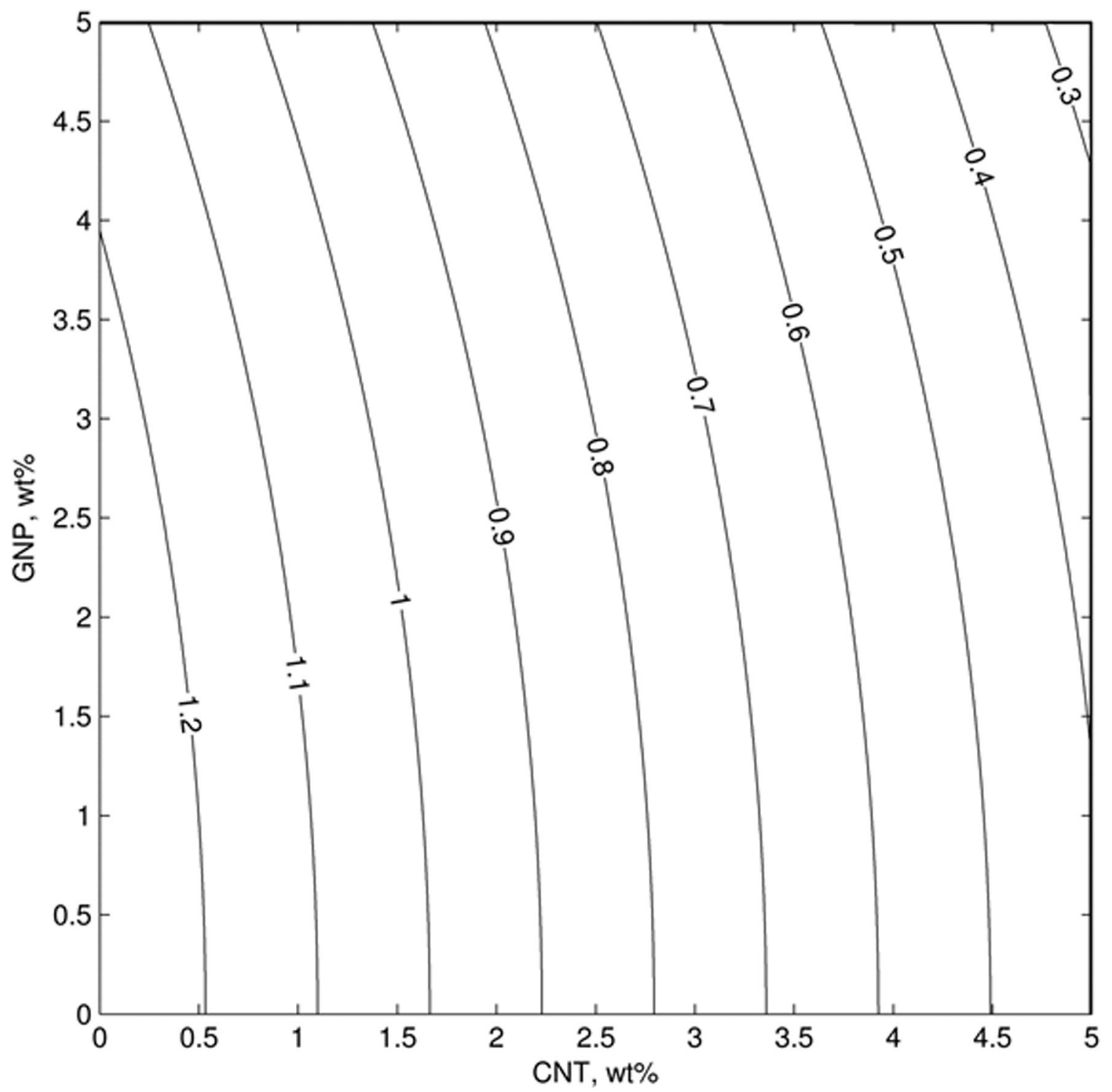

Figure 12.5. Contour Plot of Log (Log Electrical Resistivity, ohm-cm) for CNT/GNP Composites 


\section{3: Thermal Conductivity (TC) Results}

Table 12.5 shows the mean thermal conductivity, standard deviation of the thermal conductivity and number of samples that were tested for the factorial design composites containing both $\mathrm{CB}$ and $\mathrm{CNT}(\mathrm{CB} / \mathrm{CNT})$. Table 12.6 shows these same data for factorial design composites containing both $\mathrm{CB}$ and GNP (CB/GNP). Table 12.7 shows the same data for the factorial design composites containing both CNT and GNP (CNT/GNP). Results for the individual samples are given in Appendix I. We see from these tables that the best factorial design composite made roughly doubled the original PC thermal conductivity to $0.40 \mathrm{~W} / \mathrm{m} \cdot \mathrm{K}$ from $0.21 \mathrm{~W} / \mathrm{m} \cdot \mathrm{K}$.

Table 12.8 gives the results of regression analysis of thermal conductivity for the $\mathrm{CB} / \mathrm{CNT}, \mathrm{CB} / \mathrm{GNP}$ and CNT/GNP systems. Looking at the first third (CB/CNT system) we see that both fillers individually caused a statistically significant increase in composite TC. CNT caused a larger increase in TC as evidenced by the larger coefficient than $\mathrm{CB}$, but it had a diminishing effect as additional CNT is added as evidenced by the negative coefficient for the CNT squared term. The TC of the $\mathrm{CB} / \mathrm{CNT}$ system was found to be well correlated by Equation 12.4 below:

$$
\begin{gathered}
T C\left(\frac{W}{m \cdot K}\right)=0.214+0.00822 w_{C B}+0.0136 w_{C N T}-0.000846 w_{C N T}^{2} \\
R^{2}=0.992
\end{gathered}
$$

where $w_{C B}$ and $w_{C N T}$ are the weight percentage of $\mathrm{CB}$ and $\mathrm{CNT}$ respectively. 
Table 12.5: TC Results for Factorial Design Formulations Containing CB and CNT

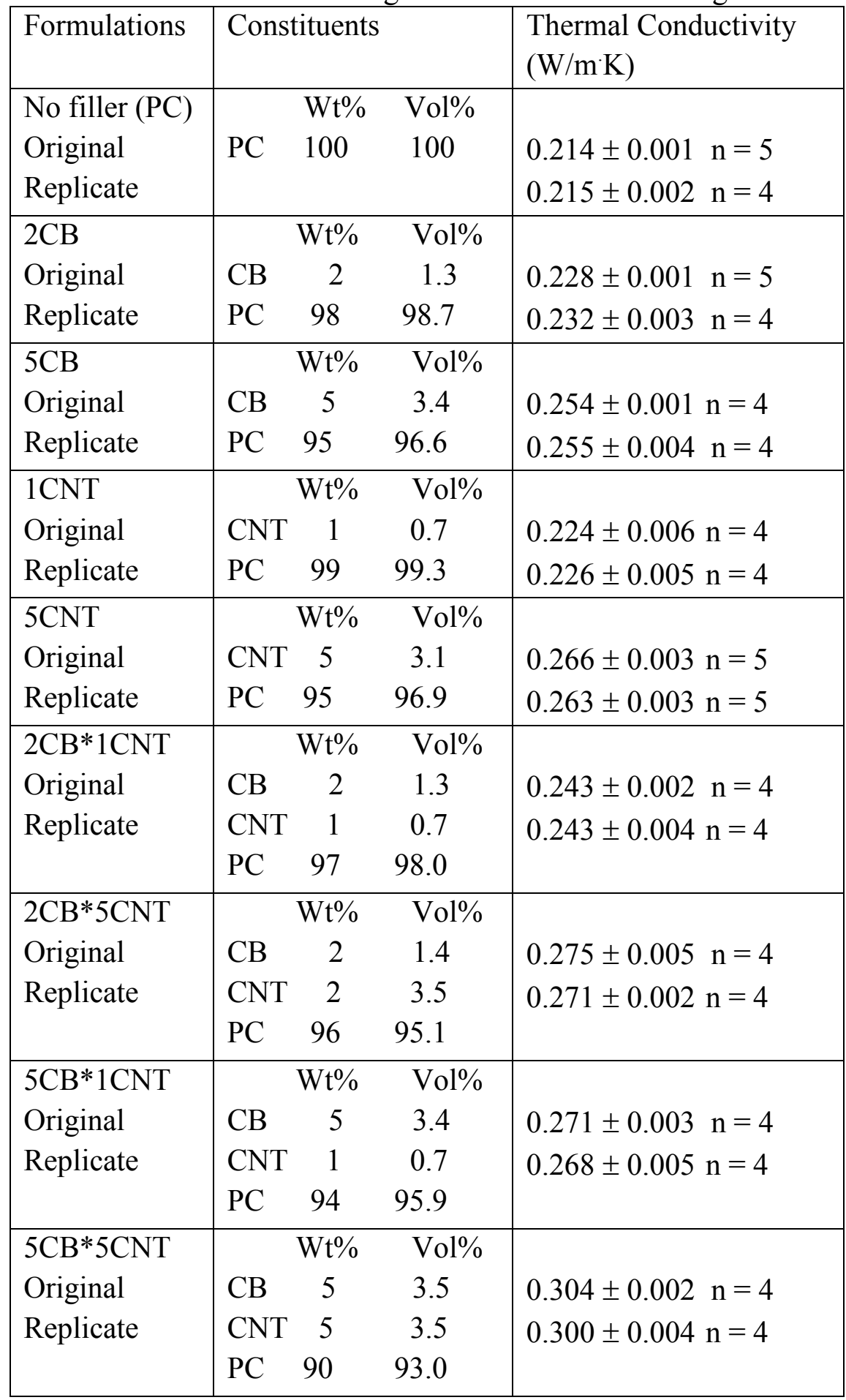


Table 12.6: TC Results for Factorial Design Formulations Containing CB and GNP

\begin{tabular}{|c|c|c|c|}
\hline Formulations & Constituent & & $\begin{array}{l}\text { Thermal Conductivity } \\
(\mathrm{W} / \mathrm{m} \cdot \mathrm{K})\end{array}$ \\
\hline $\begin{array}{l}\text { No filler (PC) } \\
\text { Original } \\
\text { Replicate }\end{array}$ & $\begin{array}{l}\mathrm{Wt} \% \\
100\end{array}$ & $\begin{array}{l}\text { Vol\% } \\
100\end{array}$ & $\begin{array}{l}0.214 \pm 0.001 \mathrm{n}=5 \\
0.215 \pm 0.002 \mathrm{n}=4\end{array}$ \\
\hline $\begin{array}{l}2 \mathrm{CB} \\
\text { Original } \\
\text { Replicate }\end{array}$ & \begin{tabular}{|rr} 
& $\mathrm{Wt} \%$ \\
$\mathrm{CB}$ & 2 \\
$\mathrm{PC}$ & 98
\end{tabular} & $\begin{array}{r}\mathrm{ol} \% \\
1.3 \\
98.7\end{array}$ & $\begin{array}{l}0.228 \pm 0.001 \mathrm{n}=5 \\
0.232 \pm 0.003 \mathrm{n}=4\end{array}$ \\
\hline $\begin{array}{l}5 \mathrm{CB} \\
\text { Original } \\
\text { Replicate }\end{array}$ & \begin{tabular}{|cc} 
& $\mathrm{Wt} \%$ \\
$\mathrm{CB}$ & 5 \\
$\mathrm{PC}$ & 95
\end{tabular} & $\begin{array}{c}\text { Vol\% } \\
3.4 \\
96.6\end{array}$ & $\begin{array}{l}0.254 \pm 0.001 \mathrm{n}=4 \\
0.255 \pm 0.004 \mathrm{n}=4\end{array}$ \\
\hline $\begin{array}{l}2 \mathrm{GNP} \\
\text { Original } \\
\text { Replicate }\end{array}$ & \begin{tabular}{|lc} 
& $\mathrm{Wt}^{\circ} \%$ \\
GNP & 2 \\
PC & 98
\end{tabular} & $\begin{array}{c}\text { Vol\% } \\
1.2 \\
98.8\end{array}$ & $\begin{array}{l}0.251 \pm 0.003 n=5 \\
0.252 \pm 0.001 \mathrm{n}=4\end{array}$ \\
\hline $\begin{array}{l}5 \mathrm{GNP} \\
\text { Original } \\
\text { Replicate }\end{array}$ & \begin{tabular}{|cc}
\multicolumn{2}{|c}{$\mathrm{Wt}^{2} \%$} \\
GNP & 5 \\
PC & 95
\end{tabular} & $\begin{array}{c}\text { Vol\% } \\
3.1 \\
96.9\end{array}$ & $\begin{array}{l}0.312 \pm 0.004 n=4 \\
0.319 \pm 0.001 \mathrm{n}=5\end{array}$ \\
\hline $\begin{array}{l}2 \mathrm{CB}^{*} 2 \mathrm{GNP} \\
\text { Original } \\
\text { Replicate }\end{array}$ & $\begin{array}{lc} & \mathrm{Wt}^{\circ} \% \\
\mathrm{CB} & 2 \\
\mathrm{GNP} & 2 \\
\mathrm{PC} & 96\end{array}$ & $\begin{array}{c}\text { Vol\% } \\
1.4 \\
1.2 \\
97.4\end{array}$ & $\begin{array}{l}0.271 \pm 0.009 \mathrm{n}=4 \\
0.269 \pm 0.004 \mathrm{n}=4\end{array}$ \\
\hline $\begin{array}{l}2 \mathrm{CB}^{*} 5 \mathrm{GNP} \\
\text { Original } \\
\text { Replicate }\end{array}$ & $\begin{array}{lc} & \mathrm{Wt} \% \\
\text { CB } & 2 \\
\text { GNP } & 5 \\
\text { PC } & 93\end{array}$ & $\begin{array}{c}\text { Vol\% } \\
1.4 \\
3.1 \\
95.5\end{array}$ & $\begin{array}{l}0.339 \pm 0.006 \mathrm{n}=4 \\
0.337 \pm 0.006 \mathrm{n}=4\end{array}$ \\
\hline $\begin{array}{l}5 \mathrm{CB}^{*} 2 \mathrm{GNP} \\
\text { Original } \\
\text { Replicate }\end{array}$ & $\begin{array}{lr} & \mathrm{Wt}^{0} \% \\
\mathrm{CB} & 5 \\
\mathrm{GNP} & 2 \\
\mathrm{PC} & 93\end{array}$ & $\begin{array}{r}\mathrm{ol} \% \\
3.4 \\
1.2 \\
95.4\end{array}$ & $\begin{array}{l}0.303 \pm 0.003 n=4 \\
0.301 \pm 0.001 \mathrm{n}=4\end{array}$ \\
\hline $\begin{array}{l}5 \mathrm{CB}^{*} 5 \mathrm{GNP} \\
\text { Original } \\
\text { Replicate }\end{array}$ & $\begin{array}{lc} & \mathrm{Wt} \% \\
\mathrm{CB} & 5 \\
\mathrm{GNP} & 5 \\
\mathrm{PC} & 90\end{array}$ & $\begin{array}{r}\text { Vol\% } \\
3.5 \\
3.1 \\
93.4\end{array}$ & $\begin{array}{l}0.357 \pm 0.001 \mathrm{n}=4 \\
0.366 \pm 0.004 \mathrm{n}=4\end{array}$ \\
\hline
\end{tabular}


Table 12.7: TC Results for Factorial Design Formulations Containing CNT and GNP

\begin{tabular}{|c|c|c|c|}
\hline Formulations & Constituent & & $\begin{array}{l}\text { Thermal Conductivity } \\
(\mathrm{W} / \mathrm{m} \cdot \mathrm{K})\end{array}$ \\
\hline $\begin{array}{l}\text { No filler }(\mathrm{PC}) \\
\text { Original } \\
\text { Replicate }\end{array}$ & $\begin{array}{ll} & \mathrm{Wt} \% \\
\mathrm{PC} & 100\end{array}$ & $\begin{array}{l}\text { Vol\% } \\
100\end{array}$ & $\begin{array}{ll}0.214 \pm 0.001 & \mathrm{n}=5 \\
0.215 \pm 0.002 & \mathrm{n}=4\end{array}$ \\
\hline $\begin{array}{l}\text { 1CNT } \\
\text { Original } \\
\text { Replicate }\end{array}$ & \begin{tabular}{lr}
\multicolumn{2}{c}{$\mathrm{Wt}_{0}$} \\
$\mathrm{CNT}$ & 1 \\
$\mathrm{PC}$ & 99
\end{tabular} & $\begin{array}{r}\mathrm{ol} \% \\
0.7 \\
99.3\end{array}$ & $\begin{array}{l}0.224 \pm 0.006 \mathrm{n}=4 \\
0.226 \pm 0.005 \mathrm{n}=4\end{array}$ \\
\hline $\begin{array}{l}5 \mathrm{CNT} \\
\text { Original } \\
\text { Replicate }\end{array}$ & \begin{tabular}{lr}
\multicolumn{2}{r}{$\mathrm{Wt}_{0}$} \\
$\mathrm{CNT}$ & 5 \\
$\mathrm{PC}$ & 95
\end{tabular} & $\begin{array}{r}\mathrm{ol} \% \\
3.1 \\
96.9\end{array}$ & $\begin{array}{l}0.266 \pm 0.003 n=5 \\
0.263 \pm 0.003 n=5\end{array}$ \\
\hline $\begin{array}{l}2 \mathrm{GNP} \\
\text { Original } \\
\text { Replicate }\end{array}$ & \begin{tabular}{lr}
\multicolumn{2}{r}{$\mathrm{Wt}^{2}$} \\
GNP & 2 \\
PC & 98
\end{tabular} & $\begin{array}{r}\mathrm{ol} \% \\
1.2 \\
98.8\end{array}$ & $\begin{array}{l}0.251 \pm 0.003 \mathrm{n}=5 \\
0.252 \pm 0.001 \mathrm{n}=4\end{array}$ \\
\hline $\begin{array}{l}5 \mathrm{GNP} \\
\text { Original } \\
\text { Replicate }\end{array}$ & $\begin{array}{lc} & \mathrm{Wt} \% \\
\text { GNP } & 5 \\
\text { PC } & 95\end{array}$ & $\begin{array}{r}\mathrm{ol} \% \\
3.1 \\
96.9\end{array}$ & $\begin{array}{l}0.312 \pm 0.004 \mathrm{n}=4 \\
0.319 \pm 0.001 \mathrm{n}=5\end{array}$ \\
\hline $\begin{array}{l}1 \mathrm{CNT}^{*} 2 \mathrm{GNP} \\
\text { Original } \\
\text { Replicate }\end{array}$ & $\begin{array}{lc} & \mathrm{Wt} \% \\
\mathrm{CNT} & 1 \\
\mathrm{GNP} & 2 \\
\mathrm{PC} & 97\end{array}$ & $\begin{array}{c}\text { Vol\% } \\
0.7 \\
1.2 \\
98.1\end{array}$ & $\begin{array}{l}0.268 \pm 0.007 \mathrm{n}=4 \\
0.264 \pm 0.006 \mathrm{n}=4\end{array}$ \\
\hline $\begin{array}{l}\text { 1CNT*5GNP } \\
\text { Original } \\
\text { Replicate }\end{array}$ & $\begin{array}{lc} & \mathrm{Wt} \% \\
\text { CNT } & 1 \\
\text { GNP } & 5 \\
\text { PC } & 94\end{array}$ & $\begin{array}{c}\mathrm{Vol} \% \\
0.7 \\
3.1 \\
96.2\end{array}$ & $\begin{array}{l}0.328 \pm 0.007 \mathrm{n}=4 \\
0.329 \pm 0.002 \mathrm{n}=4\end{array}$ \\
\hline $\begin{array}{l}5 \mathrm{CNT}^{*} 2 \mathrm{GNP} \\
\text { Original } \\
\text { Replicate }\end{array}$ & $\begin{array}{lc} & \mathrm{Wt} \% \\
\mathrm{CNT} & 5 \\
\mathrm{GNP} & 2 \\
\mathrm{PC} & 93\end{array}$ & $\begin{array}{c}\mathrm{Vol} \% \\
3.5 \\
1.2 \\
95.3\end{array}$ & $\begin{array}{l}0.318 \pm 0.006 \mathrm{n}=4 \\
0.321 \pm 0.005 \mathrm{n}=5\end{array}$ \\
\hline $\begin{array}{l}5 \mathrm{CNT} * 5 \mathrm{GNP} \\
\text { Original } \\
\text { Replicate }\end{array}$ & $\begin{array}{lc} & \mathrm{Wt} \% \\
\text { CNT } & 5 \\
\text { GNP } & 5 \\
\text { PC } & 90\end{array}$ & $\begin{array}{c}\text { Vol\% } \\
3.6 \\
3.1 \\
93.3\end{array}$ & $\begin{array}{l}0.396 \pm 0.004 \mathrm{n}=4 \\
0.397 \pm 0.007 \mathrm{n}=4\end{array}$ \\
\hline
\end{tabular}


Table 12.8: $3^{2}$ Regression Results for Thermal Conductivity (W/m.K)

\begin{tabular}{|c|c|c|c|c|}
\hline Response & Correlation Term & Coefficient & $\mathbf{t}$ & $\mathbf{p}$ \\
\hline \multirow{14}{*}{ 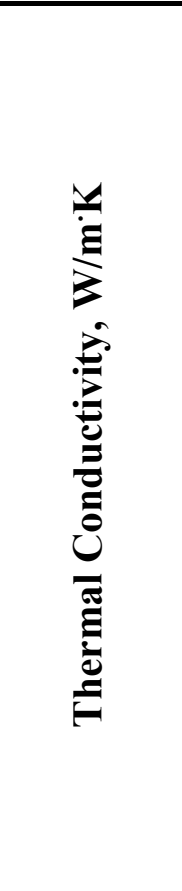 } & Correlation for $\mathrm{CB} / \mathrm{CNT}$ Composites & & & \\
\hline & Constant & 0.214 & 161.9 & 0.000 \\
\hline & $\mathrm{CB}$ & 0.00822 & 26.5 & 0.000 \\
\hline & CNT & 0.0136 & 7.1 & 0.000 \\
\hline & $\mathrm{CNT}^{2}$ & -0.000846 & -2.4 & 0.033 \\
\hline & Correlation for CB/GNP Composites & & & \\
\hline & Constant & 0.211 & 140.5 & 0.000 \\
\hline & $\mathrm{CB}$ & 0.00913 & 23.6 & 0.000 \\
\hline & GNP & 0.0211 & 54.4 & 0.000 \\
\hline & Correlation for CNT/GNP Composites & & & \\
\hline & Constant & 0.213 & 192.4 & 0.000 \\
\hline & CNT & 0.0104 & 27.6 & 0.000 \\
\hline & GNP & 0.0199 & 55.8 & 0.000 \\
\hline & $\mathrm{CNT}^{*} \mathrm{GNP}$ & 0.00126 & 10.4 & 0.000 \\
\hline
\end{tabular}

The second section of Table 12.8 (CB/GNP system) gives the results of regression analysis of TC for the $\mathrm{CB}$ and GNP system. Again each individual filler had a statistically significant increase in composite TC. For this system GNP caused a larger increase in composite $\mathrm{TC}$ than $\mathrm{CB}$. The $\mathrm{TC}$ of the $\mathrm{CB} / \mathrm{GNP}$ system was found to be correlated well by Equation 12.5 below:

$$
\begin{gathered}
T C\left(\frac{W}{m \cdot K}\right)=0.211+0.00913 w_{C B}+0.0211 w_{G N P} \\
R^{2}=0.996
\end{gathered}
$$

where $w_{C B}$ and $w_{G N P}$ are the weight percentage of CB and GNP respectively.

The last section of Table 12.8 (CNT/GNP system) gives the results of regression analysis of TC for the CNT and GNP system. As expected we see that each individual filler 
causes a statistically significant increase in composite TC. In this system GNP has a larger effect on increasing the composite TC than CNT. We also notice that there is a statistically significant interaction effect $\left(\mathrm{CNT}^{*} \mathrm{GNP}\right)$ that also increases the composite TC. This means that a composite with both CNT and GNP present would have a higher TC than would be expected solely from the additive effect of each individual filler (117). The TC of the CNT/GNP system was found to be correlated well by Equation 12.6 below:

$$
\begin{gathered}
T C\left(\frac{W}{m \cdot K}\right)=0.213+0.0104 w_{C N T}+0.0199 w_{G N P}+0.00126 w_{C N T} w_{G N P} \\
R^{2}=0.999
\end{gathered}
$$

where $w_{C N T}$ and $w_{G N P}$ are the weight percentage of CNT and GNP respectively.

For all three systems the model $\mathrm{R}^{2}$ values are very high $(>0.99)$ indicating that the majority of the data variation is within expectation for the correlations. Equations 12.4 through 12.6 could be used to estimate the $\mathrm{TC}$ of a theoretical formulation for material development purposes.

\section{4: Tensile Modulus Results}

Table 12.9 shows the mean tensile modulus, standard deviation of the tensile modulus and number of samples that were tested for the factorial design composites containing both $\mathrm{CB}$ and $\mathrm{CNT}(\mathrm{CB} / \mathrm{CNT})$. Table 12.10 shows these same data for factorial design composites containing both $\mathrm{CB}$ and GNP (CB/GNP). Table 12.11 shows the same data for the factorial design composites containing both CNT and GNP (CNT/GNP). Detailed results for this data are given in Appendix J. 
Table 12.9: Tensile Modulus Results for Factorial Design Formulations Containing CB and CNT

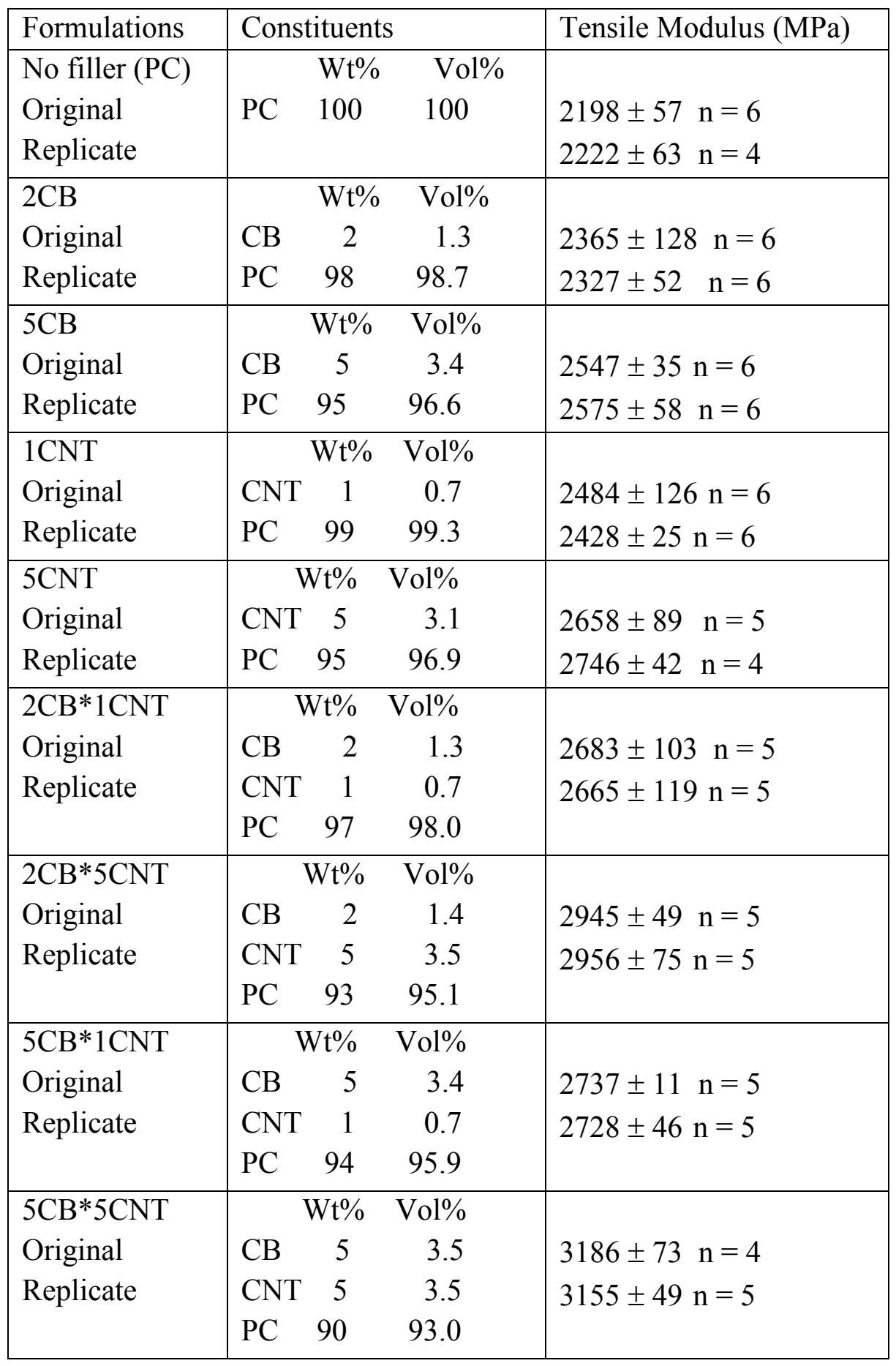


Table 12.10: Tensile Modulus Results for Factorial Design Formulations Containing CB and GNP

\begin{tabular}{|c|c|c|c|}
\hline Formulations & Constituer & & \begin{tabular}{|l} 
Tensile Modulus (MPa) \\
\end{tabular} \\
\hline $\begin{array}{l}\text { No filler (PC) } \\
\text { Original } \\
\text { Replicate }\end{array}$ & $\begin{array}{ll} & \mathrm{Wt}^{0} \\
\mathrm{PC} & 100\end{array}$ & $\begin{array}{l}\text { Vol\% } \\
100\end{array}$ & $\begin{array}{l}2198 \pm 57 \mathrm{n}=6 \\
2222 \pm 63 \mathrm{n}=4\end{array}$ \\
\hline $\begin{array}{l}2 \mathrm{CB} \\
\text { Original } \\
\text { Replicate }\end{array}$ & $\begin{array}{cc} & \mathrm{Wt}^{0} / \\
\mathrm{CB} & 2 \\
\mathrm{PC} & 98\end{array}$ & $\begin{array}{r}\text { Vol\% } \\
1.3 \\
98.7\end{array}$ & $\begin{array}{l}2365 \pm 128 \mathrm{n}=6 \\
2327 \pm 52 \mathrm{n}=6\end{array}$ \\
\hline $\begin{array}{l}5 \mathrm{CB} \\
\text { Original } \\
\text { Replicate }\end{array}$ & $\begin{array}{cc} & \mathrm{Wt}^{0} / \\
\mathrm{CB} & 5 \\
\mathrm{PC} & 95\end{array}$ & $\begin{array}{l}\text { Vol\% } \\
3.4 \\
96.6\end{array}$ & $\begin{array}{l}2547 \pm 35 \mathrm{n}=6 \\
2575 \pm 58 \mathrm{n}=6\end{array}$ \\
\hline $\begin{array}{l}\text { GNP } \\
\text { Original } \\
\text { Replicate }\end{array}$ & $\begin{array}{lr} & \mathrm{Wt}^{\circ} \\
\mathrm{GNP} & 2 \\
\mathrm{PC} & 98\end{array}$ & $\begin{array}{r}\text { Vol\% } \\
1.2 \\
98.8\end{array}$ & $\begin{array}{l}2514 \pm 55 \mathrm{n}=5 \\
2448 \pm 45 \mathrm{n}=5\end{array}$ \\
\hline $\begin{array}{l}\text { GNP } \\
\text { Original } \\
\text { Replicate }\end{array}$ & $\begin{array}{lc} & \mathrm{Wt} \% \\
\text { GNP } & 5 \\
\text { PC } & 95\end{array}$ & $\begin{array}{c}\text { Vol\% } \\
3.1 \\
96.9\end{array}$ & $\begin{array}{l}3095 \pm 64 n=5 \\
3123 \pm 68 n=7\end{array}$ \\
\hline $\begin{array}{l}2 \mathrm{CB} * 2 \mathrm{GNP} \\
\text { Original } \\
\text { Replicate }\end{array}$ & $\begin{array}{lr} & \mathrm{Wt}^{0} \% \\
\mathrm{CB} & 2 \\
\mathrm{GNP} & 2 \\
\mathrm{PC} & 96\end{array}$ & $\begin{array}{c}\text { Vol\% } \\
1.4 \\
1.2 \\
97.4\end{array}$ & $\begin{array}{l}2796 \pm 98 n=5 \\
2767 \pm 73 n=5\end{array}$ \\
\hline $\begin{array}{l}2 \mathrm{CB} * 5 \mathrm{GNP} \\
\text { Original } \\
\text { Replicate }\end{array}$ & \begin{tabular}{|lr} 
& $\mathrm{Wt} \%$ \\
CB & 2 \\
GNP & 5 \\
PC & 93
\end{tabular} & $\begin{array}{c}\text { Vol\% } \\
1.4 \\
3.1 \\
95.5\end{array}$ & $\begin{array}{l}3282 \pm 55 \mathrm{n}=5 \\
3256 \pm 36 \mathrm{n}=5\end{array}$ \\
\hline $\begin{array}{l}5 \mathrm{CB} * 2 \mathrm{GNP} \\
\text { Original } \\
\text { Replicate }\end{array}$ & 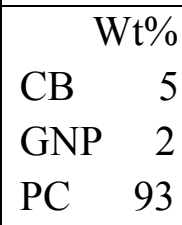 & $\begin{array}{r}\mathrm{Jol} \% \\
3.4 \\
1.2 \\
95.4\end{array}$ & $\begin{array}{l}3049 \pm 59 \mathrm{n}=5 \\
3035 \pm 76 \mathrm{n}=5\end{array}$ \\
\hline $\begin{array}{l}5 \mathrm{CB} * 5 \mathrm{GNP} \\
\text { Original } \\
\text { Replicate }\end{array}$ & 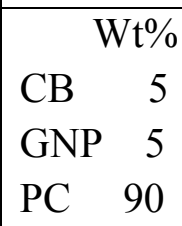 & $\begin{array}{r}\mathrm{Vol} \% \\
3.5 \\
3.1 \\
93.4\end{array}$ & $\begin{array}{l}3744 \pm 77 \mathrm{n}=5 \\
3776 \pm 137 \mathrm{n}=5\end{array}$ \\
\hline
\end{tabular}


Table 12.11: Tensile Modulus Results for Factorial Design Formulations Containing CNT and GNP

\begin{tabular}{|c|c|c|c|}
\hline Formulations & Constituent & & Tensile Modulus (MPa) \\
\hline $\begin{array}{l}\text { No filler }(\mathrm{PC}) \\
\text { Original } \\
\text { Replicate }\end{array}$ & $\begin{array}{ll} & \mathrm{Wt} \% \\
\mathrm{PC} & 100\end{array}$ & $\begin{array}{l}\text { Vol\% } \\
100\end{array}$ & $\begin{array}{l}2198 \pm 57 \mathrm{n}=6 \\
2222 \pm 63 \mathrm{n}=4\end{array}$ \\
\hline $\begin{array}{l}1 \mathrm{CNT} \\
\text { Original } \\
\text { Replicate }\end{array}$ & $\begin{array}{lc} & \mathrm{Wt} \% \\
\mathrm{CNT} & 1 \\
\mathrm{PC} & 99\end{array}$ & $\begin{array}{c}\text { Vol\% } \\
0.7 \\
99.3\end{array}$ & $\begin{array}{l}2484 \pm 126 n=6 \\
2428 \pm 25 n=6\end{array}$ \\
\hline $\begin{array}{l}5 \mathrm{CNT} \\
\text { Original } \\
\text { Replicate }\end{array}$ & $\begin{array}{lc} & \mathrm{Wt} \% \\
\mathrm{CNT} & 5 \\
\mathrm{PC} & 95\end{array}$ & $\begin{array}{c}\text { Vol\% } \\
3.1 \\
96.9\end{array}$ & $\begin{array}{ll}2658 \pm 89 & n=5 \\
2746 \pm 42 & n=4\end{array}$ \\
\hline $\begin{array}{l}\text { GNP } \\
\text { Original } \\
\text { Replicate }\end{array}$ & $\begin{array}{lc} & \mathrm{Wt} \% \\
\text { GNP } & 2 \\
\text { PC } & 98\end{array}$ & $\begin{array}{l}\text { Vol\% } \\
1.2 \\
98.8\end{array}$ & $\begin{array}{l}2514 \pm 55 \mathrm{n}=5 \\
2448 \pm 45 \mathrm{n}=5\end{array}$ \\
\hline $\begin{array}{l}5 \mathrm{GNP} \\
\text { Original } \\
\text { Replicate }\end{array}$ & $\begin{array}{lr} & \mathrm{Wt} \% \\
\mathrm{GNP} & 5 \\
\mathrm{PC} & 95\end{array}$ & $\begin{array}{c}1 \% \\
3.1 \\
96.9\end{array}$ & $\begin{array}{l}3095 \pm 64 n=5 \\
3123 \pm 68 n=7\end{array}$ \\
\hline $\begin{array}{l}\text { 1CNT*2GNP } \\
\text { Original } \\
\text { Replicate }\end{array}$ & \begin{tabular}{lr}
\multicolumn{2}{r}{$\%$} \\
CNT & 1 \\
GNP & 2 \\
PC & 97
\end{tabular} & $\begin{array}{c}\mathrm{ol} \% \\
0.7 \\
1.2 \\
98.1\end{array}$ & $\begin{array}{l}2769 \pm 77 \mathrm{n}=5 \\
2783 \pm 87 \mathrm{n}=5\end{array}$ \\
\hline $\begin{array}{l}\text { 1CNT*5GNP } \\
\text { Original } \\
\text { Replicate }\end{array}$ & \begin{tabular}{ll}
\multicolumn{3}{c}{$\mathrm{Wt}^{0} \%$} \\
CNT & 1 \\
GNP & 5 \\
94 & 96.
\end{tabular} & $\begin{array}{l}\mathrm{Ol} \% \\
\quad 0.7 \\
3.1 \mathrm{PC}\end{array}$ & $\begin{array}{l}3447 \pm 66 n=5 \\
3458 \pm 94 n=5\end{array}$ \\
\hline $\begin{array}{l}5 \mathrm{CNT} * 2 \mathrm{GNP} \\
\text { Original } \\
\text { Replicate }\end{array}$ & $\begin{array}{lc} & \mathrm{Wt} \% \\
\mathrm{CNT} & 5 \\
\mathrm{GNP} & 2 \\
\mathrm{PC} & 93\end{array}$ & $\begin{array}{c}\text { Vol\% } \\
3.5 \\
1.2 \\
95.3\end{array}$ & $\begin{array}{l}3069 \pm 100 \mathrm{n}=5 \\
3018 \pm 50 \mathrm{n}=5\end{array}$ \\
\hline $\begin{array}{l}\text { 5CNT*5GNP } \\
\text { Original } \\
\text { Replicate }\end{array}$ & \begin{tabular}{lr}
\multicolumn{2}{r}{$\%$} \\
CNT & 5 \\
GNP & 5 \\
PC & 90
\end{tabular} & $\begin{array}{r}1 \% \\
3.6 \\
3.1 \\
93.3\end{array}$ & $\begin{array}{l}3490 \pm 43 n=5 \\
3482 \pm 74 n=5\end{array}$ \\
\hline
\end{tabular}


Table 12.12. $3^{2}$ Regression Results for $3^{2}$ Regression Results for Tensile Modulus (MPa)

\begin{tabular}{|c|c|c|c|c|}
\hline Response & Correlation Term & Coefficient & $\mathbf{t}$ & $\mathbf{p}$ \\
\hline \multirow{18}{*}{ 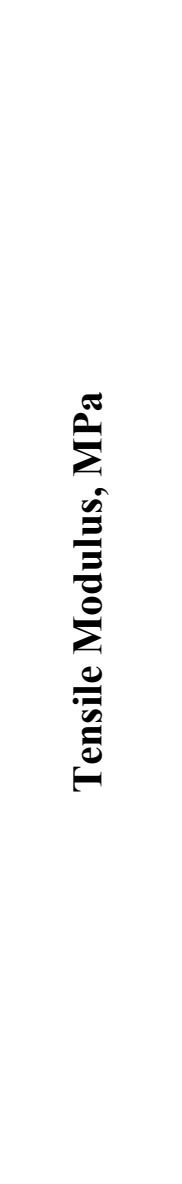 } & $\begin{array}{l}\text { Correlation for } \\
\text { CB/CNT Composites }\end{array}$ & & & \\
\hline & Constant & 2211 & 86.1 & 0.000 \\
\hline & $\mathrm{CB}$ & 107 & 5.3 & 0.000 \\
\hline & CNT & 268 & 8.5 & 0.000 \\
\hline & $\mathrm{CB}^{2}$ & -9.12 & -2.5 & 0.030 \\
\hline & $\mathrm{CNT}^{2}$ & -33.7 & -5.8 & 0.000 \\
\hline & $\mathrm{CB} * \mathrm{CNT}$ & 5.94 & 2.5 & 0.026 \\
\hline & $\begin{array}{l}\text { Correlation for } \\
\text { CB/GNP Composites }\end{array}$ & & & \\
\hline & Constant & 2192 & 87.6 & 0.000 \\
\hline & $\mathrm{CB}$ & 76.2 & 9.5 & 0.000 \\
\hline & GNP & 173 & 21.5 & 0.000 \\
\hline & CB*GNP & 12.1 & 4.7 & 0.000 \\
\hline & $\begin{array}{l}\text { Correlation for } \\
\text { CNT/GNP Composites }\end{array}$ & & & \\
\hline & Constant & 2148 & 72.3 & 0.000 \\
\hline & CNT & 361 & 9.3 & 0.000 \\
\hline & GNP & 194 & 22.8 & 0.000 \\
\hline & $\mathrm{CNT}^{2}$ & -49.9 & -6.9 & 0.000 \\
\hline & $\mathrm{CNT}^{*} \mathrm{GNP}$ & -6.96 & -2.4 & 0.031 \\
\hline
\end{tabular}

Table 12.12 gives the results of regression analysis of tensile modulus for the $\mathrm{CB} / \mathrm{CNT}$, $\mathrm{CB} / \mathrm{GNP}$ and $\mathrm{CNT} / \mathrm{GNP}$ systems. Looking at the first third (CB/CNT system) we see that both fillers individually caused a statistically significant increase in composite tensile modulus. CNT caused a larger increase in the composite tensile modulus than $\mathrm{CB}$. The tensile modulus increasing effect of both fillers was found to diminish as more of the filler is added (as seen from the negative values for the coefficient of the squared term for each filler). We also found a statistically significant interaction between the two fillers that caused the composite tensile modulus to be higher than would be expected solely 
from the additive effects of the individual fillers (117). The tensile modulus $\left(E_{t}\right)$ of the $\mathrm{CB} / \mathrm{CNT}$ system was found to be well correlated by Equation 12.7 below:

$$
\begin{gathered}
E_{t}(M P a)=2211+107 w_{C B}+268 w_{C N T}-9.12 w_{C B}^{2}-33.7 w_{C N T}^{2}+5.94 w_{C B} w_{C N T} \\
R^{2}=0.983
\end{gathered}
$$

where $w_{C B}$ and $w_{C N T}$ are the weight percentage of $\mathrm{CB}$ and $\mathrm{CNT}$ respectively. Equation 12.7 is plotted in Figure 12.6. By studying this plot we see that CNT has a larger effect on increasing the tensile modulus than CB. At higher CNT loadings, we notice that above around 2.5 vol\% CNT, additional CNT has a much smaller effect on the tensile modulus than it did at lower levels.

The second section of Table $12.12(\mathrm{CB} / \mathrm{GNP}$ system) gives the results of regression analysis of tensile modulus for the CB and GNP system. Again each individual filler had a statistically significant increase in composite tensile modulus. For this system GNP caused a larger increase in composite tensile modulus than CB. There is also a statistically significant positive interaction effect between the two fillers. This positive interaction effect means that the tensile modulus of a composite containing both $\mathrm{CB}$ and GNP would have a tensile modulus higher than would be expected just from the additive effects of the two individual fillers (117). The tensile modulus $\left(\mathrm{E}_{\mathrm{t}}\right)$ of the $\mathrm{CB} / \mathrm{GNP}$ system was found to be correlated well by Equation 12.8 below:

$$
\begin{gathered}
E_{t}(\mathrm{MPa})=2192+76.2 w_{C B}+173 w_{G N P}+12.1 w_{C B w} w_{G N P} \\
R^{2}=0.992
\end{gathered}
$$

where $w_{C B}$ and $w_{G N P}$ are the weight percentage of CB and GNP respectively. 


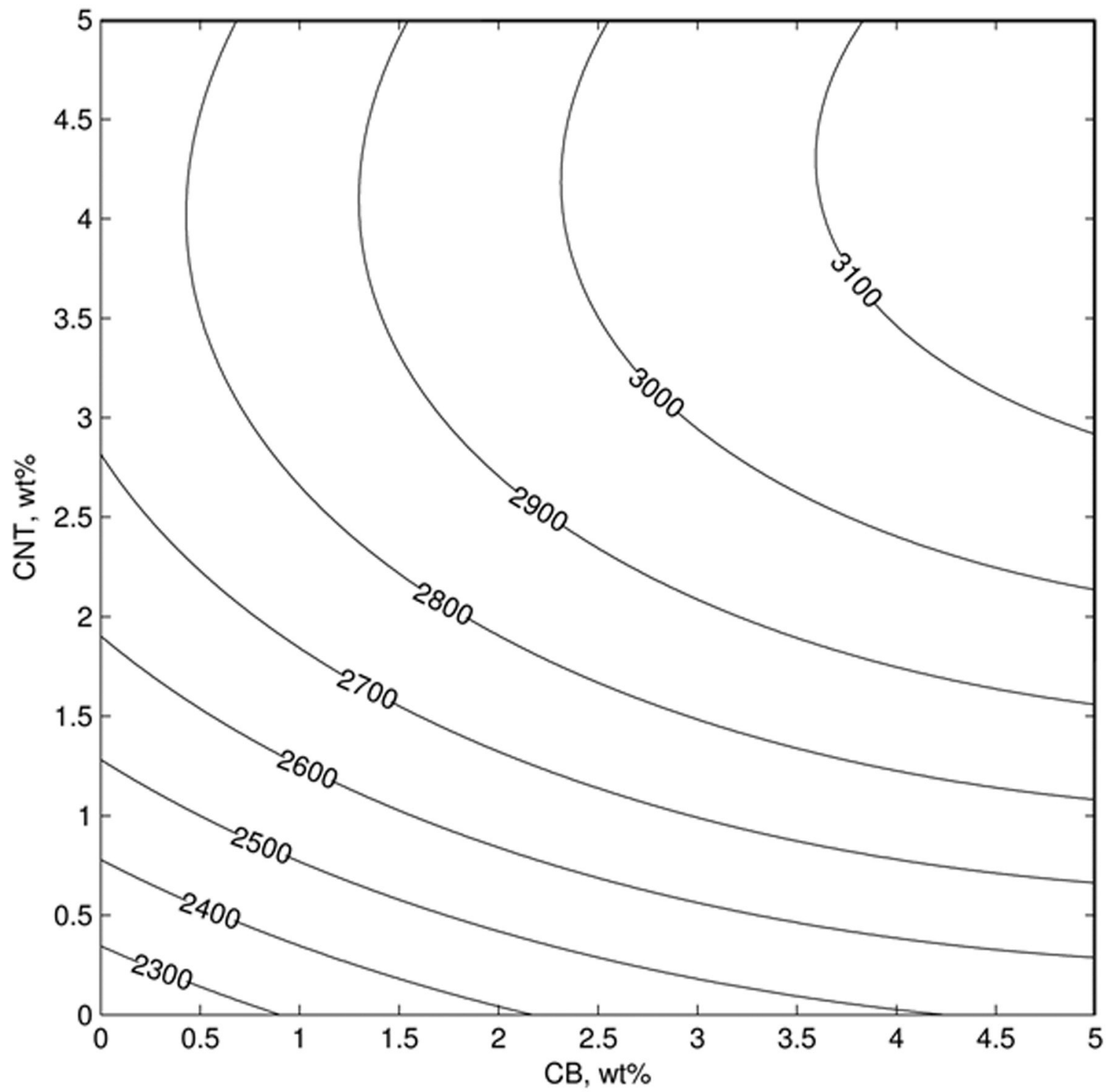

Figure 12.6: Contour Plot of Tensile Modulus (MPa) for CB/CNT Composites

Equation 12.8 is plotted in Figure 12.7. While studying this plot we can see that the GNP has a larger effect on increasing the composite tensile modulus than the CB does. 


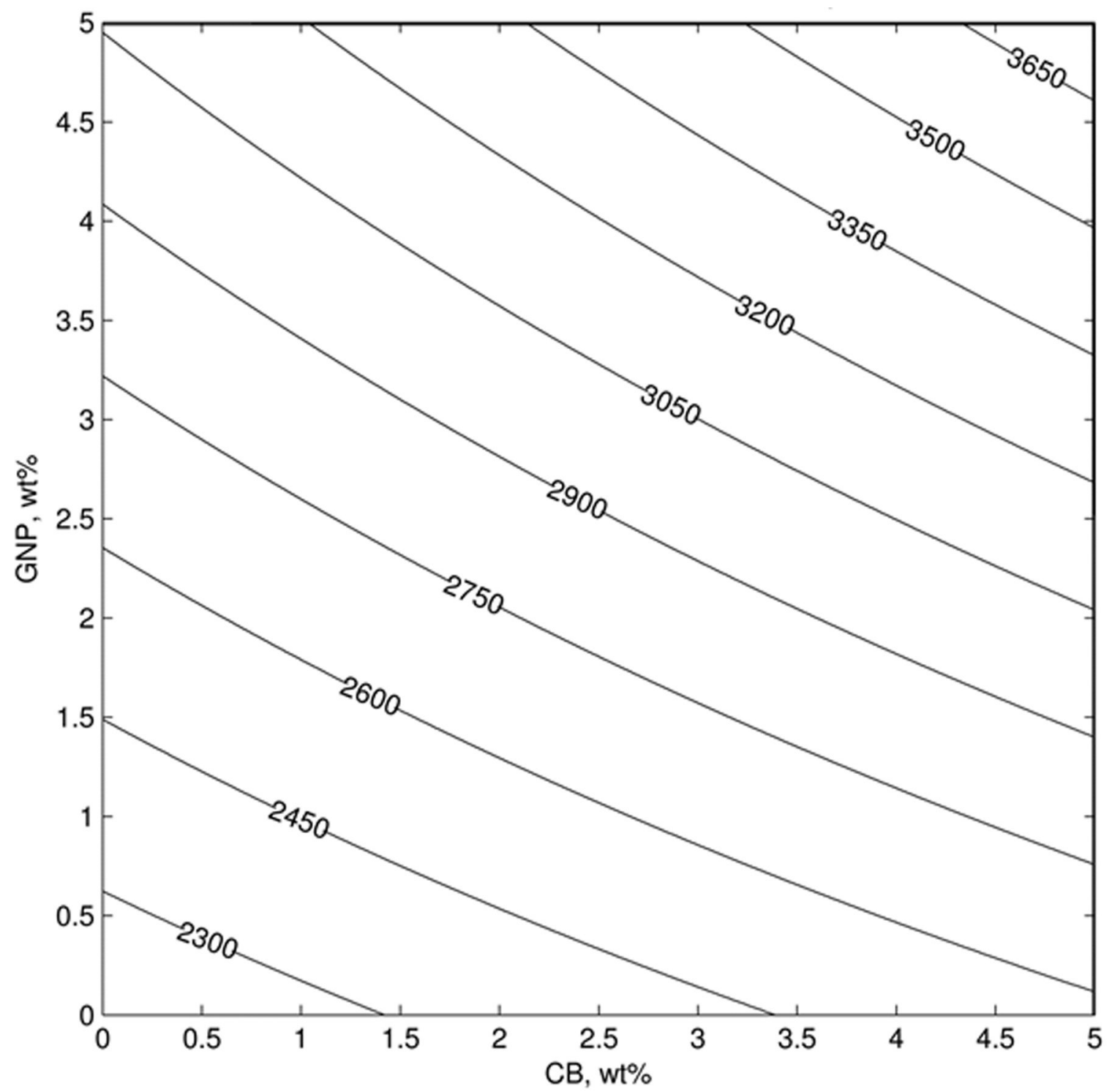

Figure 12.7: Contour Plot of Tensile Modulus (MPa) for CB/GNP Composites

The last section of Table 12.12 (CNT/GNP system) gives the results of regression analysis of tensile modulus for the CNT and GNP system. As expected we see that each individual filler causes a statistically significant increase in composite tensile modulus. In this system CNT has a larger effect on increasing the composite tensile modulus than GNP. We do notice that as the CNT loading is increased, the tensile modulus increasing effect diminishes (notice the negative coefficient on the $\mathrm{CNT}^{2}$ term). We also notice that 
there is a statistically significant negative interaction effect $\left(\mathrm{CNT}^{*} \mathrm{GNP}\right)$ that decreases the composite tensile modulus (note the negative coefficient for the CNT*GNP term). This means that a composite with both CNT and GNP present would have a lower tensile modulus than would be expected solely from the additive effect of each individual filler (117). The tensile modulus $\left(\mathrm{E}_{\mathrm{t}}\right)$ of the $\mathrm{CNT} / \mathrm{GNP}$ system was found to be correlated well by Equation 12.9 below:

$$
\begin{gathered}
E_{t}(M P a)=2148+361 w_{C N T}+194 w_{G N P}-49.9 w_{C N T}^{2}-6.96 w_{C N T} w_{G N P} \\
R^{2}=0.988
\end{gathered}
$$

where $w_{C N T}$ and $w_{G N P}$ are the weight percentage of CNT and GNP respectively. For all 3 systems the $\mathrm{R}^{2}$ values are greater than 0.98 indicating that the majority of the data variation is within expectation for the correlations. Equations 12.7 through 12.9 could be used to estimate the tensile modulus of a theoretical formulation for material development purposes.

\section{5: Flexural Modulus Results}

Table 12.13 shows the mean flexural modulus, standard deviation of the flexural modulus and number of samples that were tested for the factorial design composites containing both $\mathrm{CB}$ and $\mathrm{CNT}(\mathrm{CB} / \mathrm{CNT})$. Table 12.14 shows these same data for factorial design composites containing both $\mathrm{CB}$ and GNP (CB/GNP). Table 12.15 shows the same data for the factorial design composites containing both CNT and GNP (CNT/GNP). Detailed results for this data are given in Appendix K. 
Table 12.13: Flexural Modulus Results for Factorial Design Formulations Containing CB and CNT

\begin{tabular}{|c|c|c|c|}
\hline Formulations & Constituent & & Flexural Modulus (MPa) \\
\hline No filler (PC) & $\mathrm{Wt} \%$ & Vol\% & \\
\hline Original & PC 100 & 100 & $2479 \pm 17 \mathrm{n}=5$ \\
\hline Replicate & & & $2474 \pm 8 \mathrm{n}=5$ \\
\hline $2 \mathrm{CB}$ & $\mathrm{Wt} \%$ & Vol\% & \\
\hline Original & $\mathrm{CB}$ & 1.3 & $2599 \pm 11 \mathrm{n}=10$ \\
\hline Replicate & PC & 98.7 & $2600 \pm 14 \quad n=8$ \\
\hline $5 \mathrm{CB}$ & $\mathrm{Wt} \%$ & Vol\% & \\
\hline Original & CB & 3.4 & $2768 \pm 13 n=10$ \\
\hline Replicate & PC 95 & 96.6 & $2772 \pm 9 n=8$ \\
\hline $1 \mathrm{CNT}$ & $\mathrm{Wt} \%$ & Vol\% & \\
\hline Original & CNT 1 & 0.7 & $2606 \pm 8 n=10$ \\
\hline Replicate & PC 99 & 99.3 & $2605 \pm 14 \mathrm{n}=9$ \\
\hline $5 \mathrm{CNT}$ & $\mathrm{Wt} \%$ & Vol\% & \\
\hline Original & CNT 5 & 3.1 & $3089 \pm 24 \mathrm{n}=6$ \\
\hline Replicate & PC 95 & 96.9 & $3067 \pm 33 \mathrm{n}=5$ \\
\hline $2 \mathrm{CB} * 1 \mathrm{CNT}$ & $\mathrm{Wt} \%$ & Vol\% & \\
\hline Original & $\mathrm{CB}$ & 1.3 & $2738 \pm 37 \mathrm{n}=8$ \\
\hline Replicate & CNT 1 & 0.7 & $2743 \pm 16 n=8$ \\
\hline & PC 97 & 98.0 & \\
\hline $2 \mathrm{CB} * 5 \mathrm{CNT}$ & $\mathrm{Wt} \%$ & Vol\% & \\
\hline Original & $\mathrm{CB}$ & 1.4 & $3421 \pm 106 \mathrm{n}=7$ \\
\hline Replicate & CNT 5 & 3.5 & $3444 \pm 64 \mathrm{n}=5$ \\
\hline & PC 93 & 95.1 & \\
\hline $5 \mathrm{CB} * 1 \mathrm{CNT}$ & $\mathrm{Wt} \%$ & Vol\% & \\
\hline Original & CB & 3.4 & $2912 \pm 12 \mathrm{n}=5$ \\
\hline Replicate & CNT & 0.7 & $2916 \pm 29 n=5$ \\
\hline & PC 94 & 95.9 & \\
\hline $5 \mathrm{CB} * 5 \mathrm{CNT}$ & $\mathrm{Wt} \%$ & Vol\% & \\
\hline Original & $\mathrm{CB}$ & 3.5 & $3670 \pm 63 n=6$ \\
\hline Replicate & CNT 5 & 3.5 & $3772 \pm 71 \mathrm{n}=5$ \\
\hline & PC 90 & 93.0 & \\
\hline
\end{tabular}


Table 12.14: Flexural Modulus Results for Factorial Design Formulations Containing CB and GNP

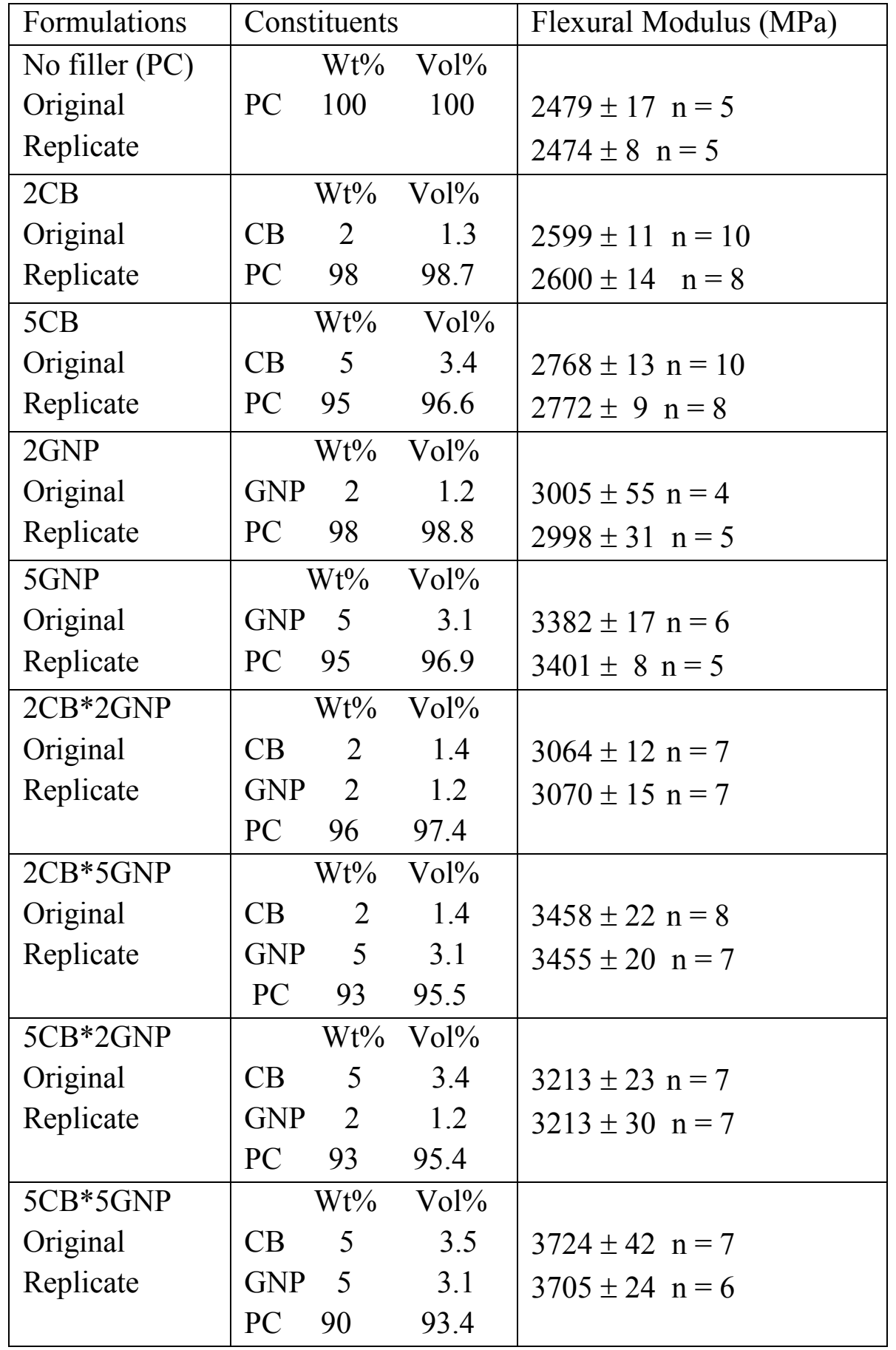


Table 12.15: Flexural Modulus Results for Factorial Design Formulations Containing CNT and GNP

\begin{tabular}{|c|c|c|c|}
\hline Formulations & Constituen & & Flexural Modulus (MPa) \\
\hline $\begin{array}{l}\text { No filler }(\mathrm{PC}) \\
\text { Original } \\
\text { Replicate }\end{array}$ & $\begin{array}{r}\mathrm{Wt} \% \\
\mathrm{PC} \quad 100\end{array}$ & $\begin{array}{l}\text { Vol\% } \\
100\end{array}$ & $\begin{array}{l}2479 \pm 17 \mathrm{n}=5 \\
2474 \pm 8 \mathrm{n}=5\end{array}$ \\
\hline $\begin{array}{l}\text { 1CNT } \\
\text { Original } \\
\text { Replicate }\end{array}$ & $\begin{array}{lr} & \mathrm{Wt} \% \\
\text { CNT } & 1 \\
\text { PC } & 99\end{array}$ & $\begin{array}{c}\text { Vol\% } \% \\
0.7 \\
99.3\end{array}$ & $\begin{array}{l}2606 \pm 8 n=10 \\
2605 \pm 14 n=9\end{array}$ \\
\hline $\begin{array}{l}5 \mathrm{CNT} \\
\text { Original } \\
\text { Replicate }\end{array}$ & $\begin{array}{lc} & \mathrm{Wt} \% \\
\text { CNT } & 5 \\
\text { PC } & 95\end{array}$ & $\begin{array}{c}\text { Vol\% } \\
3.1 \\
96.9\end{array}$ & $\begin{array}{ll}3089 \pm 24 & \mathrm{n}=6 \\
3067 \pm 33 & \mathrm{n}=5\end{array}$ \\
\hline $\begin{array}{l}2 \text { GNP } \\
\text { Original } \\
\text { Replicate }\end{array}$ & $\begin{array}{lc} & \mathrm{Wt} \% \\
\text { GNP } & 2 \\
\text { PC } & 98\end{array}$ & $\begin{array}{l}\text { Vol\% } \\
1.2 \\
98.8\end{array}$ & $\begin{array}{l}3005 \pm 55 \mathrm{n}=4 \\
2998 \pm 31 \mathrm{n}=5\end{array}$ \\
\hline $\begin{array}{l}5 \mathrm{GNP} \\
\text { Original } \\
\text { Replicate }\end{array}$ & \begin{tabular}{lr}
\multicolumn{2}{|r}{$\%$} \\
GNP $\quad 5$ \\
PC $\quad 95$
\end{tabular} & $\begin{array}{r}\text { Vol\% } \\
3.1 \\
96.9\end{array}$ & $\begin{array}{l}3382 \pm 17 \mathrm{n}=6 \\
3401 \pm 8 \mathrm{n}=5\end{array}$ \\
\hline $\begin{array}{l}1 \mathrm{CNT} * 2 \mathrm{GNP} \\
\text { Original } \\
\text { Replicate }\end{array}$ & \begin{tabular}{lr}
\multicolumn{2}{r}{$\mathrm{Wt}^{\circ}$} \\
$\mathrm{CNT}$ & 1 \\
$\mathrm{GNP}$ & 2 \\
$\mathrm{PC}$ & 97
\end{tabular} & $\begin{array}{c}\text { Vol\% } \\
0.7 \\
1.2 \\
98.1\end{array}$ & $\begin{array}{l}2938 \pm 21 n=5 \\
2925 \pm 20 n=5\end{array}$ \\
\hline $\begin{array}{l}\text { 1CNT*5GNP } \\
\text { Original } \\
\text { Replicate }\end{array}$ & \begin{tabular}{lr}
\multicolumn{2}{r}{$\mathrm{Wt} \%$} \\
$\mathrm{CNT}$ & 1 \\
$\mathrm{GNP}$ & 5 \\
$\mathrm{PC}$ & 94
\end{tabular} & $\begin{array}{r}\text { Vol\% } \\
0.7 \\
3.1 \\
96.2\end{array}$ & $\begin{array}{l}3398 \pm 83 n=5 \\
3406 \pm 47 n=5\end{array}$ \\
\hline $\begin{array}{l}5 \mathrm{CNT} * 2 \mathrm{GNP} \\
\text { Original } \\
\text { Replicate }\end{array}$ & $\begin{array}{lc} & \mathrm{Wt} \% \\
\text { CNT } & 5 \\
\text { GNP } & 2 \\
\text { PC } & 93\end{array}$ & $\begin{array}{c}\text { Vol\% } \\
3.5 \\
1.2 \\
95.3\end{array}$ & $\begin{array}{l}3592 \pm 50 \mathrm{n}=7 \\
3632 \pm 23 \mathrm{n}=6\end{array}$ \\
\hline $\begin{array}{l}5 \mathrm{CNT} * 5 \mathrm{GNP} \\
\text { Original } \\
\text { Replicate }\end{array}$ & $\begin{array}{lr} & \mathrm{Wt}^{\circ} \% \\
\mathrm{CNT} & 5 \\
\mathrm{GNP} & 5 \\
\mathrm{PC} & 90\end{array}$ & $\begin{array}{r}\text { Vol\% } \\
3.6 \\
3.1 \\
93.3\end{array}$ & $\begin{array}{l}4312 \pm 87 n=6 \\
4364 \pm 47 n=5\end{array}$ \\
\hline
\end{tabular}


Table 12.16: $3^{2}$ Regression Results for $3^{2}$ Regression Results for Flexural Modulus (MPa)

\begin{tabular}{|c|c|c|c|c|}
\hline Response & Correlation Term & Coefficient & $\mathbf{t}$ & $\mathbf{p}$ \\
\hline \multirow{17}{*}{ 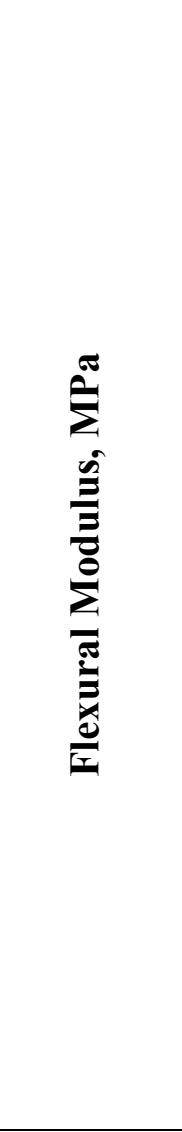 } & $\begin{array}{l}\text { Correlation for } \\
\text { CB/CNT Composites }\end{array}$ & & & \\
\hline & Constant & 2465 & 134 & 0.000 \\
\hline & $\mathrm{CB}$ & 86.3 & 5.4 & 0.000 \\
\hline & $\mathrm{CNT}$ & 127 & 22 & 0.000 \\
\hline & $\mathrm{CB}^{2}$ & -6.36 & -2.2 & 0.049 \\
\hline & $\mathrm{CB} * \mathrm{CNT}$ & 14.3 & 7.7 & 0.000 \\
\hline & $\begin{array}{l}\text { Correlation for } \\
\text { CB/GNP Composites }\end{array}$ & & & \\
\hline & Constant & 2485 & 163 & 0.000 \\
\hline & $\mathrm{CB}$ & 55.9 & 15.6 & 0.000 \\
\hline & GNP & 278 & 19.9 & 0.000 \\
\hline & $\mathrm{GNP}^{2}$ & -19.4 & -7.4 & 0.000 \\
\hline & $\begin{array}{l}\text { Correlation for } \\
\text { CNT/GNP Composites }\end{array}$ & & & \\
\hline & Constant & 2531 & 91.5 & 0.000 \\
\hline & GNP & 220 & 8.4 & 0.000 \\
\hline & $\mathrm{CNT}^{2}$ & 21.9 & 12.6 & 0.000 \\
\hline & $\mathrm{GNP}^{2}$ & -10.9 & -2.3 & 0.040 \\
\hline & $\mathrm{CNT}^{*} \mathrm{GNP}$ & 16.3 & 5.5 & 0.000 \\
\hline
\end{tabular}

Table 12.16 gives the results of regression analysis of flexural modulus for the $\mathrm{CB} / \mathrm{CNT}$, $\mathrm{CB} / \mathrm{GNP}$ and $\mathrm{CNT} / \mathrm{GNP}$ systems. Looking at the first third (CB/CNT system) we see that both fillers individually caused a statistically significant increase in composite flexural modulus. CNT caused a larger increase in the composite flexural modulus than $\mathrm{CB}$. The flexural modulus increasing effect of $\mathrm{CB}$ was found to diminish as more $\mathrm{CB}$ is added (as seen from the negative values for the coefficient of the squared term for $\mathrm{CB}$ ). We also found a statistically significant positive interaction between the two fillers that caused the composite flexural modulus to be higher than would be expected solely from 
the additive effects of the individual fillers (117). The flexural modulus $\left(\mathrm{E}_{\mathrm{f}}\right)$ of the $\mathrm{CB} / \mathrm{CNT}$ system was found to be well correlated by Equation 12.10 below:

$$
\begin{gathered}
E_{f}(M P a)=2465+86.3 w_{C B}+127 w_{C N T}-6.36 w_{C B}^{2}+14.3 w_{C B} w_{C N T} \\
R^{2}=0.994
\end{gathered}
$$

where $w_{C B}$ and $w_{C N T}$ are the weight percentage of $\mathrm{CB}$ and $\mathrm{CNT}$ respectively. Equation 12.10 is plotted in Figure 12.8. By studying this plot we see that CNT has a larger effect on increasing the flexural modulus than $\mathrm{CB}$.

The second section of Table 12.16 (CB/GNP system) gives the results of regression analysis of flexural modulus for the CB and GNP system. Again each individual filler had a statistically significant increase in composite flexural modulus. For this system GNP caused a larger increase in composite flexural modulus than CB. We see a diminishing flexural modulus increasing effect as more GNP is added (as see from the negative coefficient of the $\mathrm{GNP}^{2}$ term). The flexural modulus $\left(\mathrm{E}_{\mathrm{f}}\right)$ of the $\mathrm{CB} / \mathrm{GNP}$ system was found to be correlated well by Equation 12.11 below:

$$
\begin{gathered}
E_{f}(M P a)=2485+55.9 w_{C B}+278 w_{G N P}-19.4 w_{G N P}^{2} \\
R^{2}=0.995
\end{gathered}
$$

where $w_{C B}$ and $w_{G N P}$ are the weight percentage of CB and GNP respectively. 


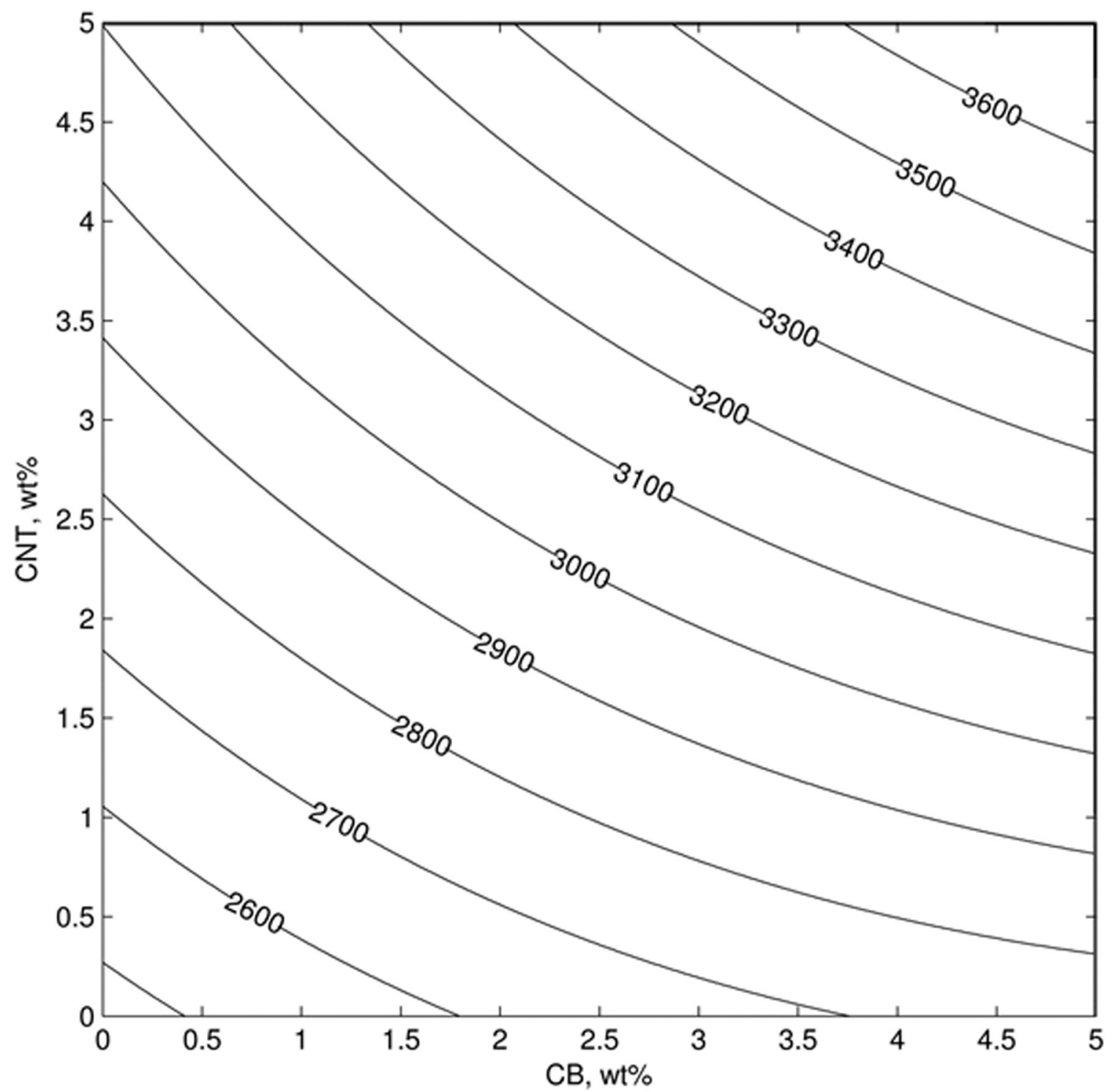

Figure 12.8: Contour Plot of Flexural Modulus (MPa) for CB/CNT Composites

Equation 12.11 is plotted in Figure 12.9. By studying this plot we see that GNP has a larger effect on increasing the flexural modulus than CB. 


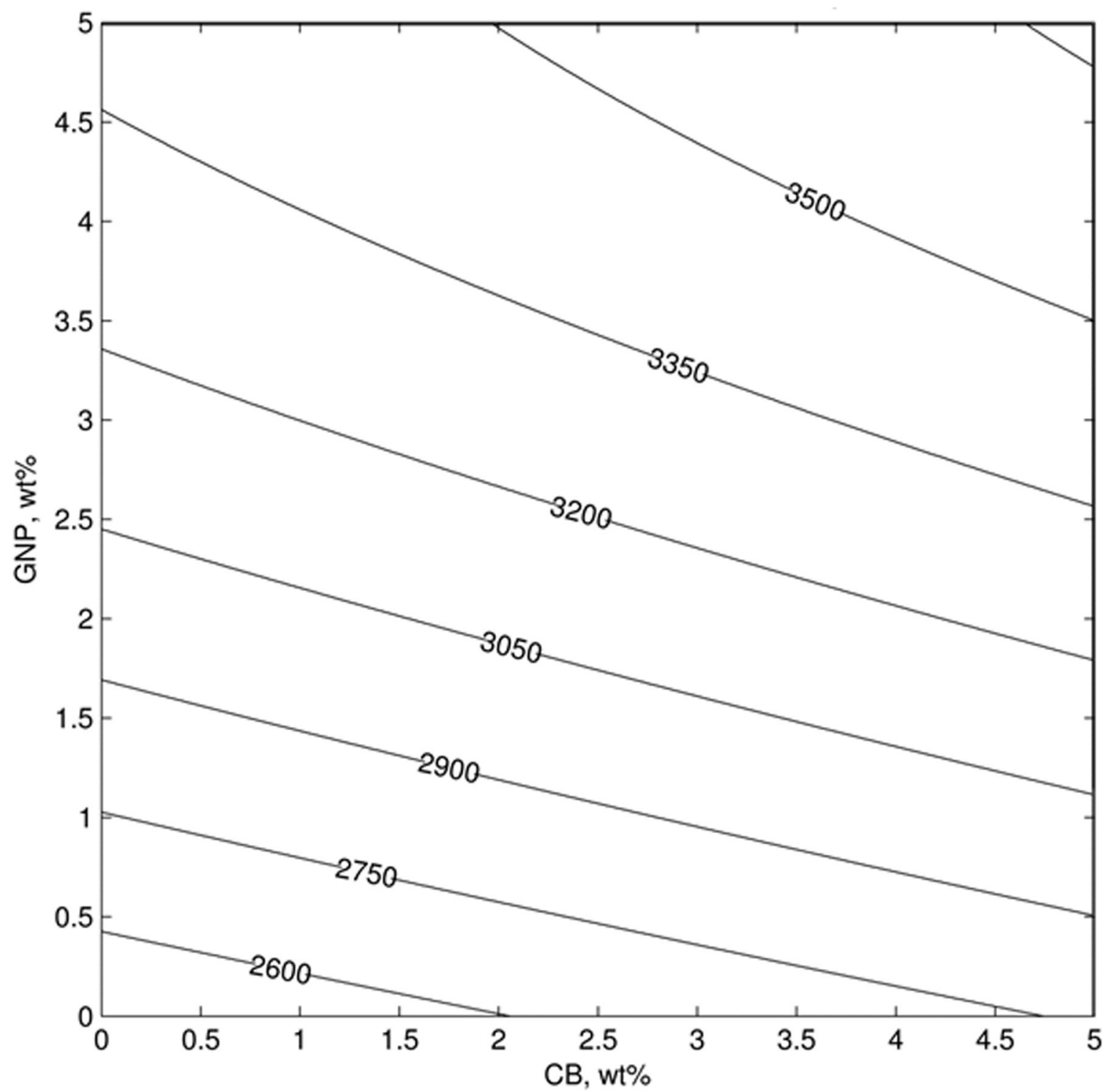

Figure 12.9: Contour Plot of Flexural Modulus (MPa) for CB/GNP Composites

The last section of Table 12.16 (CNT/GNP system) gives the results of regression analysis of flexural modulus for the CNT and GNP system. As expected we see that each individual filler causes a statistically significant increase in composite flexural modulus. In this system CNT has an increasing effect on increasing the flexural modulus as more $\mathrm{CNT}$ is added (as seen from the positive coefficient of the $\mathrm{CNT}^{2}$ tem). We do notice that as the GNP loading is increased, the flexural modulus increasing effect diminishes 
(notice the negative coefficient on the $\mathrm{GNP}^{2}$ term). We also notice that there is a statistically significant positive interaction effect $\left(\mathrm{CNT}^{*} \mathrm{GNP}\right)$ that decreases the composite flexural modulus (note the positive coefficient for the CNT*GNP term). This means that a composite with both CNT and GNP present would have a higher flexural modulus than would be expected solely from the additive effect of each individual filler (117). The flexural modulus $\left(\mathrm{E}_{\mathrm{f}}\right)$ of the $\mathrm{CNT} / \mathrm{GNP}$ system was found to be correlated well by Equation 12.12 below:

$$
\begin{gathered}
E_{t}(M P a)=2531+220 w_{G N P}+21.9 w_{C N T}^{2}-10.9 w_{G N P}^{2}+16.3 w_{C N T} w_{G N P} \\
R^{2}=0.992
\end{gathered}
$$

where $w_{C N T}$ and $w_{G N P}$ are the weight percentage of CNT and GNP respectively. For all 3 systems the $\mathrm{R}^{2}$ values are greater than 0.99 indicating that the majority of the data variation is within expectation for the correlations. Equations 12.10 through 12.12 could be used to estimate the flexural modulus of a theoretical formulation for material development purposes.

\section{6: Conclusions}

The purpose of this section of the project was to measure and model the effects of $\mathrm{CB}$, CNT and GNP on PC using three $3^{2}$ (2 factor, 3 level) factorial designs, one for each twofiller combination ( $\mathrm{CB} / \mathrm{CNT}, \mathrm{CB} / \mathrm{GNP}$ and $\mathrm{CNT} / \mathrm{GNP})$. In particular the effects on the electrical resistivity (ER), thermal conductivity (TC), tensile modulus and flexural modulus were measured and modeled. As seen in Tables 12.1 through 12.3 the filler loadings for the factorial design were 0,2 and $5 \mathrm{wt} \%$ for $\mathrm{CB}, 0,1$ and $5 \mathrm{wt} \%$ for CNT and 0,2 and $5 \mathrm{wt} \%$ for GNP. Electrically, all 3 fillers individually caused a statistically 
significant decrease in the composite ER. Of the 3 fillers, CNT caused the largest decrease in composite ER. Thermally, all 3 individual fillers caused a statistically significant increase in TC. Of the fillers, GNP had the largest effect on increasing the TC. Additionally GNP and CNT together had a significant positive interaction effect that increased the TC of the 2-filler composite more than was expected due to the additive effects of GNP and CNT. Mechanically, all 3 fillers caused statistically significant increases in both tensile and flexural modulus. Among the fillers tested, GNP caused the greatest increase in both tensile and flexural modulus. The correlatoins that were created for each property and 2-filler system could be used to create conductive composites of PC with the desired electrical, thermal and mechanical properties. 


\section{Chapter 13: Effects of Multiple Fillers on the Rheology of Polycarbonate Based Resins}

\section{1: Capillary Rheometer Results}

Figures 13.1 through 13.3 show the average values of the single filler steady-shear rheology data for the factorial design loadings. The standard deviations of these values are all less than $2 \%$ thus the error bars have been omitted for the sake of clarity. The composite with solely $5 \mathrm{wt} \% \mathrm{CB}$ generated too high of a pressure at $2000 \mathrm{~s}^{-1}$ to be measured by the rheometer and thus this data point was omitted. Additionally the $5 \mathrm{wt} \%$ CNT formulation was unable to be measured at $50 \mathrm{~s}^{-1}$ shear rate due to flow instabilities at this shear rate and the data point was thus omitted. This data, along with the two-filler data is listed in Tables 13.1 through 13.3. More detailed results are listed in Appendix M.

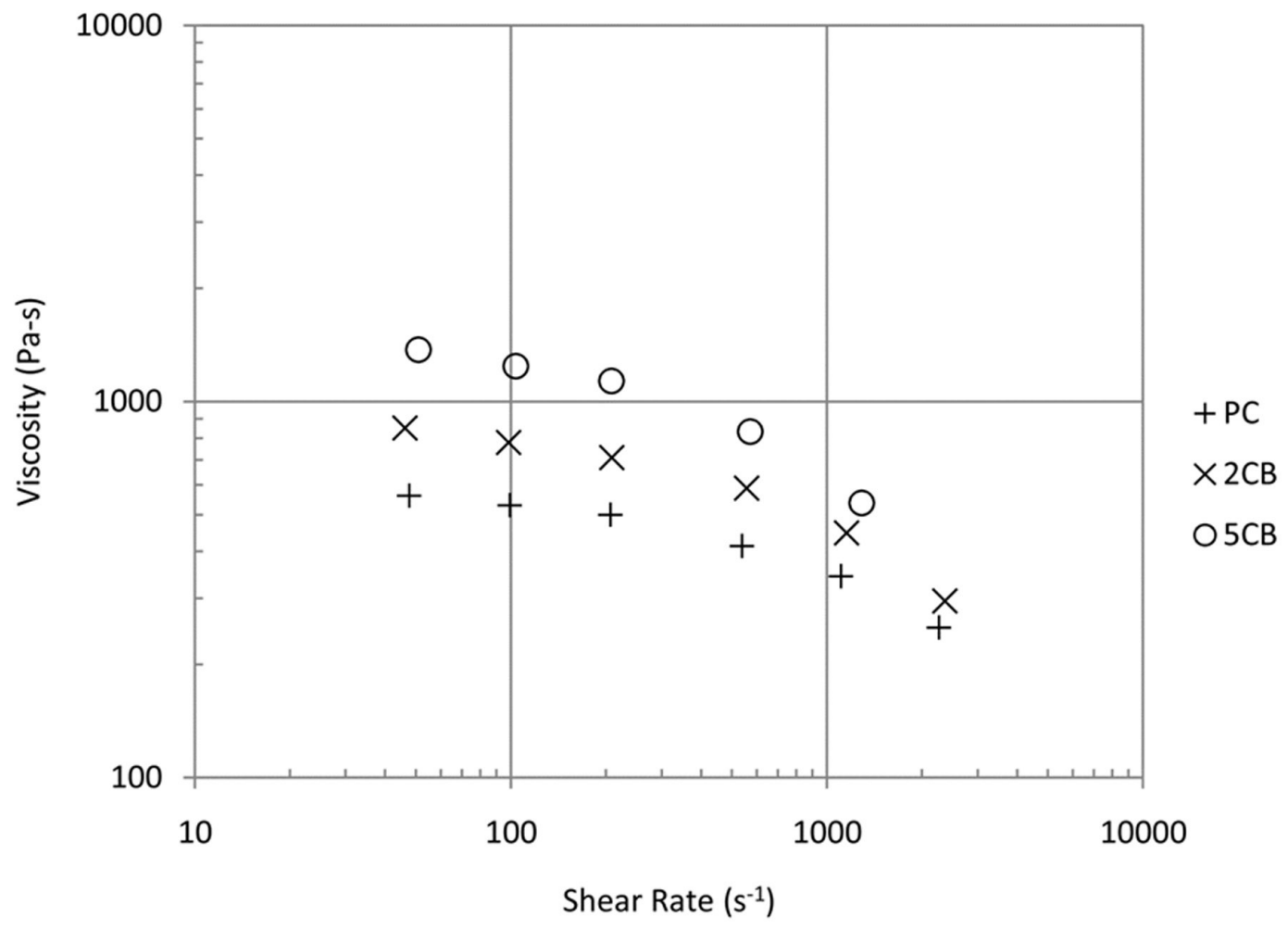

Figure 13.1: Viscosity versus Shear Rate for $\mathrm{CB} / \mathrm{PC}$ Composites at $270^{\circ} \mathrm{C}$ 


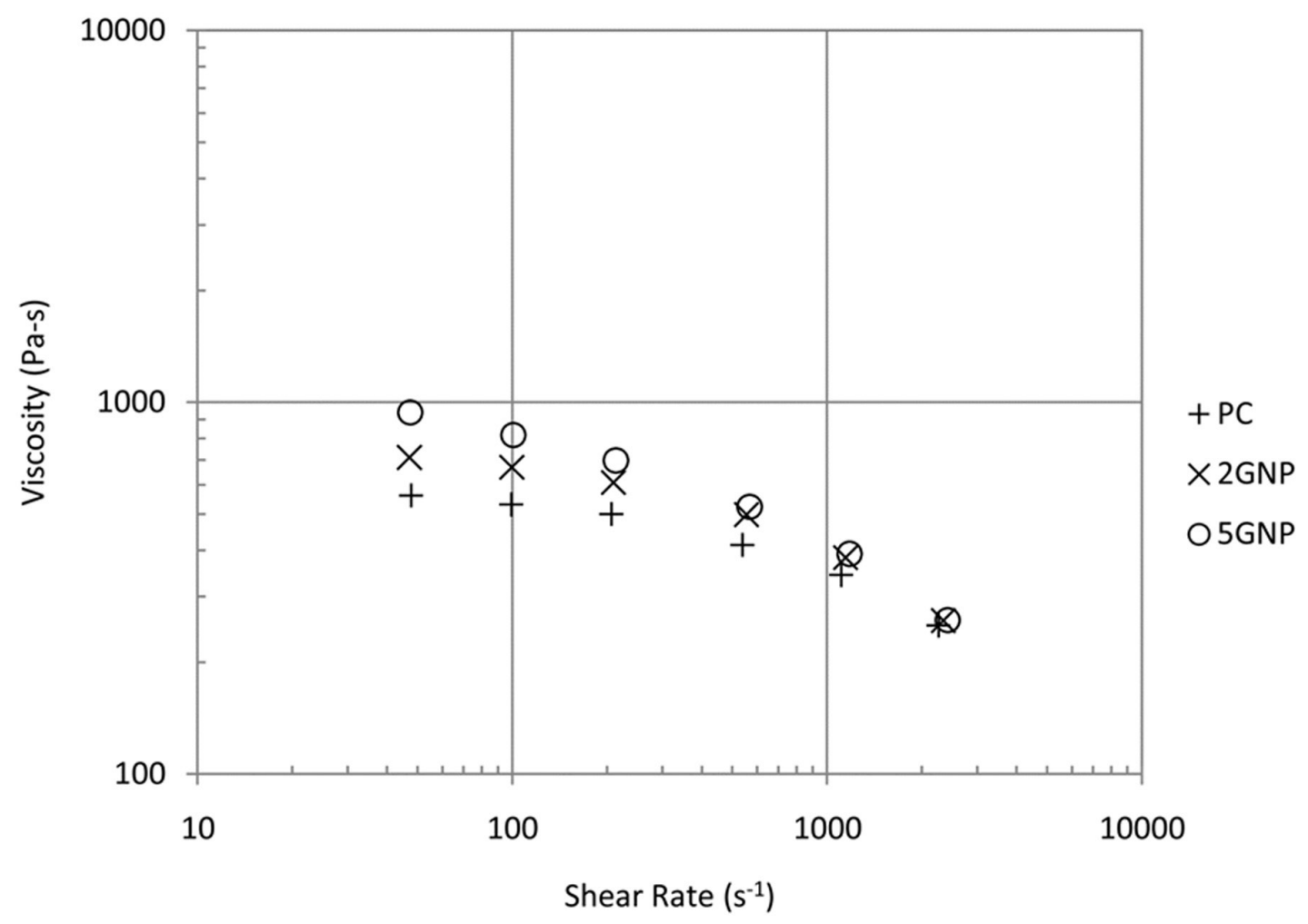

Figure 13.2: Viscosity versus Shear Rate for GNP/PC Composites at $270^{\circ} \mathrm{C}$

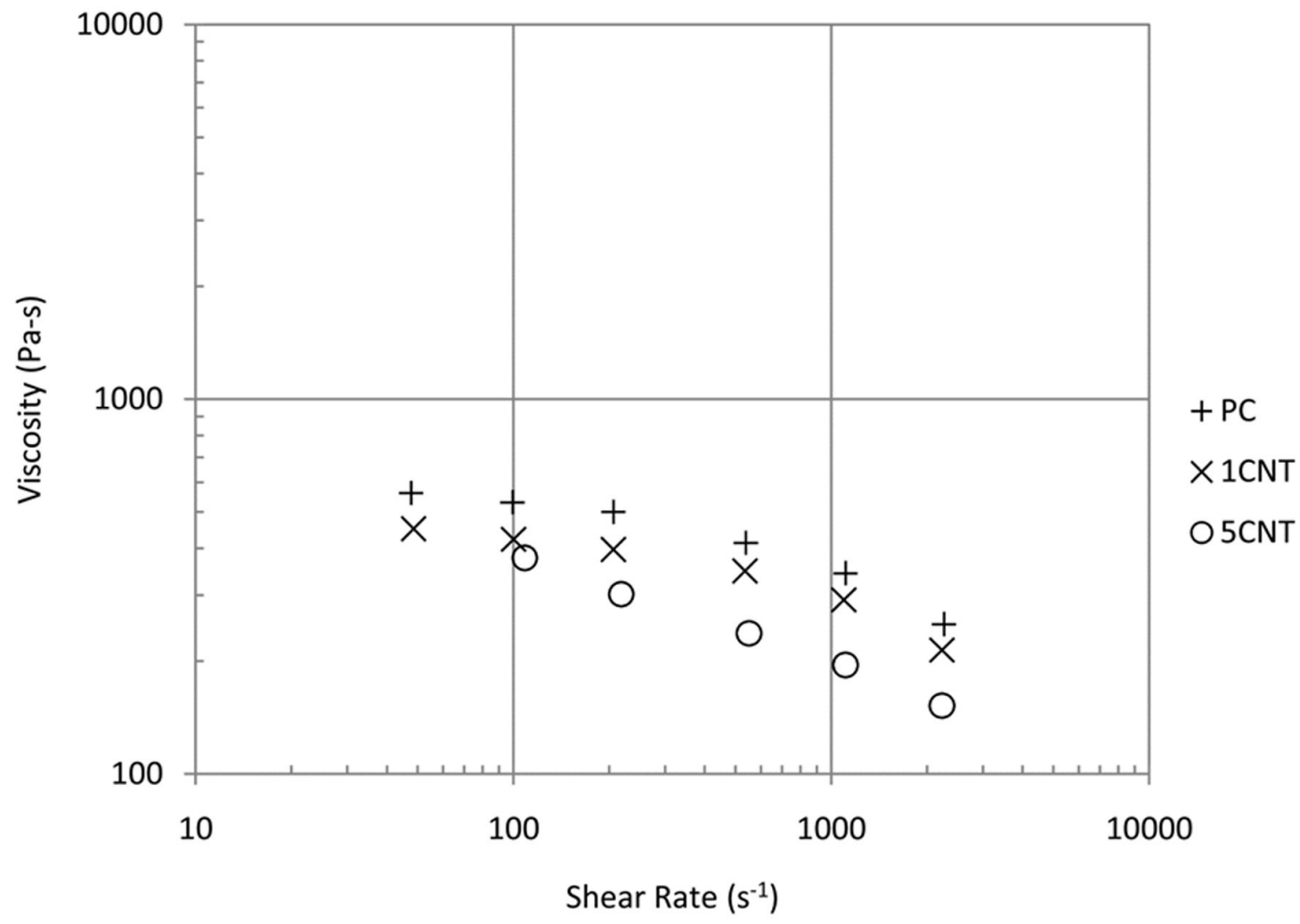

Figure 13.3: Viscosity versus Shear Rate for CNT/PC Composites at $270^{\circ} \mathrm{C}$ 
Table 13.1: Newtonian Zero-Shear Plateau Results for Factorial Design Formulations Containing CB and GNP

\begin{tabular}{|c|c|c|c|}
\hline \multirow{3}{*}{$\begin{array}{l}\text { Formulations } \\
\text { No filler (PC) }\end{array}$} & \multicolumn{2}{|l|}{ Constituents } & \multirow[t]{2}{*}{$\begin{array}{l}\text { Zero-Shear Plateau, } \\
\eta_{0}(\mathrm{~Pa}-\mathrm{s})\end{array}$} \\
\hline & $\mathrm{Wt} \%$ & Vol\% & \\
\hline & 100 & 100 & 550 \\
\hline \multirow[t]{3}{*}{$2 \mathrm{CB}$} & $\mathrm{Wt} \%$ & Vol\% & \multirow{3}{*}{850} \\
\hline & $\mathrm{CB}$ & 1.3 & \\
\hline & $\mathrm{PC}$ & 98.7 & \\
\hline \multirow[t]{3}{*}{$5 \mathrm{CB}$} & $\mathrm{Wt} \%$ & Vol\% & \multirow{3}{*}{$\geq 1400$} \\
\hline & $\mathrm{CB}$ & 3.4 & \\
\hline & PC 95 & 96.6 & \\
\hline \multirow[t]{3}{*}{ 2GNP } & $\mathrm{Wt} \%$ & Vol\% & \multirow{3}{*}{710} \\
\hline & GNP 2 & 1.2 & \\
\hline & PC 98 & 98.8 & \\
\hline \multirow[t]{3}{*}{$5 \mathrm{GNP}$} & $\mathrm{Wt} \%$ & Vol\% & \multirow{3}{*}{$\geq 940$} \\
\hline & GNP 5 & 3.1 & \\
\hline & PC 95 & 96.9 & \\
\hline \multirow[t]{4}{*}{$2 \mathrm{CB} * 2 \mathrm{GNP}$} & $\mathrm{Wt} \%$ & Vol\% & \multirow{4}{*}{$\geq 970$} \\
\hline & $\mathrm{CB}$ & 1.4 & \\
\hline & GNP 2 & 1.2 & \\
\hline & PC 96 & 97.4 & \\
\hline \multirow[t]{4}{*}{$2 \mathrm{CB} * 5 \mathrm{GNP}$} & $\mathrm{Wt} \%$ & Vol\% & \multirow{4}{*}{$\geq 1200$} \\
\hline & $\mathrm{CB}$ & 1.4 & \\
\hline & GNP 5 & $3.1 \mathrm{PC}$ & \\
\hline & 95.5 & & \\
\hline \multirow[t]{4}{*}{$5 \mathrm{CB} * 2 \mathrm{GNP}$} & $\mathrm{Wt} \%$ & Vol\% & \multirow{4}{*}{$\geq 1500$} \\
\hline & $\mathrm{CB}$ & 3.4 & \\
\hline & GNP 2 & 1.2 & \\
\hline & $\mathrm{PC}$ & 95.4 & \\
\hline \multirow[t]{4}{*}{$5 \mathrm{CB}^{*} 5 \mathrm{GNP}$} & $\mathrm{Wt} \%$ & Vol\% & \multirow{4}{*}{$\geq 1900$} \\
\hline & $\mathrm{CB}$ & 3.5 & \\
\hline & GNP 5 & 3.1 & \\
\hline & PC 90 & 93.4 & \\
\hline
\end{tabular}


Table 13.2: Electrical Resistivity and Zero-Shear Viscosity Results for Factorial Design Formulations Containing CB and CNT

\begin{tabular}{|c|c|c|c|}
\hline Formulations & Constituent & & $\begin{array}{l}\text { Zero-Shear Viscosity, } \\
\eta_{0}(\mathrm{~Pa}-\mathrm{s})\end{array}$ \\
\hline No filler (PC) & $\begin{array}{ll} & \mathrm{Wt} \% \\
\mathrm{PC} & 100\end{array}$ & $\begin{array}{l}\text { Vol\% } \\
100\end{array}$ & 550 \\
\hline $2 \mathrm{CB}$ & $\begin{array}{cc} & \mathrm{Wt} \% \\
\mathrm{CB} & 2 \\
\mathrm{PC} & 98\end{array}$ & $\begin{array}{l}\text { Vol\% } \\
1.3 \\
98.7\end{array}$ & 850 \\
\hline $5 \mathrm{CB}$ & $\begin{array}{cc} & \mathrm{Wt} \% \\
\mathrm{CB} & 5 \\
\mathrm{PC} & 95\end{array}$ & $\begin{array}{l}\text { Vol\% } \\
3.4 \\
96.6\end{array}$ & $\geq 1400$ \\
\hline $1 \mathrm{CNT}$ & $\begin{array}{lc} & \mathrm{Wt} \% \\
\mathrm{CNT} & 1 \\
\mathrm{PC} & 99\end{array}$ & $\begin{array}{l}\text { Vol\% } \\
0.7 \\
99.3\end{array}$ & 450 \\
\hline $5 \mathrm{CNT}$ & \begin{tabular}{lc}
\multicolumn{2}{c}{$\mathrm{Wt}^{2} \%$} \\
$\mathrm{CNT}$ & 5 \\
$\mathrm{PC}$ & 95
\end{tabular} & $\begin{array}{l}\text { Vol\% } \\
3.1 \\
96.9\end{array}$ & $\geq 380^{\dagger}$ \\
\hline $2 \mathrm{CB} * 1 \mathrm{CNT}$ & $\begin{array}{lr} & \mathrm{Wt} \% \\
\mathrm{CB} & 2 \\
\mathrm{CNT} & 1 \\
\mathrm{PC} & 97\end{array}$ & $\begin{array}{l}\text { Vol\% } \\
1.3 \\
0.7 \\
98.0\end{array}$ & $\geq 560$ \\
\hline $2 \mathrm{CB} * 5 \mathrm{CNT}$ & $\begin{array}{lc} & \mathrm{Wt} \% \\
\mathrm{CB} & 2 \\
\mathrm{CNT} & 2 \\
96 & 95.1\end{array}$ & $\begin{array}{l}\text { Vol\% } \\
1.4 \\
3.5 \quad \mathrm{PC}\end{array}$ & $\geq 600^{\dagger}$ \\
\hline $5 \mathrm{CB} * 1 \mathrm{CNT}$ & $\begin{array}{lc} & \mathrm{Wt} \% \\
\mathrm{CB} & 5 \\
\mathrm{CNT} & 1 \\
94 & 95.9\end{array}$ & $\begin{array}{l}\text { Vol\% } \\
3.4 \\
0.7 \mathrm{PC}\end{array}$ & $\geq 880$ \\
\hline $5 \mathrm{CB} * 5 \mathrm{CNT}$ & $\begin{array}{lr} & \mathrm{Wt} \% \\
\mathrm{CB} & 5 \\
\mathrm{CNT} & 5 \\
\mathrm{PC} & 90\end{array}$ & $\begin{array}{l}\text { Vol\% } \\
3.5 \\
3.5 \\
93.0\end{array}$ & $\geq 1100^{\dagger}$ \\
\hline
\end{tabular}

$\dagger$ indicates formulations that have significant decreases in viscosity (relative to other formulations in table) at high shear rates. 
Table 13.3: Electrical Resistivity and Zero-Shear Viscosity Results for Factorial Design Formulations Containing CNT and GNP

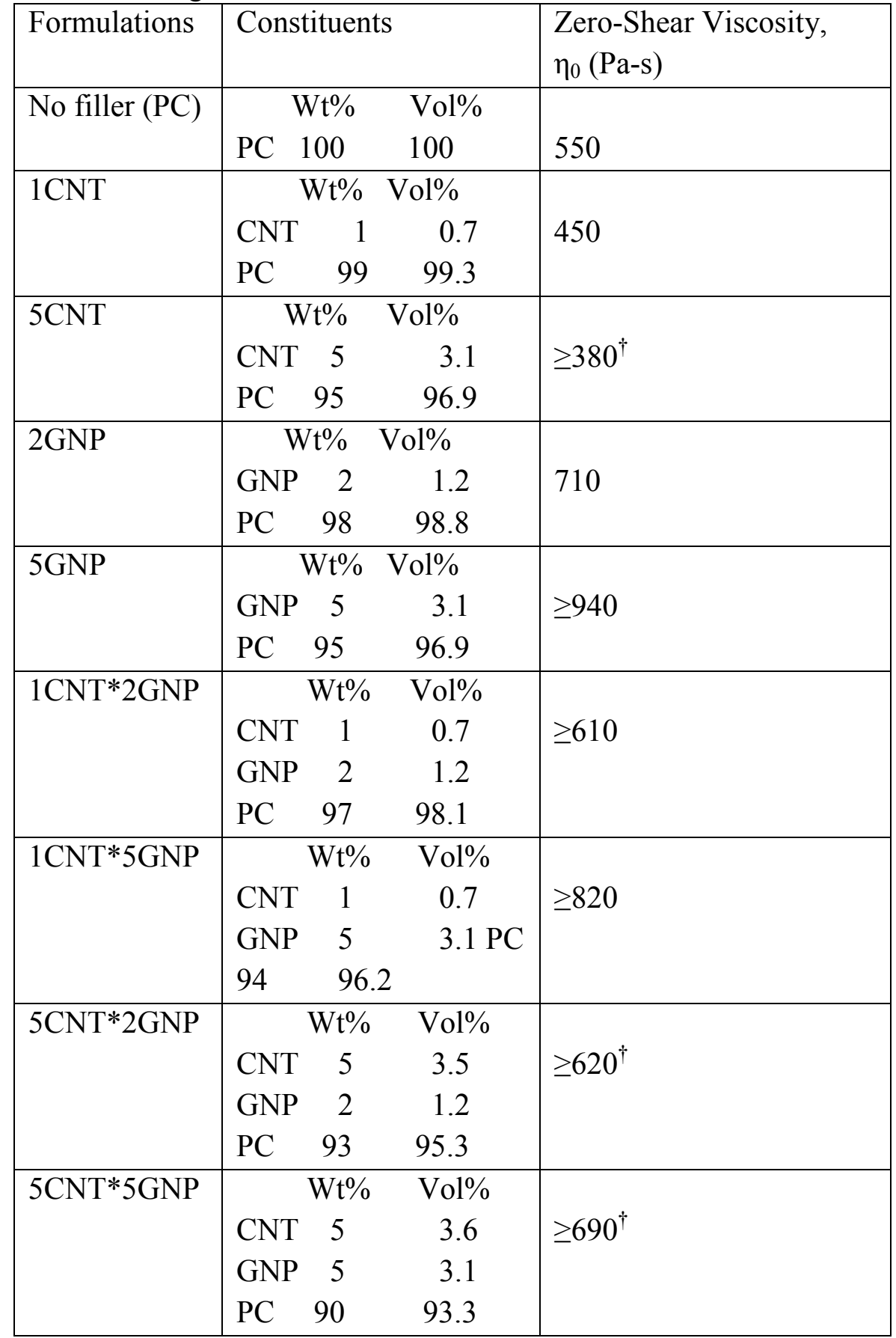

${ }^{\dagger}$ indicates formulations that have significant decreases in viscosity (relative to other formulations in table) at high shear rates.

Adding carbon fillers to thermoplastics typically increase the viscosity, especially at low shear rates (51). At higher shear rates as the polymer shear thins, the viscosity increase 
due to the fillers is usually less pronounced. Figures 13.1 and 13.2 show CB and GNP respectively following this common behavior. The addition of $2 \mathrm{wt} \% \mathrm{CB}$ increased the Newtonian zero-shear plateau to $850 \mathrm{~Pa}$-s from the $550 \mathrm{~Pa}$-s originally seen in the neat PC. When the CB loading is increased to $5 \mathrm{wt} \%$, the Newtonian zero-shear plateau is increased to 1400 Pa-s. GNP shows a similar but lesser effect with $2 \mathrm{wt} \%$ GNP only increasing the Newtonian zero-shear plateau to 710 Pa-s and $5 \mathrm{wt} \%$ GNP increasing to 940 Pa-s. These values lead to the conclusion that CB increases the viscosity of the composite $100 \%$ more than the equivalent amount of GNP does. The viscosity increase due to either of these fillers diminish as the shear-rate increases. It is noteworthy that, for GNP, there is no apparent increase in viscosity at the highest shear rates measured.

As was discussed in Chapter 8 and seen again in Figure 13.3, CNT in PC does not follow the typical carbon filled thermoplastic behavior of increased viscosity with increased filler loading. Adding $1 \mathrm{wt} \% \mathrm{CNT}$ to $\mathrm{PC}$ decreases the viscosity at all shear rates to below that of the neat PC while maintaining the shear-thinning behavior at high shear rates. Increasing the $\mathrm{CNT}$ loading to $5 \mathrm{wt} \%$ further reduces the viscosity of the composite for shear rates greater than $100 \mathrm{~s}^{-1}$. The Newtonian zero-shear plateaus of the $1 \mathrm{wt} \% \mathrm{CNT}$ and $5 \mathrm{wt} \% \mathrm{CNT}$ composites are similar, but at the highest shear rate tested, the $1 \mathrm{wt} \%$ CNT has a viscosity of $210 \mathrm{~Pa}$-s compared to then $150 \mathrm{~Pa}-\mathrm{s}$ of $5 \mathrm{wt} \% \mathrm{CNT}$. As was discussed in Chapter 8, the reduction of viscosity due to the addition of CNT is thought to be laminarization of the flow due to CNT log-rolling and flow orientation.

Combination of the two classic fillers, CB and GNP, show additive behavior and increase the viscosity of the composite in all cases as shown in Figure 13.4. When the two 
composites containing $7 \mathrm{wt} \%$ total carbon fillers are compared, we see that $\mathrm{CB}$ has a greater effect on increasing the viscosity of the composite as the $5 \mathrm{wt} \% \mathrm{CB}, 2 \mathrm{wt} \% \mathrm{GNP}$ (5CB*2GNP) composite has a higher viscosity than the $2 \mathrm{wt} \% \mathrm{CB}, 5 \mathrm{wt} \%$ GNP (2CB*5GNP) composite for all shear rates. It is also interesting to note that at the highest shear-rates measured in Figure 13.4, GNP loading had no effect on the composite viscosity (i.e. $2 \mathrm{CB} * 2 \mathrm{GNP}$ and $2 \mathrm{CB} * 5 \mathrm{GNP}$ have roughly the same viscosity).

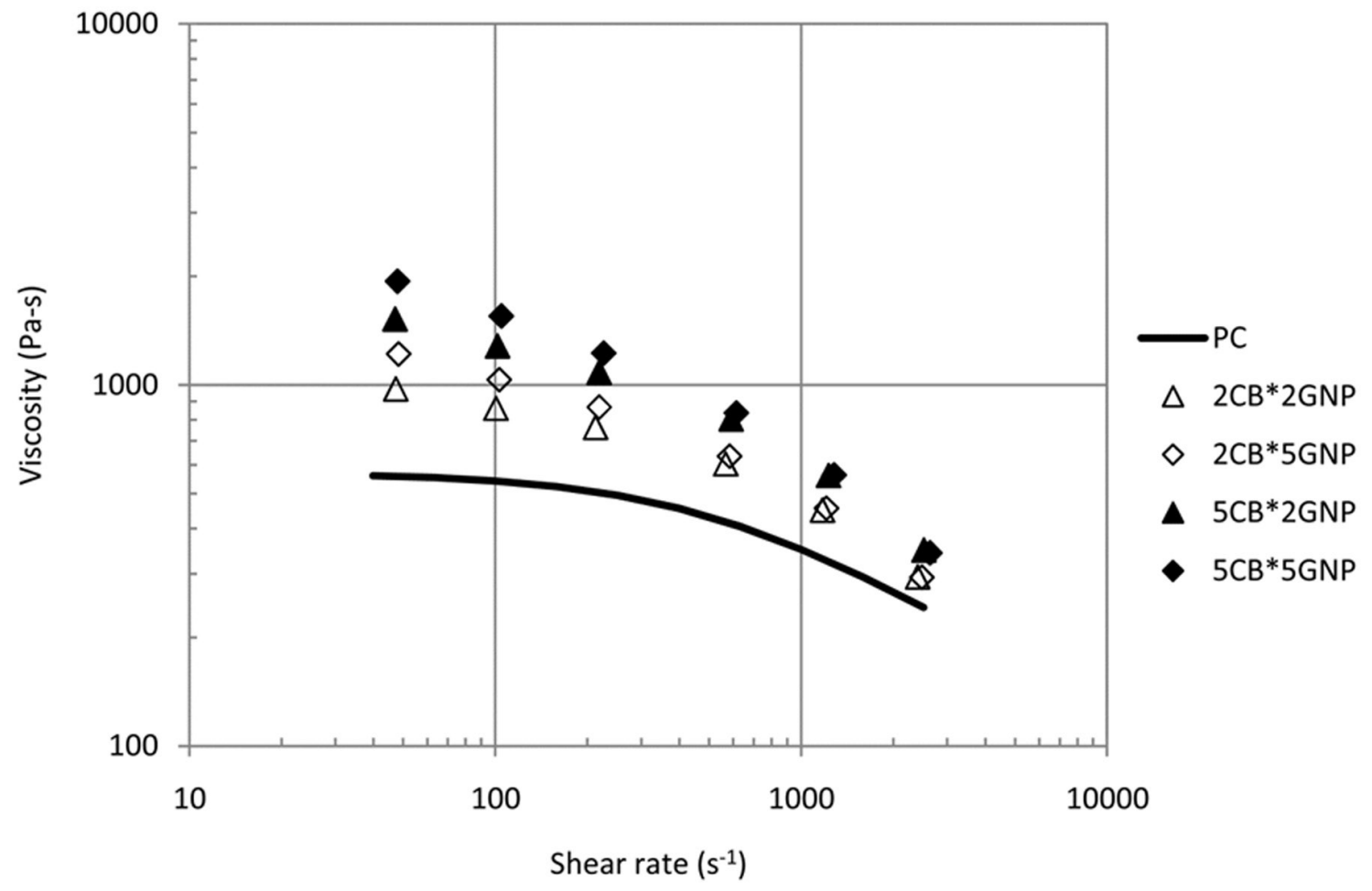

Figure 13.4: Viscosity versus Shear Rate for PC Composites with $\mathrm{CB}$ and GNP at $270^{\circ} \mathrm{C}$

Figure 13.5 shows the results of the $\mathrm{CB}$ and $\mathrm{CNT}$ two-filler composites. We see that the unusual viscosity decreasing effect is present in all 4 filled systems. Studying Figure 13.5 we see that the addition of $1 \mathrm{wt} \% \mathrm{CNT}$ to a composite with $2 \mathrm{wt} \% \mathrm{CB}$ (As seen in Figure 13.1) completely negates all of the viscosity increasing effects of the $\mathrm{CB}$ and brings the composite viscosity back down to the level of the neat PC. When the CNT 
loading is increased to $5 \mathrm{wt} \% \mathrm{CNT}$ with $2 \mathrm{wt} \% \mathrm{CB}$, the viscosity of the composite is reduced to a level well below the neat PC viscosity at higher shear-rates. The addition of $1 \mathrm{wt} \% \mathrm{CNT}$ to $5 \mathrm{wt} \% \mathrm{CB}$ caused a significant decrease in viscosity bringing the Newtonian zero-shear plateau down from 1400 Pa-s for 5CB to 880 Pa-s for 5CB*1CNT. Again, the addition of further CNT to bring the CNT loading to $5 \mathrm{wt} \% \mathrm{CNT}$ along with 5 $\mathrm{wt} \% \mathrm{CB}$ provides further viscosity reduction at higher shear rates (greater than $200 \mathrm{~s}^{-1}$ ).

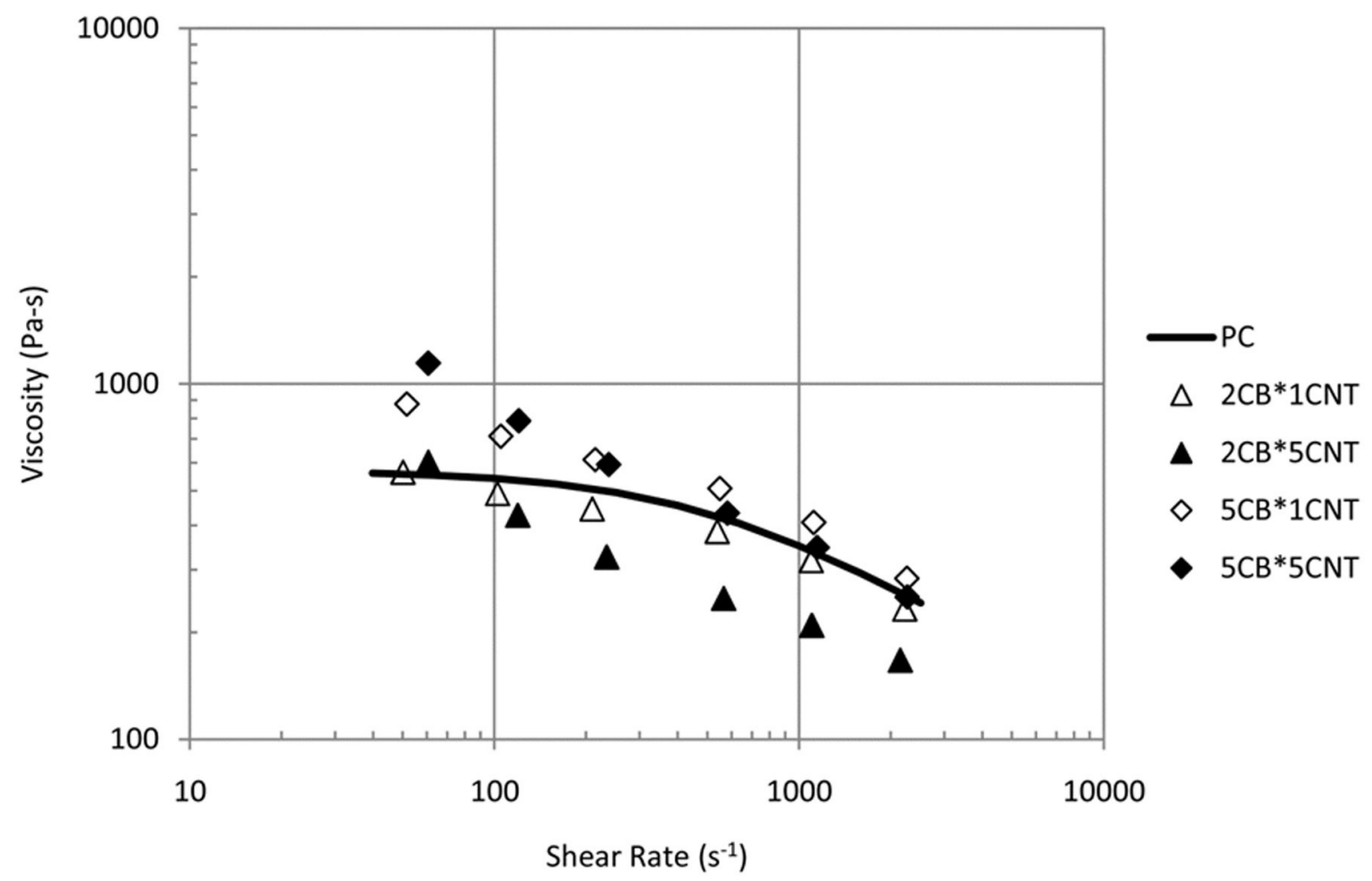

Figure 13.5: Viscosity versus Shear Rate PC Composites with $\mathrm{CB}$ and $\mathrm{CNT}$ at $270^{\circ} \mathrm{C}$

The CNT and GNP two-filler system is summarized in Figure 13.6. Similarly to the $\mathrm{CB} / \mathrm{CNT}$ system, we see that the addition of at least $1 \mathrm{wt} \% \mathrm{CNT}$ to either 2 or $5 \mathrm{wt} \%$ GNP reduces the viscosity of the resulting composite versus the original GNP filled composite (as shown in Figure 13.2). Again, similarly to the CB/CNT system, the further addition of CNT to $5 \mathrm{wt} \%$ CNT with either loading of GNP increased the viscosity reduction at higher shear rates (greater than $100 \mathrm{~s}^{-1}$ ). It is of note that both composites 187 
with $5 \mathrm{wt} \% \mathrm{CNT}(5 \mathrm{CNT} * 2 \mathrm{GNP}$ and $5 \mathrm{CNT} * 5 \mathrm{GNP})$ have viscosities lower than that of neat PC at shear rates over $100 \mathrm{~s}^{-1}$. The addition of CNT to the GNP systems has the added benefit of greatly reducing the electrical resistivity of the composites as shown in Table 12.3. For example, we see that adding just $1 \mathrm{wt} \%$ CNT to $5 \mathrm{wt} \%$ GNP can reduce the electrical resistivity from $4 \times 10^{15} \mathrm{ohm}-\mathrm{cm}$ for $5 \mathrm{GNP}$ down to $6 \times 10^{6} \mathrm{ohm}-\mathrm{cm}$ for $1 \mathrm{CNT} * 5 \mathrm{GNP}$, which falls in the electrostatic dissipative range $\left(10^{10}\right.$ to $10^{3} \mathrm{ohm}-\mathrm{cm}$. The addition of $5 \mathrm{wt} \% \mathrm{CNT}$ to $5 \mathrm{wt} \%$ GNP reduces the ER to $100 \mathrm{ohm}-\mathrm{cm}$ which places the $5 \mathrm{CNT} * 5 \mathrm{GNP}$ composite in the semiconductive range (100 to $10 \mathrm{ohm}-\mathrm{cm})$.

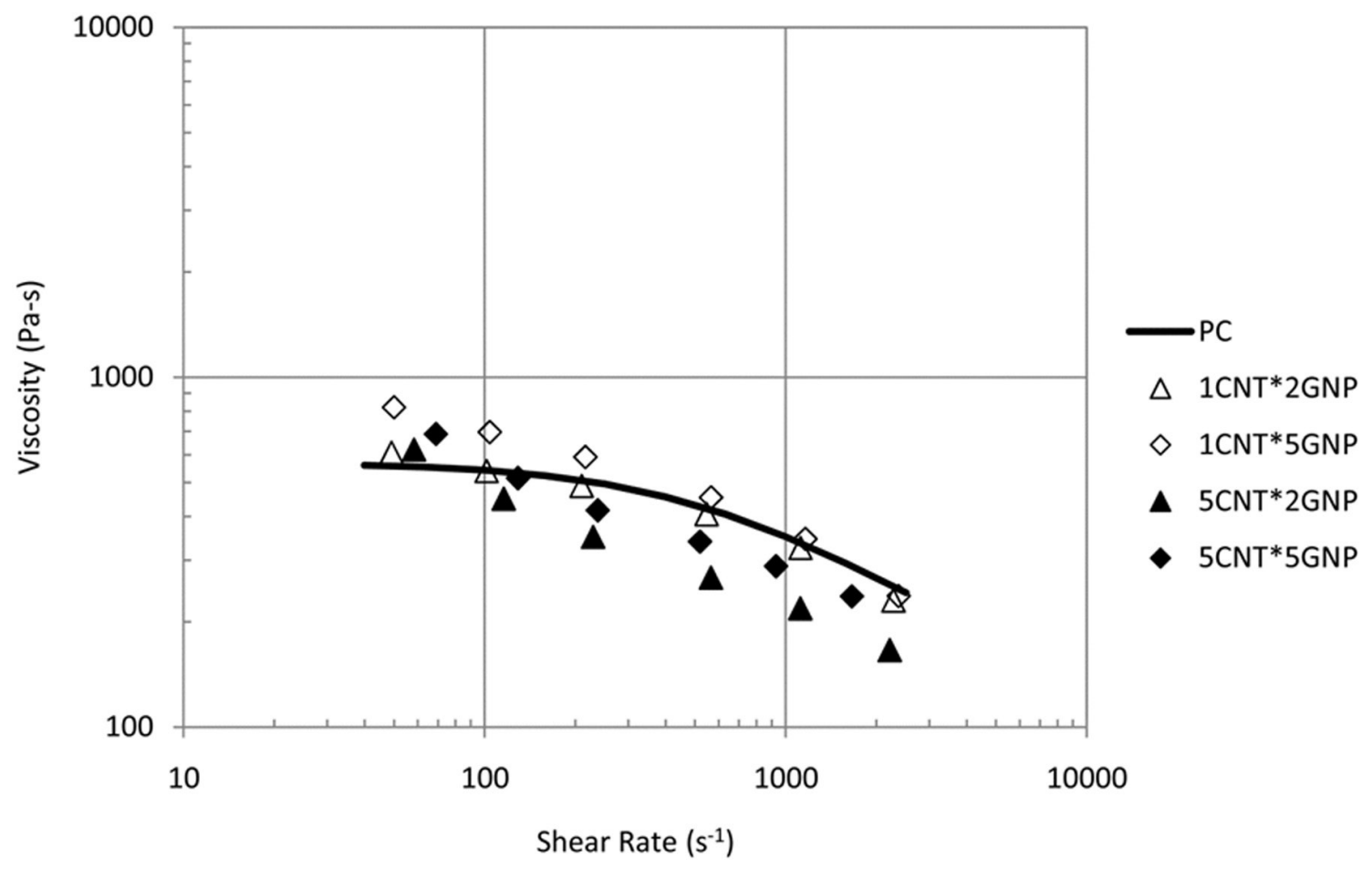

Figure 13.6: Viscosity versus Shear Rate for PC Composites with CNT and GNP at $270^{\circ} \mathrm{C}$

Both the $\mathrm{CB} / \mathrm{CNT}$ and $\mathrm{CNT} / \mathrm{GNP}$ systems illustrate the viscosity reducing effect of CNT. Even at loadings as low as $1 \mathrm{wt} \%, \mathrm{CNTs}$ can reduce the viscosity of filled or unfilled PC. Further addition of CNT up to $5 \mathrm{wt} \%$ to the systems can increase the 
viscosity reduction effect, especially at higher shear rates. Viscosity is an indicator of processability for a composite. When the viscosity is lower, the composite is typically easier to process. The addition of $1 \mathrm{wt} \% \mathrm{CNT}$ to neat PC, or PC with CB or GNP added can improve the processability of the material. It is also possible that the addition of 1 $\mathrm{wt} \% \mathrm{CNT}$ would allow for the processing of materials with loadings that are higher than are normally processable. An example of this possibility is seen in the $\mathrm{CB} / \mathrm{CNT}$ system and illustrated in Figure 13.7. The addition of $1 \mathrm{wt} \% \mathrm{CNT}$ to $2 \mathrm{wt} \% \mathrm{CB}$ brings the viscosity down to the level of neat PC. The addition of $1 \mathrm{wt} \% \mathrm{CNT}$ to $5 \mathrm{wt} \% \mathrm{CB}$ brought the viscosity to roughly that of only $2 \mathrm{wt} \% \mathrm{CB}$ in $\mathrm{PC}$, the addition of $1 \mathrm{wt} \% \mathrm{CNT}$ allowed for the addition of $3 \mathrm{wt} \% \mathrm{CB}$ with a negligible change in viscosity. The addition of the CNT has the added benefit of decreasing the ER of the resultant compounds as seen in Tables 12.1 through 12.3. Looking back at the $\mathrm{CB} / \mathrm{CNT}$ system, we see that when the addition of $1 \mathrm{wt} \% \mathrm{CNT}$ to $2 \mathrm{wt} \% \mathrm{CB}$ allowed us to increase the $\mathrm{CB}$ loading to 5 $\mathrm{wt} \%$, the ER decreased from $4 \times 10^{16} \mathrm{ohm}-\mathrm{cm}$ for $2 \mathrm{wt} \% \mathrm{CB}$ in PC to $400 \mathrm{ohm}-\mathrm{cm}$ for 1 wt $\% \mathrm{CNT}$ and $5 \mathrm{wt} \% \mathrm{CB}$ in PC. Of this 14 order of magnitude change, 13 orders of magnitude are due to the addition of the additional CB (ER of 2CB is $4 \times 10^{16} \mathrm{ohm}-\mathrm{cm}$, ER of $5 \mathrm{CB}$ is $\left.4 \times 10^{3} \mathrm{ohm}-\mathrm{cm}\right)$. 


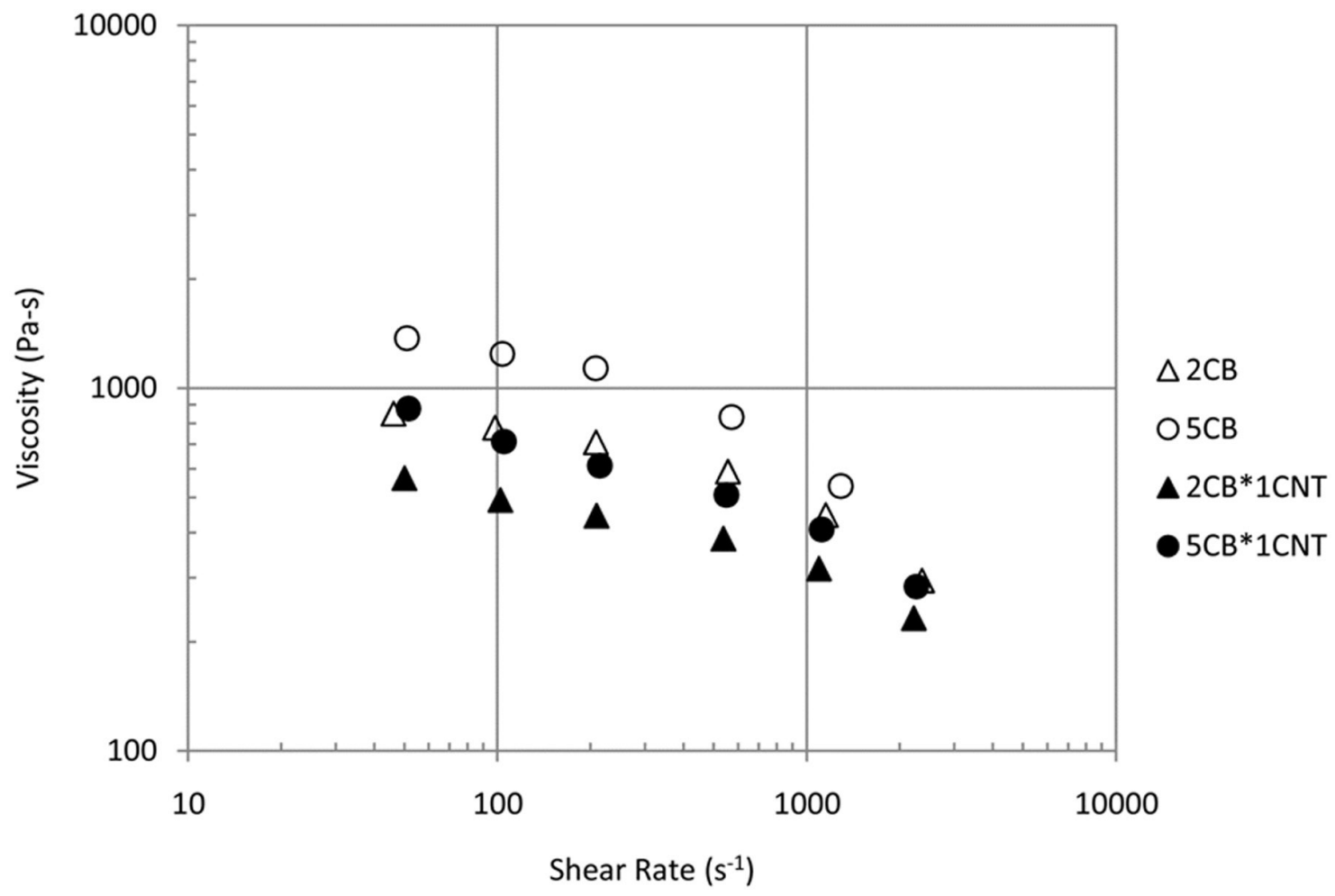

Figure 13.7: Viscosity versus Shear-Rate PC Composites with $\mathrm{CB}$ and $\mathrm{CNT}$ at $270^{\circ} \mathrm{C}$ : Illustrating the Effects of Adding $1 \mathrm{wt} \% \mathrm{CNT}$ to $\mathrm{CB} / \mathrm{PC}$ Composites

\section{2: Conclusions}

The purpose of this section of the project was to measure the effects of $\mathrm{CB}, \mathrm{CNT}$ and GNP on the viscosity of PC using three $3^{2}$ ( 2 factor, 3 level) factorial designs, one for each two-filler combination (CB/CNT, $\mathrm{CB} / \mathrm{GNP}$ and $\mathrm{CNT} / \mathrm{GNP})$ while noting electrical resistivity changes. As seen in Tables 13.1-1 through 13.1-3 the filler loadings for the factorial design were 0,2 and $5 \mathrm{wt} \%$ for $\mathrm{CB}, 0,1$ and $5 \mathrm{wt} \%$ for CNT and 0,2 and $5 \mathrm{wt} \%$ for GNP. The addition of either CB or GNP to a system increased the viscosity of the resulting composite. Comparing $\mathrm{CB}$ and $\mathrm{GNP}, \mathrm{CB}$ has a larger effect on increasing the viscosity of the composite than GNP. CNT exhibits unusual rheological behavior when added to PC. In filled or unfilled PC systems, the addition of as little as $1 \mathrm{wt} \% \mathrm{CNT}$ reduces the viscosity of the resulting composite by up to $37 \%$. The addition of further 
CNT up to a total of $5 \mathrm{wt} \%$ CNT provides additional decreases in viscosity. This behavior is beneficial in that the addition of only $1 \mathrm{wt} \% \mathrm{CNT}$ can allow for the further addition of a less expensive filler (such as CB or GNP) that follows classic behavior and increases the viscosity of the composite while maintaining high-shear processability (extrusion, injection molding, etc.). The properties of composites that are being enhanced by a filler such as CB or GNP could be enhanced by the addition of more of this filler as enabled by the addition of $1 \mathrm{wt} \% \mathrm{CNT}$. 


\section{Chapter 14: Conclusions and Future Work}

\section{1: Conclusions}

\subsection{1: Electrical Resistivity (ER)}

When conductive carbon fillers are added to polymers, they decrease the electrical conductivity of the resulting composite. The resistivity of the composite begins near the neat polymer ER. The ER of the composite slowly decreases as filler is added until a point called the percolation threshold. At the percolation threshold the ER of the composite drops several orders of magnitude over a very narrow range of filler loadings. After this percolation threshold, the ER of the composite decreases at a much slower rate as it approaches the filler ER.

Carbon black (CB), carbon nanotubes (CNT) and graphene nanoplatelets (GNP) all exhibit percolation threshold behavior when added to polycarbonate (PC). Pure PC has an ER of about $10^{17} \mathrm{ohm}-\mathrm{cm}$. CNT has the largest effect on reducing the ER of PC composites. The percolation threshold for CNT in PC is 1.0 vol\%. The lowest ER measured for a CNT/PC formulation was 8 ohm-cm for $8 \mathrm{wt} \%$ (5.6 vol\%) CNT in PC. $\mathrm{CB}$ had the next largest effect on decreasing ER. The percolation threshold for CB was about $2.4 \mathrm{vol} \%$. The lowest ER measured for a CB/PC composite was $20 \mathrm{ohm}-\mathrm{cm}$ for 10 wt\% (6.9 vol\%) CB in PC. GNP had the least effect in decreasing the ER of PC composites. The GNP/PC system percolated at around $4.0 \mathrm{vol} \%$, this is significantly higher than either $\mathrm{CNT} / \mathrm{PC}$ or $\mathrm{CB} / \mathrm{PC}$. The lowest ER value measured for a GNP/PC composite was $2.8 \times 10^{4} \mathrm{ohm}-\mathrm{cm}$ for $15 \mathrm{wt} \%$ (9.6 vol\%) GNP in PC. 
When looking at two filler systems, the majority of the behavior of the systems is dominated by single filler effects. The $\mathrm{CB} / \mathrm{GNP}$ and $\mathrm{CNT} / \mathrm{GNP}$ formulations do not deviate from this behavior in any statistically significant way. The $\mathrm{CB} / \mathrm{CNT}$ composite does exhibit an interaction effect between the two fillers. Specifically, the $\mathrm{CB} / \mathrm{CNT}$ system shows a negative interaction in reducing the electrical resistivity of the resulting composite. This means that the ER of a composite containing both $\mathrm{CB}$ and CNT will have a higher ER than one would expect from just adding the ER effects of the individual fillers together.

\subsection{2: Thermal Conductivity (TC)}

Polymers are typically low thermal conductivity materials (compared to such materials as metals and carbons). Similarly to the electrical conductivity, the thermal conductivity of polymers can be increased by adding thermally conductive fillers to the polymer. Thermal conductivity deviates from electrical conductivity behavior by not being controlled by a percolation threshold and by the values for polymer and filler varying only a couple orders of magnitude, not 12 or more as electrical conductivity does.

$\mathrm{CB}, \mathrm{CNT}$ and GNP all increase the thermal conductivity of polycarbonate. Polycarbonate itself has a TC of $0.20 \mathrm{~W} / \mathrm{m} \cdot \mathrm{K}$. GNP has the largest effect on increasing the thermal conductivity of polycarbonate composites. At $15 \mathrm{wt} \%$ (9.6 vol\%) GNP in $\mathrm{PC}$, the composite has a TC of $0.489 \mathrm{~W} / \mathrm{m} \cdot \mathrm{K}$. At $5.0 \mathrm{vol} \%$ (8 $\mathrm{wt} \%$ ) GNP increases the TC of the resulting PC composite to $0.374 \mathrm{~W} / \mathrm{m} \cdot \mathrm{K}$. CNT has the next largest effect on increasing the TC of PC composites. At the highest CNT loading, 5.6 vol\% (8 wt $\%$ ), CNT increases the TC of the resulting PC composite to $0.306 \mathrm{~W} / \mathrm{m} \cdot \mathrm{K}$, somewhat less 
than the increase given by 5.0 vol\% of GNP. CB has the smallest effect on increasing the TC of PC composites. $5.46 \mathrm{vol} \%$ ( $8 \mathrm{wt} \%$ ) CB increases the TC of PC to $0.275 \mathrm{~W} / \mathrm{m} \cdot \mathrm{K}$. At the highest CB loading level, $10 \mathrm{wt} \%$ (6.9 vol\%), CB increases the TC of PC to 0.291 $\mathrm{W} / \mathrm{m} \cdot \mathrm{K}$.

When looking at the two filler systems studied in this project, the thermal conductivity behavior of the composites is dominated by the single filler effects of the individual fillers. The $\mathrm{CB} / \mathrm{CNT}$ and $\mathrm{CB} / \mathrm{GNP}$ systems do not show any statistically significant interaction effects between the fillers. The CNT/GNP system does show a statistically significant positive interaction between the fillers. This means that when a composite contains both CNT and GNP, the TC of the composite is higher than would be expected from adding the individual effects of the single fillers together.

\subsection{3: Tensile Properties}

Adding fillers to polymers will often change the tensile properties of a material. The addition of strong and stiff fillers, such as carbons, will typically increase the tensile modulus and, to a lesser degree, tensile strength of the resulting composite.

$\mathrm{CB}, \mathrm{CNT}$ and GNP all increase the tensile modulus of PC composites. All three fillers have little effect on the tensile strength of the composite. Neat PC has a tensile modulus of about 2.2 GPa. GNP has the largest effect on the tensile modulus of the composite, increasing the tensile modulus of the resulting composite to $5.9 \mathrm{GPa}$ for the highest filled composite, $15 \mathrm{wt} \%$ (9.6 vol\%) GNP in PC. When the GNP loading is $5.0 \mathrm{vol} \%$ (8 $\mathrm{wt} \%$ ) the tensile modulus is $3.5 \mathrm{GPa}$. CNT and $\mathrm{CB}$ have similar effects on increasing the tensile modulus of PC composites. At $5.6 \mathrm{vol} \%(8 \mathrm{wt} \%) \mathrm{CNT}$, the tensile modulus of 
the PC composite is increased to $2.9 \mathrm{GPa}$. At $5.5 \mathrm{vol} \%(8 \mathrm{wt} \%) \mathrm{CB}$, the tensile modulus of the PC composite is increased to $2.8 \mathrm{GPa}$.

The two filler systems studied here all showed statistically significant interactions between the fillers in the composite. The $\mathrm{CB} / \mathrm{CNT}$ system showed each filler increasing the tensile modulus of the system with diminishing effects as more of the individual filler was added. Additionally, there was a positive interaction effect between the CB and CNT. This means that a composite containing both $\mathrm{CB}$ and $\mathrm{CNT}$ would have a tensile modulus that would be higher than would be expected from adding the individual effects of the single fillers together. The $\mathrm{CB} / \mathrm{GNP}$ system showed each filler increasing the tensile modulus of the filler as more of the filler was added. The CB/GNP system also showed a statistically significant positive interaction effect between the CB and GNP. This means that a composite containing both CB and GNP would have a tensile modulus that would be higher than would be expected from adding the individual effects of the single fillers together. The CNT/GNP system showed each filler increasing the tensile modulus of the composite with CNT exhibiting a diminishing effect as more CNT is added. The CNT/GNP system also displayed a statistically significant negative interaction between CNT and GNP. This means that a composite containing both CNT and GNP would have a tensile modulus that would be lower than would be expected from adding the individual effects of the single fillers together. 


\subsection{4: Flexural Properties}

Adding fillers to polymers will often change the flexural properties of a material. The addition of strong and stiff fillers, such as carbons, will typically increase the flexural modulus and, to a lesser degree, flexural strength of the resulting composite.

$\mathrm{CB}, \mathrm{CNT}$ and GNP all increase the flexural modulus of PC composites. All three fillers have little effect on the flexural strength of the composite. Neat PC has a flexural modulus of about 2.6 GPa. GNP has the largest effect on the flexural modulus of the composite at high loadings, increasing the flexural modulus of the resulting composite to 6.4 GPa for the highest filled composite, $15 \mathrm{wt} \%$ (9.6 vol\%) GNP in PC. When the GNP loading is $5.0 \mathrm{vol} \%(8 \mathrm{wt} \%)$ the flexural modulus is similar to that of CNT with a value of 3.6 GPa. CNT has the second largest effect on increasing the tensile modulus of PC composites. At $5.6 \mathrm{vol} \%(8 \mathrm{wt} \%) \mathrm{CNT}$, the tensile modulus of the PC composite is increased to $3.6 \mathrm{GPa}$. $\mathrm{CB}$ has the least effect on increasing the flexural modulus of Pc composites. At $5.5 \mathrm{vol} \%$ ( $8 \mathrm{wt} \%$ ) $\mathrm{CB}$, the tensile modulus of the $\mathrm{PC}$ composite is increased to 3.4 GPa, slightly less than the value for GNP or CNT.

The two filler composites all show the individual fillers increasing the flexural modulus of the resulting composites. The $\mathrm{CB} / \mathrm{CNT}$ system shows $\mathrm{CB}$ having a diminishing effect as more $\mathrm{CB}$ is added. Additionally, the $\mathrm{CB} / \mathrm{CNT}$ system exhibits a statistically significant positive interaction between $\mathrm{CB}$ and CNT. This means that a composite containing both $\mathrm{CB}$ and $\mathrm{CNT}$ would have a flexural modulus that would be higher than would be expected from adding the individual effects of the single fillers together. The $\mathrm{CB} / \mathrm{GNP}$ system shows GNP having a diminishing effect on flexural modulus as 
additional GNP is added to the composite. This system shows no statistically significant interaction effects. The CNT/GNP system shows GNP having a diminishing effect on flexural modulus as additional GNP is added. There is also a statistically significant positive interaction effect between the CNT and GNP. This means that a composite containing both CNT and GNP would have a flexural modulus that would be higher than would be expected from adding the individual effects of the single fillers together.

\subsection{5: Rheological Properties}

Pure polycarbonate shows very common shear-thinning polymeric behavior. As the shear rate applied to a polycarbonate sample increases, the viscosity of the sample decreases. As the shear rate decreases, the value of the viscosity levels out at a value known as the Newtonian zero-shear plateau. The addition of carbon fillers to a polymer typically increases the viscosity of the resulting composite. This increase in viscosity typically increases with increased filler loading. It is also common at low shear rates for carbon filled systems to exhibit a yield stress, seen on a $\log$ (stress) versus $\log ($ strain) curve as a straight line with a negative 1 slope.

$\mathrm{CB}$ and GNP show classic carbon filler behavior in PC. Both fillers increase the viscosity of the composite and exhibit yield stress behavior at low shear rates. $\mathrm{CB}$ has a larger effect on increasing the viscosity of the composite than GNP. CNT also shows a yield stress at low shear rates. At higher shear rates, CNT does not exhibit the expected carbon filler behavior. Instead, CNT reduces the viscosity of the resulting composite below the viscosity of the pure PC. This effect increases in strength with increased CNT 
loading up to about $4 \mathrm{wt} \% \mathrm{CNT}$ where the effect saturates and additional CNT does not change the high shear rate behavior of the material.

$\mathrm{CB}$ and GNP continue to act as expected in two filler systems, increasing the viscosity of the resulting composites, with larger increases for larger filler loadings. The CB/GNP system shows that both fillers act as expected for carbon fillers. The viscosity increasing effects are additive. When comparing the formulations within the $\mathrm{CB} / \mathrm{GNP}$ system, it can be determined that $\mathrm{CB}$ has a larger effect on increasing the viscosity than GNP as described in Chapter 13. CNT in two filler systems acted similarly to its behavior as a single filler. In the GNP/PC system, we can see that as little as $1 \mathrm{wt} \% \mathrm{CNT}$ can reduce the viscosity of a $2 \mathrm{wt} \%$ GNP in PC composite to below the level of the original pure PC. At high shear rates $1 \mathrm{wt} \% \mathrm{CNT}$ can even negate the effect of up to $5 \mathrm{wt} \%$ GNP bringing the viscosity of a $1 \mathrm{wt} \% \mathrm{CNT}, 5 \mathrm{wt} \% \mathrm{GNP}$ in $\mathrm{PC}$ composite down to the level of pure PC. In this system $5 \mathrm{wt} \% \mathrm{CNT}$ completely negates the effect of $5 \mathrm{wt} \%$ GNP bringing the viscosity of $5 \mathrm{wt} \% \mathrm{CNT}$ and $5 \mathrm{wt} \%$ GNP in PC to below the of the original PC for all shear rates above $10 \mathrm{~s}^{-1}$. In the $\mathrm{CB} / \mathrm{CNT}$ system, we again see CNT acting to reduce the viscosity of the composite. $1 \mathrm{wt} \% \mathrm{CNT}$ can bring the viscosity of a $2 \mathrm{wt} \% \mathrm{CB}$ in $\mathrm{PC}$ composite down below the viscosity of pure PC. Of particular note for this system, when $1 \mathrm{wt} \% \mathrm{CNT}$ is added to a $5 \mathrm{wt} \% \mathrm{CB}$ in PC composite, the resulting viscosity for all shear rates is essentially the same as $2 \mathrm{wt} \% \mathrm{CB}$ in PC. This means the addition of $1 \mathrm{wt} \% \mathrm{CNT}$ to a $2 \mathrm{wt} \% \mathrm{CB}$ in $\mathrm{PC}$ composite would allow for an additional $3 \mathrm{wt} \% \mathrm{CB}$ to be added with no change to the viscosity. 


\subsection{6: Electrical Conductivity and Tensile Modulus Modeling}

Electrical conductivity modeling for this project focused on applying the Mamunya model, additive model, and General Effective Media (GEM) model to the single filler systems. For all 3 systems the Mamunya model and additive model produced similar results. Both of these models generated significant amounts of error due to the assumption in both models that below the percolation threshold, the electrical conductivity of the composite is equal to that of the pure polymer. After the percolation threshold, both models have very good fits to the experimental data. The GEM model does not assume pure polymer electrical conductivity below the percolation threshold and was thus able to model this part of the data much better than the other two models. Above the percolation threshold the fit of the GEM model was inferior to the other two models. Overall the GEM model produced the best overall fit for all three filler systems.

Tensile modulus modeling for this project focused on applying the Halpin-Tsai models and Nielsen model to the single filler systems. Both of these models required information on the aspect ratio of the materials. In the Halpin-Tsai models, the aspect ratio is a direct input, for the Nielsen model, the aspect ratio is used to calculate the Einstein coefficient, a direct input to the model. Scanning and transmission electron microscopy of the materials showed that neither CNT nor GNP remain flat and straight in the composite. This led to ambiguity as to what the proper aspect ratio for these calculations would be. A solution was found using the previously mentioned electrical conductivity modeling. It is possible to estimate the percolation threshold of conductive fillers in a composite using an analytical model that models the fillers as simple cubic elements. Using the percolation threshold as determined from the GEM model for 
electrical conductivity, the percolation threshold models could be reworked to solve for an effective filler aspect ratio. This method was tested on the GNP/PC system as there was accurate data for the maximum packing fraction (another direct input for the Nielsen model). This effective aspect ratio, combined with a literature value for the modulus of exfoliation of graphite (the hypothesized primary failure method) produced a model that had very good agreement with the experimental data. This method was also used for the CNT/PC system to allow the 3-dimensional randomly oriented Halpin-Tsai model to fit the experimental data very well. The $\mathrm{CB} / \mathrm{PC}$ system did not require this modeling as the aspect ratio if assumed to be roughly 1 and the Einstein coefficient for spherical particles is assumed to be a constant. Overall, the percolation threshold modeling system allowed more accurate and meaningful tensile modulus modeling than has been previously seen in literature. The Halpin-Tsai model provided the best fit for CNT/PC while the Nielsen model provided the best fit for $\mathrm{CB} / \mathrm{PC}$ and GNP/PC.

\section{2: Recommendations for Future Work}

The future of this research project should focus on extending the discoveries made therein to other systems. Primarily, the viscosity reducing effects of CNT in PC, and the electrical percolation threshold modeling approach to tensile modulus modeling, need to be extended to additional systems.

In this project we have shown that the addition CNT can reduce the viscosity of PC, especially in the high shear rate domains that are consistent with extrusion and injection

molding. We have also shown that CNT can be added to PC composites that already 
have a different carbon filler to reduce the viscosity. This allows for additional amounts of the other carbon filler to be added to the composite without harming processability. Previous work with the same CNT in polypropylene (PP) did not exhibit this behavior. In CNT/PP systems, the CNT acted as a normal carbon filler to increase the viscosity of the composite. Experimentation needs to be done to determine what causes this different behavior. Looking at the structure of PC versus PP, PC contains benzene rings that are located in the backbone, as well as oxygen atoms both in the backbone, and carbonyl oxygen off the backbone while PP is fully aliphatic. This research should focus on materials that have one of these various differences to determine which of them cause the change.

Polyacrylonitrile is identical to PP except the methyl pendants are replaced with nitrile groups. If CNT reduced the viscosity of polyacrylonitrile systems, it would suggest the presence of hetero atoms in the structure cause the viscosity reduction effect.

Poly(p-xylene) contains benzene rings as a backbone element. If CNT reduced the viscosity of poly(p-xylene) systems, it would suggest the presence of benzene rings in the polymer backbone cause the viscosity reduction effect.

Polyformaldehyde contains oxygen as a backbone element. If CNT reduced the viscosity of polyformaldehyde systems, it would suggest the presence of oxygen in the polymer backbone cause the viscosity reduction effect.

Poly(phenylene oxide) has the combination of oxygen and benzene rings in the polymer backbone. If CNT reduced the viscosity of poly(phenylene oxide) systems, it would 
suggest the presence of both oxygen and benzene rings in the polymer backbone cause the viscosity reduction effect.

There are dozens of combinations and countless types of polymers that could be tested for this behavior. These are just examples of materials that could be used as part of a Design of Experiments approach to test the various differences between PC and PP to determine how the CNT interacts with the polymer matrix to reduce the viscosity of the composite.

Additionally, it is important to test other fillers to determine their compatibility with the CNT viscosity reduction effect. In this project we have shown that the effect is compatibile with nanoscale carbon fillers, particularly flake-like and spherical fillers. There are other geometries and materials that it is important to test.

Carbon nanofibers (CNF) are nanoscale rod-like fillers that would round out the knowledge of the geometries of carbon nanofillers as the CB is roughly zero dimensional, the GNP is roughly two dimensional and the carbon nanofibers are roughly one dimensional.

In addition to nanofillers, other carbon fillers should be tested. Carbon fiber is a very common reinforcement material for polymers. It is a rod-like filler like CNT or CNF, but it is on the micrometer scale. The size of the filler might change the viscosity reducing effects of CNT. 
Synthetic graphite particles are rough irregular particles. They are roughly spherical, but may have aspect ratios up to 5 . These particles are roughly 10 to 100 microns in size and again probe the interactions between CNT and larger particles.

It is possible that the CNT viscosity reduction effect could be compatible with other materials as well. Clay nanoplatelets represent a non-carbon version of the GNP studied in this project. Mica flakes represent a micrometer scale, non-carbon version of the GNP. Experimentation with both of these materials would help understand the compatibility of the CNT viscosity reduction effect.

There are other nanotubes that may exhibit this same viscosity reducing behavior. Boron nitride nanotubes in particular are quite similar to CNT in size and geometry. Experimentation with these nanotubes would help determine whether the effect is inherent to the CNT themselves, or their geometry and size.

The use of percolation threshold modeling to determine an effective aspect ratio for fillers within the composite was found to be very useful for this project. Testing the method on other systems would allow for the model to be used more universally. It would be most helpful to test the percolation threshold modeling method with other polymers and other fillers.

Similarly to the recommendations given for the CNT effect, this new tensile modeling tool should be applied to other polymer matrices. Specifically, it would be most beneficial to test it with polymer systems that are very dissimilar to polycarbonate. Polyethylene or polypropylene represent aliphatic polymers that may behave much differently than polycarbonate that contains benzene rings and oxygen atoms. 203 
Polyimides/polyamides and polysulfones contain other kinds of hetero atoms (specifically nitrogen and sulfur respectively) and could behave differently than polycarbonate. Poly(p-xylene) has benzene rings but no atoms other than carbon and hydrogen and would help bridge the gap between the behavior of the aliphatic polymers and polycarbonate. Poly(p-xylene) would also help determine whether unsaturation or hetero atoms cause any behavior difference between the polymers.

It is also important to look at different fillers when applying this tensile modulus method. Particles that are larger sized, like synthetic graphite particles or carbon fiber, may behave differently than was seen in carbon nanoparticles in polycarbonate. Also, noncarbon materials that are both nanosized, such as clay nanoplatelets and boron-nitride nanotubes, and larger, such as calcium carbonate or fiberglass, may behave differently than carbon-based materials. It is also important to test this variety of different fillers in the different polymer systems.

As the percolation threshold modeling modification to tensile modulus modeling method is validated in other systems, it can become a useful tool for tensile modeling. 


\section{Chapter 15: References}

1 Wright WM, Woodham GW. Conductive Polymers and Plastics. New York: Chapman and Hall; 1989.

2 Finan JM. Thermally conductive thermoplastic materials. In: Proceedings of the Society of Plastics Engineers Annual Technical Conference; 1999; New York. p. 1547-1550.

3 Taipalus T, Harmia M, Zhang Q, Friedrich K. The electrical conductivity of carbonfibre-reinforced polypropylene/polyaniline complex-blends: experimental characterisation and modelling. Composites Science and Technology. 2001;61(6):801814.

4 Agari Y, Uno T. Thermal-conductivity of polymer filled with carbon materials- effect of confuctive particle chains on thermal-conductivity. Journal of Applied Polymer Science. 1985;30(5):2225-2236.

5 Bigg DM. Conductive polymeric compositions. Polymer Engineering and Science. 1977;30(12):842-847.

6 Bigg DM. The effect of compounding on the conductive properties of EMI shielding compounds. Advanced Polymer Technology. 1984;4(3-4):255-266.

7 Narkis M, Lodor G, Vaxman A, Zuri J. New injection moldable electrostatic dissipative (ESD) composites based on very low carbon black loadings. Journal of Electrostatics. 1999;47(4):201-214.

8 Nagata K, Iwabuji H, Nigo H. Effect of particle size of graphites on electrical conductivity of graphite/polymer composite. Composite Interfaces. 1998;6(5):483495.

9 Demain A. Thermal Conductivity of Polymer-Chopped Carbon Fibre Composites. Louvain-la-Neuve, Belgium: Universite Catholique de Louvain; 1994.

10 King JA, Tucker KW, Meyers JD, Weber EH, Clingerman ML, Ambrosius KR. Factorial design approach applied to electrically and thermally conductive nylon 6,6. Polymer Composites. 2001;22(1):142-154. 
11 Murthy MV. Permanent EMI shielding of plastics using copper fibers. In: Proceedings of the Society of Plastics Engineers Annual Technical Conference; 1994; San Francisco. p. 1396-1401.

12 Simon RM. Thermally and electrically conductive flake filled plastics. Polymer News. 1985;11:102-108.

13 Mapleston P. Conductive composites get a growth boost from metallic fibers. Modern Plastics. 1992;69:80-83.

14 Donnet JB, Bansal RC, Wang MJ. Carbon Black. New York: Marcel Dekker; 1993.

15 Huang JC. Carbon black filled conducting polymers and polymer blends. Advances in Polymer Technology. 2002;21(4):299-313.

16 Bigg DM. The effect of chemical-exposure on the EMI shielding of conductive plastics. Polymer Composites. 1987;8(1):1-7.

17 King J, Johnson B, Via M, Ciarkowski C. Effects of carbon fillers in thermally conductive polypropylene based resins. Polymer Composites. 2010;31(3):497-506.

18 Chung DDL. Composite Materials: Science and Applications (Engineering Materials and Processes). New York: Springer; 2010.

19 King JA, Via MD, Caspary JA, Jubinski MM, Miskioglu I, Mills OP, Bogucki GR. Electrical and thermal conductivity and tensile and flexural properties of carbon nanotube/polycarbonate resins. Journal of Applied Polymer Science. 2010;118(5):2512-2520.

20 Fried JR. Polymer Science and Technology. Upper Saddle River, New Jersey: Prentice Hall; 2003.

21 Boeing 787 Dreamlinber Long-Range, Mid-Size Airliner, USA. [Internet]. [cited 2007 September 10]. Available from: http://www.aerospacetechnology.com/projects/dreamliner.

22 Bigg DM. Metal Filler Polymers: Properties and Applications. New York: Marcel Dekker Inc.; 1986. 
23 Clingerman ML, Weber EH, King JA, Schulz KH. Development of an additive equation for predicting the electrical conductivity of carbon-filled composites. Journal of Applied Polymer Science. 2003;88(9):2280-2299.

24 Broadbent SR, Hammersley JM. Percolation processes: I. Crystals and mazes. Proceedings of the Cambridge Philosophical Society. 1957;53:629-641.

25 Stauffer D. Introduction of Percolation Theory. London: Taylor and Francis; 1985.

26 Blunk RH, Lisi DJ, Yoo Y, Tucker III CL. Enhanced conductivity of fuel cell plates through controlled fiber orientation. AIChE Journal. 2003;49(1):18-29.

27 Ruschau GR, Newnham RE. Critical volume fractions in conductive composites. Journal of Composite Materials. 1992;26(18):2727-2735.

28 Bird RB, Stewart WE, Lightfoot EN. Transport Phenomena. 2nd ed. New York: Wiley; 2002.

29 Parrott JE, Stuckes AD. Thermal Conductivity of Solids. London: Pion Limited; 1975.

30 Bigg DM. Thermally conductive polymer compositions. Polymer Composites. 1986;7(3):125-140.

31 Hauser RA. Synergistic Effects and Modeling of Thermally Conductive Resins for Fuel Cell Bipolar Plate Applications. Houghton: Michigan Technological University; 2008.

32 Berman R. Thermal Conduction in Solids. Oxford: Clarendon Press; 1976.

33 Hone J, Llaguno MC, Nemes NM, Johnson AT, Fischer JE, Walters DA, Casavant MJ, Schmidt J, Smalley RE. Electrical and thermal transport properties of magnetically aligned single walt carbon nanotube films. Applied Physics Letters. 2000;77(5):666-668.

34 King JA, Johnson BA, Via MD, Ciarkowski CJ. Electrical conductivity of carbonfilled polypropylene-based resins. Journal of Applied Polymer Science. 2009;112(1):425-433. 
35 King JA, Miller MG, Barton RL, Keith JM, Hauser RA, Peterson KR, Sutter LL. Thermal and electrical conductivity of carbon-filled liquid crystal polymer composites. Journal of Applied Polymer Science. 2006;99(4):1552-1558.

36 Agari Y, Ueda A, Nagai S. Thermal-conductivities of composites in several types of dispersion-systems. Journal of Applied Polymer Science. 1991;42(6):1665-1669.

37 Gaxiola DL, Jubinski MM, Keith JM, King JA, Miskioglu I. Effects of carbon fillers on tensile and flexural properties in polypropylene-based resins. Journal of Applied Polymer Science. 2010;118(3):1620-1633.

38 Marsh H, Rodriguez-Reinoso F. Sciences of Carbon Materials. San Vincente del Raspeig, Alicante, Spain: Universidad de Alicante; 2001.

39 Gupta M, Wang KK. Fiber orientation and mechanical-properties of short-fiberreinforced injection-molded composites - Simulated and experimental results. Polymer Composites. 1993;14(5):367-382.

40 Ludwig HC, Fischer G, Becker H. A quantitative comparison of morphology and fibre orientation in push-pull processed and conventional injection-moulded parts. Composites Science and Technology. 1995;53(2):235-239.

41 Ryu SR, Lee DJ. Effects of fiber aspect ratio, fiber content, and bonding agent on tensile and tear properties of short-fiber reinforced rubber. KSME International Journal. 2001;15(1):35-43.

42 Sabic Innovative Plastics. PC Resin Product Brochure: Sabic-PLA-650. One Plastics Avenue, Pittsfield, MA, 012012008.

43 Hyperion Catalysis International. Fibrils Product Information. 38 Smith Place, Cambridge, MA 02138.

44 Spinelli F. Plastics, Additives, and Modifiers Handbook. New York: Chapman and Hall; 1996.

45 Burgess KA, Lyon F. Encyclopedia of Polymer Science and Technology. New York: John Wiley and Sons Inc.; 1985.

46 Akzo Nobel. Ketjenblack EC Product Brochure. Chicago, IL.

47 Kalaitzidou K, Fukushima H, Drzal LT. Mechanical properties and morphological 
characterization of exfoliated graphite-polypropylene nanocomposites. Composites: Part A. 2007;38(7):1675-1682.

48 Ovation Polymers, Inc. Extima GP MB PC 1530A Data Sheet. 1030 West Smith Rd, Medina, $\mathrm{OH}, 44256$.

49 American Society for Testing and Materials. ASTM D792-98: Standard Test Methods for Density and Specific Gravity (Relative Density) of Plastics by Displacement. 1998.

50 American Society for Testing and Materials. ASTM D3835-96: Standard Test Method for Determination of Properties of Polymeric Materials by Means of a Capillary Rheometer. 1996.

51 Morrison FA. Understanding Rheology. New York: Oxford University Press; 2001.

52 International Organization for Standardization. ISO 6721-99: Plastics -- Determination of dynamic mechanical properties -- Part 10: Complex shear viscosity using a parallelplate oscillatory rheometer. 1999.

53 Cox WP, Merz EH. Correlation of dynamic and steady flow viscosities. Journal of Polymer Science. 1958;28(118):619-622.

54 American Society for Testing and Materials. ASTM D257-99: Standard Test Methods for DC Resistance or Conductance of Insulating Materials. 1999.

55 American Society for Testing and Materials. ASTM D4496-04: Standard Test Method for D-C Resistance or Conductance of Moderately Conductive Materials. 2004.

56 American Society for Testing and Materials. ASTM F433-98: Standard Practice for Evaluating Thermal Conductivity of Gasket Materials. 1998.

57 Holometrix Inc. TCA 300 Operators Manual. Burlington, MA: Holometrix Inc.; 1994.

58 American Society for Testing and Materials. ASTM D638-03: Standard Test Method for Tensile Properties of Plastics. 2003.

59 American Society for Testing and Materials. ASTM D790-03: Standard Test Methods for Flexural Properties of Unreinforced and Reinforced Plastics and Electrical Insulating Materials. 2003. 
60 Weber M, Kamal MR. Estimation of the volume resistivity of electrically conductive composites. Polymer Composites. 1997;18(6):711-725.

61 Hornbostel B, Potschke P, Koz J, Roth S. Single-walled carbon nanotubes/polycarbonate composites: basic electrical and mechanical properties. Physica Status Solidi B-Basic Solid State Physics. 2006;243(13):3445-3451.

62 Potschke P, Bhattacharyya A, Janke A. Melt mixing of polycarbonate with multiwalled carbon nanotubes: microscopic studies on the state of dispersion. European Polymer Journal. 2004;40(1):137-148.

63 Potshcke P, Bhattacharyya A, Janke A. Carbon nanotube-filled polycarbonate composites produced by melt mixing and their use in blends with polyethylene. Carbon. 2004;42(5-6):965-969.

64 Pegel S, Potshke P, Petzold G, Alig I, Dudkin S, Lellinger D. Dispersion, agglomeration, and network formation of multiwalled carbon nanotubes in polycarbonate melts. Polymer. 2008;49(4):974-984.

65 Lee G, Lee J, Lee S, Park M, Kim J. Comparisons of thermal properties between inorganic filler and acid-treated multiwall nanotube/polymer composites. Journal of Materials Science. 2005;40(5):1259-1263.

66 Lopez Manchado M, Valentini L, Biagiotti J, Kenny J. Thermal and mechanical properties of single-walled carbon nanotubes-polypropylene composites prepared by melt processing. Carbon. 2005;43(7):1499-1505.

67 Fornes T, Baur J, Sabba Y, Thomas E. Morphology and properties of melt-spun polycarbonate fibers containing single- and multi-wall carbon nanotubes. Polymer. 2006;47(5):1704-1714.

68 Lee SH, Kim JH, Choi SH, Kim SY, Kim KW, Youn JR. Effects of filler geometry on internal structure and physical properties of polycarbonate composites prepared with various carbon fillers. Polymer International. 2009;58(4):354-361. 
69 Potschke P, Abdel-Goad M, Pegel S, Jehnichen D, Mark JE, Zhou D, Heinrich G. Comparisons among electrical and rheological properties of melt-mixed composites containing various carbon nanostructures. Journal of Macromolecular Science. 2010;47(1):12-19.

70 Tchoudakov R, Breuer O, Narkis M. Conductive polymer blends with low carbon black loading: Polypropylene/polycarbonate. Polymer Networks Blends. 1996;6(1):1.

71 Ezquerra TA, Martinez-Salazar J, Balta Calleja FJ. Percolation-threshold of conductive polycarbonate carbon composites as revealed by electron microscopy. Journal of Materials Science Letters. 1986;5(10):1065-1066.

72 Balta Calleja FJ, Ezquerra TA, Rueda DR. Conductive polycarbonate-carbon composites. Journal of Materials Science Letters. 1984;3(2):165-168.

73 King JA, Johnson BA, Via MD, Ciarkowski CJ. Effects of carbon fillers in thermally conductive polypropylene based resins. Polymer Composites. 2010;31(3):497-506.

74 Keith JM, King JA, Lenhart KM, Zimny B. Thermal conductivity models for carbon/liquid crystal polymer composites. Journal of Applied Polymer Science. 2007;105(6):3309-3316.

75 Huang JC, Muangchareon P, Grossman SC. Effects of carbon black on mechanical properties of polycarbonate-polypropylene blends. Journal of Polymer Engineering. 2000;20(2):111.

76 Bird RB, Armstrong RC, Hassager O. Dynamics of Polymeric Liquids. New York: Wiley; 1987.

77 King JA, Via MD, Keith JM, Morrison FA. Effects of carbon fillers on rheology of polypropylene-based resins. Journal of Composite Materials. 2009;43(25):3073-3089.

78 King JA, Morrison FA, Keith JM, Miller MG, Smith RC, Cruz M, Neuhalfen AM, Barton RL. Electrical conductivity and rheology of carbon-filled liquid crystal polymer composites. Journal of Applied Polymer Science. 2006;101(4):2680-2688. 
79 King JA, Tambling TM, Morrison FA, Keith JM, Cole AJ, Pagel RM. Effects of carbon fillers on the rheology of highly filled liquid-crystal polymer based resins. Journal of Applied Polymer Science. 2008;108(3):1646-1656.

80 Kunen E, Keith JM, Grant PW, King JA, Morrison FA. FEM calculations of capillary rheometer flow for carbon-filled liquid crystal polymer composites. Journal of Applied Polymer Science. 2007;106(1):433-438.

81 King JA, Tambling TM, Keith JM, Cole AJ, Morrison FA. Synergistic effects of multiple carbon fillers on the rheology of liquid crystal polymer based resins. Polymer Composites. 2009;30(1):111-119.

82 Shenvoy AV. Rheology of Filled Polymer Systems. Boston: Klewer Academic Publishers; 1999.

83 Kalaitzidou K, Fukushima H, Drzal LT. A new compounding method for exfoliated graphite-polypropylene nanocomposites with enhanced flexural properties and lower percolation threshold. Composites Science and Technology. 2007;67(10):2045-2051.

84 Fukushima H, Drzal LT, Rook BP, Rich MJ. Thermal conductivity of exfoliated graphite nanocomposites. Journal of Thermal Analysis and Calorimetry. 2006;85(1):235-238.

85 Kalaitzidou K, Fukushima H, Miyagawa H, Drzal LT. Flexural and tensile moduli of polypropylene nanocomposites and comparison of expermental data to Halpin-Tsai and Tandon-Wang models. Polymer Engineering and Science. 2007;47(11):17961803.

86 Maron SH, Pierce PE. Application of ree-eyring generalized flow theory to suspensions of spherical particles. Journal of Colloid Science. 1956;11(1):80-95.

87 Fisa B, Vu-Khanh T, Remillard B. Extrusion of mica filled polypropylene. Journal of Thermoplastic Composite Materials. 1988;1(4):361-370.

88 Kitano T, Kataoka T, Shirota T. An empirical-equation of the relative viscosity of polymer melts filled with various inorganic fillers. Rheologica Acta. 1981;20(2):207209. 
89 Mamunya EP, Davidenko VV, Lebedev EV. Effect of polymer-filler interface interactions on percolation conductivity of thermoplastics filled with carbon black. Composite Interfaces. 1996;4(4):169-176.

90 Keith JM, King JA, Barton RL. Electrical conductivity modeling of carbon-filled liquid-crystalline polymer composites. Journal of Applied Polymer Science. 2006;102(4):3293-3300.

91 McLachlan DS, Newman RE. Electrical resistivity of composites. Journal of the Americal Ceramic Society. 1990;73(8):2187-2203.

92 McLachlan DS. Equation for the conductivity of metal-insulator mixtures. Journal of Physics Part C: Solid State Physics. 1985;18(9):1891-1898.

93 McLachlan DS. A new interpretation of percolation conductivity results with large critical regimes. Solid State Communications. 1986;60(10):821-825.

94 Barton RL, Keith JM, King JA. Development and modeling of electrically conductive carbon filled liquid crystal polymer composites for fuel cell bipolar plate applications. Journal of New Materials for Electrochemical Systems. 2007;10(4):225-229.

95 Barton RL, Keith JM, King JA. Electrical conductivity modeling of multiple carbon fillers in liquid crystal polymer composites for fuel cell bipolar plate applications. Journal of New Materials for Electrochemical Systems. 2008;11(3):181-186.

96 Dai H, Wong EW, Lieber CM. Probing electrical transport in nanomaterials: Conductivity of individual carbon nanotubes. Science. 1996;272(5261):523-526.

97 Thess A, Lee R, Nikolaev P, Dai H, Petit P, Robert J, Xu C, Lee YH, Kim SG, Rinzler AG, et al. Crystalline ropes of metallic carbon nanotubes. Science. 1996;273(5274):483-487.

98 Grunlan JC, Liu L, Kim YS. Tunable single-walled carbon nanotube microstructure in the liquid and solid states using poly(acrylic acid). Nano Letters. 2006;6(5):911915.

99 XG Sciences Inc. xGnP Brand Graphene Nanoplatelets Product Information. East Lansing, Michigan: XG Sciences Inc.; 2010.

100 Keith JM, King JA, Johnson BA. Electrical conductivity modeling of carbon filled 
polypropylene based resins for fuel cell bipolar plate applications. Journal of New Materials for Electrochemical Systems. 2008;11(4):253-257.

101 Li J, Ma PC, Chow WS, To CK, Tang BZ, Kim JK. Correlations between percolation threshold, dispersion state, and aspect ratio of carbon nanotubes. Advanced Functional Materials. 2007;17(16):3207-3215.

$102 \mathrm{Li} \mathrm{J}$, Kim JK. Percolation threshold of conducting polymer composites containing 3D randomly distributed graphite nanoplatelets. Composites Science and Technology. 2007;67(10):2114-2120.

103 Thostenson ET, Chou TW. On the elastic properties of carbon nanotube-based composites: modelling and characterization. Journal of Physics Part D: Apllied Physics. 2003;36(5):573-582.

104 Gojny FH, Wichmann MHG, Kopke U, Fiedler B, Schulte K. Carbon nanotubereinforced epoxy-compo sites: enhanced stiffness and fracture toughness at low nanotube content. Composites Science and Technology. 2004;64(15):2363-2371.

105 Peeterbroeck S, Breugelmans L, Alandre M, Nagy JB, Viville P, Lazzaroni R, Dubois $\mathrm{P}$. The influence of the matrix polarity on the morphology and properties of ethylene vinyl acetate copolymers-carbon nanotube nanocomposites. Composites Science and Technology. 2007;67(7-8):1659-1665.

106 Nielsen LE. Polymer Rheology. New York: Marcel Dekker Inc.; 1977.

107 Rao YQ, Pochan JM. Mechanics of polymer-clay nanocomposites. Macromolecules. 2007;40(2):290-296.

108 Nielsen LE. The thermal and electrical conductivity of two-phase systems. Industrial and Engineering Chemistry Fundamentals. 1974;13(1):17-20.

109 Nielsen LE, Landel RF. Mechanical Properties of Polymers and Composites. New York: Marcel Dekker Inc.; 1994.

110 Nielsen LE. Generalized equation for the elastic moduli of composite materials. Journal of Applied Physics. 1970;41(11):4626-4627. 
111 Halpin JC, Kardos JL. The Halpin-Tsai equations: A review. Polymer Engineering and Science. 1976;16(5):344-352.

112 Agarwal BD, Broutman LJ. Analysis and Performance of Fiber Composites. New York: Wiley; 1980.

113 Mallick PK. Composites Engineering Handbook. New York: Marcel Dekker Inc.; 1997.

114 Halpin JC. Stiffness and expansion estimates for oriented short fiber composites. Journal of Composite Materials. 1969;3(4):732-734.

115 Salvetat JP, Kulik AJ, Bonard JM, Briggs GAD, Stockli T, Metenier K, Bonnamy S, Beguin F, Bunham NA, Forro L. Elastic modulus of ordered and disordered multiwalled carbon nanotubes. Advanced Materials. 1999;11(2):161-165.

116 Lu JP. Elastic properties of carbon nanotubes and nanoropes. Physical Review Letters. 1997;79(7):1297-1300.

117 Montgomery DC. Design and Analysis of Experiments. New York: John Wiley and Sons Inc.; 2001. 


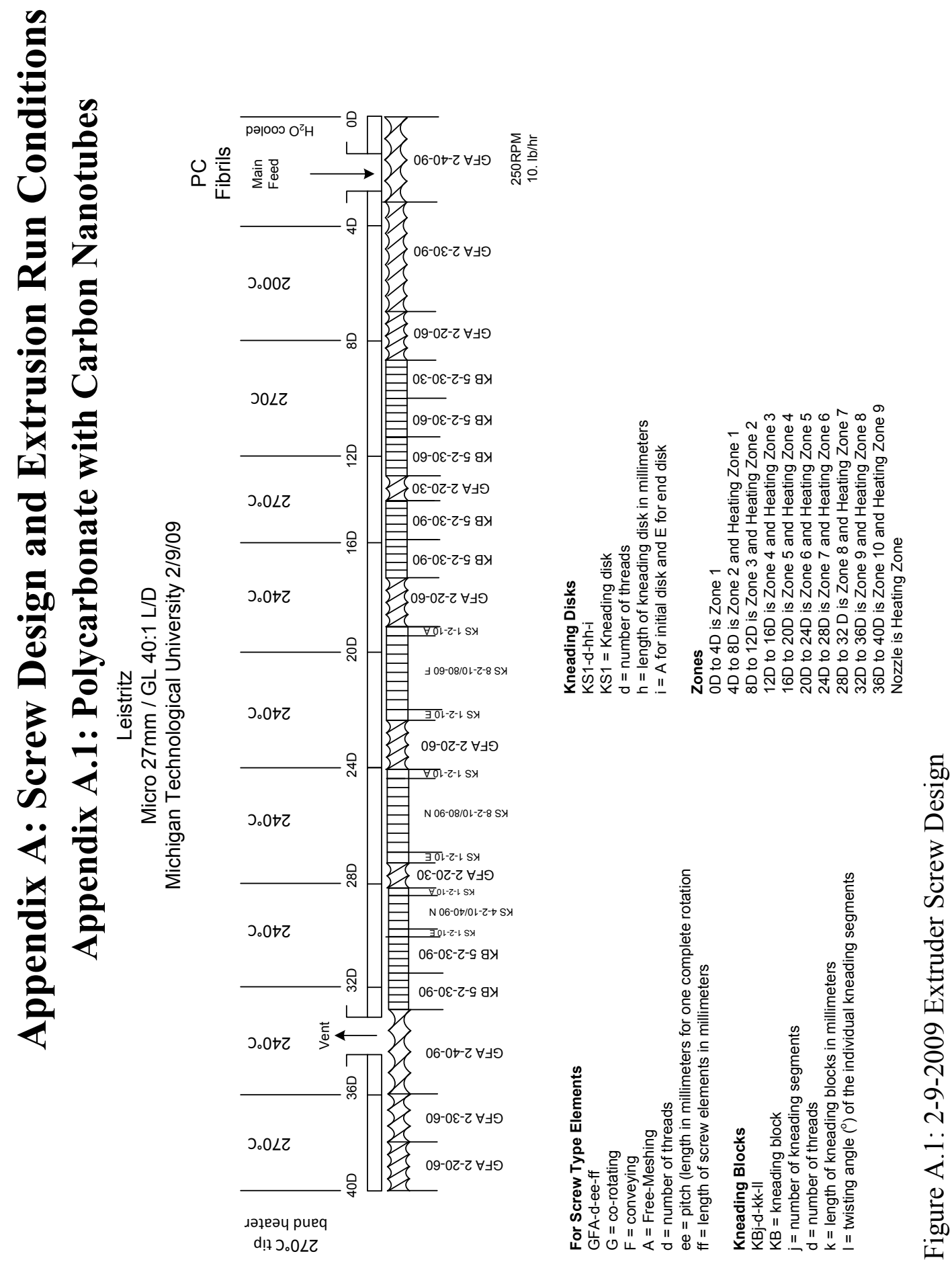




$$
\mid
$$




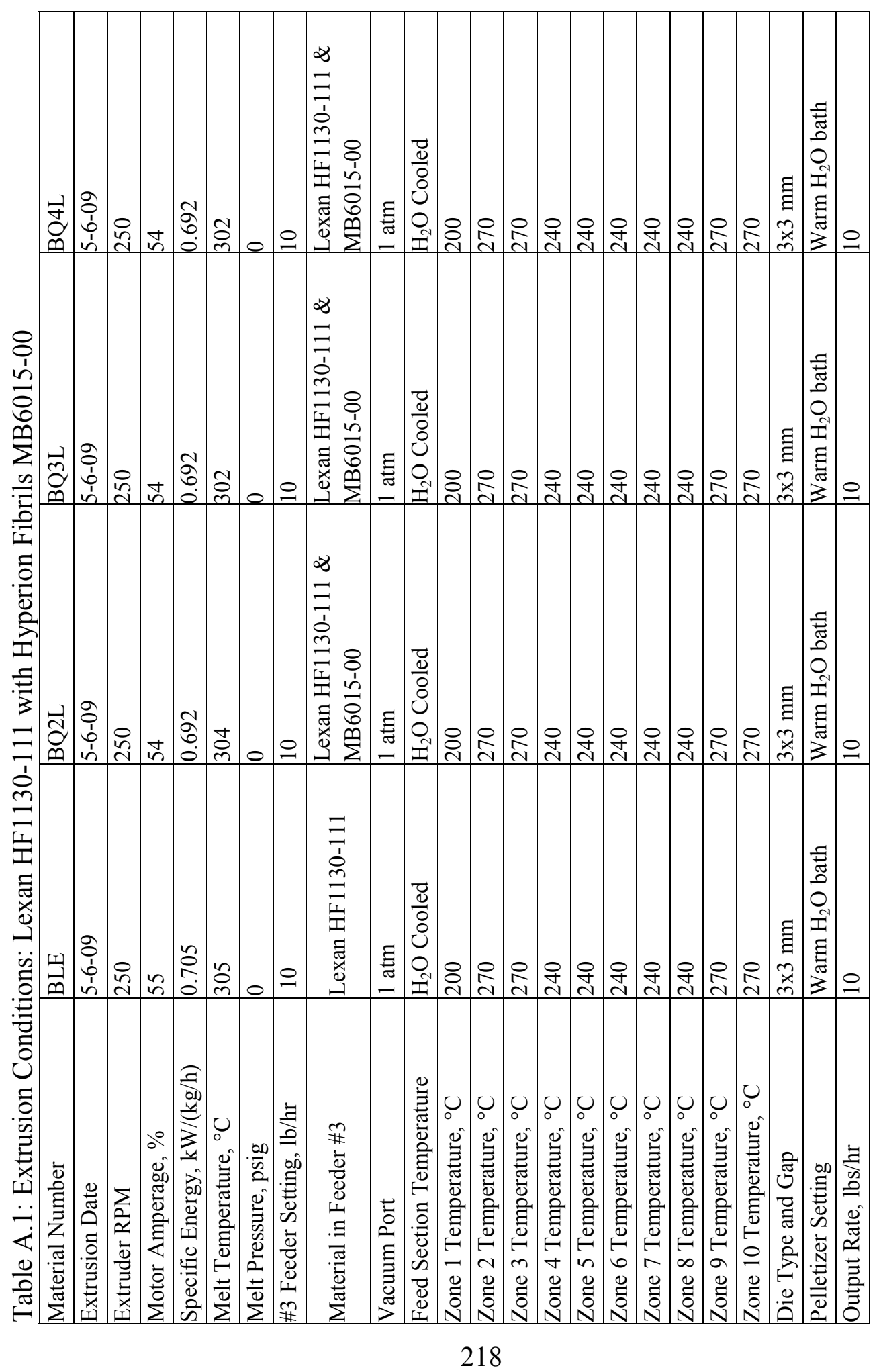




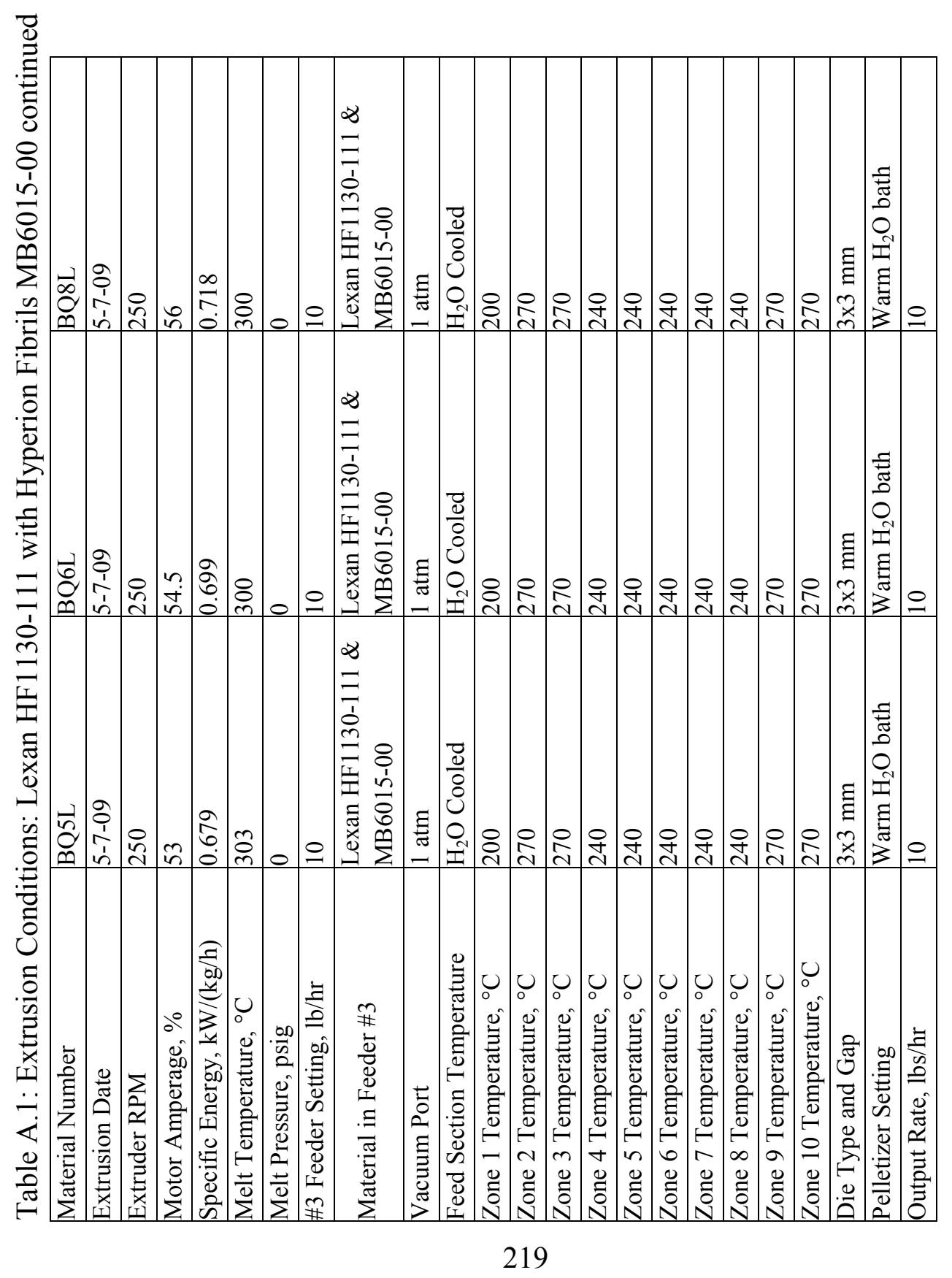




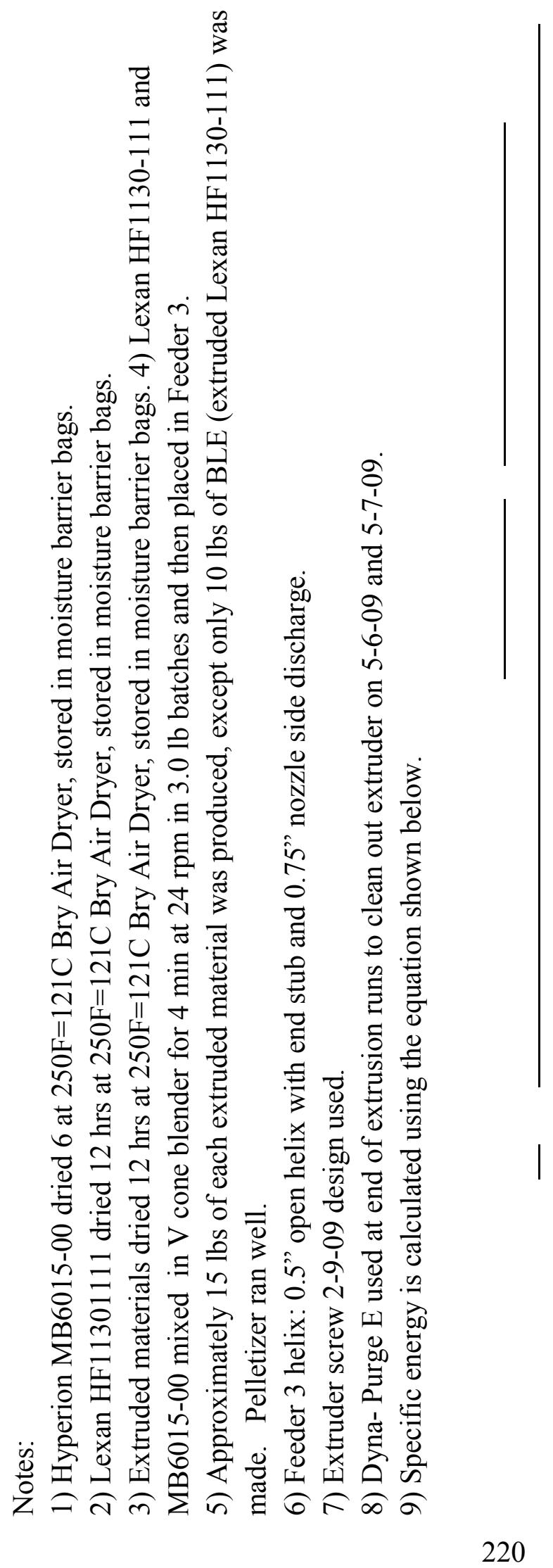



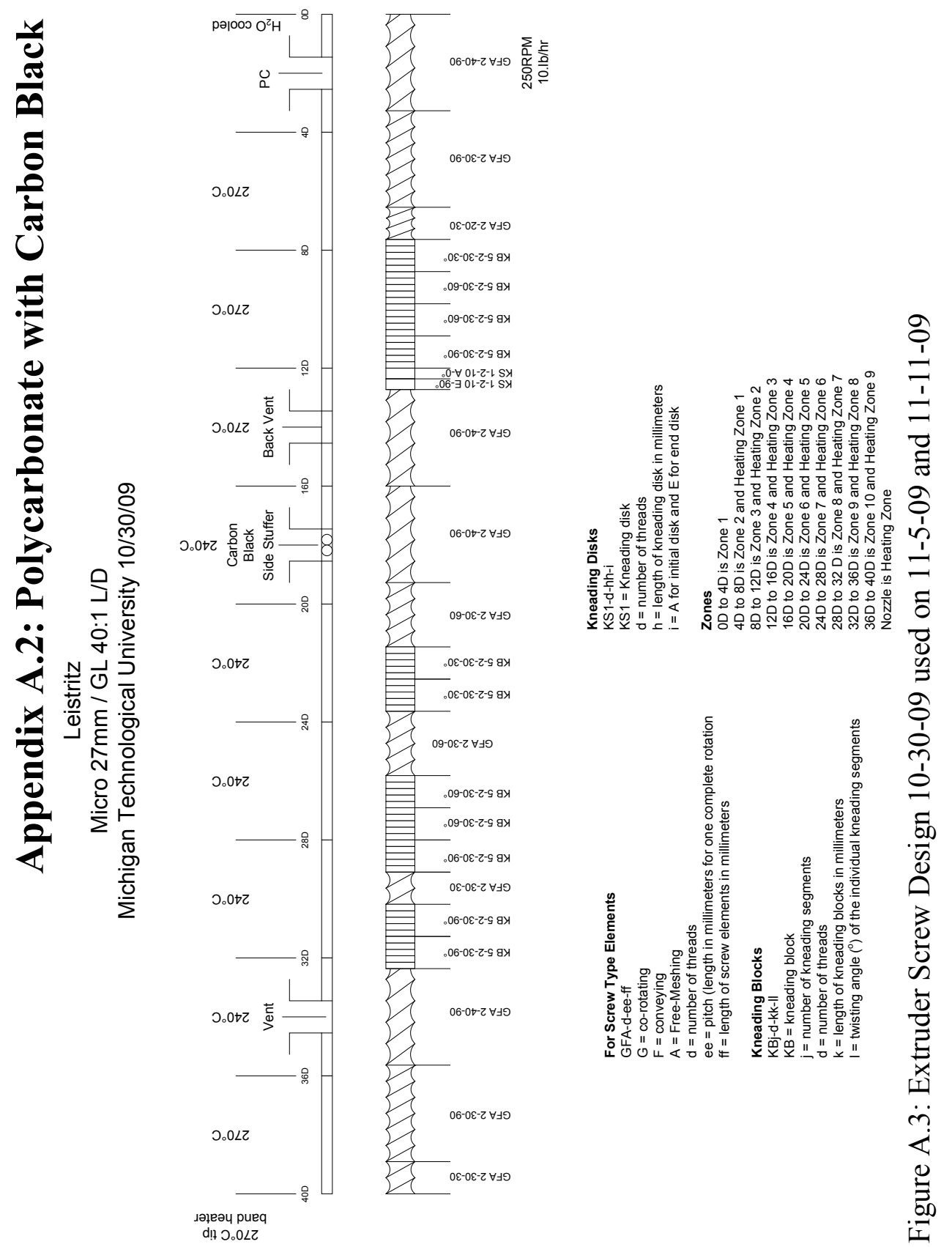


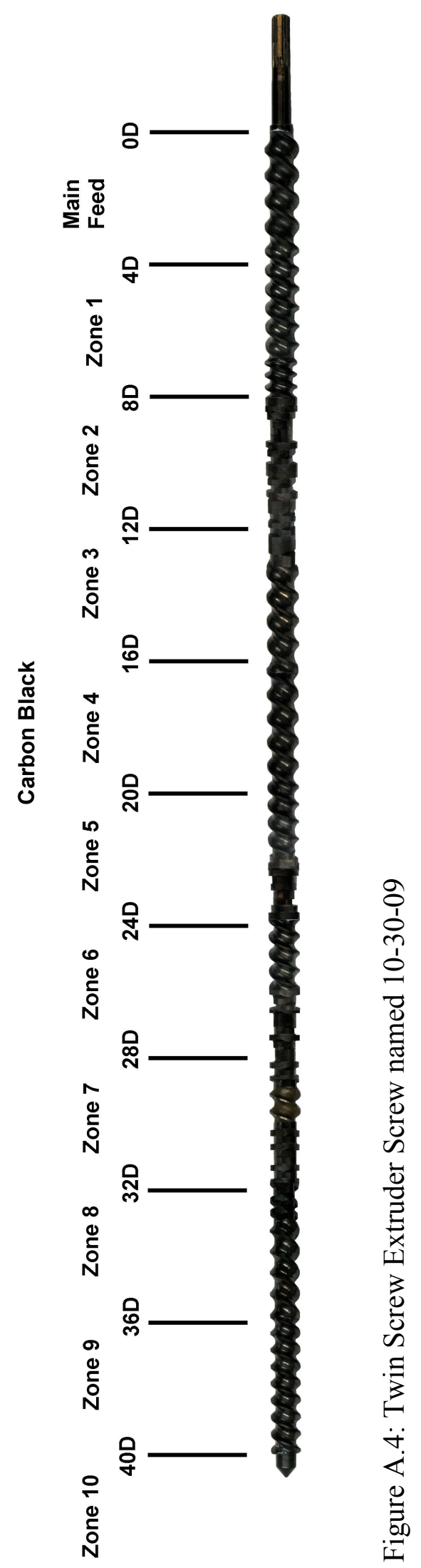




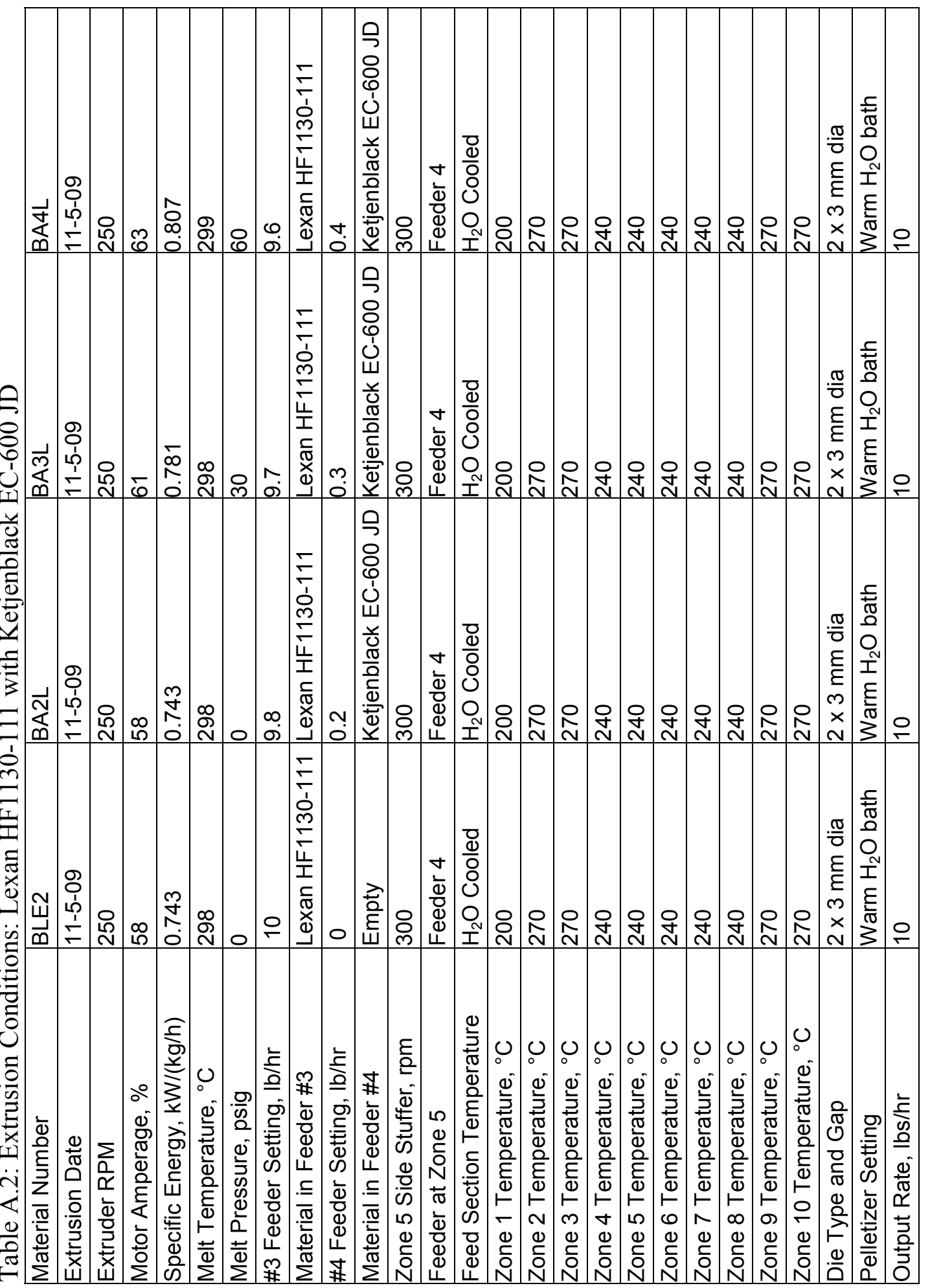




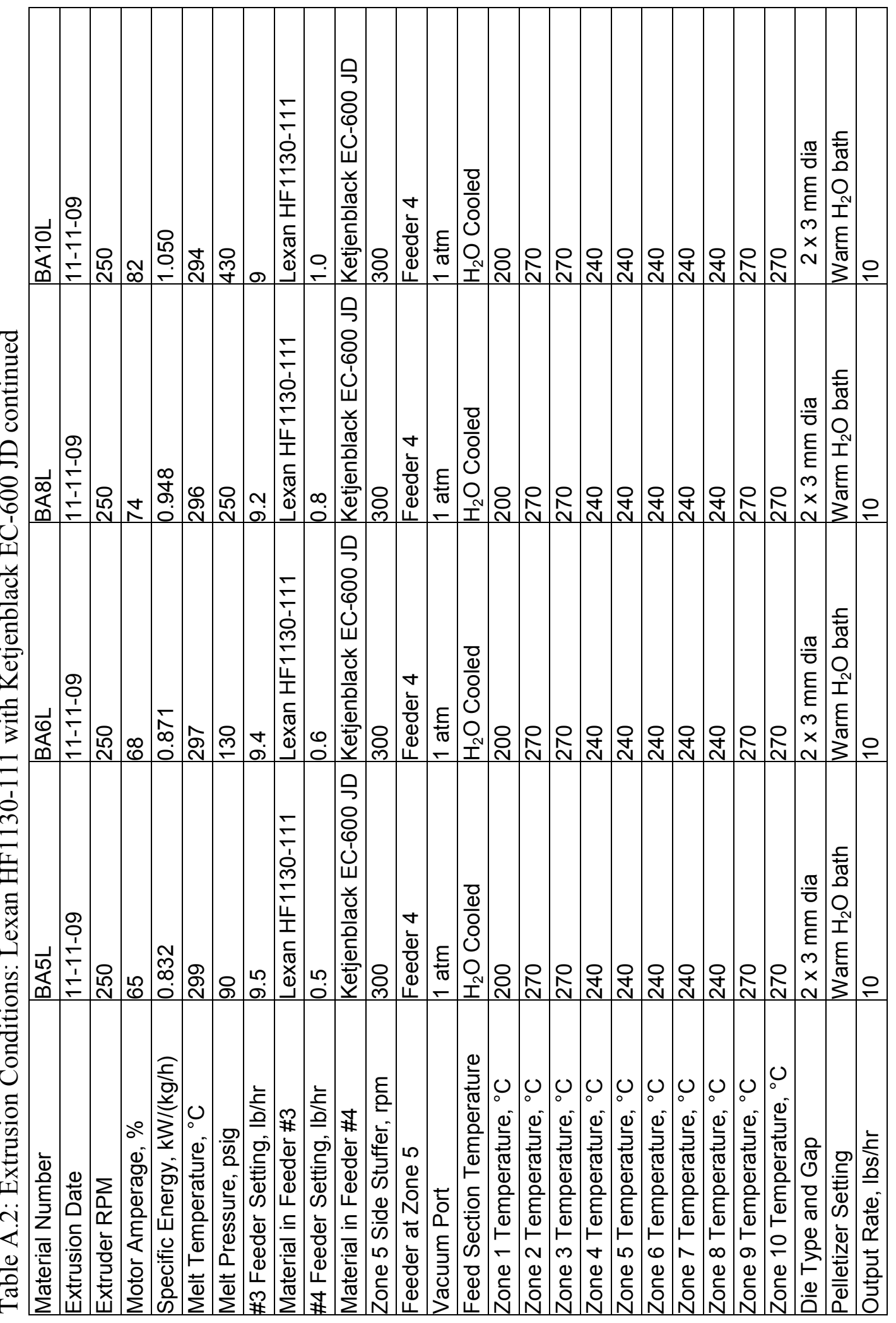




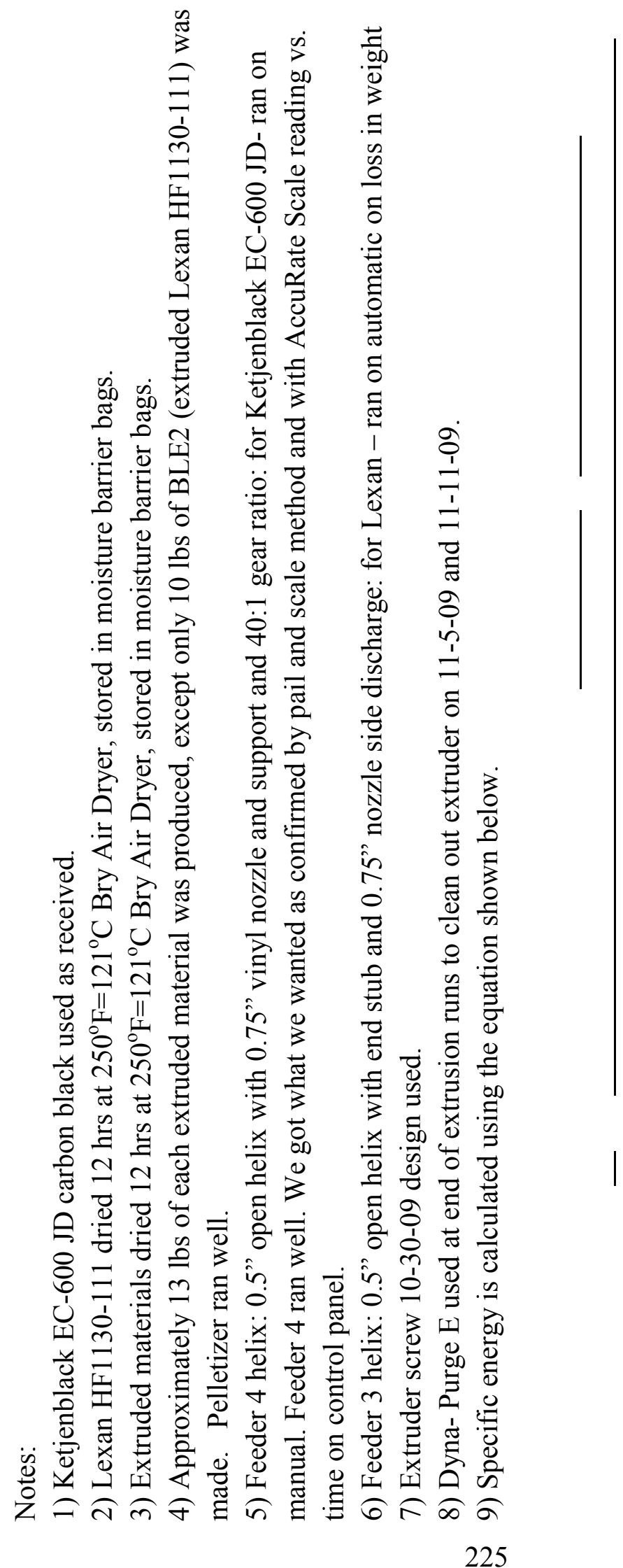



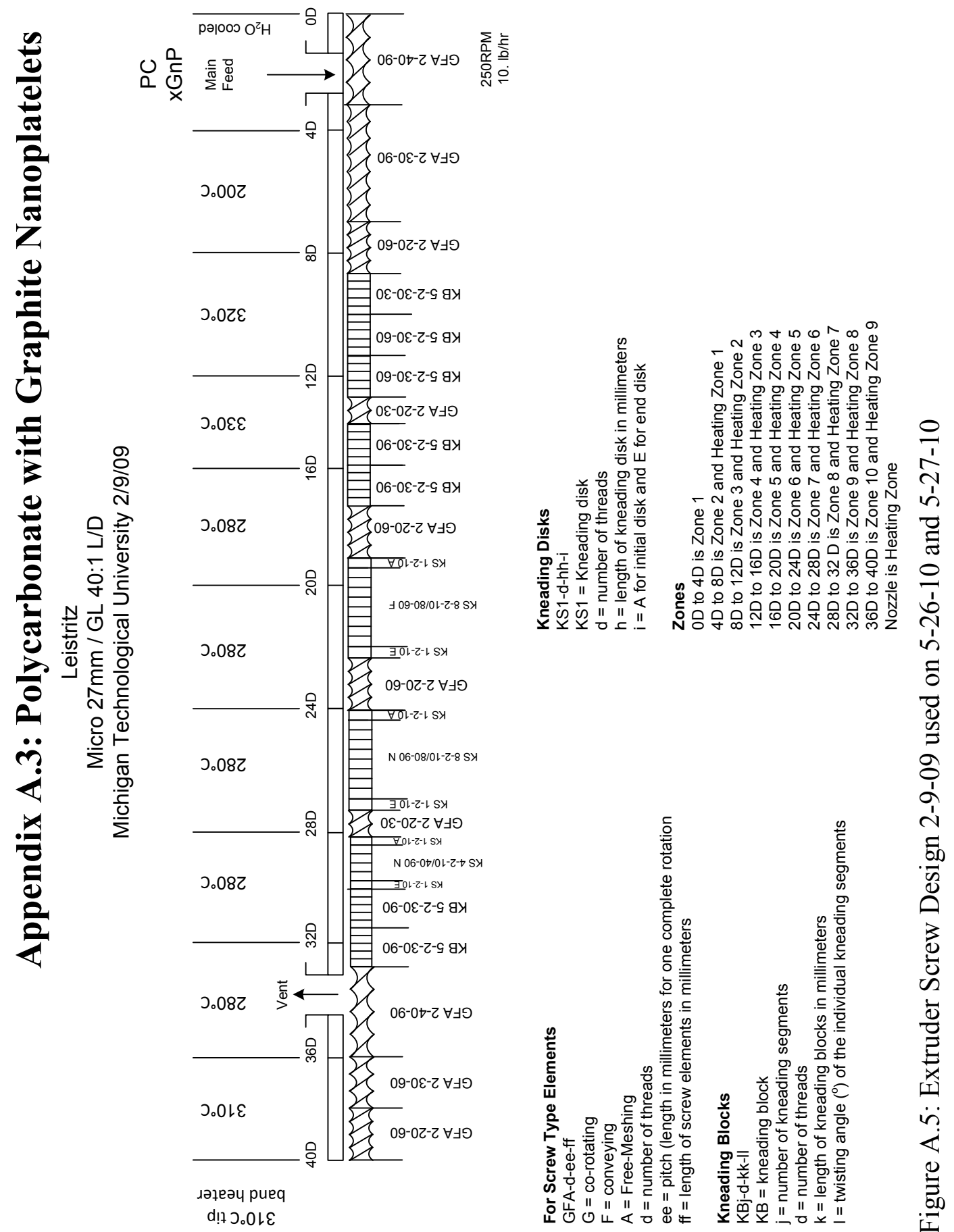


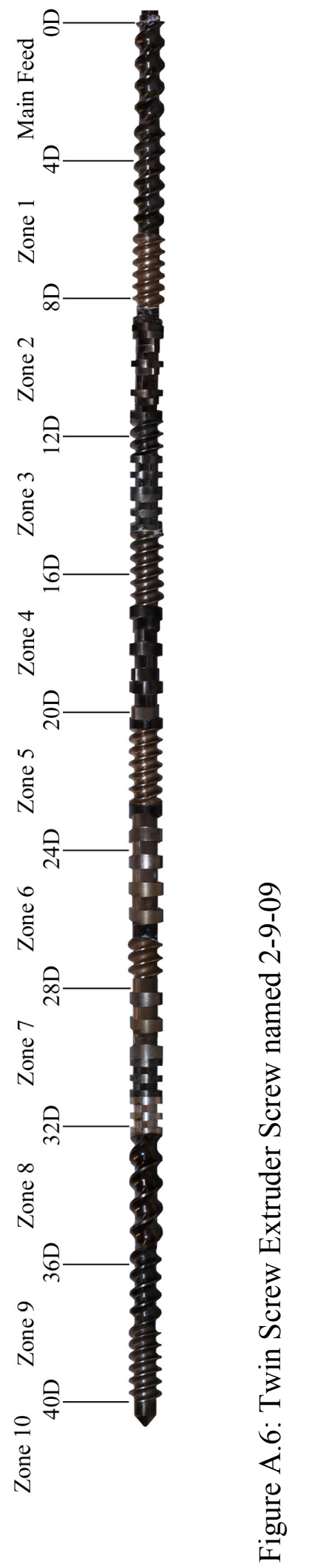




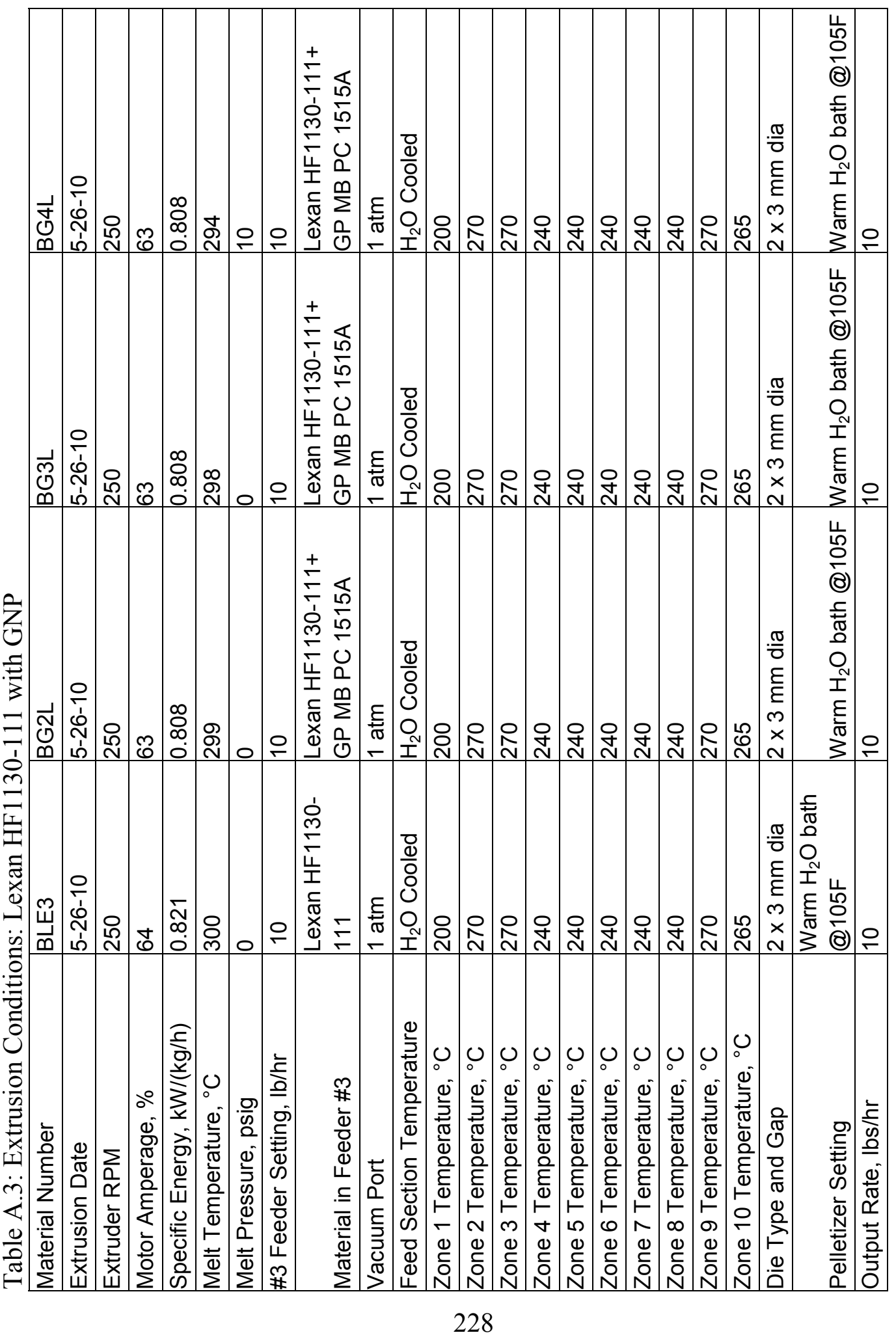




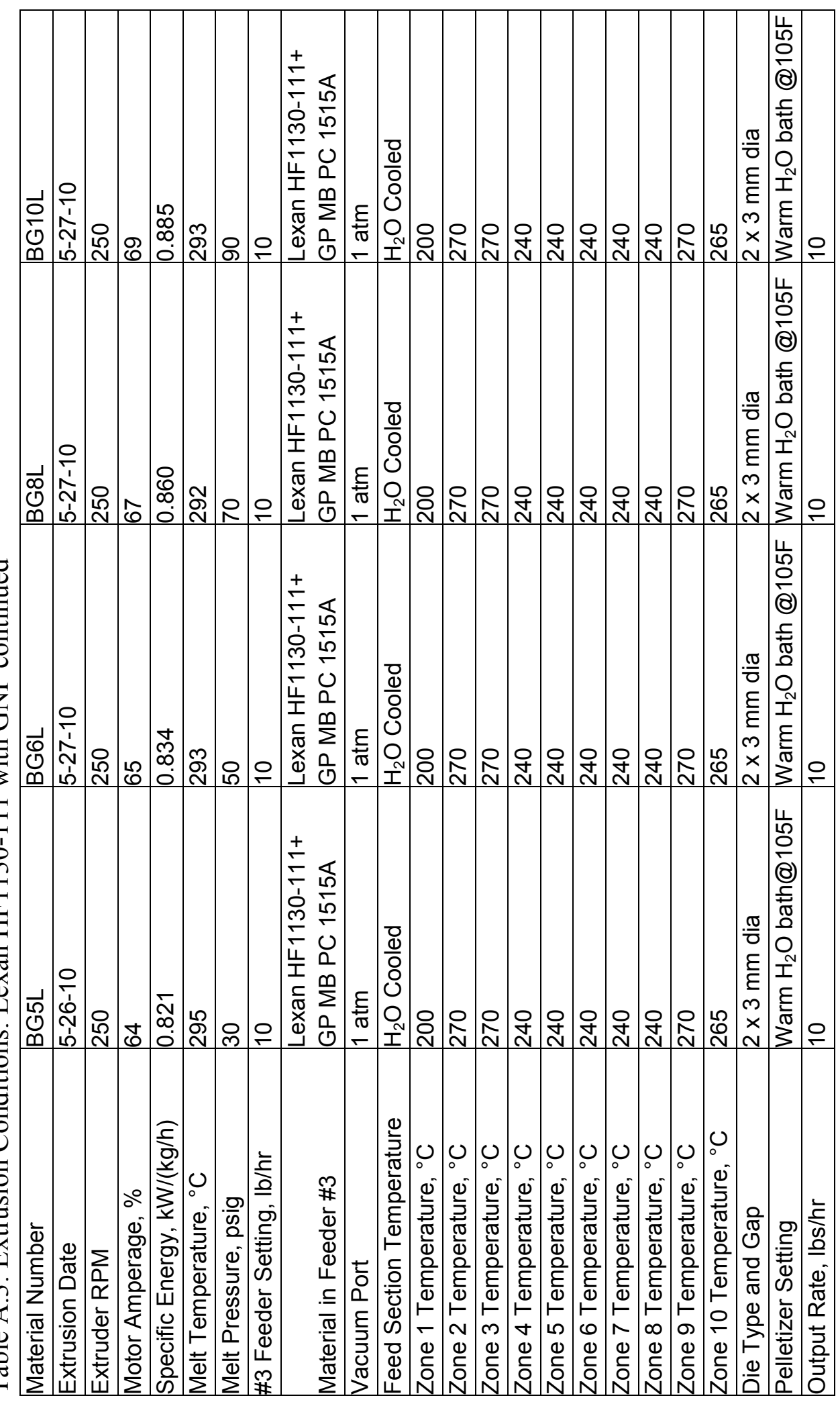




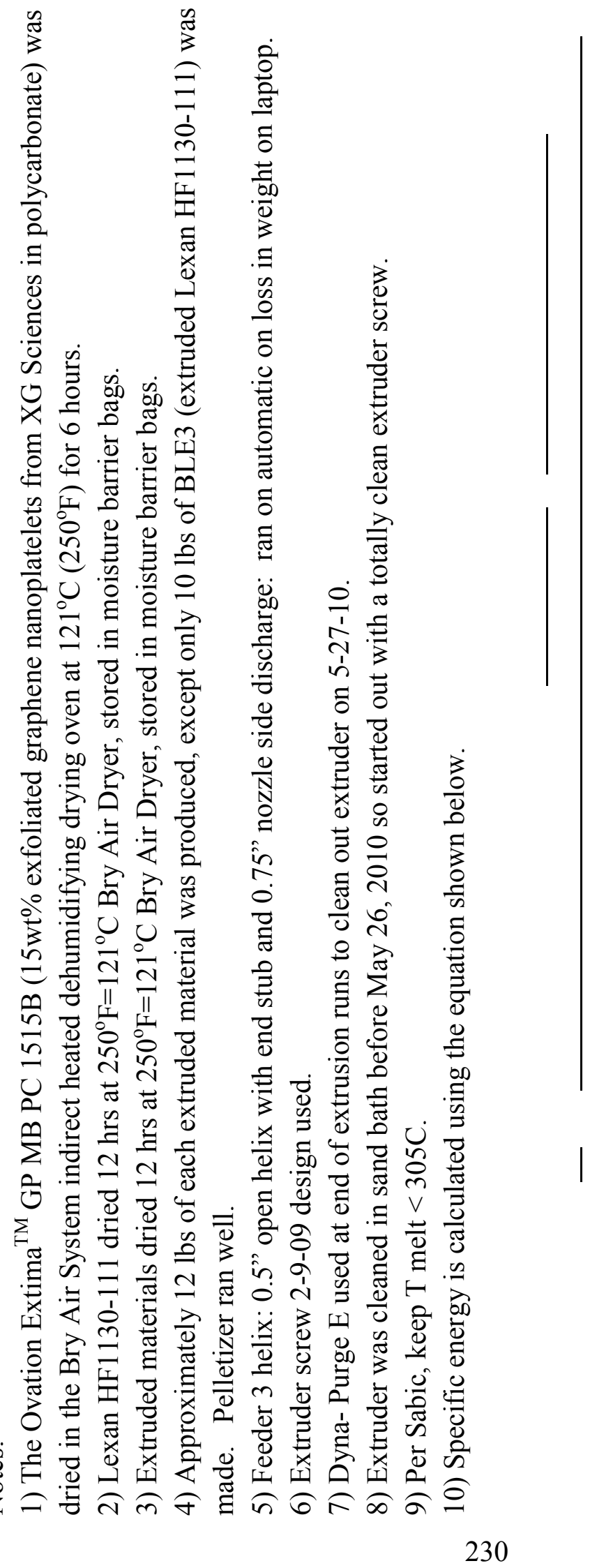




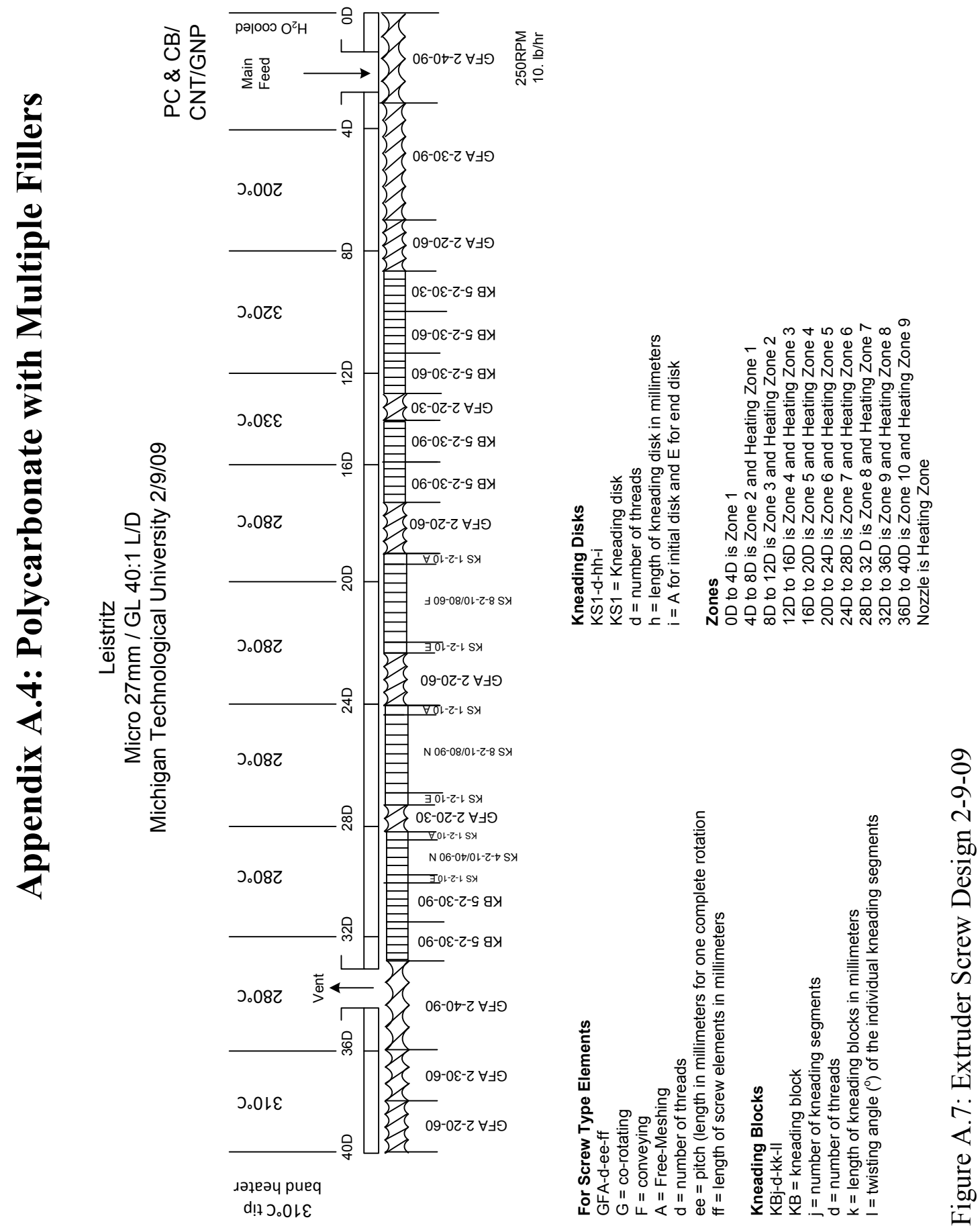




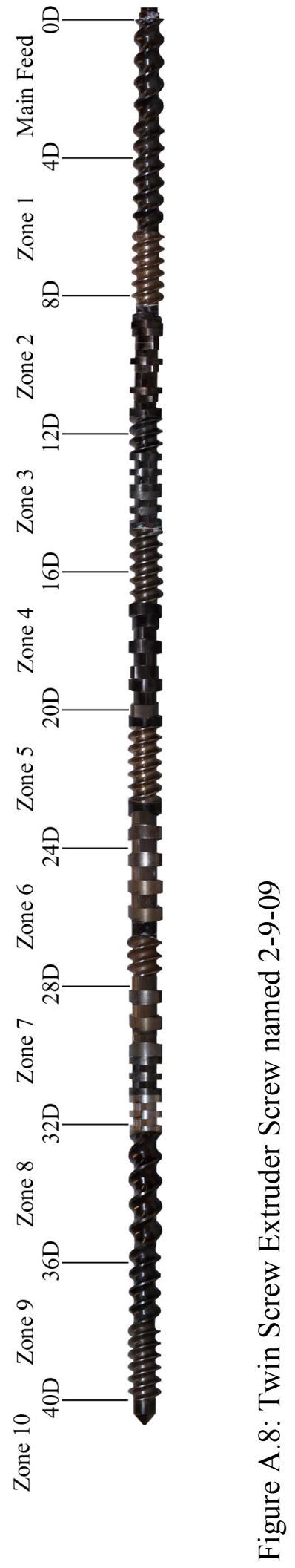


Table A.4: Extrusion Conditions: Lexan HF1130-111 with 8 wt\% Ketjenblack EC-600 JD in Lexan HF1130-111 Masterbatch (extruder screw used = 10-30-09)

\begin{tabular}{|c|c|}
\hline Material Number & BA8LR \\
\hline Extrusion Date & $4-14-10$ \\
\hline Extruder RPM & 250 \\
\hline Motor Amperage, \% & 71 \\
\hline Specific Energy, kW/(kg/h) & 0.91 \\
\hline Melt Temperature, ${ }^{\circ} \mathrm{C}$ & 296 \\
\hline Melt Pressure, psig & 250 \\
\hline \#3 Feeder Setting, lb/hr & 9.2 \\
\hline Material in Feeder \#3 & Lexan HF1130-111 \\
\hline \#4 Feeder Setting, lb/hr & 0.8 \\
\hline Material in Feeder \#4 & Ketjenblack EC-600 JD \\
\hline Vacuum Port & $1 \mathrm{~atm}$ \\
\hline Feed Section Temperature & $\mathrm{H}_{2} \mathrm{O}$ Cooled \\
\hline Zone 1 Temperature, ${ }^{\circ} \mathrm{C}$ & 200 \\
\hline Zone 2 Temperature, ${ }^{\circ} \mathrm{C}$ & 270 \\
\hline Zone 3 Temperature, ${ }^{\circ} \mathrm{C}$ & 270 \\
\hline Zone 4 Temperature, ${ }^{\circ} \mathrm{C}$ & 240 \\
\hline Zone 5 Temperature, ${ }^{\circ} \mathrm{C}$ & 240 \\
\hline Zone 6 Temperature, ${ }^{\circ} \mathrm{C}$ & 240 \\
\hline Zone 7 Temperature, ${ }^{\circ} \mathrm{C}$ & 240 \\
\hline Zone 8 Temperature, ${ }^{\circ} \mathrm{C}$ & 240 \\
\hline Zone 9 Temperature, ${ }^{\circ} \mathrm{C}$ & 270 \\
\hline Zone 10 Temperature, ${ }^{\circ} \mathrm{C}$ & 269 \\
\hline Die Type and Gap & $2 \times 3 \mathrm{~mm}$ dia \\
\hline Pelletizer Setting & Warm $\mathrm{H}_{2} \mathrm{O}$ bath @105F \\
\hline Output Rate, Ibs/hr & 10 \\
\hline
\end{tabular}


Notes:

1)The Ketjenblack EC-600 JD was used as received. The Ketjenblack EC-600 JD was used in Feeder 4 running on manual at 49\%. New carbon black was placed in Feeder 4 on April 14, 2010 (nothing was left in the feeder 4 hopper before or after the extrusion run). 2) Feeder 4 used the 0.5 " open helix with 0.75 " vinyl nozzle and support (40:1 gear ratio).

3) Lexan HF1130-111 dried $12 \mathrm{hrs}$ at $250^{\circ} \mathrm{F}=121^{\circ} \mathrm{C}$ Bry Air Dryer, stored in moisture barrier bags.

4) Extruded materials dried $12 \mathrm{hrs}$ at $250^{\circ} \mathrm{F}=121^{\circ} \mathrm{C}$ Bry Air Dryer, stored in moisture barrier bags.

5) Approximately $60 \mathrm{lbs}$ of BA8LR was produced. Pelletizer ran well.

6) Feeder 3 helix: 0.5 " open helix with end stub and 0.75 " nozzle side discharge: ran on automatic on loss in weight on laptop.

7) Extruder screw 10-30-09 design used.

8) Dyna- Purge E used at end of the extrusion run on April 14, 2010 to clean out extruder.

9) Extruder was cleaned in sand bath before April 14, 2010 so we started out with a totally clean extruder screw.

10) Per Sabic, keep T melt $<305 \mathrm{C}$.

11) Specific energy is calculated using the equation shown below. 


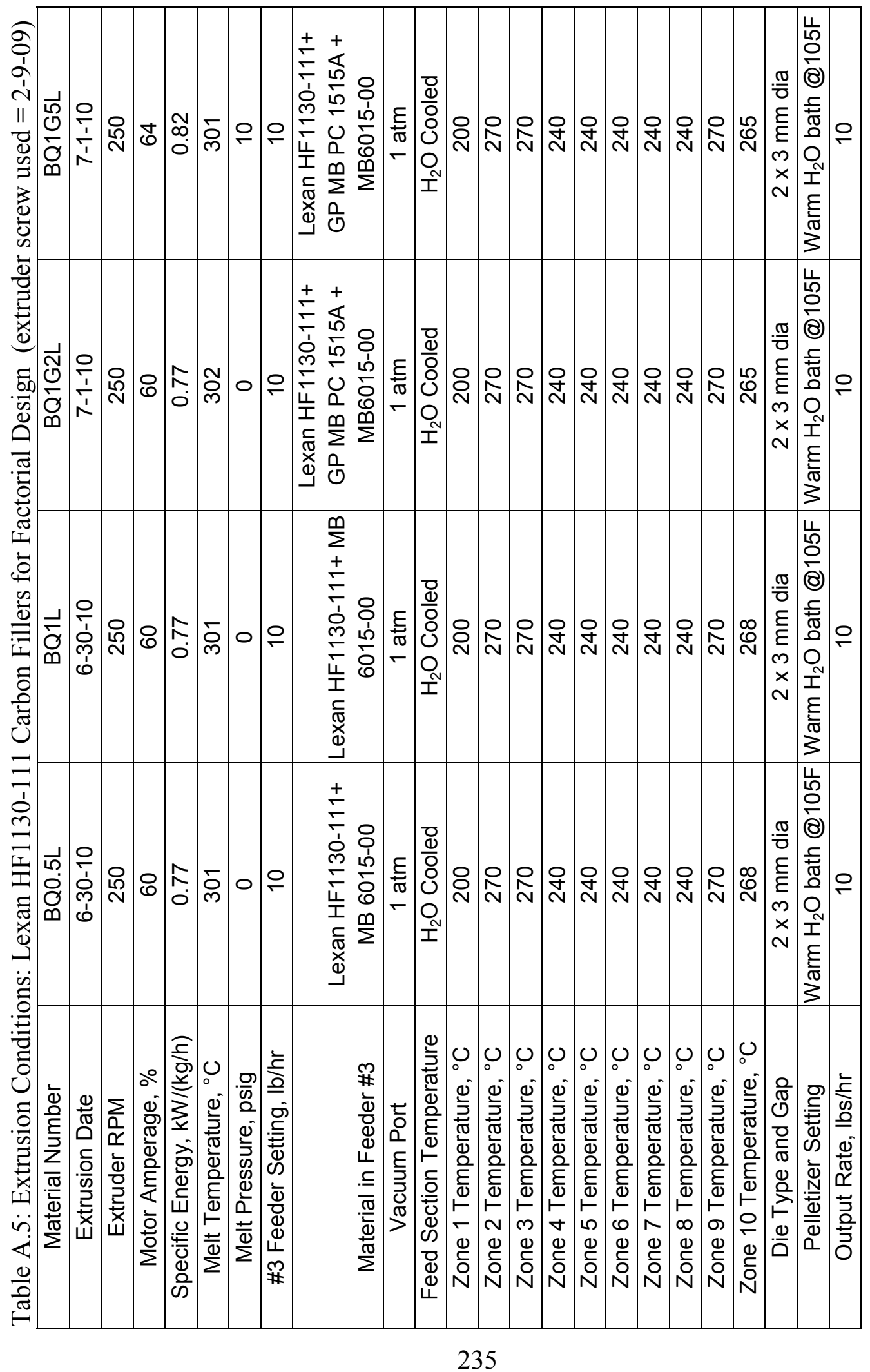




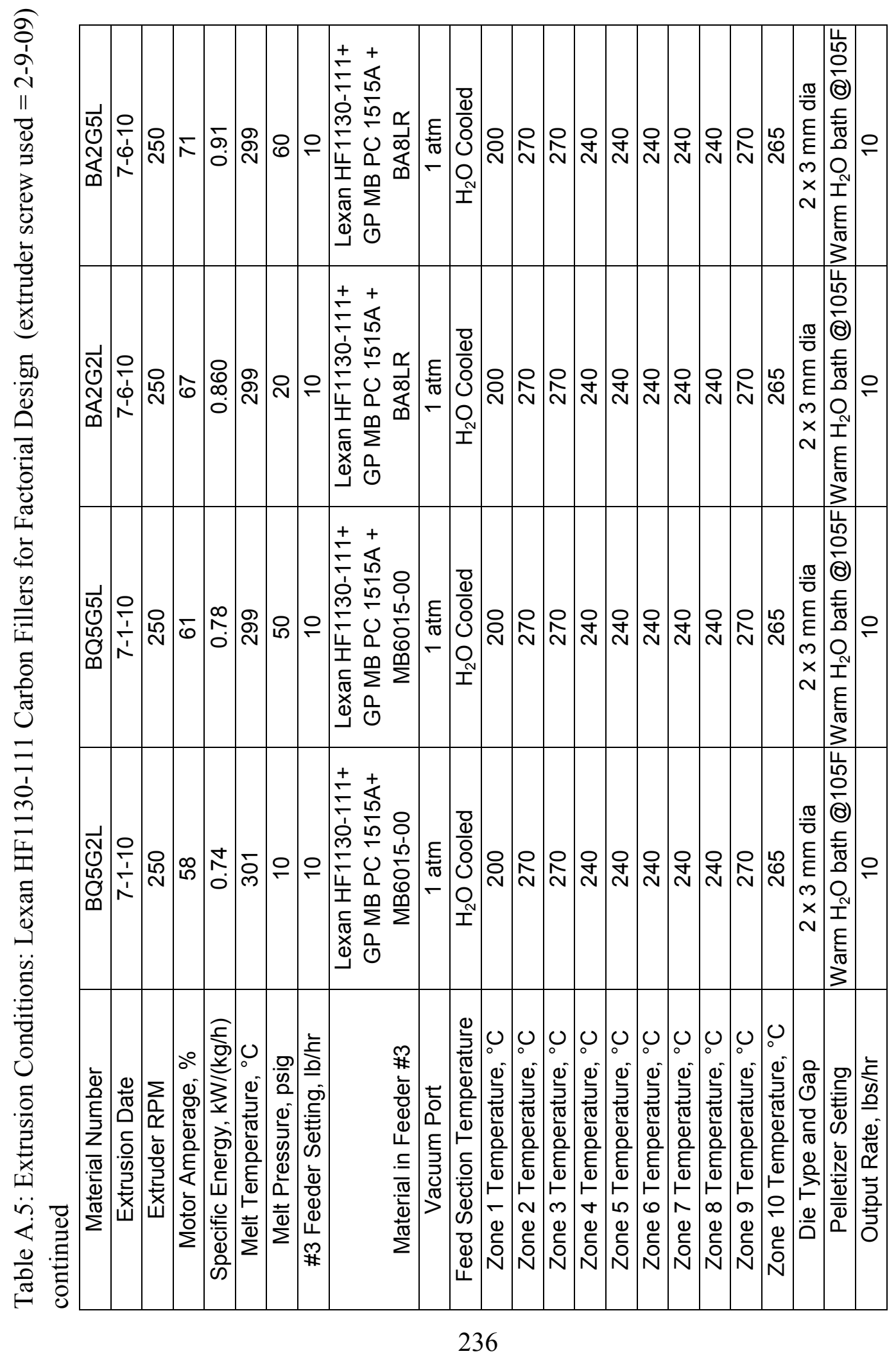




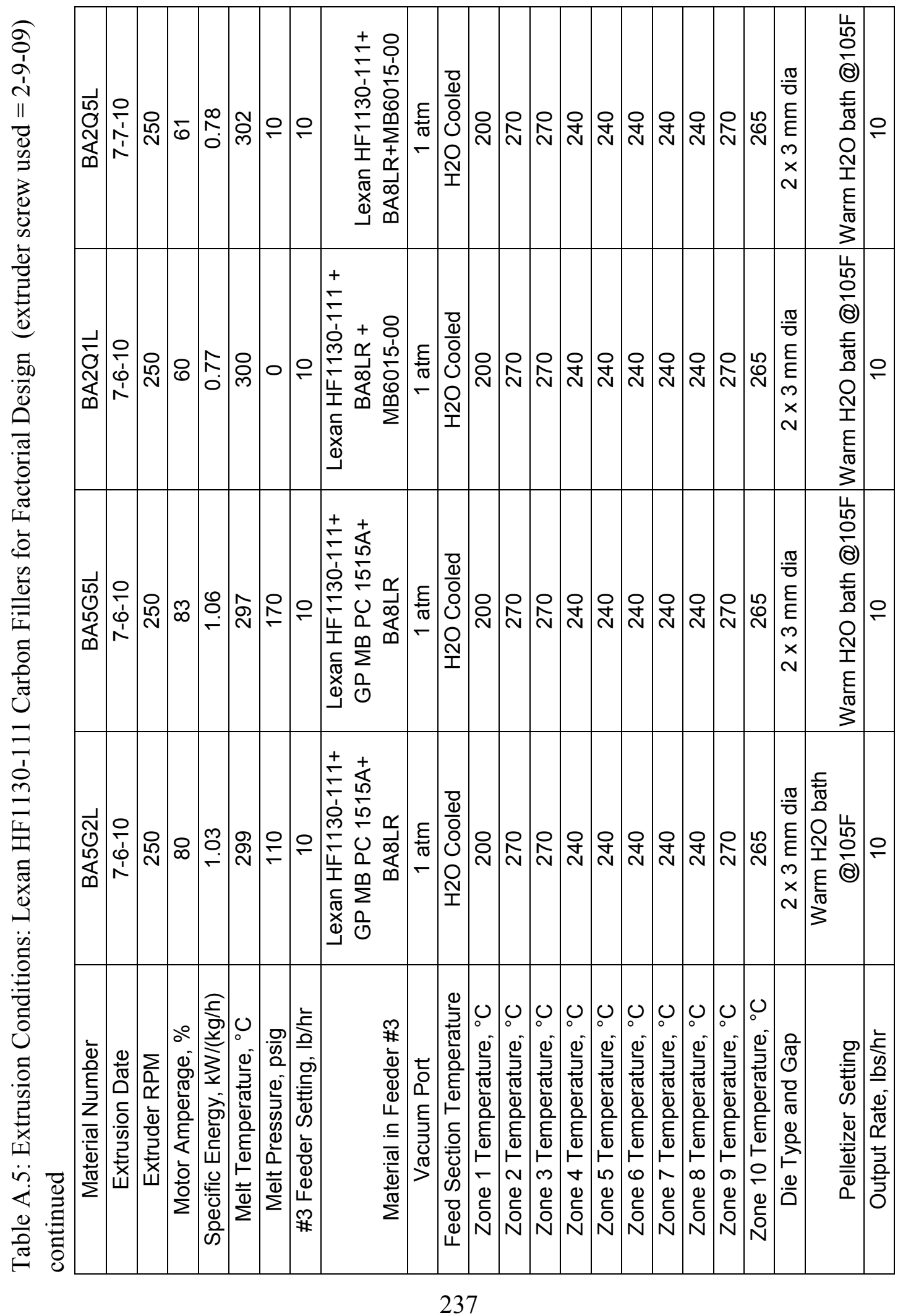




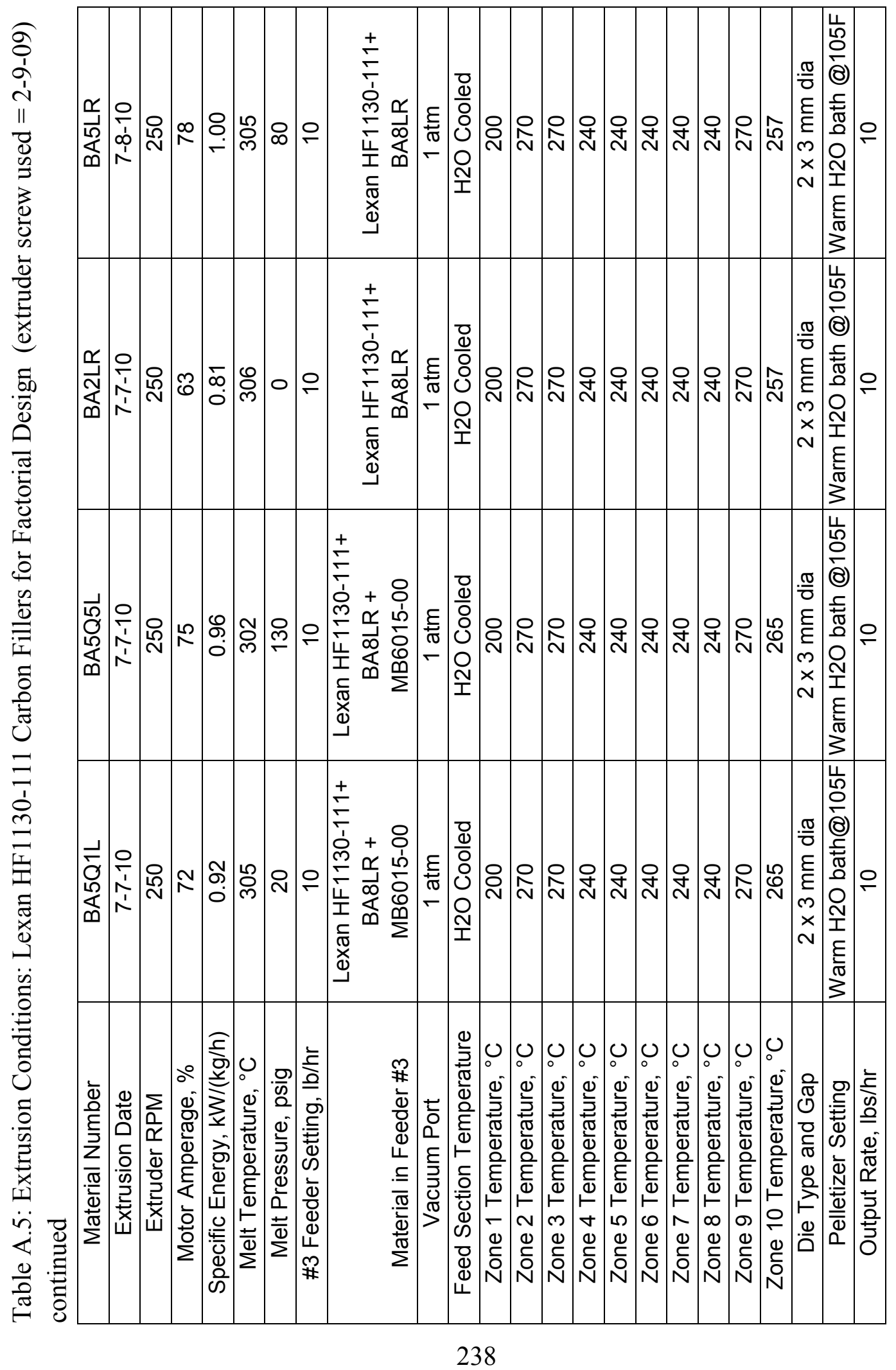




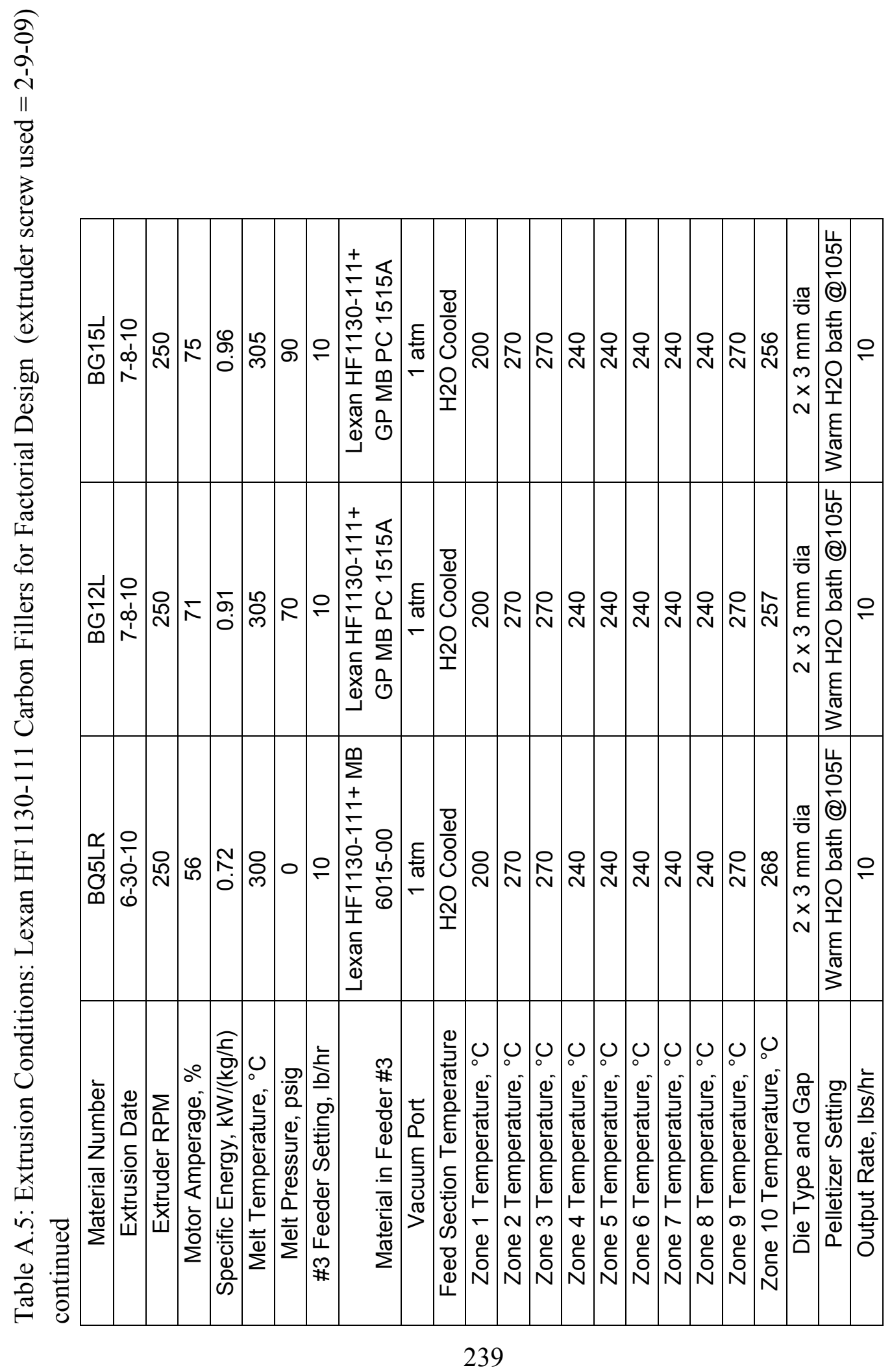




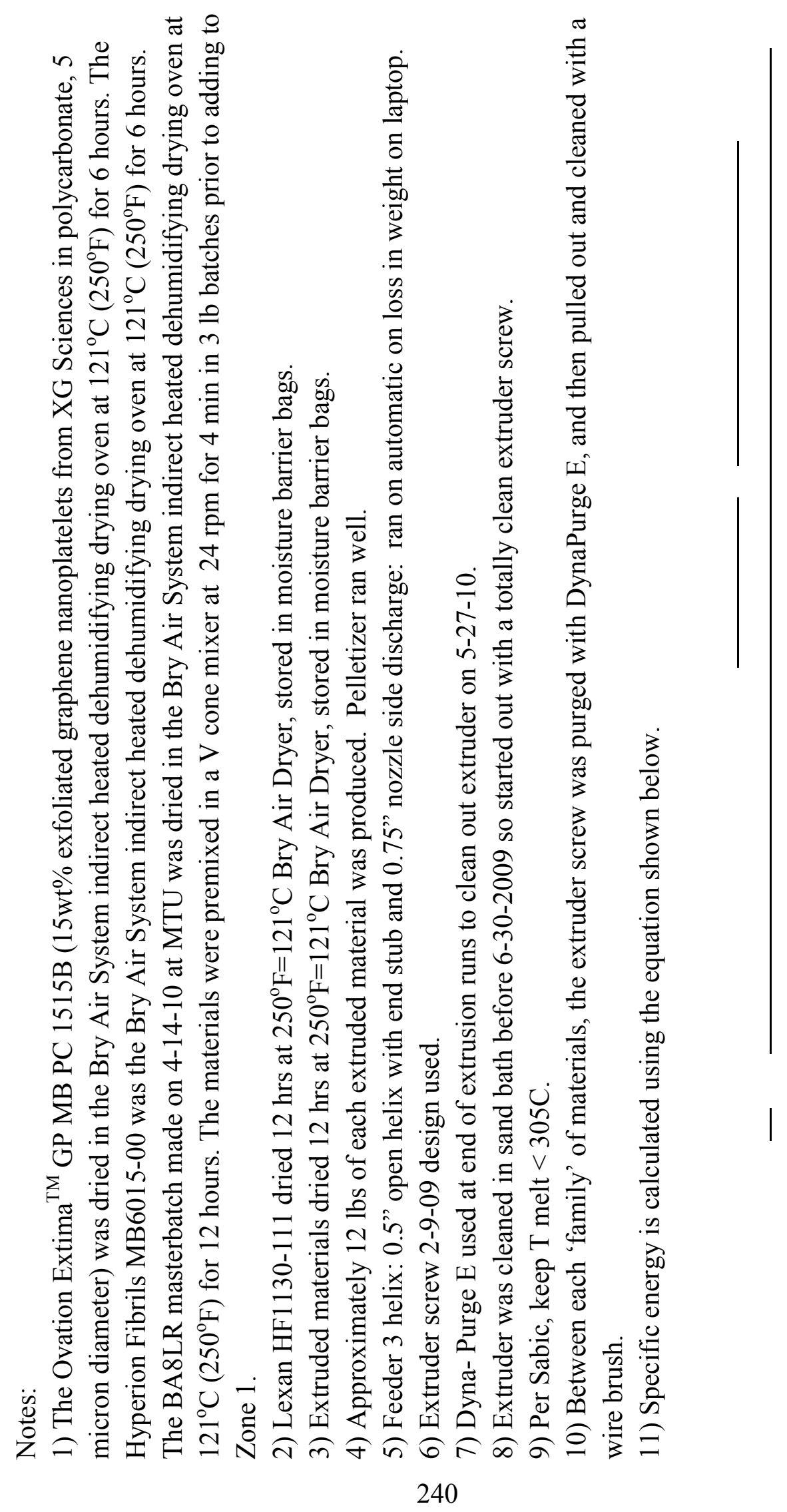




\section{Appendix B: Injection Molding Run Conditions}

\section{Appendix B.1: Polycarbonate with Carbon Nanotubes}

Table B.1: Injection Molding Conditions for CNT/PC Composites

\begin{tabular}{|c|c|c|c|c|c|}
\hline Notation & Injection Molding Conditions & $\mathrm{BL}$ & BLE & BQ2L & BQ3L \\
\hline $\mathrm{T}_{\text {mold }}$ & Mold Temperature $(\mathrm{F})$ & 190 & 190 & 190 & 190 \\
\hline $\mathrm{E} 1$ & Zone 1 Temperature (F) (nozzle) & 580 & 580 & 580 & 580 \\
\hline E2 & Zone 2 Temperature $(\mathrm{F})$ & 570 & 570 & 570 & 570 \\
\hline E3 & Zone 3 Temperature $(\mathrm{F})$ & 554 & 554 & 554 & 554 \\
\hline E4 & $\begin{array}{l}\text { Zone } 4 \text { Temperature }(F) \text { (feed } \\
\text { zone) }\end{array}$ & 536 & 536 & 536 & 536 \\
\hline $\mathrm{P} 1$ & Injection pressure (psi) & 15,827 & 15,827 & 15,827 & 15,827 \\
\hline $\mathrm{P} 2$ & Hold Pressure (psi) & 15,827 & 15,827 & 15,827 & 15,827 \\
\hline P7 & Back Pressure (psi) & 45.22 & 45.22 & 45.22 & 45.22 \\
\hline $\mathrm{S} 1$ & Shot size $(\mathrm{mm})$ & 40 & 40 & 40 & 40 \\
\hline $\mathrm{S} 2$ & Pullback before (mm) & 2.5 & 2.5 & 2.5 & 2.5 \\
\hline $\mathrm{S} 3$ & Pullback after (mm) & 5 & 5 & 5 & 5 \\
\hline S6 & Width of mold (mm) & 196 & 196 & 196 & 196 \\
\hline $\mathrm{S} 8$ & $\begin{array}{l}\text { Screw Position to Switch from } \\
\mathrm{P} 1 \text { to } \mathrm{P} 2(\mathrm{~mm})\end{array}$ & 15 & 15 & 15 & 15 \\
\hline $\mathrm{T} 1$ & Injection Time (s) & 15 & 15 & 15 & 15 \\
\hline $\mathrm{T} 2$ & Cool Time (s) & 25 & 25 & 25 & 25 \\
\hline $\mathrm{T} 3$ & Interval Time (s) & 2 & 2 & 2 & 2 \\
\hline T6 & Retraction Time (s) & 0 & 0 & 0 & 0 \\
\hline $\mathrm{T} 7$ & $\begin{array}{l}\text { Nozzle Retraction Delay Time } \\
\text { (s) }\end{array}$ & 0 & 0 & 0 & 0 \\
\hline $\mathrm{T} 8$ & Injection Delay Time (s) & 0 & 0 & 0 & 0 \\
\hline T9 & Charge Delay Time (s) & 0 & 0 & 0 & 0 \\
\hline V1 & Injection Velocity $\left(\mathrm{in}^{3} / \mathrm{s}\right)$ & 3.732 & 3.732 & 3.732 & 3.732 \\
\hline V6 & Screw Rotation (rpm) & 60 & 60 & 60 & 60 \\
\hline V9 & Retraction Velocity (\%) & 25 & 25 & 25 & 25 \\
\hline V10 & Advance Velocity (\%) & 90 & 90 & 90 & 90 \\
\hline V11 & Retraction Velocity (\%) & 90 & 90 & 90 & 90 \\
\hline $\mathrm{CF}$ & Clamp Force (US tons) & 80 & 80 & 80 & 80 \\
\hline
\end{tabular}


Table B.1: Injection Molding Conditions for CNT/PC Composites continued

\begin{tabular}{|c|c|c|c|c|c|}
\hline \multicolumn{2}{|c|}{ Notation Injection Molding Conditions } & \multirow{2}{*}{\begin{tabular}{|l|} 
BQ4L \\
190
\end{tabular}} & \multirow{2}{*}{\begin{tabular}{|l|} 
BQ5L \\
190
\end{tabular}} & \multirow{2}{*}{\begin{tabular}{|l|} 
BQ6L \\
190
\end{tabular}} & \multirow{2}{*}{\begin{tabular}{|l|} 
BQ8L \\
190
\end{tabular}} \\
\hline $\mathrm{T}_{\text {mold }}$ & Mold Temperature (F) & & & & \\
\hline E1 & Zone 1 Temperature (F) (nozzle) & 580 & 580 & 580 & 600 \\
\hline E2 & Zone 2 Temperature $(\mathrm{F})$ & 570 & 570 & 570 & 600 \\
\hline E3 & Zone 3 Temperature $(\mathrm{F})$ & 554 & 554 & 554 & 570 \\
\hline E4 & $\begin{array}{l}\text { Zone } 4 \text { Temperature }(\mathrm{F}) \text { (feed } \\
\text { zone) }\end{array}$ & 536 & 536 & 536 & 550 \\
\hline $\mathrm{P} 1$ & Injection pressure (psi) & 15,827 & 18,088 & 20,349 & 22,383 \\
\hline $\mathrm{P} 2$ & Hold Pressure (psi) & 15,827 & 15,827 & 15,827 & 15,827 \\
\hline P7 & Back Pressure (psi) & 45.22 & 45.22 & 45.22 & 45.22 \\
\hline S1 & Shot size $(\mathrm{mm})$ & 40 & 40 & 45 & 47 \\
\hline $\mathrm{S} 2$ & Pullback before (mm) & 2.5 & 2.5 & 0 & 0 \\
\hline S3 & Pullback after (mm) & 5 & 5 & 0 & 0 \\
\hline S6 & Width of mold (mm) & 196 & 196 & 196 & 196 \\
\hline $\mathrm{S} 8$ & $\begin{array}{l}\text { Screw Position to Switch from } \\
\text { P1 to P2 (mm) }\end{array}$ & 15 & 15 & 15 & 15 \\
\hline $\mathrm{T} 1$ & Injection Time (s) & 15 & 15 & 15 & 15 \\
\hline $\mathrm{T} 2$ & Cool Time (s) & 25 & 25 & 25 & 25 \\
\hline $\mathrm{T} 3$ & Interval Time (s) & 2 & 2 & 2 & 2 \\
\hline T6 & Retraction Time (s) & 0 & 0 & 0 & 0 \\
\hline $\mathrm{T} 7$ & $\begin{array}{l}\text { Nozzle Retraction Delay Time } \\
\text { (s) }\end{array}$ & 0 & 0 & 0 & 0 \\
\hline $\mathrm{T} 8$ & Injection Delay Time (s) & 0 & 0 & 0 & p \\
\hline T9 & Charge Delay Time (s) & 0 & 0 & 0 & 0 \\
\hline V1 & Injection Velocity $\left(\mathrm{in}^{3} / \mathrm{s}\right)$ & 3.732 & 3.732 & 3.732 & 3.732 \\
\hline V6 & Screw Rotation (rpm) & 60 & 60 & 60 & 60 \\
\hline V9 & Retraction Velocity (\%) & 25 & 25 & 25 & 25 \\
\hline V10 & Advance Velocity (\%) & 90 & 90 & 90 & 90 \\
\hline V11 & Retraction Velocity (\%) & 90 & 90 & 90 & 90 \\
\hline $\mathrm{CF}$ & Clamp Force (US tons) & 80 & 80 & 80 & 80 \\
\hline
\end{tabular}

Notes:

For all formulations, about 302.5 " diameter disks, 30 tensile bars, and 30 flexural bars molded.

After each formulation, the feed hopper was run until it was empty and no material was in screw. Then we discarded 5 transition shots, and then started collecting the next formulation. 


\section{Appendix B.2: Polycarbonate with Carbon Black}

Table B.2: Injection Molding Conditions for CB/PC Composites

\begin{tabular}{|c|c|c|c|c|c|}
\hline Notation & Injection Molding Conditions & $\begin{array}{l}\text { BL2 } \\
12-4-09+\end{array}$ & $\begin{array}{l}\text { BLE2 } \\
12-4-09+\end{array}$ & $\begin{array}{l}\text { BA2L } \\
12-4-09+\end{array}$ & $\begin{array}{l}\text { BA3L } \\
12-4-09+\end{array}$ \\
\hline$T_{\text {mold }}$ & Mold Temperature (F) & 160 & 160 & 160 & 160 \\
\hline E1 & Zone 1 Temperature (F) (nozzle) & 610 & 610 & 610 & 610 \\
\hline $\mathrm{E} 2$ & Zone 2 Temperature $(\mathrm{F})$ & 570 & 570 & 570 & 570 \\
\hline $\mathrm{E} 3$ & Zone 3 Temperature $(\mathrm{F})$ & 554 & 554 & 554 & 554 \\
\hline $\mathrm{E} 4$ & Zone 4 Temperature (F) (feed zone) & 536 & 536 & 536 & 536 \\
\hline P1 & Injection pressure (psi) & $\begin{array}{l}70 \% \\
=15,827\end{array}$ & $\begin{array}{l}70 \% \\
=15,827\end{array}$ & $\begin{array}{l}70 \% \\
=15,827\end{array}$ & $\begin{array}{l}70 \% \\
=15,827\end{array}$ \\
\hline $\mathrm{P} 2$ & Hold Pressure (psi) & $\begin{array}{l}55 \%= \\
12,436 \\
\end{array}$ & $\begin{array}{l}55 \%= \\
12,436 \\
\end{array}$ & $\begin{array}{l}55 \%= \\
12,436 \\
\end{array}$ & $\begin{array}{l}55 \%= \\
12,436\end{array}$ \\
\hline P7 & Back Pressure (psi) & $\begin{array}{l}0.2 \%= \\
45.2\end{array}$ & $\begin{array}{l}0.2 \%= \\
45.2\end{array}$ & $\begin{array}{l}0.2 \%= \\
45.2\end{array}$ & $\begin{array}{l}0.2 \%= \\
45.2\end{array}$ \\
\hline $\mathrm{S} 1$ & Shot size $(\mathrm{mm})$ & 20 & 20 & 20 & 20 \\
\hline $\mathrm{S} 2$ & Pullback before (mm) & 0 & 0 & 0 & 0 \\
\hline $\mathrm{S} 3$ & Pullback after (mm) & 0.1 & 0.1 & 0.1 & 0.1 \\
\hline S6 & Width of mold (mm) & 202 & 202 & 202 & 202 \\
\hline $\mathrm{S} 8$ & $\begin{array}{l}\text { Screw Position to Switch from P1 to } \\
\text { P2 (mm) }\end{array}$ & $\begin{array}{l}8 \text { for } \\
\text { tensile, } \\
10 \text { for } \\
\text { flex, } 12 \\
\text { for disk }\end{array}$ & \begin{tabular}{|l}
8 for \\
tensile, 10 \\
for flex, \\
12 for \\
disk
\end{tabular} & \begin{tabular}{|l|}
8 for \\
tensile, 10 \\
for flex, \\
12 for \\
disk
\end{tabular} & $\begin{array}{l}8 \text { for } \\
\text { tensile, } 10 \\
\text { for flex, } \\
12 \text { for } \\
\text { disk }\end{array}$ \\
\hline $\mathrm{T} 1$ & Injection Time (s) & 9 & 9 & 9 & 9 \\
\hline $\mathrm{T} 2$ & Cool Time (s) & 20 & 20 & 20 & 20 \\
\hline $\mathrm{T} 3$ & Interval Time (s) & 0.5 & 0.5 & 0.5 & 0.5 \\
\hline T6 & Retraction Time (s) & 0 & 0 & 0 & 0 \\
\hline $\mathrm{T} 7$ & Nozzle Retraction Delay Time (s) & 0 & 0 & 0 & 0 \\
\hline $\mathrm{T} 8$ & Injection Delay Time (s) & 0 & 0 & 0 & 0 \\
\hline T9 & Charge Delay Time (s) & 0 & 0 & 0 & 0 \\
\hline V1 & Injection Velocity $\left(\mathrm{in}^{3} / \mathrm{s}\right)$ & $\begin{array}{l}30 \%= \\
1.866\end{array}$ & $\begin{array}{l}30 \%= \\
1.866\end{array}$ & $\begin{array}{l}30 \%= \\
1.866\end{array}$ & $\begin{array}{l}30 \%= \\
1.866\end{array}$ \\
\hline V6 & Screw Rotation (rpm) & $19 \%=60$ & $19 \%=60$ & $19 \%=60$ & $19 \%=60$ \\
\hline V9 & Retraction Velocity (\%) & 20 & 20 & 20 & 20 \\
\hline V10 & Advance Velocity (\%) & 90 & 90 & 90 & 90 \\
\hline V11 & Retraction Velocity (\%) & 90 & 90 & 90 & 90 \\
\hline $\mathrm{CF}$ & Clamp Force (US tons) & 80 & 80 & 80 & 80 \\
\hline
\end{tabular}


Table B.2: Injection Molding Conditions for $\mathrm{CB} / \mathrm{PC}$ Composites continued

\begin{tabular}{|l|l|l|l|l|l|}
\hline & & & & \\
Notation & Injection Molding & BL2 & BLE2 & BA2L & BA3L \\
& Conditions & $12-4-09+$ & $12-4-09+$ & $12-4-09+$ & $12-4-09+$ \\
\hline P-5-1 & Inj Start, mm & -- & -- & -- & -- \\
\hline P-5-1 & Cushion, mm & -- & -- & -- & -- \\
\hline P-5-1 & P1 $\rightarrow$ P2 Fill time, sec & -- & -- & -- & -- \\
\hline P-5-1 & P1 Actual Pressure, \% & -- & -- & -- & -- \\
\hline P-5-1 & Charge time, sec & -- & -- & -- & -- \\
\hline
\end{tabular}


Table B.2: Injection Molding Conditions for $\mathrm{CB} / \mathrm{PC}$ Composites continued

\begin{tabular}{|c|c|c|c|c|c|c|}
\hline Notation & $\begin{array}{l}\text { Injection Molding } \\
\text { Conditions }\end{array}$ & $\begin{array}{l}\text { BA4L } \\
12-4-09+\end{array}$ & $\begin{array}{l}\text { BA5L } \\
12-4-09+\end{array}$ & $\begin{array}{l}\text { BA6L } \\
12-4-09+\end{array}$ & $\begin{array}{l}\text { BA8L } \\
12-4-09+\end{array}$ & $\begin{array}{l}\text { BA10L } \\
12-4-09+\end{array}$ \\
\hline $\mathrm{T}_{\text {mold }}$ & Mold Temperature (F) & 160 & 160 & 160 & 180 & 200 \\
\hline E1 & $\begin{array}{l}\text { Zone } 1 \text { Temperature }(\mathrm{F}) \\
\text { (nozzle) }\end{array}$ & 610 & 610 & 610 & 610 & 630 \\
\hline E2 & Zone 2 Temperature $(\mathrm{F})$ & 570 & 570 & 570 & 580 & 630 \\
\hline$\overline{\mathrm{E} 3}$ & Zone 3 Temperature $(\mathrm{F})$ & 554 & 554 & 554 & 570 & 610 \\
\hline E4 & $\begin{array}{l}\text { Zone } 4 \text { Temperature }(\mathrm{F}) \\
\text { (feed zone) }\end{array}$ & 536 & 536 & 536 & 560 & 610 \\
\hline P1 & Injection pressure (psi) & $\begin{array}{l}75 \% \\
=16,958\end{array}$ & $\begin{array}{l}75 \% \\
=16,958\end{array}$ & $\begin{array}{l}80 \% \\
=18,088\end{array}$ & $\begin{array}{l}90 \% \\
=20,349\end{array}$ & $\begin{array}{l}99 \% \\
=22,384\end{array}$ \\
\hline $\mathrm{P} 2$ & Hold Pressure (psi) & $\begin{array}{l}55 \%= \\
12,436\end{array}$ & $\begin{array}{l}55 \%= \\
12,436\end{array}$ & $\begin{array}{l}55 \%= \\
12,436\end{array}$ & $\begin{array}{l}55 \%= \\
12,436\end{array}$ & $\begin{array}{l}55 \%= \\
12,436\end{array}$ \\
\hline P7 & Back Pressure (psi) & $\begin{array}{l}0.2 \%= \\
45.2\end{array}$ & $\begin{array}{l}0.2 \%= \\
45.2\end{array}$ & $\begin{array}{l}0.2 \%= \\
45.2\end{array}$ & $\begin{array}{l}0.5 \%= \\
113\end{array}$ & $\begin{array}{l}0.5 \%= \\
113\end{array}$ \\
\hline S1 & Shot size $(\mathrm{mm})$ & 20 & 20 & 20 & $\begin{array}{l}20 \text { flex } \\
\text { and disk, } \\
22 \text { tensile }\end{array}$ & $\begin{array}{l}22 \text { flex } \\
\text { and } \\
\text { tensile, } 20 \\
\text { disk }\end{array}$ \\
\hline $\mathrm{S} 2$ & Pullback before (mm) & 0 & 0 & 0 & 0 & 0 \\
\hline S3 & Pullback after (mm) & 0.1 & 0.1 & 0.1 & 0.1 & 0.1 \\
\hline S6 & Width of mold (mm) & 202 & 202 & 202 & 202 & 202 \\
\hline S8 & $\begin{array}{l}\text { Screw Position to Switch } \\
\text { from P1 to P2 (mm) }\end{array}$ & $\begin{array}{l}8 \text { for } \\
\text { tensile, } 10 \\
\text { for flex, } \\
12 \text { for disk }\end{array}$ & $\begin{array}{l}8 \text { for } \\
\text { tensile, } 10 \\
\text { for flex, } \\
12 \text { for disk }\end{array}$ & $\begin{array}{l}8 \text { for } \\
\text { tensile, } 10 \\
\text { for flex, } \\
12 \text { for disk }\end{array}$ & $\begin{array}{l}7 \text { for } \\
\text { tensile, } 10 \\
\text { for flex, } \\
12 \text { for disk }\end{array}$ & $\begin{array}{l}12 \text { for } \\
\text { tensile, } 14 \\
\text { for flex, } \\
18 \text { for disk }\end{array}$ \\
\hline $\mathrm{T} 1$ & Injection Time (s) & 8* & $8 *$ & $8^{*}$ & 8* & $8 *$ \\
\hline $\mathrm{T} 2$ & Cool Time (s) & $16^{*}$ & $16^{*}$ & $16^{*}$ & $16^{*}$ & $16^{*}$ \\
\hline T3 & Interval Time (s) & $0.1^{*}$ & $0.1^{*}$ & $0.1^{*}$ & $0.1^{*}$ & $0.1^{*}$ \\
\hline T6 & Retraction Time (s) & 0 & 0 & 0 & 0 & 0 \\
\hline $\mathrm{T} 7$ & $\begin{array}{l}\text { Nozzle Retraction Delay } \\
\text { Time }(\mathrm{s})\end{array}$ & 0 & 0 & 0 & 0 & 0 \\
\hline $\mathrm{T} 8$ & Injection Delay Time (s) & 0 & 0 & 0 & 0 & 0 \\
\hline T9 & Charge Delay Time (s) & 0 & 0 & 0 & 0 & 0 \\
\hline V1 & Injection Velocity $\left(\mathrm{in}^{3} / \mathrm{s}\right)$ & $\begin{array}{l}30 \%= \\
1.866\end{array}$ & $\begin{array}{l}30 \%= \\
1.866\end{array}$ & $\begin{array}{l}10 \%= \\
0.622\end{array}$ & $\begin{array}{l}10 \%= \\
0.622\end{array}$ & $\begin{array}{l}10 \%= \\
0.622\end{array}$ \\
\hline V6 & Screw Rotation (rpm) & $19 \%=60$ & $19 \%=60$ & $19 \%=60$ & $19 \%=60$ & $19 \%=60$ \\
\hline V9 & Retraction Velocity (\%) & 20 & 20 & 20 & 20 & 20 \\
\hline V10 & Advance Velocity (\%) & 90 & 90 & 50 & 50 & 50 \\
\hline V11 & Retraction Velocity (\%) & 90 & 90 & 90 & 90 & 90 \\
\hline
\end{tabular}


Table B.2: Injection Molding Conditions for CB/PC Composites continued

\begin{tabular}{|c|c|c|c|c|c|c|}
\hline Notation & $\begin{array}{l}\text { Injection Molding } \\
\text { Conditions }\end{array}$ & $\begin{array}{l}\text { BA4L } \\
12-4-09+\end{array}$ & $\begin{array}{l}\text { BA5L } \\
12-4-09+\end{array}$ & \begin{tabular}{|l} 
BA6L \\
$12-4-09+$
\end{tabular} & \begin{tabular}{|l} 
BA8L \\
$12-4-09+$
\end{tabular} & $\begin{array}{l}\text { BA10L } \\
12-4-09+\end{array}$ \\
\hline $\mathrm{CF}$ & Clamp Force (US tons) & 80 & 80 & 80 & 80 & 80 \\
\hline P-5-1 & Inj Start, mm & 21 & \begin{tabular}{|l}
20.8 \\
tensile \\
20.8 flex \\
20.9 disk
\end{tabular} & $\begin{array}{l}21 \text { tensile } \\
21 \text { flex } \\
20.9 \text { disk }\end{array}$ & $\begin{array}{l}22.5 \\
\text { tensile } \\
20.1 \text { flex } \\
20.3 \text { disk }\end{array}$ & $\begin{array}{l}22.1 \\
\text { tensile } \\
22.1 \text { flex } \\
20.1 \text { disk }\end{array}$ \\
\hline P-5-1 & Cushion, mm & 2.5 & $\begin{array}{l}2.1 \text { tensile } \\
3.9 \text { flex } \\
3.1 \text { disk }\end{array}$ & $\begin{array}{l}2.3 \text { tensile } \\
4 \text { flex } \\
3.2 \text { disk }\end{array}$ & $\begin{array}{l}3.9 \text { tensile } \\
3.0 \text { flex } \\
2.8 \text { disk }\end{array}$ & $\begin{array}{l}2.0 \text { tensile } \\
2.0 \text { flex } \\
2.0 \text { disk }\end{array}$ \\
\hline P-5-1 & $\mathrm{P} 1 \rightarrow \mathrm{P} 2$ Fill time, sec & 0.75 & $\begin{array}{l}1.0 \text { tensile } \\
0.65 \text { flex } \\
0.56 \text { disk }\end{array}$ & $\begin{array}{l}2.4 \text { tensile } \\
2.1 \text { flex } \\
1.7 \text { disk }\end{array}$ & $\begin{array}{l}2.81 \\
\text { tensile } \\
1.94 \text { flex } \\
1.6 \text { disk }\end{array}$ & $\begin{array}{l}1.81 \\
\text { tensile } \\
1.53 \mathrm{flex} \\
0.56 \mathrm{disk}\end{array}$ \\
\hline$P-5-1$ & P1 Actual Pressure, \% & 68 & $\begin{array}{l}60 \text { tensile } \\
60 \text { flex } \\
48 \text { disk }\end{array}$ & $\begin{array}{l}57 \text { tensile } \\
47 \text { flex } \\
36 \text { disk }\end{array}$ & $\begin{array}{l}86 \text { tensile } \\
51 \text { flex } \\
38 \text { disk }\end{array}$ & $\begin{array}{l}48 \text { tensile } \\
39 \text { flex } \\
17 \text { disk }\end{array}$ \\
\hline P-5-1 & Charge time, sec & 3.3 & $\begin{array}{l}3.3 \text { tensile } \\
2.9 \text { flex } \\
3.0 \text { disk }\end{array}$ & $\begin{array}{l}3.4 \text { tensile } \\
3.4 \text { flex } \\
3.3 \text { disk }\end{array}$ & $\begin{array}{l}3.7 \text { tensile } \\
3.5 \text { flex } \\
3.4 \text { disk }\end{array}$ & $\begin{array}{l}3.6 \text { tensile } \\
3.2 \text { flex } \\
3.4 \text { disk }\end{array}$ \\
\hline
\end{tabular}

Notes:

1) For all formulations, about 302.5 " diameter disks, 30 tensile bars, and 30 flexural bars molded. Each sample was individually molded. The injection molding machine ran on the fully automatic mode all day on Dec 4, 2009.

2) After each formulation, the feed hopper was run until it was empty and no material was in screw. Then we discarded 5 transition shots, and then started collecting the next formulation.

3) *Can use for $\mathrm{T} 1=$ injection time $=8$ seconds, $\mathrm{T} 2=\mathrm{Cool}$ time $=16$ seconds, and $\mathrm{T} 3=$ time between shots $=0.1$ seconds for all inj molding done on Dec 4, 2009. This reduces cycle time.

4) Did not record P-5-1 values until started BA4L formulation.

5) Formulation BA10L did not mold 'pretty samples' but it was the best we could do. Formulation is too viscous.

6) Formulation BA8L was starting to get much more viscous too. We had to make several temperature changes, etc.

7) $\dagger$ is injection molding date 


\section{Appendix B.3: Polycarbonate with Graphite Nanoplatelets}

Table B.3: Injection Molding conditions for GNP/PC composites

\begin{tabular}{|c|c|c|c|c|c|c|}
\hline Notation & $\begin{array}{l}\text { Injection Molding } \\
\text { Conditions }\end{array}$ & BL3 & BLE3 & BG2L & BG3L & BG4L \\
\hline $\mathrm{T}_{\text {mold }}$ & Mold Temperature (F) & 160 & 160 & 160 & 160 & 160 \\
\hline E1 & $\begin{array}{l}\text { Zone } 1 \text { Temperature }(\mathrm{F}) \\
\text { (nozzle) }\end{array}$ & 610 & 610 & 610 & 610 & 610 \\
\hline E2 & Zone 2 Temperature $(\mathrm{F})$ & 570 & 570 & 570 & 570 & 570 \\
\hline E3 & Zone 3 Temperature $(\mathrm{F})$ & 554 & 554 & 554 & 554 & 554 \\
\hline E4 & $\begin{array}{l}\text { Zone } 4 \text { Temperature }(\mathrm{F}) \\
\text { (feed zone) }\end{array}$ & 536 & 536 & 536 & 536 & 536 \\
\hline $\mathrm{P} 1$ & Injection pressure (psi) & $\begin{aligned} & 70 \% \\
= & 15,827\end{aligned}$ & $\begin{aligned} & 70 \% \\
= & 15,827\end{aligned}$ & $\begin{aligned} & 70 \% \\
= & 15,827\end{aligned}$ & $\begin{aligned} & 70 \% \\
= & 15,827\end{aligned}$ & $\begin{aligned} & 70 \% \\
= & 15,827\end{aligned}$ \\
\hline $\mathrm{P} 2$ & Hold Pressure (psi) & $\begin{array}{l}55 \%= \\
12,436\end{array}$ & $\begin{array}{l}55 \%= \\
12,436\end{array}$ & $\begin{array}{l}55 \%= \\
12,436\end{array}$ & $\begin{array}{l}55 \%= \\
12,436\end{array}$ & $\begin{array}{l}55 \%= \\
12,436\end{array}$ \\
\hline P7 & Back Pressure (psi) & $\begin{array}{c}0.2 \%= \\
45.2\end{array}$ & $\begin{array}{c}0.2 \%= \\
45.2\end{array}$ & $\begin{array}{c}0.2 \%= \\
45.2\end{array}$ & $\begin{array}{c}0.2 \%= \\
45.2\end{array}$ & $\begin{array}{c}0.2 \%= \\
45.2\end{array}$ \\
\hline S1 & Shot size $(\mathrm{mm})$ & 20 & 20 & 20 & 20 & 20 \\
\hline S2 & Pullback before (mm) & 0 & $\overline{0}$ & 0 & 0 & $\overline{0}$ \\
\hline S3 & Pullback after (mm) & 0.1 & 0.1 & 0.1 & 0.1 & 0.1 \\
\hline S6 & Width of mold (mm) & 202 & 202 & 202 & 202 & 202 \\
\hline S8 & $\begin{array}{l}\text { Screw Position to Switch } \\
\text { from P1 to P2 (mm) }\end{array}$ & $\begin{array}{c}8 \text { for } \\
\text { tensile, } 10 \\
\text { for flex, } \\
12 \text { for disk }\end{array}$ & $\begin{array}{c}8 \text { for } \\
\text { tensile, } 10 \\
\text { for flex, } \\
12 \text { for disk }\end{array}$ & $\begin{array}{c}8 \text { for } \\
\text { tensile, } 10 \\
\text { for flex, } \\
12 \text { for disk }\end{array}$ & $\begin{array}{c}8 \text { for } \\
\text { tensile, } 10 \\
\text { for flex, } \\
12 \text { for disk }\end{array}$ & $\begin{array}{c}8 \text { for } \\
\text { tensile, } 10 \\
\text { for flex, } \\
12 \text { for disk }\end{array}$ \\
\hline T1 & Injection Time (s) & 8 & 8 & 8 & 8 & 8 \\
\hline $\mathrm{T} 2$ & Cool Time (s) & 16 & 16 & 16 & 16 & 16 \\
\hline T3 & Interval Time (s) & 0.1 & 0.1 & 0.1 & 0.1 & 0.1 \\
\hline T6 & Retraction Time (s) & 0 & 0 & 0 & 0 & 0 \\
\hline $\mathrm{T} 7$ & $\begin{array}{c}\text { Nozzle Retraction Delay } \\
\text { Time (s) }\end{array}$ & 0 & 0 & 0 & 0 & 0 \\
\hline T8 & Injection Delay Time (s) & 0 & 0 & 0 & 0 & 0 \\
\hline T9 & Charge Delay Time (s) & 0 & 0 & 0 & 0 & 0 \\
\hline V1 & Injection Velocity $\left(\mathrm{in}^{3} / \mathrm{s}\right)$ & $\begin{array}{c}30 \%= \\
1.866\end{array}$ & $\begin{array}{c}30 \%= \\
1.866\end{array}$ & $\begin{array}{c}30 \%= \\
1.866\end{array}$ & $\begin{array}{c}30 \%= \\
1.866\end{array}$ & $\begin{array}{c}30 \%= \\
1.866\end{array}$ \\
\hline V6 & Screw Rotation (rpm) & $19 \%=60$ & $19 \%=60$ & $19 \%=60$ & $19 \%=60$ & $19 \%=60$ \\
\hline V9 & Retraction Velocity (\%) & 20 & 20 & 20 & 20 & 20 \\
\hline V10 & Advance Velocity (\%) & 50 & 50 & 50 & 50 & 50 \\
\hline V11 & Retraction Velocity (\%) & 90 & 90 & 90 & 90 & 90 \\
\hline
\end{tabular}


Table B.3: Injection Molding conditions for GNP/PC composites continued

\begin{tabular}{|c|c|c|c|c|c|c|}
\hline Notation & $\begin{array}{l}\text { Injection Molding } \\
\text { Conditions }\end{array}$ & BL3 & BLE3 & BG2L & BG3L & BG4L \\
\hline $\mathrm{CF}$ & Clamp For & 80 & 80 & 80 & 80 & 80 \\
\hline P-5-1 & Actual Cycle Time, sec & $\begin{array}{c}30.6 \\
\text { tensile } \\
30.6 \text { flex } \\
30.8 \text { disk }\end{array}$ & $\begin{array}{c}30.9 \\
\text { tensile } \\
30.9 \text { flex } \\
31.0 \text { disk }\end{array}$ & \begin{tabular}{|c}
31.1 \\
tensile \\
31.1 flex \\
31.1 disk
\end{tabular} & $\begin{array}{c}31.3 \\
\text { tensile } \\
31.1 \text { flex } \\
31.1 \text { disk }\end{array}$ & $\begin{array}{c}31.5 \\
\text { tensile } \\
31.5 \text { flex } \\
31.5 \text { disk }\end{array}$ \\
\hline P-5-1 & Inj Start, mm & $\begin{array}{c}20.1 \\
\text { tensile } \\
20.1 \text { flex } \\
20.2 \text { disk }\end{array}$ & $\begin{array}{c}20.3 \\
\text { tensile } \\
20.7 \text { flex } \\
20.7 \text { disk }\end{array}$ & $\begin{array}{c}20.3 \\
\text { tensile } \\
20.8 \text { flex } \\
20.9 \text { disk }\end{array}$ & $\begin{array}{c}20.8 \\
\text { tensile } \\
20.8 \text { flex } \\
20.9 \text { disk }\end{array}$ & $\begin{array}{c}20.8 \\
\text { tensile } \\
20.8 \text { flex } \\
20.9 \text { disk }\end{array}$ \\
\hline P-5-1 & Cushion, mm & $\begin{array}{c}1.2 \text { tensile } \\
3.0 \text { flex } \\
2.0 \text { disk }\end{array}$ & $\begin{array}{c}1.9 \text { tensile } \\
3.6 \text { flex } \\
2.2 \text { disk }\end{array}$ & $\begin{array}{c}1.5 \text { tensile } \\
3.8 \text { flex } \\
2.9 \text { disk }\end{array}$ & $\begin{array}{c}2.2 \text { tensile } \\
3.9 \text { flex } \\
3.3 \text { disk }\end{array}$ & $\begin{array}{c}2.1 \text { tensile } \\
3.9 \text { flex } \\
3.1 \text { disk }\end{array}$ \\
\hline P-5-1 & $\mathrm{P} 1 \rightarrow \mathrm{P} 2$ Fill time, sec & $\begin{array}{c}0.68 \\
\text { tensile } \\
0.58 \text { flex } \\
0.48 \text { disk }\end{array}$ & $\begin{array}{c}0.70 \\
\text { tensile } \\
0.60 \text { flex } \\
0.48 \text { disk }\end{array}$ & $\begin{array}{c}0.68 \\
\text { tensile } \\
0.61 \text { flex } \\
0.53 \text { disk }\end{array}$ & $\begin{array}{c}0.70 \\
\text { tensile } \\
0.63 \text { flex } \\
0.55 \text { disk }\end{array}$ & $\begin{array}{c}0.70 \\
\text { tensile } \\
0.66 \text { flex } \\
0.51 \text { disk }\end{array}$ \\
\hline P-5-1 & $\begin{array}{c}\text { P1 Actual Pressure P1 } \rightarrow \\
\text { P2 }, \mathrm{kg} / \mathrm{cm}^{2}\end{array}$ & $\begin{array}{c}52 \text { tensile } \\
46 \text { flex } \\
36 \text { disk }\end{array}$ & $\begin{array}{c}55 \text { tensile } \\
49 \text { flex } \\
40 \text { disk }\end{array}$ & $\begin{array}{c}57 \text { tensile } \\
51 \text { flex } \\
40 \text { disk }\end{array}$ & $\begin{array}{c}61 \text { tensile } \\
60 \text { flex } \\
58 \text { disk }\end{array}$ & $\begin{array}{c}63 \text { tensile } \\
61 \text { flex } \\
50 \text { disk }\end{array}$ \\
\hline P-5-1 & Charge time, sec & $\begin{array}{c}4.1 \text { tensile } \\
3.3 \text { flex } \\
3.5 \text { disk }\end{array}$ & $\begin{array}{c}3.4 \text { tensile } \\
3.1 \text { flex } \\
3.5 \text { disk }\end{array}$ & $\begin{array}{c}3.6 \text { tensile } \\
3.2 \text { flex } \\
3.3 \text { disk }\end{array}$ & $\begin{array}{c}4.0 \text { tensile } \\
3.6 \text { flex } \\
3.5 \text { disk }\end{array}$ & $\begin{array}{c}4.0 \text { tensile } \\
4.0 \text { flex } \\
4.0 \text { disk }\end{array}$ \\
\hline P-5-1 & $\begin{array}{c}\text { Injection Peak Pressure P1, } \\
\mathrm{kg} / \mathrm{cm}^{2}\end{array}$ & -- & -- & -- & 83 tensile & $\begin{array}{c}99 \text { tensile } \\
96 \text { flex } \\
50 \text { disk }\end{array}$ \\
\hline
\end{tabular}


Table B.3: Injection Molding conditions for GNP/PC composites continued

\begin{tabular}{|c|c|c|c|c|c|}
\hline Notation & $\begin{array}{c}\text { Injection Molding } \\
\text { Conditions }\end{array}$ & BG5L & BG6L & BG8L & BG10L \\
\hline $\mathrm{T}_{\text {mold }}$ & Mold Temperature (F) & 160 & 160 & 180 & 200 \\
\hline E1 & $\begin{array}{c}\text { Zone } 1 \text { Temperature }(\mathrm{F}) \\
\text { (nozzle) }\end{array}$ & 610 & 620 & $\begin{array}{|lr|}\text { Tensile } & 630 \\
\text { Flex, disk } & 620 \\
\end{array}$ & 630 \\
\hline E2 & Zone 2 Temperature (F) & $\begin{array}{c}\text { Tensile } 580 \\
\text { Flex, disk } \\
570\end{array}$ & 590 & $\begin{array}{lr}\text { Tensile } & 630 \\
\text { Flex, disk } & 590\end{array}$ & 630 \\
\hline E3 & Zone 3 Temperature (F) & 554 & 570 & $\begin{array}{c}\text { Tensile } 600 \\
\text { Flex, disk } 570\end{array}$ & 610 \\
\hline E4 & $\begin{array}{c}\text { Zone } 4 \text { Temperature (F) } \\
\text { (feed zone) }\end{array}$ & 536 & 536 & $\begin{array}{c}\text { Tensile } 550 \\
\text { Flex, disk } 536\end{array}$ & 550 \\
\hline $\mathrm{P} 1$ & Injection pressure (psi) & $\begin{aligned} & 70 \% \\
= & 15,827\end{aligned}$ & $\begin{aligned} & 75 \% \\
= & 16,958\end{aligned}$ & $\begin{array}{c}\text { Tensile } 90 \% \\
=20,349 \\
\text { Flex, disks } \\
75 \%=16,958\end{array}$ & $\begin{aligned} & 99 \% \\
= & 22,384\end{aligned}$ \\
\hline P2 & Hold Pressure (psi) & $\begin{array}{l}55 \%= \\
12,436\end{array}$ & $\begin{array}{l}55 \%= \\
12,436\end{array}$ & $55 \%=12,436$ & $\begin{array}{l}55 \%= \\
12,436\end{array}$ \\
\hline P7 & Back Pressure (psi) & $0.2 \%=45.2$ & $0.5 \%=113$ & $0.5 \%=113$ & $0.5 \%=113$ \\
\hline S1 & Shot size $(\mathrm{mm})$ & 20 & 20 & 20 & 20 \\
\hline S2 & Pullback before (mm) & 0 & 0 & 0 & 0 \\
\hline S3 & Pullback after (mm) & 0.1 & 0.1 & 0.1 & 0.1 \\
\hline S6 & Width of mold (mm) & 202 & 202 & 202 & 202 \\
\hline S8 & $\begin{array}{l}\text { Screw Position to Switch } \\
\text { from P1 to P2 (mm) }\end{array}$ & $\begin{array}{c}8 \text { for tensile, } \\
10 \text { for flex, } \\
12 \text { for disk }\end{array}$ & \begin{tabular}{|c|}
8 for \\
tensile, 10 \\
for flex, 12 \\
for disk \\
\end{tabular} & $\begin{array}{c}8 \text { for tensile, } 10 \\
\text { for flex, } 12 \text { for } \\
\text { disk }\end{array}$ & \begin{tabular}{|c|}
8 for \\
tensile, 10 \\
for flex, 12 \\
for disk \\
\end{tabular} \\
\hline $\mathrm{T} 1$ & Injection Time (s) & 8 & 8 & 8 & 8 \\
\hline $\mathrm{T} 2$ & Cool Time (s) & 16 & 16 & 16 & 16 \\
\hline T3 & Interval Time (s) & 0.1 & 0.1 & 0.1 & 0.1 \\
\hline T6 & Retraction Time (s) & 0 & 0 & 0 & 0 \\
\hline $\mathrm{T} 7$ & $\begin{array}{c}\text { Nozzle Retraction Delay } \\
\text { Time (s) }\end{array}$ & 0 & 0 & 0 & 0 \\
\hline T8 & Injection Delay Time (s) & 0 & 0 & 0 & 0 \\
\hline T9 & Charge Delay Time (s) & 0 & 0 & 0 & 0 \\
\hline V1 & Injection Velocity $\left(\mathrm{in}^{3} / \mathrm{s}\right)$ & $30 \%=1.866$ & $\begin{array}{c}30 \%= \\
1.866\end{array}$ & $\begin{array}{c}\text { Tensile } 10 \%= \\
0.622 \\
\text { Flex, disk } 30 \% \\
=1.866\end{array}$ & $\begin{array}{l}10 \%= \\
0.622\end{array}$ \\
\hline V6 & Screw Rotation (rpm) & $19 \%=60$ & $19 \%=60$ & $19 \%=60$ & $19 \%=60$ \\
\hline V9 & Retraction Velocity (\%) & 20 & 20 & 20 & 20 \\
\hline
\end{tabular}


Table B.3: Injection Molding conditions for GNP/PC composites continued

\begin{tabular}{|c|c|c|c|c|c|}
\hline Notation & $\begin{array}{l}\text { Injection Molding } \\
\text { Conditions }\end{array}$ & BG5L & BG6L & BG8L & BG10L \\
\hline V10 & Advance Velocity (\%) & 50 & 50 & 50 & 50 \\
\hline V11 & $\begin{array}{c}\text { Retraction Velocity } \\
(\%)\end{array}$ & 90 & 90 & 90 & 90 \\
\hline $\mathrm{CF}$ & Clamp Force (US tons) & 80 & 80 & 80 & 80 \\
\hline P-5-1 & $\begin{array}{l}\text { Actual Cycle Time, } \\
\text { sec }\end{array}$ & $\begin{array}{c}30.4 \text { tensile } \\
31.8 \text { flex } \\
31.6 \text { disk }\end{array}$ & $\begin{array}{c}30.7 \text { tensile } \\
30.6 \text { flex } \\
30.6 \text { disk }\end{array}$ & $\begin{array}{c}30.5 \text { tensile } \\
30.7 \text { flex } \\
30.6 \text { disk }\end{array}$ & $\begin{array}{c}30.7 \text { tensile } \\
30.4 \text { flex } \\
30.4 \text { disk }\end{array}$ \\
\hline P-5-1 & Inj Start, mm & $\begin{array}{c}20.8 \text { tensile } \\
20.8 \text { flex } \\
20.9 \text { disk }\end{array}$ & $\begin{array}{c}20.5 \text { tensile } \\
20.7 \text { flex } \\
20.6 \text { disk }\end{array}$ & $\begin{array}{c}20.9 \text { tensile } \\
20.9 \text { flex } \\
20.9 \text { disk }\end{array}$ & $\begin{array}{c}20.7 \text { tensile } \\
20.7 \text { flex } \\
20.7 \text { disk }\end{array}$ \\
\hline P-5-1 & Cushion, mm & $\begin{array}{l}2.1 \text { tensile } \\
3.9 \text { flex } \\
3.1 \text { disk }\end{array}$ & $\begin{array}{c}2.1 \text { tensile } \\
3.5 \text { flex } \\
2.9 \text { disk }\end{array}$ & $\begin{array}{l}1.6 \text { tensile } \\
3.8 \text { flex } \\
2.8 \text { disk }\end{array}$ & $\begin{array}{c}1.6 \text { tensile } \\
3.3 \text { flex } \\
2.5 \text { disk }\end{array}$ \\
\hline$P-5-1$ & $\mathrm{P} 1 \rightarrow \mathrm{P} 2$ Fill time, sec & $\begin{array}{c}1.0 \text { tensile } \\
0.65 \text { flex } \\
0.56 \text { disk }\end{array}$ & $\begin{array}{c}0.70 \text { tensile } \\
0.61 \text { flex } \\
0.51 \text { disk }\end{array}$ & $\begin{array}{c}2.0 \text { tensile } \\
0.6 \text { flex } \\
0.51 \text { disk }\end{array}$ & $\begin{array}{c}1.99 \text { tensile } \\
1.70 \text { flex } \\
1.41 \text { disk }\end{array}$ \\
\hline P-5-1 & $\begin{array}{l}\text { P1 Actual Pressure } \\
\mathrm{P} 1 \rightarrow \mathrm{P} 2, \mathrm{~kg} / \mathrm{cm}^{2}\end{array}$ & $\begin{array}{c}60 \text { tensile } \\
60 \text { flex } \\
48 \text { disk }\end{array}$ & $\begin{array}{c}57 \text { tensile } \\
46 \text { flex } \\
39 \text { disk }\end{array}$ & $\begin{array}{c}34 \text { tensile } \\
50 \text { flex } \\
42 \text { disk }\end{array}$ & $\begin{array}{c}35 \text { tensile } \\
29 \text { flex } \\
22 \text { disk }\end{array}$ \\
\hline P-5-1 & Charge time, sec & $\begin{array}{c}3.3 \text { tensile } \\
2.9 \text { flex } \\
3.0 \text { disk }\end{array}$ & $\begin{array}{l}3.8 \text { tensile } \\
3.2 \text { flex } \\
3.4 \text { disk }\end{array}$ & $\begin{array}{c}3.2 \text { tensile } \\
3.2 \text { flex } \\
3.2 \text { disk }\end{array}$ & $\begin{array}{c}3.1 \text { tensile } \\
2.8 \text { flex } \\
2.9 \text { disk }\end{array}$ \\
\hline P-5-1 & $\begin{array}{c}\text { Injection Peak } \\
\text { Pressure P1, } \mathrm{kg} / \mathrm{cm}^{2}\end{array}$ & $\begin{array}{c}98 \text { tensile } \\
56 \text { flex } \\
46 \text { disk }\end{array}$ & $\begin{array}{c}57 \text { tensile } \\
46 \text { flex } \\
39 \text { disk }\end{array}$ & $\begin{array}{c}34 \text { tensile } \\
50 \text { flex } \\
42 \text { disk }\end{array}$ & $\begin{array}{c}35 \text { tensile } \\
29 \text { flex } \\
22 \text { disk }\end{array}$ \\
\hline
\end{tabular}

Notes:

1) For all formulations, about 302.5 " diameter disks, 30 tensile bars, and 20 flexural bars molded. Each sample was individually molded. The injection molding machine ran on the fully automatic mode.

2) After each formulation, the feed hopper was run until it was empty and no material was in screw. Then we discarded 5 transition shots, and then started collecting the next formulation.

3) If injection mold again, try different conditions for BG5L and BG6L. Maybe increase P1 to $90 \%$, etc. 


\section{Appendix B.4: Polycarbonate with Multiple Fillers}

Table B.4: Injection Molding Conditions for Combinations in PC

\begin{tabular}{|c|c|c|c|c|}
\hline Notation & $\begin{array}{l}\text { Injection Molding } \\
\text { Conditions }\end{array}$ & BL4 & $\mathrm{BQ} 0.5 \mathrm{~L}$ & BQ1L \\
\hline $\mathrm{T}_{\text {mold }}$ & Mold Temperature $(\mathrm{F})$ & 160 & 160 & 160 \\
\hline E1 & $\begin{array}{c}\text { Zone } 1 \text { Temperature }(\mathrm{F}) \\
\text { (nozzle) }\end{array}$ & 610 & 610 & 610 \\
\hline E2 & Zone 2 Temperature $(\mathrm{F})$ & 570 & 570 & 570 \\
\hline E3 & Zone 3 Temperature $(\mathrm{F})$ & 554 & 554 & 554 \\
\hline E4 & $\begin{array}{c}\text { Zone } 4 \text { Temperature }(\mathrm{F}) \\
\text { (feed zone) }\end{array}$ & 536 & 536 & 536 \\
\hline P1 & Injection pressure (psi) & $70 \%=15,827$ & $70 \%=15,827$ & $70 \%=15,827$ \\
\hline $\mathrm{P} 2$ & Hold Pressure (psi) & $55 \%=12,436$ & $55 \%=12,436$ & $55 \%=12,436$ \\
\hline P7 & Back Pressure (psi) & $0.2 \%=45.2$ & $0.2 \%=45.2$ & $0.2 \%=45.2$ \\
\hline S1 & Shot size $(\mathrm{mm})$ & 20 & 20 & 20 \\
\hline S2 & Pullback before (mm) & 0 & 0 & 0 \\
\hline S3 & Pullback after (mm) & 0.1 & 0.1 & 0.1 \\
\hline S6 & Width of mold (mm) & 202 & 202 & 202 \\
\hline S8 & $\begin{array}{l}\text { Screw Position to Switch } \\
\text { from P1 to P2 (mm) }\end{array}$ & $\begin{array}{c}8 \text { for tensile, } 10 \\
\text { for flex, } 12 \text { for } \\
\text { disk }\end{array}$ & $\begin{array}{l}8 \text { for tensile, } \\
10 \text { for flex, } 12 \\
\text { for disk }\end{array}$ & $\begin{array}{c}8 \text { for tensile, } 10 \\
\text { for flex, } 12 \text { for } \\
\text { disk }\end{array}$ \\
\hline $\mathrm{T} 1$ & Injection Time (s) & 8 & 8 & 8 \\
\hline $\mathrm{T} 2$ & Cool Time (s) & 16 & 16 & 16 \\
\hline $\mathrm{T} 3$ & Interval Time (s) & 0.1 & 0.1 & 0.1 \\
\hline T6 & Retraction Time (s) & 0 & 0 & 0 \\
\hline $\mathrm{T} 7$ & $\begin{array}{c}\text { Nozzle Retraction Delay } \\
\text { Time (s) }\end{array}$ & 0 & 0 & 0 \\
\hline $\mathrm{T} 8$ & Injection Delay Time (s) & 0 & 0 & 0 \\
\hline T9 & Charge Delay Time (s) & 0 & 0 & 0 \\
\hline V1 & Injection Velocity $\left(\mathrm{in}^{3} / \mathrm{s}\right)$ & $30 \%=1.866$ & $\begin{array}{c}20 \% \text { for disk, } \\
30 \% \text { for rest }\end{array}$ & $\begin{array}{c}20 \% \text { for disk } \\
30 \%=1.866 \text { for } \\
\text { tensile, flex }\end{array}$ \\
\hline V6 & Screw Rotation (rpm) & $19 \%=60$ & $19 \%=60$ & $19 \%=60$ \\
\hline V9 & Retraction Velocity (\%) & 20 & 20 & 20 \\
\hline V10 & Advance Velocity (\%) & 50 & 50 & 50 \\
\hline V11 & Retraction Velocity (\%) & 90 & 90 & 90 \\
\hline $\mathrm{CF}$ & Clamp Force (US tons) & 80 & 80 & 80 \\
\hline
\end{tabular}


Table B.4: Injection Molding Conditions for Combinations in PC continued

\begin{tabular}{|c|c|c|c|c|}
\hline & Injection Molding & & & \\
& Conditions & BL4 & BQ0.5L & BQ1L \\
\hline & & 30.8 tensile & 30.9 tensile & 31.1 tensile \\
P-5-1 & Actual Cycle Time, sec & 30.8 flex & 30.9 flex & 31.1 flex \\
& & 20.1 densile & 20.7 tensile & 20.8 tensile \\
P-5-1 & Inj Start, mm & 20.1 flex & 20.5 flex & 20.8 flex \\
& & 20.1 disk & 20.4 disk & 20.9 disk \\
\hline & & 1.3 tensile & 1.6 tensile & 1.9 tensile \\
P-5-1 & Cushion, mm & 2.0 flex & 3.3 flex & 3.8 flex \\
& & 0.68 & 2.9 disk & 2.9 disk \\
& & tensile & 0.70 tensile & 0.68 tensile \\
& & 0.58 flex & 0.60 flex & 0.61 flex \\
P-5-1 & P1 P2 Fill time, sec & 0.50 disk & 0.73 disk & 0.73 disk \\
\hline & & 73 tensile & 67 tensile & 64 tensile \\
& P1 Actual Pressure P1 $\rightarrow$ & 64 flex & 63 flex & 59 flex \\
P-5-1 & P2, $\mathrm{kg} / \mathrm{cm}^{2}$ & 63 disk & 49 disk & 47 disk \\
\hline & & 3.9 tensile & 3.6 tensile & 3.6 tensile \\
& & 3.9 flex & 3.1 flex & 3.2 flex \\
P-5-1 & Charge time, sec & 5.1 disk & 3.1 disk & 3.3 disk \\
\hline & & 73 tensile & 67 tensile & 64 tensile \\
& Injection Peak Pressure P1, & 64 flex & 63 flex & 59 flex \\
P-5-1 & kg/cm ${ }^{2}$ & 63 disk & 49 disk & 47 disk \\
\hline
\end{tabular}


Table B.4: Injection Molding Conditions for Combinations in PC continued

\begin{tabular}{|c|c|c|c|c|}
\hline Notation & $\begin{array}{l}\text { Injection Molding } \\
\text { Conditions }\end{array}$ & $\begin{array}{l}\text { BA2LR } \\
7-26-10^{*}\end{array}$ & $\begin{array}{l}\text { BA5LR } \\
7-26-10 *\end{array}$ & $\begin{array}{l}\text { BA2Q1L } \\
7-26-10 *\end{array}$ \\
\hline $\mathrm{T}_{\text {mold }}$ & Mold Temperature $(\mathrm{F})$ & 160 & 160 & 160 \\
\hline E1 & $\begin{array}{c}\text { Zone } 1 \text { Temperature }(\mathrm{F}) \\
\text { (nozzle) }\end{array}$ & 610 & 610 & 610 \\
\hline E2 & Zone 2 Temperature (F) & 570 & 570 & 570 \\
\hline E3 & Zone 3 Temperature (F) & 554 & 554 & 554 \\
\hline E4 & $\begin{array}{c}\text { Zone } 4 \text { Temperature }(\mathrm{F}) \\
\text { (feed zone) }\end{array}$ & 536 & 536 & 536 \\
\hline $\mathrm{P} 1$ & Injection pressure (psi) & $70 \%=15,827$ & $75 \%=16,958$ & $75 \%=16,958$ \\
\hline $\mathrm{P} 2$ & Hold Pressure (psi) & $55 \%=12,436$ & $55 \%=12,436$ & $55 \%=12,436$ \\
\hline P7 & Back Pressure (psi) & $0.2 \%=45.2$ & $0.2 \%=45.2$ & $0.2 \%=45.2$ \\
\hline S1 & Shot size $(\mathrm{mm})$ & 20 & 20 & 20 \\
\hline S2 & Pullback before (mm) & 0 & 0 & 0 \\
\hline S3 & Pullback after (mm) & 0.1 & 0.1 & 0.1 \\
\hline S6 & Width of mold (mm) & 202 & 202 & 202 \\
\hline S8 & $\begin{array}{l}\text { Screw Position to Switch } \\
\text { from P1 to P2 (mm) }\end{array}$ & $\begin{array}{l}8 \text { for tensile, } \\
10 \text { for flex, } 12 \\
\text { for disk }\end{array}$ & $\begin{array}{c}8 \text { for tensile, } 10 \\
\text { for flex, } 12 \text { for } \\
\text { disk }\end{array}$ & $\begin{array}{c}8 \text { for tensile, } 10 \\
\text { for flex, } 12 \text { for } \\
\text { disk }\end{array}$ \\
\hline $\mathrm{T} 1$ & Injection Time (s) & 8 & 8 & 8 \\
\hline $\mathrm{T} 2$ & Cool Time (s) & 16 & 16 & 16 \\
\hline $\mathrm{T} 3$ & Interval Time (s) & 0.1 & 0.1 & 0.1 \\
\hline T6 & Retraction Time (s) & 0 & 0 & 0 \\
\hline $\mathrm{T} 7$ & $\begin{array}{c}\text { Nozzle Retraction Delay } \\
\text { Time (s) }\end{array}$ & 0 & 0 & 0 \\
\hline $\mathrm{T} 8$ & Injection Delay Time (s) & 0 & 0 & 0 \\
\hline T9 & Charge Delay Time (s) & 0 & 0 & 0 \\
\hline V1 & Injection Velocity $\left(\mathrm{in}^{3} / \mathrm{s}\right)$ & $30 \%=1.866$ & $30 \%=1.866$ & $30 \%=1.866$ \\
\hline V6 & Screw Rotation (rpm) & $19 \%=60$ & $19 \%=60$ & $19 \%=60$ \\
\hline V9 & Retraction Velocity (\%) & 20 & 20 & 20 \\
\hline V10 & Advance Velocity (\%) & 50 & 50 & 50 \\
\hline V11 & Retraction Velocity (\%) & 90 & 90 & 90 \\
\hline $\mathrm{CF}$ & Clamp Force (US tons) & 80 & 80 & 80 \\
\hline
\end{tabular}


Table B.4: Injection Molding Conditions for Combinations in PC continued

\begin{tabular}{|c|c|c|c|c|}
\hline Notation & $\begin{array}{l}\text { Injection Molding } \\
\text { Conditions }\end{array}$ & $\begin{array}{l}\text { BA2LR } \\
7-26-10^{*}\end{array}$ & $\begin{array}{l}\text { BA5LR } \\
7-26-10^{*}\end{array}$ & $\begin{array}{l}\text { BA2Q1L } \\
7-26-10 *\end{array}$ \\
\hline P-5-1 & Actual Cycle Time, sec & $\begin{array}{c}30.9 \text { tensile } \\
30.7 \text { flex } \\
30.6 \text { disk }\end{array}$ & $\begin{array}{c}30.7 \text { tensile } \\
30.8 \text { flex } \\
30.6 \text { disk }\end{array}$ & $\begin{array}{c}30.9 \text { tensile } \\
30.7 \text { flex } \\
30.6 \text { disk }\end{array}$ \\
\hline P-5-1 & Inj Start, mm & $\begin{array}{c}30.9 \text { tensile } \\
30.7 \text { flex } \\
30.6 \text { disk }\end{array}$ & $\begin{array}{c}30.7 \text { tensile } \\
30.8 \text { flex } \\
30.6 \text { disk }\end{array}$ & $\begin{array}{c}30.9 \text { tensile } \\
30.7 \text { flex } \\
30.6 \text { disk }\end{array}$ \\
\hline P-5-1 & Cushion, mm & $\begin{array}{c}20.8 \text { tensile } \\
20.8 \text { flex } \\
20.9 \text { disk }\end{array}$ & $\begin{array}{c}20.0 \text { tensile } \\
20.0 \text { flex } \\
20.0 \text { disk }\end{array}$ & $\begin{array}{c}20.8 \text { tensile } \\
20.8 \text { flex } \\
20.9 \text { disk }\end{array}$ \\
\hline P-5-1 & $\mathrm{P} 1 \rightarrow \mathrm{P} 2$ Fill time, sec & $\begin{array}{c}2.0 \text { tensile } \\
3.9 \text { flex } \\
3.3 \text { disk }\end{array}$ & $\begin{array}{l}1.7 \text { tensile } \\
3.1 \mathrm{flex} \\
2.7 \mathrm{disk}\end{array}$ & $\begin{array}{c}1.6 \text { tensile } \\
3.3 .3 \text { flex } \\
2.5 \text { disk }\end{array}$ \\
\hline P-5-1 & $\begin{array}{c}\text { P1 Actual Pressure P1 } \rightarrow \\
\text { P2, } \mathrm{kg} / \mathrm{cm}^{2}\end{array}$ & $\begin{array}{c}0.70 \text { tensile } \\
0.60 \text { flex } \\
0.51 \text { disk }\end{array}$ & $\begin{array}{c}0.70 \text { tensile } \\
0.61 \text { flex } \\
0.51 \text { disk }\end{array}$ & $\begin{array}{c}0.70 \text { tensile } \\
0.60 \text { flex } \\
0.51 \text { disk }\end{array}$ \\
\hline P-5-1 & Charge time, sec & $\begin{array}{l}70 \text { tensile } \\
65 \text { flex } \\
58 \text { disk }\end{array}$ & $\begin{array}{l}81 \text { tensile } \\
71 \text { flex } \\
63 \text { disk }\end{array}$ & $\begin{array}{l}52 \text { tensile } \\
44 \text { flex } \\
38 \text { disk }\end{array}$ \\
\hline P-5-1 & $\begin{array}{l}\text { Injection Peak Pressure P1, } \\
\qquad \mathrm{kg} / \mathrm{cm}^{2}\end{array}$ & $\begin{array}{c}4.0 \text { tensile } \\
3.6 \text { flex } \\
3.5 \text { disk }\end{array}$ & $\begin{array}{l}5.5 \text { tensile } \\
5.3 \text { flex } \\
4.1 \text { disk }\end{array}$ & $\begin{array}{l}3.4 \text { tensile } \\
2.9 \text { flex } \\
3.2 \text { disk }\end{array}$ \\
\hline P-5-1 & Inj Start, mm & $\begin{array}{l}70 \text { tensile } \\
65 \text { flex } \\
58 \text { disk }\end{array}$ & $\begin{array}{c}81 \text { tensile } \\
71 \text { flex } \\
63 \text { disk }\end{array}$ & $\begin{array}{l}52 \text { tensile } \\
44 \text { flex } \\
38 \text { disk }\end{array}$ \\
\hline
\end{tabular}


Table B.4: Injection Molding Conditions for Combinations in PC continued

\begin{tabular}{|c|c|c|c|c|}
\hline Notation & Injection Molding Conditions & \begin{tabular}{|c|} 
BA5Q1L \\
$7-26-10$ for disk \\
and flex*
\end{tabular} & $\begin{array}{c}\text { BA5Q1L } \\
\text { 8-25-10 and } \\
\text { 8-6-10 tensile } \\
\text { only* }\end{array}$ & $\begin{array}{c}\text { BA2Q5L } \\
8-6-10 \text { and } \\
8-25-10 *\end{array}$ \\
\hline $\mathrm{T}_{\text {mold }}$ & Mold Temperature (F) & 160 & 160 & 160 \\
\hline E1 & $\begin{array}{l}\text { Zone } 1 \text { Temperature }(\mathrm{F}) \\
\text { (nozzle) }\end{array}$ & 610 & 590 & 590 \\
\hline E2 & Zone 2 Temperature $(\mathrm{F})$ & 570 & 570 & 570 \\
\hline E3 & Zone 3 Temperature $(\mathrm{F})$ & 554 & 554 & 554 \\
\hline E4 & $\begin{array}{c}\text { Zone } 4 \text { Temperature (F) (feed } \\
\text { zone) }\end{array}$ & 536 & 536 & 536 \\
\hline P1 & Injection pressure (psi) & $75 \%=16,958$ & $\begin{array}{c}75 \%=16,958, \\
80 \%=18,088 \\
\text { worked too }\end{array}$ & $\begin{array}{c}\text { Tensile, flex } 75 \% \\
=16,958 \\
\text { disks } 50 \%=11,305\end{array}$ \\
\hline P2 & Hold Pressure (psi) & $55 \%=12,436$ & $55 \%=12,436$ & $\begin{aligned} & 55 \%= 12,436 \text { flex } \\
& \text { tensile } \\
& 45 \%=10,175 \text { disks }\end{aligned}$ \\
\hline P7 & Back Pressure (psi) & $0.2 \%=45.2$ & $0.2 \%$ or $5 \%$ & \begin{tabular}{|c|} 
Set point $5.0 \%$ \\
$=1100 \mathrm{but}$ actual \\
was $1 \mathrm{kgf} / \mathrm{cm}^{2}$
\end{tabular} \\
\hline S1 & Shot size $(\mathrm{mm})$ & 20 & 22 & 22 \\
\hline S2 & Pullback before (mm) & 0 & 0 & 0 \\
\hline S3 & Pullback after (mm) & 0.1 & 0.4 & 0.4 \\
\hline S6 & Width of mold (mm) & 202 & 202 & 202 \\
\hline S8 & $\begin{array}{l}\text { Screw Position to Switch } \\
\text { from P1 to P2 (mm) }\end{array}$ & $\begin{array}{l}10 \text { for flex, } 12 \\
\text { for disk }\end{array}$ & 7 for tensile & $\begin{array}{l}7 \text { for tensile, } 10 \text { for } \\
\text { flex, } 12 \text { for disk }\end{array}$ \\
\hline $\mathrm{T} 1$ & Injection Time (s) & 8 & 8 & 8 \\
\hline $\mathrm{T} 2$ & Cool Time (s) & 16 & 16 or 20 & 16 or 20 \\
\hline T3 & Interval Time (s) & 0.1 & 0.1 & 0.1 \\
\hline T6 & Retraction Time (s) & 0 & 0 & 0 \\
\hline $\mathrm{T} 7$ & $\begin{array}{c}\text { Nozzle Retraction Delay } \\
\text { Time (s) }\end{array}$ & 0 & 0 & 0 \\
\hline $\mathrm{T} 8$ & Injection Delay Time (s) & 0 & 0 & 0 \\
\hline T9 & Charge Delay Time (s) & 0 & 0 & 0 \\
\hline V1 & Injection Velocity $\left(\mathrm{in}^{3} / \mathrm{s}\right)$ & $30 \%=1.866$ & $30 \%=1.866$ & $30 \%=1.866$ \\
\hline V6 & Screw Rotation (rpm) & $19 \%=60$ & $19 \%=60$ & $19 \%=60$ \\
\hline V9 & Retraction Velocity (\%) & 20 & 20 & 20 \\
\hline V10 & Advance Velocity (\%) & 50 & 50 & 50 \\
\hline V11 & Retraction Velocity (\%) & 90 & 90 & 90 \\
\hline $\mathrm{CF}$ & Clamp Force (US tons) & 80 & 80 & 80 \\
\hline
\end{tabular}


Table B.4: Injection Molding Conditions for Combinations in PC continued

\begin{tabular}{|c|c|c|c|c|}
\hline Notation & Injection Molding Conditions & \begin{tabular}{|c|} 
BA5Q1L \\
7-26-10 for disk \\
and flex*
\end{tabular} & $\begin{array}{c}\text { BA5Q1L } \\
\text { 8-25-10 and } \\
\text { 8-6-10 tensile } \\
\text { only* }\end{array}$ & $\begin{array}{l}\text { BA2Q5L } \\
8-6-10 \text { and } \\
8-25-10^{*}\end{array}$ \\
\hline P-5-1 & Actual Cycle Time, sec & $\begin{array}{l}31.8 \text { flex } \\
31.6 \text { disk }\end{array}$ & $\begin{array}{l}35.1 \text { or } 31.3 \\
\text { tensile }\end{array}$ & \begin{tabular}{|c|}
35.1 or 31.3 tensile \\
35.1 flex \\
35.1 disk
\end{tabular} \\
\hline P-5-1 & Inj Start, mm & $\begin{array}{l}20.8 \text { flex } \\
20.9 \text { disk }\end{array}$ & 22.4 tensile & $\begin{array}{l}22.5 \text { tensile } \\
22.5 \text { flex } \\
22.5 \text { disk }\end{array}$ \\
\hline P-5-1 & Cushion, mm & $\begin{array}{l}3.5 \text { flex } \\
2.7 \text { disk }\end{array}$ & 3.5 tensile & $\begin{array}{c}3.3 \text { tensile } \\
5.0 \text { flex } \\
4.0 \text { disk }\end{array}$ \\
\hline P-5-1 & $\mathrm{P} 1 \rightarrow \mathrm{P} 2$ Fill time, sec & $\begin{array}{l}0.6 \text { flex } \\
0.5 \text { disk }\end{array}$ & 0.80 tensile & $\begin{array}{c}0.78 \text { tensile } \\
0.65 \text { flex } \\
0.56 \text { disk }\end{array}$ \\
\hline P-5-1 & $\begin{array}{c}\text { P1 Actual Pressure } \mathrm{P} 1 \rightarrow \mathrm{P} 2 \text {, } \\
\mathrm{kgf} / \mathrm{cm} 2\end{array}$ & $\begin{array}{l}56 \text { flex } \\
48 \text { disk }\end{array}$ & 65 tensile & $\begin{array}{c}42 \text { tensile } \\
33 \text { flex } \\
26 \text { disk }\end{array}$ \\
\hline P-5-1 & Charge time, sec & $\begin{array}{l}3.3 \text { flex } \\
3.5 \text { disk }\end{array}$ & 3.8 tensile & $\begin{array}{l}3.2 \text { tensile } \\
3.2 \text { flex } \\
3.2 \text { disk }\end{array}$ \\
\hline P-5-1 & $\begin{array}{l}\text { Injection Peak Pressure P1, } \\
\qquad \mathrm{kg} / \mathrm{cm} 2\end{array}$ & $\begin{array}{l}56 \text { flex } \\
48 \text { disk }\end{array}$ & 65 tensile & $\begin{array}{l}42 \text { tensile } \\
33 \text { flex } \\
26 \text { disk }\end{array}$ \\
\hline
\end{tabular}


Table B.4: Injection Molding Conditions for Combinations in PC continued

\begin{tabular}{|c|c|c|c|c|}
\hline Notation & Injection Molding Conditions & $\begin{array}{l}\text { BA5Q5L } \\
8-6-10 \text { and } \\
8-25-10^{*}\end{array}$ & $\begin{array}{c}\text { BG2L } \\
\text { 9-9-10Tensile } \\
\text { and flex only* }\end{array}$ & $\begin{array}{c}\text { BG5L } \\
\text { 9-9-10 tensile } \\
\text { and flex* }\end{array}$ \\
\hline $\mathrm{T}_{\text {mold }}$ & Mold Temperature (F) & 160 & 160 & 160 \\
\hline E1 & $\begin{array}{c}\text { Zone } 1 \text { Temperature }(\mathrm{F}) \\
\text { (nozzle) }\end{array}$ & 620 & 610 & 620 \\
\hline $\bar{E} 2$ & Zone 2 Temperature $(\mathrm{F})$ & 590 & 570 & 590 \\
\hline E3 & Zone 3 Temperature $(\mathrm{F})$ & 560 & 554 & 560 \\
\hline E4 & $\begin{array}{c}\text { Zone } 4 \text { Temperature (F) (feed } \\
\text { zone) }\end{array}$ & 550 & 536 & 550 \\
\hline P1 & Injection pressure (psi) & $\begin{array}{c}\text { Tensile, flex } 75 \% \\
=16,958 \\
\text { disks } \\
50 \%=11,305\end{array}$ & $70 \%=15,827$ & $75 \%=16,958$ \\
\hline $\mathrm{P} 2$ & Hold Pressure (psi) & $\begin{array}{c}55 \%=12,436 \\
\text { flex, tensile } \\
45 \%=10,175 \\
\text { disks }\end{array}$ & $55 \%=12,436$ & $55 \%=12,436$ \\
\hline P7 & Back Pressure (psi) & $\begin{array}{c}\text { Set point } 5.0 \% \\
=1100 \text { but actual } \\
\text { was } 1 \mathrm{kgf} / \mathrm{cm}^{2}\end{array}$ & $\begin{array}{c}\text { Set point } 5.0 \% \\
=1100 \mathrm{but} \text { actual } \\
\text { was } 1 \mathrm{kgf} / \mathrm{cm}^{2}\end{array}$ & $\begin{array}{l}\text { Set point } 5.0 \% \\
=1100 \text { but actual } \\
\text { was } 1 \mathrm{kgf} / \mathrm{cm}^{2}\end{array}$ \\
\hline S1 & Shot size $(\mathrm{mm})$ & 22 & 22 & 22 \\
\hline S2 & Pullback before (mm) & 0 & 0 & 0 \\
\hline S3 & Pullback after (mm) & 0.4 & 0.4 & 0.4 \\
\hline S6 & Width of mold (mm) & 202 & 202 & 202 \\
\hline S8 & $\begin{array}{l}\text { Screw Position to Switch } \\
\text { from P1 to P2 (mm) }\end{array}$ & $\begin{array}{l}7 \text { for tensile, } 10 \\
\text { for flex, } 12 \text { for } \\
\text { disk }\end{array}$ & $\begin{array}{l}7 \text { for tensile, } 10 \\
\text { for flex }\end{array}$ & $\begin{array}{c}7 \text { for tensile, } 10 \\
\text { for flex }\end{array}$ \\
\hline T1 & Injection Time (s) & 8 & 8 & 8 \\
\hline $\mathrm{T} 2$ & Cool Time (s) & 24 & 16 & 20 \\
\hline T3 & Interval Time (s) & 0.1 & 0.1 & 0.1 \\
\hline T6 & Retraction Time (s) & 0 & 0 & 0 \\
\hline $\mathrm{T} 7$ & $\begin{array}{c}\text { Nozzle Retraction Delay } \\
\text { Time (s) }\end{array}$ & 0 & 0 & 0 \\
\hline $\mathrm{T} 8$ & Injection Delay Time (s) & 0 & 0 & 0 \\
\hline T9 & Charge Delay Time (s) & 0 & 0 & 0 \\
\hline V1 & Injection Velocity $\left(\mathrm{in}^{3} / \mathrm{s}\right)$ & $\begin{array}{c}55 \%=3.69 \text { tensile } \\
30 \%=1.866 \text { disk } \\
\text { flex }\end{array}$ & $30 \%=1.866$ & $8 \%=0.54$ \\
\hline V6 & Screw Rotation (rpm) & $19 \%=60$ & $19 \%=60$ & $19 \%=60$ \\
\hline
\end{tabular}


Table B.4: Injection Molding Conditions for Combinations in PC continued

\begin{tabular}{|c|c|c|c|c|}
\hline Notation & $\begin{array}{l}\text { Injection Molding } \\
\text { Conditions }\end{array}$ & $\begin{array}{l}\text { BA5Q5L } \\
8-6-10 \text { and } \\
8-25-10^{*}\end{array}$ & $\begin{array}{c}\text { BG2L } \\
\text { 9-9-10 } \\
\text { Tensile and flex } \\
\text { only* }\end{array}$ & $\begin{array}{c}\text { BG5L } \\
\text { 9-9-10 tensile } \\
\text { and flex* }\end{array}$ \\
\hline V9 & Retraction Velocity (\%) & 20 & 20 & 20 \\
\hline V10 & Advance Velocity (\%) & 50 & 50 & 50 \\
\hline V11 & Retraction Velocity (\%) & 90 & 90 & 90 \\
\hline $\mathrm{CF}$ & Clamp Force (US tons) & 80 & 80 & 80 \\
\hline P-5-1 & Actual Cycle Time, sec & $\begin{array}{c}38.7 \text { tensile } \\
38.7 \text { flex } \\
38.7 \text { disk }\end{array}$ & $\begin{array}{l}30.9 \text { tensile } \\
30.6 \text { flex }\end{array}$ & 34.8 \\
\hline P-5-1 & Inj Start, mm & $\begin{array}{c}22.5 \text { tensile } \\
22.5 \text { flex } \\
22.5 \text { disk }\end{array}$ & 22.4 & 22.5 \\
\hline P-5-1 & Cushion, mm & $\begin{array}{c}3.3 \text { tensile } \\
5.0 \text { flex } \\
4.0 \text { disk }\end{array}$ & $\begin{array}{l}3.5 \text { tensile } \\
5.3 \text { flex }\end{array}$ & $\begin{array}{l}3.5 \text { tensile } \\
5.3 \text { flex }\end{array}$ \\
\hline P-5-1 & $\mathrm{P} 1 \rightarrow \mathrm{P} 2$ Fill time, sec & $\begin{array}{c}0.50 \text { tensile } \\
0.65 \text { flex } \\
0.56 \text { disk }\end{array}$ & $\begin{array}{l}0.8 \text { tensile } \\
0.68 \text { flex }\end{array}$ & $\begin{array}{l}3.6 \text { tensile } \\
2.8 \text { flex }\end{array}$ \\
\hline P-5-1 & $\begin{array}{c}\text { P1 Actual Pressure } \mathrm{P} 1 \rightarrow \\
\mathrm{P} 2, \mathrm{~kg}_{\mathrm{f}} / \mathrm{cm}^{2}\end{array}$ & $\begin{array}{l}64 \text { tensile } \\
46 \text { flex } \\
34 \text { disk }\end{array}$ & $\begin{array}{l}67 \text { tensile } \\
54 \text { flex }\end{array}$ & $\begin{array}{l}47 \text { tensile } \\
35 \text { flex }\end{array}$ \\
\hline P-5-1 & Charge time, sec & $\begin{array}{c}3.9 \text { tensile } \\
3.5 \text { flex } \\
3.5 \text { disk }\end{array}$ & $\begin{array}{l}3.5 \text { tensile } \\
3.3 \text { flex }\end{array}$ & $\begin{array}{l}3.3 \text { tensile } \\
2.9 \text { flex }\end{array}$ \\
\hline $\mathrm{P}-5-1$ & $\begin{array}{c}\text { Injection Peak Pressure P1, } \\
\mathrm{kg} / \mathrm{cm}^{2}\end{array}$ & $\begin{array}{c}64 \text { tensile } \\
46 \text { flex } \\
34 \text { disk }\end{array}$ & $\begin{array}{l}67 \text { tensile } \\
54 \text { flex }\end{array}$ & $\begin{array}{l}47 \text { tensile } \\
35 \text { flex }\end{array}$ \\
\hline
\end{tabular}


Table B.4: Injection Molding Conditions for Combinations in PC continued

\begin{tabular}{|c|c|c|c|c|}
\hline Notation & $\begin{array}{l}\text { Injection Molding } \\
\text { Conditions }\end{array}$ & $\begin{array}{c}\text { BG12L } \\
8-18-10^{*}\end{array}$ & $\begin{array}{c}\text { BG15L } \\
8-18-10^{*}\end{array}$ & $\begin{array}{c}\text { BQ5LR } \\
8-25-10^{*}\end{array}$ \\
\hline$\overline{T_{\text {mold }}}$ & Mold Temperature $(\mathrm{F})$ & 200 & 200 & 160 \\
\hline E1 & $\begin{array}{c}\text { Zone } 1 \text { Temperature }(\mathrm{F}) \\
\text { (nozzle) }\end{array}$ & 630 & 640 & 590 \\
\hline $\bar{E} 2$ & Zone 2 Temperature $(\mathrm{F})$ & 640 & 650 & 570 \\
\hline E3 & Zone 3 Temperature $(\mathrm{F})$ & 610 & 610 & 554 \\
\hline E4 & $\begin{array}{c}\text { Zone } 4 \text { Temperature }(\mathrm{F}) \\
\text { (feed zone) }\end{array}$ & 550 & 550 & 536 \\
\hline $\mathrm{P} 1$ & Injection pressure (psi) & $99 \%=22,384$ & $99 \%=22,384$ & $\begin{array}{c}\text { Tensile, flex } \\
70 \%=15,827 \\
\text { disks } 50 \%=11,305\end{array}$ \\
\hline $\mathrm{P} 2$ & Hold Pressure (psi) & $55 \%=12,436$ & $\begin{array}{c}55 \%=12,436 \\
\text { flex, tensile } \\
45 \% \text { disks }\end{array}$ & $\begin{array}{c}55 \%=12,436 \\
\text { flex, tensile } \\
45 \% \text { disks }\end{array}$ \\
\hline P7 & Back Pressure (psi) & $\begin{array}{c}\text { Set point } 5.0 \% \\
=1100 \mathrm{but} \text { actual } \\
\text { was } 1 \mathrm{kgf} / \mathrm{cm}^{2}\end{array}$ & $\begin{array}{c}\text { Set point } 5.0 \% \\
=1100 \mathrm{but} \text { actual } \\
\text { was } 1 \mathrm{kgf} / \mathrm{cm}^{2}\end{array}$ & $\begin{array}{c}\text { Set point } 5.0 \% \\
=1100 \mathrm{but} \text { actual } \\
\text { was } 1 \mathrm{kgf} / \mathrm{cm}^{2}\end{array}$ \\
\hline S1 & Shot size $(\mathrm{mm})$ & 22 & 22 & 22 \\
\hline S2 & Pullback before (mm) & 0 & 0 & 0 \\
\hline S3 & Pullback after (mm) & 0.4 & 0.4 & 0.4 \\
\hline S6 & Width of mold (mm) & 202 & 202 & 202 \\
\hline S8 & $\begin{array}{l}\text { Screw Position to Switch } \\
\text { from P1 to P2 (mm) }\end{array}$ & $\begin{array}{l}7 \text { for tensile } \\
10 \text { for flex } \\
12 \text { for disk }\end{array}$ & $\begin{array}{c}7 \text { for tensile, } 10 \\
\text { for flex, } 12 \text { for } \\
\text { disk }\end{array}$ & $\begin{array}{c}7 \text { for tensile, } 10 \\
\text { for flex, } 12 \text { for } \\
\text { disk }\end{array}$ \\
\hline $\mathrm{T} 1$ & Injection Time (s) & 8 & 8 & 8 \\
\hline $\mathrm{T} 2$ & Cool Time (s) & 16 & 16 & 16 \\
\hline T3 & Interval Time (s) & 0.1 & 0.1 & 0.1 \\
\hline T6 & Retraction Time (s) & 0 & 0 & 0 \\
\hline $\mathrm{T} 7$ & $\begin{array}{c}\text { Nozzle Retraction Delay } \\
\text { Time (s) }\end{array}$ & 0 & 0 & 0 \\
\hline $\mathrm{T} 8$ & Injection Delay Time (s) & 0 & 0 & 0 \\
\hline T9 & Charge Delay Time (s) & 0 & 0 & 0 \\
\hline V1 & Injection Velocity $\left(\mathrm{in}^{3} / \mathrm{s}\right)$ & $8 \%=0.54$ & $8 \%=0.54$ & $30 \%=1.866$ \\
\hline V6 & Screw Rotation (rpm) & $19 \%=60$ & $19 \%=60$ & $19 \%=60$ \\
\hline V9 & Retraction Velocity (\%) & 20 & 20 & 20 \\
\hline $\mathrm{V} 10$ & Advance Velocity (\%) & 50 & 50 & 50 \\
\hline V11 & Retraction Velocity (\%) & 90 & 90 & 90 \\
\hline
\end{tabular}




\begin{tabular}{|l|l|l|l|l|} 
CF & Clamp Force (US tons) & 80 & 80 & 80 \\
\hline
\end{tabular}

Table B.4: Injection Molding Conditions for Combinations in PC continued

\begin{tabular}{|c|c|c|c|c|}
\hline Notation & $\begin{array}{l}\text { Injection Molding } \\
\text { Conditions }\end{array}$ & $\begin{array}{l}\text { BG12L } \\
8-18-10 *\end{array}$ & $\begin{array}{c}\text { BG15L } \\
8-18-10^{*}\end{array}$ & $\begin{array}{l}\text { BQ5LR } \\
8-25-10 *\end{array}$ \\
\hline P-5-1 & $\begin{array}{c}\text { Actual Cycle Time, } \\
\text { sec }\end{array}$ & 31 & 31 & 31 \\
\hline P-5-1 & Inj Start, mm & 22.4 & 22.4 & 22.5 \\
\hline$P-5-1$ & Cushion, mm & $\begin{array}{c}3.0 \text { tensile } \\
4.9 \text { flex } \\
4.1 \text { disk }\end{array}$ & $\begin{array}{c}3.3 \text { tensile } \\
5.0 \text { flex } \\
4.0 \text { disk }\end{array}$ & $\begin{array}{c}3.4 \text { tensile } \\
5.0 \text { flex } \\
4.0 \text { disk }\end{array}$ \\
\hline P-5-1 & $\mathrm{P} 1 \rightarrow \mathrm{P} 2$ Fill time, sec & $\begin{array}{c}3.5 \text { tensile } \\
2.9 \text { flex } \\
2.4 \text { disk }\end{array}$ & $\begin{array}{c}3.5 \text { tensile } \\
2.9 \text { flex } \\
2.4 \text { disk }\end{array}$ & $\begin{array}{c}0.78 \text { tensile } \\
0.65 \text { flex } \\
0.56 \text { disk }\end{array}$ \\
\hline P-5-1 & $\begin{array}{l}\text { P1 Actual Pressure } \\
\mathrm{P} 1 \rightarrow \mathrm{P} 2, \mathrm{~kg} / \mathrm{cm}^{2}\end{array}$ & $\begin{array}{c}42 \text { tensile } \\
30 \text { flex } \\
19 \text { disk }\end{array}$ & $\begin{array}{c}40 \text { tensile } \\
28 \text { flex } \\
17 \text { disk }\end{array}$ & $\begin{array}{c}42 \text { tensile } \\
37 \text { flex } \\
22 \text { disk }\end{array}$ \\
\hline P-5-1 & Charge time, sec & 3.0 & $\begin{array}{c}3.2 \text { tensile } \\
3.2 \text { flex } \\
3.2 \text { disk }\end{array}$ & 3.5 \\
\hline P-5-1 & $\begin{array}{c}\text { Injection Peak } \\
\text { Pressure } \mathrm{P} 1, \mathrm{~kg} / \mathrm{cm}^{2}\end{array}$ & $\begin{array}{c}2 \text { tensile } \\
30 \text { flex } \\
19 \text { disk }\end{array}$ & $\begin{array}{c}40 \text { tensile } \\
28 \text { flex } \\
17 \text { disk }\end{array}$ & $\begin{array}{c}42 \text { tensile } \\
37 \text { flex } \\
22 \text { disk }\end{array}$ \\
\hline
\end{tabular}


Table B.4: Injection Molding Conditions for Combinations in PC continued

\begin{tabular}{|c|c|c|c|c|}
\hline Notation & $\begin{array}{l}\text { Injection Molding } \\
\text { Conditions }\end{array}$ & $\begin{array}{c}\text { BA2G2L } \\
8-18-10 \text { and } \\
8-6-10 *\end{array}$ & $\begin{array}{c}\text { BA2G5L } \\
8-18-10 \text { and } \\
8-6-10^{*}\end{array}$ & $\begin{array}{c}\text { BA5G2L } \\
8-18-10 \text { and } \\
8-6-10^{*}\end{array}$ \\
\hline $\mathrm{T}_{\text {mold }}$ & Mold Temperature (F) & 160 & 160 & 160 \\
\hline E1 & $\begin{array}{c}\text { Zone } 1 \text { Temperature }(\mathrm{F}) \\
\text { (nozzle) }\end{array}$ & 620 & 620 & 620 \\
\hline E2 & Zone 2 Temperature (F) & 590 & 590 & 590 \\
\hline E3 & Zone 3 Temperature $(\mathrm{F})$ & 560 & 560 & 560 \\
\hline E4 & $\begin{array}{c}\text { Zone } 4 \text { Temperature }(\mathrm{F}) \\
\text { (feed zone) }\end{array}$ & 550 & 550 & 550 \\
\hline $\mathrm{P} 1$ & Injection pressure (psi) & $75 \%=16,958$ & $\begin{array}{c}75 \%=16,958 \\
\text { flex, tensile } \\
50 \%=11,305 \mathrm{disk}\end{array}$ & $\begin{array}{c}75 \%=16,958 \text { flex } \\
\text { tensile } \\
50 \%=11,305 \text { disk }\end{array}$ \\
\hline $\mathrm{P} 2$ & Hold Pressure (psi) & $55 \%=12,436$ & $\begin{array}{c}55 \%=12,436 \\
\text { tensile, flex } \\
45 \% \text { disk }\end{array}$ & $\begin{array}{c}55 \%=12,436 \\
\text { tensile, flex } \\
45 \% \text { disk }\end{array}$ \\
\hline P7 & Back Pressure (psi) & $\begin{array}{c}\text { Set point } 5.0 \% \\
=1100 \text { but actual } \\
\text { was } 1 \mathrm{kgf} / \mathrm{cm}^{2}\end{array}$ & $\begin{array}{c}\text { Set point } 5.0 \% \\
=1100 \mathrm{but} \text { actual } \\
\text { was } 1 \mathrm{kgf} / \mathrm{cm}^{2}\end{array}$ & $\begin{array}{c}\text { Set point } 5.0 \% \\
=1100 \mathrm{but} \text { actual } \\
\text { was } 1 \mathrm{kgf} / \mathrm{cm}^{2}\end{array}$ \\
\hline S1 & Shot size $(\mathrm{mm})$ & 22 & 22 & 22 \\
\hline S2 & Pullback before (mm) & 0 & 0 & 0 \\
\hline S3 & Pullback after (mm) & 0.4 & 0.4 & 0.4 \\
\hline S6 & Width of mold (mm) & 202 & 202 & 202 \\
\hline S8 & $\begin{array}{l}\text { Screw Position to Switch } \\
\text { from P1 to P2 (mm) }\end{array}$ & $\begin{array}{l}7 \text { for tensile } \\
10 \text { for flex } \\
12 \text { for disk }\end{array}$ & $\begin{array}{l}7 \text { for tensile } \\
10 \text { for flex } \\
12 \text { for disk }\end{array}$ & $\begin{array}{l}7 \text { for tensile, } 10 \text { for } \\
\text { flex, } 12 \text { for disk }\end{array}$ \\
\hline T1 & Injection Time (s) & 8 & 8 & 8 \\
\hline $\mathrm{T} 2$ & Cool Time (s) & 16 & 16 & 16 \\
\hline T3 & Interval Time (s) & 0.1 & 0.1 & 0.1 \\
\hline T6 & Retraction Time (s) & 0 & 0 & 0 \\
\hline $\mathrm{T} 7$ & $\begin{array}{c}\text { Nozzle Retraction Delay } \\
\text { Time (s) }\end{array}$ & 0 & 0 & 0 \\
\hline $\mathrm{T} 8$ & Injection Delay Time (s) & 0 & 0 & 0 \\
\hline T9 & Charge Delay Time (s) & 0 & 0 & 0 \\
\hline V1 & Injection Velocity $\left(\mathrm{in}^{3} / \mathrm{s}\right)$ & $\begin{array}{c}30 \%=1.866 \text { disk } \\
55 \%=3.69 \text { flex } \\
\text { and tensile }\end{array}$ & $\begin{array}{c}30 \% \text { disk }=1.866 \\
55 \%=3.69 \text { flex } \\
\text { and tensile }\end{array}$ & $\begin{array}{c}8 \%=0.54 \text { tensile } \\
\text { flex } \\
30 \%=1.866 \mathrm{disk}\end{array}$ \\
\hline V6 & Screw Rotation (rpm) & $19 \%=60$ & $19 \%=60$ & $19 \%=60$ \\
\hline V9 & Retraction Velocity (\%) & 20 & 20 & 20 \\
\hline V10 & Advance Velocity (\%) & 50 & 50 & 50 \\
\hline
\end{tabular}


Table B.4: Injection Molding Conditions for Combinations in PC continued

\begin{tabular}{|c|c|c|c|c|}
\hline Notation & $\begin{array}{l}\text { Injection Molding } \\
\text { Conditions }\end{array}$ & $\begin{array}{c}\text { BA2G2L } \\
8-18-10 \text { and } \\
8-6-10^{*}\end{array}$ & $\begin{array}{c}\text { BA2G5L } \\
8-18-10 \text { and } \\
8-6-10^{*}\end{array}$ & $\begin{array}{c}\text { BA5G2L } \\
8-18-10 \text { and } \\
8-6-10^{*}\end{array}$ \\
\hline V11 & Retraction Velocity (\%) & 90 & 90 & 90 \\
\hline $\mathrm{CF}$ & Clamp Force (US tons) & 80 & 80 & 80 \\
\hline P-5-1 & Actual Cycle Time, sec & 31 & 31 & 31 \\
\hline P-5-1 & Inj Start, mm & 22.4 & 22.4 & 22.4 \\
\hline P-5-1 & Cushion, mm & $\begin{array}{c}3.0 \text { tensile } \\
4.9 \text { flex } \\
4.1 \text { disk }\end{array}$ & $\begin{array}{l}4.1 \text { tensile } \\
4.9 \text { flex } \\
4.1 \text { disk }\end{array}$ & $\begin{array}{l}4.0 \text { tensile } \\
5.7 \text { flex } \\
5.8 \text { disk }\end{array}$ \\
\hline P-5-1 & $\mathrm{P} 1 \rightarrow \mathrm{P} 2$ Fill time, sec & $\begin{array}{c}0.5 \text { tensile } \\
0.4 \text { flex } \\
0.36 \text { disk }\end{array}$ & $\begin{array}{c}0.56 \text { tensile } \\
0.43 \text { flex } \\
0.51 \text { disk }\end{array}$ & $\begin{array}{c}3.5 \text { tensile } \\
2.9 \text { flex } \\
0.6 \text { disk }\end{array}$ \\
\hline $\mathrm{P}-5-1$ & $\begin{array}{c}\text { P1 Actual Pressure P1 } \rightarrow \\
\text { P2, } \mathrm{kg} / \mathrm{cm}^{2}\end{array}$ & $\begin{array}{c}83 \text { tensile } \\
70 \text { flex } \\
53 \text { disk }\end{array}$ & $\begin{array}{c}85 \text { tensile } \\
72 \text { flex } \\
40 \text { disk }\end{array}$ & $\begin{array}{c}63 \text { tensile } \\
48 \text { flex } \\
46 \text { disk }\end{array}$ \\
\hline P-5-1 & Charge time, sec & 3.0 & 3.0 & $\begin{array}{l}3.2 \text { tensile } \\
3.2 \text { flex } \\
3.2 \text { disk }\end{array}$ \\
\hline P-5-1 & $\begin{array}{c}\text { Injection Peak Pressure P1, } \\
\mathrm{kg} / \mathrm{cm}^{2}\end{array}$ & $\begin{array}{c}83 \text { tensile } \\
70 \text { flex } \\
53 \text { disk }\end{array}$ & $\begin{array}{c}85 \text { tensile } \\
72 \text { flex } \\
40 \text { disk }\end{array}$ & $\begin{array}{c}63 \text { tensile } \\
48 \text { flex } \\
46 \text { disk }\end{array}$ \\
\hline
\end{tabular}


Table B.4: Injection Molding Conditions for Combinations in PC continued

\begin{tabular}{|c|c|c|c|c|}
\hline Notation & $\begin{array}{l}\text { Injection Molding } \\
\text { Conditions }\end{array}$ & $\begin{array}{c}\text { BA5G5L } \\
8-18-10 \text { and } \\
8-6-10 *\end{array}$ & $\begin{array}{c}\text { BQ1G2L } \\
8-18-10 \text { and } \\
8-6-10^{*}\end{array}$ & $\begin{array}{c}\text { BQ1G5L } \\
8-18-10 \text { and 8-6- } \\
10 *\end{array}$ \\
\hline $\mathrm{T}_{\text {mold }}$ & Mold Temperature (F) & 160 & 160 & 160 \\
\hline E1 & $\begin{array}{l}\text { Zone } 1 \text { Temperature }(\mathrm{F}) \\
\text { (nozzle) }\end{array}$ & 620 & 620 & 620 \\
\hline E2 & Zone 2 Temperature $(\mathrm{F})$ & 590 & 590 & 590 \\
\hline E3 & Zone 3 Temperature (F) & 560 & 560 & 560 \\
\hline E4 & $\begin{array}{c}\text { Zone } 4 \text { Temperature }(\mathrm{F}) \\
\text { (feed zone) }\end{array}$ & 550 & 550 & 550 \\
\hline P1 & Injection pressure (psi) & $\begin{array}{c}\text { Tensile, flex } \\
70 \%=15,827 \\
\text { disks } \\
50 \%=11,305\end{array}$ & $75 \%=16,958$ & $75 \%=16,958$ \\
\hline $\mathrm{P} 2$ & Hold Pressure (psi) & $\begin{array}{c}55 \%=12,436 \\
\text { flex, tensile } \\
45 \% \text { disks }\end{array}$ & $55 \%=12,436$ & $55 \%=12,436$ \\
\hline P7 & Back Pressure (psi) & $\begin{array}{c}\text { Set point } 5.0 \% \\
=1100 \text { but actual } \\
\text { was } 1 \mathrm{kgf} / \mathrm{cm}^{2}\end{array}$ & $\begin{array}{c}\text { Set point } 5.0 \% \\
=1100 \mathrm{but} \text { actual } \\
\text { was } 1 \mathrm{kgf} / \mathrm{cm}^{2}\end{array}$ & $\begin{array}{c}\text { Set point } 5.0 \% \\
=1100 \mathrm{but} \text { actual } \\
\text { was } 1 \mathrm{kgf} / \mathrm{cm}^{2}\end{array}$ \\
\hline S1 & Shot size $(\mathrm{mm})$ & 22 & 22 & 22 \\
\hline S2 & Pullback before (mm) & 0 & 0 & 0 \\
\hline S3 & Pullback after (mm) & 0.4 & 0.4 & 0.4 \\
\hline S6 & Width of mold (mm) & 202 & 202 & 202 \\
\hline S8 & $\begin{array}{l}\text { Screw Position to Switch } \\
\text { from P1 to P2 (mm) }\end{array}$ & $\begin{array}{c}7 \text { for tensile, } 10 \\
\text { for flex, } 12 \text { for } \\
\text { disk }\end{array}$ & $\begin{array}{l}7 \text { for tensile } \\
10 \text { for flex } \\
12 \text { for disk }\end{array}$ & $\begin{array}{l}7 \text { for tensile } \\
10 \text { for flex } \\
12 \text { for disk }\end{array}$ \\
\hline T1 & Injection Time (s) & 8 & 8 & 8 \\
\hline $\mathrm{T} 2$ & Cool Time (s) & 16 & 16 & 16 \\
\hline $\mathrm{T} 3$ & Interval Time (s) & 0.1 & 0.1 & 0.1 \\
\hline T6 & Retraction Time (s) & 0 & 0 & 0 \\
\hline $\mathrm{T} 7$ & $\begin{array}{c}\text { Nozzle Retraction Delay } \\
\text { Time (s) }\end{array}$ & 0 & 0 & 0 \\
\hline $\mathrm{T} 8$ & Injection Delay Time (s) & 0 & 0 & 0 \\
\hline T9 & Charge Delay Time (s) & 0 & 0 & 0 \\
\hline V1 & Injection Velocity $\left(\mathrm{in}^{3} / \mathrm{s}\right)$ & $8 \%=0.54$ & $8 \%=0.54$ & $8 \%=0.54$ \\
\hline V6 & Screw Rotation (rpm) & $19 \%=60$ & $19 \%=60$ & $19 \%=60$ \\
\hline V9 & Retraction Velocity (\%) & 20 & 20 & 20 \\
\hline V10 & Advance Velocity (\%) & 50 & 50 & 50 \\
\hline V11 & Retraction Velocity (\%) & 90 & 90 & 90 \\
\hline $\mathrm{CF}$ & Clamp Force (US tons) & 80 & 80 & 80 \\
\hline
\end{tabular}


Table B.4: Injection Molding Conditions for Combinations in PC continued

\begin{tabular}{|c|c|c|c|c|}
\hline Notation & $\begin{array}{l}\text { Injection Molding } \\
\text { Conditions }\end{array}$ & $\begin{array}{c}\text { BA5G5L } \\
8-18-10 \text { and } \\
8-6-10^{*}\end{array}$ & $\begin{array}{c}\text { BQ1G2L } \\
8-18-10 \text { and } \\
8-6-10^{*}\end{array}$ & $\begin{array}{c}\text { BQ1G5L } \\
8-18-10 \text { and 8-6- } \\
10^{*}\end{array}$ \\
\hline P-5-1 & Actual Cycle Time, sec & 31 & 31 & 31 \\
\hline $\mathrm{P}-5-1$ & Inj Start, mm & 22.5 & 22.4 & 22.8 \\
\hline P-5-1 & Cushion, mm & $\begin{array}{l}3.0 \text { tensile } \\
5.0 \text { flex } \\
4.0 \text { disk }\end{array}$ & $\begin{array}{l}3.0 \text { tensile } \\
4.9 \text { flex } \\
4.1 \text { disk }\end{array}$ & $\begin{array}{l}3.6 \text { tensile } \\
5.3 \text { flex } \\
4.5 \text { disk }\end{array}$ \\
\hline P-5-1 & $\mathrm{P} 1 \rightarrow \mathrm{P} 2$ Fill time, sec & $\begin{array}{l}3.5 \text { tensile } \\
2.9 \text { flex } \\
2.4 \text { disk }\end{array}$ & $\begin{array}{l}3.5 \text { tensile } \\
2.8 \text { flex } \\
2.4 \text { disk }\end{array}$ & $\begin{array}{l}3.5 \text { tensile } \\
2.8 \text { flex } \\
2.4 \text { disk }\end{array}$ \\
\hline P-5-1 & $\begin{array}{c}\text { P1 Actual Pressure } \mathrm{P} 1 \rightarrow \\
\mathrm{P} 2, \mathrm{~kg} / \mathrm{cm}^{2}\end{array}$ & $\begin{array}{c}65 \text { tensile } \\
48 \text { flex } \\
31 \text { disk }\end{array}$ & $\begin{array}{c}34 \text { tensile } \\
26 \text { flex } \\
17 \text { disk }\end{array}$ & $\begin{array}{l}1 \text { tensile } \\
30 \text { flex } \\
18 \text { disk }\end{array}$ \\
\hline P-5-1 & Charge time, sec & 3.5 & 3.0 & 3.0 \\
\hline P-5-1 & $\begin{array}{l}\text { Injection Peak Pressure P1, } \\
\mathrm{kg} / \mathrm{cm}^{2}\end{array}$ & $\begin{array}{c}65 \text { tensile } \\
48 \text { flex } \\
31 \text { disk }\end{array}$ & $\begin{array}{c}34 \text { tensile } \\
26 \text { flex } \\
17 \text { disk }\end{array}$ & $\begin{array}{c}41 \text { tensile } \\
30 \text { flex } \\
18 \text { disk }\end{array}$ \\
\hline
\end{tabular}


Table B.4: Injection Molding Conditions for Combinations in PC continued

\begin{tabular}{|c|c|c|c|c|}
\hline Notation & $\begin{array}{l}\text { Injection Molding } \\
\text { Conditions }\end{array}$ & $\begin{array}{c}\text { BQ2LR } \\
\text { 7-26-10 flex } \\
\text { only* }\end{array}$ & $\begin{array}{c}\text { BQ3LR } \\
\text { 7-26-10 flex } \\
\text { only* }\end{array}$ & $\begin{array}{c}\text { BQ5G2L } \\
9-9-10 \text { and 8-6- } \\
10^{*}\end{array}$ \\
\hline $\mathrm{T}_{\text {mold }}$ & Mold Temperature (F) & 160 & 160 & 160 \\
\hline E1 & $\begin{array}{c}\text { Zone } 1 \text { Temperature (F) } \\
\text { (nozzle) }\end{array}$ & 610 & 610 & 620 \\
\hline E2 & Zone 2 Temperature (F) & 570 & 570 & 590 \\
\hline E3 & Zone 3 Temperature (F) & 554 & 554 & 560 \\
\hline E4 & $\begin{array}{c}\text { Zone } 4 \text { Temperature }(\mathrm{F}) \\
\text { (feed zone) }\end{array}$ & 536 & 536 & 550 \\
\hline $\mathrm{P} 1$ & Injection pressure (psi) & $70 \%=15,827$ & $70 \%=15,827$ & $75 \%=16,958$ \\
\hline $\mathrm{P} 2$ & Hold Pressure (psi) & $55 \%=12,436$ & $55 \%=12,436$ & $55 \%=12,436$ \\
\hline P7 & Back Pressure (psi) & $0.2 \%=45.2$ & $0.2 \%=45.2$ & $\begin{array}{c}\text { Set point } 5.0 \% \\
=1100 \mathrm{but} \text { actual } \\
\text { was } 1 \mathrm{kgf} / \mathrm{cm}^{2}\end{array}$ \\
\hline S1 & Shot size $(\mathrm{mm})$ & 20 & 20 & 22 \\
\hline S2 & Pullback before (mm) & 0 & 0 & 0 \\
\hline S3 & Pullback after (mm) & 0.1 & 0.1 & 0.4 \\
\hline S6 & Width of mold (mm) & 202 & 202 & 202 \\
\hline S8 & $\begin{array}{l}\text { Screw Position to Switch } \\
\text { from P1 to P2 (mm) }\end{array}$ & 10 flex & 10 flex & $\begin{array}{c}7 \text { for tensile } \\
10 \text { for flex, } 12 \\
\text { disk }\end{array}$ \\
\hline $\mathrm{T} 1$ & Injection Time (s) & 8 & 8 & 8 \\
\hline $\mathrm{T} 2$ & Cool Time (s) & 16 & 16 & 20 \\
\hline $\mathrm{T} 3$ & Interval Time (s) & 0.1 & 0.1 & 0.1 \\
\hline T6 & Retraction Time (s) & 0 & 0 & 0 \\
\hline $\mathrm{T} 7$ & $\begin{array}{c}\text { Nozzle Retraction Delay } \\
\text { Time (s) }\end{array}$ & 0 & 0 & 0 \\
\hline T8 & Injection Delay Time (s) & 0 & 0 & 0 \\
\hline T9 & Charge Delay Time (s) & 0 & 0 & 0 \\
\hline V1 & Injection Velocity $\left(\mathrm{in}^{3} / \mathrm{s}\right)$ & $30 \%=1.866$ & $30 \%=1.866$ & $8 \%=0.54$ \\
\hline V6 & Screw Rotation (rpm) & $19 \%=60$ & $19 \%=60$ & $19 \%=60$ \\
\hline V9 & Retraction Velocity (\%) & 20 & 20 & 20 \\
\hline V10 & Advance Velocity (\%) & 50 & 50 & 50 \\
\hline V11 & Retraction Velocity (\%) & 90 & 90 & 90 \\
\hline $\mathrm{CF}$ & Clamp Force (US tons) & 80 & 80 & 80 \\
\hline
\end{tabular}


Table B.4: Injection Molding Conditions for Combinations in PC continued

\begin{tabular}{|c|c|c|c|c|}
\hline Notation & $\begin{array}{c}\text { Injection Molding } \\
\text { Conditions }\end{array}$ & $\begin{array}{c}\text { BQ2LR } \\
\text { 7-26-10 flex } \\
\text { only* }\end{array}$ & $\begin{array}{c}\text { BQ3LR } \\
\text { 7-26-10 flex } \\
\text { only* }\end{array}$ & $\begin{array}{c}\text { BQ5G2L } \\
\text { 9-9-10 and 8-6- } \\
10^{*}\end{array}$ \\
\hline P-5-1 & Actual Cycle Time, sec & 31 flex & 31 flex & 35 \\
\hline P-5-1 & Inj Start, mm & 20.6 & 20.6 & 22.4 \\
\hline P-5-1 & Cushion, mm & 3.4 & 3.4 & $\begin{array}{c}3.2 \text { tensile } \\
4.8 \text { flex }\end{array}$ \\
\hline P-5-1 & P1 $\rightarrow$ P2 Fill time, sec & 0.61 & 0.61 & $\begin{array}{c}3.6 \text { tensile } \\
2.9 \text { flex }\end{array}$ \\
\hline P-5-1 & $\begin{array}{c}\text { P1 Actual Pressure P1 } \rightarrow \\
\text { P2, kg/cm }\end{array}$ & 57 & 57 & 27 tensile \\
\hline P-5-1 & Charge time, sec & 2.9 & 2.9 & 3.9 \\
\hline & $\begin{array}{c}\text { Injection Peak Pressure } \\
\text { P1, } \mathrm{kg} / \mathrm{cm}^{2}\end{array}$ & 57 & 57 & 27 tensile \\
P-5-1 & & & & 21 flex \\
\hline
\end{tabular}


Table B.4: Injection Molding Conditions for Combinations in PC continued

\begin{tabular}{|c|c|c|c|c|}
\hline Notation & Injection Molding Conditions & $\begin{array}{c}\text { BQ5G5L } \\
9-9-10 \text { and 8- } \\
6-10^{*}\end{array}$ & $\begin{array}{c}\text { BQ4LR } \\
\text { 8-25-10 flex } \\
\text { only* }\end{array}$ & $\begin{array}{c}\text { BQ6LR } \\
\text { 8-25-10 flex } \\
\text { only* }\end{array}$ \\
\hline $\mathrm{T}_{\text {mold }}$ & Mold Temperature (F) & 160 & 160 & 160 \\
\hline E1 & Zone 1 Temperature (F) (nozzle) & 620 & 610 & 610 \\
\hline E2 & Zone 2 Temperature $(\mathrm{F})$ & 590 & 570 & 570 \\
\hline E3 & Zone 3 Temperature $(\mathrm{F})$ & 560 & 554 & 554 \\
\hline E4 & $\begin{array}{l}\text { Zone } 4 \text { Temperature }(\mathrm{F}) \text { (feed } \\
\text { zone) }\end{array}$ & 550 & 536 & 536 \\
\hline P1 & Injection pressure (psi) & $75 \%=16,958$ & $70 \%=15,827$ & $70 \%=15,827$ \\
\hline $\mathrm{P} 2$ & Hold Pressure (psi) & $55 \%=12,436$ & $55 \%=12,436$ & $55 \%=12,436$ \\
\hline P7 & Back Pressure (psi) & $\begin{array}{c}\text { Set point } 5.0 \% \\
=1100 \text { but } \\
\text { actual was } 1 \\
\mathrm{kgf} / \mathrm{cm}^{2}\end{array}$ & $\begin{array}{c}\text { Set point } 5.0 \% \\
=1100 \text { but } \\
\text { actual was } 1 \\
\mathrm{kgf} / \mathrm{cm}^{2}\end{array}$ & $\begin{array}{c}\text { Set point } 5.0 \% \\
=1100 \text { but } \\
\text { actual was } 1 \\
\mathrm{kgf} / \mathrm{cm}^{2}\end{array}$ \\
\hline S1 & Shot size $(\mathrm{mm})$ & 22 & 22 & 22 \\
\hline S2 & Pullback before (mm) & 0 & 0 & 0 \\
\hline S3 & Pullback after $(\mathrm{mm})$ & 0.4 & 0.4 & 0.4 \\
\hline S6 & Width of mold (mm) & 202 & 202 & 202 \\
\hline S8 & $\begin{array}{c}\text { Screw Position to Switch from P1 } \\
\text { to P2 (mm) }\end{array}$ & $\begin{array}{c}7 \text { for tensile } \\
10 \text { for flex, } 12 \\
\text { disk }\end{array}$ & 10 flex & 10 flex \\
\hline T1 & Injection Time (s) & 8 & 8 & 8 \\
\hline $\mathrm{T} 2$ & Cool Time (s) & 20 & 16 & 20 \\
\hline $\mathrm{T} 3$ & Interval Time (s) & 0.1 & 0.1 & 0.1 \\
\hline T6 & Retraction Time (s) & 0 & 0 & 0 \\
\hline $\mathrm{T} 7$ & Nozzle Retraction Delay Time (s) & 0 & 0 & 0 \\
\hline $\mathrm{T} 8$ & Injection Delay Time (s) & 0 & 0 & 0 \\
\hline T9 & Charge Delay Time (s) & 0 & 0 & 0 \\
\hline V1 & Injection Velocity $\left(\mathrm{in}^{3} / \mathrm{s}\right)$ & $8 \%=0.54$ & $30 \%=1.866$ & $30 \%=1.866$ \\
\hline V6 & Screw Rotation (rpm) & $19 \%=60$ & $19 \%=60$ & $19 \%=60$ \\
\hline V9 & Retraction Velocity (\%) & 20 & 20 & 20 \\
\hline V10 & Advance Velocity (\%) & 50 & 50 & 50 \\
\hline V11 & Retraction Velocity (\%) & 90 & 90 & 90 \\
\hline $\mathrm{CF}$ & Clamp Force (US tons) & 80 & 80 & 80 \\
\hline
\end{tabular}


Table B.4: Injection Molding Conditions for Combinations in PC continued

\begin{tabular}{|c|c|c|c|c|}
\hline Notation & $\begin{array}{l}\text { Injection Molding } \\
\text { Conditions }\end{array}$ & $\begin{array}{c}\text { BQ5G5L } \\
9-9-10 \text { and 8-6- } \\
10 *\end{array}$ & $\begin{array}{c}\text { BQ4LR } \\
\text { 8-25-10 flex } \\
\text { only* }\end{array}$ & $\begin{array}{c}\text { BQ6LR } \\
8-25-10 \text { flex only* }\end{array}$ \\
\hline P-5-1 & Actual Cycle Time, sec & 35 & 31 flex only & 31 flex only \\
\hline $\mathrm{P}-5-1$ & Inj Start, mm & 22.4 & 22.4 & 22.4 \\
\hline P-5-1 & Cushion, mm & $\begin{array}{l}3.0 \text { tensile } \\
4.9 \text { flex }\end{array}$ & 5.1 & 5.1 \\
\hline P-5-1 & $\mathrm{P} 1 \rightarrow \mathrm{P} 2$ Fill time, sec & $\begin{array}{l}3.7 \text { tensile } \\
3.0 \text { flex }\end{array}$ & 0.66 flex & 0.66 flex \\
\hline P-5-1 & $\begin{array}{c}\text { P1 Actual Pressure P1 } \rightarrow \\
\text { P2, } \mathrm{kg} / \mathrm{cm}^{2}\end{array}$ & $\begin{array}{l}32 \text { tensile } \\
26 \text { flex }\end{array}$ & 40 flex & 40 flex \\
\hline $\mathrm{P}-5-1$ & Charge time, sec & 3.7 & 3.2 & 3.2 \\
\hline P-5-1 & $\begin{array}{l}\text { Injection Peak Pressure P1, } \\
\qquad \mathrm{kg} / \mathrm{cm}^{2}\end{array}$ & $\begin{array}{l}32 \text { tensile } \\
26 \text { flex }\end{array}$ & 40 flex & 40 flex \\
\hline
\end{tabular}


Table B.4: Injection Molding Conditions for Combinations in PC continued

\begin{tabular}{|c|c|c|}
\hline Notation & Injection Molding Conditions & $\begin{array}{c}\text { BQ8LR } \\
8-25-10 \text { flex only* }\end{array}$ \\
\hline $\mathrm{T}_{\text {mold }}$ & Mold Temperature (F) & 180 \\
\hline E1 & Zone 1 Temperature (F) (nozzle) & 610 \\
\hline E2 & Zone 2 Temperature $(\mathrm{F})$ & 570 \\
\hline E3 & Zone 3 Temperature $(\mathrm{F})$ & 554 \\
\hline E4 & Zone 4 Temperature (F) (feed zone) & 536 \\
\hline $\mathrm{P} 1$ & Injection pressure (psi) & $80 \%=18,088$ \\
\hline $\mathrm{P} 2$ & Hold Pressure (psi) & $55 \%=12,436$ \\
\hline P7 & Back Pressure (psi) & $\begin{array}{c}\text { Set point } 5.0 \%=1100 \text { but actual was } 1 \\
\mathrm{kgf} / \mathrm{cm}^{2}\end{array}$ \\
\hline S1 & Shot size $(\mathrm{mm})$ & 22 \\
\hline S2 & Pullback before (mm) & 0 \\
\hline S3 & Pullback after (mm) & 0.4 \\
\hline S6 & Width of mold (mm) & 202 \\
\hline S8 & $\begin{array}{l}\text { Screw Position to Switch from P1 to } \\
\text { P2 (mm) }\end{array}$ & 10 flex \\
\hline $\mathrm{T} 1$ & Injection Time (s) & 8 \\
\hline $\mathrm{T} 2$ & Cool Time (s) & 20 \\
\hline $\mathrm{T} 3$ & Interval Time (s) & 0.1 \\
\hline T6 & Retraction Time (s) & 0 \\
\hline $\mathrm{T} 7$ & Nozzle Retraction Delay Time (s) & 0 \\
\hline $\mathrm{T} 8$ & Injection Delay Time (s) & 0 \\
\hline T9 & Charge Delay Time (s) & 0 \\
\hline V1 & Injection Velocity (in 3 s) & $30 \%=1.866$ \\
\hline V6 & Screw Rotation (rpm) & $19 \%=60$ \\
\hline V9 & Retraction Velocity (\%) & 20 \\
\hline V10 & Advance Velocity (\%) & 50 \\
\hline V11 & Retraction Velocity (\%) & 90 \\
\hline $\mathrm{CF}$ & Clamp Force (US tons) & 80 \\
\hline $\mathrm{P}-5-1$ & Actual Cycle Time, sec & 35 flex only \\
\hline $\mathrm{P}-5-1$ & Inj Start, mm & 22.4 \\
\hline $\mathrm{P}-5-1$ & Cushion, mm & 5.1 \\
\hline $\mathrm{P}-5-1$ & $\mathrm{P} 1 \rightarrow \mathrm{P} 2$ Fill time, sec & 0.66 flex \\
\hline $\mathrm{P}-5-1$ & $\mathrm{P} 1$ Actual Pressure $\mathrm{P} 1 \rightarrow \mathrm{P} 2, \mathrm{~kg} / \mathrm{cm}^{2}$ & 35 flex \\
\hline $\mathrm{P}-5-1$ & Charge time, sec & 3.2 \\
\hline $\mathrm{P}-5-1$ & Injection Peak Pressure $\mathrm{P} 1, \mathrm{~kg} / \mathrm{cm}^{2}$ & 35 flex \\
\hline
\end{tabular}


Notes:

1) For each formulation, typically about 302.5 " diameter disks, 30 tensile bars, and 20 flexural bars molded. Each sample was individually molded. The injection molding machine ran on the fully automatic mode.

2) After each formulation, the feed hopper was run until it was empty and no material was in screw. Then we discarded 5 transition shots, and then started collecting the next formulation.

3) Between each 'family', we cleaned with DynaPurge E, and then Lexan HF1130111.

4) * is injection molding date 


\section{Appendix C: Density Data}

\section{Appendix C.1: Polycarbonate with Carbon Nanotubes}

Table C.1: Density Results for Lexan HF1130-111:BL: Injection Molded May 26, 2009

\begin{tabular}{|l|l|l|l|}
\hline Test Date & Sample Number & $\begin{array}{l}\text { Theoretical } \\
\text { Density }(\mathrm{g} / \mathrm{ml})\end{array}$ & $\begin{array}{l}\text { Actual } \\
\text { Density } \\
(\mathrm{g} / \mathrm{ml})\end{array}$ \\
\hline $6 / 26 / 2009$ & BL-TC-15 & 1.200 & 1.195 \\
\hline $6 / 26 / 2009$ & BL-TC-18 & 1.200 & 1.192 \\
\hline $6 / 26 / 2009$ & BL-TC-21 & 1.200 & 1.191 \\
\hline $6 / 26 / 2009$ & BL-TC-26 & 1.200 & 1.192 \\
\hline $6 / 26 / 2009$ & BL-TC-31 & 1.200 & 1.191 \\
\hline & & Average & 1.1923 \\
\cline { 3 - 4 } & $\begin{array}{l}\text { Standard } \\
\text { Deviation }\end{array}$ & 0.0016 \\
\cline { 3 - 4 } & $\begin{array}{l}\text { Number of } \\
\text { Samples }\end{array}$ & 5 \\
\cline { 3 - 4 } & &
\end{tabular}

Table C.2: Density Results for Extruded Lexan HF1130-111:BLE: Injection Molded May 26, 2009

\begin{tabular}{|c|c|c|c|}
\hline Test Date & Sample Number & $\begin{array}{l}\text { Theoretical } \\
\text { Density }(\mathrm{g} / \mathrm{ml})\end{array}$ & $\begin{array}{l}\text { Actual } \\
\text { Density } \\
(\mathrm{g} / \mathrm{ml})\end{array}$ \\
\hline $6 / 26 / 2009$ & BLE-TC-12 & 1.200 & 1.193 \\
\hline $6 / 26 / 2009$ & BLE-TC-14 & 1.200 & 1.194 \\
\hline $6 / 26 / 2009$ & BLE-TC-19 & 1.200 & 1.193 \\
\hline $6 / 26 / 2009$ & BLE-TC-22 & 1.200 & 1.195 \\
\hline \multirow[t]{4}{*}{$6 / 26 / 2009$} & BLE-TC-24 & 1.200 & 1.194 \\
\hline & & Average & 1.1938 \\
\hline & & $\begin{array}{l}\text { Standard } \\
\text { Deviation }\end{array}$ & 0.0008 \\
\hline & & $\begin{array}{l}\text { Number of } \\
\text { Samples }\end{array}$ & 5 \\
\hline
\end{tabular}


Table C.3: Density Results for $2 \mathrm{wt} \%$ fibrils in Lexan HF1130-111: BQ2L: Injection Molded May 26, 2009

\begin{tabular}{|l|l|l|l|}
\hline Test Date & Sample Number & $\begin{array}{l}\text { Theoretical } \\
\text { Density }(\mathrm{g} / \mathrm{ml})\end{array}$ & $\begin{array}{l}\text { Actual } \\
\text { Density } \\
(\mathrm{g} / \mathrm{ml})\end{array}$ \\
\hline $5 / 26 / 2009$ & BQ2L-TC-19 & 1.210 & 1.202 \\
\hline $5 / 26 / 2009$ & BQ2L-TC-14 & 1.210 & 1.201 \\
\hline $5 / 26 / 2009$ & BQ2L-TC-16 & 1.210 & 1.201 \\
\hline $5 / 26 / 2009$ & BQ2L-TC-13 & 1.210 & 1.202 \\
\hline $5 / 26 / 2009$ & BQ2L-TC-29 & 1.210 & 1.201 \\
\hline $5 / 26 / 2009$ & BQ2L-TC-31 & 1.210 & 1.202 \\
\hline $5 / 26 / 2009$ & BQ2L-TC-17 & 1.210 & 1.201 \\
\hline $5 / 26 / 2009$ & BQ2L-TC-27 & 1.210 & 1.202 \\
\hline & & Average & 1.2015 \\
\cline { 3 - 4 } & & $\begin{array}{l}\text { Standard } \\
\text { Deviation }\end{array}$ & 0.0005 \\
\cline { 3 - 4 } & $\begin{array}{l}\text { Number of } \\
\text { Samples }\end{array}$ & 8 \\
\cline { 3 - 4 } & &
\end{tabular}

Table C.4: Density Results for $3 \mathrm{wt} \%$ fibrils in Lexan HF1130-111: BQ3L: Injection Molded May 26, 2009

\begin{tabular}{|l|l|l|l|}
\hline Test Date & Sample Number & $\begin{array}{l}\text { Theoretical } \\
\text { Density }(\mathrm{g} / \mathrm{ml})\end{array}$ & $\begin{array}{l}\text { Actual } \\
\text { Density } \\
(\mathrm{g} / \mathrm{ml})\end{array}$ \\
\hline $5 / 26 / 2009$ & BQ3L-TC-21 & 1.215 & 1.206 \\
\hline $5 / 26 / 2009$ & BQ3L-TC-26 & 1.215 & 1.204 \\
\hline $5 / 26 / 2009$ & BQ3L-TC-11 & 1.215 & 1.204 \\
\hline $5 / 26 / 2009$ & BQ3L-TC-22 & 1.215 & 1.205 \\
\hline $5 / 26 / 2009$ & BQ3L-TC-12 & 1.215 & 1.206 \\
\hline $5 / 26 / 2009$ & BQ3L-TC-17 & 1.215 & 1.206 \\
\hline & Average & 1.2052 \\
\cline { 3 - 4 } & & $\begin{array}{l}\text { Standard } \\
\text { Deviation }\end{array}$ & 0.0010 \\
\cline { 3 - 4 } & $\begin{array}{l}\text { Number of } \\
\text { Samples }\end{array}$ & 6 \\
\cline { 3 - 4 } & & & \\
& &
\end{tabular}


Table C.5: Density Results for 4 wt\% fibrils in Lexan HF1130-111: BQ4L: Injection Molded May 26, 2009

\begin{tabular}{|l|l|l|l|}
\hline \multirow{2}{*}{ Test Date } & Sample Number & $\begin{array}{l}\text { Theoretical } \\
\text { Density }(\mathrm{g} / \mathrm{ml})\end{array}$ & $\begin{array}{l}\text { Actual } \\
\text { Density } \\
(\mathrm{g} / \mathrm{ml})\end{array}$ \\
\hline $6 / 26 / 2009$ & BQ4L-TC-13 & 1.220 & 1.213 \\
\hline $6 / 26 / 2009$ & BQ4L-TC-18 & 1.220 & 1.210 \\
\hline $6 / 26 / 2009$ & BQ4L-TC-24 & 1.220 & 1.213 \\
\hline $6 / 26 / 2009$ & BQ4L-TC-28 & 1.220 & 1.213 \\
\hline $6 / 26 / 2009$ & BQ4L-TC-32 & 1.220 & 1.213 \\
\hline & & Average & 1.2123 \\
\cline { 3 - 4 } & & $\begin{array}{l}\text { Standard } \\
\text { Deviation }\end{array}$ & 0.0013 \\
\cline { 3 - 4 } & & $\begin{array}{l}\text { Number of } \\
\text { Samples }\end{array}$ & 5 \\
\cline { 3 - 4 } & &
\end{tabular}

Table C.6: Density Results for 5 wt\% fibrils in Lexan HF1130-111: BQ5L: Injection Molded May 26, 2009

\begin{tabular}{|l|l|l|l|}
\hline \multirow{2}{*}{ Test Date } & Sample Number & $\begin{array}{l}\text { Theoretical } \\
\text { Density }(\mathrm{g} / \mathrm{ml})\end{array}$ & $\begin{array}{l}\text { Actual } \\
\text { Density } \\
(\mathrm{g} / \mathrm{ml})\end{array}$ \\
\hline $6 / 26 / 2009$ & BQ5L-TC-12 & 1.225 & 1.217 \\
\hline $6 / 26 / 2009$ & BQ5L-TC-18 & 1.225 & 1.217 \\
\hline $6 / 26 / 2009$ & BQ5L-TC-20 & 1.225 & 1.216 \\
\hline $6 / 26 / 2009$ & BQ5L-TC-28 & 1.225 & 1.217 \\
\hline $6 / 26 / 2009$ & BQ5L-TC-32 & 1.225 & 1.217 \\
\hline & & Average & 1.2168 \\
\cline { 3 - 4 } & & $\begin{array}{l}\text { Standard } \\
\text { Deviation }\end{array}$ & 0.0004 \\
\cline { 3 - 4 } & & $\begin{array}{l}\text { Number of } \\
\text { Samples }\end{array}$ & 5 \\
\cline { 3 - 4 } & & & 5 \\
\cline { 3 - 4 } & & & \\
& & &
\end{tabular}


Table C.7: Density Results for $6 \mathrm{wt} \%$ fibrils in Lexan HF1130-111: BQ6L: Injection Molded May 26, 2009

\begin{tabular}{|l|l|l|l|}
\hline Test Date & Sample Number & $\begin{array}{l}\text { Theoretical } \\
\text { Density }(\mathrm{g} / \mathrm{ml})\end{array}$ & $\begin{array}{l}\text { Actual } \\
\text { Density } \\
(\mathrm{g} / \mathrm{ml})\end{array}$ \\
\hline $6 / 26 / 2009$ & BQ6L-TC-12 & 1.224 & 1.221 \\
\hline $6 / 26 / 2009$ & BQ6L-TC-20 & 1.224 & 1.222 \\
\hline $6 / 26 / 2009$ & BQ6L-TC-26 & 1.224 & 1.222 \\
\hline $6 / 26 / 2009$ & BQ6L-TC-29 & 1.224 & 1.221 \\
\hline $6 / 26 / 2009$ & BQ6L-TC-33 & 1.224 & 1.221 \\
\hline & Average & 1.2214 \\
\cline { 3 - 4 } & $\begin{array}{l}\text { Standard } \\
\text { Deviation }\end{array}$ & 0.0004 \\
\cline { 3 - 4 } & $\begin{array}{l}\text { Number of } \\
\text { Samples }\end{array}$ & 5 \\
\cline { 3 - 4 } & &
\end{tabular}

Table C.8: Density Results for $8 \mathrm{wt} \%$ fibrils in Lexan HF1130-111: BQ8L: Injection Molded May 26, 2009

\begin{tabular}{|l|l|l|l|}
\hline Test Date & Sample Number & $\begin{array}{l}\text { Theoretical } \\
\text { Density }(\mathrm{g} / \mathrm{ml})\end{array}$ & $\begin{array}{l}\text { Actual } \\
\text { Density } \\
(\mathrm{g} / \mathrm{ml})\end{array}$ \\
\hline $6 / 26 / 2009$ & BQ8L-TC-11 & 1.225 & 1.231 \\
\hline $6 / 26 / 2009$ & BQ8L-TC-19 & 1.225 & 1.232 \\
\hline $6 / 26 / 2009$ & BQ8L-TC-24 & 1.225 & 1.231 \\
\hline $6 / 26 / 2009$ & BQ8L-TC-29 & 1.225 & 1.231 \\
\hline $6 / 26 / 2009$ & BQ8L-TC-33 & 1.225 & 1.232 \\
\hline & & Average & 1.2313 \\
\cline { 3 - 4 } & $\begin{array}{l}\text { Standard } \\
\text { Deviation }\end{array}$ & 0.0005 \\
\cline { 3 - 4 } & $\begin{array}{l}\text { Number of } \\
\text { Samples }\end{array}$ & 5 \\
\cline { 3 - 4 } & &
\end{tabular}




\section{Appendix C.2: Polycarbonate with Carbon Black}

Table C.9: Density Results for Lexan HF1130-111:BL2: Injection Molded December 4, 2009

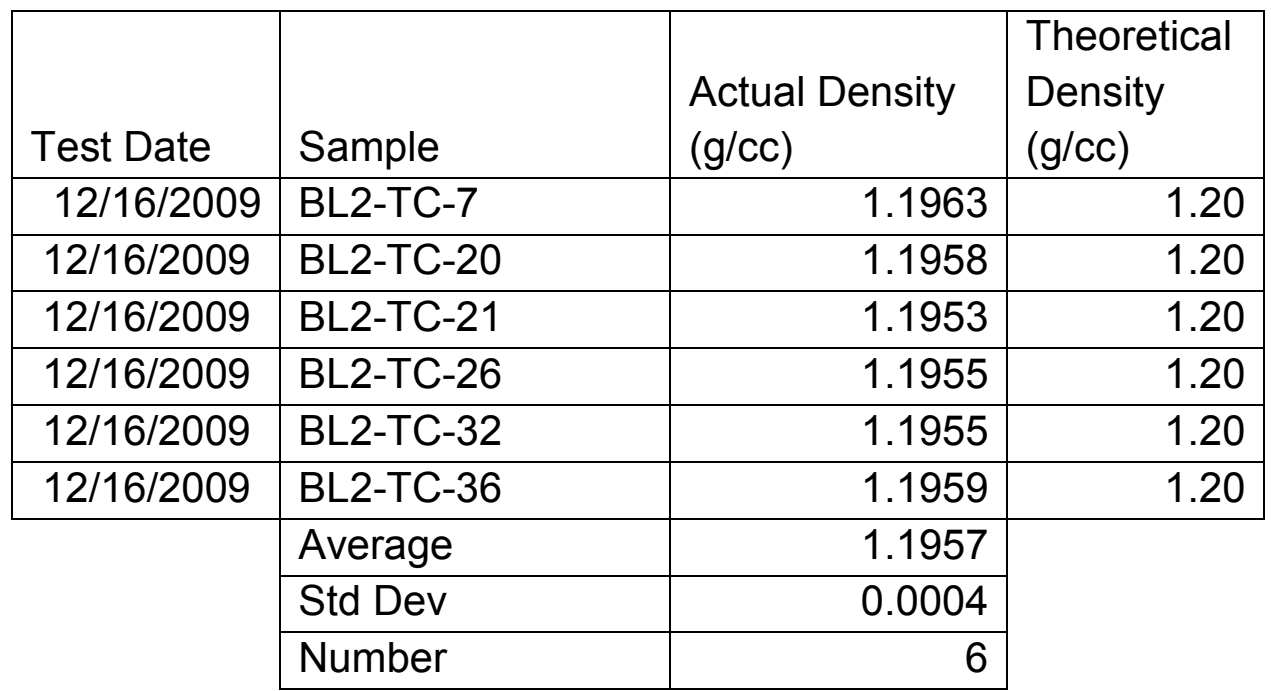

Table C.10: Density Results for Extruded Lexan HF1130-111:BLE2: Extruded Nov 5, 2009; Injection Molded December 4, 2009

\begin{tabular}{|c|c|c|c|}
\hline Test Date & Sample & $\begin{array}{l}\text { Actual Density } \\
(\mathrm{g} / \mathrm{cc})\end{array}$ & $\begin{array}{l}\text { Theoretical } \\
\text { Density } \\
\text { (g/cc) }\end{array}$ \\
\hline $12 / 16 / 2009$ & BLE2-TC-9 & 1.1957 & 1.20 \\
\hline 12/16/2009 & BLE2-TC-11 & 1.1957 & 1.20 \\
\hline 12/16/2009 & BLE2-TC-13 & 1.1957 & 1.20 \\
\hline 12/16/2009 & BLE2-TC-14 & 1.1960 & 1.20 \\
\hline 12/16/2009 & BLE2-TC-19 & 1.1962 & 1.20 \\
\hline $12 / 16 / 2009$ & BLE2-TC-20 & 1.1958 & 1.20 \\
\hline $12 / 16 / 2009$ & BLE2-TC-25 & 1.1957 & 1.20 \\
\hline & Average & 1.1958 & \\
\hline & Std Dev & 0.0002 & \\
\hline & Number & 7 & \\
\hline
\end{tabular}


Table C.11: Density Results for $2 \mathrm{wt} \%$ Ketjenblack EC-600 JD in Lexan HF1130-111: BA2L: Extruded November 5, 2009; Injection Molded December 4, 2009

\begin{tabular}{|c|c|c|c|c|}
\hline Test Date & Sample & $\begin{array}{l}\text { Actual } \\
\text { Density } \\
\text { (g/cc) }\end{array}$ & $\begin{array}{l}\text { Theoretical } \\
\text { Density }(\mathrm{g} / \mathrm{cc}) \text { * }\end{array}$ & $\begin{array}{l}\text { Theoretical } \\
\text { Density }(\mathrm{g} / \mathrm{cc})^{\star *}\end{array}$ \\
\hline $12 / 16 / 2009$ & BA2L-TC-11 & 1.2037 & 1.208 & 1.204 \\
\hline $12 / 16 / 2009$ & BA2L-TC-19 & 1.2030 & 1.208 & 1.204 \\
\hline $12 / 16 / 2009$ & BA2L-TC-22 & 1.2036 & 1.208 & 1.204 \\
\hline $12 / 16 / 2009$ & BA2L-TC-25 & 1.2026 & 1.208 & 1.204 \\
\hline $12 / 16 / 2009$ & BA2L-TC-27 & 1.2032 & 1.208 & 1.204 \\
\hline $12 / 16 / 2009$ & BA2L-TC-28 & 1.2033 & 1.208 & 1.204 \\
\hline $12 / 16 / 2009$ & BA2L-TC-30 & 1.2034 & 1.208 & 1.204 \\
\hline & Average & 1.2032 & & \\
\hline & Std Dev & 0.0004 & & \\
\hline & Number & 7 & & \\
\hline \multicolumn{5}{|c|}{ * using $1.20 \mathrm{~g} / \mathrm{cc}$ for Lexan } \\
\hline$* *$ using $1.1 \mathrm{c}$ & $6 \mathrm{~g} / \mathrm{cc}$ for & & & \\
\hline
\end{tabular}

Table C.12: Density Results for $3 \mathrm{wt} \%$ Ketjenblack EC-600 JD in Lexan HF1130-111: BA3L: Extruded November 5, 2009; Injection Molded December 4, 2009

\begin{tabular}{|c|c|c|c|c|}
\hline Test Date & Sample & $\begin{array}{l}\text { Actual } \\
\text { Density } \\
\text { (g/cc) }\end{array}$ & $\begin{array}{l}\text { Theoretical } \\
\text { Density }(\mathrm{g} / \mathrm{cc}) \text { * }\end{array}$ & $\begin{array}{l}\text { Theoretical } \\
\text { Density } \\
(\mathrm{g} / \mathrm{cc})^{* *}\end{array}$ \\
\hline $12 / 16 / 2009$ & BA3L-TC-14 & 1.2081 & 1.212 & 1.208 \\
\hline $12 / 16 / 2009$ & BA3L-TC-16 & 1.2079 & 1.212 & 1.208 \\
\hline $12 / 16 / 2009$ & BA3L-TC-18 & 1.2077 & 1.212 & 1.208 \\
\hline $12 / 16 / 2009$ & BA3L-TC-21 & 1.2082 & 1.212 & 1.208 \\
\hline $12 / 16 / 2009$ & BA3L-TC-24 & 1.2080 & 1.212 & 1.208 \\
\hline \multirow[t]{4}{*}{$12 / 16 / 2009$} & BA3L-TC-29 & 1.2080 & 1.212 & 1.208 \\
\hline & Average & 1.2080 & & \\
\hline & Std Dev & 0.0001 & & \\
\hline & Number & 6 & & \\
\hline
\end{tabular}

* using $1.20 \mathrm{~g} / \mathrm{cc}$ for Lexan

** using $1.196 \mathrm{~g} / \mathrm{cc}$ for Lexan 
Table C.13: Density Results for 4 wt\% Ketjenblack EC-600 JD in Lexan HF1130-111: BA4L: Extruded November 5, 2009; Injection Molded December 4, 2009

\begin{tabular}{|c|c|c|c|c|}
\hline Test Date & Sample & $\begin{array}{l}\text { Actual } \\
\text { Density } \\
\text { (g/cc) }\end{array}$ & $\begin{array}{l}\text { Theoretical } \\
\text { Density }(\mathrm{g} / \mathrm{cc}) \text { * }\end{array}$ & $\begin{array}{l}\text { Theoretical } \\
\text { Density }(\mathrm{g} / \mathrm{cc})^{\star *}\end{array}$ \\
\hline $12 / 16 / 2009$ & BA4L-TC-10 & 1.2113 & 1.216 & 1.212 \\
\hline $12 / 16 / 2009$ & BA4L-TC-16 & 1.2123 & 1.216 & 1.212 \\
\hline $12 / 16 / 2009$ & BA4L-TC-18 & 1.2113 & 1.216 & 1.212 \\
\hline $12 / 16 / 2009$ & BA4L-TC-24 & 1.2126 & 1.216 & 1.212 \\
\hline $12 / 16 / 2009$ & BA4L-TC-27 & 1.2115 & 1.216 & 1.212 \\
\hline $12 / 16 / 2009$ & BA4L-TC-30 & 1.2126 & 1.216 & 1.212 \\
\hline & Average & 1.2119 & & \\
\hline & Std Dev & 0.0007 & & \\
\hline & Number & 6 & & \\
\hline \multicolumn{5}{|c|}{ * using $1.20 \mathrm{~g} / \mathrm{cc}$ for Lexan } \\
\hline 1.19 & $g / c c$ for & & & \\
\hline
\end{tabular}

Table C.15: Density Results for 5 wt\% Ketjenblack EC-600 JD in Lexan HF1130-111: BA5L: Extruded November 11, 2009; Injection Molded December 4, 2009

\begin{tabular}{|c|c|c|c|c|}
\hline Test Date & Sample & $\begin{array}{l}\text { Actual } \\
\text { Density } \\
\text { (g/cc) }\end{array}$ & $\begin{array}{l}\text { Theoretical } \\
\text { Density }(\mathrm{g} / \mathrm{cc}) \text { * }\end{array}$ & $\begin{array}{l}\text { Theoretical } \\
\text { Density }(\mathrm{g} / \mathrm{cc})^{\star *}\end{array}$ \\
\hline $12 / 16 / 2009$ & BA5L-TC-15 & 1.2155 & 1.220 & 1.216 \\
\hline $12 / 16 / 2009$ & BA5L-TC-16 & 1.2153 & 1.220 & 1.216 \\
\hline $12 / 16 / 2009$ & BA5L-TC-22 & 1.2151 & 1.220 & 1.216 \\
\hline $12 / 16 / 2009$ & BA5L-TC-25 & 1.2153 & 1.220 & 1.216 \\
\hline $12 / 16 / 2009$ & BA5L-TC-26 & 1.2153 & 1.220 & 1.216 \\
\hline $12 / 16 / 2009$ & BA5L-TC-30 & 1.2155 & 1.220 & 1.216 \\
\hline & Average & 1.2153 & & \\
\hline & Std Dev & 0.0001 & & \\
\hline & Number & 6 & & \\
\hline \multicolumn{5}{|c|}{ * using $1.20 \mathrm{~g} / \mathrm{cc}$ for Lexan } \\
\hline 1.19 & g/cc for & & & \\
\hline
\end{tabular}


Table C.14: Density Results for 6 wt\% Ketjenblack EC-600 JD in Lexan HF1130-111: BA6L: Extruded November 11, 2009; Injection Molded December 4, 2009

\begin{tabular}{|c|c|c|c|c|}
\hline Test Date & Sample & $\begin{array}{l}\text { Actual } \\
\text { Density } \\
\text { (g/cc) }\end{array}$ & $\begin{array}{l}\text { Theoretical } \\
\text { Density }(\mathrm{g} / \mathrm{cc}) \text { * }\end{array}$ & $\begin{array}{l}\text { Theoretical } \\
\text { Density }(\mathrm{g} / \mathrm{cc})^{\star *}\end{array}$ \\
\hline $12 / 16 / 2009$ & BA6L-TC-12 & 1.2184 & 1.224 & 1.221 \\
\hline $12 / 16 / 2009$ & BA6L-TC-16 & 1.2188 & 1.224 & 1.221 \\
\hline $12 / 16 / 2009$ & BA6L-TC-18 & 1.2190 & 1.224 & 1.221 \\
\hline $12 / 16 / 2009$ & BA6L-TC-23 & 1.2189 & 1.224 & 1.221 \\
\hline $12 / 16 / 2009$ & BA6L-TC-24 & 1.2193 & 1.224 & 1.221 \\
\hline & Average & 1.2189 & & \\
\hline & Std Dev & 0.0004 & & \\
\hline & Number & 5 & & \\
\hline \multicolumn{5}{|c|}{ * using $1.20 \mathrm{~g} / \mathrm{cc}$ for Lexan } \\
\hline
\end{tabular}

Table C.16: Density Results for 8 wt\% Ketjenblack EC-600 JD in Lexan HF1130-111: BA8L: Extruded November 11, 2009; Injection Molded December 4, 2009

\begin{tabular}{|c|c|c|c|c|}
\hline Test Date & Sample & $\begin{array}{l}\text { Actual } \\
\text { Density } \\
\text { (g/cc) }\end{array}$ & $\begin{array}{l}\text { Theoretical } \\
\text { Density }(\mathrm{g} / \mathrm{cc}) \text { * }\end{array}$ & $\begin{array}{l}\text { Theoretical } \\
\text { Density }(\mathrm{g} / \mathrm{cc})^{\star *}\end{array}$ \\
\hline $12 / 16 / 2009$ & BA8L-TC-20 & 1.2265 & 1.233 & 1.229 \\
\hline $12 / 16 / 2009$ & BA8L-TC-24 & 1.2268 & 1.233 & 1.229 \\
\hline $12 / 16 / 2009$ & BA8L-TC-26 & 1.2272 & 1.233 & 1.229 \\
\hline $12 / 16 / 2009$ & BA8L-TC-28 & 1.2268 & 1.233 & 1.229 \\
\hline $12 / 16 / 2009$ & BA8L-TC-30 & 1.2266 & 1.233 & 1.229 \\
\hline & Average & 1.2268 & & \\
\hline & Std Dev & 0.0002 & & \\
\hline & Number & 5 & & \\
\hline \multicolumn{5}{|c|}{ * using $1.20 \mathrm{~g} / \mathrm{cc}$ for Lexan } \\
\hline ** using 1.1 & g/cc for & & & \\
\hline
\end{tabular}


Table C.17: Density Results for 10 wt\% Ketjenblack EC-600 JD in Lexan HF1130-111: BA10L: Extruded November 11, 2009; Injection Molded December 4, 2009

\begin{tabular}{|c|c|c|c|c|}
\hline Test Date & Sample & $\begin{array}{l}\text { Actual } \\
\text { Density } \\
(\mathrm{g} / \mathrm{cc})\end{array}$ & $\begin{array}{l}\text { Theoretical } \\
\text { Density }(\mathrm{g} / \mathrm{cc}) \text { * }\end{array}$ & $\begin{array}{l}\text { Theoretical } \\
\text { Density }(\mathrm{g} / \mathrm{cc})^{\star *}\end{array}$ \\
\hline $12 / 16 / 2009$ & BA10L-TC-12 & 1.2372 & 1.241 & 1.238 \\
\hline $12 / 16 / 2009$ & BA10L-TC-16 & 1.2372 & 1.241 & 1.238 \\
\hline $12 / 16 / 2009$ & BA10L-TC-24 & 1.2374 & 1.241 & 1.238 \\
\hline $12 / 16 / 2009$ & BA10L-TC-27 & 1.2378 & 1.241 & 1.238 \\
\hline \multirow[t]{4}{*}{$12 / 16 / 2009$} & BA10L-TC-29 & 1.2372 & 1.241 & 1.238 \\
\hline & Average & 1.2373 & & \\
\hline & Std Dev & 0.0003 & & \\
\hline & Number & 5 & & \\
\hline
\end{tabular}

* using $1.20 \mathrm{~g} / \mathrm{cc}$ for Lexan

** using $1.196 \mathrm{~g} / \mathrm{cc}$ for Lexan 


\section{Appendix C.3: Polycarbonate with Graphene Nanoplatelets}

Table C.18: Density Results for Lexan HF1130-111:BL3: Injection Molded June 3, 2010

\begin{tabular}{|c|l|r|r|}
\hline \multirow{2}{*}{ Test Date } & Sample Number & $\begin{array}{l}\text { Theoretical } \\
\text { Density }(\mathrm{g} / \mathrm{ml})\end{array}$ & $\begin{array}{l}\text { Actual } \\
\text { Density } \\
(\mathrm{g} / \mathrm{ml})\end{array}$ \\
\hline $6 / 4 / 2010$ & BL3-TC-20 & 1.200 & 1.1957 \\
\hline $6 / 4 / 2010$ & BL3-TC-15 & 1.200 & 1.1938 \\
\hline $6 / 4 / 2010$ & BL3-TC-18 & 1.200 & 1.1956 \\
\hline $6 / 4 / 2010$ & BL3-TC-7 & 1.200 & 1.1941 \\
\hline $6 / 4 / 2010$ & BL3-TC-25 & 1.200 & 1.1941 \\
\hline & & Average & 1.1947 \\
\cline { 2 - 4 } & $\begin{array}{l}\text { Standard } \\
\text { Deviation }\end{array}$ \\
\cline { 2 - 4 } & & $\begin{array}{l}\text { Number of } \\
\text { Samples }\end{array}$ \\
\cline { 3 - 4 } & & & 0.0009 \\
\cline { 3 - 4 } & & &
\end{tabular}

Table C.19: Density Results for Extruded Lexan HF1130-111:BLE3: Extruded May 26, 2010; Injection Molded June 3, 2010

\begin{tabular}{|c|l|r|r|}
\hline \multirow{2}{*}{ Test Date } & Sample Number & $\begin{array}{l}\text { Theoretical } \\
\text { Density }(\mathrm{g} / \mathrm{ml})\end{array}$ & $\begin{array}{l}\text { Actual } \\
\text { Density } \\
(\mathrm{g} / \mathrm{ml})\end{array}$ \\
\hline $6 / 4 / 2010$ & BLE3-TC-17 & 1.200 & 1.1940 \\
\hline $6 / 4 / 2010$ & BLE3-TC-16 & 1.200 & 1.1946 \\
\hline $6 / 4 / 2010$ & BLE3-TC-12 & 1.200 & 1.1938 \\
\hline $6 / 4 / 2010$ & BLE3-TC-20 & 1.200 & 1.1938 \\
\hline $6 / 4 / 2010$ & BLE3-TC-18 & 1.200 & 1.1946 \\
\hline & & Average & 1.1942 \\
\cline { 2 - 4 } & $\begin{array}{l}\text { Standard } \\
\text { Deviation }\end{array}$ \\
\cline { 3 - 4 } & & $\begin{array}{l}\text { Number of } \\
\text { Samples }\end{array}$ \\
\cline { 3 - 4 } & &
\end{tabular}


Table C.20: Density Results for $2 \mathrm{wt} \% \mathrm{xGnP}$ in Lexan HF1130-111: BG2L: Extruded May 26, 2010; Injection Molded June 3, 2010

\begin{tabular}{|r|l|l|r|r|}
\hline Test Date & $\begin{array}{l}\text { Sample } \\
\text { Number }\end{array}$ & $\begin{array}{l}\text { Theoretical } \\
\text { Density }(\mathrm{g} / \mathrm{ml})^{*}\end{array}$ & $\begin{array}{l}\text { Theoretical } \\
\text { Density }(\mathrm{g} / \mathrm{ml})^{\star *}\end{array}$ & $\begin{array}{l}\text { Actual } \\
\text { Density } \\
(\mathrm{g} / \mathrm{ml})\end{array}$ \\
\hline $6 / 4 / 2010$ & BG2L-TC-8 & 1.2097 & 1.2044 & 1.2041 \\
\hline $6 / 4 / 2010$ & BG2L-TC-13 & 1.2097 & 1.2044 & 1.2029 \\
\hline $6 / 4 / 2010$ & BG2L-TC-29 & 1.2097 & 1.2044 & 1.2029 \\
\hline $6 / 4 / 2010$ & BG2L-TC-19 & 1.2097 & 1.2044 & 1.2018 \\
\hline $6 / 4 / 2010$ & BG2L-TC-11 & \multicolumn{1}{|c|}{1.2097} & 1.2044 & 1.2021 \\
\hline & Average & & 1.2027 \\
\cline { 3 - 5 } & $\begin{array}{l}\text { Standard } \\
\text { Deviation }\end{array}$ & \\
\cline { 3 - 5 } & $\begin{array}{l}\text { Number of } \\
\text { Samples }\end{array}$ & & \\
\cline { 3 - 5 } & & & \\
\end{tabular}

* using $1.20 \mathrm{~g} / \mathrm{cc}$ for Lexan

** using $1.1947 \mathrm{~g} / \mathrm{cc}$ for Lexan

Table C.21: Density Results for $3 \mathrm{wt} \% \mathrm{xGnP}$ in Lexan HF1130-111: BG3L: Extruded May 26, 2010; Injection Molded June 3, 2010

\begin{tabular}{|c|c|c|c|c|}
\hline Test Date & Sample Number & $\begin{array}{l}\text { Theoretical } \\
\text { Density }(\mathrm{g} / \mathrm{ml})^{*}\end{array}$ & $\begin{array}{l}\text { Theoretical } \\
\text { Density }(\mathrm{g} / \mathrm{ml})^{* *}\end{array}$ & $\begin{array}{l}\text { Actual } \\
\text { Density } \\
(\mathrm{g} / \mathrm{ml})\end{array}$ \\
\hline 6/4/2010 & BG3L-TC-8 & 1.2146 & 1.2093 & 1.2059 \\
\hline $6 / 4 / 2010$ & BG3L-TC-10 & 1.2146 & 1.2093 & 1.2060 \\
\hline $6 / 4 / 2010$ & BG3L-TC-28 & 1.2146 & 1.2093 & 1.2063 \\
\hline $6 / 4 / 2010$ & BG3L-TC-20 & 1.2146 & 1.2093 & 1.2061 \\
\hline $6 / 4 / 2010$ & BG3L-TC-16 & 1.2146 & 1.2093 & 1.2075 \\
\hline $6 / 4 / 2010$ & BG3L-TC-23 & 1.2146 & 1.2093 & 1.2056 \\
\hline & & Average & & 1.2062 \\
\hline & & $\begin{array}{l}\text { Standard } \\
\text { Deviation }\end{array}$ & & 0.0007 \\
\hline & & $\begin{array}{l}\text { Number of } \\
\text { Samples }\end{array}$ & & 6 \\
\hline
\end{tabular}

* using $1.20 \mathrm{~g} / \mathrm{cc}$ for Lexan

** using $1.1947 \mathrm{~g} / \mathrm{cc}$ for Lexan 
Table C.22: Density Results for $4 \mathrm{wt} \% \mathrm{xGnP}$ in Lexan HF1130-111: BG4L: Extruded May 26, 2010; Injection Molded June 3, 2010

\begin{tabular}{|c|c|c|c|c|}
\hline Test Date & Sample Number & $\begin{array}{l}\text { Theoretical } \\
\text { Density } \\
(\mathrm{g} / \mathrm{ml})^{\star}\end{array}$ & $\begin{array}{l}\text { Theoretical } \\
\text { Density }(\mathrm{g} / \mathrm{ml})^{\star *}\end{array}$ & $\begin{array}{l}\text { Actual } \\
\text { Density } \\
(\mathrm{g} / \mathrm{ml})\end{array}$ \\
\hline $6 / 4 / 2010$ & BG4L-TC-11 & 1.2195 & 1.2143 & 1.2104 \\
\hline $6 / 4 / 2010$ & BG4L-TC-24 & 1.2195 & 1.2143 & 1.2110 \\
\hline $6 / 4 / 2010$ & BG4L-TC-14 & 1.2195 & 1.2143 & 1.2107 \\
\hline $6 / 4 / 2010$ & BG4L-TC-18 & 1.2195 & 1.2143 & 1.2108 \\
\hline $6 / 4 / 2010$ & BG4L-TC-21 & 1.2195 & 1.2143 & 1.2125 \\
\hline $6 / 4 / 2010$ & BG4L-TC-15 & 1.2195 & 1.2143 & 1.2069 \\
\hline & & Average & & 1.2104 \\
\hline & & $\begin{array}{l}\text { Standard } \\
\text { Deviation }\end{array}$ & & 0.0019 \\
\hline & & $\begin{array}{l}\text { Number of } \\
\text { Samples }\end{array}$ & & 6 \\
\hline
\end{tabular}

* using $1.20 \mathrm{~g} / \mathrm{cc}$ for Lexan

** using $1.1947 \mathrm{~g} / \mathrm{cc}$ for Lexan

Table C.23: Density Results for $5 \mathrm{wt} \% \mathrm{xGnP}$ in Lexan HF1130-111: BG5L: Extruded May 26, 2010; Injection Molded June 3, 2010

\begin{tabular}{|r|l|l|r|r|}
\hline Test Date & Sample Number & $\begin{array}{l}\text { Theoretical } \\
\text { Density } \\
(\mathrm{g} / \mathrm{ml})^{*}\end{array}$ & $\begin{array}{l}\text { Theoretical } \\
\text { Density }(\mathrm{g} / \mathrm{ml})^{* *}\end{array}$ & $\begin{array}{l}\text { Actual } \\
\text { Density } \\
(\mathrm{g} / \mathrm{ml})\end{array}$ \\
\hline $6 / 4 / 2010$ & BG5L-TC-30 & 1.2245 & 1.2192 & 1.2156 \\
\hline $6 / 4 / 2010$ & BG5L-TC-20 & 1.2245 & 1.2192 & 1.2152 \\
\hline $6 / 4 / 2010$ & BG5L-TC-15 & 1.2245 & 1.2192 & 1.2156 \\
\hline $6 / 4 / 2010$ & BG5L-TC-26 & 1.2245 & 1.2192 & 1.2159 \\
\hline $6 / 4 / 2010$ & BG5L-TC-10 & 1.2245 & 1.2192 & 1.2146 \\
\hline & Average & & 1.2154 \\
\cline { 3 - 5 } & $\begin{array}{l}\text { Standard } \\
\text { Deviation }\end{array}$ & \\
\cline { 3 - 5 } & $\begin{array}{l}\text { Number of } \\
\text { Samples }\end{array}$ & & \\
\cline { 3 - 5 } & & & & \\
& & & & \\
\end{tabular}

* using $1.20 \mathrm{~g} / \mathrm{cc}$ for Lexan

** using $1.1947 \mathrm{~g} / \mathrm{cc}$ for Lexan 
Table C.24: Density Results for $6 \mathrm{wt} \% \mathrm{xGnP}$ in Lexan HF1130-111: BG5L: Extruded May 27, 2010; Injection Molded June 8, 2010

\begin{tabular}{|c|c|c|c|c|}
\hline Test Date & Sample Number & $\begin{array}{l}\text { Theoretical } \\
\text { Density } \\
(\mathrm{g} / \mathrm{ml})^{*}\end{array}$ & $\begin{array}{l}\text { Theoretical } \\
\text { Density } \\
(\mathrm{g} / \mathrm{ml})^{* *}\end{array}$ & $\begin{array}{l}\text { Actual } \\
\text { Density }(\mathrm{g} / \mathrm{ml})\end{array}$ \\
\hline $6 / 8 / 2009$ & BG6L-TC-11 & 1.2295 & 1.2243 & 1.2224 \\
\hline $6 / 8 / 2009$ & BG6L-TC-16 & 1.2295 & 1.2243 & 1.2232 \\
\hline $6 / 8 / 2009$ & BG6L-TC-19 & 1.2295 & 1.2243 & 1.2226 \\
\hline $6 / 8 / 2009$ & BG6L-TC-29 & 1.2295 & 1.2243 & 1.2224 \\
\hline $6 / 8 / 2009$ & BG6L-TC-23 & 1.2295 & 1.2243 & 1.2237 \\
\hline & & Average & & 1.2229 \\
\hline & & $\begin{array}{l}\text { Standard } \\
\text { Deviation }\end{array}$ & & 0.0006 \\
\hline & & $\begin{array}{l}\text { Number of } \\
\text { Samples }\end{array}$ & & 5 \\
\hline
\end{tabular}

* using $1.20 \mathrm{~g} / \mathrm{cc}$ for Lexan

** using $1.1947 \mathrm{~g} / \mathrm{cc}$ for Lexan

Table C.25: Density Results for 8 wt\% xGnP in Lexan HF1130-111: BG5L: Extruded May 27, 2010; Injection Molded June 8, 2010

\begin{tabular}{|r|l|r|r|r|}
\hline & & $\begin{array}{l}\text { Theoretical } \\
\text { Density } \\
\text { Test Date }\end{array}$ & $\begin{array}{l}\text { Theoretical } \\
\text { Density } \\
(\mathrm{g} / \mathrm{ml})^{* *}\end{array}$ & $\begin{array}{l}\text { Actual } \\
\text { Density }(\mathrm{g} / \mathrm{ml})\end{array}$ \\
\hline $6 / 8 / 2009$ & Sample Number & 1.2397 & 1.2345 & 1.2308 \\
\hline $6 / 8 / 2009$ & BG8L-TC-15 & 1.2397 & 1.2345 & 1.2307 \\
\hline $6 / 8 / 2009$ & BG8L-TC-20 & 1.2397 & 1.2345 & 1.2311 \\
\hline $6 / 8 / 2009$ & BG8L-TC-9 & 1.2397 & 1.2345 & 1.2298 \\
\hline $6 / 8 / 2009$ & BG8L-TC-25 & 1.2397 & 1.2345 & 1.2306 \\
\hline & Average & & 1.2306 \\
\cline { 2 - 5 } & $\begin{array}{l}\text { Standard } \\
\text { Deviation }\end{array}$ & 0.0005 \\
\cline { 2 - 5 } & $\begin{array}{l}\text { Number of } \\
\text { Samples }\end{array}$ \\
\cline { 3 - 5 } & & & \\
\cline { 3 - 5 } & & & \\
\end{tabular}

* using $1.20 \mathrm{~g} / \mathrm{cc}$ for Lexan

** using $1.1947 \mathrm{~g} / \mathrm{cc}$ for Lexan 
Table C.26: Density Results for 10 wt\% xGnP in Lexan HF1130-111: BG5L: Extruded May 27, 2010; Injection Molded June 8, 2010

\begin{tabular}{|c|c|c|c|c|}
\hline Test Date & Sample Number & $\begin{array}{l}\text { Theoretical } \\
\text { Density } \\
(\mathrm{g} / \mathrm{ml})^{*}\end{array}$ & $\begin{array}{l}\text { Theoretical } \\
\text { Density } \\
(\mathrm{g} / \mathrm{ml})^{* *}\end{array}$ & $\begin{array}{l}\text { Actual } \\
\text { Density } \\
(\mathrm{g} / \mathrm{ml})\end{array}$ \\
\hline 6/8/2009 & BG10L-TC-33 & 1.2500 & 1.2448 & 1.2411 \\
\hline 6/8/2009 & BG10L-TC-41 & 1.2500 & 1.2448 & 1.2411 \\
\hline $6 / 8 / 2009$ & BG10L-TC-19 & 1.2500 & 1.2448 & 1.2395 \\
\hline $6 / 8 / 2009$ & BG10L-TC-32 & 1.2500 & 1.2448 & 1.2396 \\
\hline $6 / 8 / 2009$ & BG10L-TC-11 & 1.2500 & 1.2448 & 1.2399 \\
\hline & & Average & & 1.2402 \\
\hline & & $\begin{array}{l}\text { Standard } \\
\text { Deviation }\end{array}$ & & 0.0008 \\
\hline & & $\begin{array}{l}\text { Number of } \\
\text { Samples }\end{array}$ & & 5 \\
\hline
\end{tabular}

* using $1.20 \mathrm{~g} / \mathrm{cc}$ for Lexan

** using $1.1947 \mathrm{~g} / \mathrm{cc}$ for Lexan 


\section{Appendix C.4: Polycarbonate with Multiple Fillers}

Table C.27: Density Results for Lexan HF1130-111:BL4: Injection Molded July 26, 2010

\begin{tabular}{|c|c|c|c|}
\hline Test Date & Sample Number & $\begin{array}{l}\text { Theoretical } \\
\text { Density }(\mathrm{g} / \mathrm{ml})\end{array}$ & $\begin{array}{l}\text { Actual Density } \\
(\mathrm{g} / \mathrm{ml})\end{array}$ \\
\hline $7 / 30 / 2010$ & BL4-TC-3 & 1.200 & 1.1935 \\
\hline $7 / 30 / 2010$ & BL4-TC-9 & 1.200 & 1.1924 \\
\hline $7 / 30 / 2010$ & BL4-TC-4 & 1.200 & 1.1927 \\
\hline $7 / 30 / 2010$ & BL4-TC-6 & 1.200 & 1.1930 \\
\hline $7 / 30 / 2010$ & BL4-TC-1 & 1.200 & 1.1942 \\
\hline $7 / 30 / 2010$ & BL4-TC-5 & 1.200 & 1.1929 \\
\hline $7 / 30 / 2010$ & BL4-TC-2 & 1.200 & 1.1922 \\
\hline & & Average & 1.1930 \\
\hline & & $\begin{array}{l}\text { Standard } \\
\text { Deviation }\end{array}$ & 0.0007 \\
\hline & & $\begin{array}{l}\text { Number of } \\
\text { Samples }\end{array}$ & 7 \\
\hline
\end{tabular}

Table C.28: Density Results for BA2LR: Extruded 7-8-10; Injection Molded 7-26-10

\begin{tabular}{|c|c|c|c|}
\hline Test Date & $\begin{array}{l}\text { Sample } \\
\text { Number }\end{array}$ & $\begin{array}{l}\text { Theoretical } \\
\text { Density }(\mathrm{g} / \mathrm{ml})\end{array}$ & $\begin{array}{l}\text { Actual Density } \\
(\mathrm{g} / \mathrm{ml})\end{array}$ \\
\hline $7 / 30 / 2010$ & BA2LR-TC-9 & 1.2011 & 1.2022 \\
\hline $7 / 30 / 2010$ & BA2LR-TC-5 & 1.2011 & 1.2019 \\
\hline $7 / 30 / 2010$ & BA2LR-TC-15 & 1.2011 & 1.2017 \\
\hline $7 / 30 / 2010$ & BA2LR-TC-23 & 1.2011 & 1.2016 \\
\hline $7 / 30 / 2010$ & BA2LR-TC-28 & 1.2011 & 1.2017 \\
\hline $7 / 30 / 2010$ & BA2LR-TC-32 & 1.2011 & 1.2019 \\
\hline $7 / 30 / 2010$ & BA2LR-TC-13 & 1.2011 & 1.2017 \\
\hline & & Average & 1.2018 \\
\hline & & $\begin{array}{l}\text { Standard } \\
\text { Deviation }\end{array}$ & 0.0002 \\
\hline & & $\begin{array}{l}\text { Number of } \\
\text { Samples }\end{array}$ & 7 \\
\hline
\end{tabular}


Table C29: Density Results for BA5LR: Extruded 7-8-10; Injection Molded 7-26-10

\begin{tabular}{|c|l|r|r|}
\hline \multirow{2}{*}{ Test Date } & $\begin{array}{l}\text { Sample } \\
\text { Number }\end{array}$ & $\begin{array}{l}\text { Theoretical } \\
\text { Density }(\mathrm{g} / \mathrm{ml})\end{array}$ & $\begin{array}{l}\text { Actual Density } \\
(\mathrm{g} / \mathrm{ml})\end{array}$ \\
\hline $7 / 30 / 2010$ & BA5LR-TC-21 & 1.2135 & 1.2142 \\
\hline $7 / 30 / 2010$ & BA5LR-TC-27 & 1.2135 & 1.2144 \\
\hline $7 / 30 / 2010$ & BA5LR-TC-13 & 1.2135 & 1.2138 \\
\hline $7 / 30 / 2010$ & BA5LR-TC-30 & 1.2135 & 1.2148 \\
\hline $7 / 30 / 2010$ & BA5LR-TC-14 & 1.2135 & 1.2132 \\
\hline $7 / 30 / 2010$ & BA5LR-TC-10 & 1.2135 & 1.2129 \\
\hline $7 / 30 / 2010$ & BA5LR-TC-25 & 1.2135 & 1.2141 \\
\hline \multirow{5}{*}{} & Average & 1.2139 \\
\cline { 2 - 4 } & $\begin{array}{l}\text { Standard } \\
\text { Deviation }\end{array}$ \\
\cline { 2 - 4 } & $\begin{array}{l}\text { Number of } \\
\text { Samples }\end{array}$ \\
\cline { 3 - 4 } & & & \\
& & & \\
& &
\end{tabular}

Table C.30: Density Results for BG12L: Extruded 7-8-10; Injection Molded 8-18-10

\begin{tabular}{|r|l|l|l|}
\hline \multirow{2}{*}{ Test Date } & $\begin{array}{l}\text { Sample } \\
\text { Number }\end{array}$ & $\begin{array}{l}\text { Theoretical } \\
\text { Density }(\mathrm{g} / \mathrm{ml})\end{array}$ & $\begin{array}{l}\text { Actual Density } \\
(\mathrm{g} / \mathrm{ml})\end{array}$ \\
\hline $8 / 31 / 2010$ & BG12L-TC-22 & 1.2537 & 1.2519 \\
\hline $8 / 31 / 2010$ & BG12L-TC-28 & 1.2537 & 1.2520 \\
\hline $8 / 31 / 2010$ & BG12L-TC-30 & 1.2537 & 1.2523 \\
\hline $8 / 31 / 2010$ & BG12L-TC-11 & 1.2537 & 1.2521 \\
\hline $8 / 31 / 2010$ & BG12L-TC-12 & 1.2537 & 1.2523 \\
\hline & Average & 1.2521 \\
\cline { 2 - 4 } & $\begin{array}{l}\text { Standard } \\
\text { Deviation }\end{array}$ & 0.0002 \\
\cline { 2 - 4 } & $\begin{array}{l}\text { Number of } \\
\text { Samples }\end{array}$ \\
\cline { 3 - 4 } & & \\
\end{tabular}


Table C.31: Density Results for BG15L: Extruded 7-8-10; Injection Molded 8-18-10

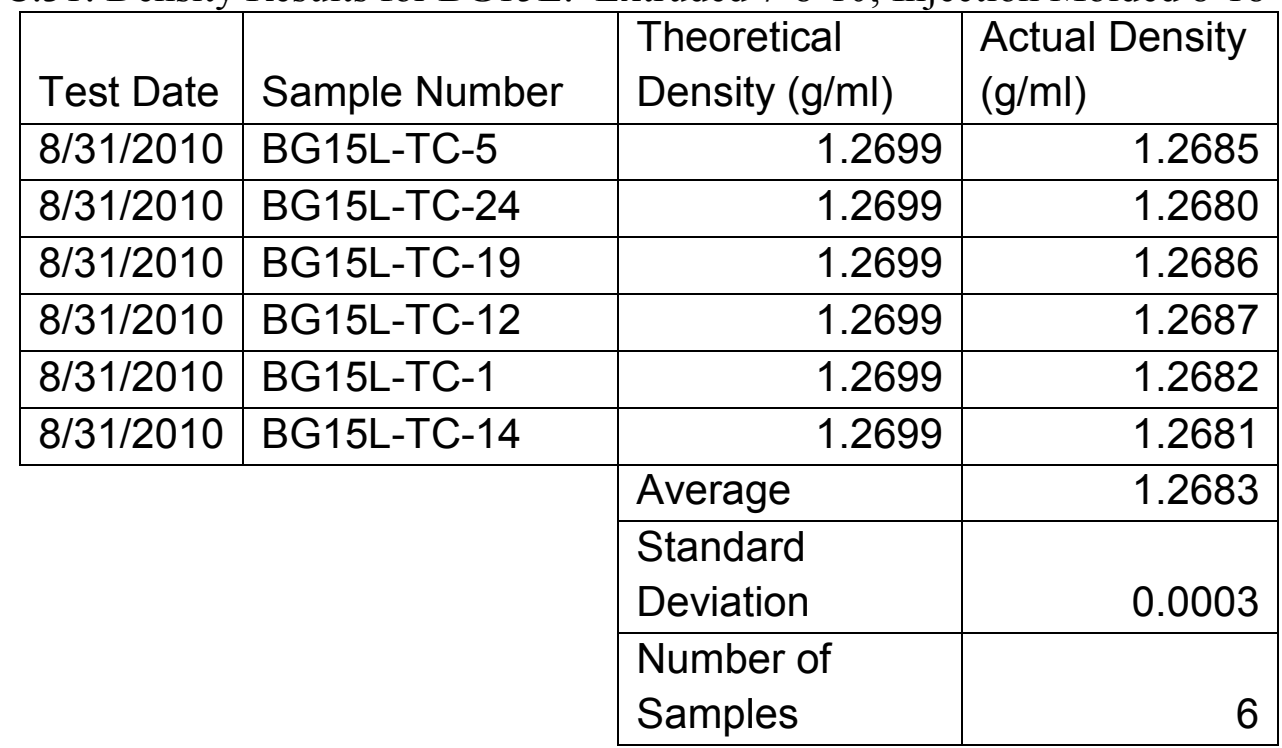

Table C.32: Density Results for BA2Q1L: Extruded 7-7-10; Injection Molded June 26, 2010

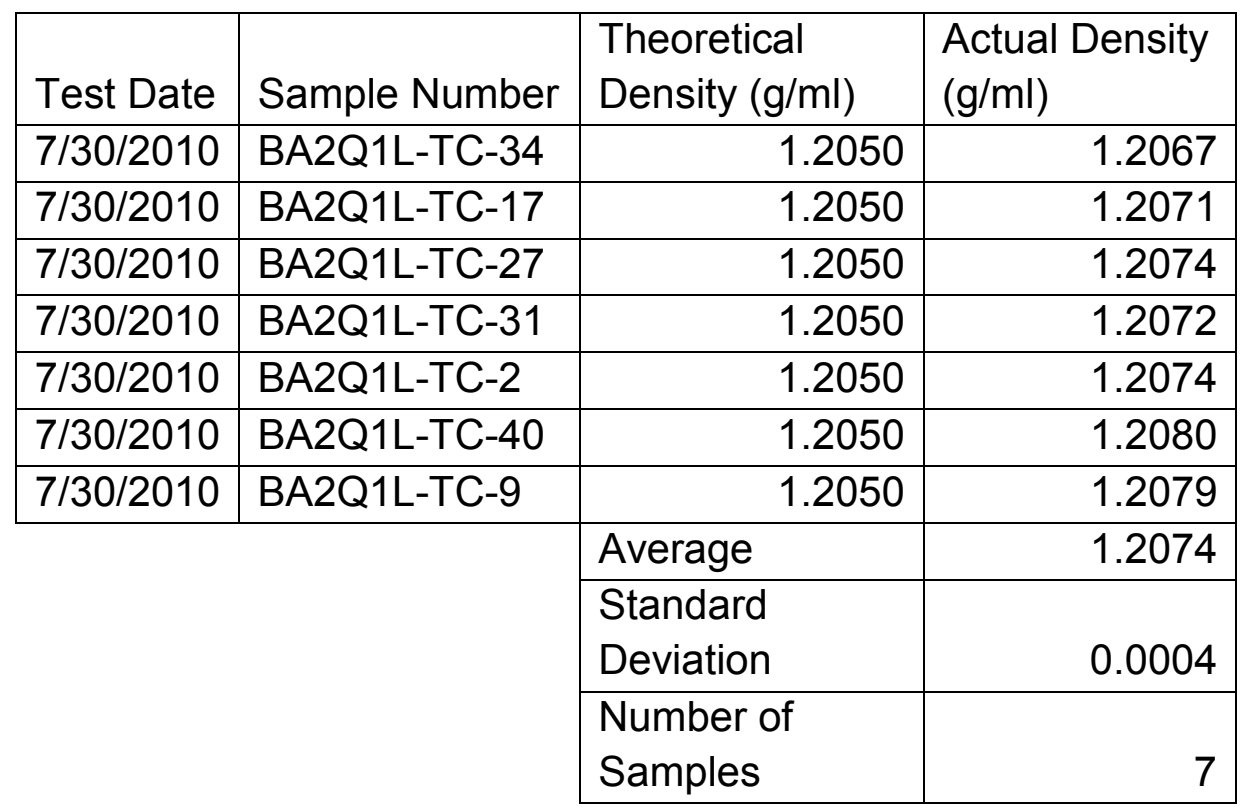


Table C.33: Density Results for BA2Q5L: Extruded 7-7-10; Injection Molded 8-25-10

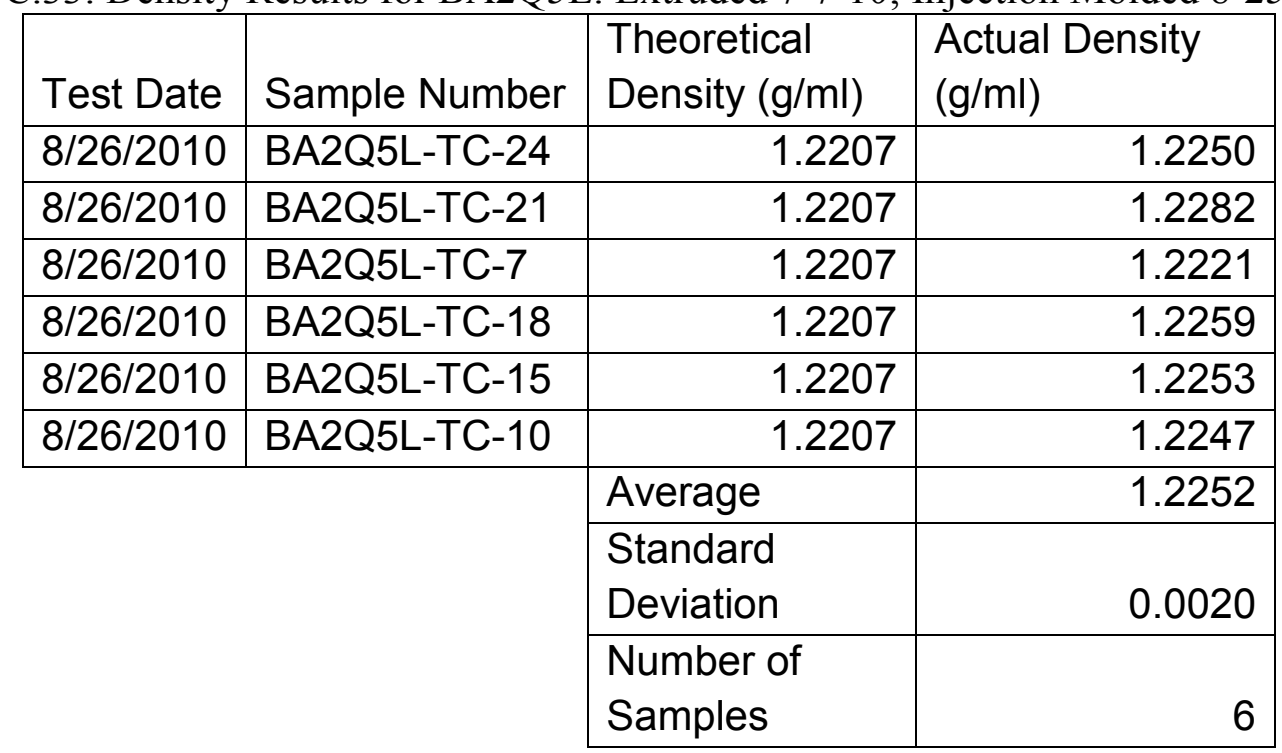

Table C.34: Density Results for BA5Q1L: Extruded 7-7-10; Injection Molded 7-26-10

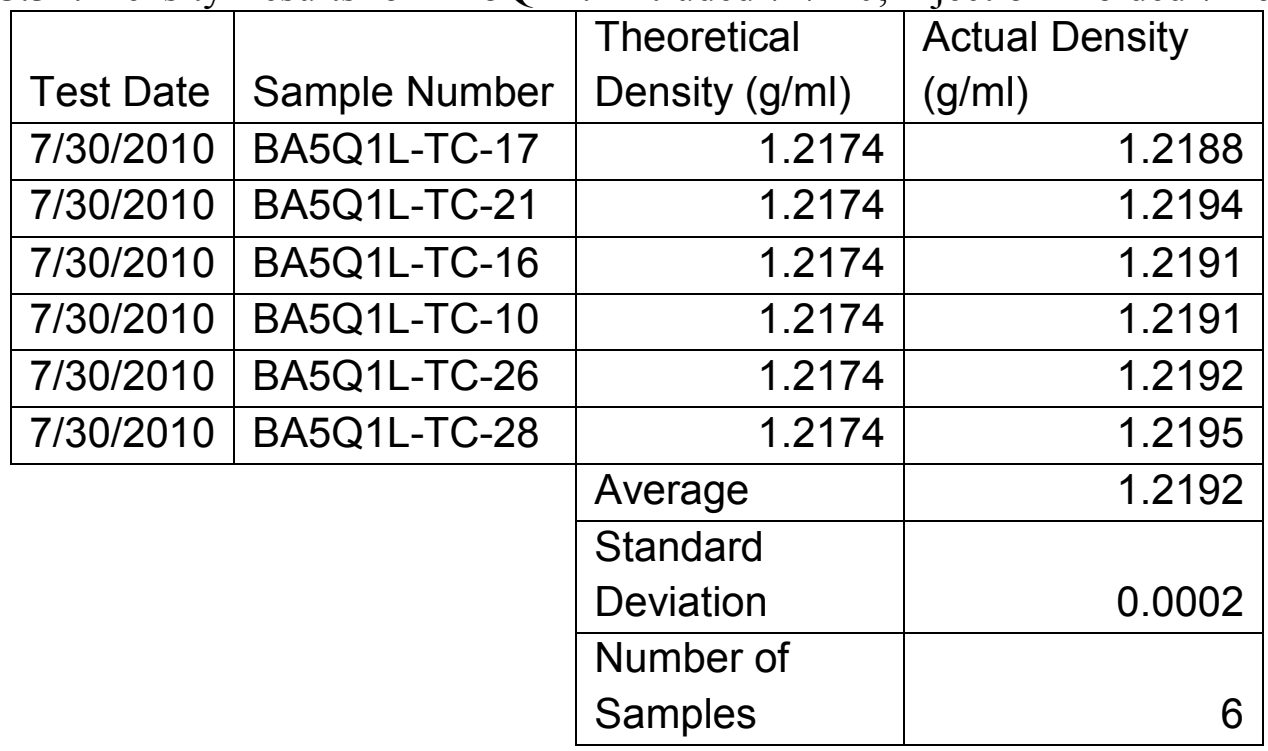


Table C.35: Density Results for BA5Q5L: Extruded 7-7-10; Injection Molded 8-25-10

\begin{tabular}{|l|l|l|r|}
\hline Test Date & Sample Number & $\begin{array}{l}\text { Theoretical } \\
\text { Density }(\mathrm{g} / \mathrm{ml})\end{array}$ & $\begin{array}{l}\text { Actual Density } \\
(\mathrm{g} / \mathrm{ml})\end{array}$ \\
\hline $8 / 26 / 2010$ & BA5Q5L-TC-9 & 1.2334 & 1.2353 \\
\hline $8 / 26 / 2010$ & BA5Q5L-TC-17 & 1.2334 & 1.2384 \\
\hline $8 / 26 / 2010$ & BA5Q5L-TC-23 & 1.2334 & 1.2388 \\
\hline $8 / 26 / 2010$ & BA5Q5L-TC-25 & 1.2334 & 1.2384 \\
\hline $8 / 26 / 2010$ & BA5Q5L-TC-15 & 1.2334 & 1.2385 \\
\hline & Average & 1.2379 \\
\cline { 3 - 4 } & $\begin{array}{l}\text { Standard } \\
\text { Deviation }\end{array}$ & 0.0015 \\
\cline { 3 - 4 } & $\begin{array}{l}\text { Number of } \\
\text { Samples }\end{array}$ \\
\cline { 3 - 4 } & & \\
\end{tabular}

Table C.36: Density Results for BA2G2L: Extruded 7-6-10; Injection Molded 8-18-10

\begin{tabular}{|r|l|r|r|}
\hline \multirow{2}{*}{ Test Date } & Sample Number & $\begin{array}{l}\text { Theoretical } \\
\text { Density }(\mathrm{g} / \mathrm{ml})\end{array}$ & $\begin{array}{l}\text { Actual Density } \\
(\mathrm{g} / \mathrm{ml})\end{array}$ \\
\hline $8 / 18 / 2010$ & BA2G2L-TC-12 & 1.2109 & 1.2095 \\
\hline $8 / 18 / 2010$ & BA2G2L-TC-6 & 1.2109 & 1.2098 \\
\hline $8 / 18 / 2010$ & BA2G2L-TC-1 & 1.2109 & 1.2091 \\
\hline $8 / 18 / 2010$ & BA2G2L-TC-20 & 1.2109 & 1.2090 \\
\hline $8 / 18 / 2010$ & BA2G2L-TC-13 & 1.2109 & 1.2090 \\
\hline $8 / 18 / 2010$ & BA2G2L-TC-4 & 1.2109 & 1.2096 \\
\hline \multirow{5}{*}{} & Average & 1.2093 \\
\cline { 2 - 4 } & $\begin{array}{l}\text { Standard } \\
\text { Deviation }\end{array}$ \\
\cline { 2 - 4 } & $\begin{array}{l}\text { Number of } \\
\text { Samples }\end{array}$ \\
\cline { 3 - 4 } & &
\end{tabular}


Table C.37: Density Results for BA2G5L: Extruded 7-6-10; Injection Molded 8-18-10

\begin{tabular}{|c|l|l|r|}
\hline \multirow{2}{*}{ Test Date } & Sample Number & $\begin{array}{l}\text { Theoretical } \\
\text { Density }(\mathrm{g} / \mathrm{ml})\end{array}$ & $\begin{array}{l}\text { Actual Density } \\
(\mathrm{g} / \mathrm{ml})\end{array}$ \\
\hline $8 / 18 / 2010$ & BA2G5L-TC-7 & 1.2260 & 1.2240 \\
\hline $8 / 18 / 2010$ & BA2G5L-TC-30 & 1.2260 & 1.2259 \\
\hline $8 / 18 / 2010$ & BA2G5L-TC-20 & 1.2260 & 1.2256 \\
\hline $8 / 18 / 2010$ & BA2G5L-TC-17 & 1.2260 & 1.2249 \\
\hline $8 / 18 / 2010$ & BA2G5L-TC-6 & 1.2260 & 1.2254 \\
\hline & Average & 1.2252 \\
\cline { 3 - 4 } & $\begin{array}{l}\text { Standard } \\
\text { Deviation }\end{array}$ \\
\cline { 3 - 4 } & $\begin{array}{l}\text { Number of } \\
\text { Samples }\end{array}$ \\
\cline { 3 - 4 } & & \\
& &
\end{tabular}

Table C.38: Density Results for BA5G2L: Extruded 7-6-10; Injection Molded 8-18-10

\begin{tabular}{|c|l|l|r|}
\hline \multirow{2}{*}{ Test Date } & Sample Number & $\begin{array}{l}\text { Theoretical } \\
\text { Density }(\mathrm{g} / \mathrm{ml})\end{array}$ & $\begin{array}{l}\text { Actual Density } \\
(\mathrm{g} / \mathrm{ml})\end{array}$ \\
\hline $8 / 18 / 2010$ & BA5G2L-TC-24 & 1.2235 & 1.2238 \\
\hline $8 / 18 / 2010$ & BA5G2L-TC-17 & 1.2235 & 1.2234 \\
\hline $8 / 18 / 2010$ & BA5G2L-TC-3 & 1.2235 & 1.2258 \\
\hline $8 / 18 / 2010$ & BA5G2L-TC-10 & 1.2235 & 1.2242 \\
\hline $8 / 18 / 2010$ & BA5G2L-TC-25 & 1.2235 & 1.2235 \\
\hline $8 / 18 / 2010$ & BA5G2L-TC-14 & \multicolumn{1}{|c|}{1.2235} & 1.2245 \\
\hline & Average & 1.2241 \\
\cline { 2 - 4 } & $\begin{array}{l}\text { Standard } \\
\text { Deviation }\end{array}$ \\
\cline { 2 - 4 } & $\begin{array}{l}\text { Number of } \\
\text { Samples }\end{array}$ \\
\cline { 3 - 4 } & & & 0.0010 \\
\hline
\end{tabular}


Table C.39: Density Results for BA5G5L: Extruded 7-6-10; Injection Molded 8-18-10

\begin{tabular}{|c|l|r|r|}
\hline \multirow{2}{*}{ Test Date } & Sample Number & \multicolumn{1}{|l|}{$\begin{array}{l}\text { Theoretical } \\
\text { Density }(\mathrm{g} / \mathrm{ml})\end{array}$} & $\begin{array}{l}\text { Actual } \\
\text { Density }(\mathrm{g} / \mathrm{ml})\end{array}$ \\
\hline $8 / 18 / 2010$ & BA5G5L-TC-19 & 1.2389 & 1.2387 \\
\hline $8 / 18 / 2010$ & BA5G5L-TC-7 & 1.2389 & 1.2381 \\
\hline $8 / 18 / 2010$ & BA5G5L-TC-16 & 1.2389 & 1.2377 \\
\hline $8 / 18 / 2010$ & BA5G5L-TC-28 & \multicolumn{1}{|c|}{1.2389} & 1.2388 \\
\hline & Average & 1.2382 \\
\cline { 3 - 4 } & $\begin{array}{l}\text { Standard } \\
\text { Deviation }\end{array}$ & 0.0005 \\
\cline { 3 - 4 } & $\begin{array}{l}\text { Number of } \\
\text { Samples }\end{array}$ \\
\cline { 3 - 4 } & &
\end{tabular}

Table C.40: Density Results for BQ1G2L: Extruded 7-1-10; Injection Molded 8-18-10

\begin{tabular}{|c|l|l|r|}
\hline \multirow{2}{*}{ Test Date } & Sample Number & \multicolumn{1}{|l|}{$\begin{array}{l}\text { Theoretical } \\
\text { Density }(\mathrm{g} / \mathrm{ml})\end{array}$} & $\begin{array}{l}\text { Actual } \\
\text { Density }(\mathrm{g} / \mathrm{ml})\end{array}$ \\
\hline $8 / 18 / 2010$ & BQ1G2L-TC-8 & 1.2066 & 1.2050 \\
\hline $8 / 18 / 2010$ & BQ1G2L-TC-4 & 1.2066 & 1.2053 \\
\hline $8 / 18 / 2010$ & BQ1G2L-TC-11 & 1.2066 & 1.2057 \\
\hline $8 / 18 / 2010$ & BQ1G2L-TC-23 & \multicolumn{1}{|c|}{1.2066} & 1.2047 \\
\hline & Average & 1.2053 \\
\cline { 2 - 4 } & $\begin{array}{l}\text { Standard } \\
\text { Deviation }\end{array}$ & 0.0004 \\
\cline { 2 - 4 } & $\begin{array}{l}\text { Number of } \\
\text { Samples }\end{array}$ \\
\cline { 2 - 4 } & &
\end{tabular}


Table C.41: Density Results for BQ1G5L: Extruded 7-1-10; Injection Molded 8-18-10

\begin{tabular}{|c|l|r|r|}
\hline \multirow{2}{*}{ Test Date } & Sample Number & \multicolumn{1}{|l|}{$\begin{array}{l}\text { Theoretical } \\
\text { Density }(\mathrm{g} / \mathrm{ml})\end{array}$} & $\begin{array}{l}\text { Actual Density } \\
(\mathrm{g} / \mathrm{ml})\end{array}$ \\
\hline $8 / 18 / 2010$ & BQ1G5L-TC-19 & 1.2215 & 1.2192 \\
\hline $8 / 18 / 2010$ & BQ1G5L-TC-4 & 1.2215 & 1.2195 \\
\hline $8 / 18 / 2010$ & BQ1G5L-TC-10 & 1.2215 & 1.2197 \\
\hline $8 / 18 / 2010$ & BQ1G5L-TC-27 & \multicolumn{1}{|c|}{1.2215} & 1.2193 \\
\hline & Average & 1.2194 \\
\cline { 3 - 4 } & $\begin{array}{l}\text { Standard } \\
\text { Deviation }\end{array}$ & 0.0002 \\
\cline { 3 - 4 } & $\begin{array}{l}\text { Number of } \\
\text { Samples }\end{array}$ \\
\cline { 3 - 4 } & &
\end{tabular}

Table C.42: Density Results for BQ5G2L: Extruded 7-1-10; Injection Molded 8-18-10

\begin{tabular}{|c|c|c|c|}
\hline Test Date & Sample Number & $\begin{array}{l}\text { Theoretical } \\
\text { Density }(\mathrm{g} / \mathrm{ml})\end{array}$ & $\begin{array}{l}\text { Actual Density } \\
(\mathrm{g} / \mathrm{ml})\end{array}$ \\
\hline $8 / 19 / 2010$ & BQ5G2L-TC-22 & 1.2223 & 1.2231 \\
\hline $8 / 19 / 2010$ & BQ5G2L-TC-14 & 1.2223 & 1.2222 \\
\hline $8 / 19 / 2010$ & BQ5G2L-TC-9 & 1.2223 & 1.2233 \\
\hline $8 / 19 / 2010$ & BQ5G2L-TC-3 & 1.2223 & 1.2224 \\
\hline $8 / 19 / 2010$ & BQ5G2L-TC-17 & 1.2223 & 1.2236 \\
\hline $8 / 19 / 2010$ & BQ5G2L-TC-25 & 1.2223 & 1.2228 \\
\hline & & Average & 1.2229 \\
\hline & & $\begin{array}{l}\text { Standard } \\
\text { Deviation }\end{array}$ & 0.0006 \\
\hline & & $\begin{array}{l}\text { Number of } \\
\text { Samples }\end{array}$ & 6 \\
\hline
\end{tabular}


Table C.43: Density Results for BQ5G5L: Extruded 7-1-10; Injection Molded 8-18-10

\begin{tabular}{|r|l|l|r|}
\hline \multirow{2}{*}{ Test Date } & Sample Number & $\begin{array}{l}\text { Theoretical } \\
\text { Density }(\mathrm{g} / \mathrm{ml})\end{array}$ & $\begin{array}{l}\text { Actual Density } \\
(\mathrm{g} / \mathrm{ml})\end{array}$ \\
\hline $8 / 19 / 2010$ & BQ5G5L-TC-12 & 1.2377 & 1.2370 \\
\hline $8 / 19 / 2010$ & BQ5G5L-TC-23 & 1.2377 & 1.2378 \\
\hline $8 / 19 / 2010$ & BQ5G5L-TC-30 & 1.2377 & 1.2379 \\
\hline $8 / 19 / 2010$ & BQ5G5L-TC-16 & 1.2377 & 1.2380 \\
\hline & Average & 1.2376 \\
\cline { 2 - 4 } & $\begin{array}{l}\text { Standard } \\
\text { Deviation }\end{array}$ & 0.0005 \\
\cline { 2 - 4 } & $\begin{array}{l}\text { Number of } \\
\text { Samples }\end{array}$ \\
\cline { 3 - 4 } & & 4 \\
\hline
\end{tabular}




\section{Appendix D: Melt Flow Indexing Results}

\section{Appendix D.1: Polycarbonate with Carbon Nanotubes}

Table D.1: MFI Results for BL at $300^{\circ} \mathrm{C}$

\begin{tabular}{|l|l|l|}
\hline BL & $\begin{array}{l}\text { weight } \\
(\mathbf{g})\end{array}$ & $\begin{array}{l}\text { MFI } \\
(\mathbf{g} / \mathbf{1 0} \mathbf{m i n})\end{array}$ \\
\hline 1 & 0.7886 & 31.544 \\
\hline & & \\
2 & 0.8187 & 32.748 \\
\hline 3 & 0.8087 & 32.348 \\
\hline 4 & 0.8021 & 32.084 \\
\hline 5 & 0.8392 & 33.568 \\
\hline 6 & 0.7541 & 30.164 \\
\hline 7 & 0.8046 & 32.184 \\
\hline Average & 0.80 & 32.09 \\
\hline Stdev & 0.03 & 1.06 \\
\hline Count & 7 & 7 \\
\hline
\end{tabular}

Conditions

Dwell time $\quad 360 \quad \mathrm{~s}$

Collect

Time $\quad 15 \quad \mathrm{~s}$

Temp $\quad 300 \quad{ }^{\circ} \mathrm{C}$

Mass $\quad 1.25 \quad \mathrm{~kg}$

Table D.2: MFI Results for BLE at $300^{\circ} \mathrm{C}$

\begin{tabular}{|l|l|l|}
\hline BLE & $\begin{array}{l}\text { weight } \\
(\mathbf{g})\end{array}$ & $\begin{array}{l}\text { MFI } \\
(\mathbf{g} / \mathbf{1 0 m i n})\end{array}$ \\
\hline 1 & 0.8777 & 35.108 \\
\hline 2 & 0.8479 & 33.916 \\
\hline 3 & 0.8965 & 35.86 \\
\hline 4 & 0.876 & 35.04 \\
\hline 5 & 0.8273 & 33.092 \\
\hline 6 & 0.852 & 34.08 \\
\hline 7 & 0.8868 & 35.472 \\
\hline Average & 0.87 & 34.65 \\
\hline Stdev & 0.02 & 0.98 \\
\hline Count & 7 & 7 \\
\hline
\end{tabular}

Conditions

Dwell

time $\quad 360 \quad \mathrm{~s}$

Collect

Time $\quad 15 \quad \mathrm{~s}$

Temp $\quad 300 \quad{ }^{\circ} \mathrm{C}$

Mass $\quad 1.25 \quad \mathrm{~kg}$ 
Table D.3: MFI Results for BQ2L at $320^{\circ} \mathrm{C}$

\begin{tabular}{|l|l|l|l|}
\hline BQ2L & weight $(\mathbf{g})$ & $\begin{array}{l}\text { collection time } \\
(\mathbf{s})\end{array}$ & $\begin{array}{l}\text { MFI } \\
(\mathbf{g} / \mathbf{1 0 m i n})\end{array}$ \\
\hline 1 & 7.0399 & 16.10 & 262.36 \\
\hline 2 & 7.5509 & 16.36 & 276.93 \\
\hline 3 & 6.8234 & 12.77 & 320.60 \\
\hline 4 & 6.4848 & 11.80 & 329.74 \\
\hline 5 & 7.377 & 16.41 & 269.73 \\
\hline Average & 7.0552 & 14.69 & 291.87 \\
\hline Stdev & 0.4267889 & 2.22 & 31.00 \\
\hline Count & 5 & 5 & 5 \\
\hline
\end{tabular}

Conditions

Dwell time $360 \quad \mathrm{~s}$

Temp $\quad 320 \quad{ }^{\circ} \mathrm{C}$

Mass $5 \quad \mathrm{~kg}$

Table D.4: MFI Results for BQ3L at $320^{\circ} \mathrm{C}$

\begin{tabular}{|c|c|c|c|c|c|}
\hline BQ3L & weight (g) & $\begin{array}{l}\text { collection time } \\
\text { (s) }\end{array}$ & $\begin{array}{l}\text { MFI } \\
(\mathrm{g} / 10 \mathrm{~min})\end{array}$ & Conditions & \\
\hline 1 & 7.5219 & 19.21 & 234.94 & Dwell time & 360 \\
\hline 2 & 7.1056 & 16.29 & 261.72 & Temp & 320 \\
\hline 3 & 7.7166 & 18.21 & 254.25 & Mass & \\
\hline 4 & 7.0722 & 16.89 & 251.23 & & \\
\hline 5 & 7.2726 & 17.98 & 242.69 & & \\
\hline 6 & 7.2386 & 19.58 & 221.82 & & \\
\hline 7 & 7.1151 & 16.80 & 254.11 & & \\
\hline Average & 7.2918 & 17.85 & 245.82 & & \\
\hline Stdev & 0.2417704 & 1.25 & 13.70 & & \\
\hline Count & 7 & 7 & 7 & & \\
\hline
\end{tabular}


Table D.5: MFI Results for BQ4L at $320^{\circ} \mathrm{C}$

\begin{tabular}{|l|l|l|l|}
\hline BQ4L & weight $\mathbf{( g )}$ & $\begin{array}{l}\text { collection time } \\
(\mathbf{s})\end{array}$ & $\begin{array}{l}\text { MFI } \\
(\mathbf{g} / \mathbf{1 0 m i n})\end{array}$ \\
\hline 1 & 7.8128 & 31.32 & 149.67 \\
\hline 2 & 7.6709 & 25.35 & 181.56 \\
\hline 3 & 7.8292 & 25.12 & 187.00 \\
\hline 4 & 7.9561 & 26.52 & 180.00 \\
\hline 5 & 7.5923 & 25.76 & 176.84 \\
\hline 6 & 7.7082 & 27.97 & 165.35 \\
\hline 7 & 7.786 & 27.80 & 168.04 \\
\hline Average & 7.7650714 & 27.12 & 172.64 \\
\hline Stdev & 0.1192148 & 2.17 & 12.65 \\
\hline Count & 7 & 7 & 7 \\
\hline
\end{tabular}

Conditions

Dwell time $\quad 360 \mathrm{~s}$

Temp

$320{ }^{\circ} \mathrm{C}$

Mass

$5 \quad \mathrm{~kg}$

Table D.6: MFI Results for BQ5L at $320^{\circ} \mathrm{C}$

\begin{tabular}{|l|l|l|l|}
\hline BQ5L & weight & $\begin{array}{l}\text { collection time } \\
(\mathbf{s})\end{array}$ & $\begin{array}{l}\text { MFI } \\
(\mathbf{g} / \mathbf{1 0 m i n})\end{array}$ \\
\hline 1 & 8.7469 & 50.05 & 104.86 \\
\hline 2 & 8.5612 & 42.52 & 120.81 \\
\hline 3 & 8.5034 & 42.63 & 119.68 \\
\hline 4 & 8.4834 & 45.44 & 112.02 \\
\hline 5 & 8.5521 & 42.87 & 119.69 \\
\hline 6 & 8.551 & 40.96 & 125.26 \\
\hline 7 & 6.8488 & 35.87 & 114.56 \\
\cline { 2 - 4 } 8 & 8.5386 & 46.39 & 110.44 \\
\cline { 2 - 4 } & 8.7448 & 50.91 & 103.06 \\
\hline 9 & 8.3922444 & 44.18 & 114.49 \\
\hline Average & 0.5866946 & 4.64 & 7.55 \\
\hline Stdev & 9 & 9 & 9 \\
\hline Count & 9 & \multicolumn{2}{|l}{} \\
\hline
\end{tabular}

Conditions

Dwell time $360 \mathrm{~s}$

Temp

$320{ }^{\circ} \mathrm{C}$

Mass

$5 \quad \mathrm{~kg}$ 
Table D.7: MFI Results for BQ6L at $320^{\circ} \mathrm{C}$

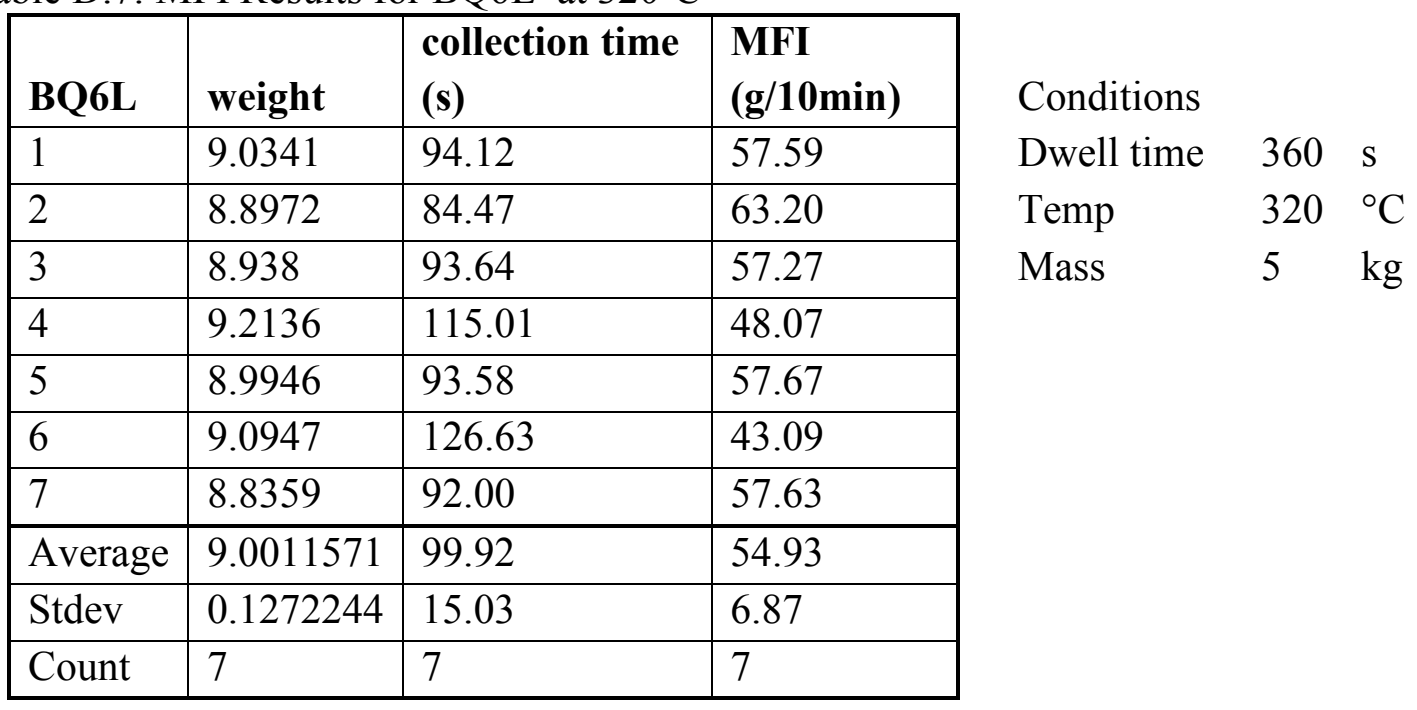




\section{Appendix D.2: Polycarbonate with Carbon Black}

Table D.8: MFI Results for BA2L at $320^{\circ} \mathrm{C}$

\begin{tabular}{|r|r|r|r|}
\hline \multicolumn{1}{|l|}{ BA2L } & \multicolumn{1}{l|}{$\begin{array}{l}\text { Weight } \\
\text { (g) }\end{array}$} & $\begin{array}{l}\text { Collection time } \\
\text { (s) }\end{array}$ & $\begin{array}{l}\text { MFI } \\
\text { (g/10min) }\end{array}$ \\
\hline 1 & 7.424 & 30.43 & 146.38 \\
\hline 2 & 7.5022 & 31.75 & 141.77 \\
\hline 3 & 5.3803 & 20.97 & 153.94 \\
\hline 4 & 7.4205 & 30.17 & 147.57 \\
\hline 5 & 4.1336 & 16.45 & 150.77 \\
\hline Average & & & $\mathbf{1 4 8 . 0 9}$ \\
\hline Stdev & & & $\mathbf{4 . 6 0}$ \\
\hline Count & & & $\mathbf{5}$ \\
\hline
\end{tabular}

Conditions

Dwell time $\quad 360 \quad \mathrm{~s}$

Temp $\quad 320{ }^{\circ} \mathrm{C}$

$\mathrm{Wt}$

$5 \mathrm{~kg}$

Table D.9: MFI Results for BA3L at $320^{\circ} \mathrm{C}$

\begin{tabular}{|r|r|r|r|}
\hline \multicolumn{1}{|l|}{ BA3L } & $\begin{array}{l}\text { Weight } \\
(\mathbf{g})\end{array}$ & $\begin{array}{l}\text { Collection time } \\
\text { (s) }\end{array}$ & $\begin{array}{l}\text { MFI } \\
\text { (g/10min) }\end{array}$ \\
\hline 1 & 6.1856 & 34.64 & 107.14 \\
\hline 2 & 6.4552 & 34.73 & 111.52 \\
\hline 3 & 4.468 & 22.33 & 120.05 \\
\hline 4 & 6.824 & 35.28 & 116.05 \\
\hline 5 & 6.0563 & 32.1 & 113.20 \\
\hline 6 & 6.1082 & 35.36 & 103.65 \\
\hline Average & & & $\mathbf{1 1 1 . 9 4}$ \\
\hline Stdev & & & $\mathbf{5 . 9 4}$ \\
\hline Count & & & $\mathbf{6}$ \\
\hline
\end{tabular}

Conditions

Dwell time $\quad 360 \mathrm{~s}$

Temp

$320{ }^{\circ} \mathrm{C}$

Weight

$5 \mathrm{~kg}$ 
Table D.10: MFI Results for BA4L at $320^{\circ} \mathrm{C}$

\begin{tabular}{|r|r|r|r|}
\hline \multicolumn{1}{|l|}{ BA4L } & $\begin{array}{l}\text { Weight } \\
(\mathbf{g})\end{array}$ & $\begin{array}{l}\text { Collection time } \\
\text { (s) }\end{array}$ & $\begin{array}{l}\text { MFI } \\
\text { (g/10min) }\end{array}$ \\
\hline 1 & 7.7919 & 54.44 & 85.88 \\
\hline 2 & 6.3183 & 42.21 & 89.81 \\
\hline 3 & 7.0031 & 47.26 & 88.91 \\
\hline 4 & 6.4195 & 45.20 & 85.21 \\
\hline 5 & 7.2145 & 50.7 & 85.38 \\
\hline 6 & 7.5645 & 50.37 & 90.11 \\
\hline Average & & & $\mathbf{8 7 . 5 5}$ \\
\hline Stdev & & & $\mathbf{2 . 3 0}$ \\
\hline Count & & & $\mathbf{6}$ \\
\hline
\end{tabular}

Conditions

Dwell time $\quad 360 \mathrm{~s}$

Temp

$320{ }^{\circ} \mathrm{C}$

Weight

$5 \mathrm{~kg}$

Table D.11: MFI Results for BA5L at $320^{\circ} \mathrm{C}$

\begin{tabular}{|r|r|r|r|}
\hline \multicolumn{1}{|l|}{ BA5L } & \multicolumn{1}{l|}{$\begin{array}{l}\text { Weight } \\
\text { (g) }\end{array}$} & $\begin{array}{l}\text { Collection time } \\
\text { (s) }\end{array}$ & $\begin{array}{l}\text { MFI } \\
\text { (g/10min) }\end{array}$ \\
\hline 1 & 1.6637 & 15.0 & 66.55 \\
\hline 2 & 6.2791 & 61.63 & 61.13 \\
\hline 3 & 2.0807 & 20.0 & 62.42 \\
\hline 4 & 1.6109 & 15.0 & 64.44 \\
\hline 5 & 2.1543 & 20.0 & 64.63 \\
\hline 6 & 2.1407 & 20.32 & 62.15 \\
\hline Average & & & $\mathbf{6 3 . 5 5}$ \\
\hline Stdev & & & $\mathbf{2 . 0 0}$ \\
\hline Count & & & $\mathbf{6}$ \\
\hline
\end{tabular}

Conditions

Dwell time $\quad 360 \mathrm{~s}$

Temp

$320{ }^{\circ} \mathrm{C}$

Weight

$5 \mathrm{~kg}$

Table D.12: MFI Results for BA6L at $320^{\circ} \mathrm{C}$

\begin{tabular}{|r|r|r|r|}
\hline \multicolumn{1}{|l|}{ BA6L } & \multicolumn{1}{l|}{$\begin{array}{l}\text { Weight } \\
\text { (g) }\end{array}$} & $\begin{array}{l}\text { Collection time } \\
\text { (s) }\end{array}$ & $\begin{array}{l}\text { MFI } \\
\text { (g/10min) }\end{array}$ \\
\hline 1 & 7.7246 & 131.3 & 35.30 \\
\hline 2 & 6.5882 & 102.6 & 38.53 \\
\hline 3 & 5.5827 & 98.7 & 33.94 \\
\hline 4 & 6.3849 & 98.2 & 39.01 \\
\hline 5 & 6.2436 & 99.38 & 37.69 \\
\hline 6 & 7.6590 & 120.0 & 38.30 \\
\hline Average & & & $\mathbf{3 7 . 1 3}$ \\
\hline Stdev & & & $\mathbf{2 . 0 3}$ \\
\hline Count & & & $\mathbf{6}$ \\
\hline
\end{tabular}

Conditions

Dwell time $\quad 360 \mathrm{~s}$

Temp

Weight

$320{ }^{\circ} \mathrm{C}$

$5 \mathrm{~kg}$ 


\section{Appendix D.3: Polycarbonate with Graphene Nanoplatelets}

Table D.13: MFI Results for BLE3 at $300^{\circ} \mathrm{C}$

\begin{tabular}{|c|c|c|c|c|}
\hline BLE3 & $\begin{array}{l}\text { Weight } \\
\text { (g) }\end{array}$ & MFI (g/10min) & \multirow{3}{*}{$\begin{array}{l}\text { Conditions } \\
\text { Dwell time } \\
\text { Collect }\end{array}$} & \multirow[b]{2}{*}{360} \\
\hline 1 & 0.8653 & 34.612 & & \\
\hline 2 & 0.8647 & 34.588 & & 15 \\
\hline 3 & 0.823 & 32.92 & Temp & 300 \\
\hline 4 & 0.8309 & 33.236 & Weight & 1.25 \\
\hline 5 & 0.8847 & 35.388 & & \\
\hline 6 & 0.8153 & 32.612 & & \\
\hline 7 & 0.864 & 34.56 & & \\
\hline Average & 0.85 & 33.99 & & \\
\hline Stdev & 0.03 & 1.05 & & \\
\hline Count & 7 & 7 & & \\
\hline
\end{tabular}

Table D.14: MFI Results for BG2L at $320^{\circ} \mathrm{C}$

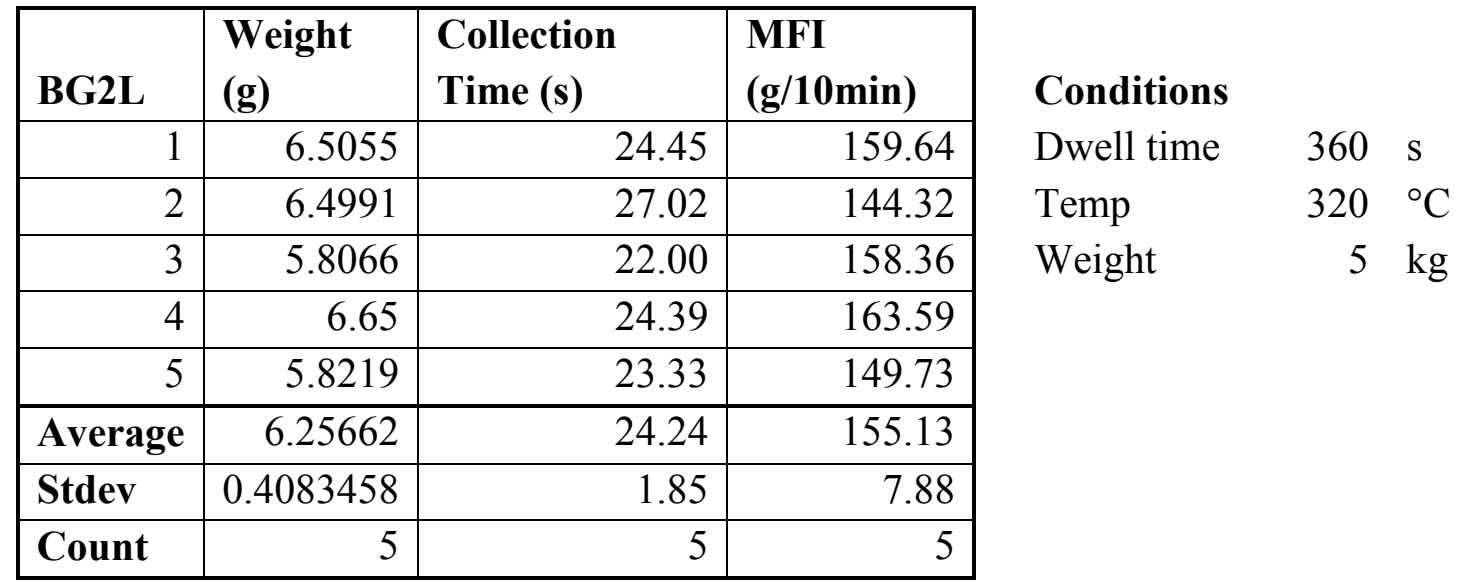


Table D.15: MFI Results for BG3L at $320^{\circ} \mathrm{C}$

\begin{tabular}{|r|r|r|r|}
\hline \multicolumn{1}{|l|l|l|}{$\begin{array}{l}\text { Weight } \\
\text { BGL }\end{array}$} & \multicolumn{1}{l|}{$\begin{array}{l}\text { Collection } \\
\text { Time (s) }\end{array}$} & $\begin{array}{l}\text { MFI } \\
\text { (g/10min) }\end{array}$ \\
\hline 1 & 6.7809 & 27.86 & 146.04 \\
\hline 2 & 6.3482 & 27.15 & 140.29 \\
\hline 3 & 6.4 & 27.32 & 140.56 \\
\hline 4 & 6.564 & 27.20 & 144.79 \\
\hline Average & 6.523275 & 27.38 & 142.92 \\
\hline Stdev & 0.1948308 & 0.33 & 2.93 \\
\hline Count & 4 & 4 & 4 \\
\hline
\end{tabular}

\section{Conditions}

Dwell time $\quad 360 \mathrm{~s}$

Temp

$320{ }^{\circ} \mathrm{C}$

Weight

$5 \mathrm{~kg}$

Table D.16: MFI Results for BG4L at $320^{\circ} \mathrm{C}$

\begin{tabular}{|r|r|r|r|}
\hline \multicolumn{1}{|l|l|l|}{$\begin{array}{l}\text { Weight } \\
\text { BG4L }\end{array}$} & $\begin{array}{l}\text { Collection } \\
\text { Time (s) }\end{array}$ & $\begin{array}{l}\text { MFI } \\
\text { (g/10min) }\end{array}$ \\
\hline 1 & 6.5626 & 30.49 & 129.14 \\
\hline 2 & 6.249 & 30.48 & 123.01 \\
\hline 3 & 6.268 & 29.19 & 128.84 \\
\hline 4 & 6.2607 & 28.72 & 130.79 \\
\hline 5 & 6.2837 & 29.75 & 126.73 \\
\hline Average & 6.3248 & 29.73 & 127.70 \\
\hline Stdev & 0.1335278 & 0.78 & 2.99 \\
\hline Count & 5 & 5 & 5 \\
\hline
\end{tabular}

\section{Conditions}

Dwell time $\quad 360 \mathrm{~s}$

Temp

$320{ }^{\circ} \mathrm{C}$

Weight

$5 \mathrm{~kg}$

Table D.17: MFI Results for BG5L at $320^{\circ} \mathrm{C}$

\begin{tabular}{|r|r|r|r|}
\hline \multicolumn{1}{|l|l|l|}{$\begin{array}{l}\text { Weight } \\
\text { BG5 }\end{array}$} & $\begin{array}{l}\text { Collection } \\
\text { Time (s) }\end{array}$ & $\begin{array}{l}\text { MFI } \\
\text { (g/10min) }\end{array}$ \\
\hline 1 & 6.7978 & 35.31 & 115.51 \\
\hline 2 & 6.3043 & 31.22 & 121.16 \\
\hline 3 & 6.2818 & 32.87 & 114.67 \\
\hline 4 & 6.2872 & 31.97 & 118.00 \\
\hline 5 & 6.0184 & 31.81 & 113.52 \\
\hline Average & 6.3379 & 32.64 & 116.57 \\
\hline Stdev & 0.2830349 & 1.61 & 3.05 \\
\hline Count & 5 & 5 & 5 \\
\hline
\end{tabular}

\section{Conditions}

Dwell time $\quad 360 \mathrm{~s}$

Temp

$320{ }^{\circ} \mathrm{C}$

Weight

$5 \mathrm{~kg}$ 
Table D.18: MFI Results for BG6L at $320^{\circ} \mathrm{C}$

\begin{tabular}{|r|r|r|r|}
\hline \multicolumn{1}{|l|}{ BG6L } & \multicolumn{1}{l|}{$\begin{array}{l}\text { Weight } \\
\text { (g) }\end{array}$} & \multicolumn{1}{l|}{$\begin{array}{l}\text { Collection } \\
\text { Time (s) }\end{array}$} & $\begin{array}{l}\text { MFI } \\
\text { (g/10min) }\end{array}$ \\
\hline 1 & 6.5994 & 38.82 & 102.00 \\
\hline 2 & 6.5142 & 36.64 & 106.67 \\
\hline 3 & 6.6678 & 36.62 & 109.25 \\
\hline 4 & 6.3793 & 35.26 & 108.55 \\
\hline 5 & 6.7822 & 37.86 & 107.48 \\
\hline Average & 6.58858 & 37.04 & 106.79 \\
\hline Stdev & 0.1526533 & 1.36 & 2.85 \\
\hline Count & 5 & 5 & 5 \\
\hline
\end{tabular}

Conditions

Dwell time $\quad 360 \mathrm{~s}$

Temp

Weight

$320{ }^{\circ} \mathrm{C}$

$5 \mathrm{~kg}$

Table D.19: MFI Results for BG8L at $320^{\circ} \mathrm{C}$

\begin{tabular}{|r|r|r|r|}
\hline \multicolumn{1}{|l|l|l|}{$\begin{array}{l}\text { Weight } \\
\text { BGL }\end{array}$} & \multicolumn{1}{l|}{$\begin{array}{l}\text { Collection } \\
\text { Time (s) }\end{array}$} & $\begin{array}{l}\text { MFI } \\
\text { (g/10min) }\end{array}$ \\
\hline 1 & 7.2204 & 55.29 & 78.35 \\
\hline 2 & 6.8595 & 47.21 & 87.18 \\
\hline 3 & 6.8792 & 50.51 & 81.72 \\
\hline 4 & 7.0451 & 48.53 & 87.10 \\
\hline 5 & 6.8555 & 48.87 & 84.17 \\
\hline Average & 6.97194 & 50.08 & 83.70 \\
\hline Stdev & 0.1595983 & 3.14 & 3.75 \\
\hline Count & 5 & 5 & 5 \\
\hline
\end{tabular}

Conditions

Dwell time $\quad 360 \mathrm{~s}$

Temp

$320{ }^{\circ} \mathrm{C}$

Weight

$5 \mathrm{~kg}$

Table D.20: MFI Results for BG10L at $320^{\circ} \mathrm{C}$

\begin{tabular}{|c|c|c|c|}
\hline BG10L & $\begin{array}{l}\text { Weight } \\
\text { (g) }\end{array}$ & $\begin{array}{l}\text { Collection } \\
\text { Time (s) }\end{array}$ & $\begin{array}{l}\text { MFI } \\
(\mathrm{g} / \mathbf{1 0 m i n})\end{array}$ \\
\hline 1 & 6.9704 & 61.05 & 68.51 \\
\hline 2 & 6.7229 & 57.80 & 69.79 \\
\hline 3 & 7.2966 & 61.44 & 71.26 \\
\hline 4 & 6.6696 & 55.99 & 71.47 \\
\hline 5 & 6.7842 & 56.92 & 71.51 \\
\hline Average & 6.88874 & 58.64 & 70.51 \\
\hline Stdev & 0.254692 & 2.47 & 1.33 \\
\hline Count & 5 & 5 & 5 \\
\hline
\end{tabular}

Conditions

Dwell time $\quad 360 \mathrm{~s}$

Temp

Weight

$320{ }^{\circ} \mathrm{C}$

$5 \mathrm{~kg}$ 


\section{Appendix D.4: Polycarbonate with Multiple Fillers}

Table D.21: MFI Results for BA2LR

\begin{tabular}{|r|r|r|r|}
\hline BA2LR & Mass (g) & $\begin{array}{l}\text { Collection Time } \\
\text { (s) }\end{array}$ & $\begin{array}{l}\text { MFI } \\
\text { (g/10min) }\end{array}$ \\
\hline 1 & 5.6484 & 22.48 & 150.758 \\
\hline 2 & 5.7247 & 23.49 & 146.225 \\
\hline 3 & 6.5661 & 27.01 & 145.859 \\
\hline 4 & 7.3149 & 31.02 & 141.487 \\
\hline 5 & 5.9090 & 25.14 & 141.026 \\
\hline Average & 6.2326 & 16.14 & 145.071 \\
\hline STDEV & 0.7047 & 3.37 & 3.984 \\
\hline Count & 5 & 5 & 5 \\
\hline
\end{tabular}

Conditions:

Dwell

Time: $\quad 360 \mathrm{~s}$

Melt Temp: $\quad 320{ }^{\circ} \mathrm{C}$

Mass: $\quad 5 \mathrm{~kg}$

Table D.22: MFI Results for BA5LR

\begin{tabular}{|r|r|r|r|}
\hline BA5LR & Mass (g) & $\begin{array}{l}\text { Collection Time } \\
\text { (s) }\end{array}$ & $\begin{array}{l}\text { MFI } \\
\text { (g/10min) }\end{array}$ \\
\hline 1 & 6.7137 & 58.25 & 69.154 \\
\hline 2 & 7.5117 & 68.78 & 65.528 \\
\hline 3 & 7.0857 & 61.70 & 68.905 \\
\hline 4 & 7.3767 & 61.49 & 71.980 \\
\hline Average & 7.1720 & 16.14 & 68.892 \\
\hline STDEV & 0.3535 & 4.44 & 2.641 \\
\hline Count & 4 & 4 & 4 \\
\hline
\end{tabular}

\section{Conditions:}

Dwell

Time: $\quad 360 \mathrm{~s}$

Melt Temp: $\quad 320{ }^{\circ} \mathrm{C}$

Mass: $\quad 5 \mathrm{~kg}$

Table D.23: MFI Results for BQ0.5L

\begin{tabular}{|r|r|r|r|}
\hline BQ0.5L & weight (g) & $\begin{array}{l}\text { collection time } \\
\text { (s) }\end{array}$ & $\begin{array}{l}\text { MFI } \\
\text { (g/10min) }\end{array}$ \\
\hline 1 & 5.6229 & 10.35 & 325.97 \\
\hline 2 & 5.0695 & 10.27 & 296.17 \\
\hline 3 & 4.8193 & 9.10 & 317.76 \\
\hline 4 & 4.1398 & 7.51 & 330.74 \\
\hline 5 & 5.7201 & 9.57 & 358.63 \\
\hline & & & \\
Average & 5.07432 & 9.36 & 325.85 \\
\hline Stdev & 0.6434978 & 1.16 & 22.61 \\
\hline Count & 5 & \multicolumn{3}{|c|}{303} \\
\hline
\end{tabular}

Conditions

Dwell time $\quad 360 \mathrm{~s}$

Temp $\quad 320{ }^{\circ} \mathrm{C}$

Mass $\quad 5 \mathrm{~kg}$

extruded 6-30-10

2-9-09

screw 
Table D.24: MFI Results for BQ1L

\begin{tabular}{|r|r|r|r|}
\hline \multicolumn{1}{|l|}{ BQ1L } & weight $(\mathbf{g})$ & $\begin{array}{l}\text { collection time } \\
\text { (s) }\end{array}$ & $\begin{array}{l}\text { MFI } \\
\text { (g/10min) }\end{array}$ \\
\hline 1 & 5.4763 & 11.86 & 277.05 \\
\hline 2 & 5.3404 & 10.10 & 317.25 \\
\hline 3 & 6.1525 & 10.31 & 358.05 \\
\hline 4 & 4.9803 & 9.25 & 323.05 \\
\hline 5 & 5.1941 & 9.54 & 326.67 \\
\hline & & & \\
Average & 5.42872 & 10.21 & 320.41 \\
\hline Stdev & 0.4444234 & 1.01 & 28.95 \\
\hline Count & 5 & 5 & 5 \\
\hline
\end{tabular}

Conditions

Dwell time $360 \mathrm{~s}$

Temp $\quad 320{ }^{\circ} \mathrm{C}$

Mass $\quad 5 \mathrm{~kg}$

extruded 6-30-10

2-9-09

screw

Table D.25: MFI Results for BG12L

\begin{tabular}{|r|l|r|r|}
\hline BG12L & $\begin{array}{l}\text { Mass } \\
(\mathbf{g})\end{array}$ & $\begin{array}{l}\text { Collection Time } \\
(\mathbf{s})\end{array}$ & $\begin{array}{l}\text { MFI } \\
(\mathbf{g} / \mathbf{1 0 m i n})\end{array}$ \\
\hline 1 & 6.7200 & 73.43 & 54.909 \\
\hline 2 & 7.0295 & 75.12 & 56.146 \\
\hline 3 & 7.0785 & 75.42 & 56.313 \\
\hline 4 & 6.8060 & 74.12 & 55.094 \\
\hline 5 & 6.6946 & 70.58 & 56.911 \\
\hline Average & $\mathbf{6 . 8 6 5 7}$ & $\mathbf{7 3 . 7 3}$ & $\mathbf{5 5 . 8 7 5}$ \\
\hline STDEV & $\mathbf{0 . 1 7 7 6}$ & $\mathbf{1 . 9 3}$ & $\mathbf{0 . 8 4 8}$ \\
\hline Count & $\mathbf{5}$ & $\mathbf{5}$ & $\mathbf{5}$ \\
\hline
\end{tabular}

\section{Conditions:}

Dwell

Time: $\quad 360$ s

Melt Temp: $\quad 320{ }^{\circ} \mathrm{C}$

Mass: $\quad 5 \mathrm{~kg}$

Table D.26: MFI Results for BG15L

\begin{tabular}{|r|l|r|r|}
\hline BG15L & $\begin{array}{l}\text { Mass } \\
(\mathbf{g})\end{array}$ & $\begin{array}{l}\text { Collection Time } \\
\text { (s) }\end{array}$ & $\begin{array}{l}\text { MFI } \\
\text { (g/10min) }\end{array}$ \\
\hline 1 & 7.9222 & 149.03 & 31.895 \\
\hline 2 & 8.4803 & 152.44 & 33.378 \\
\hline 3 & 8.2269 & 165.56 & 29.815 \\
\hline 4 & 8.0704 & 162.99 & 29.709 \\
\hline 5 & 7.1327 & 126.01 & 33.963 \\
\hline Average & $\mathbf{7 . 9 6 6 5}$ & $\mathbf{1 5 1 . 2 1}$ & $\mathbf{3 1 . 7 5 2}$ \\
\hline STDEV & $\mathbf{0 . 5 0 9 8}$ & $\mathbf{1 5 . 7 0}$ & $\mathbf{1 . 9 6 7}$ \\
\hline Count & $\mathbf{5}$ & $\mathbf{5}$ & $\mathbf{5}$ \\
\hline
\end{tabular}

\section{Conditions:}

Dwell

Time: $\quad 360 \mathrm{~s}$

Melt Temp: $\quad 320{ }^{\circ} \mathrm{C}$

Mass: $\quad 5 \mathrm{~kg}$ 
Table D.27: MFI Results for BA2Q1L

\begin{tabular}{|r|l|r|r|}
\hline BA2Q1L & $\begin{array}{l}\text { Mass } \\
(\mathbf{g})\end{array}$ & $\begin{array}{l}\text { Collection Time } \\
\text { (s) }\end{array}$ & $\begin{array}{l}\text { MFI } \\
\mathbf{( g / 1 0 m i n})\end{array}$ \\
\hline 1 & 4.0725 & 8.43 & 289.858 \\
\hline 2 & 6.2589 & 12.80 & 293.386 \\
\hline 3 & 6.4543 & 13.50 & 286.858 \\
\hline 4 & 6.1503 & 12.67 & 291.253 \\
\hline 5 & 6.3000 & 12.48 & 302.885 \\
\hline Average & 5.8472 & 11.98 & 292.848 \\
\hline STDEV & 0.9981 & 2.02 & 6.091 \\
\hline Count & 5 & 5 & 5 \\
\hline
\end{tabular}

Table D.28: MFI Results for BA2Q5L

\begin{tabular}{|r|l|r|r|}
\hline BA2Q5L & $\begin{array}{l}\text { Mass } \\
(\mathbf{g})\end{array}$ & $\begin{array}{l}\text { Collection Time } \\
\text { (s) }\end{array}$ & $\begin{array}{l}\text { MFI } \\
(\mathbf{g} / \mathbf{1 0 m i n})\end{array}$ \\
\hline 1 & 7.2307 & 67.97 & 63.828 \\
\hline 2 & 6.3634 & 52.12 & 73.255 \\
\hline 3 & 6.5801 & 53.02 & 74.464 \\
\hline 4 & 6.7000 & 64.42 & 62.403 \\
\hline 5 & 6.1862 & 50.02 & 74.205 \\
\hline Average & 6.6121 & 57.51 & 69.631 \\
\hline STDEV & 0.3983 & 8.10 & 5.986 \\
\hline Count & 5 & 5 & 5 \\
\hline
\end{tabular}

Table D.29: MFI Results for BA5Q1L

\begin{tabular}{|r|r|r|r|}
\hline BA5Q1L & Mass (g) & $\begin{array}{l}\text { Collection Time } \\
\text { (s) }\end{array}$ & $\begin{array}{l}\text { MFI } \\
\text { (g/10min) }\end{array}$ \\
\hline 1 & 6.4642 & 30.47 & 127.290 \\
\hline 2 & 6.9321 & 35.70 & 116.506 \\
\hline 3 & 6.5939 & 34.18 & 115.750 \\
\hline 4 & 6.1080 & 31.90 & 114.884 \\
\hline 5 & 7.1207 & 35.45 & 120.520 \\
\hline Average & 6.6438 & 33.54 & 118.990 \\
\hline STDEV & 0.3976 & 2.28 & 5.117 \\
\hline Count & 5 & 5 & 5 \\
\hline
\end{tabular}

\section{Conditions:}

Dwell

Time: $\quad 360$ s

Melt Temp: $320{ }^{\circ} \mathrm{C}$

Mass: $\quad 5 \mathrm{~kg}$ $\begin{array}{lrl}\text { Conditions: } & & \\ \text { Dwell } & & \\ \text { Time: } & 360 & \mathrm{~s} \\ \text { Melt Temp: } & 320 & { }^{\circ} \mathrm{C} \\ \text { Mass: } & 5 & \mathrm{~kg}\end{array}$

\section{Conditions:}

Dwell

Time: $\quad 360 \mathrm{~s}$

Melt Temp: $\quad 320{ }^{\circ} \mathrm{C}$

Mass: $\quad 5 \mathrm{~kg}$ 
Table D.30: MFI Results for BA2G2L

\begin{tabular}{|r|r|r|r|}
\hline BA2G2L & Mass (g) & $\begin{array}{l}\text { Collection Time } \\
\text { (s) }\end{array}$ & $\begin{array}{l}\text { MFI } \\
\text { (g/10min) }\end{array}$ \\
\hline 1 & 5.5844 & 31.42 & 106.640 \\
\hline 2 & 4.7933 & 24.67 & 116.578 \\
\hline 3 & 5.4900 & 30.17 & 109.181 \\
\hline 4 & 5.9189 & 31.92 & 111.258 \\
\hline 5 & 5.4177 & 31.00 & 104.859 \\
\hline Average & 5.4409 & 29.84 & 109.703 \\
\hline STDEV & 0.4097 & 2.96 & 4.550 \\
\hline Count & 5 & 5 & 5 \\
\hline
\end{tabular}

Conditions:

Dwell

Time: $\quad 360 \mathrm{~s}$

Melt Temp: $\quad 320{ }^{\circ} \mathrm{C}$

Mass: $\quad 5 \mathrm{~kg}$

Table D.31: MFI Results for BA2G5L

\begin{tabular}{|r|r|r|r|}
\hline BA2G5L & Mass (g) & $\begin{array}{l}\text { Collection Time } \\
\text { (s) }\end{array}$ & $\begin{array}{l}\text { MFI } \\
\text { (g/10min) }\end{array}$ \\
\hline 1 & 6.1636 & 45.47 & 81.332 \\
\hline 2 & 6.3866 & 46.83 & 81.827 \\
\hline 3 & 5.4733 & 41.41 & 79.304 \\
\hline 4 & 6.1589 & 44.56 & 82.930 \\
\hline 5 & 5.3509 & 41.10 & 78.115 \\
\hline Average & 5.9067 & 43.87 & 80.702 \\
\hline STDEV & 0.4628 & 2.53 & 1.954 \\
\hline Count & 5 & 5 & 5 \\
\hline
\end{tabular}

Table D.32: MFI Results for BA5G2L

\begin{tabular}{|c|c|c|c|c|c|}
\hline BA5G2L & Mass (g) & $\begin{array}{l}\text { Collection Time } \\
\text { (s) }\end{array}$ & $\begin{array}{l}\text { MFI } \\
(\mathrm{g} / 10 \mathrm{~min})\end{array}$ & \multirow{2}{*}{$\begin{array}{l}\text { Conditions: } \\
\text { Dwell } \\
\text { Time: }\end{array}$} & \multirow[b]{2}{*}{360} \\
\hline 1 & 7.8549 & 99.25 & 47.486 & & \\
\hline 2 & 8.0947 & 102.83 & 47.232 & Melt Temp: & 320 \\
\hline 3 & 8.1574 & 95.66 & 51.165 & Mass: & 5 \\
\hline 4 & 7.9282 & 95.30 & 49.915 & & \\
\hline 5 & 7.1263 & 81.76 & 52.297 & & \\
\hline$\overline{\text { Average }}$ & 7.8323 & 94.96 & 49.619 & & \\
\hline STDEV & 0.4131 & 7.99 & 2.230 & & \\
\hline Count & 5 & 5 & 5 & & \\
\hline
\end{tabular}

Conditions:

Dwell

Time: $\quad 360 \mathrm{~s}$

Melt Temp: $320{ }^{\circ} \mathrm{C}$

Mass: $\quad 5 \mathrm{~kg}$

Conditions :

Time: $\quad 360 \mathrm{~s}$

Mass: $\quad 5 \mathrm{~kg}$ 
Table D.33: MFI Results for BA5G5L

\begin{tabular}{|r|r|r|r|}
\hline BA5G5L & Mass (g) & $\begin{array}{l}\text { Collection Time } \\
\text { (s) }\end{array}$ & $\begin{array}{l}\text { MFI } \\
\text { (g/10min) }\end{array}$ \\
\hline 1 & 6.5516 & 197.51 & 19.903 \\
\hline 2 & 7.4600 & 172.18 & 25.996 \\
\hline 3 & 8.1355 & 214.36 & 22.772 \\
\hline 4 & 8.6637 & 248.38 & 20.928 \\
\hline 5 & 7.1263 & 208.62 & 20.496 \\
\hline Average & 7.5874 & 208.21 & 22.019 \\
\hline STDEV & 0.8307 & 27.68 & 2.468 \\
\hline Count & 5 & 5 & 5 \\
\hline
\end{tabular}

Conditions:

Dwell

Time: $\quad 360 \mathrm{~s}$

Melt Temp: $\quad 320{ }^{\circ} \mathrm{C}$

Mass: $\quad 5 \mathrm{~kg}$

Table D.34: MFI Results for BQ1G2L

\begin{tabular}{|r|r|r|r|}
\hline BQ1G2L & Mass (g) & $\begin{array}{l}\text { Collection Time } \\
\text { (s) }\end{array}$ & $\begin{array}{l}\text { MFI } \\
\text { (g/10min) }\end{array}$ \\
\hline 1 & 4.9825 & 14.82 & 201.721 \\
\hline 2 & 4.5854 & 12.10 & 227.375 \\
\hline 3 & 4.9918 & 12.98 & 230.746 \\
\hline 4 & 4.8379 & 12.63 & 229.829 \\
\hline 5 & 5.1504 & 15.09 & 204.787 \\
\hline Average & 4.9096 & 16.14 & 218.892 \\
\hline STDEV & 0.2123 & 1.35 & 14.369 \\
\hline Count & 5 & 5 & 5 \\
\hline
\end{tabular}

\section{Conditions:}

Dwell

Time: $\quad 360 \mathrm{~s}$

Melt Temp: $320{ }^{\circ} \mathrm{C}$

Mass: $\quad 5 \mathrm{~kg}$

Table D.35: MFI Results for BQ1G5L

\begin{tabular}{|r|r|r|r|}
\hline BQ1G5L & Mass (g) & $\begin{array}{l}\text { Collection Time } \\
\text { (s) }\end{array}$ & $\begin{array}{l}\text { MFI } \\
\text { (g/10min) }\end{array}$ \\
\hline 1 & 5.5200 & 20.55 & 161.168 \\
\hline 2 & 5.3902 & 20.27 & 159.552 \\
\hline 3 & 5.7949 & 21.77 & 159.712 \\
\hline 4 & 5.8422 & 21.23 & 165.112 \\
\hline 5 & 4.9440 & 18.93 & 156.704 \\
\hline Average & 5.4983 & 16.14 & 160.450 \\
\hline STDEV & 0.3625 & 1.08 & 3.067 \\
\hline Count & 5 & 5 & 5 \\
\hline
\end{tabular}

\section{Conditions:}

Dwell

Time: $\quad 360 \mathrm{~s}$

Melt Temp: $320{ }^{\circ} \mathrm{C}$

Mass: $\quad 5 \mathrm{~kg}$ 
Table D.36: MFI Results for BQ5G2L

\begin{tabular}{|r|r|r|r|}
\hline BQ5G2L & Mass (g) & $\begin{array}{l}\text { Collection Time } \\
\text { (s) }\end{array}$ & $\begin{array}{l}\text { MFI } \\
\text { (g/10min) }\end{array}$ \\
\hline 1 & 7.0572 & 32.06 & 132.075 \\
\hline 2 & 6.7910 & 27.72 & 146.991 \\
\hline 3 & 7.0427 & 27.84 & 151.782 \\
\hline 4 & 6.4428 & 27.54 & 140.366 \\
\hline 5 & 7.1681 & 28.98 & 148.408 \\
\hline Average & 6.9004 & 16.14 & 143.924 \\
\hline STDEV & 0.2906 & 1.89 & 7.816 \\
\hline Count & 5 & 5 & 5 \\
\hline
\end{tabular}

\section{Conditions:}

Dwell

Time: $\quad 360 \mathrm{~s}$

Melt Temp: $\quad 320{ }^{\circ} \mathrm{C}$

Mass: $\quad 5 \mathrm{~kg}$

Table D.37: MFI Results for BQ5G5L

\begin{tabular}{|r|r|r|r|}
\hline BQ5G5L & Mass (g) & $\begin{array}{l}\text { Collection Time } \\
\text { (s) }\end{array}$ & $\begin{array}{l}\text { MFI } \\
\text { (g/10min) }\end{array}$ \\
\hline 1 & 7.0087 & 72.03 & 58.382 \\
\hline 2 & 7.3604 & 70.47 & 62.668 \\
\hline 3 & 7.4759 & 74.70 & 60.047 \\
\hline 4 & 7.2946 & 80.42 & 54.424 \\
\hline 5 & 6.7267 & 65.68 & 61.450 \\
\hline Average & 7.1733 & 16.14 & 59.394 \\
\hline STDEV & 0.3032 & 5.44 & 3.206 \\
\hline Count & 5 & 5 & 5 \\
\hline
\end{tabular}

\section{Conditions:}

Dwell

Time: $\quad 360 \mathrm{~s}$

Melt Temp: $\quad 320{ }^{\circ} \mathrm{C}$

Mass:

$5 \mathrm{~kg}$ 


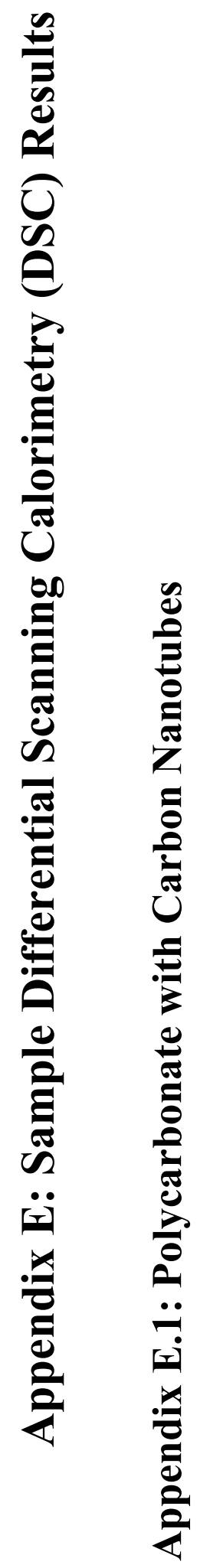




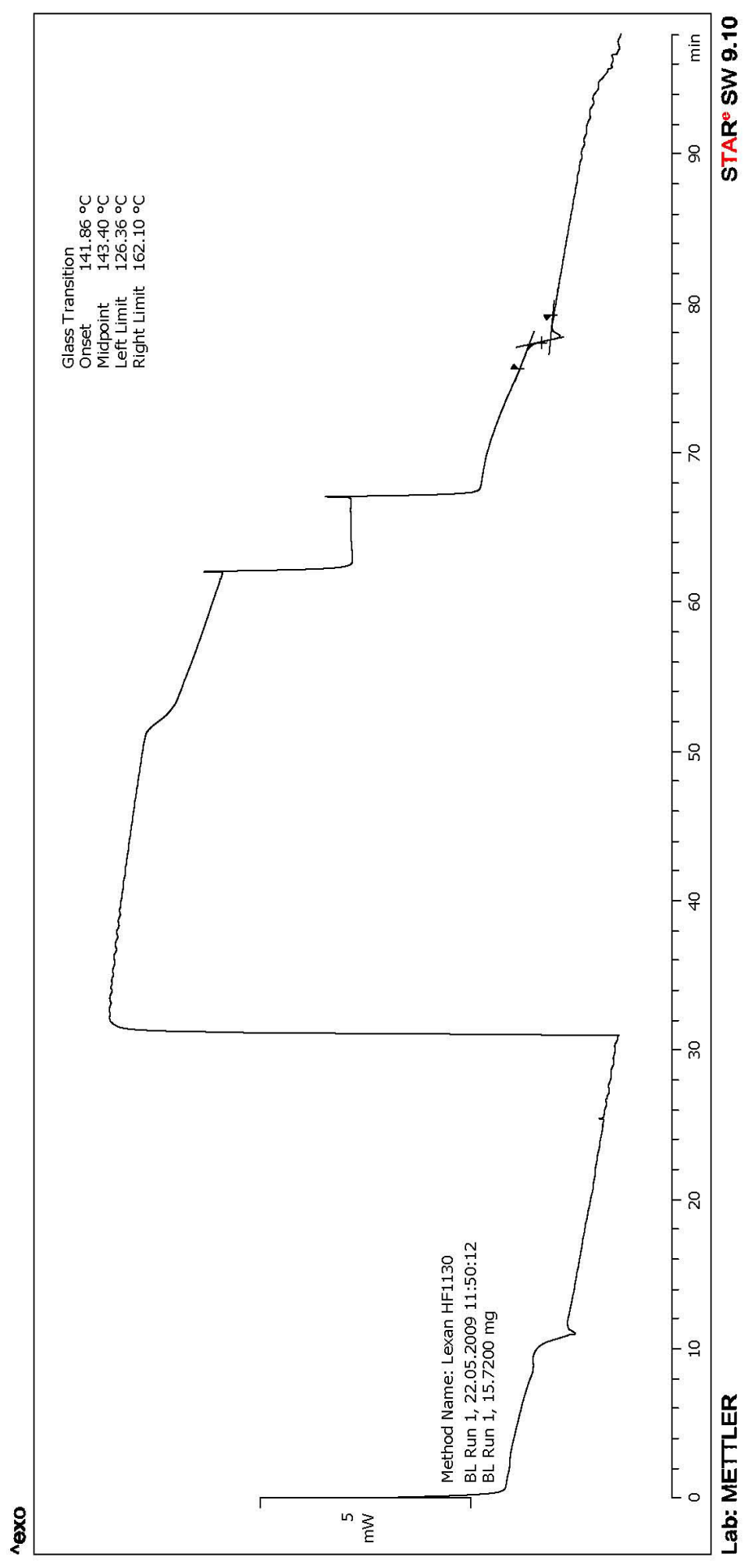

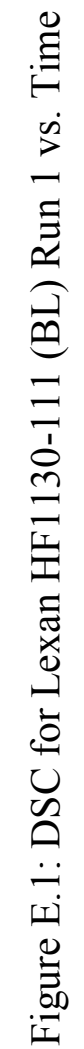




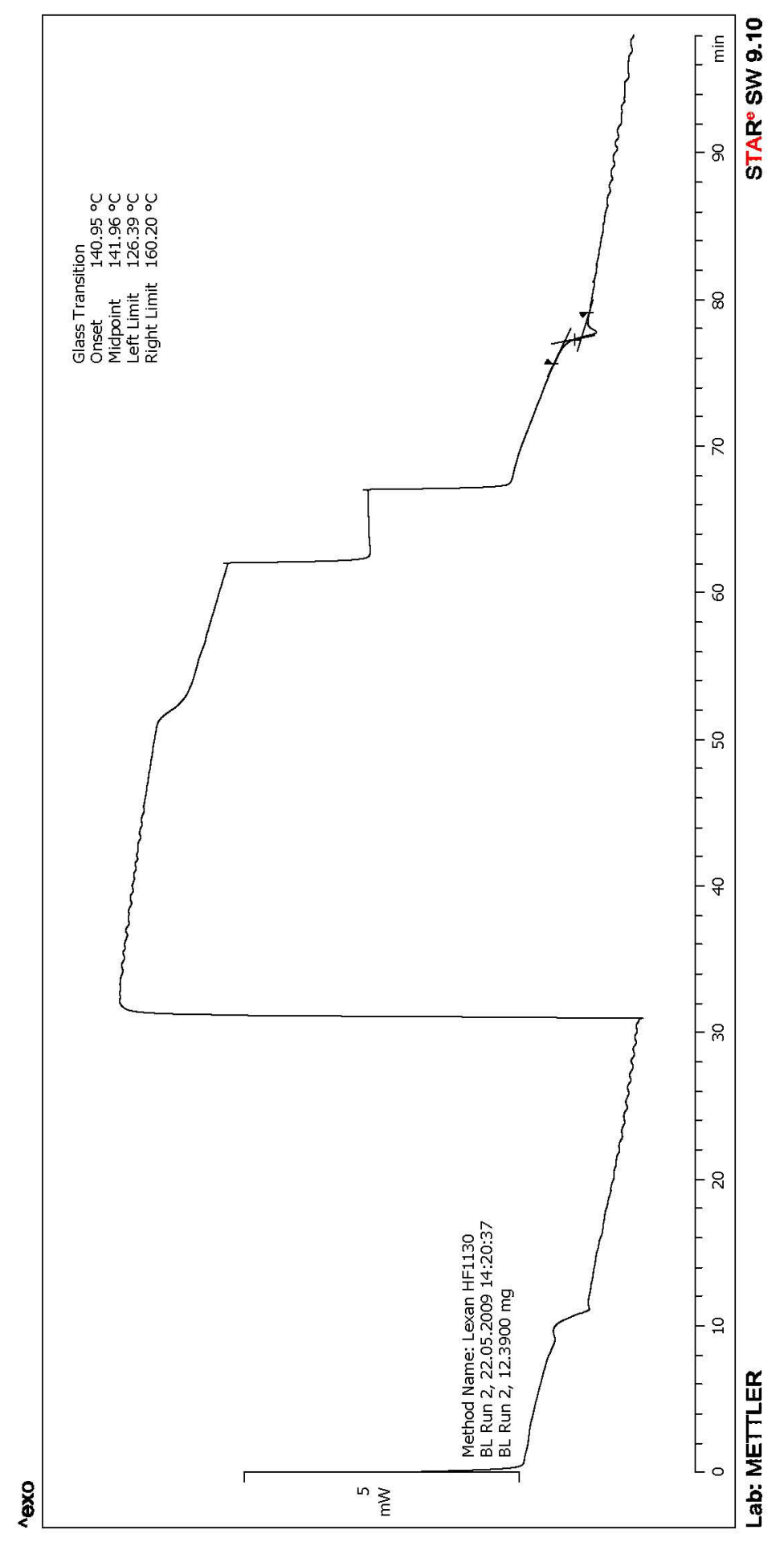

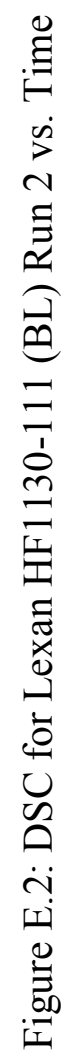




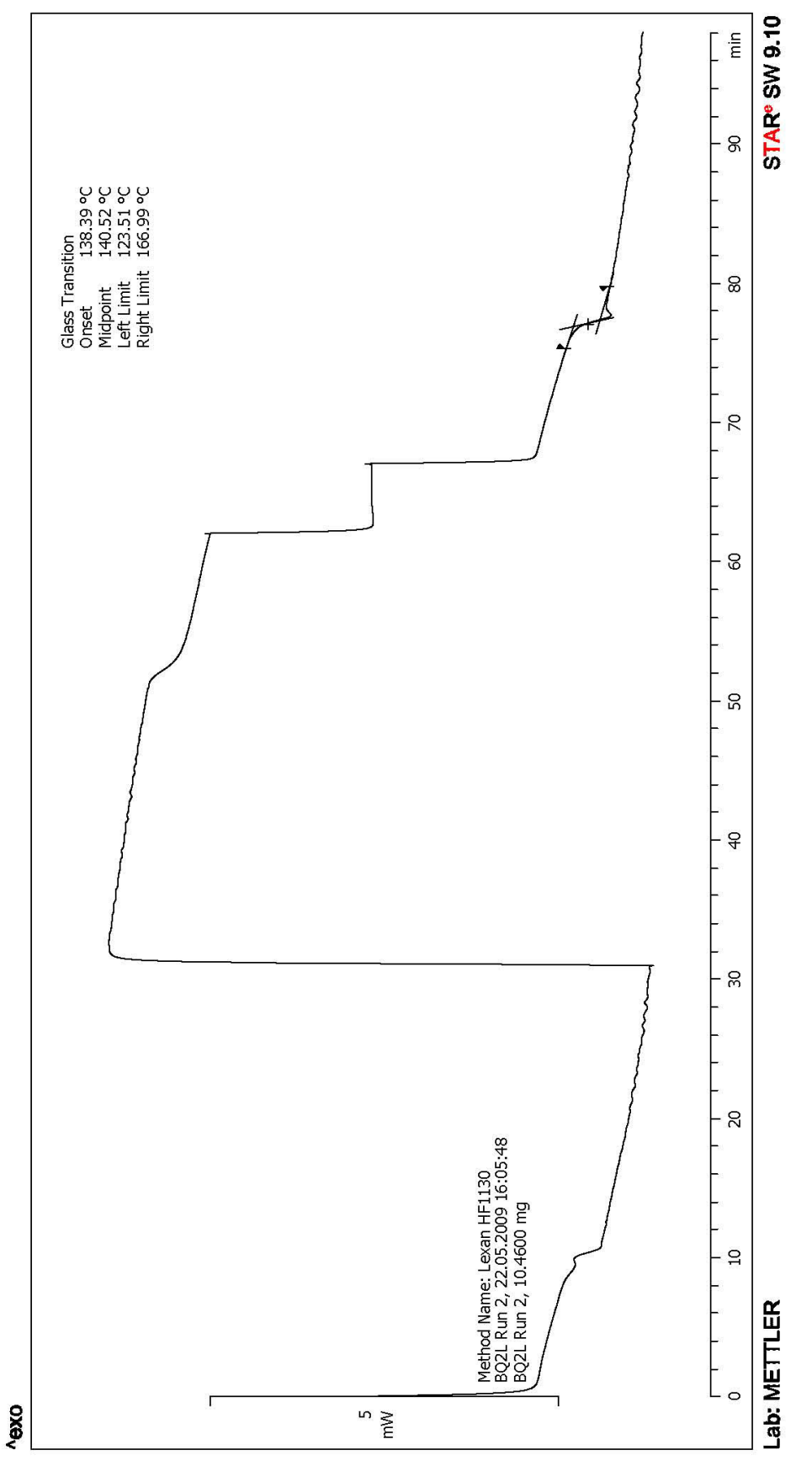

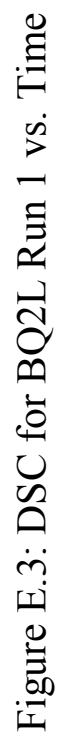




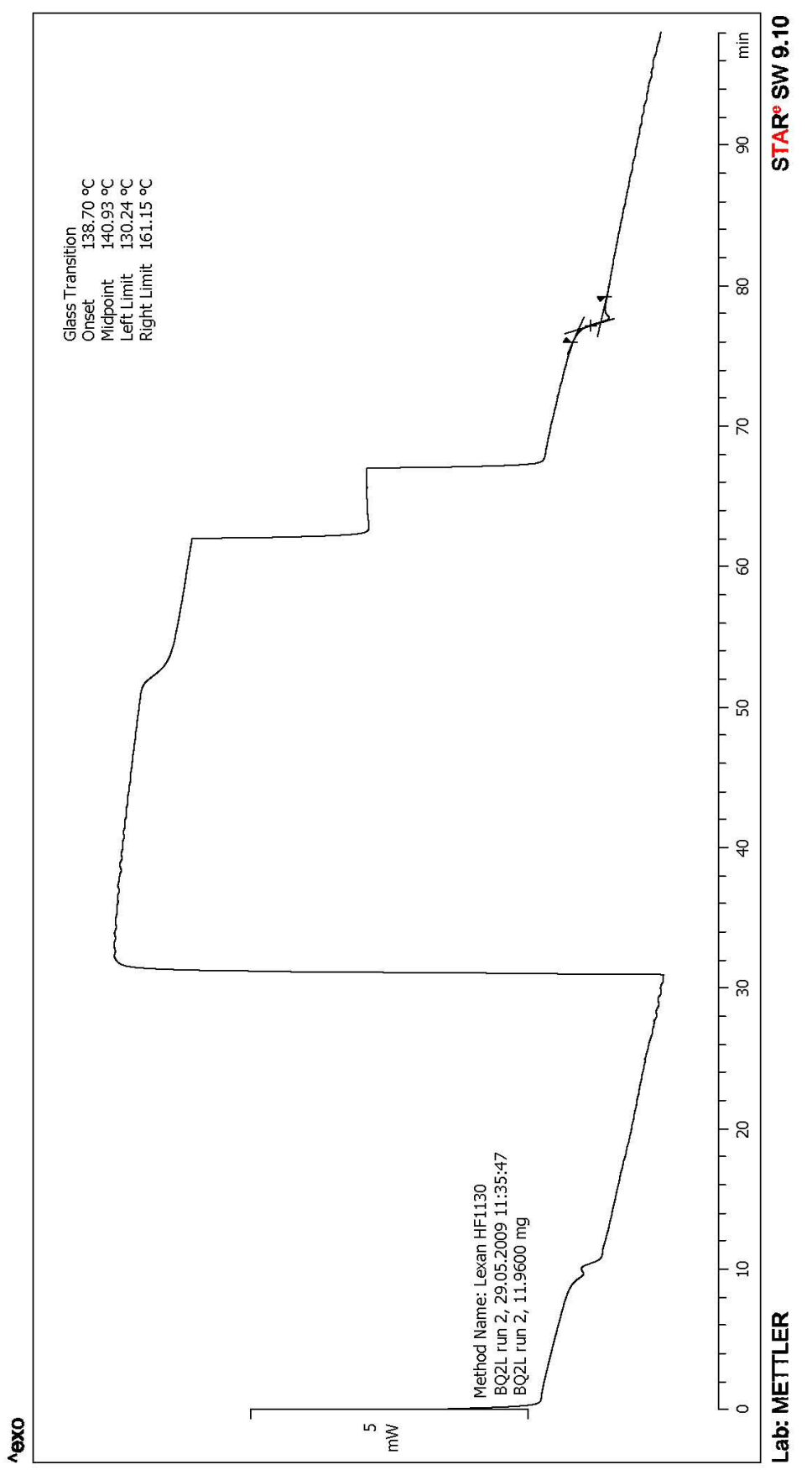

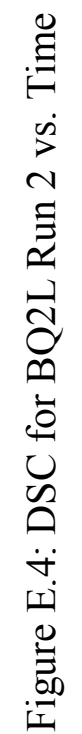




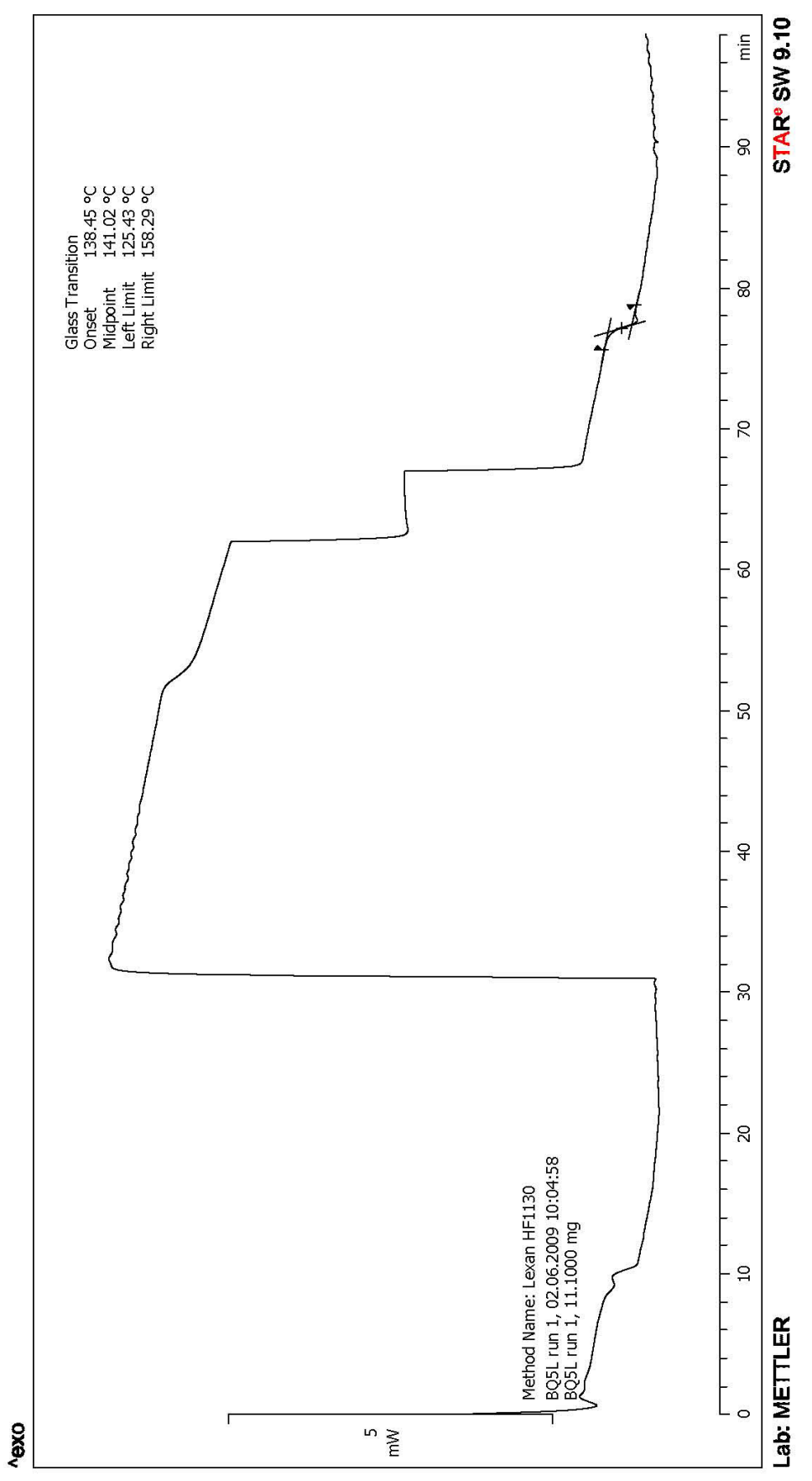

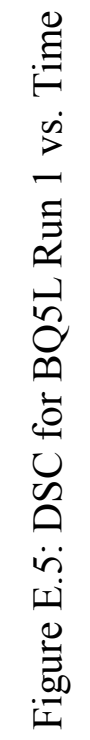




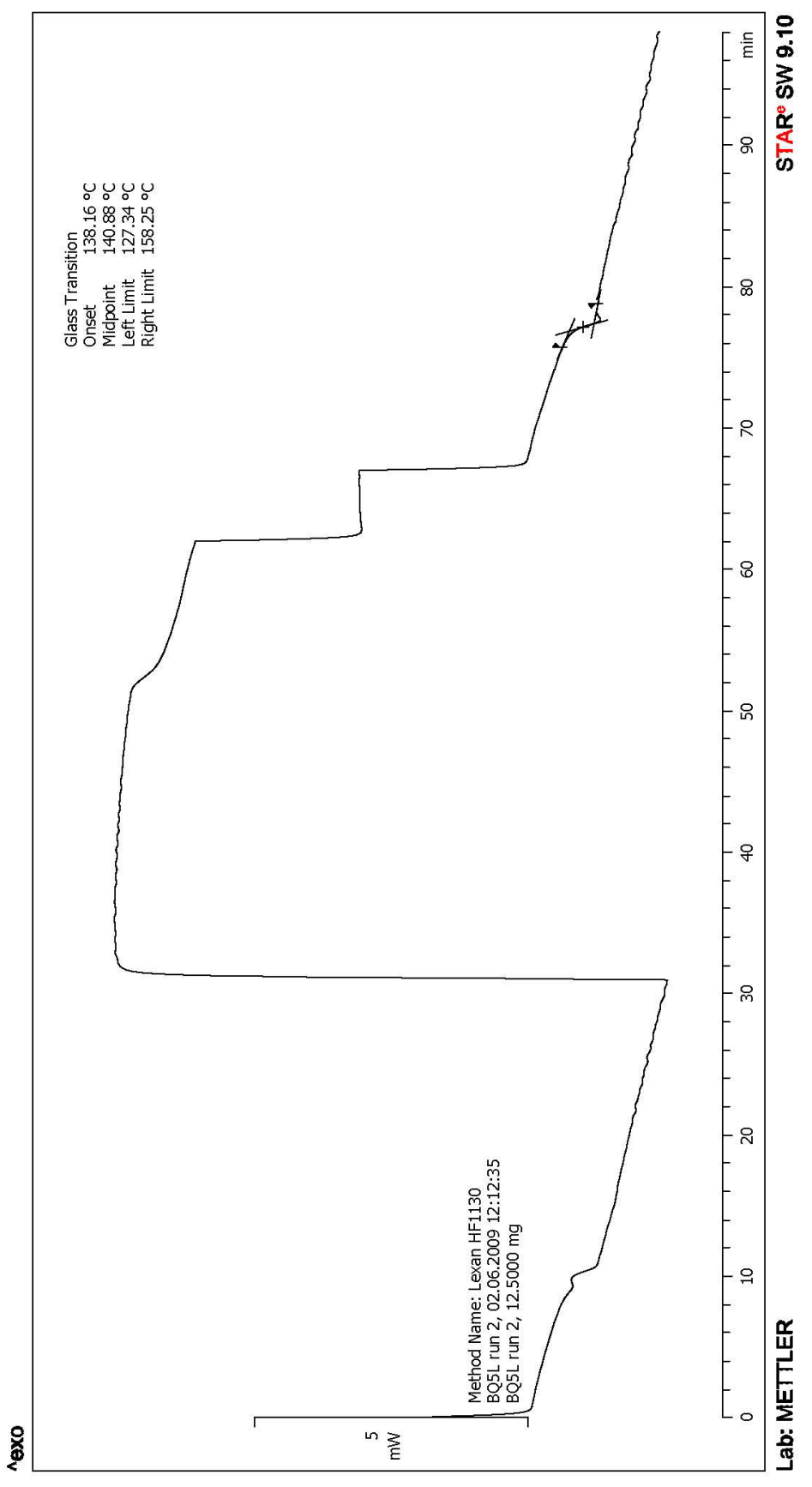

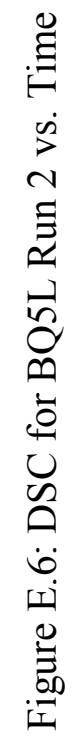




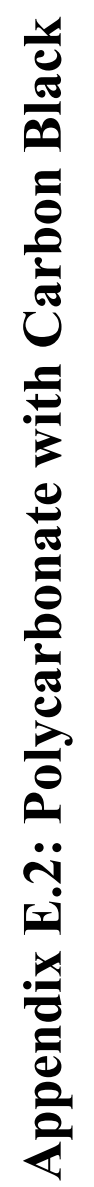




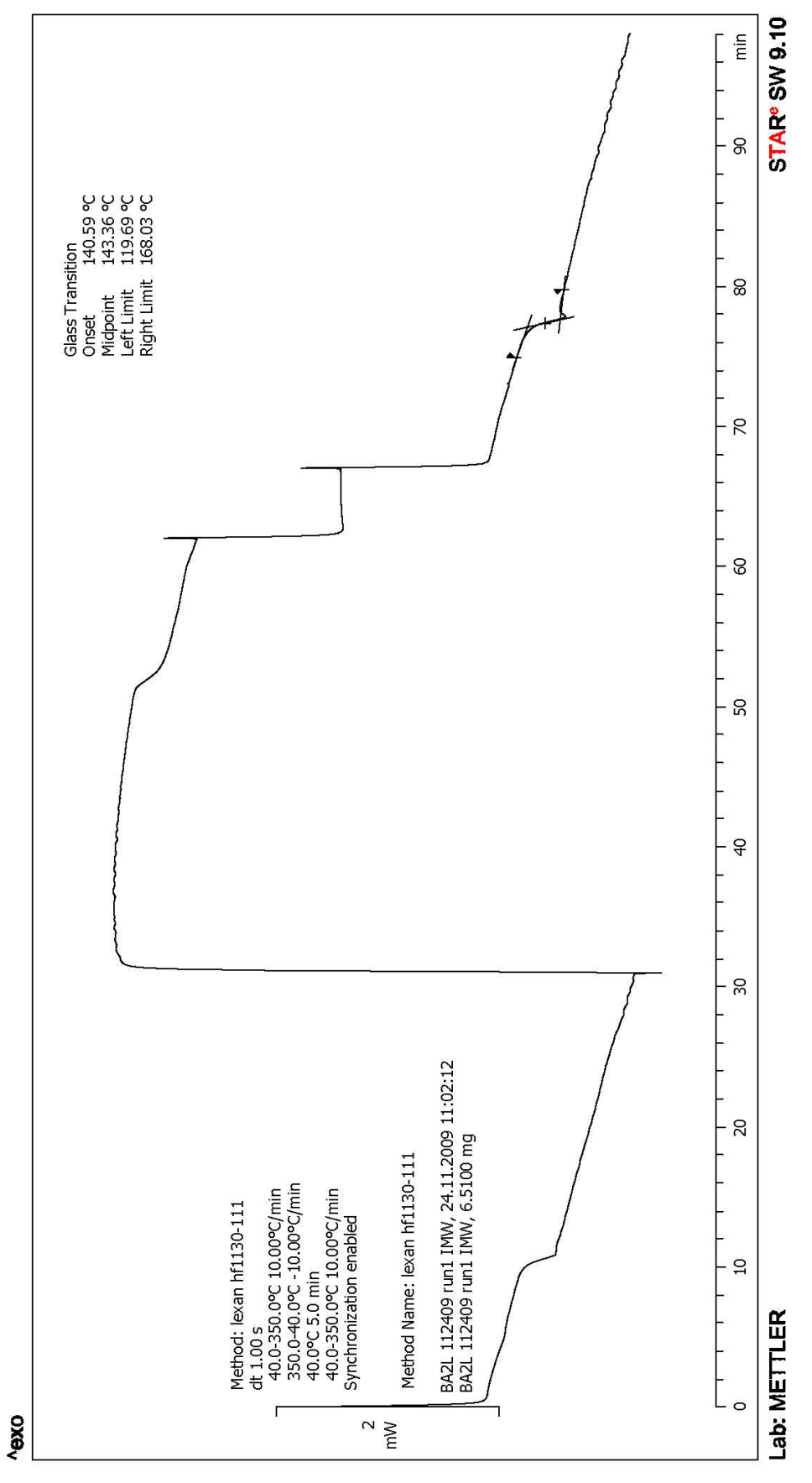

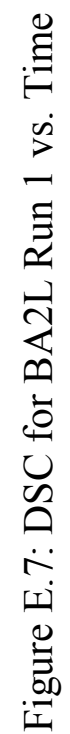




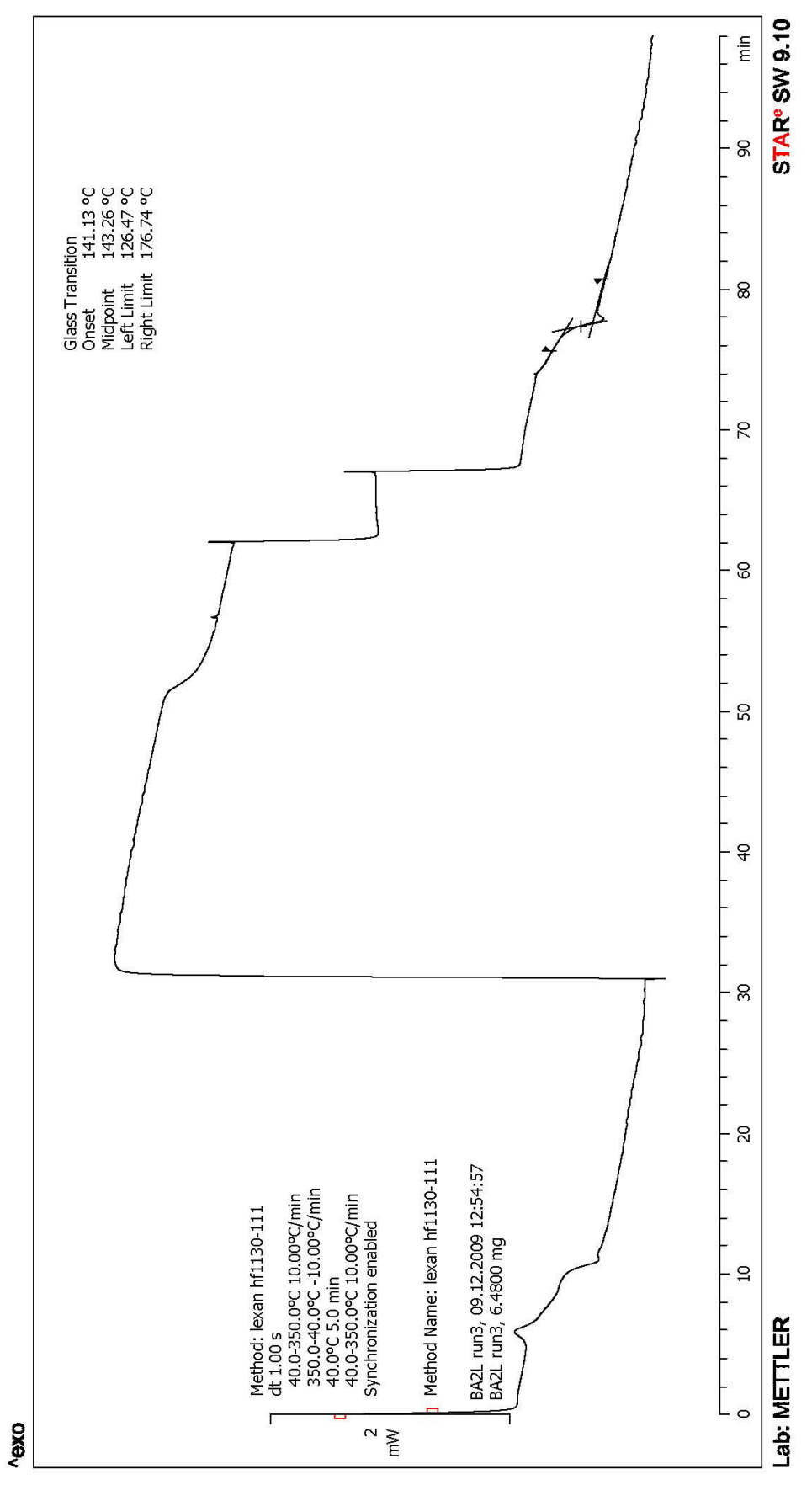

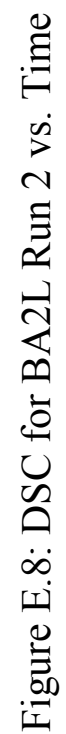




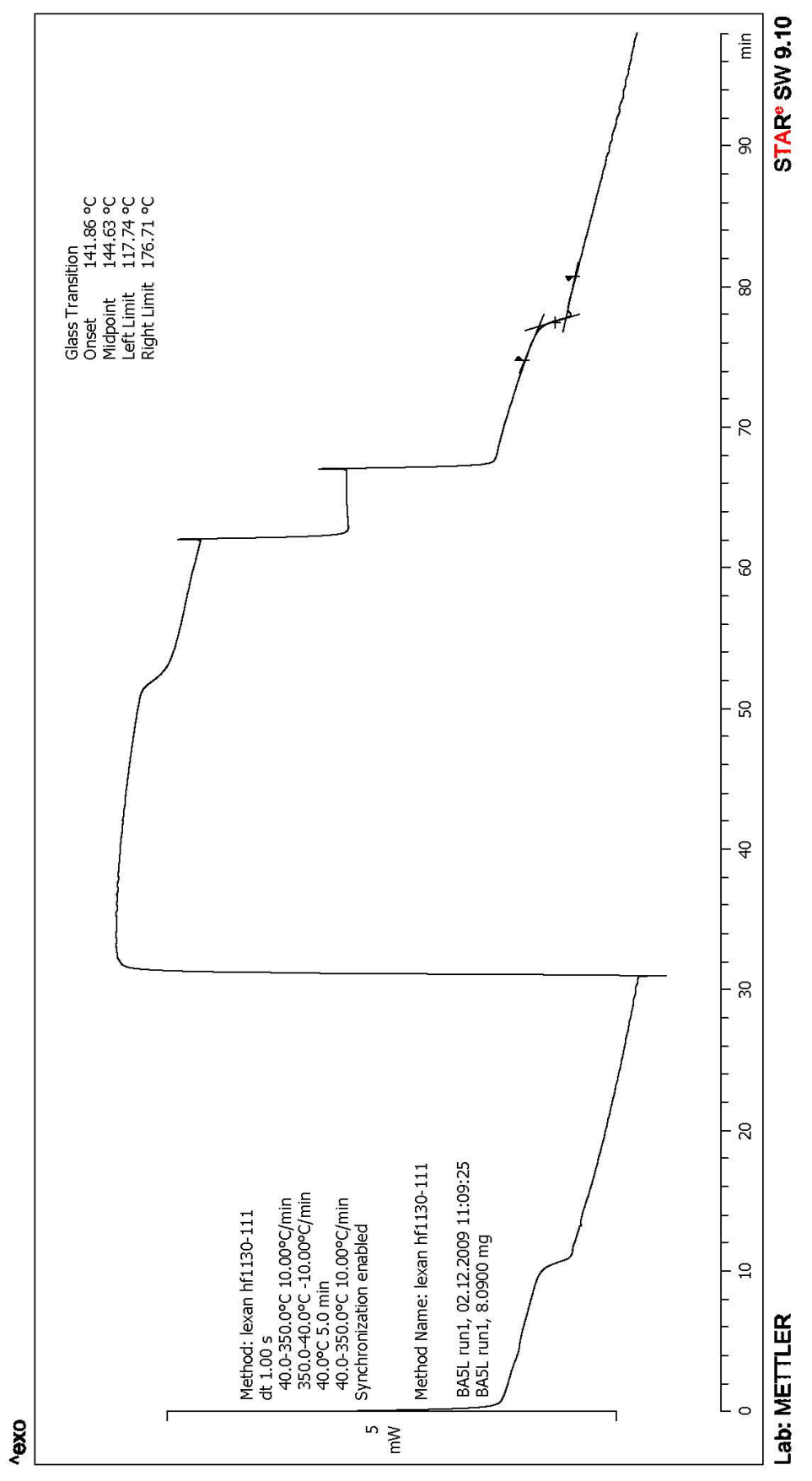

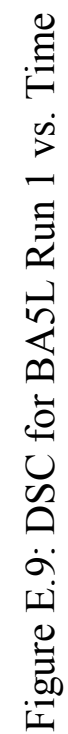




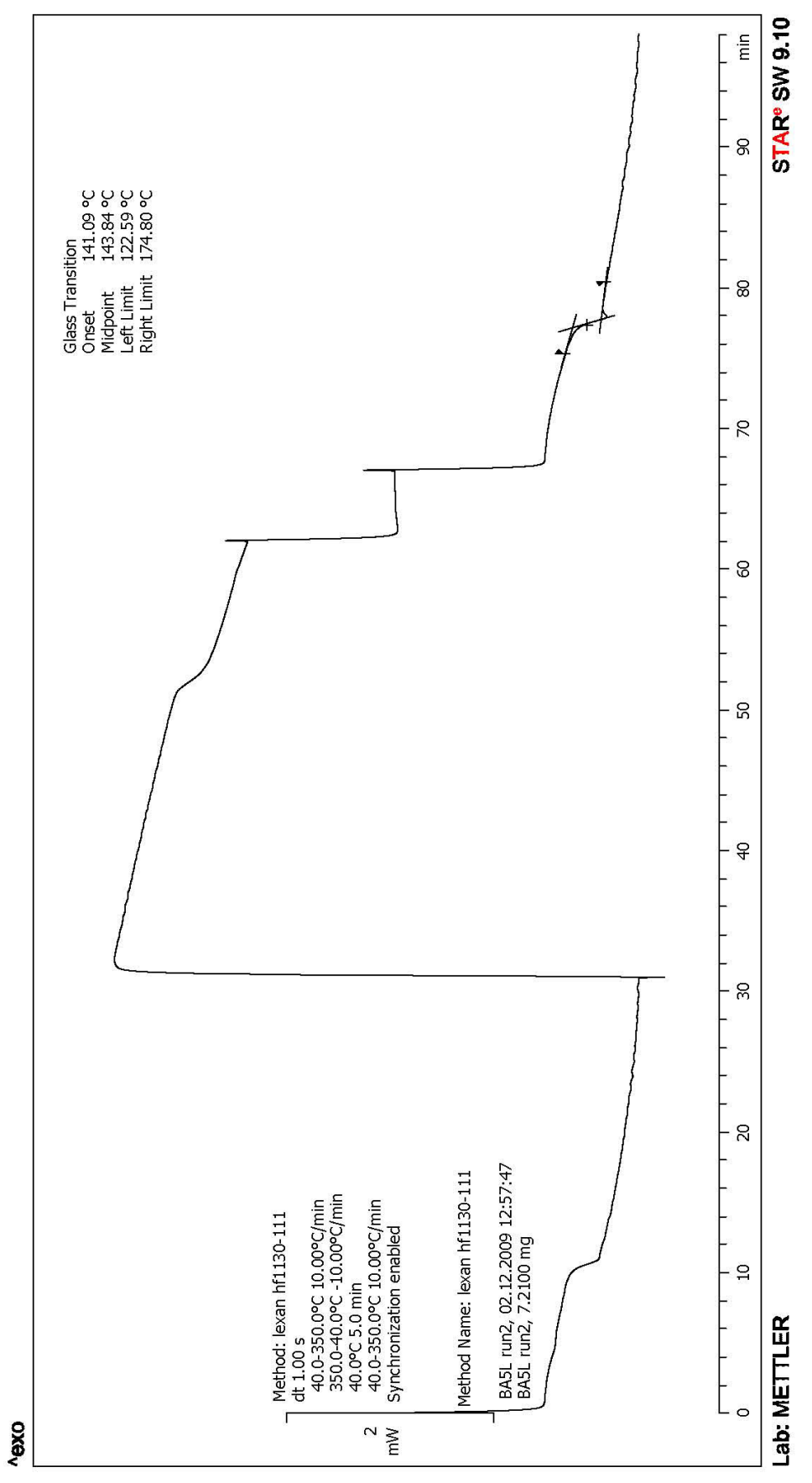

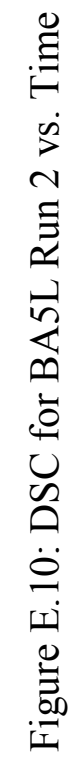




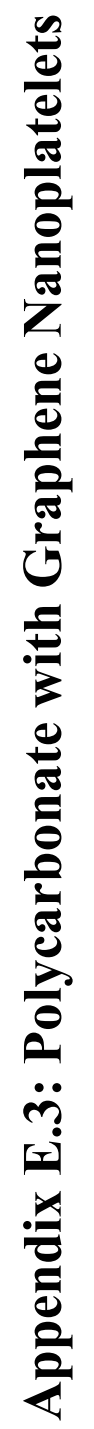




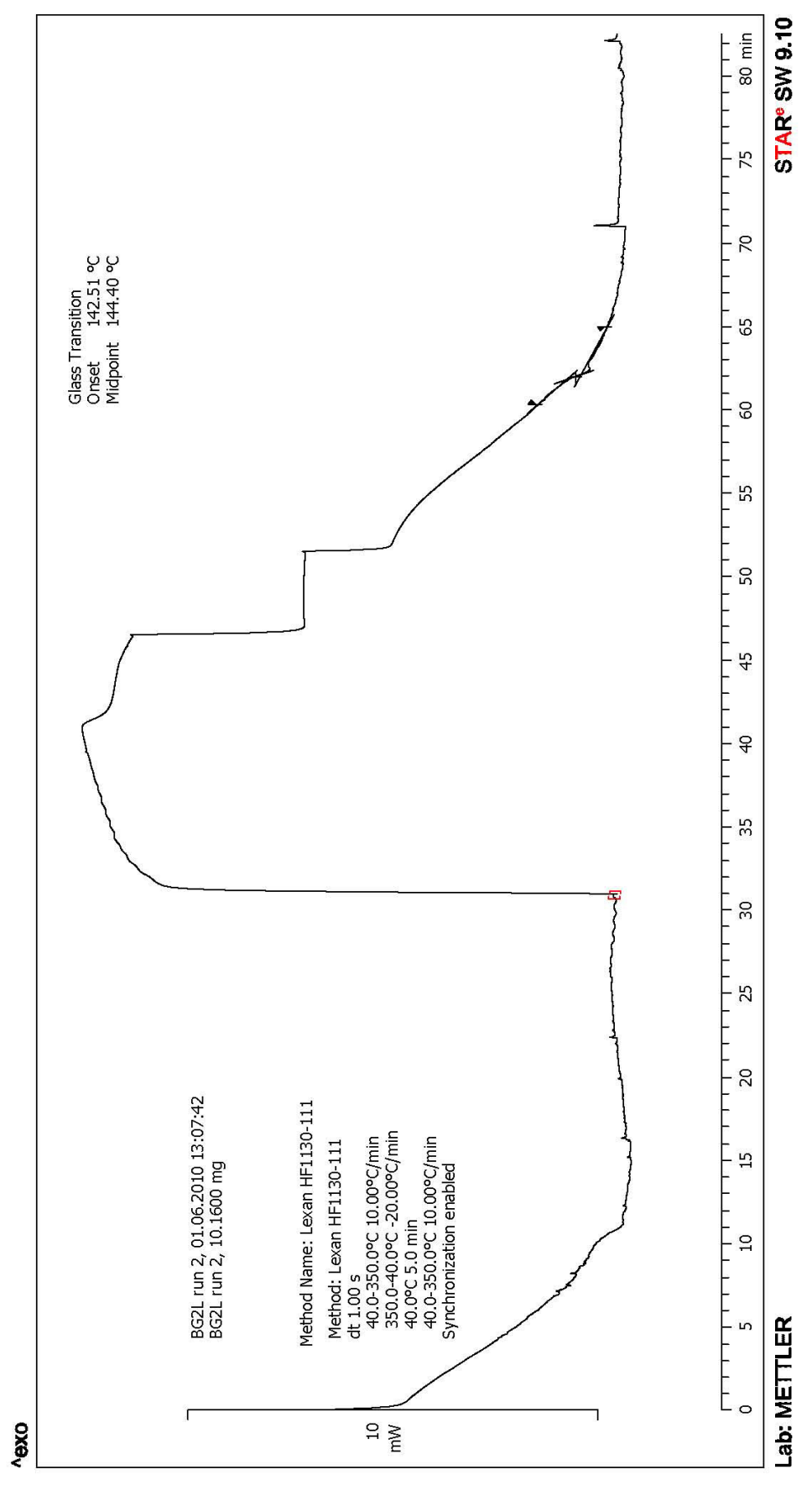

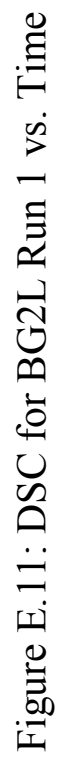




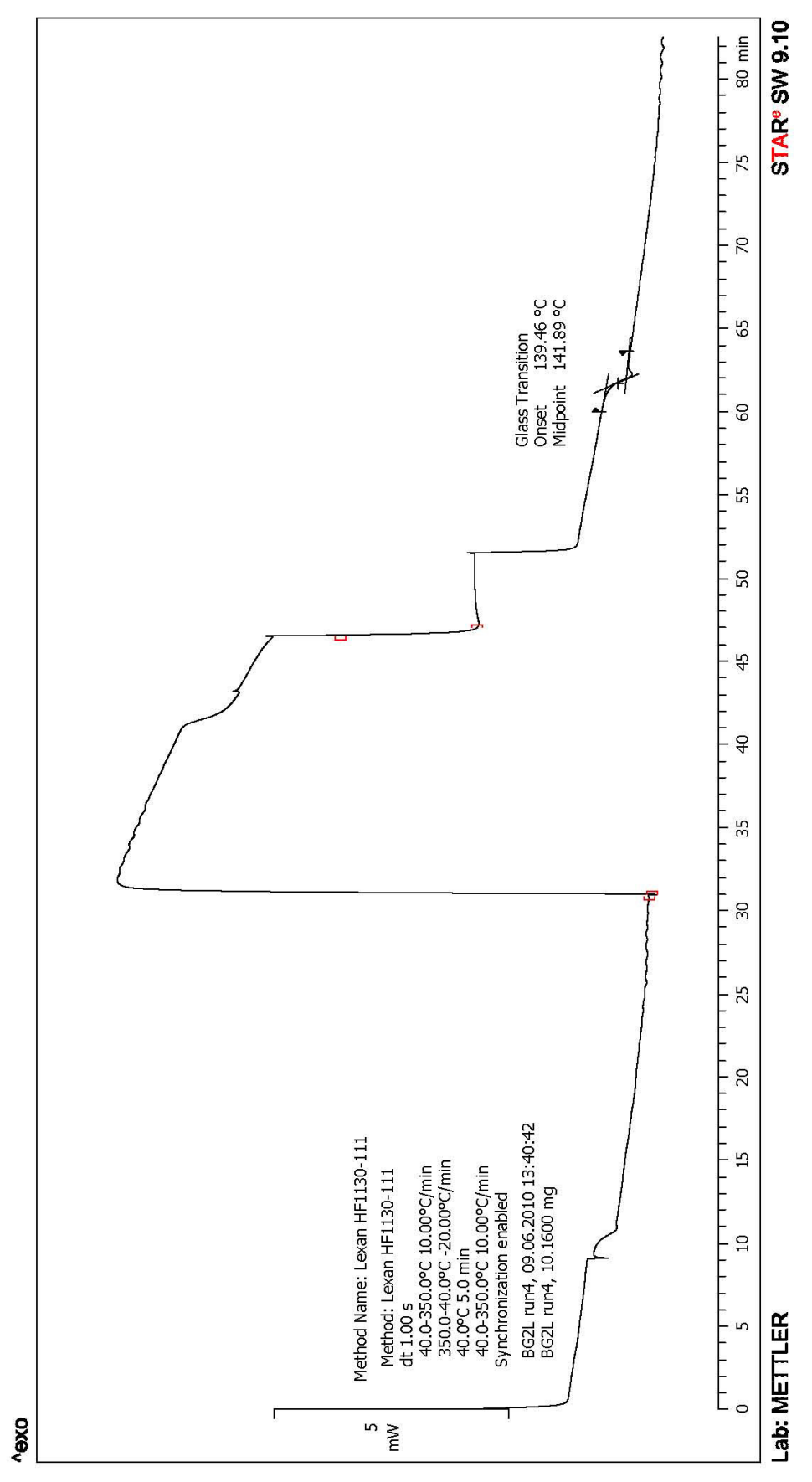

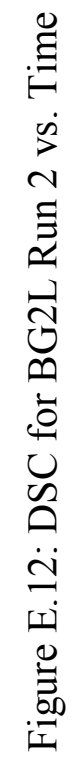




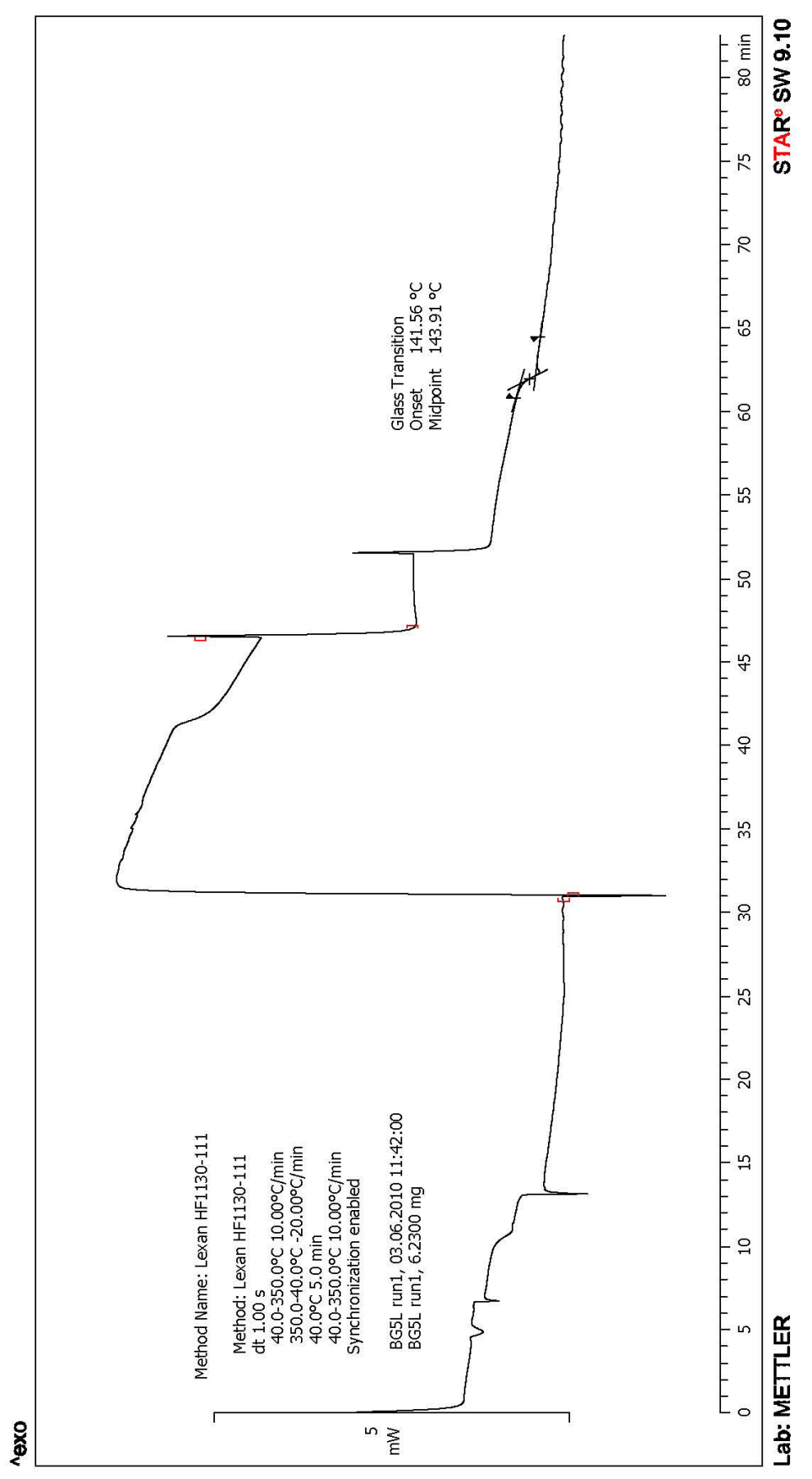

尚 


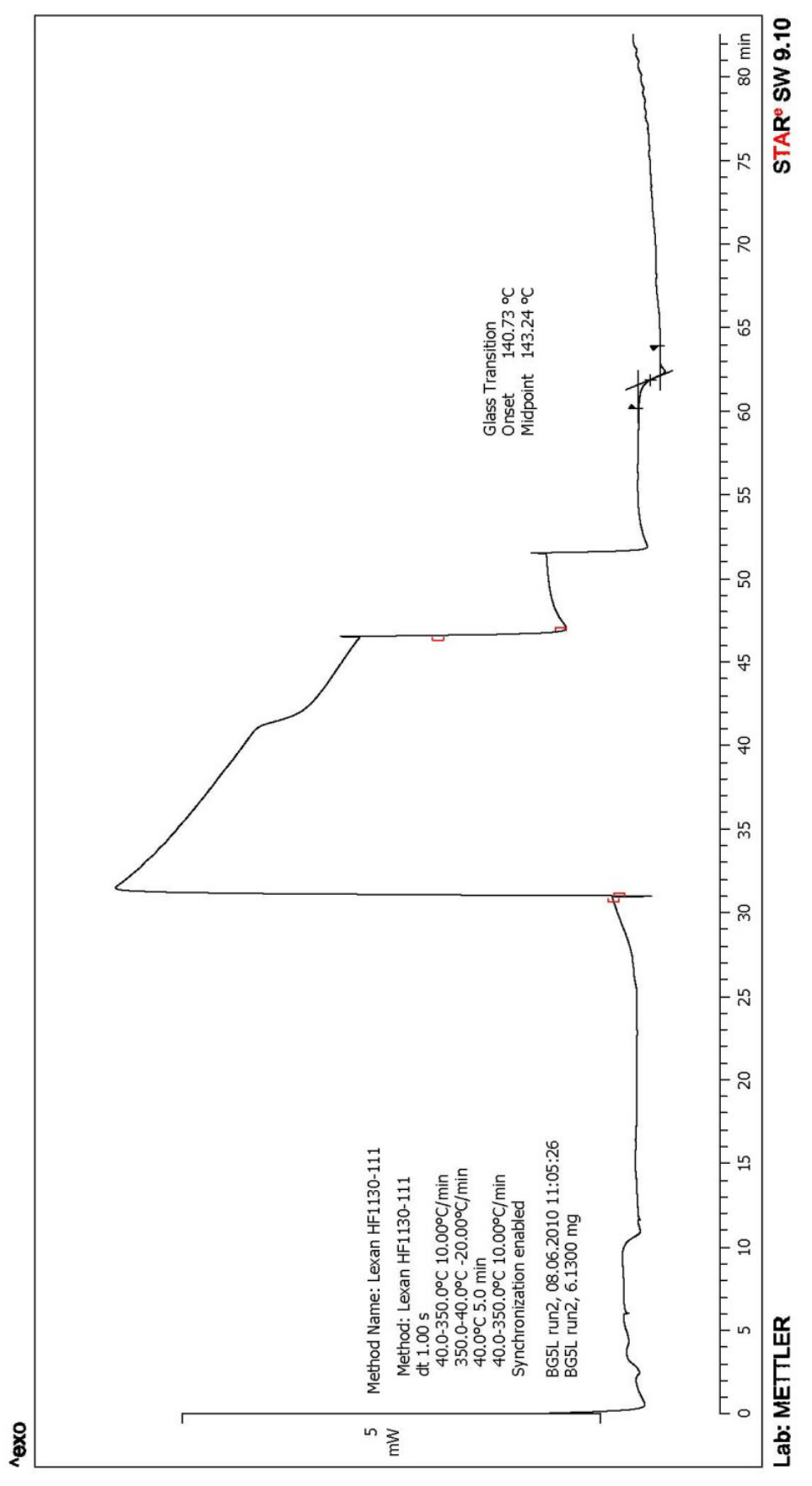

ఏ 


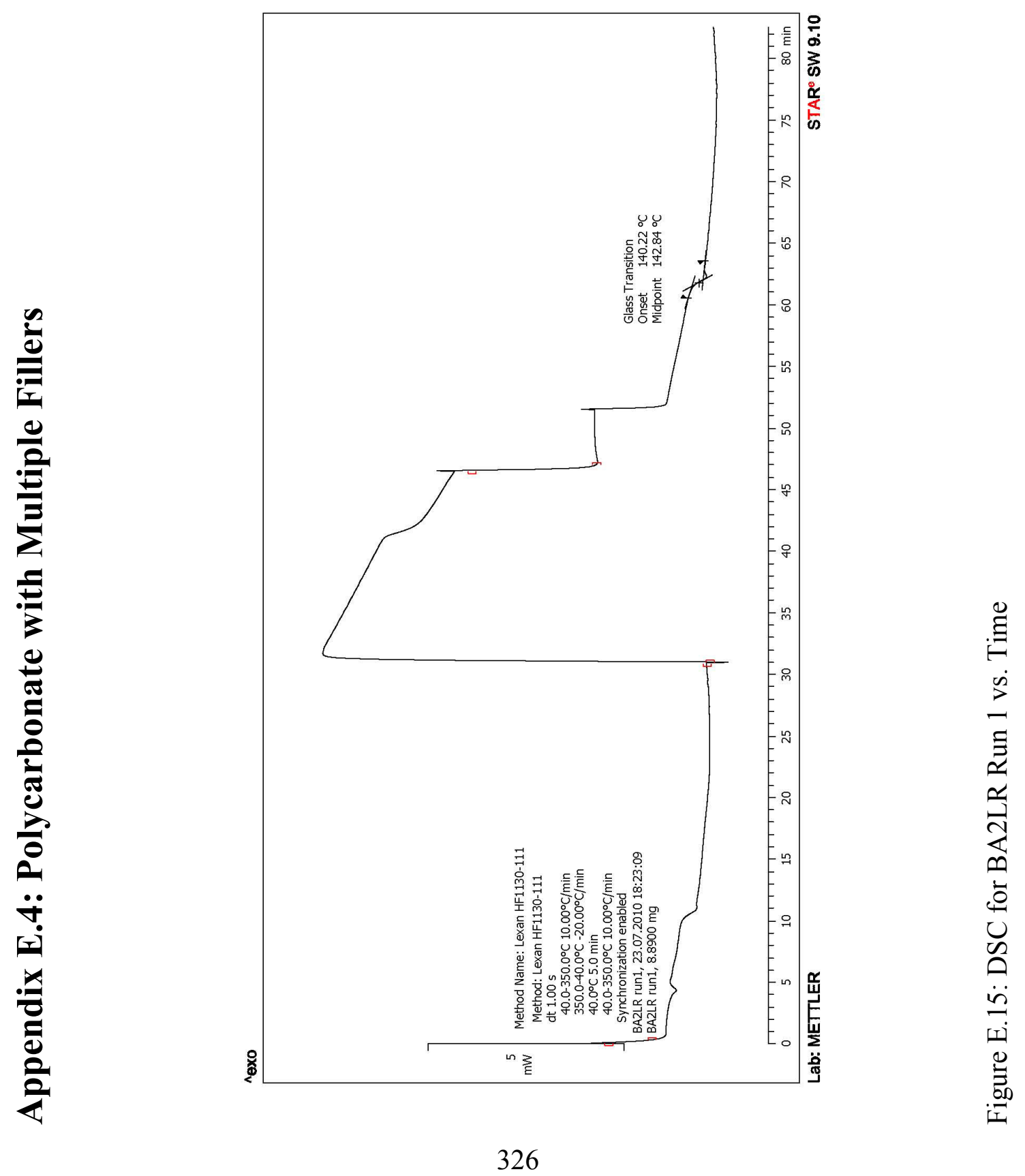




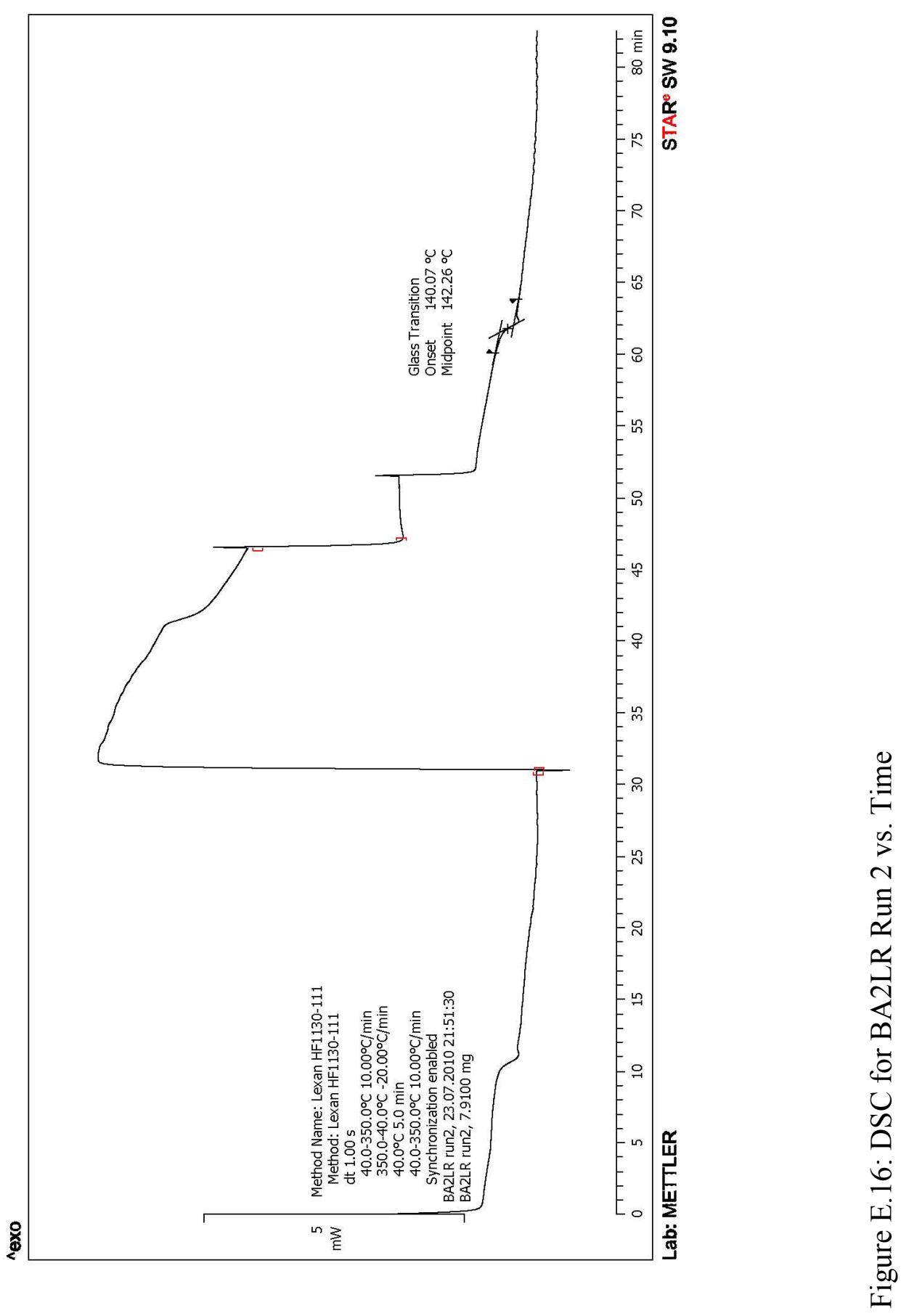




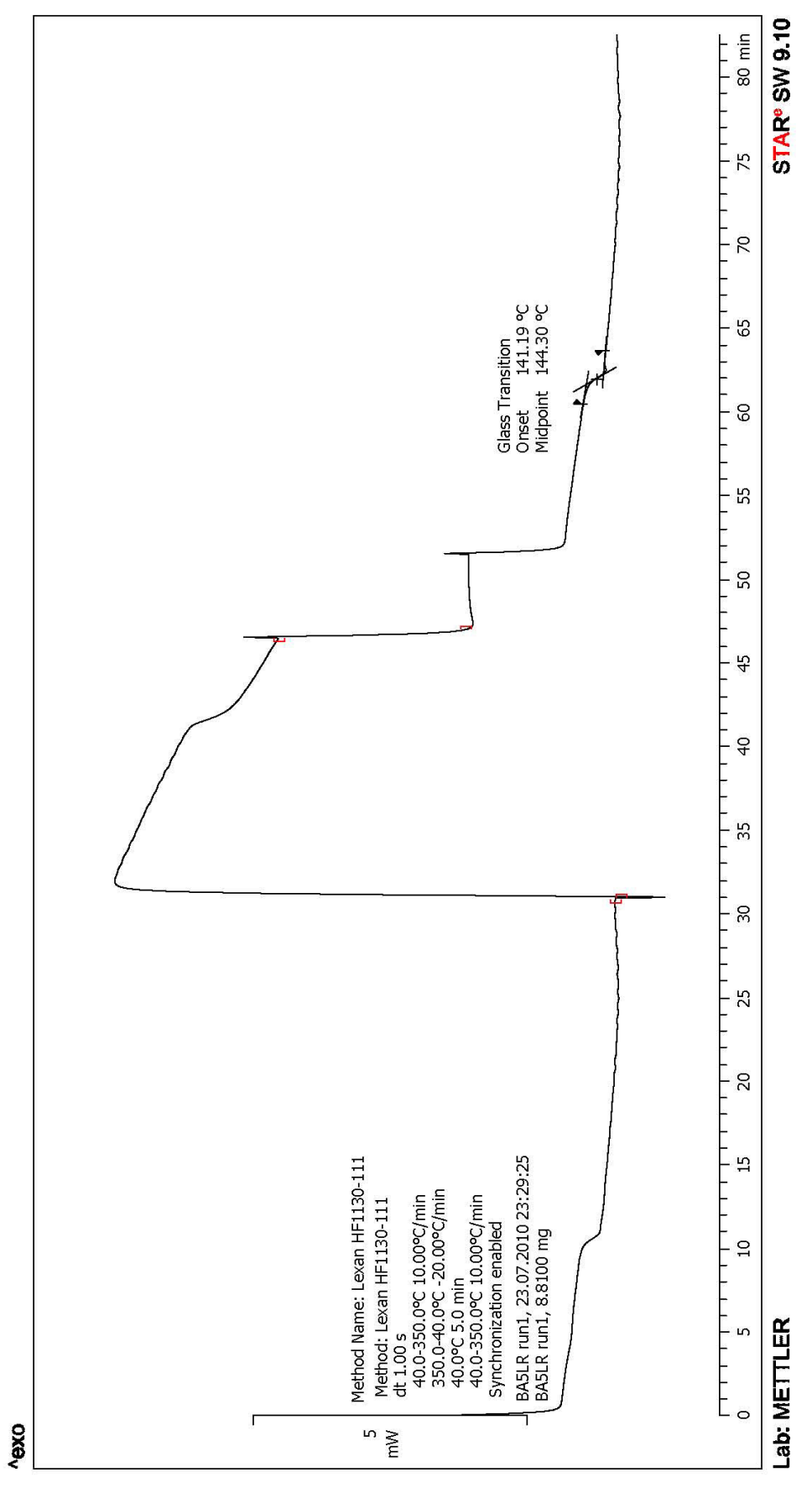

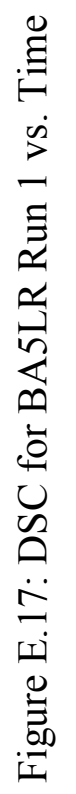




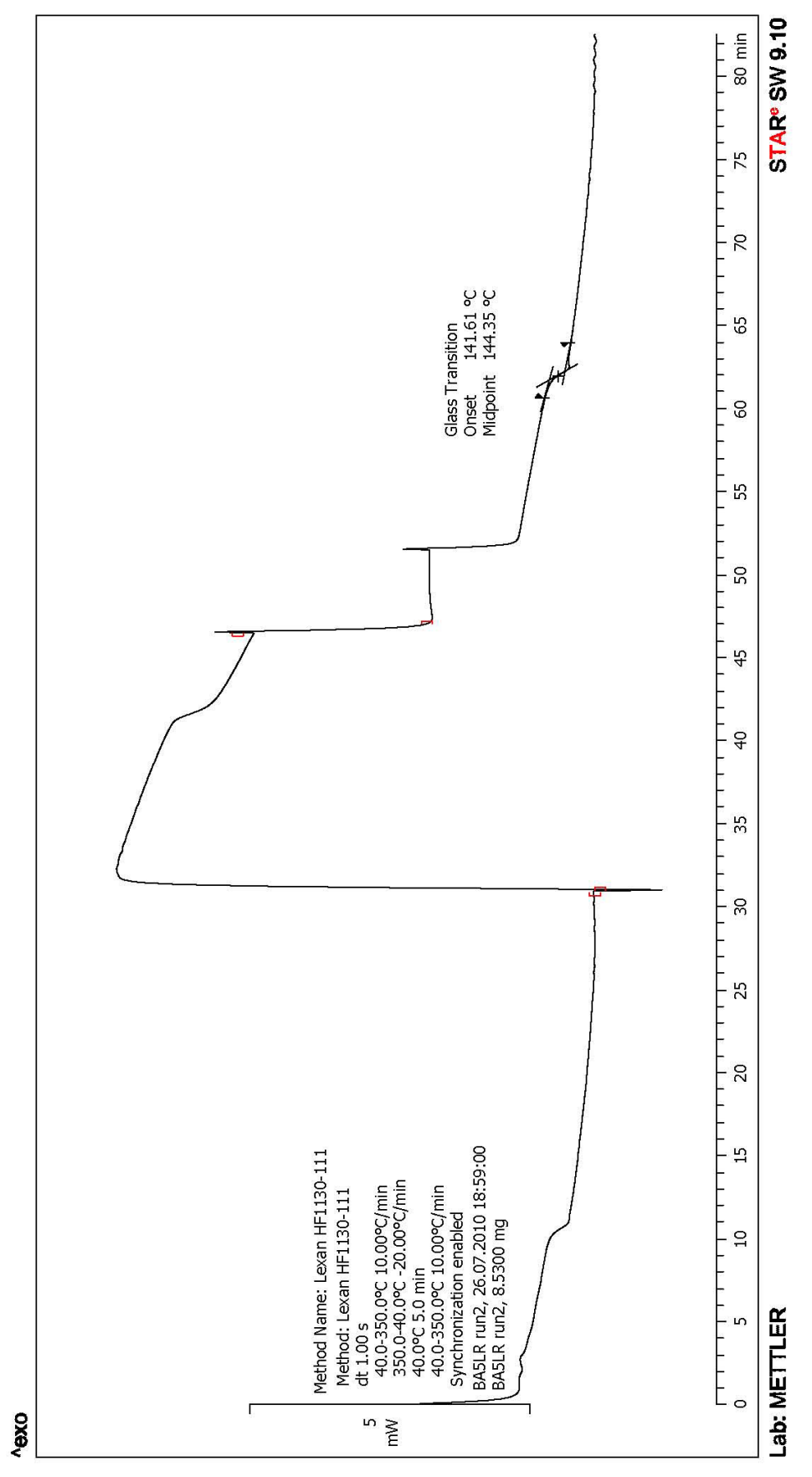

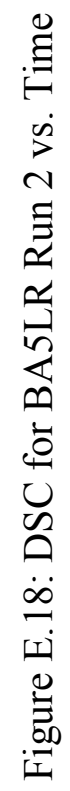




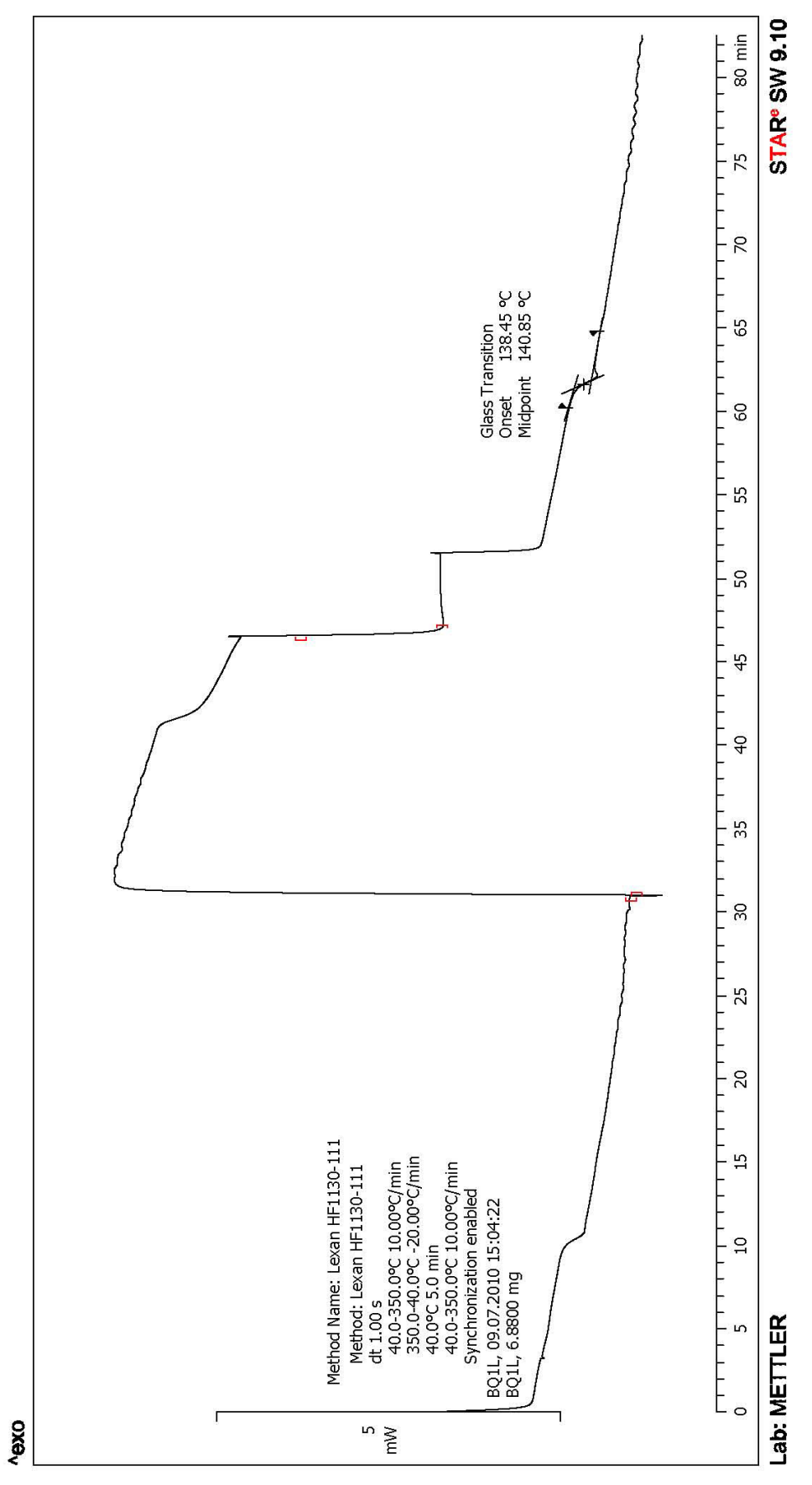

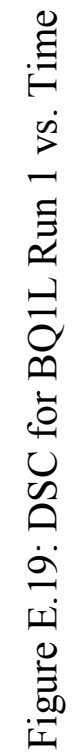




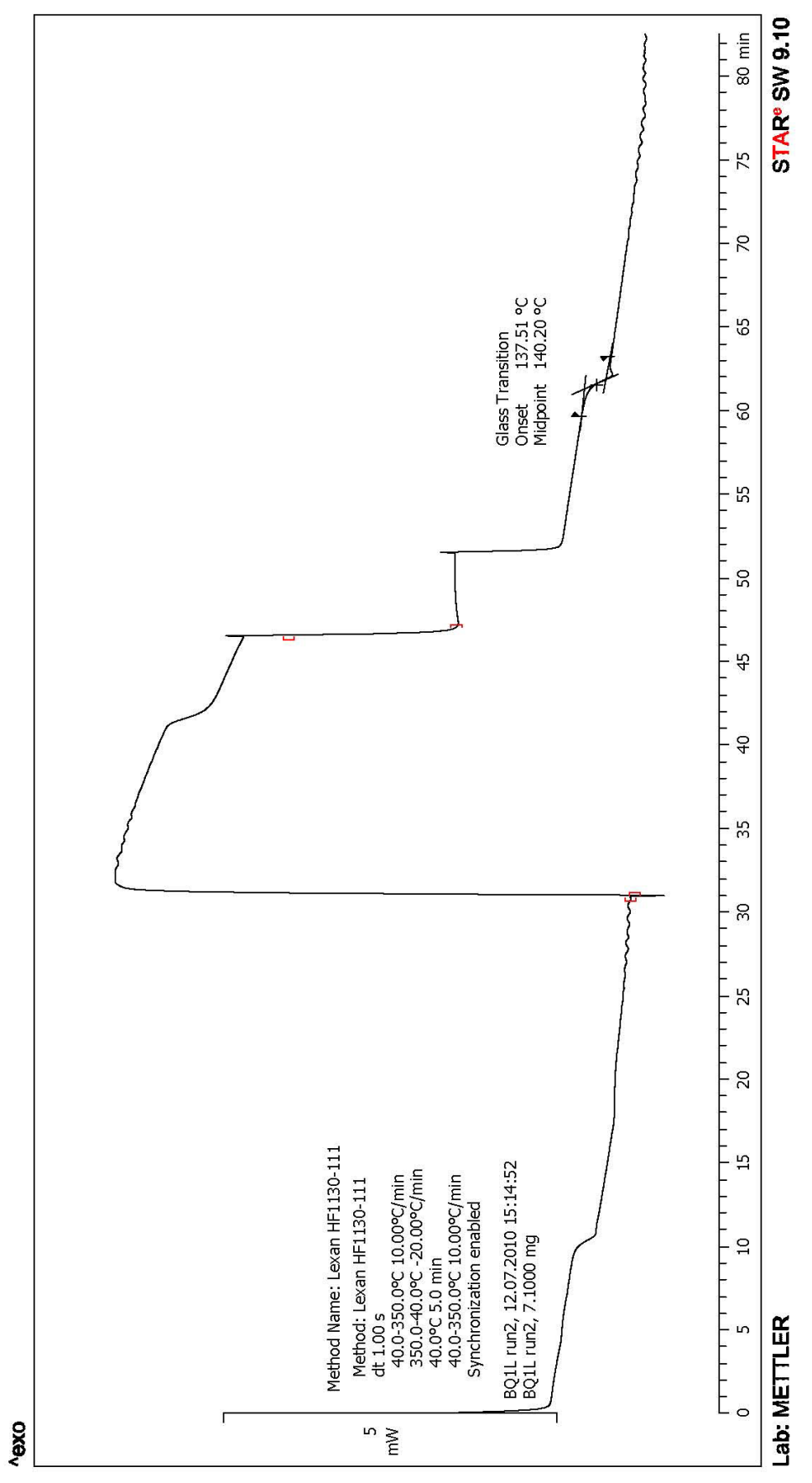

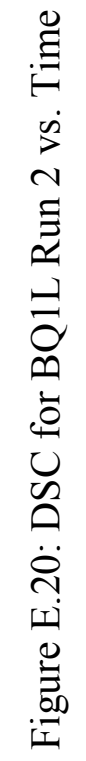




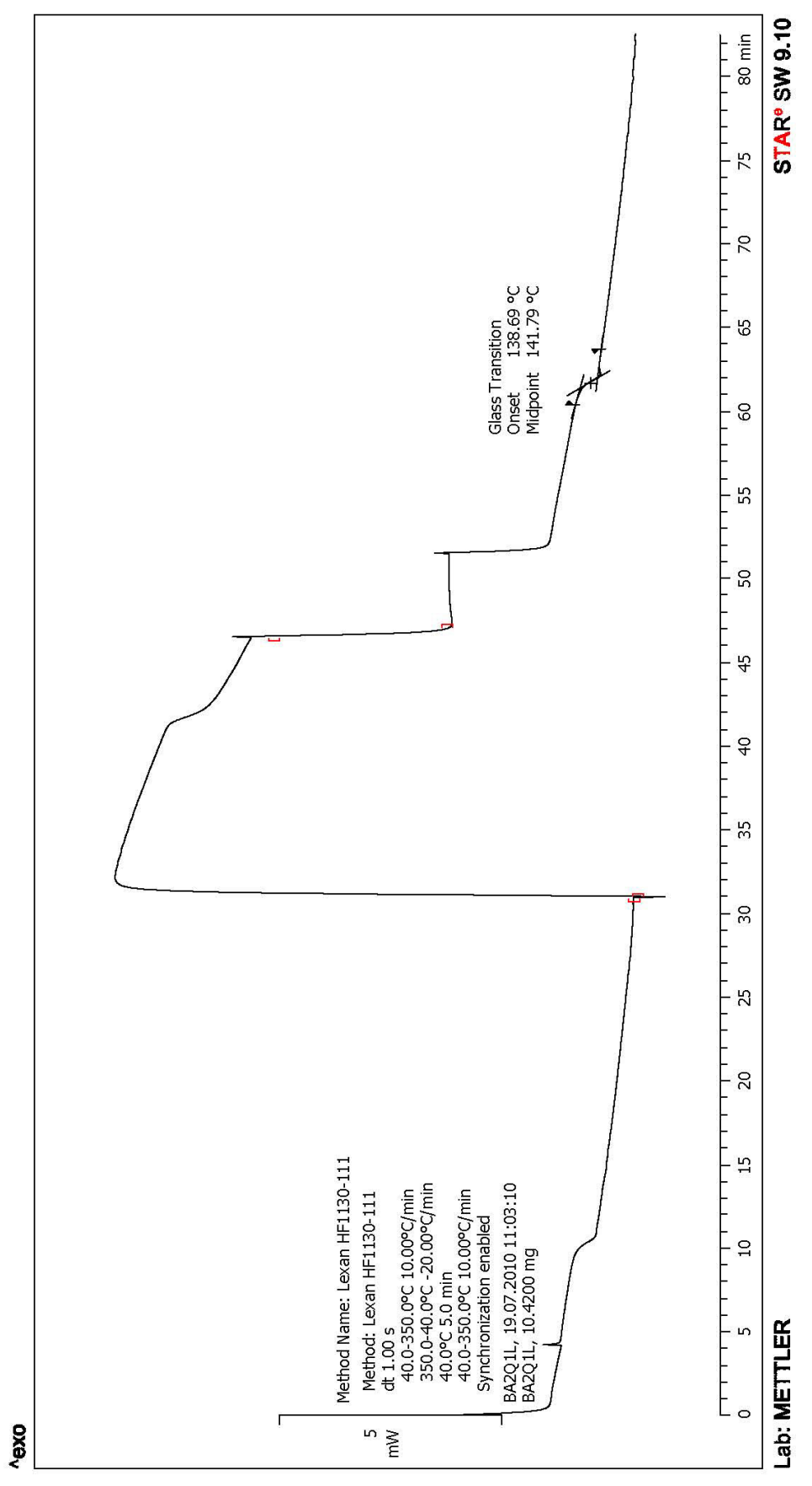

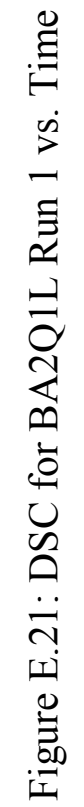




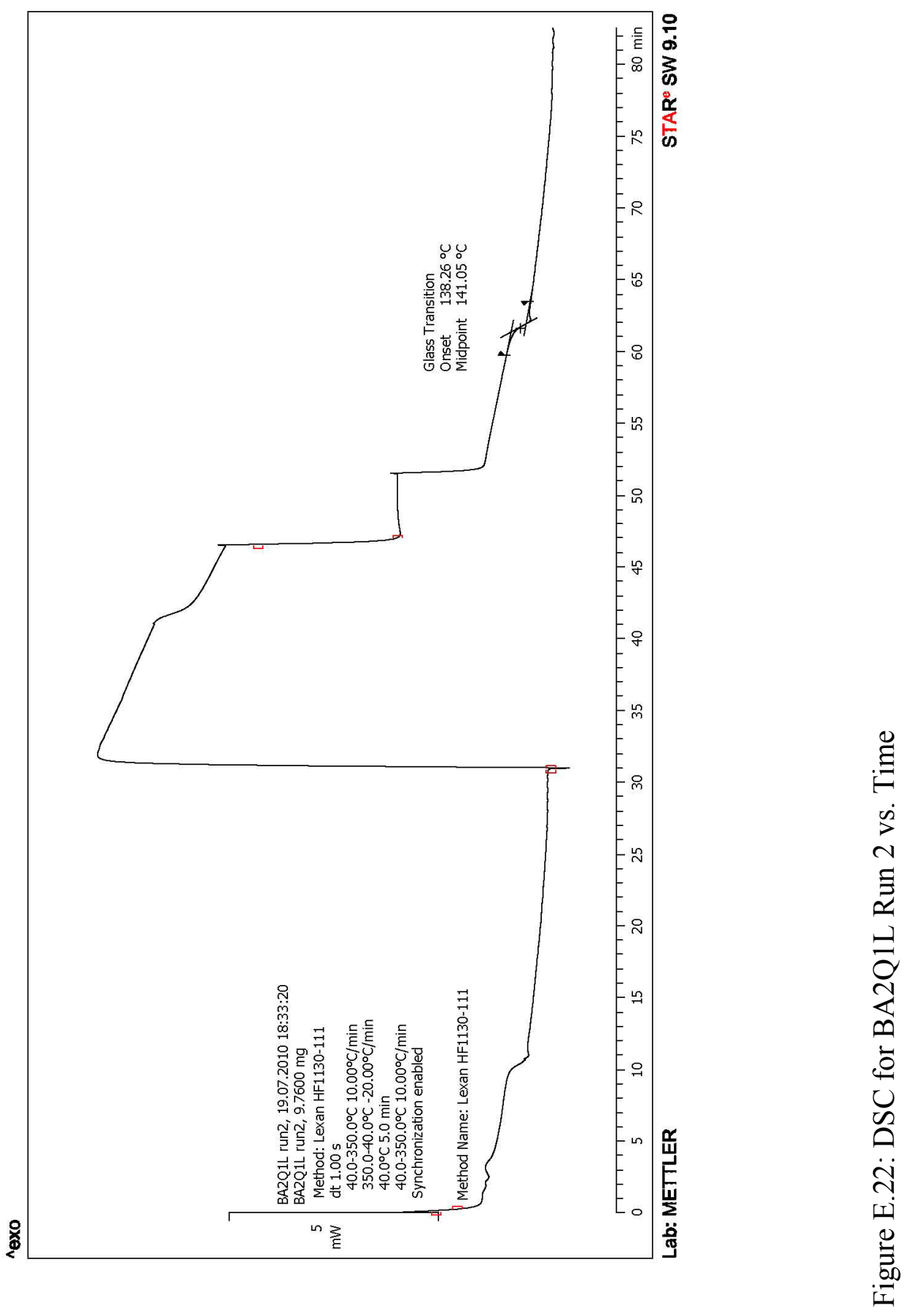



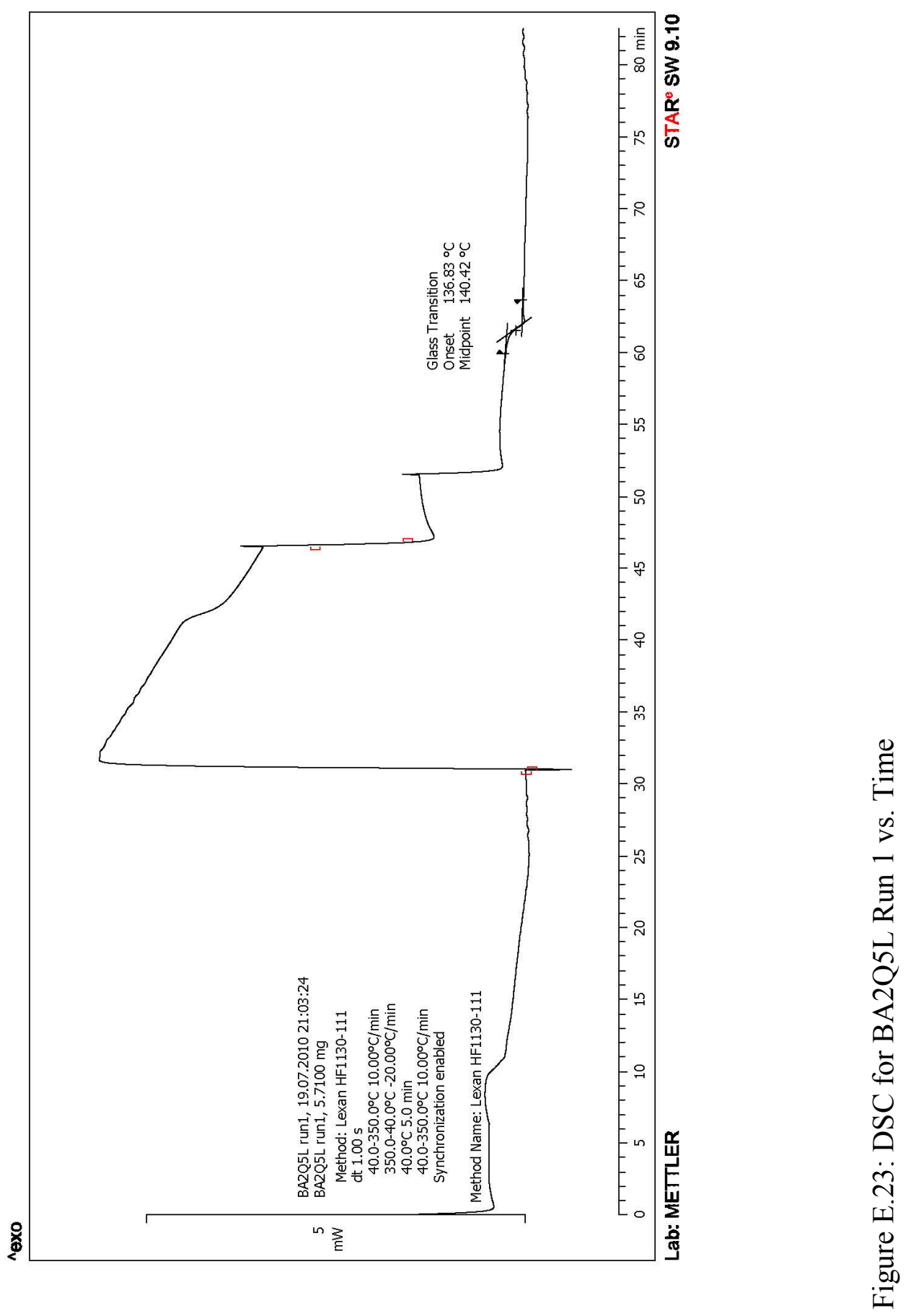


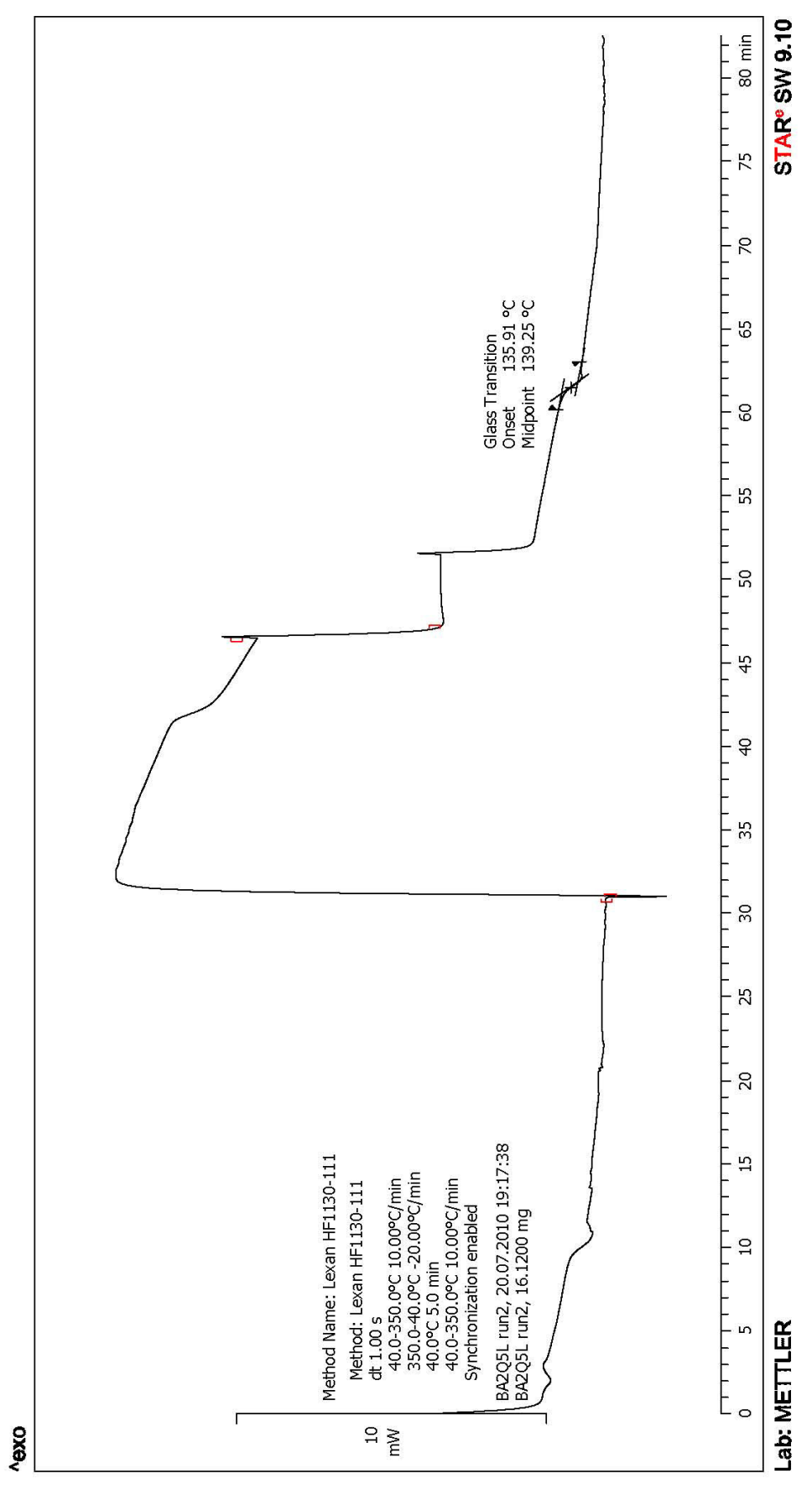

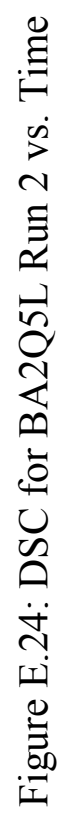




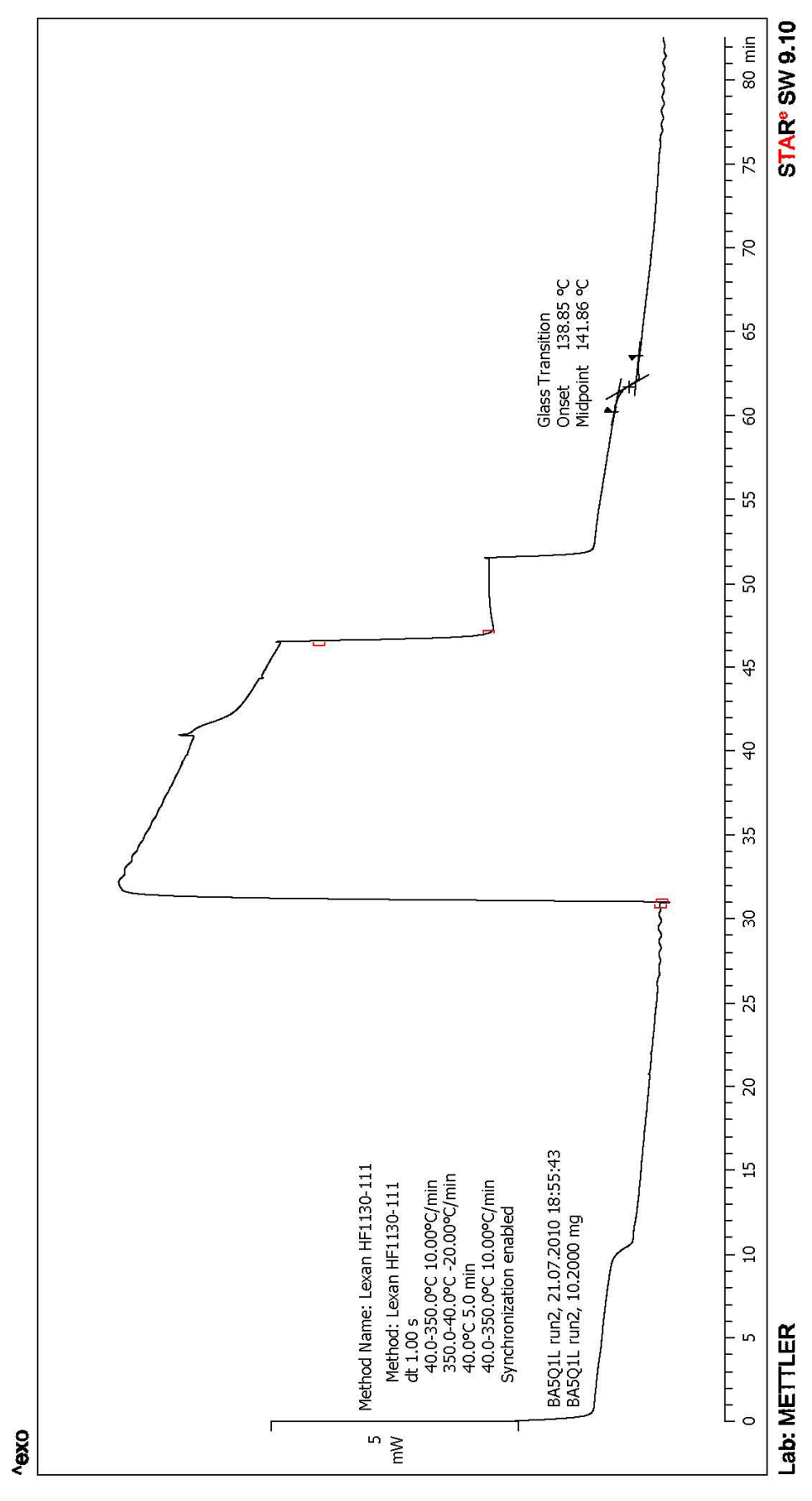

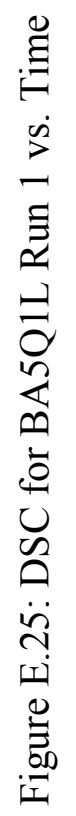




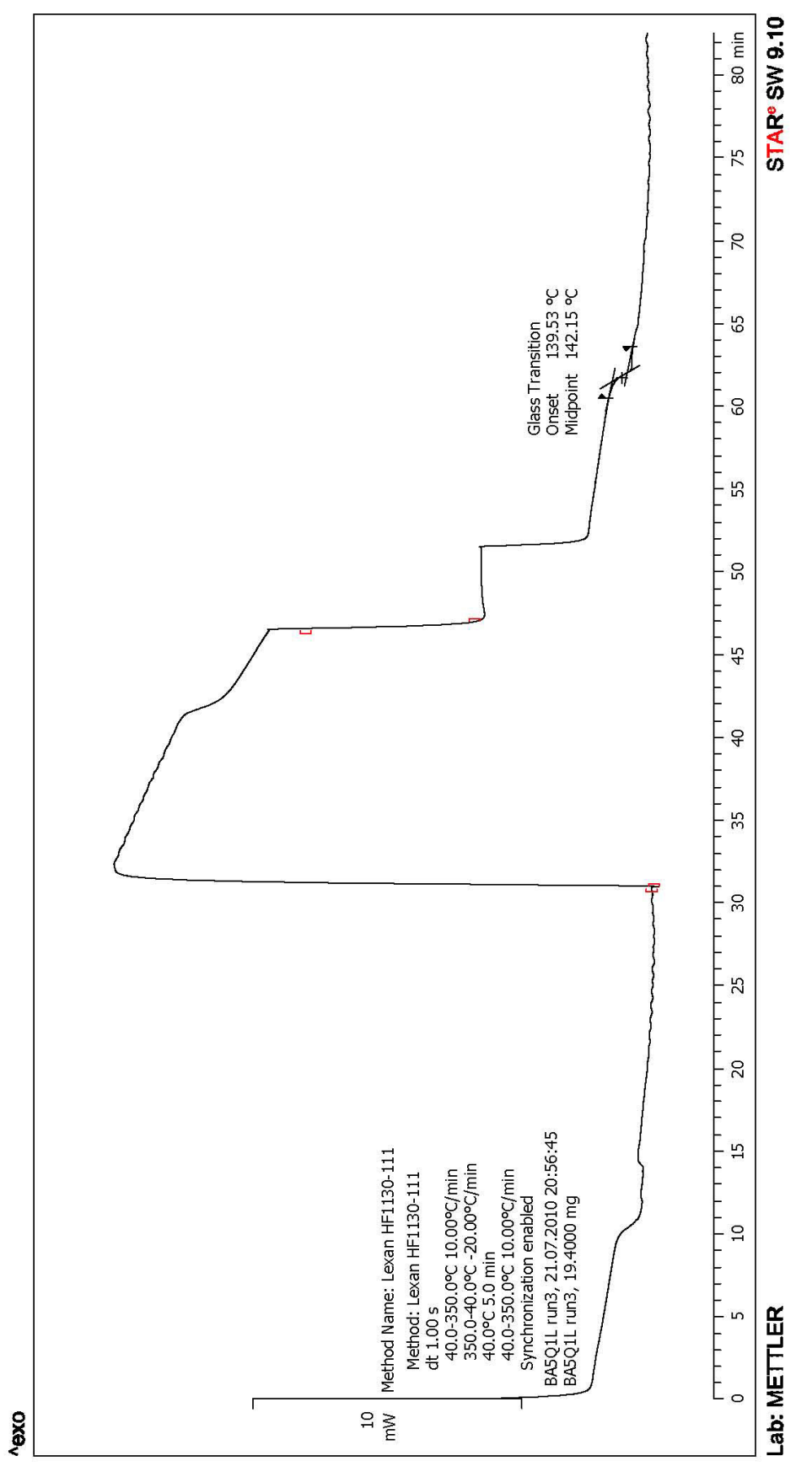

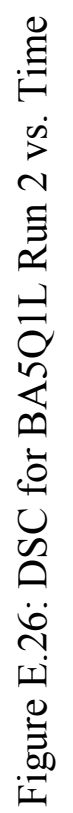




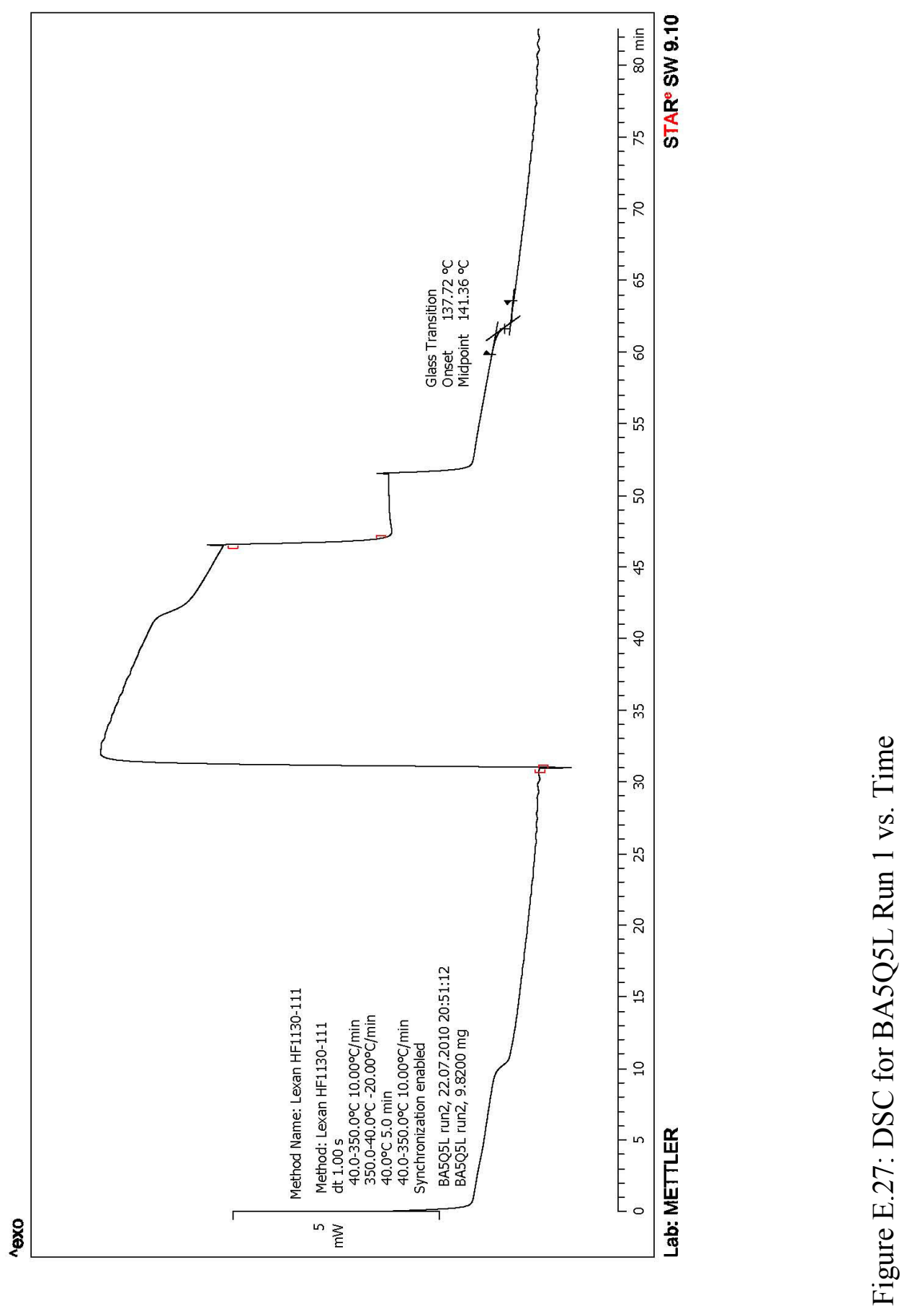




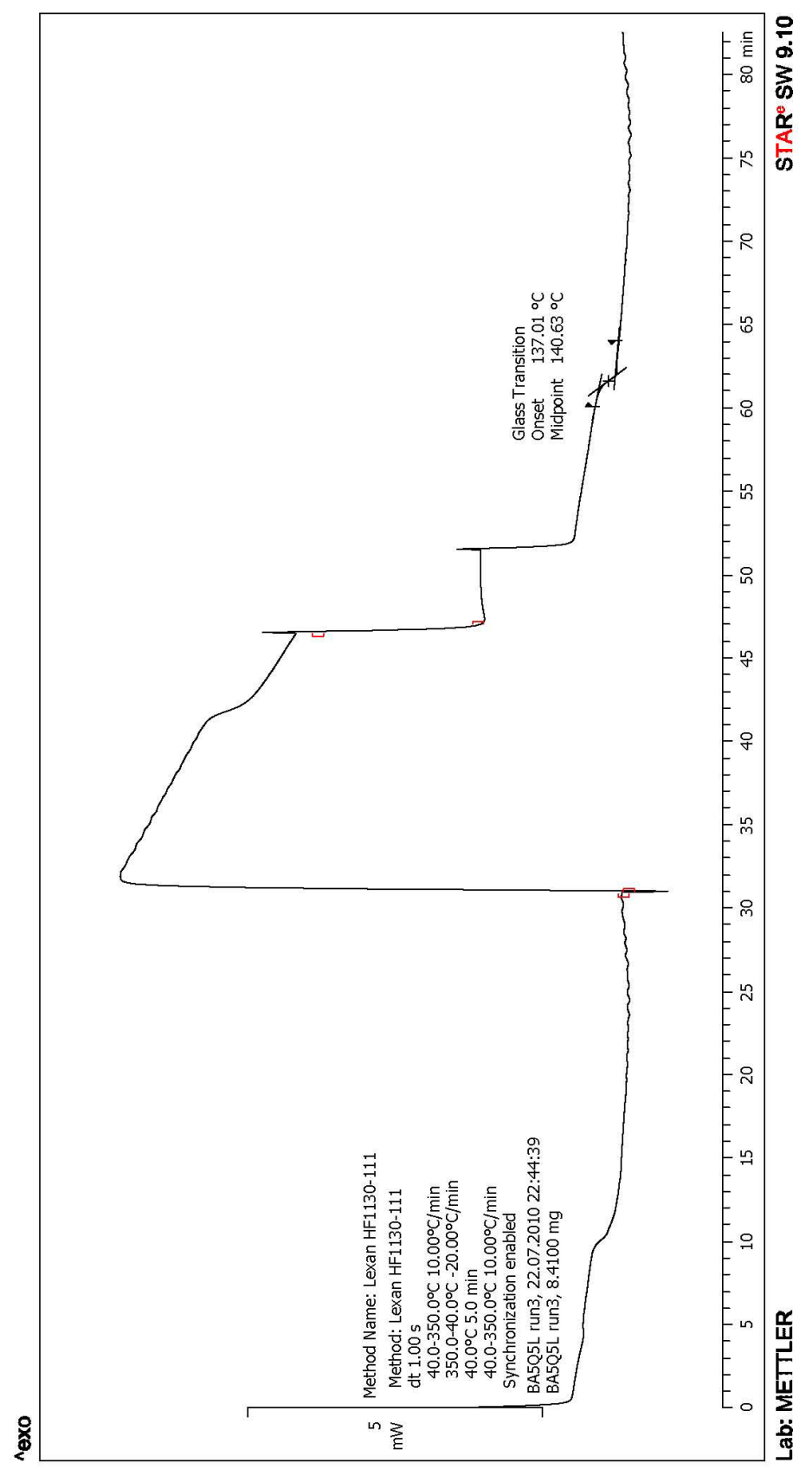

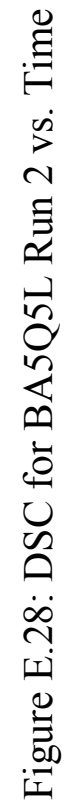




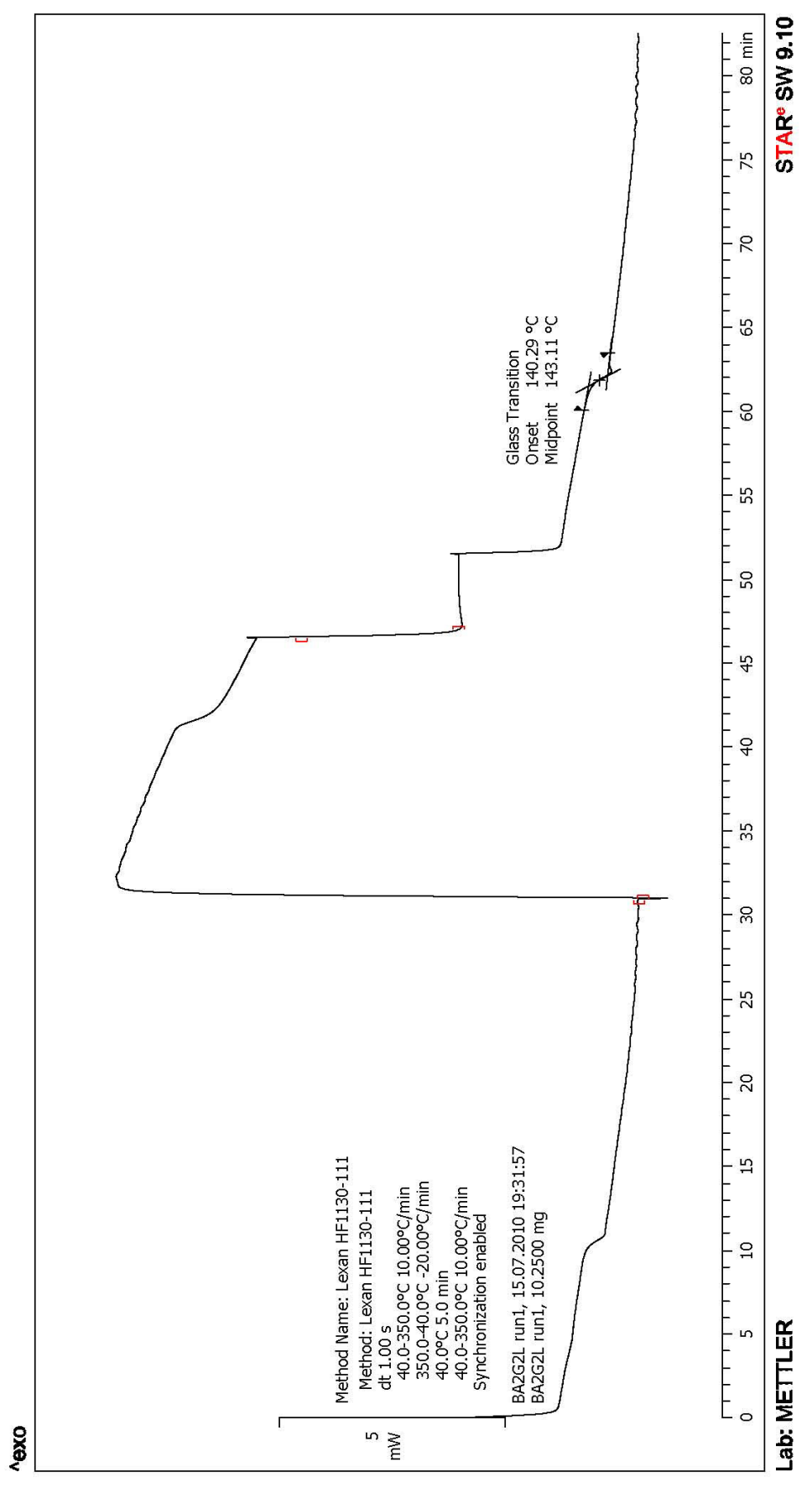

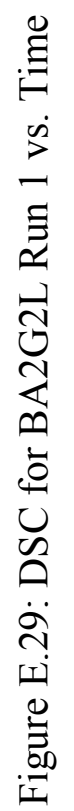




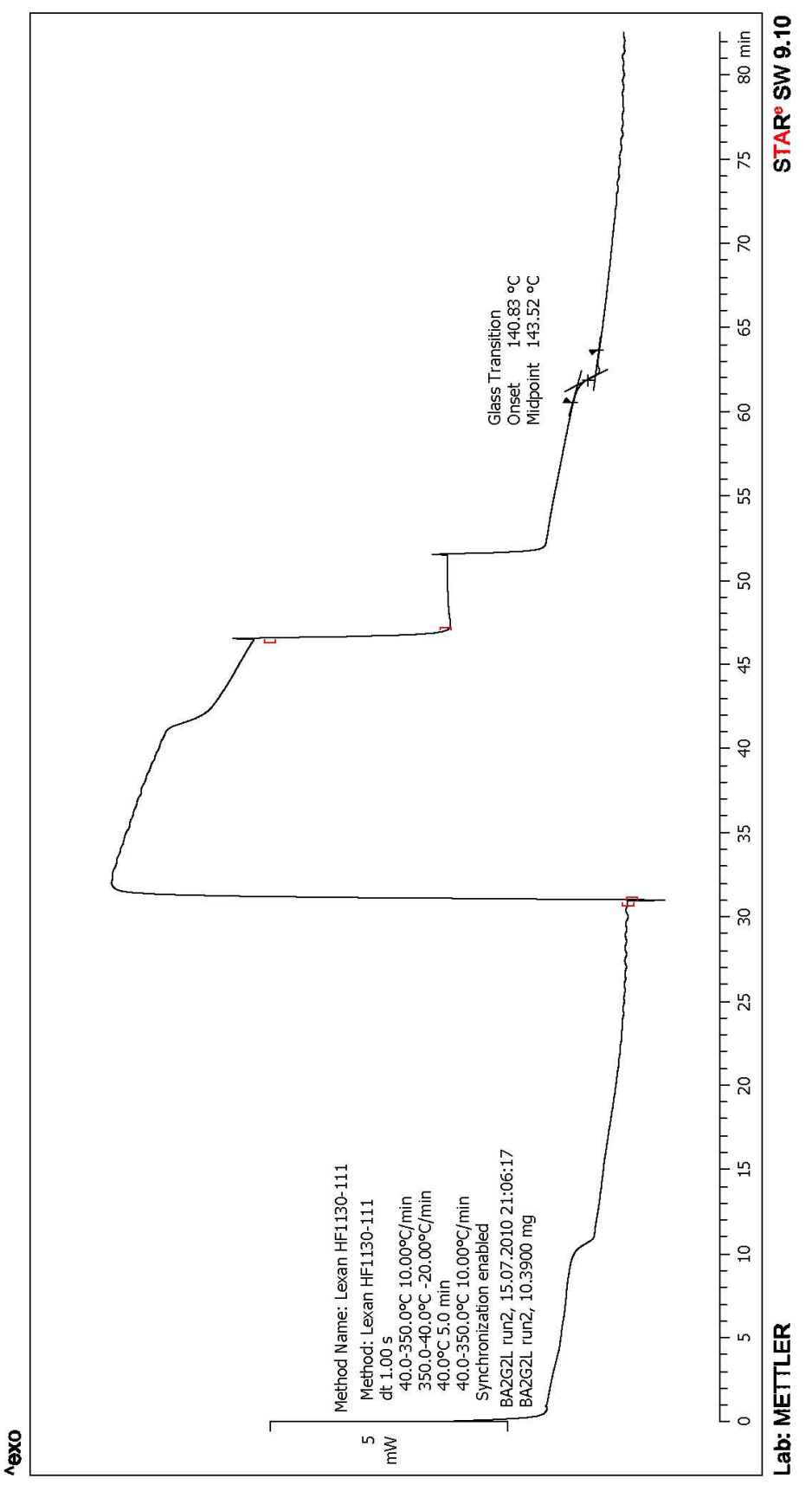

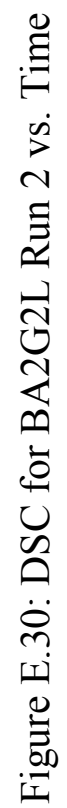




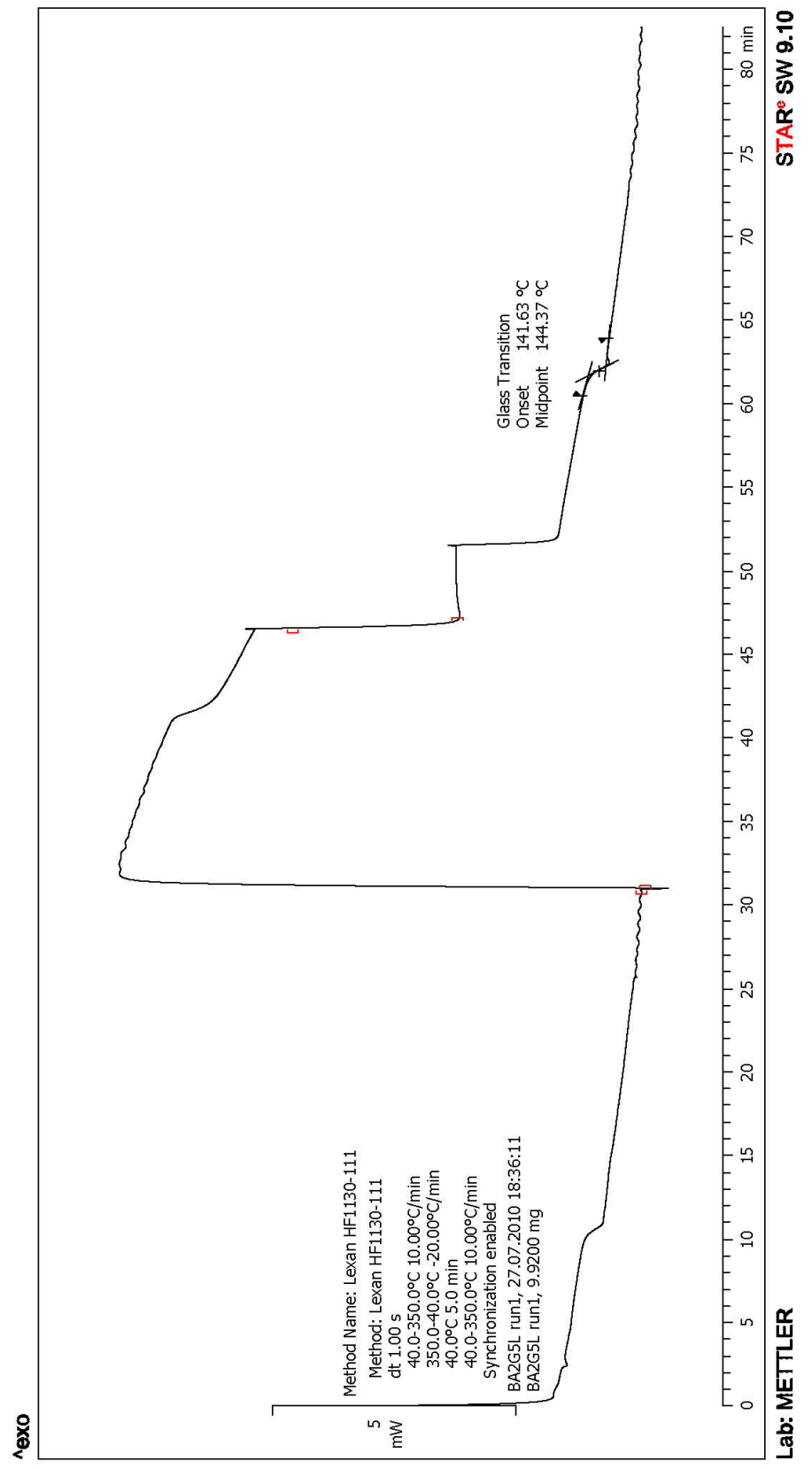

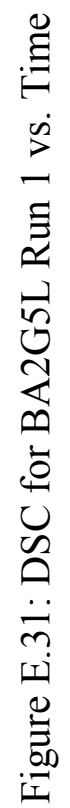




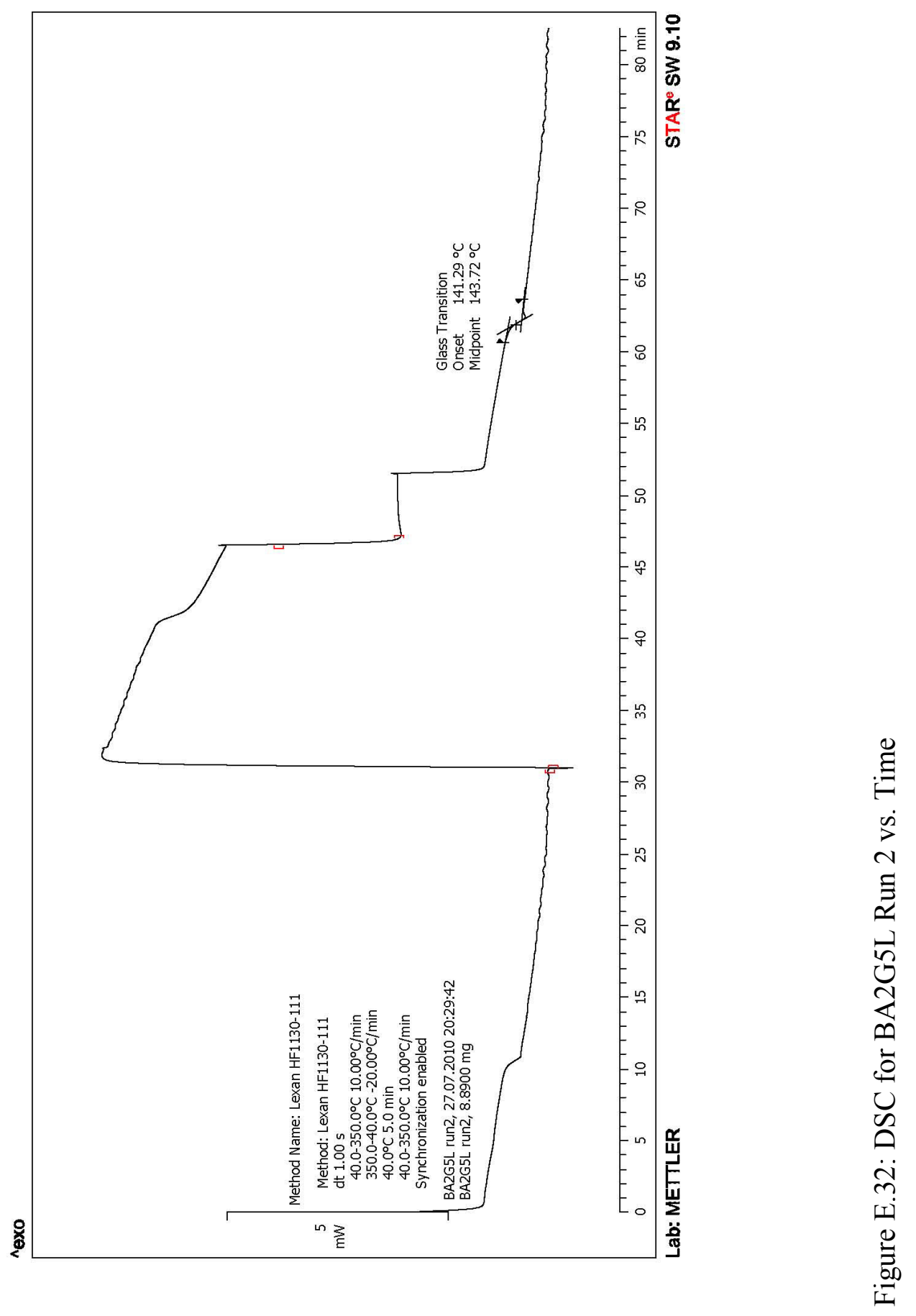




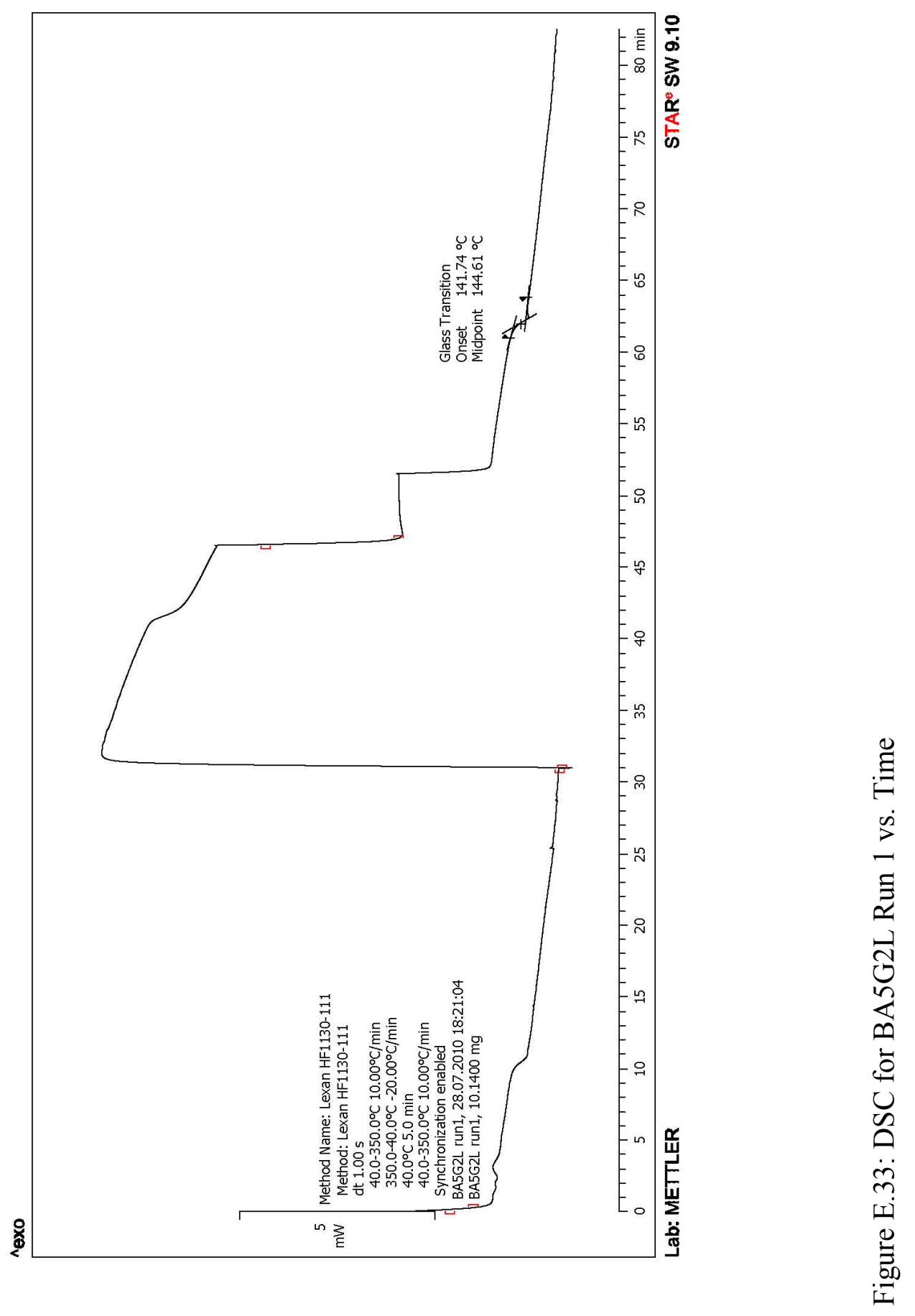




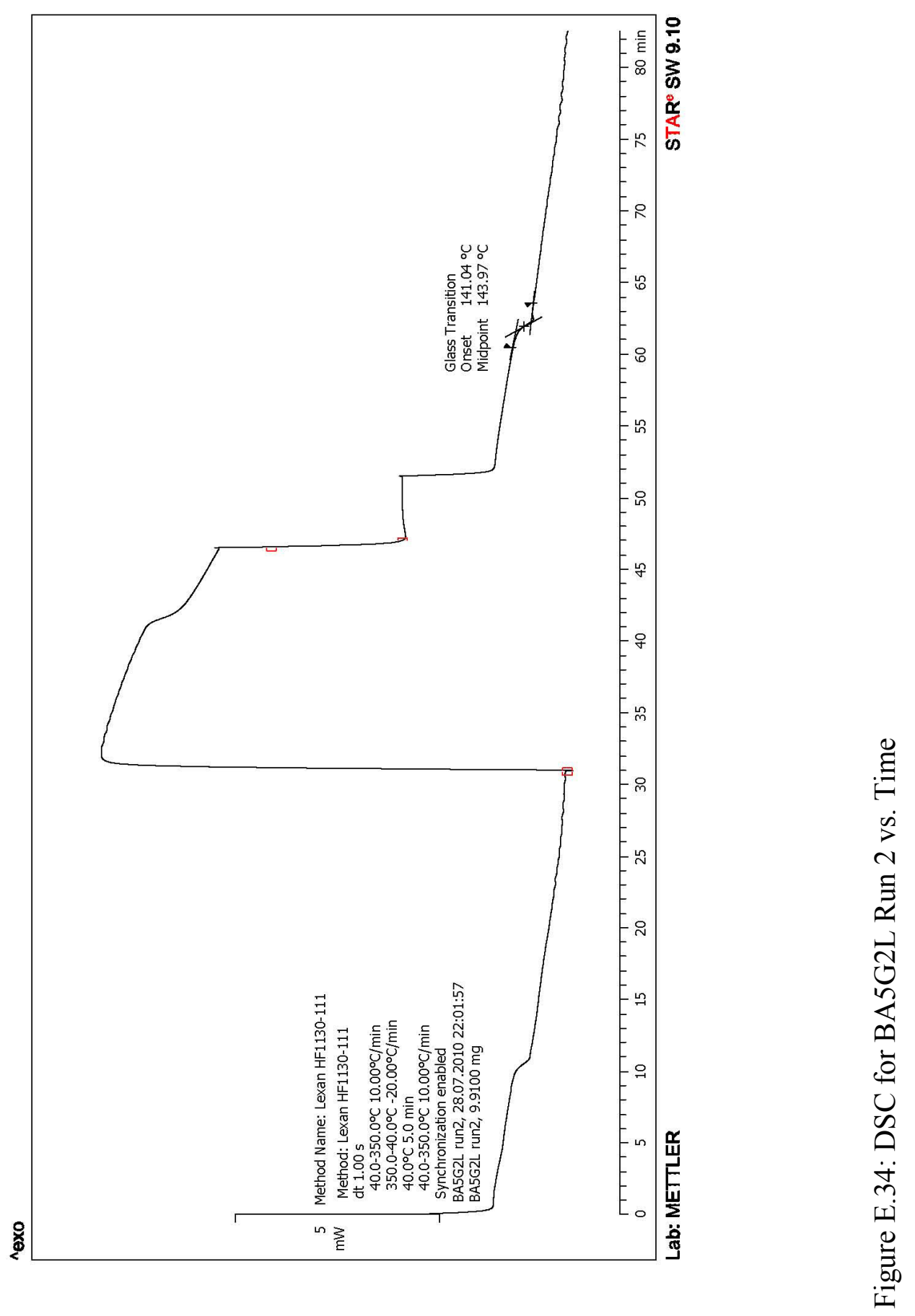




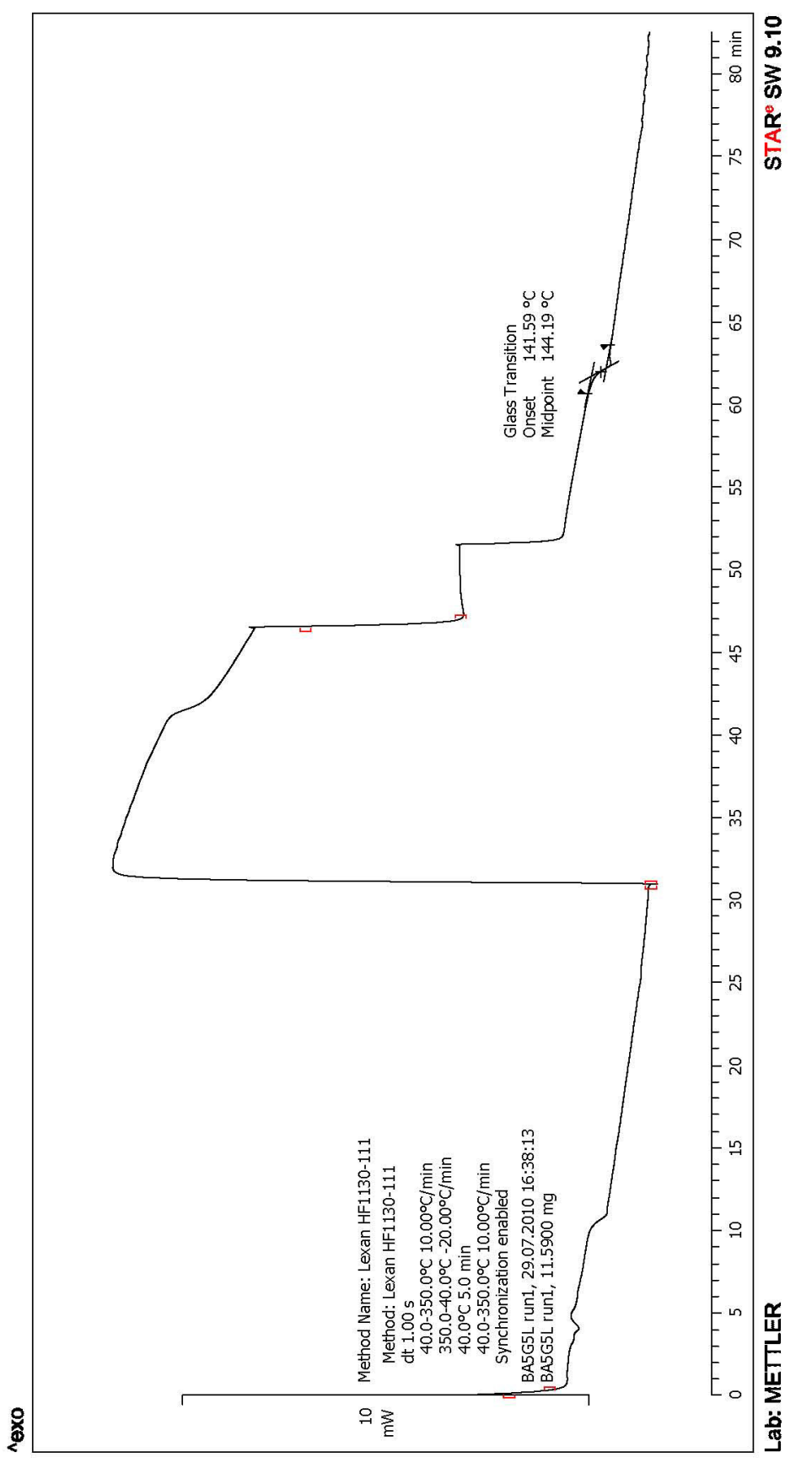

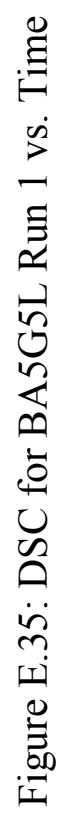




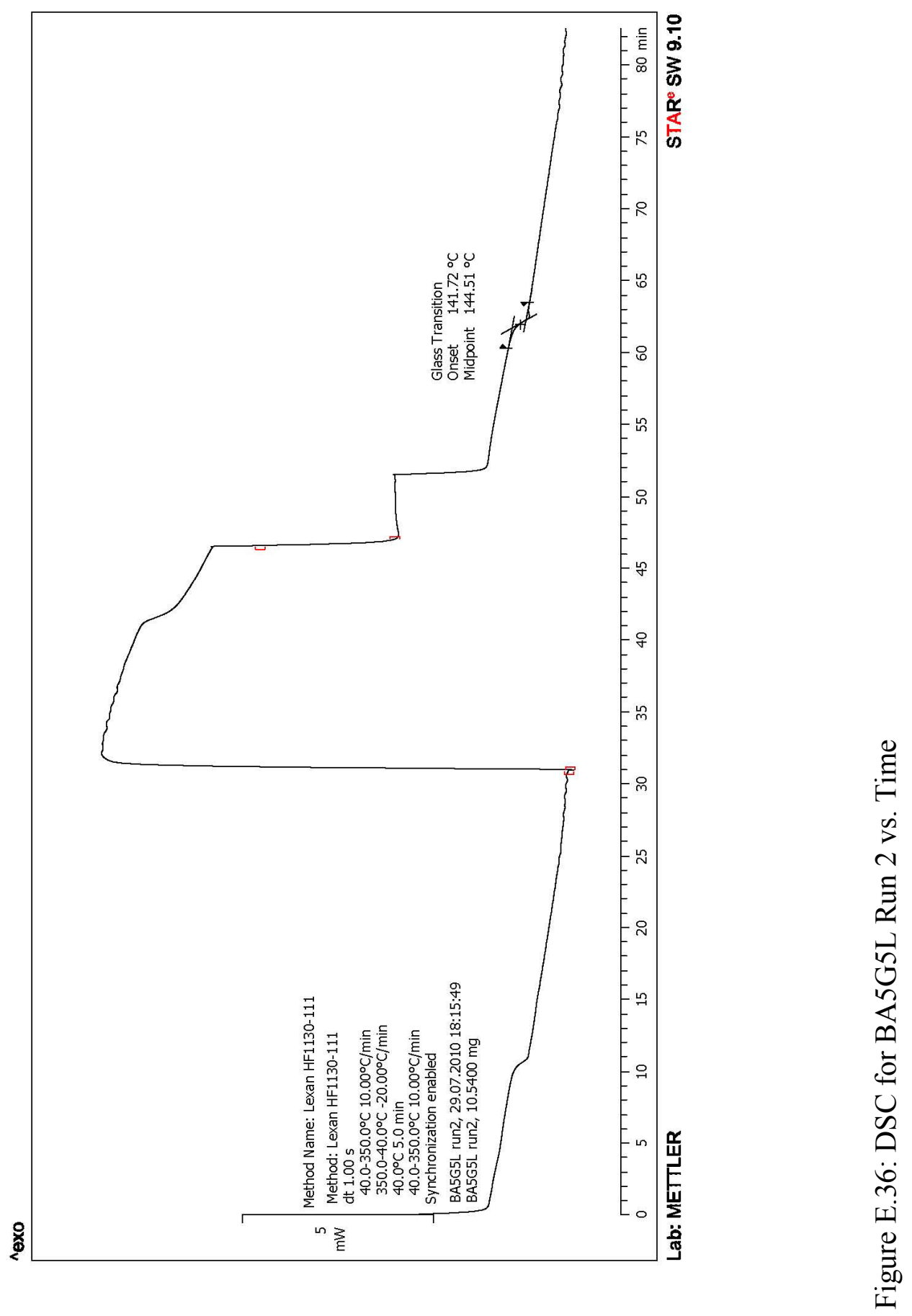




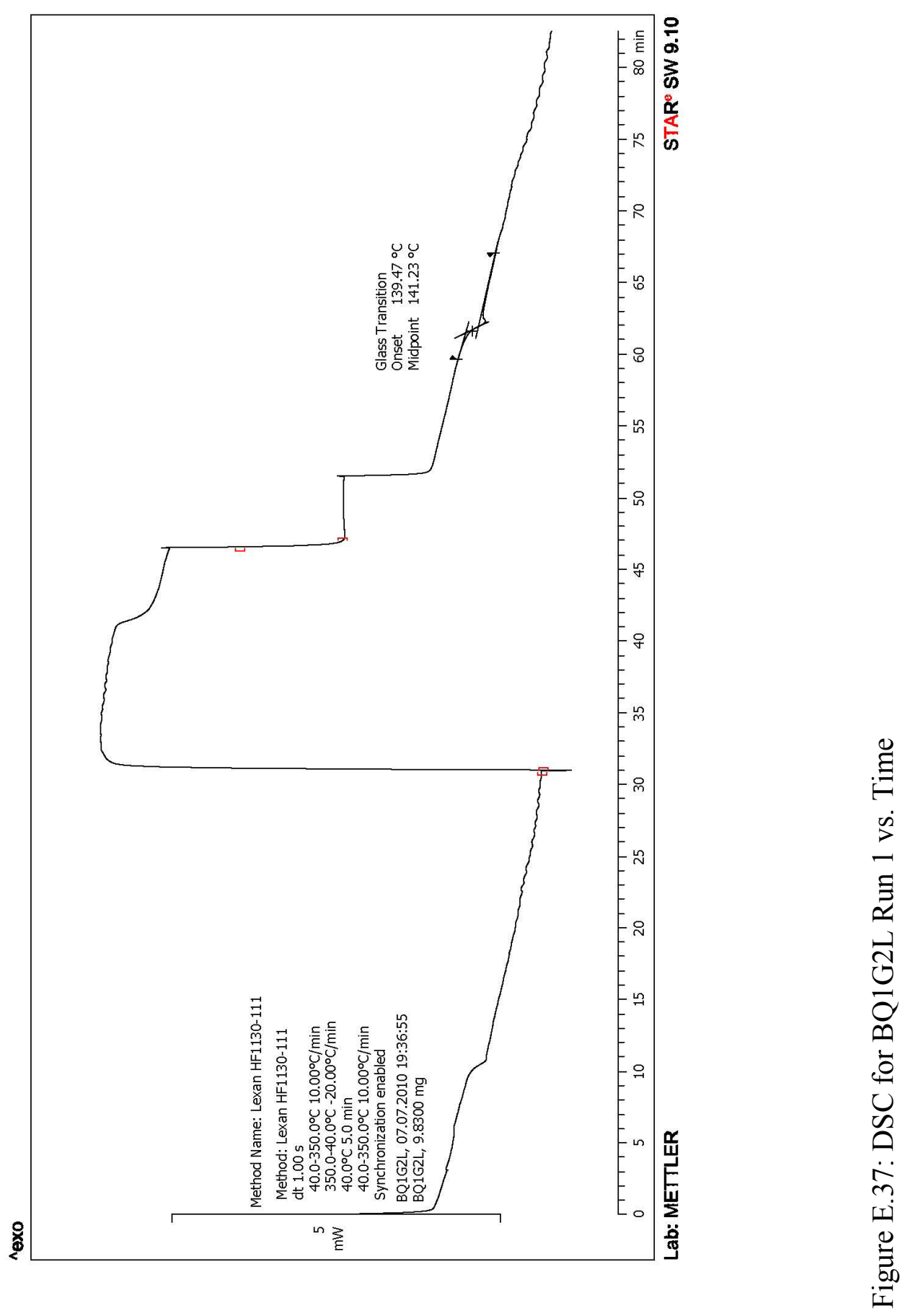




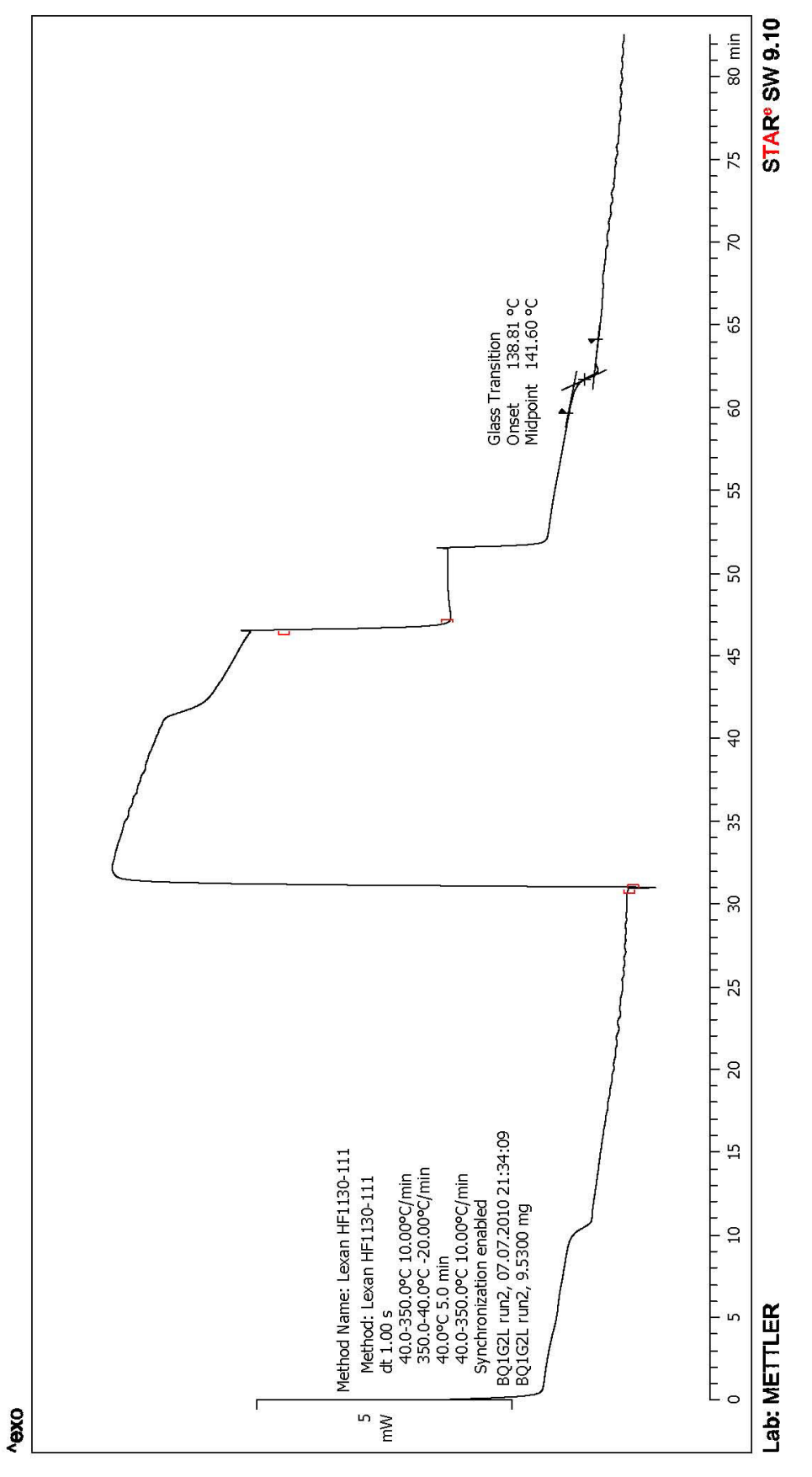

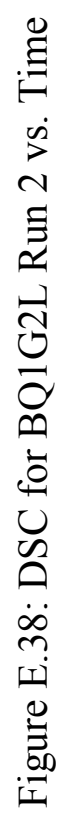




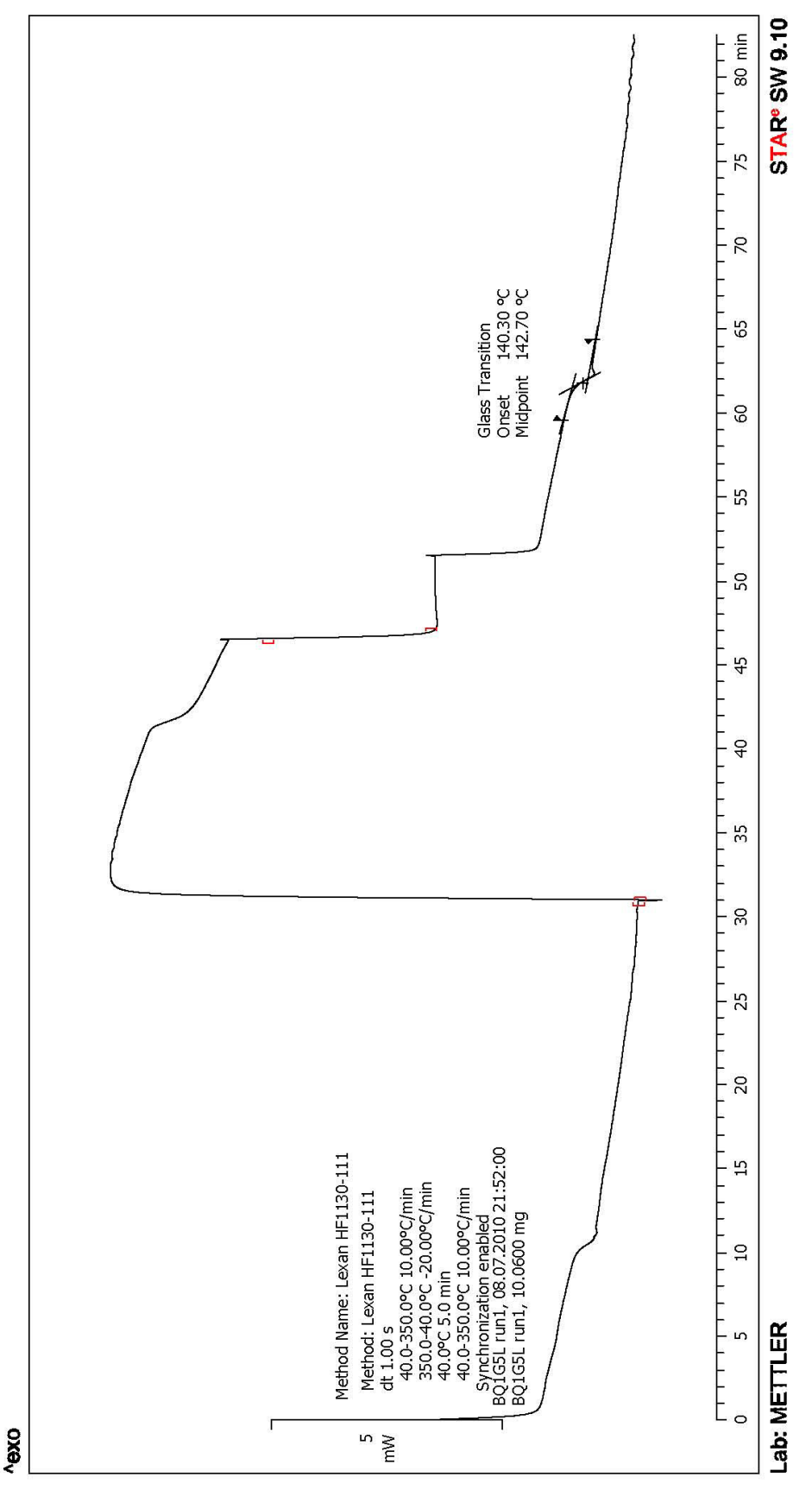

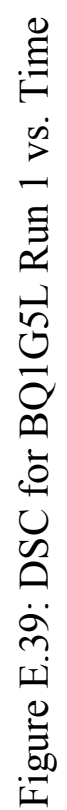




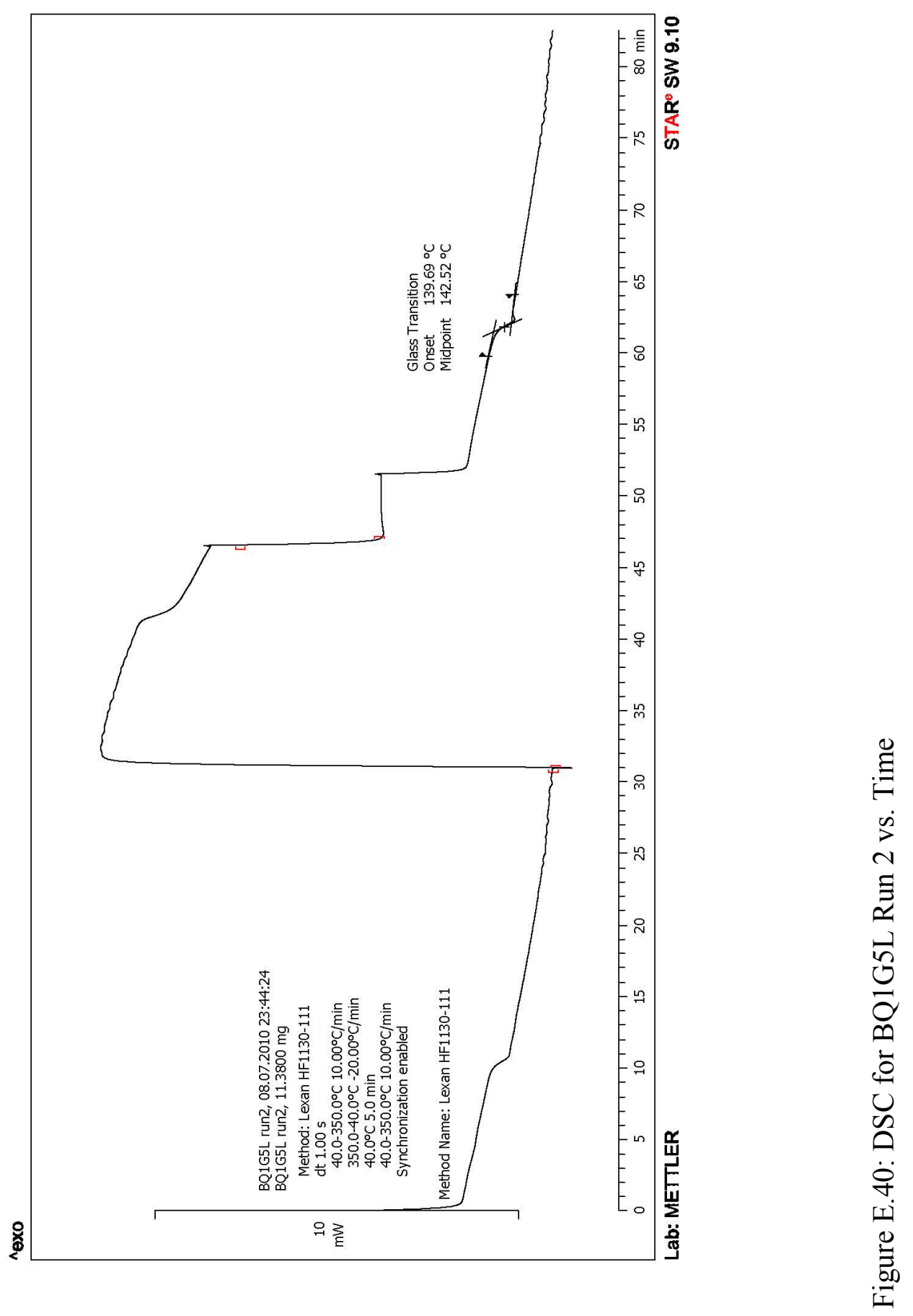




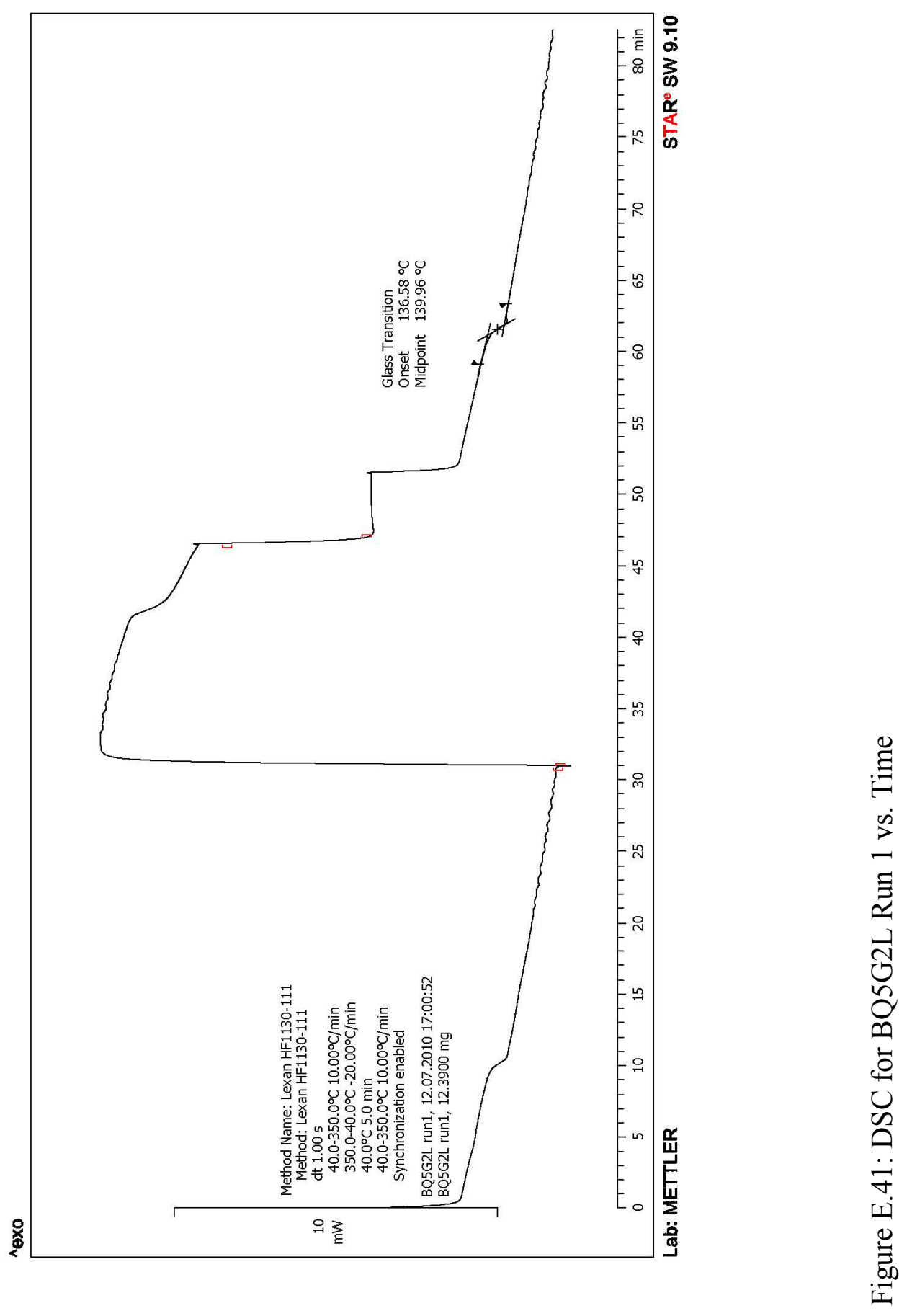




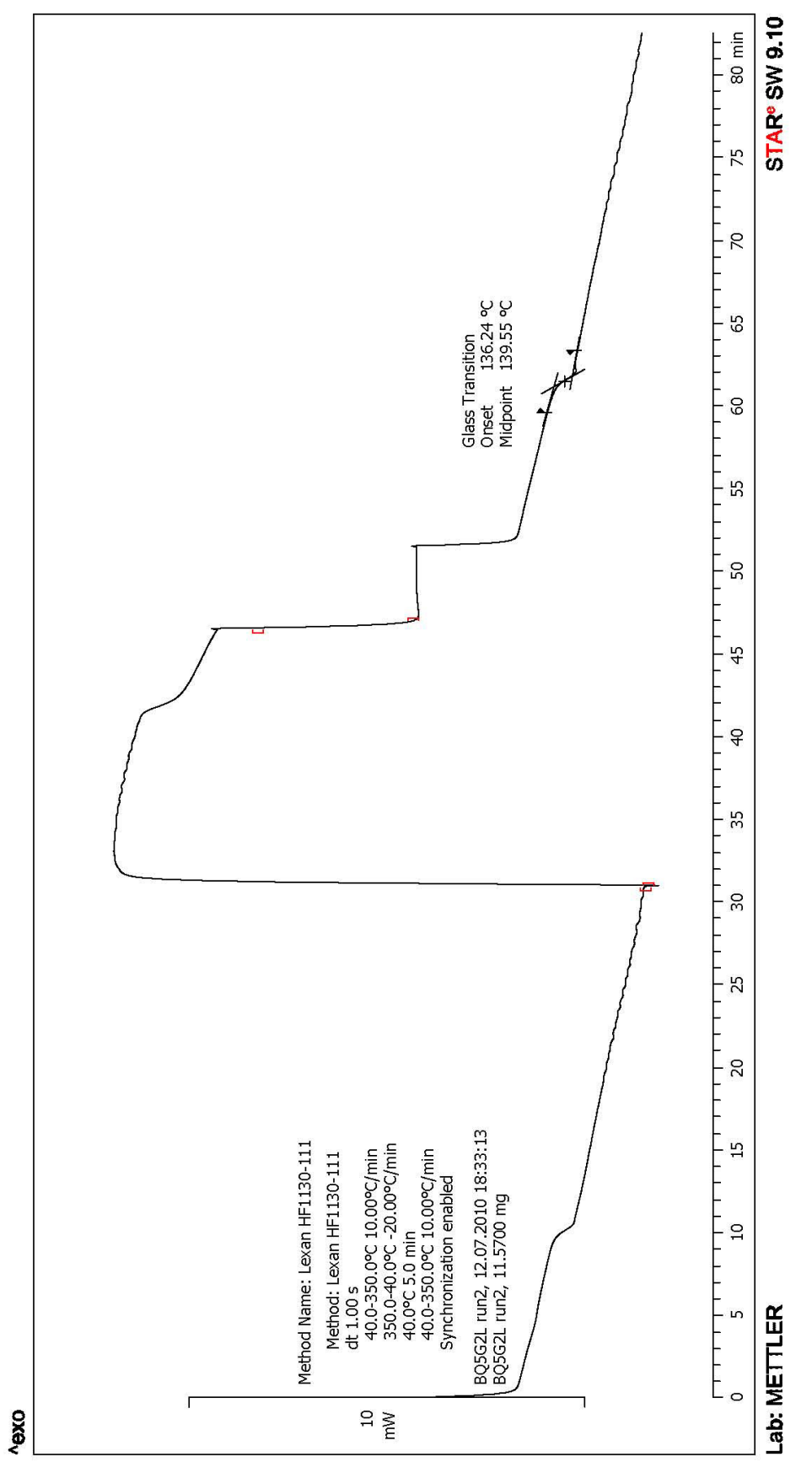

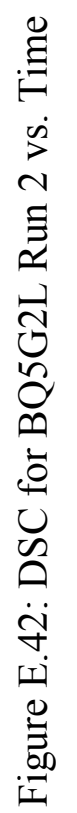




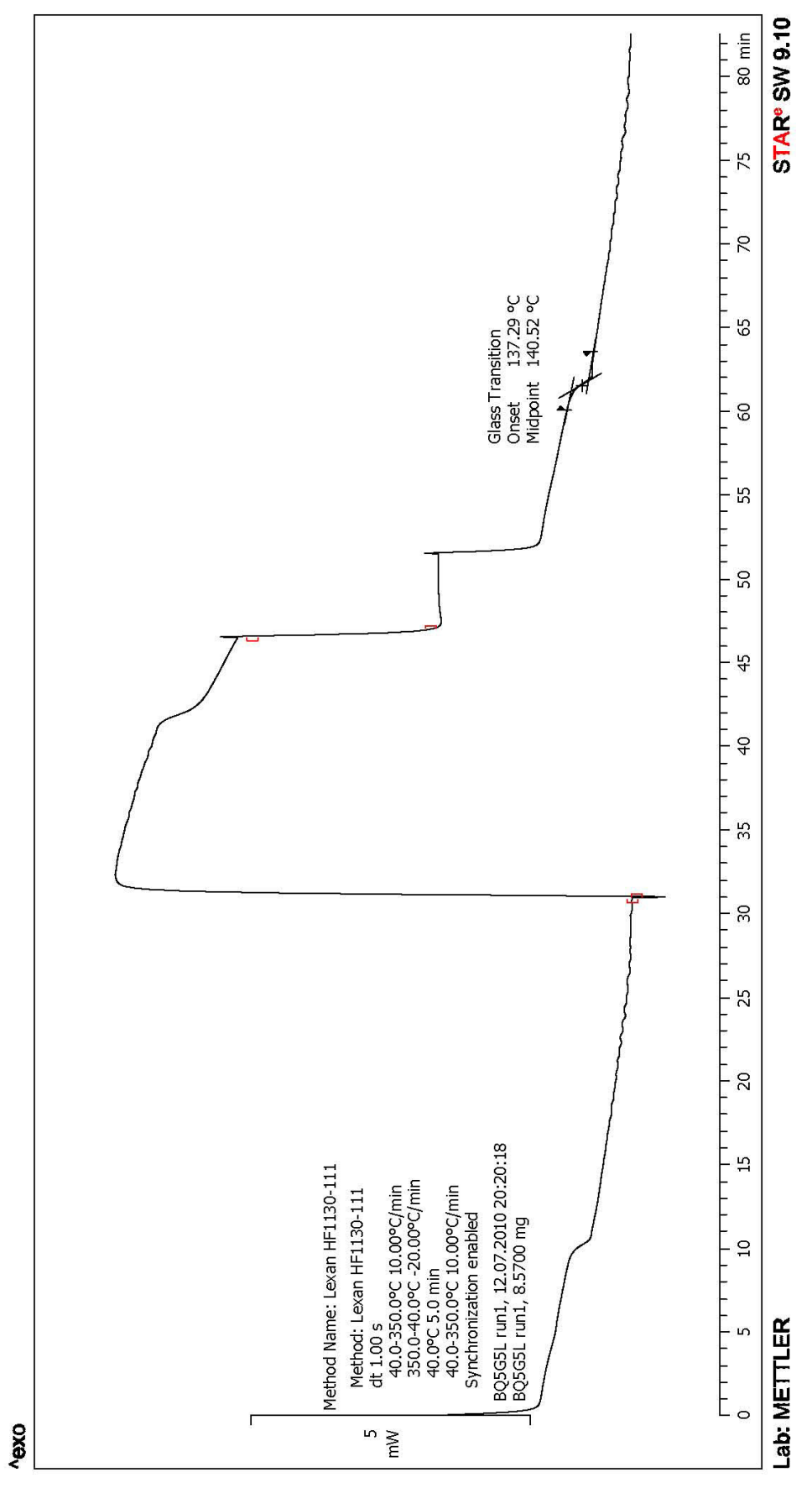

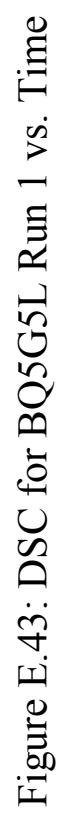




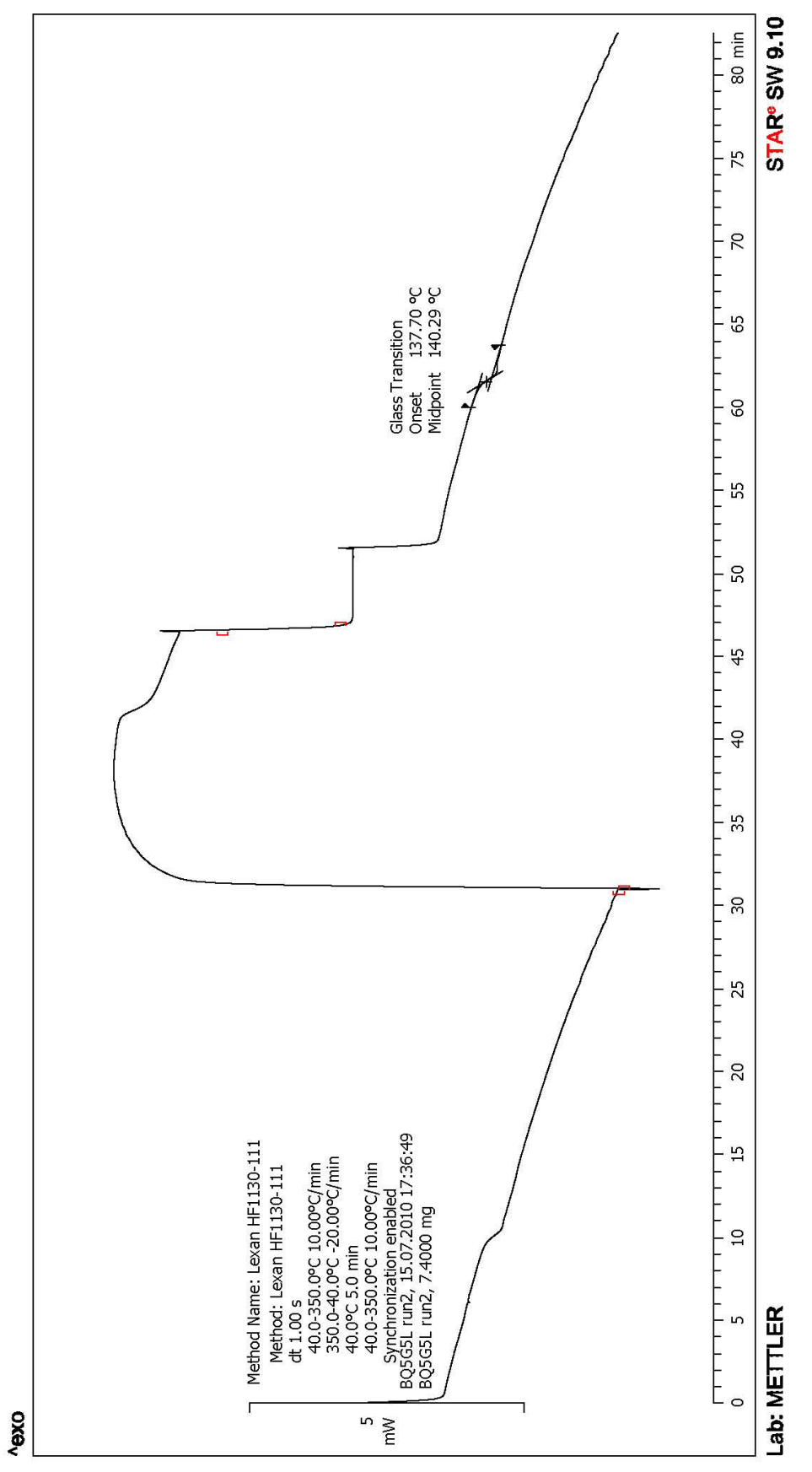

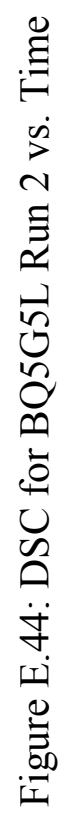




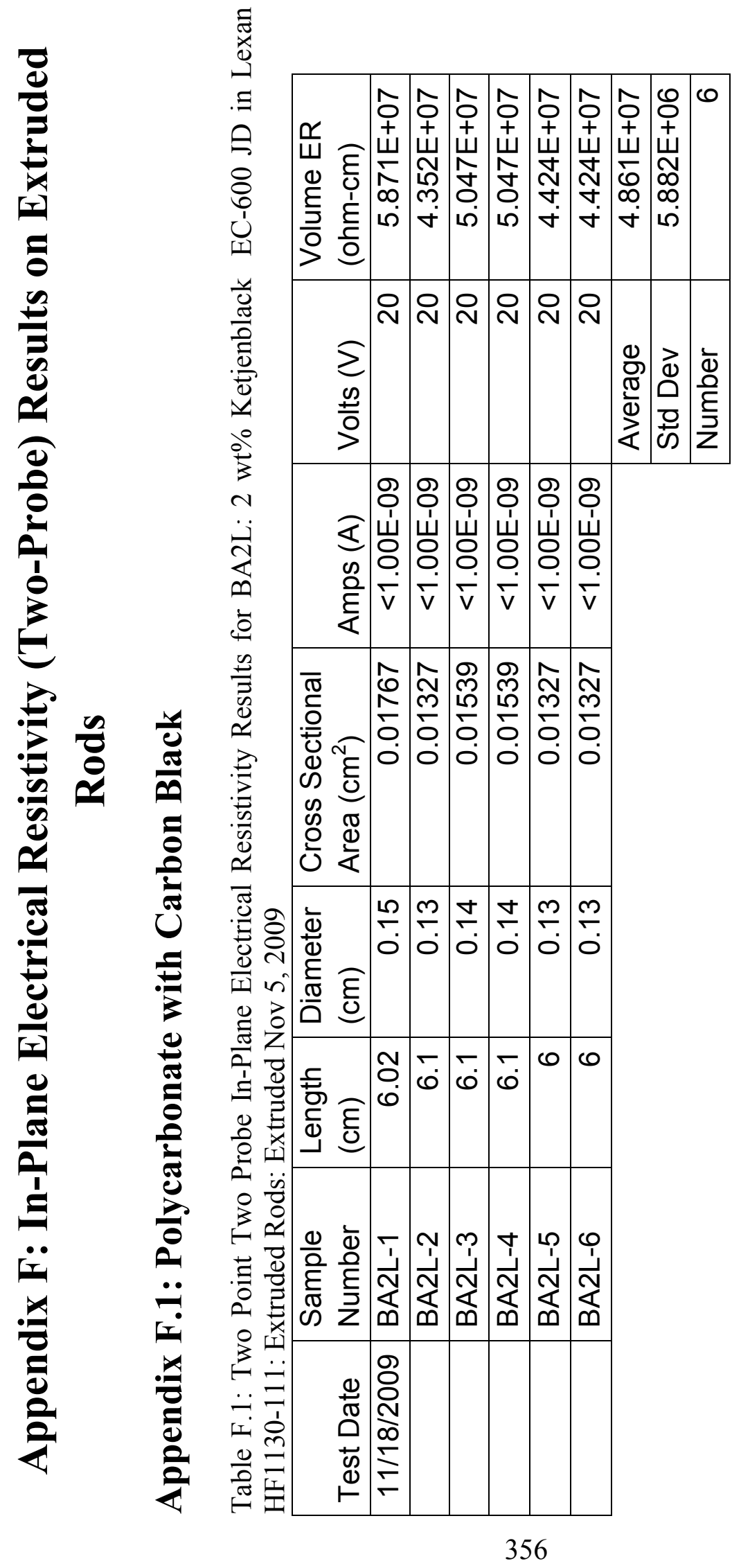




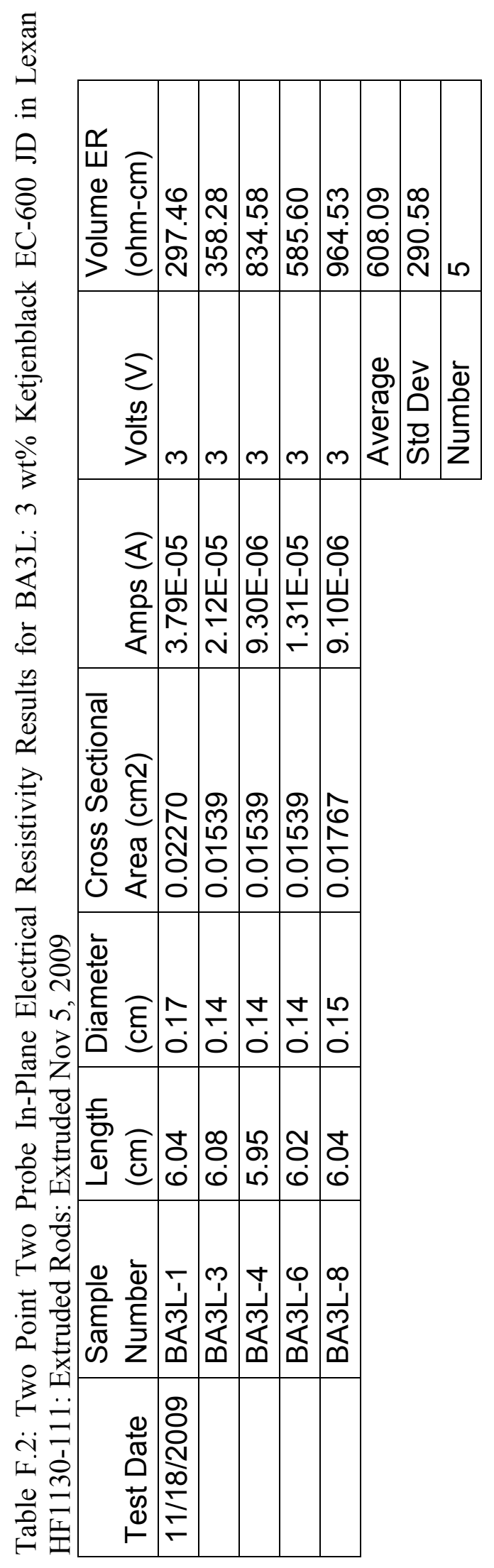




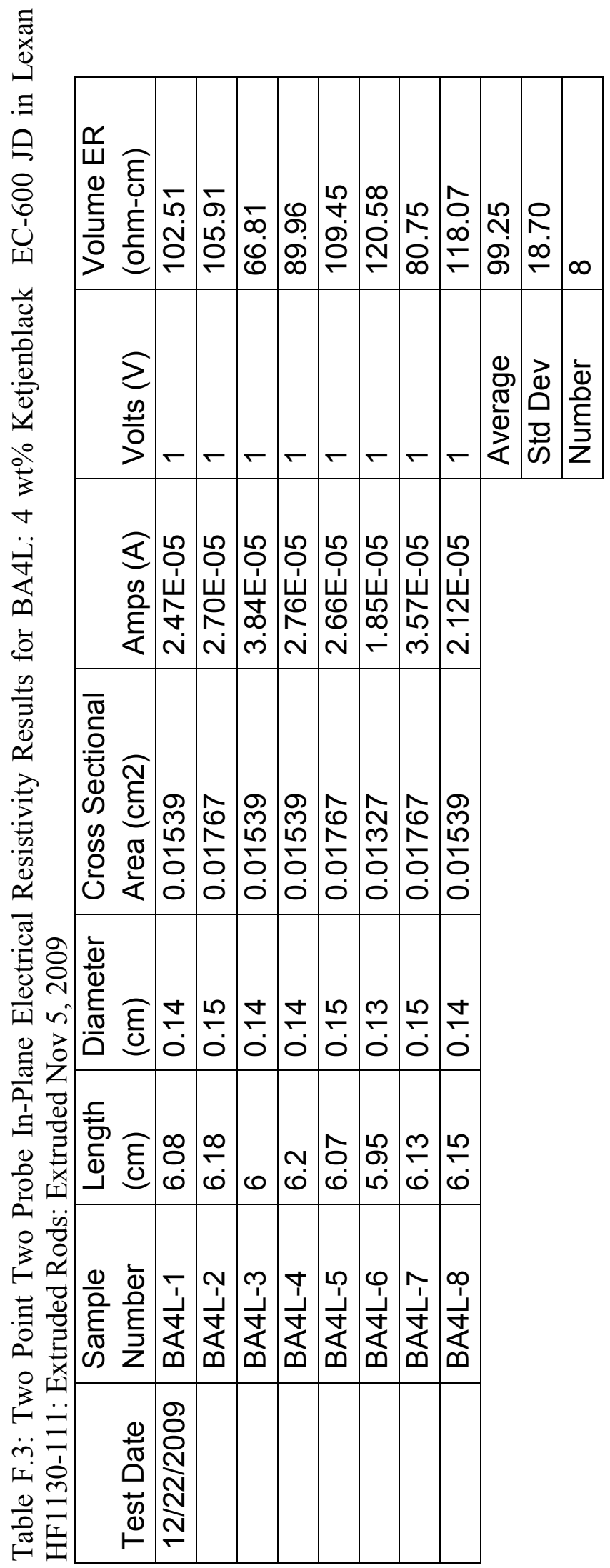




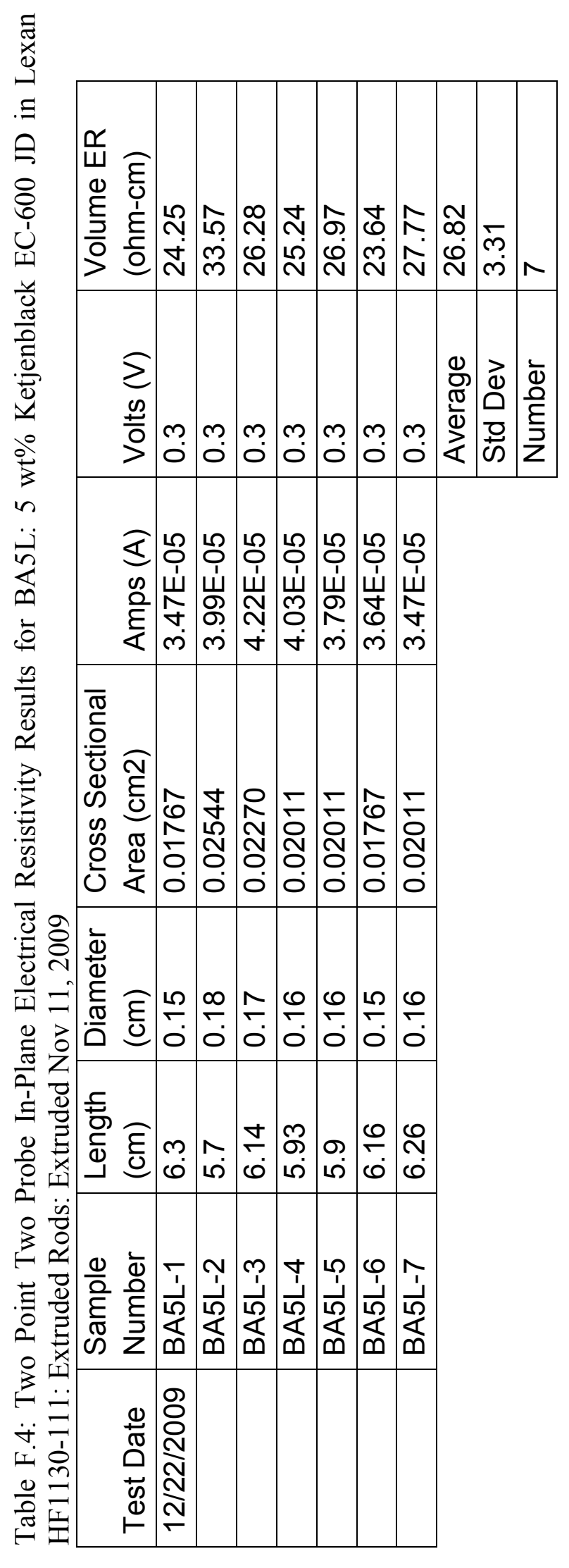




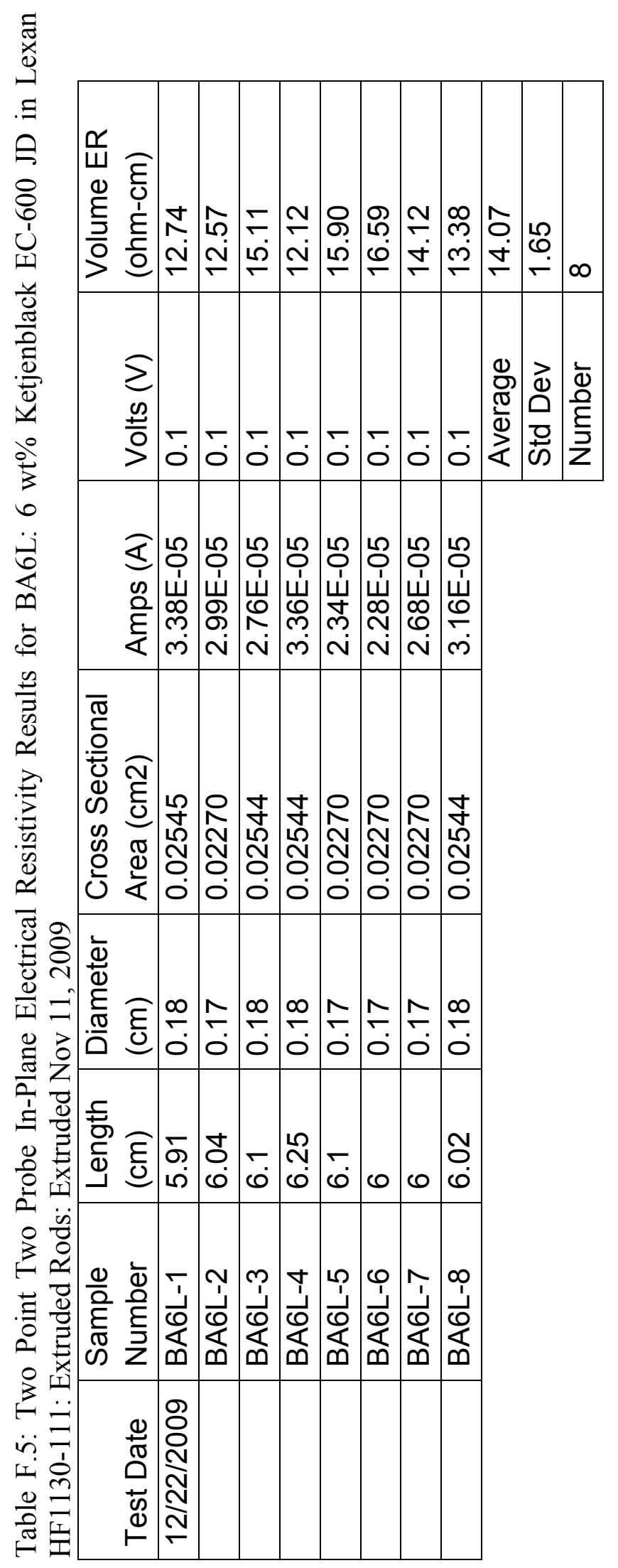




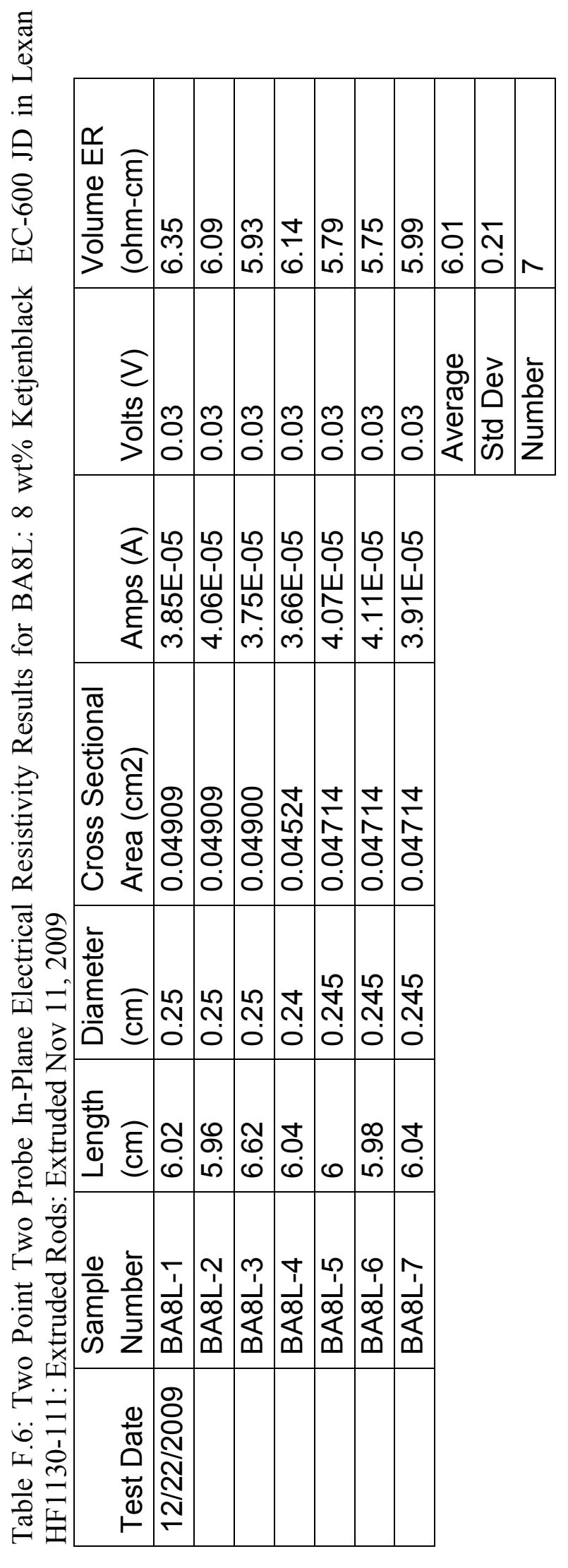




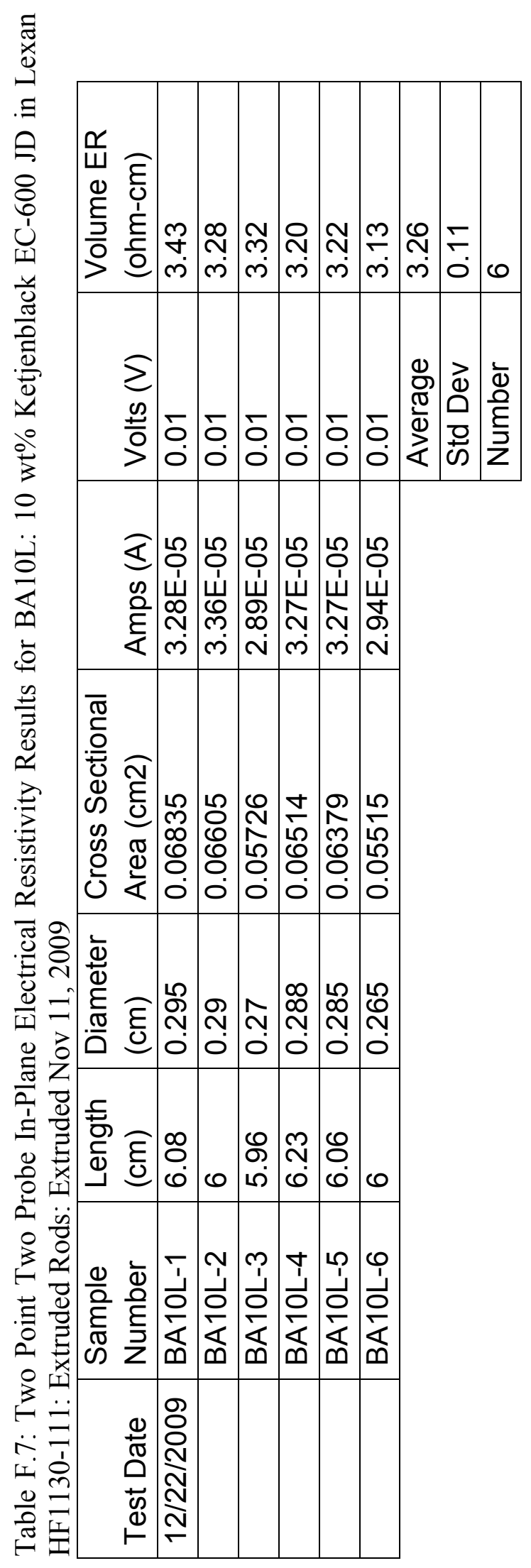




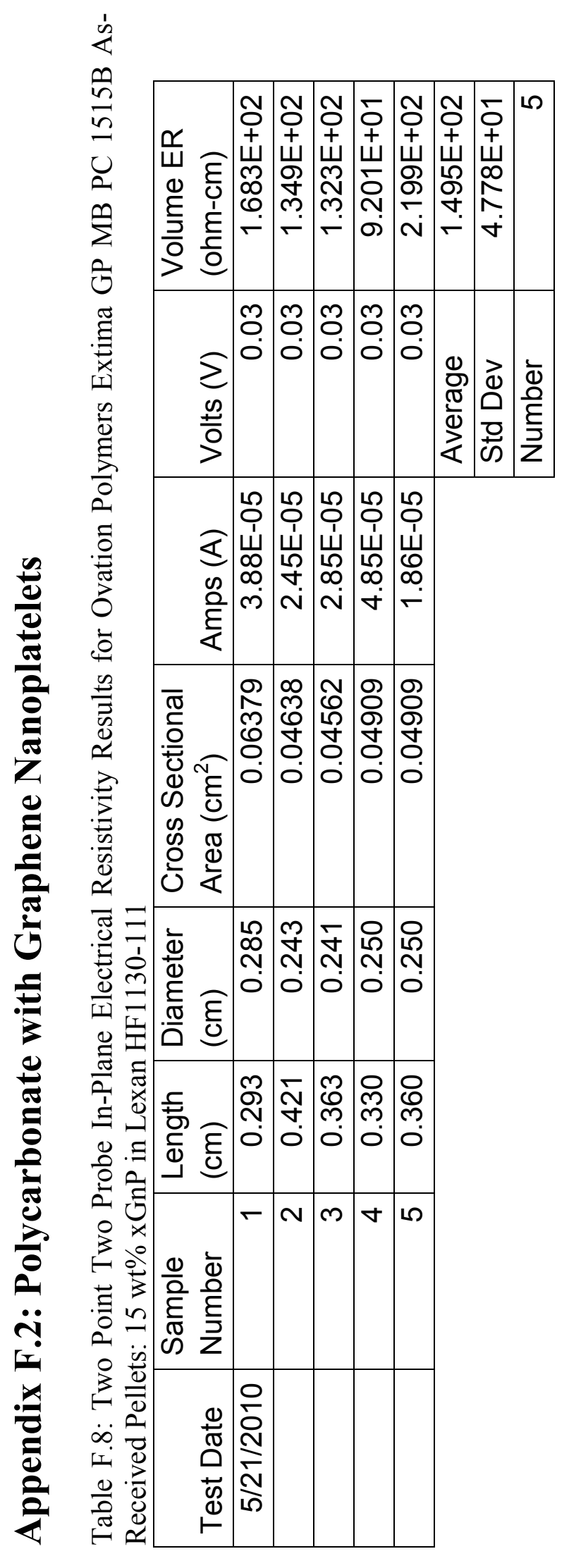




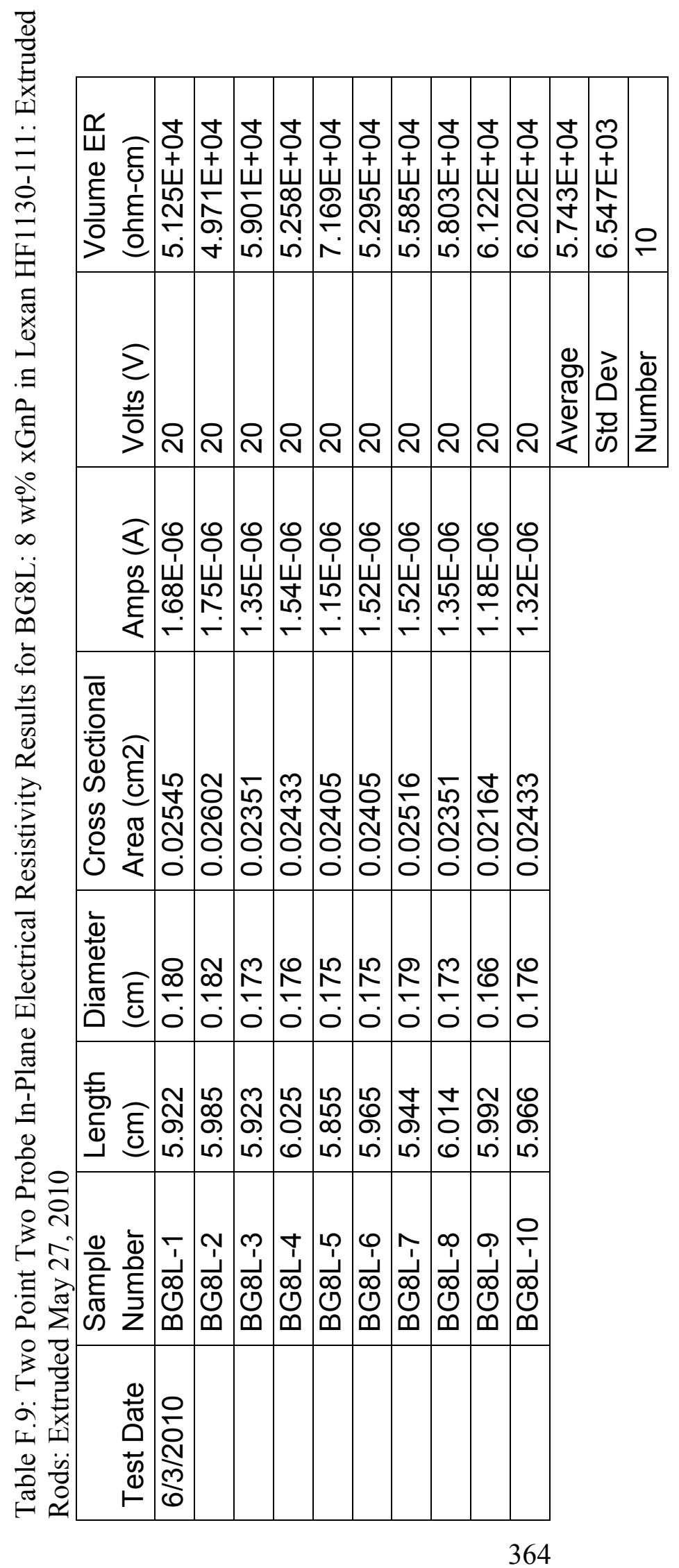




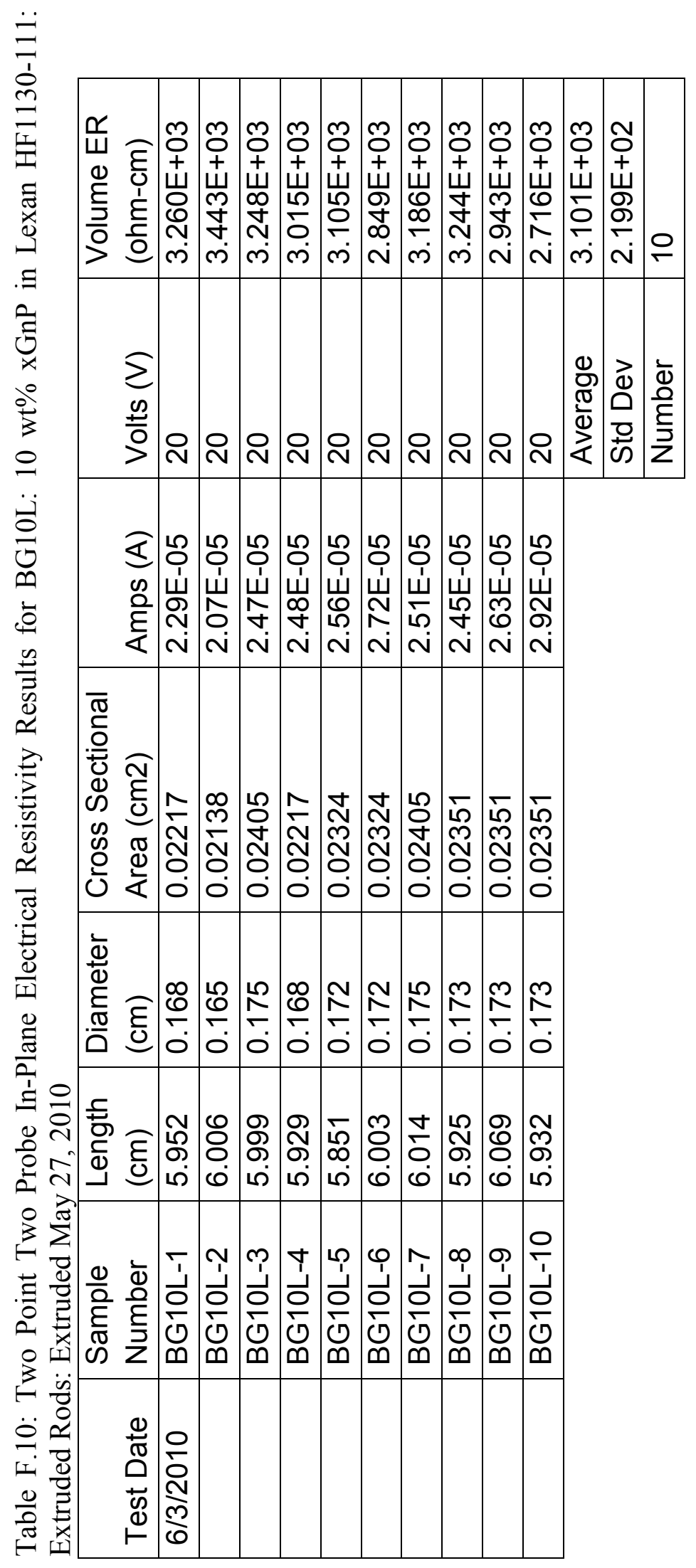




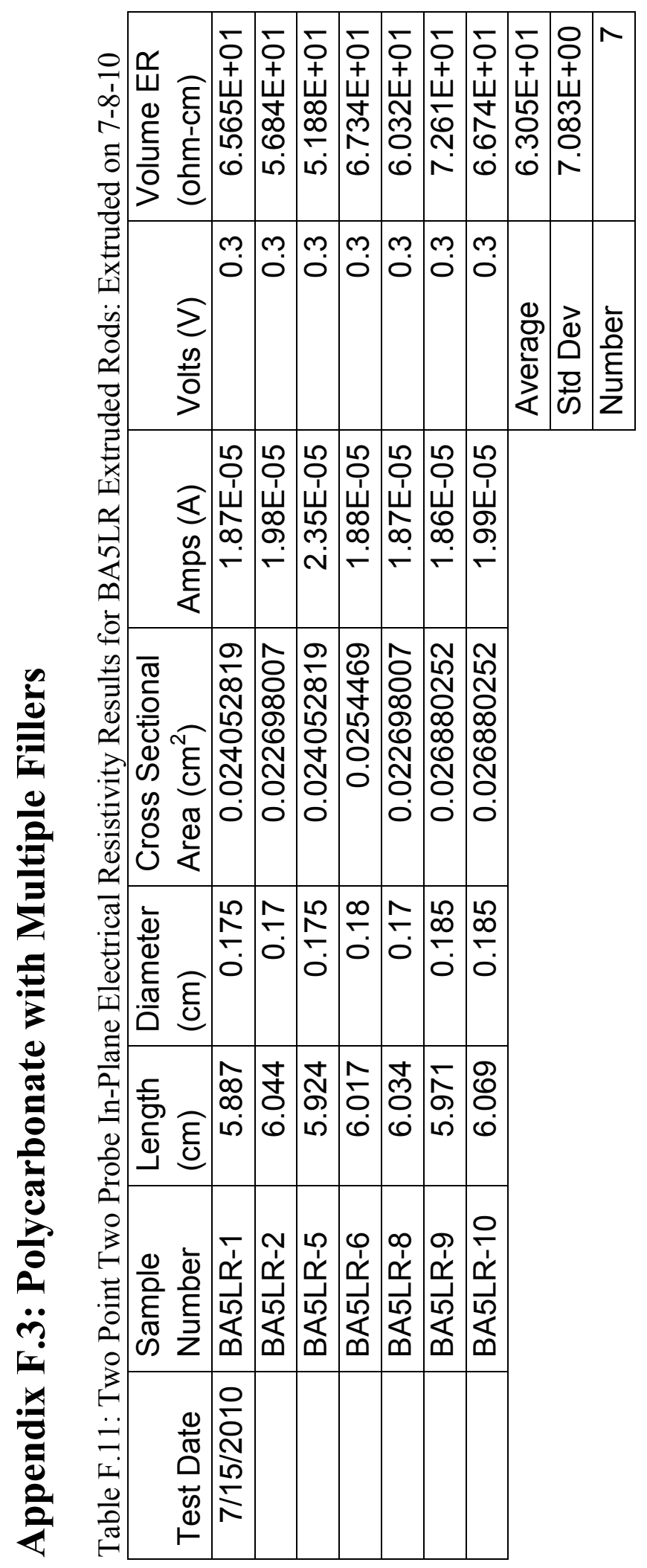




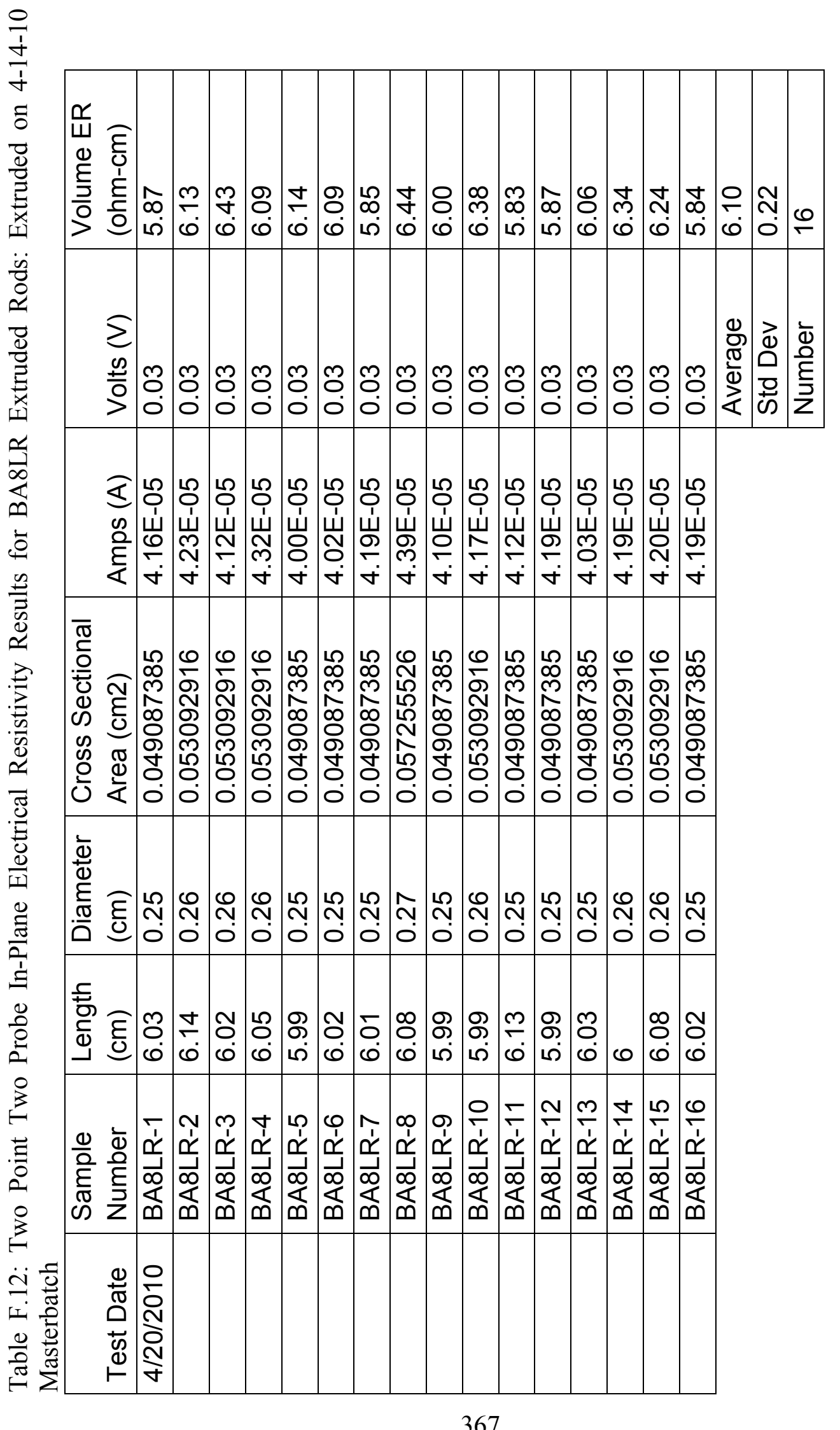




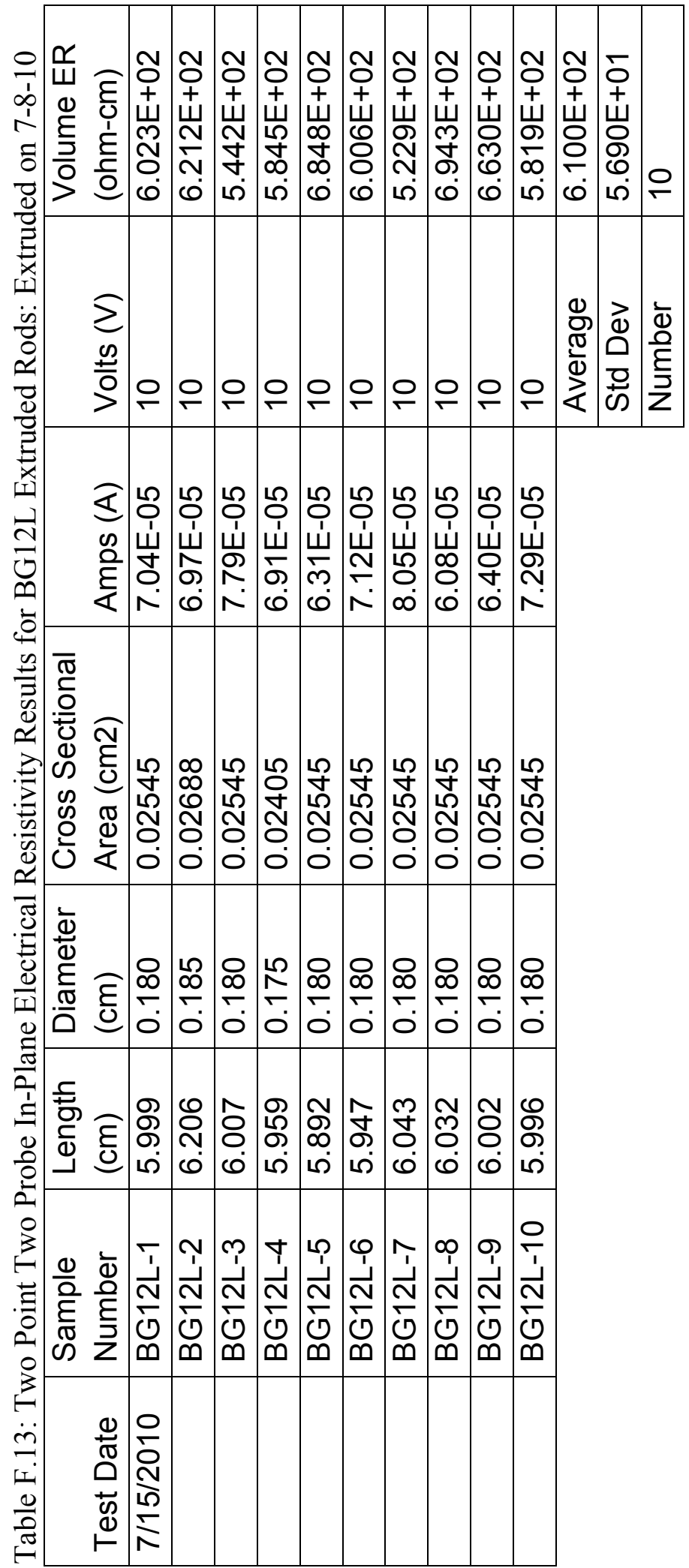




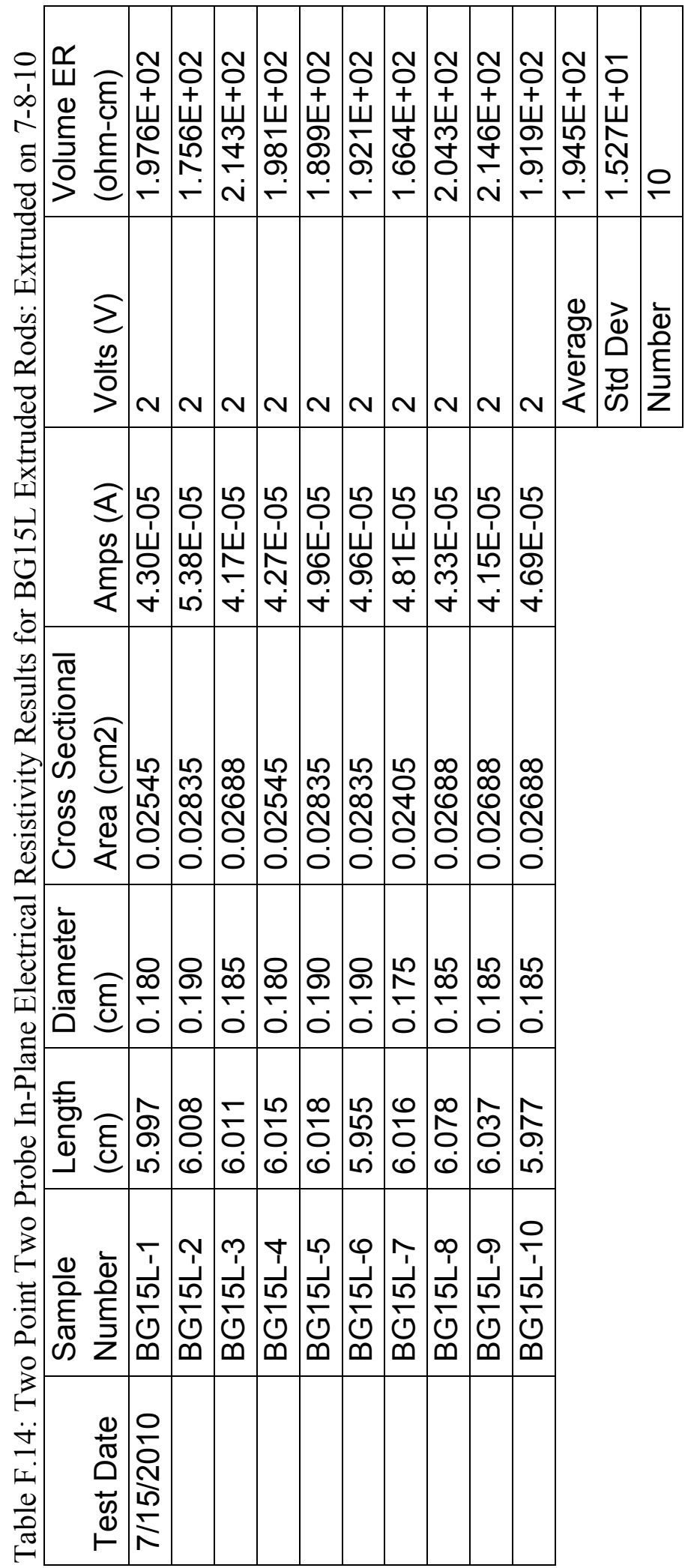



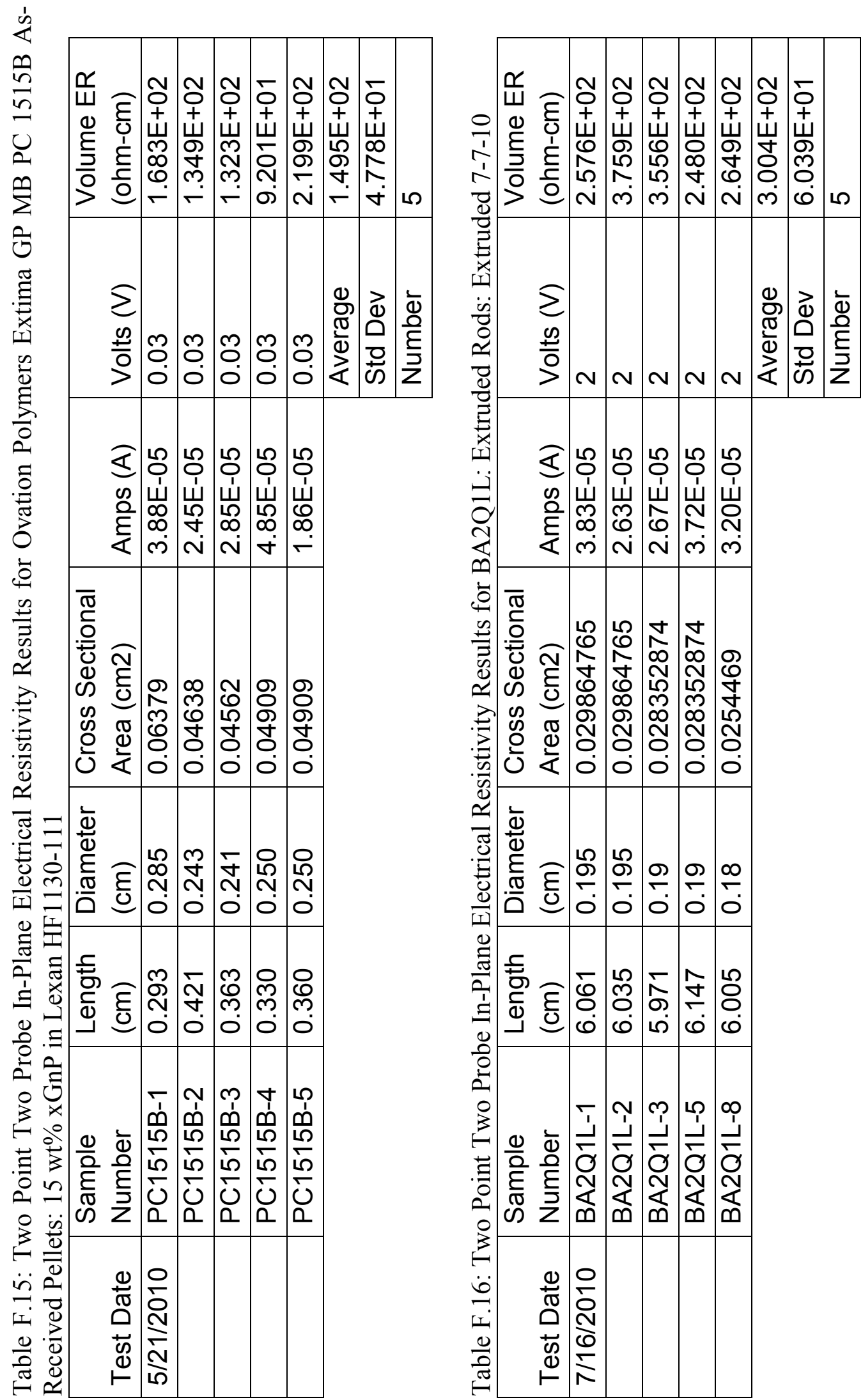


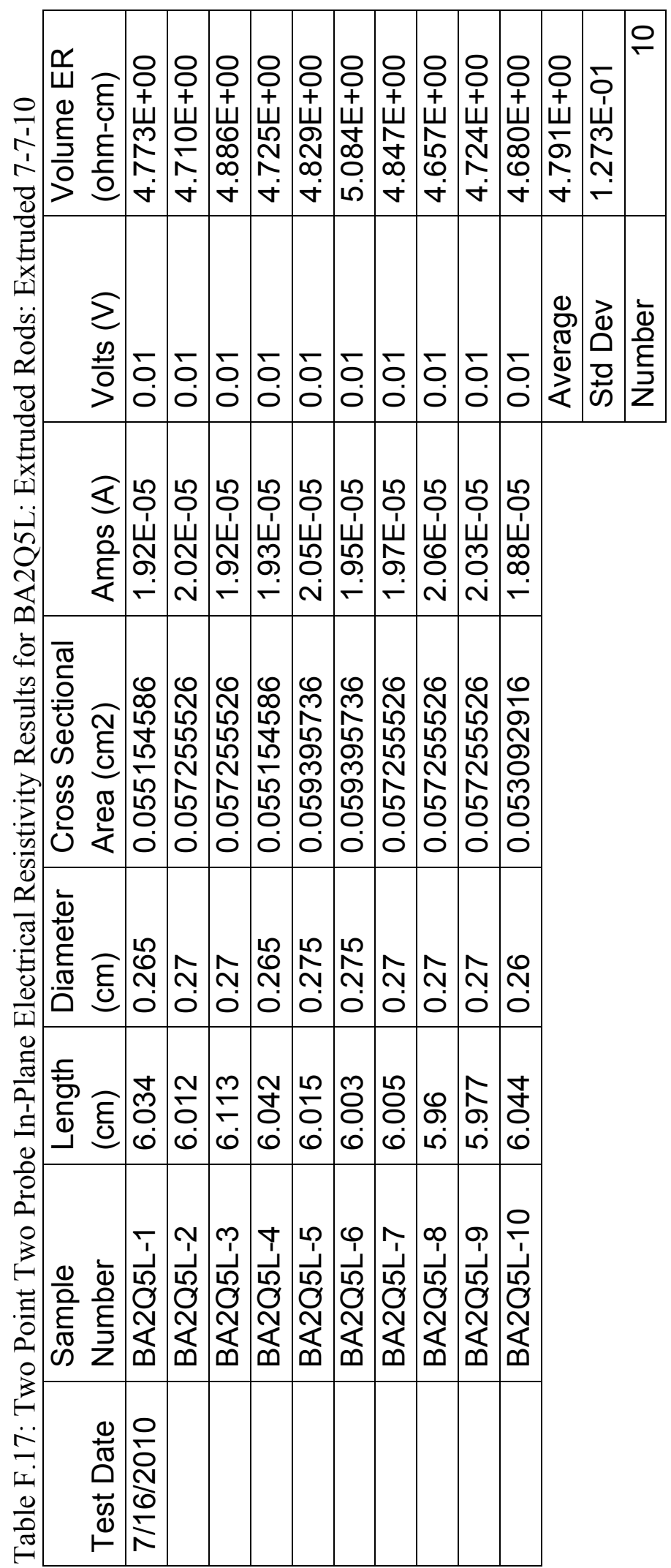




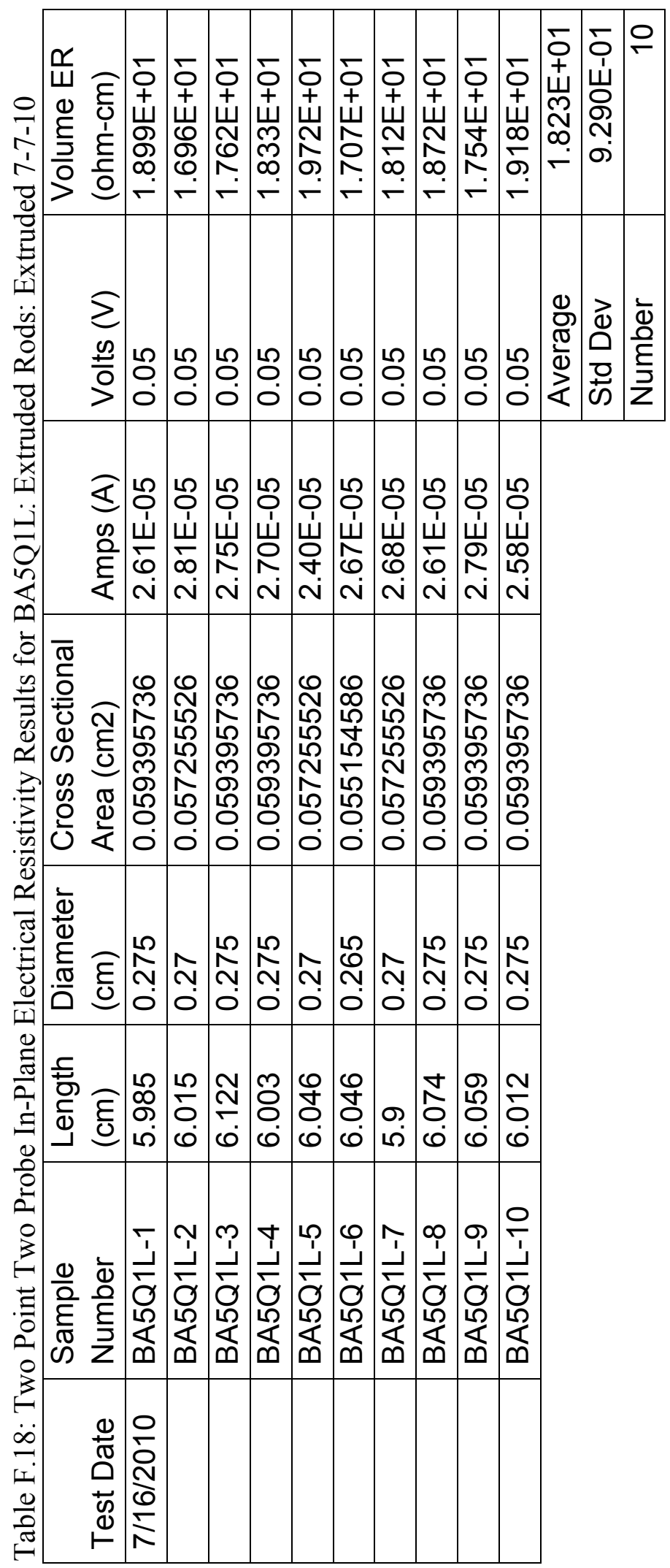




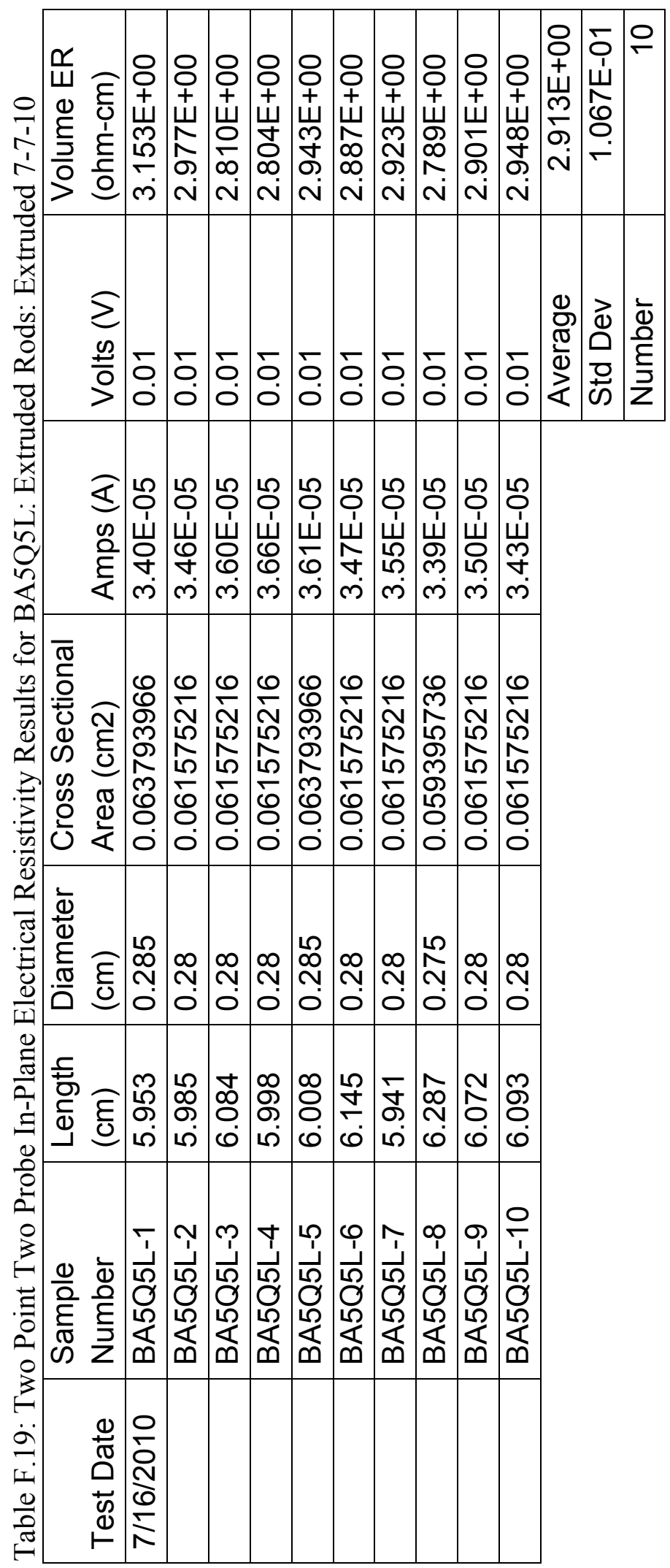




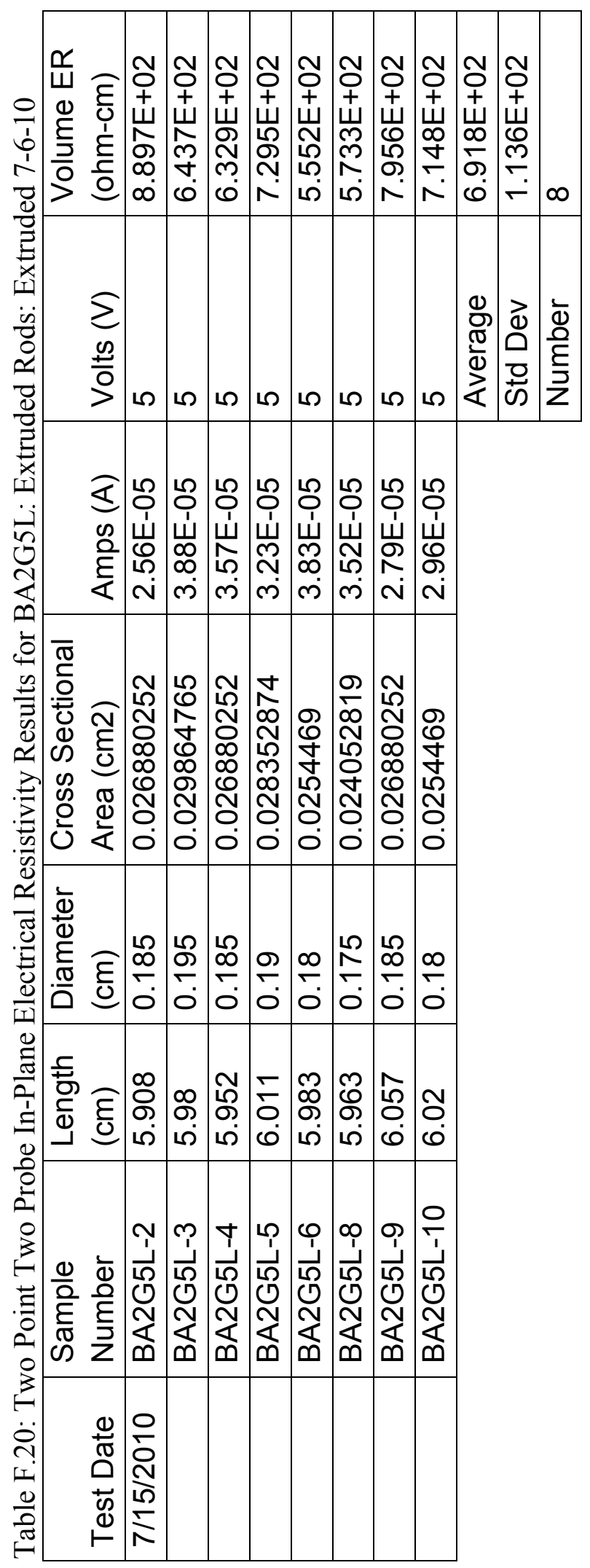




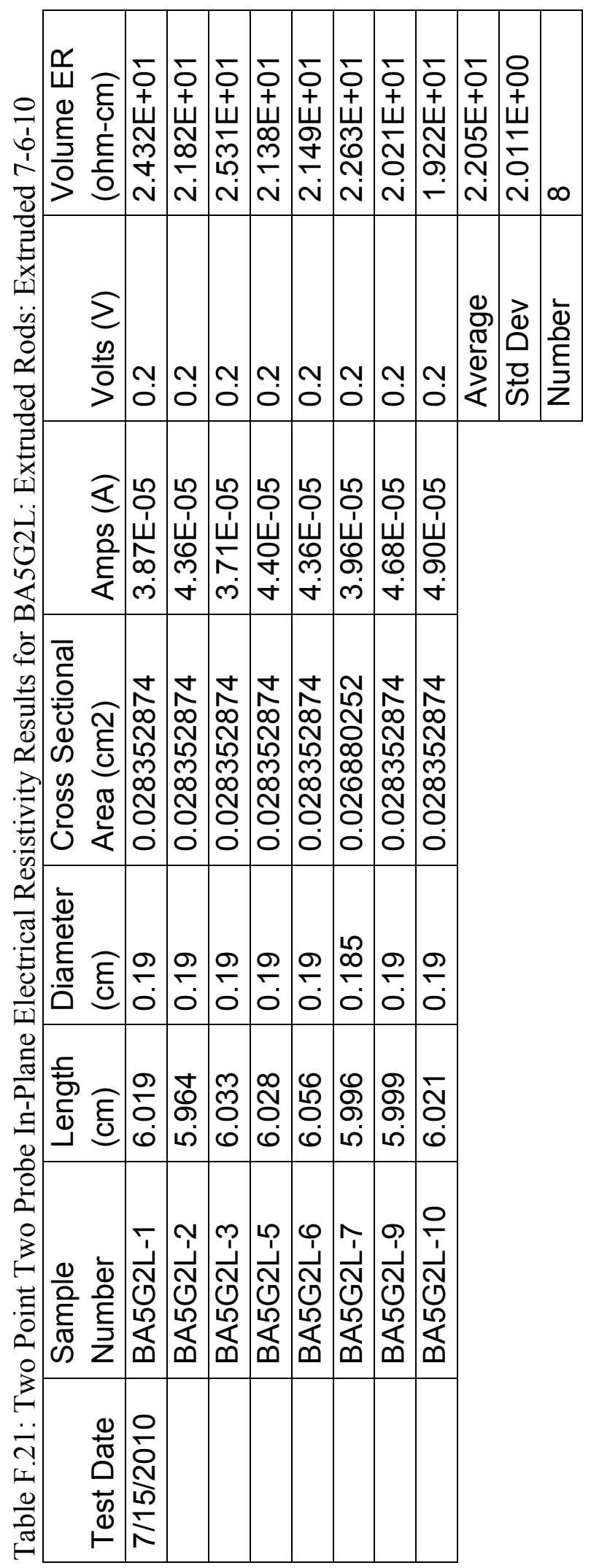




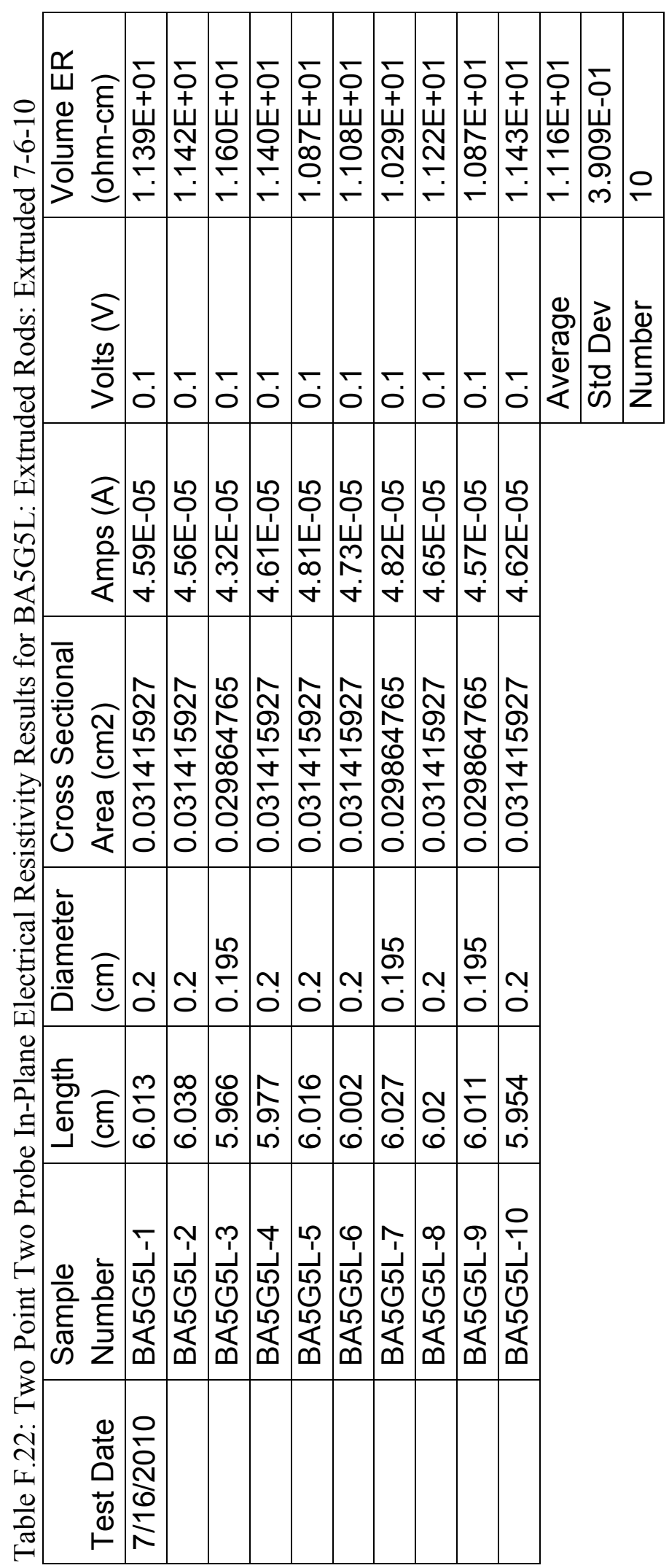




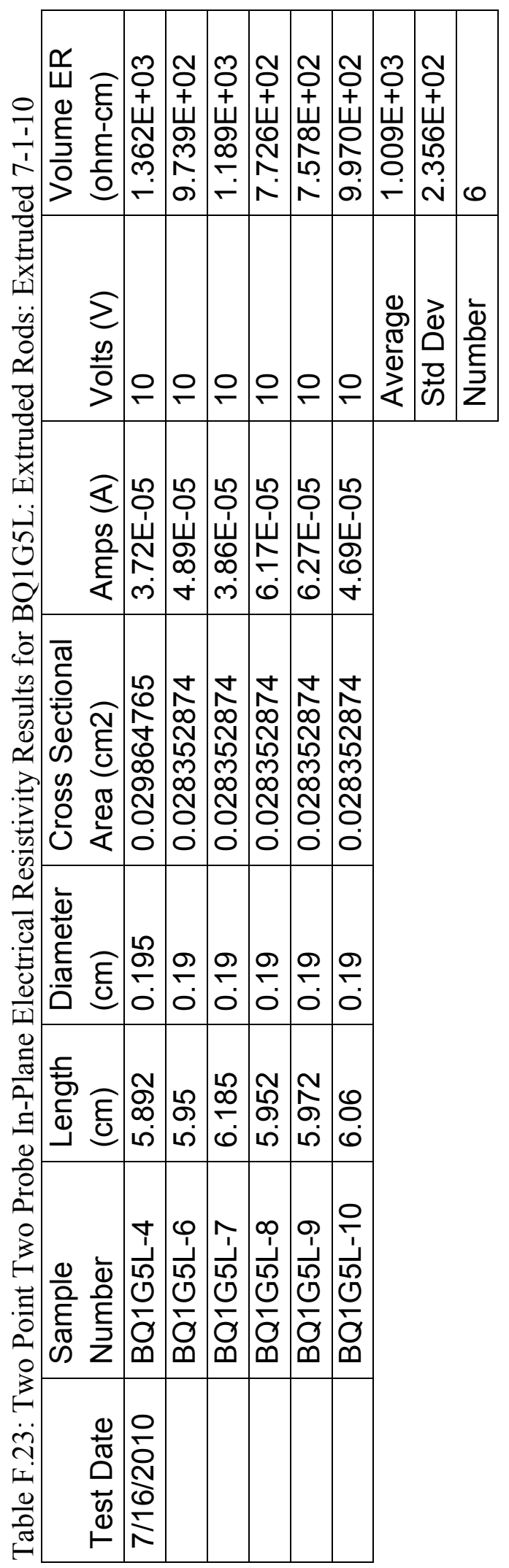




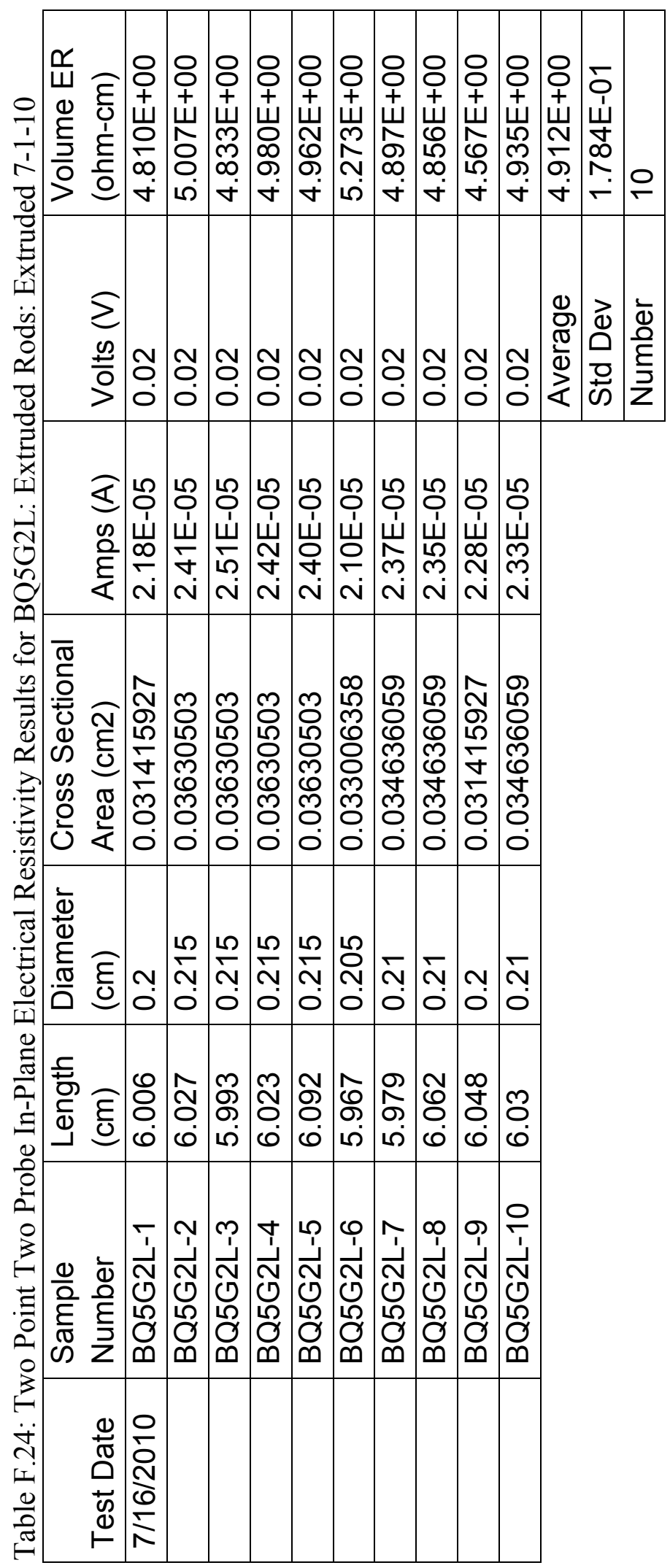




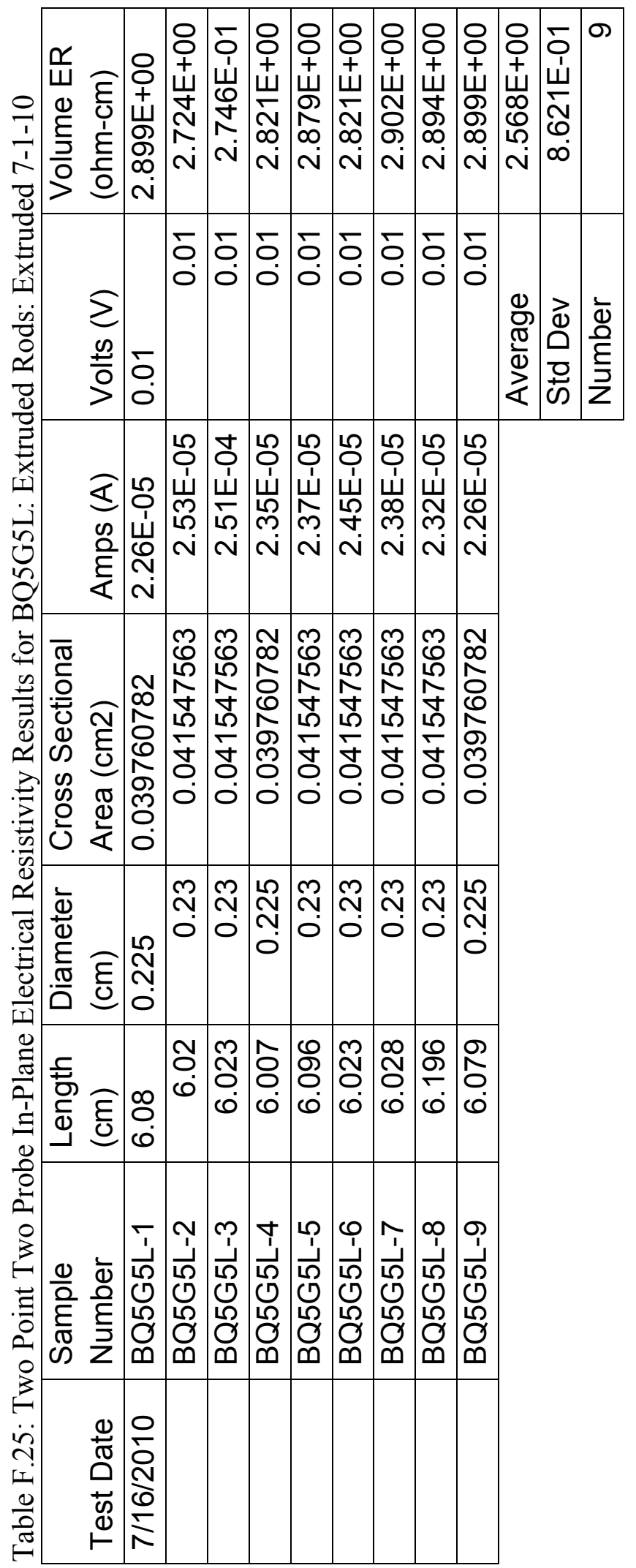




\section{Appendix G: Through Plane Electrical Resistivity (ASTM D257) Results}

\section{Appendix G.1: Polycarbonate with Carbon Nanotubes}

Table G.1: ASTM D257 Through Plane Electrical Resistivity Results for BL: Lexan HF1130-111: Injection Molded May 26, 2009

\begin{tabular}{|c|c|c|c|c|}
\hline Test Date & $\begin{array}{l}\text { Sample } \\
\text { Number }\end{array}$ & $\begin{array}{l}\text { Applied Voltage } \\
\text { (V) }\end{array}$ & $\begin{array}{l}\text { Through- } \\
\text { Plane } \\
\text { Surface } \\
\text { Electrical } \\
\text { Resistivity } \\
\text { ( } \Omega \text { /square) }\end{array}$ & $\begin{array}{l}\text { Through- } \\
\text { Plane } \\
\text { Volume } \\
\text { Electrical } \\
\text { Resistivity } \\
(\Omega-\mathrm{cm})\end{array}$ \\
\hline $6 / 10 / 2009$ & BL-TC-12 & 100 & $1.33 \mathrm{E}+16$ & $1.50 \mathrm{E}+16$ \\
\hline $6 / 10 / 2009$ & BL-TC-15 & 100 & $2.44 \mathrm{E}+16$ & $3.00 E+16$ \\
\hline $6 / 10 / 2009$ & BL-TC-17 & 100 & $1.53 \mathrm{E}+17$ & $1.79 \mathrm{E}+17$ \\
\hline $6 / 10 / 2009$ & BL-TC-21 & 100 & $4.26 \mathrm{E}+16$ & $5.38 E+16$ \\
\hline $6 / 19 / 2009$ & BL-TC-13 & 100 & $1.59 \mathrm{E}+17$ & $1.74 \mathrm{E}+17$ \\
\hline $6 / 19 / 2009$ & BL-TC-30 & 100 & $6.44 \mathrm{E}+16$ & $7.96 \mathrm{E}+16$ \\
\hline $6 / 10 / 2009$ & BL-TC-31 & 100 & 1.67E+17 & $2.09 \mathrm{E}+17$ \\
\hline & & Average & $8.918 E+16$ & 1.057E+17 \\
\hline & & $\begin{array}{l}\text { Standard } \\
\text { Deviation }\end{array}$ & $6.806 E+16$ & $7.963 E+16$ \\
\hline & & $\begin{array}{l}\text { Number of } \\
\text { Samples }\end{array}$ & 7 & 7 \\
\hline
\end{tabular}


Table G.2: ASTM D257 Through Plane Electrical Resistivity Results for BLE: Extruded Lexan HF1130-111: Injection Molded May 26, 2009

\begin{tabular}{|c|c|c|c|c|}
\hline Test Date & $\begin{array}{l}\text { Sample } \\
\text { Number }\end{array}$ & $\begin{array}{l}\text { Applied Voltage } \\
\text { (V) }\end{array}$ & $\begin{array}{l}\text { Through- } \\
\text { Plane } \\
\text { Surface } \\
\text { Electrical } \\
\text { Resistivity } \\
\text { ( } \Omega \text { /square) }\end{array}$ & $\begin{array}{l}\text { Through- } \\
\text { Plane } \\
\text { Volume } \\
\text { Electrical } \\
\text { Resistivity } \\
(\Omega-\mathrm{cm})\end{array}$ \\
\hline $6 / 10 / 2009$ & BLE-TC-13 & 100 & $1.94 \mathrm{E}+16$ & $2.39 \mathrm{E}+16$ \\
\hline $6 / 11 / 2009$ & BLE-TC-16 & 100 & $6.78 \mathrm{E}+16$ & $8.48 \mathrm{E}+16$ \\
\hline $6 / 11 / 2009$ & BLE-TC-17 & 100 & $4.88 \mathrm{E}+16$ & $5.92 \mathrm{E}+16$ \\
\hline $6 / 11 / 2009$ & BLE-TC-20 & 100 & $1.18 \mathrm{E}+17$ & $1.50 \mathrm{E}+17$ \\
\hline $6 / 11 / 2009$ & BLE-TC-22 & 100 & $2.67 E+16$ & $3.19 E+16$ \\
\hline $6 / 11 / 2009$ & BLE-TC-24 & 100 & $6.12 \mathrm{E}+16$ & $7.48 \mathrm{E}+16$ \\
\hline & & Average & $5.703 E+16$ & $7.068 \mathrm{E}+16$ \\
\hline & & $\begin{array}{l}\text { Standard } \\
\text { Deviation }\end{array}$ & $3.546 E+16$ & $4.530 E+16$ \\
\hline & & $\begin{array}{c}\text { Number of } \\
\text { Samples }\end{array}$ & 6 & 6 \\
\hline
\end{tabular}




\section{Appendix G.2: Polycarbonate with Carbon Black}

Table G.3: ASTM D257 Through Plane Electrical Resistivity Results for BL2: Lexan HF1130-111: Injection Molded December 4, 2009

\begin{tabular}{|c|c|c|c|c|}
\hline Test Date & $\begin{array}{l}\text { Sample } \\
\text { Number }\end{array}$ & $\begin{array}{l}\text { Applied Voltage } \\
\text { (V) }\end{array}$ & $\begin{array}{l}\text { Through- } \\
\text { Plane } \\
\text { Surface } \\
\text { Electrical } \\
\text { Resistivity } \\
\text { ( } \Omega / \text { square) }\end{array}$ & $\begin{array}{l}\text { Through- } \\
\text { Plane } \\
\text { Volume } \\
\text { Electrical } \\
\text { Resistivity } \\
(\Omega \text {-cm) }\end{array}$ \\
\hline $12 / 7 / 2009$ & BL2-TC-40 & 100 & $8.8010 \mathrm{E}+16$ & $9.6089 \mathrm{E}+16$ \\
\hline $12 / 7 / 2009$ & BL2-TC-22 & 100 & $5.5567 \mathrm{E}+16$ & $1.4034 \mathrm{E}+17$ \\
\hline $12 / 7 / 2009$ & BL2-TC-9 & 100 & $1.7208 \mathrm{E}+17$ & $8.6133 \mathrm{E}+16$ \\
\hline $12 / 7 / 2009$ & BL2-TC-25 & 100 & $1.0728 \mathrm{E}+17$ & $1.3089 \mathrm{E}+17$ \\
\hline $12 / 7 / 2009$ & BL2-TC-29 & 100 & $1.0565 \mathrm{E}+17$ & $1.2183 \mathrm{E}+17$ \\
\hline \multirow[t]{4}{*}{$12 / 7 / 2009$} & BL2-TC-27 & 100 & $1.3064 \mathrm{E}+17$ & $1.7982 \mathrm{E}+17$ \\
\hline & & Average & $1.0987 \mathrm{E}+17$ & $1.2585 \mathrm{E}+17$ \\
\hline & & $\begin{array}{l}\text { Standard } \\
\text { Deviation }\end{array}$ & $3.9377 \mathrm{E}+16$ & $3.3548 \mathrm{E}+16$ \\
\hline & & $\begin{array}{l}\text { Number of } \\
\text { Samples }\end{array}$ & 6 & 6 \\
\hline
\end{tabular}


Table G.4: ASTM D257 Through Plane Electrical Resistivity Results for BLE2: Extruded Lexan HF1130-111: Extruded Nov 5, 2009; Injection Molded December 4, 2009

\begin{tabular}{|c|c|c|c|c|}
\hline Test Date & $\begin{array}{l}\text { Sample } \\
\text { Number }\end{array}$ & $\begin{array}{l}\text { Applied Voltage } \\
\text { (V) }\end{array}$ & $\begin{array}{l}\text { Through- } \\
\text { Plane } \\
\text { Surface } \\
\text { Electrical } \\
\text { Resistivity } \\
\text { ( } \Omega / \text { square) }\end{array}$ & $\begin{array}{l}\text { Through- } \\
\text { Plane } \\
\text { Volume } \\
\text { Electrical } \\
\text { Resistivity } \\
(\Omega-\mathrm{cm})\end{array}$ \\
\hline $12 / 17 / 2009$ & BLE2-TC-14 & 100 & $2.3979 E+16$ & $4.0569 E+17$ \\
\hline $12 / 17 / 2009$ & BLE2-TC-11 & 100 & $3.4927 E+16$ & $1.2131 \mathrm{E}+17$ \\
\hline $12 / 17 / 2009$ & BLE2-TC-7 & 100 & $2.5512 E+16$ & $2.6558 E+17$ \\
\hline $12 / 17 / 2009$ & BLE2-TC-17 & 100 & $2.0976 E+16$ & $4.4475 E+17$ \\
\hline $12 / 17 / 2009$ & BLE2-TC-9 & 100 & $3.0226 E+16$ & 1.3335E+17 \\
\hline \multirow[t]{4}{*}{$12 / 17 / 2009$} & BLE2-TC-21 & 100 & $9.5520 E+15$ & $1.8589 E+17$ \\
\hline & & Average & $2.4195 E+16$ & $2.5943 E+17$ \\
\hline & & $\begin{array}{l}\text { Standard } \\
\text { Deviation }\end{array}$ & 8.6961E+15 & $1.3868 \mathrm{E}+17$ \\
\hline & & $\begin{array}{l}\text { Number of } \\
\text { Samples }\end{array}$ & 6 & 6 \\
\hline
\end{tabular}

Table G.5: ASTM D257 Through Plane Electrical Resistivity Results for BA2L: 2 wt\% Ketjenblack EC-600 JD in Lexan HF1130-111: Injection Molded December 4, 2009

\begin{tabular}{|c|c|c|c|c|}
\hline Test Date & $\begin{array}{l}\text { Sample } \\
\text { Number }\end{array}$ & $\begin{array}{l}\text { Applied Voltage } \\
\text { (V) }\end{array}$ & $\begin{array}{l}\text { Through- } \\
\text { Plane } \\
\text { Surface } \\
\text { Electrical } \\
\text { Resistivity } \\
\text { ( } \Omega \text { /square) }\end{array}$ & $\begin{array}{l}\text { Through- } \\
\text { Plane } \\
\text { Volume } \\
\text { Electrical } \\
\text { Resistivity } \\
(\Omega \text {-cm) }\end{array}$ \\
\hline $12 / 18 / 2009$ & BA2L-TC-24 & 100 & $2.4674 \mathrm{E}+17$ & $2.5957 E+16$ \\
\hline $12 / 18 / 2009$ & BA2L-TC-27 & 100 & $3.1627 E+16$ & $1.7383 E+16$ \\
\hline $12 / 18 / 2009$ & BA2L-TC-5 & 100 & $1.1845 E+16$ & $5.9889 E+16$ \\
\hline $12 / 18 / 2009$ & BA2L-TC-9 & 100 & $2.4993 E+16$ & $1.5829 E+16$ \\
\hline $12 / 18 / 2009$ & BA2L-TC-30 & 100 & $1.1959 \mathrm{E}+16$ & $8.2963 E+16$ \\
\hline $12 / 18 / 2009$ & BA2L-TC-19 & 100 & $1.1826 \mathrm{E}+16$ & $4.1130 E+16$ \\
\hline & & Average & $5.6498 E+16$ & $4.0525 E+16$ \\
\hline & & $\begin{array}{l}\text { Standard } \\
\text { Deviation }\end{array}$ & $9.3570 \mathrm{E}+16$ & $2.6586 E+16$ \\
\hline & & $\begin{array}{l}\text { Number of } \\
\text { Samples }\end{array}$ & 6 & 6 \\
\hline
\end{tabular}


Table G.6: ASTM D257 Through Plane Electrical Resistivity Results for BA3L: 3 wt\% Ketjenblack EC-600 JD in Lexan HF1130-111: Injection Molded December 4, 2009

\begin{tabular}{|c|c|c|c|c|}
\hline Test Date & $\begin{array}{l}\text { Sample } \\
\text { Number }\end{array}$ & $\begin{array}{l}\text { Applied Voltage } \\
\text { (V) }\end{array}$ & $\begin{array}{l}\text { Through- } \\
\text { Plane } \\
\text { Surface } \\
\text { Electrical } \\
\text { Resistivity } \\
\text { ( } \Omega / \text { square) }\end{array}$ & $\begin{array}{l}\text { Through- } \\
\text { Plane } \\
\text { Volume } \\
\text { Electrical } \\
\text { Resistivity } \\
(\Omega-\mathrm{cm})\end{array}$ \\
\hline $12 / 18 / 2009$ & BA3L-TC-28 & 100 & $6.2420 E+15$ & $2.3907 E+15$ \\
\hline $12 / 18 / 2009$ & BA3L-TC-24 & 100 & $3.6602 E+15$ & $2.5925 E+15$ \\
\hline $12 / 18 / 2009$ & BA3L-TC-20 & 100 & $2.6216 E+15$ & $2.8664 \mathrm{E}+15$ \\
\hline $12 / 18 / 2009$ & BA3L-TC-7 & 100 & $3.9430 E+15$ & $2.4510 E+15$ \\
\hline $12 / 18 / 2009$ & BA3L-TC-11 & 100 & $2.3223 E+15$ & $3.3838 E+15$ \\
\hline \multirow[t]{4}{*}{$12 / 18 / 2009$} & BA3L-TC-4 & 100 & $2.6606 E+15$ & $3.4235 E+15$ \\
\hline & & Average & $3.5750 \mathrm{E}+15$ & $2.8513 E+15$ \\
\hline & & $\begin{array}{l}\text { Standard } \\
\text { Deviation }\end{array}$ & 1.4540E+15 & $4.5838 E+14$ \\
\hline & & $\begin{array}{l}\text { Number of } \\
\text { Samples }\end{array}$ & 6 & 6 \\
\hline
\end{tabular}




\section{Appendix G.3: Polycarbonate with Graphite Nanoparticles}

Table G.7: ASTM D257 Through Plane Electrical Resistivity Results for BL3: Lexan HF1130-111: Injection Molded 6-3-10

\begin{tabular}{|c|c|c|c|c|}
\hline Test Date & $\begin{array}{l}\text { Sample } \\
\text { Number }\end{array}$ & $\begin{array}{l}\text { Applied } \\
\text { Voltage (V) }\end{array}$ & $\begin{array}{l}\text { Through-Plane } \\
\text { Surface Electrical } \\
\text { Resistivity } \\
\text { ( } \Omega / \text { square) }\end{array}$ & $\begin{array}{l}\text { Through-Plane } \\
\text { Volume Electrical } \\
\text { Resistivity ( } \Omega \text { - } \\
\mathrm{cm})\end{array}$ \\
\hline $6 / 4 / 2010$ & BL3-TC-12 & 100 & $8.9746 E+16$ & 1.1695E+17 \\
\hline $6 / 4 / 2010$ & BL3-TC-23 & 100 & $8.9015 E+14$ & $9.4655 E+16$ \\
\hline 6/8/2010 & BL3-TC-18 & 100 & $3.0826 E+16$ & 1.1713E+17 \\
\hline $6 / 8 / 2010$ & BL3-TC-27 & 100 & $2.6199 E+17$ & $6.9047 E+16$ \\
\hline $6 / 8 / 2010$ & BL3-TC-28 & 100 & $3.6130 E+17$ & $8.5692 E+16$ \\
\hline $6 / 8 / 2010$ & BL3-TC-29 & 100 & $5.5683 E+17$ & $7.8509 E+16$ \\
\hline & & Average & $2.1693 E+17$ & $9.3664 \mathrm{E}+16$ \\
\hline & & $\begin{array}{l}\text { Standard } \\
\text { Deviation }\end{array}$ & $2.1720 E+17$ & $1.9965 E+16$ \\
\hline & & $\begin{array}{l}\text { Number of } \\
\text { Samples }\end{array}$ & 6 & 6 \\
\hline
\end{tabular}


Table G.8: ASTM D257 Through Plane Electrical Resistivity Results for BLE3: Extruded Lexan HF1130-111: Extruded 5-26-10; Injection Molded 6-3-10

\begin{tabular}{|c|c|c|c|c|}
\hline Test Date & $\begin{array}{l}\text { Sample } \\
\text { Number }\end{array}$ & $\begin{array}{l}\text { Applied } \\
\text { Voltage (V) }\end{array}$ & $\begin{array}{l}\text { Through-Plane } \\
\text { Surface } \\
\text { Electrical } \\
\text { Resistivity } \\
\text { ( } \Omega \text { /square) }\end{array}$ & $\begin{array}{l}\text { Through-Plane } \\
\text { Volume } \\
\text { Electrical } \\
\text { Resistivity ( } \Omega \text { - } \\
\mathrm{cm} \text { ) }\end{array}$ \\
\hline $6 / 8 / 2010$ & BLE3-TC-5 & 100 & $6.3781 \mathrm{E}+15$ & $6.3695 E+16$ \\
\hline $6 / 8 / 2010$ & BLE3-TC-6 & 100 & $2.0715 E+17$ & $9.9061 \mathrm{E}+16$ \\
\hline $6 / 8 / 2010$ & BLE3-TC-7 & 100 & 1.2488E+17 & $9.7042 E+16$ \\
\hline $6 / 8 / 2010$ & BLE3-TC-8 & 100 & $2.4365 E+17$ & $9.2231 \mathrm{E}+16$ \\
\hline $6 / 8 / 2010$ & BLE3-TC-9 & 100 & $5.6273 E+16$ & $1.0422 \mathrm{E}+17$ \\
\hline $6 / 8 / 2010$ & BLE3-TC-4 & 100 & $5.0379 E+16$ & $2.0189 \mathrm{E}+17$ \\
\hline $6 / 8 / 2010$ & BLE3-TC-3 & 100 & $2.1701 \mathrm{E}+17$ & $9.8937 E+16$ \\
\hline $6 / 8 / 2010$ & BLE3-TC-11 & 100 & 1.2729E+16 & $7.7092 E+16$ \\
\hline & & Average & $1.1481 \mathrm{E}+17$ & $1.0427 \mathrm{E}+17$ \\
\hline & & $\begin{array}{l}\text { Standard } \\
\text { Deviation }\end{array}$ & $9.6680 E+16$ & $4.1692 E+16$ \\
\hline & & $\begin{array}{l}\text { Number of } \\
\text { Samples }\end{array}$ & 8 & 0 \\
\hline
\end{tabular}

Table G.9: ASTM D257 Through Plane Electrical Resistivity Results for BG2L: 2 wt\% $\mathrm{xGnP}$ in Lexan HF1130-111: Injection Molded 6-3-10

\begin{tabular}{|c|c|c|c|c|}
\hline Test Date & $\begin{array}{l}\text { Sample } \\
\text { Number }\end{array}$ & $\begin{array}{l}\text { Applied } \\
\text { Voltage (V) }\end{array}$ & $\begin{array}{l}\text { Through-Plane } \\
\text { Surface } \\
\text { Electrical } \\
\text { Resistivity } \\
(\Omega / \text { square) }\end{array}$ & $\begin{array}{l}\text { Through-Plane } \\
\text { Volume } \\
\text { Electrical } \\
\text { Resistivity ( } \Omega \text { - } \\
\mathrm{cm} \text { ) }\end{array}$ \\
\hline $6 / 8 / 2010$ & BG2L-TC-15 & 100 & $9.6444 \mathrm{E}+14$ & $5.0799 E+16$ \\
\hline $6 / 8 / 2010$ & BG2L-TC-13 & 100 & $4.3584 \mathrm{E}+16$ & $5.4010 E+16$ \\
\hline $6 / 8 / 2010$ & BG2L-TC-24 & 100 & $9.8499 E+16$ & $5.3507 E+16$ \\
\hline $6 / 8 / 2010$ & BG2L-TC-23 & 100 & $5.6887 E+16$ & $6.2724 E+16$ \\
\hline $6 / 8 / 2010$ & BG2L-TC-22 & 100 & 1.6322E+17 & $4.9213 E+16$ \\
\hline $6 / 8 / 2010$ & BG2L-TC-21 & 100 & $3.1959 E+16$ & $5.7554 \mathrm{E}+16$ \\
\hline & & Average & $6.5852 E+16$ & $5.4635 E+16$ \\
\hline & & $\begin{array}{l}\text { Standard } \\
\text { Deviation }\end{array}$ & $5.7414 \mathrm{E}+16$ & 4.8934E+15 \\
\hline & & $\begin{array}{l}\text { Number of } \\
\text { Samples }\end{array}$ & 6 & 6 \\
\hline
\end{tabular}


Table G.10: ASTM D257 Through Plane Electrical Resistivity Results for BG3L: 3 wt\% xGnP in Lexan HF1130-111: Injection Molded 6-3-10

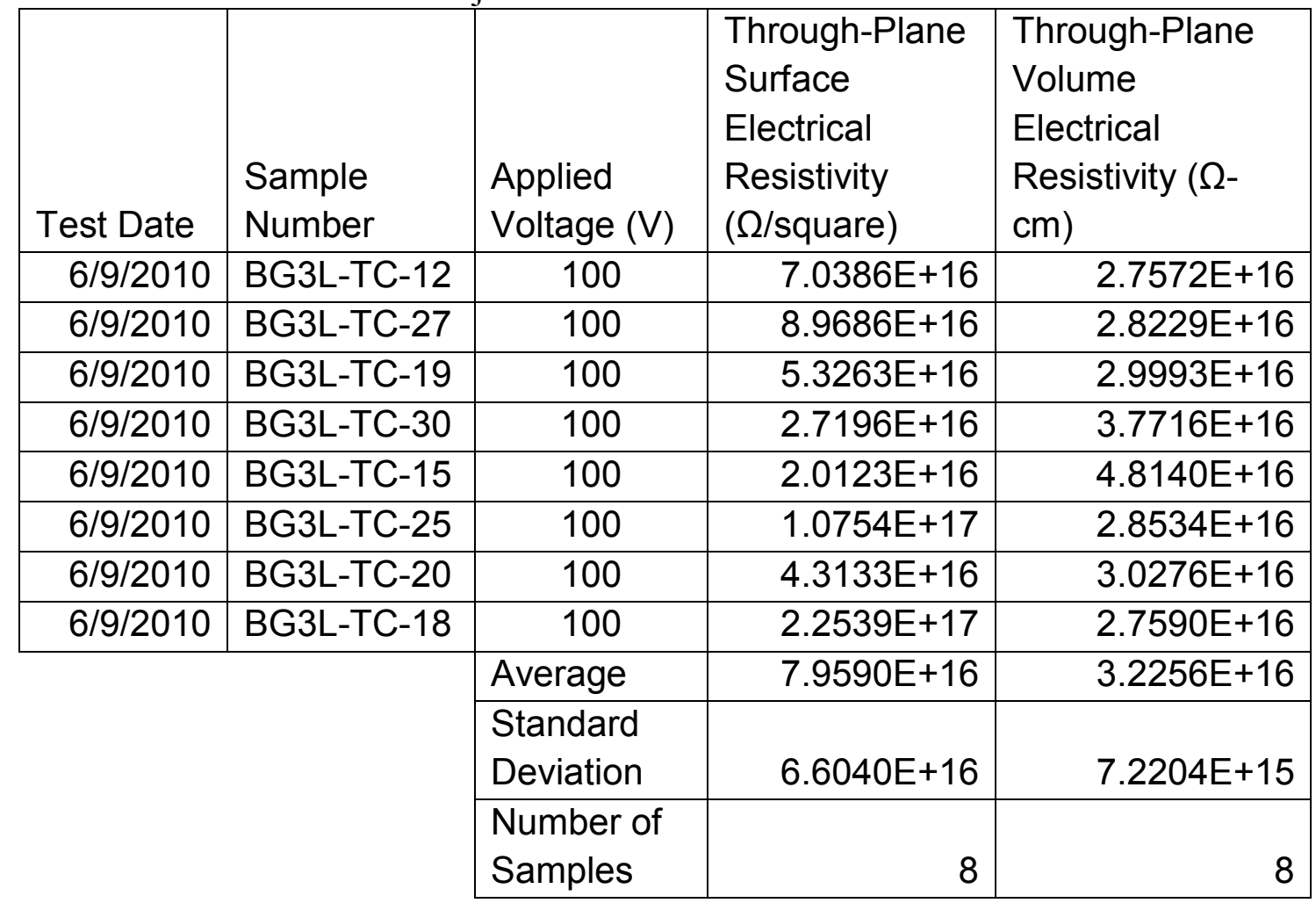

Table G.11: ASTM D257 Through Plane Electrical Resistivity Results for BG4L: 4 wt\% xGnP in Lexan HF1130-111: Injection Molded 6-3-10

\begin{tabular}{|c|c|c|c|c|}
\hline Test Date & $\begin{array}{l}\text { Sample } \\
\text { Number }\end{array}$ & $\begin{array}{l}\text { Applied } \\
\text { Voltage (V) }\end{array}$ & $\begin{array}{l}\text { Through-Plane } \\
\text { Surface } \\
\text { Electrical } \\
\text { Resistivity } \\
(\Omega / \text { square) }\end{array}$ & $\begin{array}{l}\text { Through-Plane } \\
\text { Volume } \\
\text { Electrical } \\
\text { Resistivity ( } \Omega \text { - } \\
\mathrm{cm})\end{array}$ \\
\hline $6 / 9 / 2010$ & BG4L-TC-21 & 100 & $9.4799 \mathrm{E}+16$ & $1.1575 E+16$ \\
\hline $6 / 9 / 2010$ & BG4L-TC-20 & 100 & $7.5057 \mathrm{E}+16$ & $1.1796 \mathrm{E}+16$ \\
\hline $6 / 9 / 2010$ & BG4L-TC-19 & 100 & $9.0056 \mathrm{E}+16$ & $1.2164 \mathrm{E}+16$ \\
\hline 6/9/2010 & BG4L-TC-18 & 100 & $9.2462 \mathrm{E}+16$ & $1.2126 \mathrm{E}+16$ \\
\hline $6 / 9 / 2010$ & BG4L-TC-16 & 100 & $5.5037 \mathrm{E}+16$ & $1.2539 \mathrm{E}+16$ \\
\hline \multirow[t]{4}{*}{ 6/9/2010 } & BG4L-TC-13 & 100 & $9.1579 \mathrm{E}+16$ & $1.1732 \mathrm{E}+16$ \\
\hline & & Average & $8.3165 \mathrm{E}+16$ & $1.1989 \mathrm{E}+16$ \\
\hline & & $\begin{array}{l}\text { Standard } \\
\text { Deviation }\end{array}$ & $1.5472 \mathrm{E}+16$ & $3.5400 \mathrm{E}+14$ \\
\hline & & $\begin{array}{l}\text { Number of } \\
\text { Samples }\end{array}$ & 6 & 6 \\
\hline
\end{tabular}


Table G.12: ASTM D257 Through Plane Electrical Resistivity Results for BG5L: 5 wt\% $\mathrm{xGnP}$ in Lexan HF1130-111: Injection Molded 6-3-10

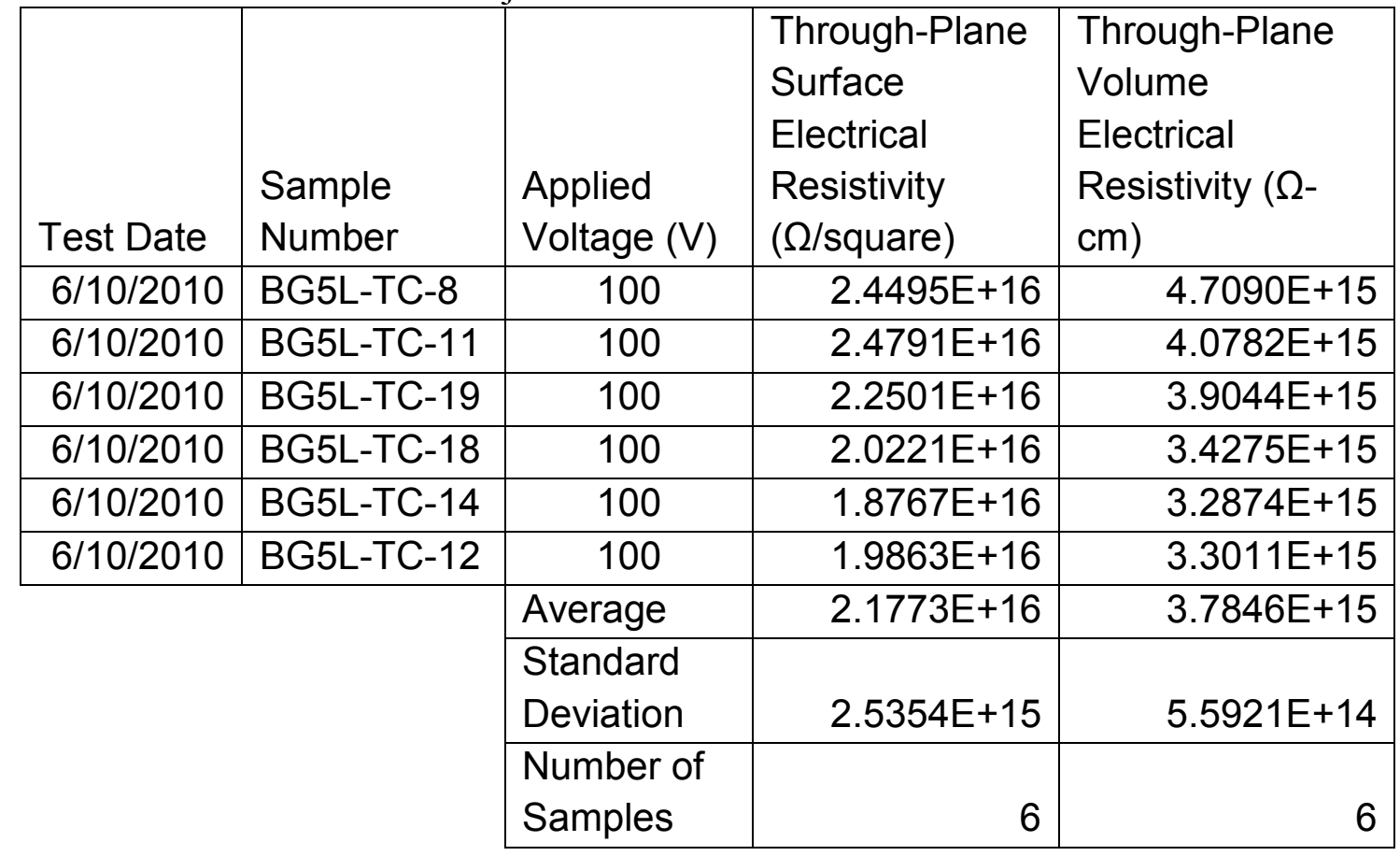

Table G.13: ASTM D257 Through Plane Electrical Resistivity Results for BG6L: 6 wt\% $\mathrm{xGnP}$ in Lexan HF1130-111: Injection Molded 6-8-10

\begin{tabular}{|c|c|c|c|c|}
\hline Test Date & $\begin{array}{l}\text { Sample } \\
\text { Number }\end{array}$ & $\begin{array}{l}\text { Applied } \\
\text { Voltage (V) }\end{array}$ & $\begin{array}{l}\text { Through-Plane } \\
\text { Surface } \\
\text { Electrical } \\
\text { Resistivity } \\
(\Omega / \text { square) }\end{array}$ & $\begin{array}{l}\text { Through-Plane } \\
\text { Volume } \\
\text { Electrical } \\
\text { Resistivity ( } \Omega \text { - } \\
\mathrm{cm} \text { ) }\end{array}$ \\
\hline $6 / 10 / 2010$ & BG6L-TC-22 & 100 & $1.7140 \mathrm{E}+15$ & $2.0798 \mathrm{E}+14$ \\
\hline $6 / 10 / 2010$ & BG6L-TC-26 & 100 & $3.4737 \mathrm{E}+15$ & $1.9682 \mathrm{E}+14$ \\
\hline $6 / 10 / 2010$ & BG6L-TC-19 & 100 & $3.2758 \mathrm{E}+15$ & $1.9705 \mathrm{E}+14$ \\
\hline $6 / 10 / 2010$ & BG6L-TC-16 & 100 & $3.3674 \mathrm{E}+15$ & $2.0585 \mathrm{E}+14$ \\
\hline $6 / 10 / 2010$ & BG6L-TC-23 & 100 & $3.6406 \mathrm{E}+15$ & $1.9690 \mathrm{E}+14$ \\
\hline $6 / 10 / 2010$ & BG6L-TC-11 & 100 & $3.0990 \mathrm{E}+15$ & 1.9927E+14 \\
\hline & & Average & $3.0951 \mathrm{E}+15$ & $2.0065 \mathrm{E}+14$ \\
\hline & & $\begin{array}{l}\text { Standard } \\
\text { Deviation }\end{array}$ & $7.0074 \mathrm{E}+14$ & $4.9873 E+12$ \\
\hline & & $\begin{array}{l}\text { Number of } \\
\text { Samples }\end{array}$ & 6 & 6 \\
\hline
\end{tabular}




\section{Appendix G.4: Polycarbonate with Multiple Fillers}

Table G.14: ASTM D257 Through Plane Electrical Resistivity Results for BA2LR: Extruded 7-8-10, Injection Molded 7-26-10

\begin{tabular}{|c|c|c|c|c|}
\hline Test Date & $\begin{array}{l}\text { Sample } \\
\text { Number }\end{array}$ & $\begin{array}{l}\text { Applied } \\
\text { Voltage } \\
\text { (V) }\end{array}$ & $\begin{array}{l}\text { Through-Plane } \\
\text { Surface } \\
\text { Electrical } \\
\text { Resistivity } \\
(\Omega / \text { square })\end{array}$ & $\begin{array}{l}\text { Through-Plane } \\
\text { Volume } \\
\text { Electrical } \\
\text { Resistivity ( } \Omega \text { - } \\
\mathrm{cm} \text { ) }\end{array}$ \\
\hline $8 / 2 / 2010$ & BA2LR-TC-11 & 100 & 1.3994E+16 & $1.0845 E+16$ \\
\hline $8 / 2 / 2010$ & BA2LR-TC-9 & 100 & $2.8471 E+16$ & $1.5032 E+16$ \\
\hline $8 / 2 / 2010$ & BA2LR-TC-20 & 100 & $2.8633 E+16$ & 1.3669E+16 \\
\hline $8 / 2 / 2010$ & BA2LR-TC-8 & 100 & $1.3855 E+16$ & $3.9620 \mathrm{E}+16$ \\
\hline $8 / 2 / 2010$ & BA2LR-TC-19 & 100 & $1.1641 \mathrm{E}+16$ & $2.5462 E+16$ \\
\hline $8 / 2 / 2010$ & BA2LR-TC-27 & 100 & $3.6494 E+16$ & $3.7168 E+16$ \\
\hline & & Average & $2.2181 \mathrm{E}+16$ & $2.3633 E+16$ \\
\hline & & $\begin{array}{l}\text { Standard } \\
\text { Deviation }\end{array}$ & $1.0330 \mathrm{E}+16$ & $1.2481 \mathrm{E}+16$ \\
\hline & & $\begin{array}{l}\text { Number of } \\
\text { Samples }\end{array}$ & 6 & 6 \\
\hline
\end{tabular}

Table G.15: ASTM D257 Through Plane Electrical Resistivity Results for BQ0.5L: Extruded 6-30-10, Injection Molded 7-26-10

\begin{tabular}{|c|c|c|c|c|}
\hline Test Date & $\begin{array}{l}\text { Sample } \\
\text { Number }\end{array}$ & $\begin{array}{l}\text { Applied } \\
\text { Voltage } \\
\text { (V) }\end{array}$ & $\begin{array}{l}\text { Through-Plane } \\
\text { Surface } \\
\text { Electrical } \\
\text { Resistivity } \\
\text { ( } \Omega \text { /square) }\end{array}$ & $\begin{array}{l}\text { Through-Plane } \\
\text { Volume } \\
\text { Electrical } \\
\text { Resistivity ( } \Omega \text { - } \\
\mathrm{cm} \text { ) }\end{array}$ \\
\hline $7 / 30 / 2010$ & BQ0.5L-TC-26 & 100 & $7.637 \mathrm{E}+16$ & $4.097 \mathrm{E}+16$ \\
\hline $7 / 30 / 2010$ & BQ0.5L-TC-15 & 100 & $3.670 E+16$ & $6.188 E+16$ \\
\hline $7 / 30 / 2010$ & BQ0.5L-TC-18 & 100 & $4.716 E+16$ & $5.720 \mathrm{E}+16$ \\
\hline $7 / 30 / 2010$ & BQ0.5L-TC-31 & 100 & $6.643 E+16$ & $7.594 E+16$ \\
\hline $7 / 30 / 2010$ & BQ0.5L-TC-11 & 100 & $2.304 E+17$ & $6.609 E+16$ \\
\hline $7 / 30 / 2010$ & BQ0.5L-TC-9 & 100 & $2.114 \mathrm{E}+17$ & $6.921 \mathrm{E}+16$ \\
\hline & & Average & $1.114 \mathrm{E}+17$ & $6.188 \mathrm{E}+16$ \\
\hline & & $\begin{array}{l}\text { Standard } \\
\text { Deviation }\end{array}$ & $8.616 E+16$ & $1.207 E+16$ \\
\hline & & $\begin{array}{l}\text { Number of } \\
\text { Samples }\end{array}$ & 6 & 6 \\
\hline
\end{tabular}


Table G.16: ASTM D257 Through Plane Electrical Resistivity Results for BQ1L Set 1: Extruded 6-30-10, Injection Molded 7-26-10

\begin{tabular}{|c|c|c|c|c|}
\hline Test Date & $\begin{array}{l}\text { Sample } \\
\text { Number }\end{array}$ & $\begin{array}{l}\text { Applied } \\
\text { Voltage } \\
\text { (V) }\end{array}$ & $\begin{array}{l}\text { Through-Plane } \\
\text { Surface } \\
\text { Electrical } \\
\text { Resistivity } \\
\text { ( } \Omega / \text { square) }\end{array}$ & $\begin{array}{l}\text { Through-Plane } \\
\text { Volume } \\
\text { Electrical } \\
\text { Resistivity ( } \Omega \text { - } \\
\mathrm{cm} \text { ) }\end{array}$ \\
\hline $7 / 30 / 2010$ & BQ1L-TC-24 & 100 & $1.146 \mathrm{E}+16$ & $1.802 \mathrm{E}+16$ \\
\hline $7 / 30 / 2010$ & BQ1L-TC-29 & 100 & $1.719 \mathrm{E}+16$ & $2.028 \mathrm{E}+16$ \\
\hline $7 / 30 / 2010$ & BQ1L-TC-4 & 100 & $2.461 \mathrm{E}+16$ & $2.410 \mathrm{E}+16$ \\
\hline $7 / 30 / 2010$ & BQ1L-TC-22 & 100 & $1.440 \mathrm{E}+16$ & $2.999 E+16$ \\
\hline $7 / 30 / 2010$ & BQ1L-TC-27 & 100 & $1.273 E+16$ & $2.218 \mathrm{E}+16$ \\
\hline $7 / 30 / 2010$ & BQ1L-TC-15 & 100 & $4.375 \mathrm{E}+15$ & $7.540 \mathrm{E}+15$ \\
\hline & & Average & $1.413 \mathrm{E}+16$ & $2.035 \mathrm{E}+16$ \\
\hline & & $\begin{array}{l}\text { Standard } \\
\text { Deviation }\end{array}$ & $6.686 \mathrm{E}+15$ & $7.482 E+15$ \\
\hline & & $\begin{array}{l}\text { Number of } \\
\text { Samples }\end{array}$ & 6 & 6 \\
\hline
\end{tabular}

Table G.17: ASTM D257 Through Plane Electrical Resistivity Results for BQ1L Set 2: Extruded 6-30-10, Injection Molded 7-26-10

\begin{tabular}{|c|c|c|c|c|}
\hline Test Date & $\begin{array}{l}\text { Sample } \\
\text { Number }\end{array}$ & $\begin{array}{l}\text { Applied } \\
\text { Voltage } \\
\text { (V) }\end{array}$ & $\begin{array}{l}\text { Through-Plane } \\
\text { Surface } \\
\text { Electrical } \\
\text { Resistivity } \\
\text { ( } \Omega / \text { square) }\end{array}$ & $\begin{array}{l}\text { Through-Plane } \\
\text { Volume } \\
\text { Electrical } \\
\text { Resistivity ( } \Omega \text { - } \\
\mathrm{cm})\end{array}$ \\
\hline $8 / 16 / 2010$ & BQ1L-TC-19 & 100 & $2.187 \mathrm{E}+15$ & $1.180 \mathrm{E}+16$ \\
\hline $8 / 16 / 2010$ & BQ1L-TC-1 & 100 & $3.631 \mathrm{E}+15$ & $1.364 \mathrm{E}+16$ \\
\hline $8 / 16 / 2010$ & BQ1L-TC-5 & 100 & $1.987 \mathrm{E}+16$ & $2.968 \mathrm{E}+16$ \\
\hline $8 / 16 / 2010$ & BQ1L-TC-8 & 100 & $4.156 \mathrm{E}+15$ & $2.217 \mathrm{E}+16$ \\
\hline $8 / 16 / 2010$ & BQ1L-TC-12 & 100 & $2.853 E+15$ & $2.359 \mathrm{E}+16$ \\
\hline $8 / 16 / 2010$ & BQ1L-TC-20 & 100 & $1.994 \mathrm{E}+15$ & $2.054 \mathrm{E}+16$ \\
\hline & & Average & $5.782 \mathrm{E}+15$ & $2.024 \mathrm{E}+16$ \\
\hline & & $\begin{array}{l}\text { Standard } \\
\text { Deviation }\end{array}$ & $6.950 \mathrm{E}+15$ & $6.618 \mathrm{E}+15$ \\
\hline & & $\begin{array}{l}\text { Number of } \\
\text { Samples }\end{array}$ & 6 & 6 \\
\hline
\end{tabular}


Table G.18: ASTM D257 Through Plane Electrical Resistivity Results for BG2L Set 2: Extruded 5-26-10, Injection Molded 6-3-10

\begin{tabular}{|c|c|c|c|c|}
\hline Test Date & $\begin{array}{l}\text { Sample } \\
\text { Number }\end{array}$ & $\begin{array}{l}\text { Applied } \\
\text { Voltage } \\
\text { (V) }\end{array}$ & $\begin{array}{l}\text { Through-Plane } \\
\text { Surface } \\
\text { Electrical } \\
\text { Resistivity } \\
(\Omega / \text { square }) \\
\end{array}$ & $\begin{array}{l}\text { Through-Plane } \\
\text { Volume } \\
\text { Electrical } \\
\text { Resistivity ( } \Omega \text { - } \\
\mathrm{cm} \text { ) }\end{array}$ \\
\hline $8 / 17 / 2010$ & BG2L-TC-17 & 100 & $6.5513 \mathrm{E}+16$ & $4.8041 \mathrm{E}+16$ \\
\hline $8 / 17 / 2010$ & BG2L-TC-28 & 100 & $3.1168 \mathrm{E}+16$ & $8.3672 \mathrm{E}+16$ \\
\hline $8 / 17 / 2010$ & BG2L-TC-27 & 100 & $7.8210 \mathrm{E}+16$ & $5.2731 \mathrm{E}+16$ \\
\hline $8 / 17 / 2010$ & BG2L-TC-10 & 100 & $4.0170 \mathrm{E}+15$ & $2.8324 \mathrm{E}+16$ \\
\hline $8 / 17 / 2010$ & BG2L-TC-16 & 100 & $3.4634 \mathrm{E}+16$ & $7.1192 \mathrm{E}+16$ \\
\hline $8 / 17 / 2010$ & BG2L-TC-12 & 100 & $3.4313 \mathrm{E}+15$ & $2.7823 \mathrm{E}+16$ \\
\hline & & Average & $3.6162 \mathrm{E}+16$ & $5.1964 \mathrm{E}+16$ \\
\hline & & $\begin{array}{l}\text { Standard } \\
\text { Deviation }\end{array}$ & $3.0859 E+16$ & $2.2507 \mathrm{E}+16$ \\
\hline & & $\begin{array}{l}\text { Number of } \\
\text { Samples }\end{array}$ & 6 & 6 \\
\hline
\end{tabular}

Table G.19: ASTM D257 Through Plane Electrical Resistivity Results for BG5L Set 2: Extruded 5-26-10, Injection Molded 6-3-10

\begin{tabular}{|c|c|c|c|c|}
\hline Test Date & $\begin{array}{l}\text { Sample } \\
\text { Number }\end{array}$ & $\begin{array}{l}\text { Applied } \\
\text { Voltage } \\
\text { (V) }\end{array}$ & $\begin{array}{l}\text { Through-Plane } \\
\text { Surface } \\
\text { Electrical } \\
\text { Resistivity } \\
(\Omega / \text { square) }\end{array}$ & $\begin{array}{l}\text { Through-Plane } \\
\text { Volume } \\
\text { Electrical } \\
\text { Resistivity }(\Omega- \\
\mathrm{cm})\end{array}$ \\
\hline $8 / 17 / 2010$ & BG5L-TC-13 & 100 & $1.1369 \mathrm{E}+16$ & $3.6390 \mathrm{E}+15$ \\
\hline $8 / 17 / 2010$ & BG5L-TC-15 & 100 & $1.7158 \mathrm{E}+16$ & $3.6826 \mathrm{E}+15$ \\
\hline $8 / 17 / 2010$ & BG5L-TC-17 & 100 & $7.6844 \mathrm{E}+15$ & $4.3248 \mathrm{E}+15$ \\
\hline $8 / 17 / 2010$ & BG5L-TC-21 & 100 & $1.9709 \mathrm{E}+16$ & $3.6472 \mathrm{E}+15$ \\
\hline $8 / 17 / 2010$ & BG5L-TC-24 & 100 & $1.6271 \mathrm{E}+16$ & $3.5443 \mathrm{E}+15$ \\
\hline $8 / 17 / 2010$ & BG5L-TC-27 & 100 & $1.6329 \mathrm{E}+16$ & $3.7105 E+15$ \\
\hline & & Average & $1.4753 \mathrm{E}+16$ & $3.7581 \mathrm{E}+15$ \\
\hline & & $\begin{array}{l}\text { Standard } \\
\text { Deviation }\end{array}$ & $4.3943 E+15$ & $2.8331 \mathrm{E}+14$ \\
\hline & & $\begin{array}{l}\text { Number of } \\
\text { Samples }\end{array}$ & 6 & 6 \\
\hline
\end{tabular}


Table G.20: ASTM D257 Through Plane Electrical Resistivity Results for BA2G2L set 1: Extruded 7-6-10, Injection Molded 8-18-10

\begin{tabular}{|c|c|c|c|c|}
\hline Test Date & $\begin{array}{l}\text { Sample } \\
\text { Number }\end{array}$ & $\begin{array}{l}\text { Applied } \\
\text { Voltage } \\
\text { (V) }\end{array}$ & $\begin{array}{l}\text { Through-Plane } \\
\text { Surface } \\
\text { Electrical } \\
\text { Resistivity } \\
\text { ( } \Omega / \text { square) }\end{array}$ & $\begin{array}{l}\text { Through-Plane } \\
\text { Volume } \\
\text { Electrical } \\
\text { Resistivity ( } \Omega \text { - } \\
\mathrm{cm} \text { ) }\end{array}$ \\
\hline $8 / 20 / 2010$ & BA2G2L-TC-6 & 100 & $9.4365 E+15$ & $3.7396 \mathrm{E}+15$ \\
\hline $8 / 20 / 2010$ & BA2G2L-TC-14 & 100 & $8.0971 \mathrm{E}+14$ & $2.3171 \mathrm{E}+15$ \\
\hline $8 / 20 / 2010$ & BA2G2L-TC-11 & 100 & $1.2262 \mathrm{E}+16$ & $4.4191 \mathrm{E}+15$ \\
\hline $8 / 20 / 2010$ & BA2G2L-TC-1 & 100 & $4.5653 \mathrm{E}+15$ & $3.9580 \mathrm{E}+15$ \\
\hline $8 / 20 / 2010$ & BA2G2L-TC-13 & 100 & $4.7833 \mathrm{E}+15$ & $4.8149 \mathrm{E}+15$ \\
\hline $8 / 20 / 2010$ & BA2G2L-TC-3 & 100 & $6.5848 \mathrm{E}+15$ & $4.7114 \mathrm{E}+15$ \\
\hline & & Average & $6.4069 \mathrm{E}+15$ & $3.9934 \mathrm{E}+15$ \\
\hline & & $\begin{array}{l}\text { Standard } \\
\text { Deviation }\end{array}$ & $4.0212 \mathrm{E}+15$ & $9.2166 \mathrm{E}+14$ \\
\hline & & $\begin{array}{l}\text { Number of } \\
\text { Samples }\end{array}$ & 6 & 6 \\
\hline
\end{tabular}

Table G.21: ASTM D257 Through Plane Electrical Resistivity Results for BA2G2L set 2: Extruded 7-6-10, Injection Molded 8-18-10

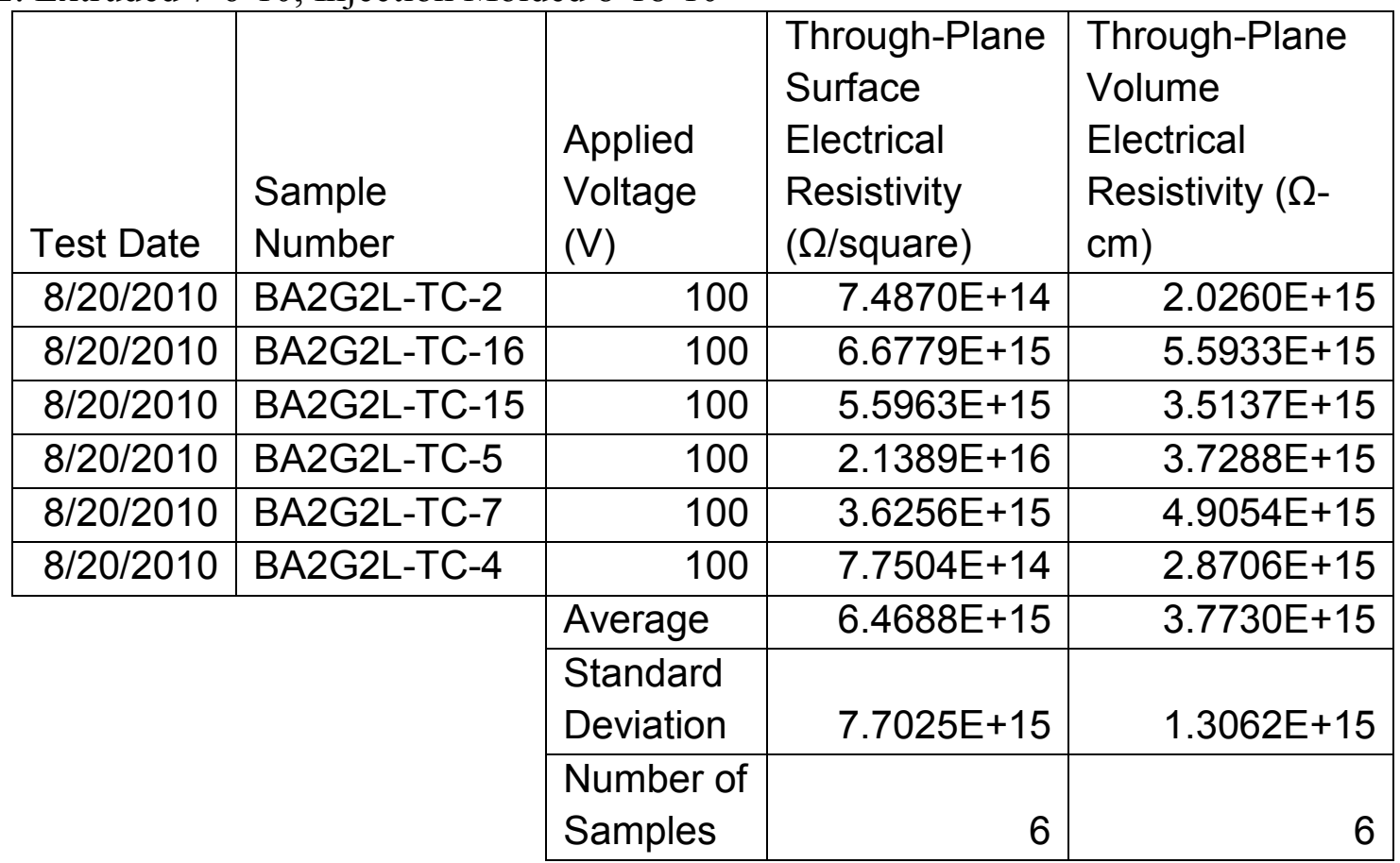


Table G.22: ASTM D257 Through Plane Electrical Resistivity Results for BQ1G2L set 1: Extruded 7-1-10, Injection Molded 8-18-10

\begin{tabular}{|c|c|c|c|c|}
\hline Test Date & Sample Number & $\begin{array}{l}\text { Applied } \\
\text { Voltage } \\
\text { (V) }\end{array}$ & $\begin{array}{l}\text { Through-Plane } \\
\text { Surface } \\
\text { Electrical } \\
\text { Resistivity } \\
(\Omega / \text { square })\end{array}$ & $\begin{array}{l}\text { Through-Plane } \\
\text { Volume } \\
\text { Electrical } \\
\text { Resistivity ( } \Omega \text { - } \\
\mathrm{cm})\end{array}$ \\
\hline $8 / 20 / 2010$ & BQ1G2L-TC-17 & 100 & 1.9206E+15 & $1.7656 \mathrm{E}+15$ \\
\hline $8 / 20 / 2010$ & BQ1G2L-TC-18 & 100 & $2.9728 E+15$ & $5.6134 \mathrm{E}+15$ \\
\hline $8 / 20 / 2010$ & BQ1G2L-TC-19 & 100 & $2.9754 \mathrm{E}+15$ & $3.2043 E+15$ \\
\hline $8 / 20 / 2010$ & BQ1G2L-TC-24 & 100 & $1.4475 E+15$ & $6.4033 E+15$ \\
\hline $8 / 20 / 2010$ & BQ1G2L-TC-23 & 100 & $5.4451 \mathrm{E}+14$ & $2.9981 E+15$ \\
\hline $8 / 20 / 2010$ & BQ1G2L-TC-25 & 100 & $5.3023 E+14$ & $3.8702 E+15$ \\
\hline $8 / 20 / 2010$ & BQ1G2L-TC-9 & 100 & $3.8504 \mathrm{E}+15$ & $5.0674 \mathrm{E}+15$ \\
\hline & & Average & $2.0345 E+15$ & $4.1318 E+15$ \\
\hline & & $\begin{array}{l}\text { Standard } \\
\text { Deviation }\end{array}$ & $1.2848 E+15$ & $1.6352 E+15$ \\
\hline & & $\begin{array}{l}\text { Number of } \\
\text { Samples }\end{array}$ & 7 & 7 \\
\hline
\end{tabular}

Table G.23: ASTM D257 Through Plane Electrical Resistivity Results for BQ1G2L set 2: Extruded 7-1-10, Injection Molded 8-18-10

\begin{tabular}{|c|c|c|c|c|}
\hline Test Date & Sample Number & $\begin{array}{l}\text { Applied } \\
\text { Voltage } \\
\text { (V) }\end{array}$ & $\begin{array}{l}\text { Through-Plane } \\
\text { Surface } \\
\text { Electrical } \\
\text { Resistivity } \\
(\Omega / \text { square })\end{array}$ & $\begin{array}{l}\text { Through-Plane } \\
\text { Volume } \\
\text { Electrical } \\
\text { Resistivity ( } \Omega \text { - } \\
\mathrm{cm})\end{array}$ \\
\hline $8 / 20 / 2010$ & BQ1G2L-TC-12 & 100 & $2.6538 E+15$ & $4.0712 E+15$ \\
\hline $8 / 20 / 2010$ & BQ1G2L-TC-11 & 100 & $1.2402 E+15$ & 7.3240E+15 \\
\hline $8 / 20 / 2010$ & BQ1G2L-TC-13 & 100 & $4.9092 E+15$ & $3.5794 \mathrm{E}+15$ \\
\hline $8 / 20 / 2010$ & BQ1G2L-TC-16 & 100 & $1.8394 \mathrm{E}+15$ & $7.4054 \mathrm{E}+15$ \\
\hline $8 / 20 / 2010$ & BQ1G2L-TC-14 & 100 & $4.3601 E+15$ & $4.1251 \mathrm{E}+15$ \\
\hline $8 / 20 / 2010$ & BQ1G2L-TC-21 & 100 & $1.9085 E+15$ & $3.6652 E+15$ \\
\hline & & Average & $2.8185 \mathrm{E}+15$ & $5.0284 E+15$ \\
\hline & & $\begin{array}{l}\text { Standard } \\
\text { Deviation }\end{array}$ & $1.4868 E+15$ & 1.8226E+15 \\
\hline & & $\begin{array}{l}\text { Number of } \\
\text { Samples }\end{array}$ & 6 & 6 \\
\hline
\end{tabular}




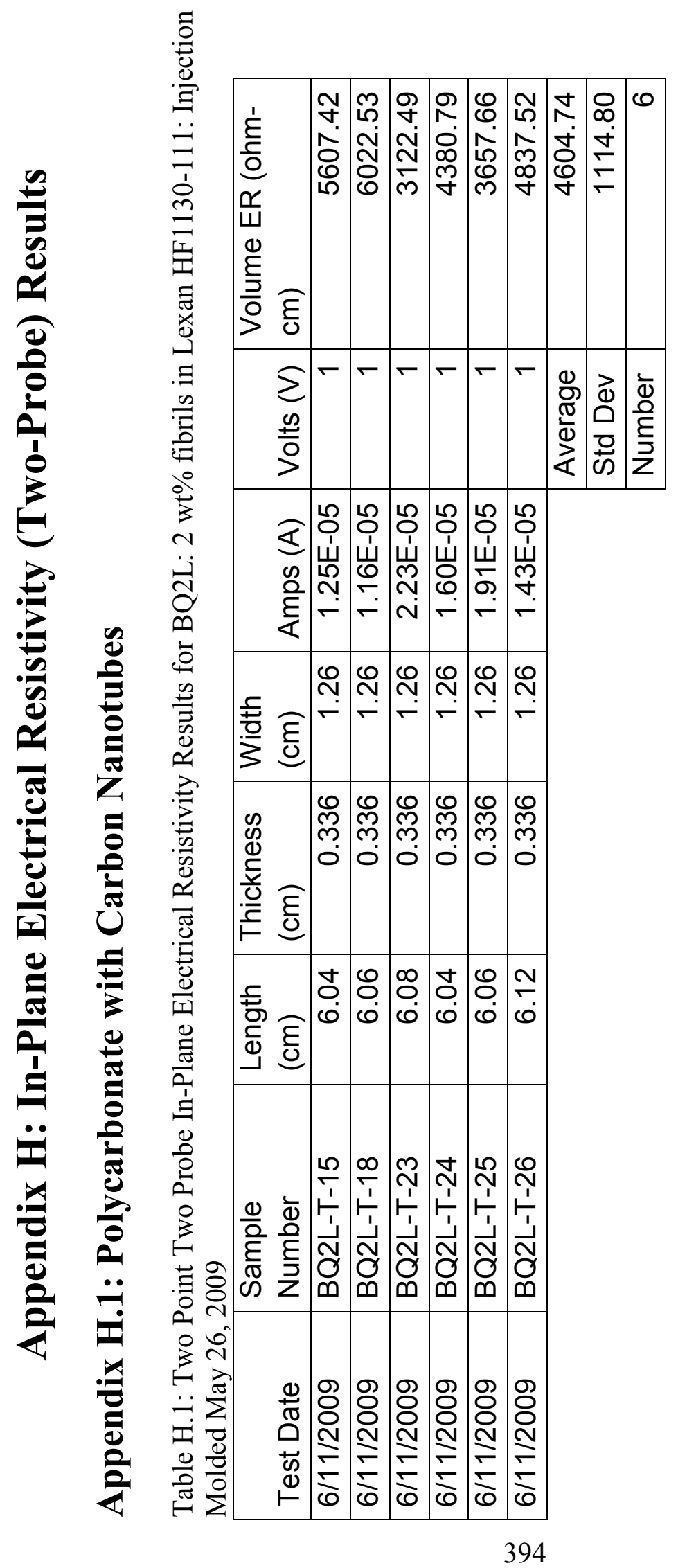



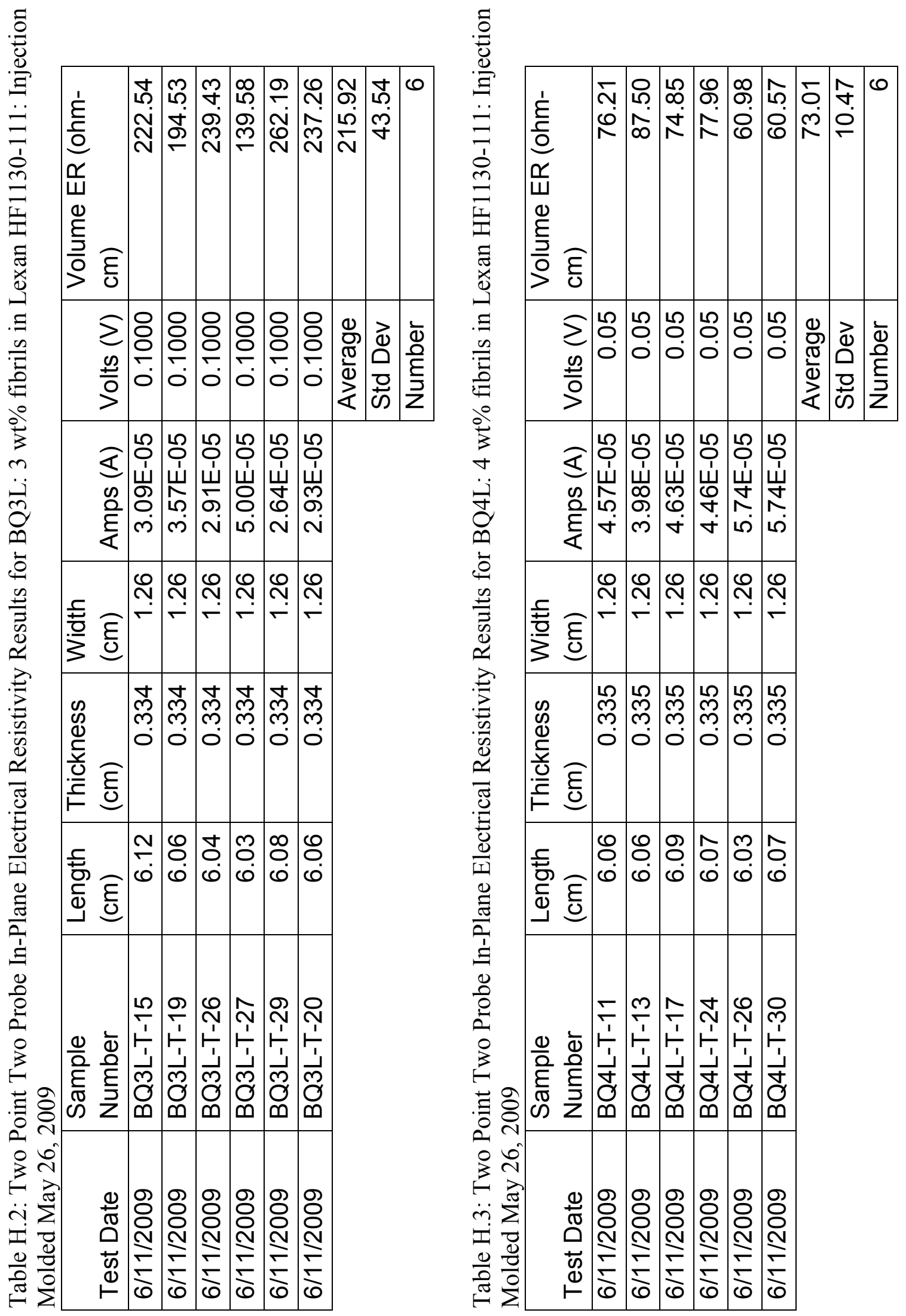

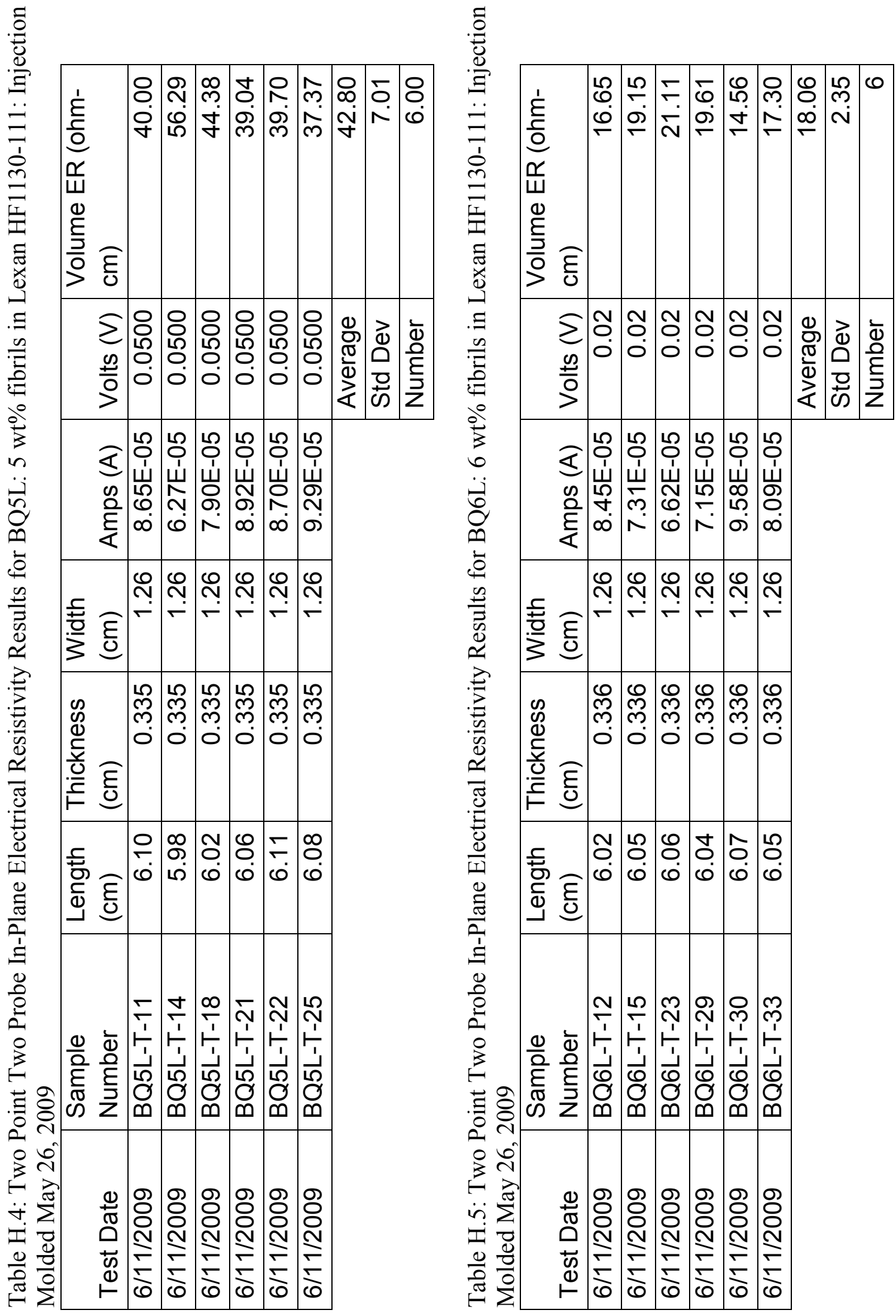


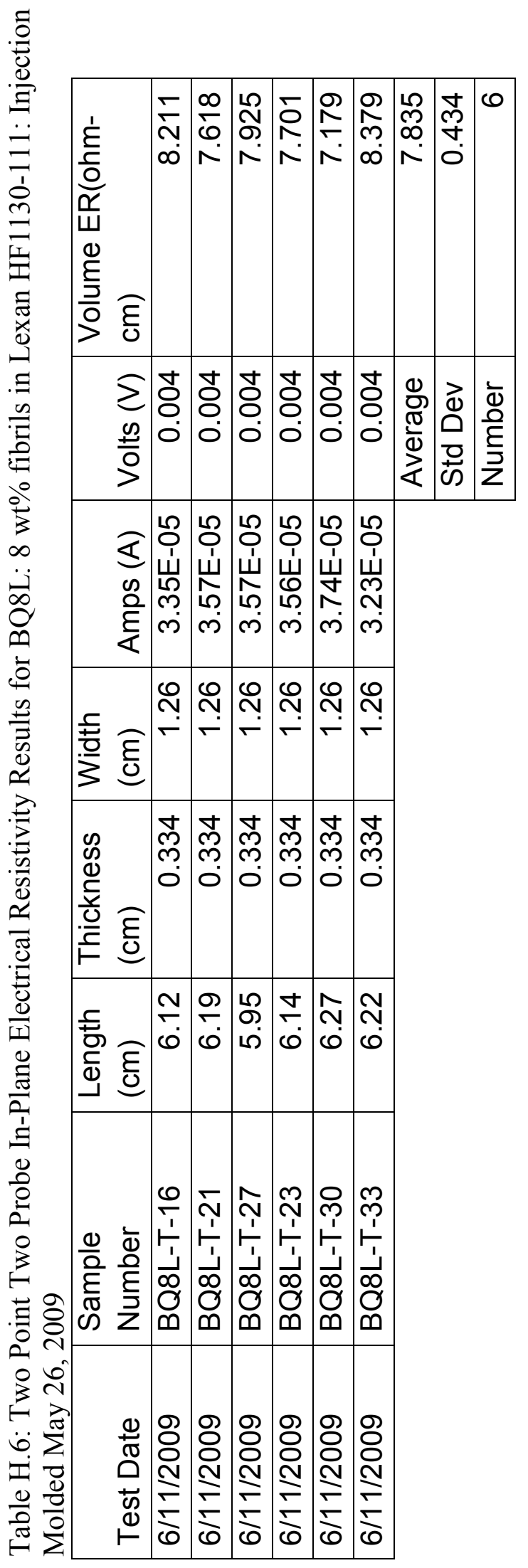




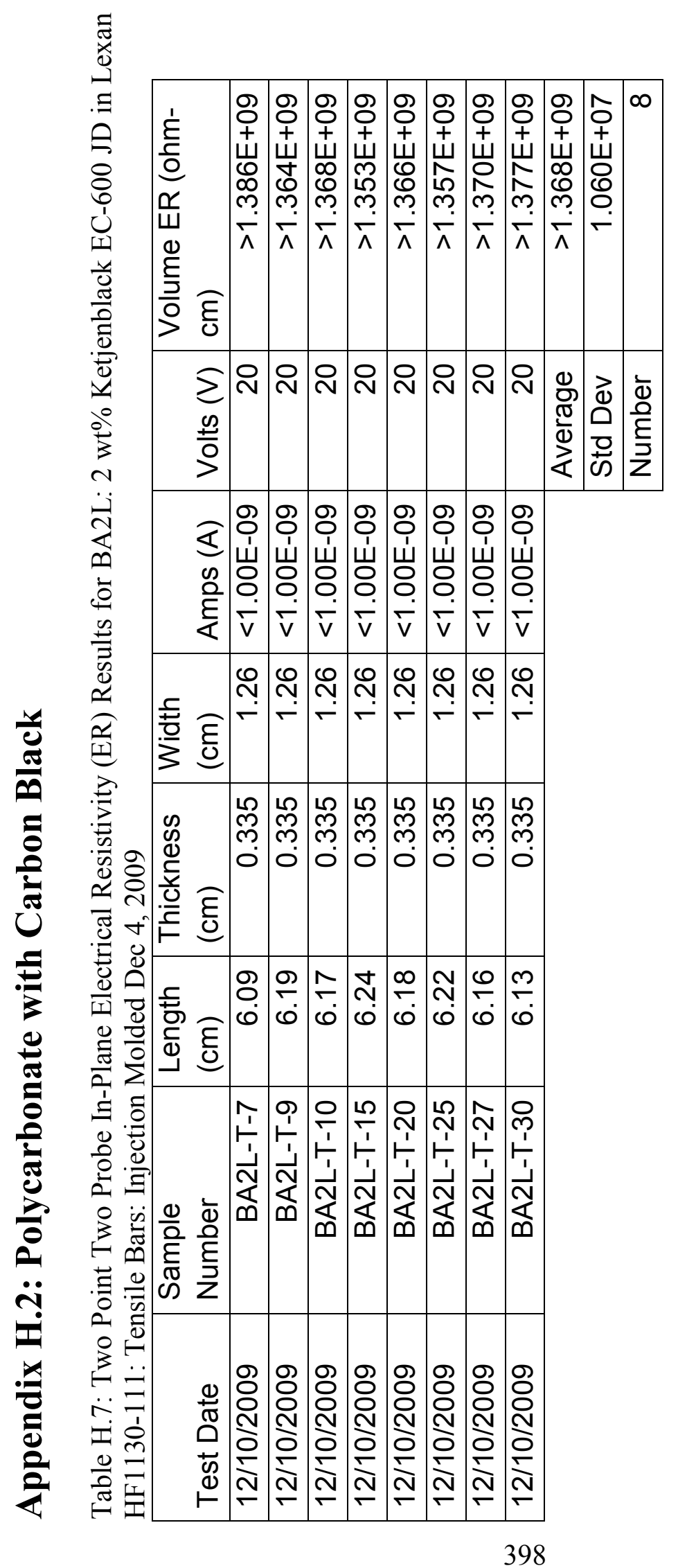




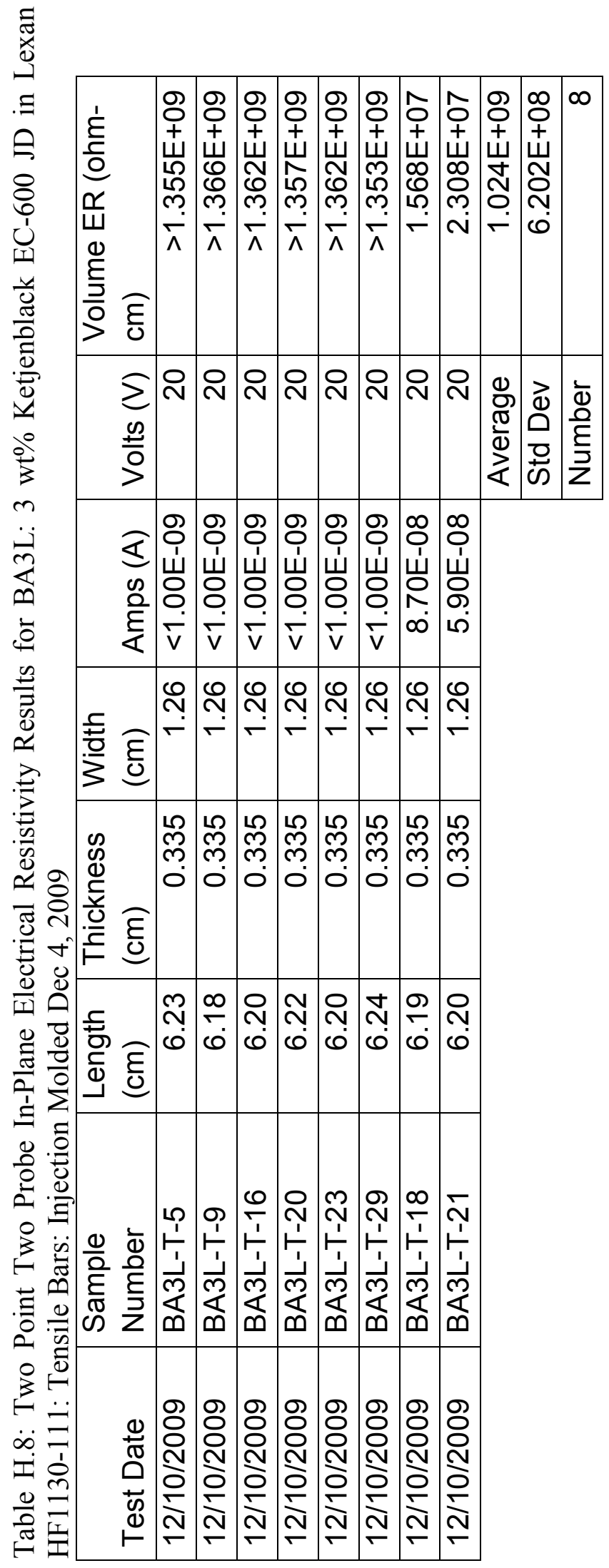




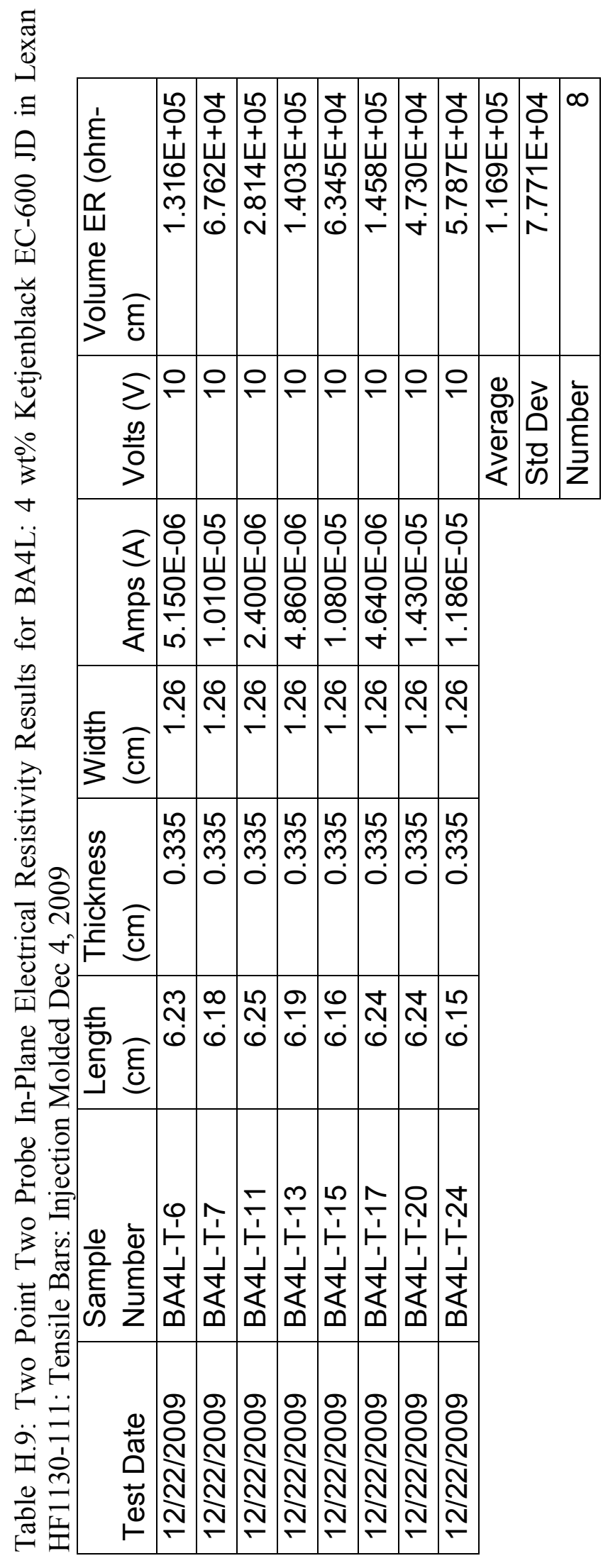




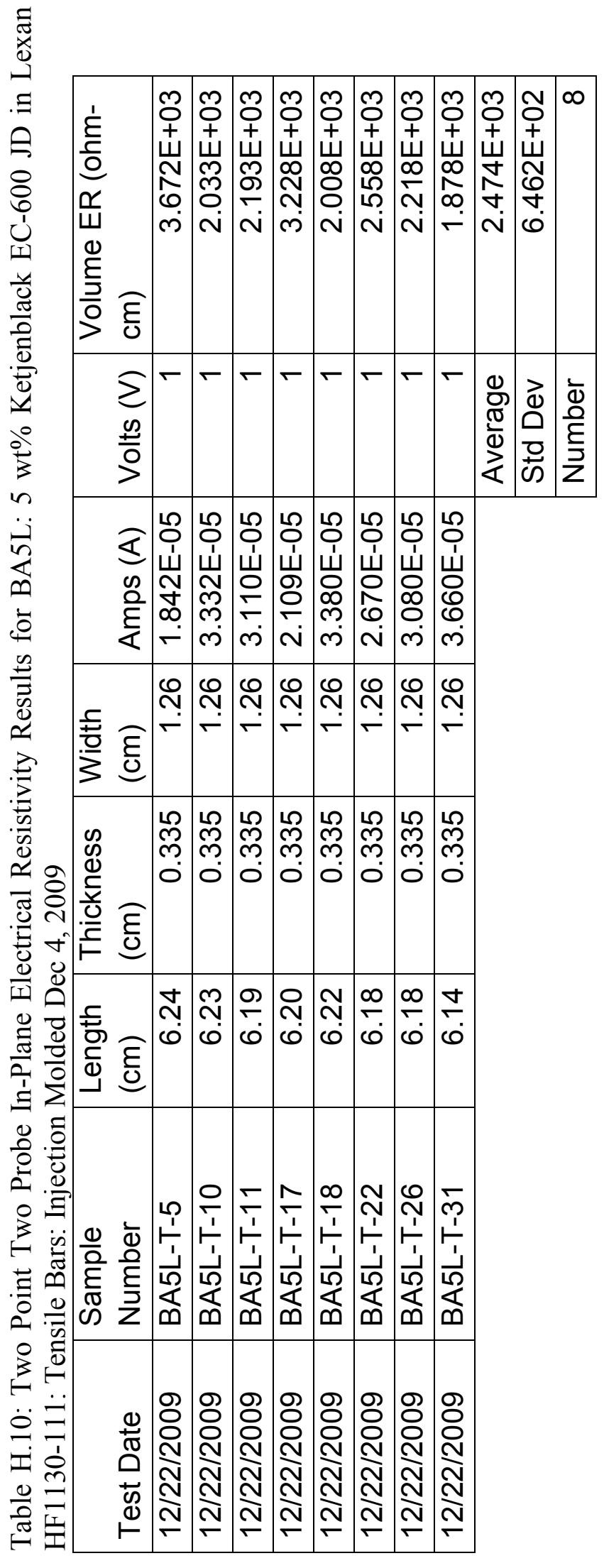




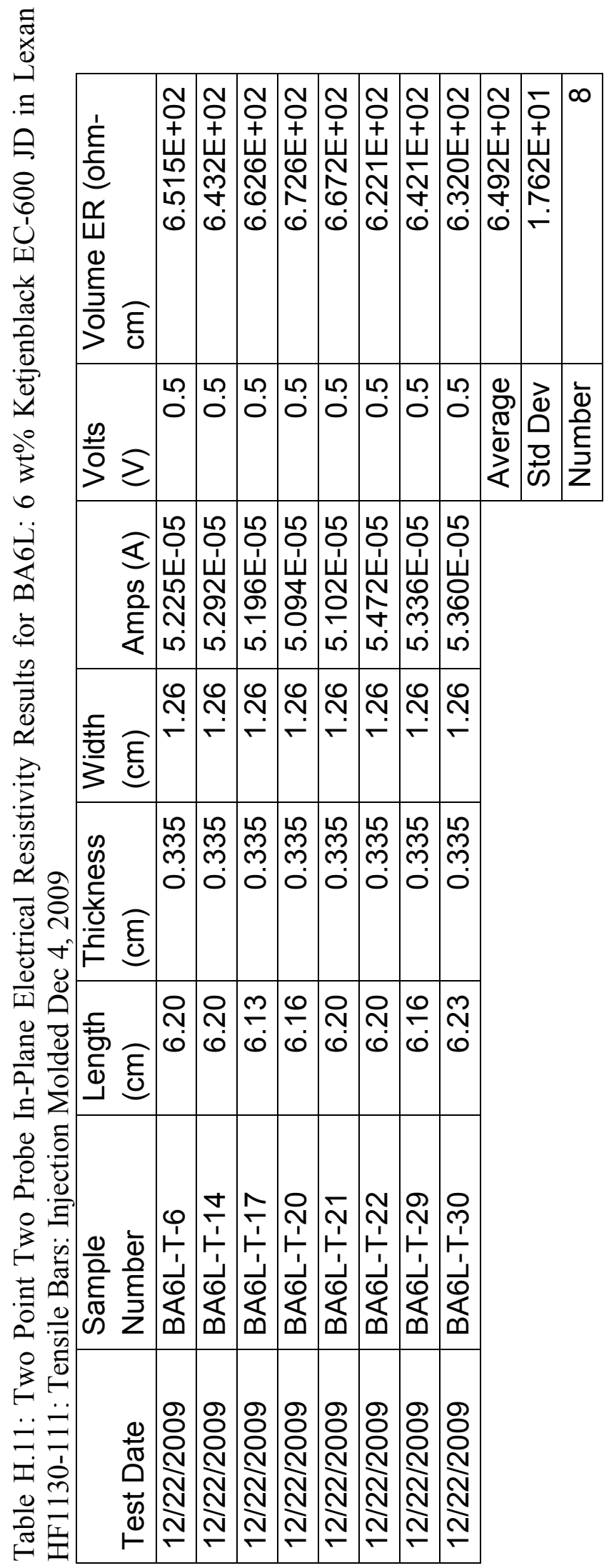




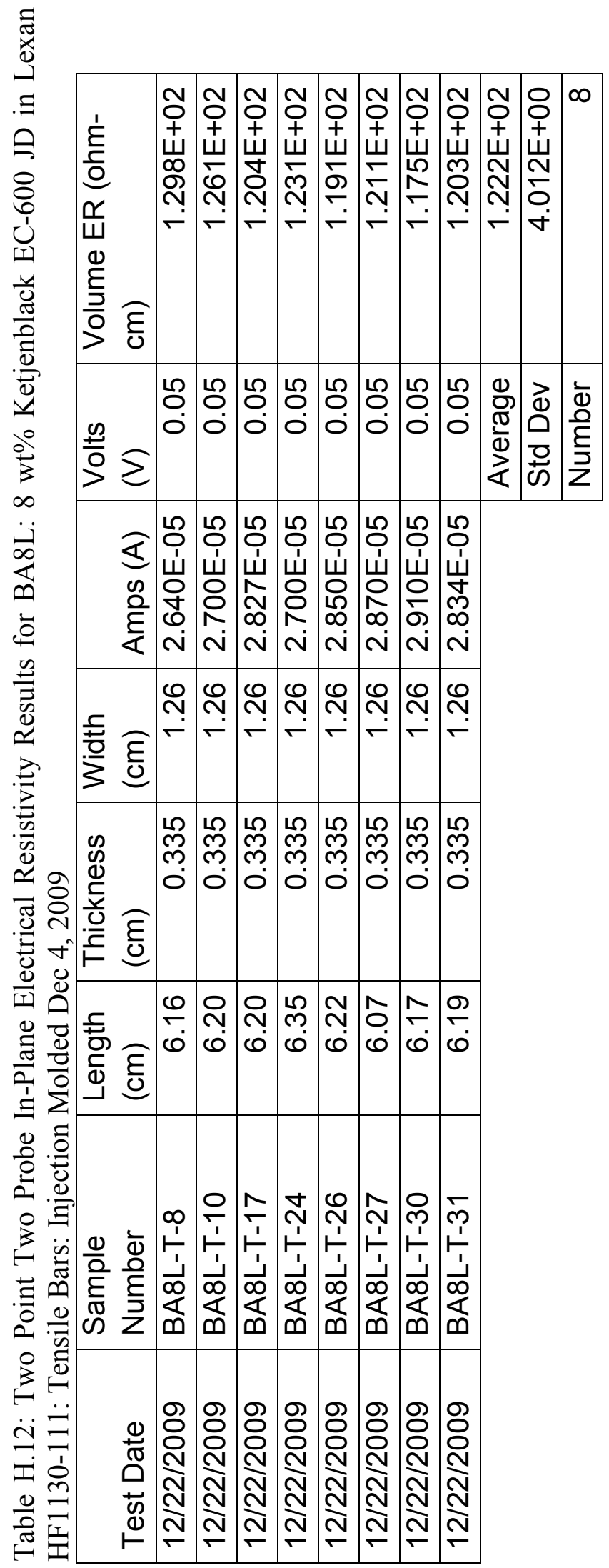




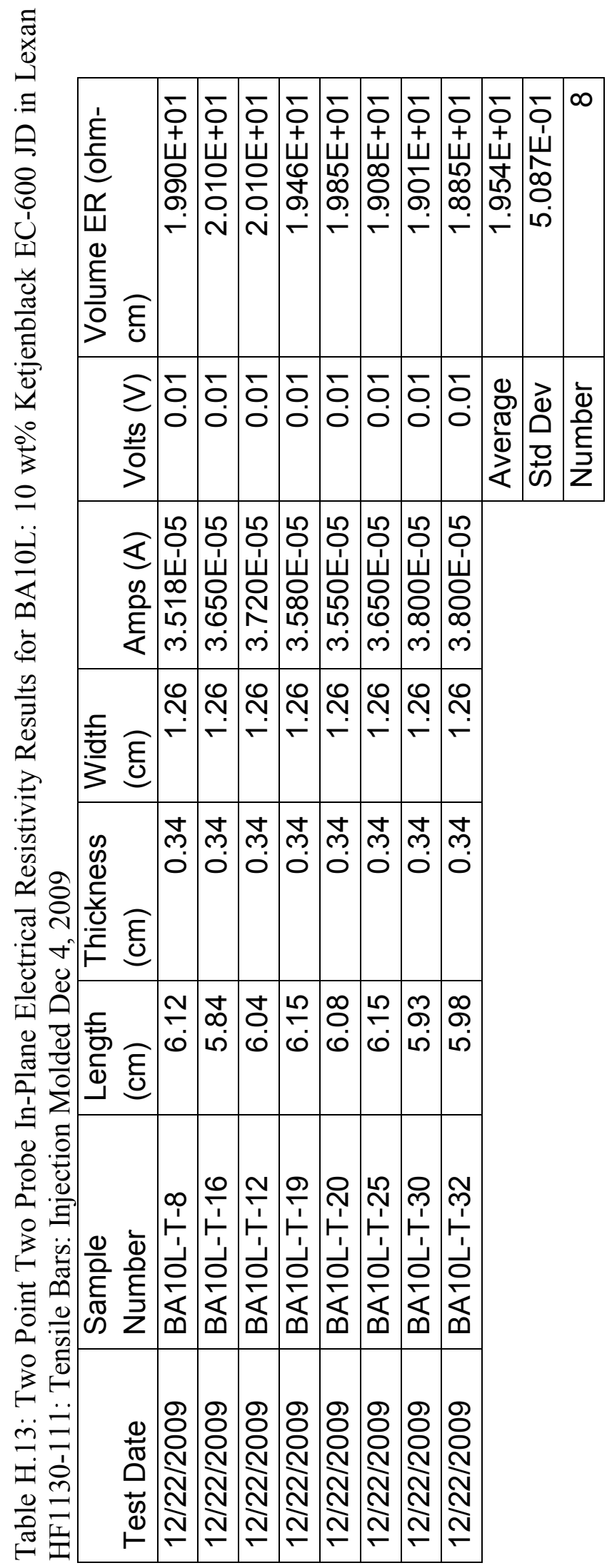




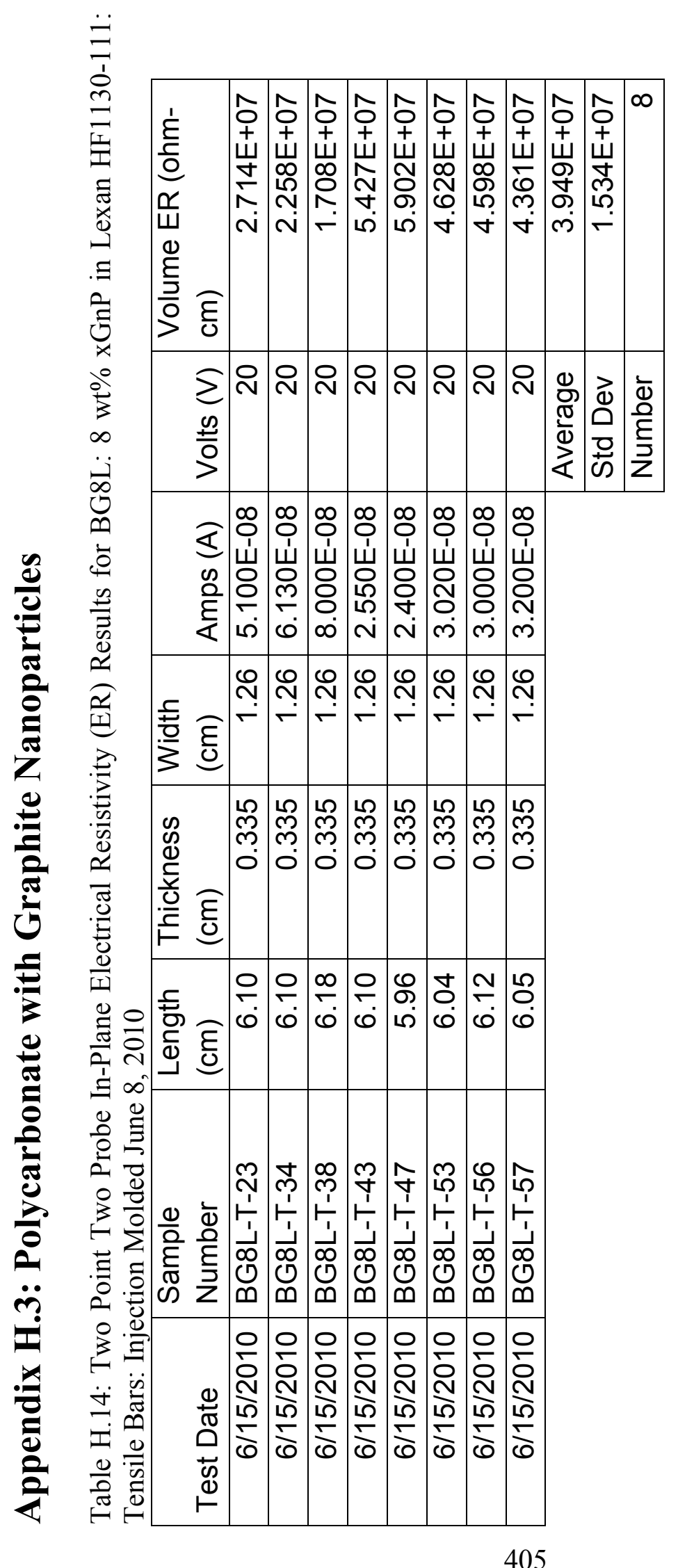




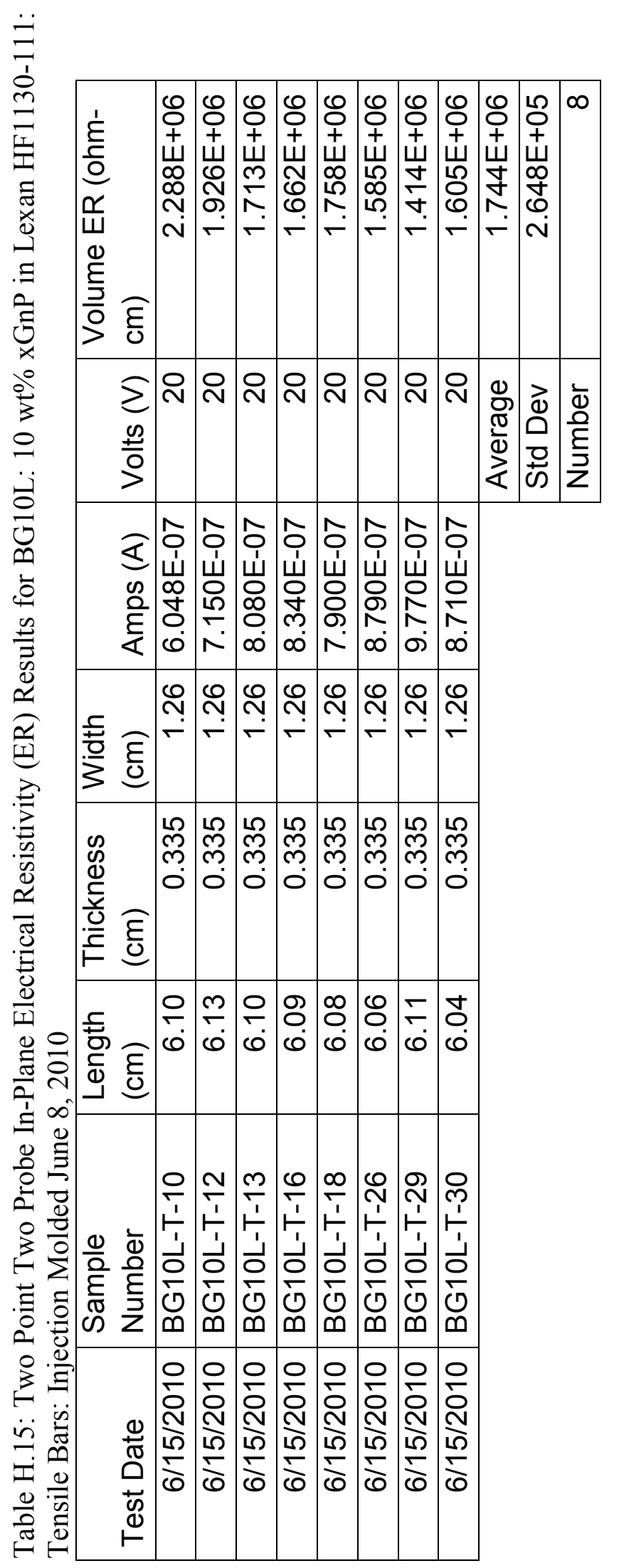




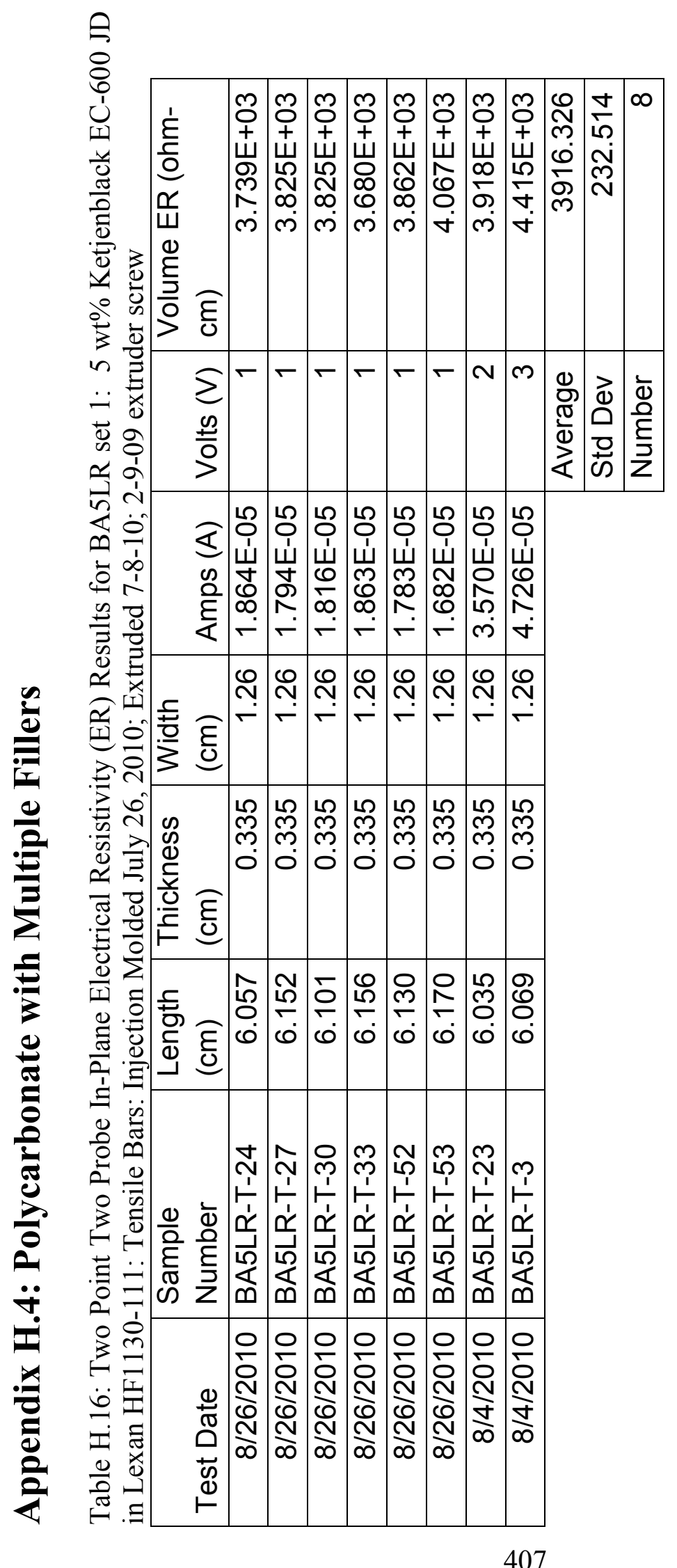




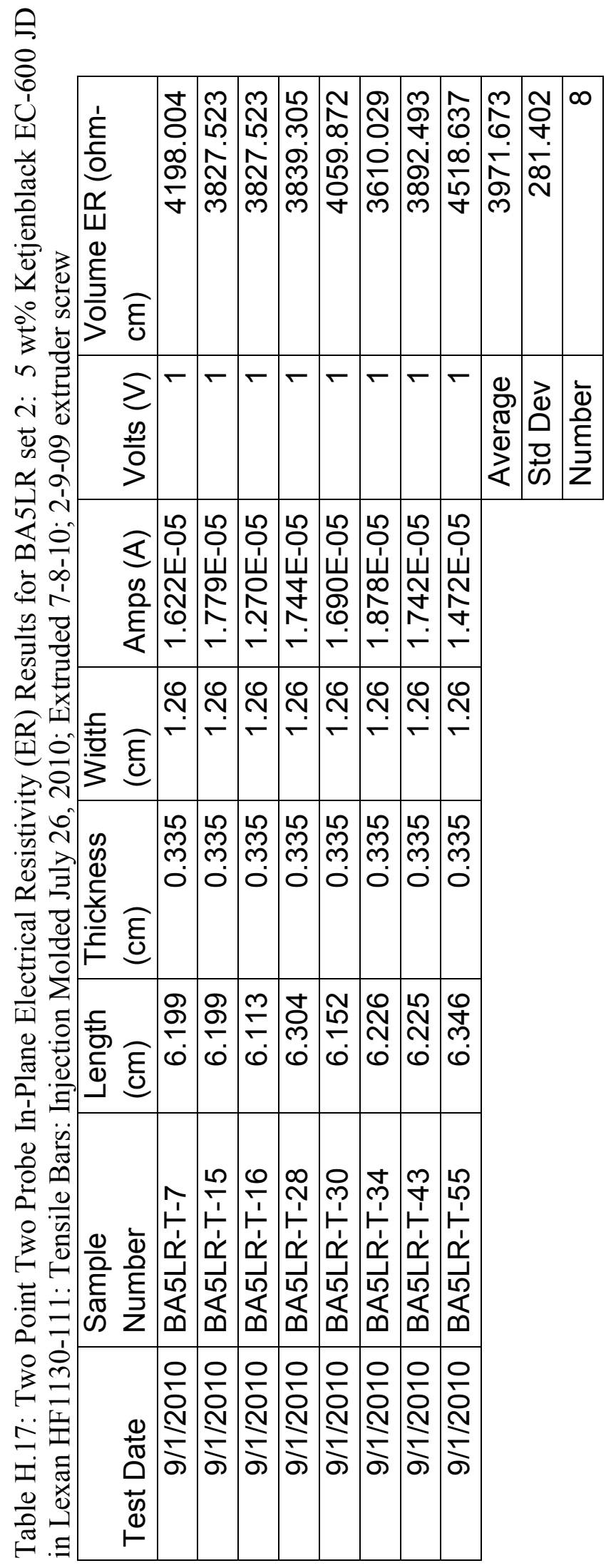



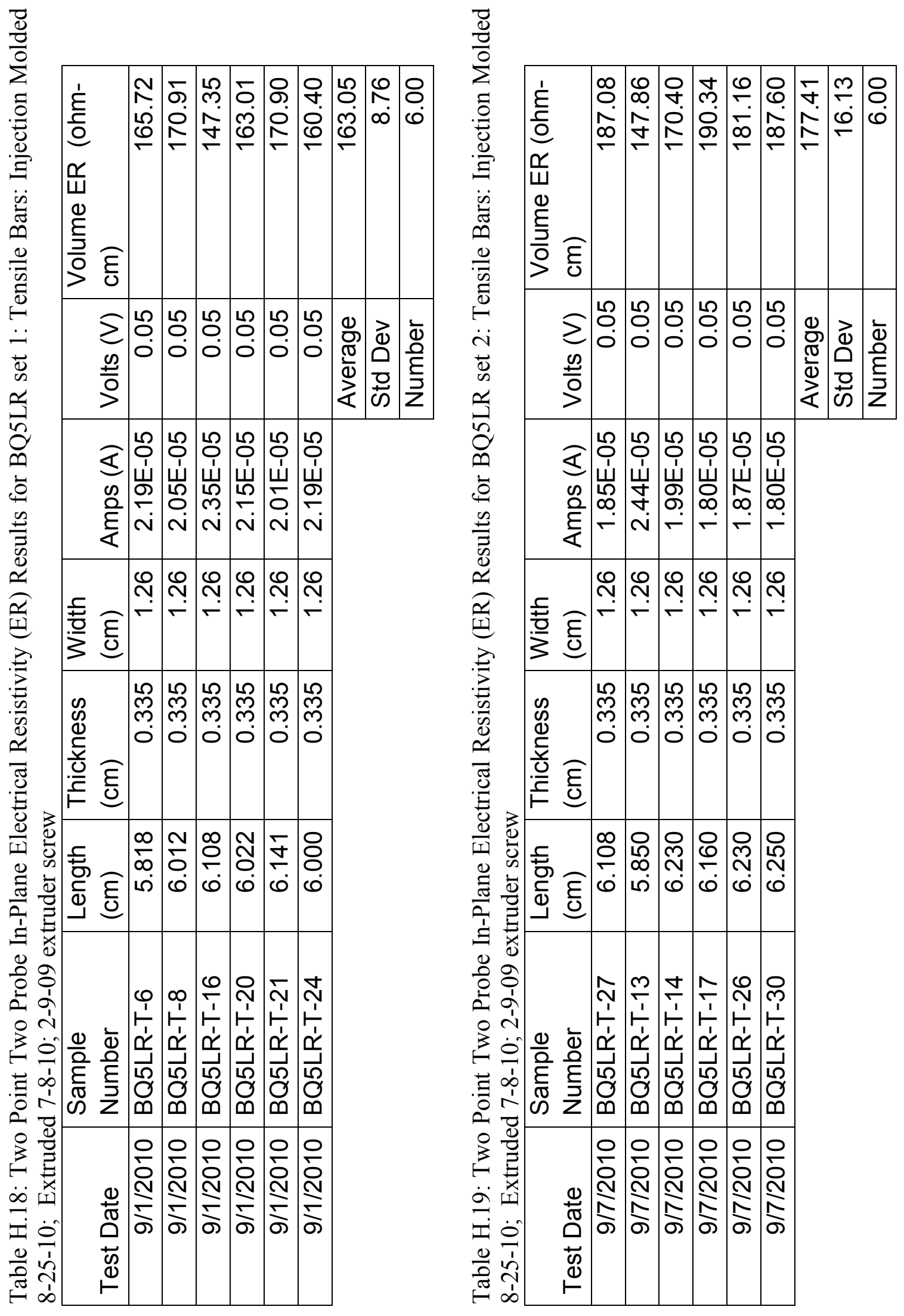


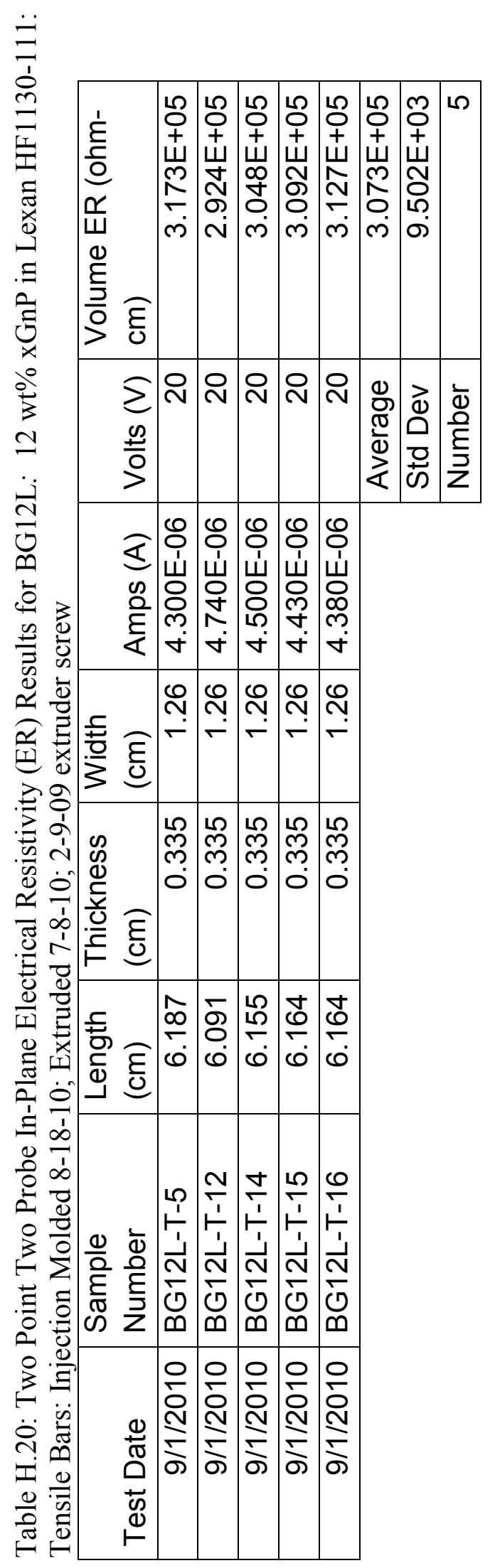




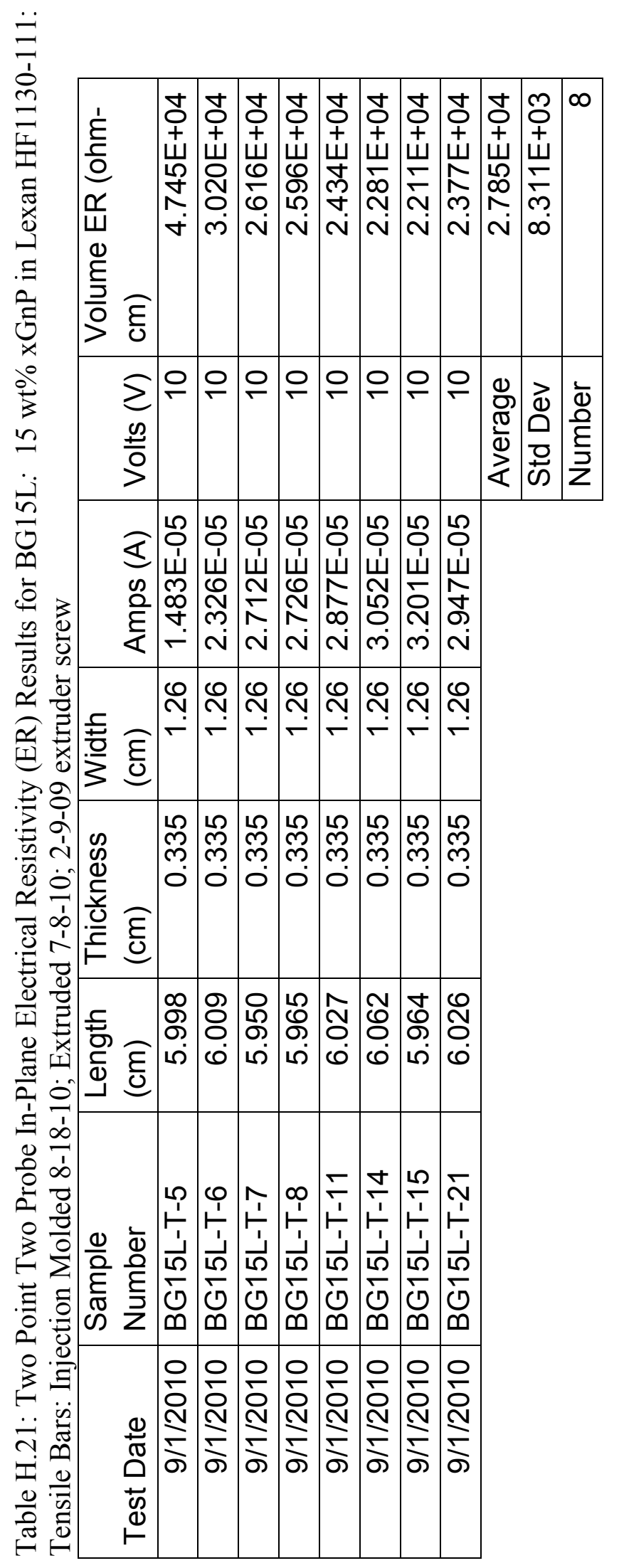




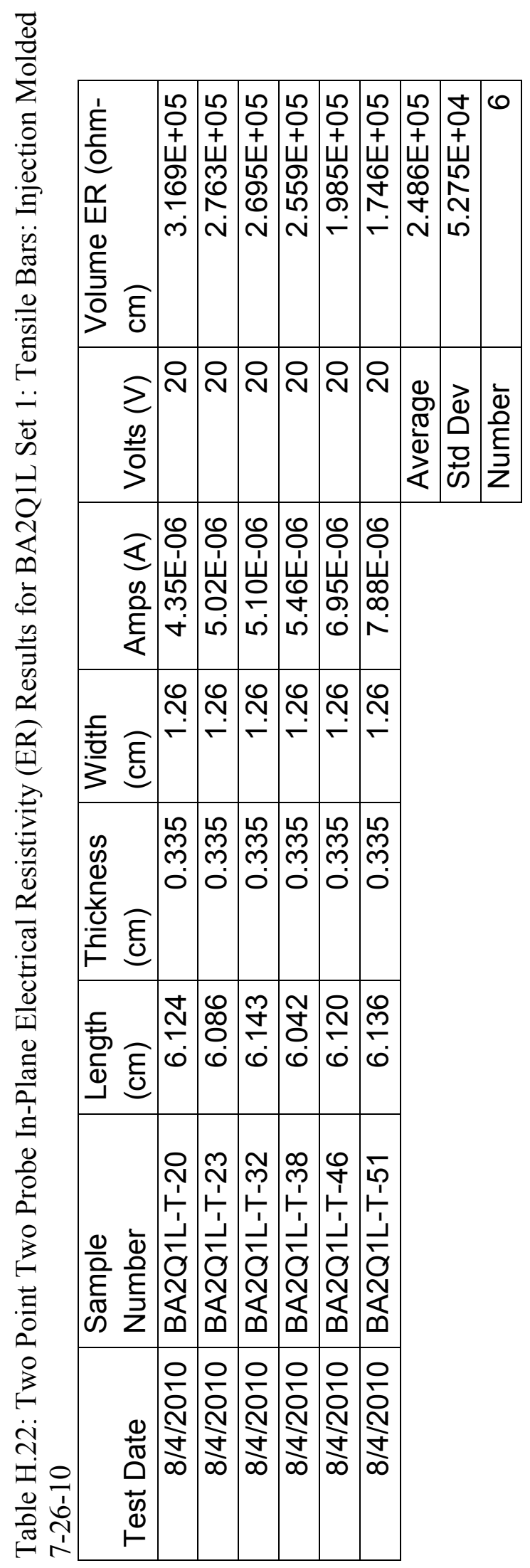




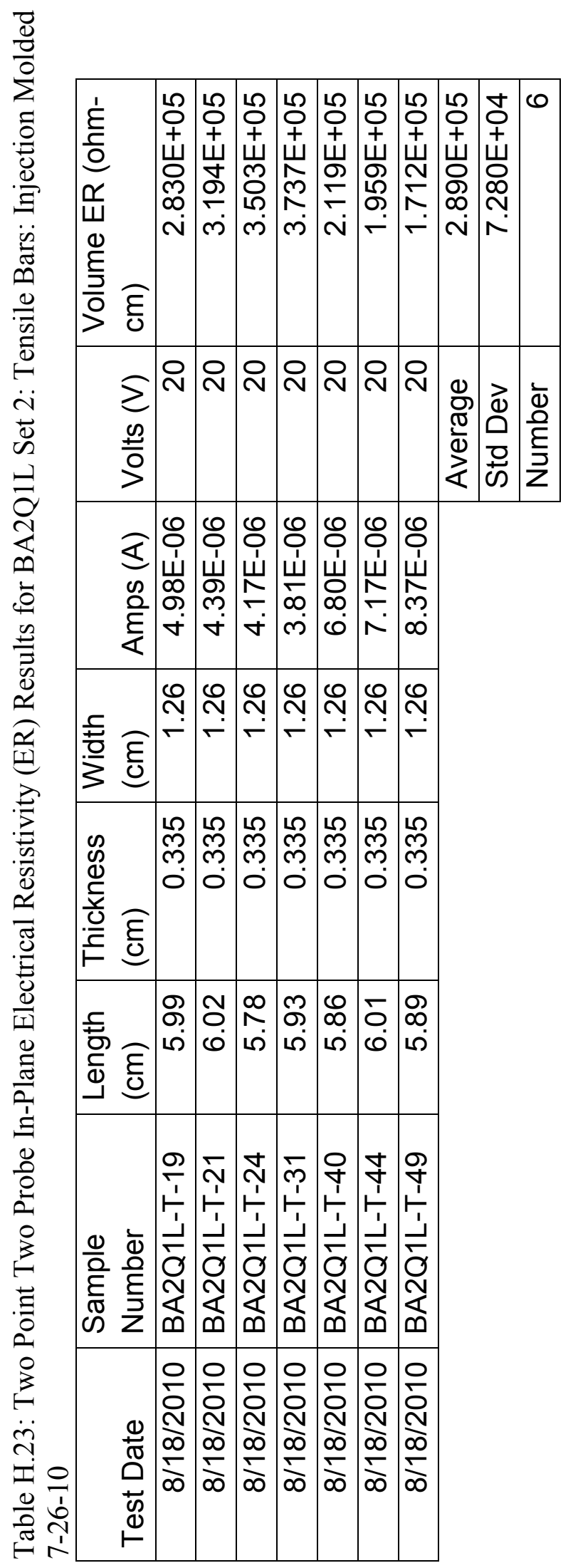



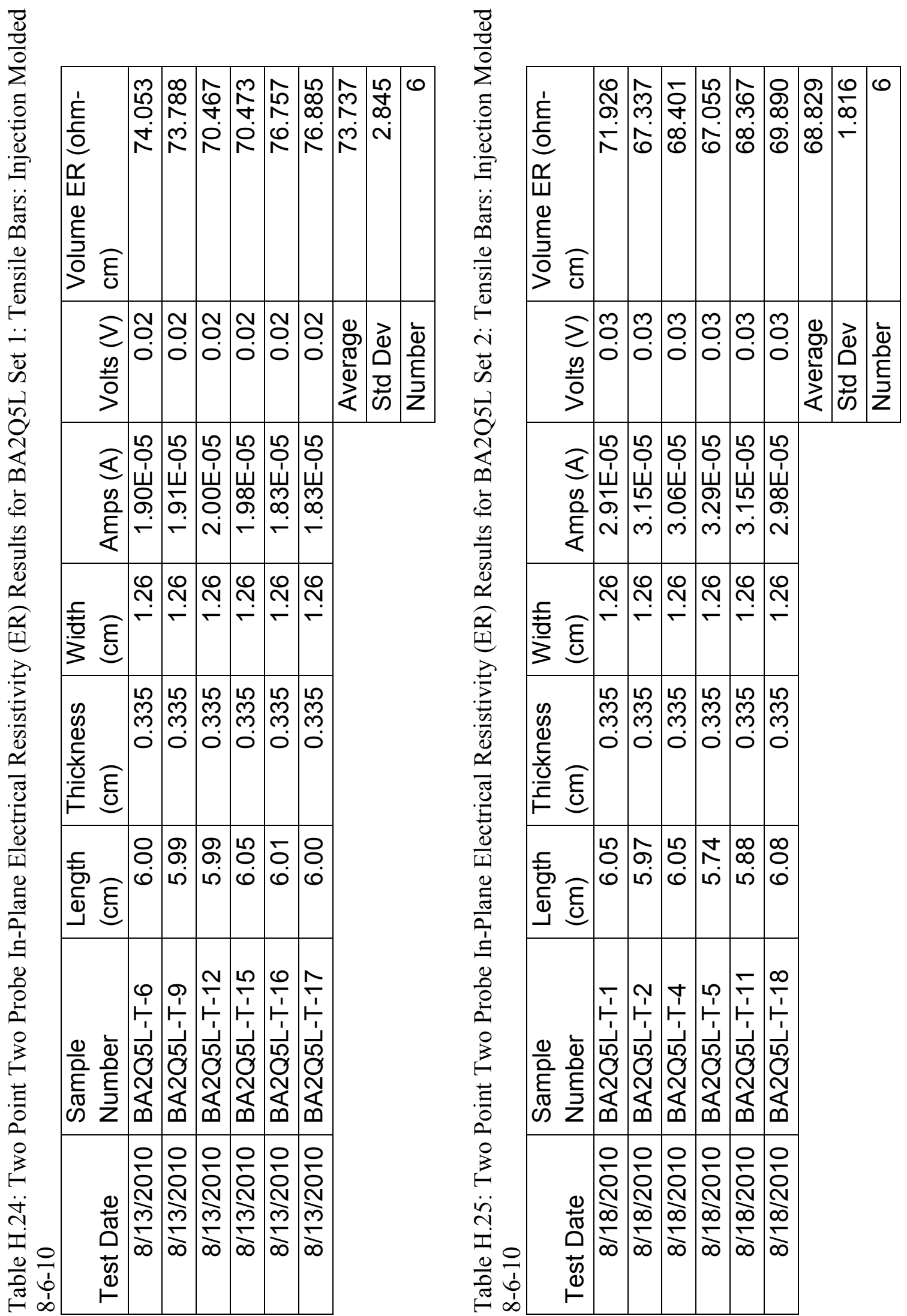

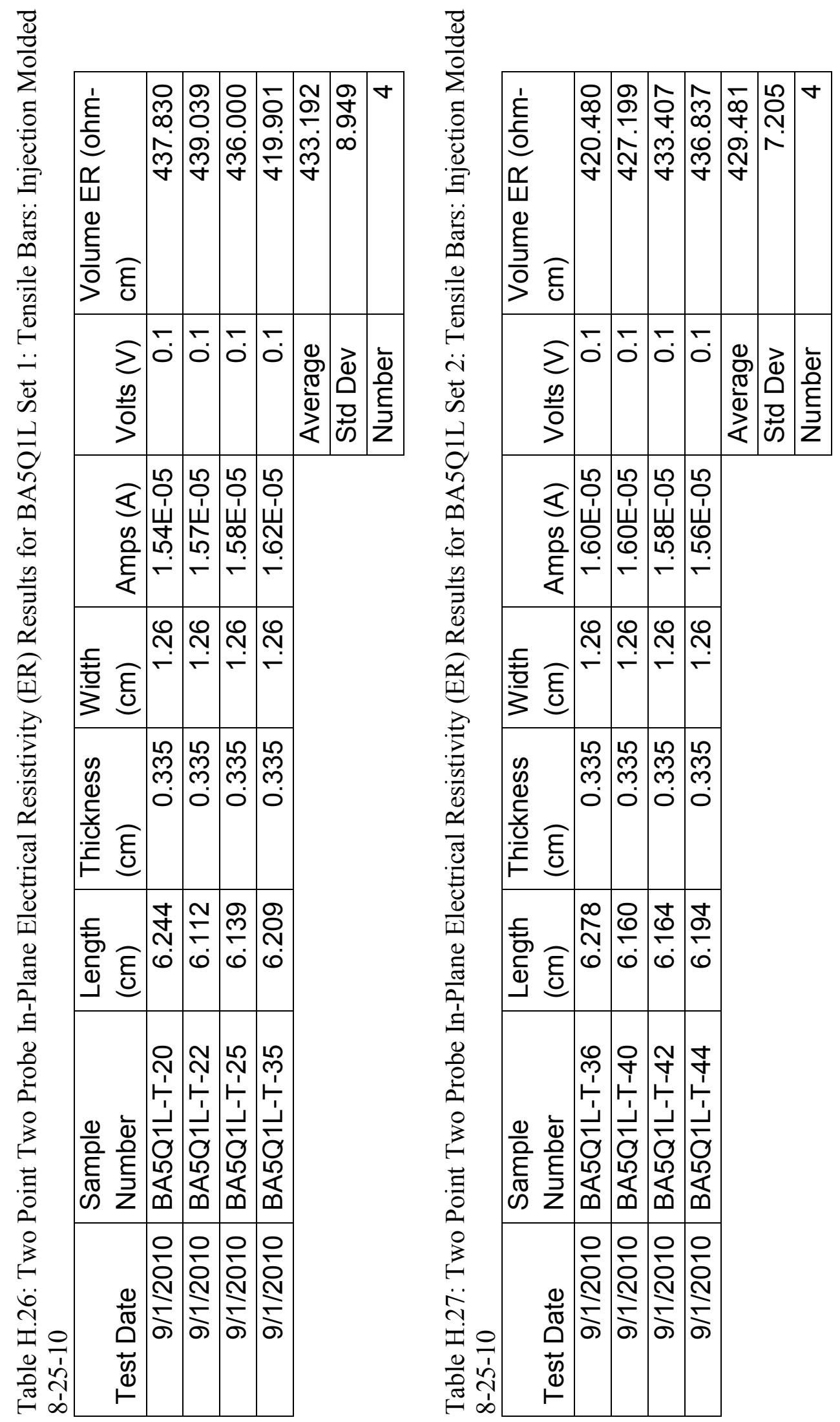


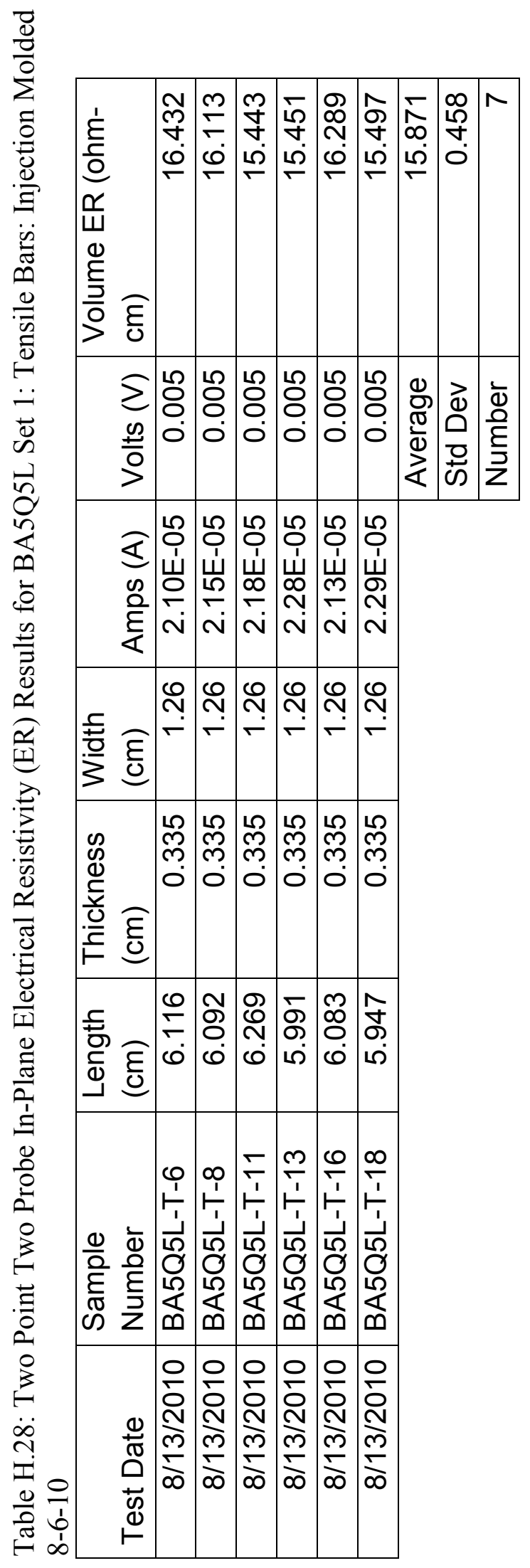



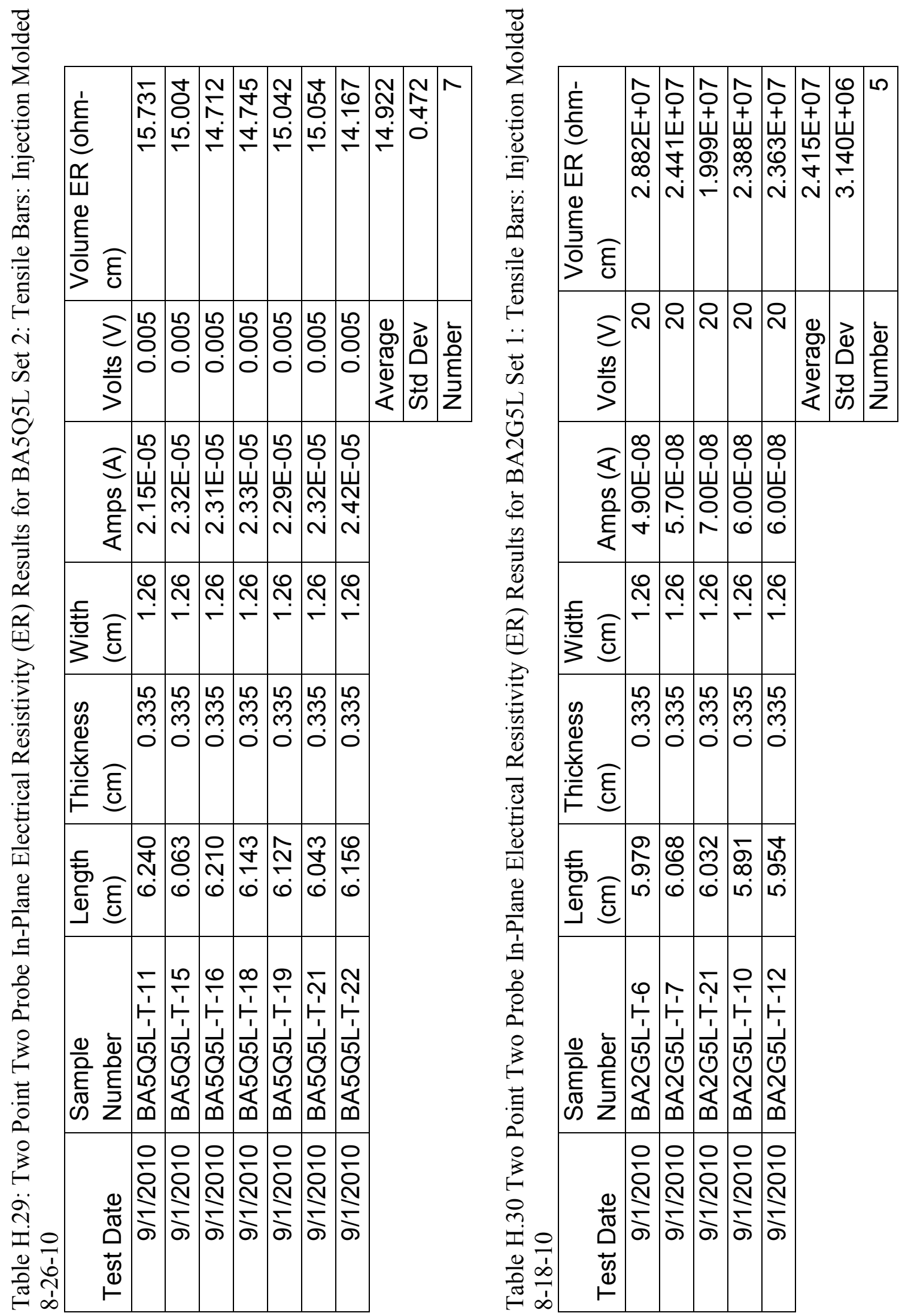

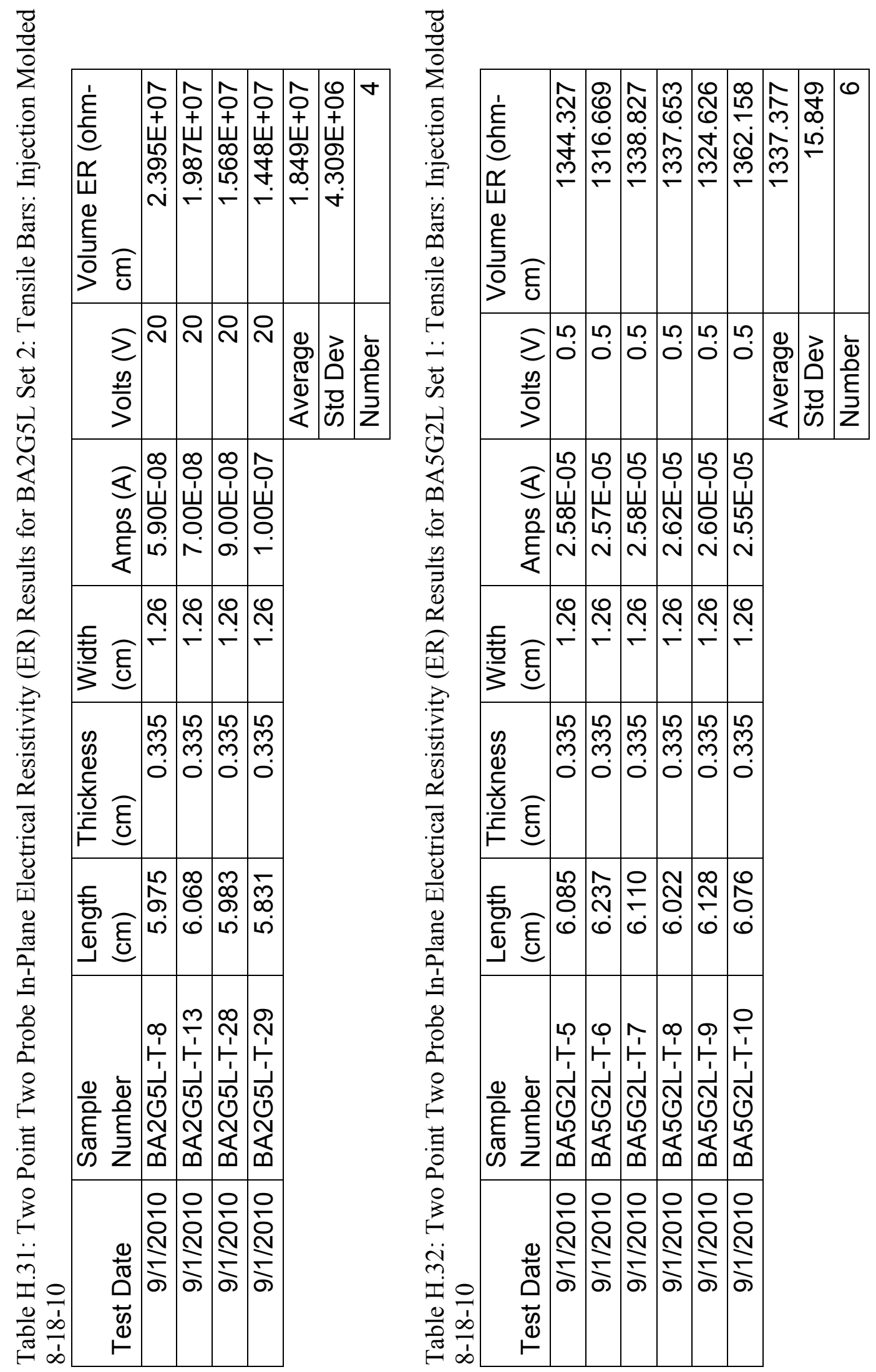

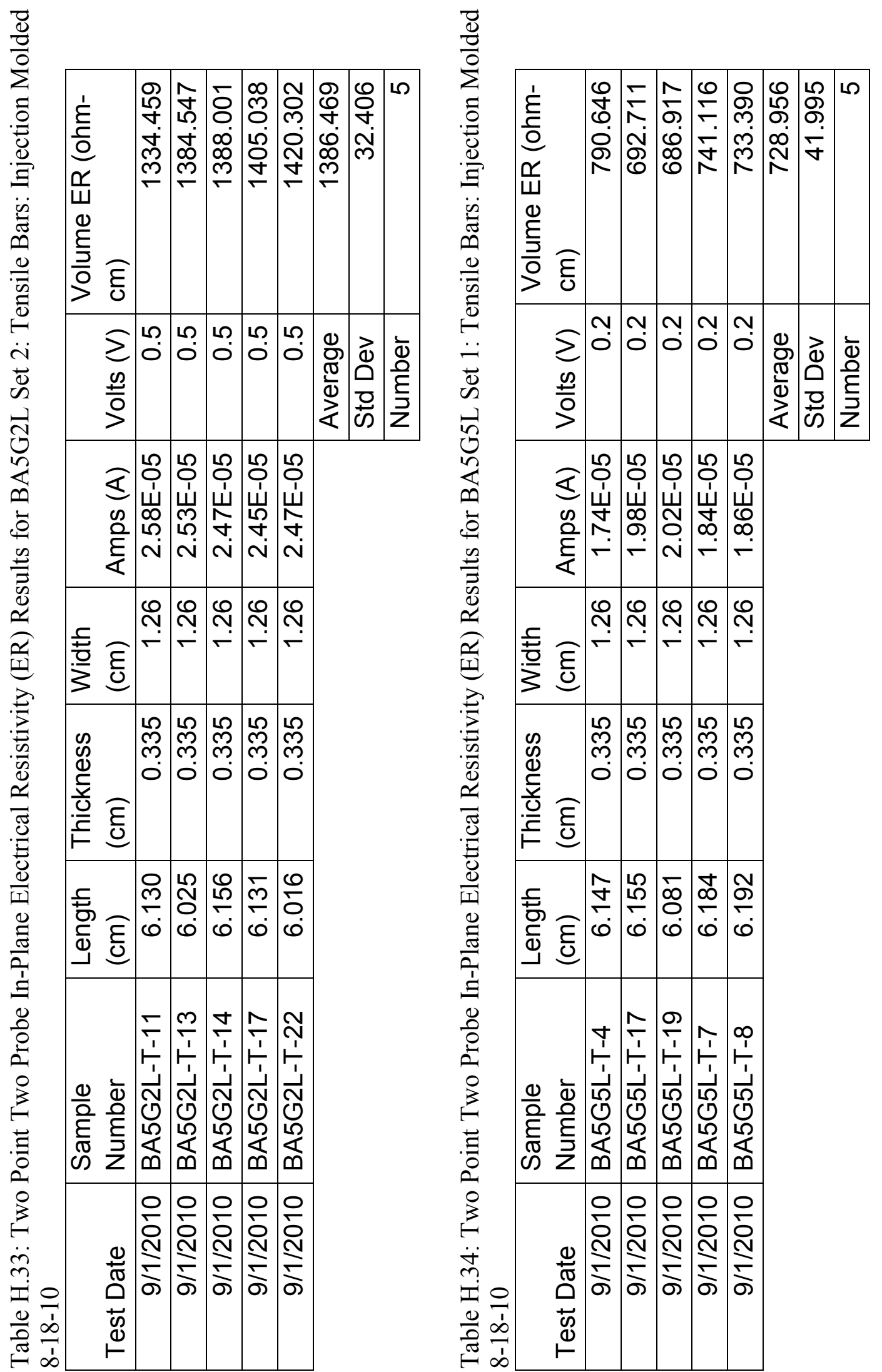


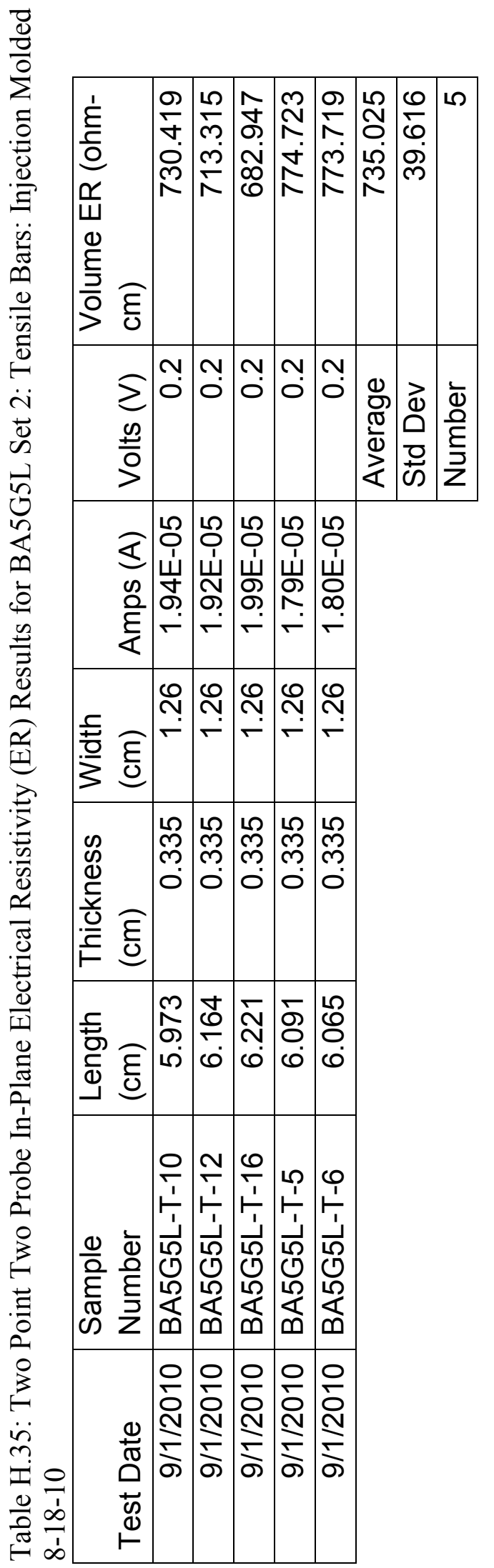

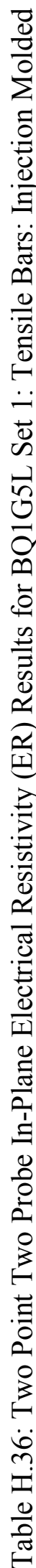

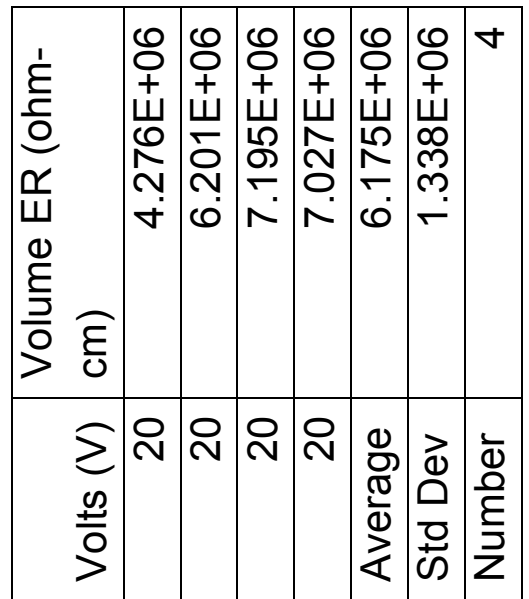

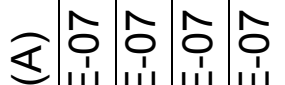

ఱ

ह

m $\sim \sim$

\begin{tabular}{ll|l|l|l|} 
& 0 \\
& 0 & 0 & 0 \\
\hline
\end{tabular}

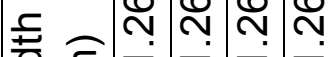

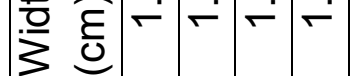

密 $>$

m ल

ल ल ल

孚

0

를

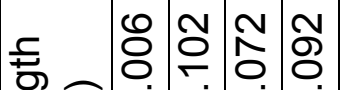

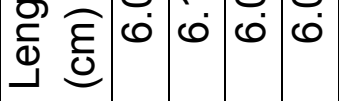

.

$\stackrel{\frac{1}{1}}{\frac{1}{1}} \frac{r_{1}}{r_{1}}$

(1) बे

을 을

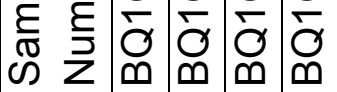

3 은 읃읃읃

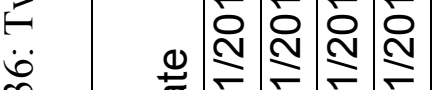

范

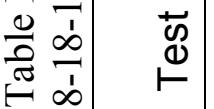



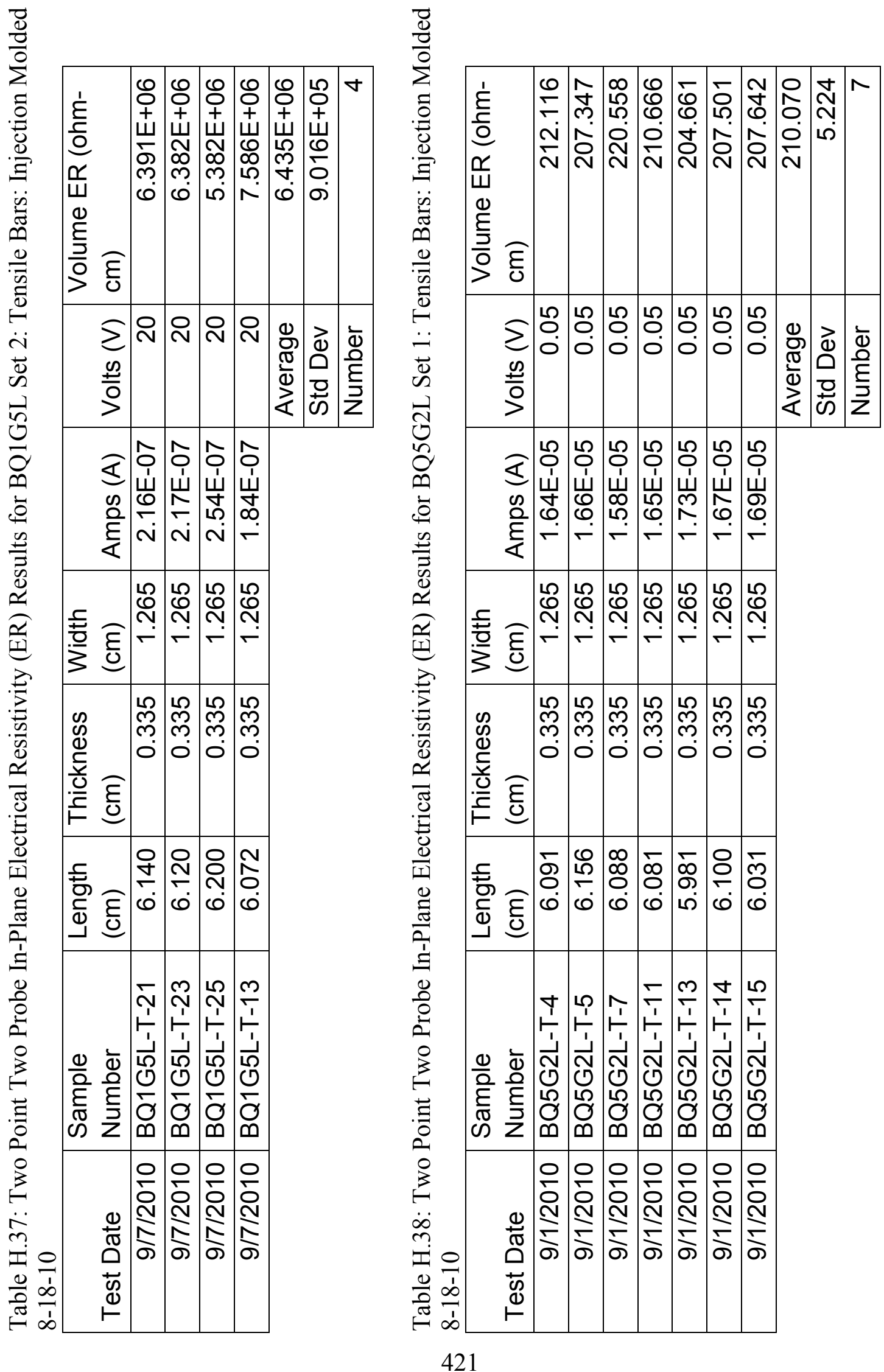

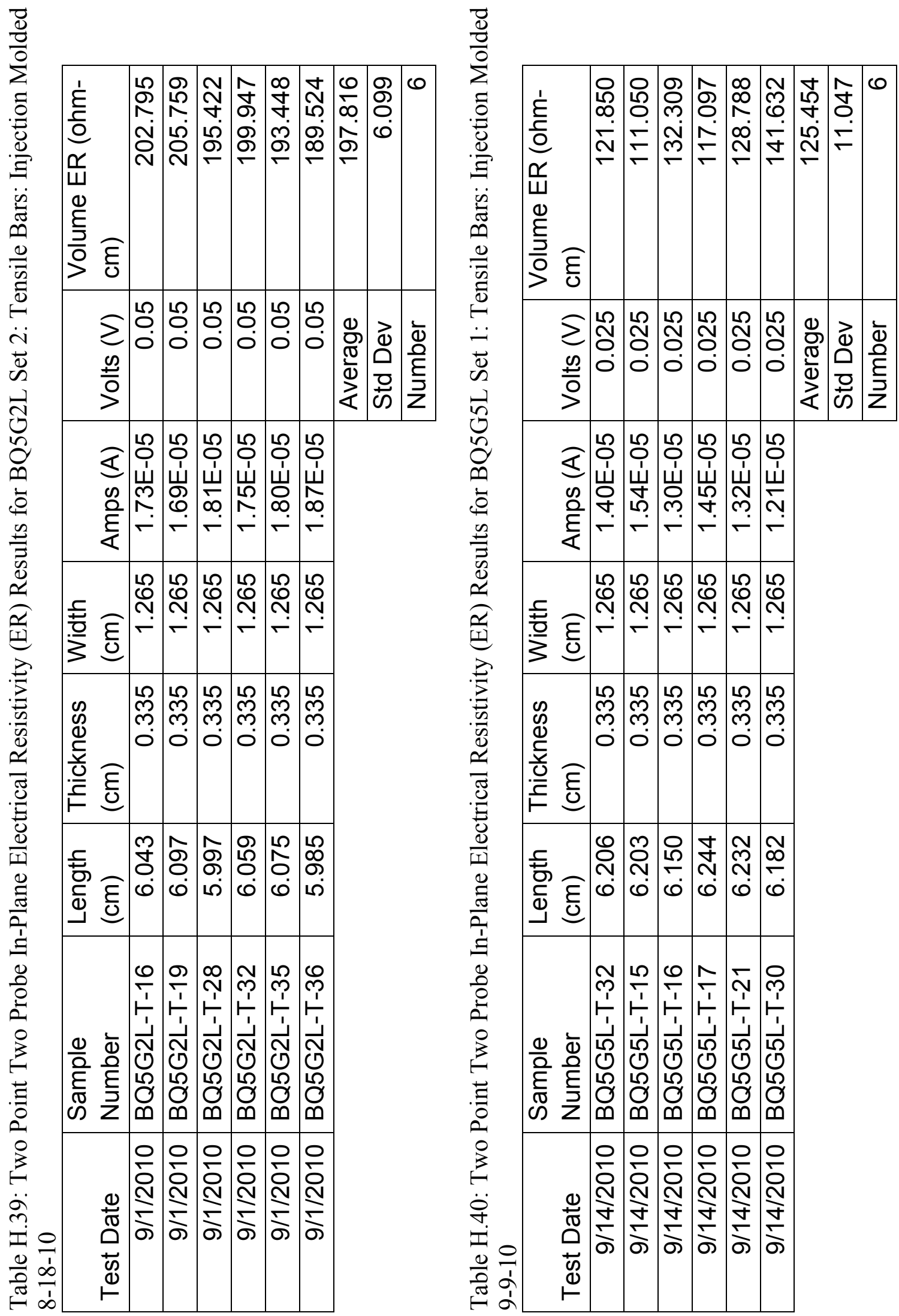


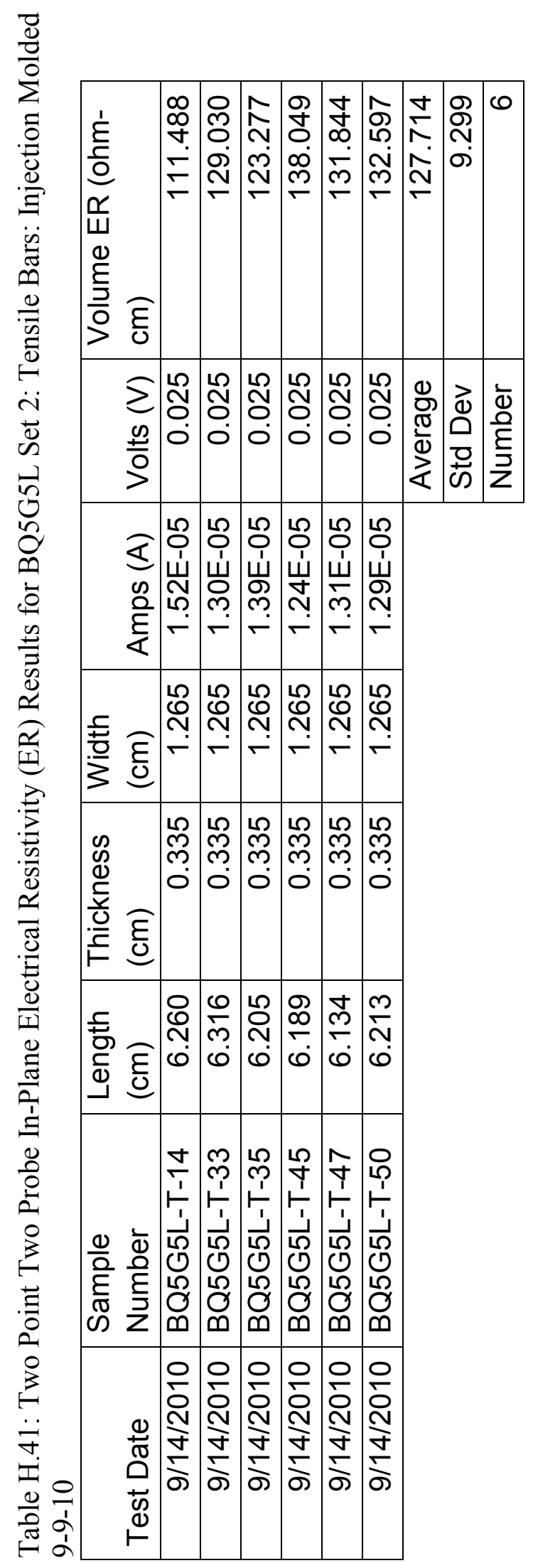




\section{Appendix I: Through-Plane Thermal Conductivity (ASTM F433) Test Results}

\section{Appendix I.1: Polycarbonate with Carbon Nanotubes}

Table I.1: Through-Plane Thermal Conductivity at $55^{\circ} \mathrm{C}$ using Holometrix TCA-300 Guarded Heat Flow Meter Method for Lexan HF1130-111 (BL): Injection Molded May 26,2009

\begin{tabular}{|c|c|c|}
\hline Test Date & Sample Number & $\begin{array}{c}\text { Through Plane Thermal } \\
\text { Conductivity }(\mathrm{W} / \mathrm{m} \cdot \mathrm{K})\end{array}$ \\
\hline $7 / 2 / 2009$ & BL-TC-10 & 0.2181 \\
\hline $7 / 2 / 2009$ & BL-TC-16 & 0.2167 \\
\hline $7 / 2 / 2009$ & BL-TC-22 & 0.2174 \\
\hline $7 / 2 / 2009$ & BL-TC-34 & 0.2181 \\
\hline $7 / 2 / 2009$ & BL-TC-37 & 0.2206 \\
\hline & Average & 0.2182 \\
\cline { 2 - 3 } & Standard Deviation & 0.0015 \\
\cline { 2 - 3 } & Number of Samples & 5 \\
\hline
\end{tabular}

Table I.2: Through-Plane Thermal Conductivity at $55^{\circ} \mathrm{C}$ using Holometrix TCA-300 Guarded Heat Flow Meter Method for Extruded Lexan HF1130-111 (BLE): Injection Molded May 26, 2009

\begin{tabular}{|c|c|c|}
\hline Test Date & Sample Number & $\begin{array}{c}\text { Through Plane Thermal } \\
\text { Conductivity }(\mathrm{W} / \mathrm{m} \cdot \mathrm{K})\end{array}$ \\
\hline $7 / 2 / 2009$ & BLE-TC-13 & 0.2161 \\
\hline $7 / 2 / 2009$ & BLE-TC-21 & 0.2161 \\
\hline $7 / 6 / 2009$ & BLE-TC-22 & 0.2180 \\
\hline $7 / 2 / 2009$ & BLE-TC-24 & 0.2170 \\
\hline $7 / 2 / 2009$ & BLE-TC-26 & 0.2178 \\
\hline & Average & 0.2170 \\
\cline { 2 - 3 } & Standard Deviation & 0.0009 \\
\cline { 2 - 3 } & Number of Samples & 5 \\
\hline
\end{tabular}


Table I.3: Through-Plane Thermal Conductivity at $55^{\circ} \mathrm{C}$ using Holometrix TCA-300 Guarded Heat Flow Meter Method for $2 \mathrm{wt} \%$ Hyperion Fibrils in Lexan HF1130-111 (BQ2L): Injection Molded May 26, 2009

\begin{tabular}{|c|c|c|}
\hline Test Date & Sample Number & $\begin{array}{c}\text { Through Plane Thermal } \\
\text { Conductivity }(\mathrm{W} / \mathrm{m} \cdot \mathrm{K})\end{array}$ \\
\hline $7 / 1 / 2009$ & BQ2L-TC-16 & 0.2293 \\
\hline $7 / 1 / 2009$ & BQ2L-TC-19 & 0.2334 \\
\hline $7 / 6 / 2009$ & BQ2L-TC-21 & 0.2307 \\
\hline $7 / 6 / 2009$ & BQ2L-TC-29 & 0.2333 \\
\hline $7 / 2 / 2009$ & BQ2L-TC-30 & 0.2329 \\
\hline & Average & 0.2319 \\
\cline { 2 - 3 } & Standard Deviation & 0.0018 \\
\cline { 2 - 3 } & Number of Samples & 5 \\
\hline
\end{tabular}

Table I.4: Through-Plane Thermal Conductivity at $55^{\circ} \mathrm{C}$ using Holometrix TCA-300 Guarded Heat Flow Meter Method for 3 wt $\%$ Hyperion Fibrils in Lexan HF1130-111 (BQ3L): Injection Molded May 26, 2009

\begin{tabular}{|c|c|c|}
\hline Test Date & Sample Number & $\begin{array}{c}\text { Through Plane Thermal } \\
\text { Conductivity }(\mathrm{W} / \mathrm{m} \cdot \mathrm{K})\end{array}$ \\
\hline $7 / 1 / 2009$ & BQ3L-TC-13 & 0.2372 \\
\hline $7 / 1 / 2009$ & BQ3L-TC-16 & 0.2373 \\
\hline $7 / 1 / 2009$ & BQ3L-TC-19 & 0.2411 \\
\hline $7 / 1 / 2009$ & BQ3L-TC-20 & 0.2431 \\
\hline $7 / 1 / 2009$ & BQ3L-TC-32 & 0.2445 \\
\hline & Average & 0.2406 \\
\cline { 2 - 3 } & Standard Deviation & 0.0033 \\
\cline { 2 - 3 } & Number of Samples & 5 \\
\hline
\end{tabular}


Table I.5: Through-Plane Thermal Conductivity at $55^{\circ} \mathrm{C}$ using Holometrix TCA-300 Guarded Heat Flow Meter Method for $4 \mathrm{wt} \%$ Hyperion Fibrils in Lexan HF1130-111 (BQ4L): Injection Molded May 26, 2009

\begin{tabular}{|c|c|c|}
\hline Test Date & Sample Number & $\begin{array}{c}\text { Through Plane Thermal } \\
\text { Conductivity }(\mathrm{W} / \mathrm{m} \cdot \mathrm{K})\end{array}$ \\
\hline $7 / 1 / 2009$ & BQ4L-TC-13 & 0.2533 \\
\hline $7 / 6 / 2009$ & BQ4L-TC-19 & 0.2540 \\
\hline $7 / 1 / 2009$ & BQ4L-TC-24 & 0.2560 \\
\hline $7 / 1 / 2009$ & BQ4L-TC-29 & 0.2542 \\
\hline $7 / 1 / 2009$ & BQ4L-TC-32 & 0.2565 \\
\hline & Average & 0.2548 \\
\cline { 2 - 3 } & Standard Deviation & 0.0014 \\
\cline { 2 - 3 } & Number of Samples & 5 \\
\hline
\end{tabular}

Table I.6: Through-Plane Thermal Conductivity at $55^{\circ} \mathrm{C}$ using Holometrix TCA-300 Guarded Heat Flow Meter Method for 5 wt $\%$ Hyperion Fibrils in Lexan HF1130-111 (BQ5L): Injection Molded May 26, 2009

\begin{tabular}{|c|c|c|}
\hline Test Date & Sample Number & $\begin{array}{c}\text { Through Plane Thermal } \\
\text { Conductivity }(\mathrm{W} / \mathrm{m} \cdot \mathrm{K})\end{array}$ \\
\hline $6 / 30 / 2009$ & BQ5L-TC-15 & 0.2684 \\
\hline $7 / 6 / 2009$ & BQ5L-TC-19 & 0.2612 \\
\hline $6 / 30 / 2009$ & BQ5L-TC-21 & 0.2700 \\
\hline $6 / 30 / 2009$ & BQ5L-TC-27 & 0.2646 \\
\hline $6 / 30 / 2009$ & BQ5L-TC-30 & 0.2660 \\
\hline & Average & 0.2660 \\
\cline { 2 - 3 } & Standard Deviation & 0.0034 \\
\cline { 2 - 3 } & Number of Samples & 5 \\
\hline
\end{tabular}


Table I.7: Through-Plane Thermal Conductivity at $55^{\circ} \mathrm{C}$ using Holometrix TCA-300 Guarded Heat Flow Meter Method for 6 wt\% Hyperion Fibrils in Lexan HF1130-111 (BQ6L): Injection Molded May 26, 2009

\begin{tabular}{|c|c|c|}
\hline Test Date & Sample Number & $\begin{array}{c}\text { Through Plane Thermal } \\
\text { Conductivity }(\mathrm{W} / \mathrm{m} \cdot \mathrm{K})\end{array}$ \\
\hline $6 / 30 / 2009$ & BQ6L-TC-12 & 0.2717 \\
\hline $6 / 30 / 2009$ & BQ6L-TC-15 & 0.2721 \\
\hline $6 / 30 / 2009$ & BQ6L-TC-20 & 0.2772 \\
\hline $6 / 30 / 2009$ & BQ6L-TC-29 & 0.2766 \\
\hline $6 / 30 / 2009$ & BQ6L-TC-33 & 0.2761 \\
\hline & Average & 0.2747 \\
\cline { 2 - 3 } & Standard Deviation & 0.0026 \\
\cline { 2 - 3 } & Number of Samples & 5 \\
\hline
\end{tabular}

Table I.8: Through-Plane Thermal Conductivity at $55^{\circ} \mathrm{C}$ using Holometrix TCA-300 Guarded Heat Flow Meter Method for $8 \mathrm{wt} \%$ Hyperion Fibrils in Lexan HF1130-111 (BQ8L): Injection Molded May 26, 2009

\begin{tabular}{|c|c|c|}
\hline Test Date & Sample Number & $\begin{array}{c}\text { Through Plane Thermal } \\
\text { Conductivity }(\mathrm{W} / \mathrm{m} \cdot \mathrm{K})\end{array}$ \\
\hline $7 / 6 / 2009$ & BQ8L-TC-18 & 0.3085 \\
\hline $6 / 30 / 2009$ & BQ8L-TC-21 & 0.3079 \\
\hline $6 / 30 / 2009$ & BQ8L-TC-22 & 0.3040 \\
\hline $6 / 30 / 2009$ & BQ8L-TC-26 & 0.3008 \\
\hline $6 / 30 / 2009$ & BQ8L-TC-35 & 0.3078 \\
\hline & Average & 0.3058 \\
\cline { 2 - 3 } & Standard Deviation & 0.0033 \\
\cline { 2 - 3 } & Number of Samples & 5 \\
\hline
\end{tabular}




\section{Appendix I.2: Polycarbonate with Carbon Black}

Table I.9: Through-Plane Thermal Conductivity at $55^{\circ} \mathrm{C}$ using Holometrix TCA-300 Guarded Heat Flow Meter Method for Lexan HF1130-111 (BL2): Injection Molded December 4, 2009

\begin{tabular}{|c|c|c|}
\hline Test Date & Sample Number & $\begin{array}{c}\text { Through Plane Thermal } \\
\text { Conductivity }(\mathrm{W} / \mathrm{m} \cdot \mathrm{K})\end{array}$ \\
\hline $12 / 21 / 2009$ & BL2-TC-19 & 0.2149 \\
\hline $12 / 21 / 2009$ & BL2-TC-37 & 0.2126 \\
\hline $12 / 21 / 2009$ & BL2-TC-10 & 0.2126 \\
\hline $12 / 21 / 2009$ & BL2-TC-24 & 0.2141 \\
\hline $12 / 21 / 2009$ & BL2-TC-30 & 0.2136 \\
\hline & Average & 0.2136 \\
\cline { 2 - 3 } & Standard Deviation & 0.0010 \\
\cline { 2 - 3 } & Number of Samples & 5 \\
\hline
\end{tabular}

Table I.10: Through-Plane Thermal Conductivity at $55^{\circ} \mathrm{C}$ using Holometrix TCA-300 Guarded Heat Flow Meter Method for Extruded Lexan HF1130-111 (BLE2): Injection Molded Dec 4, 2009

\begin{tabular}{|c|c|c|}
\hline Test Date & Sample Number & $\begin{array}{c}\text { Through Plane Thermal } \\
\text { Conductivity }(\mathrm{W} / \mathrm{m} \bullet \mathrm{K})\end{array}$ \\
\hline $12 / 21 / 2009$ & BLE2-TC-12 & 0.2140 \\
\hline $12 / 21 / 2009$ & BLE2-TC-16 & 0.2150 \\
\hline $12 / 21 / 2009$ & BLE2-TC-23 & 0.2151 \\
\hline $12 / 21 / 2009$ & BLE2-TC-22 & 0.2157 \\
\hline $12 / 21 / 2009$ & BLE2-TC-13 & 0.2124 \\
\hline & Average & 0.2144 \\
\cline { 2 - 3 } & Standard Deviation & 0.0013 \\
\cline { 2 - 3 } & Number of Samples & 5 \\
\hline
\end{tabular}


Table I.11: Through-Plane Thermal Conductivity at $55^{\circ} \mathrm{C}$ using Holometrix TCA-300 Guarded Heat Flow Meter Method for 2 wt\% Ketjenblack EC-600 JD in Lexan HF1130111 (BA2L): Injection Molded Dec 4, 2009

\begin{tabular}{|c|c|c|}
\hline Test Date & Sample Number & $\begin{array}{c}\text { Through Plane Thermal } \\
\text { Conductivity }(\mathrm{W} / \mathrm{m} \cdot \mathrm{K})\end{array}$ \\
\hline $12 / 22 / 2009$ & BA2L-TC-26 & 0.2268 \\
\hline $12 / 22 / 2009$ & BA2L-TC-16 & 0.2276 \\
\hline $12 / 22 / 2009$ & BA2L-TC-15 & 0.2291 \\
\hline $12 / 22 / 2009$ & BA2L-TC-29 & 0.2282 \\
\hline $12 / 22 / 2009$ & BA2L-TC-12 & 0.2284 \\
\hline & Average & 0.2280 \\
\cline { 2 - 3 } & Standard Deviation & 0.0009 \\
\cline { 2 - 3 } & Number of Samples & 5 \\
\hline
\end{tabular}

Table I.12: Through-Plane Thermal Conductivity at $55^{\circ} \mathrm{C}$ using Holometrix TCA-300 Guarded Heat Flow Meter Method for 3 wt\% Ketjenblack EC-600 JD in Lexan HF1130111 (BA3L): Injection Molded Dec 4, 2009

\begin{tabular}{|c|c|c|}
\hline Test Date & Sample Number & $\begin{array}{c}\text { Through Plane Thermal } \\
\text { Conductivity }(\mathrm{W} / \mathrm{m} \cdot \mathrm{K})\end{array}$ \\
\hline $12 / 22 / 2009$ & BA3L-TC-10 & 0.2363 \\
\hline $12 / 22 / 2009$ & BA3L-TC-19 & 0.2319 \\
\hline $12 / 22 / 2009$ & BA3L-TC-12 & 0.2331 \\
\hline $12 / 22 / 2009$ & BA3L-TC-27 & 0.2355 \\
\hline & Average & 0.2342 \\
\cline { 2 - 3 } & Standard Deviation & 0.0020 \\
\cline { 2 - 3 } & Number of Samples & 4 \\
\hline
\end{tabular}

Table I.13: Through-Plane Thermal Conductivity at $55^{\circ} \mathrm{C}$ using Holometrix TCA-300 Guarded Heat Flow Meter Method for 4 wt\% Ketjenblack EC-600 JD in Lexan HF1130111 (BA4L): Injection Molded Dec 4, 2009

\begin{tabular}{|c|c|c|}
\hline Test Date & Sample Number & $\begin{array}{c}\text { Through Plane Thermal } \\
\text { Conductivity }(\mathrm{W} / \mathrm{m} \cdot \mathrm{K})\end{array}$ \\
\hline $12 / 23 / 2009$ & BA4L-TC-11 & 0.2453 \\
\hline $12 / 23 / 2009$ & BA4L-TC-15 & 0.2451 \\
\hline $12 / 23 / 2009$ & BA4L-TC-17 & 0.2444 \\
\hline $12 / 23 / 2009$ & BA4L-TC-22 & 0.2448 \\
\hline & Average & 0.2449 \\
\cline { 2 - 3 } & Standard Deviation & 0.0004 \\
\cline { 2 - 3 } & Number of Samples & 4 \\
\hline
\end{tabular}


Table I.14: Through-Plane Thermal Conductivity at $55^{\circ} \mathrm{C}$ using Holometrix TCA-300 Guarded Heat Flow Meter Method for 5 wt\% Ketjenblack EC-600 JD in Lexan HF1130111 (BA5L): Injection Molded Dec 4, 2009

\begin{tabular}{|c|c|c|}
\hline Test Date & Sample Number & $\begin{array}{c}\text { Through Plane Thermal } \\
\text { Conductivity }(\mathrm{W} / \mathrm{m} \cdot \mathrm{K})\end{array}$ \\
\hline $12 / 23 / 2009$ & BA5L-TC-13 & 0.2541 \\
\hline $12 / 23 / 2009$ & BA5L-TC-20 & 0.2551 \\
\hline $12 / 23 / 2009$ & BA5L-TC-23 & 0.2525 \\
\hline $12 / 23 / 2009$ & BA5L-TC-26 & 0.2533 \\
\hline & Average & 0.2538 \\
\cline { 2 - 3 } & Standard Deviation & 0.0011 \\
\cline { 2 - 3 } & Number of Samples & 4 \\
\hline
\end{tabular}

Table I.15: Through-Plane Thermal Conductivity at $55^{\circ} \mathrm{C}$ using Holometrix TCA-300 Guarded Heat Flow Meter Method for 6 wt\% Ketjenblack EC-600 JD in Lexan HF1130111 (BA6L): Injection Molded Dec 4, 2009

\begin{tabular}{|c|c|c|}
\hline Test Date & Sample Number & $\begin{array}{c}\text { Through Plane Thermal } \\
\text { Conductivity }(\mathrm{W} / \mathrm{m} \cdot \mathrm{K})\end{array}$ \\
\hline $1 / 4 / 2010$ & BA6L-TC-18 & 0.2599 \\
\hline $1 / 4 / 2010$ & BA6L-TC-23 & 0.2595 \\
\hline $1 / 4 / 2010$ & BA6L-TC-28 & 0.2601 \\
\hline $1 / 4 / 2010$ & BA6L-TC-31 & 0.2614 \\
\hline & Average & 0.2602 \\
\cline { 2 - 3 } & Standard Deviation & 0.0008 \\
\cline { 2 - 3 } & Number of Samples & 4 \\
\hline
\end{tabular}

Table I.16: Through-Plane Thermal Conductivity at $55^{\circ} \mathrm{C}$ using Holometrix TCA-300 Guarded Heat Flow Meter Method for 8 wt\% Ketjenblack EC-600 JD in Lexan HF1130111 (BA8L): Injection Molded Dec 4, 2009

\begin{tabular}{|c|c|c|}
\hline Test Date & Sample Number & $\begin{array}{c}\text { Through Plane Thermal } \\
\text { Conductivity }(\mathrm{W} / \mathrm{m} \cdot \mathrm{K})\end{array}$ \\
\hline $1 / 4 / 2010$ & BA8L-TC-13 & 0.2765 \\
\hline $1 / 4 / 2010$ & BA8L-TC-18 & 0.2767 \\
\hline $1 / 4 / 2010$ & BA8L-TC-20 & 0.2709 \\
\hline $1 / 4 / 2010$ & BA8L-TC-25 & 0.2774 \\
\hline & Average & 0.2754 \\
\cline { 2 - 3 } & Standard Deviation & 0.0030 \\
\cline { 2 - 3 } & Number of Samples & 4 \\
\hline
\end{tabular}


Table I.17: Through-Plane Thermal Conductivity at $55^{\circ} \mathrm{C}$ using Holometrix TCA-300 Guarded Heat Flow Meter Method for $10 \mathrm{wt} \%$ Ketjenblack EC-600 JD in Lexan HF1130-111 (BA10L): Injection Molded Dec 4, 2009

\begin{tabular}{|c|c|c|}
\hline Test Date & Sample Number & $\begin{array}{c}\text { Through Plane Thermal } \\
\text { Conductivity }(\mathrm{W} / \mathrm{m} \cdot \mathrm{K})\end{array}$ \\
\hline $1 / 5 / 2010$ & BA10L-TC-19 & 0.2888 \\
\hline $1 / 5 / 2010$ & BA10L-TC-21 & 0.2944 \\
\hline $1 / 5 / 2010$ & BA10L-TC-24 & 0.2892 \\
\hline $1 / 5 / 2010$ & BA10L-TC-27 & 0.2908 \\
\hline & Average & 0.2908 \\
\cline { 2 - 3 } & Standard Deviation & 0.0026 \\
\cline { 2 - 3 } & Number of Samples & 4 \\
\hline
\end{tabular}




\section{Appendix I.3: Polycarbonate with Graphite Nanoparticles}

Table I.18: Through-Plane Thermal Conductivity at $55^{\circ} \mathrm{C}$ using Holometrix TCA-300 Guarded Heat Flow Meter Method for Lexan HF1130-111 (BL3): Injection Molded June 3,2010

\begin{tabular}{|c|c|c|}
\hline Test Date & Sample Number & $\begin{array}{c}\text { Through Plane Thermal } \\
\text { Conductivity }(\mathrm{W} / \mathrm{m} \cdot \mathrm{K})\end{array}$ \\
\hline $6 / 15 / 2010$ & BL3-TC-10 & 0.2126 \\
\hline $6 / 15 / 2010$ & BL3-TC-16 & 0.2181 \\
\hline $6 / 15 / 2010$ & BL3-TC-19 & 0.2158 \\
\hline $6 / 15 / 2010$ & BL3-TC-8 & 0.2136 \\
\hline & Average & 0.2150 \\
\cline { 2 - 3 } & Standard Deviation & 0.0024 \\
\cline { 2 - 3 } & Number of Samples & 4 \\
\hline
\end{tabular}

Table I.19: Through-Plane Thermal Conductivity at $55^{\circ} \mathrm{C}$ using Holometrix TCA-300 Guarded Heat Flow Meter Method for Extruded Lexan HF1130-111 (BLE3): Injection Molded June 3, 2010

\begin{tabular}{|c|c|c|}
\hline Test Date & Sample Number & $\begin{array}{c}\text { Through Plane Thermal } \\
\text { Conductivity }(\mathrm{W} / \mathrm{m} \bullet \mathrm{K})\end{array}$ \\
\hline $6 / 15 / 2010$ & BLE3-TC-5 & 0.2141 \\
\hline $6 / 15 / 2010$ & BLE3-TC-6 & 0.2176 \\
\hline $6 / 15 / 2010$ & BLE3-TC-18 & 0.2167 \\
\hline $6 / 15 / 2010$ & BLE3-TC-2 & 0.2182 \\
\hline $6 / 15 / 2010$ & BLE3-TC-3 & 0.2187 \\
\hline & Average & 0.2171 \\
\cline { 2 - 3 } & Standard Deviation & 0.0018 \\
\cline { 2 - 3 } & Number of Samples & 5 \\
\hline
\end{tabular}


Table I.20: Through-Plane Thermal Conductivity at $55^{\circ} \mathrm{C}$ using Holometrix TCA-300 Guarded Heat Flow Meter Method for $2 \mathrm{wt} \% \mathrm{xGnP}$ in Lexan HF1130-111 (BG2L): Injection Molded June 3, 2010

\begin{tabular}{|c|c|c|}
\hline Test Date & Sample Number & $\begin{array}{c}\text { Through Plane Thermal } \\
\text { Conductivity }(\mathrm{W} / \mathrm{m} \bullet \mathrm{K})\end{array}$ \\
\hline $6 / 16 / 2010$ & BG2L-TC-23 & 0.2527 \\
\hline $6 / 16 / 2010$ & BG2L-TC-13 & 0.2498 \\
\hline $6 / 16 / 2010$ & BG2L-TC-21 & 0.2479 \\
\hline $6 / 16 / 2010$ & BG2L-TC-22 & 0.2479 \\
\hline $6 / 16 / 2010$ & BG2L-TC-24 & 0.254 \\
\hline & Average & 0.2505 \\
\cline { 2 - 3 } & Standard Deviation & 0.0028 \\
\cline { 2 - 3 } & Number of Samples & 5 \\
\hline
\end{tabular}

Table I.21: Through-Plane Thermal Conductivity at $55^{\circ} \mathrm{C}$ using Holometrix TCA-300 Guarded Heat Flow Meter Method for $3 \mathrm{wt} \%$ xGnP in Lexan HF1130-111 (BG3L): Injection Molded June 3, 2010

\begin{tabular}{|c|c|c|}
\hline Test Date & Sample Number & $\begin{array}{c}\text { Through Plane Thermal } \\
\text { Conductivity }(\mathrm{W} / \mathrm{m} \cdot \mathrm{K})\end{array}$ \\
\hline $6 / 16 / 2010$ & BG3L-TC-18 & 0.278 \\
\hline $6 / 16 / 2010$ & BG3L-TC-20 & 0.2781 \\
\hline $6 / 16 / 2010$ & BG3L-TC-25 & 0.2695 \\
\hline $6 / 16 / 2010$ & BG3L-TC-29 & 0.2676 \\
\hline $6 / 16 / 2010$ & BG3L-TC-30 & 0.2695 \\
\hline & Average & 0.2725 \\
\cline { 2 - 3 } & Standard Deviation & 0.0051 \\
\cline { 2 - 3 } & Number of Samples & 5 \\
\hline
\end{tabular}


Table I.22: Through-Plane Thermal Conductivity at $55^{\circ} \mathrm{C}$ using Holometrix TCA-300 Guarded Heat Flow Meter Method for $4 \mathrm{wt} \% \mathrm{xGnP}$ in Lexan HF1130-111 (BG4L): Injection Molded June 3, 2010

\begin{tabular}{|c|c|c|}
\hline Test Date & Sample Number & $\begin{array}{c}\text { Through Plane Thermal } \\
\text { Conductivity }(\mathrm{W} / \mathrm{m} \bullet \mathrm{K})\end{array}$ \\
\hline $6 / 16 / 2010$ & BG4L-TC-16 & 0.301 \\
\hline $6 / 16 / 2010$ & BG4L-TC-19 & 0.2931 \\
\hline $6 / 16 / 2010$ & BG4L-TC-18 & 0.2971 \\
\hline $6 / 16 / 2010$ & BG4L-TC-20 & 0.2985 \\
\hline $6 / 16 / 2010$ & BG4L-TC-21 & 0.3008 \\
\hline & Average & 0.2981 \\
\cline { 2 - 3 } & Standard Deviation & 0.0032 \\
\cline { 2 - 3 } & Number of Samples & 5 \\
\hline
\end{tabular}

Table I.23: Through-Plane Thermal Conductivity at $55^{\circ} \mathrm{C}$ using Holometrix TCA-300 Guarded Heat Flow Meter Method 5 wt\% xGnP in Lexan HF1130-111 (BG5L): Injection Molded June 3, 2010

\begin{tabular}{|c|c|c|}
\hline Test Date & Sample Number & $\begin{array}{c}\text { Through Plane Thermal } \\
\text { Conductivity }(\mathrm{W} / \mathrm{m} \cdot \mathrm{K})\end{array}$ \\
\hline $6 / 17 / 2010$ & BG5L-TC-8 & 0.3148 \\
\hline $6 / 17 / 2010$ & BG5L-TC-14 & 0.3172 \\
\hline $6 / 17 / 2010$ & BG5L-TC-18 & 0.3067 \\
\hline $6 / 17 / 2010$ & BG5L-TC-12 & 0.3083 \\
\hline $6 / 17 / 2010$ & BG5L-TC-11 & 0.3123 \\
\hline & Average & 0.3119 \\
\cline { 2 - 3 } & Standard Deviation & 0.0044 \\
\cline { 2 - 3 } & Number of Samples & 5 \\
\hline
\end{tabular}


Table I.24: Through-Plane Thermal Conductivity at $55^{\circ} \mathrm{C}$ using Holometrix TCA-300 Guarded Heat Flow Meter Method for $6 \mathrm{wt} \% \mathrm{xGnP}$ in Lexan HF1130-111 (BG6L): Injection Molded June 8, 2010

\begin{tabular}{|c|c|c|}
\hline Test Date & Sample Number & $\begin{array}{c}\text { Through Plane Thermal } \\
\text { Conductivity }(\mathrm{W} / \mathrm{m} \bullet \mathrm{K})\end{array}$ \\
\hline $6 / 17 / 2010$ & BG6L-TC-23 & 0.3438 \\
\hline $6 / 17 / 2010$ & BG6L-TC-22 & 0.3459 \\
\hline $6 / 17 / 2010$ & BG6L-TC-26 & 0.3427 \\
\hline $6 / 18 / 2010$ & BG6L-TC-16 & 0.3405 \\
\hline $6 / 18 / 2010$ & BG6L-TC-19 & 0.3449 \\
\hline & Average & 0.3436 \\
\cline { 2 - 3 } & Standard Deviation & 0.0021 \\
\cline { 2 - 3 } & Number of Samples & 5 \\
\hline
\end{tabular}

Table I.25: Through-Plane Thermal Conductivity at $55^{\circ} \mathrm{C}$ using Holometrix TCA-300 Guarded Heat Flow Meter Method for for $8 \mathrm{wt} \%$ xGnP in Lexan HF1130-111 (BG8L): Injection Molded June 8, 2010

\begin{tabular}{|c|c|c|}
\hline Test Date & Sample Number & $\begin{array}{c}\text { Through Plane Thermal } \\
\text { Conductivity }(\mathrm{W} / \mathrm{m} \cdot \mathrm{K})\end{array}$ \\
\hline $6 / 18 / 2010$ & BG8L-TC-23 & 0.371 \\
\hline $6 / 18 / 2010$ & BG8L-TC-26 & 0.3817 \\
\hline $6 / 18 / 2010$ & BG8L-TC-24 & 0.3694 \\
\hline $6 / 18 / 2010$ & BG8L-TC-14 & 0.3822 \\
\hline $6 / 18 / 2010$ & BG8L-TC-12 & 0.3679 \\
\hline & Average & 0.3744 \\
\cline { 2 - 3 } & Standard Deviation & 0.0069 \\
\cline { 2 - 3 } & Number of Samples & 5 \\
\hline
\end{tabular}


Table I.26: Through-Plane Thermal Conductivity at $55^{\circ} \mathrm{C}$ using Holometrix TCA-300 Guarded Heat Flow Meter Method for $10 \mathrm{wt} \%$ xGnP in Lexan HF1130-111 (BG10L): Injection Molded June 8, 2010

\begin{tabular}{|c|c|c|}
\hline Test Date & Sample Number & $\begin{array}{c}\text { Through Plane Thermal } \\
\text { Conductivity }(\mathrm{W} / \mathrm{m} \bullet \mathrm{K})\end{array}$ \\
\hline $6 / 18 / 2010$ & BG10L-TC-39 & 0.4229 \\
\hline $6 / 18 / 2010$ & BG10L-TC-26 & 0.4171 \\
\hline $6 / 18 / 2010$ & BG10L-TC-20 & 0.4176 \\
\hline $6 / 18 / 2010$ & BG10L-TC-15 & 0.419 \\
\hline $6 / 18 / 2010$ & BG10L-TC-14 & 0.4209 \\
\hline & Average & 0.4195 \\
\cline { 2 - 3 } & Standard Deviation & 0.0024 \\
\cline { 2 - 3 } & Number of Samples & 5 \\
\hline
\end{tabular}




\section{Appendix I.4: Polycarbonate with Multiple Fillers}

Table I.27: Through-Plane Thermal Conductivity at $55^{\circ} \mathrm{C}$ using Holometrix TCA-300 Guarded Heat Flow Meter Method for BQ0.5L: Injection Molded 7-26-10

\begin{tabular}{|c|c|c|}
\hline Test Date & Sample Number & $\begin{array}{c}\text { Through Plane Thermal } \\
\text { Conductivity }(\mathrm{W} / \mathrm{m} \cdot \mathrm{K})\end{array}$ \\
\hline $8 / 13 / 2010$ & BQ0.5L-TC-25 & 0.2176 \\
\hline $8 / 13 / 2010$ & BQ0.5L-TC-26 & 0.2213 \\
\hline $8 / 13 / 2010$ & BQ0.5L-TC-29 & 0.2172 \\
\hline $8 / 13 / 2010$ & BQ0.5L-TC-15 & 0.2173 \\
\hline & Average & 0.2184 \\
\cline { 2 - 3 } & Standard Deviation & 0.0020 \\
\cline { 2 - 3 } & Number of Samples & 4 \\
\hline
\end{tabular}

Table I.28: Through-Plane Thermal Conductivity at $55^{\circ} \mathrm{C}$ using Holometrix TCA-300 Guarded Heat Flow Meter Method for BQ1L Set 1: Injection Molded 7-26-10

\begin{tabular}{|c|c|c|}
\hline Test Date & Sample Number & $\begin{array}{c}\text { Through Plane Thermal } \\
\text { Conductivity }(\mathrm{W} / \mathrm{m} \cdot \mathrm{K})\end{array}$ \\
\hline $8 / 13 / 2010$ & BQ1L-TC-21 & 0.2167 \\
\hline $8 / 13 / 2010$ & BQ1L-TC-9 & 0.2206 \\
\hline $8 / 13 / 2010$ & BQ1L-TC-29 & 0.2296 \\
\hline $8 / 13 / 2010$ & BQ1L-TC-7 & 0.2281 \\
\hline & Average & 0.2238 \\
\cline { 2 - 3 } & Standard Deviation & 0.0061 \\
\cline { 2 - 3 } & Number of Samples & 4 \\
\hline
\end{tabular}

Table I.29: Through-Plane Thermal Conductivity at $55^{\circ} \mathrm{C}$ using Holometrix TCA-300 Guarded Heat Flow Meter Method for BQ1L Set 2: Injection Molded 7-26-10

\begin{tabular}{|c|c|c|}
\hline Test Date & Sample Number & $\begin{array}{c}\text { Through Plane Thermal } \\
\text { Conductivity }(\mathrm{W} / \mathrm{m} \cdot \mathrm{K})\end{array}$ \\
\hline $8 / 13 / 2010$ & BQ1L-TC-30 & 0.2304 \\
\hline $8 / 13 / 2010$ & BQ1L-TC-3 & 0.2202 \\
\hline $8 / 13 / 2010$ & BQ1L-TC-23 & 0.2232 \\
\hline $8 / 13 / 2010$ & BQ1L-TC-24 & 0.2282 \\
\hline & Average & 0.2255 \\
\cline { 2 - 3 } & Standard Deviation & 0.0046 \\
\cline { 2 - 3 } & Number of Samples & 4 \\
\hline
\end{tabular}


Table I.30: Through-Plane Thermal Conductivity at $55^{\circ} \mathrm{C}$ using Holometrix TCA-300 Guarded Heat Flow Meter Method for BQ5L Set 2

\begin{tabular}{|c|c|c|}
\hline Test Date & Sample Number & $\begin{array}{c}\text { Through Plane Thermal } \\
\text { Conductivity }(\mathrm{W} / \mathrm{m} \bullet \mathrm{K})\end{array}$ \\
\hline $8 / 17 / 2010$ & BQ5L-TC-10 & 0.2608 \\
\hline $8 / 17 / 2010$ & BQ5L-TC-11 & 0.265 \\
\hline $8 / 17 / 2010$ & BQ5L-TC-19 & 0.2639 \\
\hline $8 / 17 / 2010$ & BQ5L-TC-20 & 0.2656 \\
\hline $8 / 18 / 2010$ & BQ5L-TC-44 & 0.2595 \\
\hline & Average & 0.2630 \\
\cline { 2 - 3 } & Standard Deviation & 0.0027 \\
\cline { 2 - 3 } & Number of Samples & 5 \\
\hline
\end{tabular}

Table I.31: Through-Plane Thermal Conductivity at $55^{\circ} \mathrm{C}$ using Holometrix TCA-300 Guarded Heat Flow Meter Method for BG2L Set 2: Injection Molded 6-3-10

\begin{tabular}{|c|c|c|}
\hline Test Date & Sample Number & $\begin{array}{c}\text { Through Plane Thermal } \\
\text { Conductivity }(\mathrm{W} / \mathrm{m} \cdot \mathrm{K})\end{array}$ \\
\hline $8 / 17 / 2010$ & BG2L-TC-9 & 0.259 \\
\hline $8 / 17 / 2010$ & BG2L-TC-14 & 0.258 \\
\hline $8 / 17 / 2010$ & BG2L-TC-18 & 0.2498 \\
\hline $8 / 17 / 2010$ & BG2L-TC-20 & 0.2416 \\
\hline & Average & 0.2521 \\
\cline { 2 - 3 } & Standard Deviation & 0.0081 \\
\cline { 2 - 3 } & Number of Samples & 4 \\
\hline
\end{tabular}

Table I.32: Through-Plane Thermal Conductivity at $55^{\circ} \mathrm{C}$ using Holometrix TCA-300 Guarded Heat Flow Meter Method for BG5L Set 2: Injection Molded 6-3-10

\begin{tabular}{|c|c|c|}
\hline Test Date & Sample Number & $\begin{array}{c}\text { Through Plane Thermal } \\
\text { Conductivity }(\mathrm{W} / \mathrm{m} \cdot \mathrm{K})\end{array}$ \\
\hline $8 / 17 / 2010$ & BG5L-TC-22 & 0.3184 \\
\hline $8 / 17 / 2010$ & BG5L-TC-23 & 0.3166 \\
\hline $8 / 17 / 2010$ & BG5L-TC-9 & 0.3184 \\
\hline $8 / 17 / 2010$ & BG5L-TC-29 & 0.3197 \\
\hline $8 / 17 / 2010$ & BG5L-TC-20 & 0.3198 \\
\hline & Average & 0.3186 \\
\cline { 2 - 3 } & Standard Deviation & 0.0013 \\
\cline { 2 - 3 } & Number of Samples & 5 \\
\hline
\end{tabular}


Table I.33: Through-Plane Thermal Conductivity at $55^{\circ} \mathrm{C}$ using Holometrix TCA-300 Guarded Heat Flow Meter Method for BG12L: Injection Molded 8-18-10

\begin{tabular}{|c|c|c|}
\hline Test Date & Sample Number & $\begin{array}{c}\text { Through Plane Thermal } \\
\text { Conductivity }(\mathrm{W} / \mathrm{m} \cdot \mathrm{K})\end{array}$ \\
\hline $8 / 20 / 2010$ & BG12L-TC-1 & 0.4729 \\
\hline $8 / 20 / 2010$ & BG12L-TC-4 & 0.4527 \\
\hline $8 / 20 / 2010$ & BG12L-TC-5 & 0.4667 \\
\hline $8 / 20 / 2010$ & BG12L-TC-6 & 0.4379 \\
\hline & Average & 0.4576 \\
\cline { 2 - 3 } & Standard Deviation & 0.0156 \\
\cline { 2 - 3 } & Number of Samples & 4 \\
\hline
\end{tabular}

Table I.34: Through-Plane Thermal Conductivity at $55^{\circ} \mathrm{C}$ using Holometrix TCA-300 Guarded Heat Flow Meter Method for BG15L: Injection Molded 8-18-10

\begin{tabular}{|c|c|c|}
\hline Test Date & Sample Number & $\begin{array}{c}\text { Through Plane Thermal } \\
\text { Conductivity }(\mathrm{W} / \mathrm{m} \cdot \mathrm{K})\end{array}$ \\
\hline $8 / 20 / 2010$ & BG15L-TC-23 & 0.4895 \\
\hline $8 / 20 / 2010$ & BG15L-TC-6 & 0.4727 \\
\hline $8 / 20 / 2010$ & BG15L-TC-15 & 0.5098 \\
\hline $8 / 20 / 2010$ & BG15L-TC-18 & 0.4832 \\
\hline & Average & 0.4888 \\
\cline { 2 - 3 } & Standard Deviation & 0.0156 \\
\cline { 2 - 3 } & Number of Samples & 4 \\
\hline
\end{tabular}

Table I.35: Through-Plane Thermal Conductivity at $55^{\circ} \mathrm{C}$ using Holometrix TCA-300 Guarded Heat Flow Meter Method for BA2Q1L set 1: Injection Molded 7-26-10

\begin{tabular}{|c|c|c|}
\hline Test Date & Sample Number & $\begin{array}{c}\text { Through Plane Thermal } \\
\text { Conductivity }(\mathrm{W} / \mathrm{m} \cdot \mathrm{K})\end{array}$ \\
\hline $8 / 13 / 2010$ & BA2Q1L-TC-17 & 0.2401 \\
\hline $8 / 13 / 2010$ & BA2Q1L-TC-31 & 0.2436 \\
\hline $8 / 13 / 2010$ & BA2Q1L-TC-2 & 0.2442 \\
\hline $8 / 13 / 2010$ & BA2Q1L-TC-12 & 0.2444 \\
\hline & Average & 0.2431 \\
\cline { 2 - 3 } & Standard Deviation & 0.0020 \\
\cline { 2 - 3 } & Number of Samples & 4 \\
\hline
\end{tabular}


Table I.36: Through-Plane Thermal Conductivity at $55^{\circ} \mathrm{C}$ using Holometrix TCA-300 Guarded Heat Flow Meter Method for BA2Q1L set 2: Injection Molded 7-26-10

\begin{tabular}{|c|c|c|}
\hline Test Date & Sample Number & $\begin{array}{c}\text { Through Plane Thermal } \\
\text { Conductivity }(\mathrm{W} / \mathrm{m} \bullet \mathrm{K})\end{array}$ \\
\hline $8 / 13 / 2010$ & BA2Q1L-TC-35 & 0.2432 \\
\hline $8 / 13 / 2010$ & BA2Q1L-TC-25 & 0.2429 \\
\hline $8 / 13 / 2010$ & BA2Q1L-TC-10 & 0.2437 \\
\hline $8 / 13 / 2010$ & BA2Q1L-TC-33 & 0.2428 \\
\hline & Average & 0.2432 \\
\cline { 2 - 3 } & Standard Deviation & 0.0004 \\
\cline { 2 - 3 } & Number of Samples & 4 \\
\hline
\end{tabular}

Table I.37: Through-Plane Thermal Conductivity at $55^{\circ} \mathrm{C}$ using Holometrix TCA-300 Guarded Heat Flow Meter Method for BA2Q5L set 1: Injection Molded 8-25-10

\begin{tabular}{|c|c|c|}
\hline Test Date & Sample Number & $\begin{array}{c}\text { Through Plane Thermal } \\
\text { Conductivity }(\mathrm{W} / \mathrm{m} \cdot \mathrm{K})\end{array}$ \\
\hline $9 / 2 / 2010$ & BA2Q5L-TC-7 & 0.2718 \\
\hline & BA2Q5L-TC-13 & 0.2718 \\
\hline & BA2Q5L-TC-11 & 0.2833 \\
\hline & BA2Q5L-TC-15 & 0.2746 \\
& Average & 0.2754 \\
\cline { 2 - 3 } & Standard Deviation & 0.0054 \\
\cline { 2 - 3 } & Number of Samples & 4 \\
\hline
\end{tabular}

Table I.38: Through-Plane Thermal Conductivity at $55^{\circ} \mathrm{C}$ using Holometrix TCA-300 Guarded Heat Flow Meter Method for BA2Q5L set 2: Injection Molded 8-25-10

\begin{tabular}{|c|c|c|}
\hline Test Date & Sample Number & $\begin{array}{c}\text { Through Plane Thermal } \\
\text { Conductivity }(\mathrm{W} / \mathrm{m} \cdot \mathrm{K})\end{array}$ \\
\hline $9 / 2 / 2010$ & BA2Q5L-TC-22 & 0.2707 \\
\hline & BA2Q5L-TC-8 & 0.2688 \\
\hline & BA2Q5L-TC-23 & 0.274 \\
\hline & BA2Q5L-TC-18 & 0.2719 \\
\hline & Average & 0.2714 \\
\cline { 2 - 3 } & Standard Deviation & 0.0022 \\
\cline { 2 - 3 } & Number of Samples & 4 \\
\hline
\end{tabular}


Table I.4-39: Through-Plane Thermal Conductivity at $55^{\circ} \mathrm{C}$ using Holometrix TCA-300 Guarded Heat Flow Meter Method for BA5Q1L set 1: Injection Molded 7-26-10

\begin{tabular}{|c|c|c|}
\hline Test Date & Sample Number & $\begin{array}{c}\text { Through Plane Thermal } \\
\text { Conductivity }(\mathrm{W} / \mathrm{m} \cdot \mathrm{K})\end{array}$ \\
\hline $8 / 13 / 2010$ & BA5Q1L-TC-24 & 0.2666 \\
\hline $8 / 13 / 2010$ & BA5Q1L-TC-30 & 0.2722 \\
\hline $8 / 13 / 2010$ & BA5Q1L-TC-19 & 0.2715 \\
\hline $8 / 13 / 2010$ & BA5Q1L-TC-16 & 0.2718 \\
\hline & Average & 0.2705 \\
\cline { 2 - 3 } & Standard Deviation & 0.0026 \\
\cline { 2 - 3 } & Number of Samples & 4 \\
\hline
\end{tabular}

Table I.4-40: Through-Plane Thermal Conductivity at $55^{\circ} \mathrm{C}$ using Holometrix TCA-300 Guarded Heat Flow Meter Method for BA5Q1L set 2: Injection Molded 7-26-10

\begin{tabular}{|c|c|c|}
\hline Test Date & Sample Number & $\begin{array}{c}\text { Through Plane Thermal } \\
\text { Conductivity }(\mathrm{W} / \mathrm{m} \cdot \mathrm{K})\end{array}$ \\
\hline $8 / 13 / 2010$ & BA5Q1L-TC-23 & 0.2708 \\
\hline $8 / 13 / 2010$ & BA5Q1L-TC-28 & 0.2701 \\
\hline $8 / 13 / 2010$ & BA5Q1L-TC-13 & 0.2709 \\
\hline $8 / 13 / 2010$ & BA5Q1L-TC-9 & 0.2605 \\
\hline & Average & 0.2681 \\
\cline { 2 - 3 } & Standard Deviation & 0.0051 \\
\cline { 2 - 3 } & Number of Samples & 4 \\
\hline
\end{tabular}

Table I.41: Through-Plane Thermal Conductivity at $55^{\circ} \mathrm{C}$ using Holometrix TCA-300 Guarded Heat Flow Meter Method for BA5Q5L set 1: Injection Molded 8-25-10

\begin{tabular}{|c|c|c|}
\hline Test Date & Sample Number & $\begin{array}{c}\text { Through Plane Thermal } \\
\text { Conductivity }(\mathrm{W} / \mathrm{m} \cdot \mathrm{K})\end{array}$ \\
\hline $9 / 3 / 2010$ & BA5Q5L-TC-15 & 0.3041 \\
\hline & BA5Q5L-TC-27 & 0.3034 \\
\hline & BA5Q5L-TC-16 & 0.3015 \\
\hline & BA5Q5L-TC-24 & 0.306 \\
\hline & Average & 0.3038 \\
\cline { 2 - 3 } & Standard Deviation & 0.0019 \\
\cline { 2 - 3 } & Number of Samples & 4 \\
\hline
\end{tabular}


Table I.42: Through-Plane Thermal Conductivity at $55^{\circ} \mathrm{C}$ using Holometrix TCA-300 Guarded Heat Flow Meter Method for BA5Q5L set 2: Injection Molded 8-25-10

\begin{tabular}{|c|c|c|}
\hline Test Date & Sample Number & $\begin{array}{c}\text { Through Plane Thermal } \\
\text { Conductivity }(\mathrm{W} / \mathrm{m} \cdot \mathrm{K})\end{array}$ \\
\hline $9 / 3 / 2010$ & BA5Q5L-TC-9 & 0.2959 \\
\hline & BA5Q5L-TC-20 & 0.3064 \\
\hline & BA5Q5L-TC-11 & 0.299 \\
\hline & BA5Q5L-TC-21 & 0.2997 \\
\hline & Average & 0.3003 \\
\cline { 2 - 3 } & Standard Deviation & 0.0044 \\
\cline { 2 - 3 } & Number of Samples & 4 \\
\hline
\end{tabular}

Table I.43: Through-Plane Thermal Conductivity at $55^{\circ} \mathrm{C}$ using Holometrix TCA-300 Guarded Heat Flow Meter Method for BA2G2L set 1: Injection Molded 8-18-10

\begin{tabular}{|c|c|c|}
\hline Test Date & Sample Number & $\begin{array}{c}\text { Through Plane Thermal } \\
\text { Conductivity }(\mathrm{W} / \mathrm{m} \cdot \mathrm{K})\end{array}$ \\
\hline $8 / 23 / 2010$ & BA2G2L-TC-6 & 0.2799 \\
\hline $8 / 23 / 2010$ & BA2G2L-TC-9 & 0.2766 \\
\hline $8 / 23 / 2010$ & BA2G2L-TC-19 & 0.2616 \\
\hline $8 / 23 / 2010$ & BA2G2L-TC-15 & 0.2667 \\
\hline & Average & 0.2712 \\
\cline { 2 - 3 } & Standard Deviation & 0.0085 \\
\cline { 2 - 3 } & Number of Samples & 4 \\
\hline
\end{tabular}

Table I.44: Through-Plane Thermal Conductivity at $55^{\circ} \mathrm{C}$ using Holometrix TCA-300 Guarded Heat Flow Meter Method for BA2G2L set 2: Injection Molded 8-18-10

\begin{tabular}{|c|c|c|}
\hline Test Date & Sample Number & $\begin{array}{c}\text { Through Plane Thermal } \\
\text { Conductivity }(\mathrm{W} / \mathrm{m} \cdot \mathrm{K})\end{array}$ \\
\hline $8 / 23 / 2010$ & BA2G2L-TC-10 & 0.2732 \\
\hline $8 / 23 / 2010$ & BA2G2L-TC-7 & 0.2704 \\
\hline $8 / 23 / 2010$ & BA2G2L-TC-16 & 0.2641 \\
\hline $8 / 23 / 2010$ & BA2G2L-TC-8 & 0.2684 \\
\hline & Average & 0.2690 \\
\cline { 2 - 3 } & Standard Deviation & 0.0038 \\
\cline { 2 - 3 } & Number of Samples & 4 \\
\hline
\end{tabular}


Table I.45: Through-Plane Thermal Conductivity at $55^{\circ} \mathrm{C}$ using Holometrix TCA-300

Guarded Heat Flow Meter Method for BA2G5L set 1: Injection Molded 8-18-10

\begin{tabular}{|c|c|c|}
\hline Test Date & Sample Number & $\begin{array}{c}\text { Through Plane Thermal } \\
\text { Conductivity }(\mathrm{W} / \mathrm{m} \cdot \mathrm{K})\end{array}$ \\
\hline $8 / 20 / 2010$ & BA2G5L-TC-4 & 0.3346 \\
\hline $8 / 20 / 2010$ & BA2G5L-TC-7 & 0.3312 \\
\hline $8 / 20 / 2010$ & BA2G5L-TC-9 & 0.3443 \\
\hline $8 / 20 / 2010$ & BA2G5L-TC-10 & 0.3426 \\
\hline $8 / 20 / 2010$ & BA2G5L-TC-17 & 0.3429 \\
\hline & Average & 0.3391 \\
\cline { 2 - 3 } & Standard Deviation & 0.0058 \\
\cline { 2 - 3 } & Number of Samples & 5 \\
\hline
\end{tabular}

Table I.46: Through-Plane Thermal Conductivity at $55^{\circ} \mathrm{C}$ using Holometrix TCA-300 Guarded Heat Flow Meter Method for BA2G5L set 2: Injection Molded 8-18-10

\begin{tabular}{|c|c|c|}
\hline Test Date & Sample Number & $\begin{array}{c}\text { Through Plane Thermal } \\
\text { Conductivity }(\mathrm{W} / \mathrm{m} \cdot \mathrm{K})\end{array}$ \\
\hline $8 / 20 / 2010$ & BA2G5L-TC-21 & 0.3339 \\
\hline $8 / 20 / 2010$ & BA2G5L-TC-28 & 0.3417 \\
\hline $8 / 20 / 2010$ & BA2G5L-TC-29 & 0.3419 \\
\hline $8 / 20 / 2010$ & BA2G5L-TC-30 & 0.3291 \\
\hline & Average & 0.3367 \\
\cline { 2 - 3 } & Standard Deviation & 0.0063 \\
\cline { 2 - 3 } & Number of Samples & 4 \\
\hline
\end{tabular}

Table I.47: Through-Plane Thermal Conductivity at $55^{\circ} \mathrm{C}$ using Holometrix TCA-300 Guarded Heat Flow Meter Method for BA5G2L set 1: Injection Molded 8-18-10

\begin{tabular}{|c|c|c|}
\hline Test Date & Sample Number & $\begin{array}{c}\text { Through Plane Thermal } \\
\text { Conductivity }(\mathrm{W} / \mathrm{m} \cdot \mathrm{K})\end{array}$ \\
\hline $8 / 24 / 2010$ & BA5G2L-TC-8 & 0.3076 \\
\hline $8 / 24 / 2010$ & BA5G2L-TC-18 & 0.3015 \\
\hline $8 / 24 / 2010$ & BA5G2L-TC-12 & 0.3015 \\
\hline $8 / 24 / 2010$ & BA5G2L-TC-15 & 0.3028 \\
\hline & Average & 0.3034 \\
\cline { 2 - 3 } & Standard Deviation & 0.0029 \\
\cline { 2 - 3 } & Number of Samples & 4 \\
\hline
\end{tabular}


Table I.48: Through-Plane Thermal Conductivity at $55^{\circ} \mathrm{C}$ using Holometrix TCA-300 Guarded Heat Flow Meter Method for BA5G2L set 2: Injection Molded 8-18-10

\begin{tabular}{|c|c|c|}
\hline Test Date & Sample Number & $\begin{array}{c}\text { Through Plane Thermal } \\
\text { Conductivity }(\mathrm{W} / \mathrm{m} \bullet \mathrm{K})\end{array}$ \\
\hline $8 / 24 / 2010$ & BA5G2L-TC-25 & 0.3008 \\
\hline $8 / 24 / 2010$ & BA5G2L-TC-14 & 0.3016 \\
\hline $8 / 24 / 2010$ & BA5G2L-TC-24 & 0.2996 \\
\hline $8 / 24 / 2010$ & BA5G2L-TC-21 & 0.3025 \\
\hline & Average & 0.3011 \\
\cline { 2 - 3 } & Standard Deviation & 0.0012 \\
\cline { 2 - 3 } & Number of Samples & 4 \\
\hline
\end{tabular}

Table I.49: Through-Plane Thermal Conductivity at $55^{\circ} \mathrm{C}$ using Holometrix TCA-300 Guarded Heat Flow Meter Method for BA5G5L set 1: Injection Molded 8-18-10

\begin{tabular}{|c|c|c|}
\hline Test Date & Sample Number & $\begin{array}{c}\text { Through Plane Thermal } \\
\text { Conductivity }(\mathrm{W} / \mathrm{m} \cdot \mathrm{K})\end{array}$ \\
\hline $8 / 26 / 2010$ & BA5G5L-TC-10 & 0.3708 \\
\hline $8 / 26 / 2010$ & BA5G5L-TC-22 & 0.3518 \\
\hline $8 / 26 / 2010$ & BA5G5L-TC-23 & 0.3526 \\
\hline $8 / 26 / 2010$ & BA5G5L-TC-9 & 0.3511 \\
\hline & Average & 0.3566 \\
\cline { 2 - 3 } & Standard Deviation & 0.0095 \\
\cline { 2 - 3 } & Number of Samples & 4 \\
\hline
\end{tabular}

Table I.50: Through-Plane Thermal Conductivity at $55^{\circ} \mathrm{C}$ using Holometrix TCA-300 Guarded Heat Flow Meter Method for BA5G5L set 2: Injection Molded 8-18-10

\begin{tabular}{|c|c|c|}
\hline Test Date & Sample Number & $\begin{array}{c}\text { Through Plane Thermal } \\
\text { Conductivity }(\mathrm{W} / \mathrm{m} \cdot \mathrm{K})\end{array}$ \\
\hline $8 / 26 / 2010$ & BA5G5L-TC-25 & 0.3687 \\
\hline $8 / 26 / 2010$ & BA5G5L-TC-15 & 0.3683 \\
\hline $8 / 26 / 2010$ & BA5G5L-TC-6 & 0.3599 \\
\hline $8 / 26 / 2010$ & BA5G5L-TC-14 & 0.3667 \\
\hline & Average & 0.3659 \\
\cline { 2 - 3 } & Standard Deviation & 0.0041 \\
\cline { 2 - 3 } & Number of Samples & 4 \\
\hline
\end{tabular}


Table I.51: Through-Plane Thermal Conductivity at $55^{\circ} \mathrm{C}$ using Holometrix TCA-300

Guarded Heat Flow Meter Method for BQ1G2L set 1: Injection Molded 8-18-10

\begin{tabular}{|c|c|c|}
\hline Test Date & Sample Number & $\begin{array}{c}\text { Through Plane Thermal } \\
\text { Conductivity }(\mathrm{W} / \mathrm{m} \cdot \mathrm{K})\end{array}$ \\
\hline $8 / 27 / 2010$ & BQ1G2L-TC-16 & 0.258 \\
\hline $8 / 27 / 2010$ & BQ1G2L-TC-18 & 0.2704 \\
\hline $8 / 27 / 2010$ & BQ1G2L-TC-19 & 0.2732 \\
\hline $8 / 27 / 2010$ & BQ1G2L-TC-10 & 0.2682 \\
\hline & Average & 0.2675 \\
\cline { 2 - 3 } & Standard Deviation & 0.0066 \\
\cline { 2 - 3 } & Number of Samples & 4 \\
\hline
\end{tabular}

Table I.52: Through-Plane Thermal Conductivity at $55^{\circ} \mathrm{C}$ using Holometrix TCA-300 Guarded Heat Flow Meter Method for BQ1G2L set 2: Injection Molded 8-18-10

\begin{tabular}{|c|c|c|}
\hline Test Date & Sample Number & $\begin{array}{c}\text { Through Plane Thermal } \\
\text { Conductivity }(\mathrm{W} / \mathrm{m} \cdot \mathrm{K})\end{array}$ \\
\hline $8 / 27 / 2010$ & BQ1G2L-TC-23 & 0.2726 \\
\hline $8 / 27 / 2010$ & BQ1G2L-TC-11 & 0.261 \\
\hline $8 / 27 / 2010$ & BQ1G2L-TC-25 & 0.2607 \\
\hline $8 / 27 / 2010$ & BQ1G2L-TC-22 & 0.263 \\
\hline & Average & 0.2643 \\
\cline { 2 - 3 } & Standard Deviation & 0.0056 \\
\cline { 2 - 3 } & Number of Samples & 4 \\
\hline
\end{tabular}

Table I-53: Through-Plane Thermal Conductivity at $55^{\circ} \mathrm{C}$ using Holometrix TCA-300 Guarded Heat Flow Meter Method for BQ1G5L set 1: Injection Molded 8-18-10

\begin{tabular}{|c|c|c|}
\hline Test Date & Sample Number & $\begin{array}{c}\text { Through Plane Thermal } \\
\text { Conductivity }(\mathrm{W} / \mathrm{m} \cdot \mathrm{K})\end{array}$ \\
\hline $8 / 30 / 2010$ & BQ1G5L-TC-6 & 0.329 \\
\hline & BQ1G5L-TC-19 & 0.3254 \\
\hline & BQ1G5L-TC-14 & 0.3357 \\
\hline & BQ1G5L-TC-13 & 0.3201 \\
\hline & Average & 0.3276 \\
\cline { 2 - 3 } & Standard Deviation & 0.0065 \\
\cline { 2 - 3 } & Number of Samples & 4 \\
\hline
\end{tabular}


Table I.54: Through-Plane Thermal Conductivity at $55^{\circ} \mathrm{C}$ using Holometrix TCA-300 Guarded Heat Flow Meter Method for BQ1G5L set 2: Injection Molded 8-18-10

\begin{tabular}{|c|c|c|}
\hline Test Date & Sample Number & $\begin{array}{c}\text { Through Plane Thermal } \\
\text { Conductivity }(\mathrm{W} / \mathrm{m} \bullet \mathrm{K})\end{array}$ \\
\hline $8 / 30 / 2010$ & BQ1G5L-TC-29 & 0.3278 \\
\hline & BQ1G5L-TC-15 & 0.3261 \\
\hline & BQ1G5L-TC-17 & 0.3304 \\
\hline & BQ1G5L-TC-25 & 0.3311 \\
\hline & Average & 0.3289 \\
\cline { 2 - 3 } & Standard Deviation & 0.0023 \\
\cline { 2 - 3 } & Number of Samples & 4 \\
\hline
\end{tabular}

Table I.55: Through-Plane Thermal Conductivity at $55^{\circ} \mathrm{C}$ using Holometrix TCA-300 Guarded Heat Flow Meter Method for BQ5G2L set 1: Injection Molded 8-18-10

\begin{tabular}{|c|c|c|}
\hline Test Date & Sample Number & $\begin{array}{c}\text { Through Plane Thermal } \\
\text { Conductivity }(\mathrm{W} / \mathrm{m} \cdot \mathrm{K})\end{array}$ \\
\hline $8 / 31 / 2010$ & BQ5G2L-TC-25 & 0.3194 \\
\hline & BQ5G2L-TC-21 & 0.3165 \\
\hline & BQ5G2L-TC-14 & 0.3116 \\
\hline & BQ5G2L-TC-24 & 0.3254 \\
& Average & 0.3182 \\
\cline { 2 - 3 } & Standard Deviation & 0.0058 \\
\cline { 2 - 3 } & Number of Samples & 4 \\
\hline
\end{tabular}

Table I.56: Through-Plane Thermal Conductivity at $55^{\circ} \mathrm{C}$ using Holometrix TCA-300 Guarded Heat Flow Meter Method for BQ5G2L set 2: Injection Molded 8-18-10

\begin{tabular}{|c|c|c|}
\hline Test Date & Sample Number & $\begin{array}{c}\text { Through Plane Thermal } \\
\text { Conductivity }(\mathrm{W} / \mathrm{m} \bullet \mathrm{K})\end{array}$ \\
\hline $8 / 31 / 2010$ & BQ5G2L-TC-15 & 0.319 \\
\hline & BQ5G2L-TC-13 & 0.3147 \\
\hline & BQ5G2L-TC-19 & 0.3279 \\
\hline & BQ5G2L-TC-20 & 0.3231 \\
\hline & BQ5G2L-TC-11 & 0.3196 \\
\hline & Average & 0.3209 \\
\cline { 2 - 3 } & Standard Deviation & 0.0049 \\
\cline { 2 - 3 } & Number of Samples & 5 \\
\hline
\end{tabular}


Table I.57: Through-Plane Thermal Conductivity at $55^{\circ} \mathrm{C}$ using Holometrix TCA-300 Guarded Heat Flow Meter Method for BQ5G5L set 1: Injection Molded 8-18-10

\begin{tabular}{|c|c|c|}
\hline Test Date & Sample Number & $\begin{array}{c}\text { Through Plane Thermal } \\
\text { Conductivity }(\mathrm{W} / \mathrm{m} \cdot \mathrm{K})\end{array}$ \\
\hline $9 / 1 / 2010$ & BQ5G5L-TC-17 & 0.3908 \\
\hline & BQ5G5L-TC-21 & 0.3986 \\
\hline & BQ5G5L-TC-24 & 0.3965 \\
\hline & BQ5G5L-TC-5 & 0.3977 \\
\hline & Average & 0.3959 \\
\cline { 2 - 3 } & Standard Deviation & 0.0035 \\
\cline { 2 - 3 } & Number of Samples & 4 \\
\hline
\end{tabular}

Table I.58: Through-Plane Thermal Conductivity at $55^{\circ} \mathrm{C}$ using Holometrix TCA-300 Guarded Heat Flow Meter Method for BQ5G5L set 2: Injection Molded 8-18-10

\begin{tabular}{|c|c|c|}
\hline Test Date & Sample Number & $\begin{array}{c}\text { Through Plane Thermal } \\
\text { Conductivity }(\mathrm{W} / \mathrm{m} \bullet \mathrm{K})\end{array}$ \\
\hline 9/1/2010 & BQ5G5L-TC-27 & 0.4017 \\
\hline & BQ5G5L-TC-19 & 0.3899 \\
\hline & BQ5G5L-TC-10 & 0.4042 \\
\hline & BQ5G5L-TC-15 & 0.3905 \\
\hline & Average & 0.3966 \\
\cline { 2 - 3 } & Standard Deviation & 0.0074 \\
\cline { 2 - 3 } & Number of Samples & 4 \\
\hline
\end{tabular}




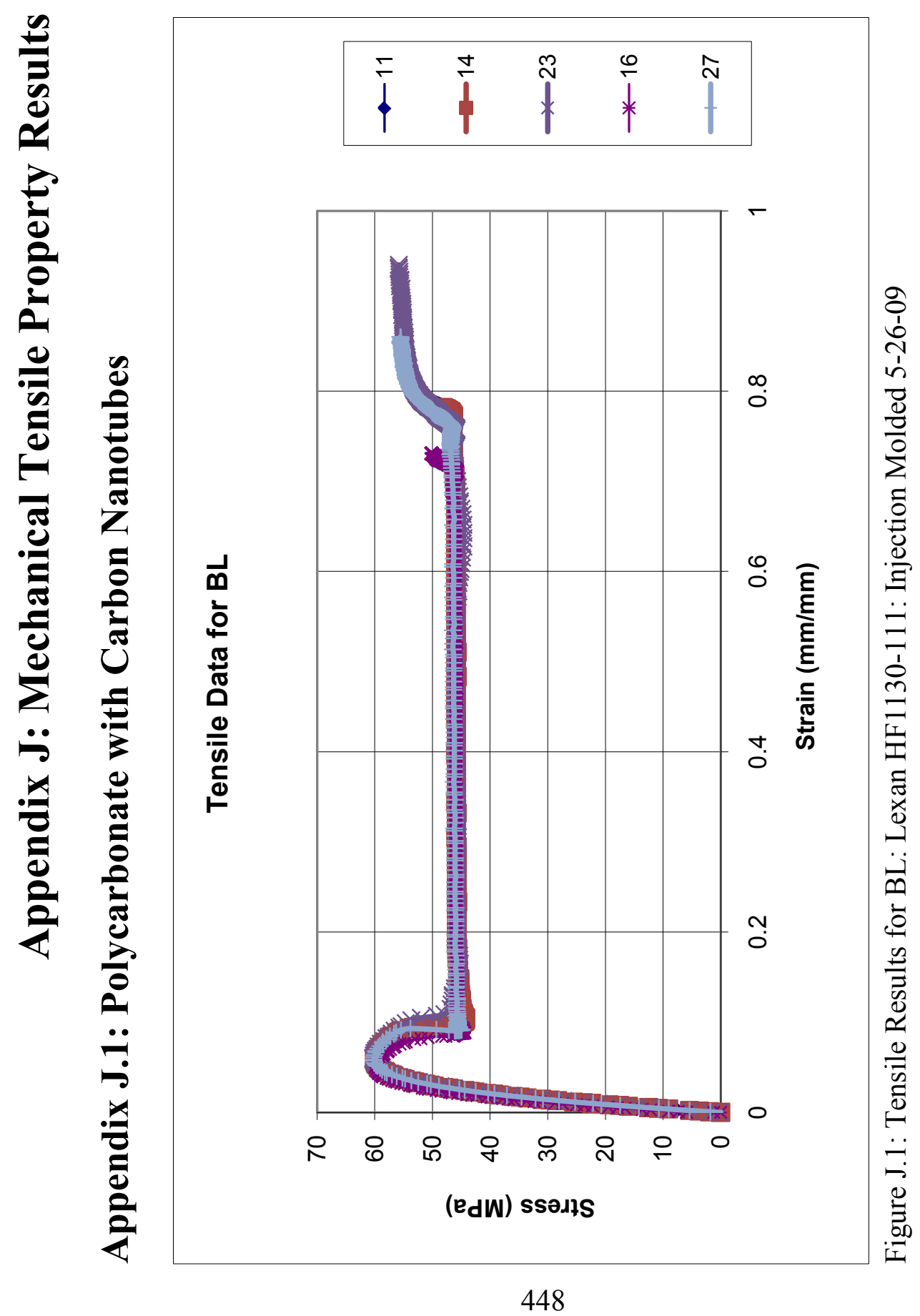




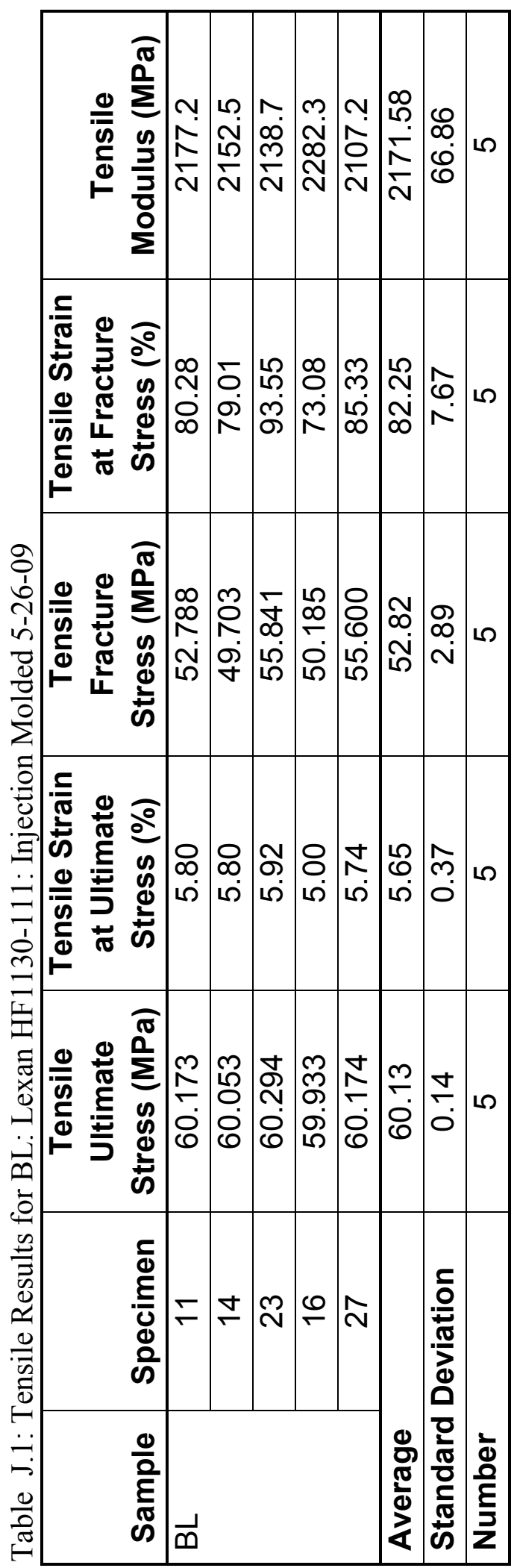




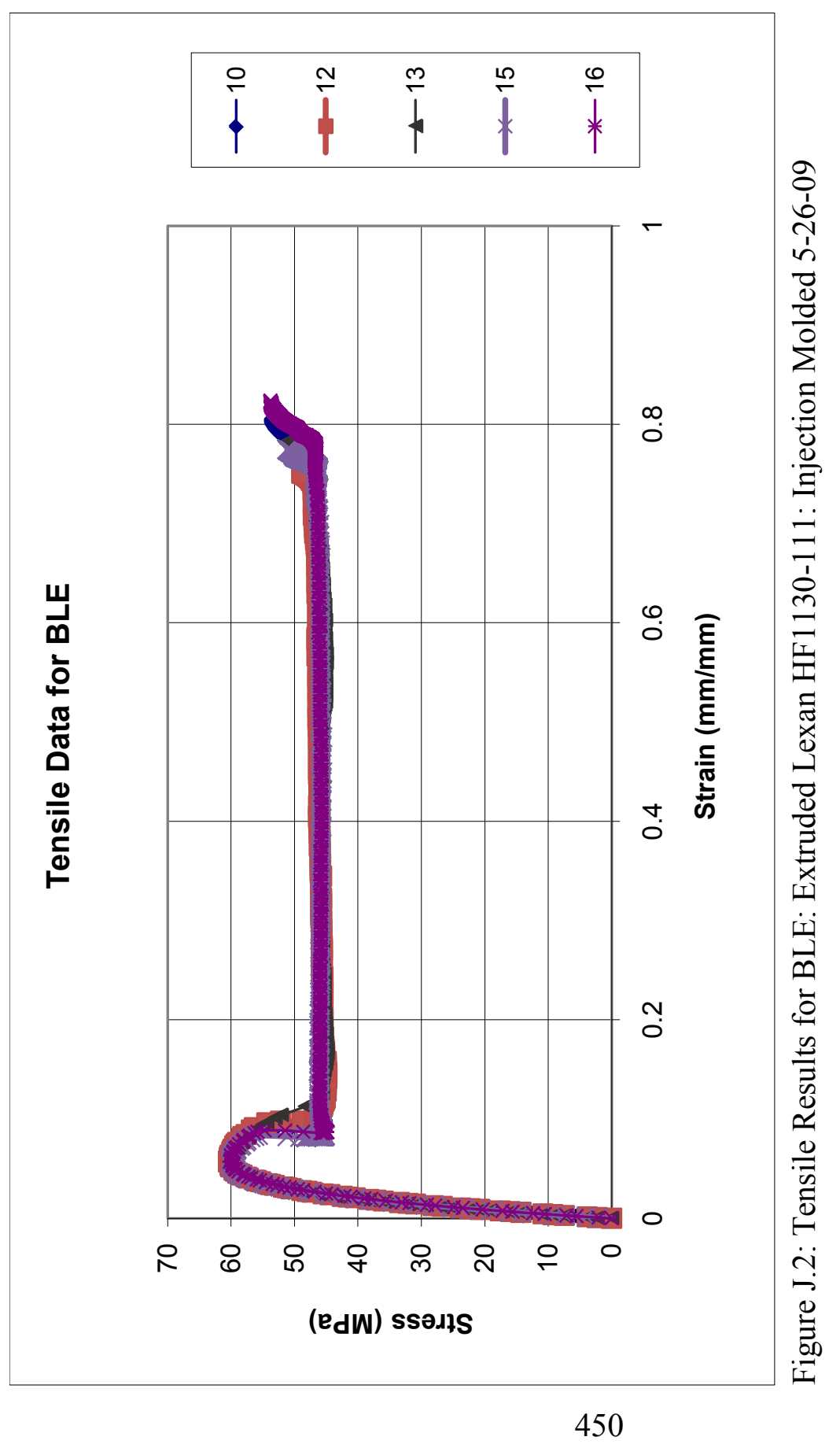




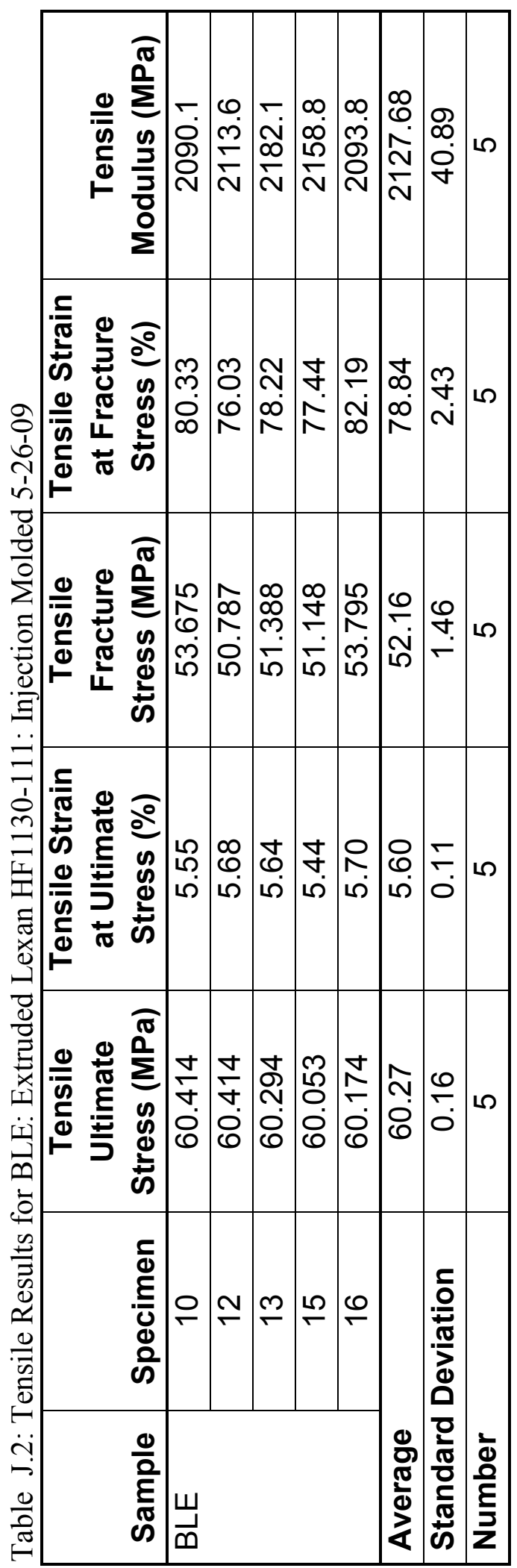




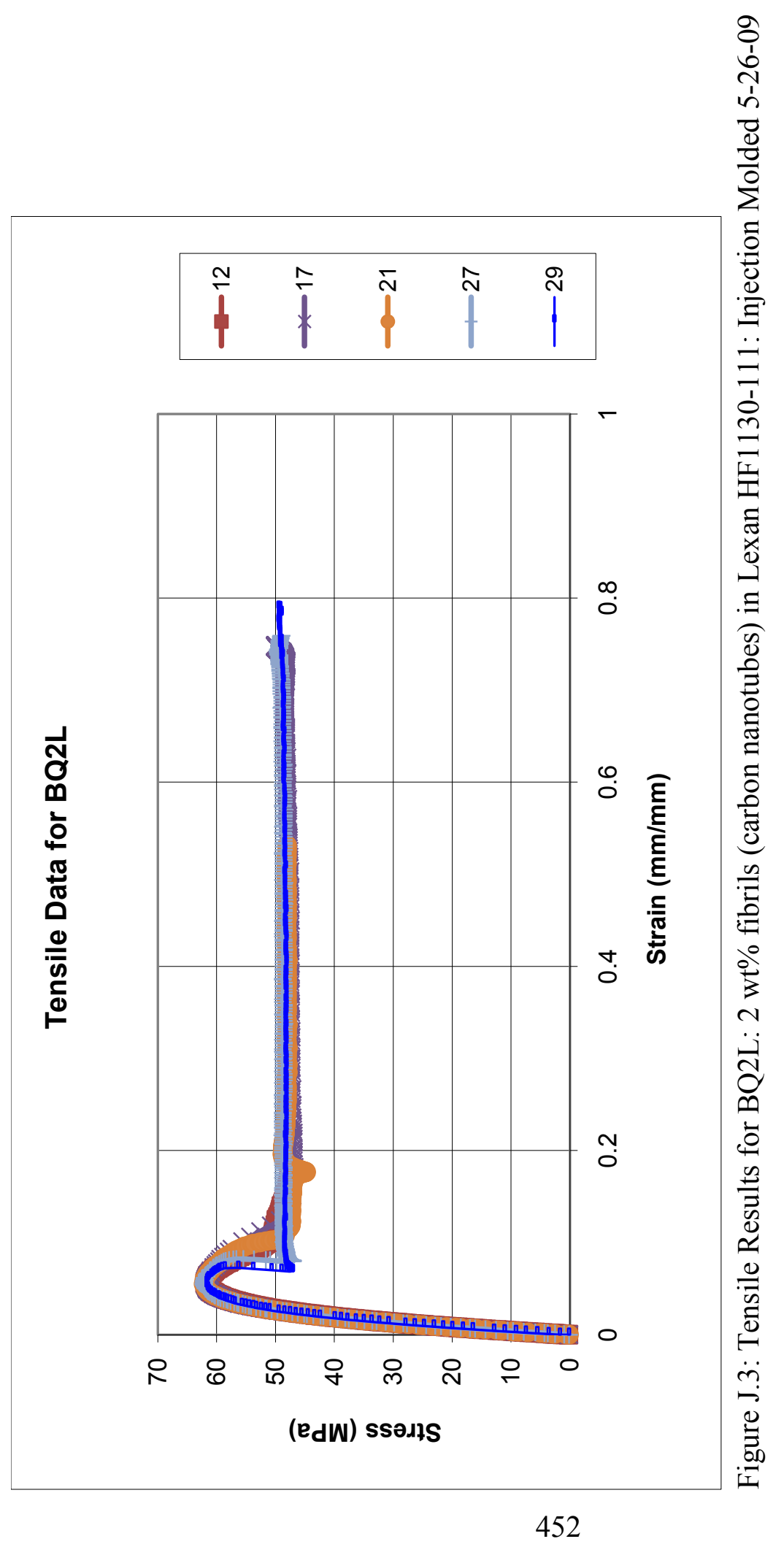




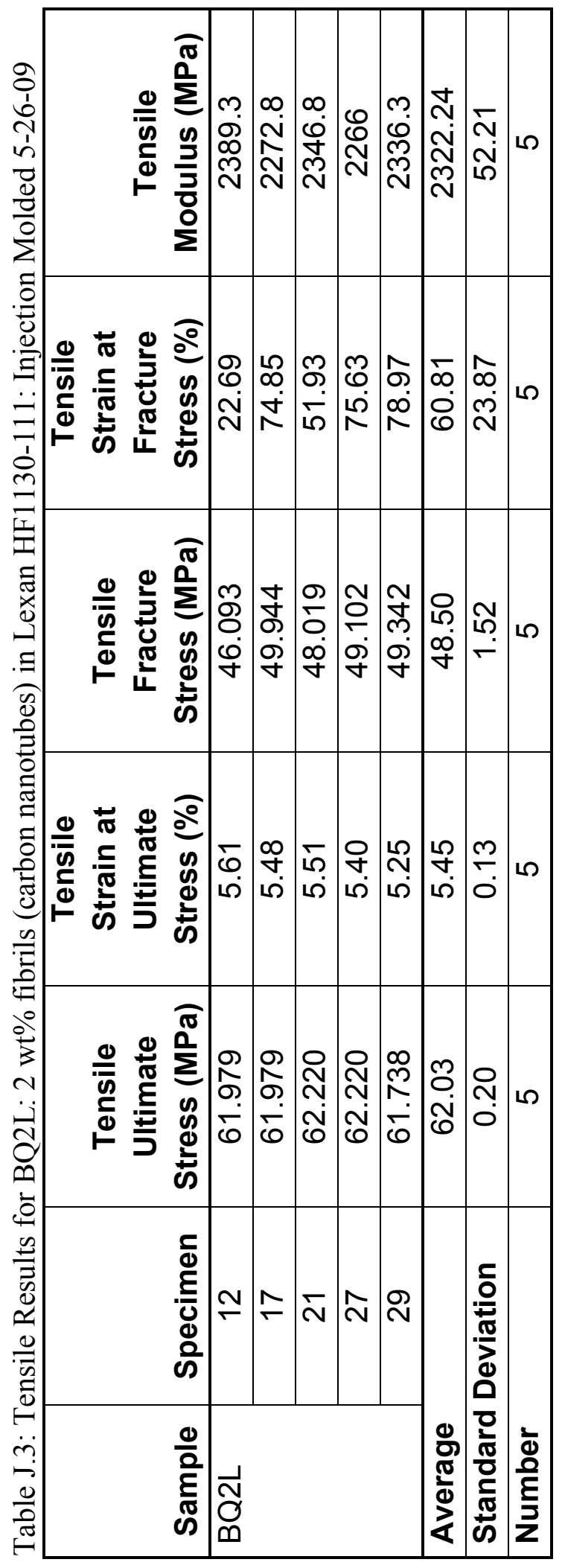




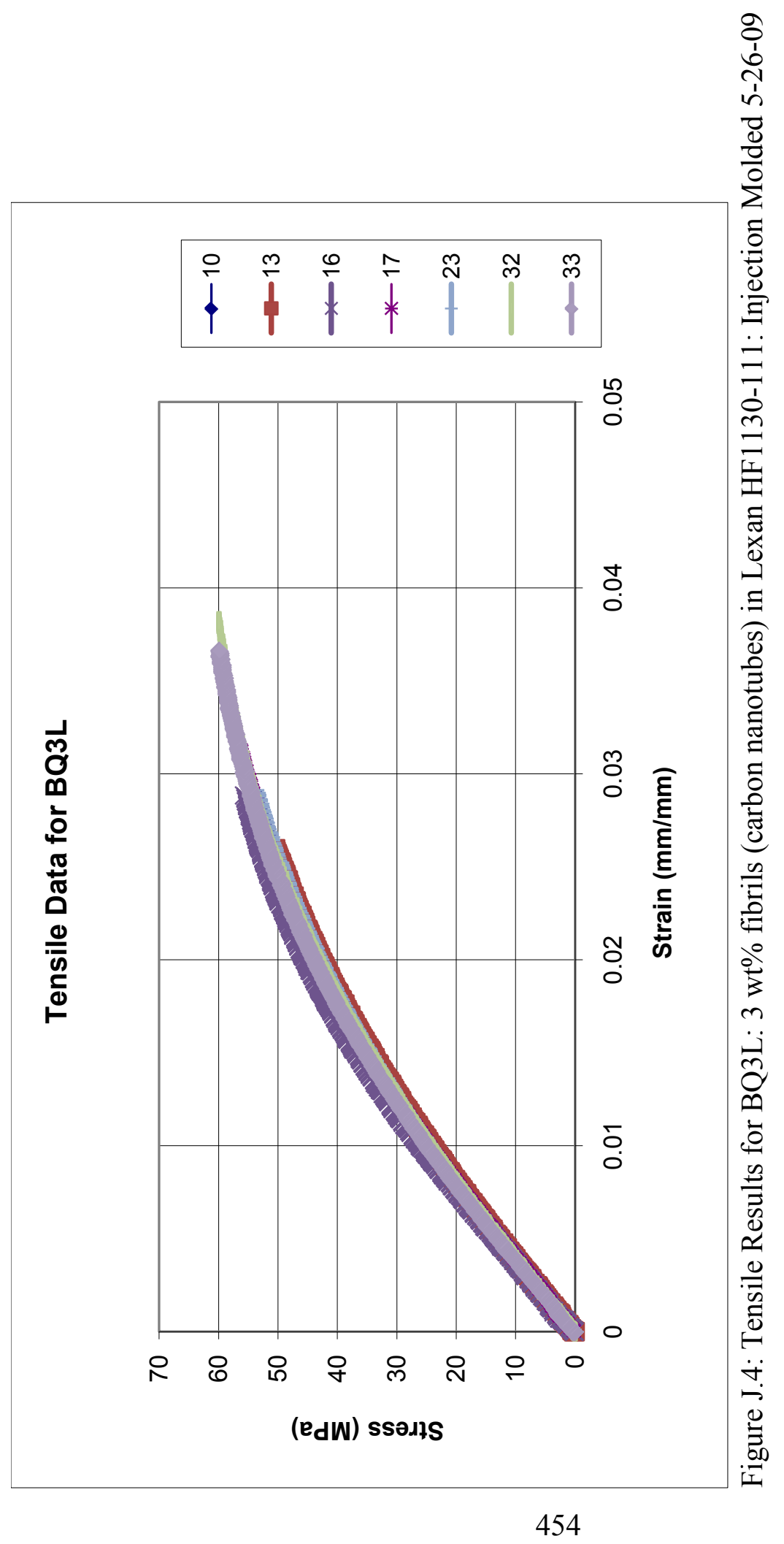




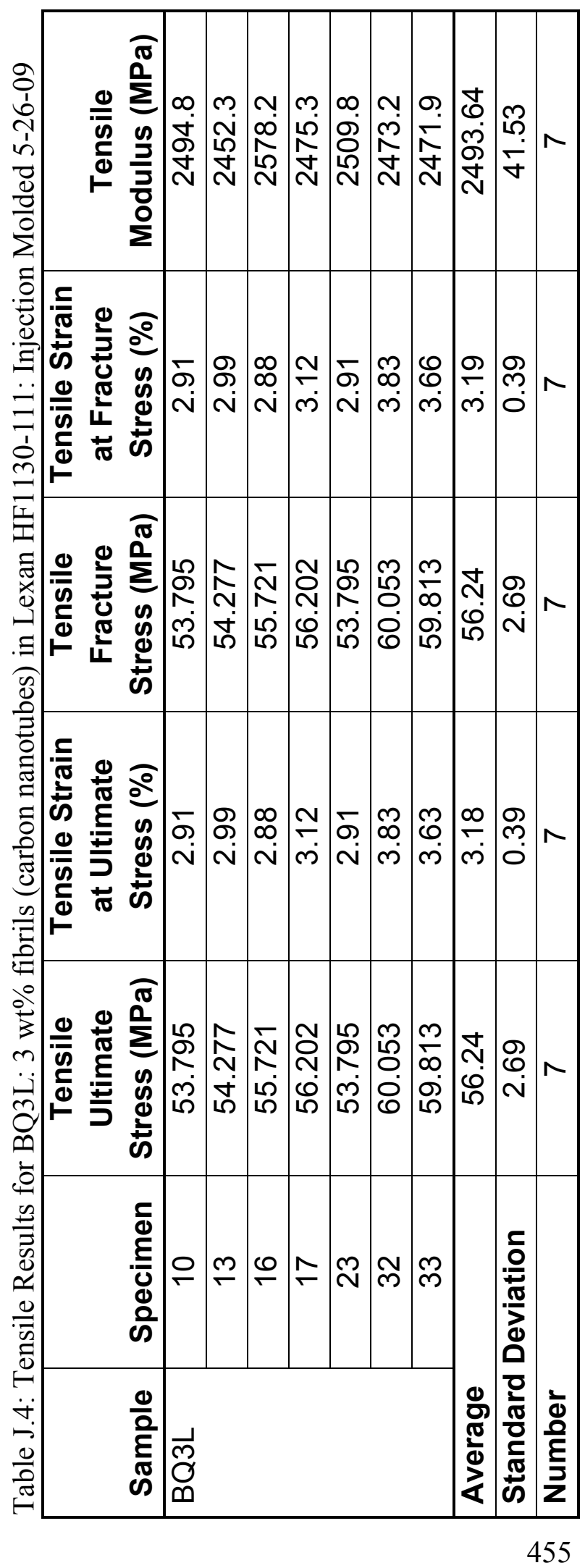




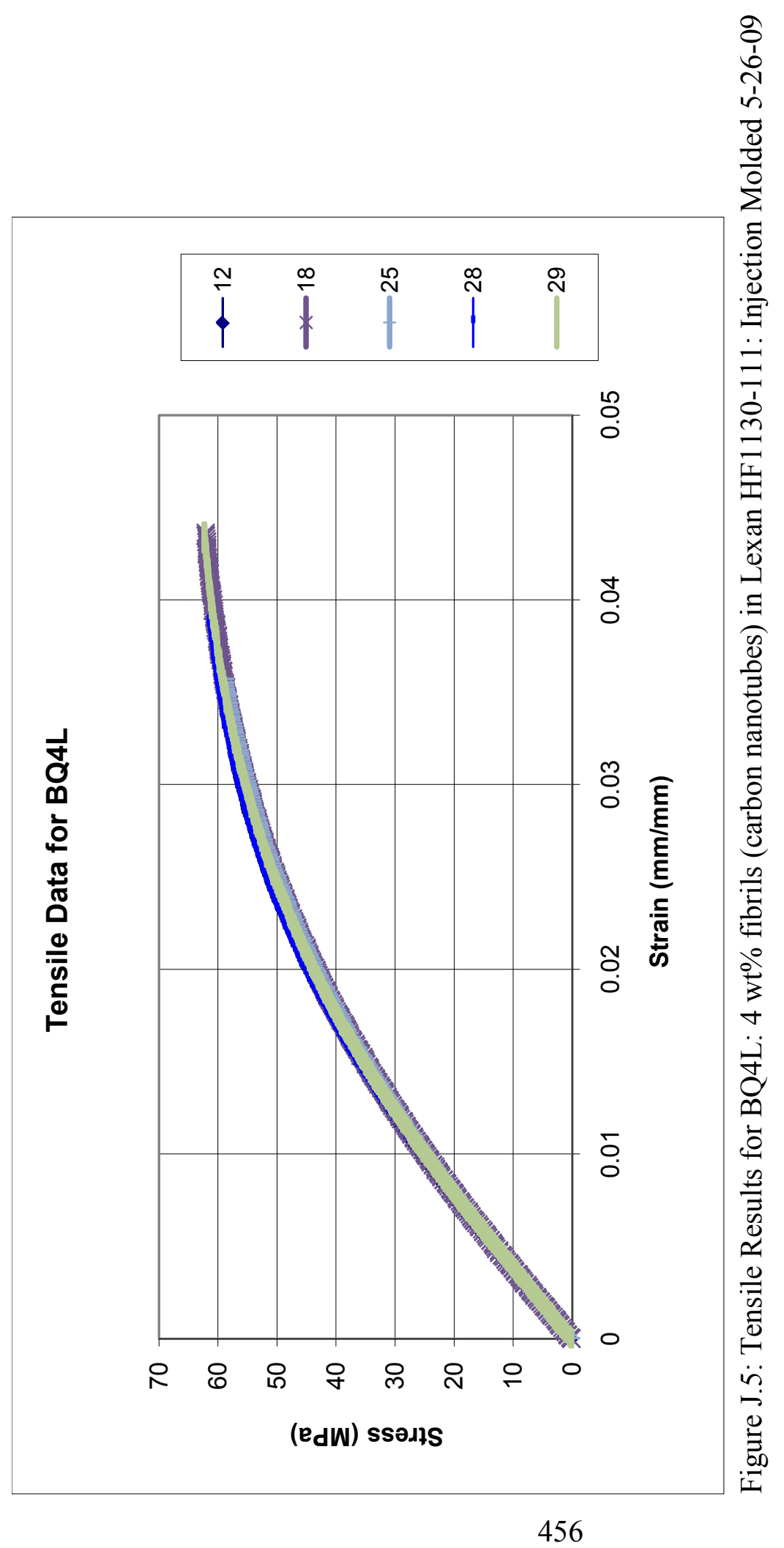




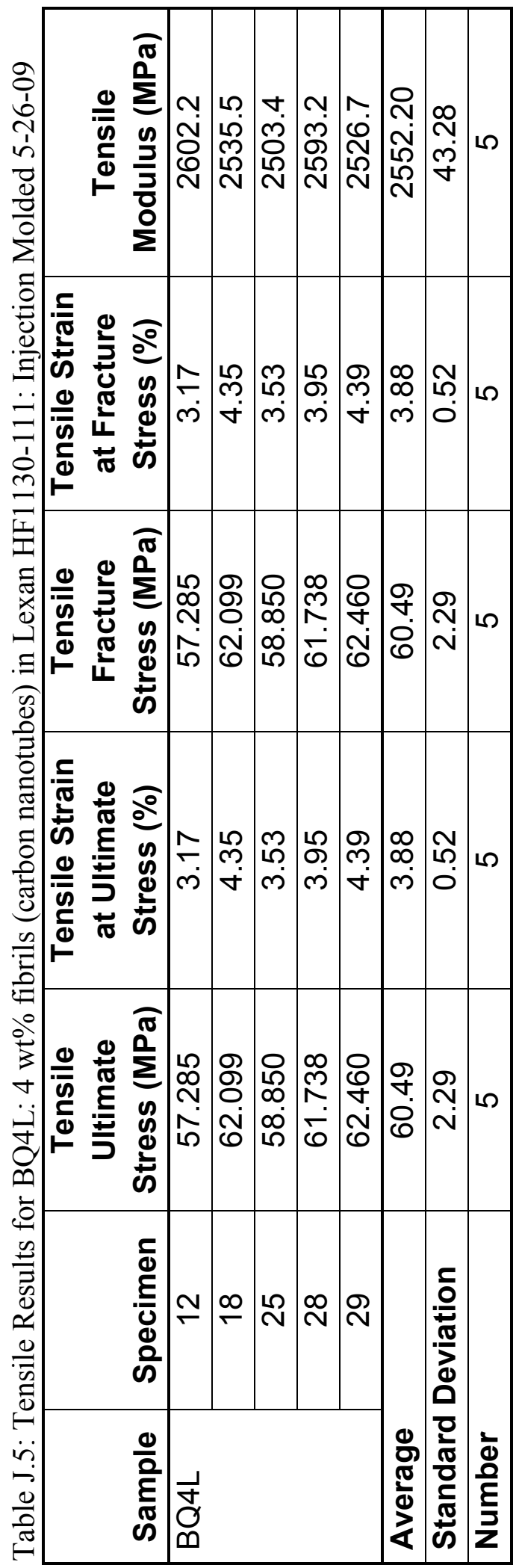




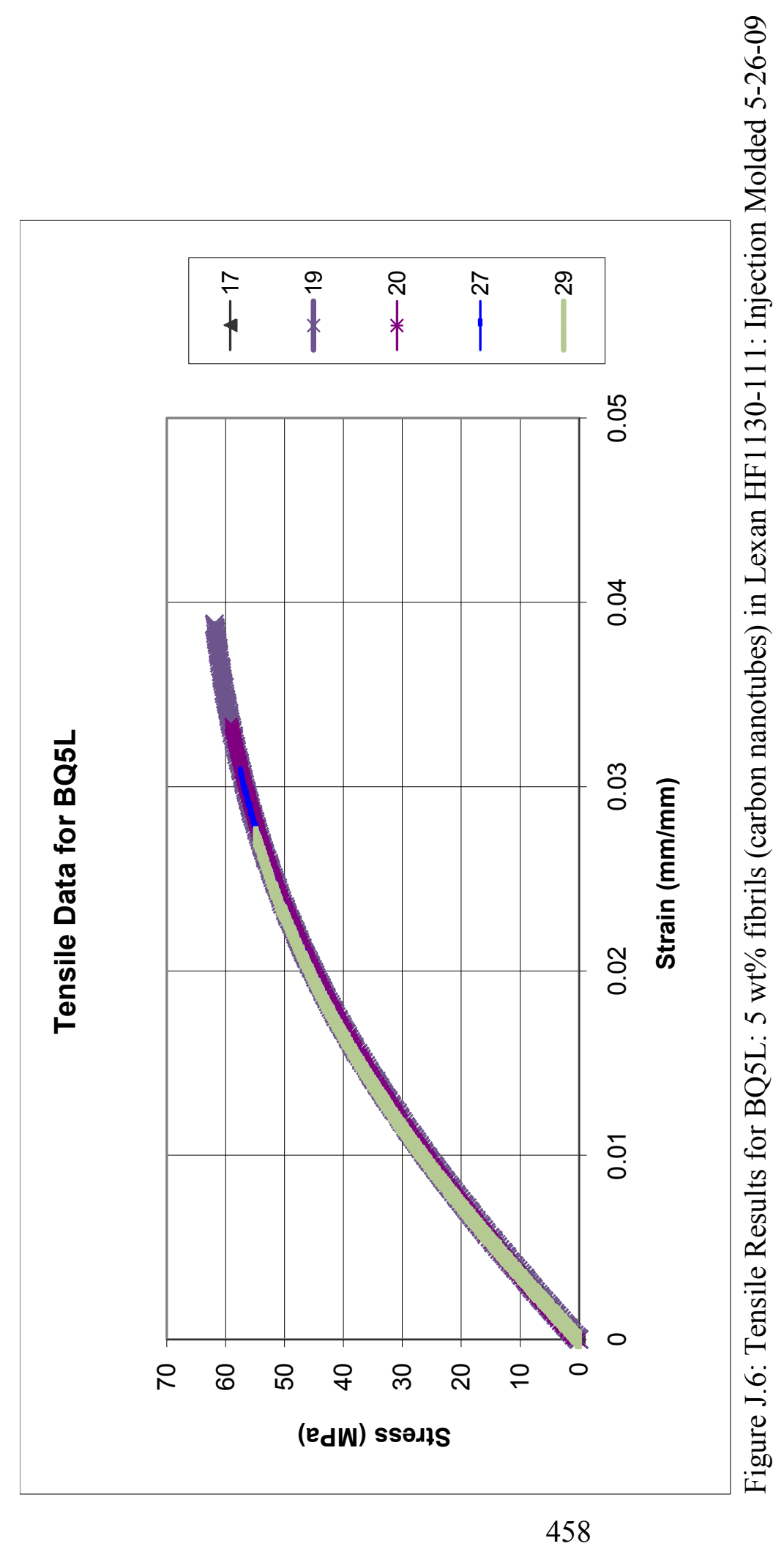




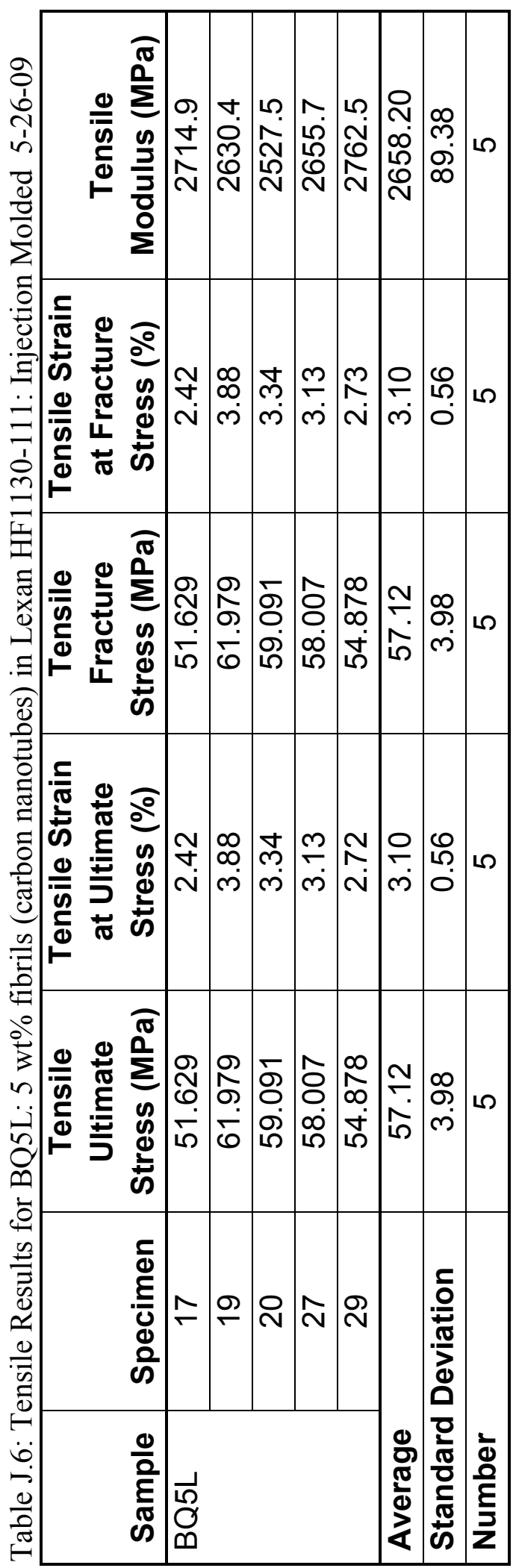




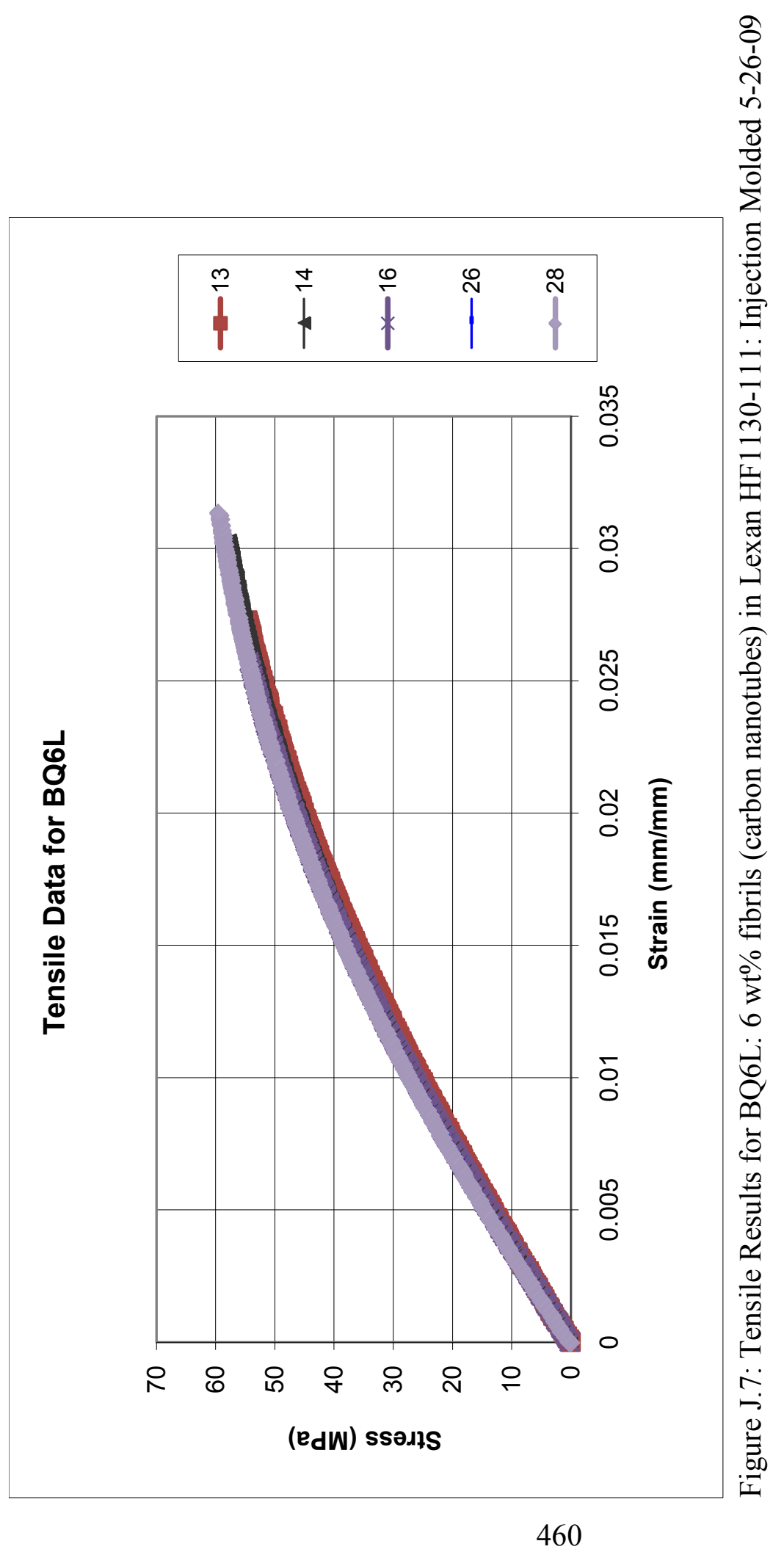




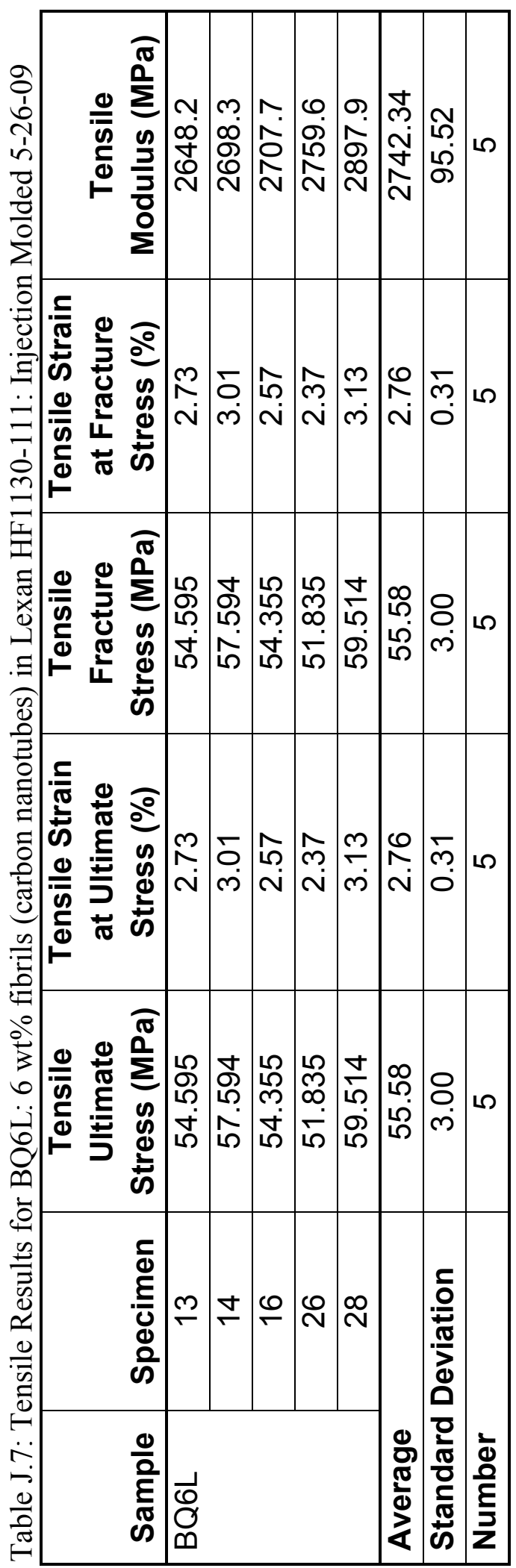




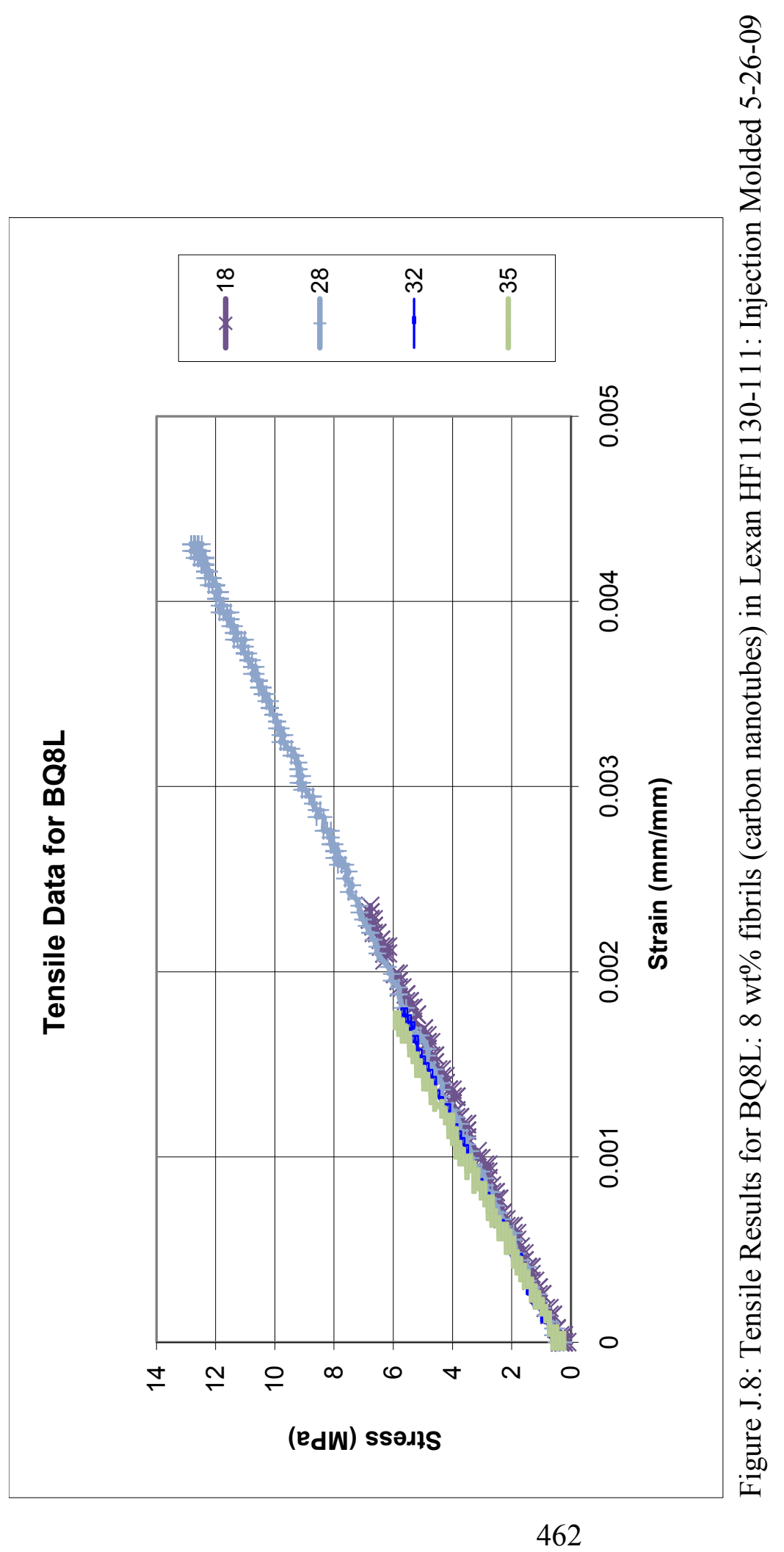




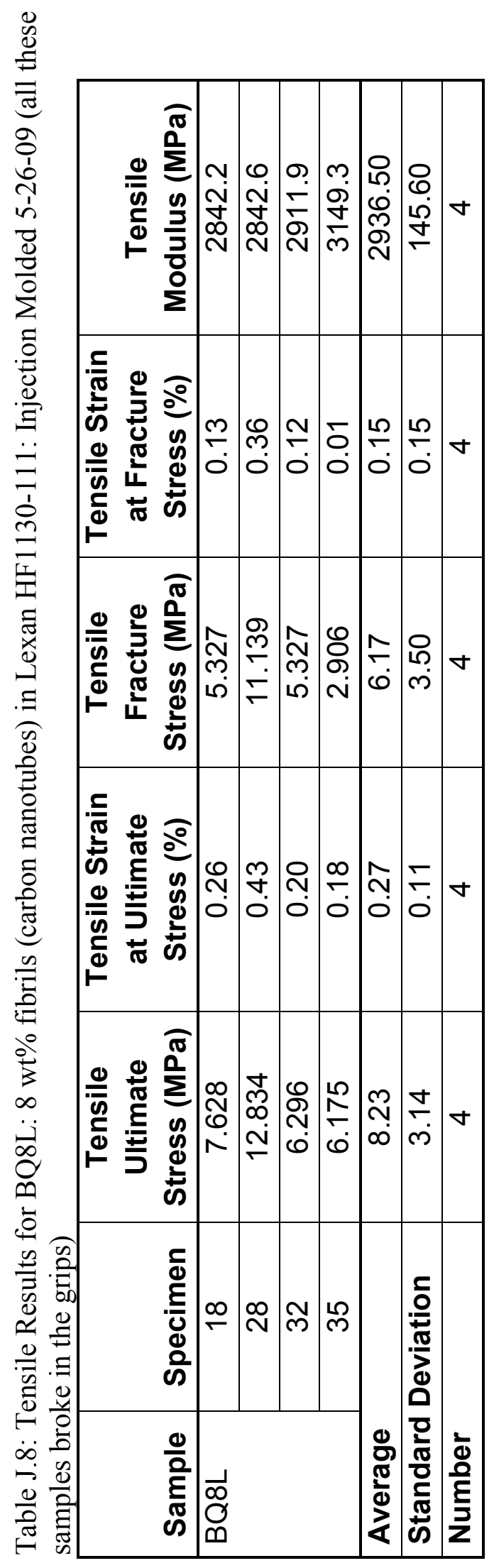




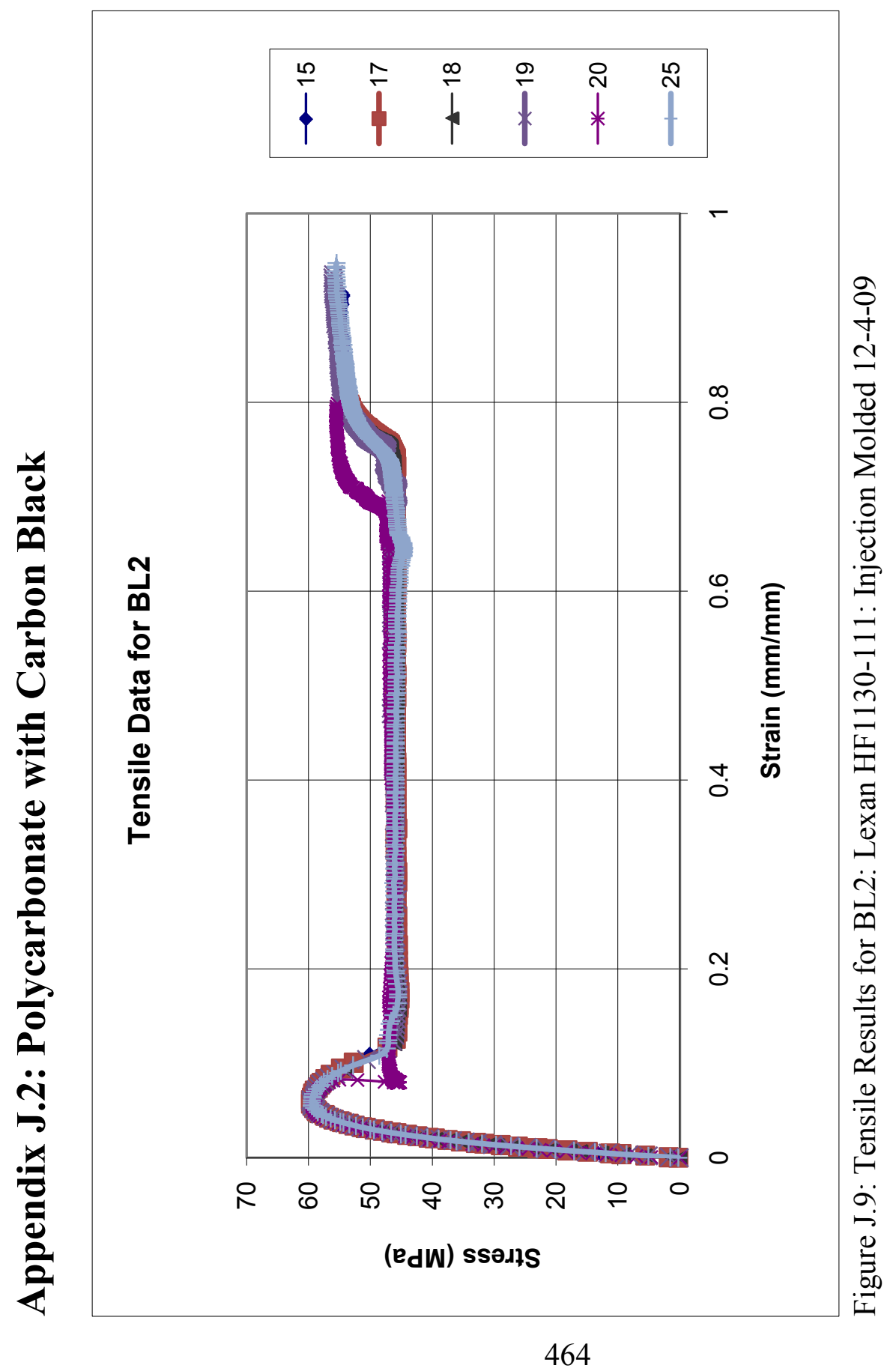




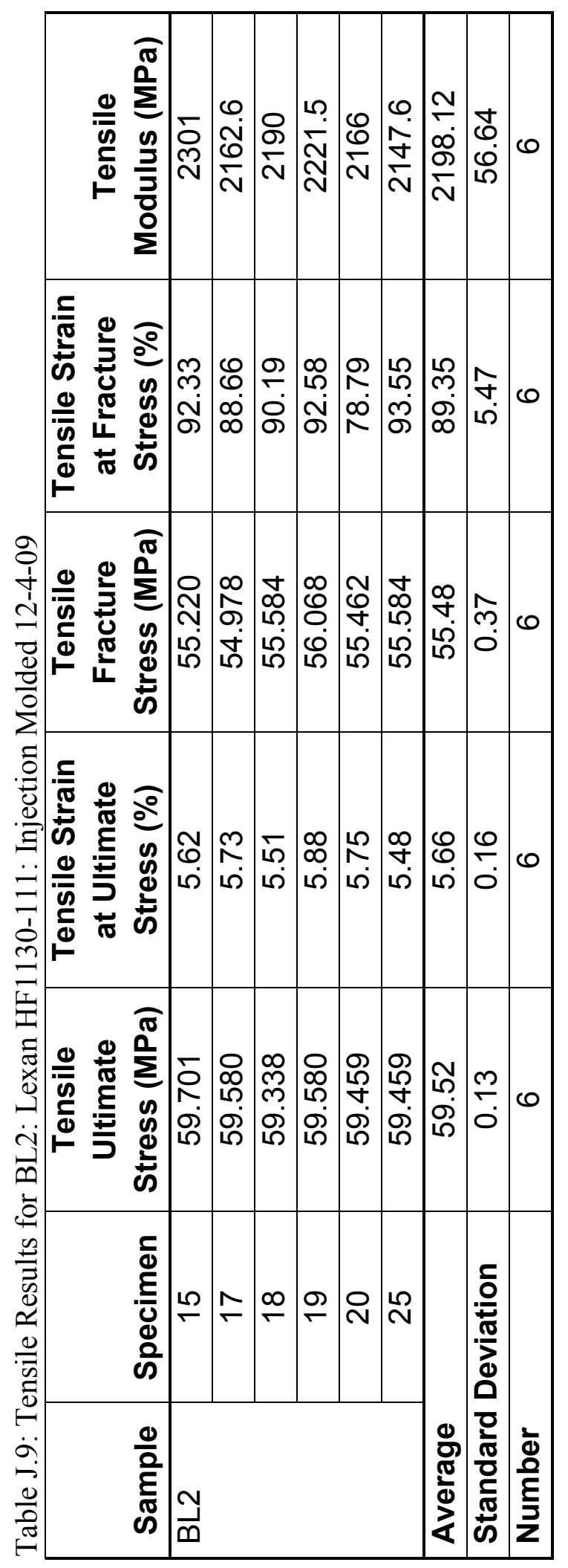




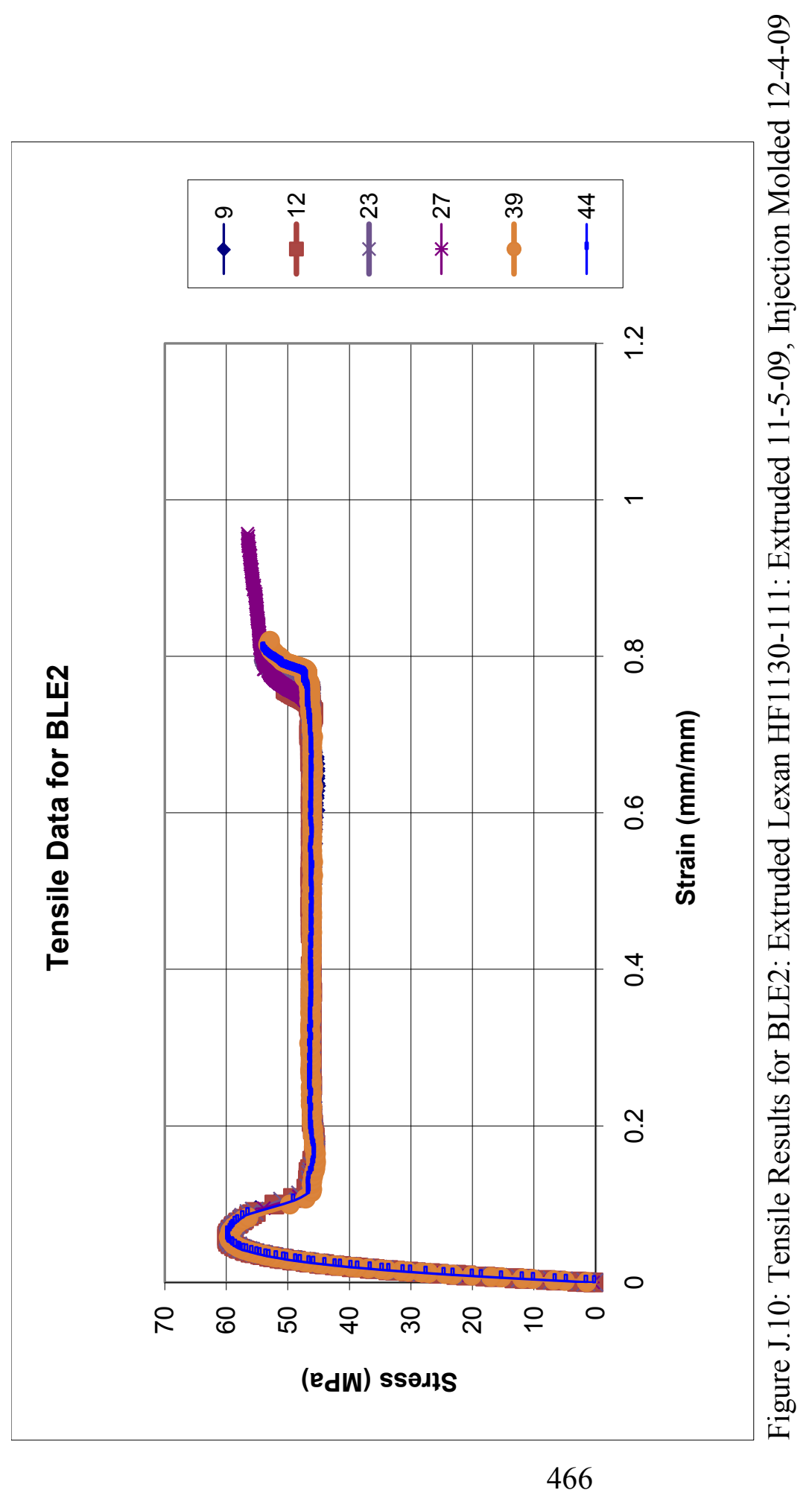




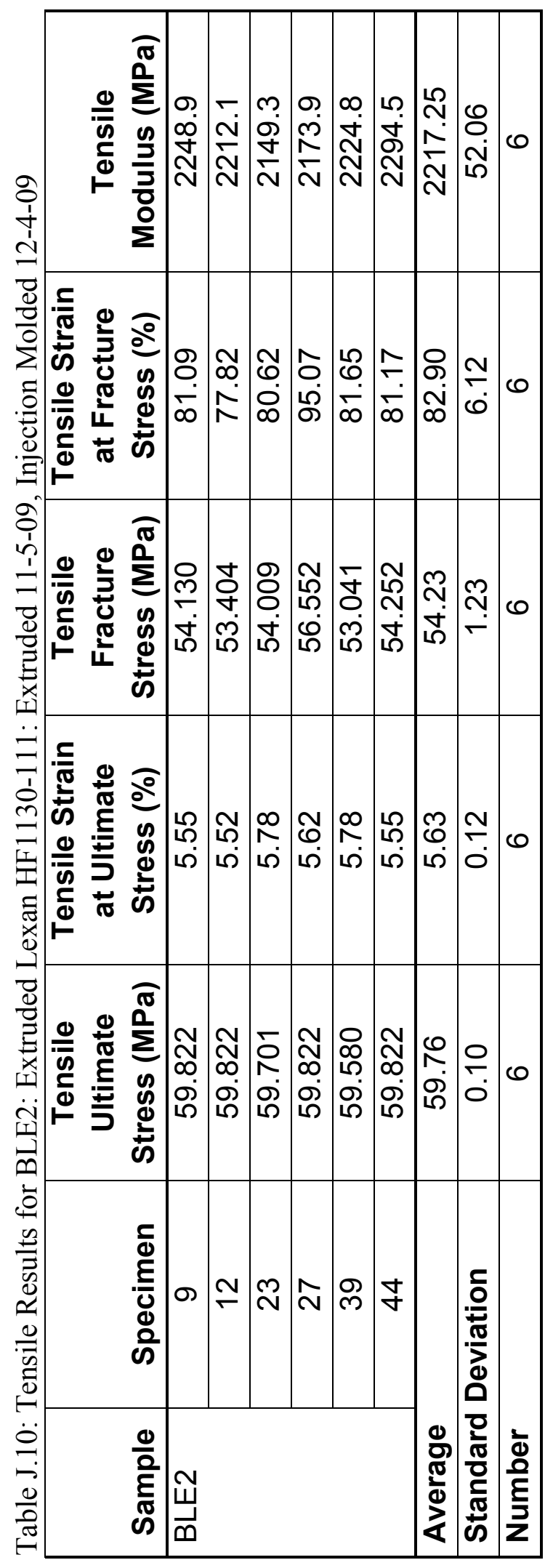




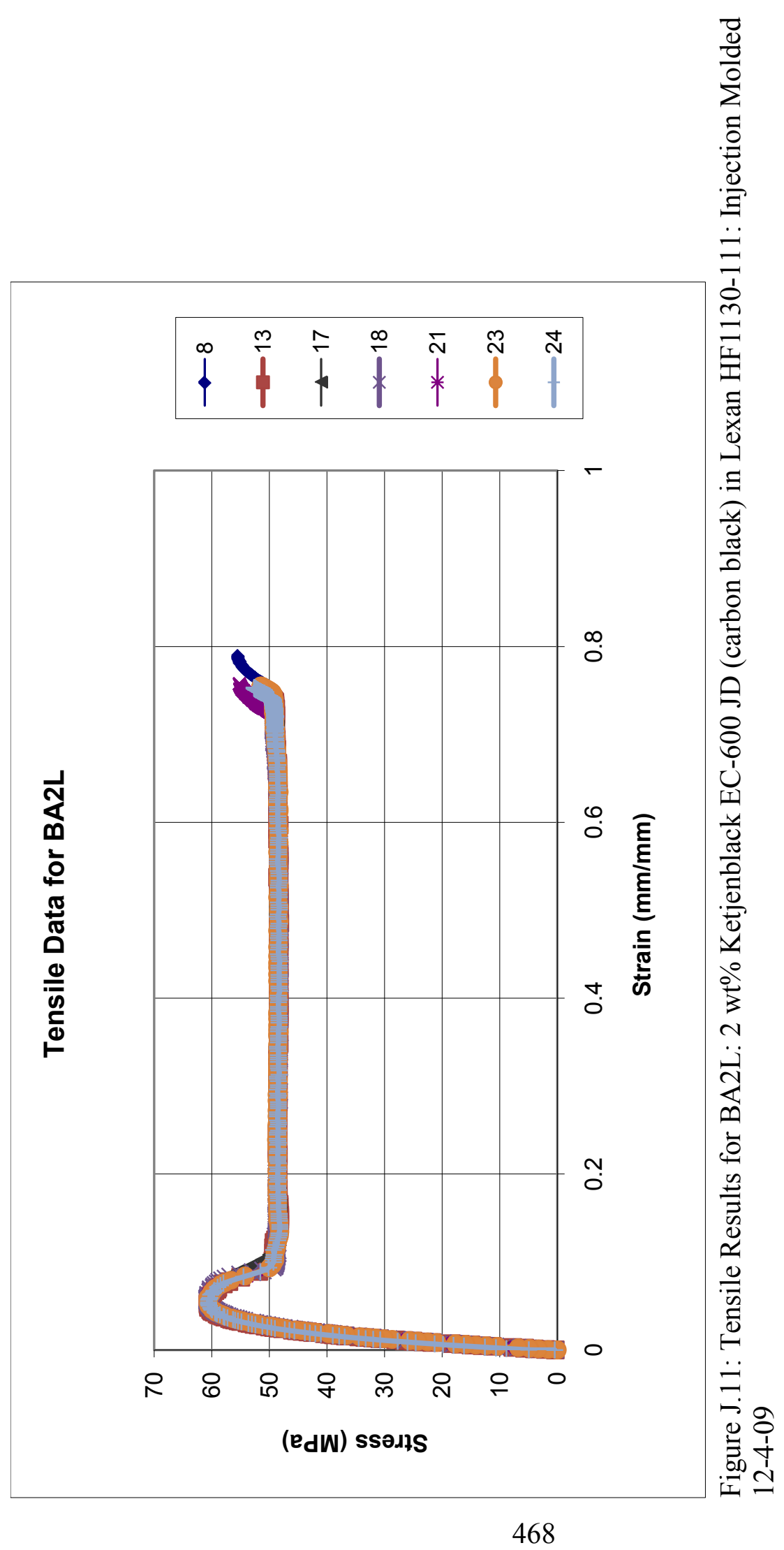




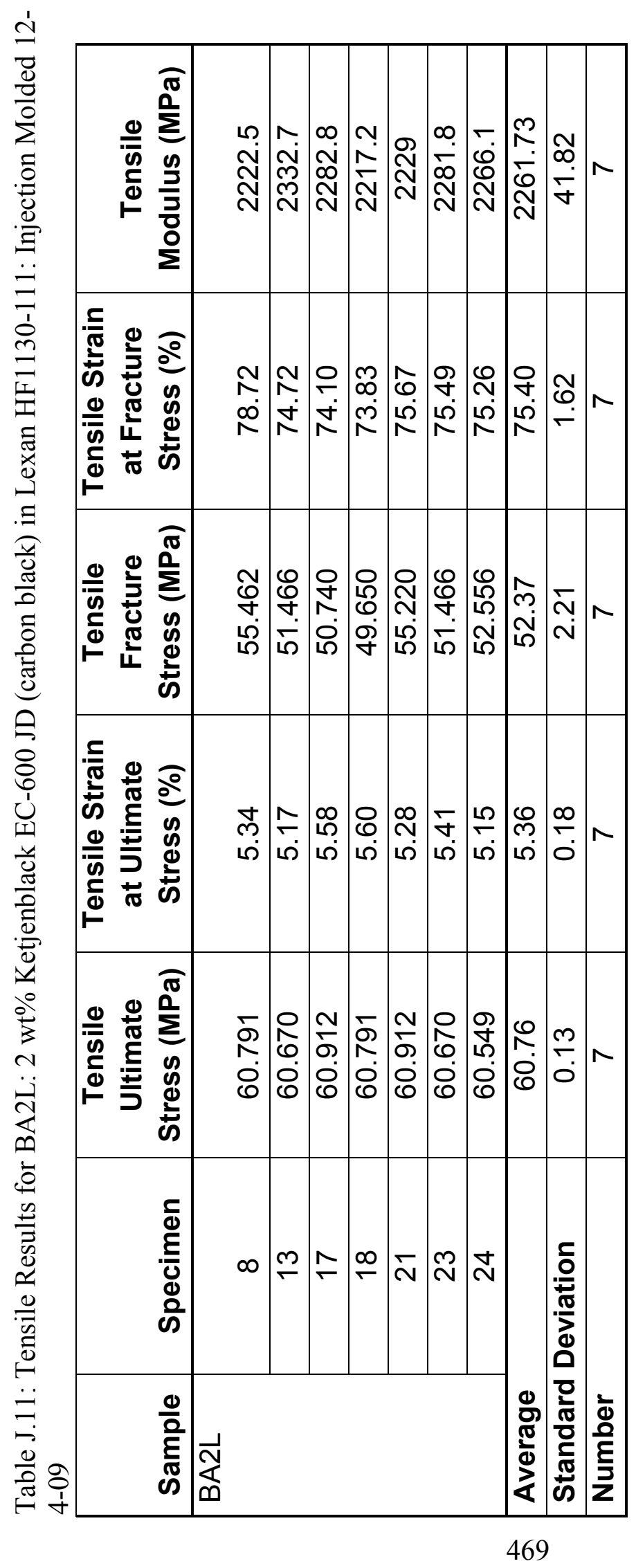




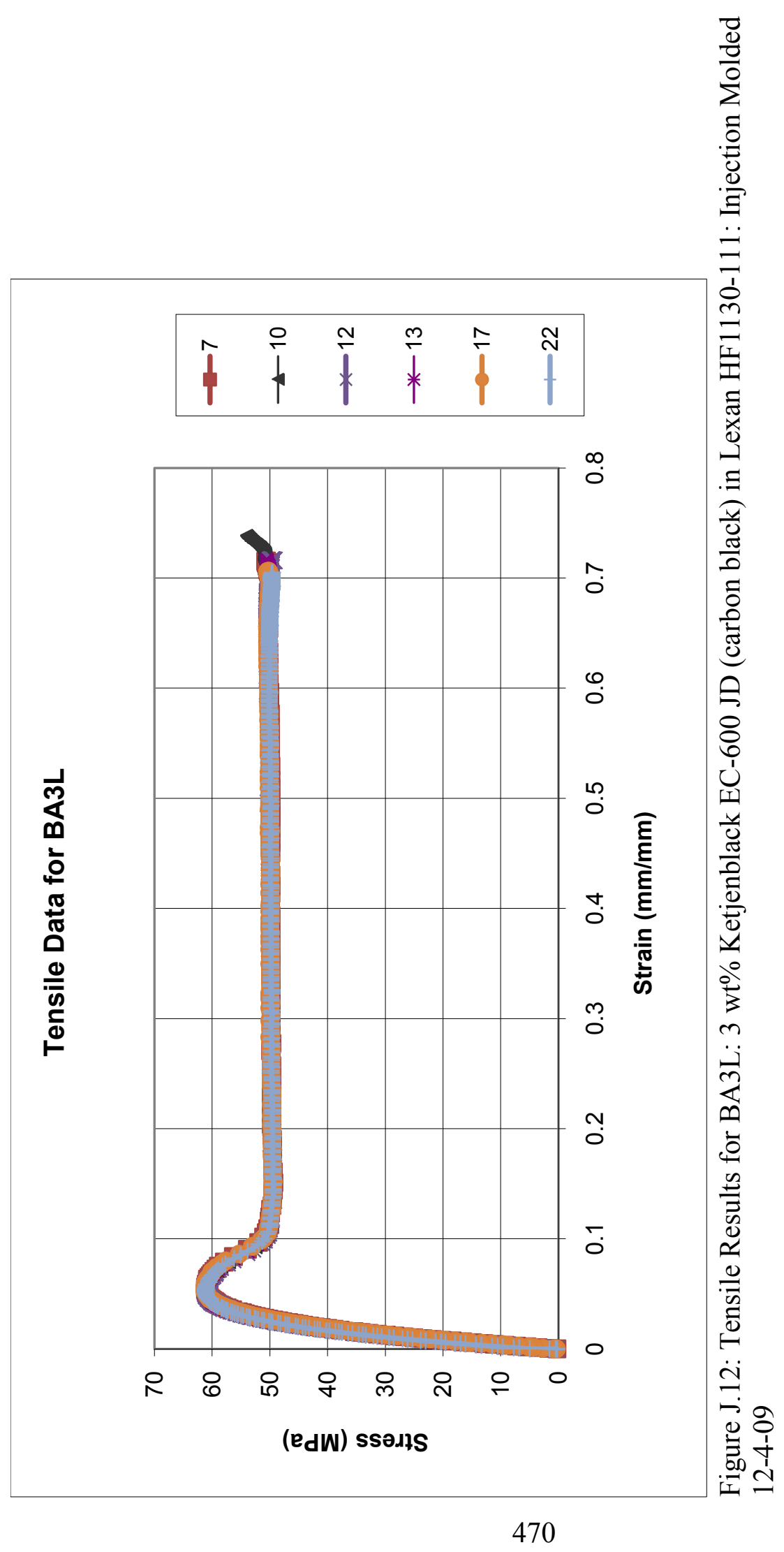




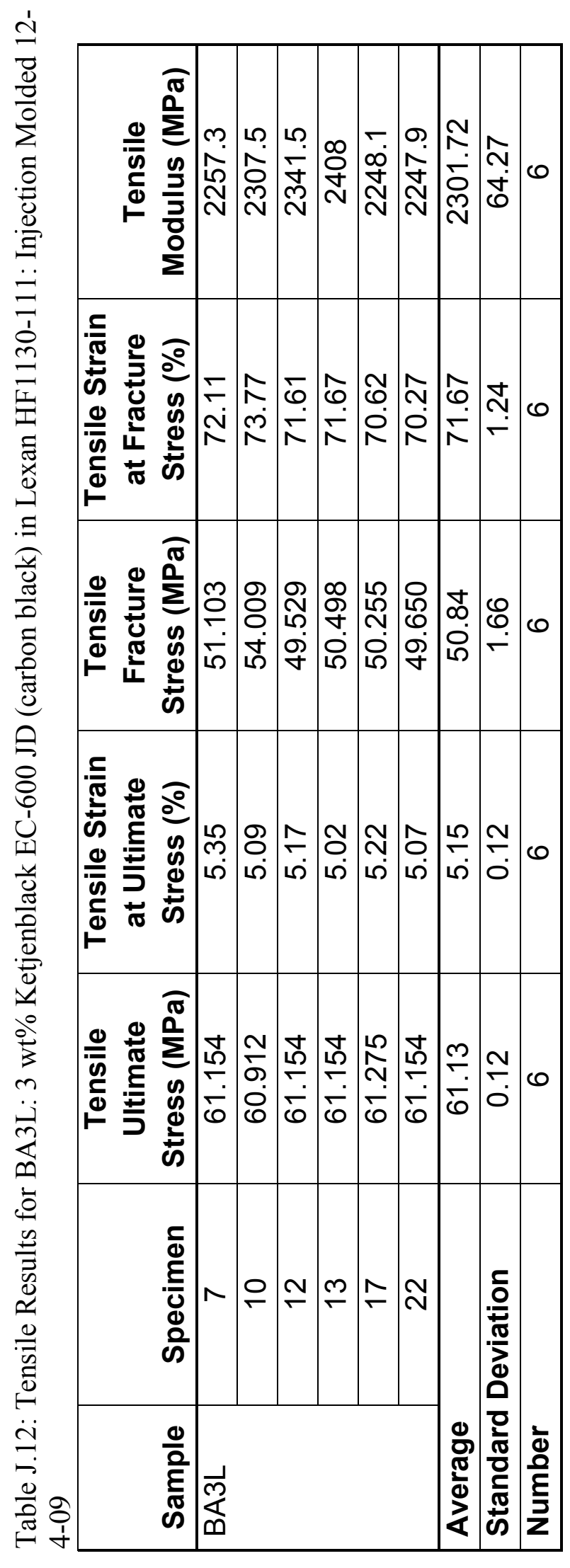




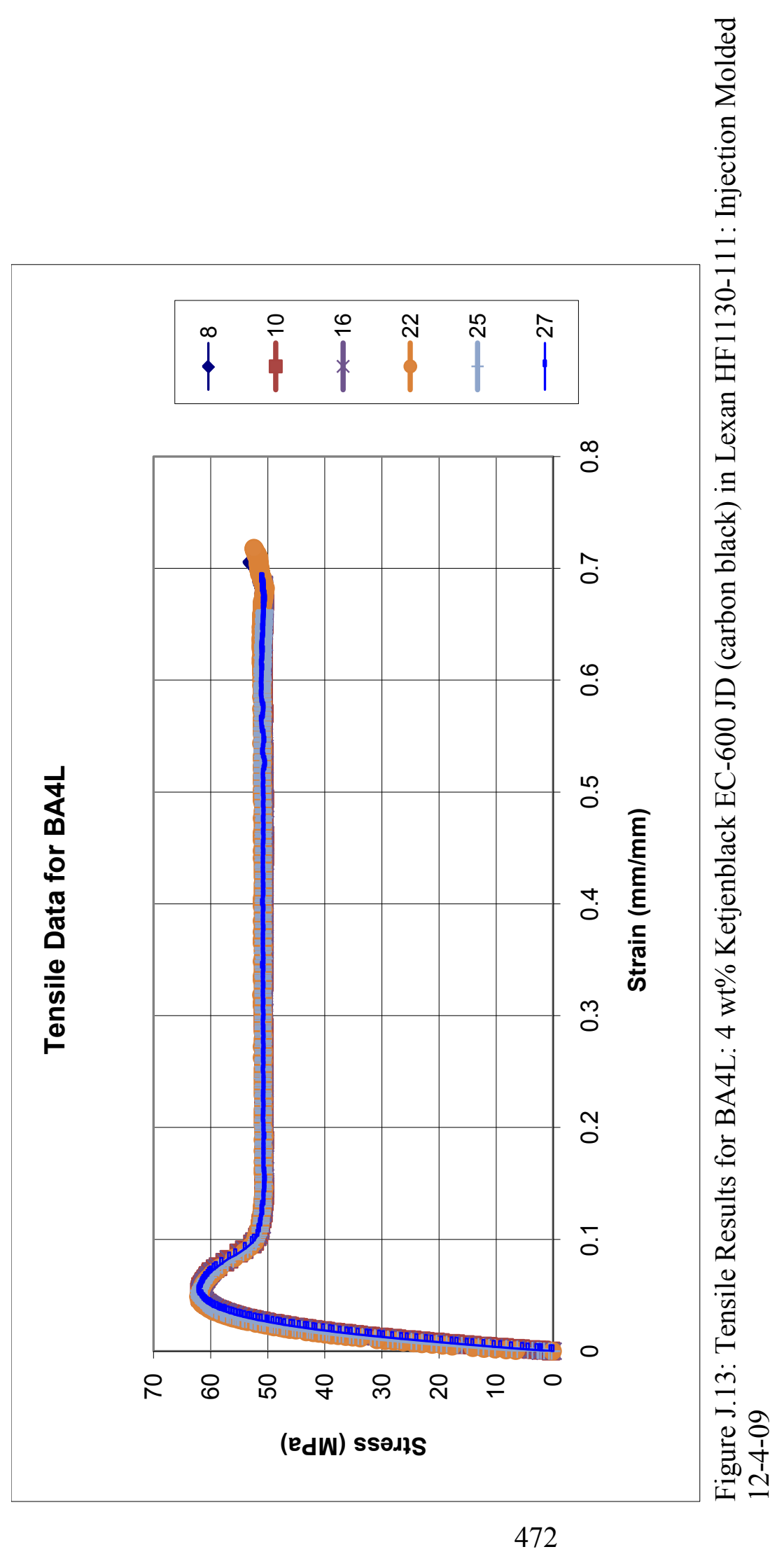




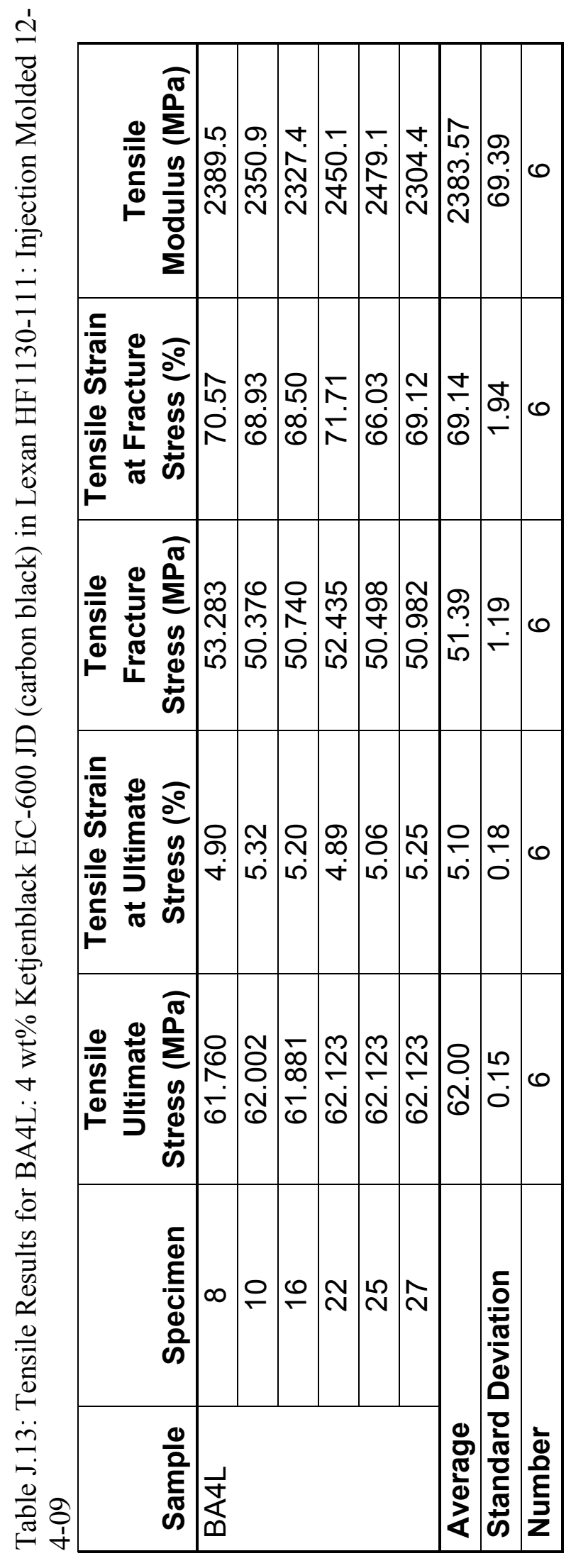




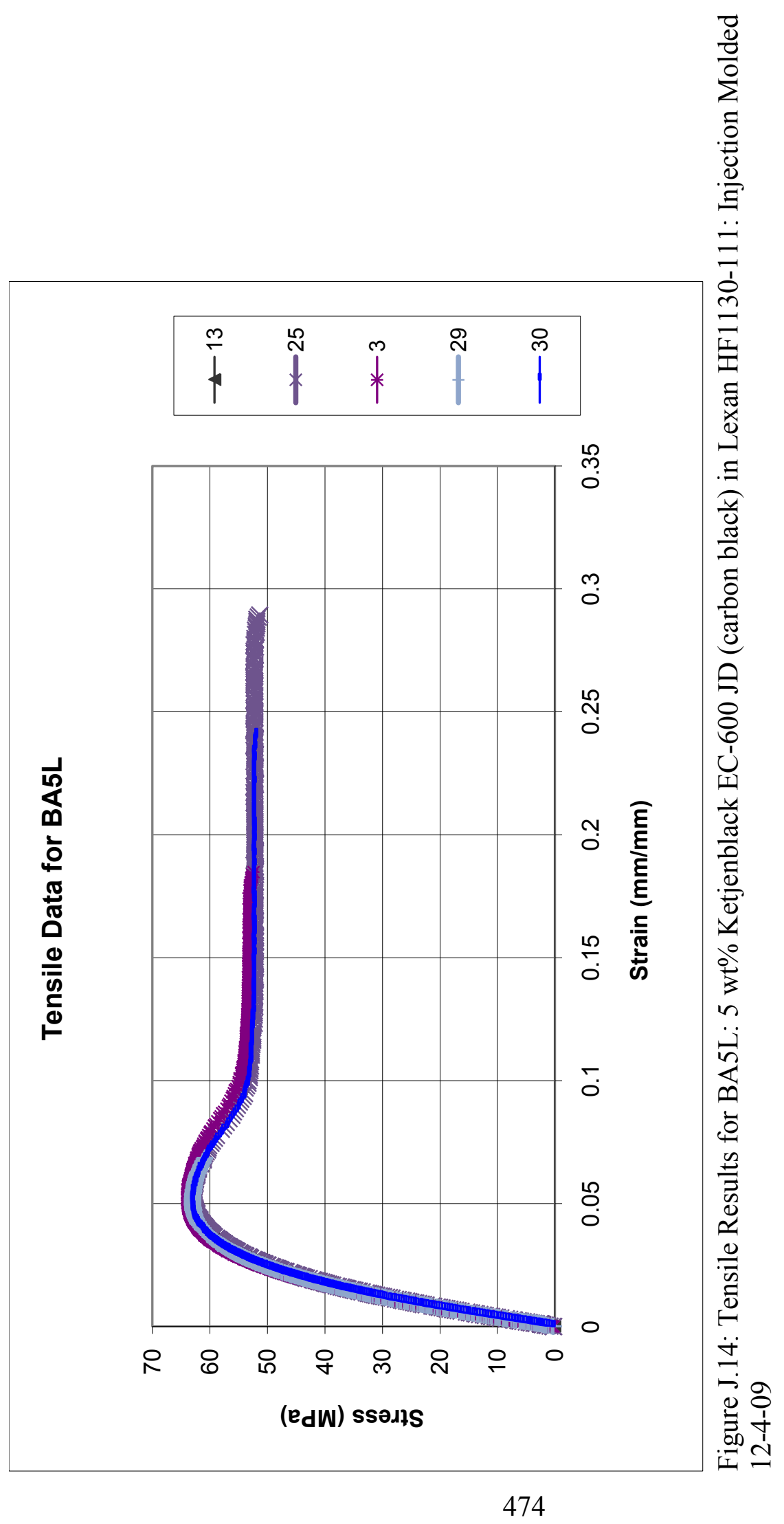




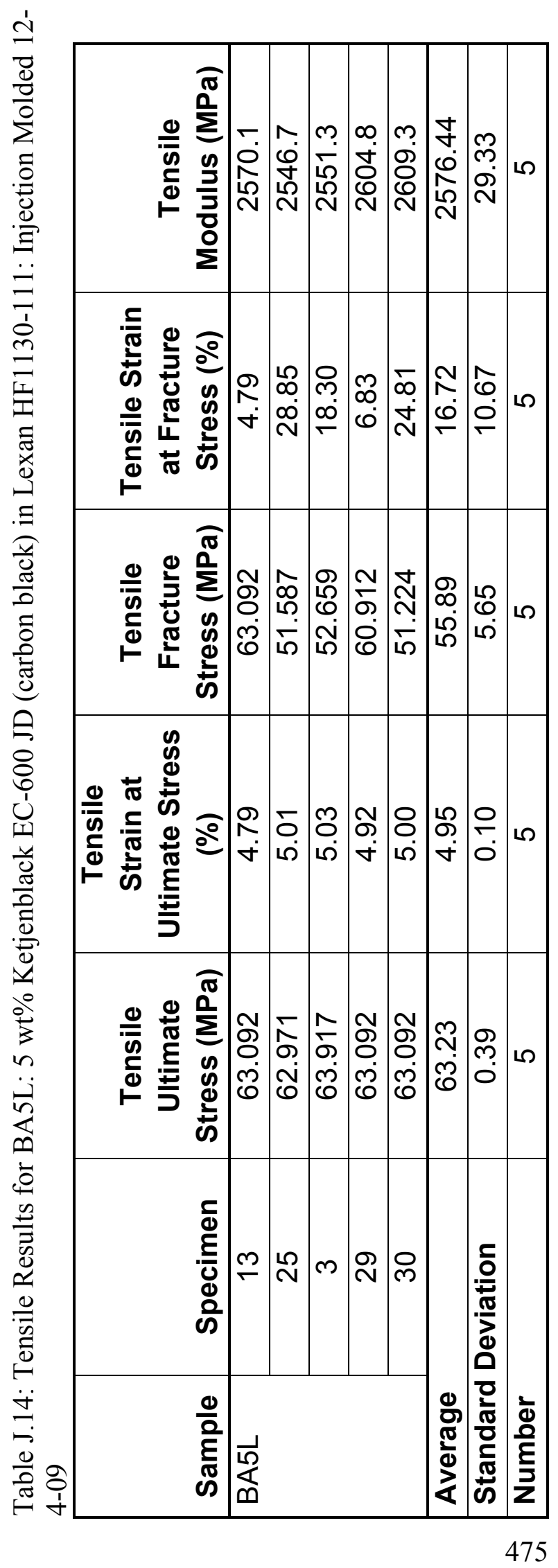




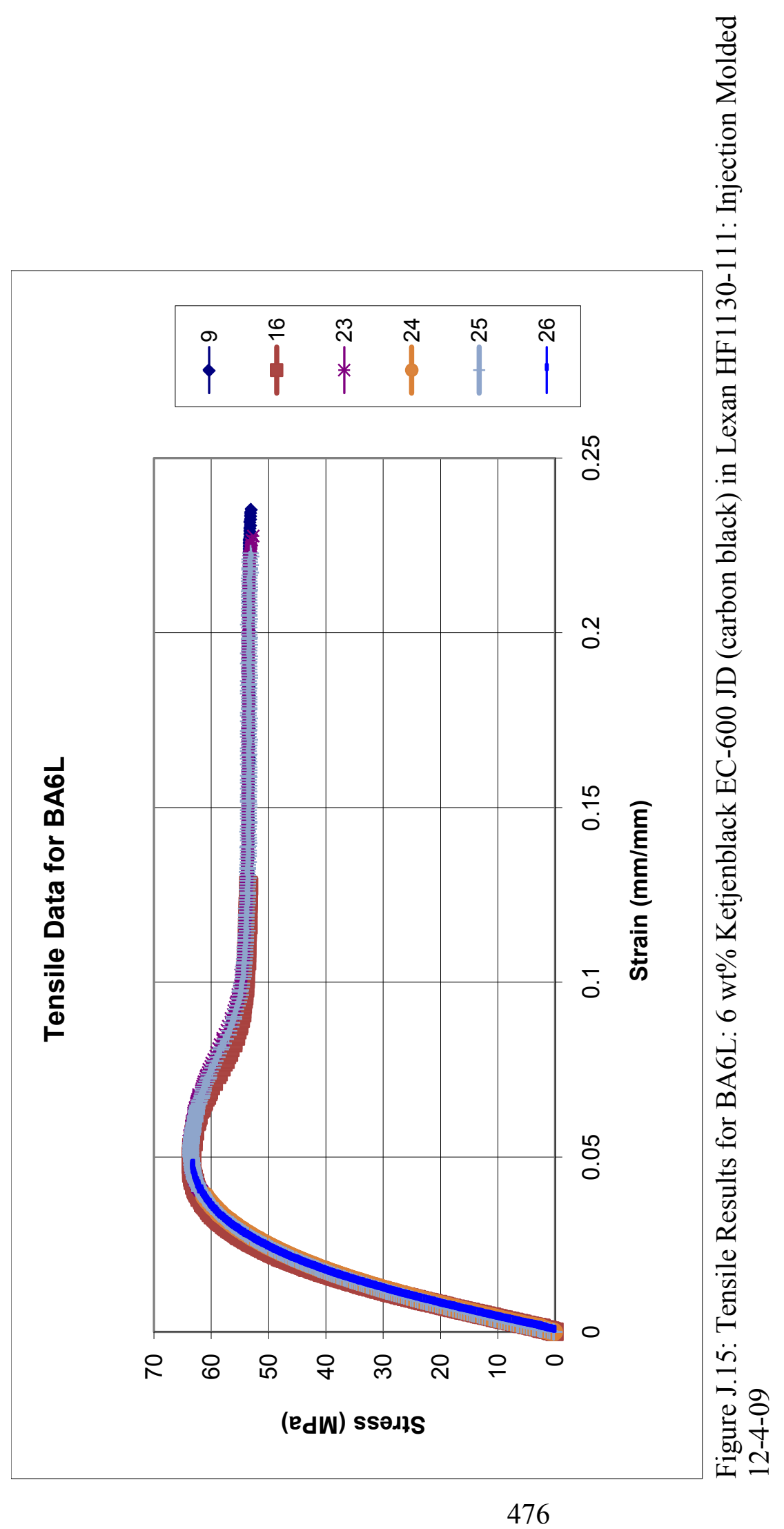




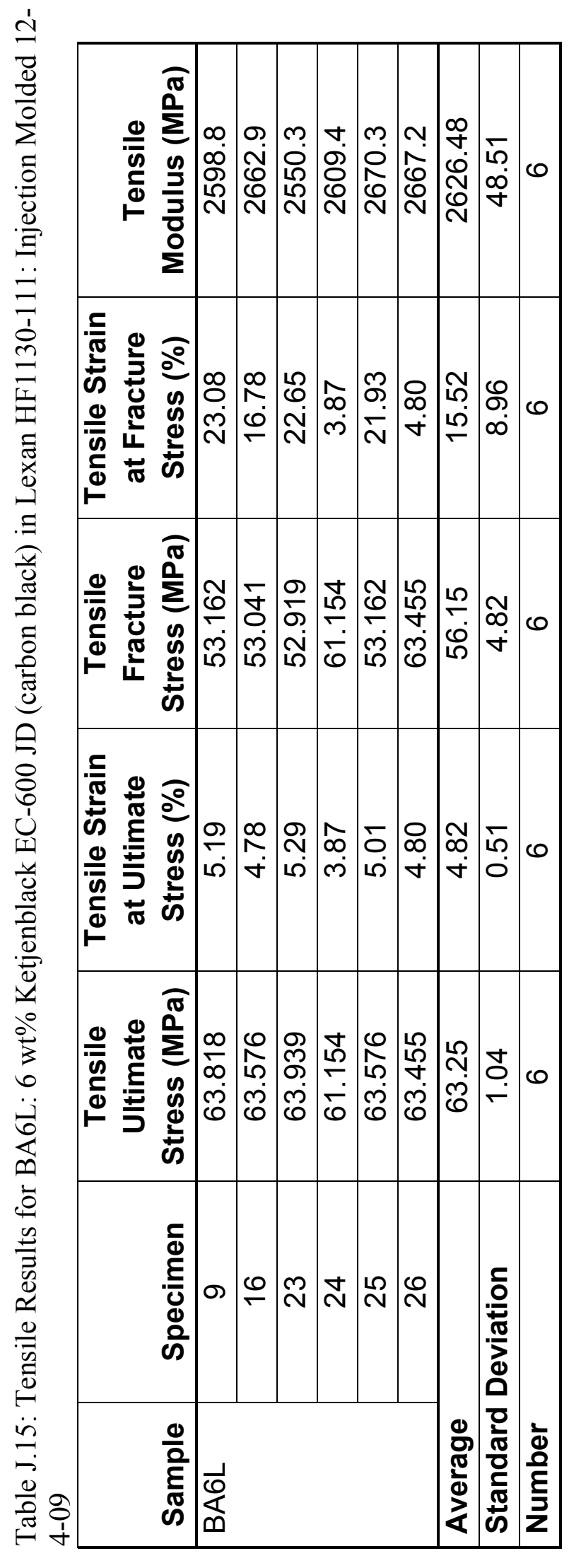




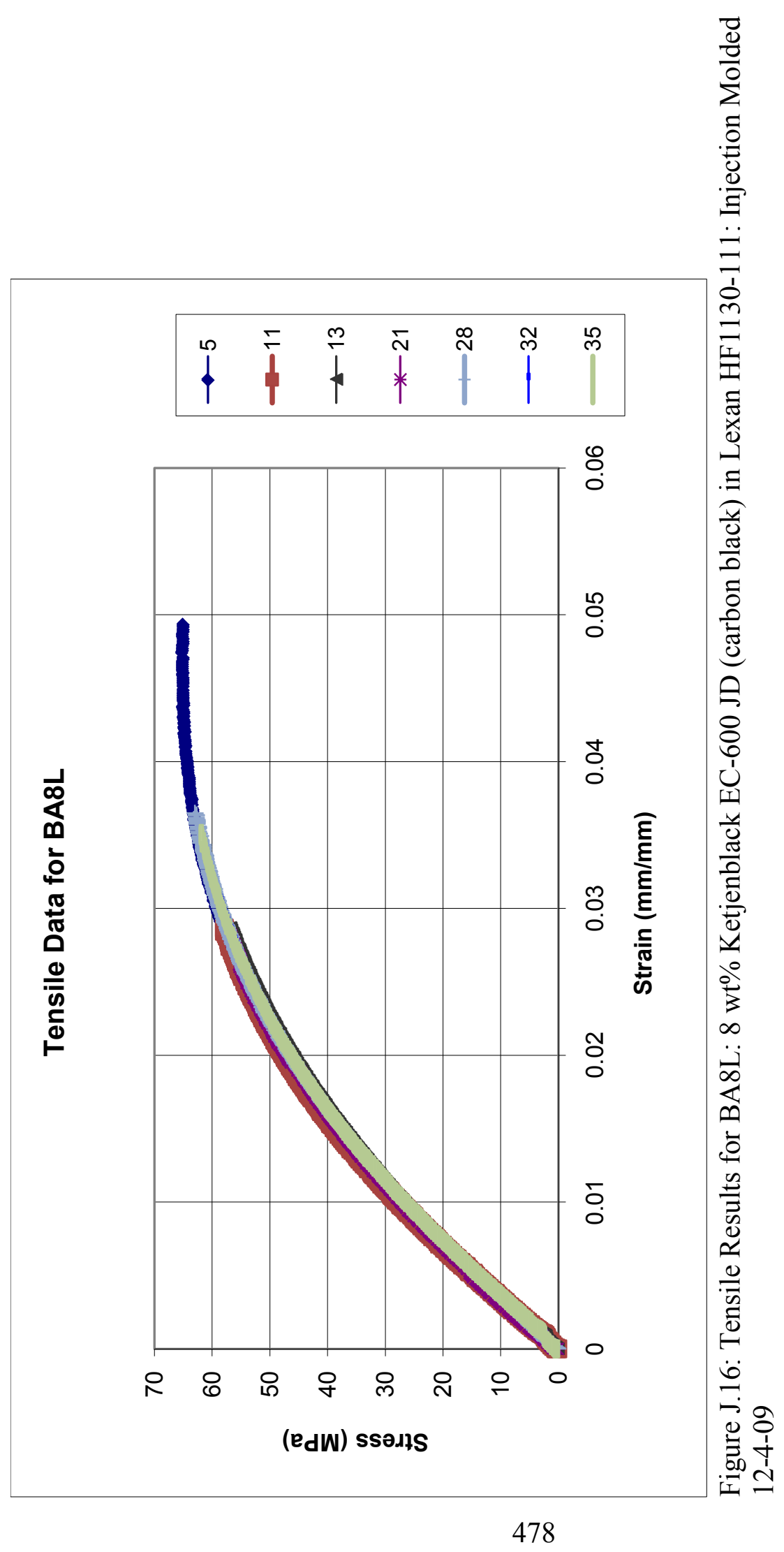




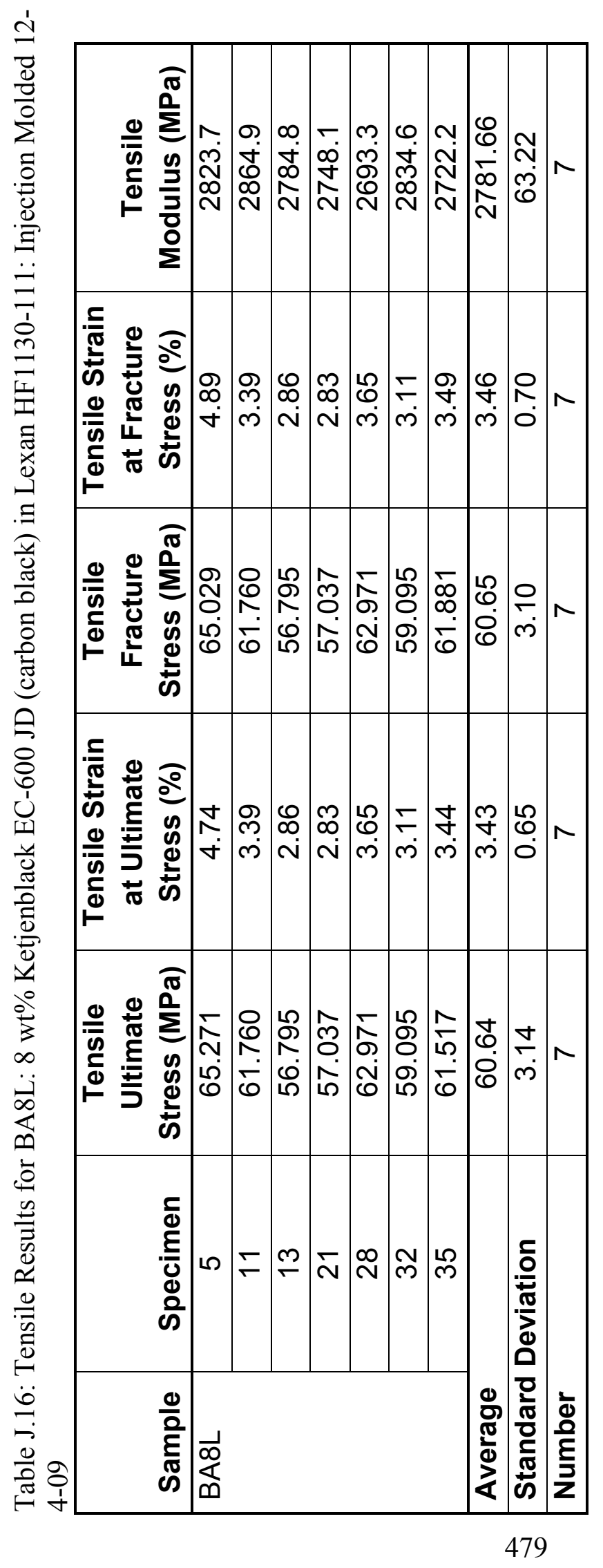




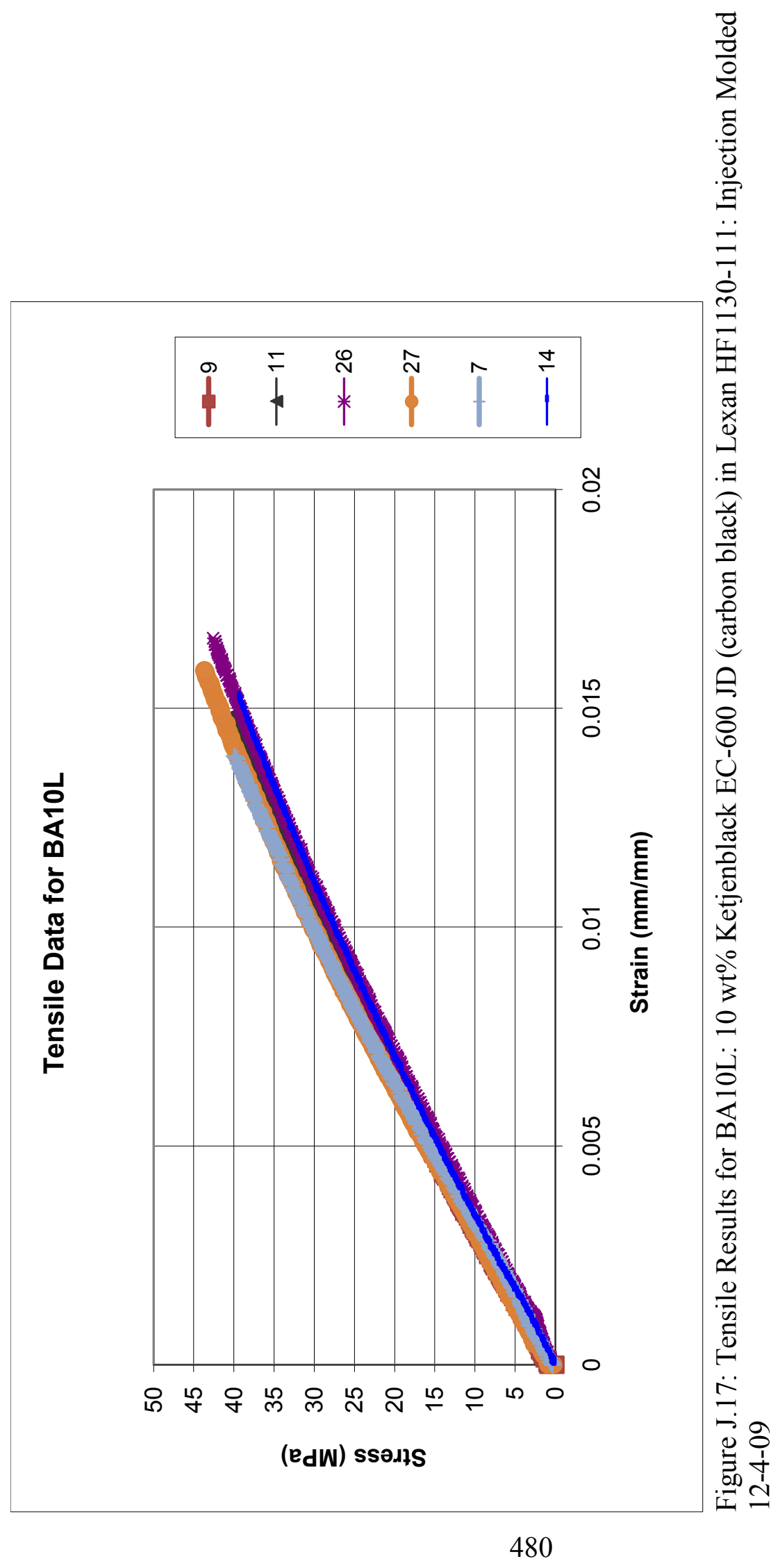




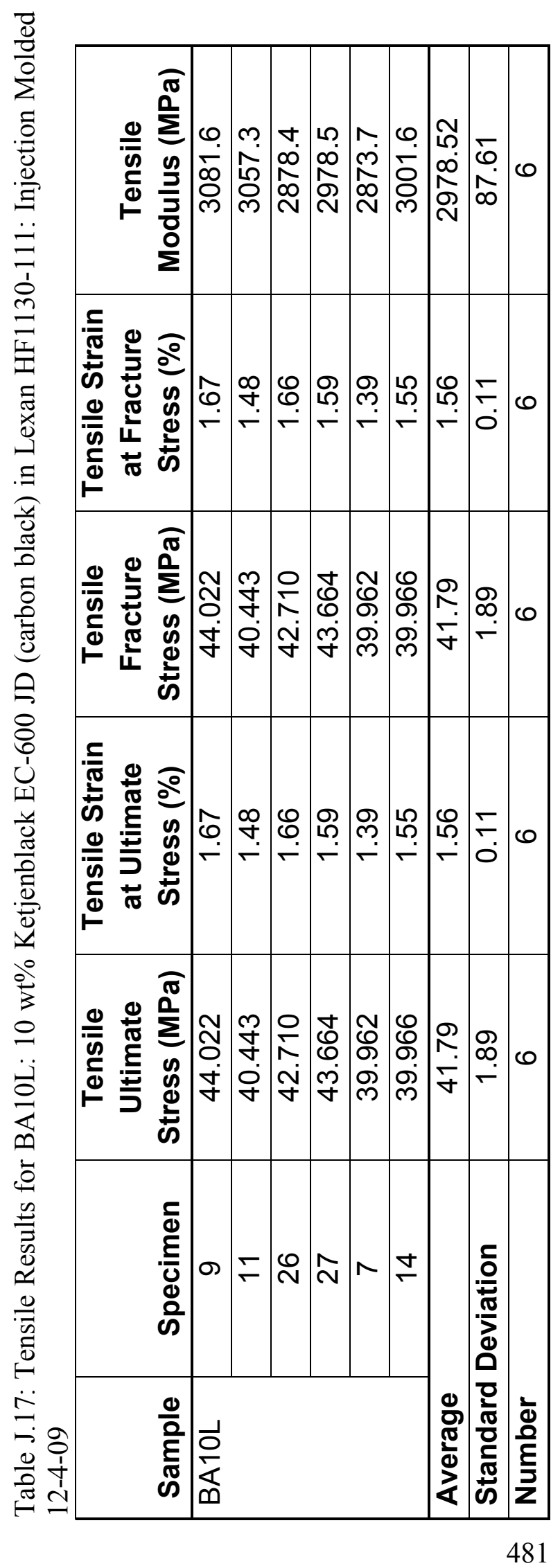




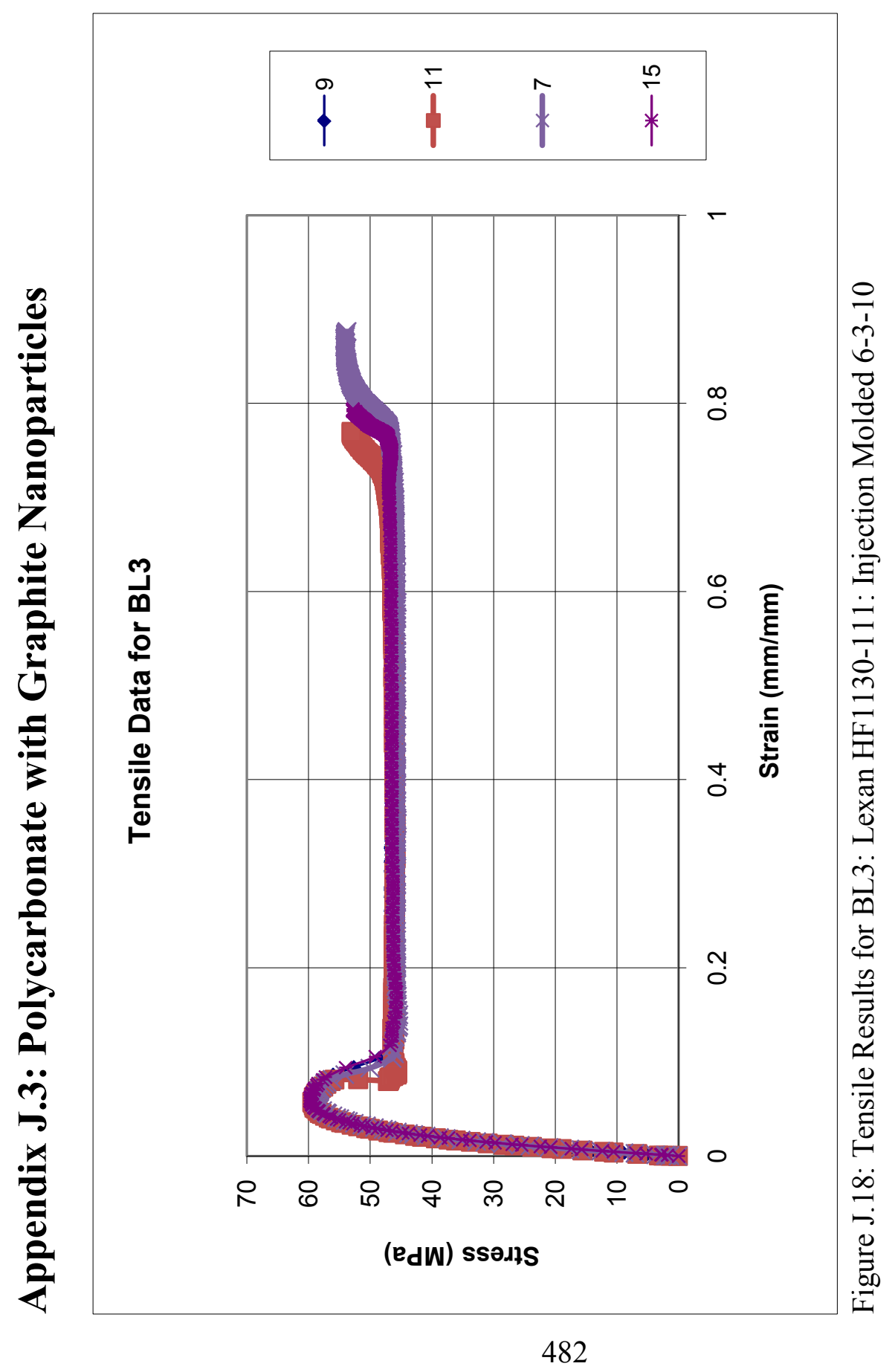




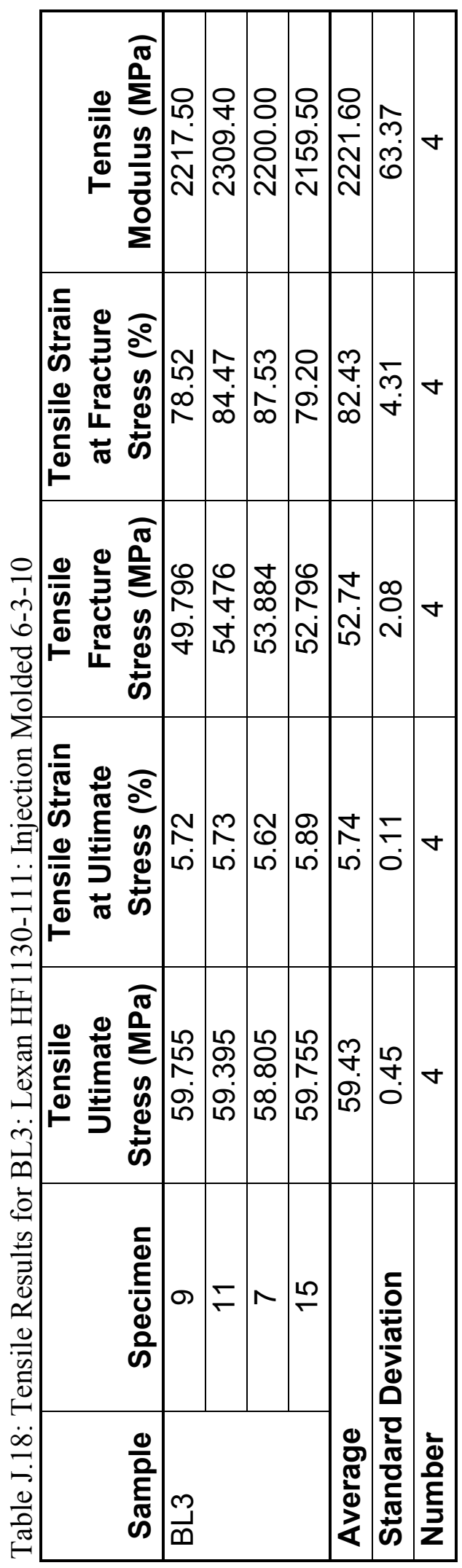




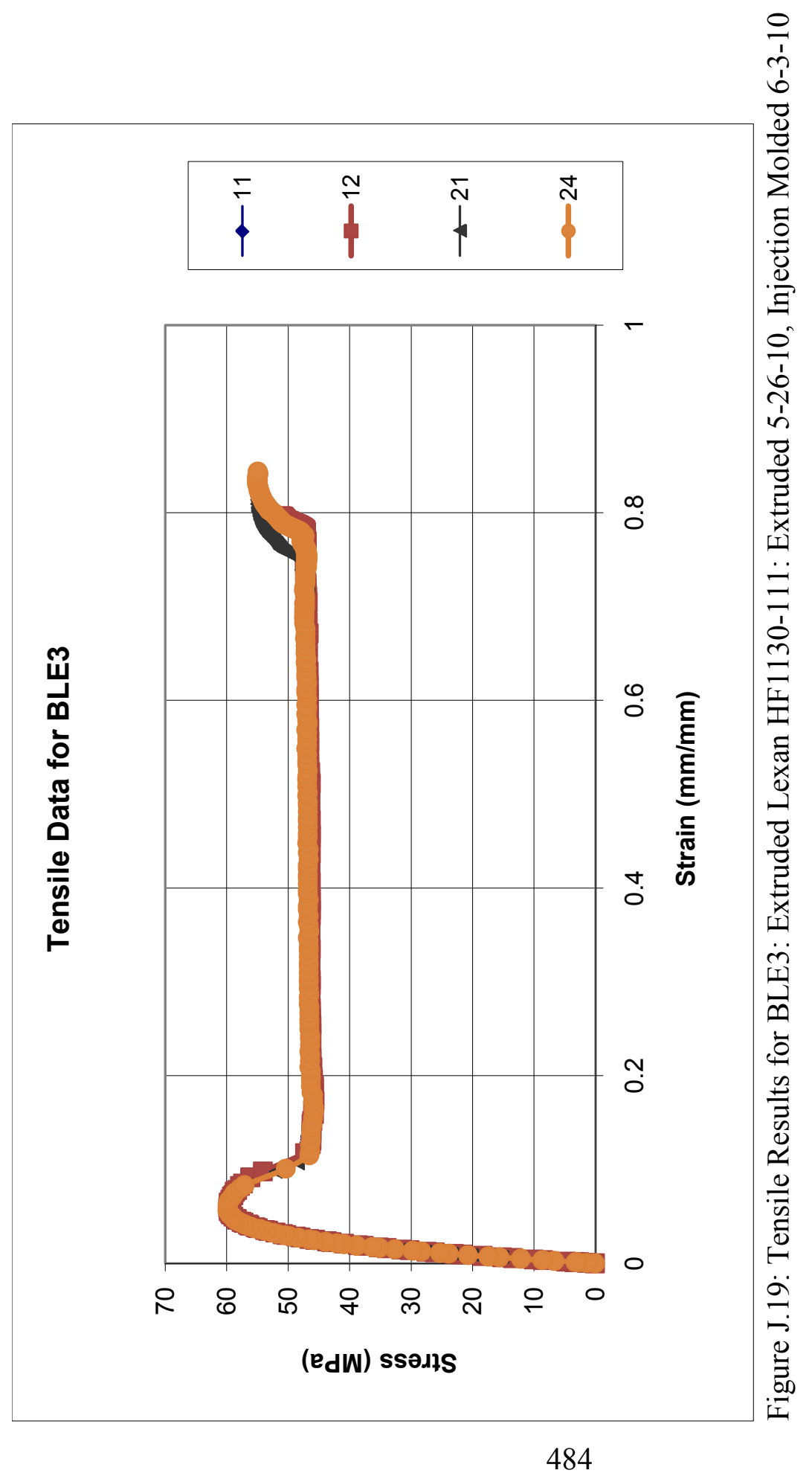




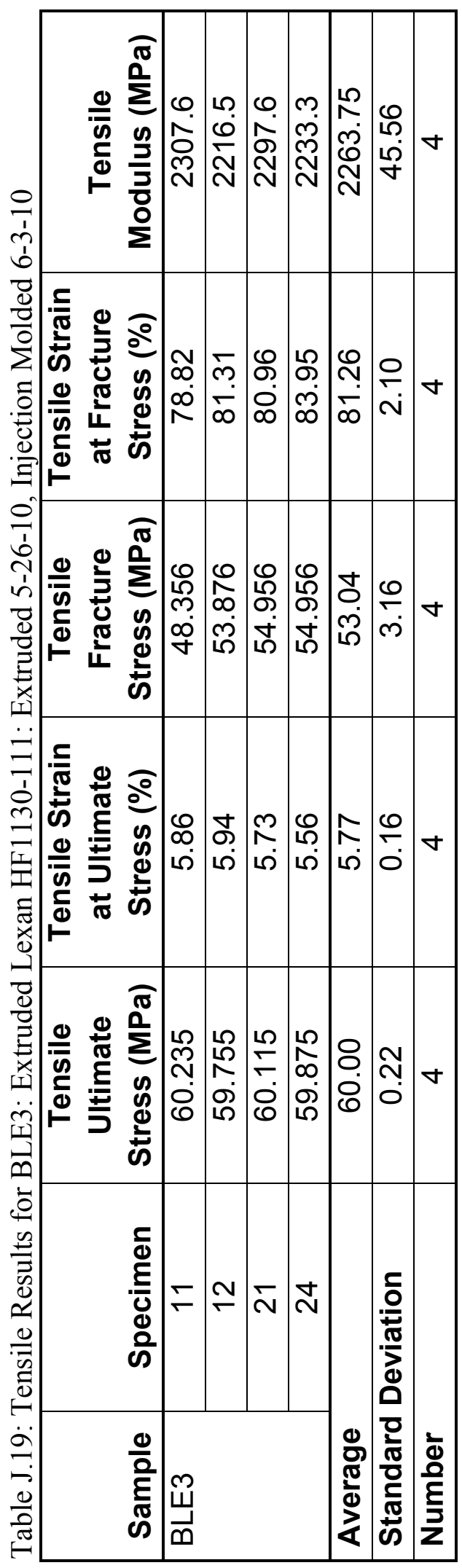




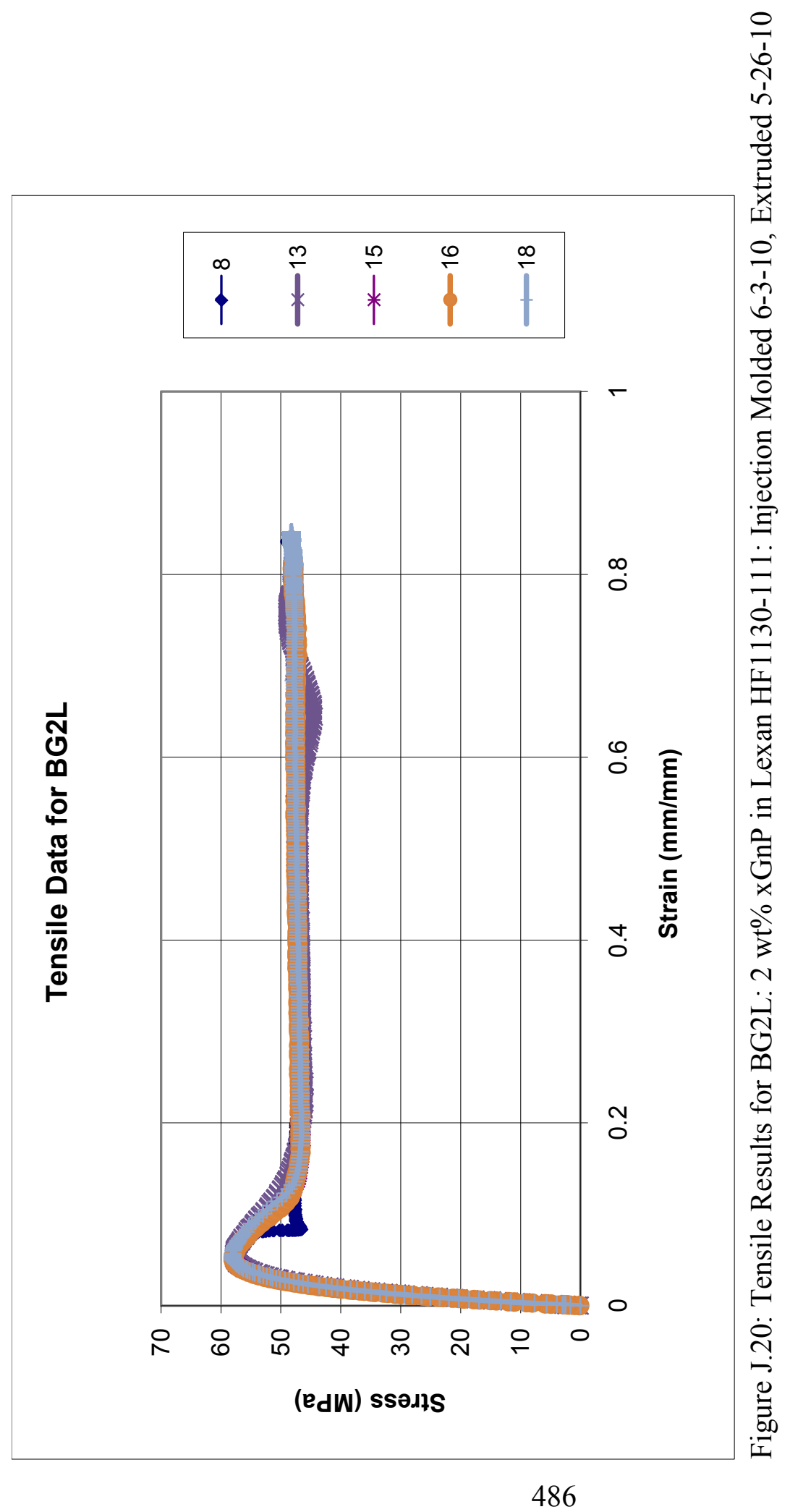




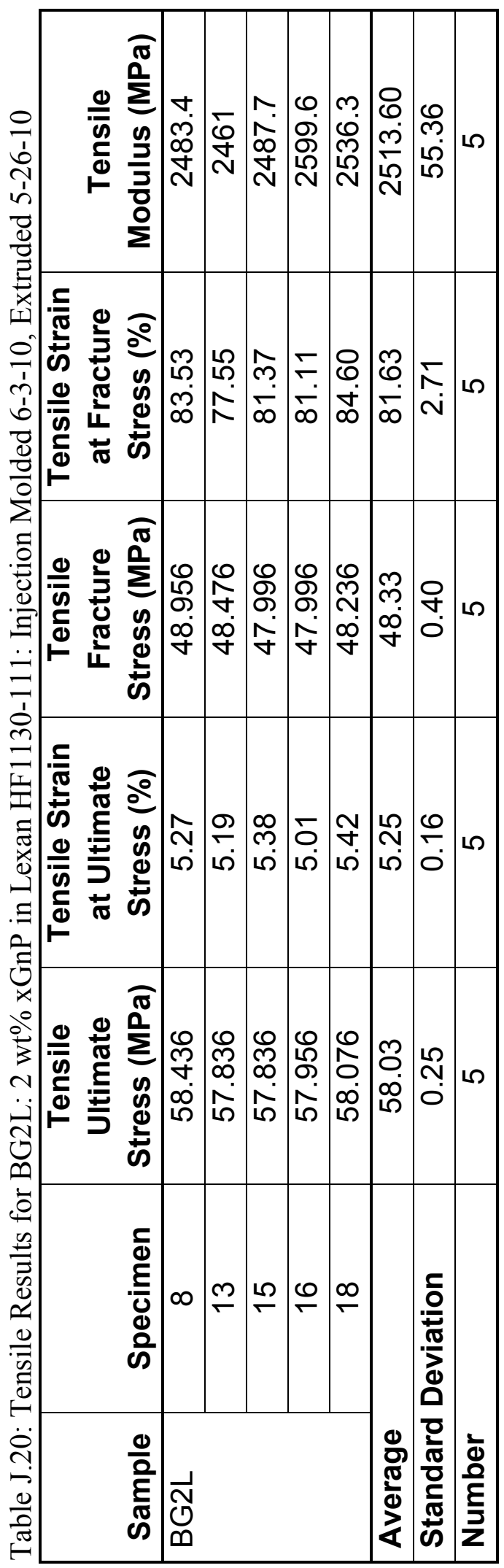




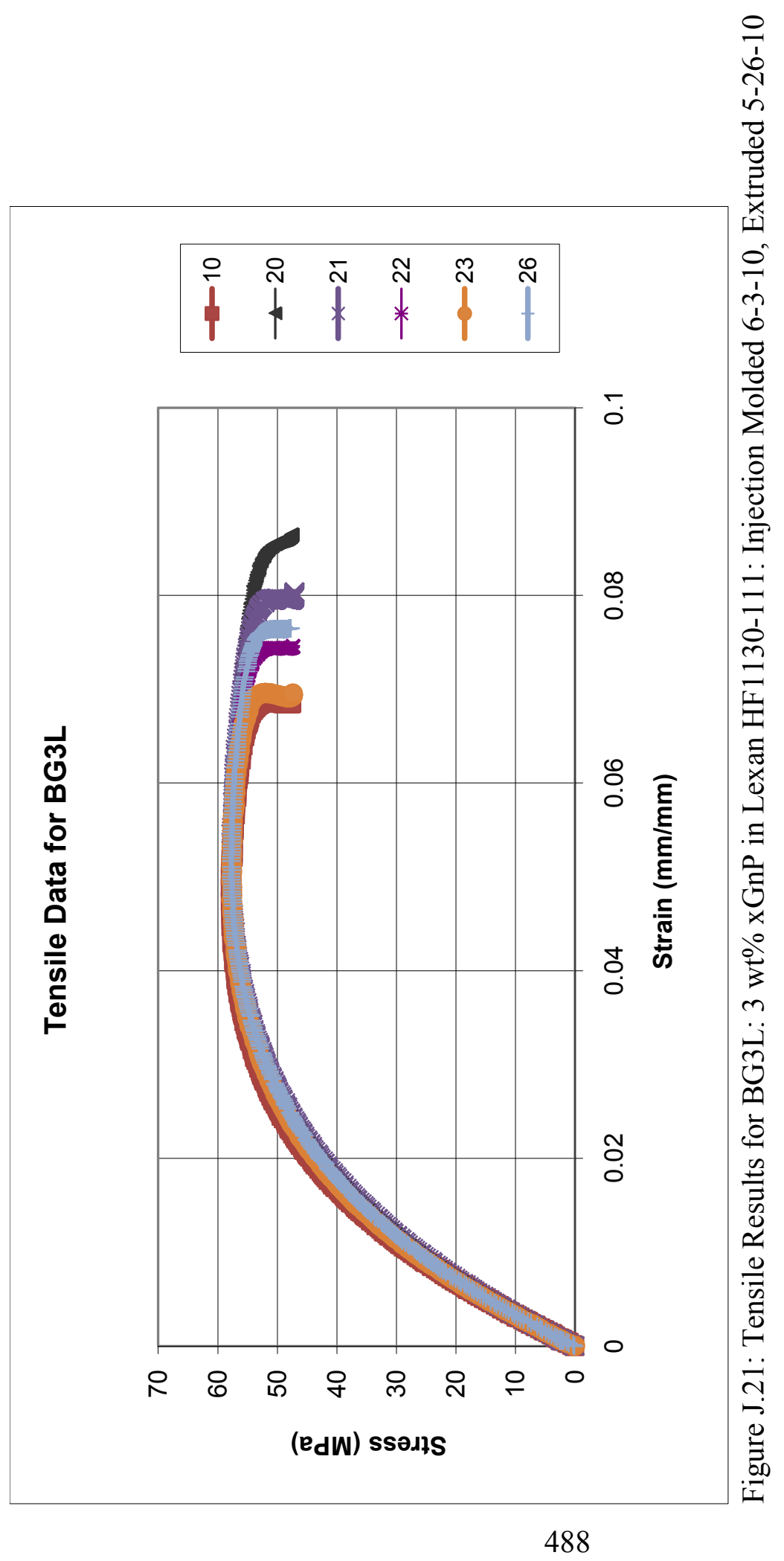




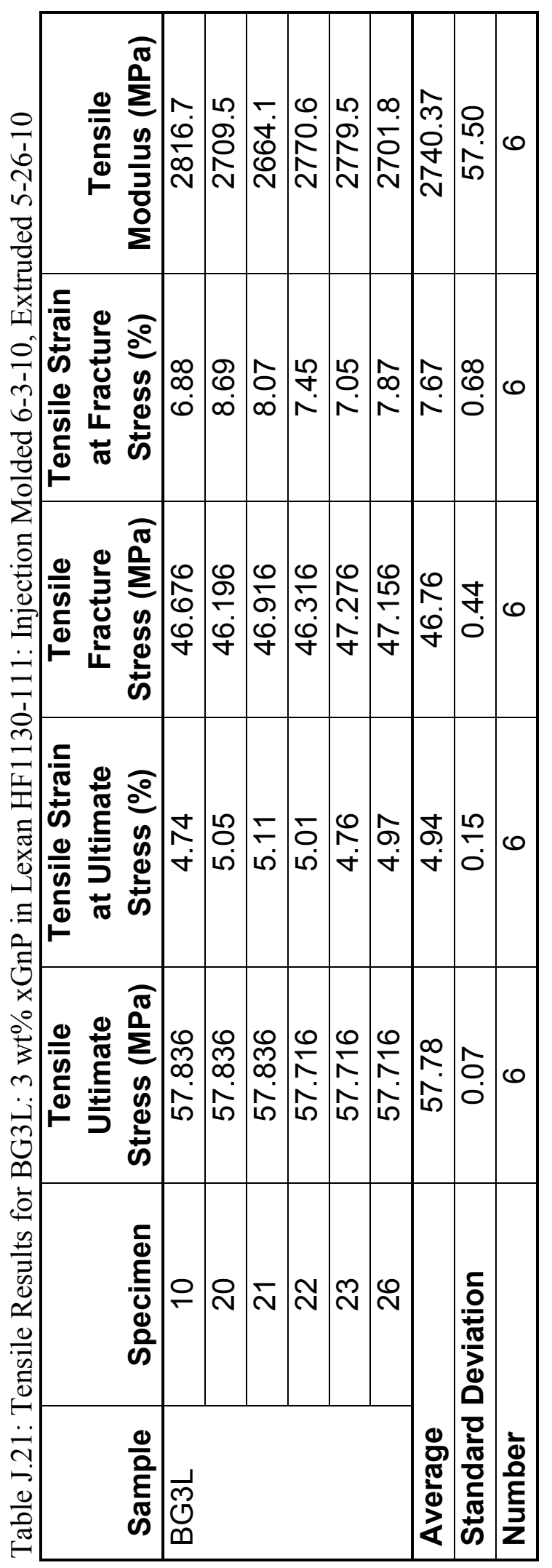




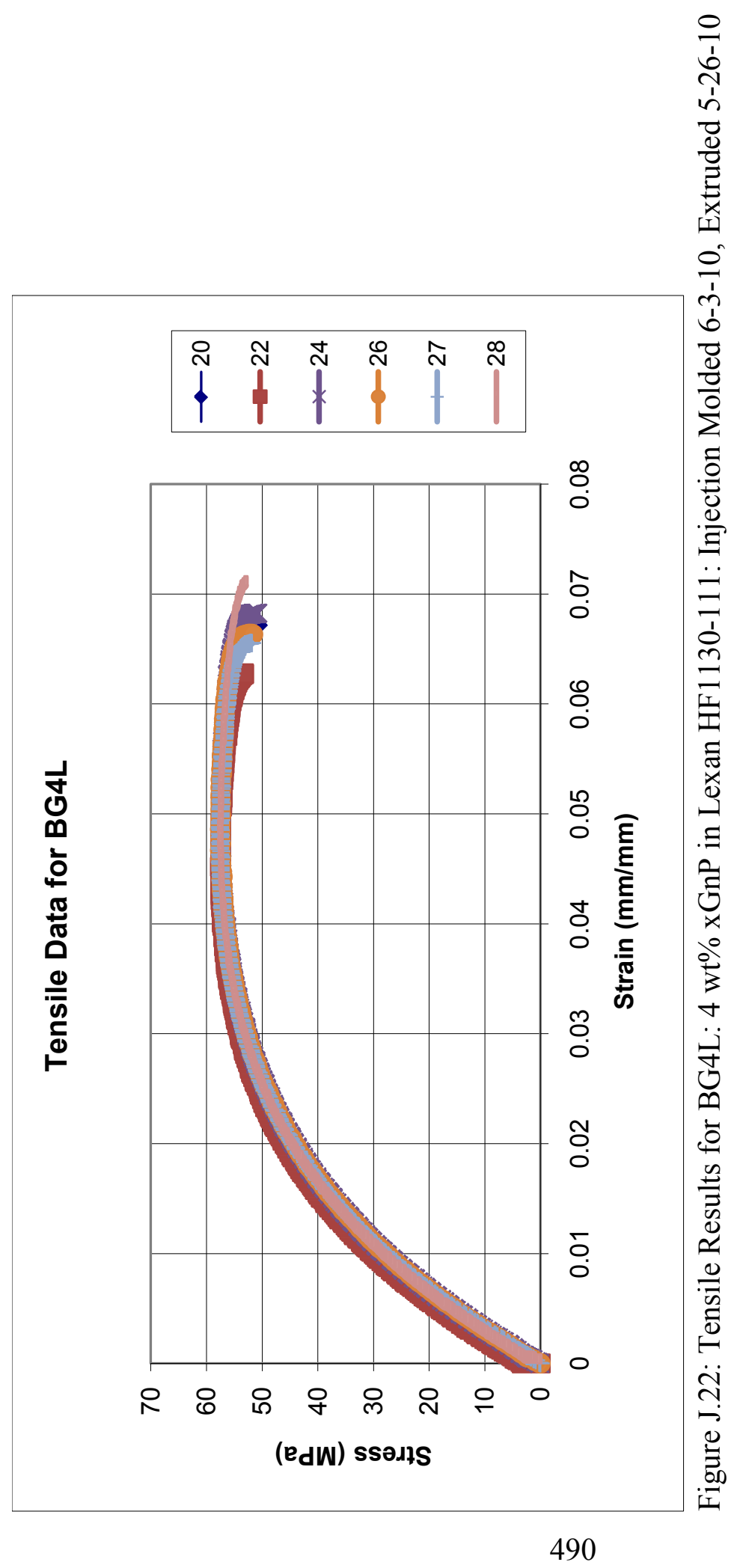




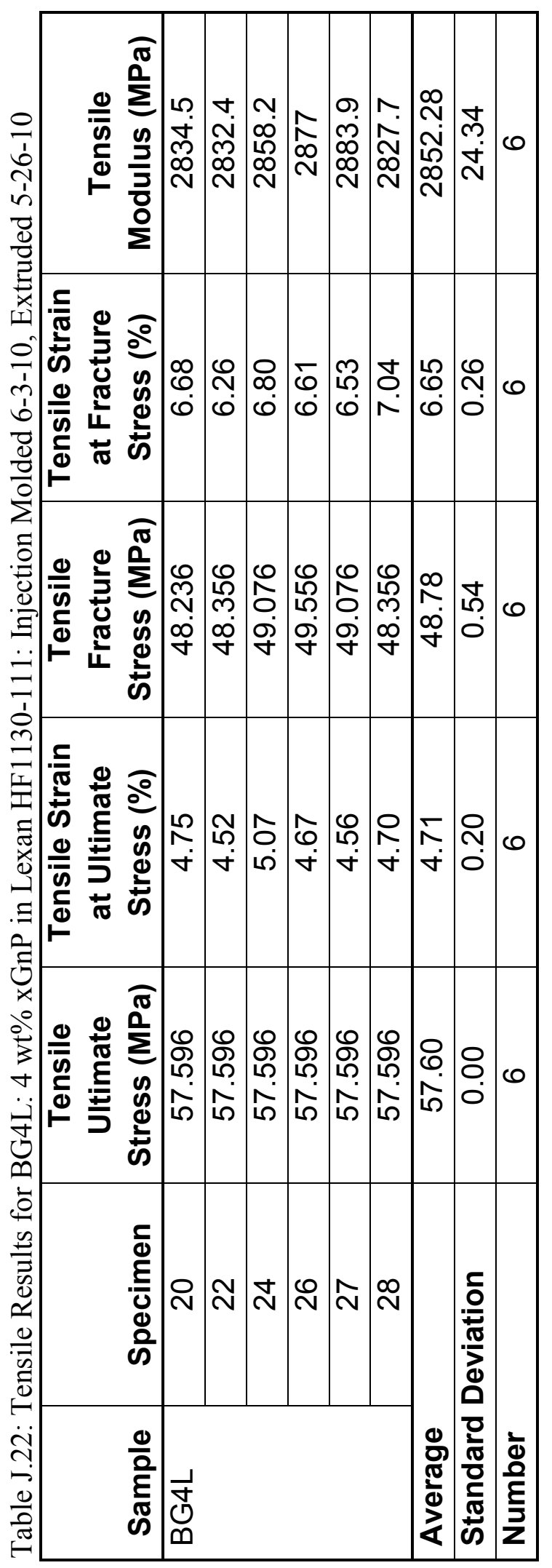




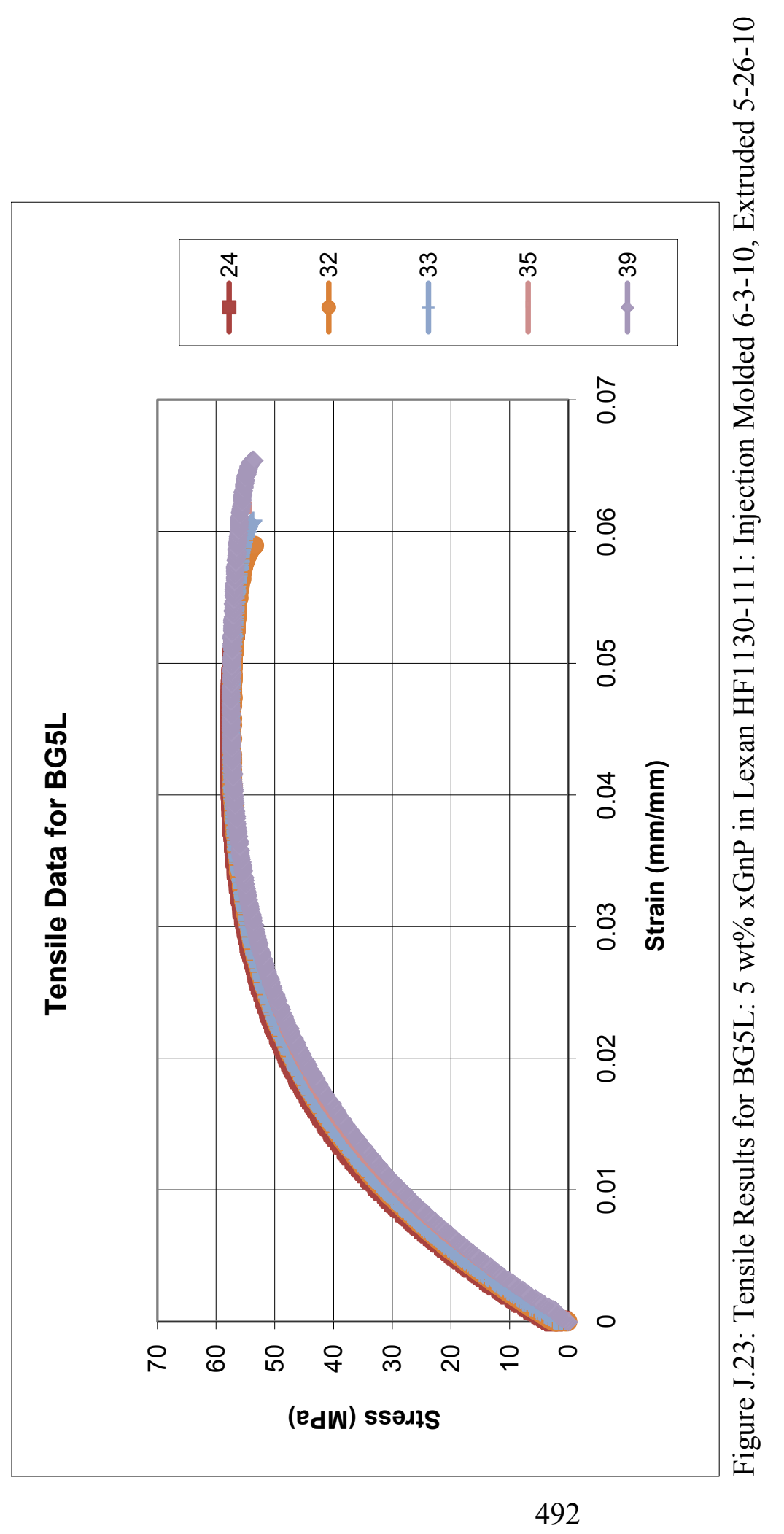




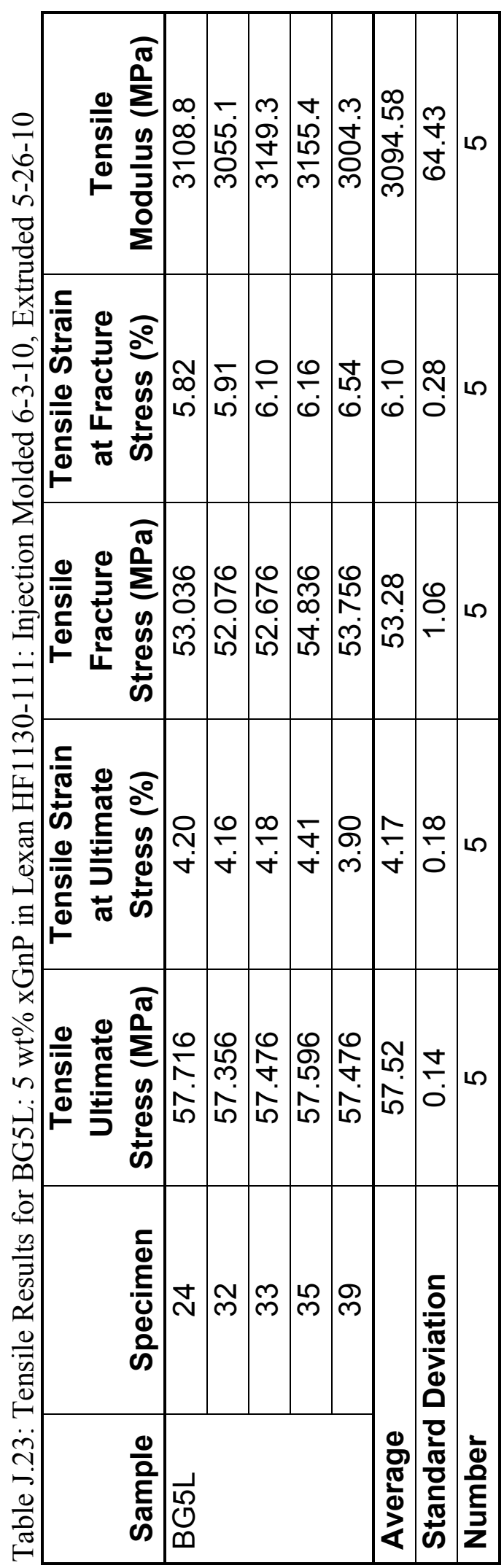




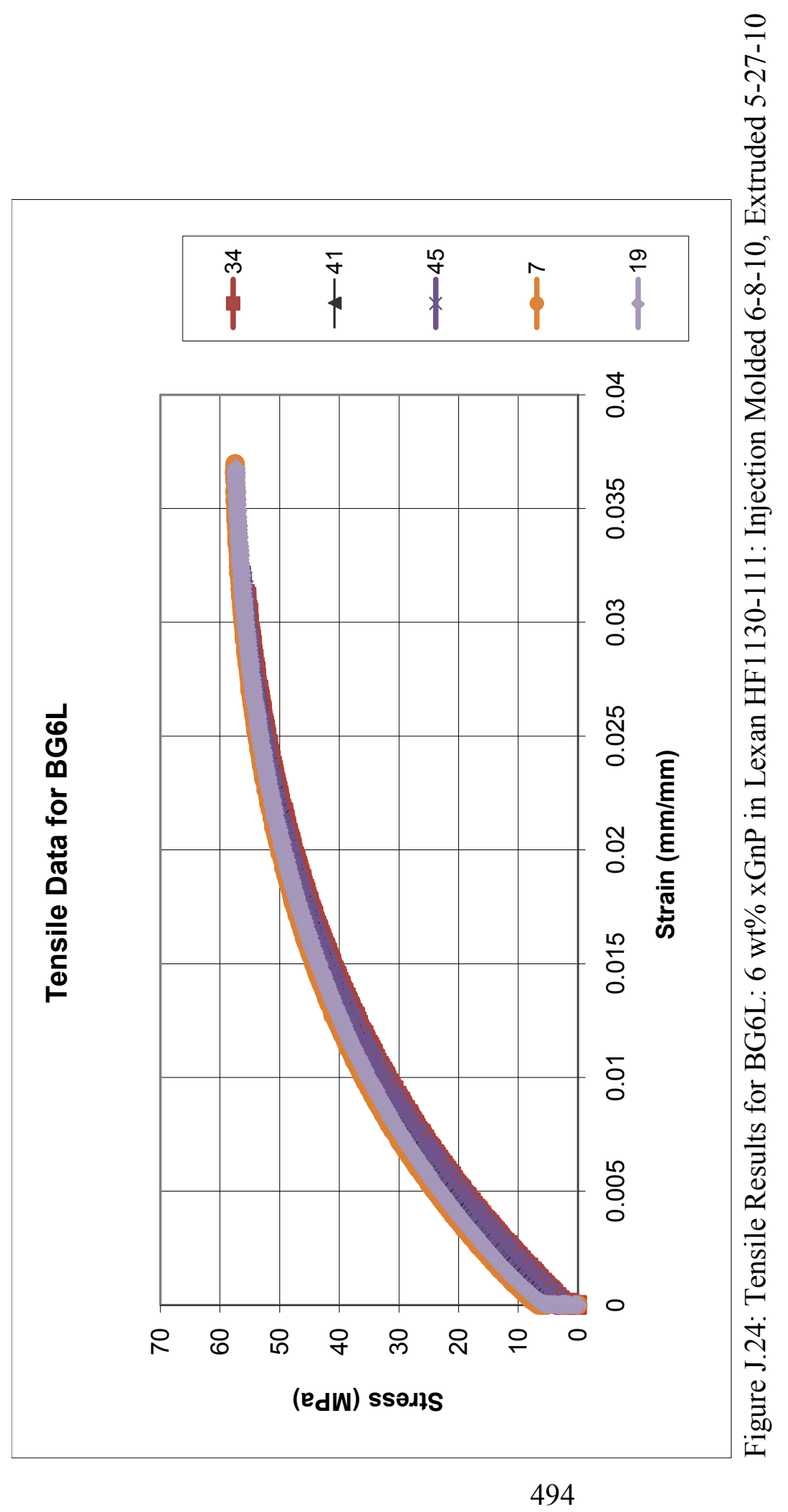




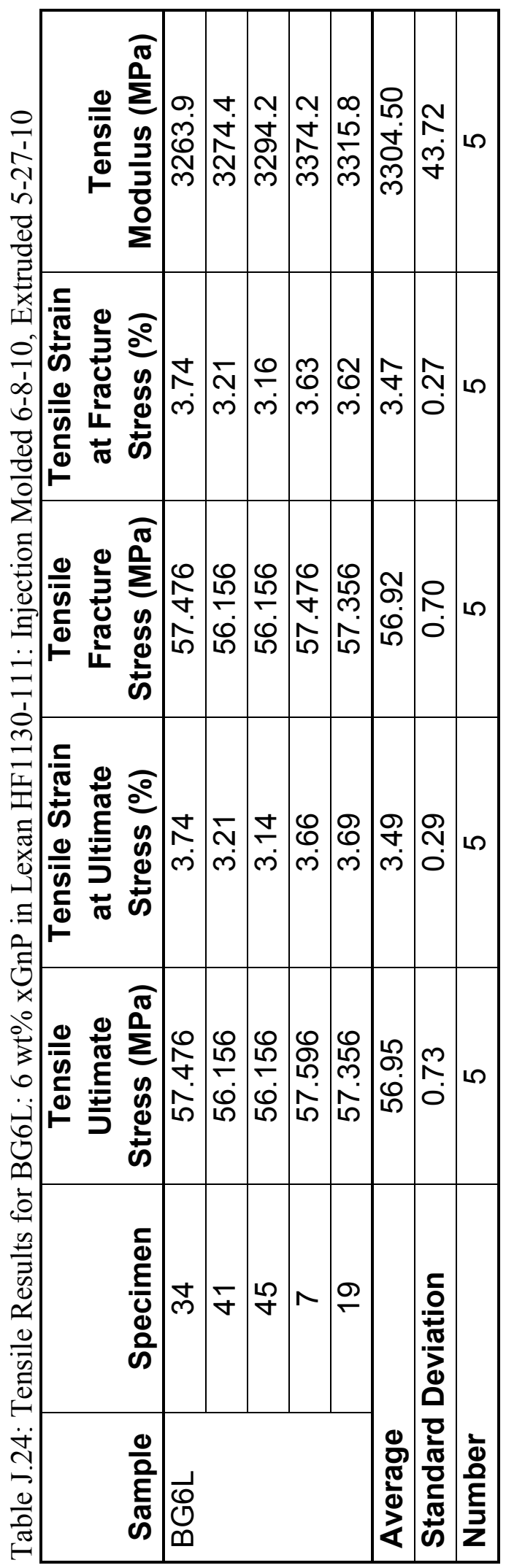




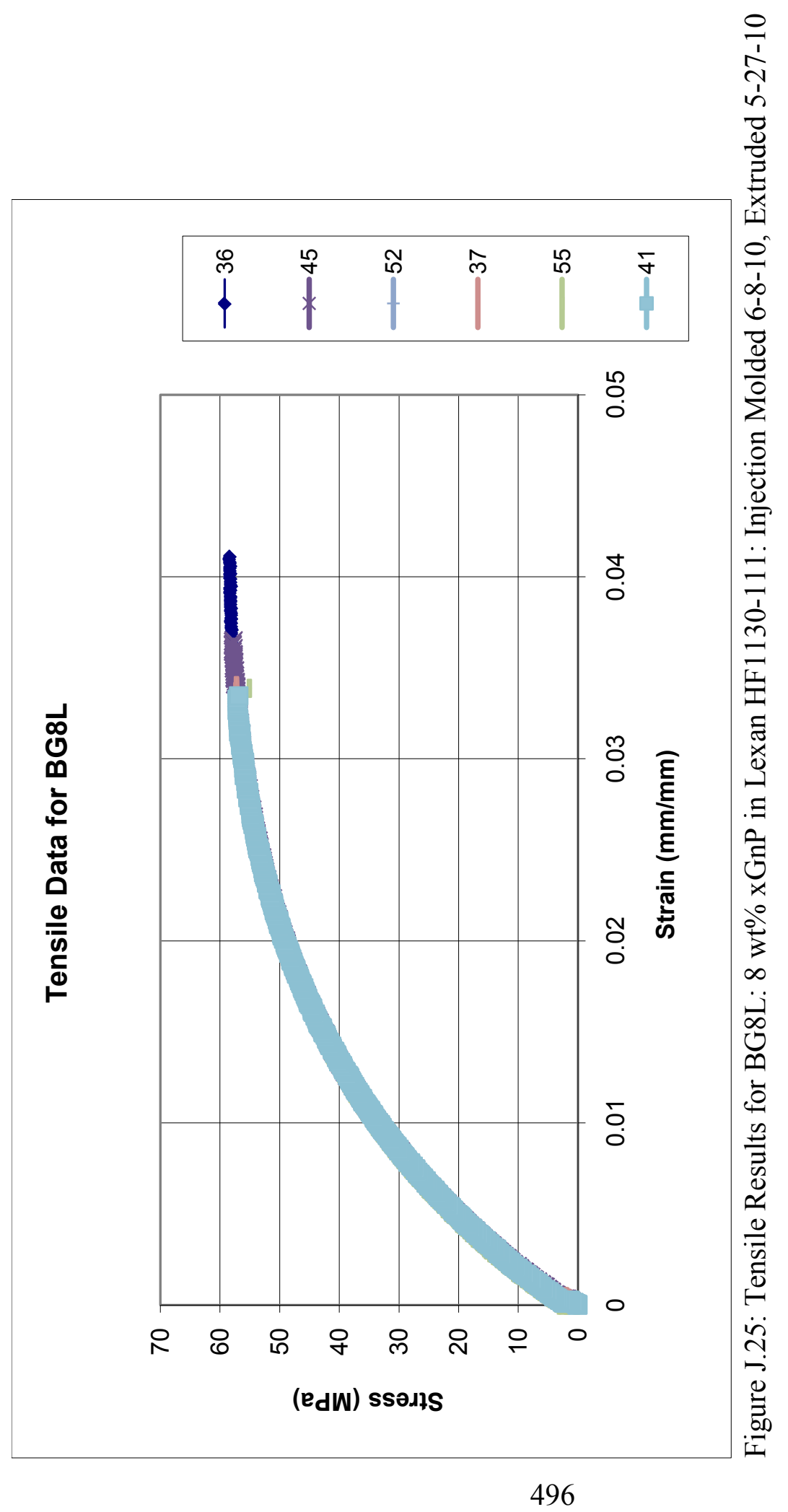




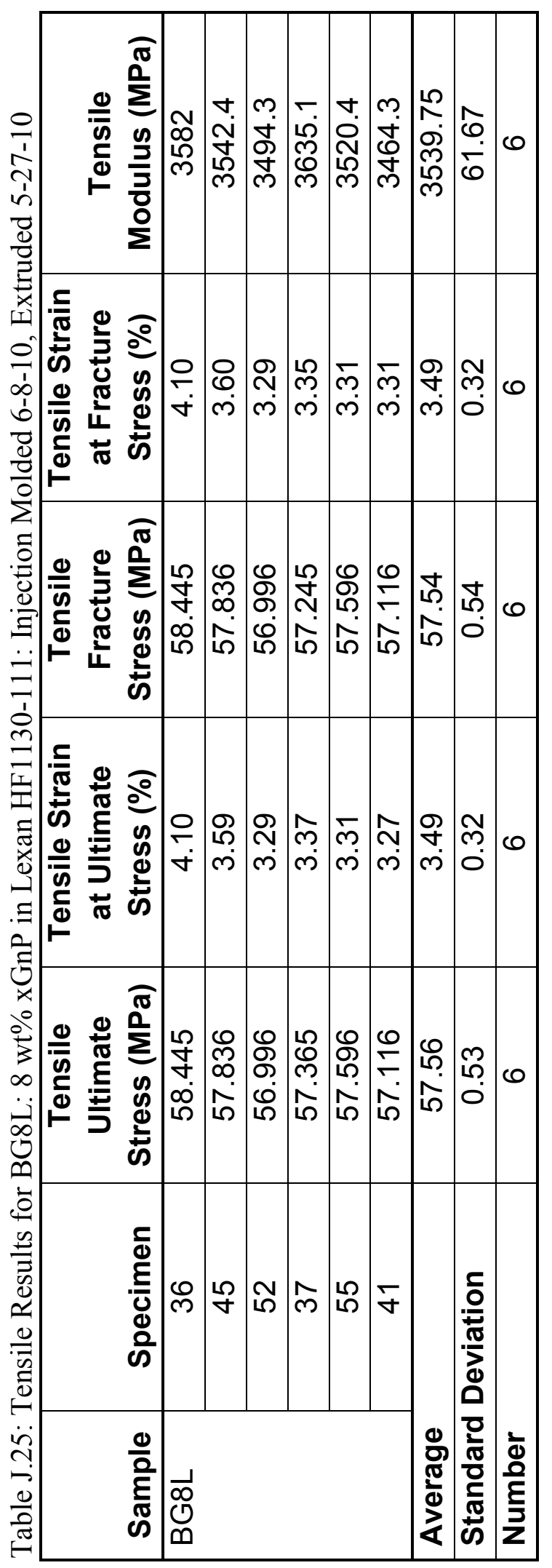




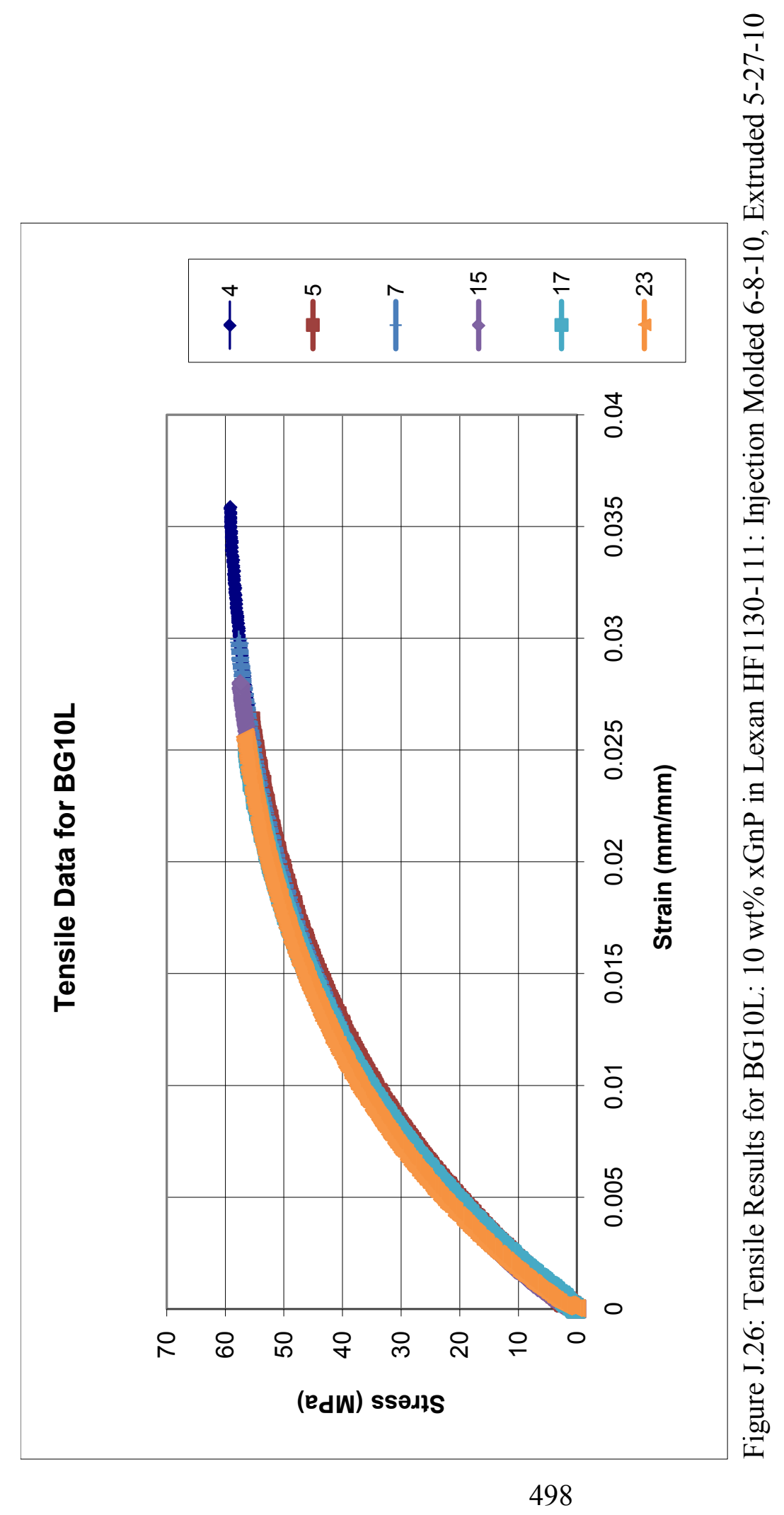




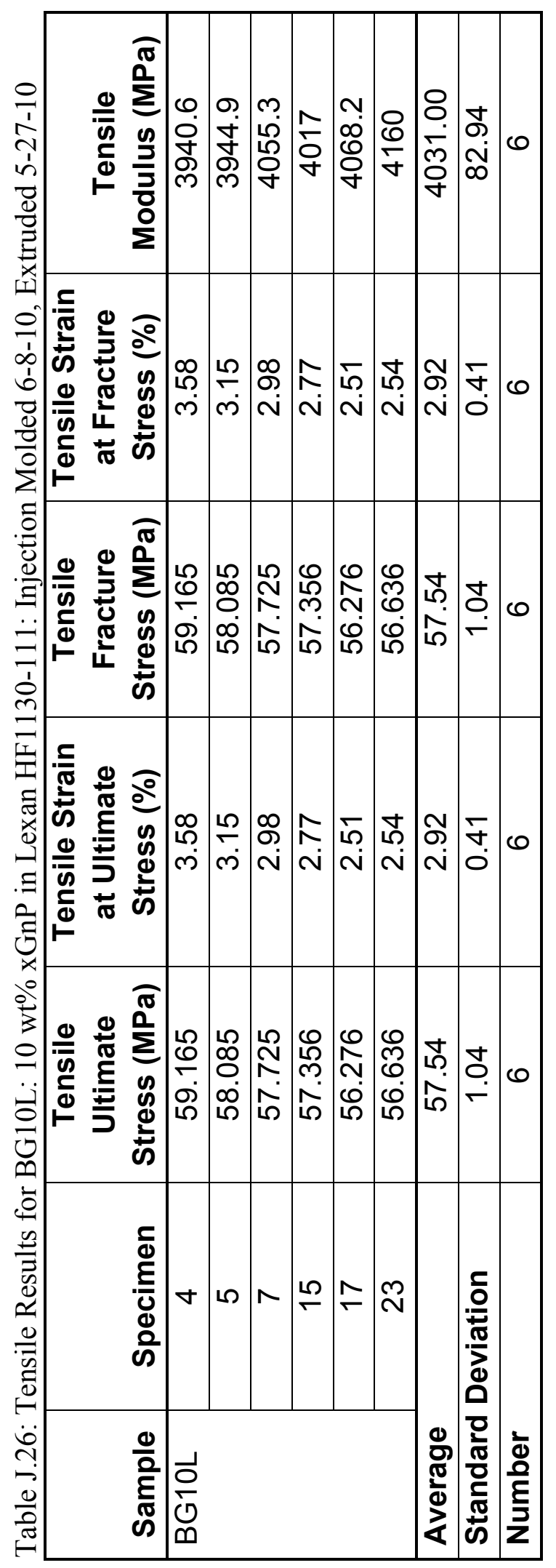




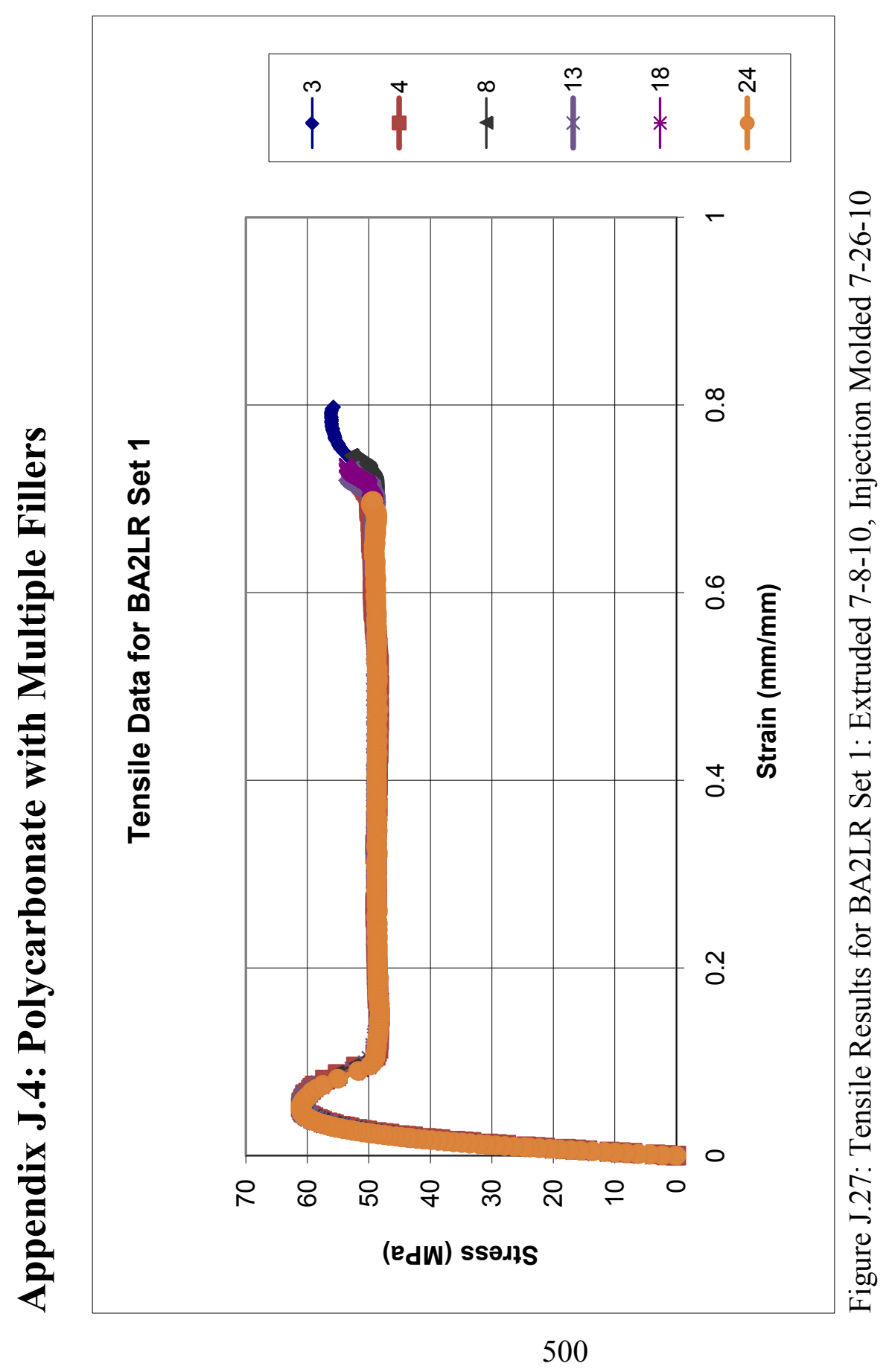




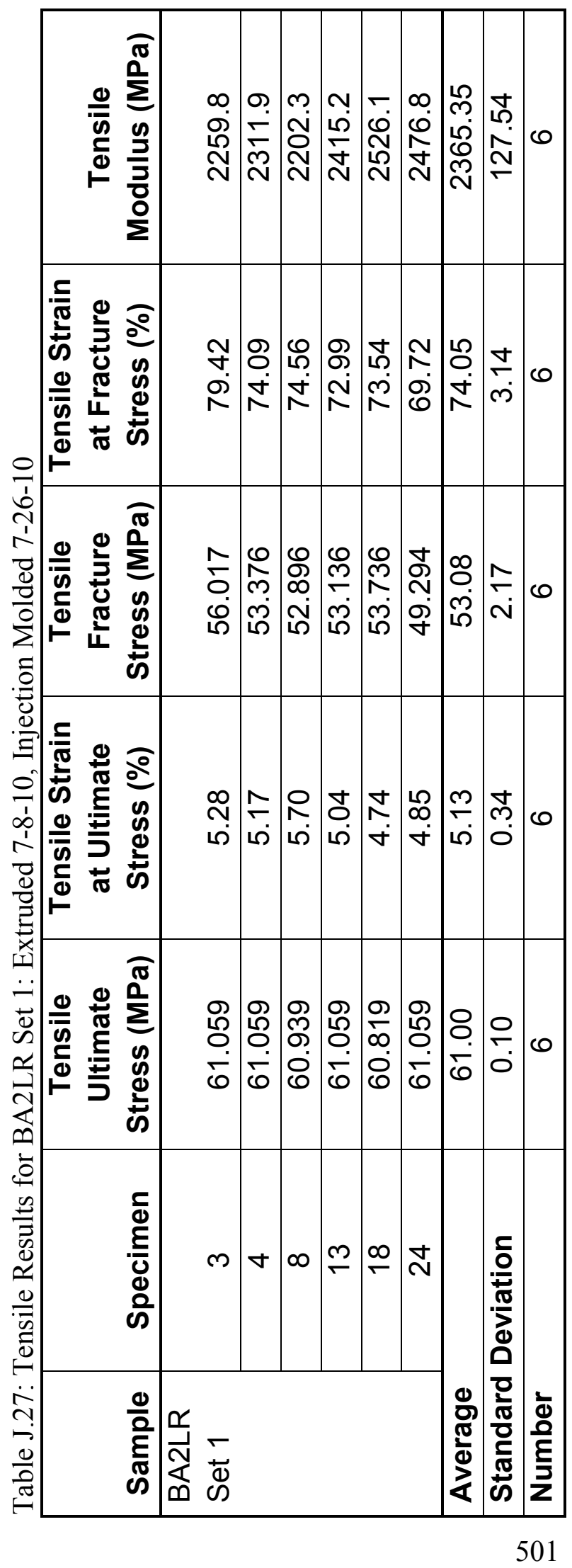




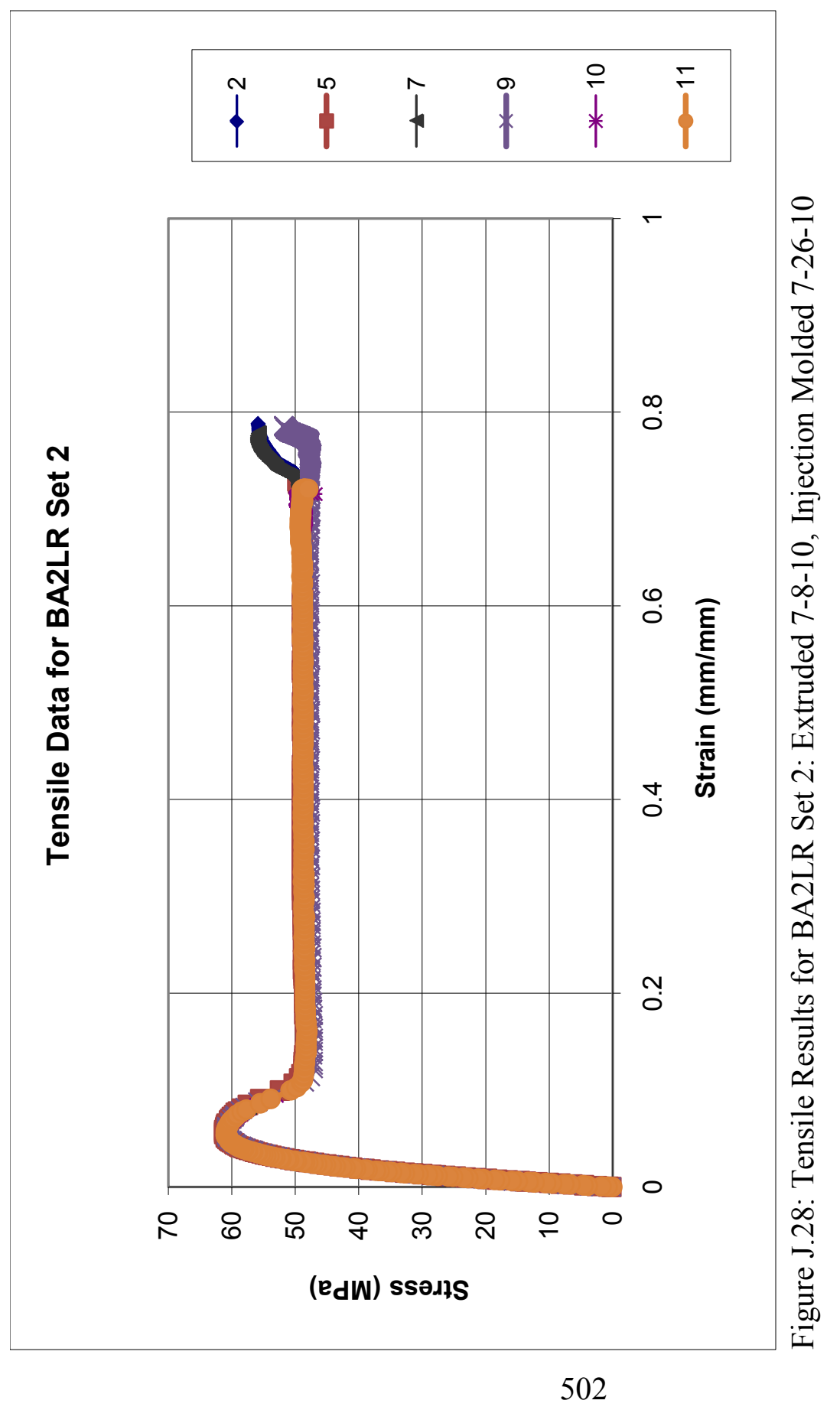




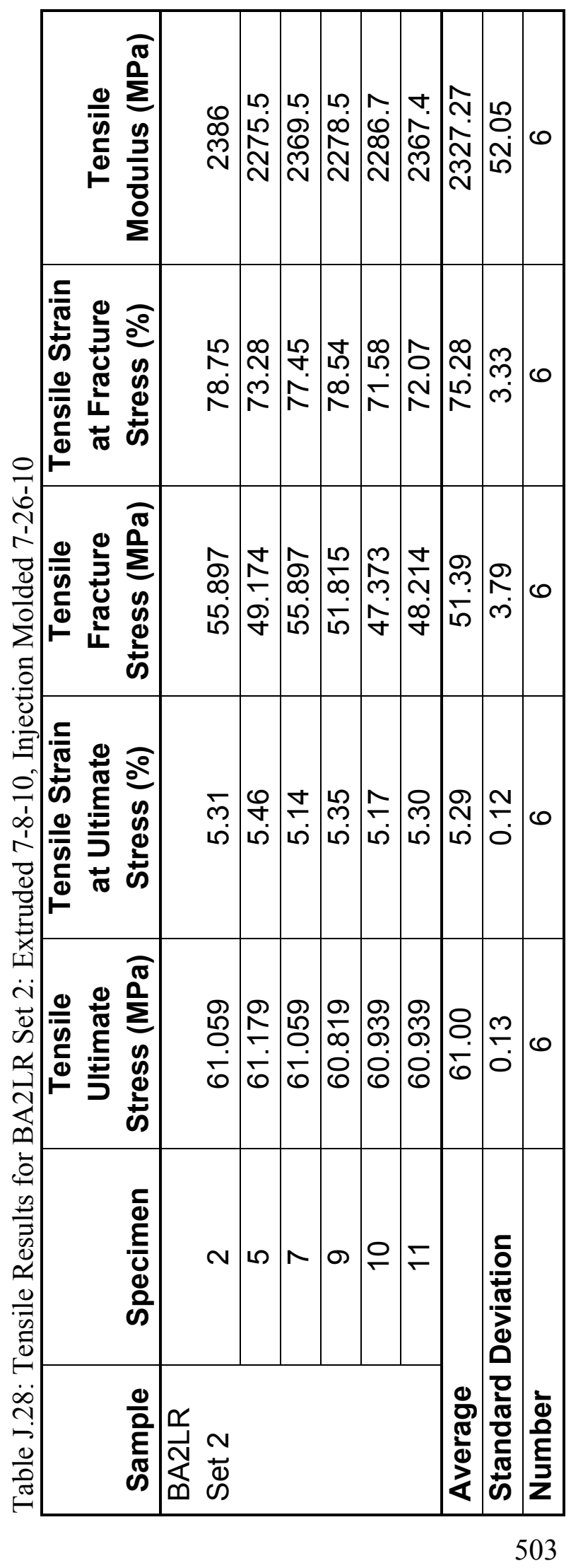




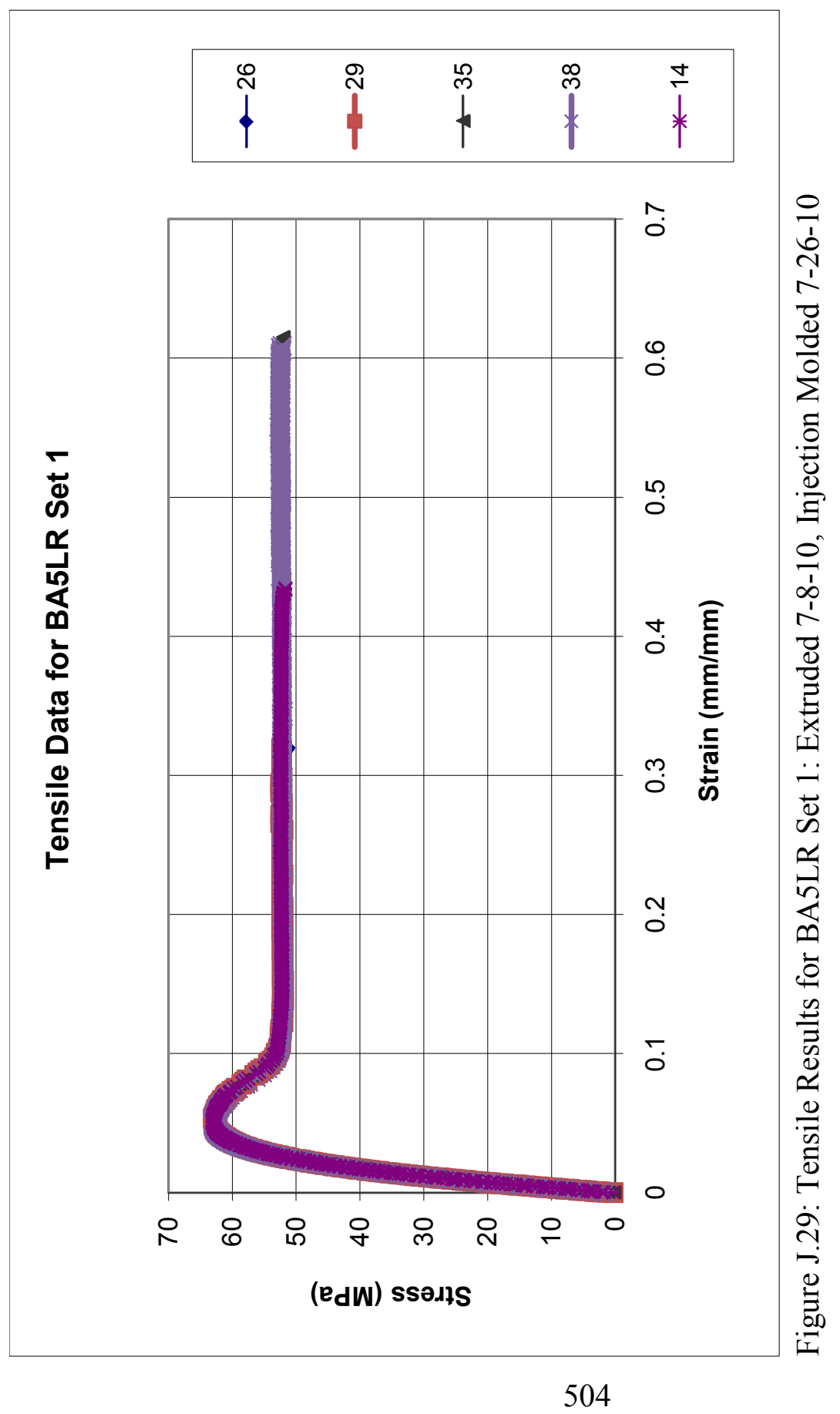




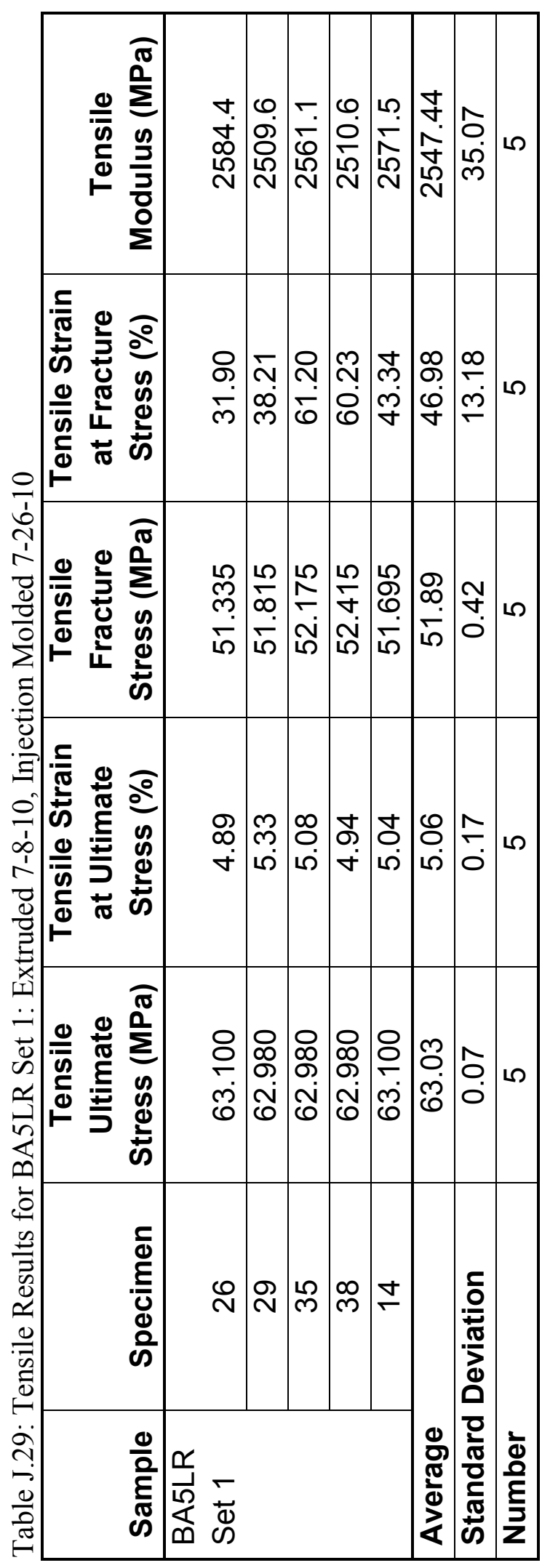




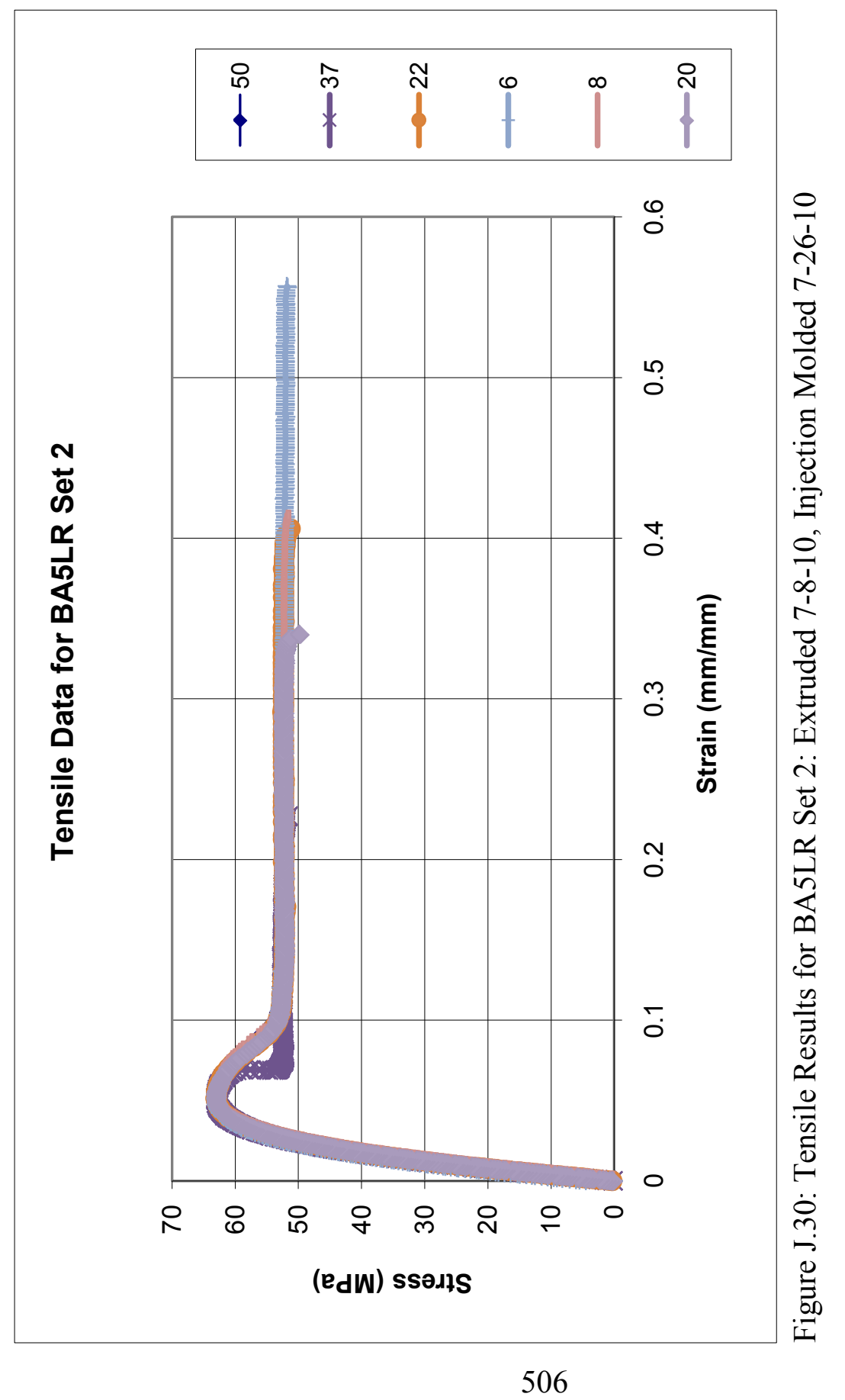




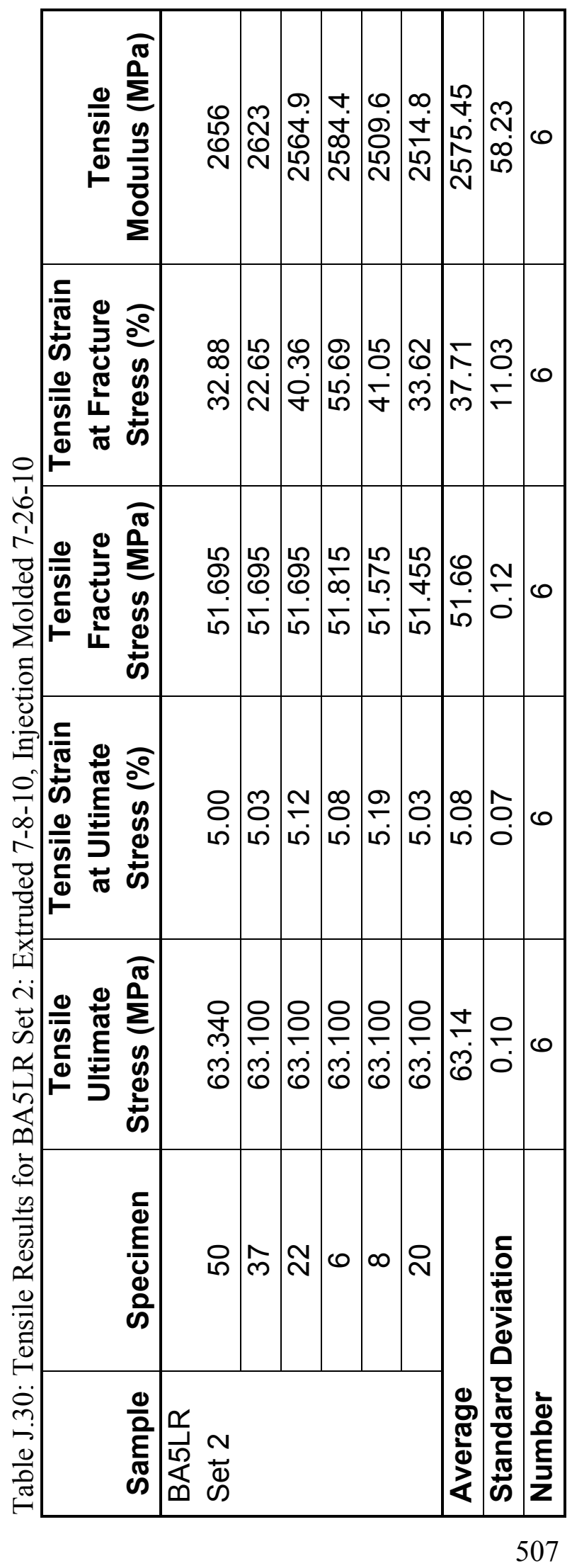




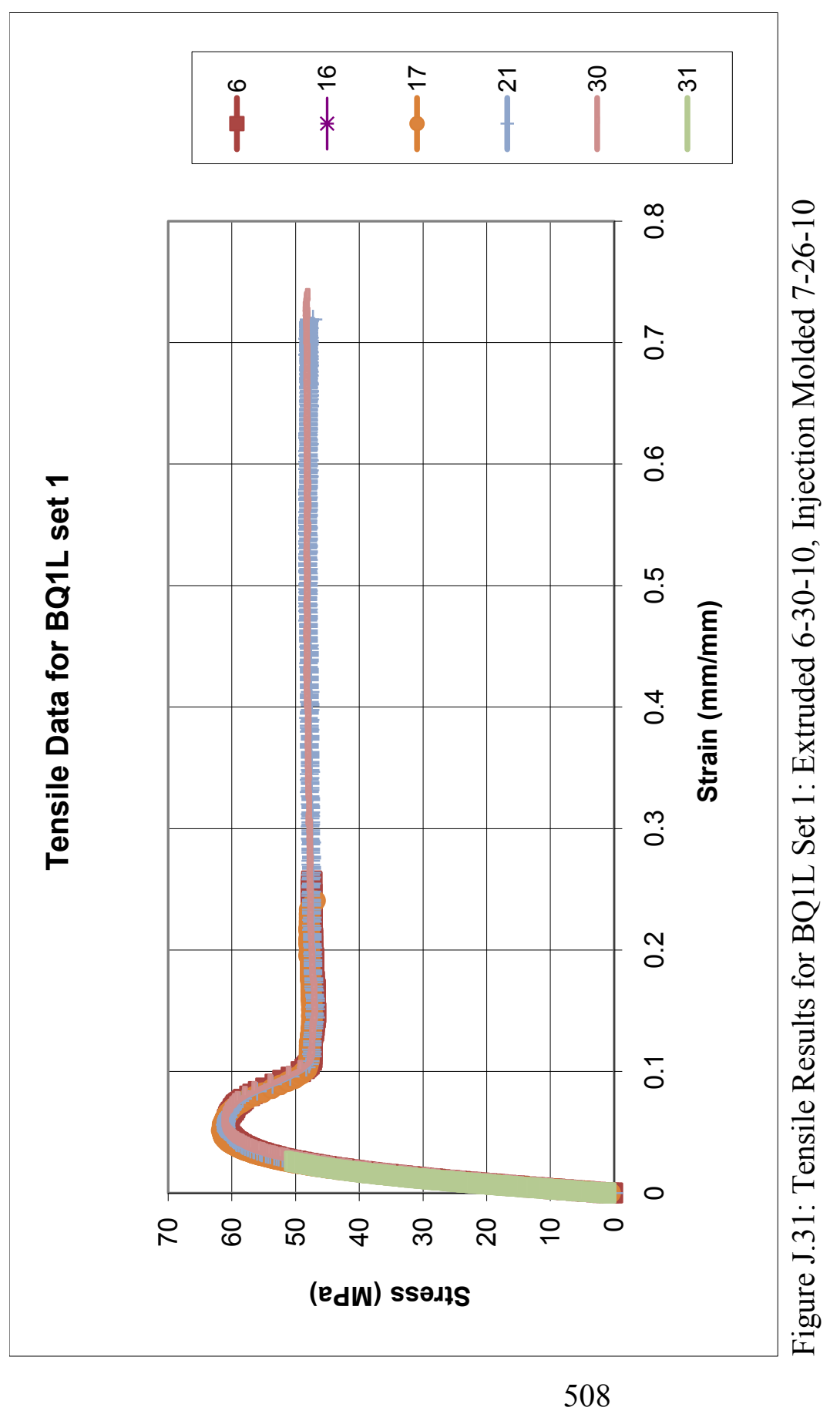




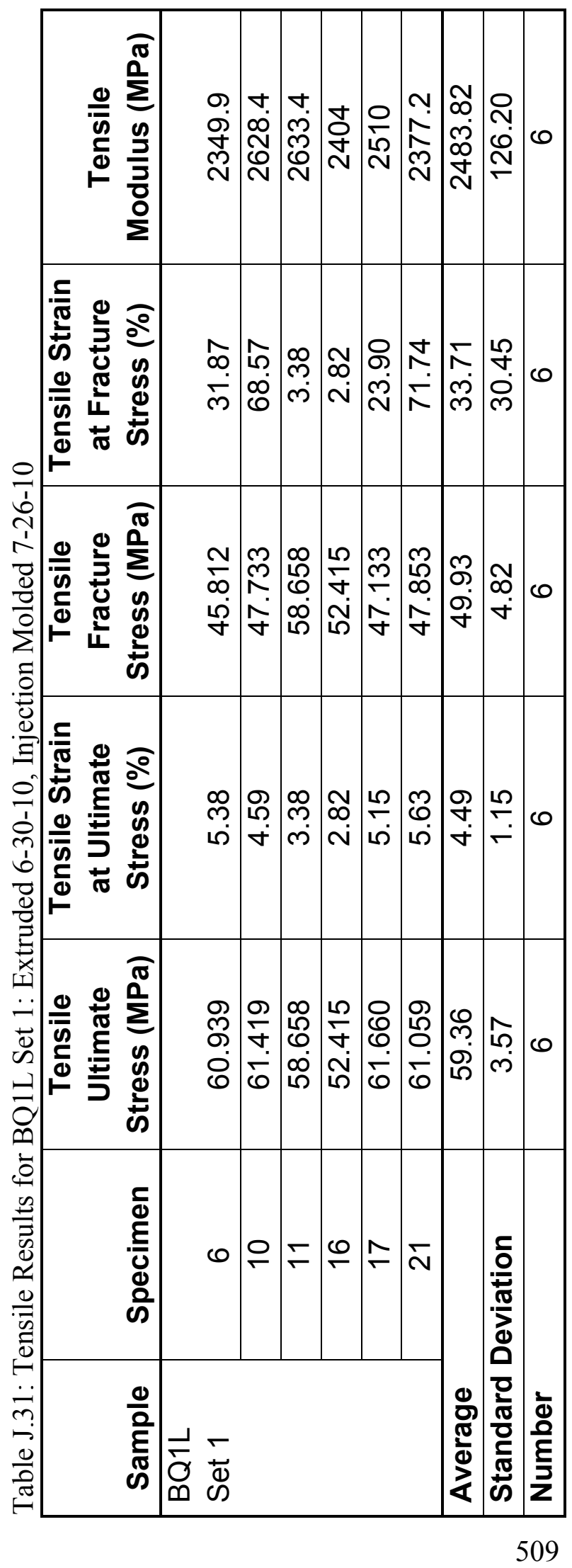




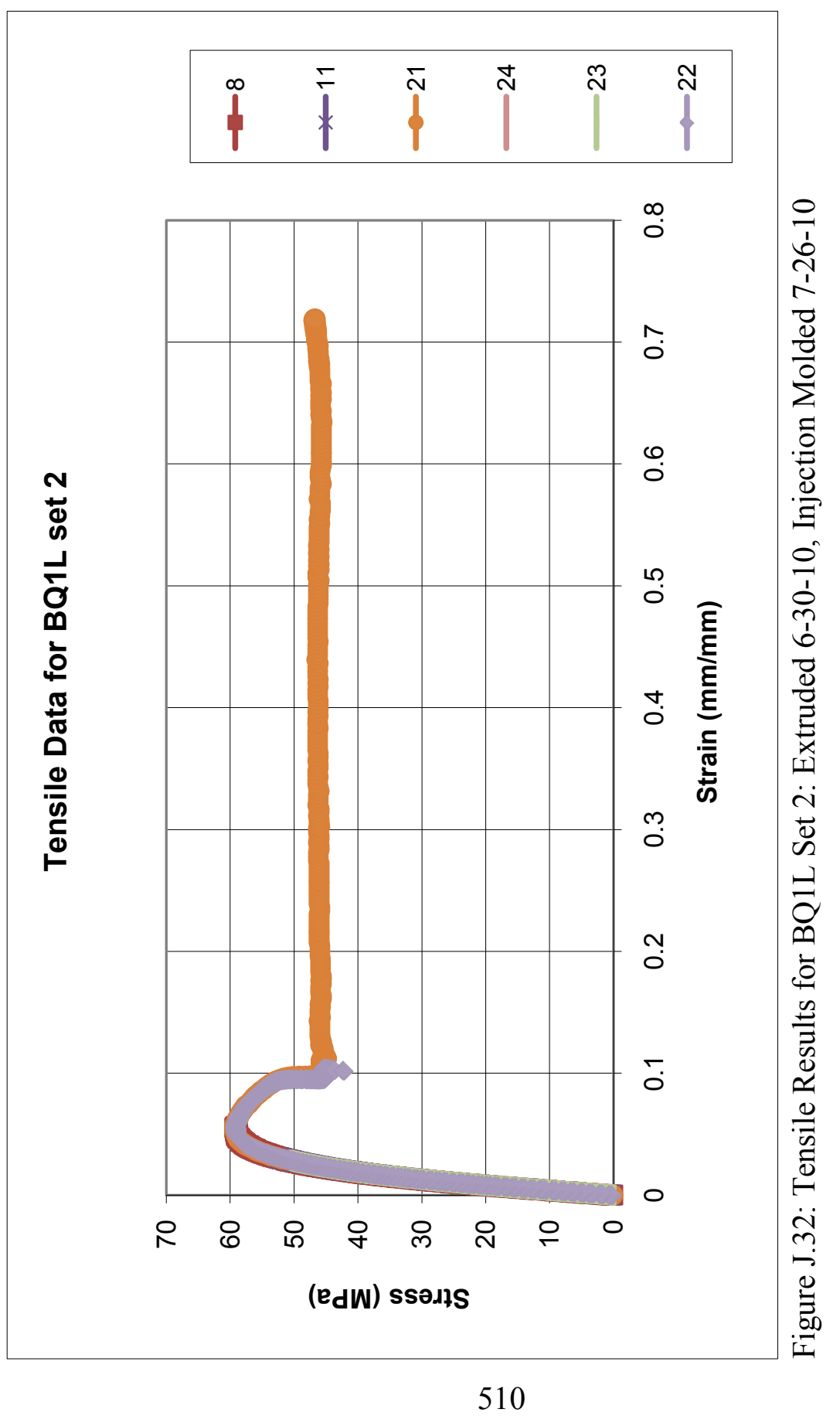




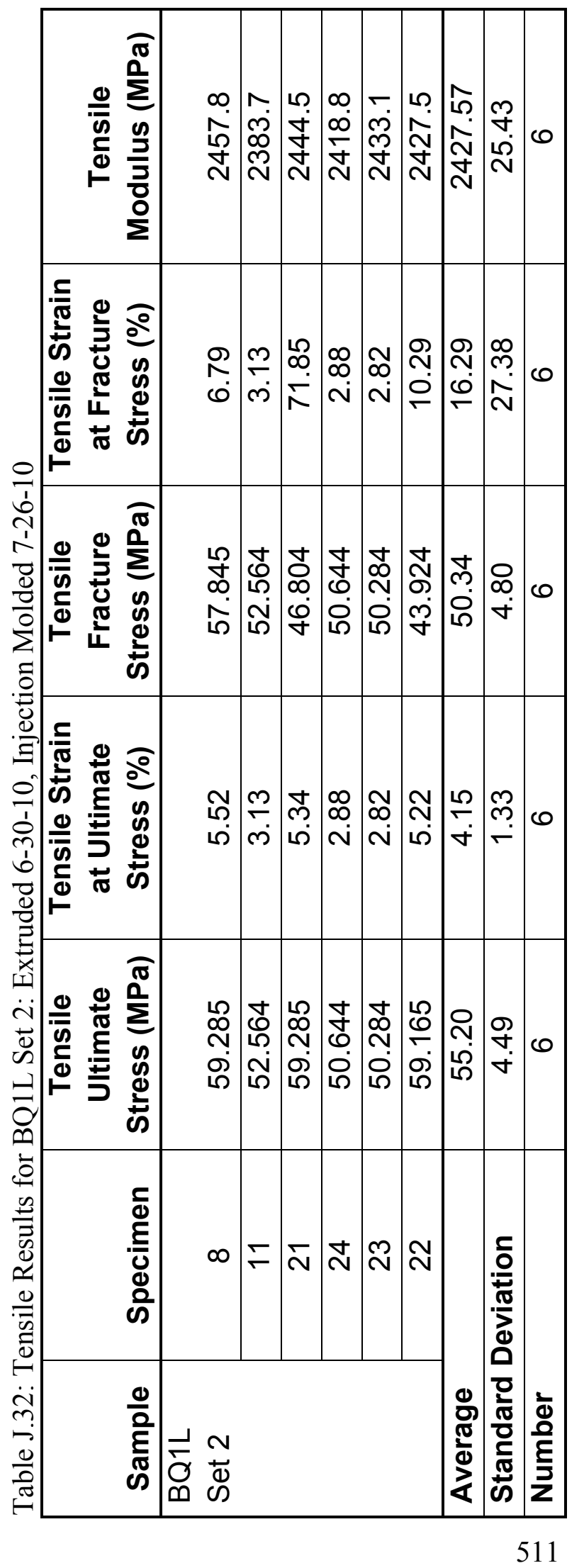




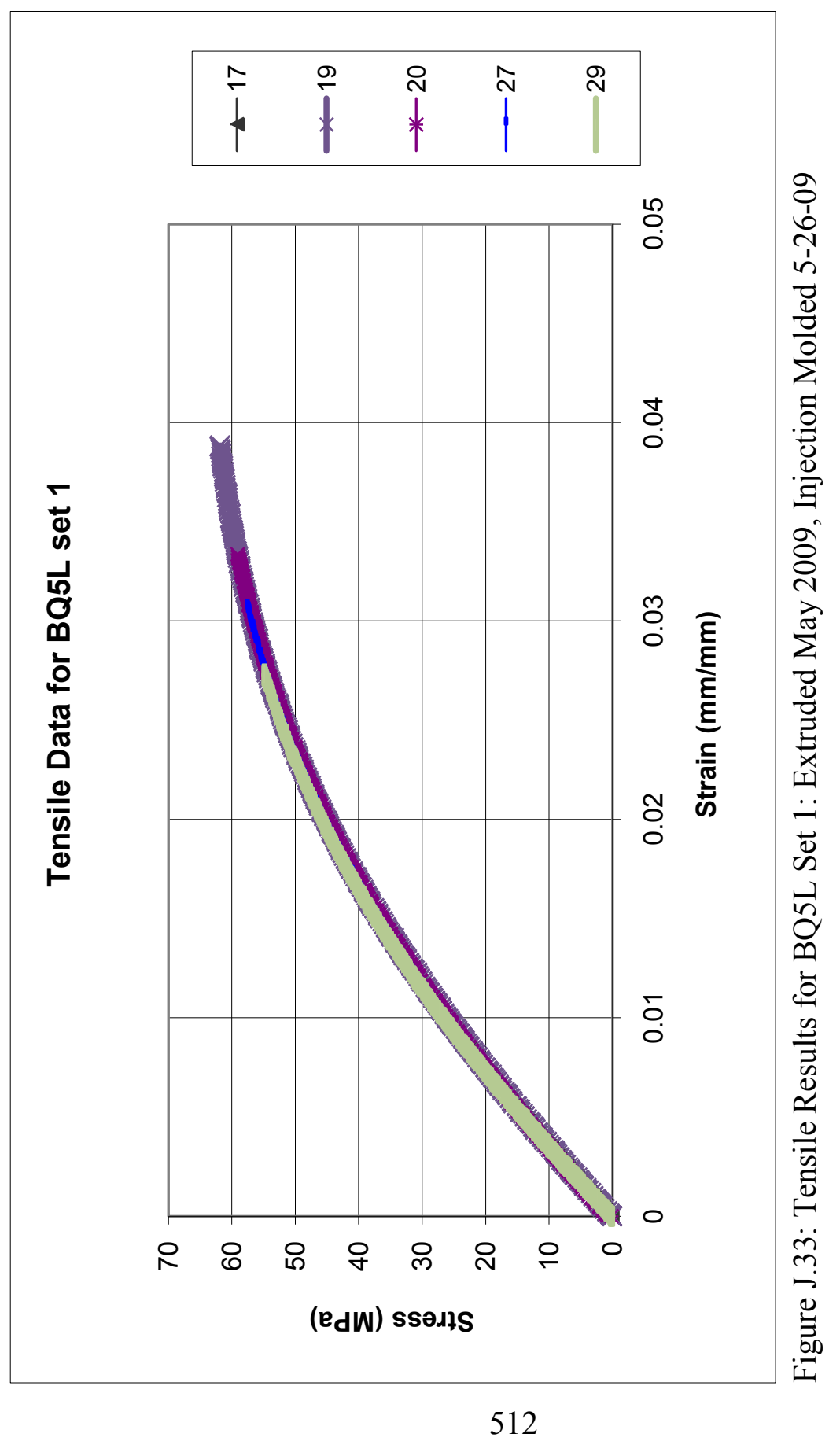




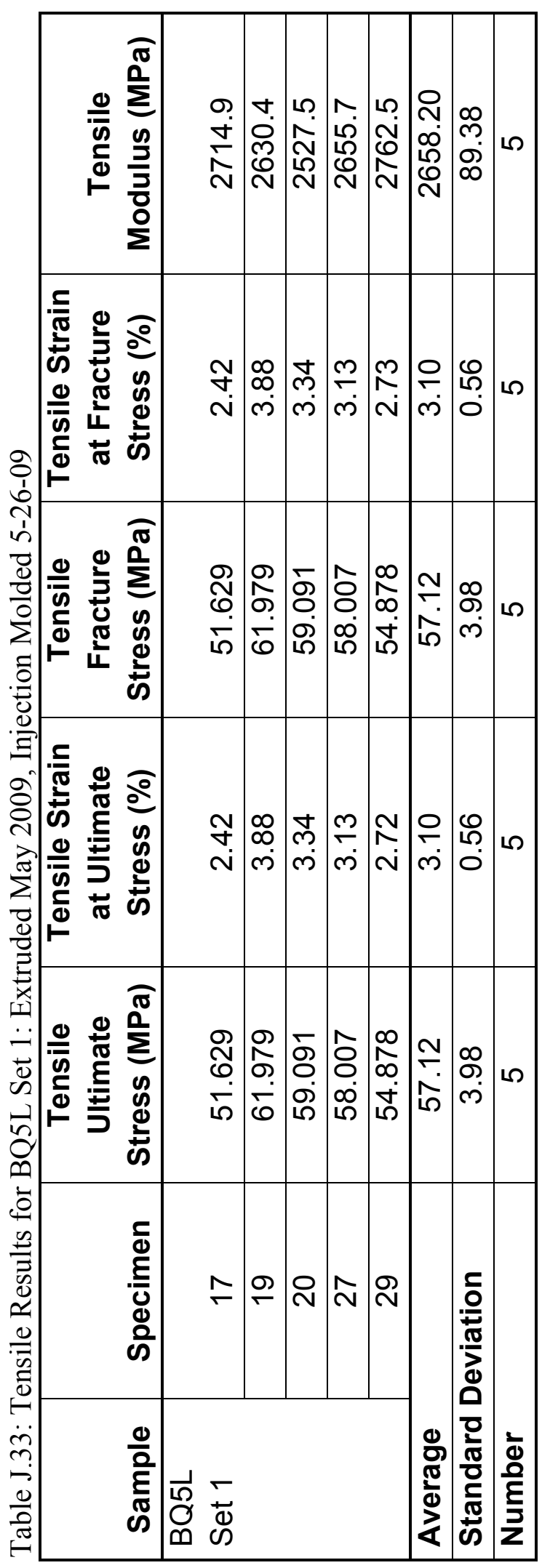




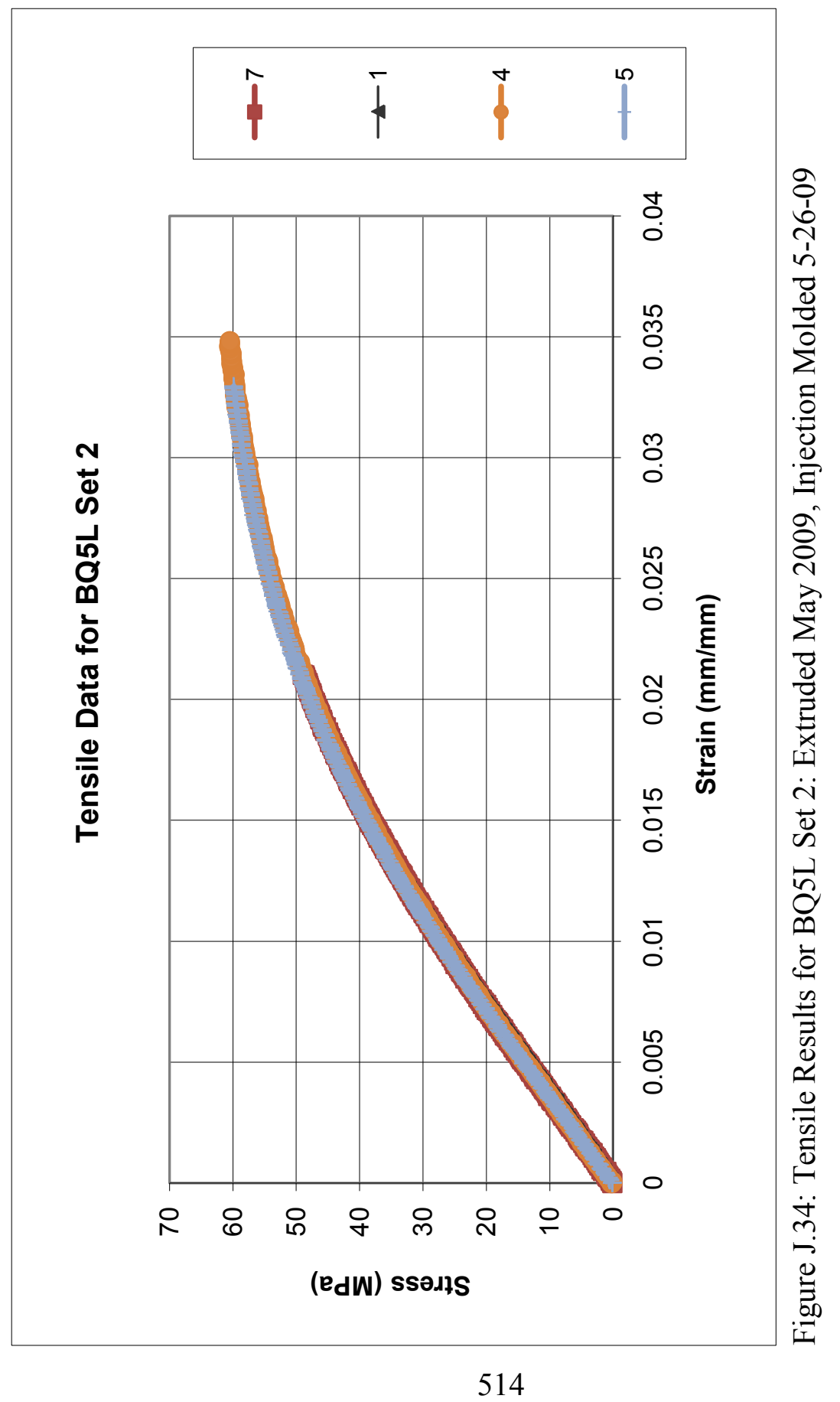




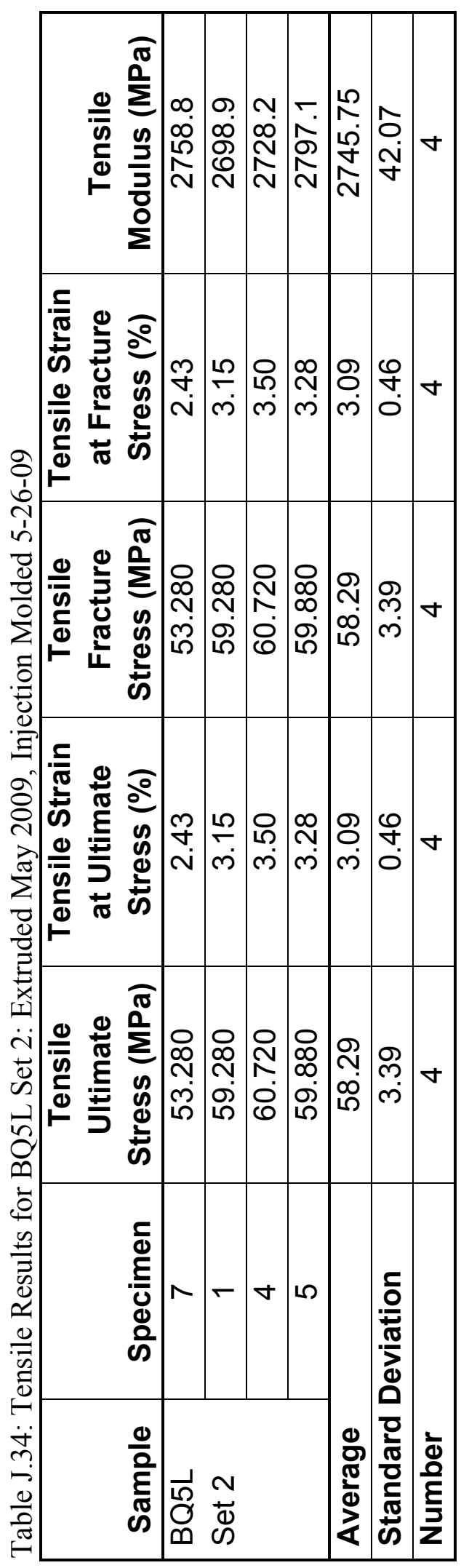




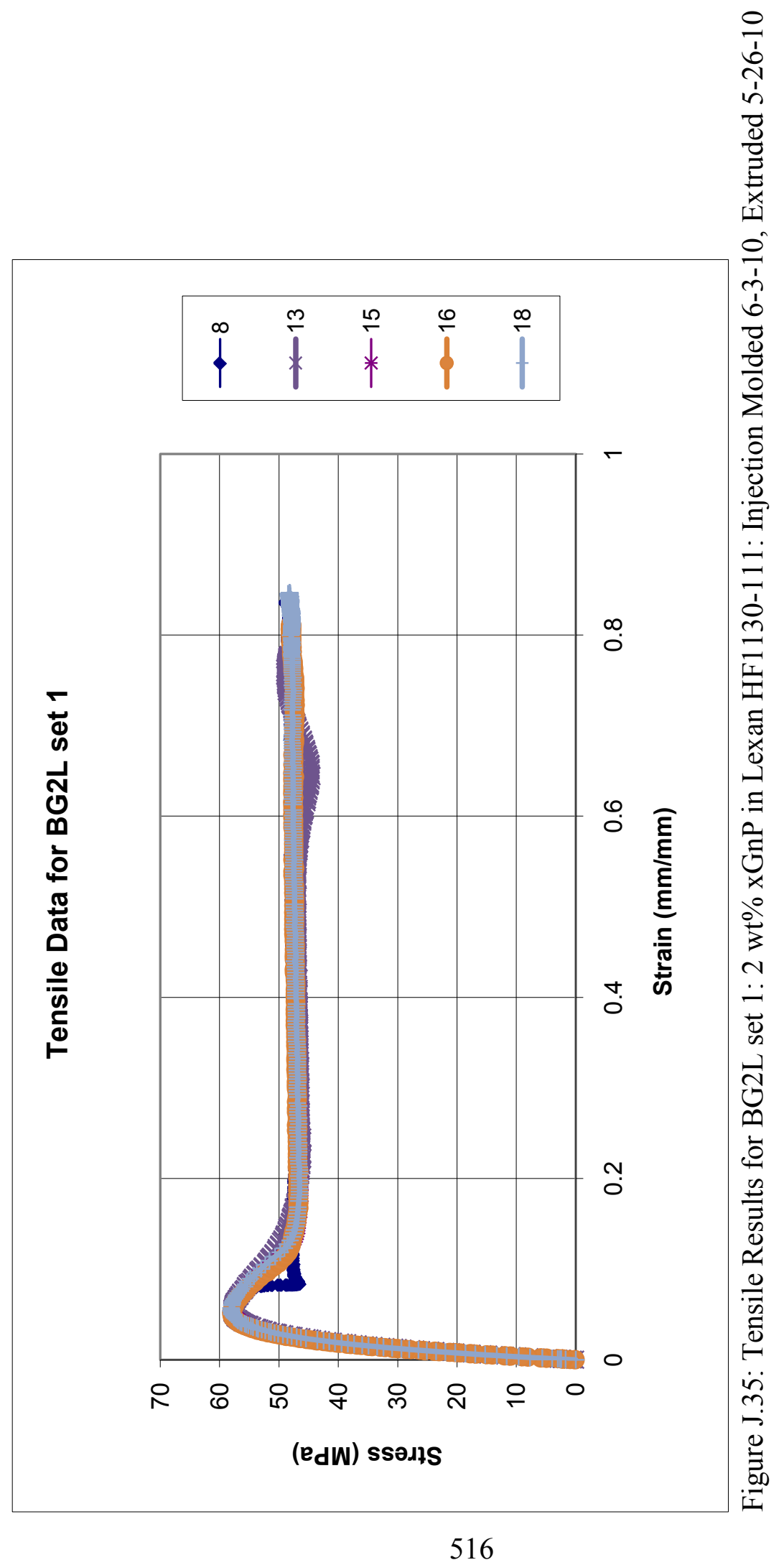




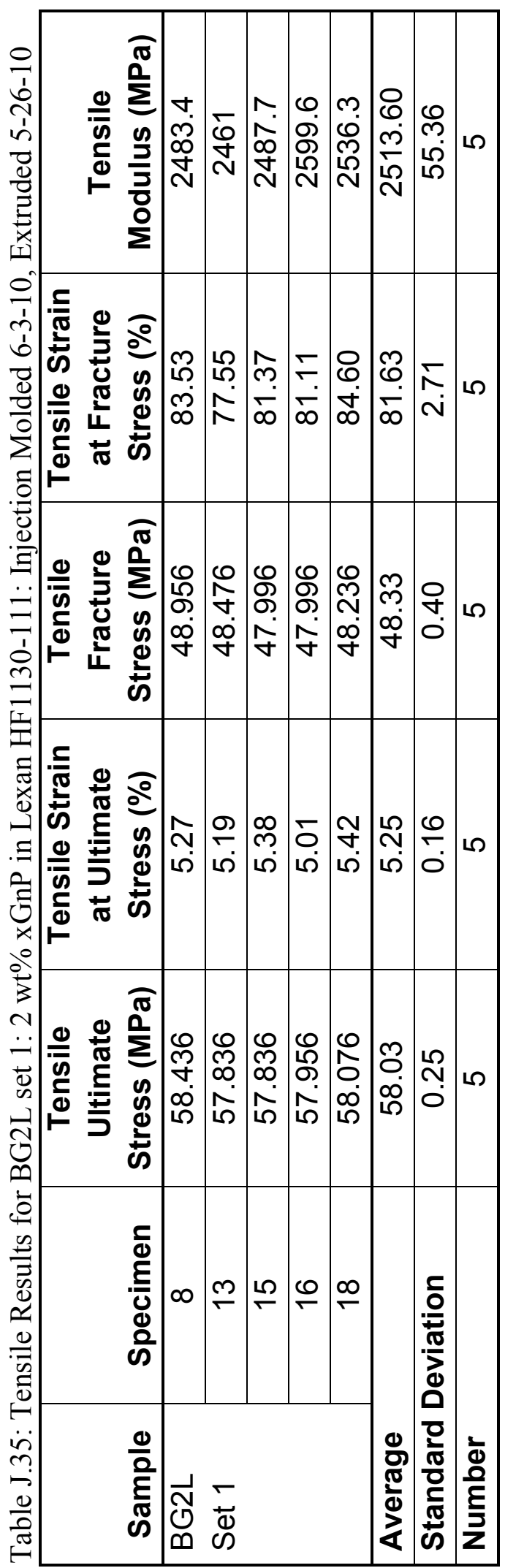




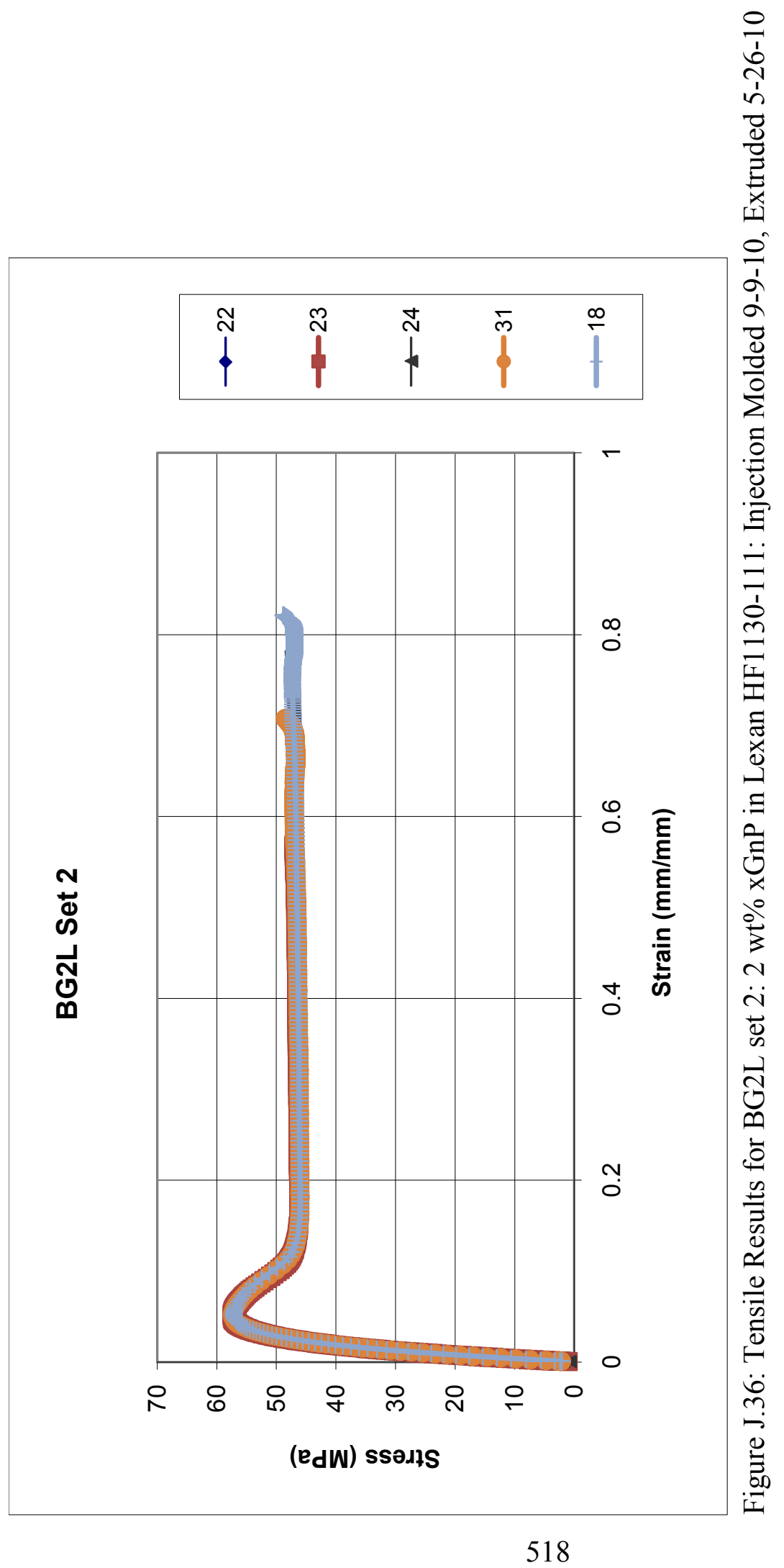




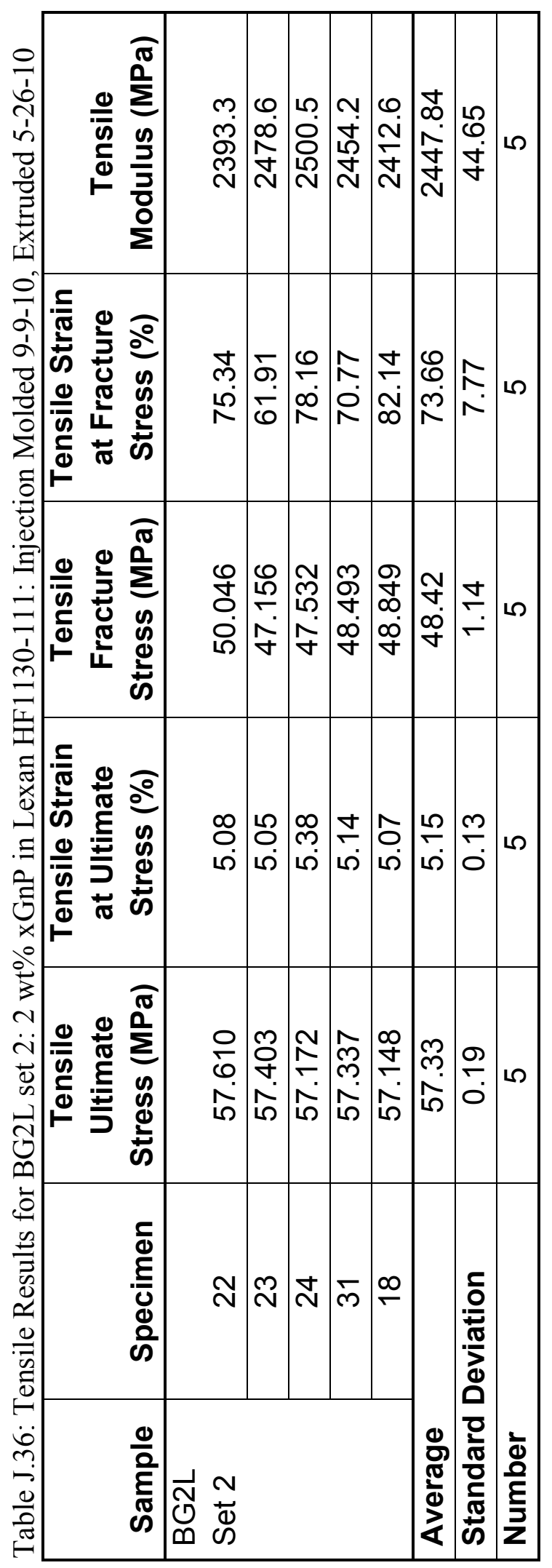




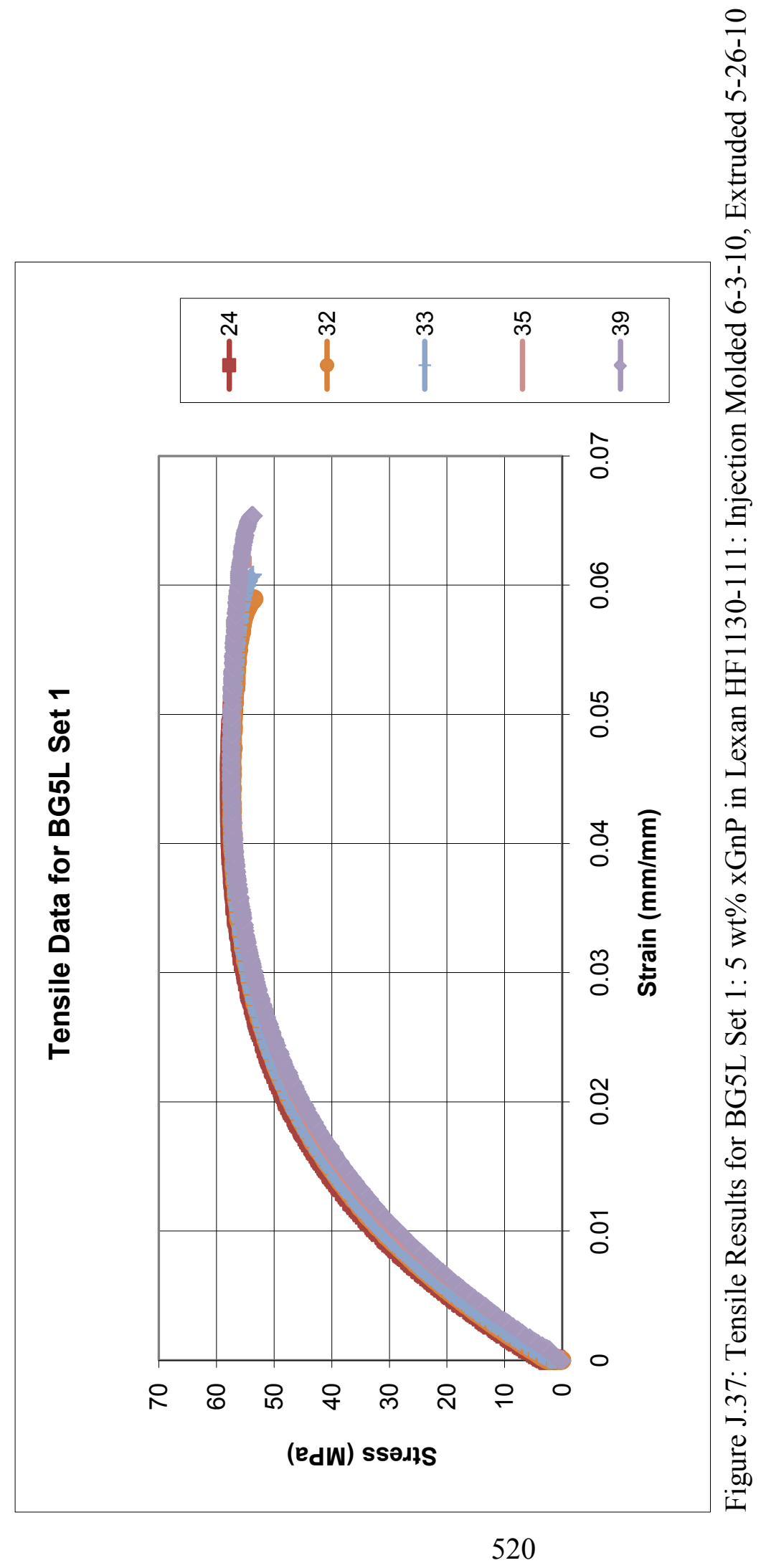




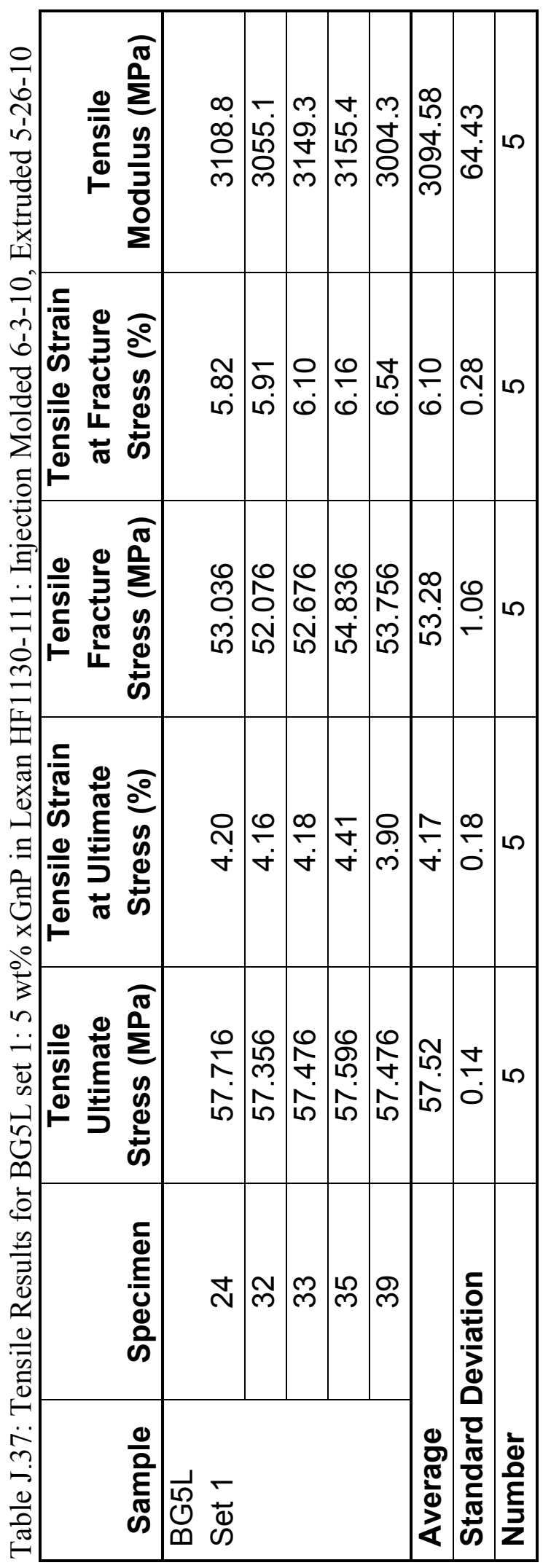




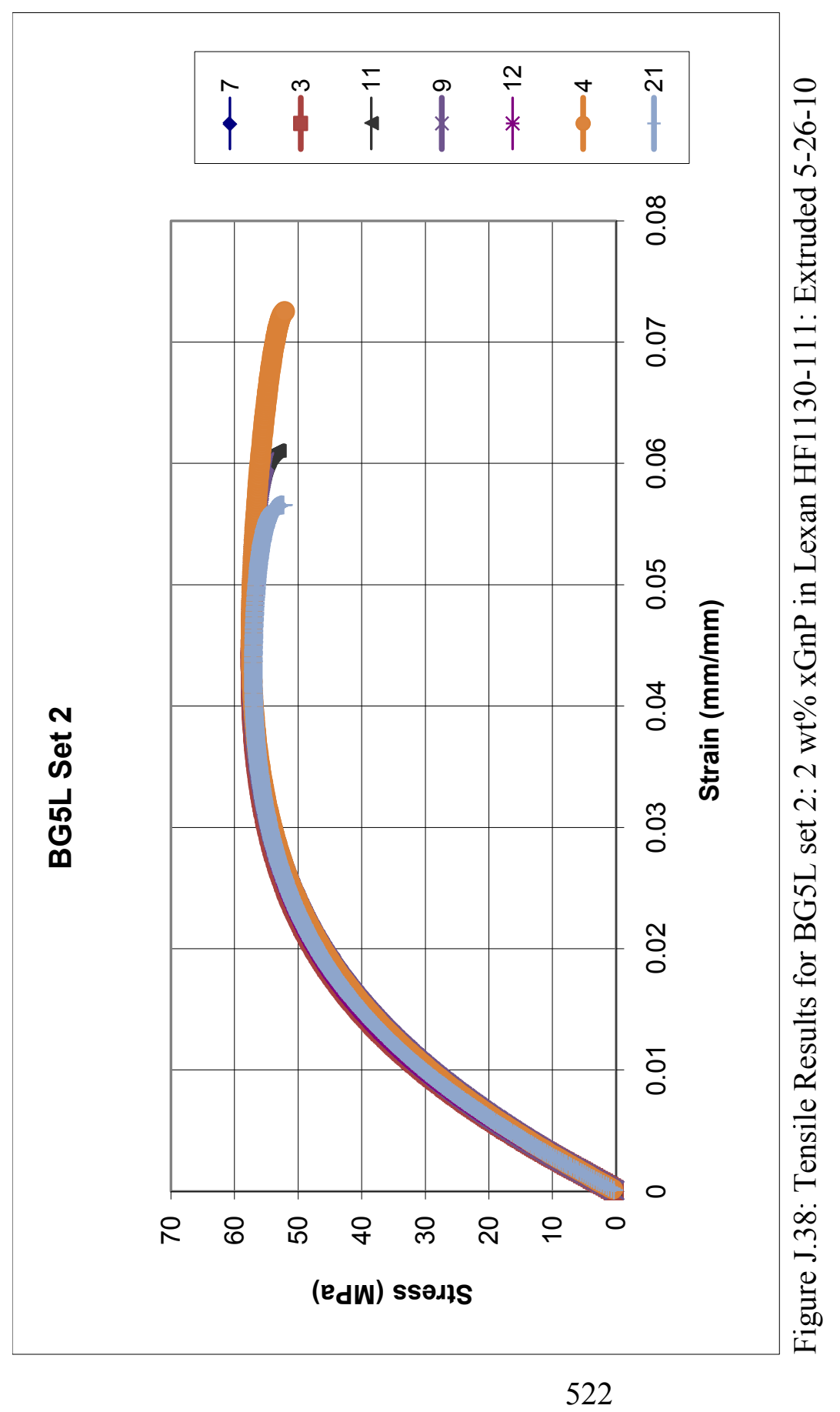




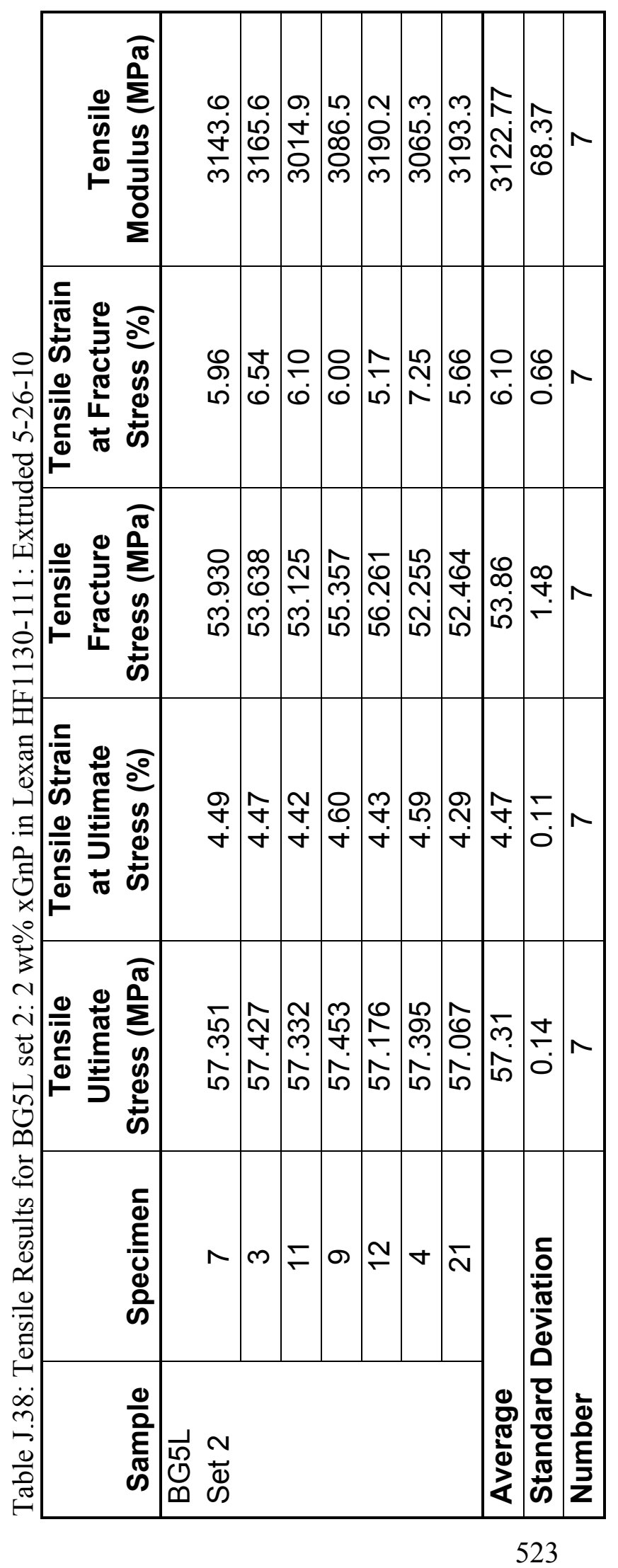




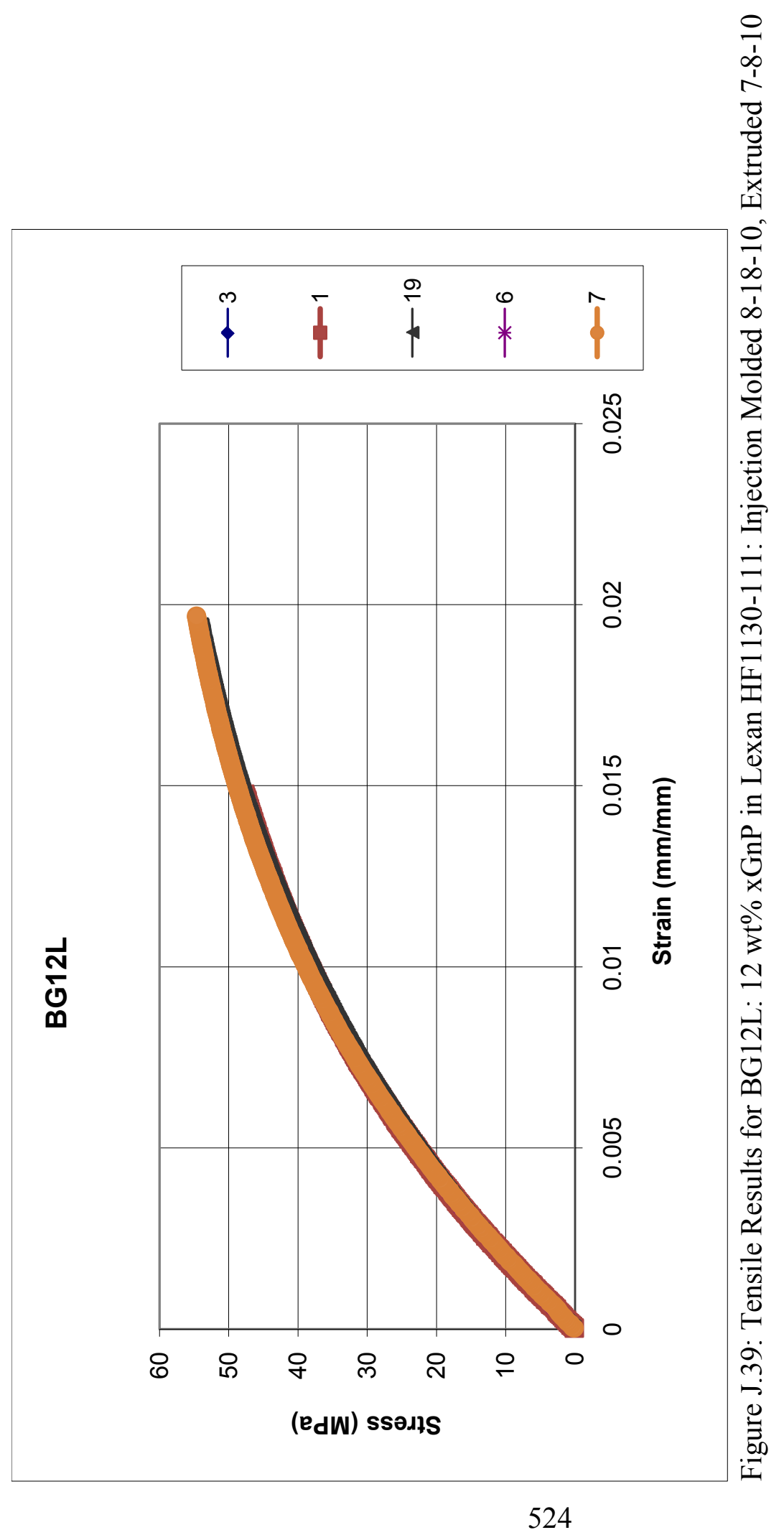




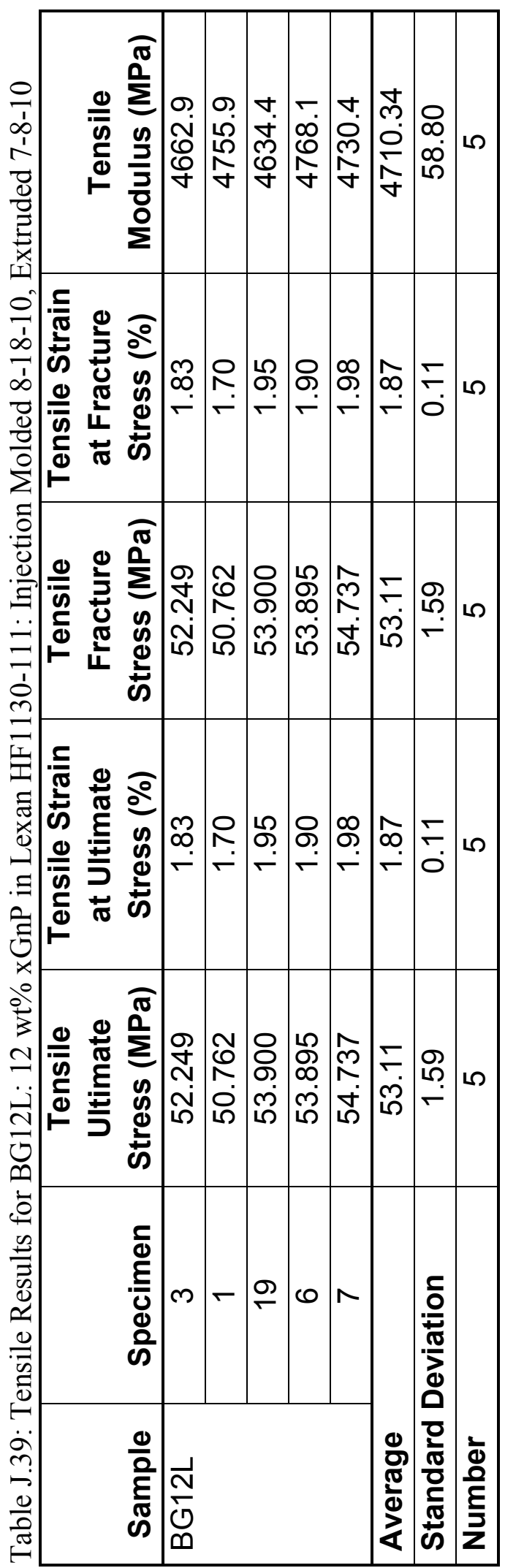




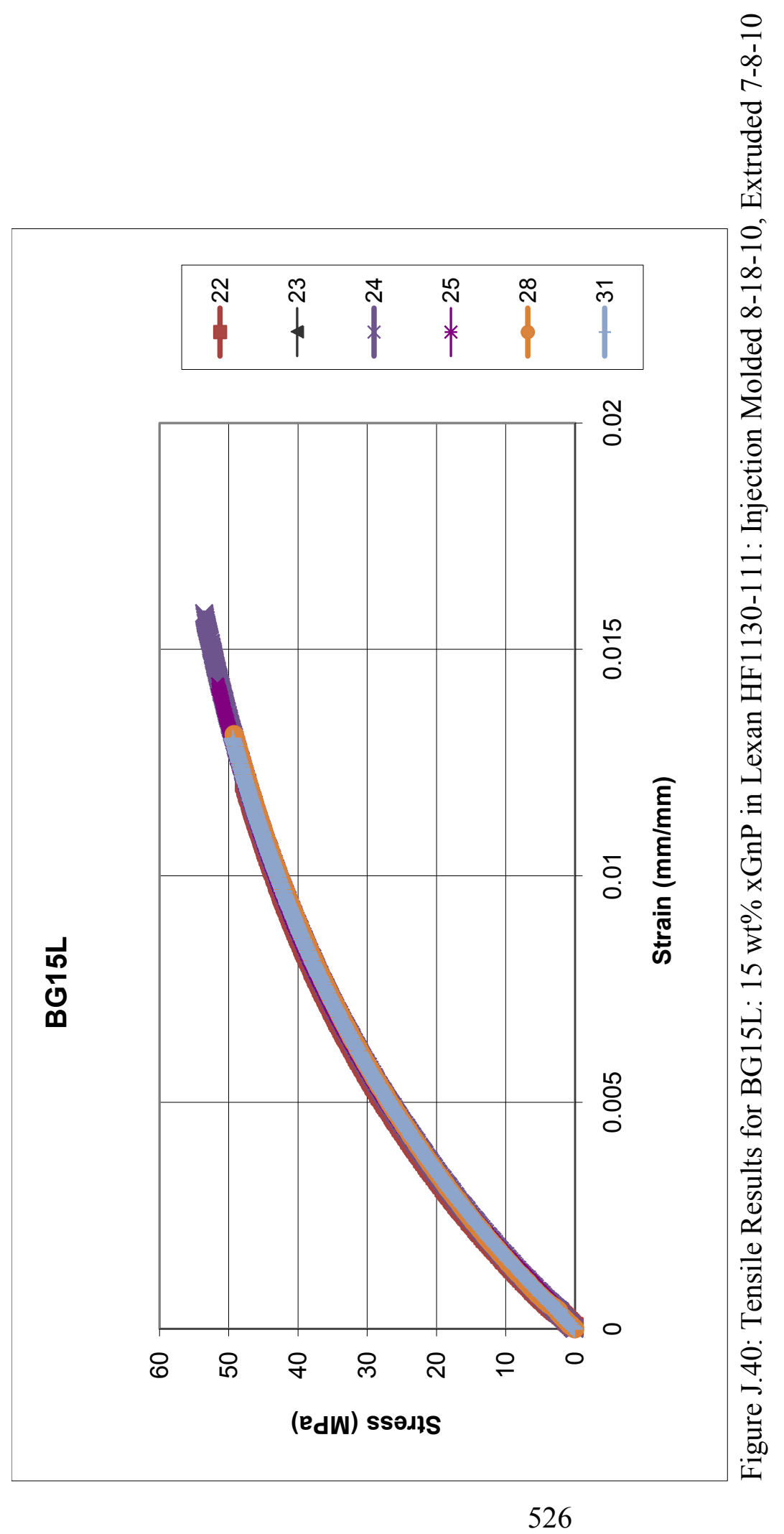




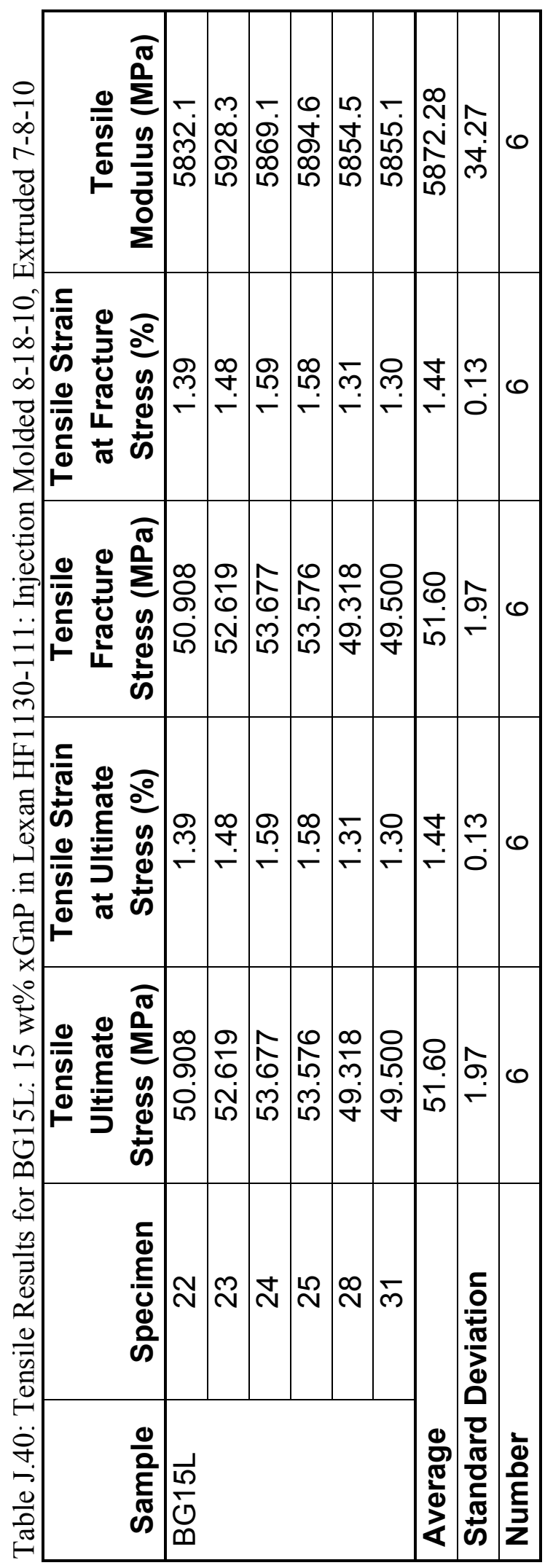




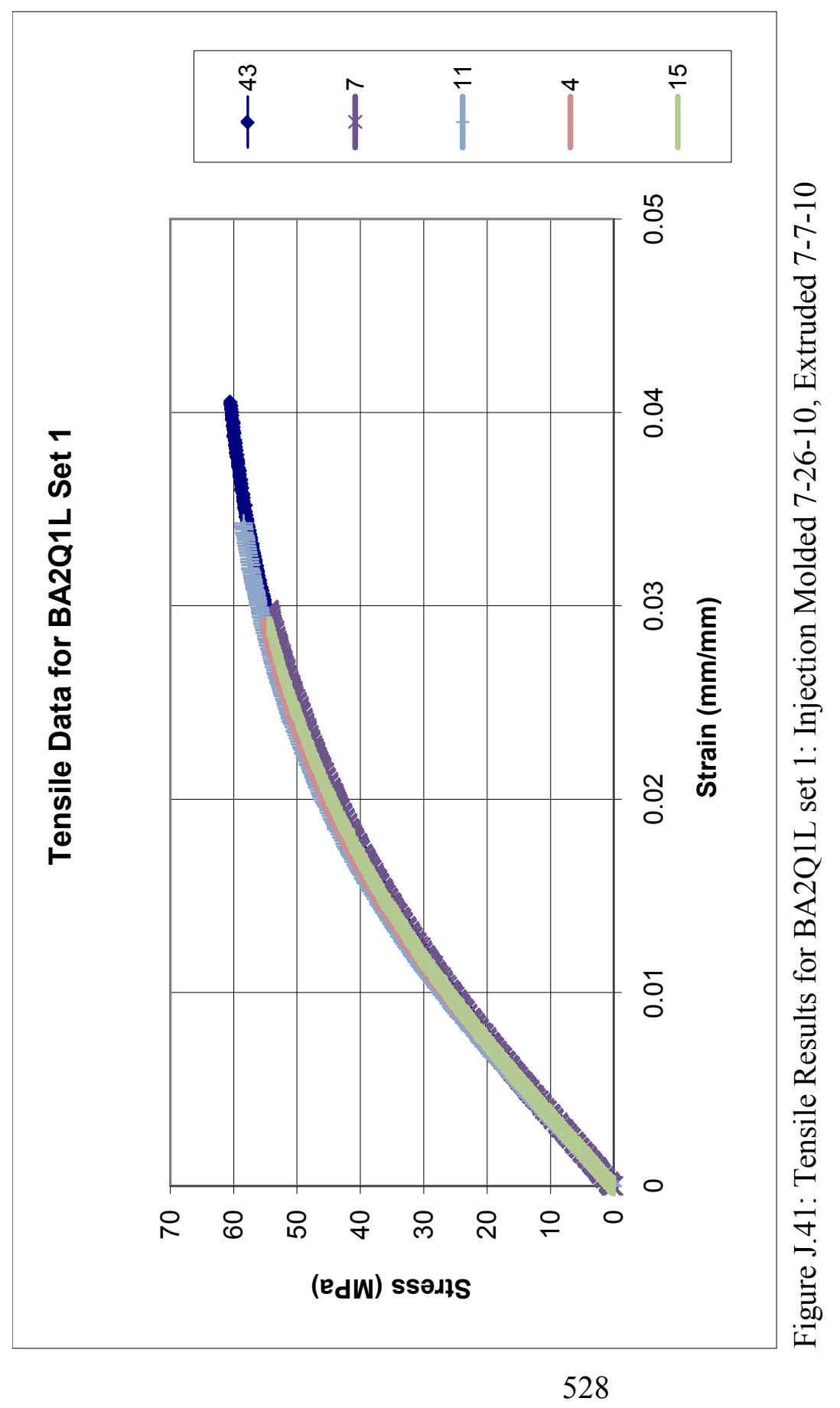




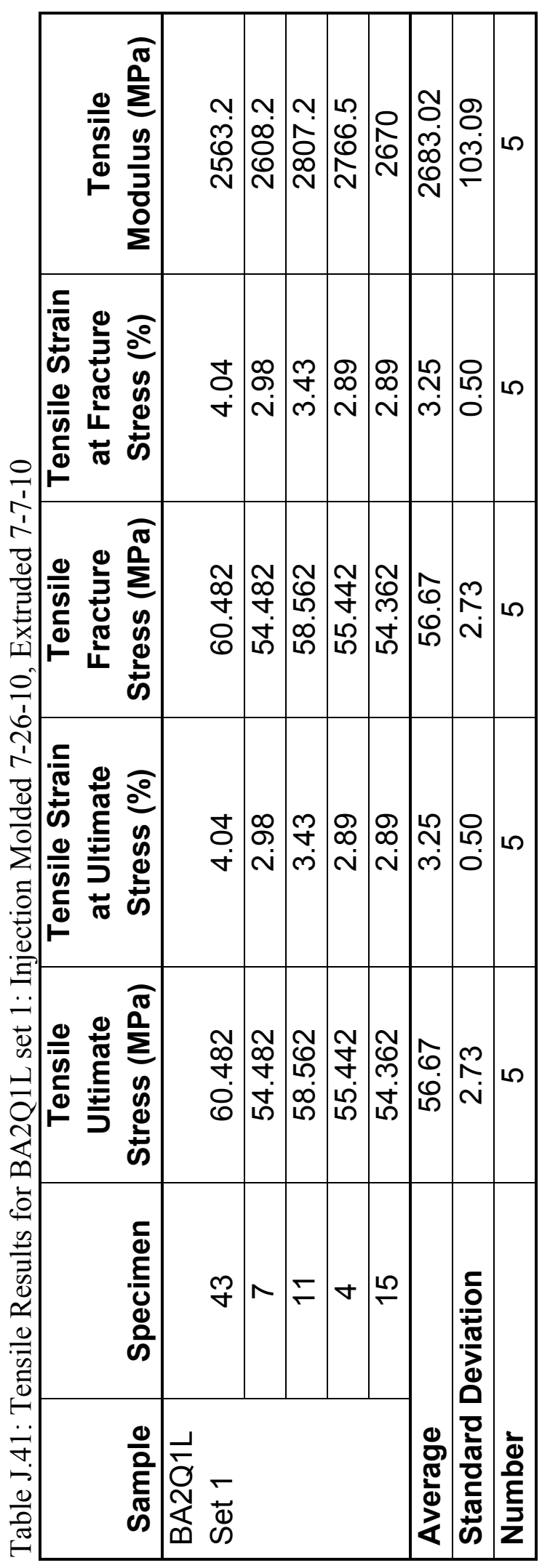




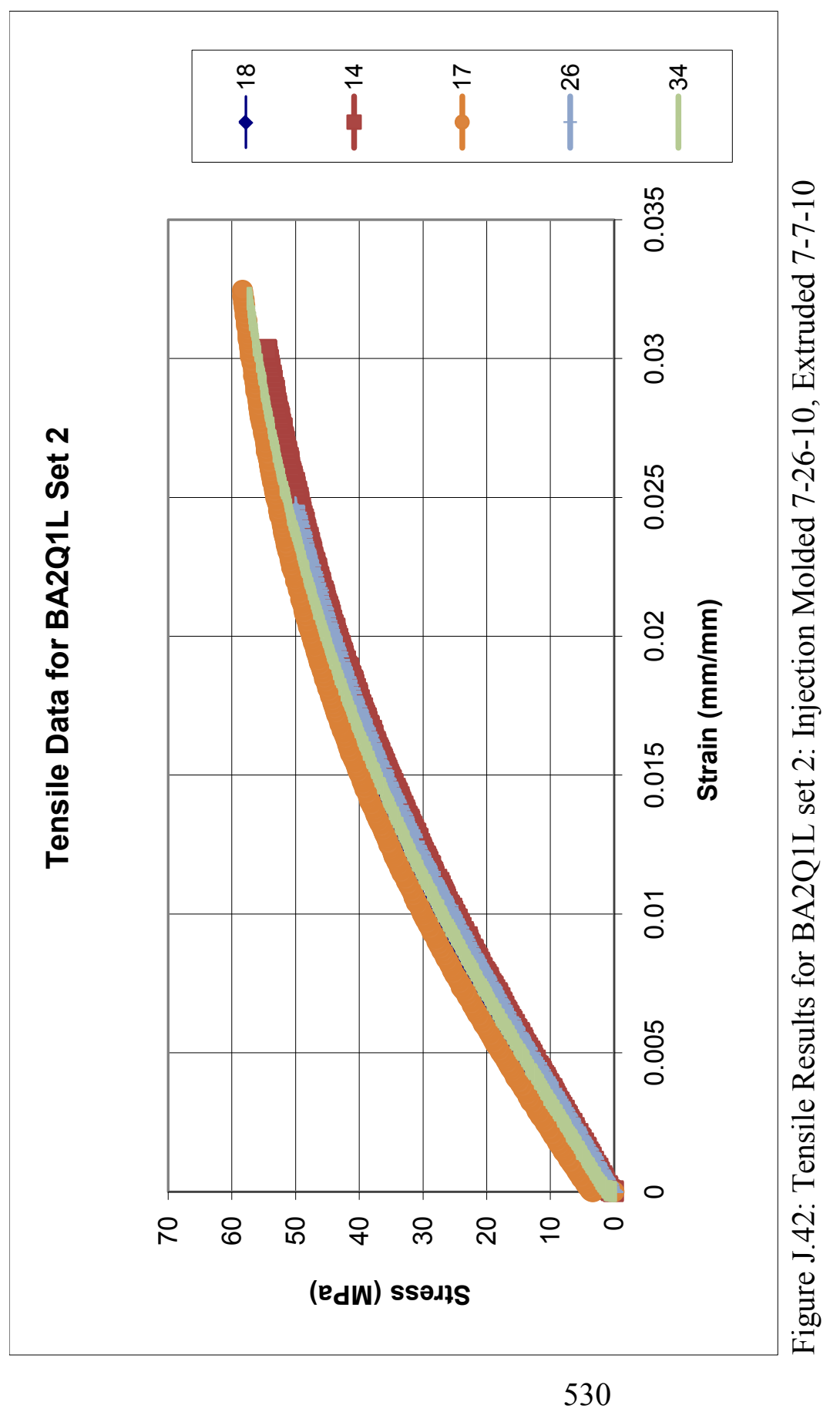




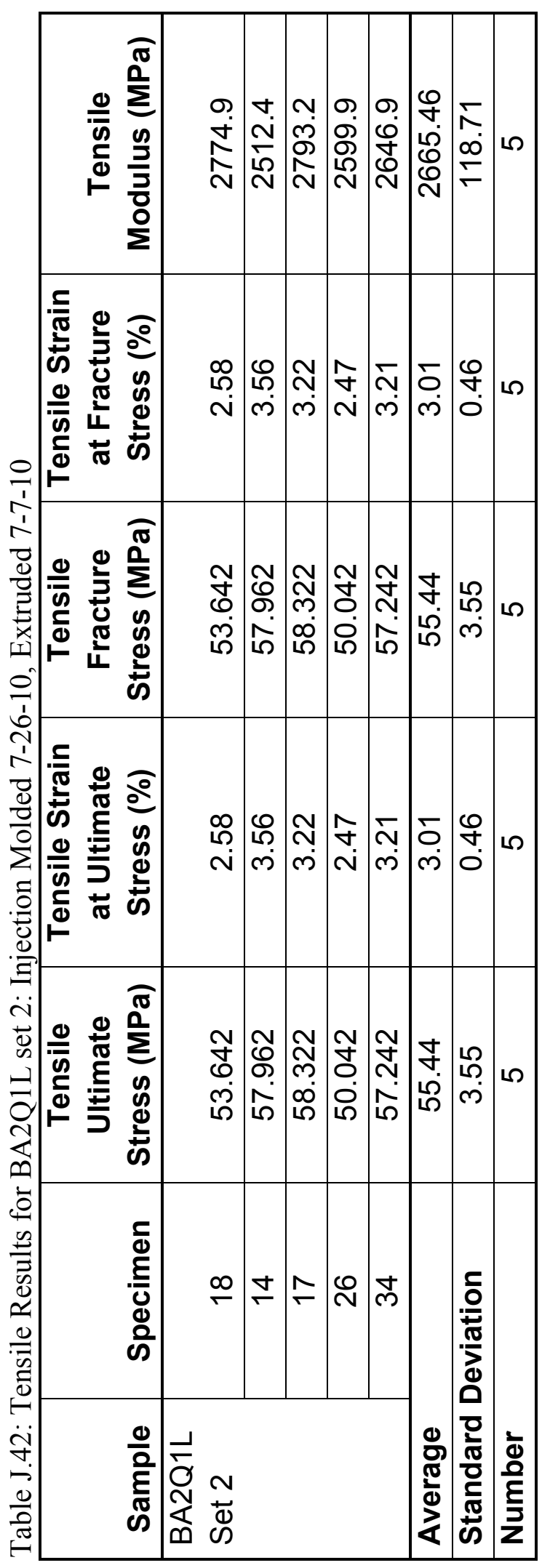




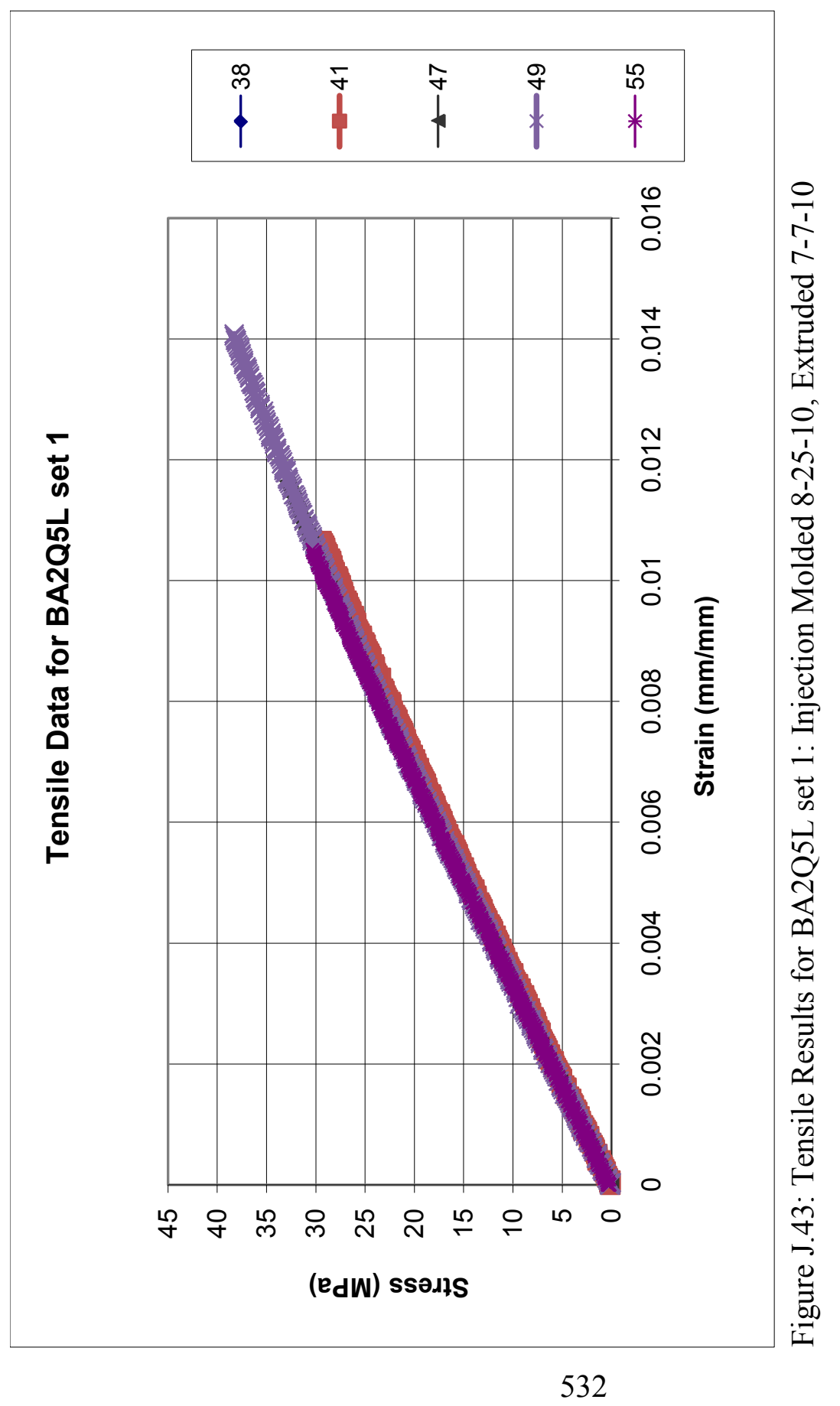




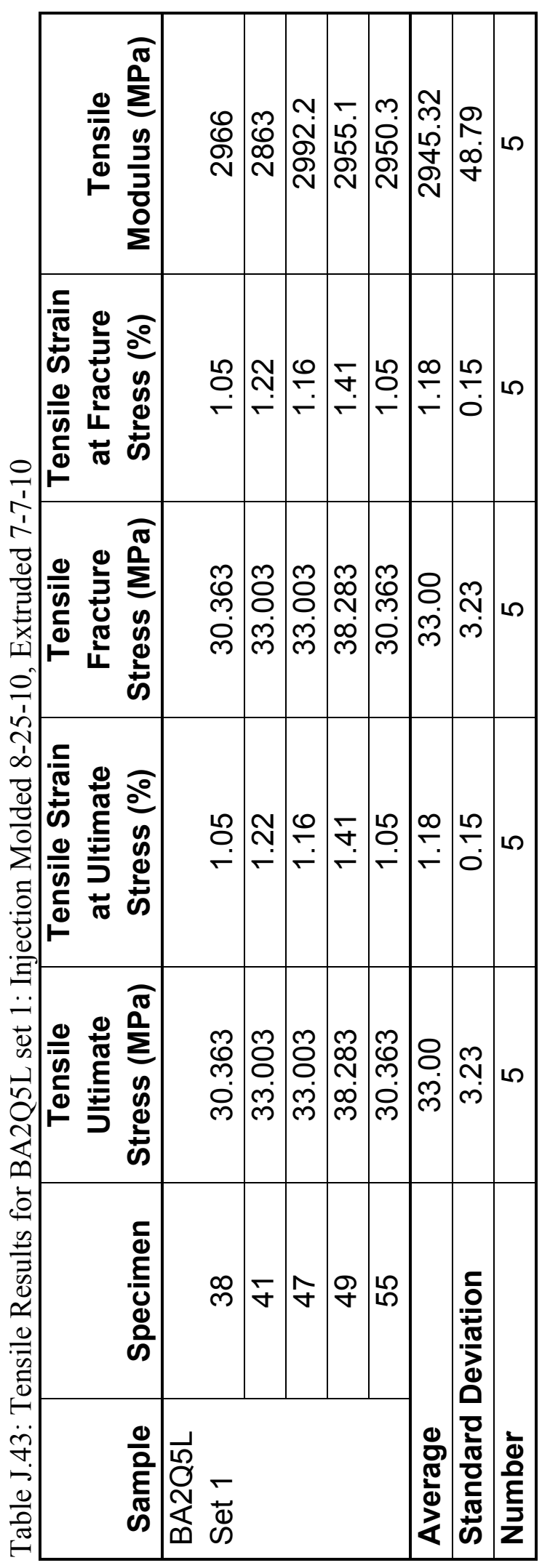




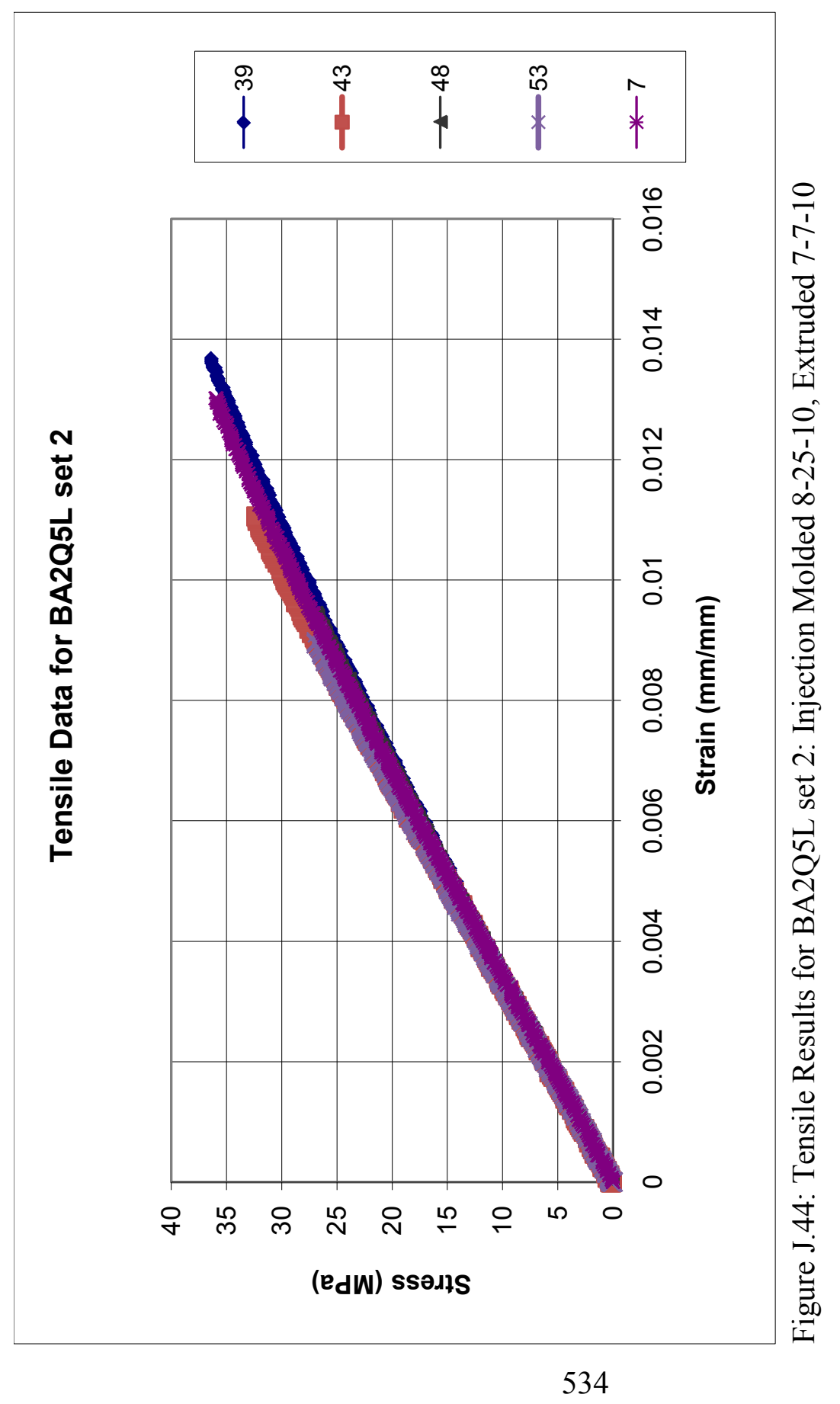




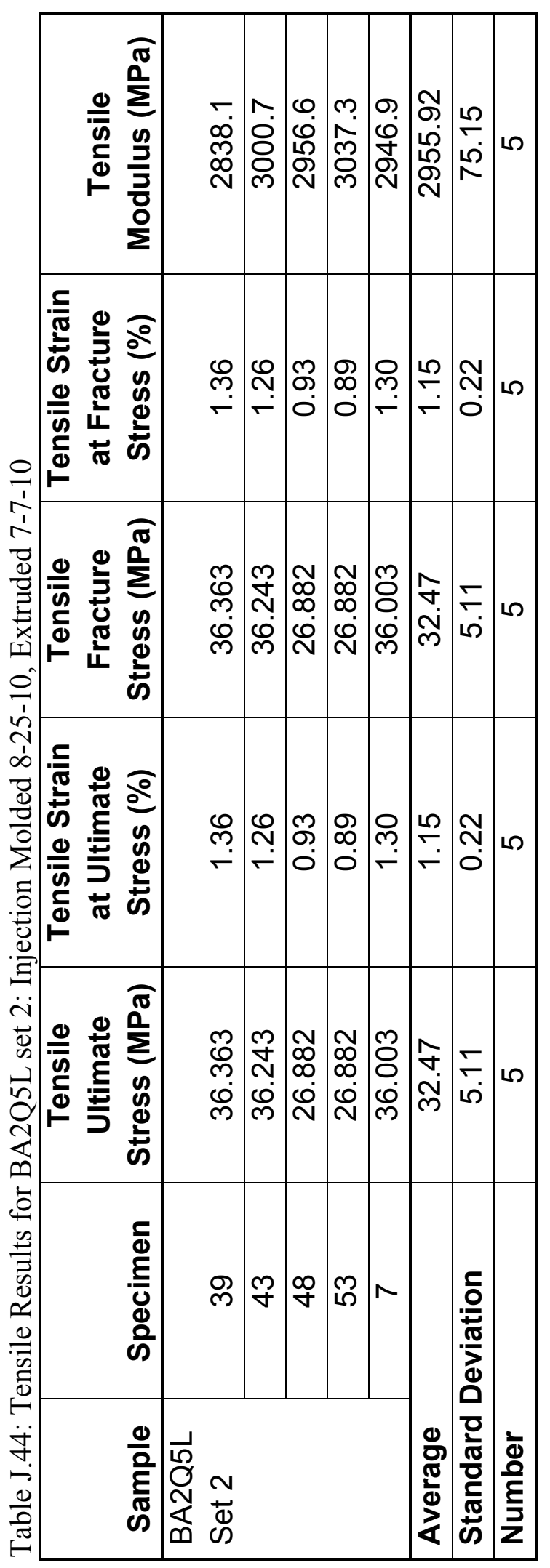




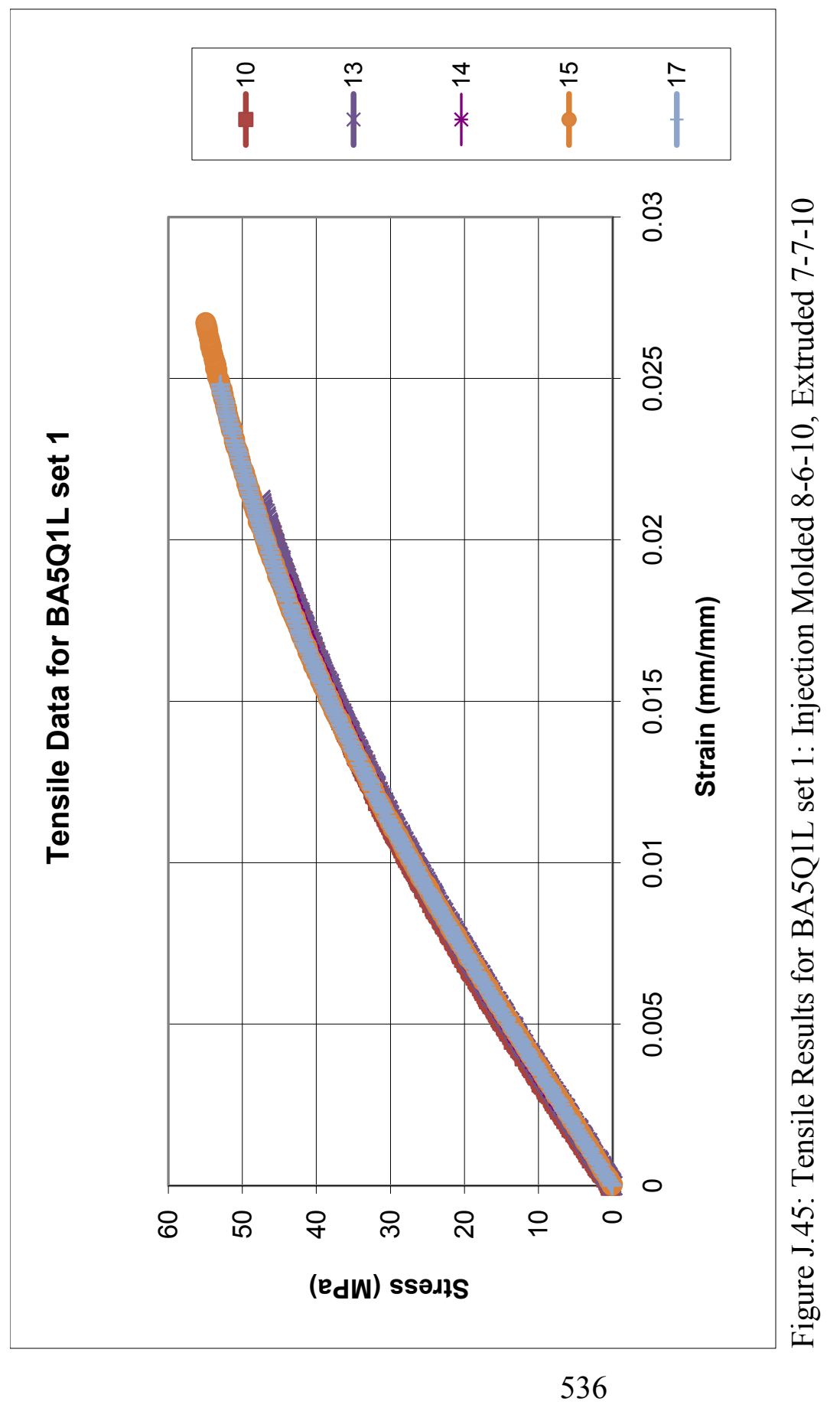




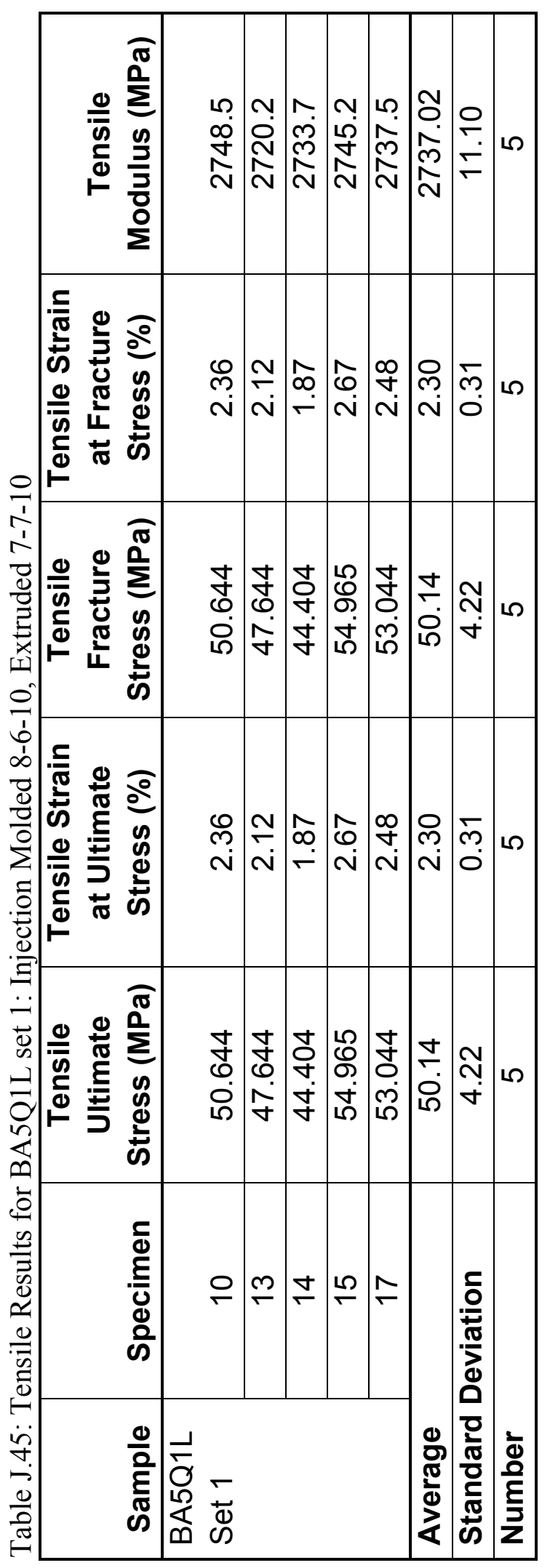




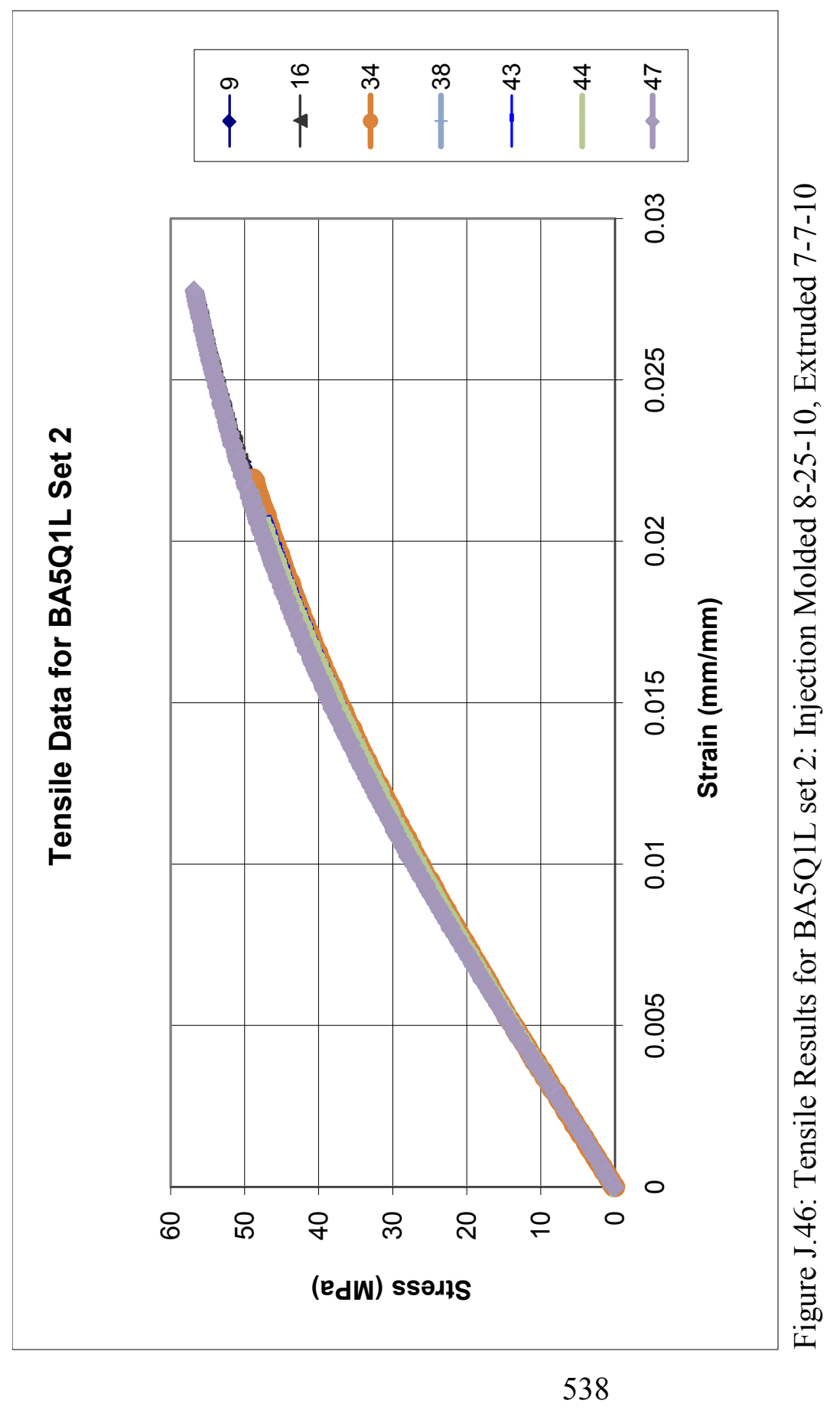




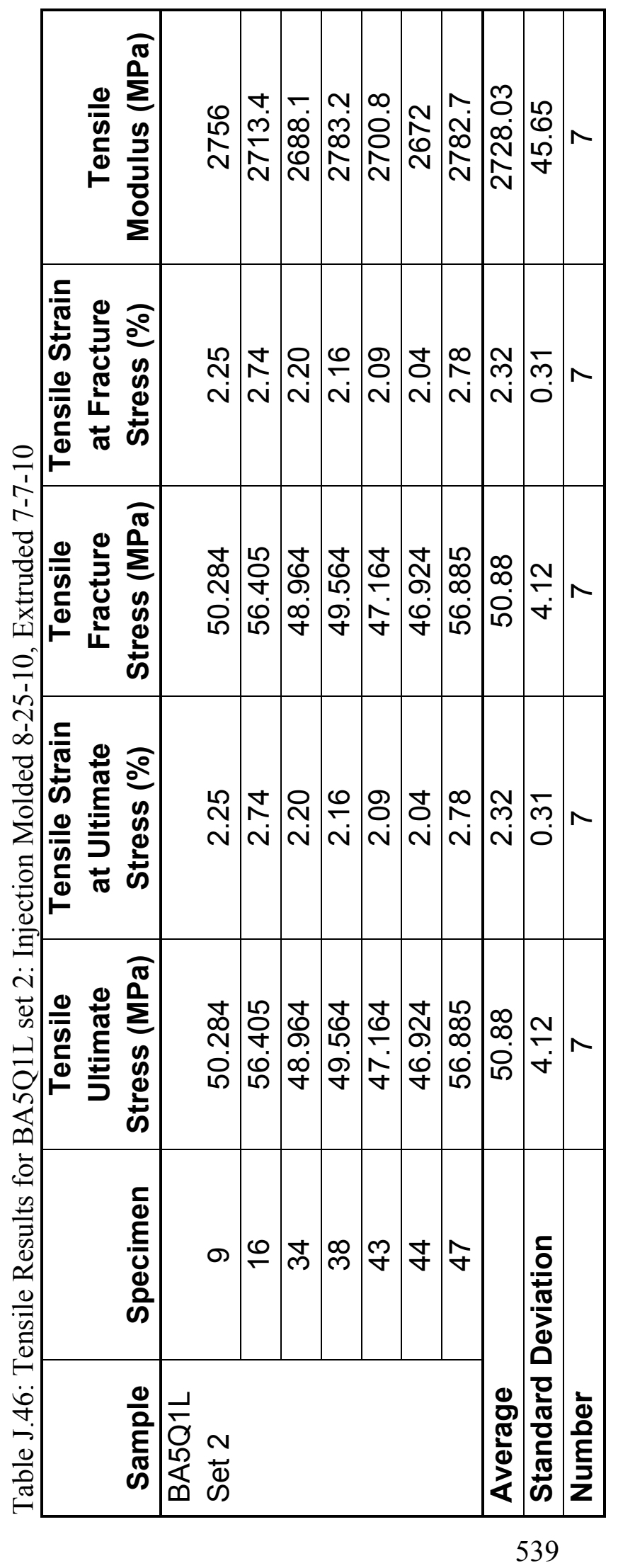




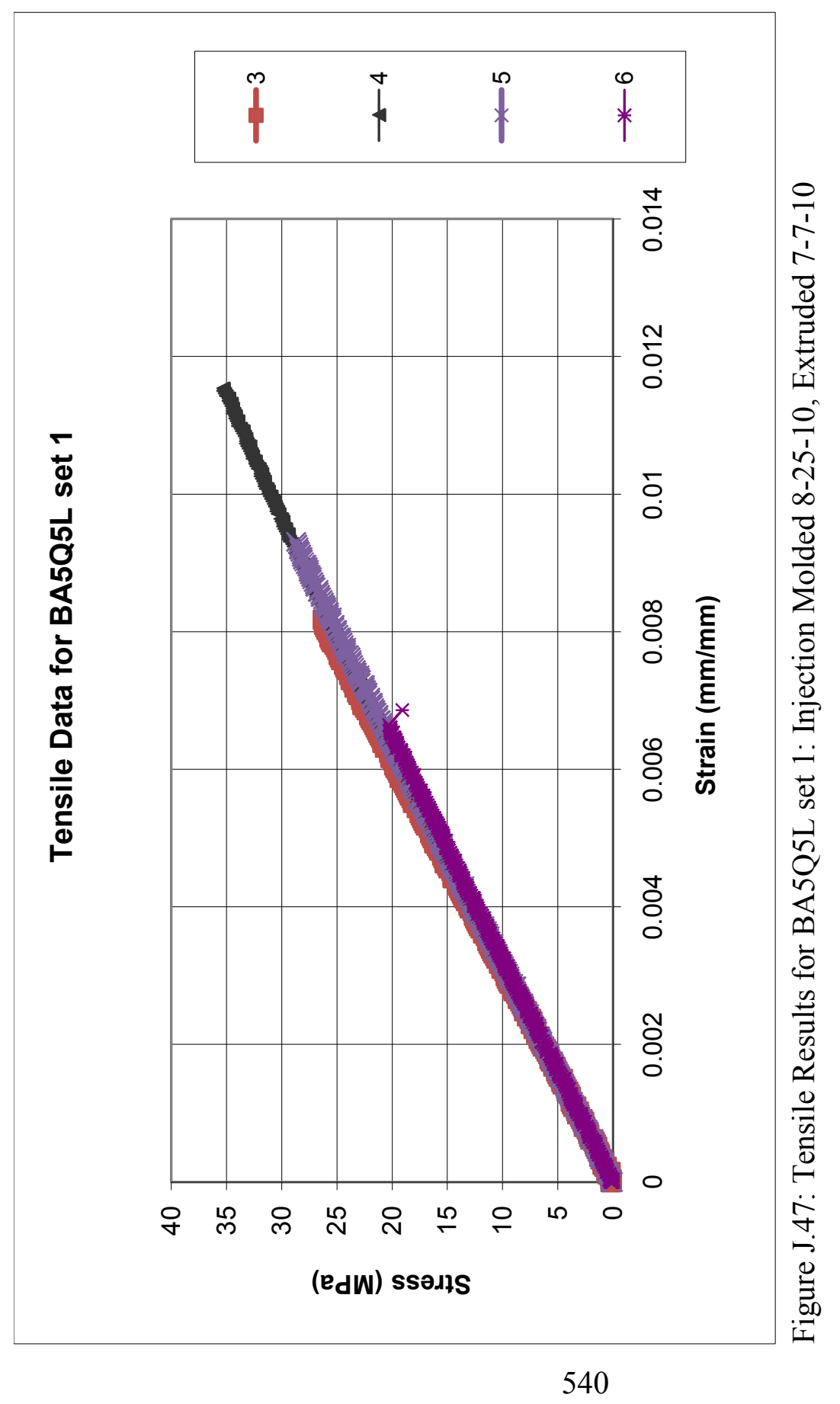




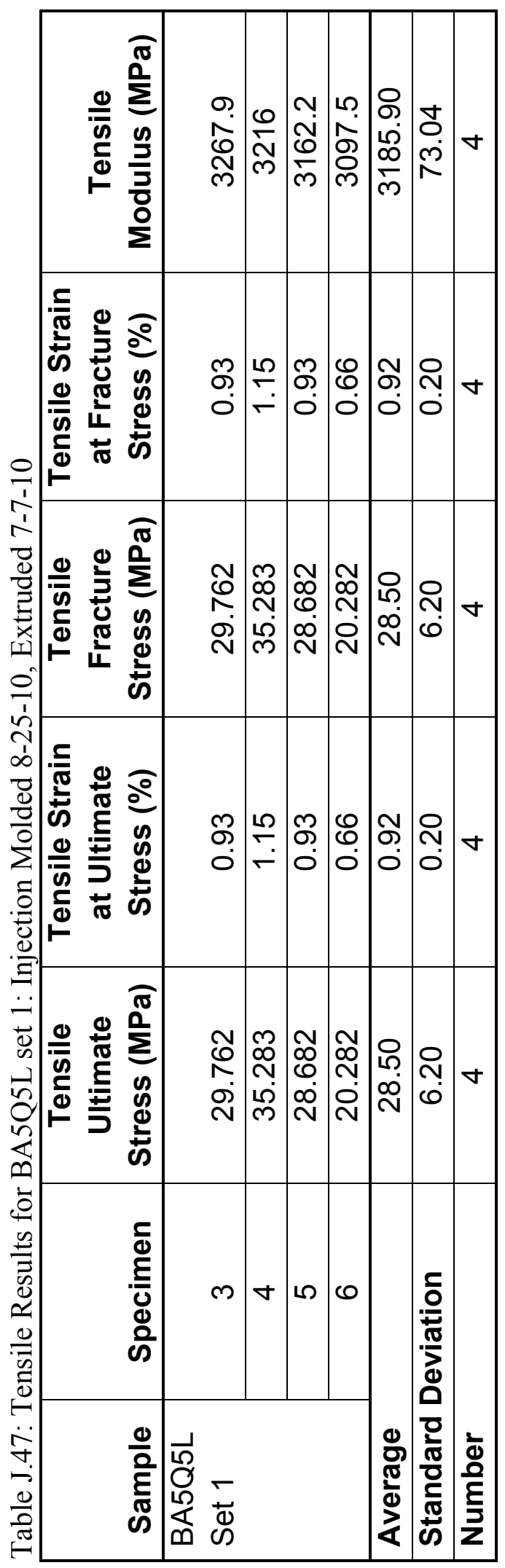




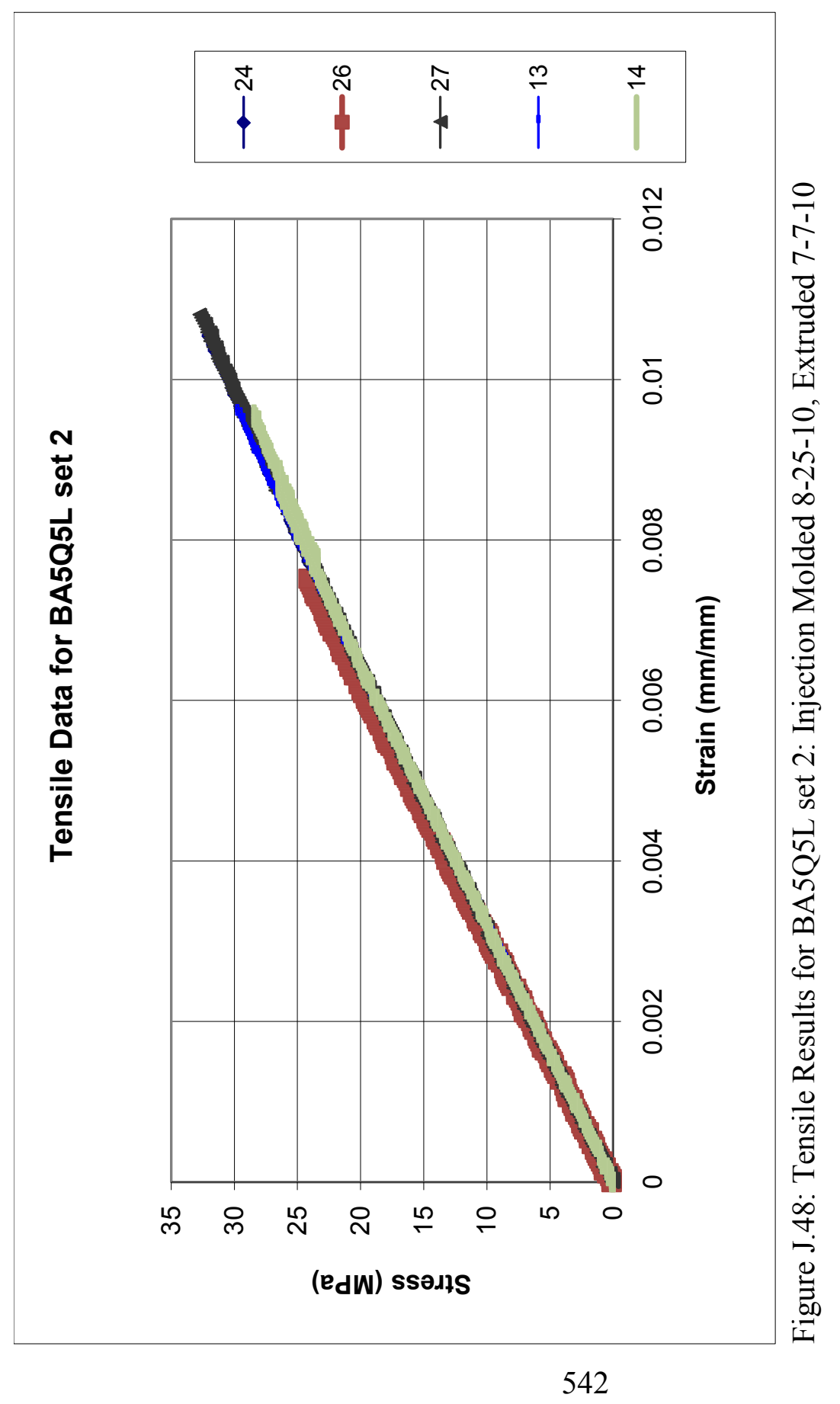




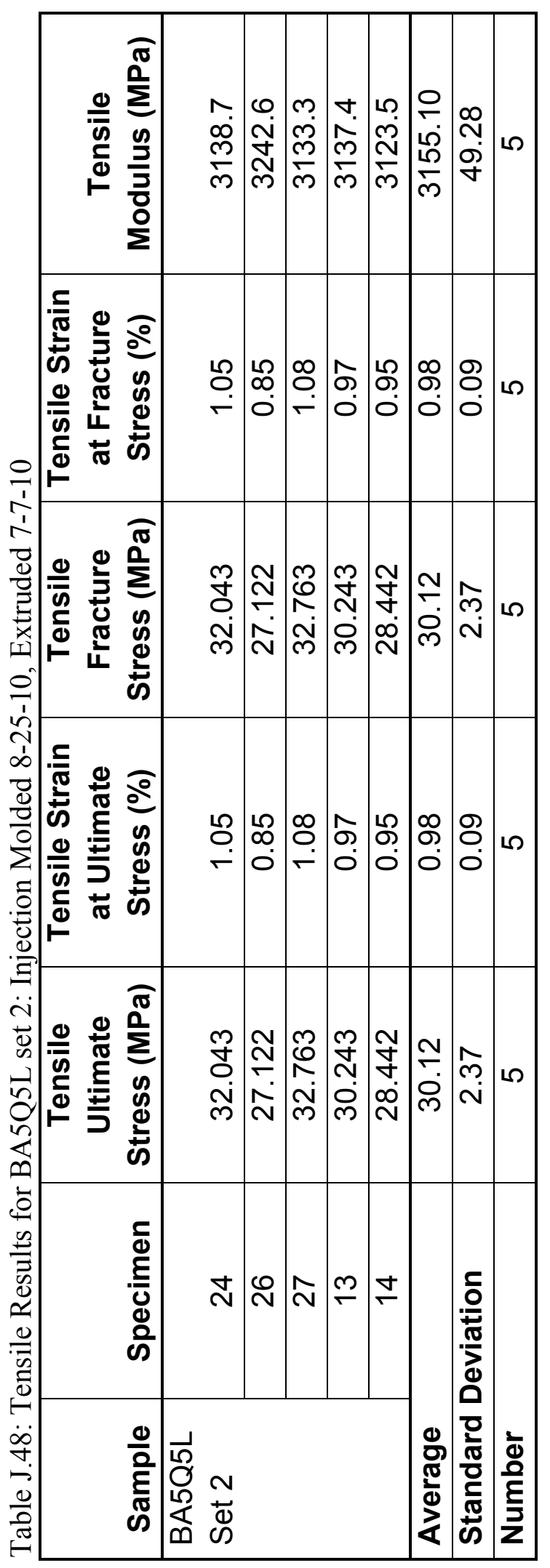




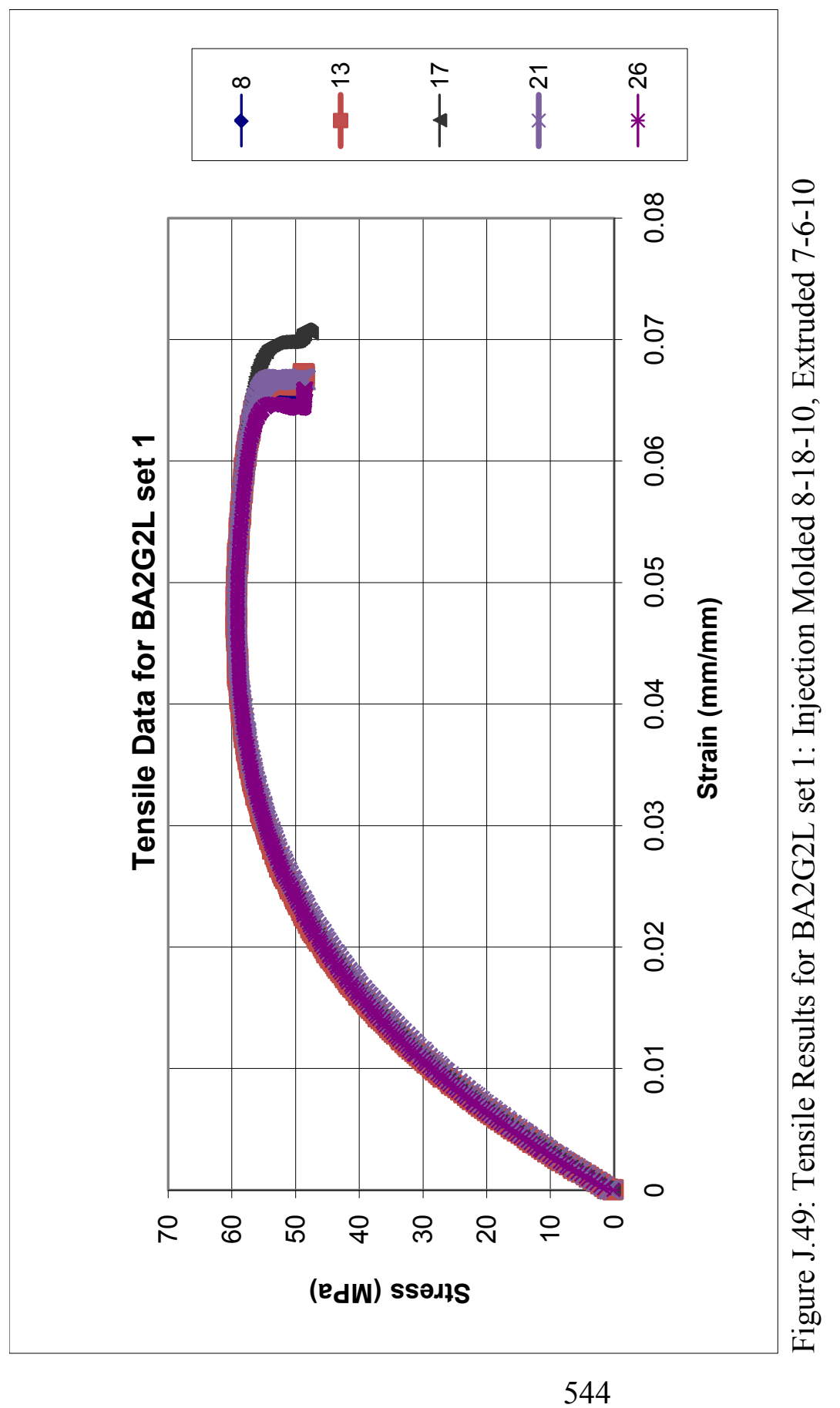




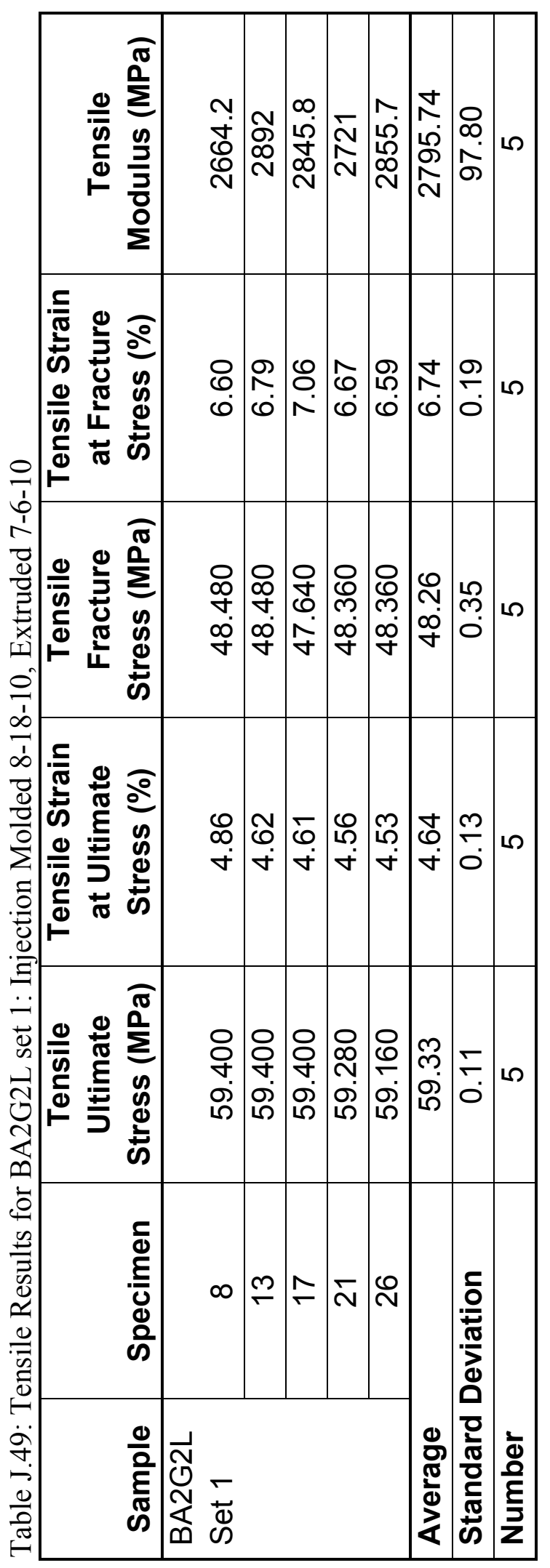




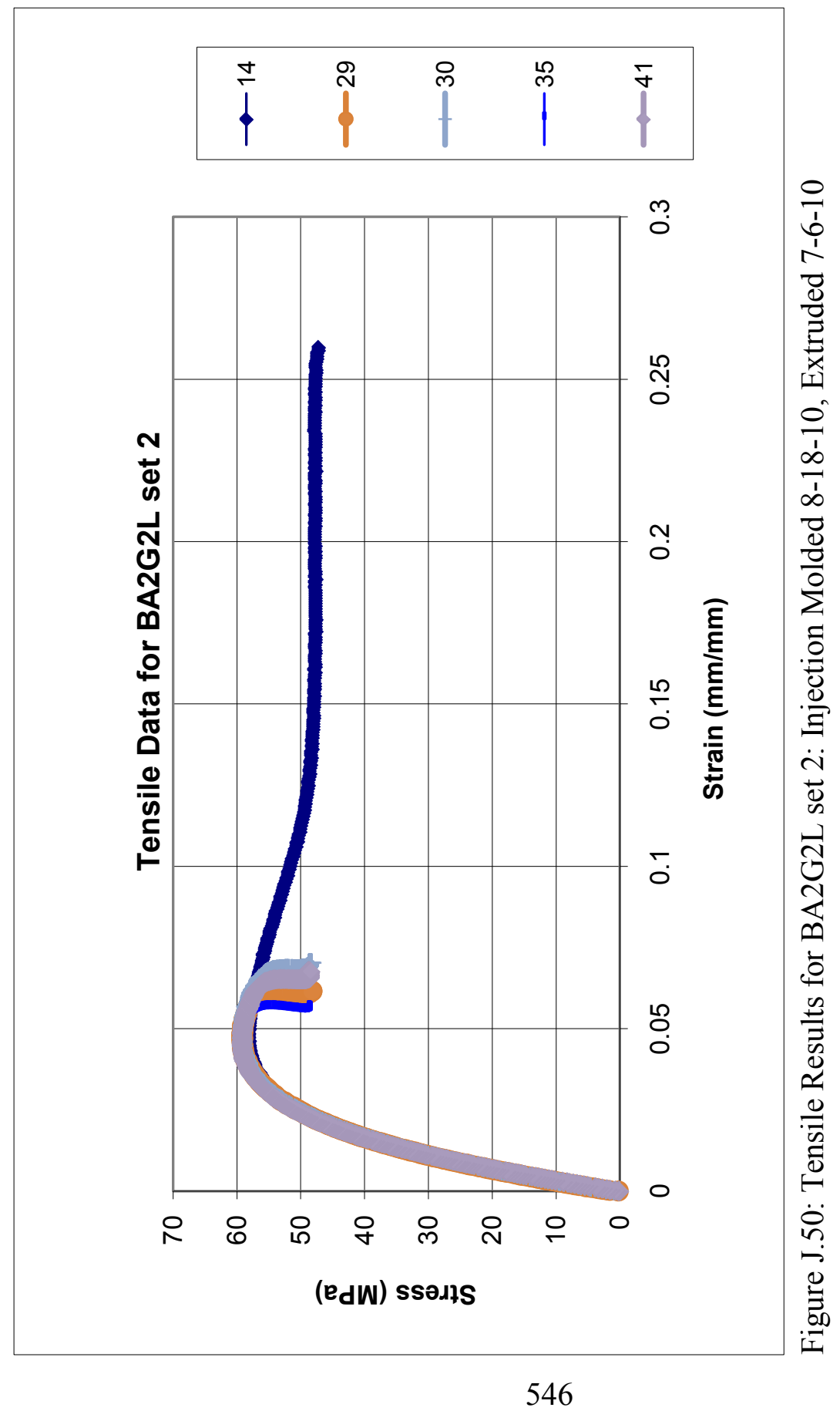




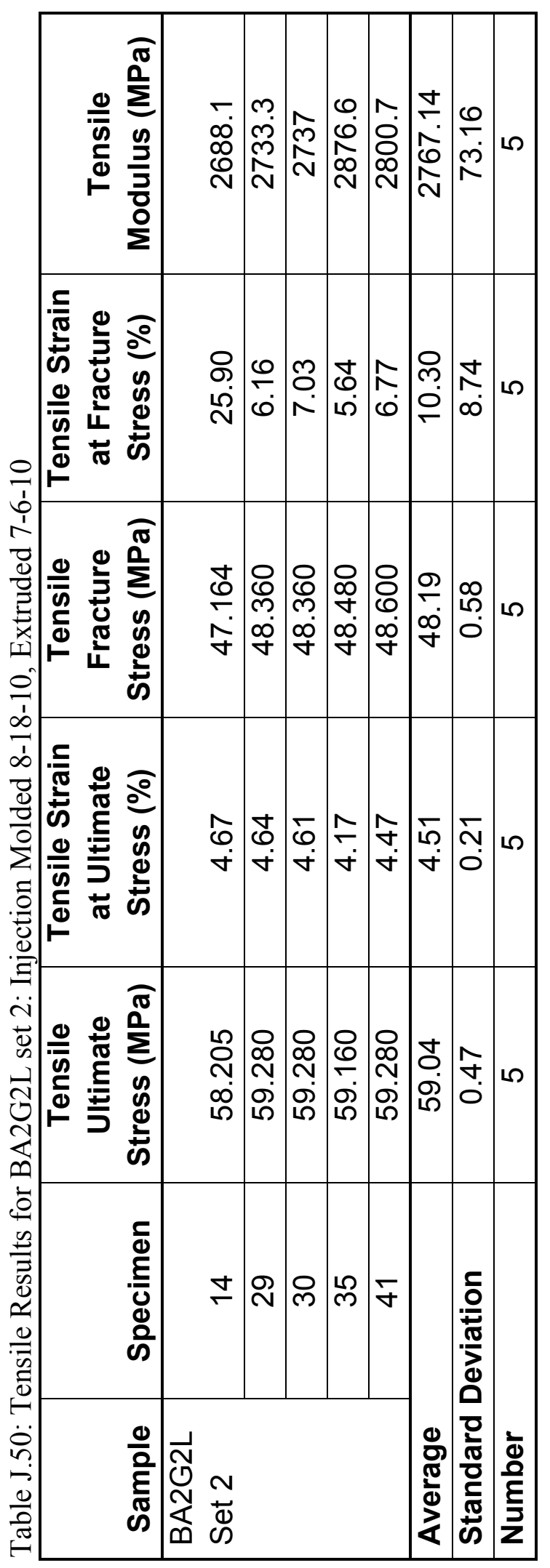




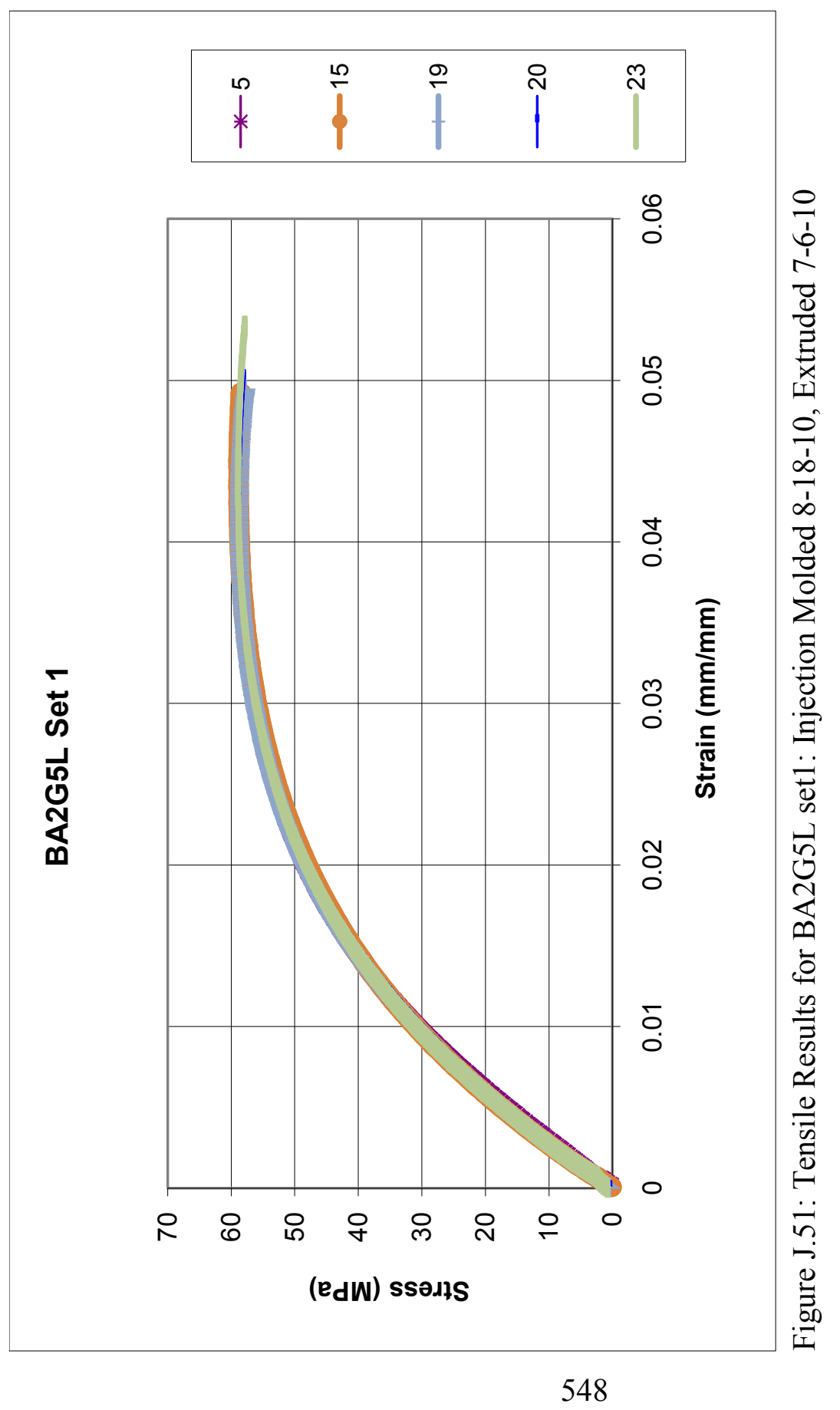




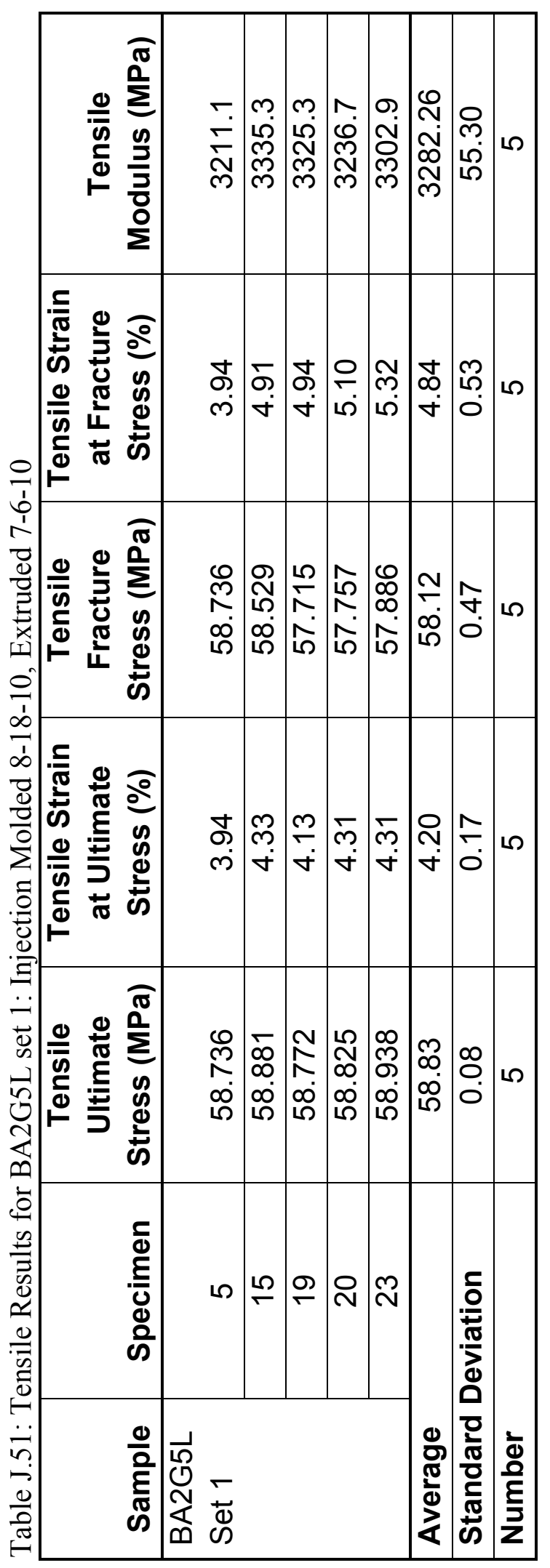




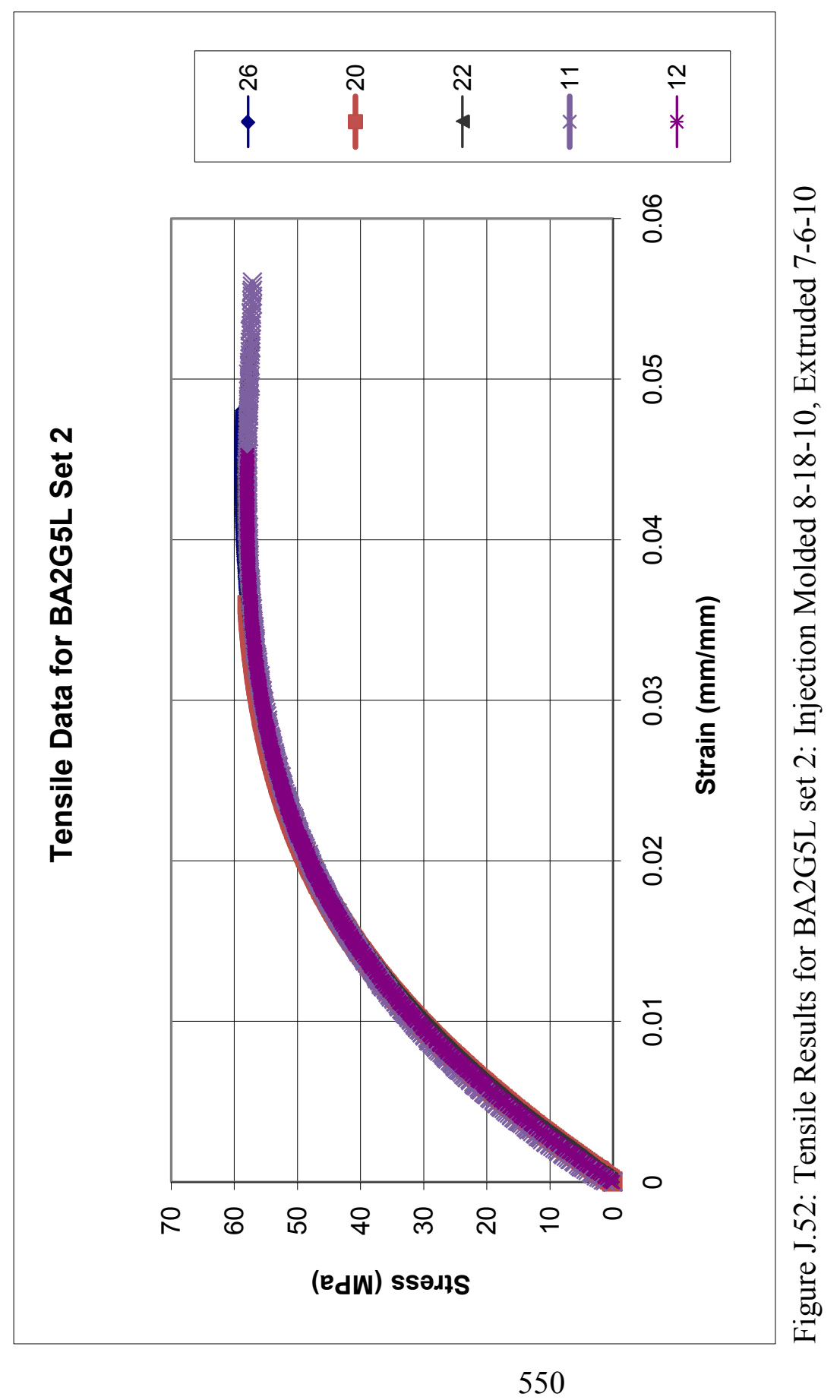




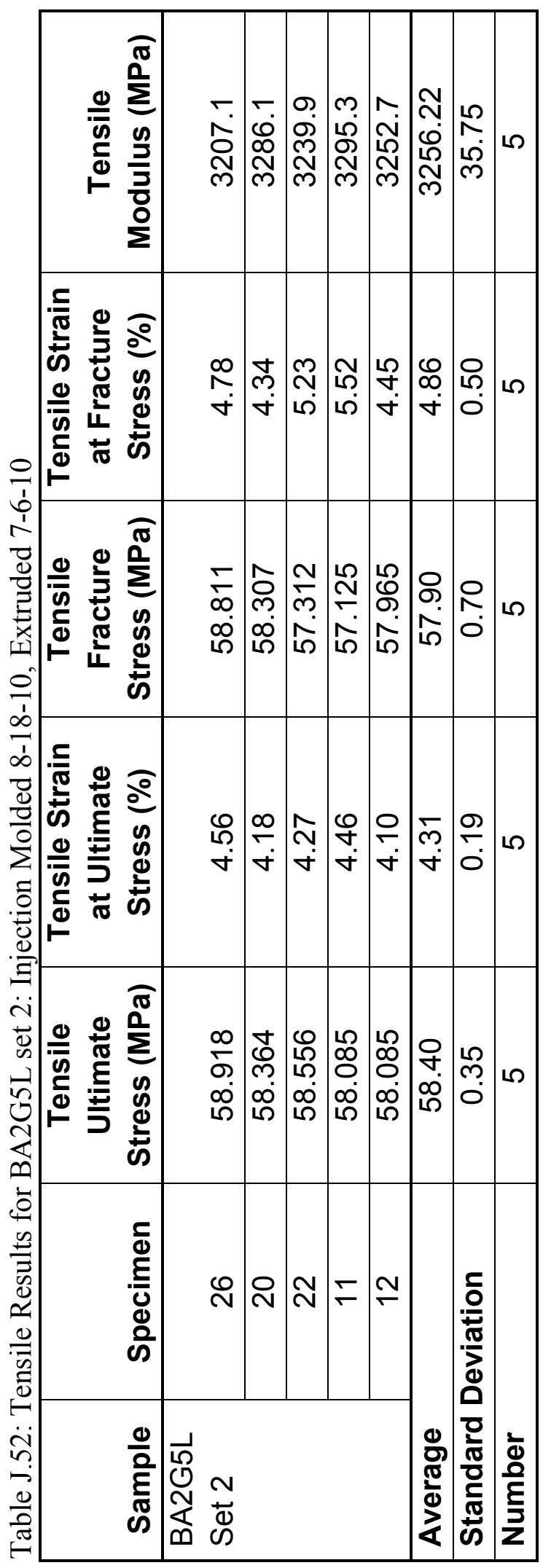




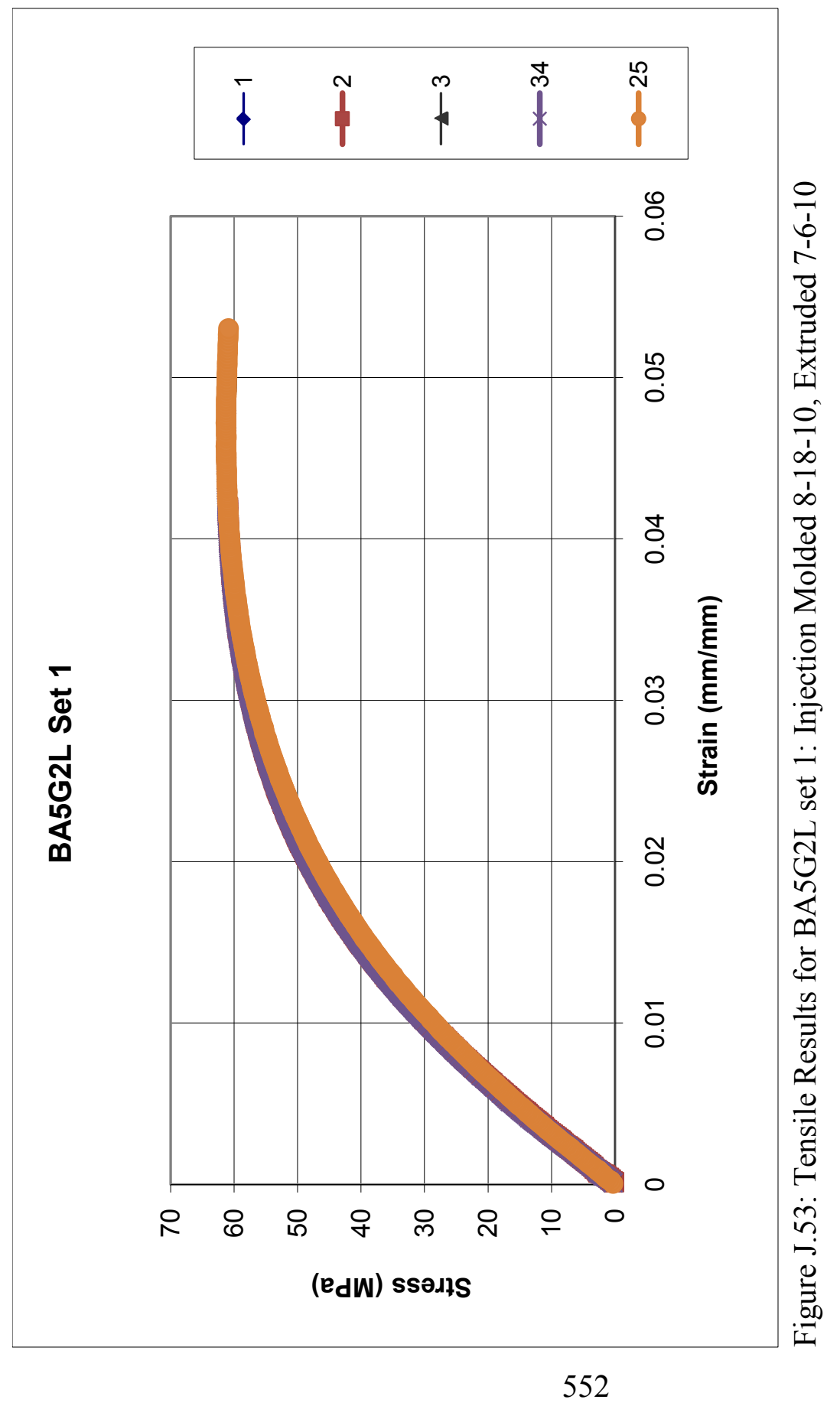




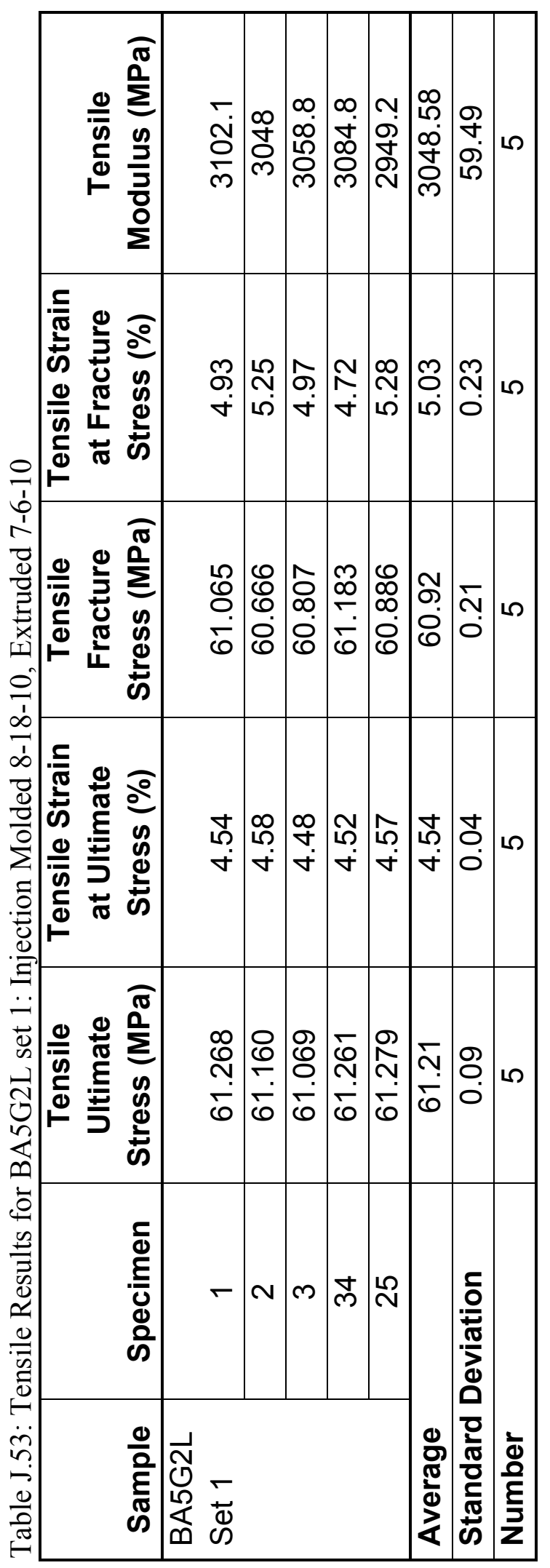




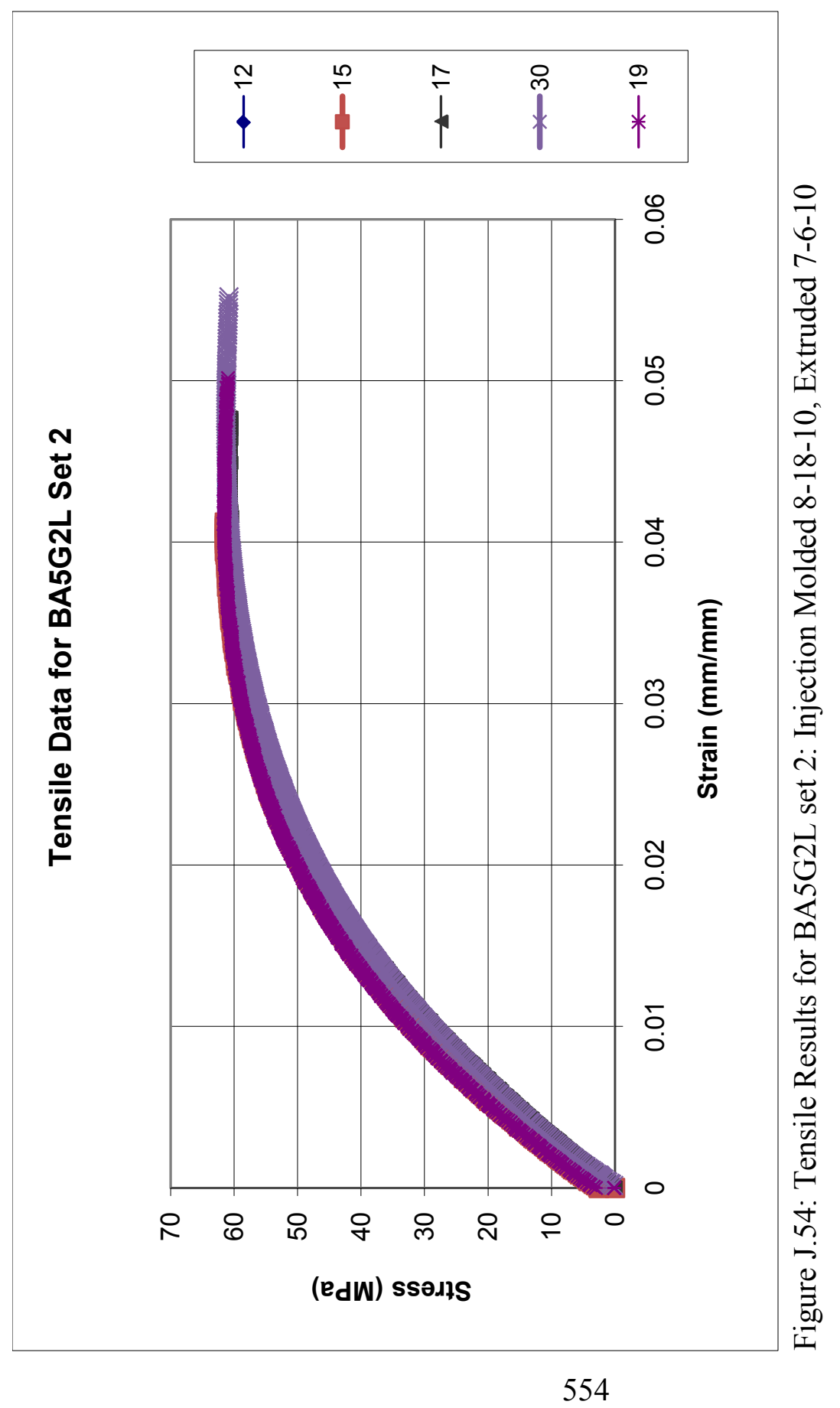




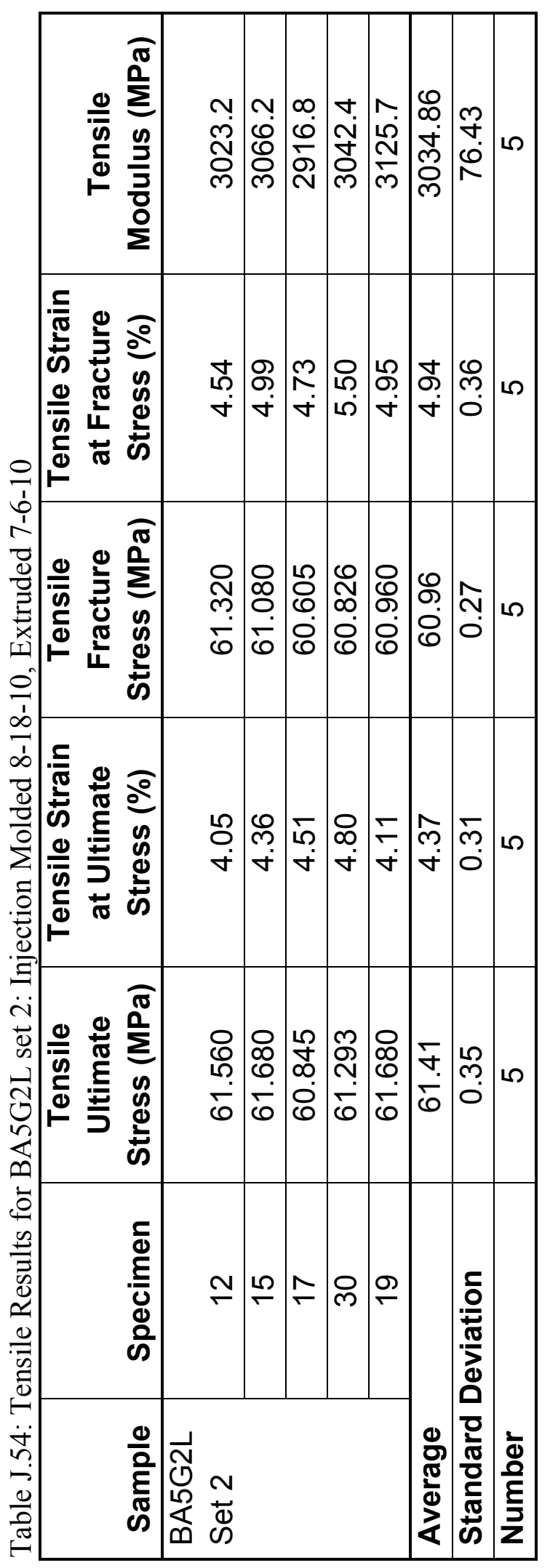




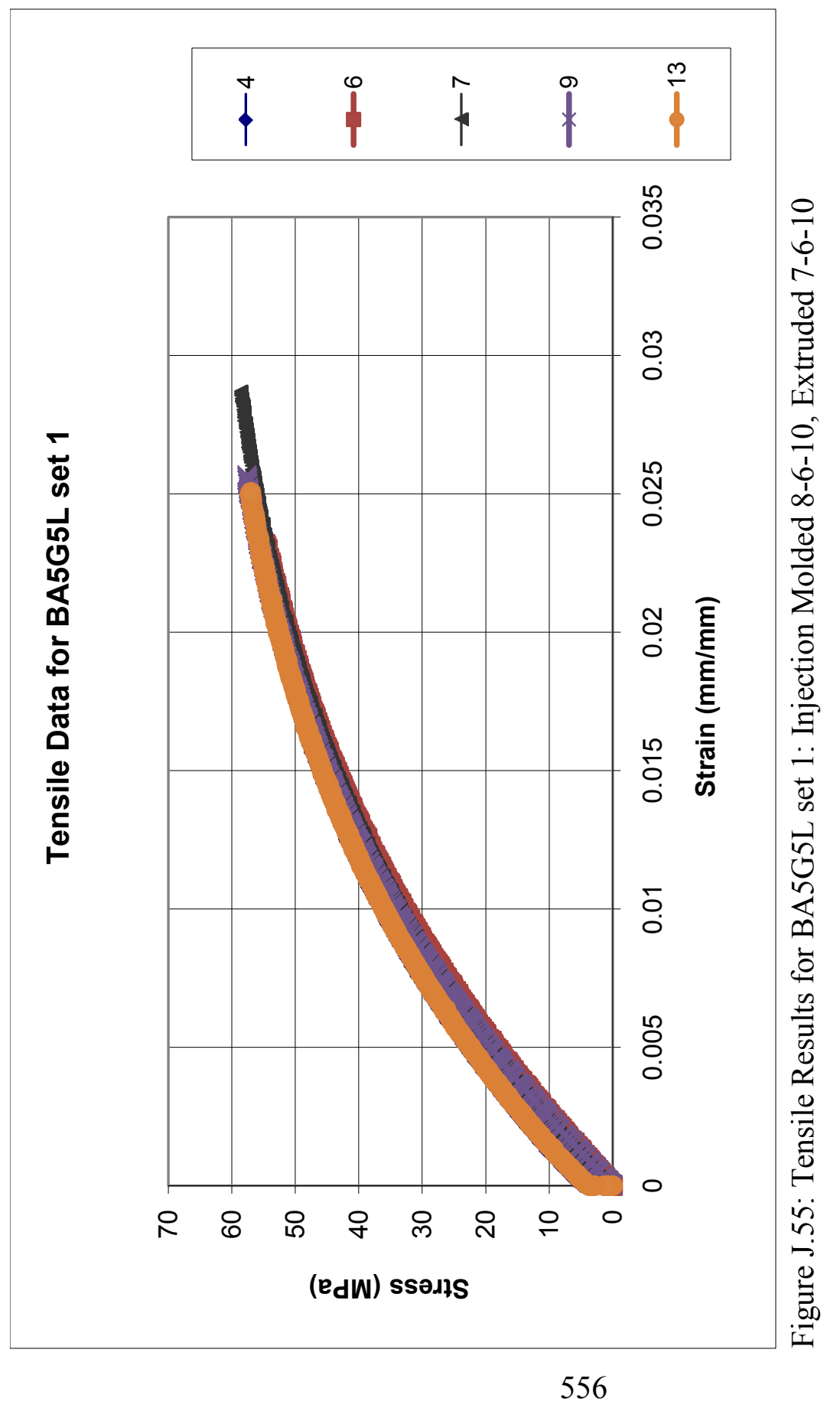




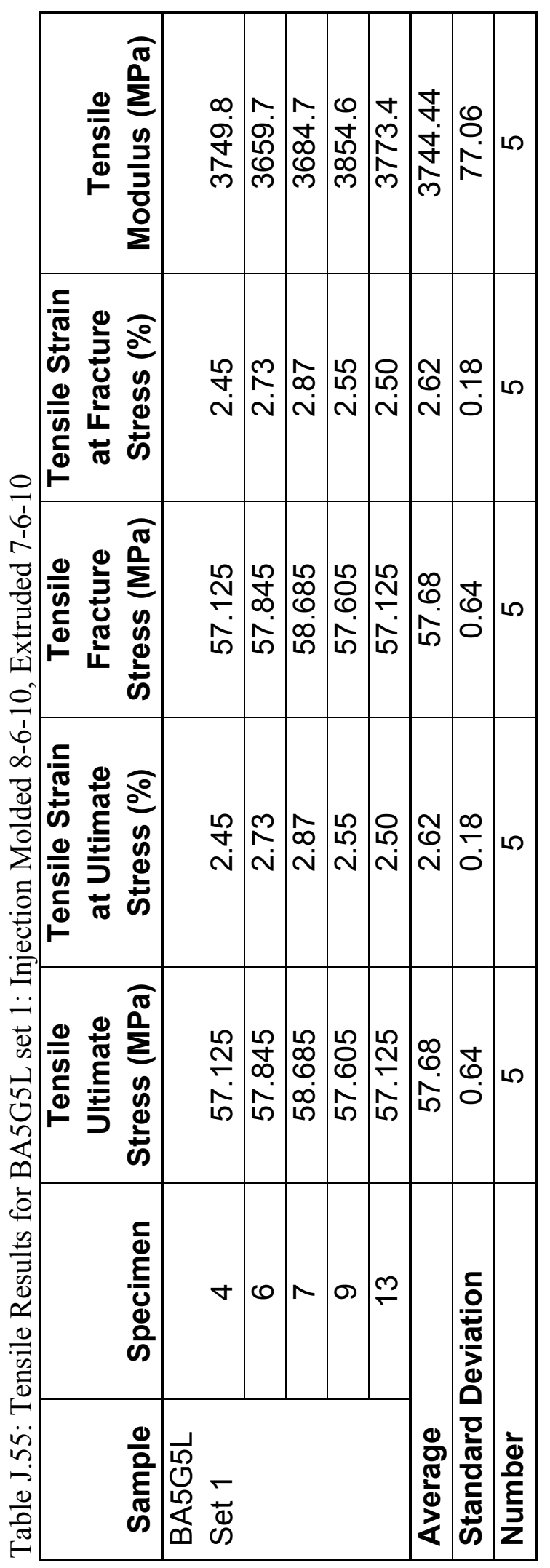




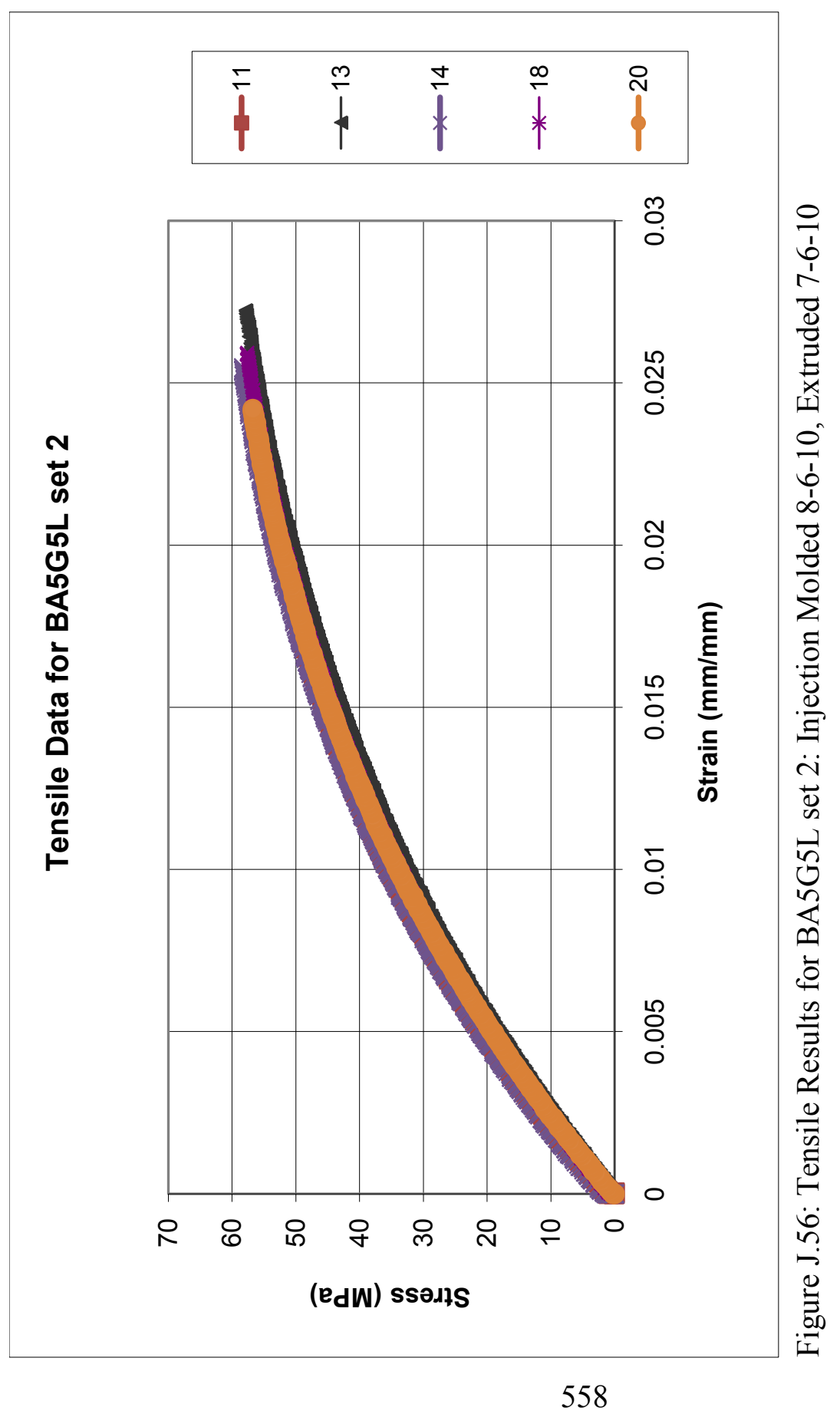




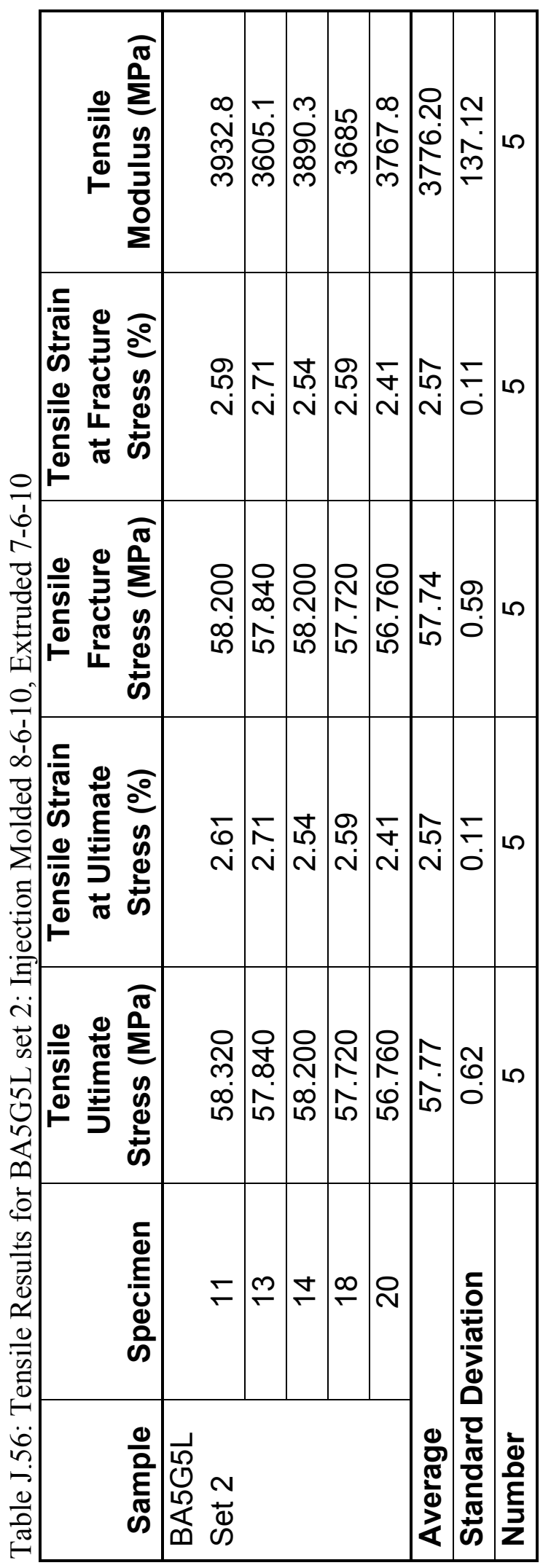




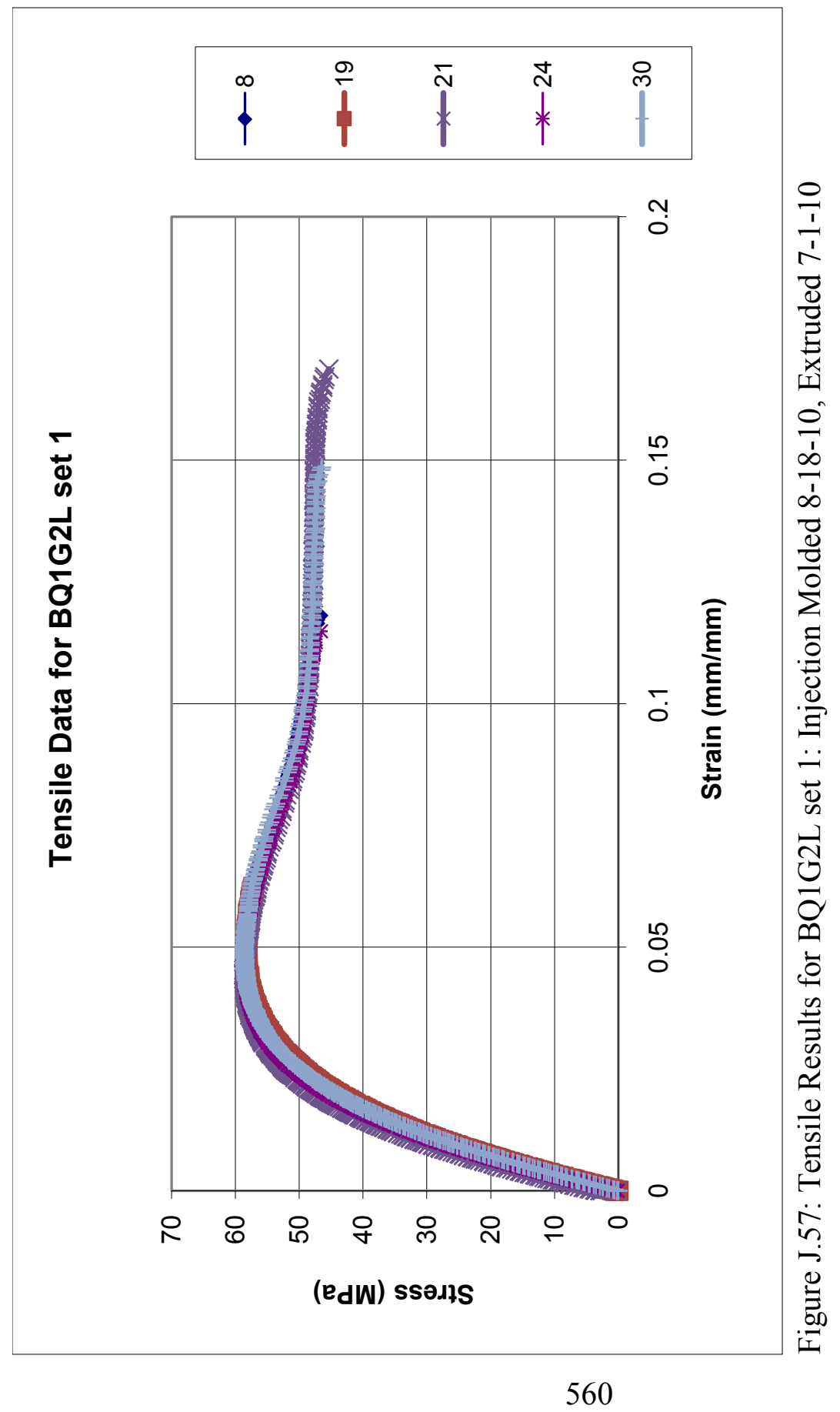




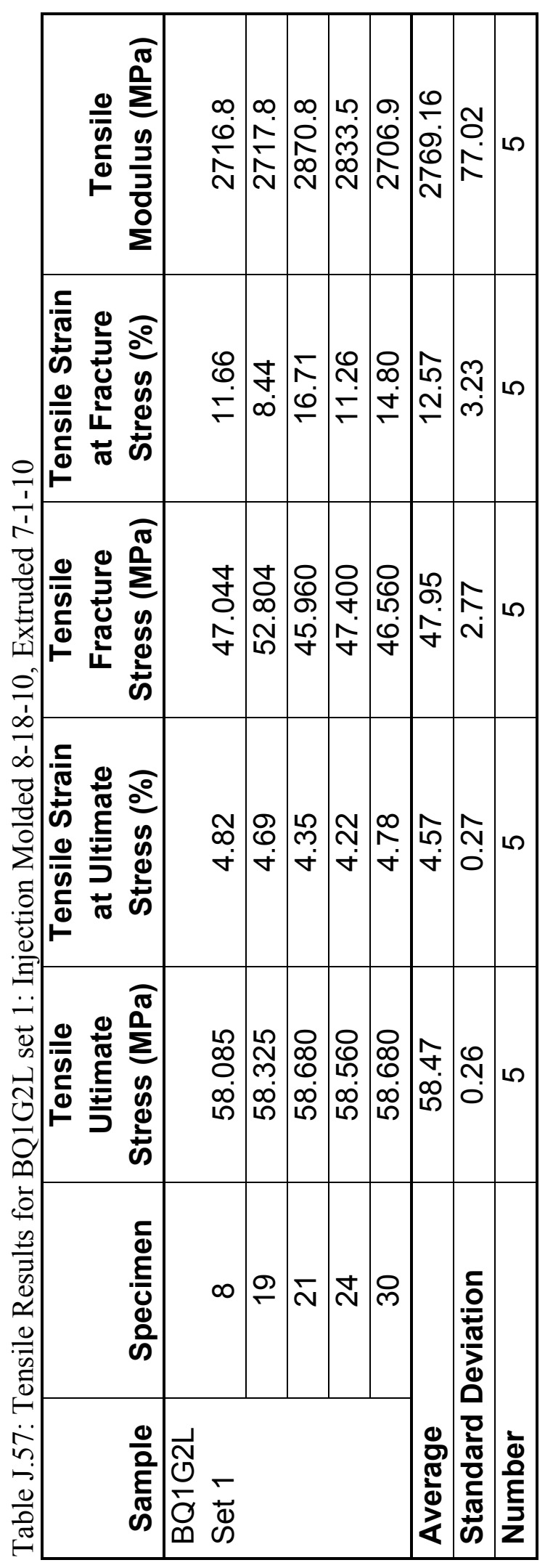




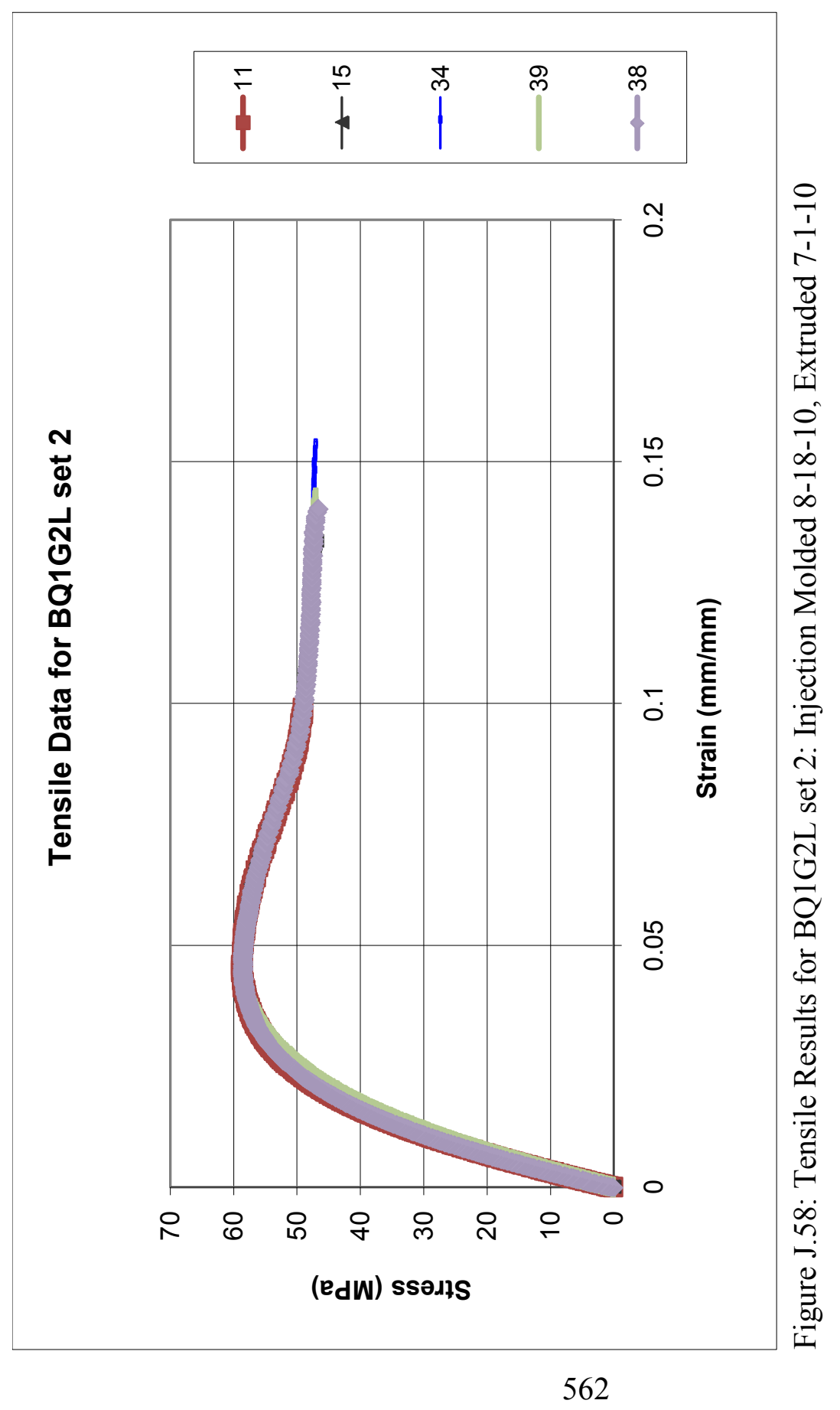




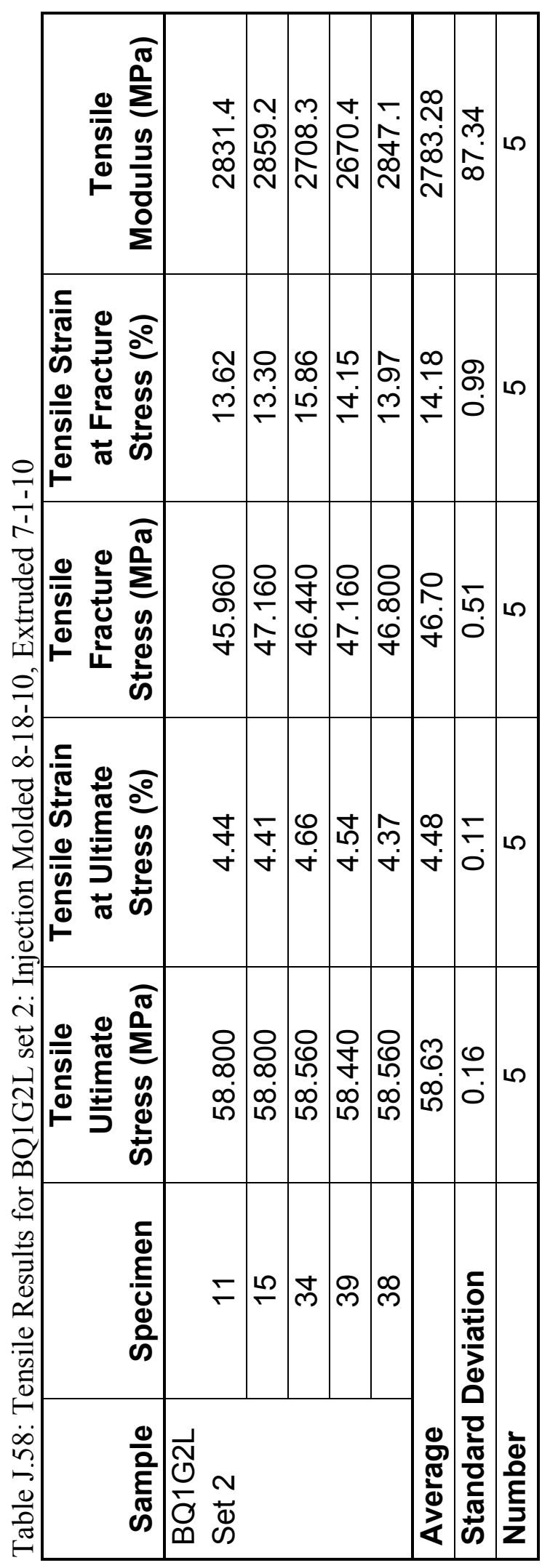




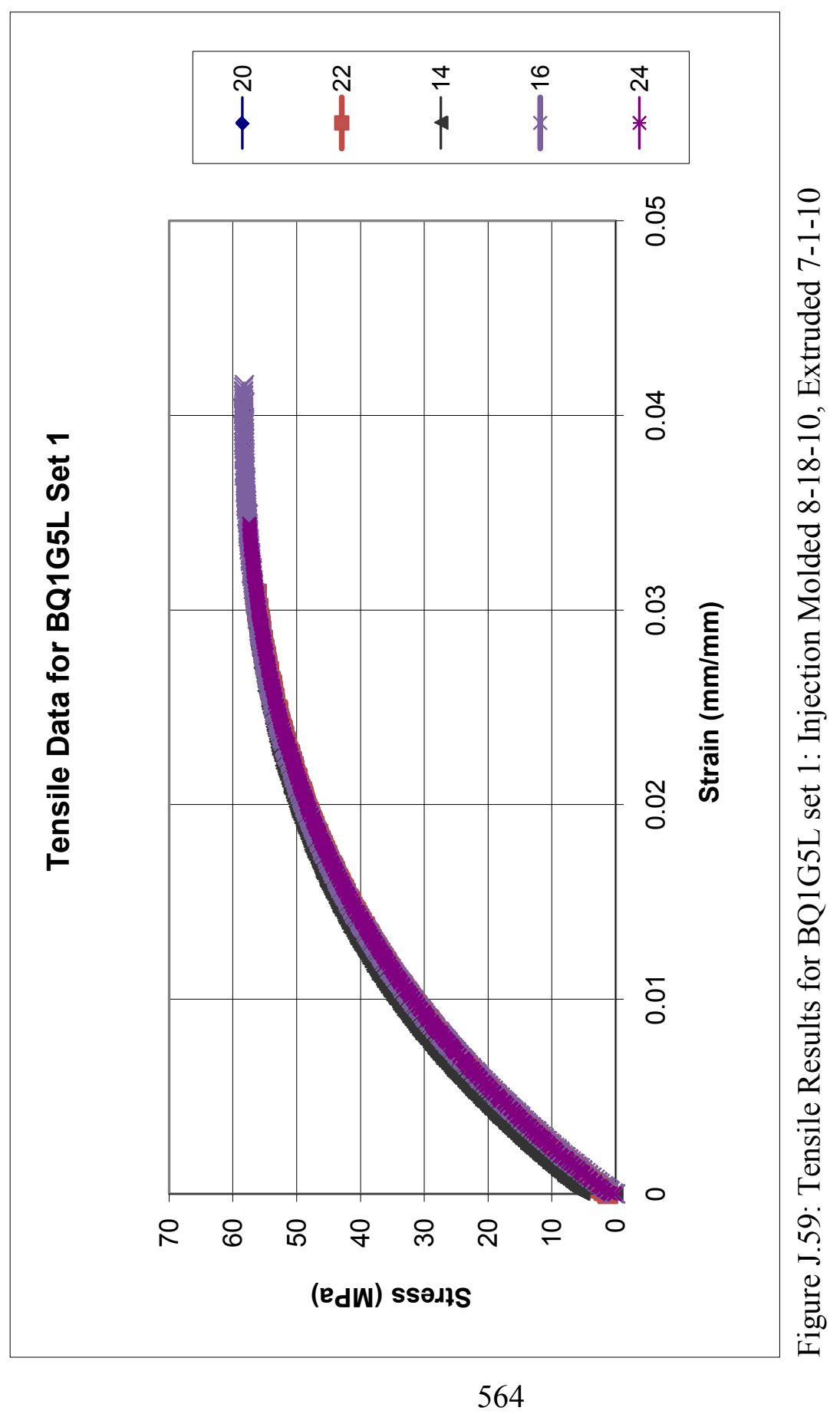




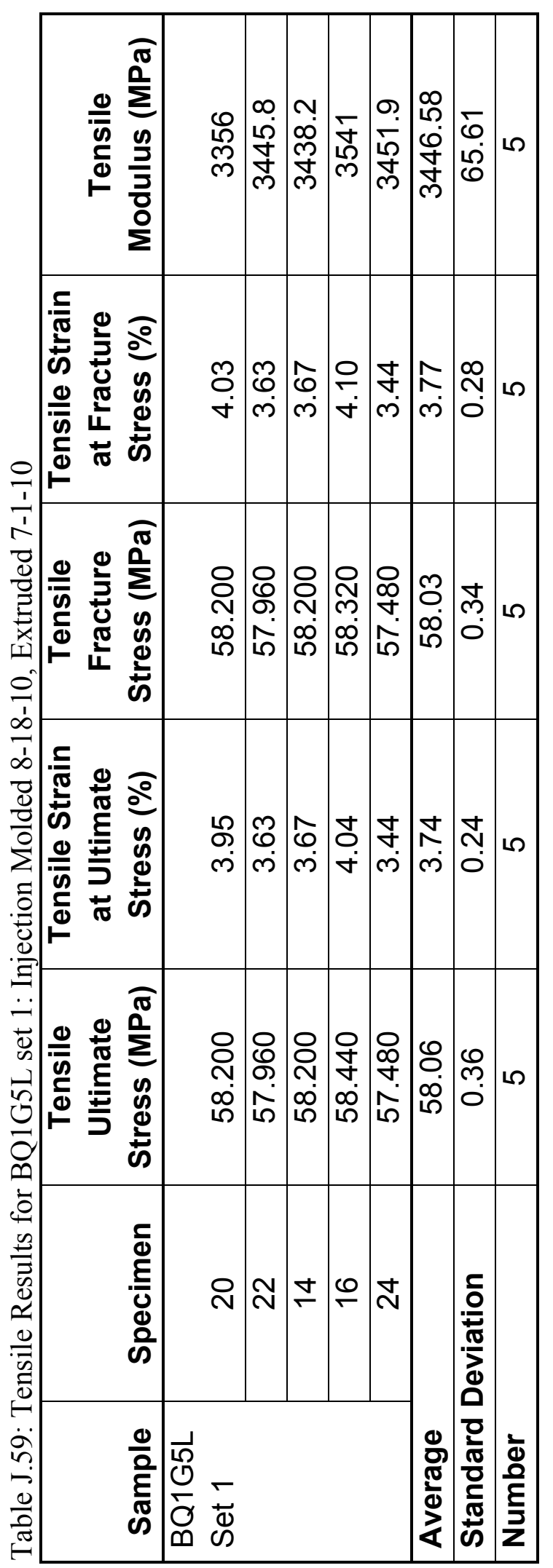




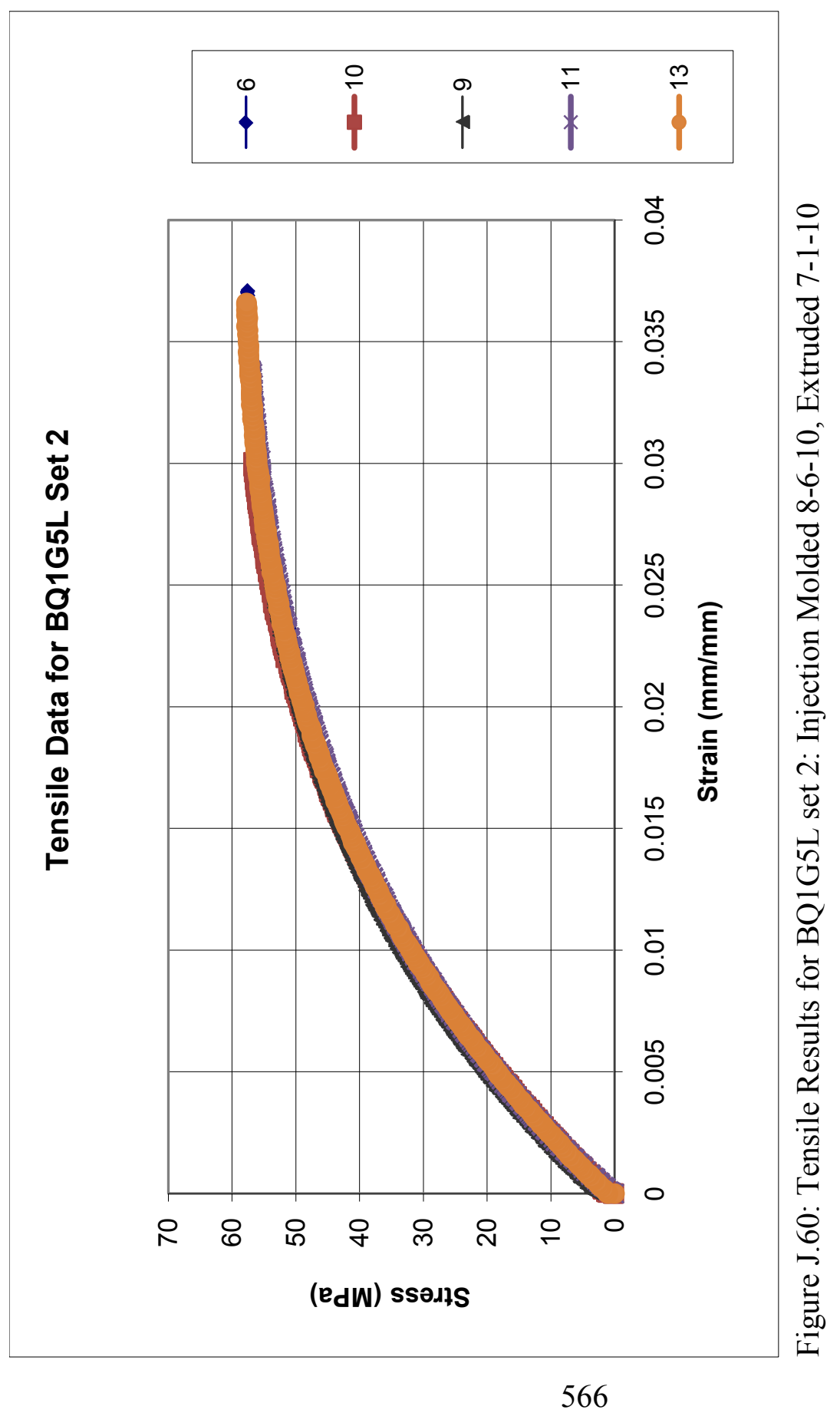




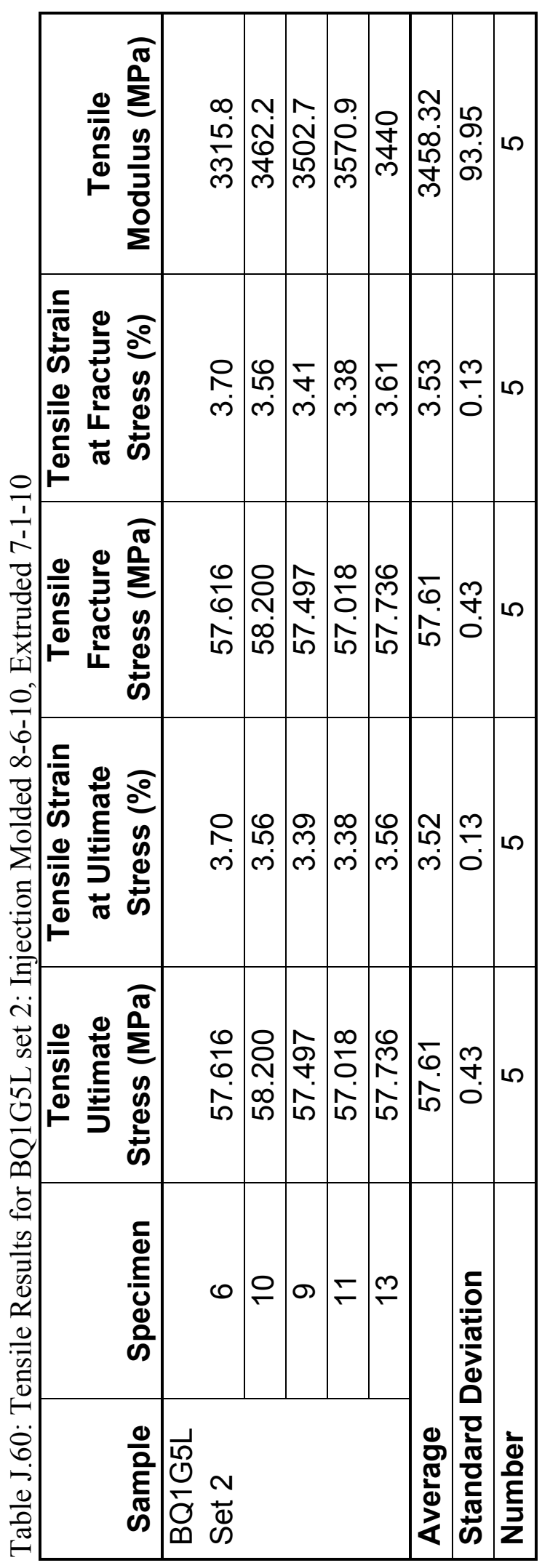




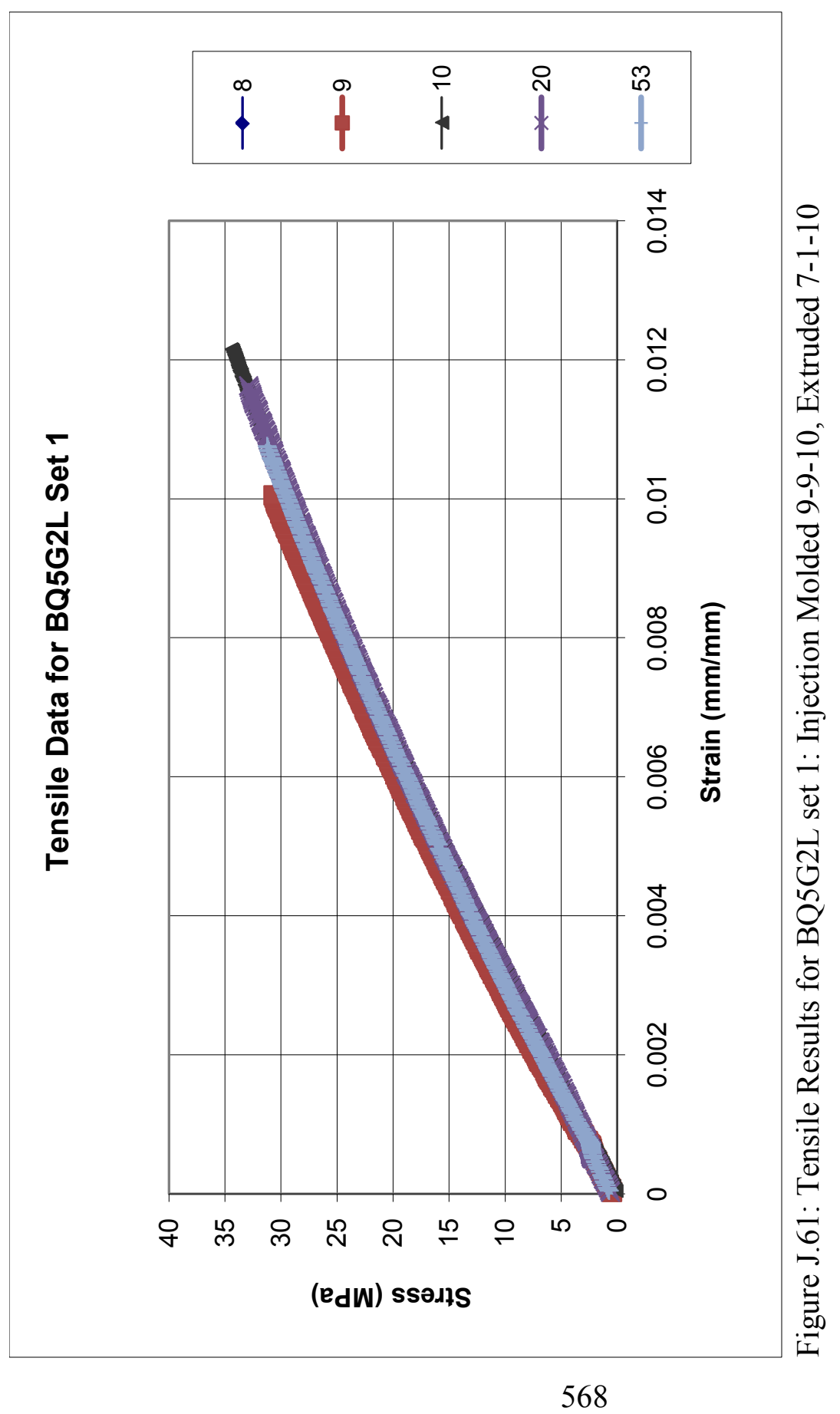




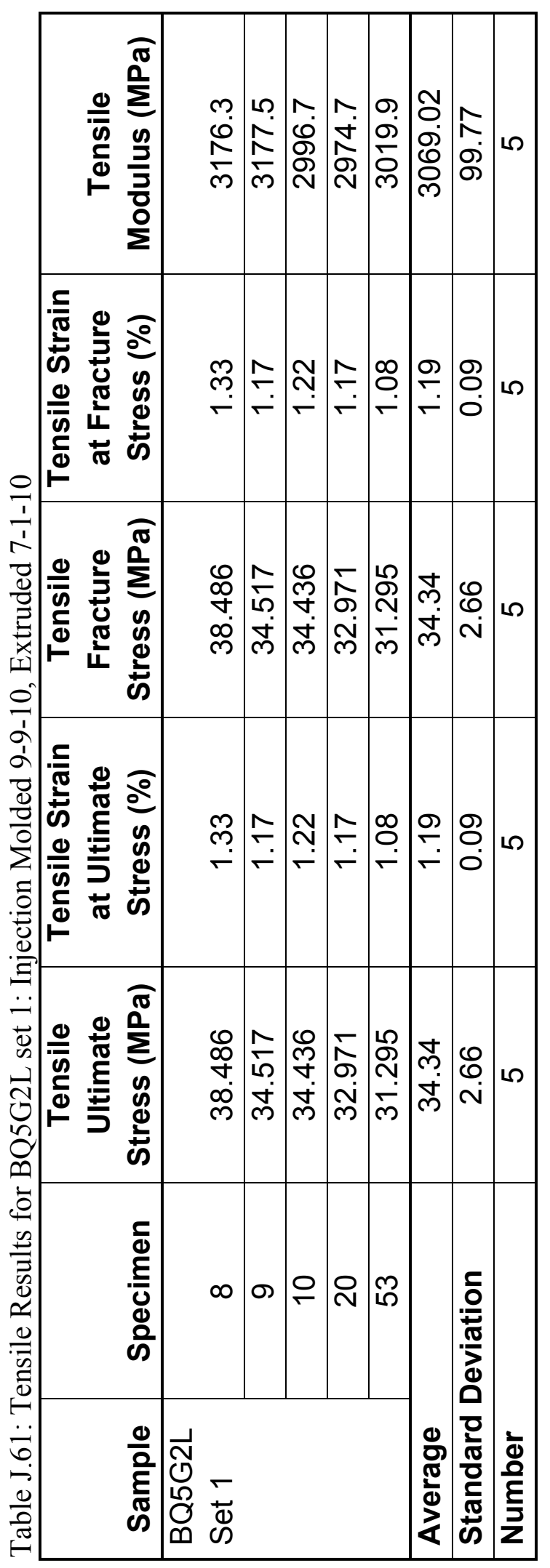




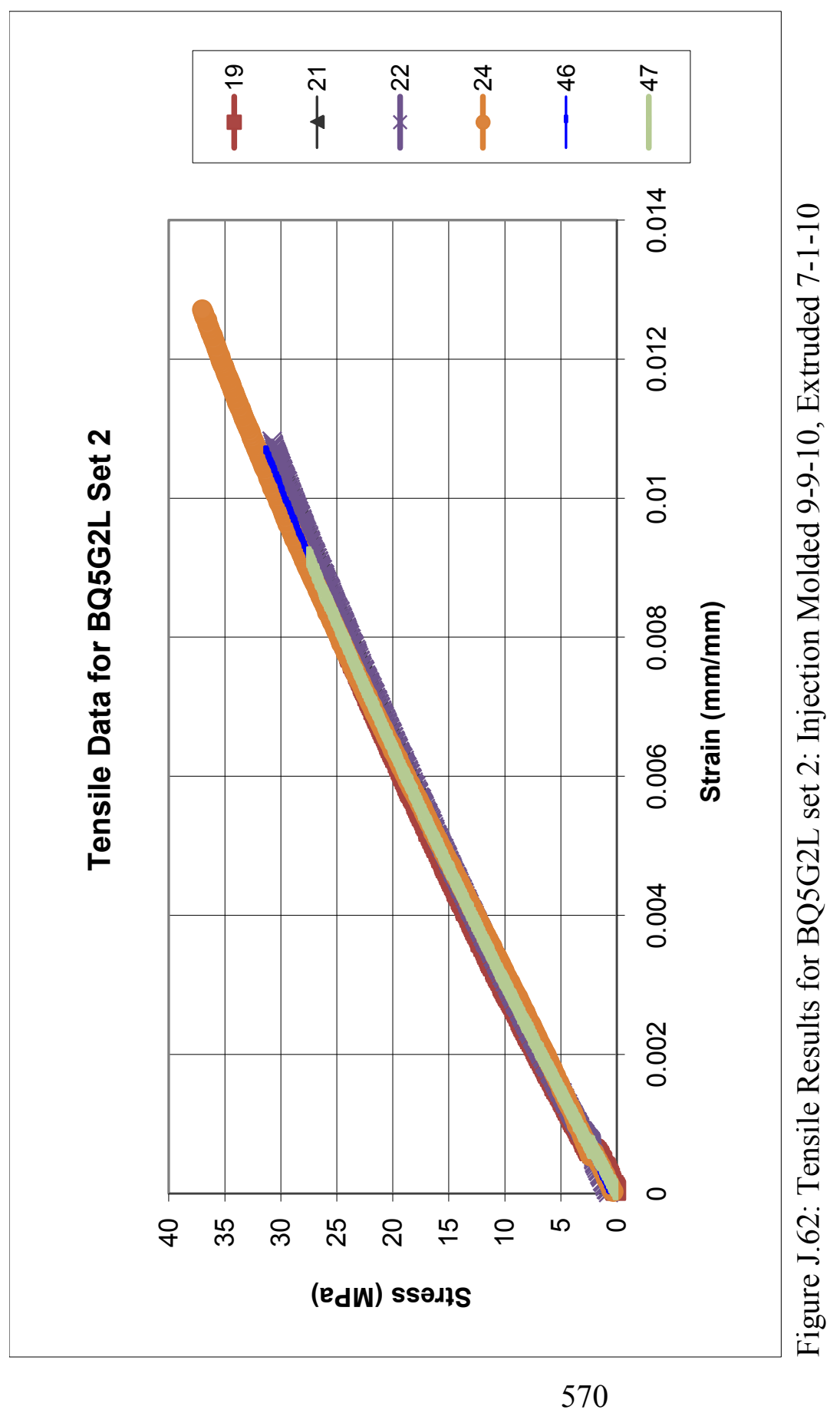




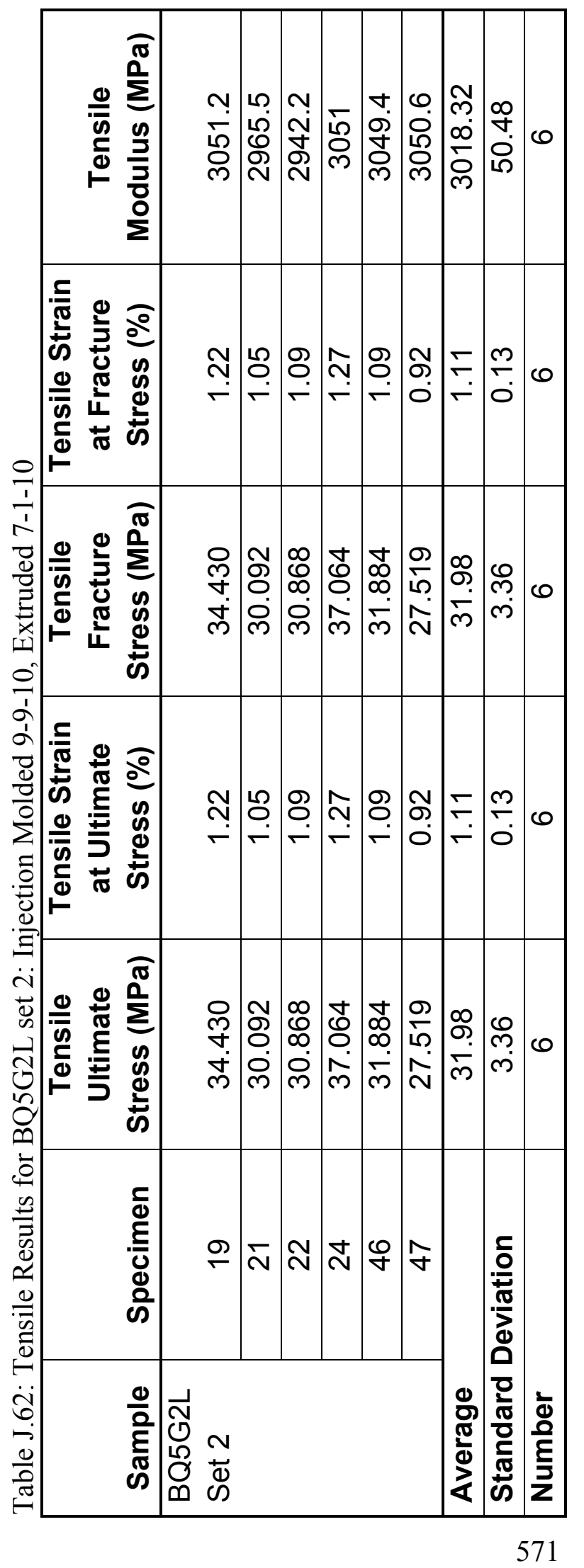




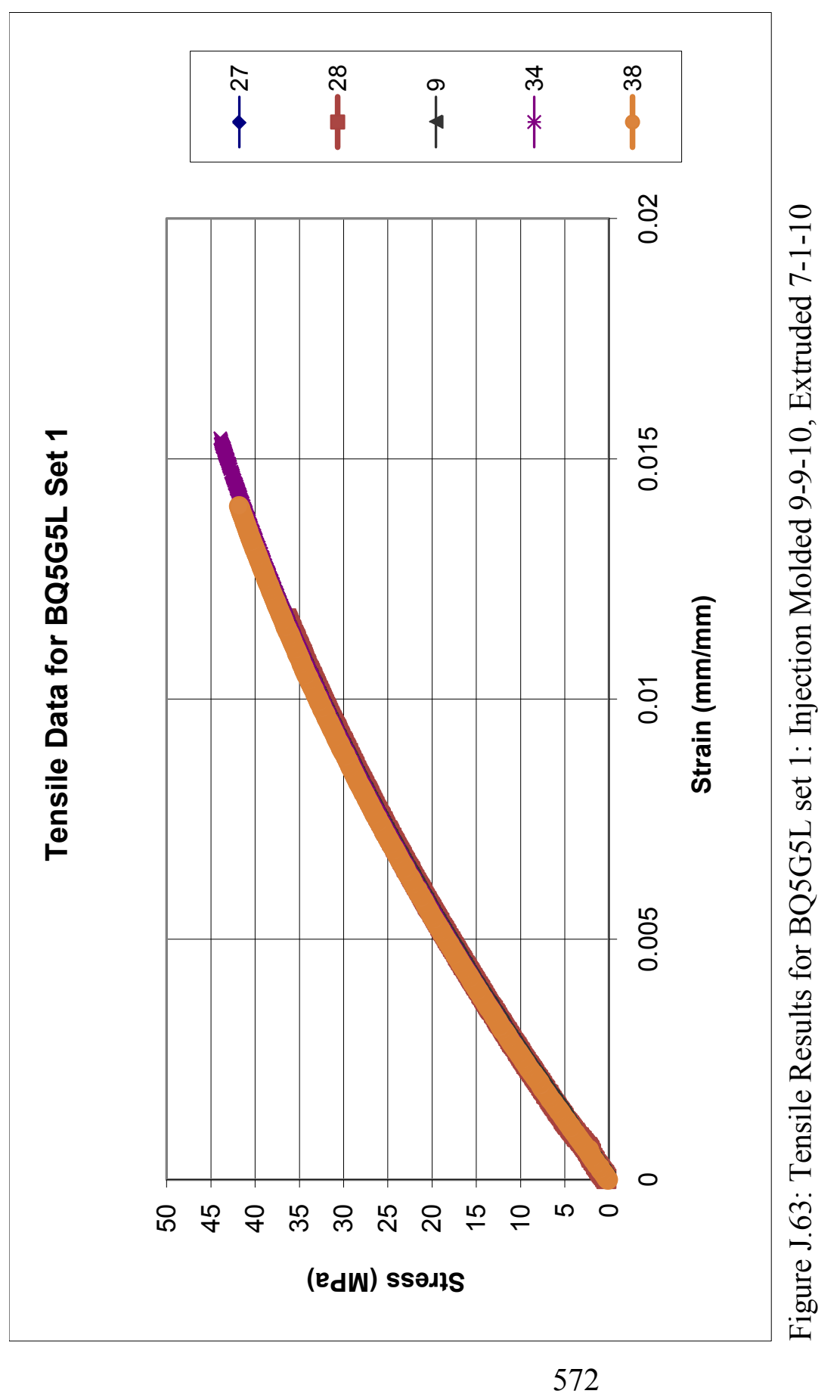




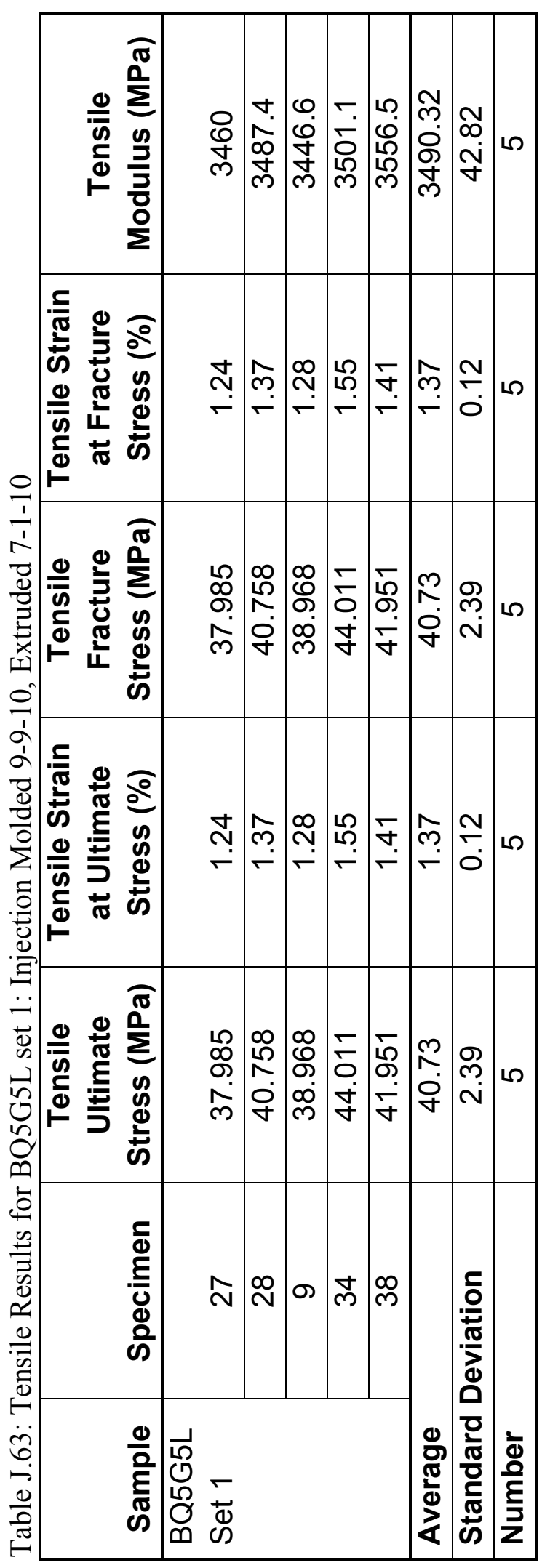




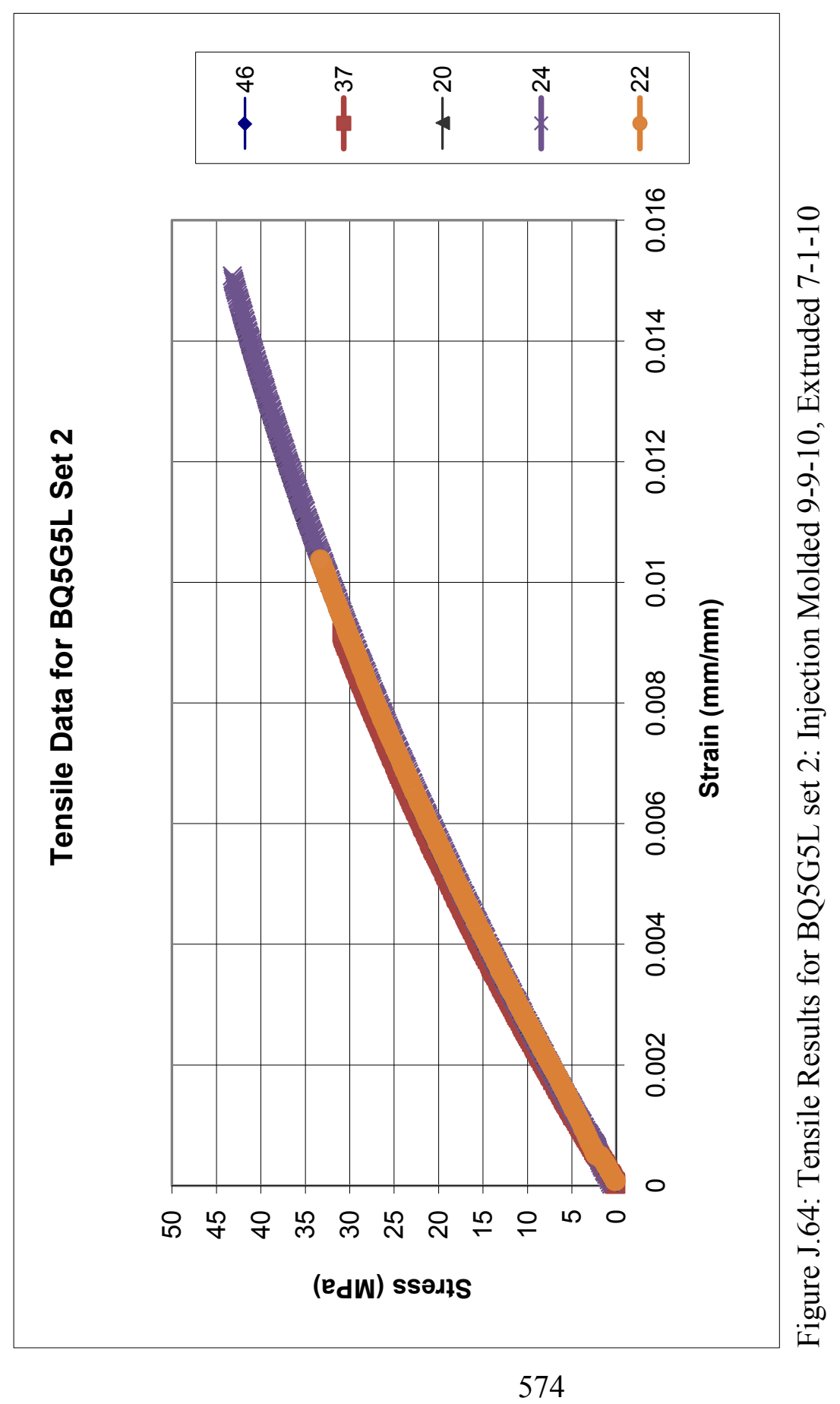




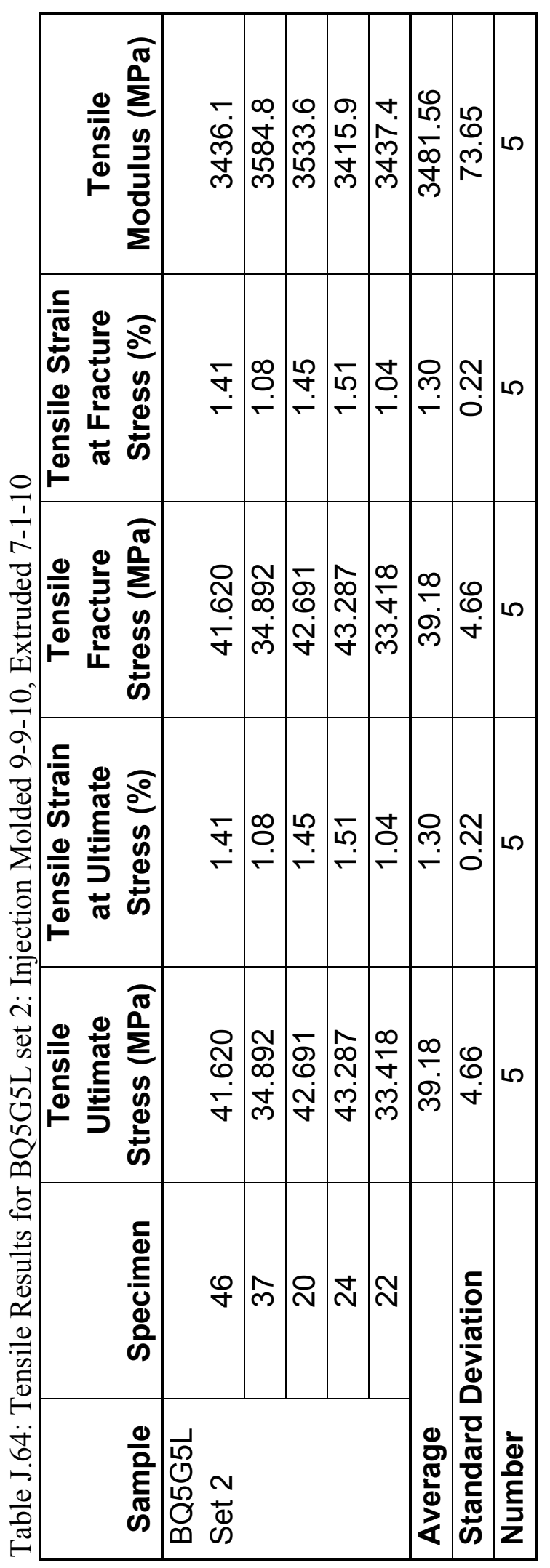




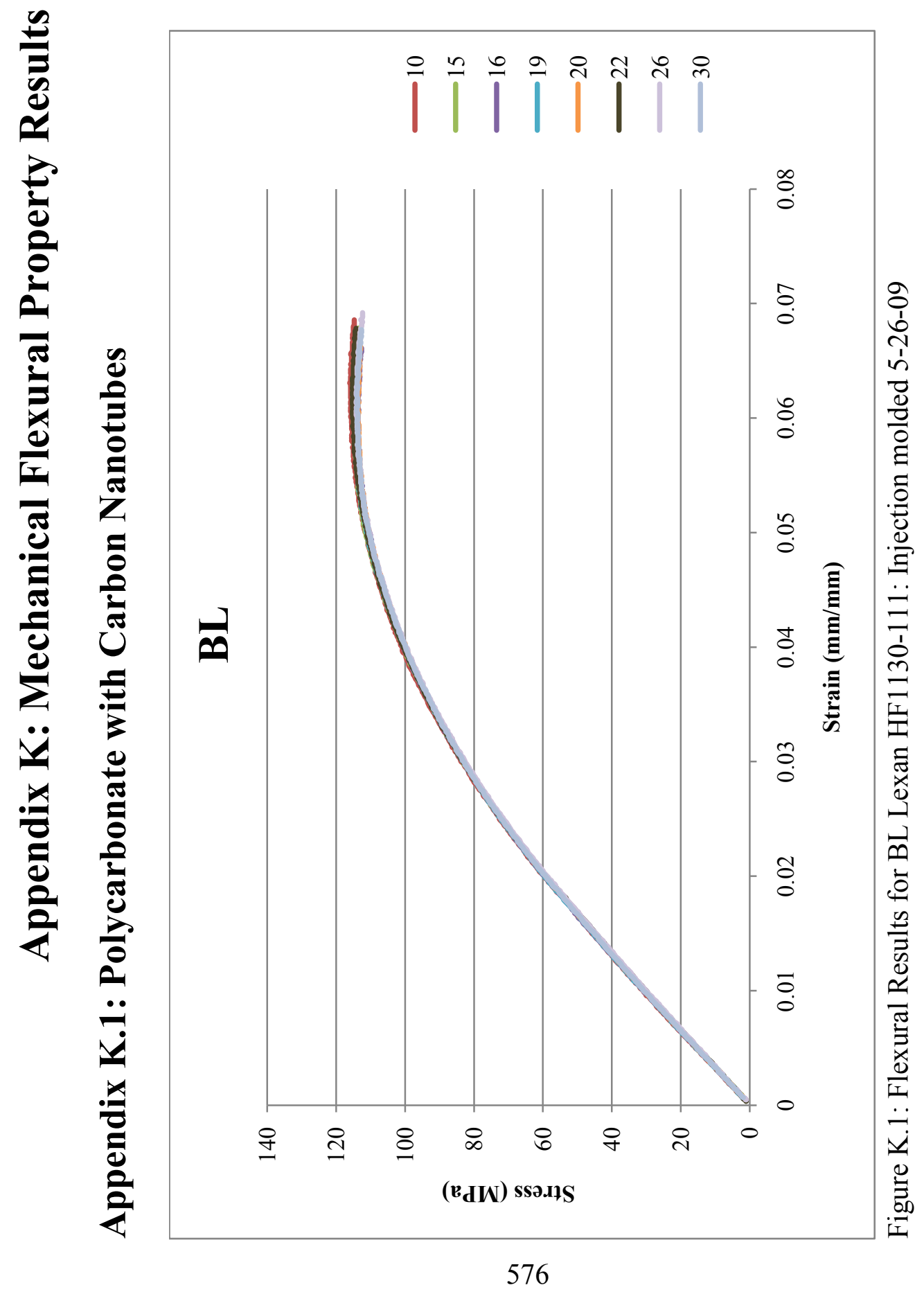




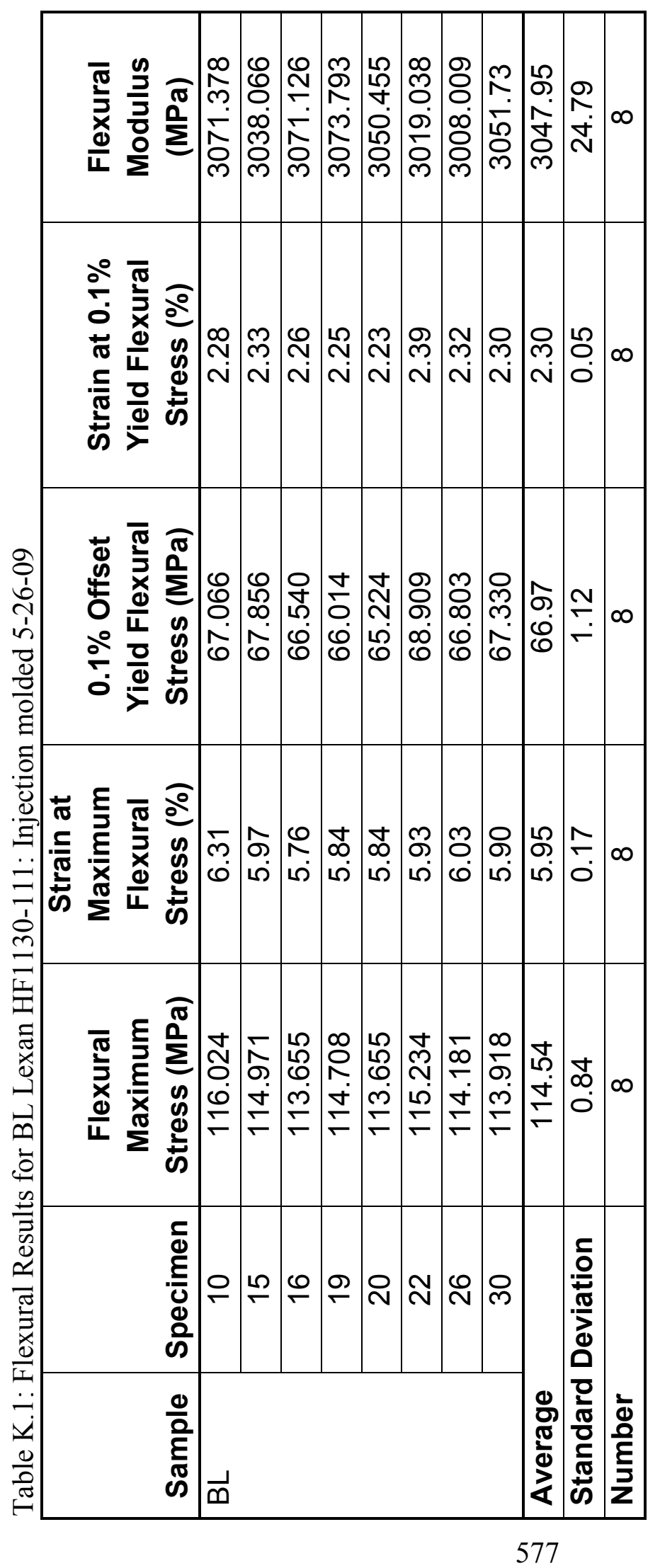




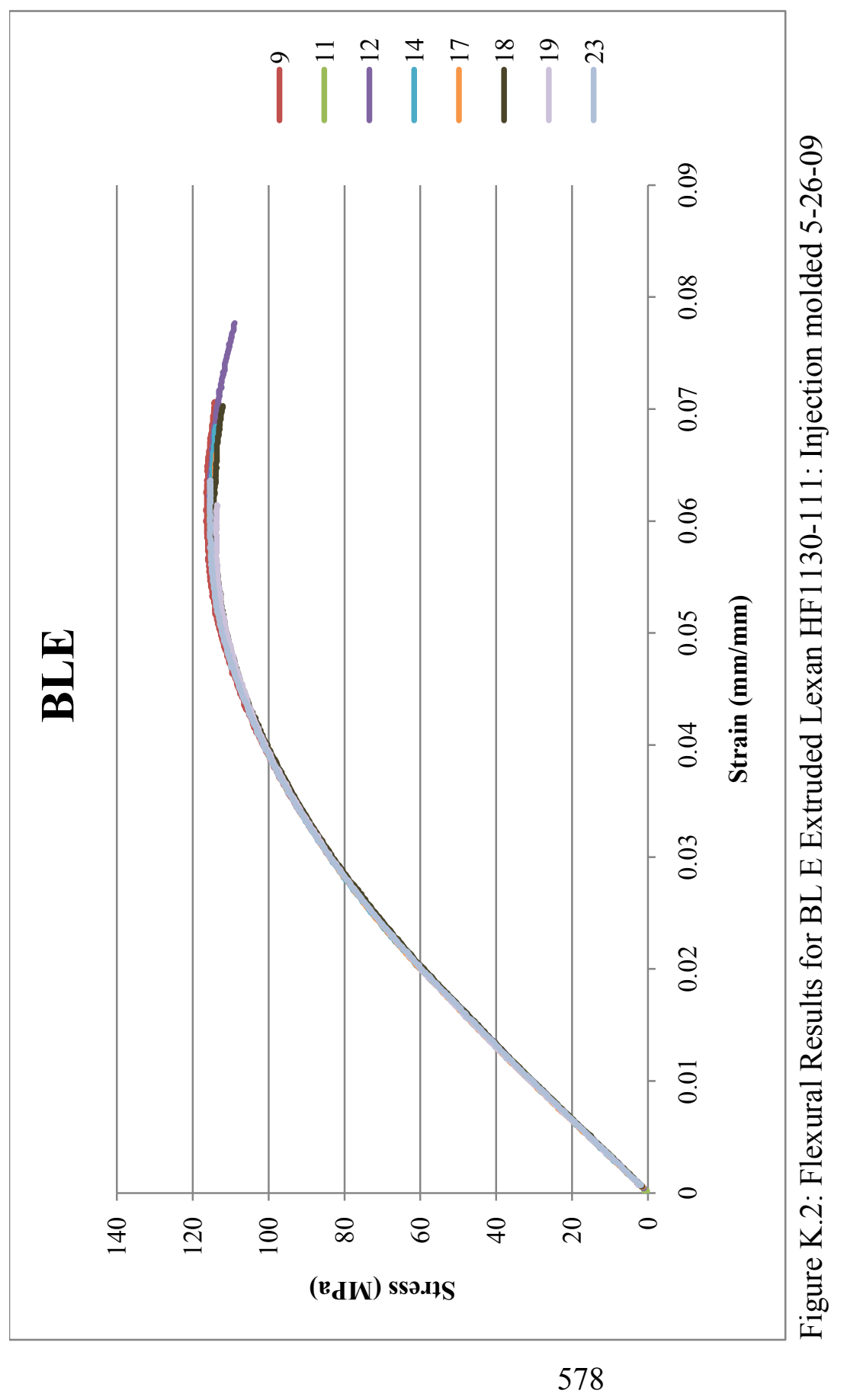




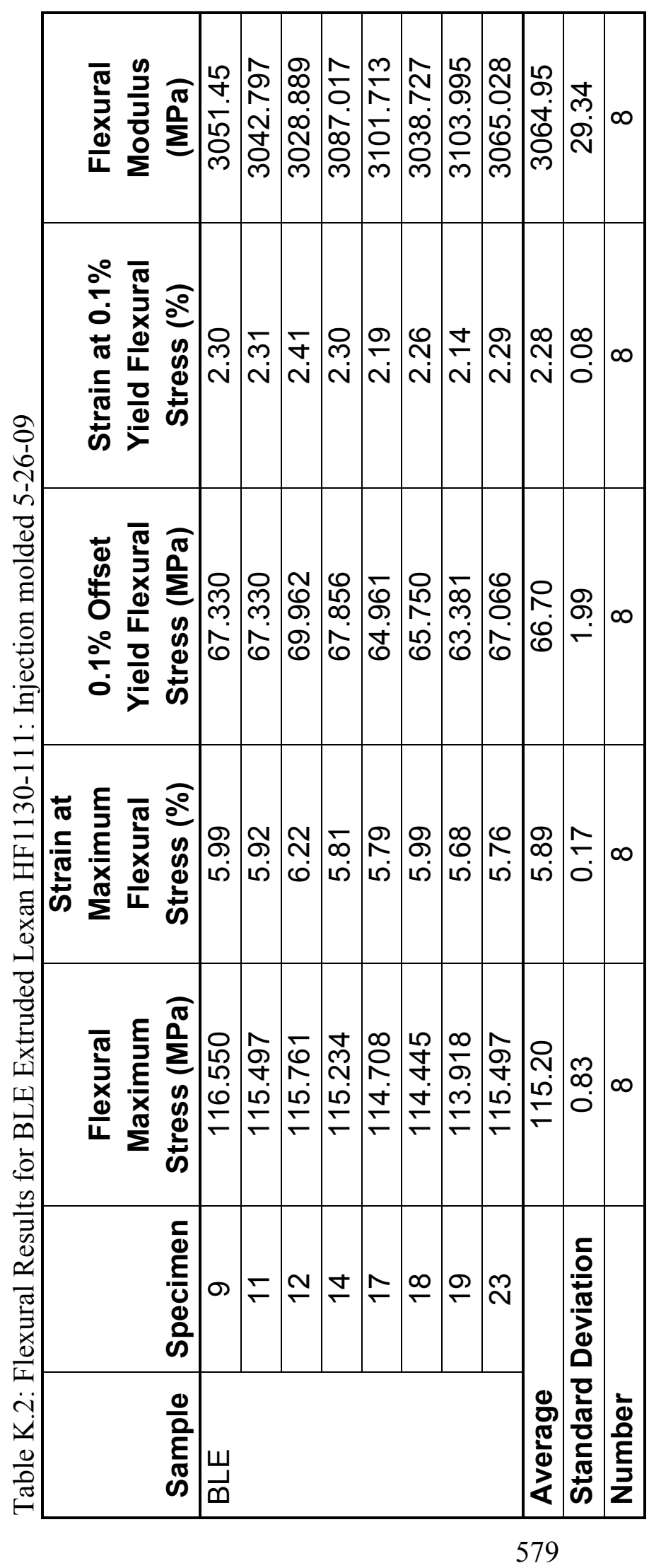




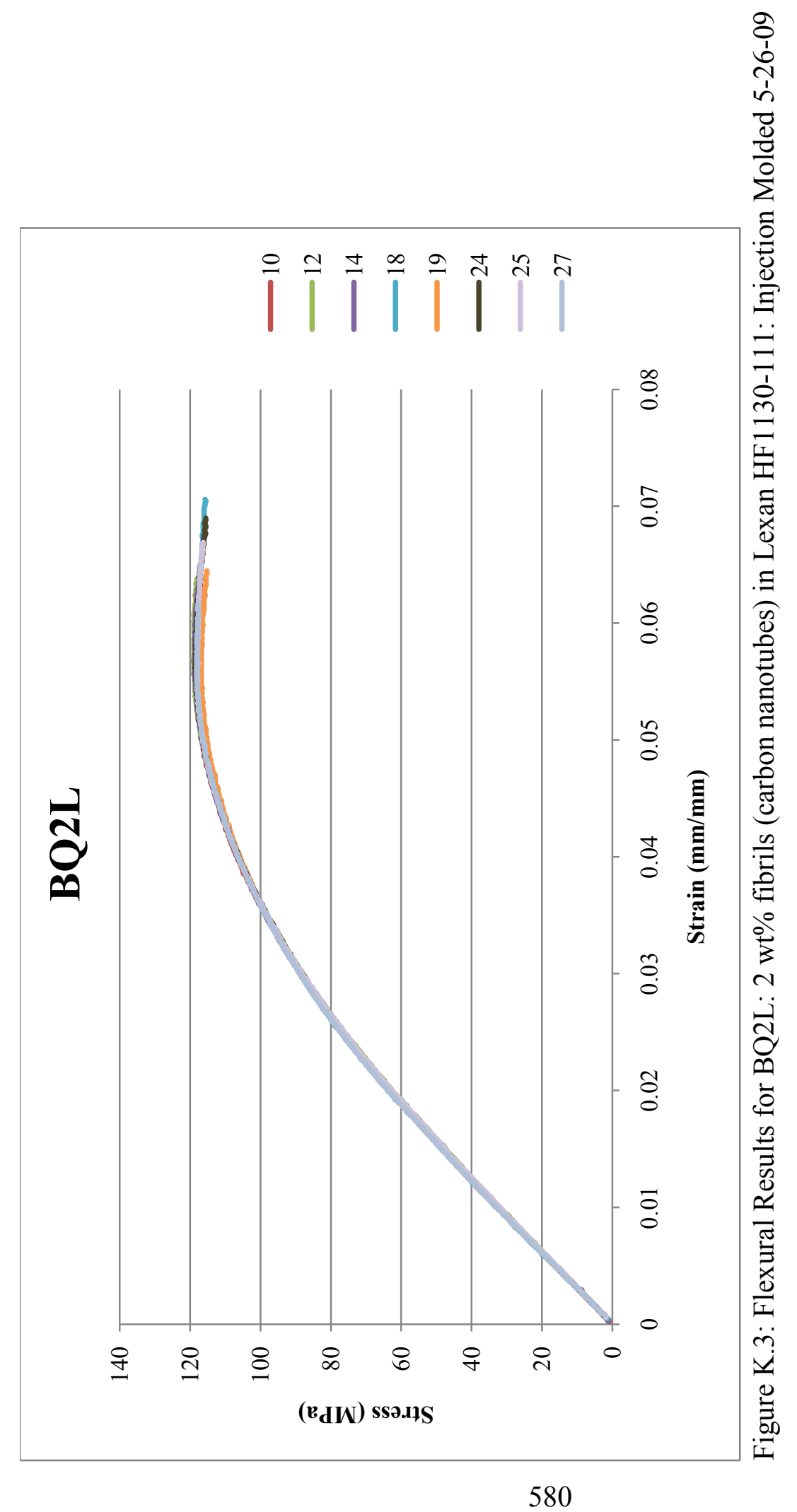




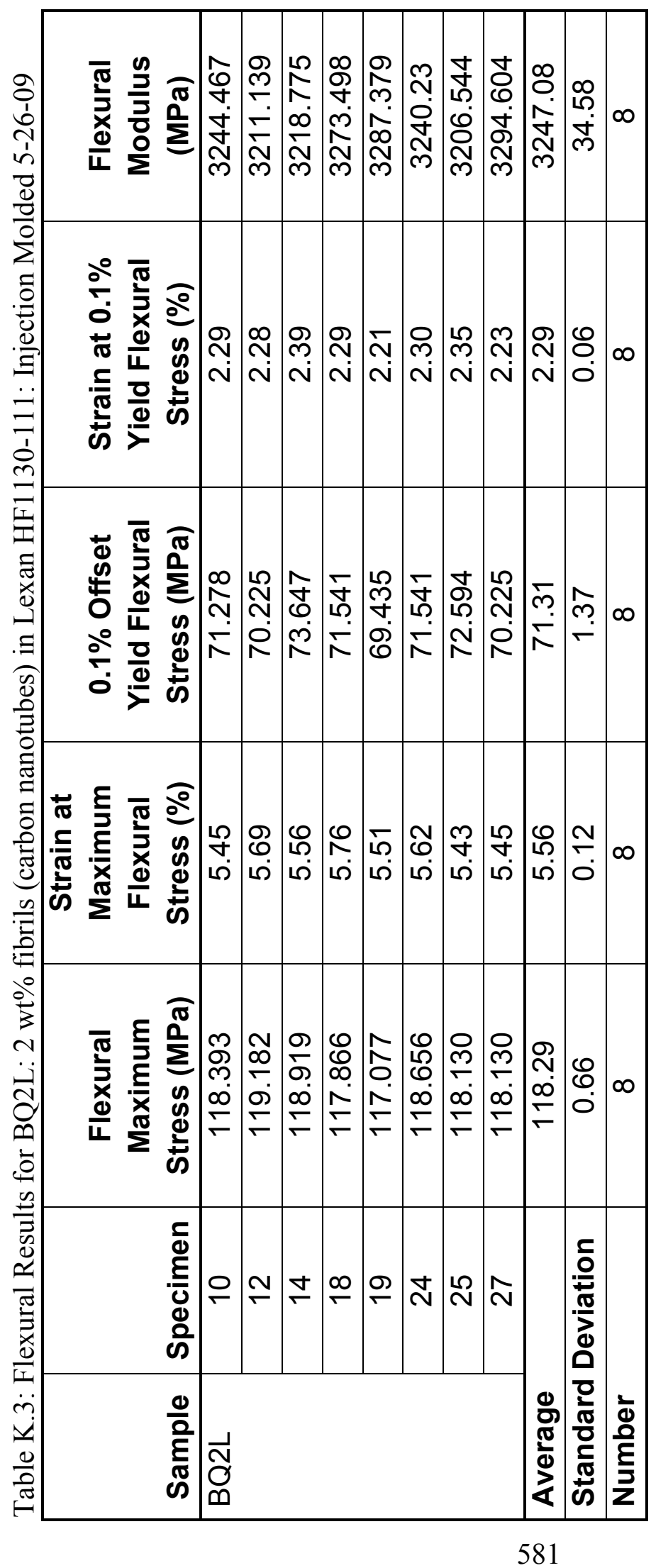




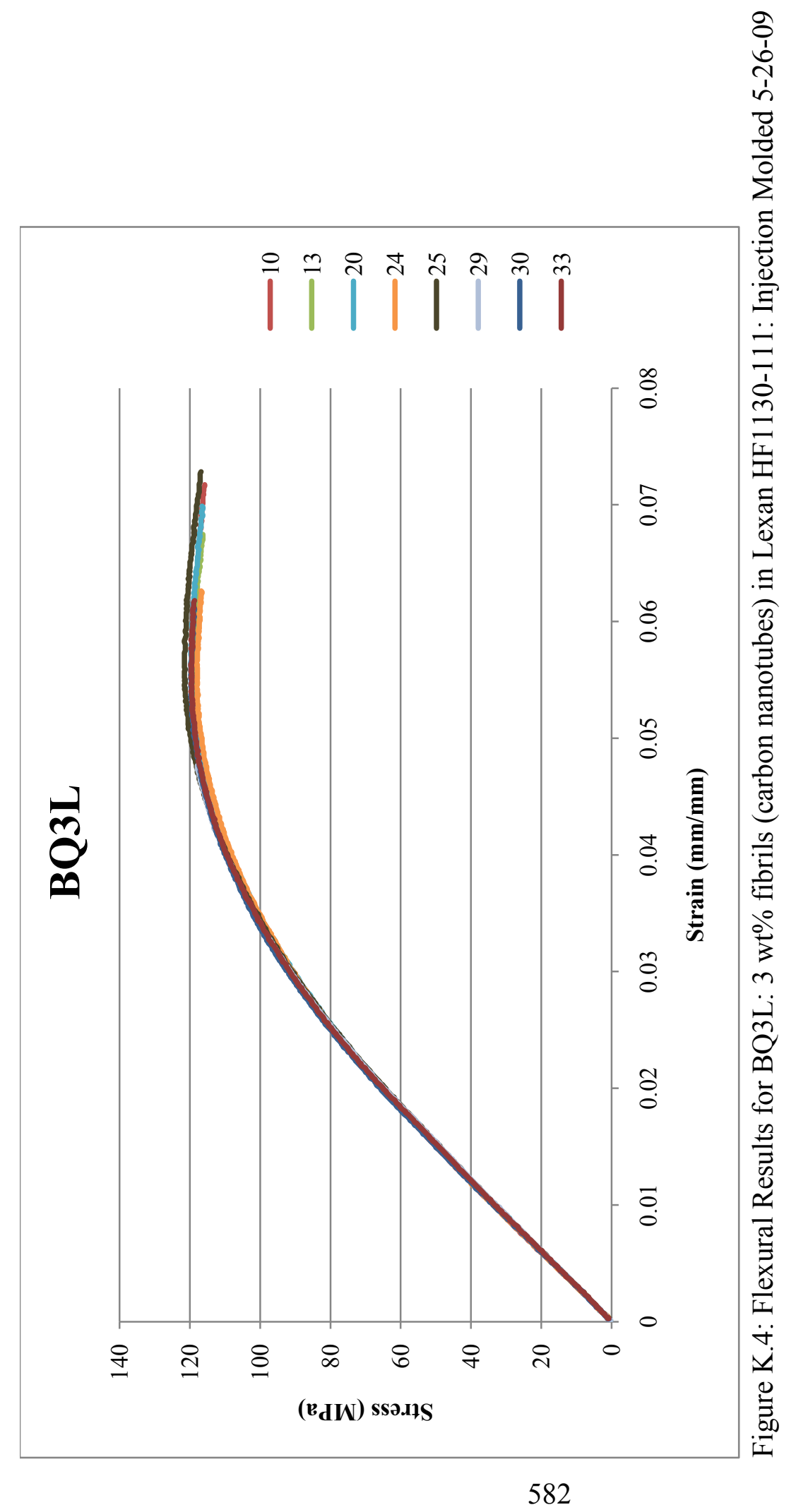




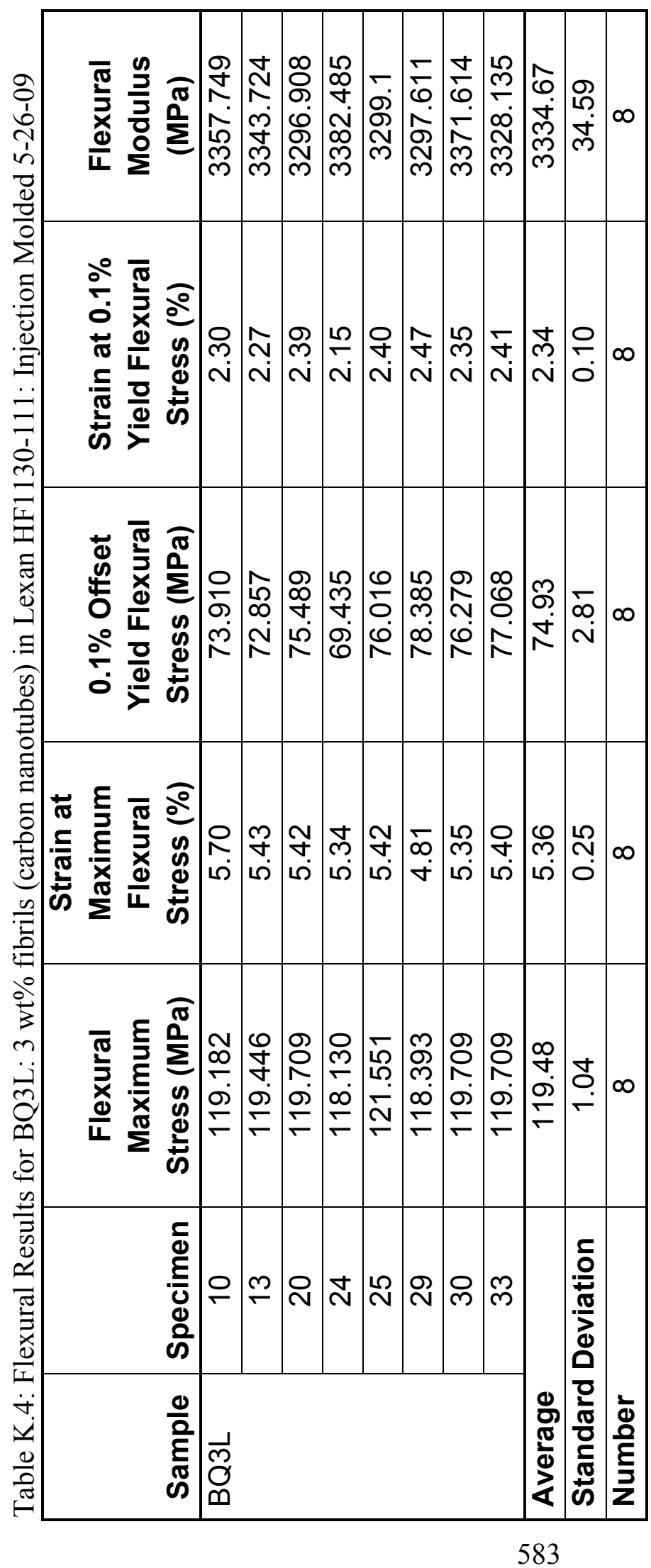




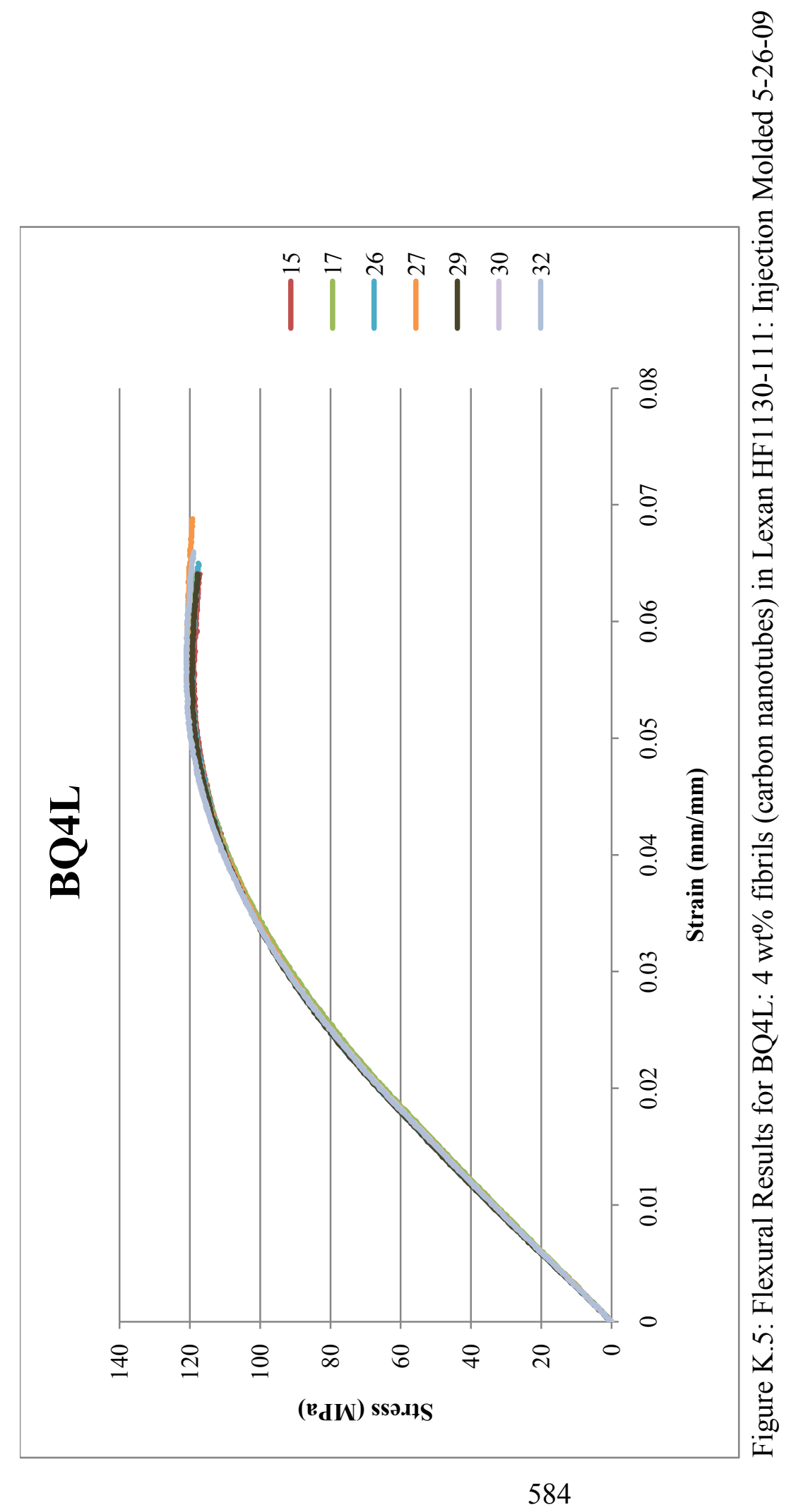




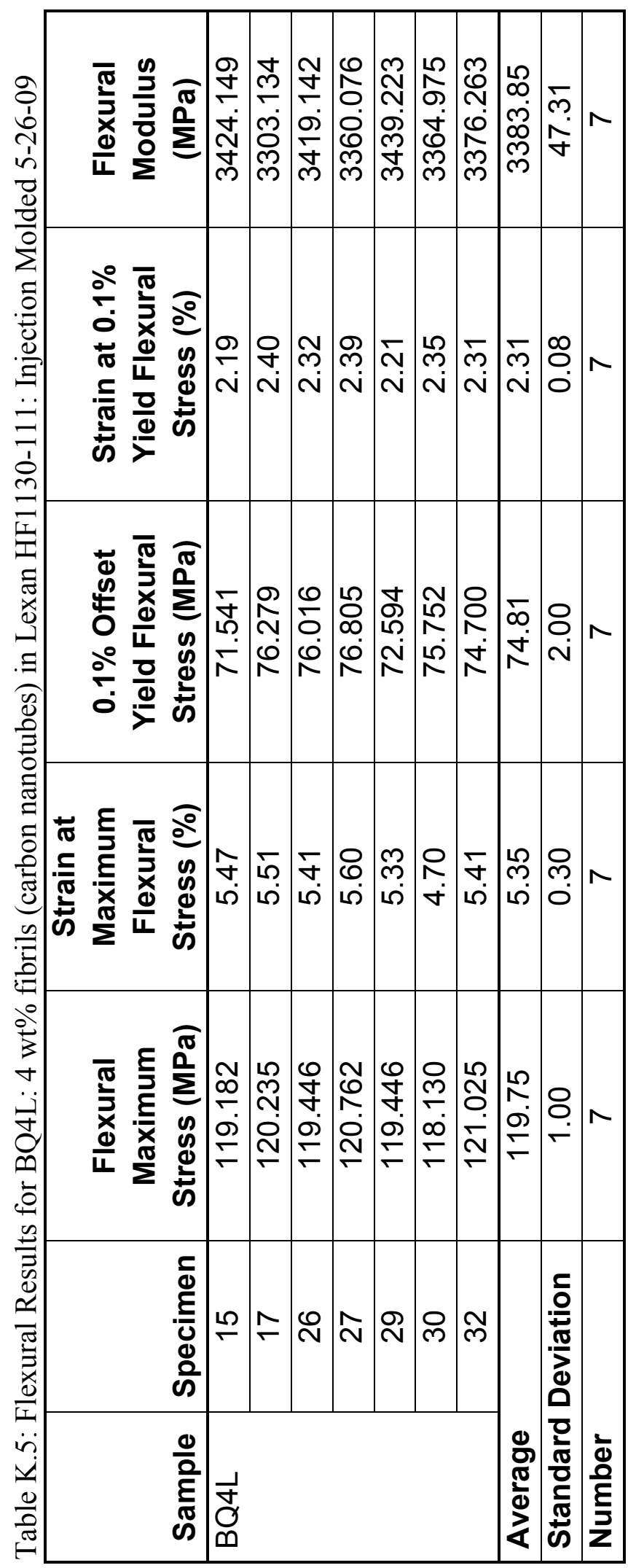




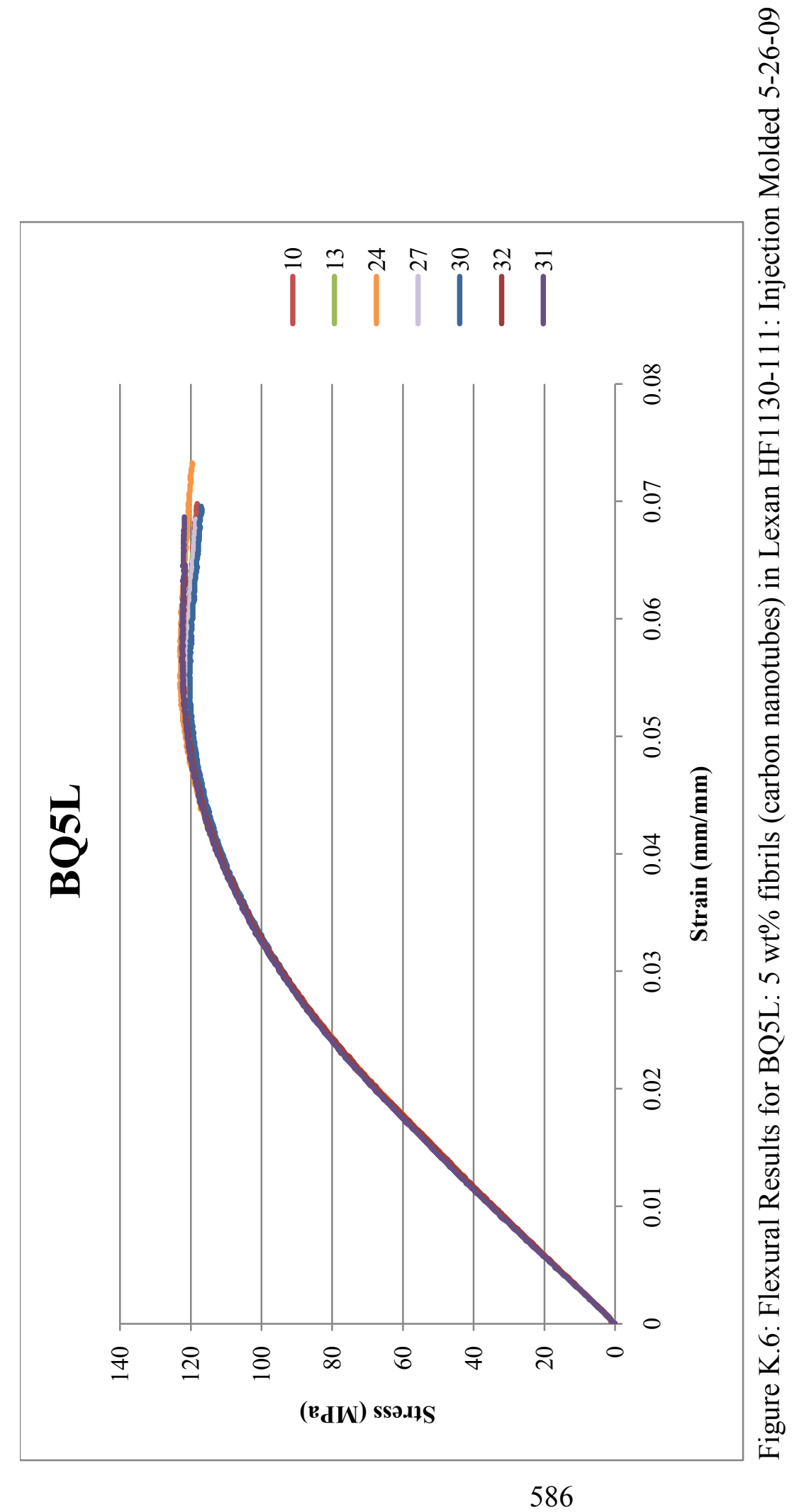




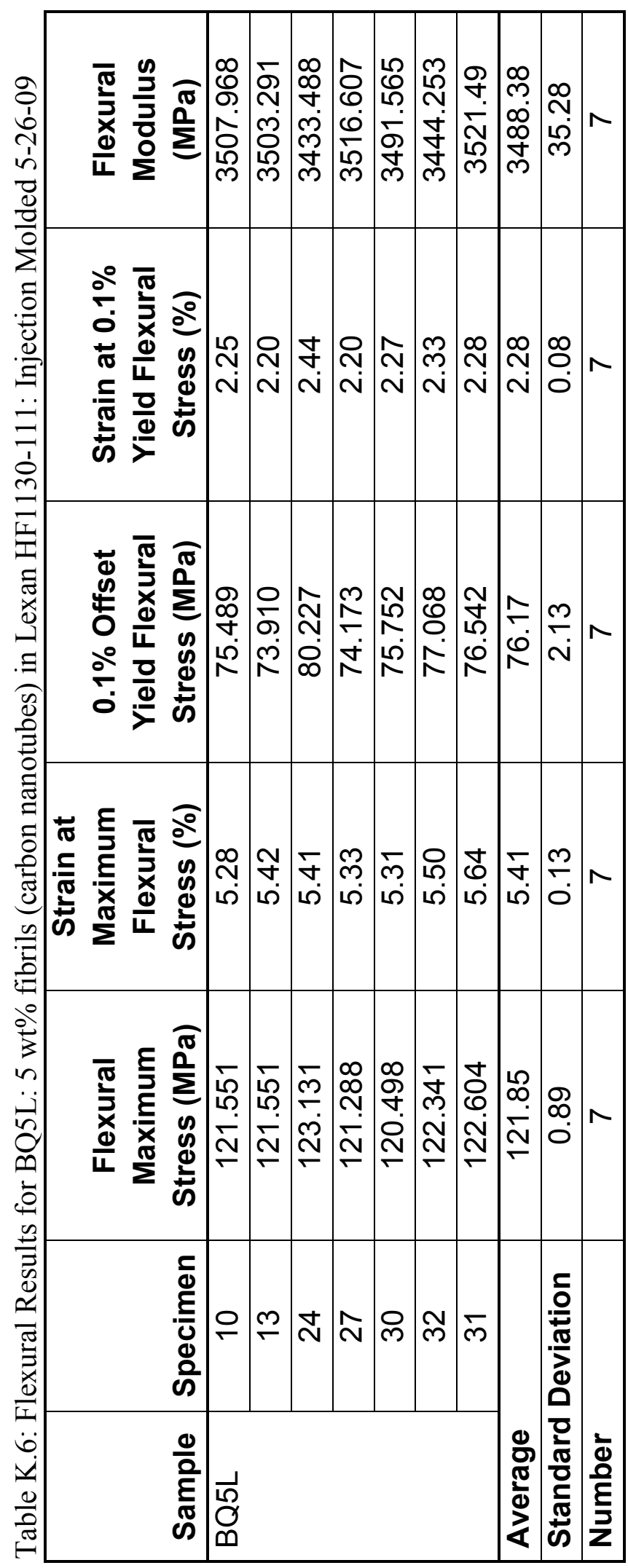




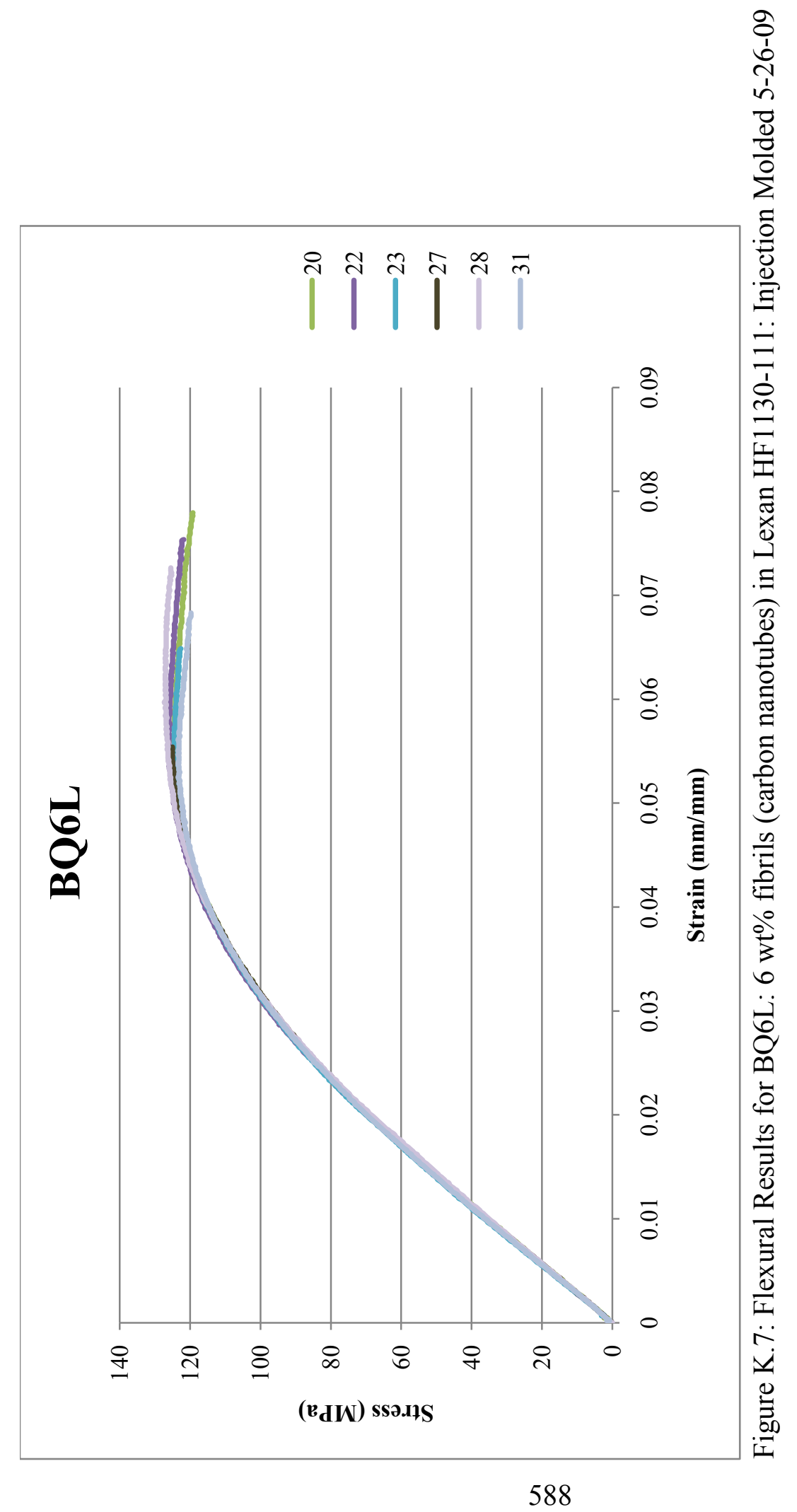




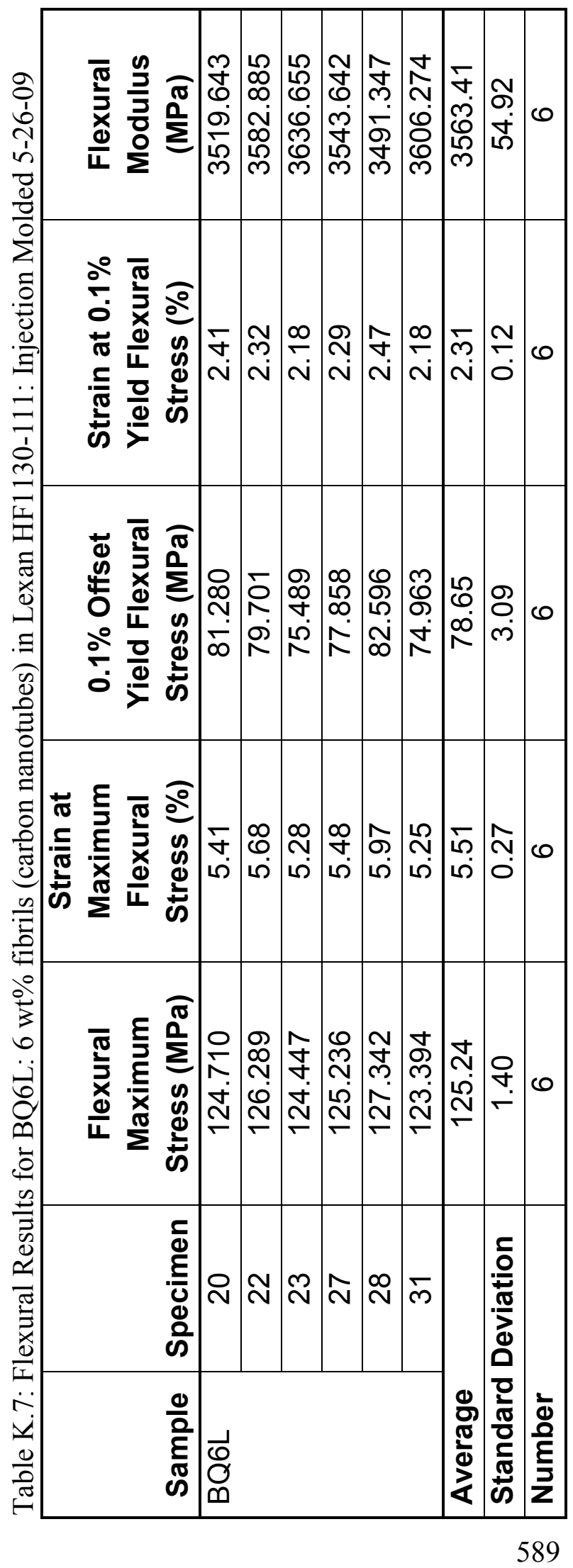




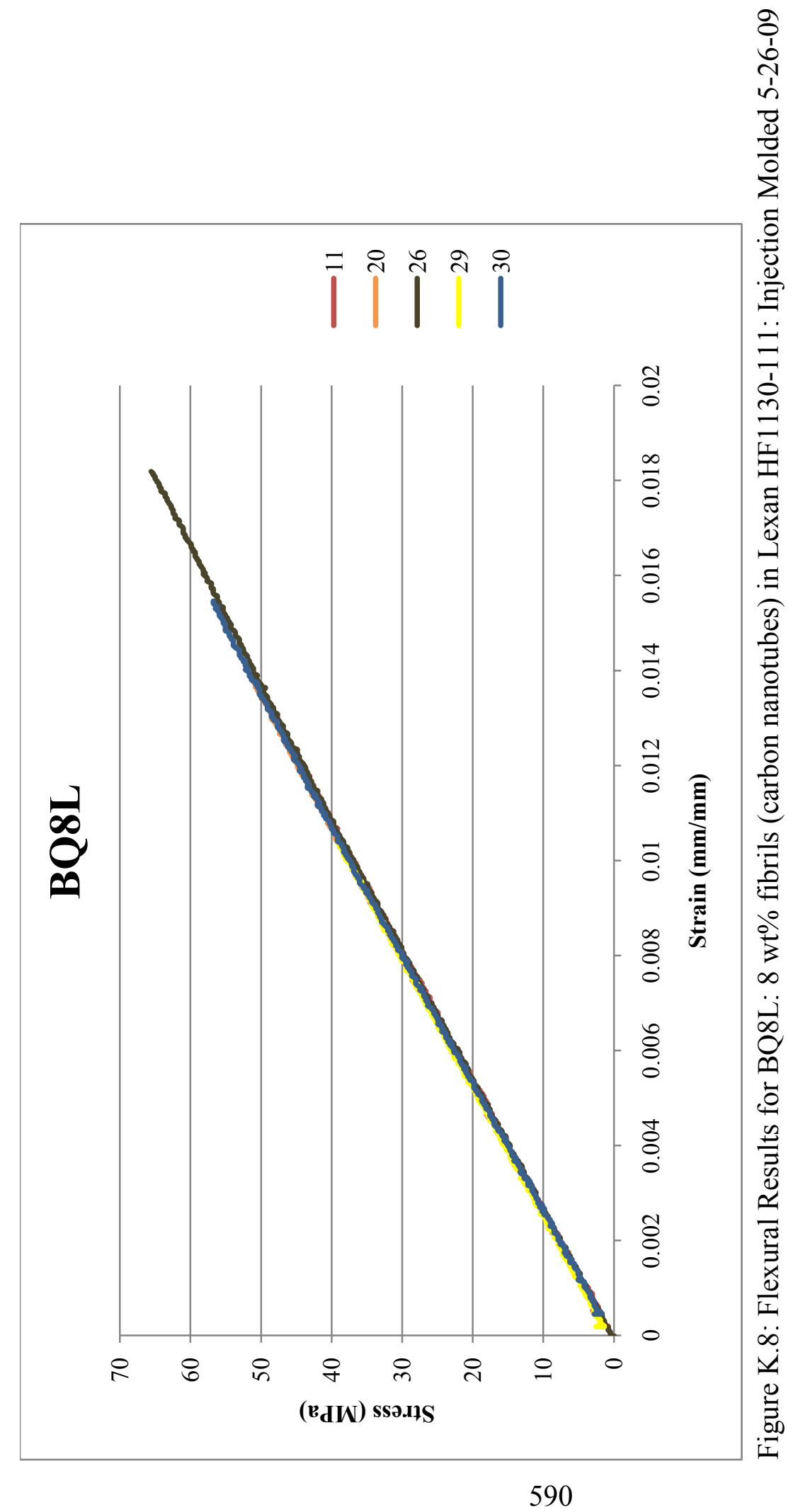




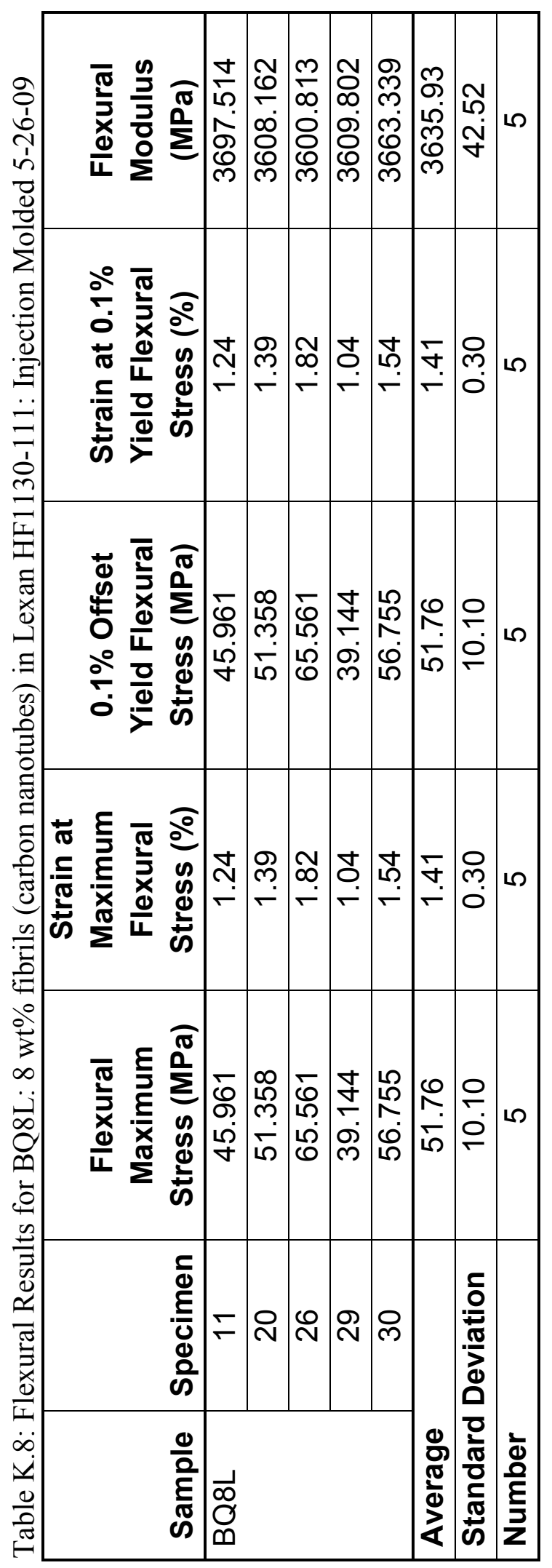




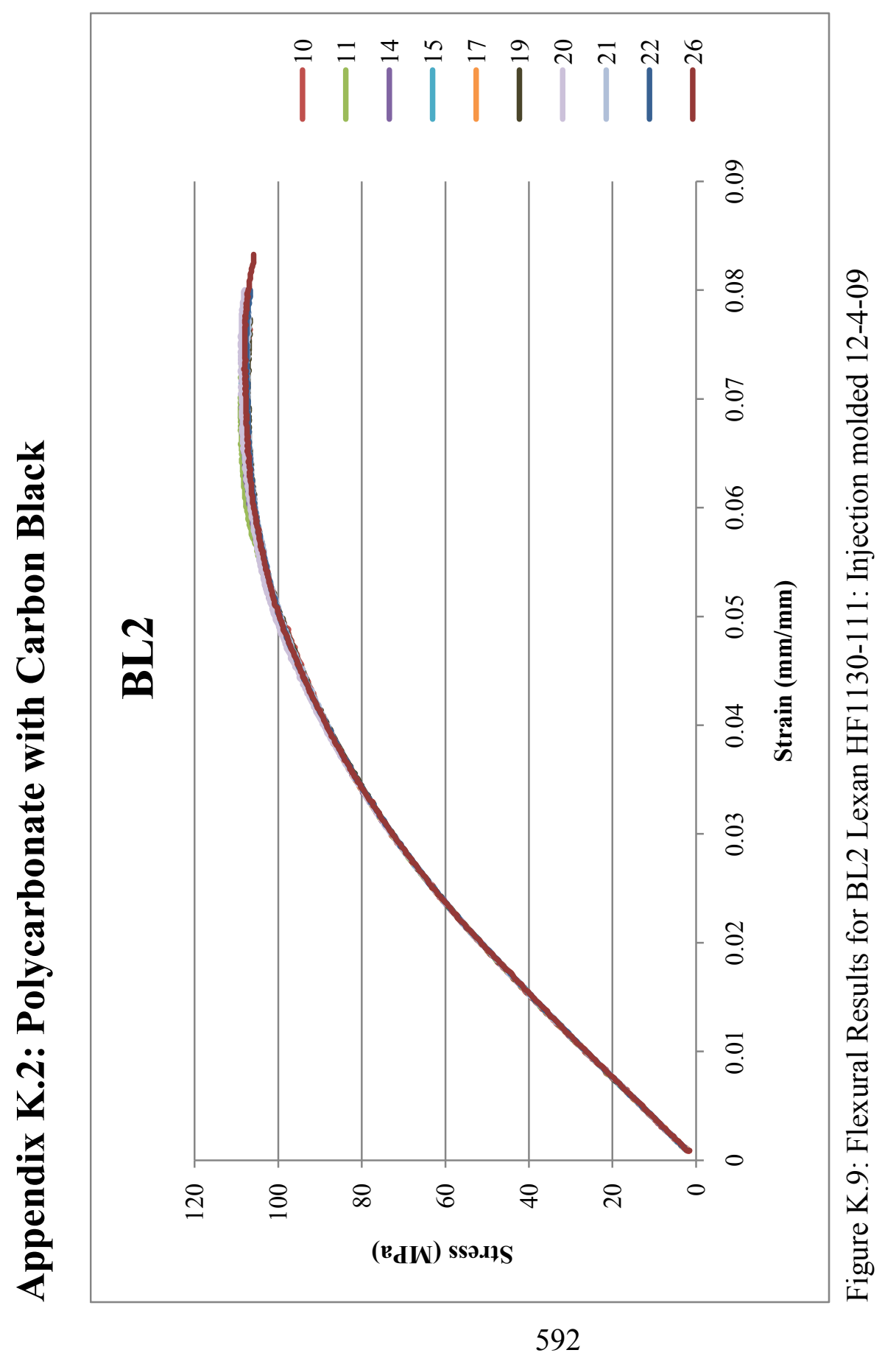




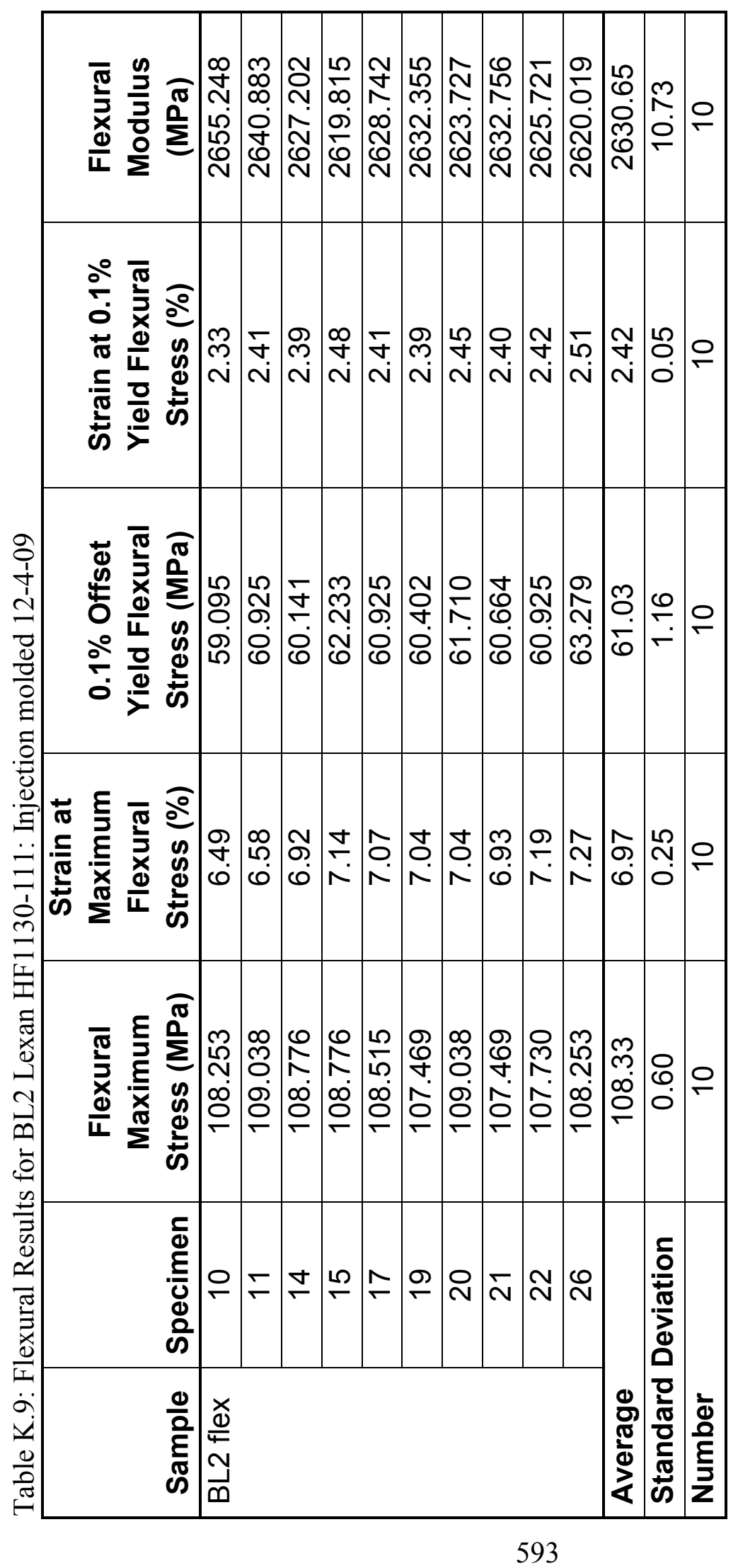




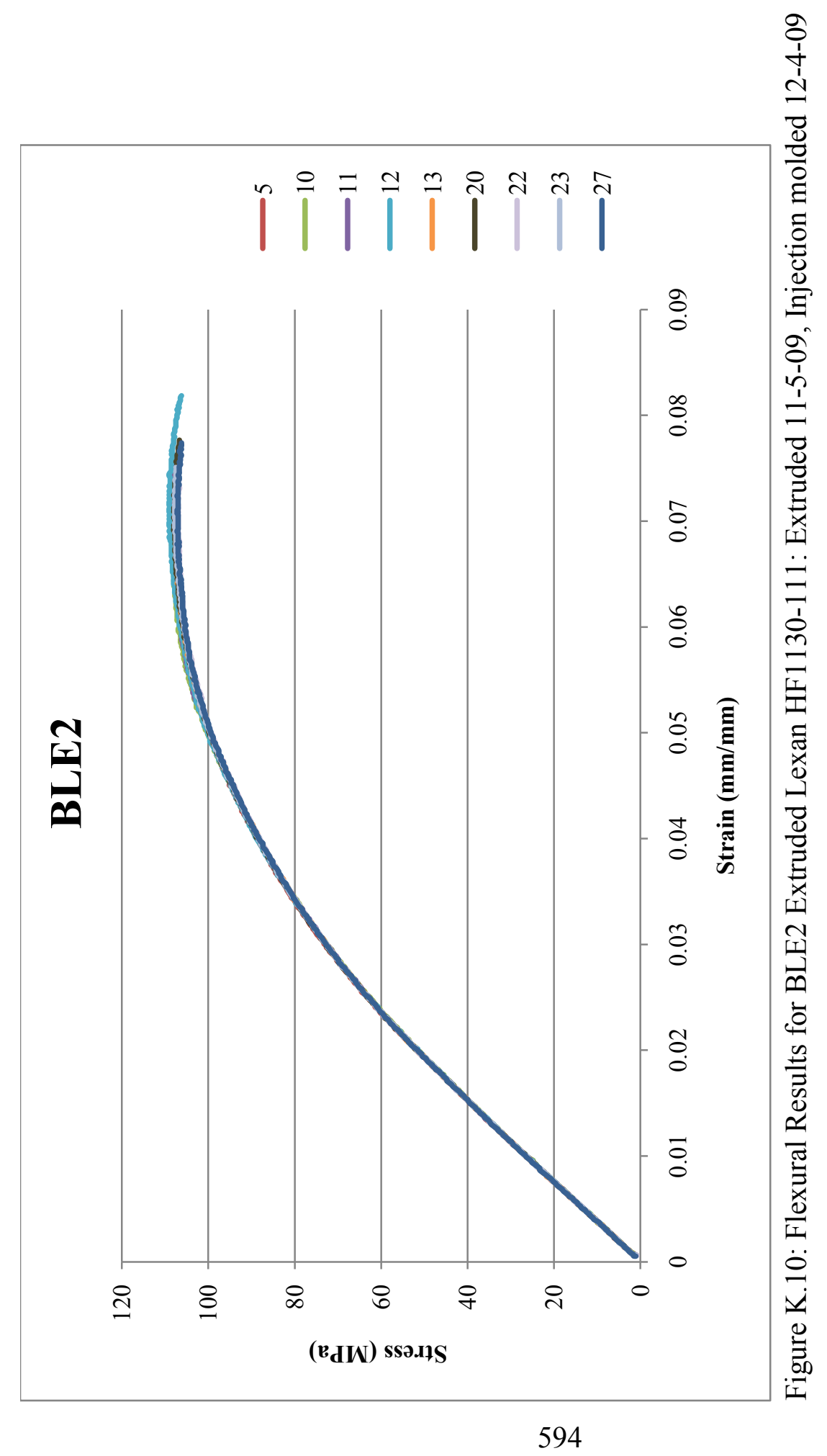




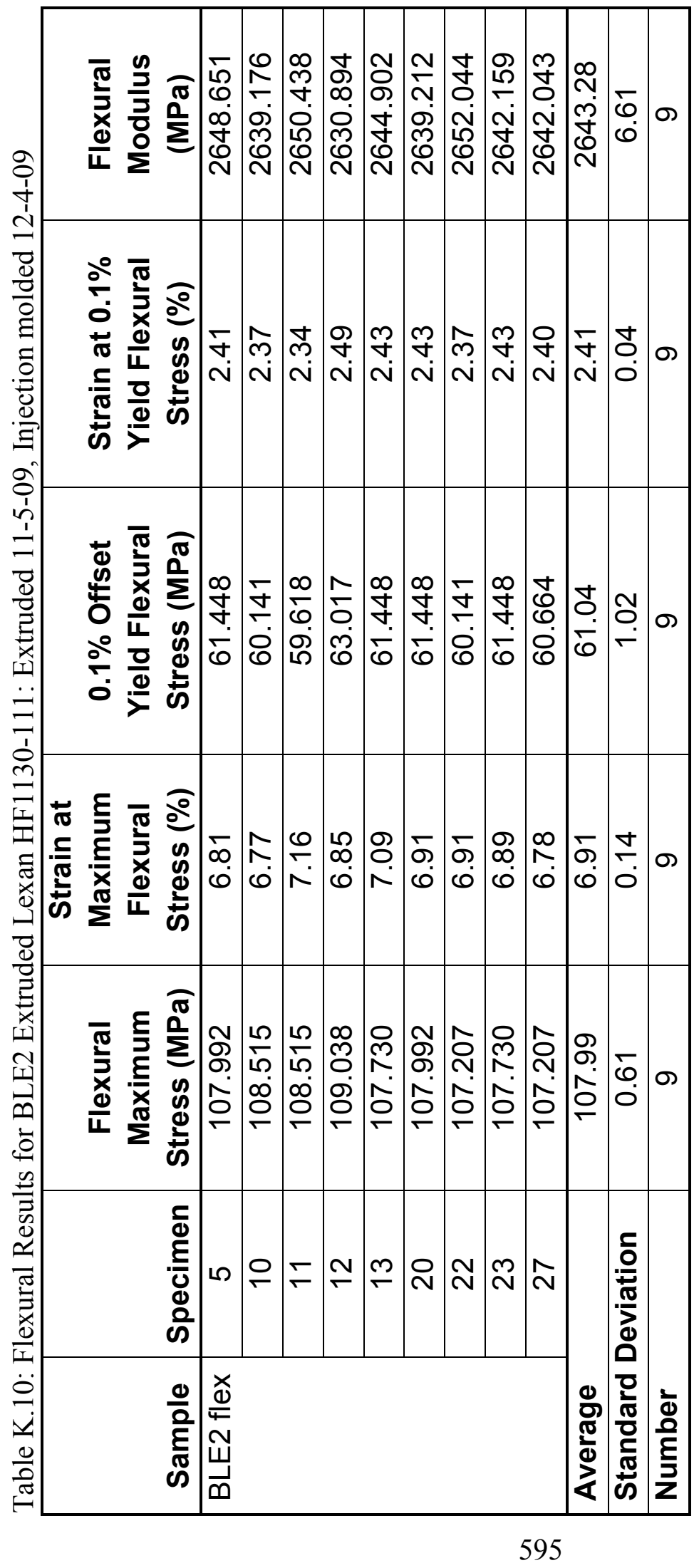




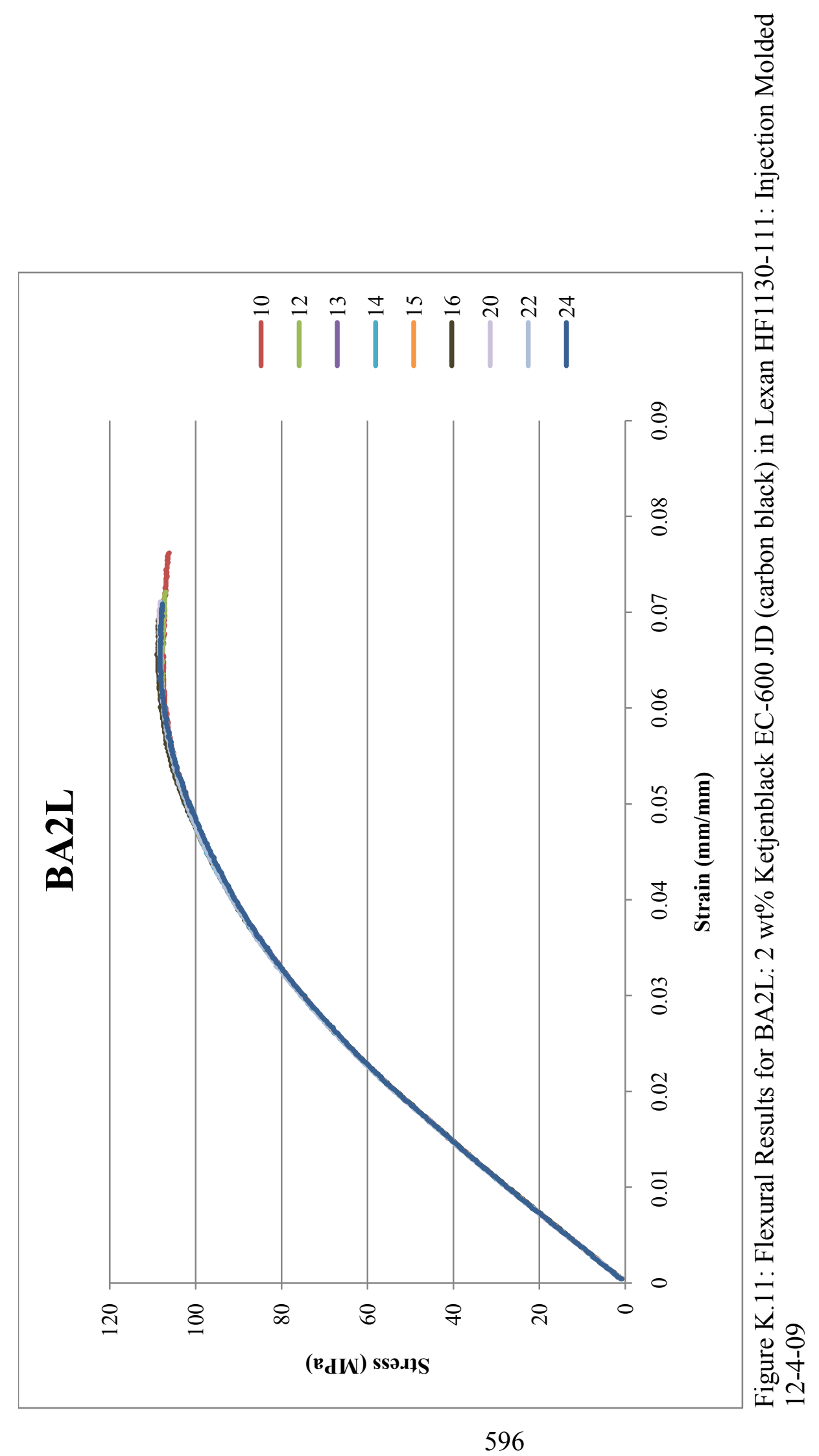




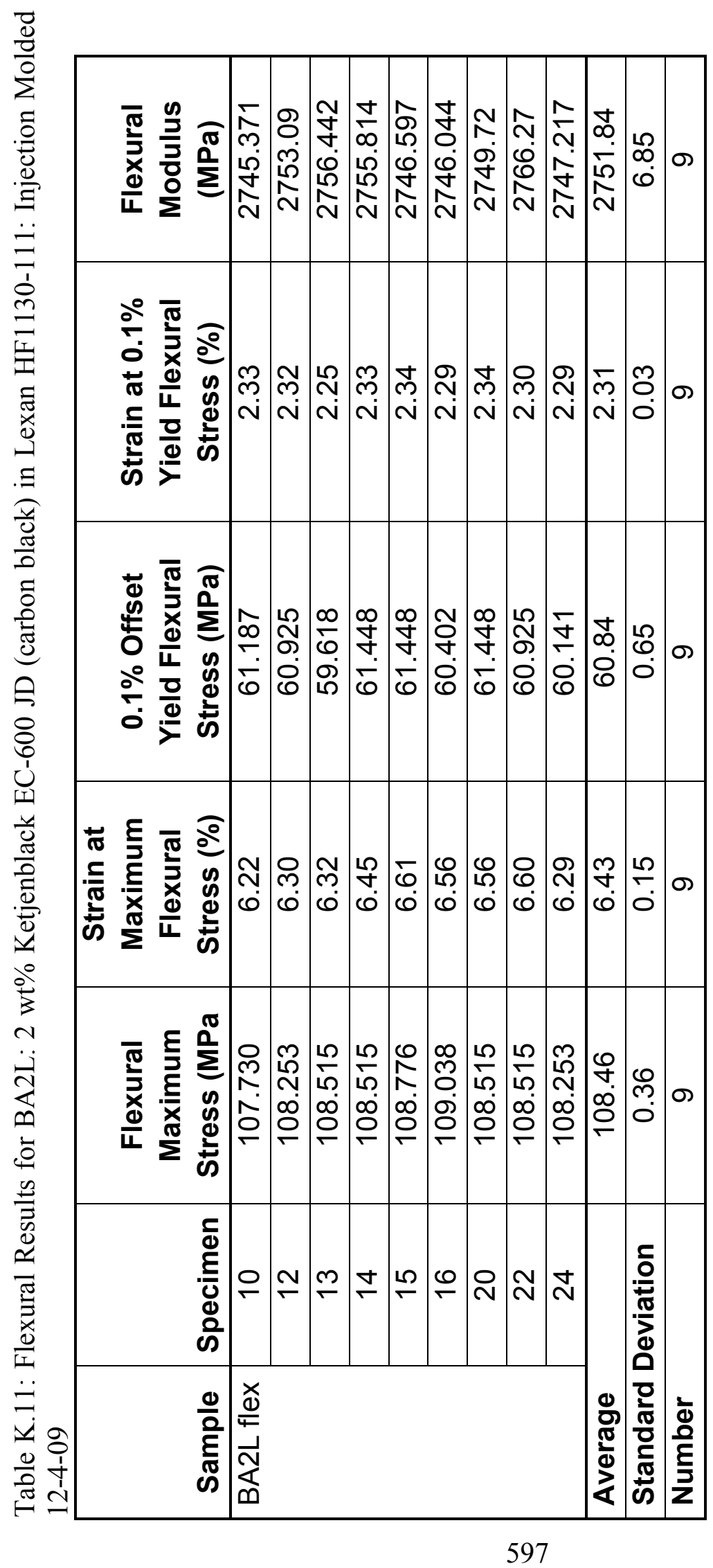




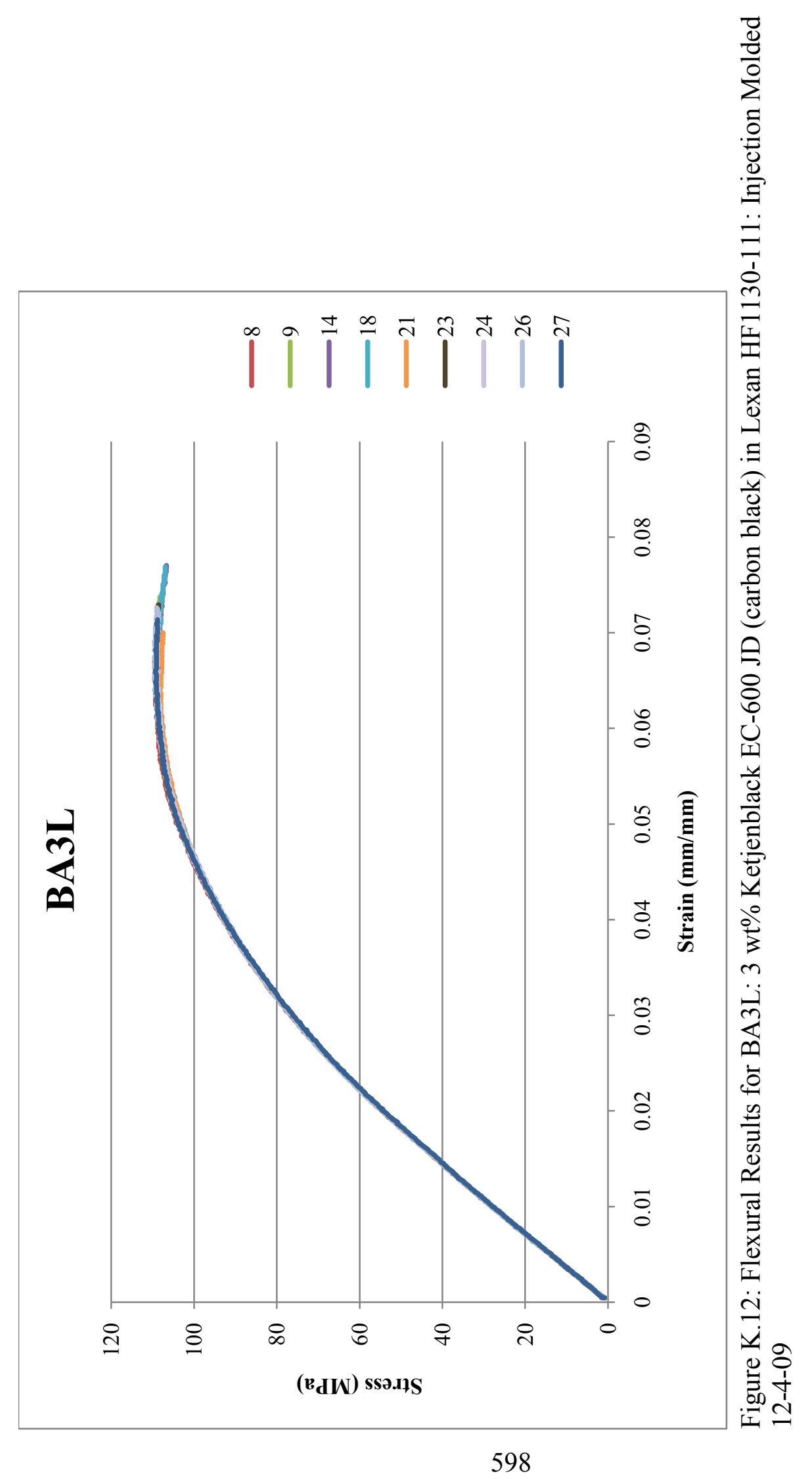




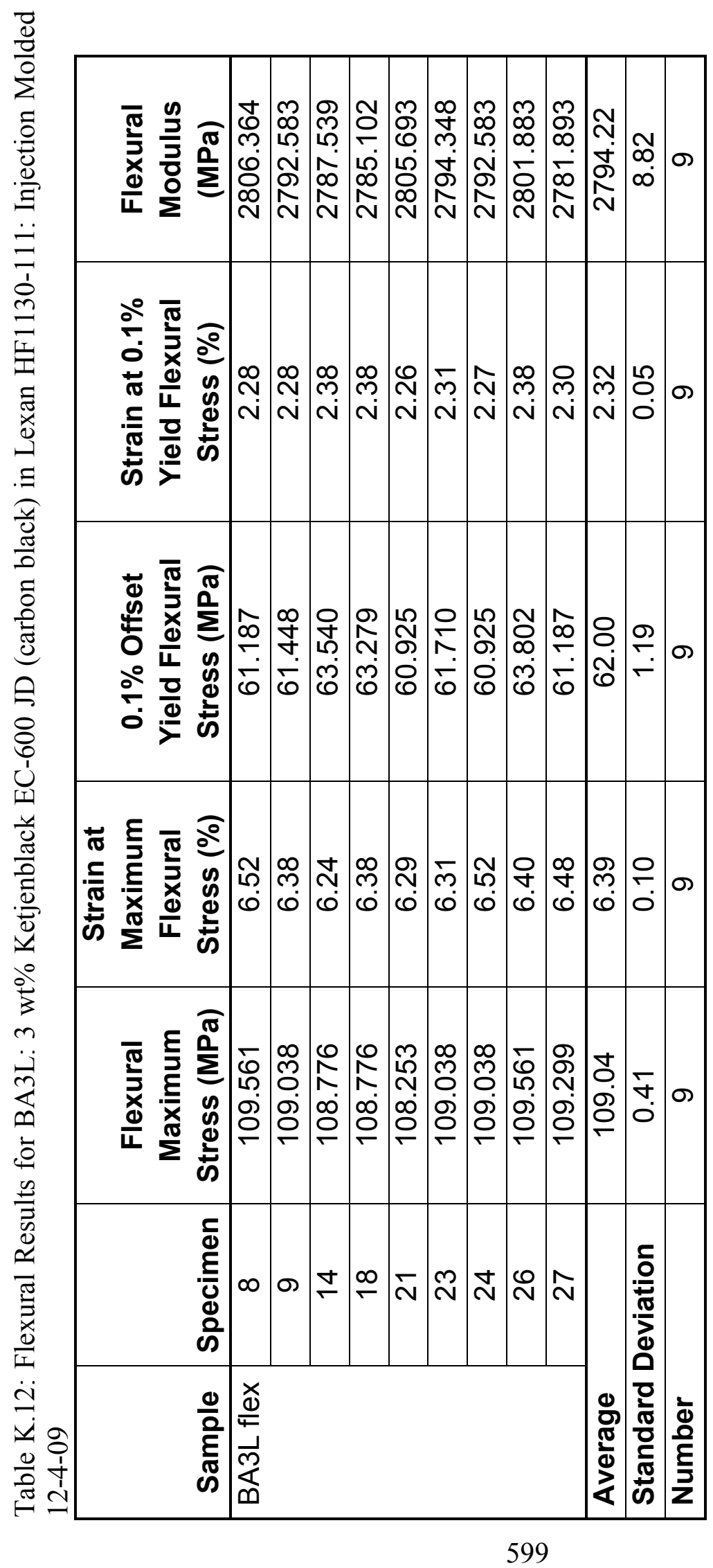




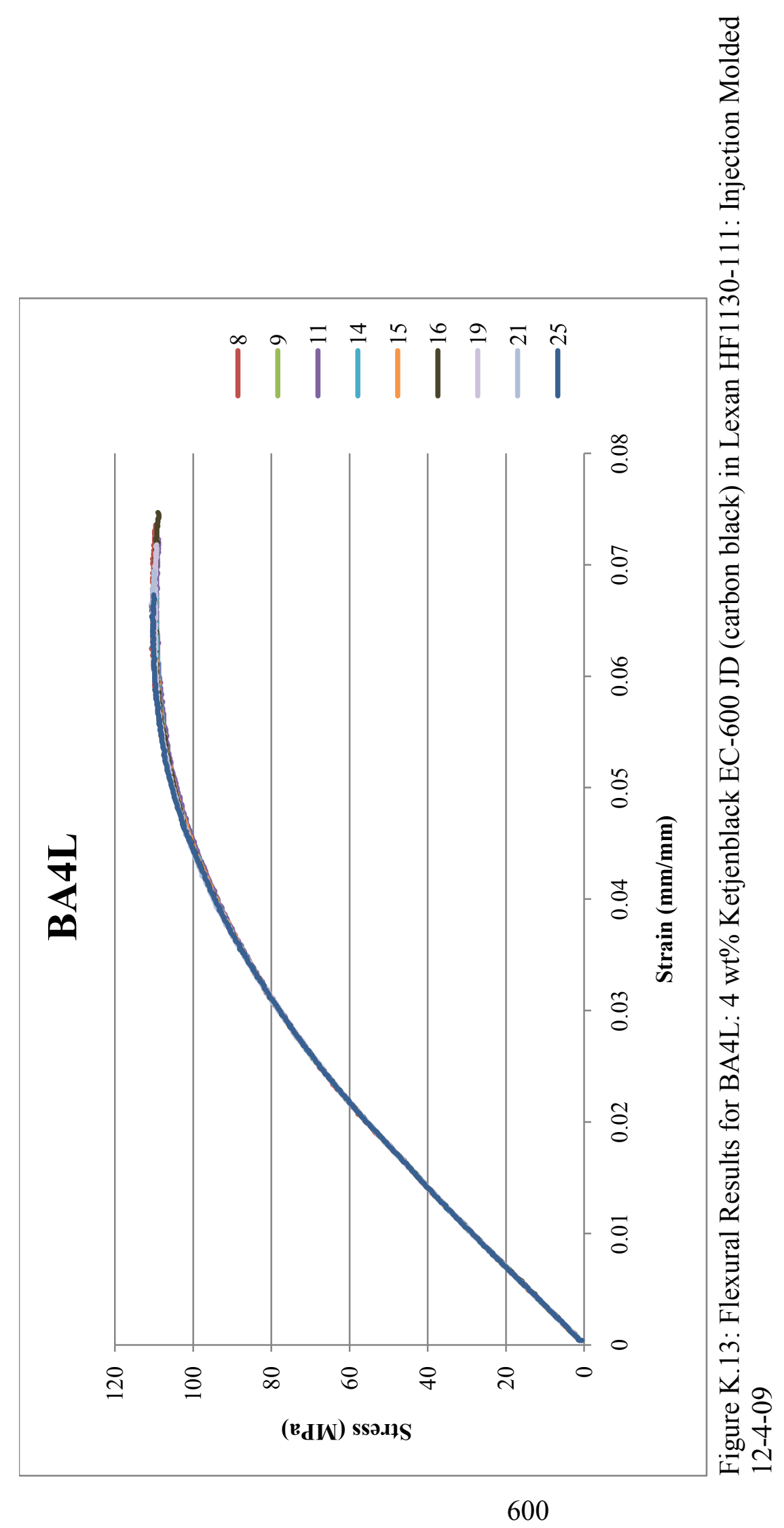




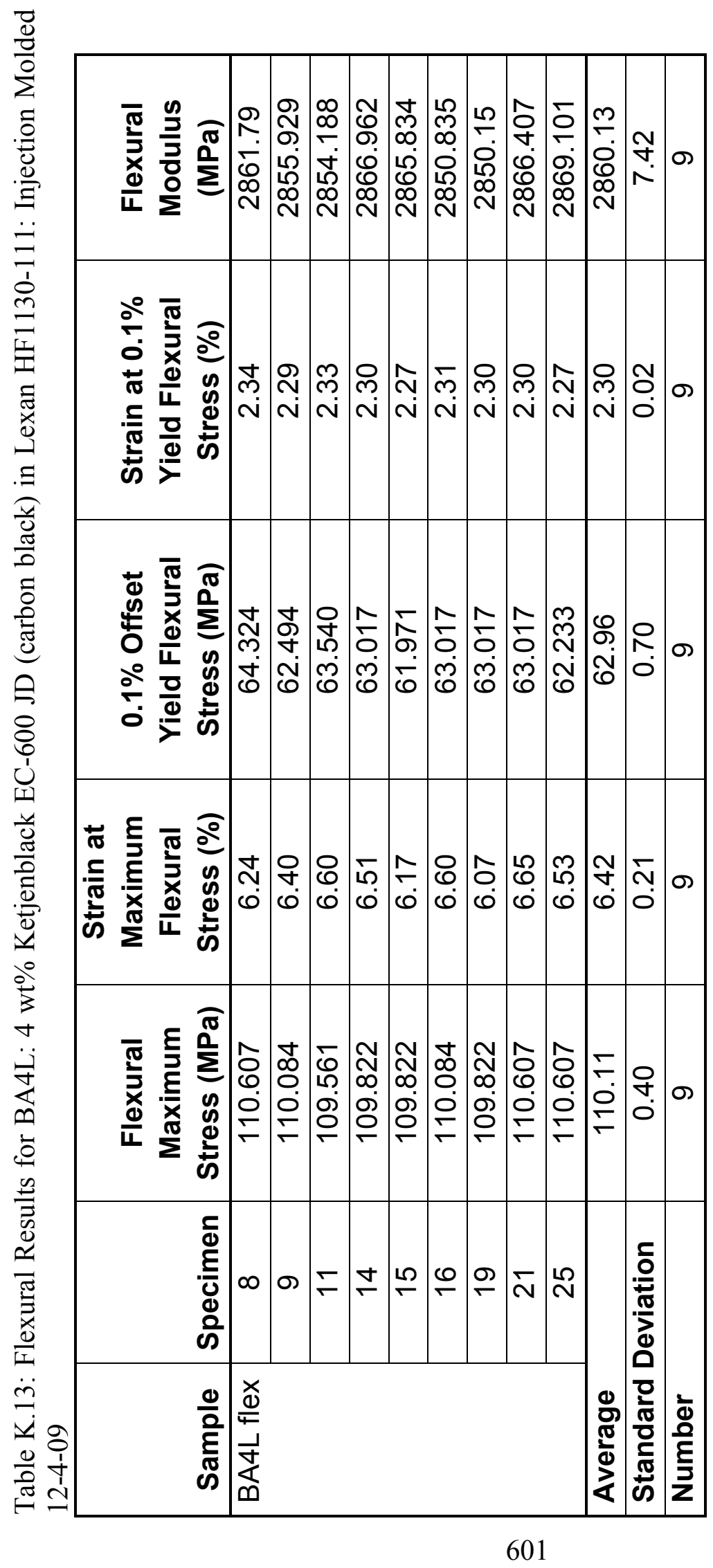




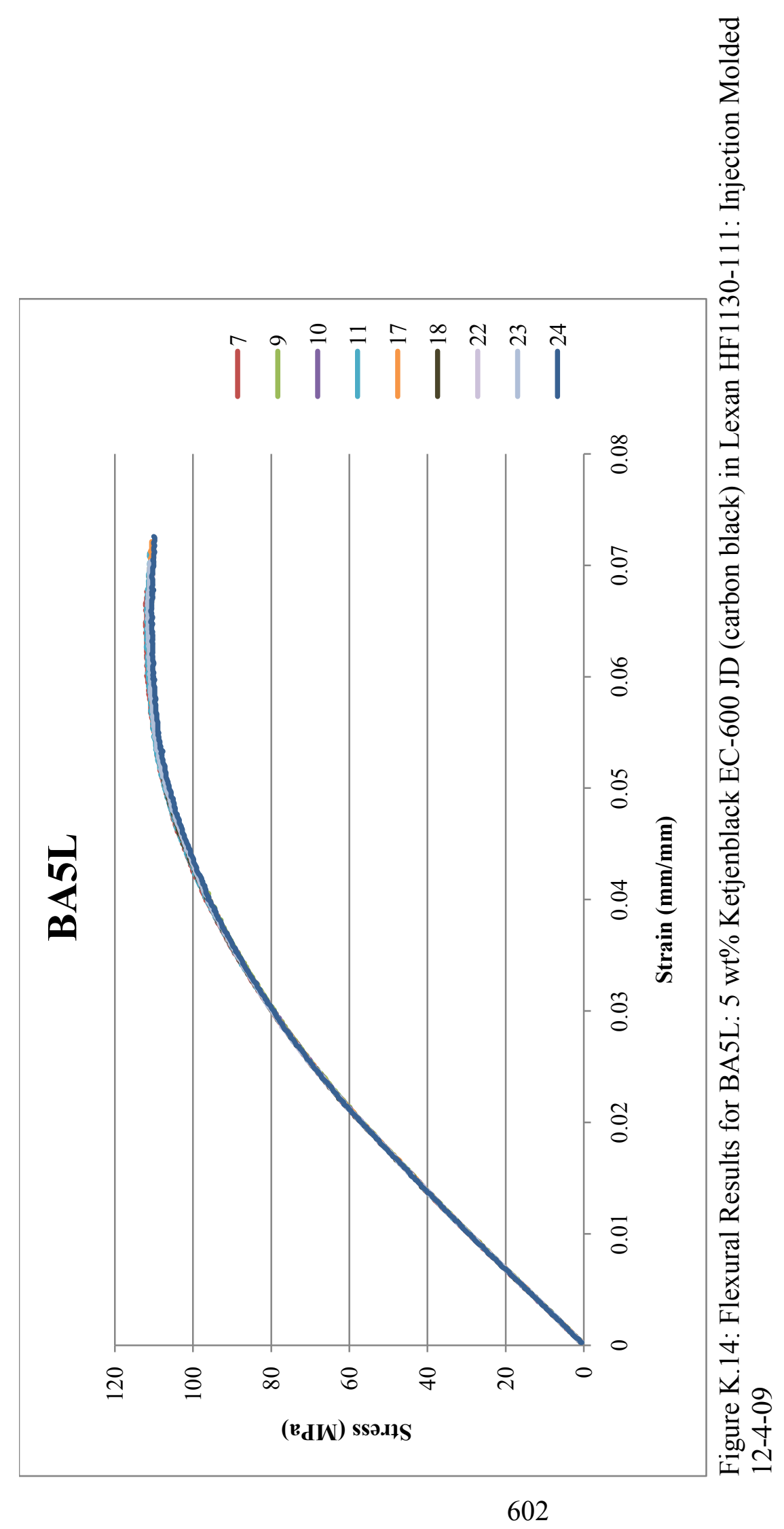




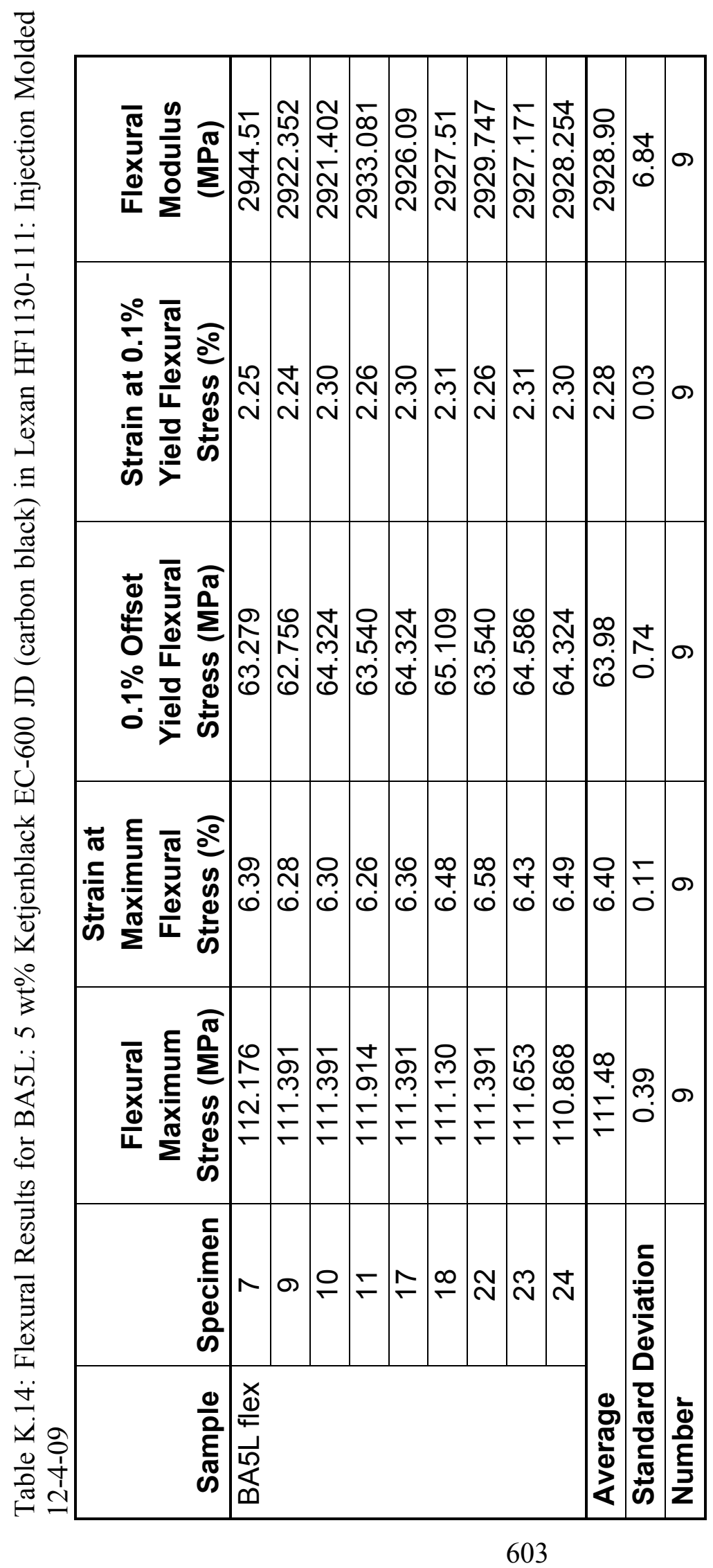




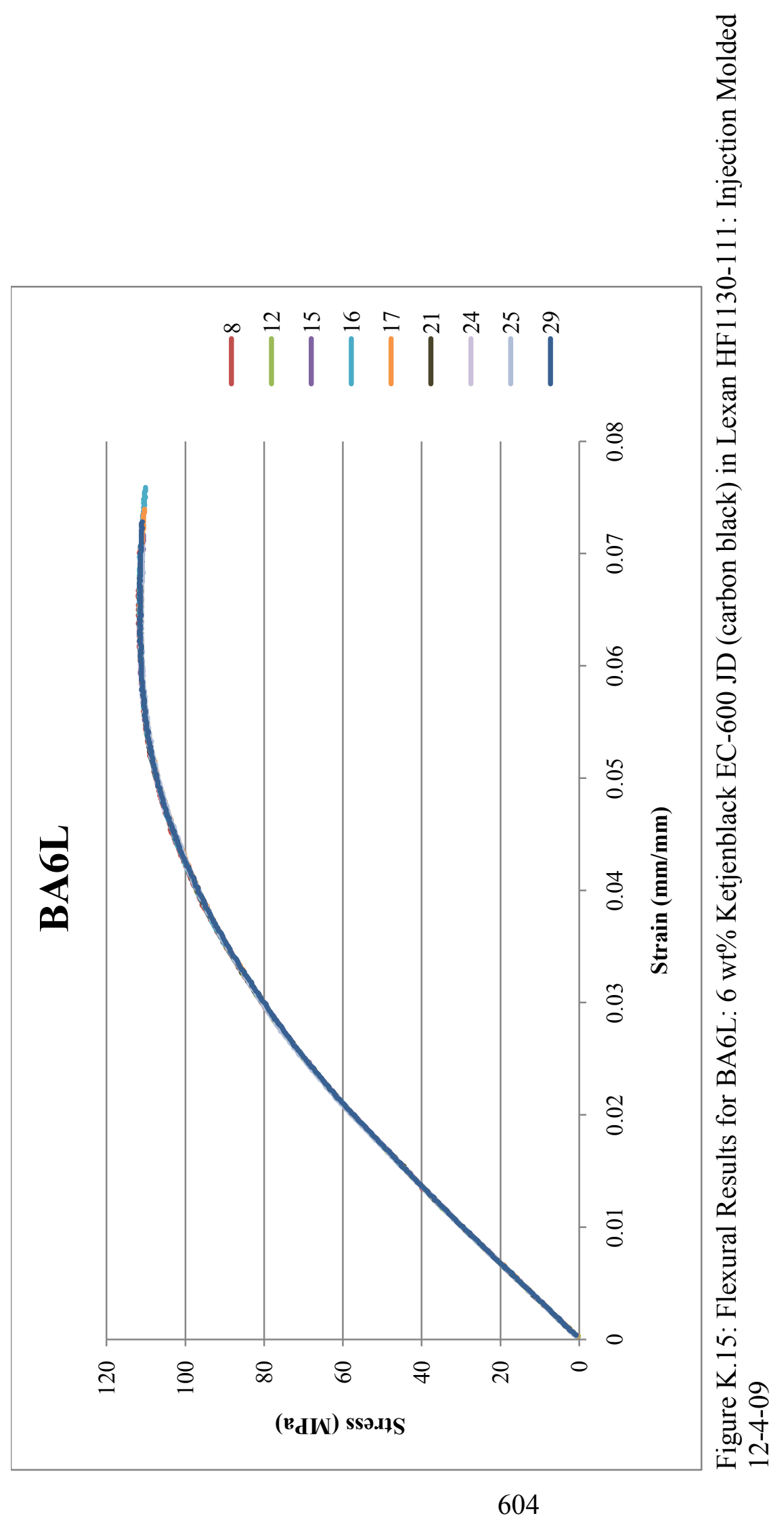




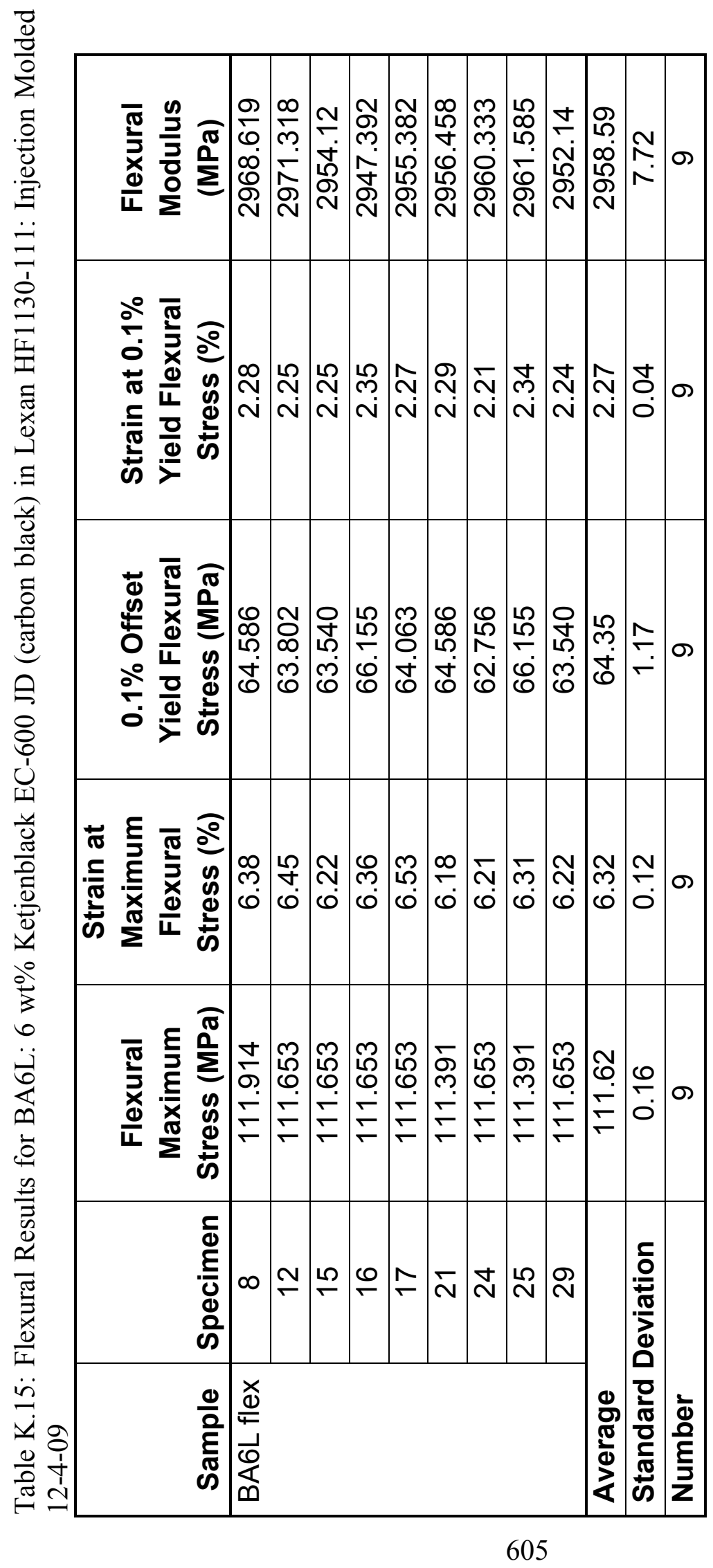




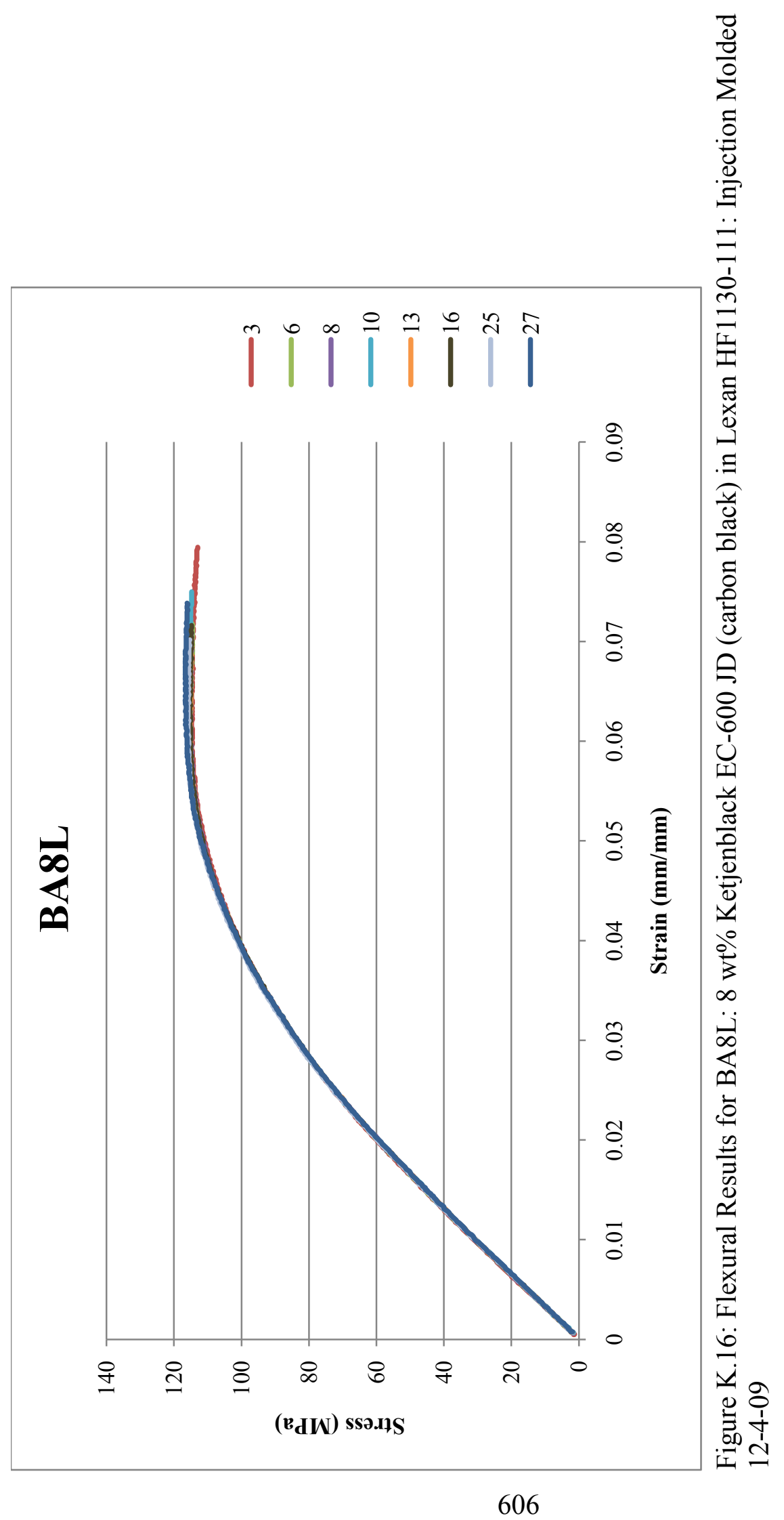




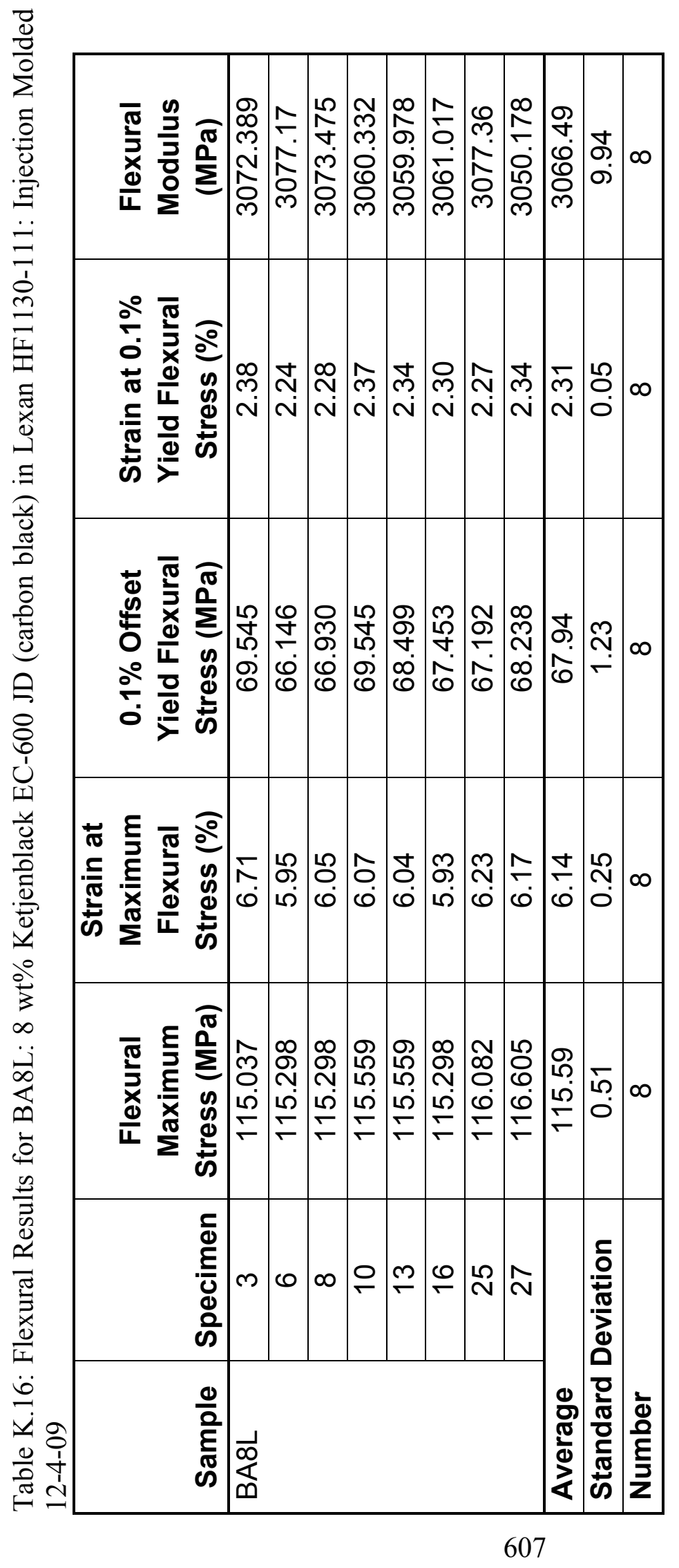




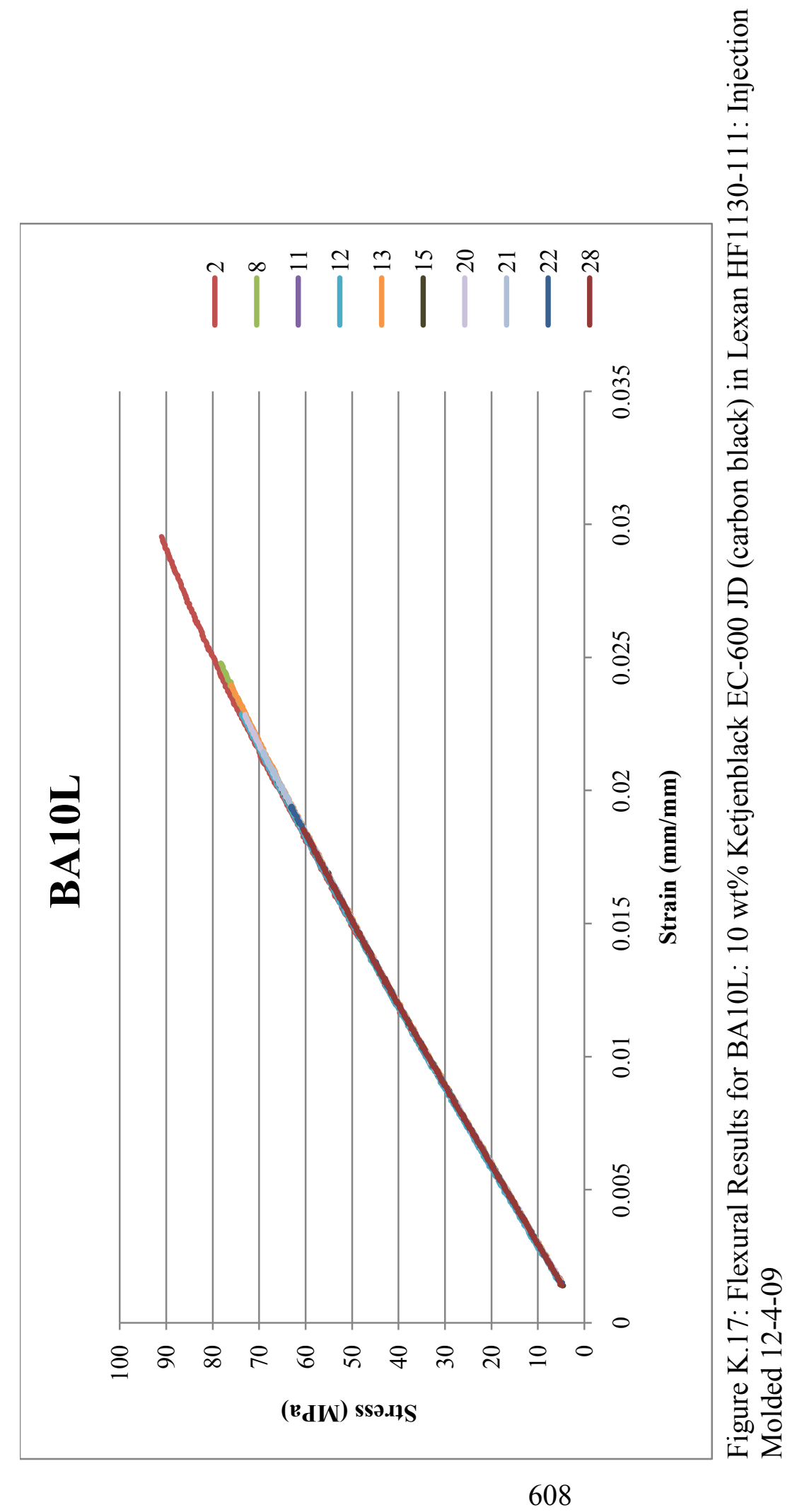




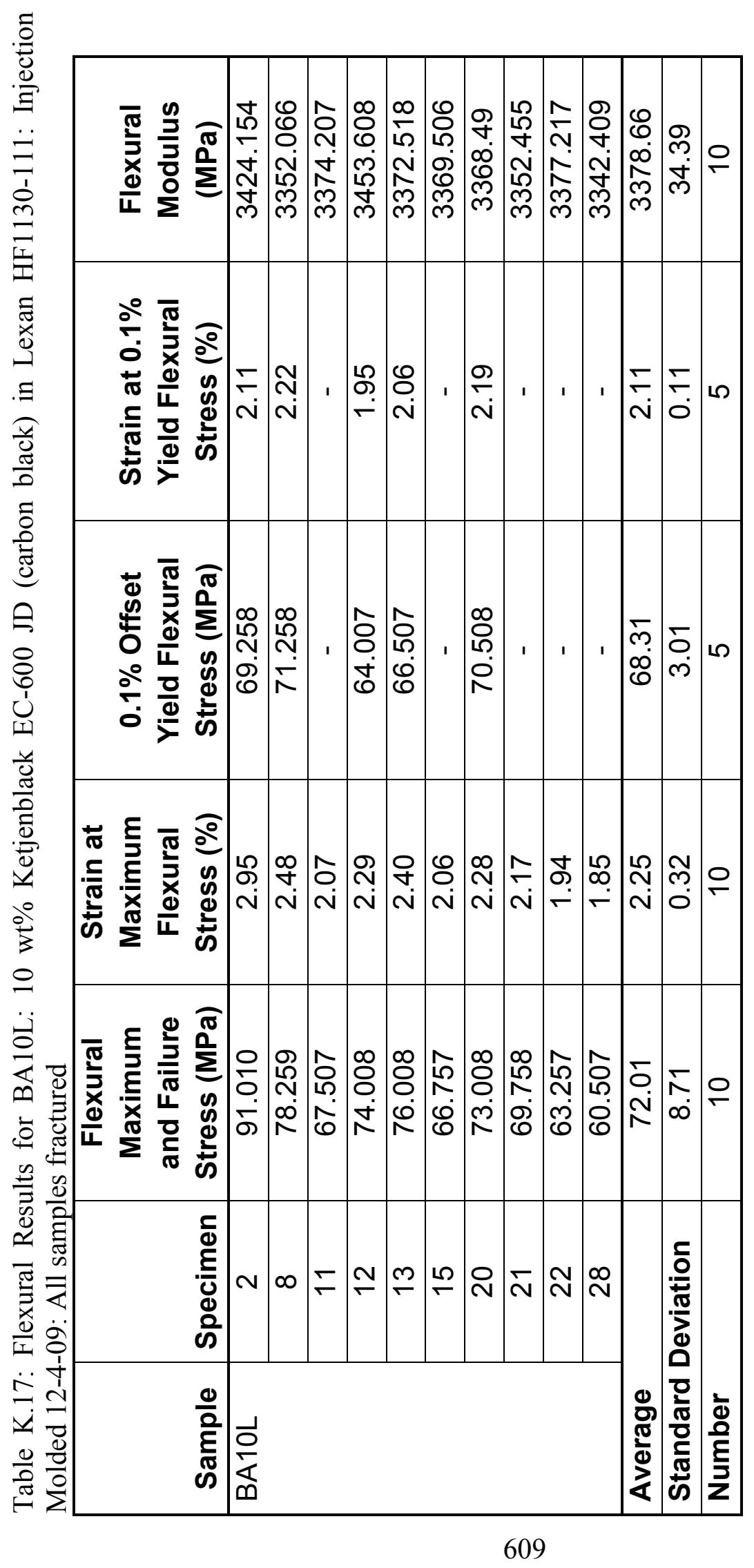




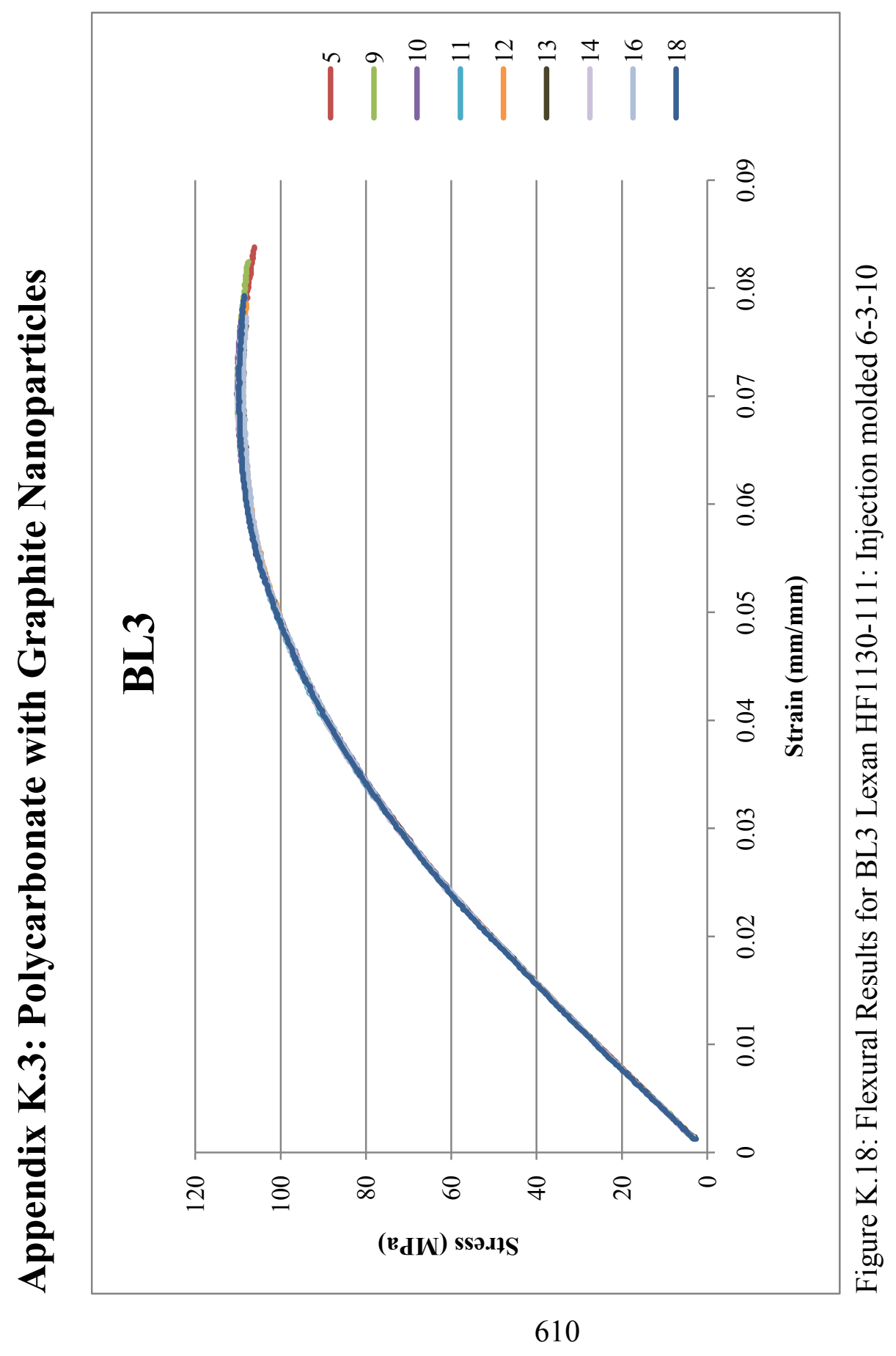




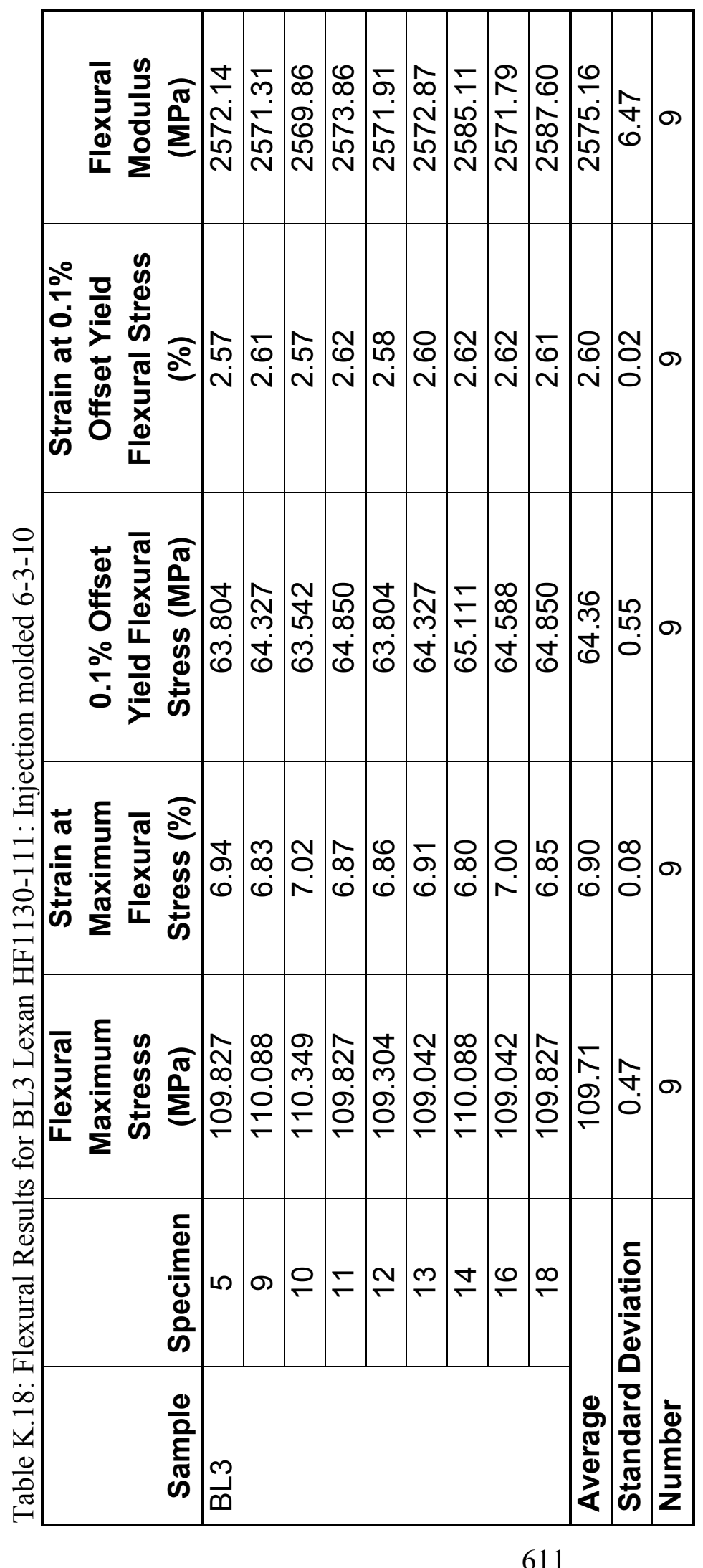




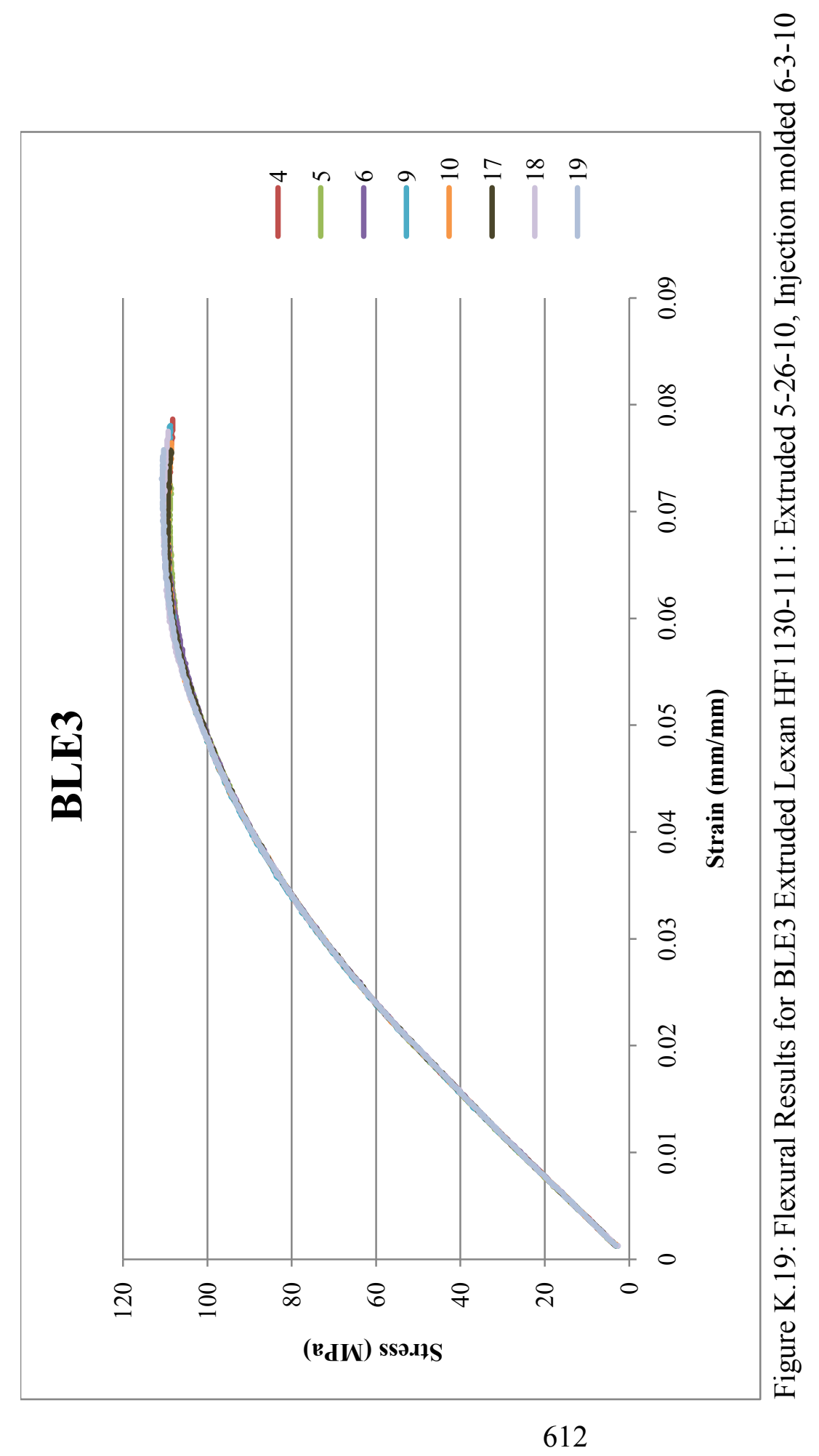




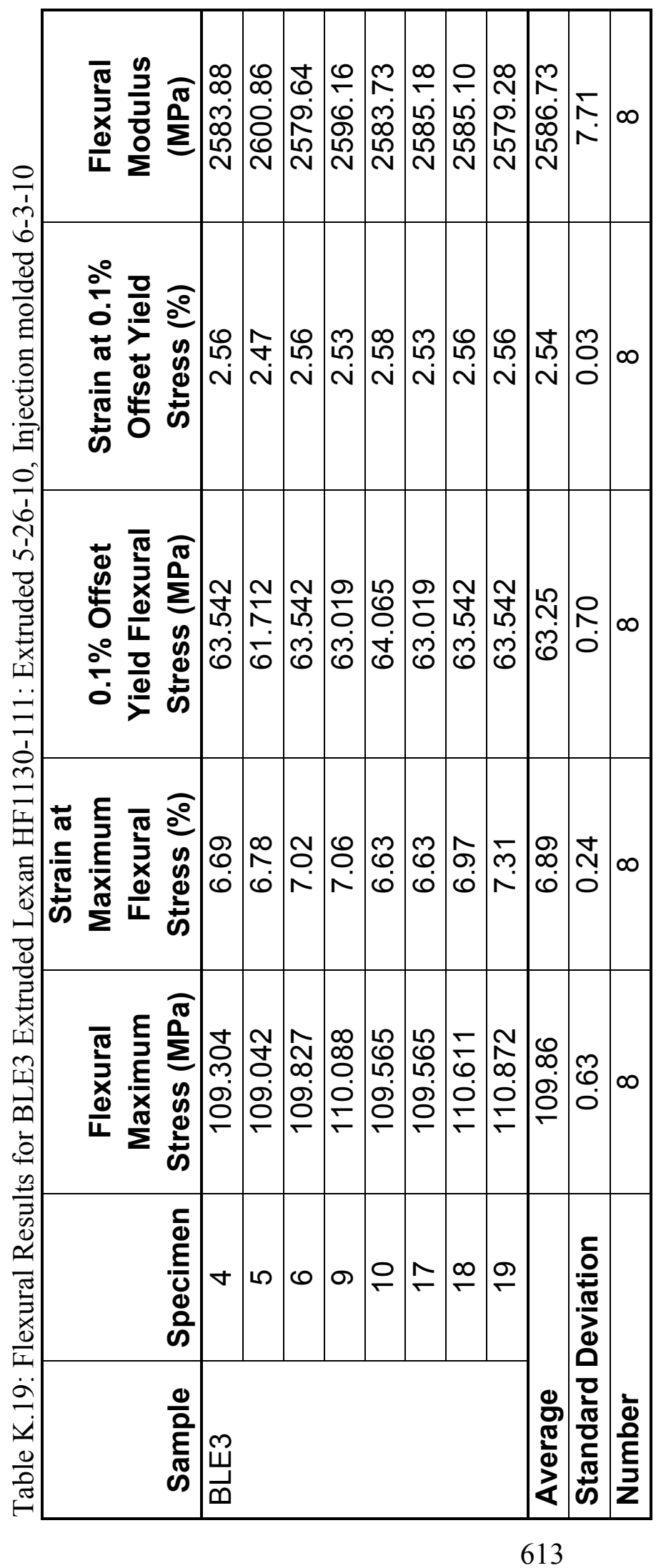




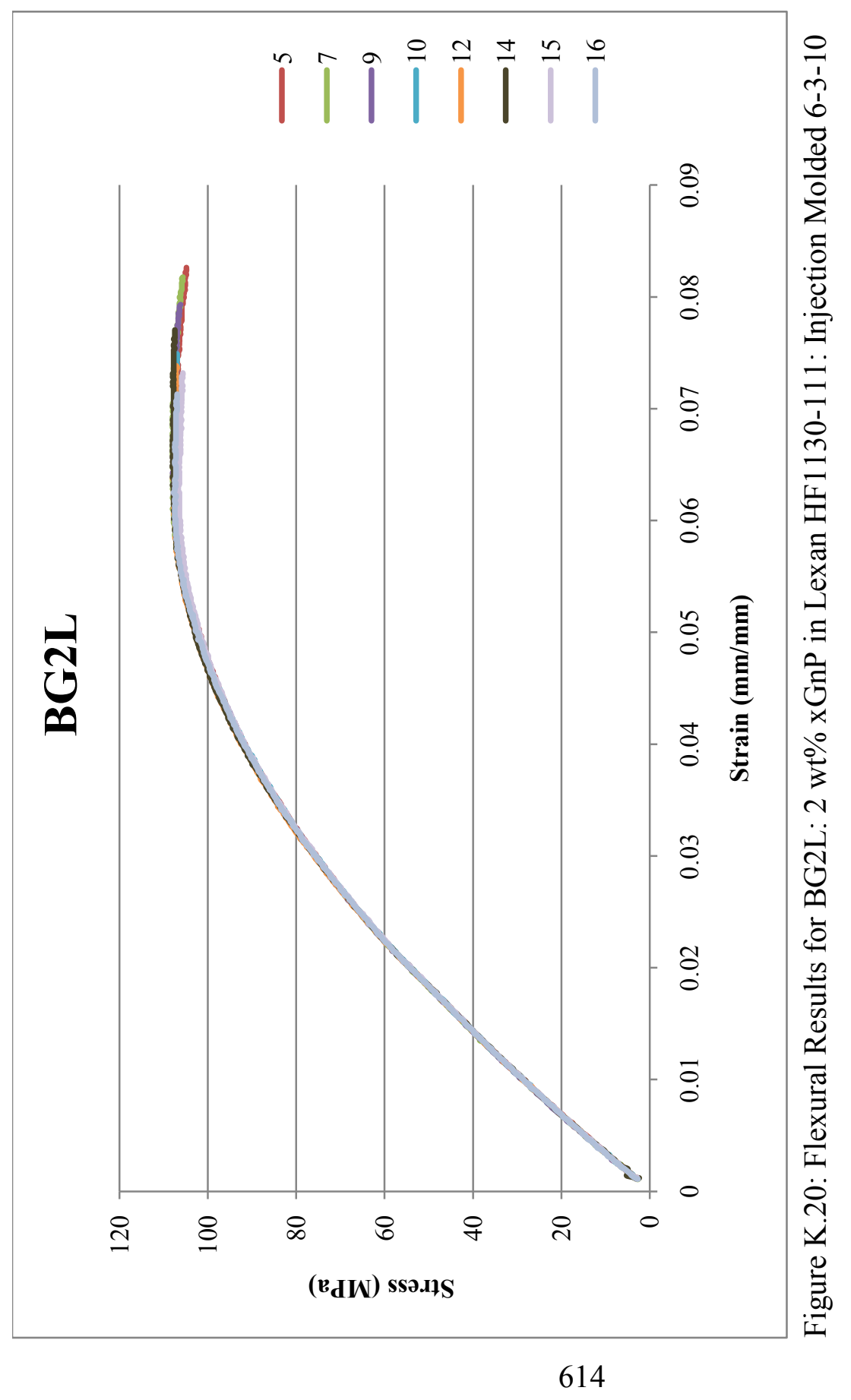




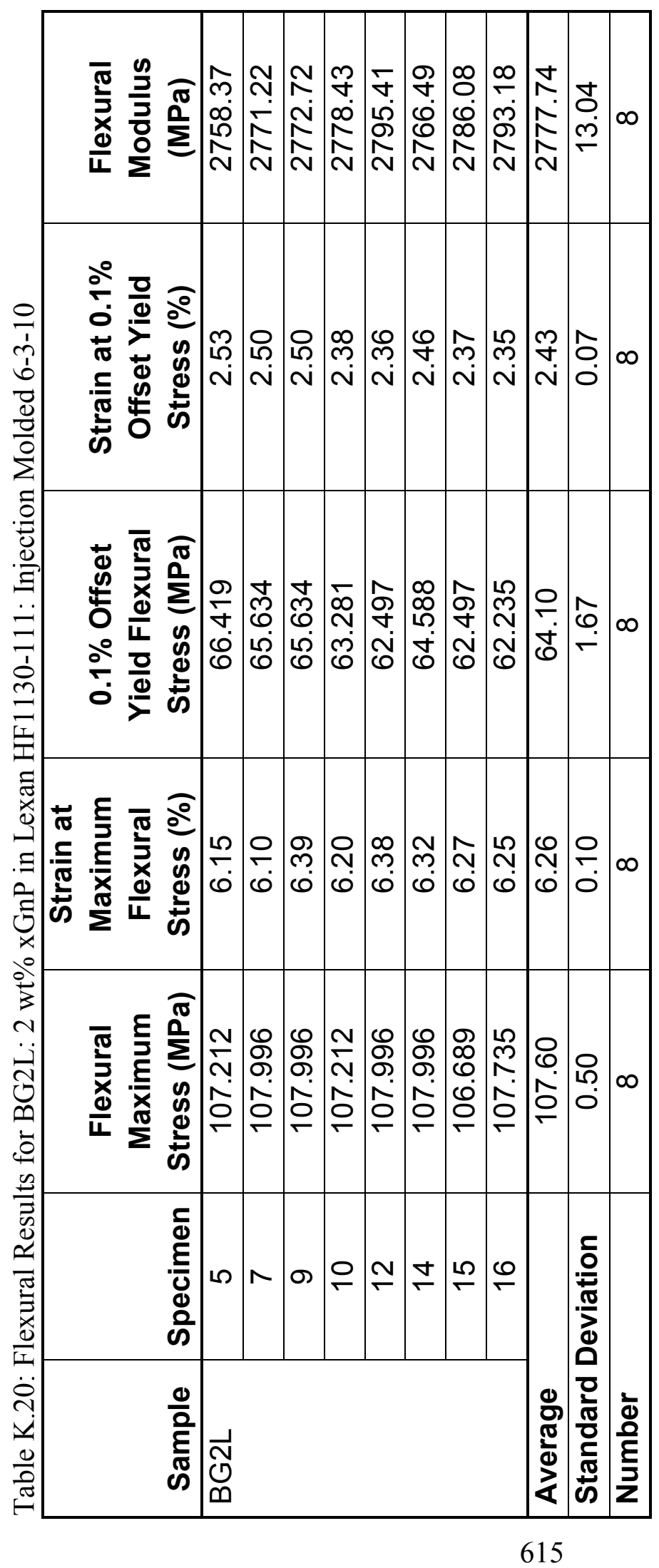




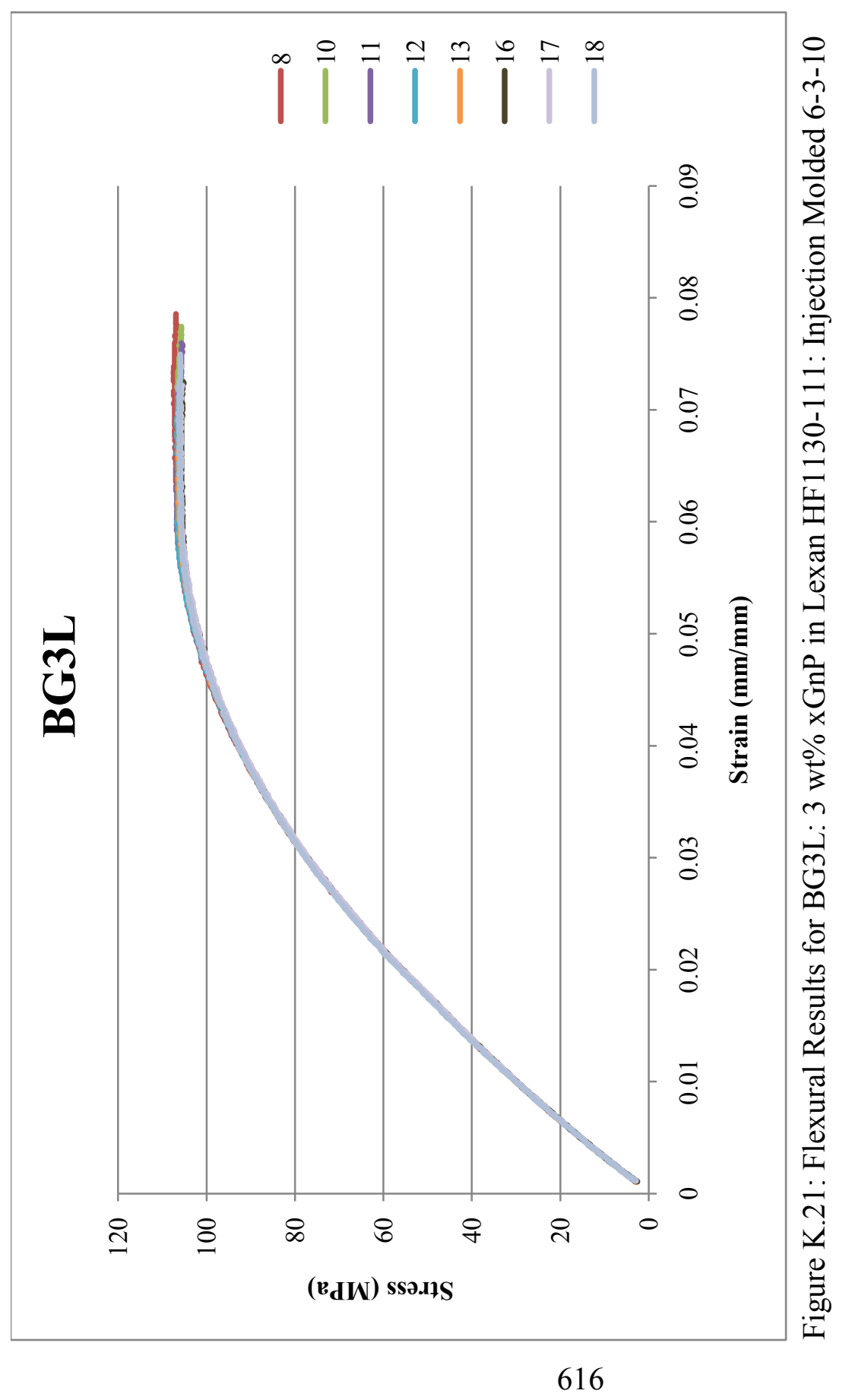




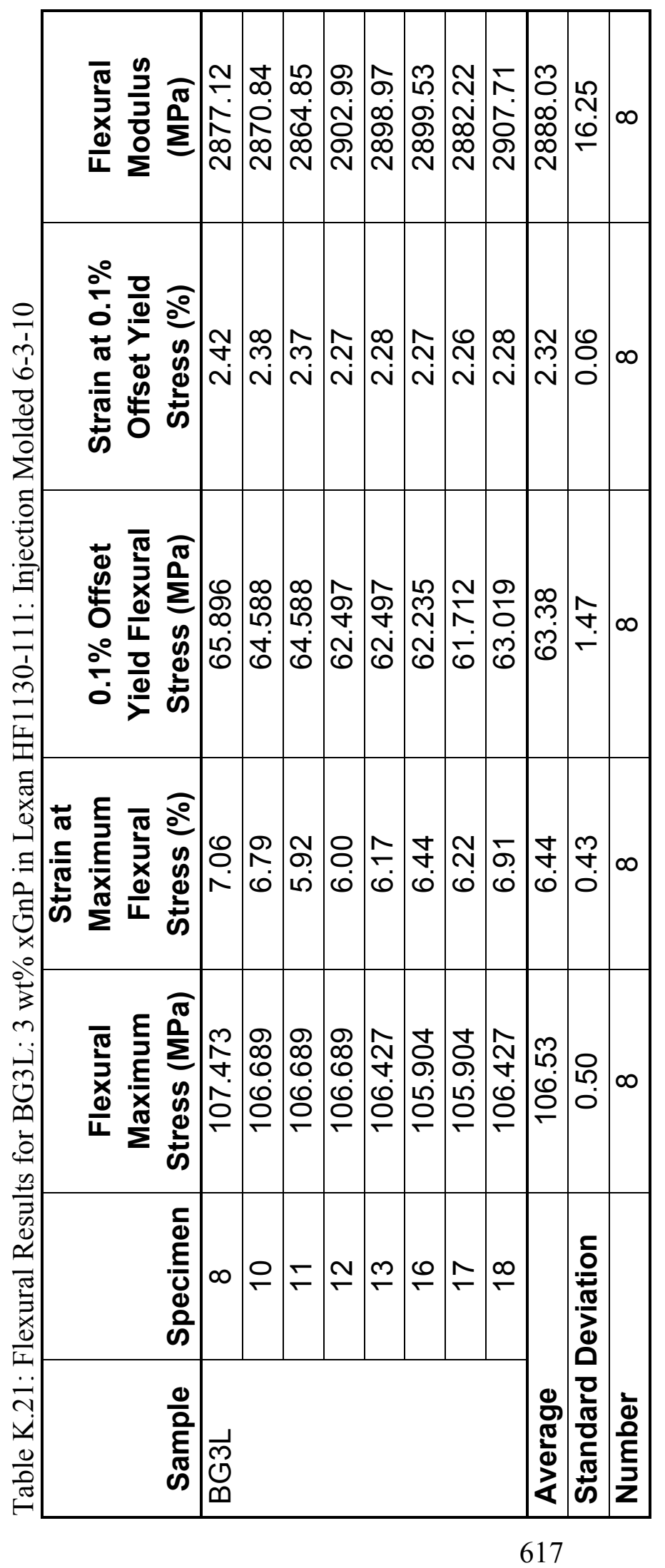




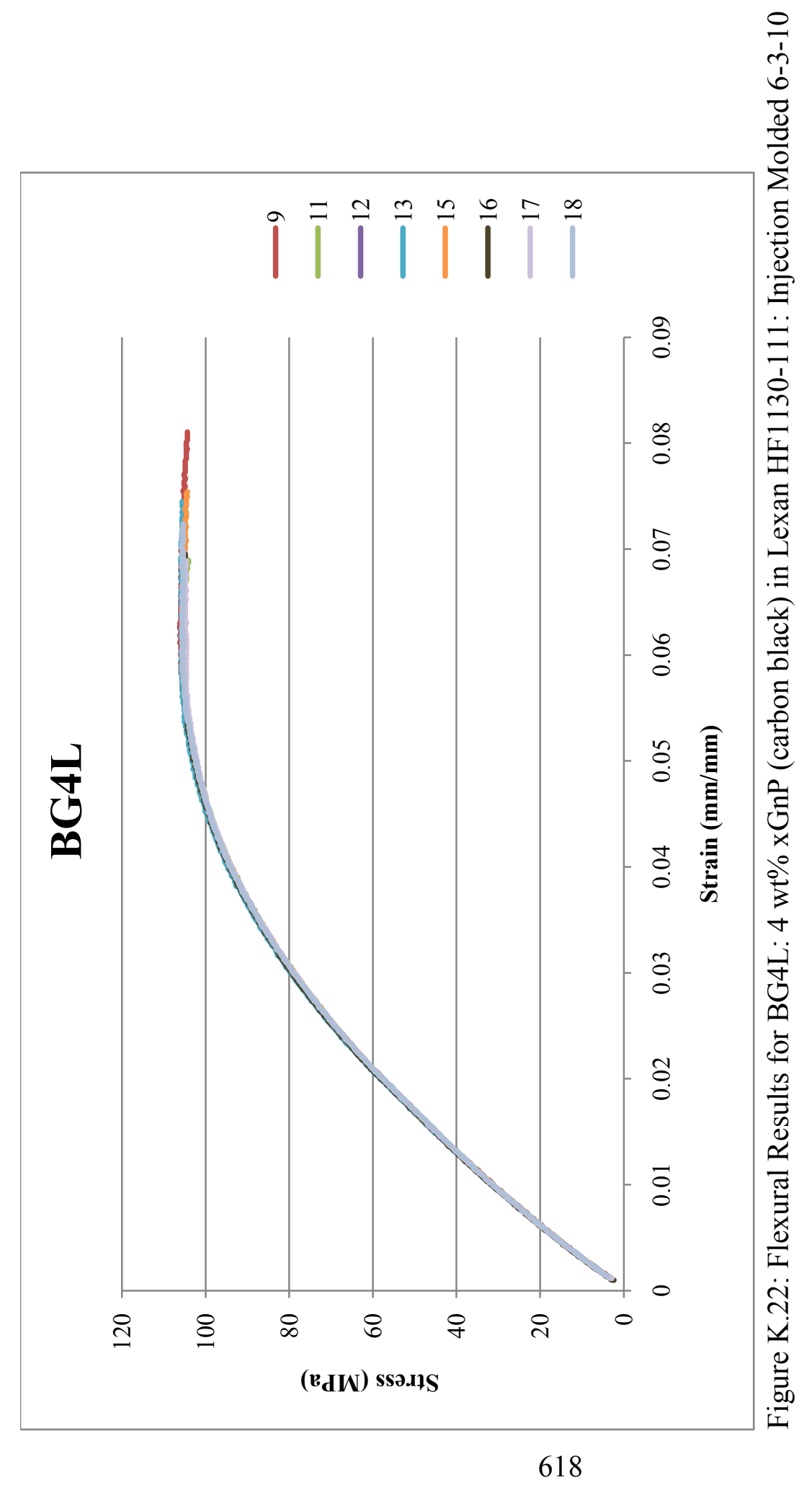




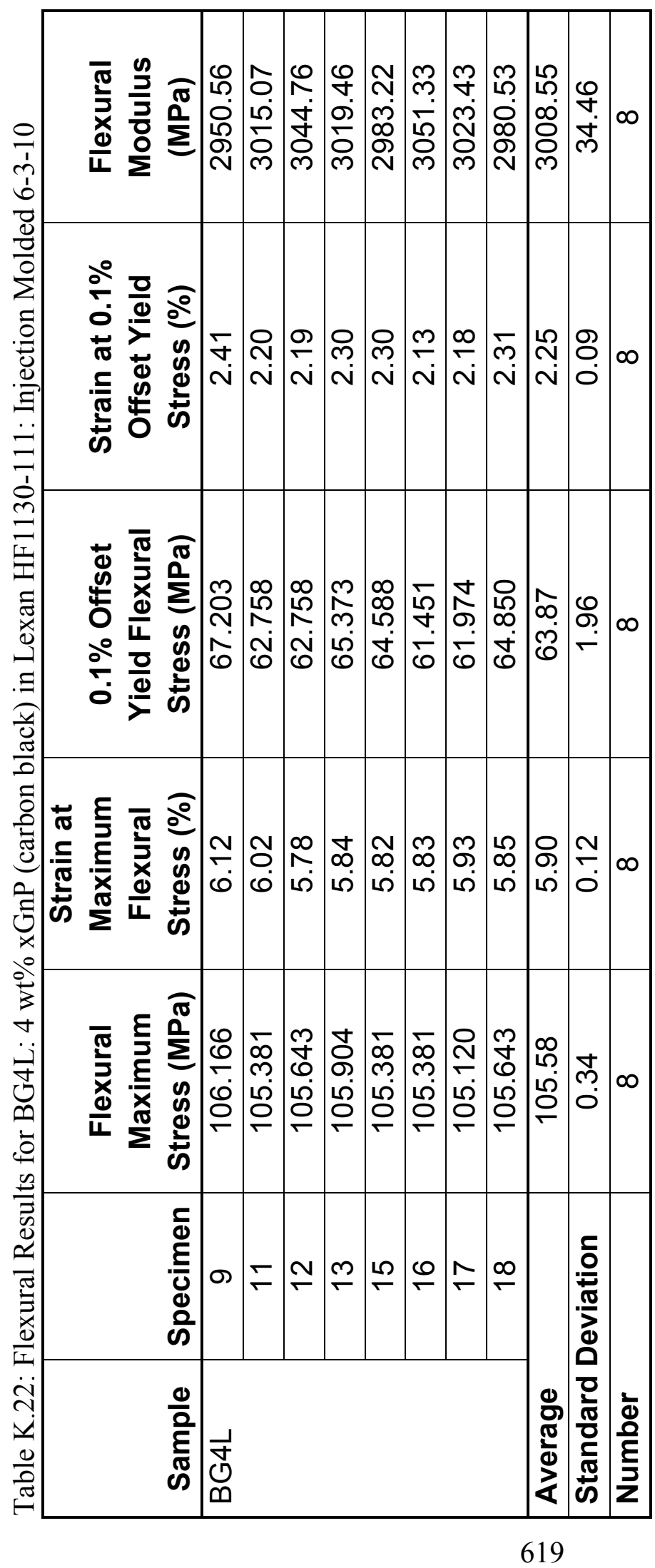




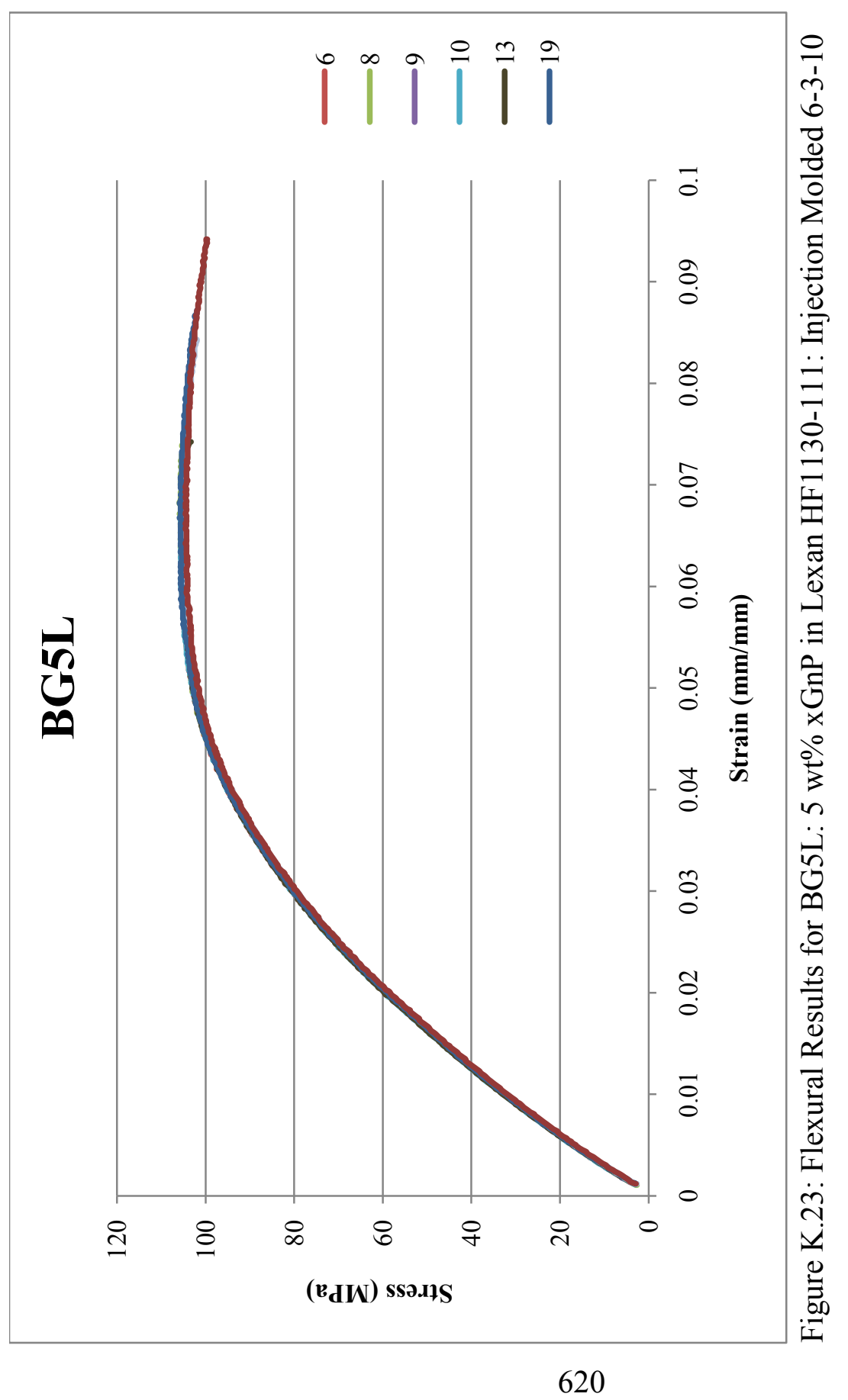




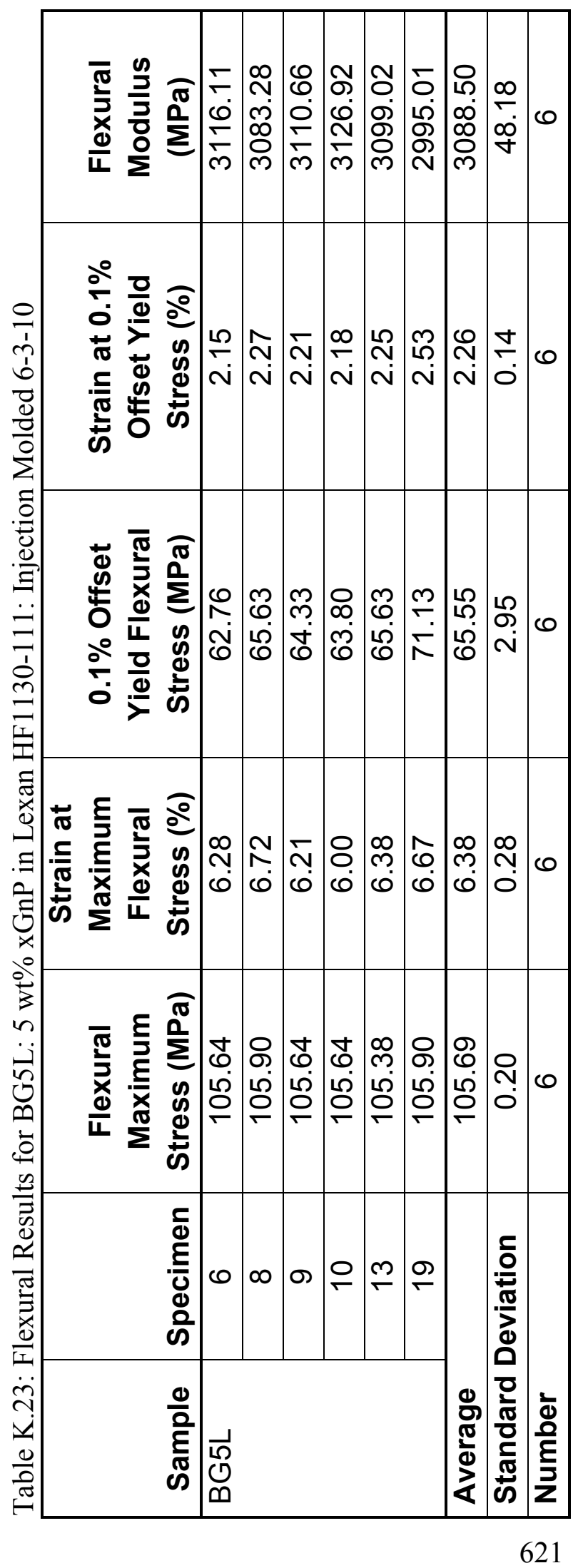




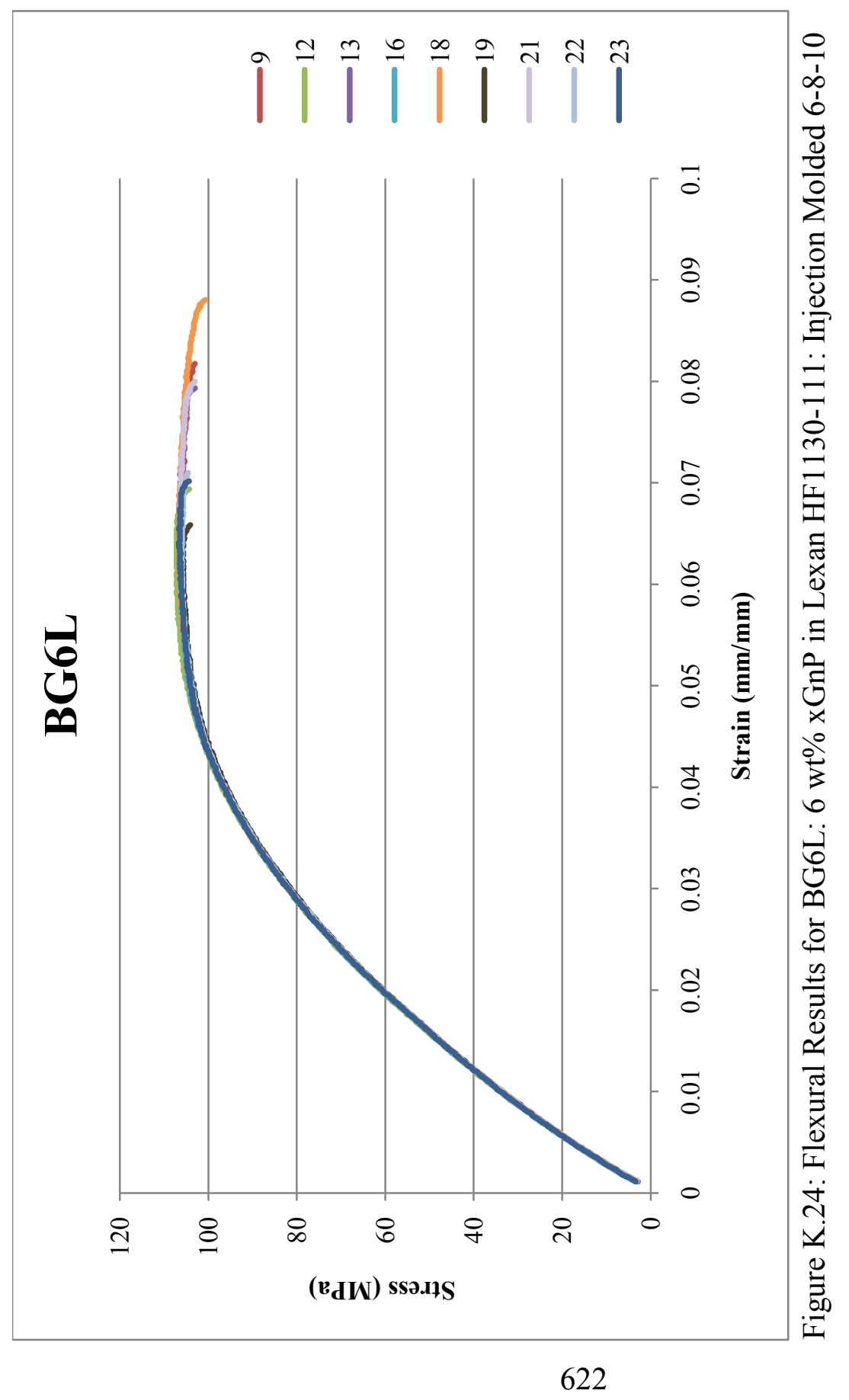




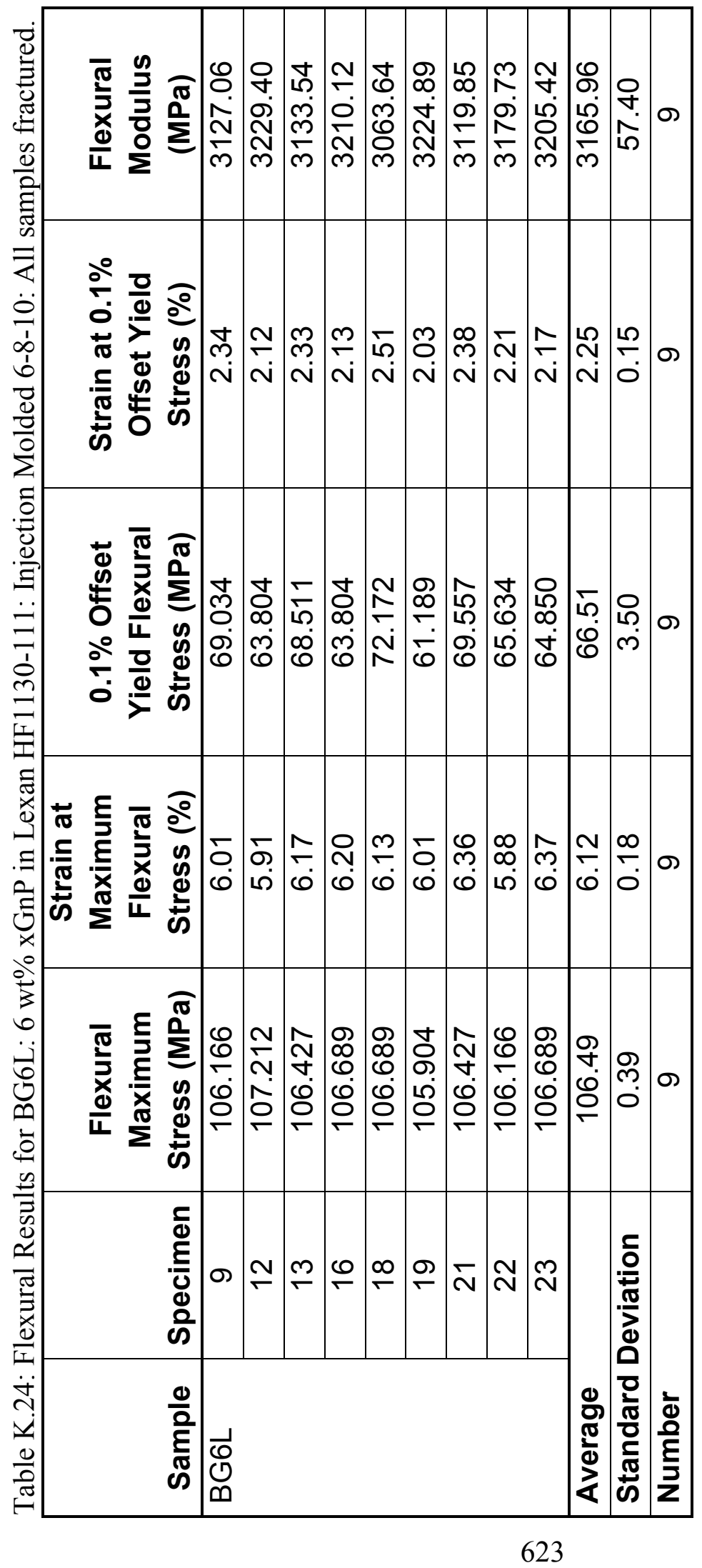




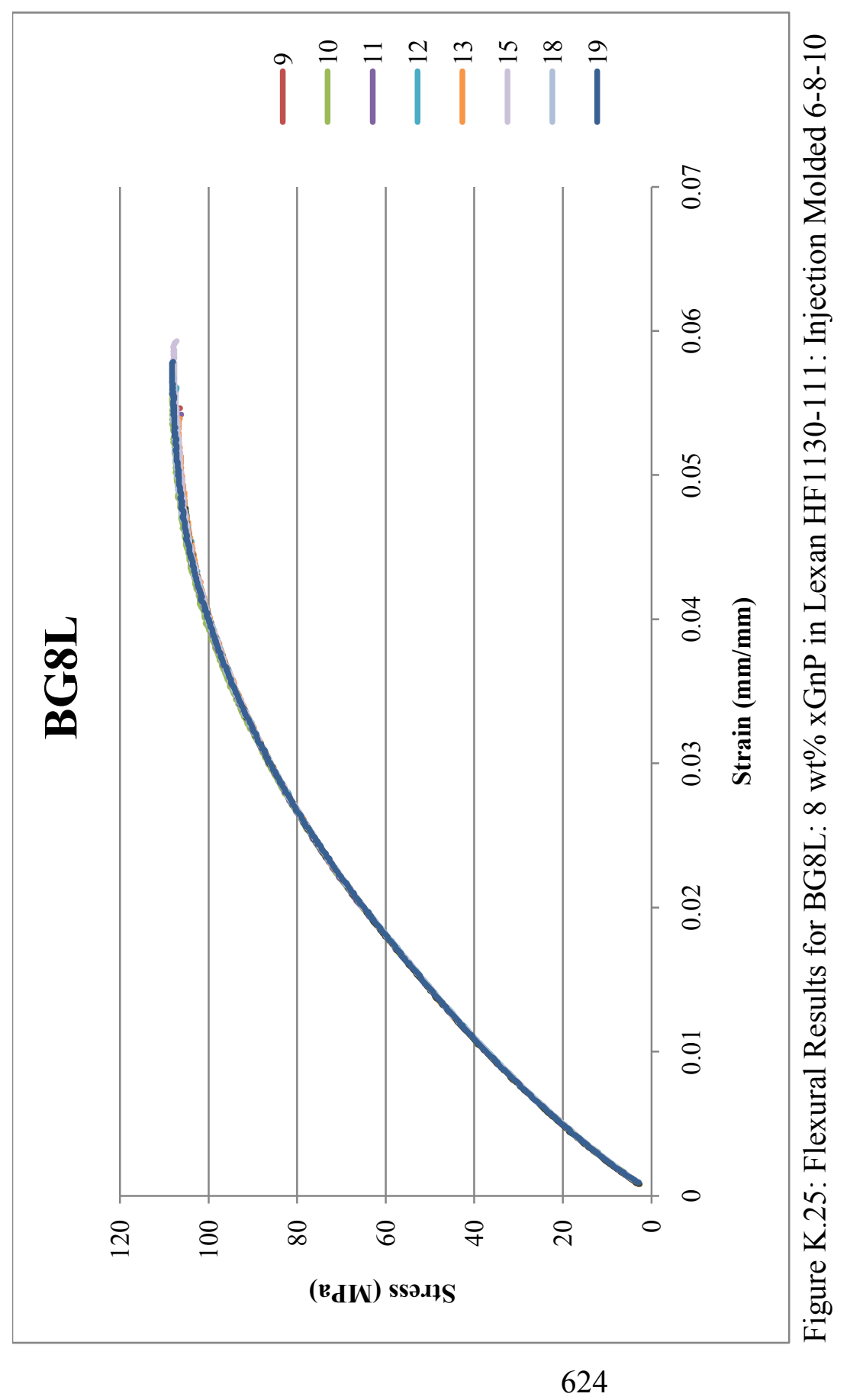




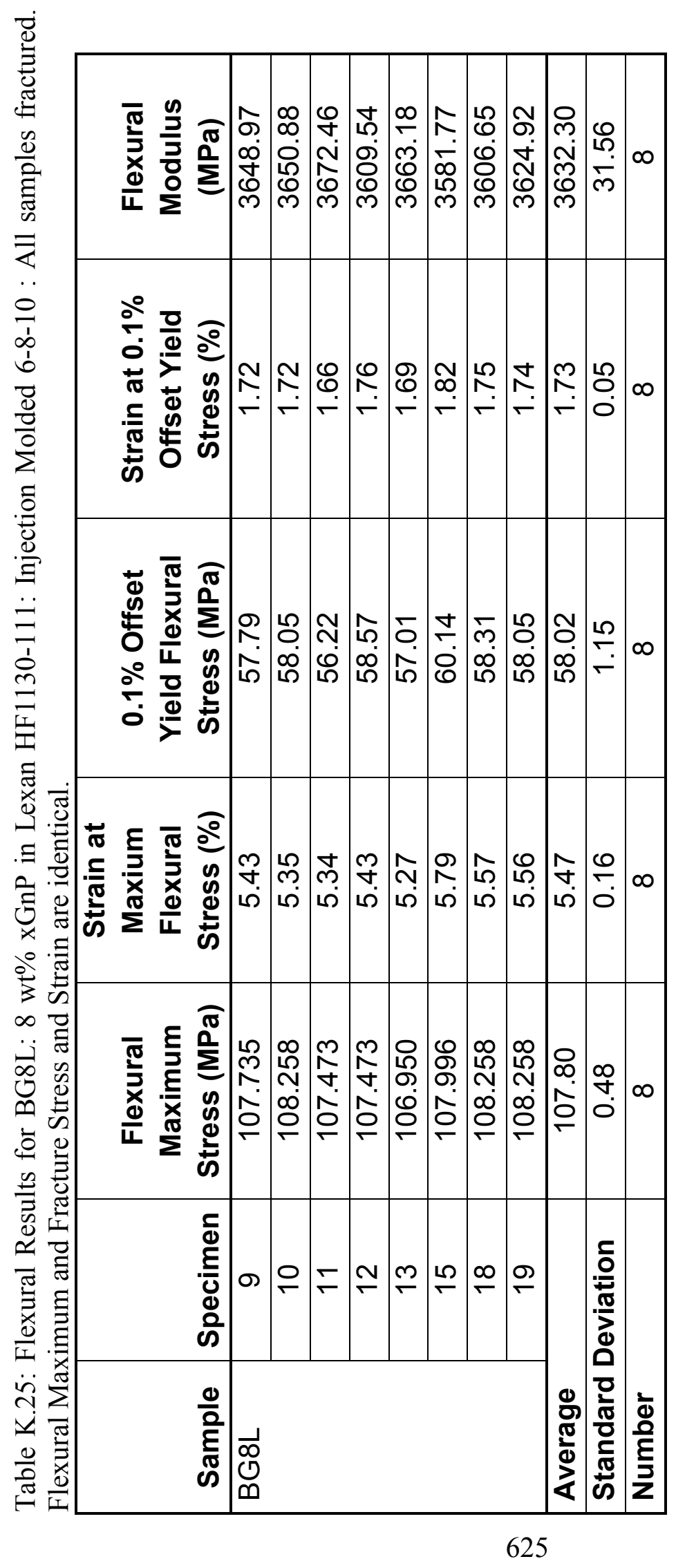




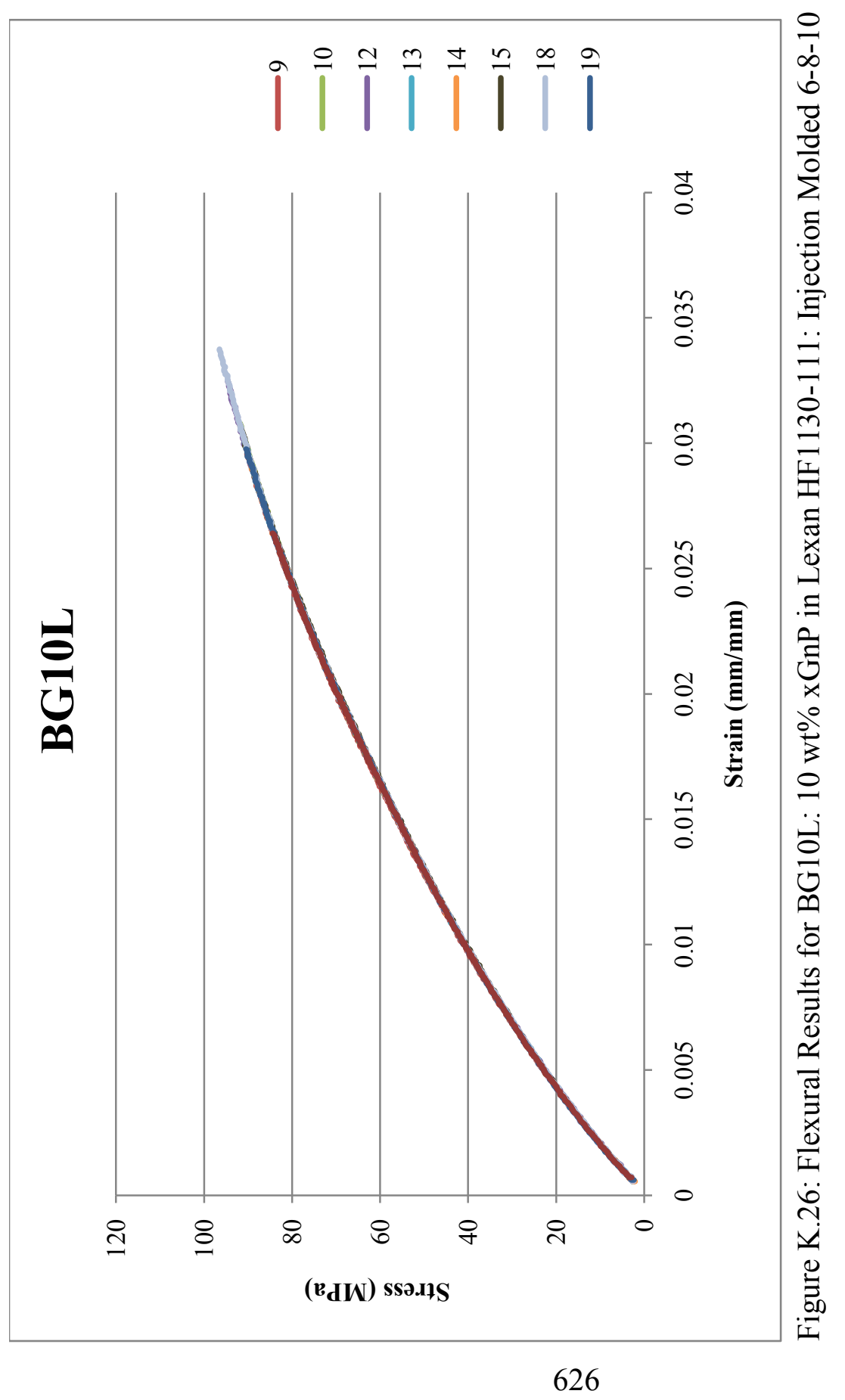




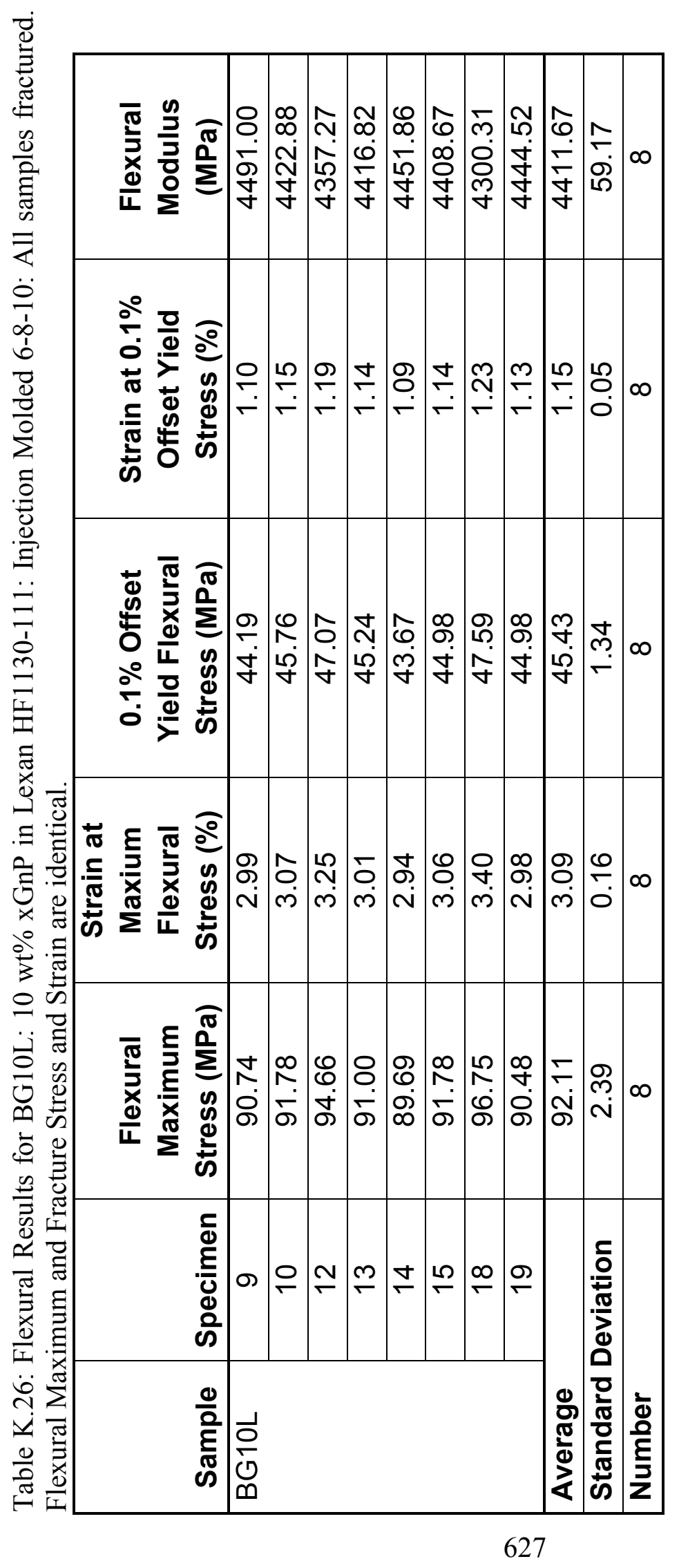




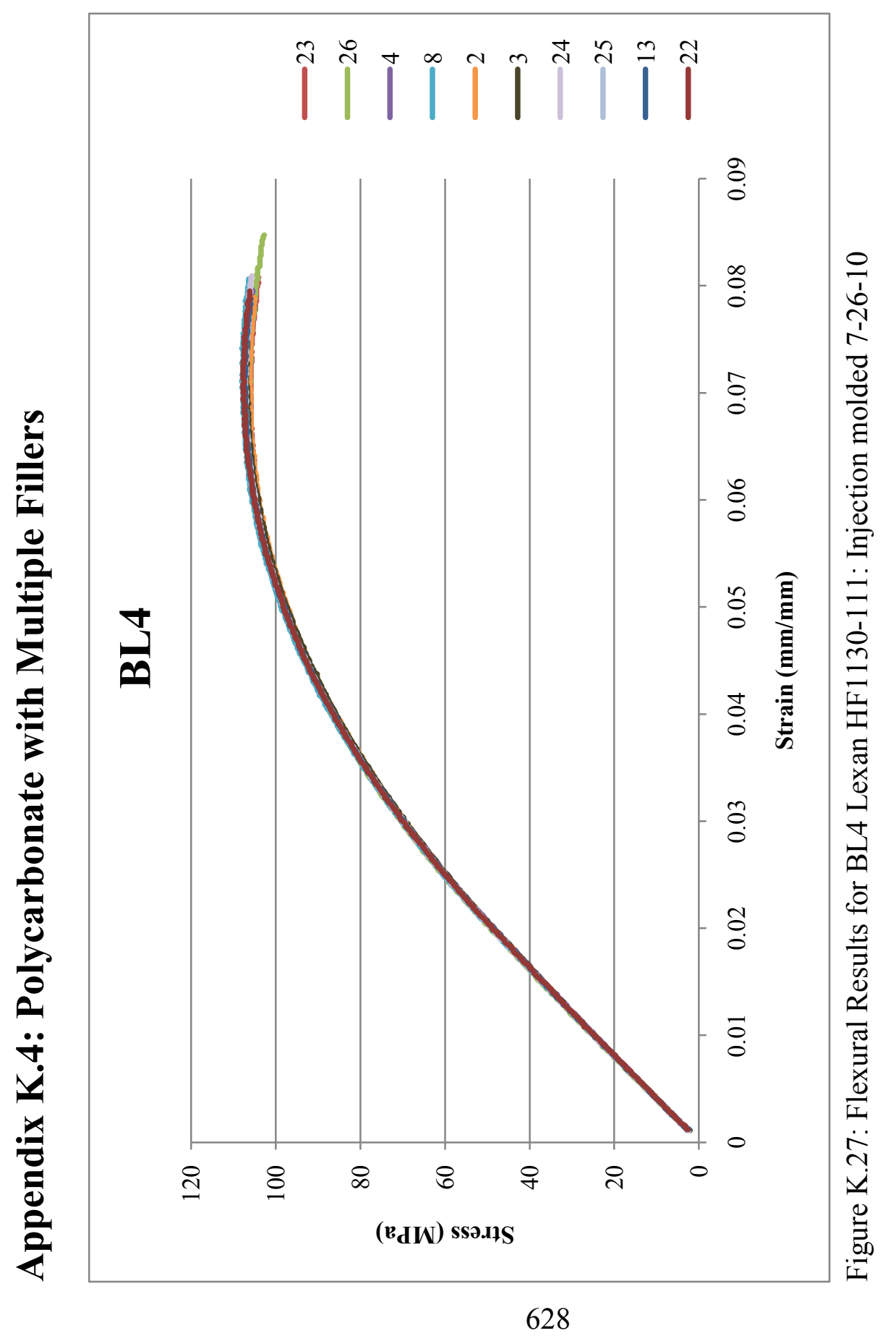




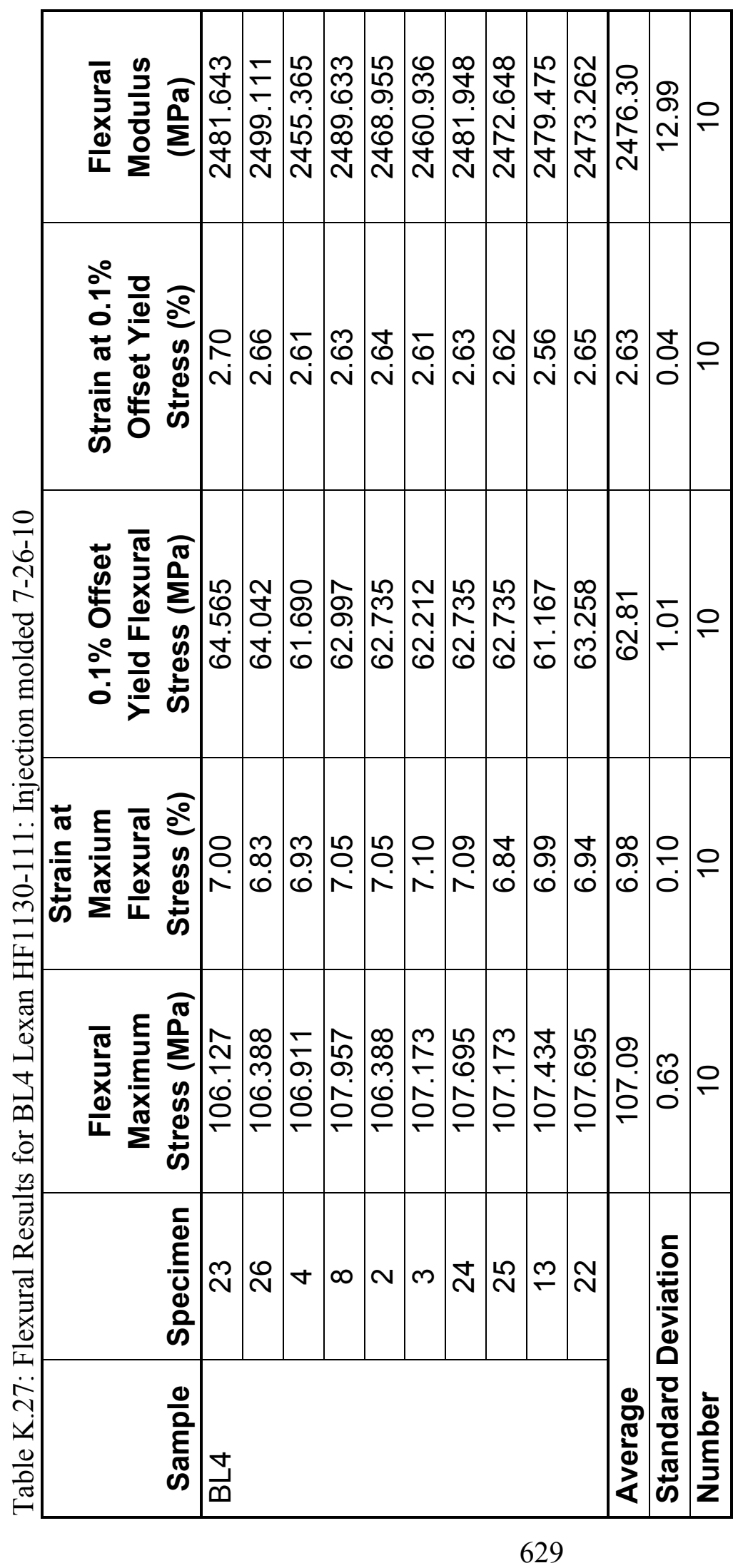




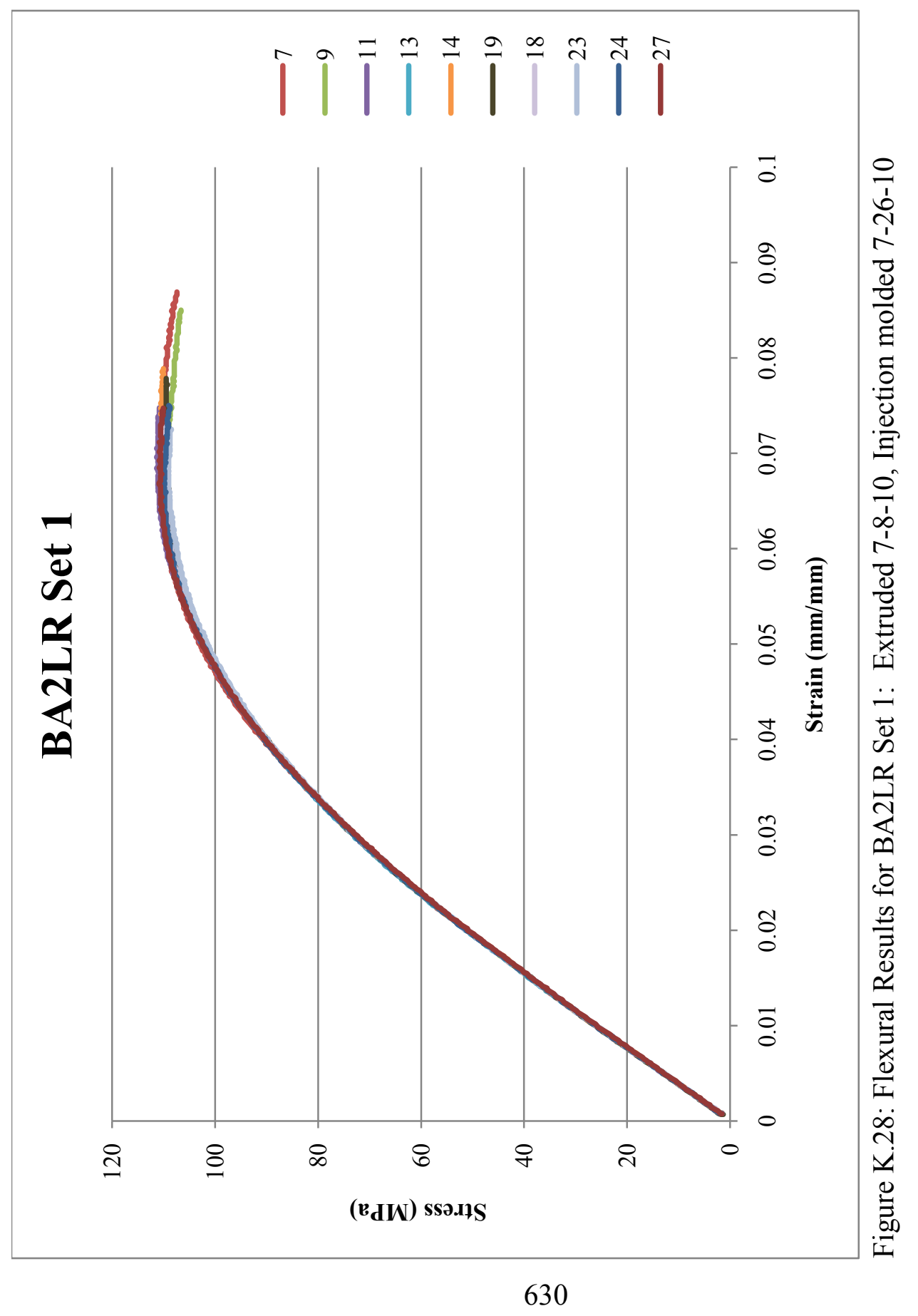




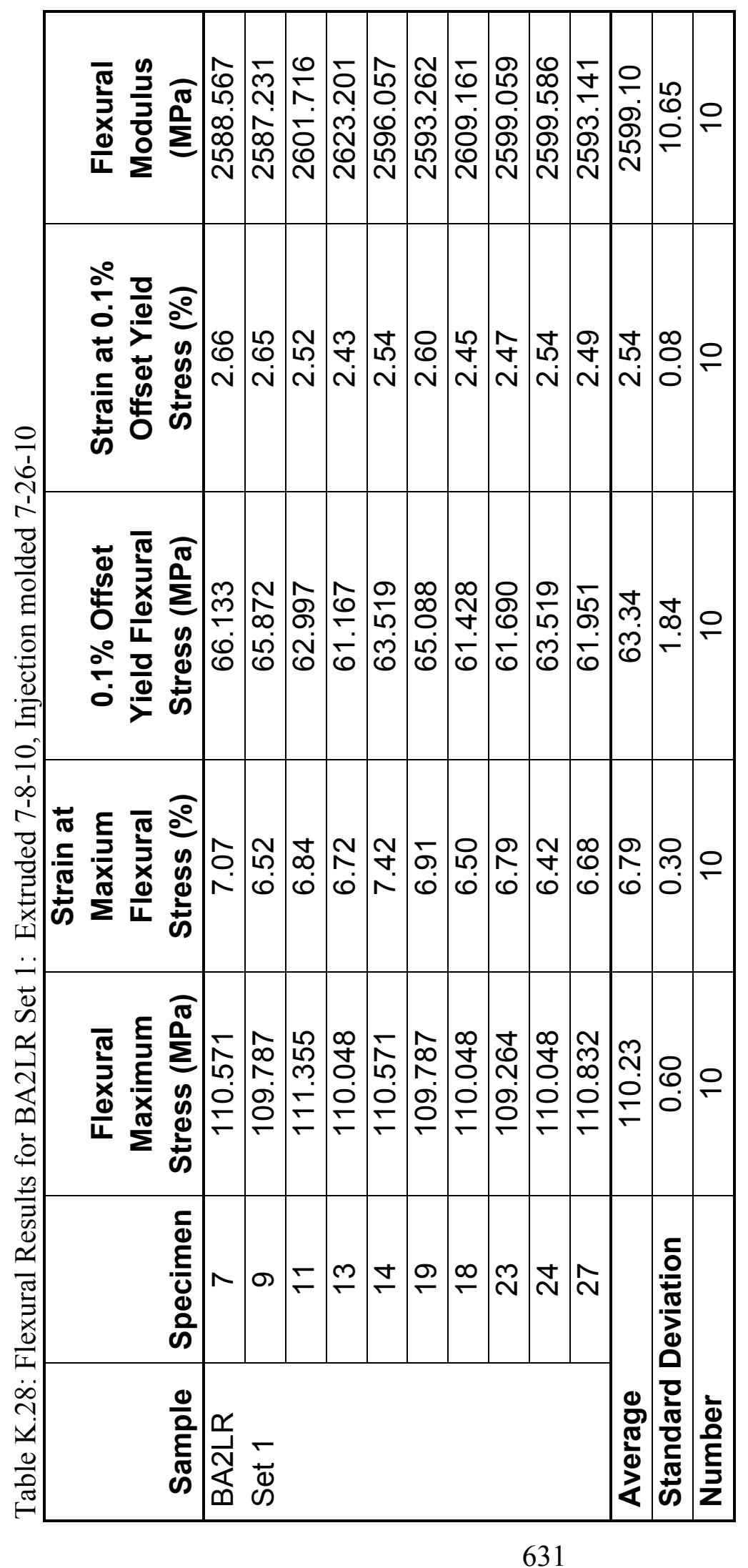




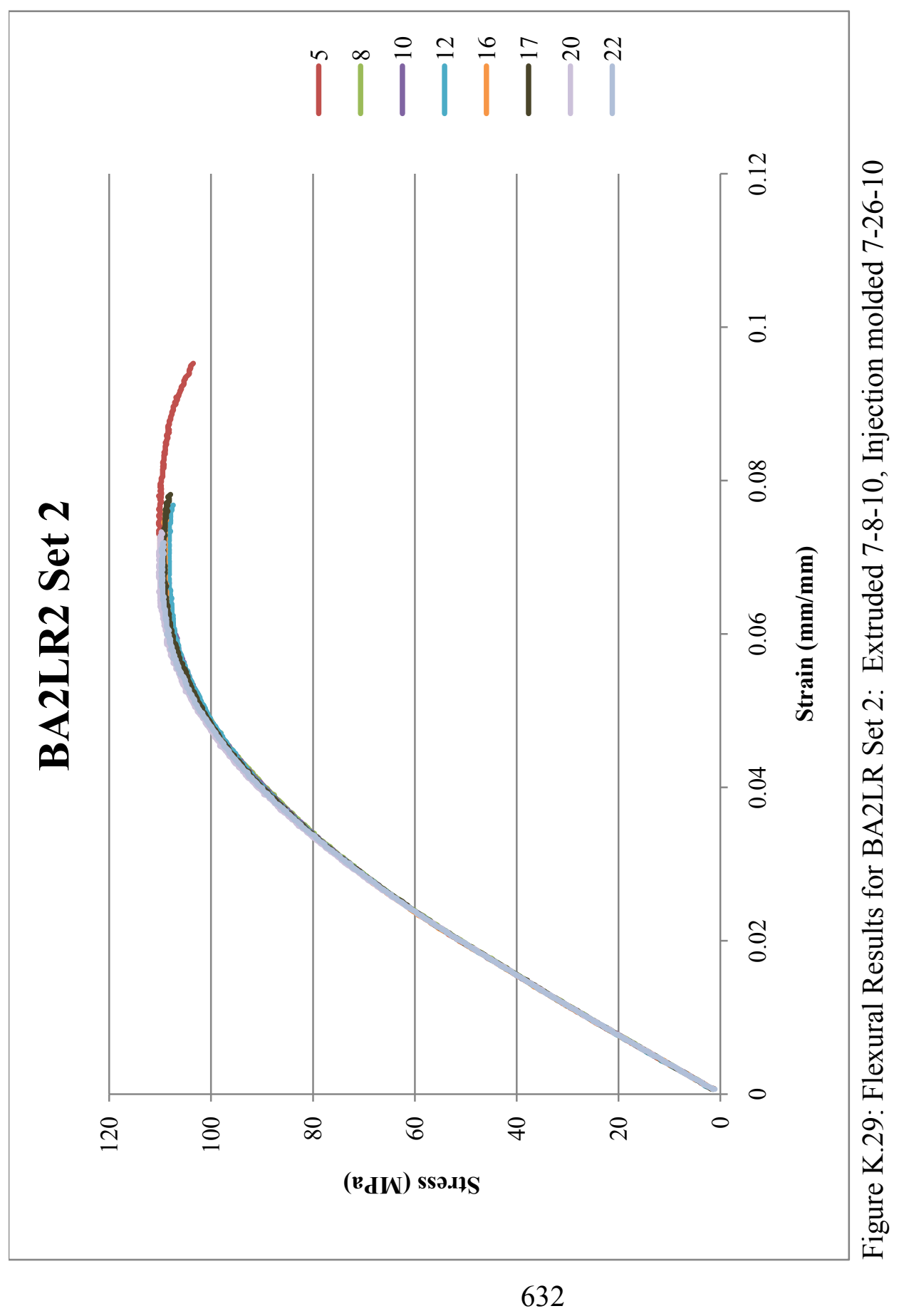




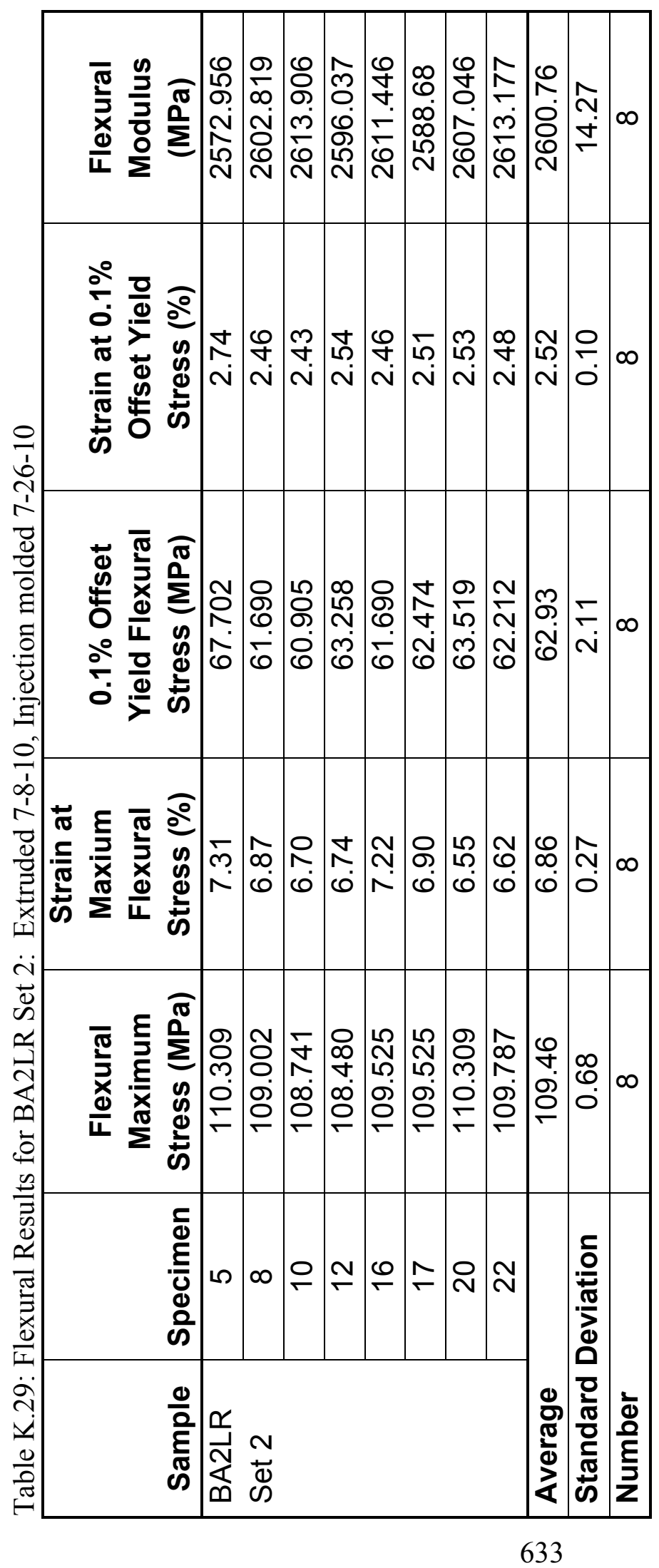




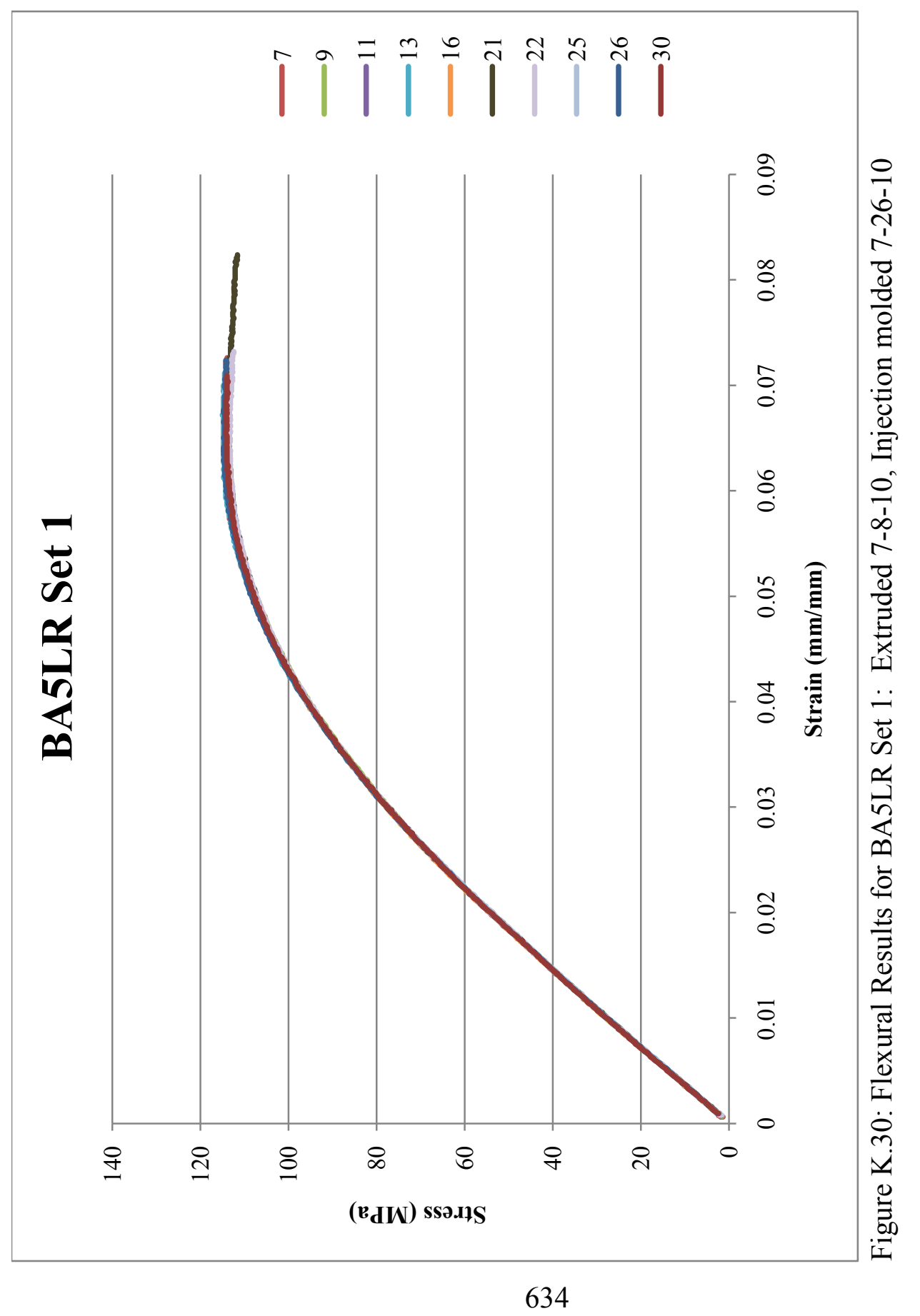




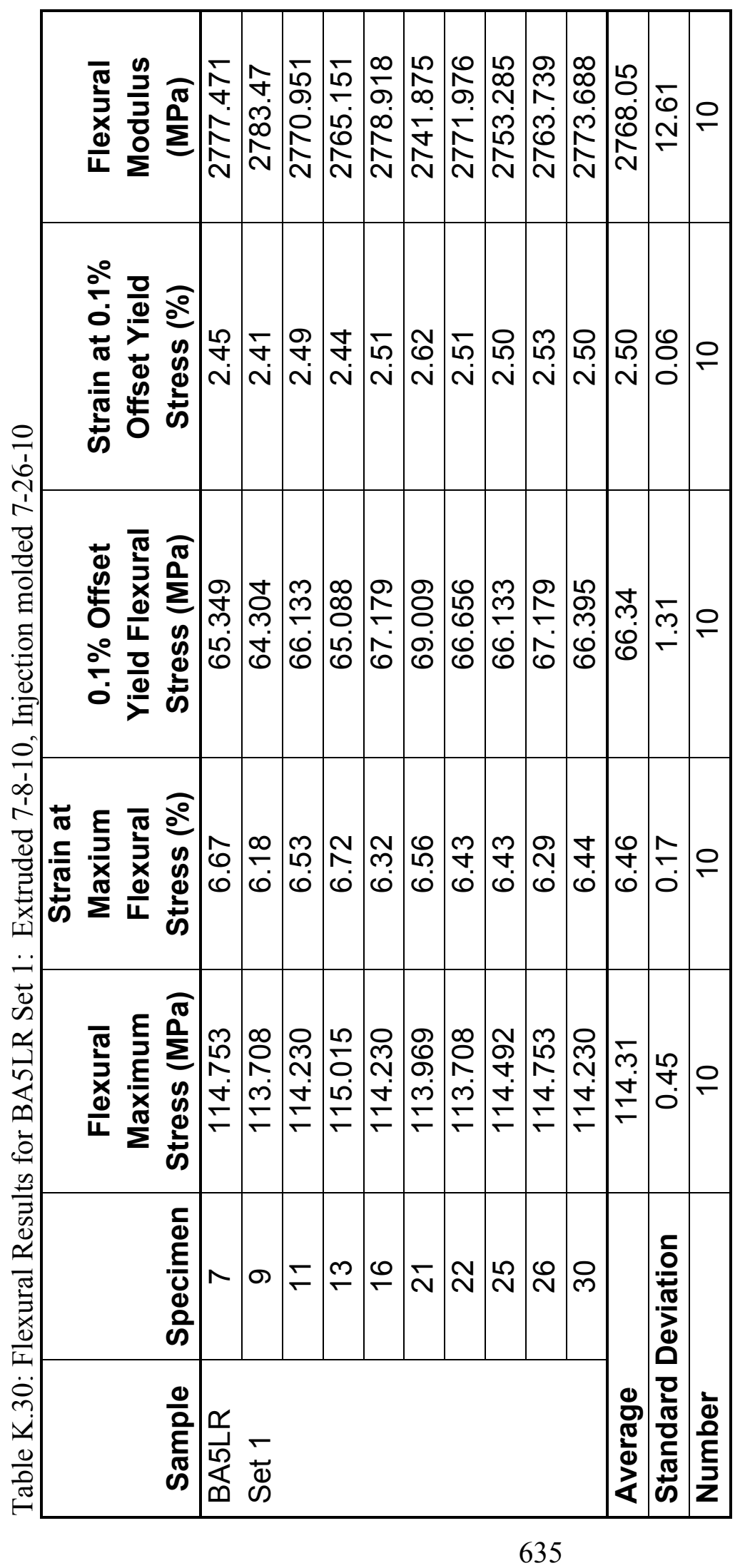




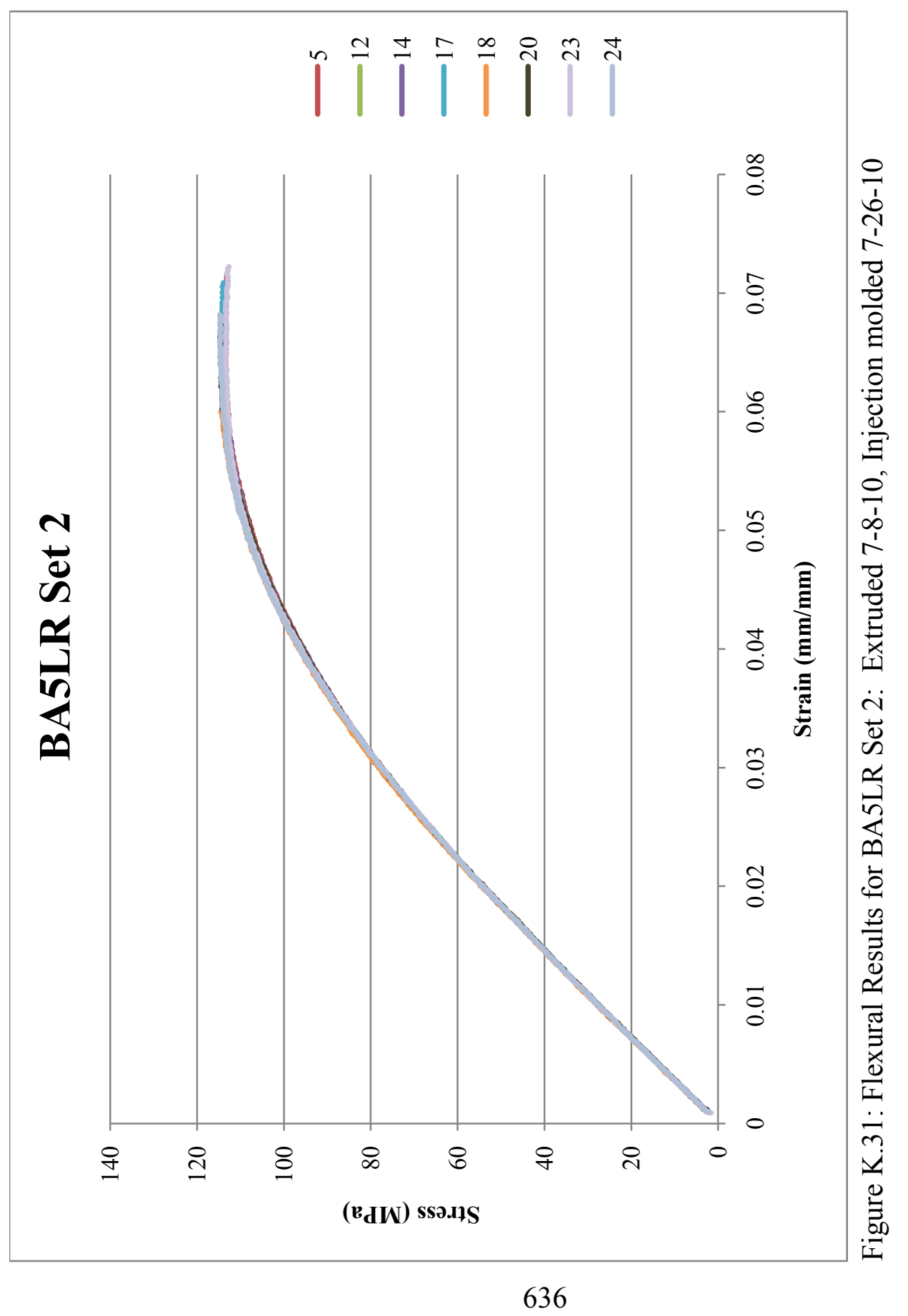




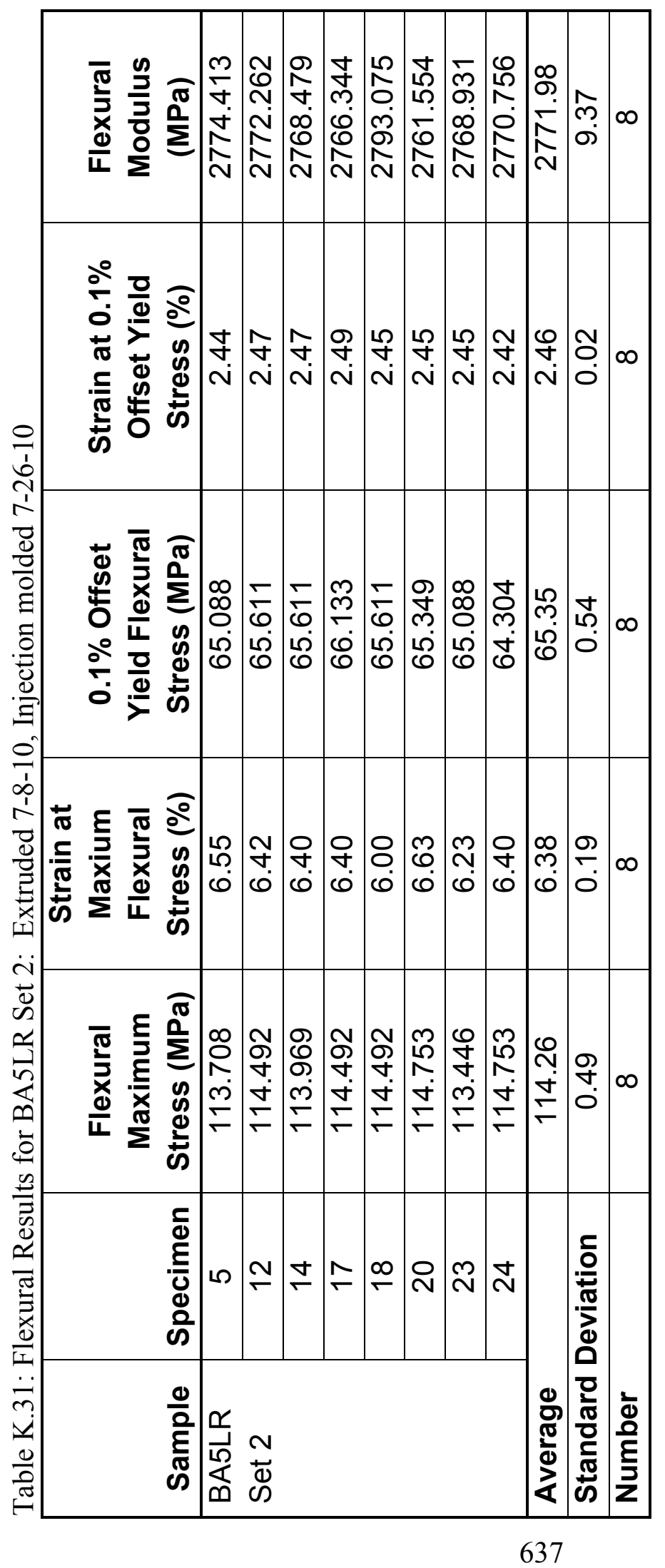




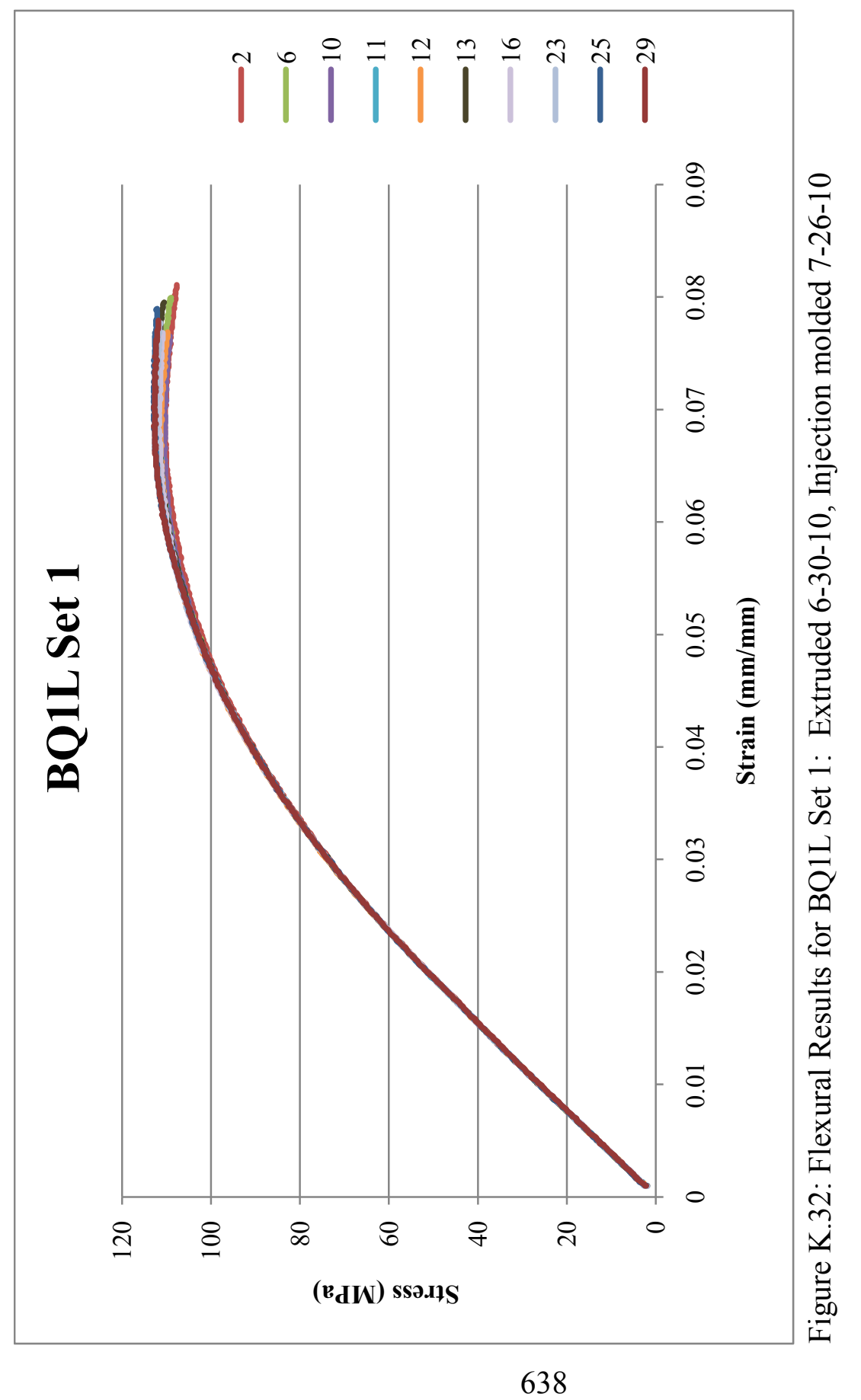




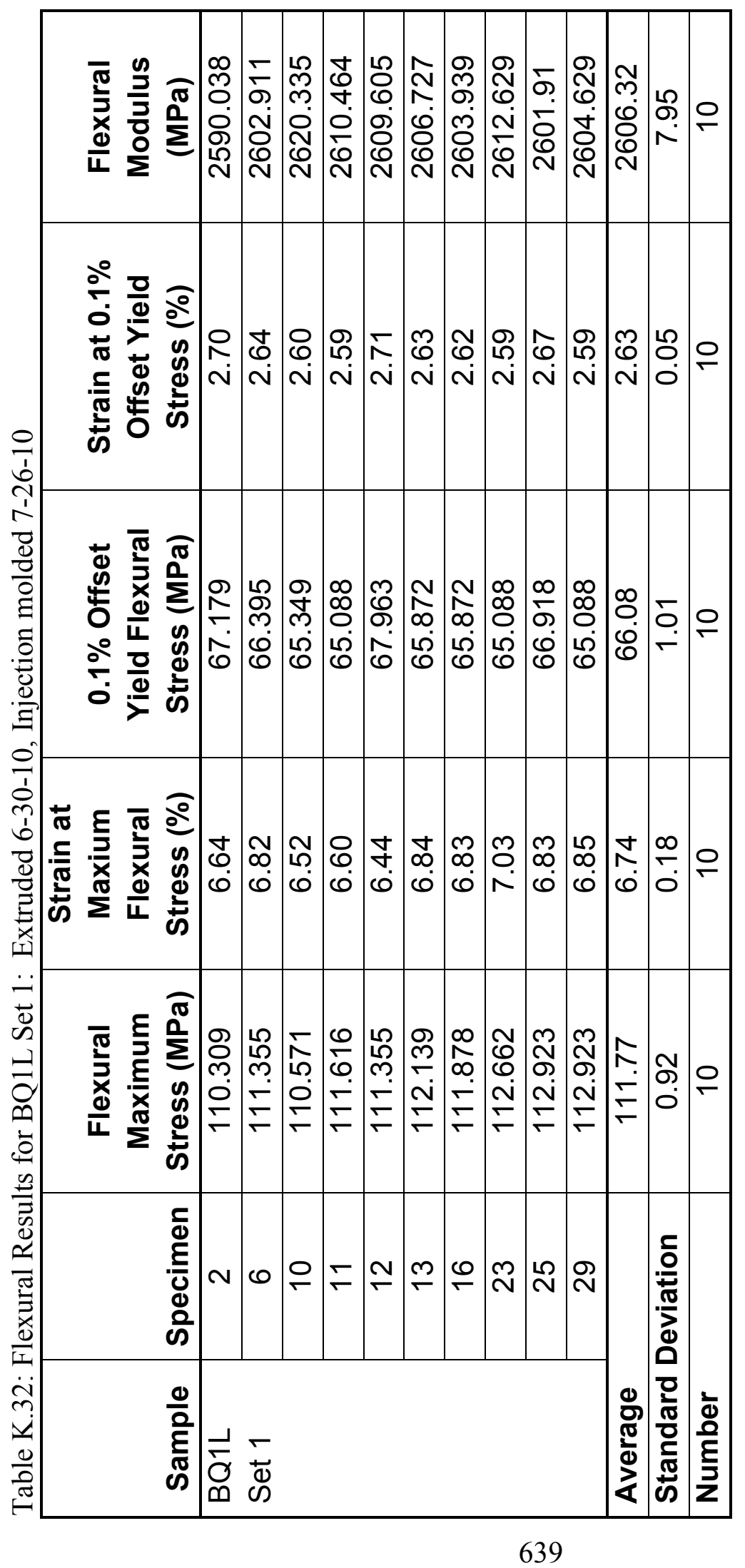




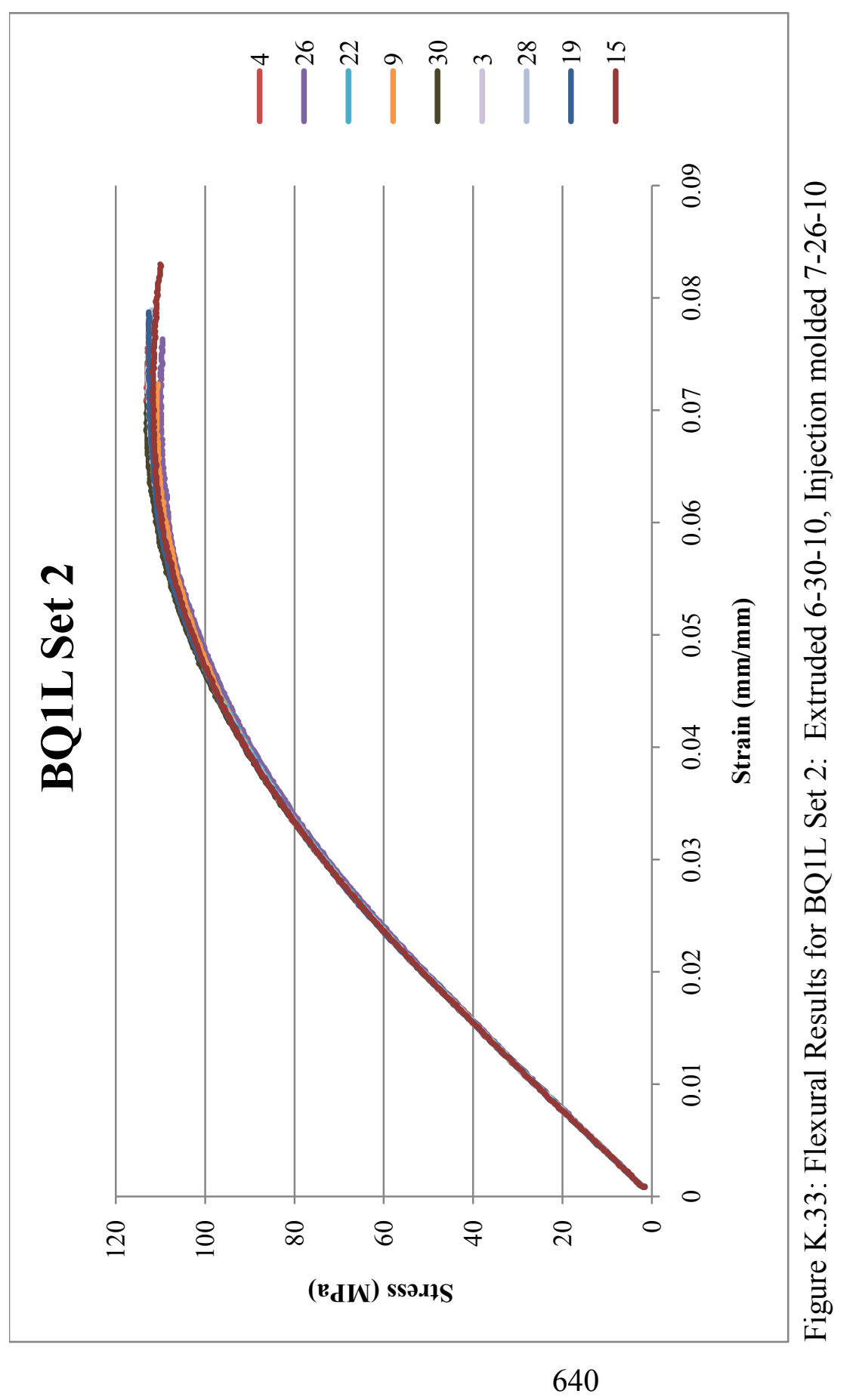




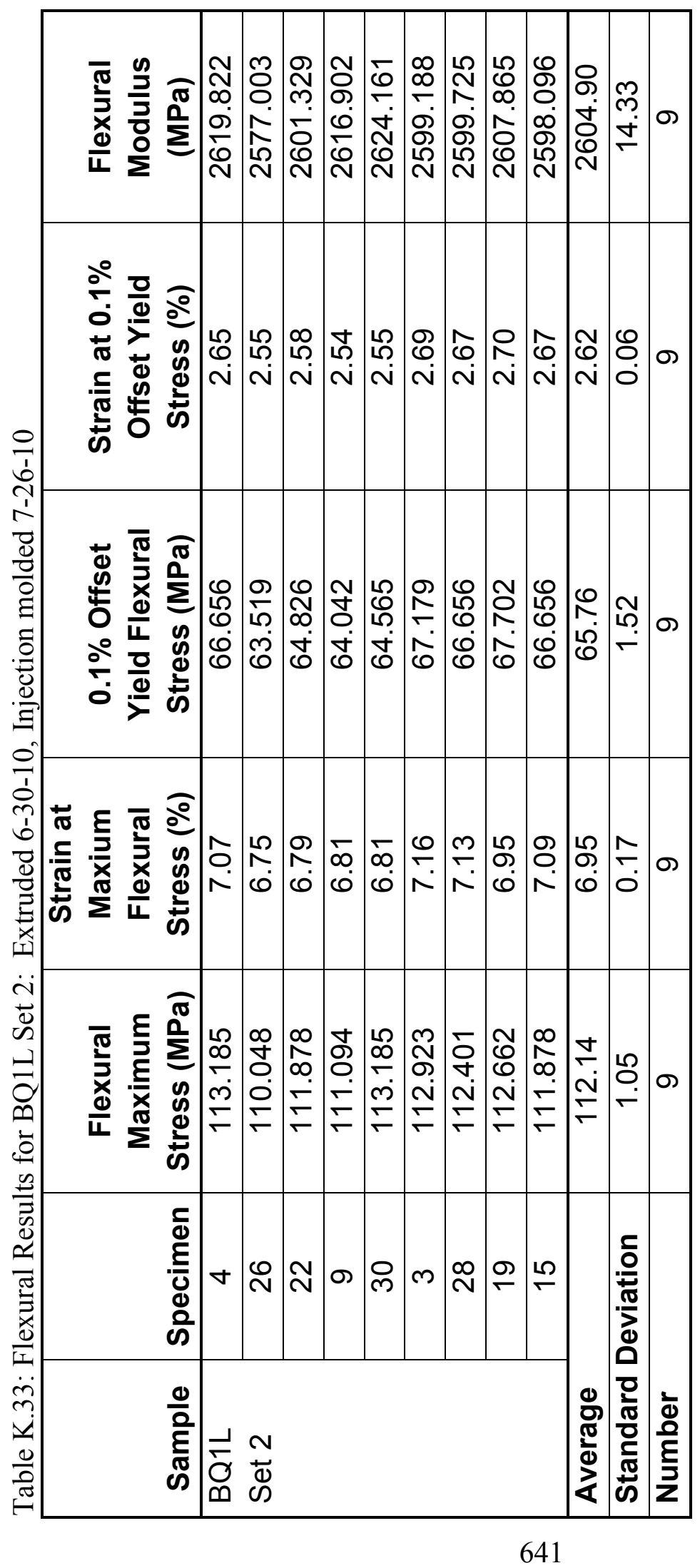




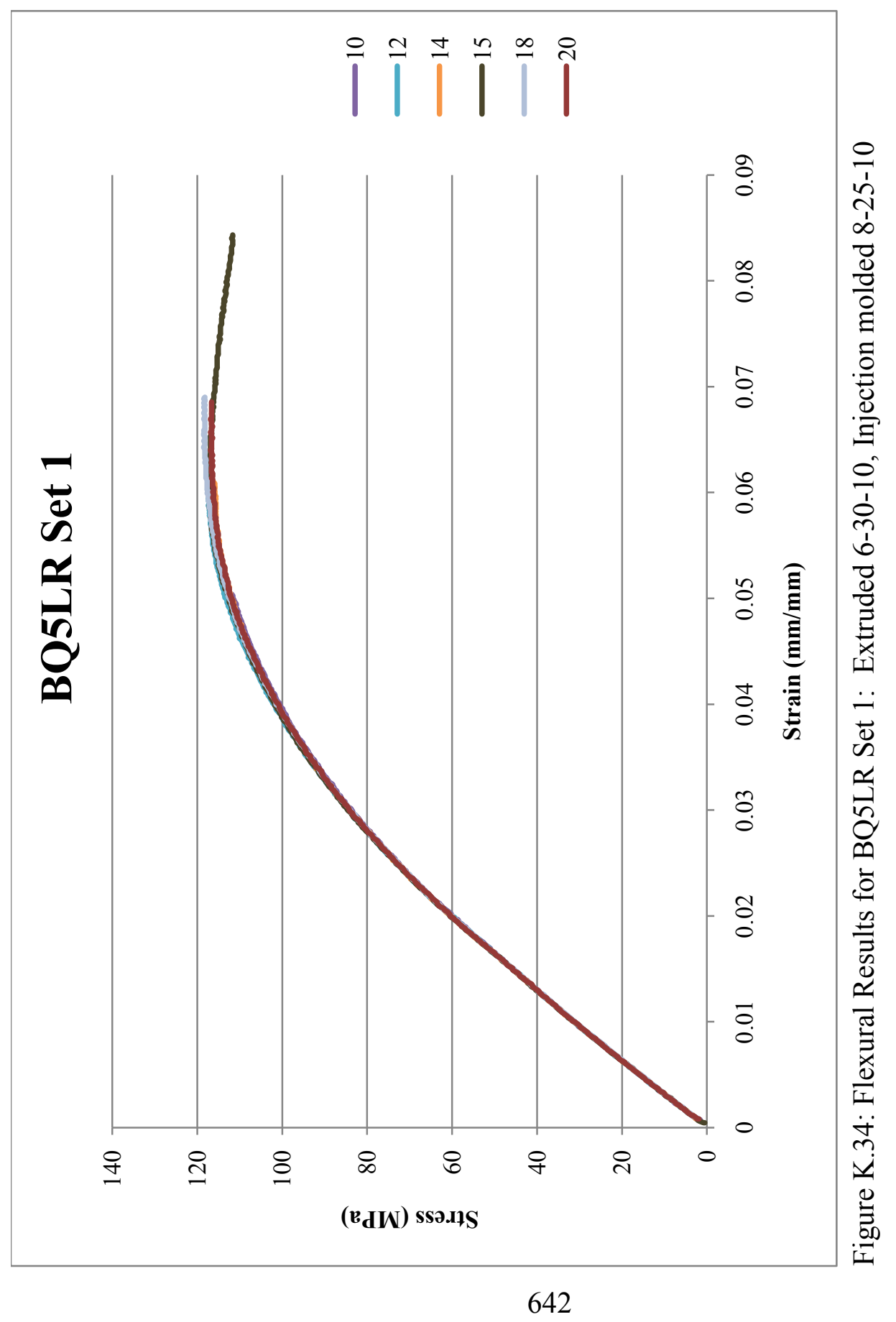




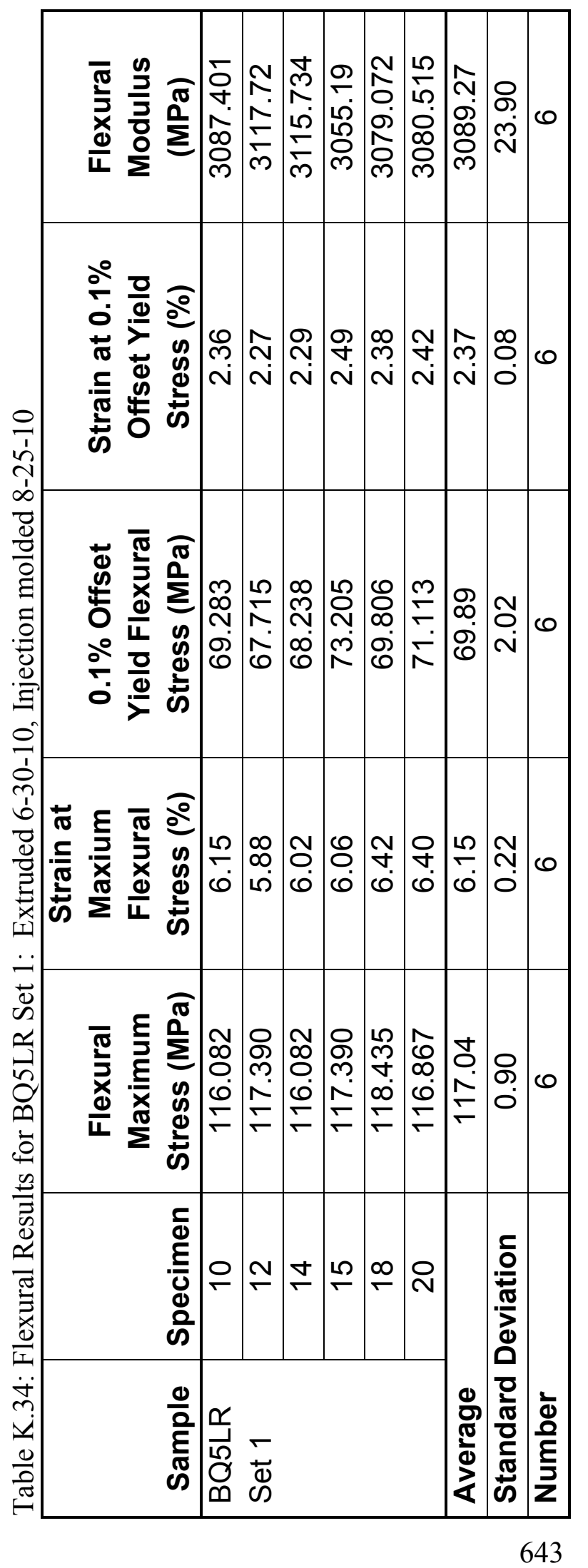




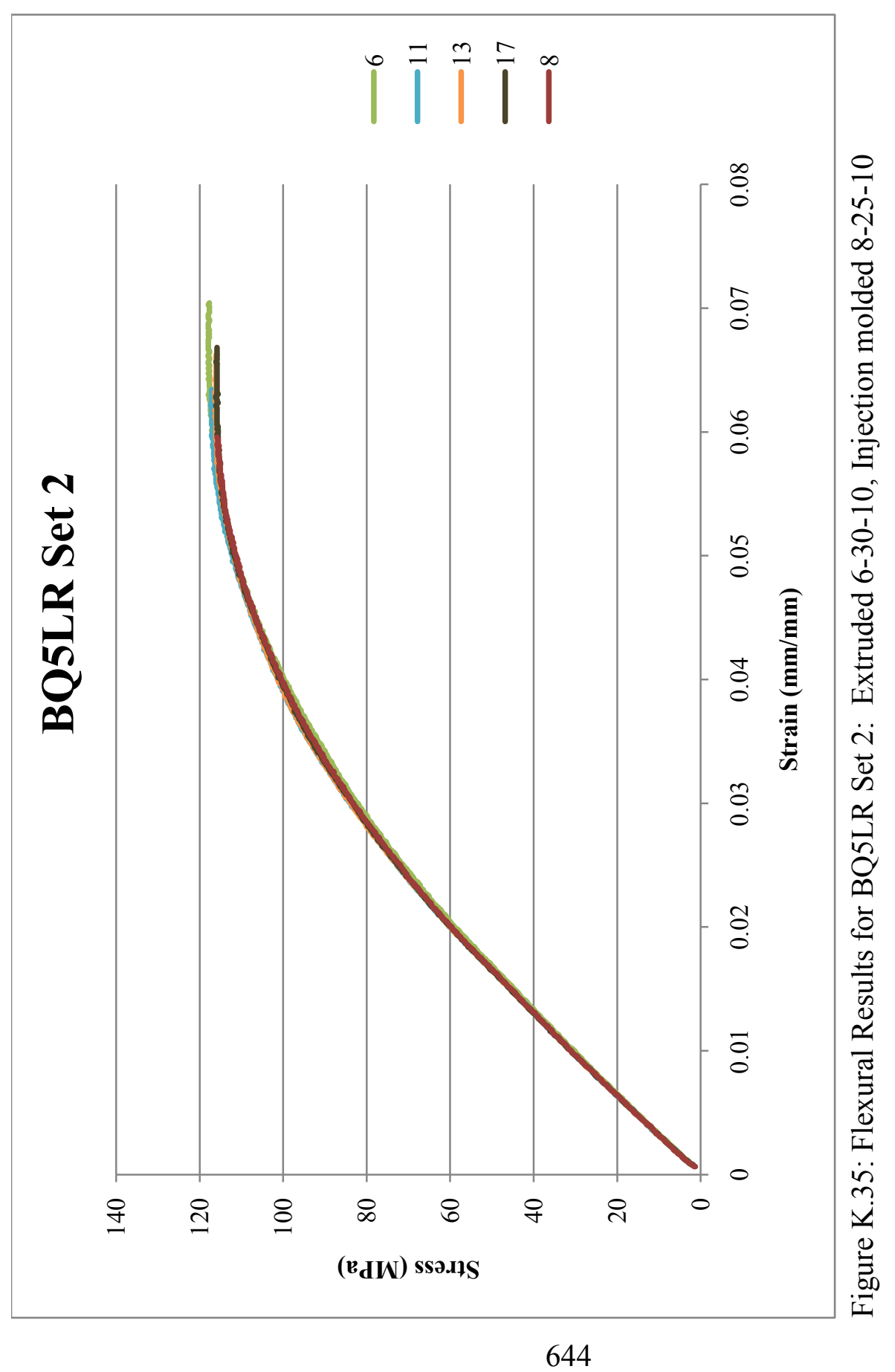




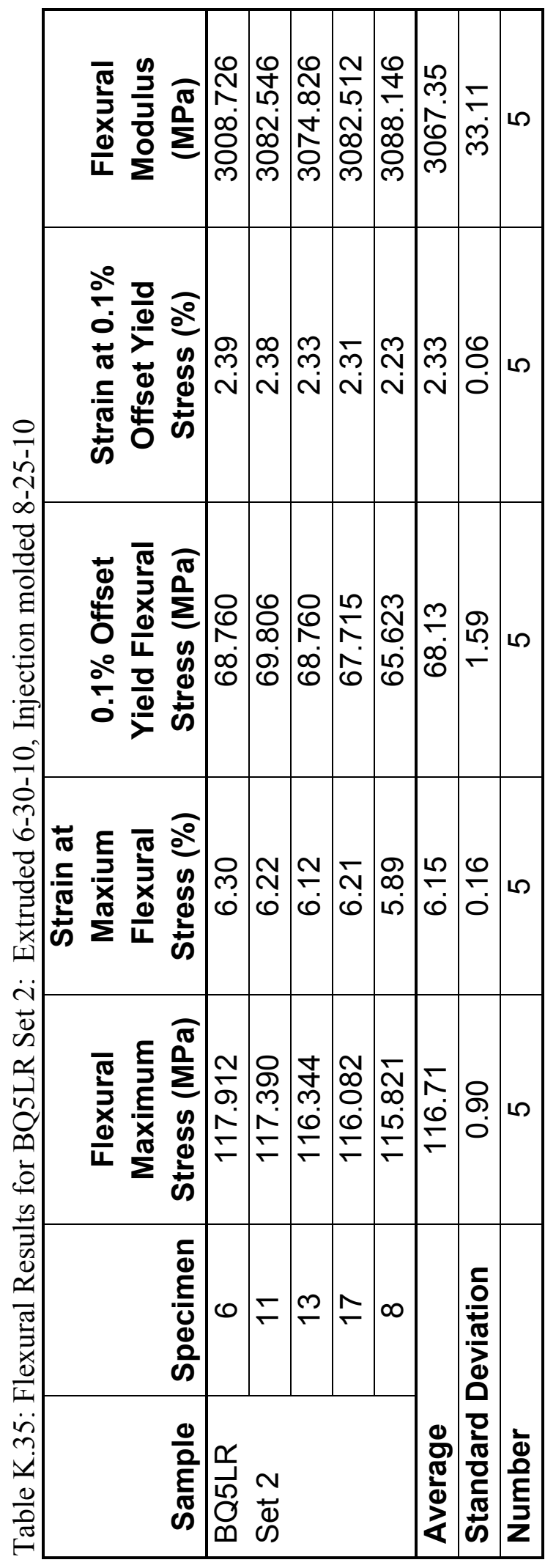




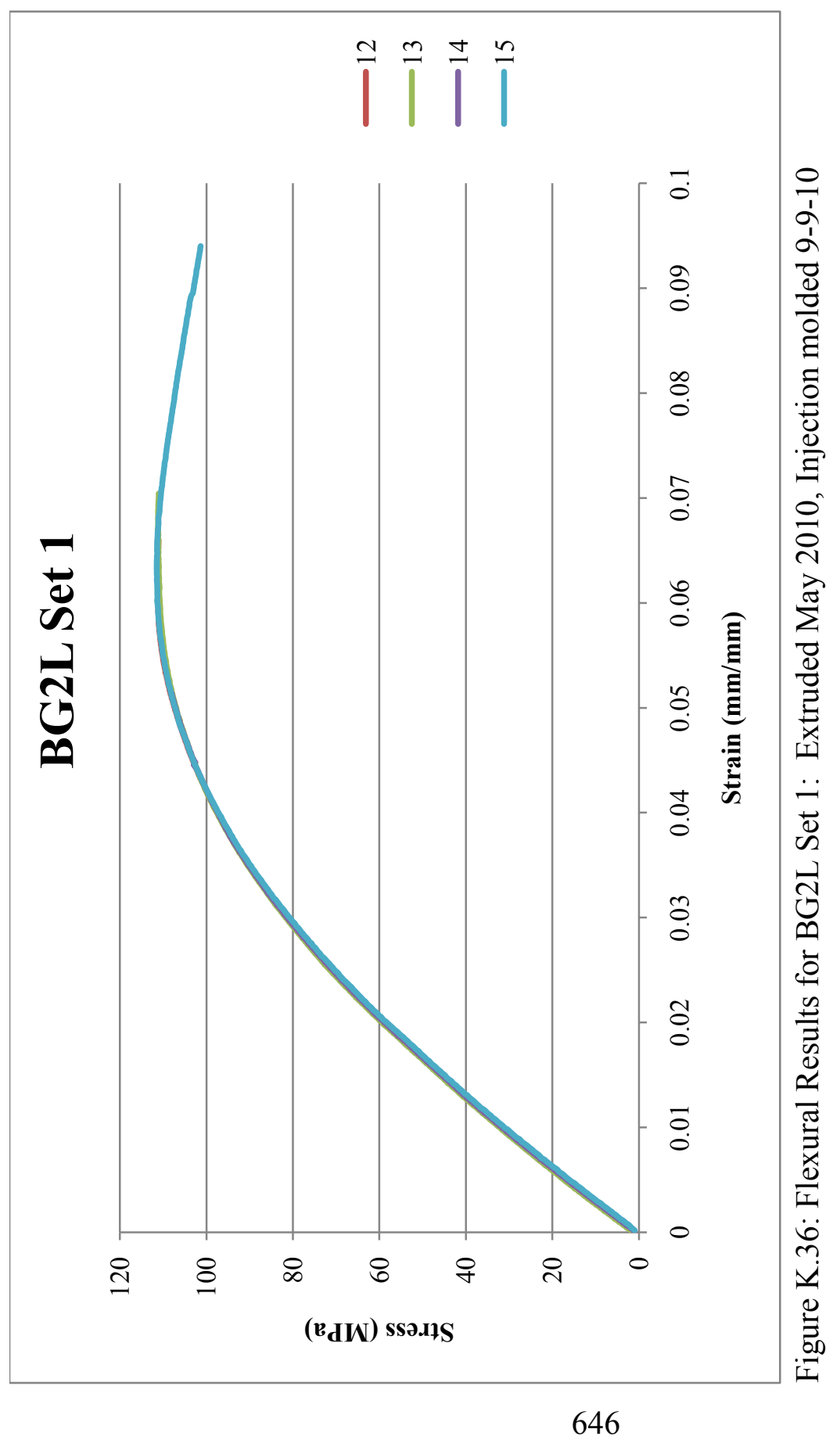




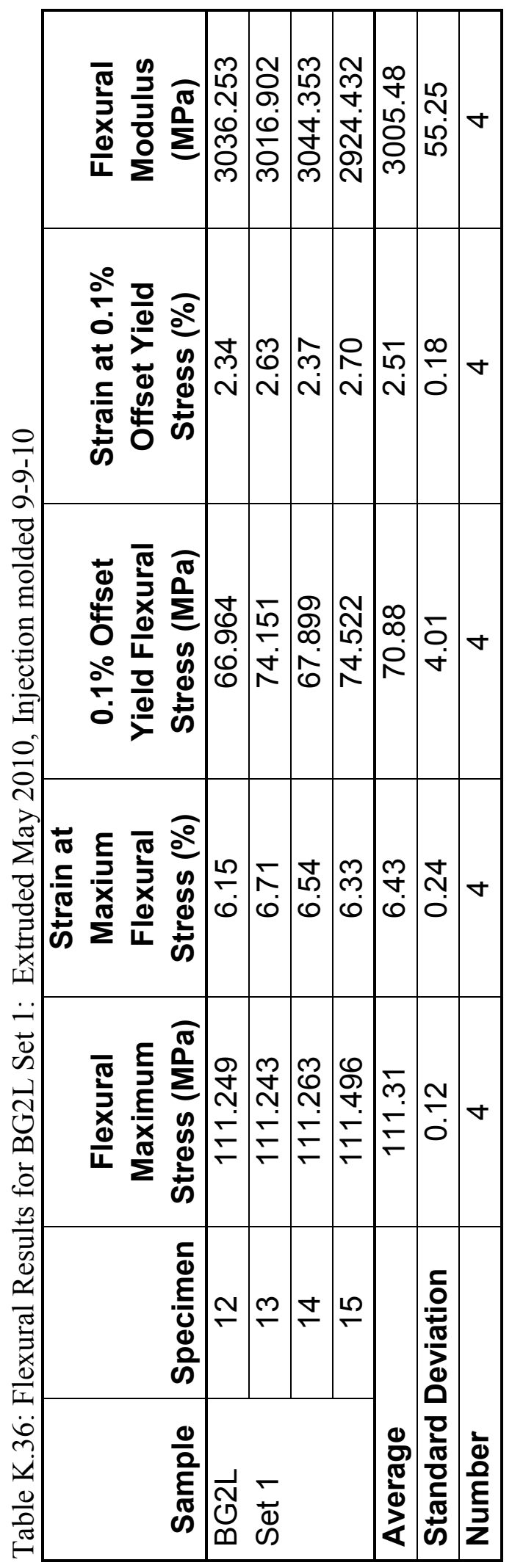




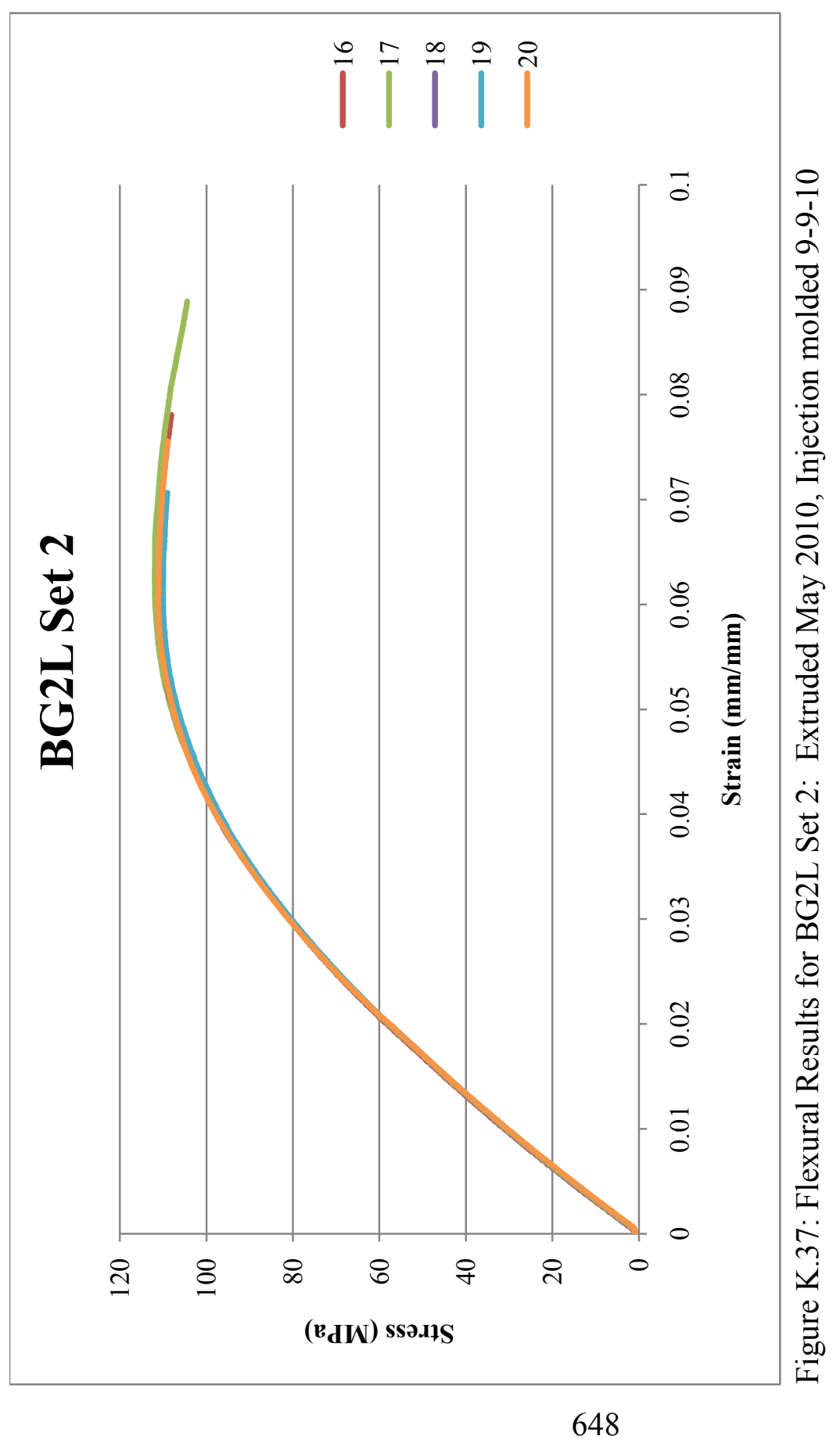




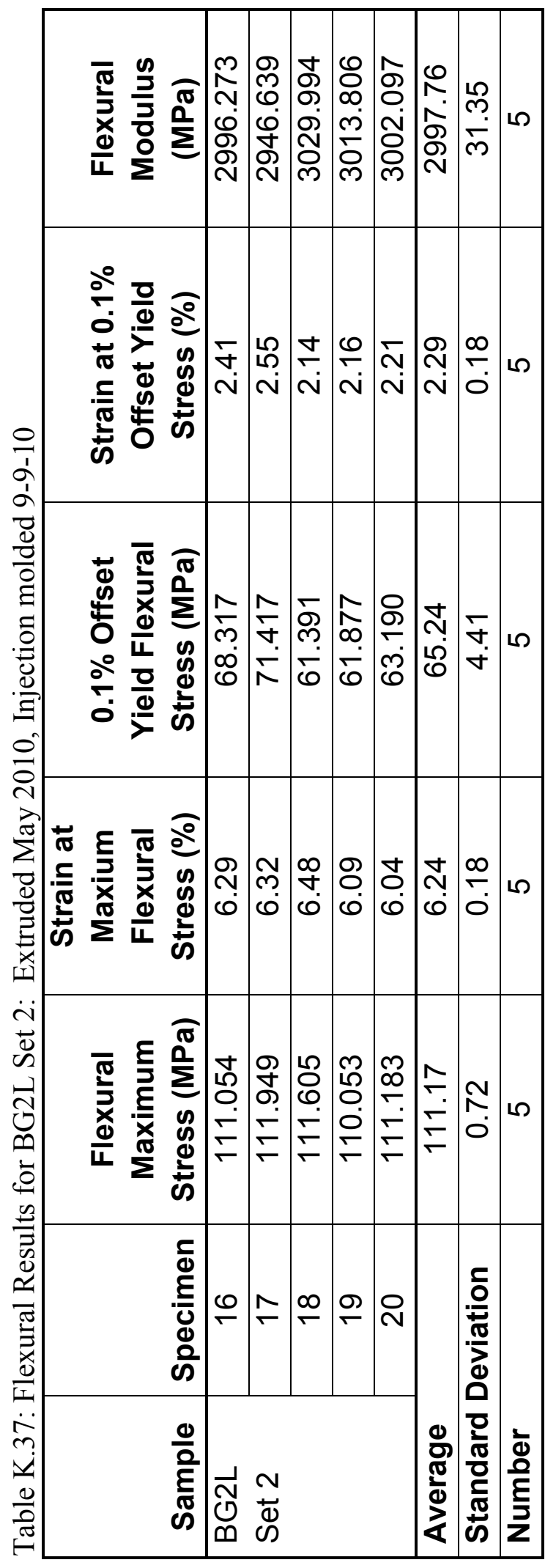




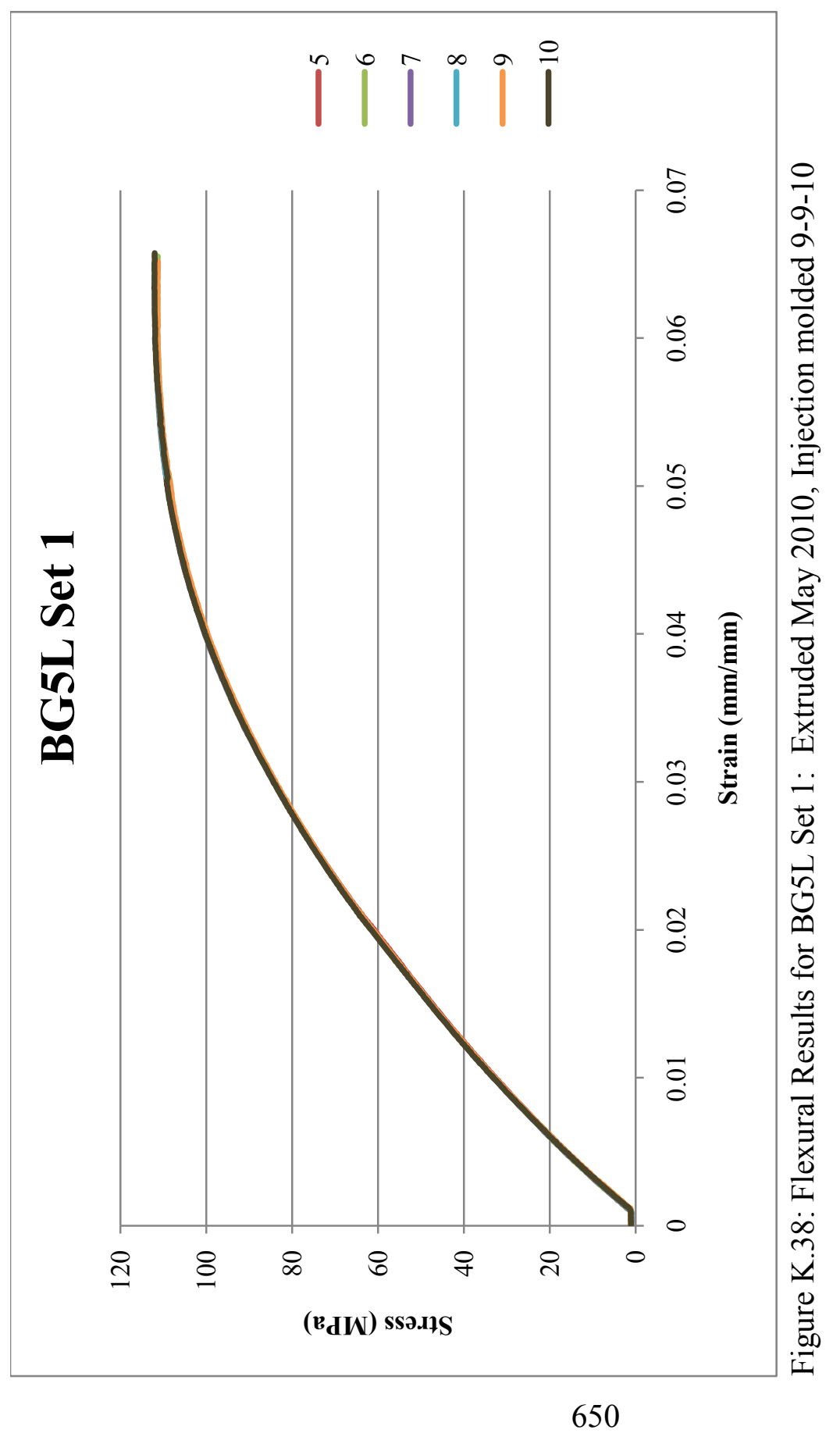




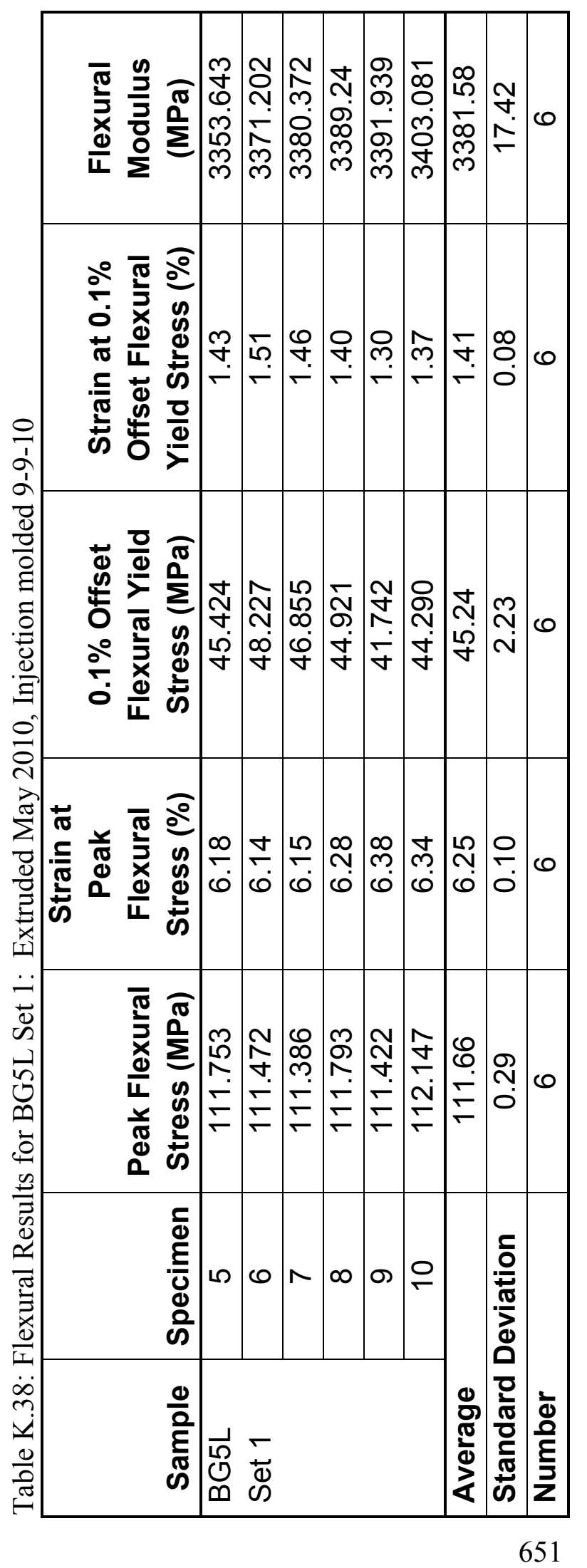




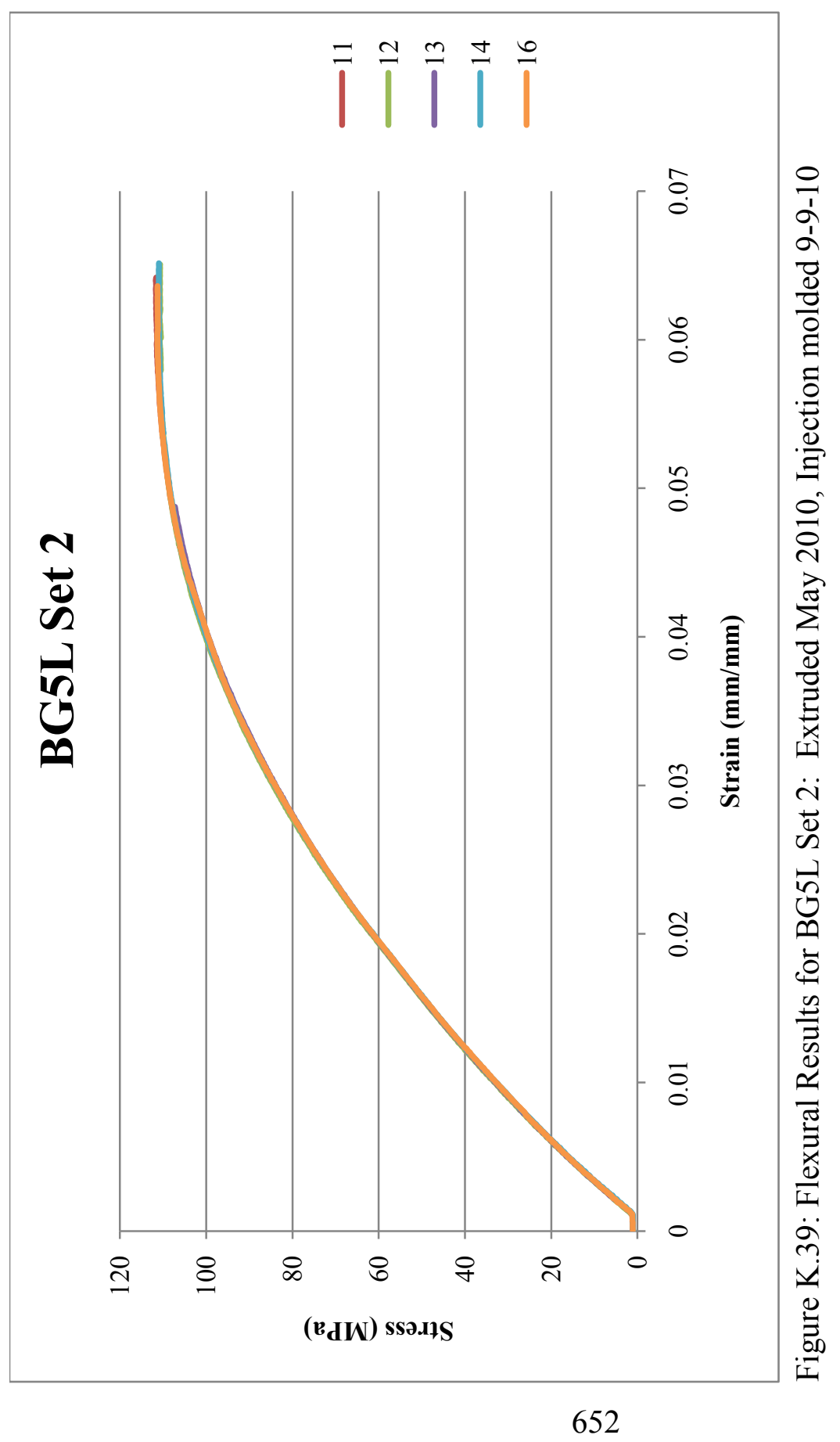




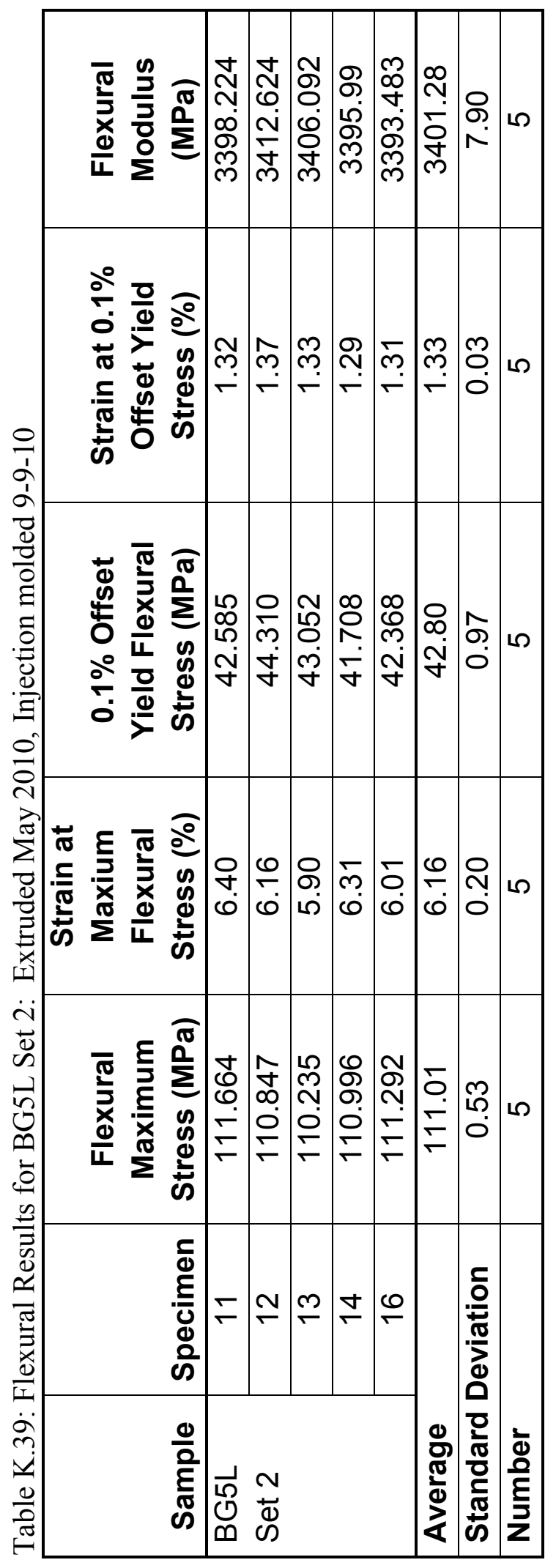




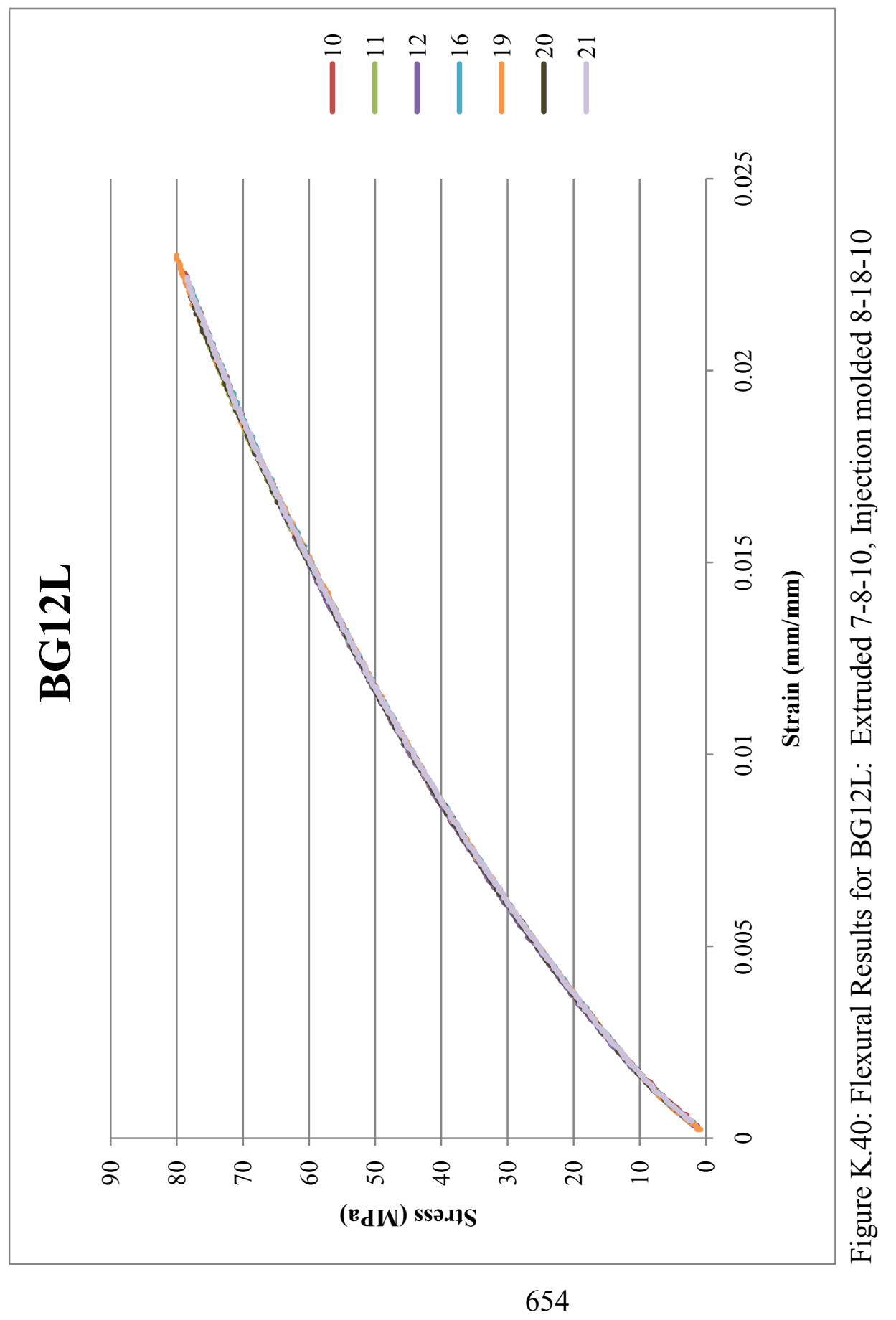




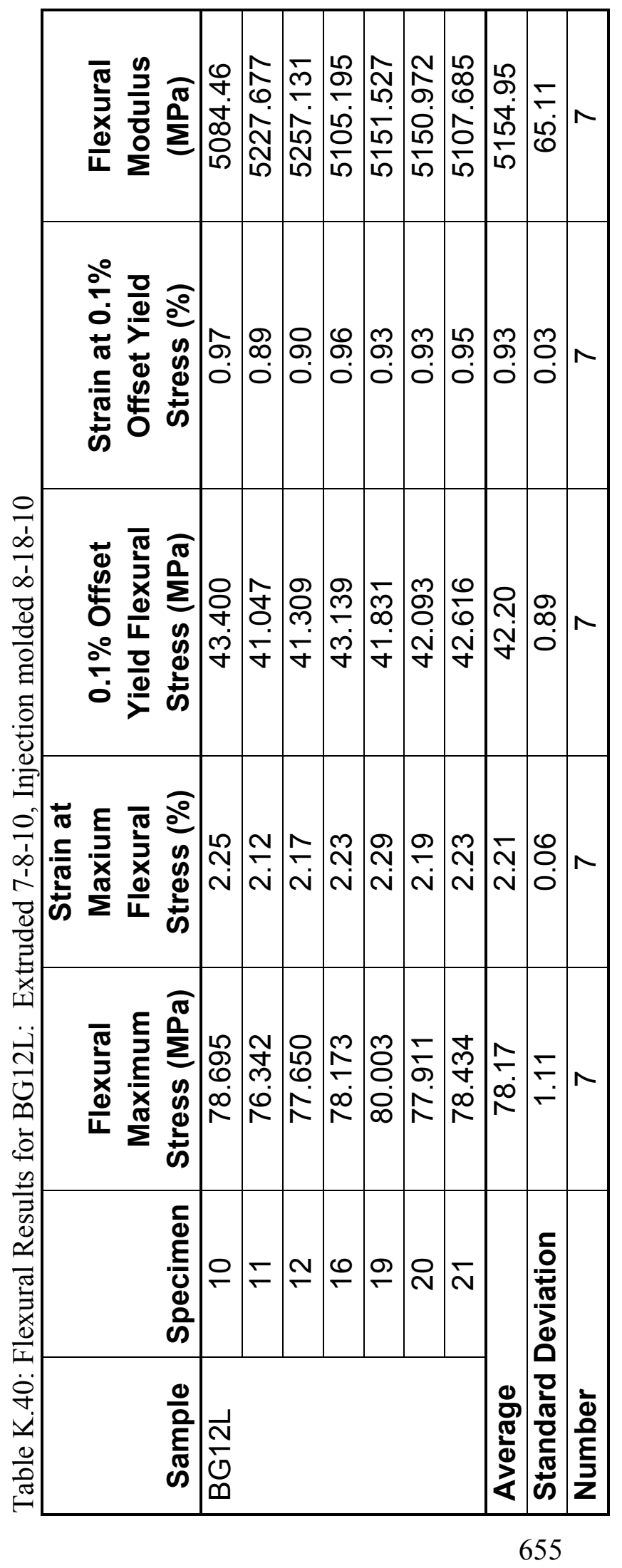




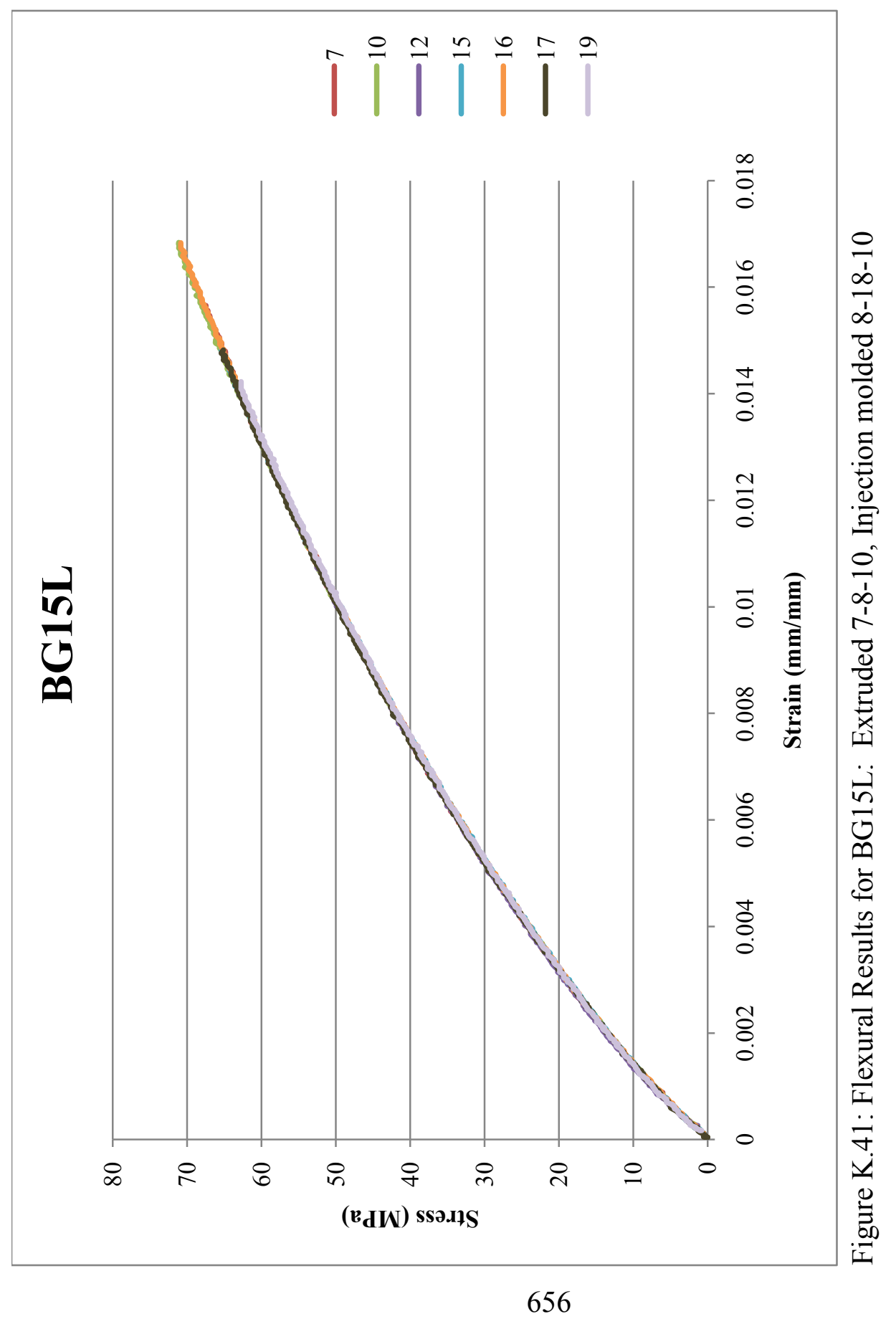




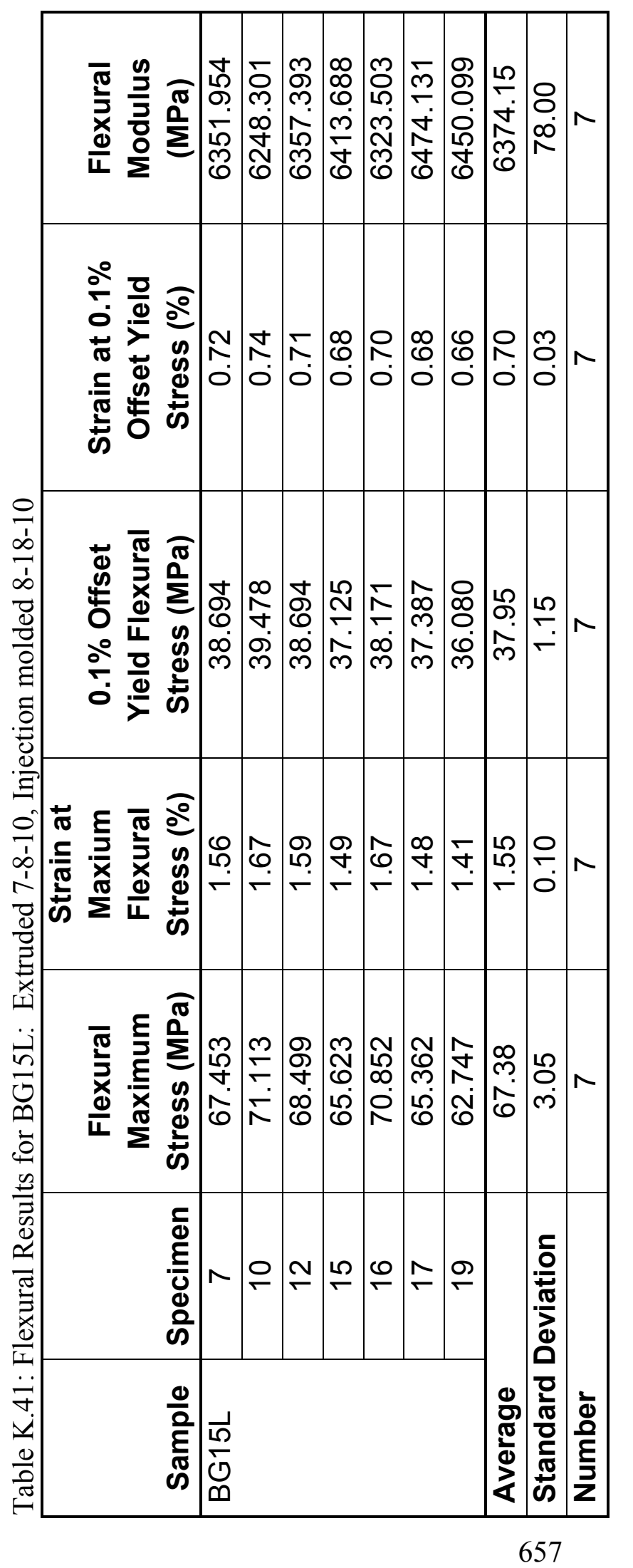




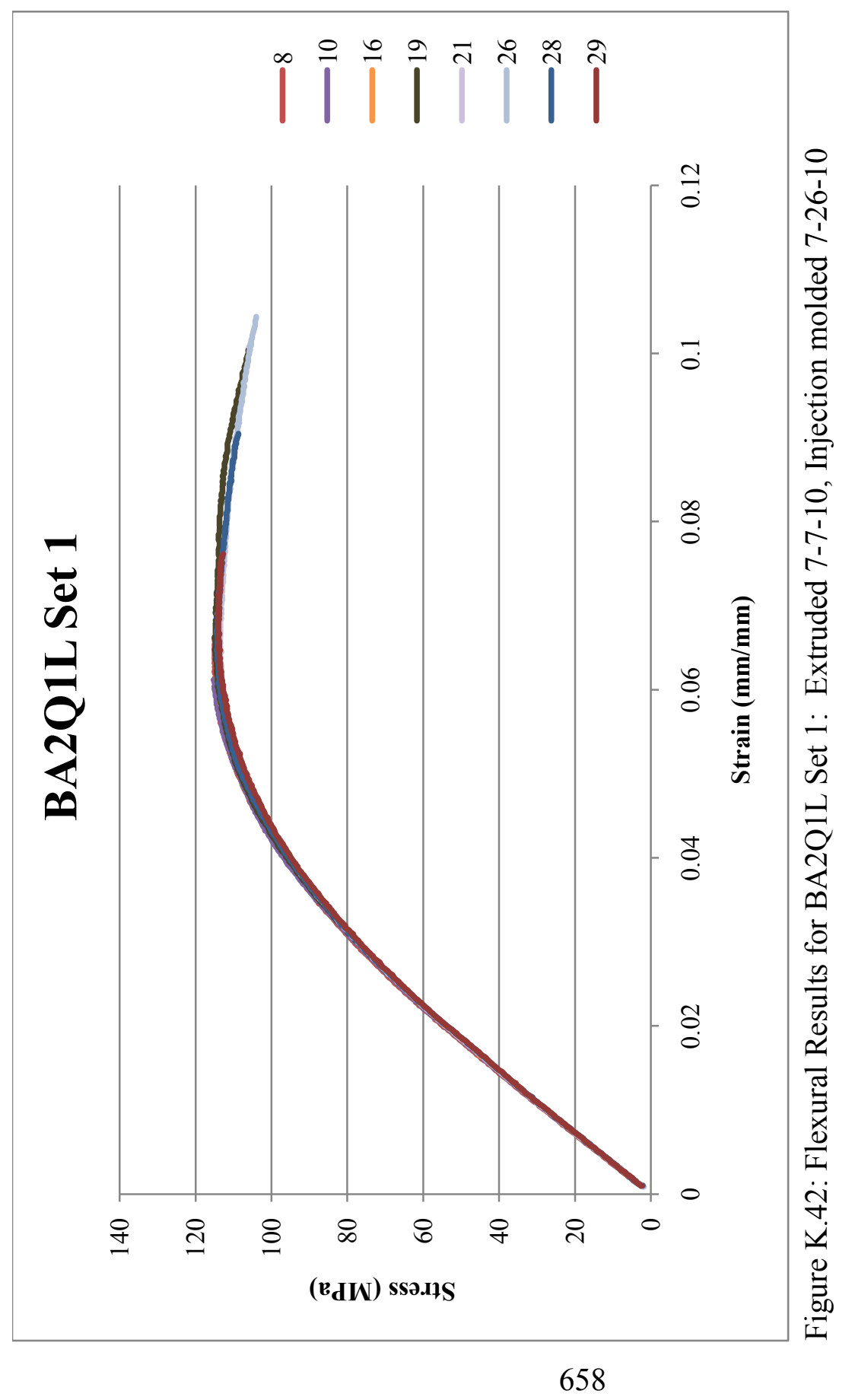




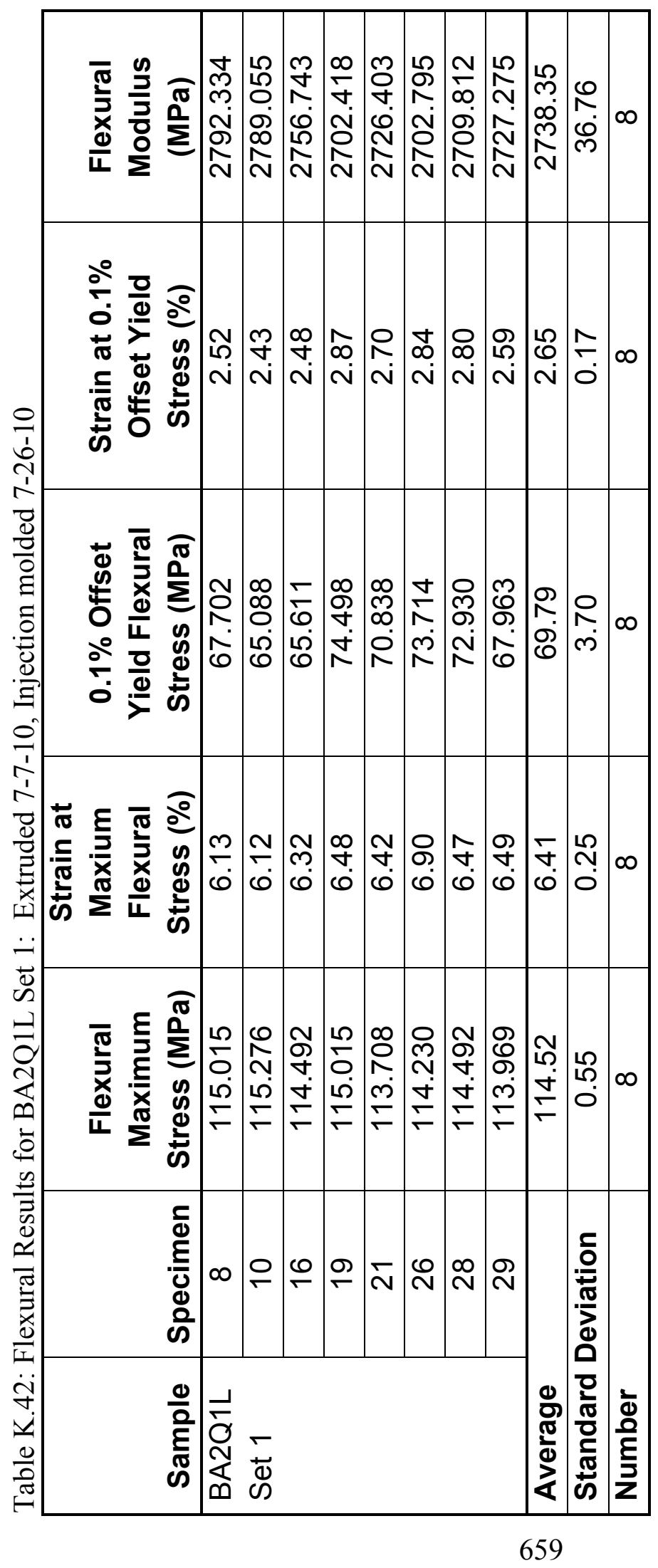




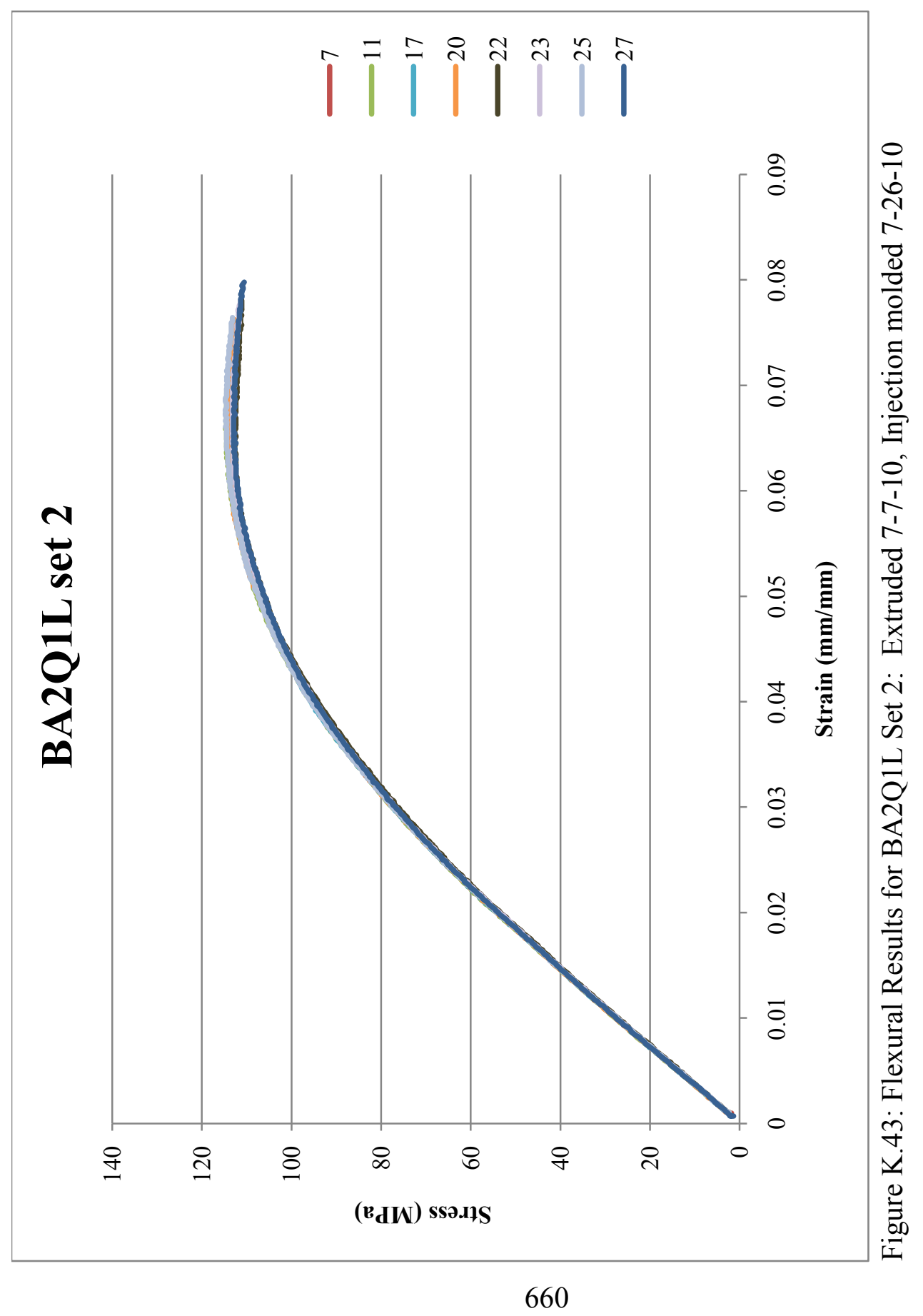




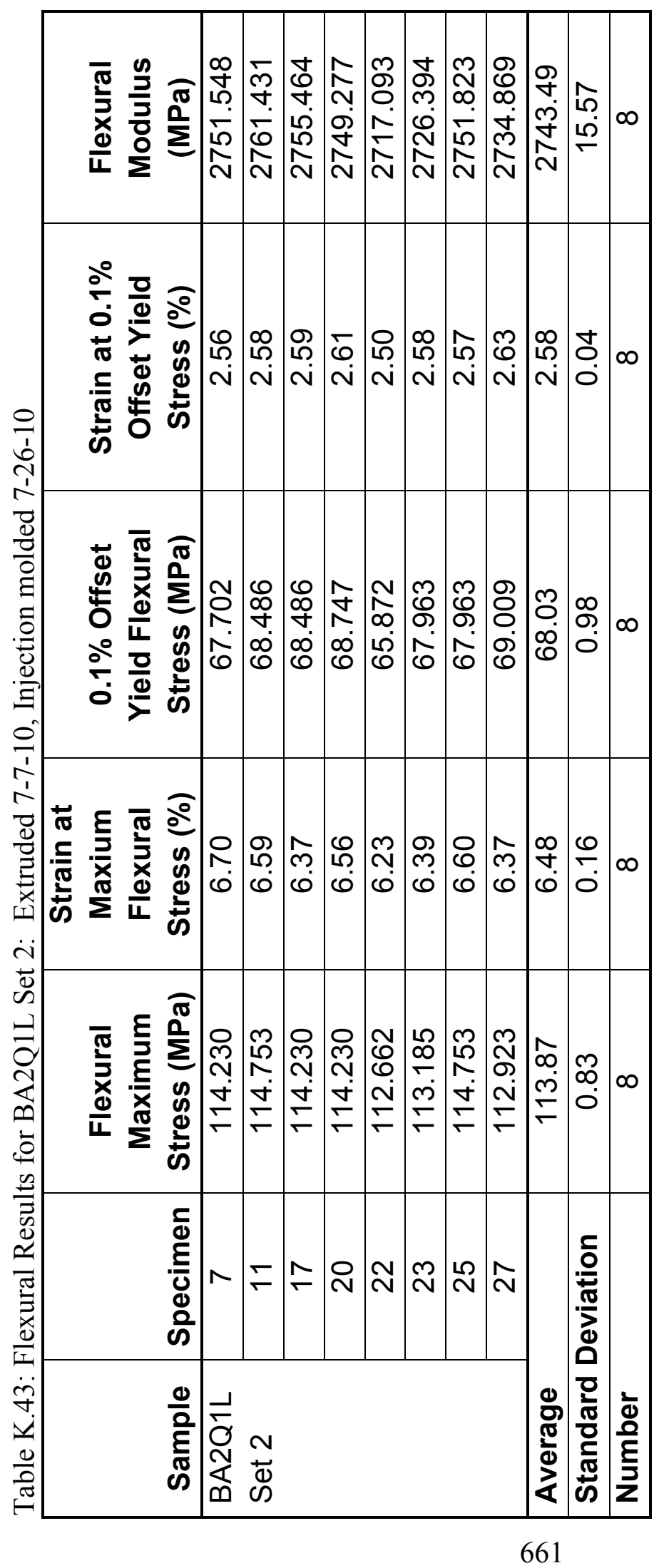




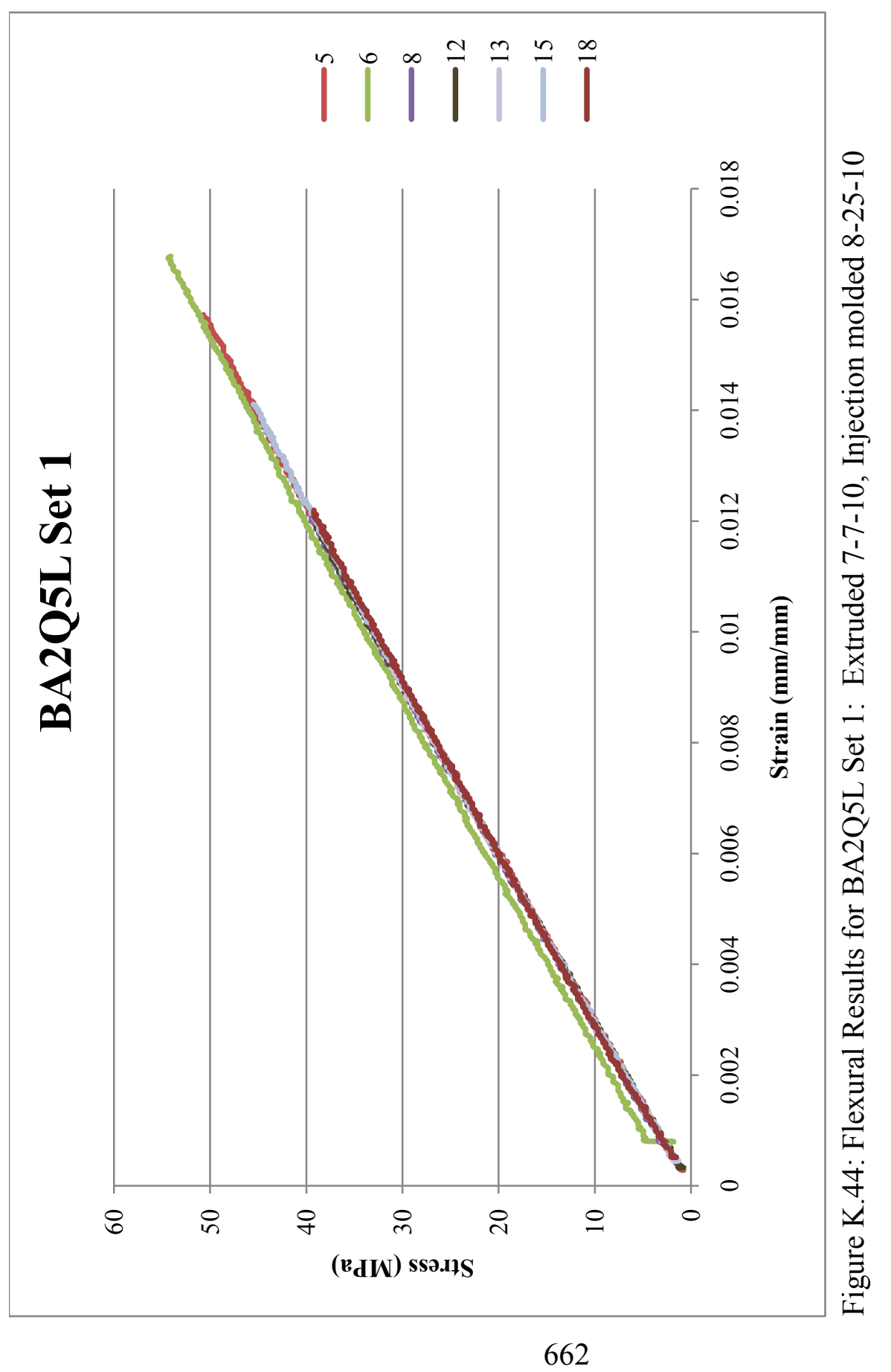




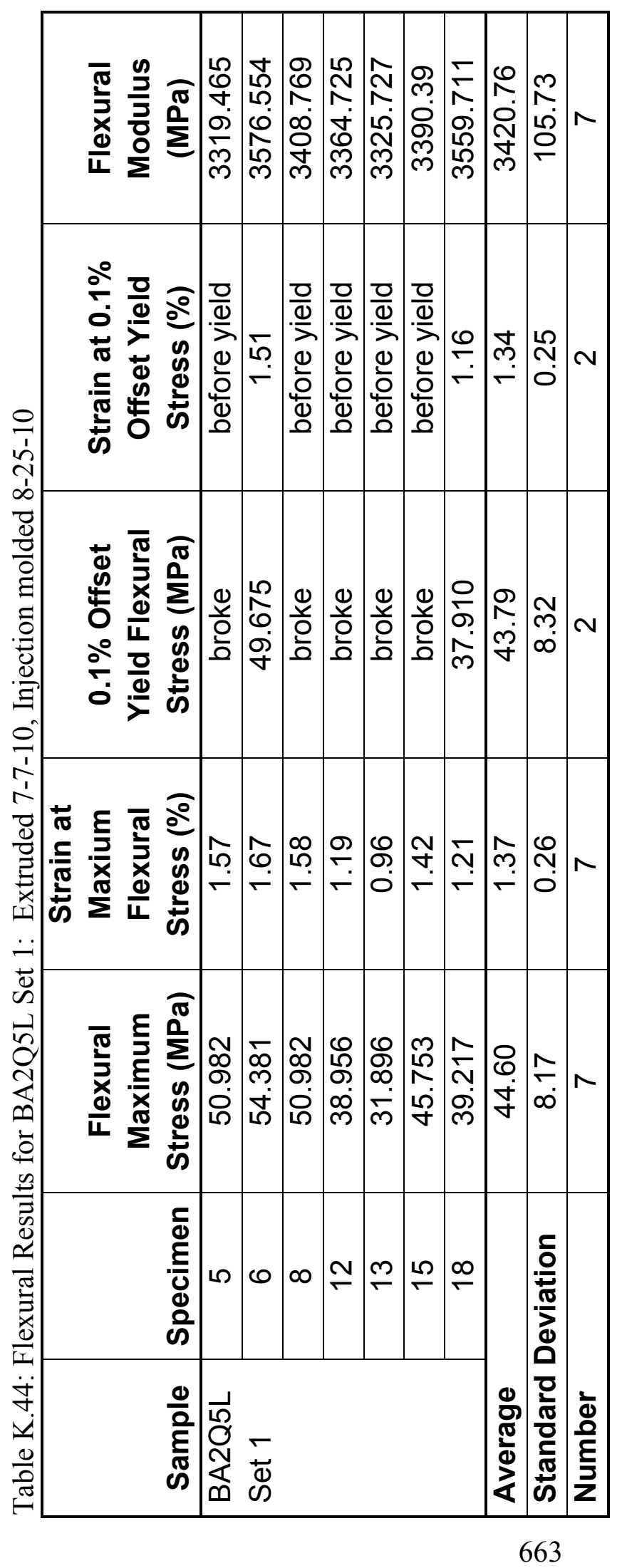




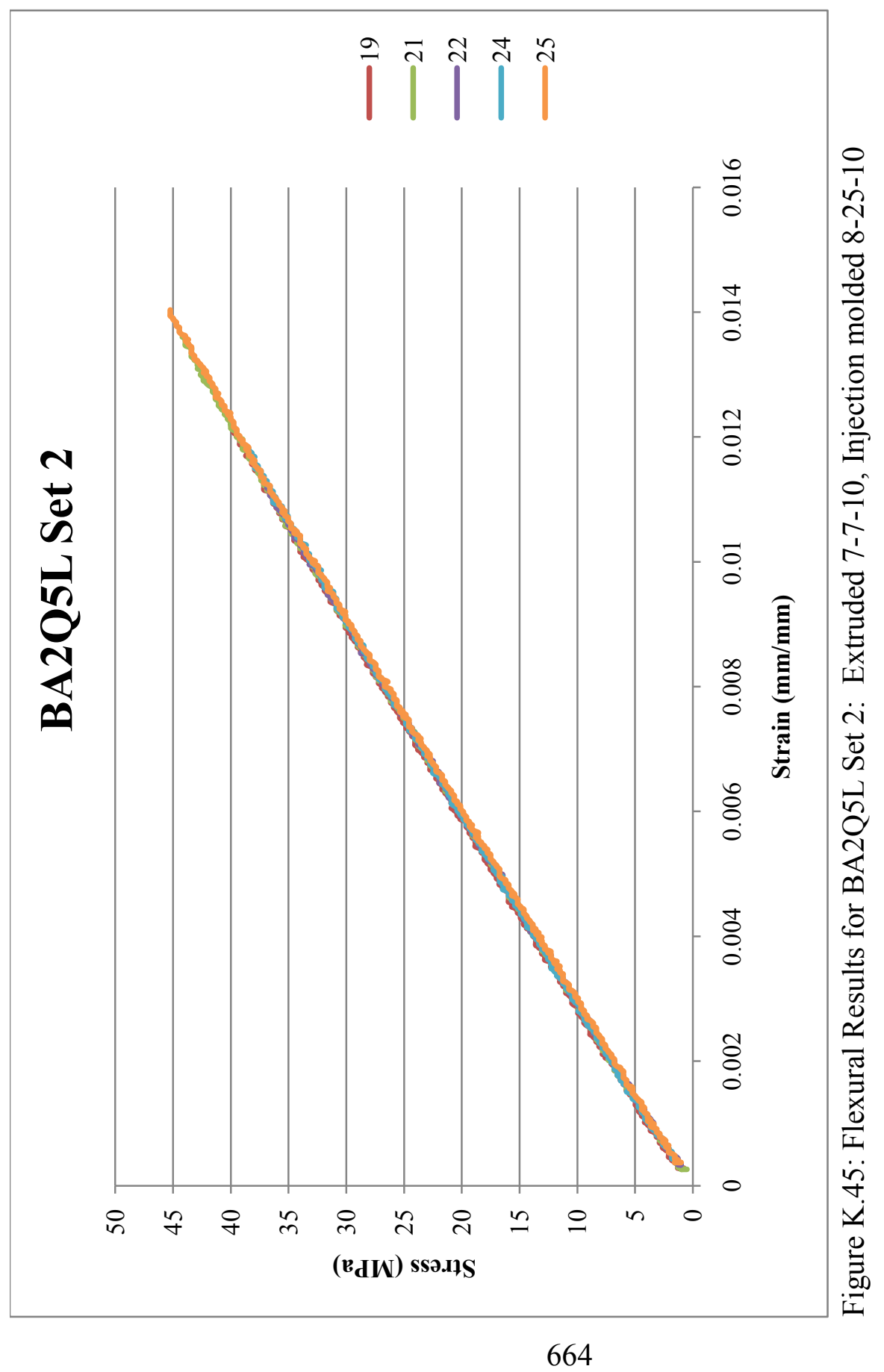




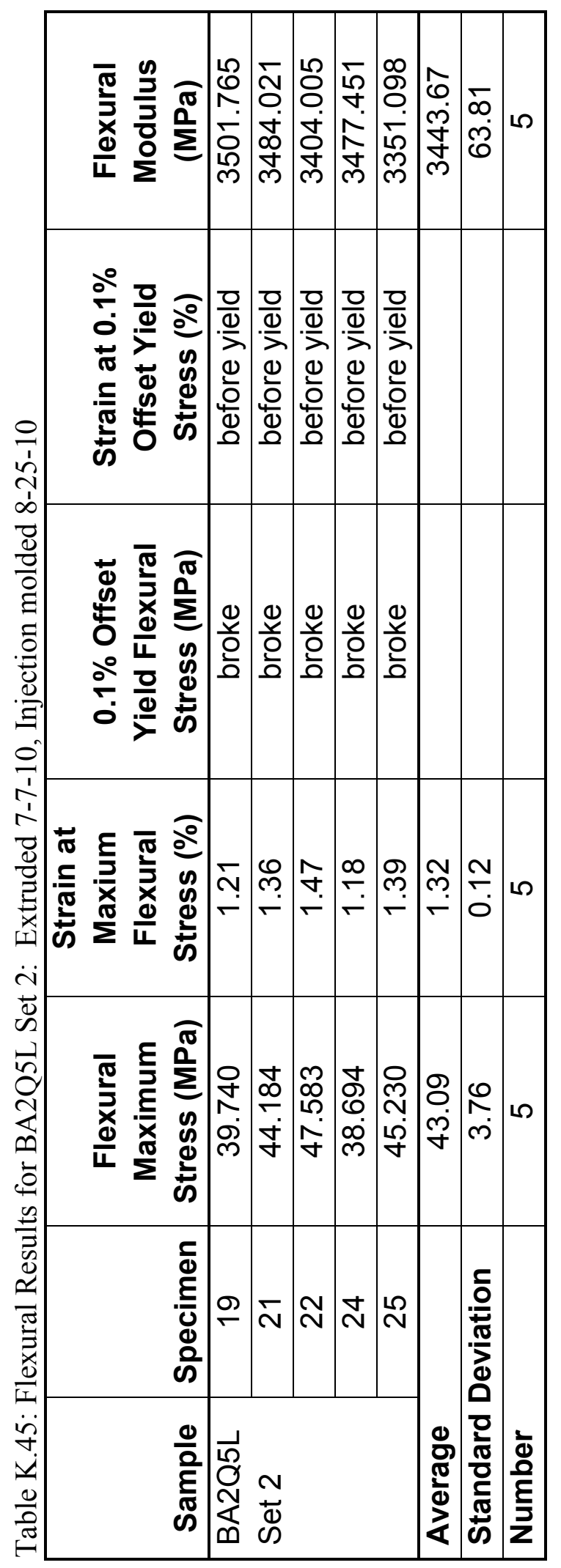




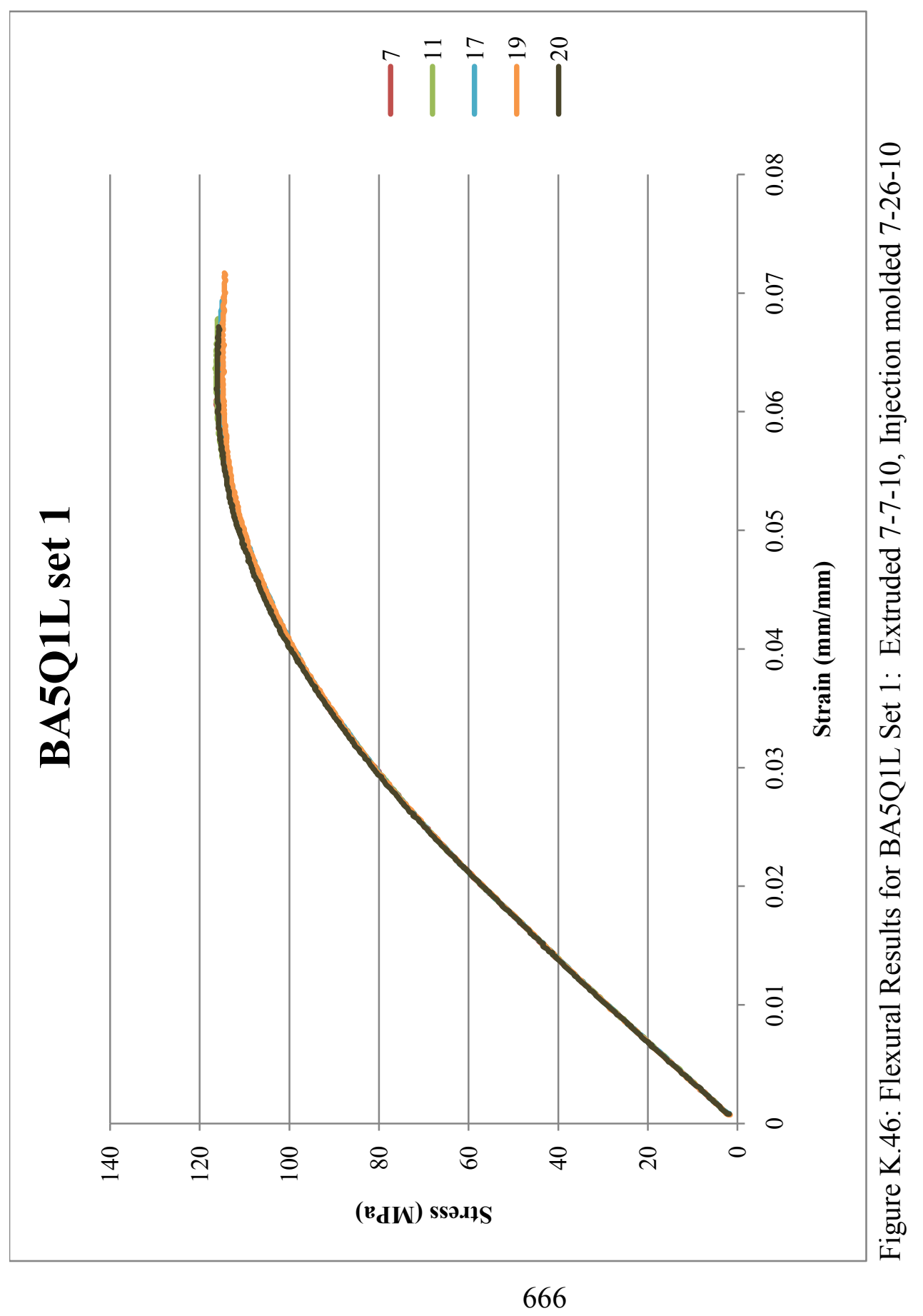




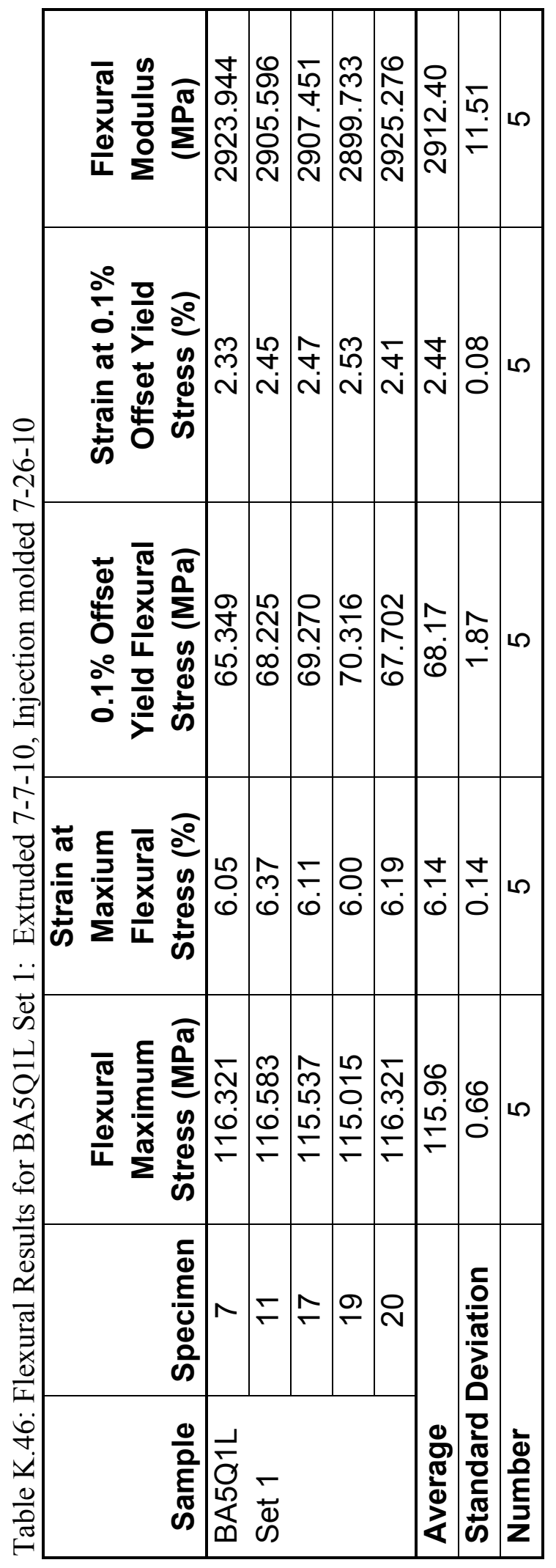




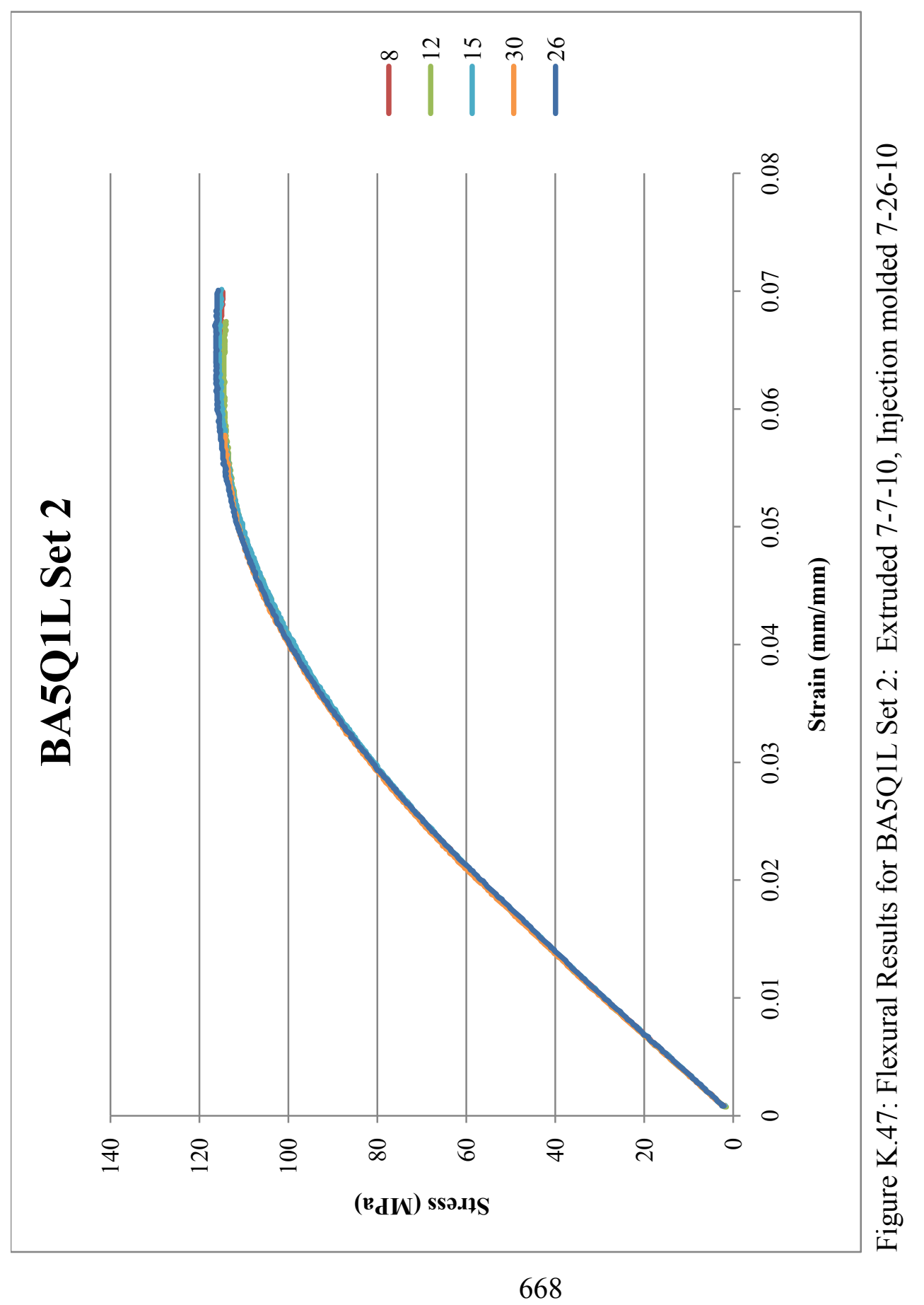




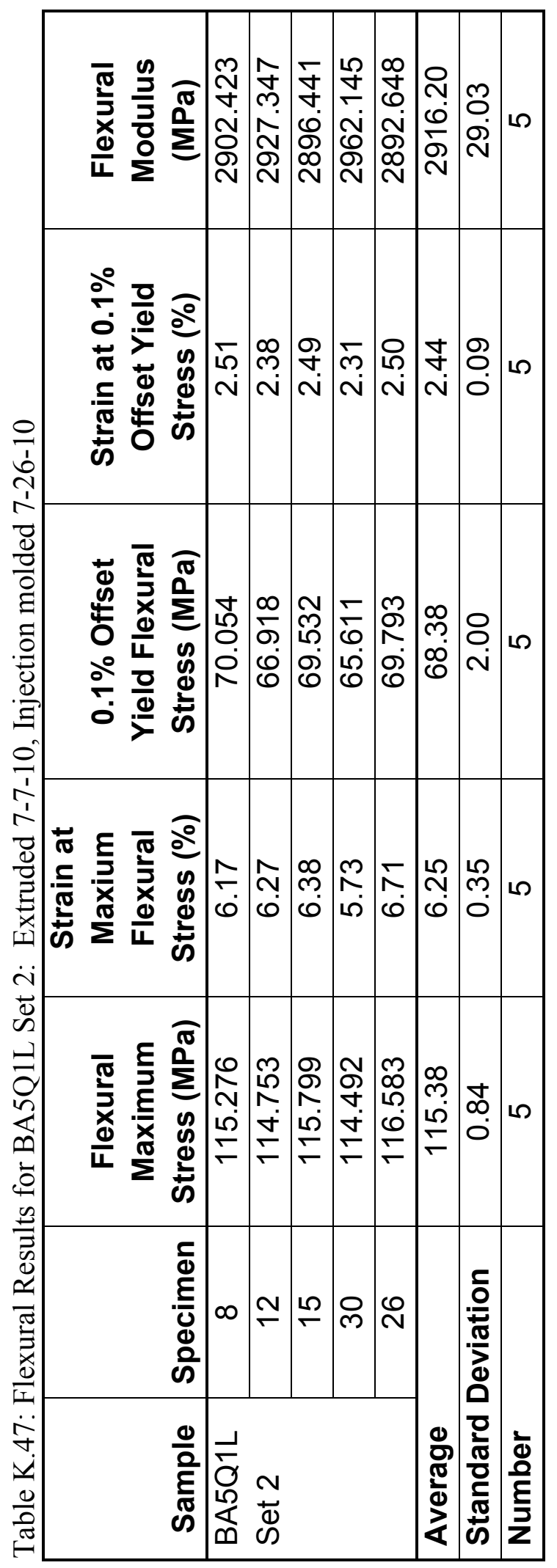




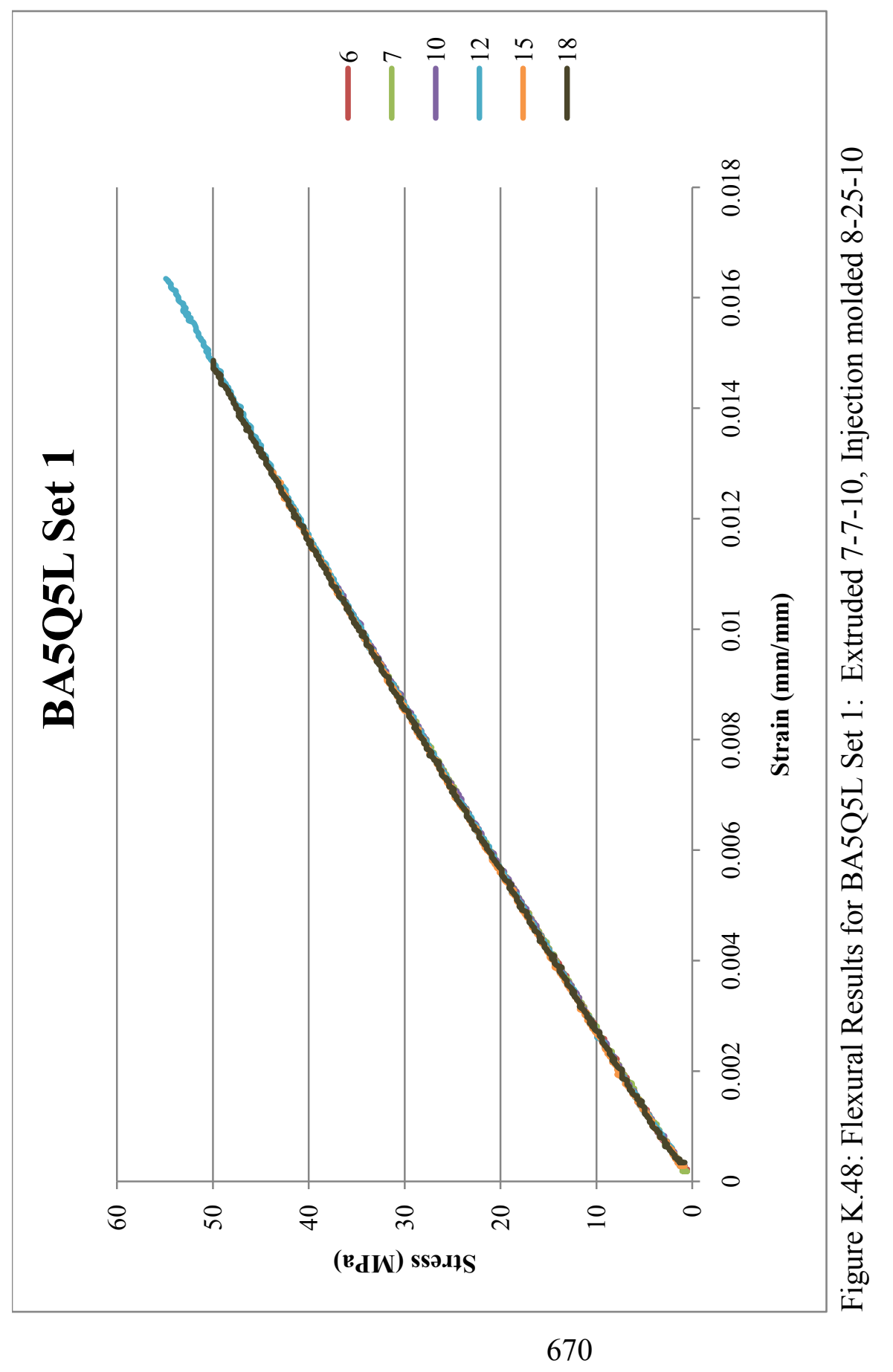




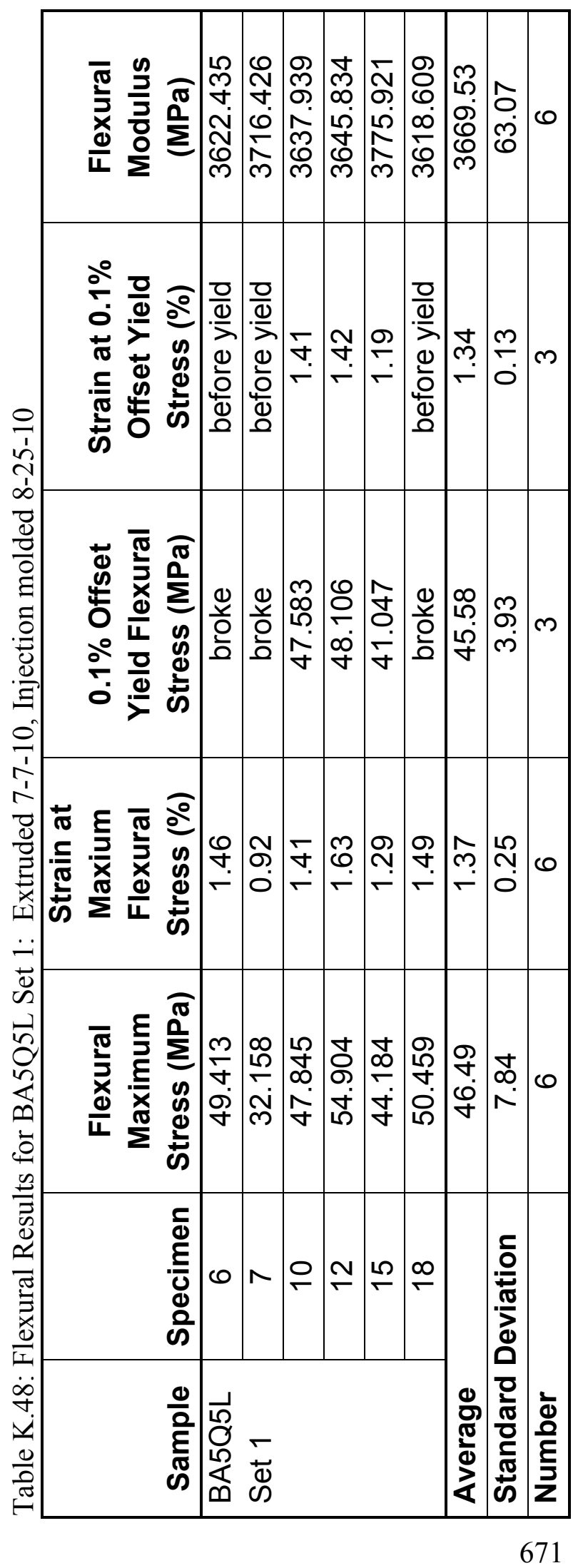




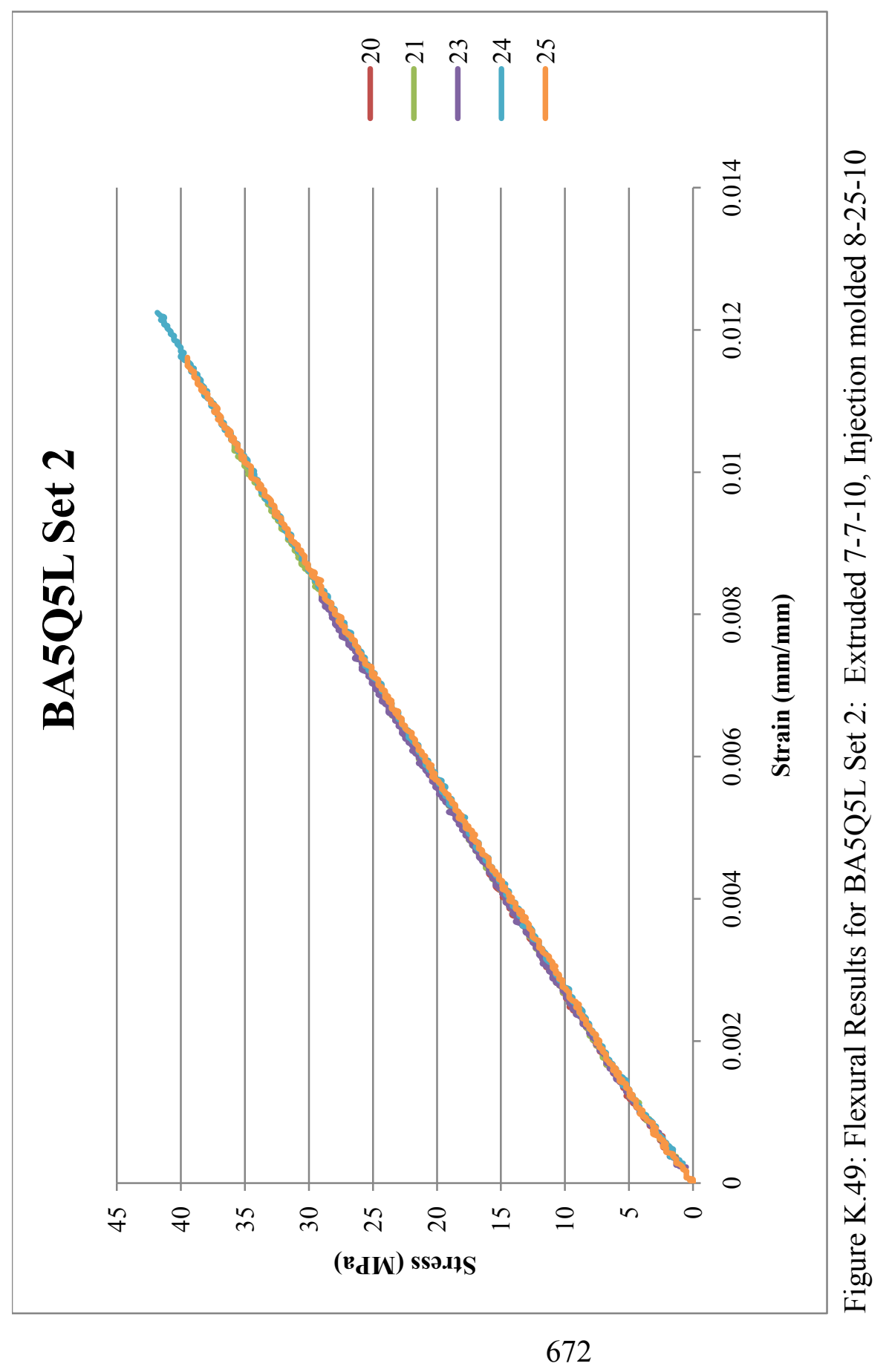




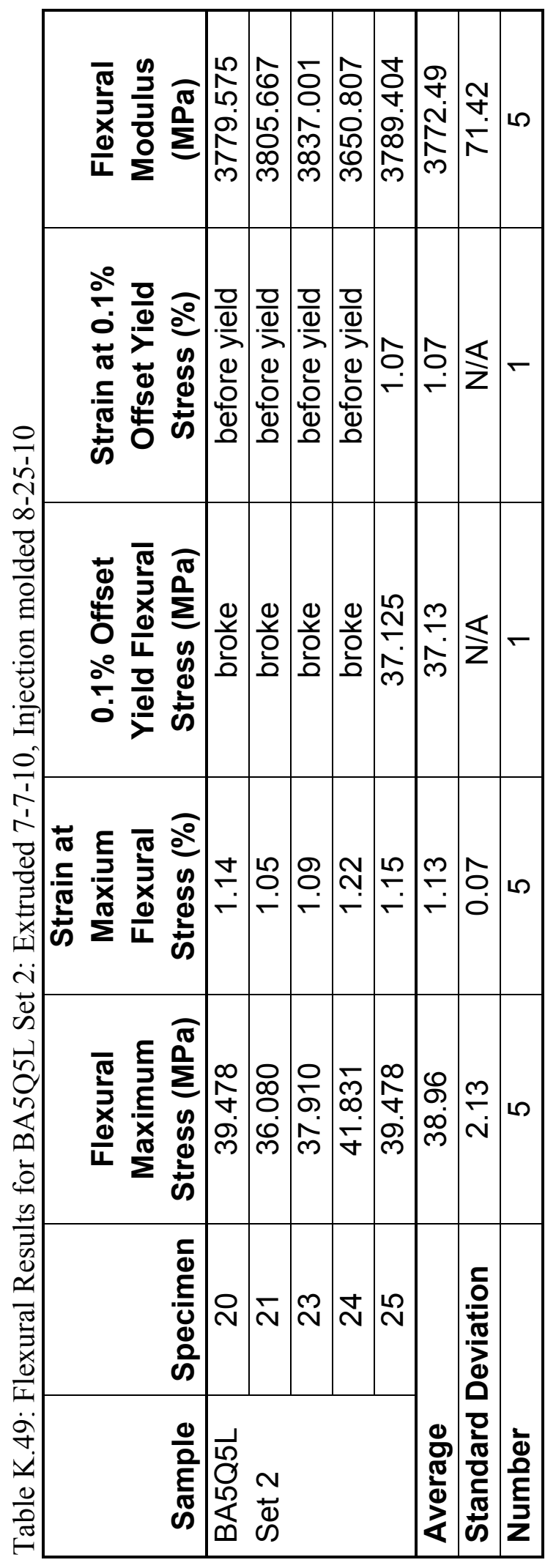




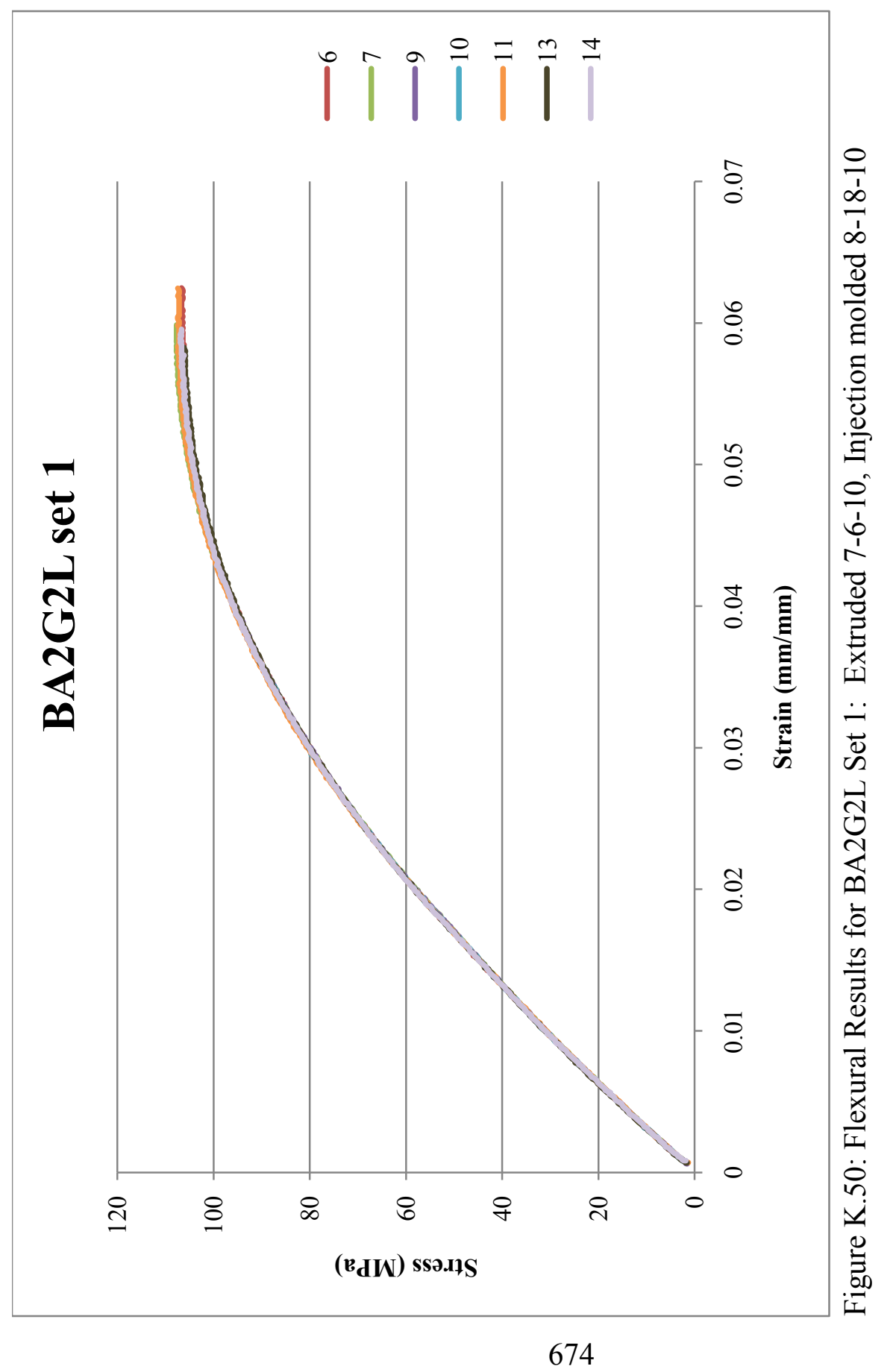




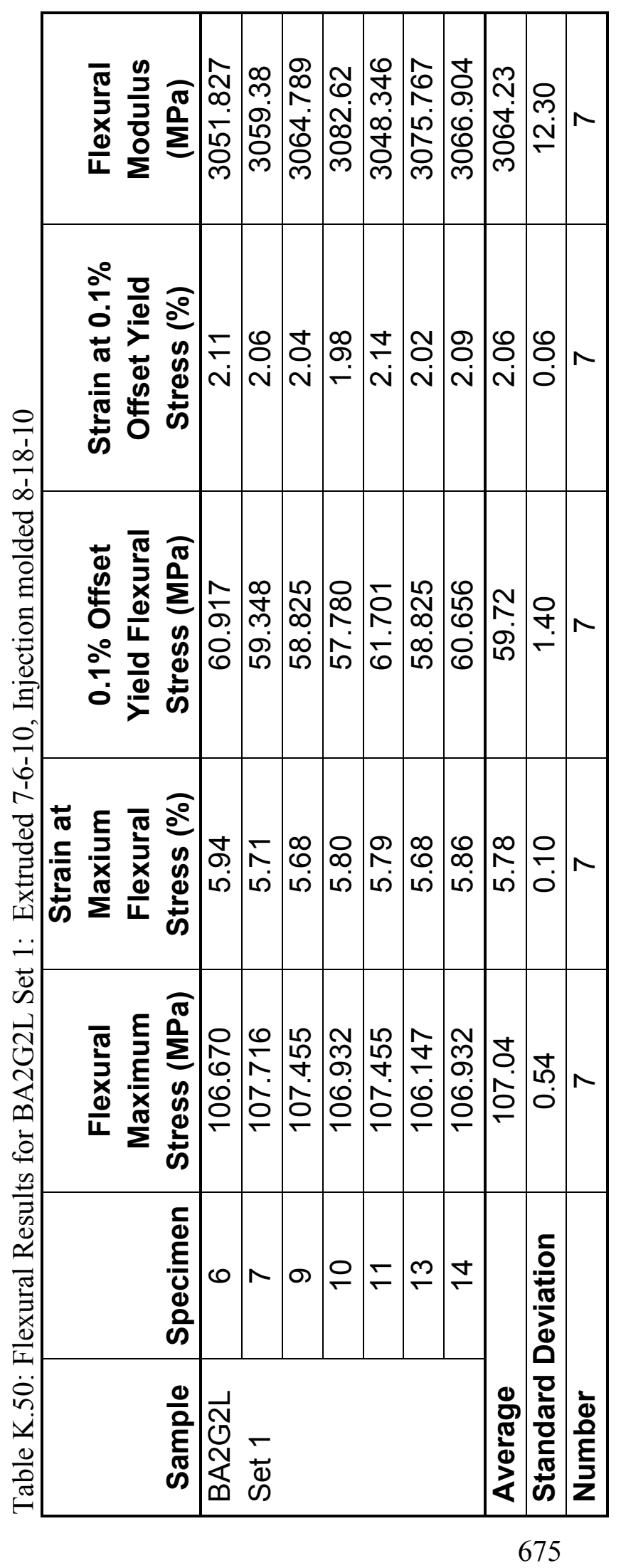




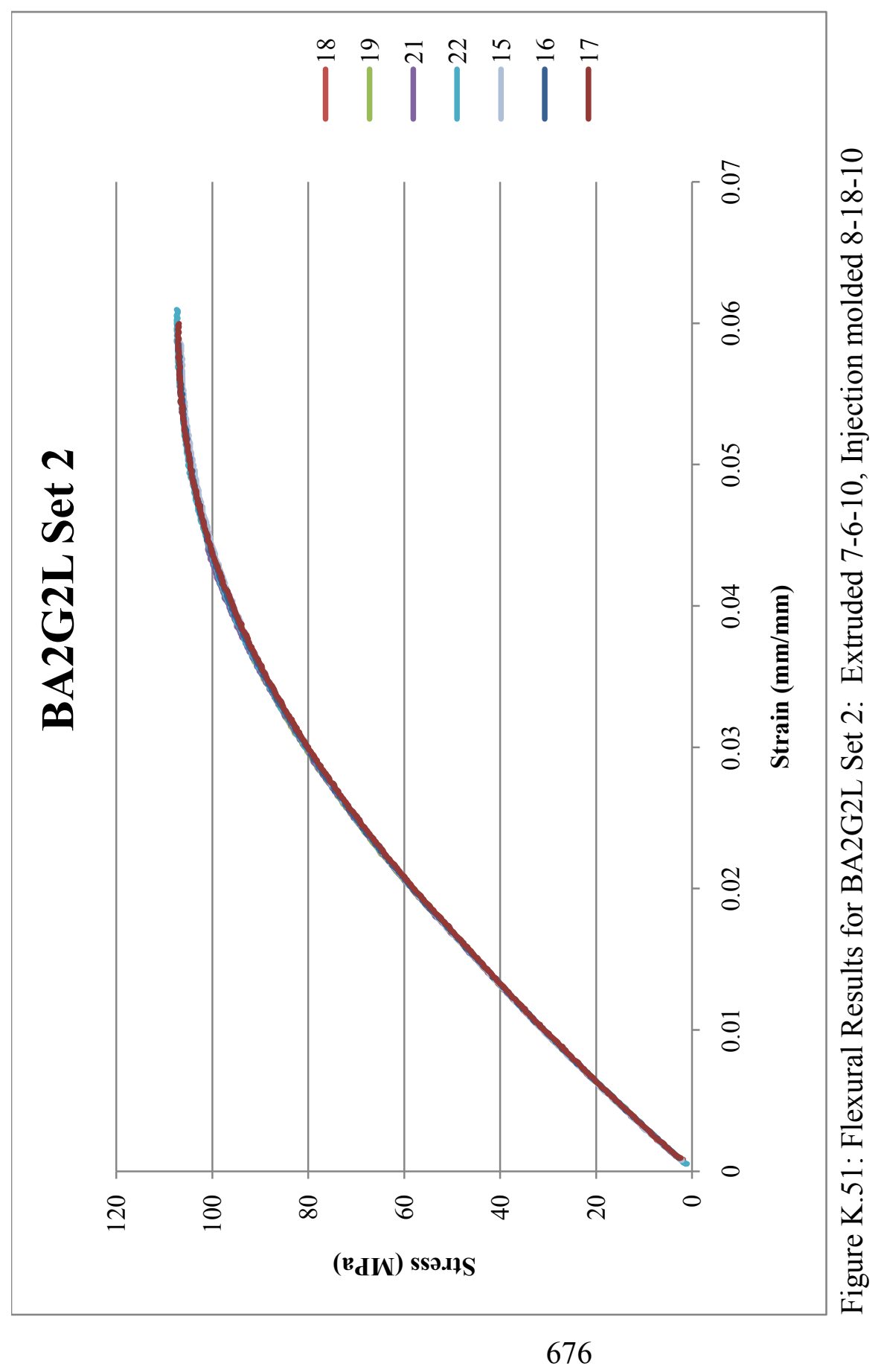




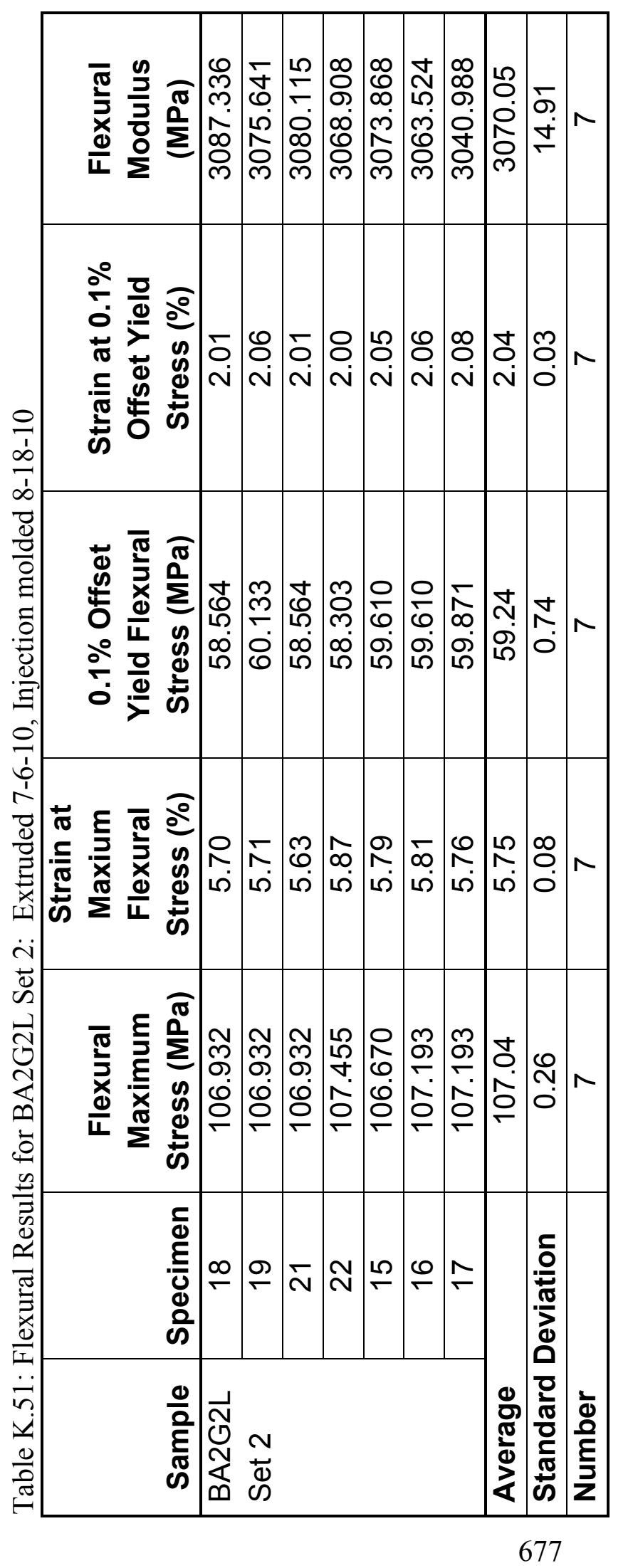




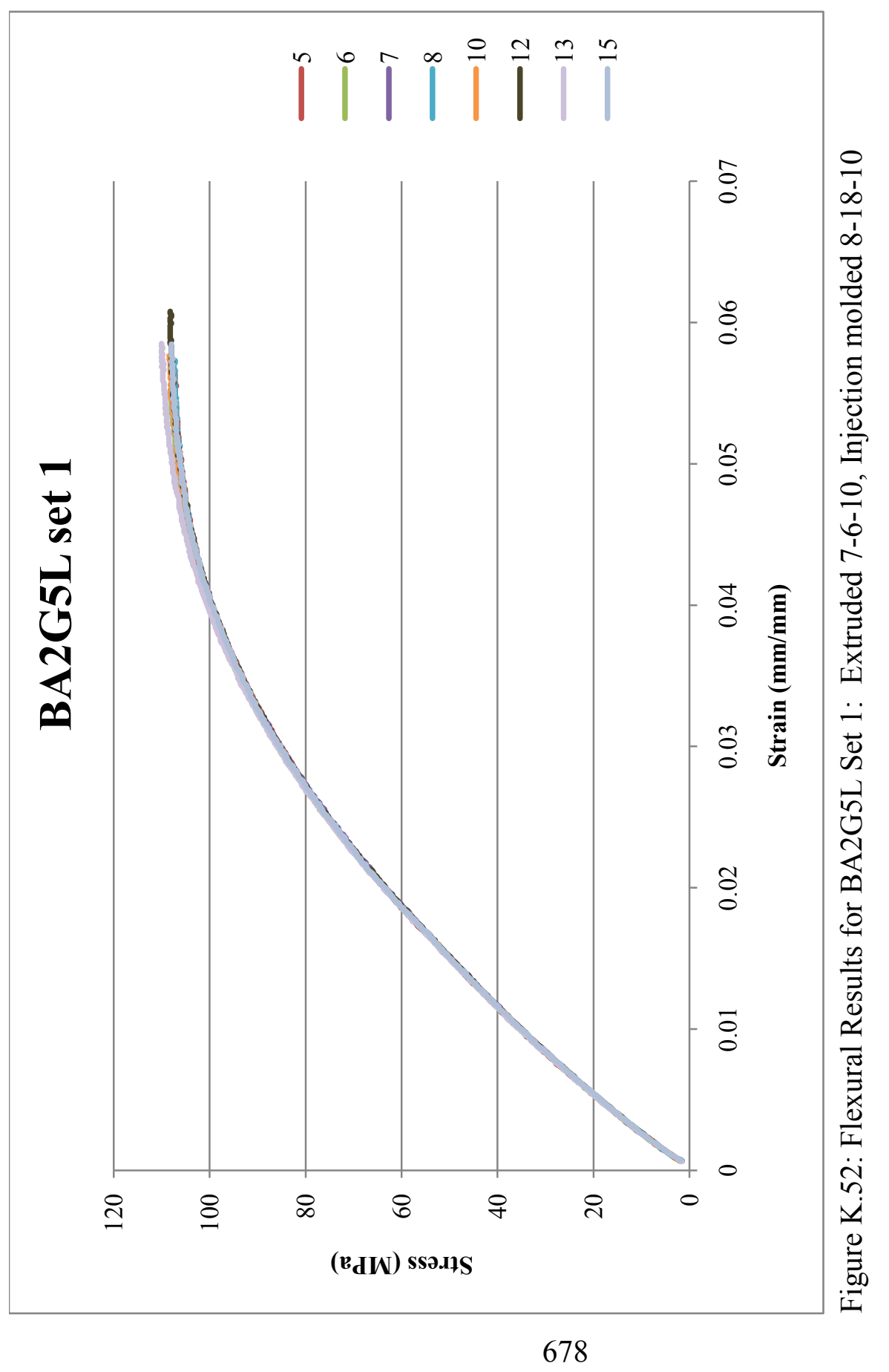




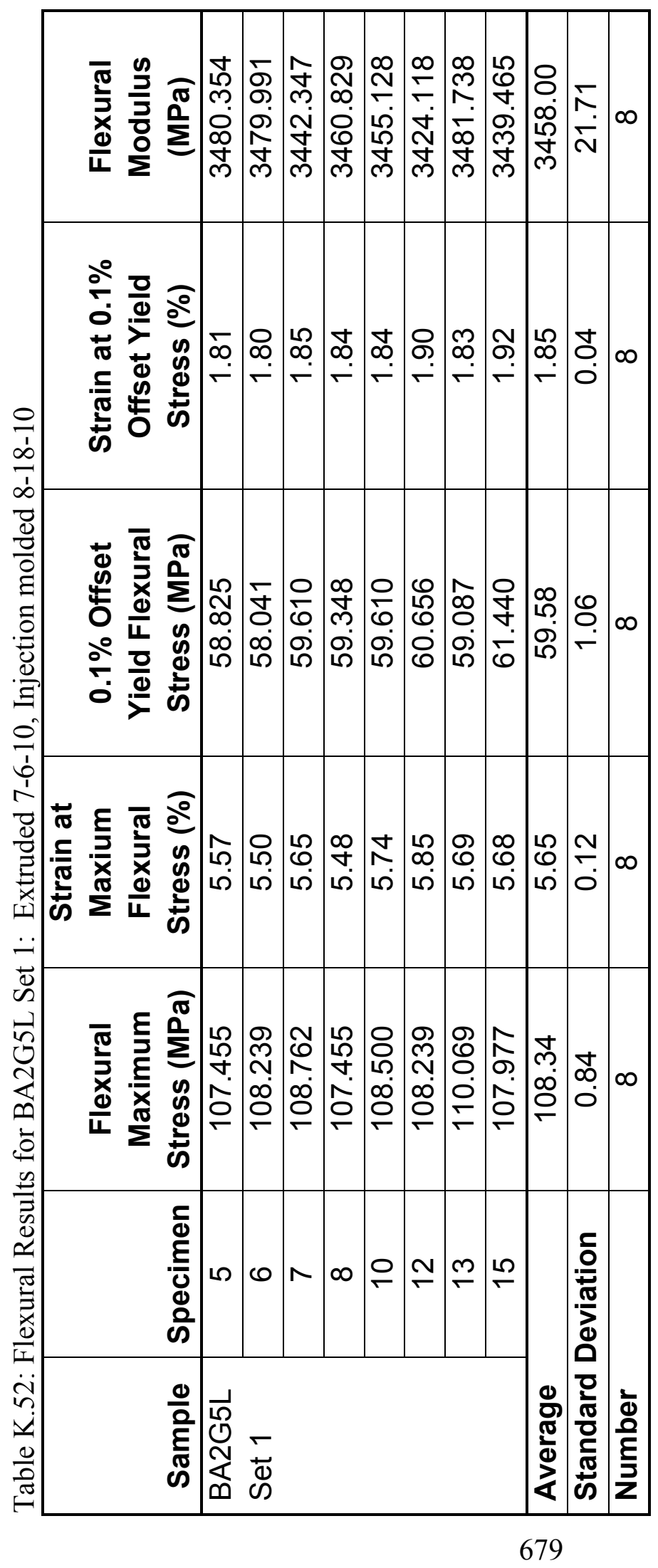




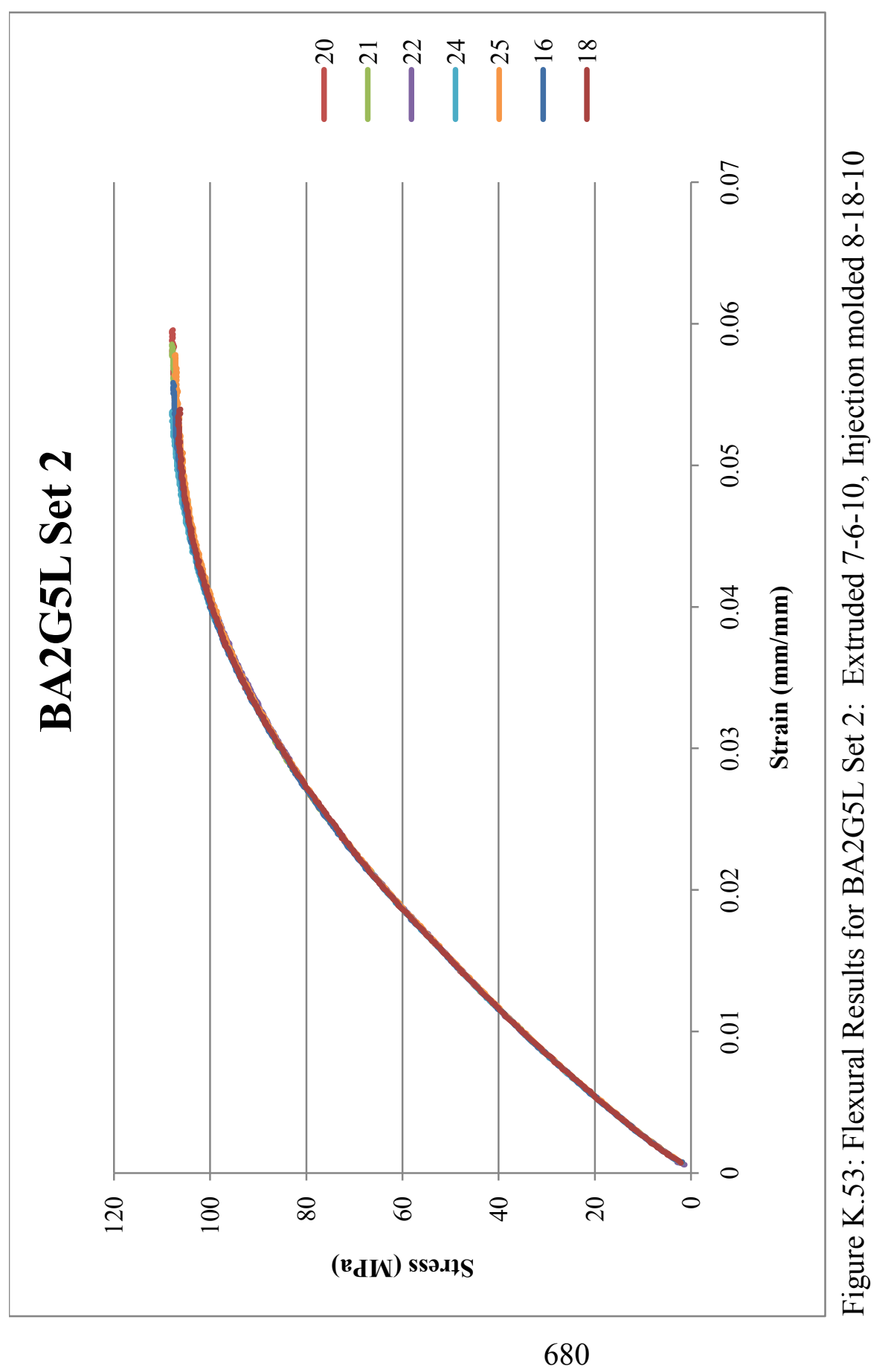




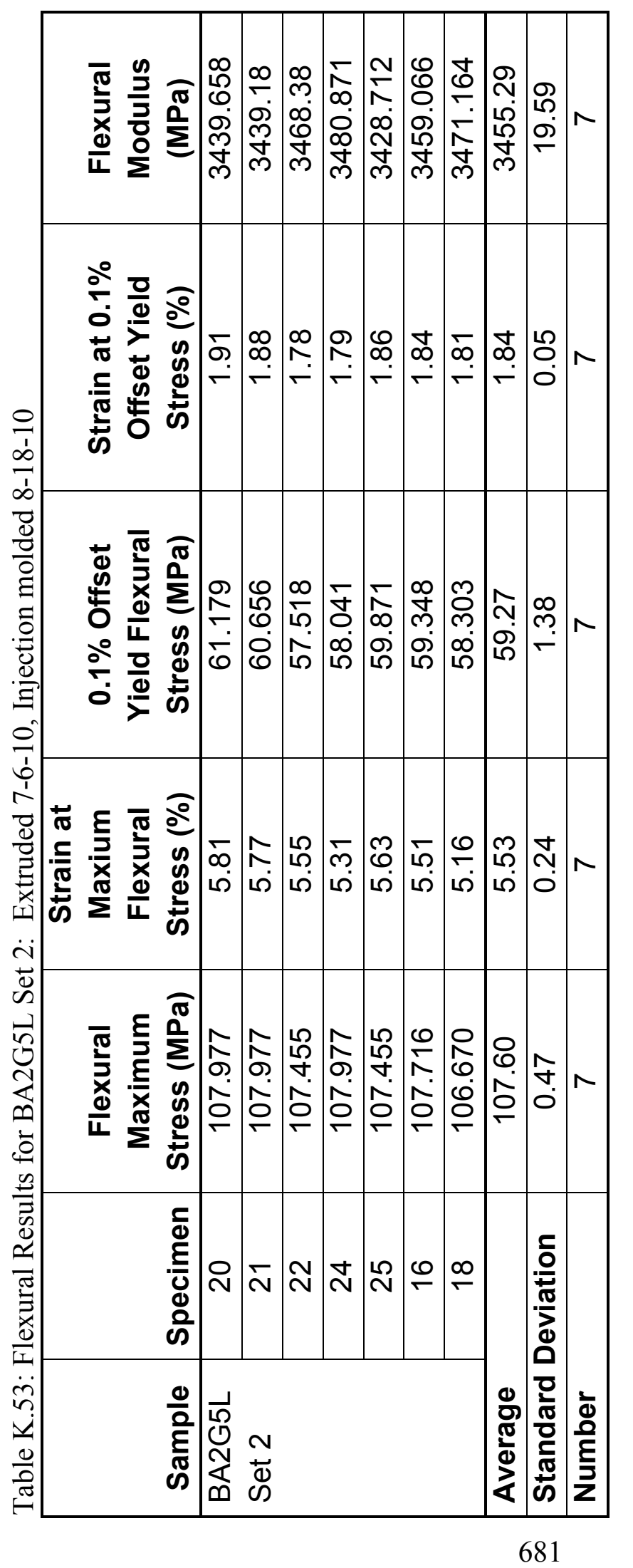




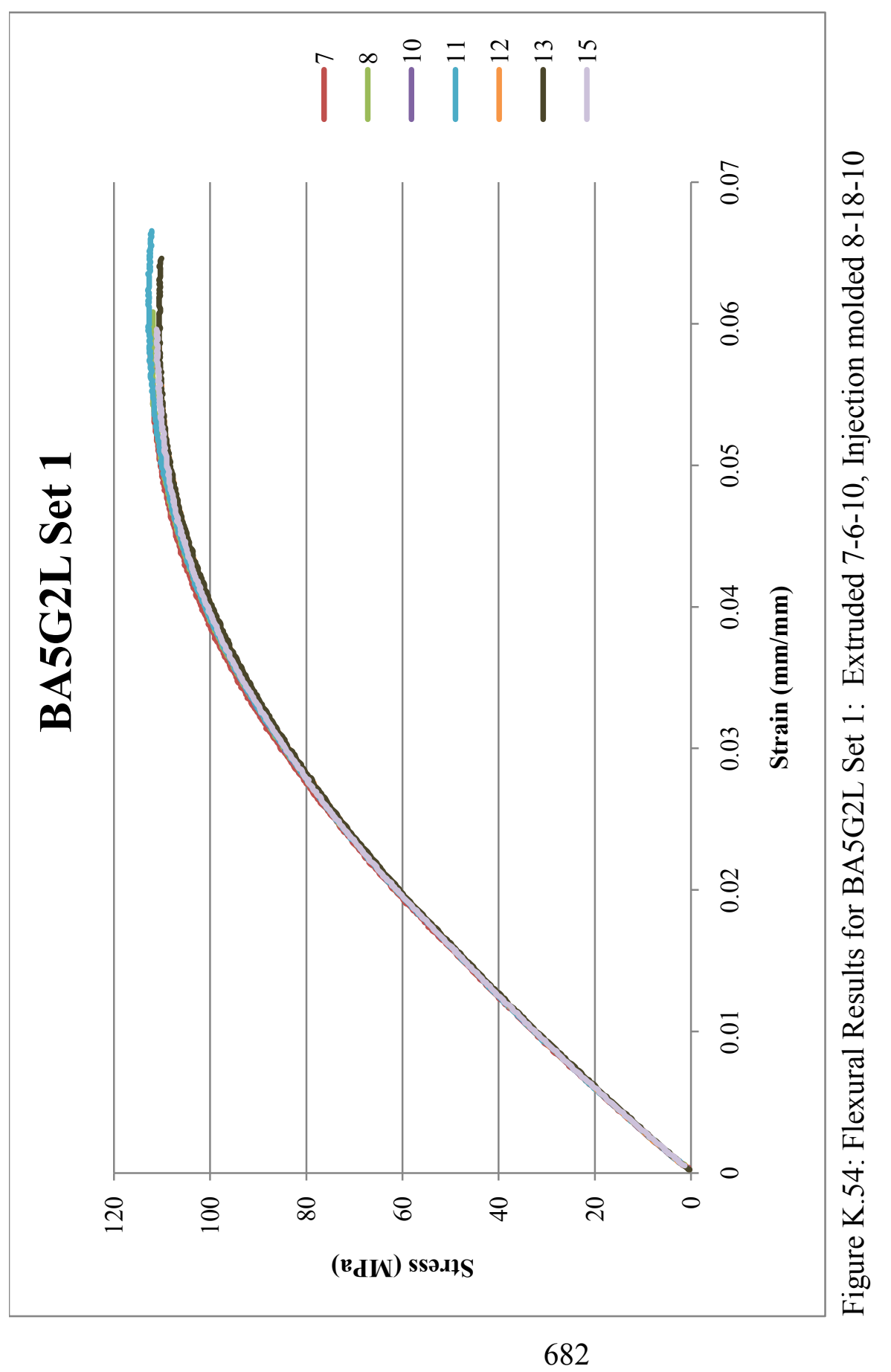




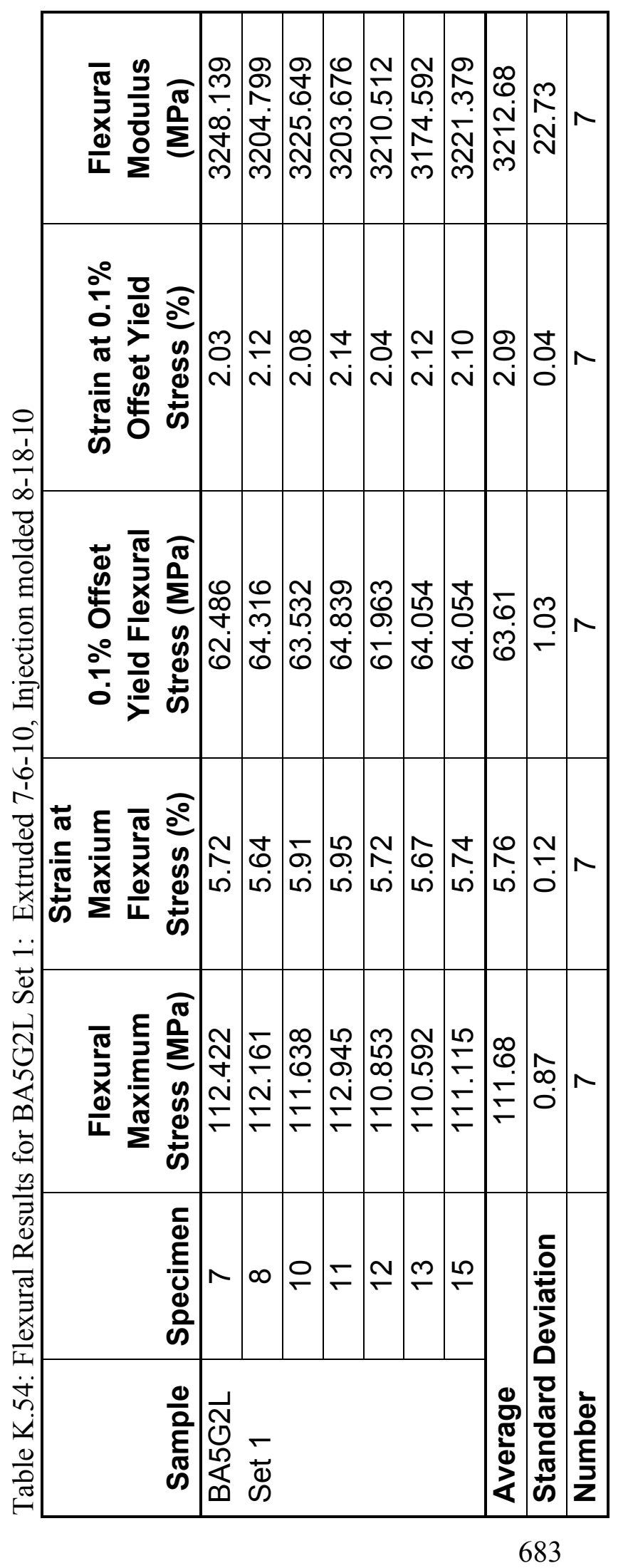




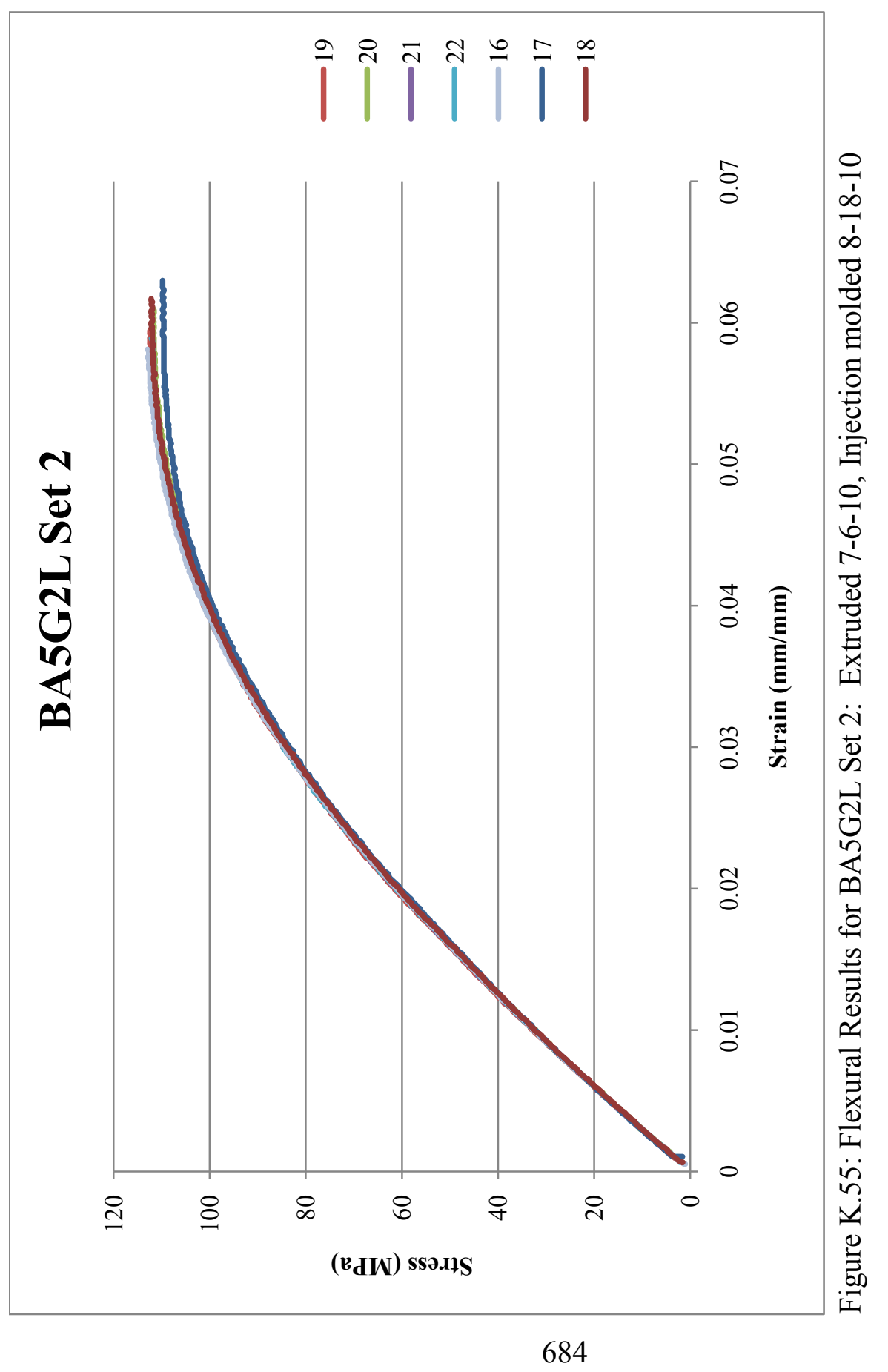




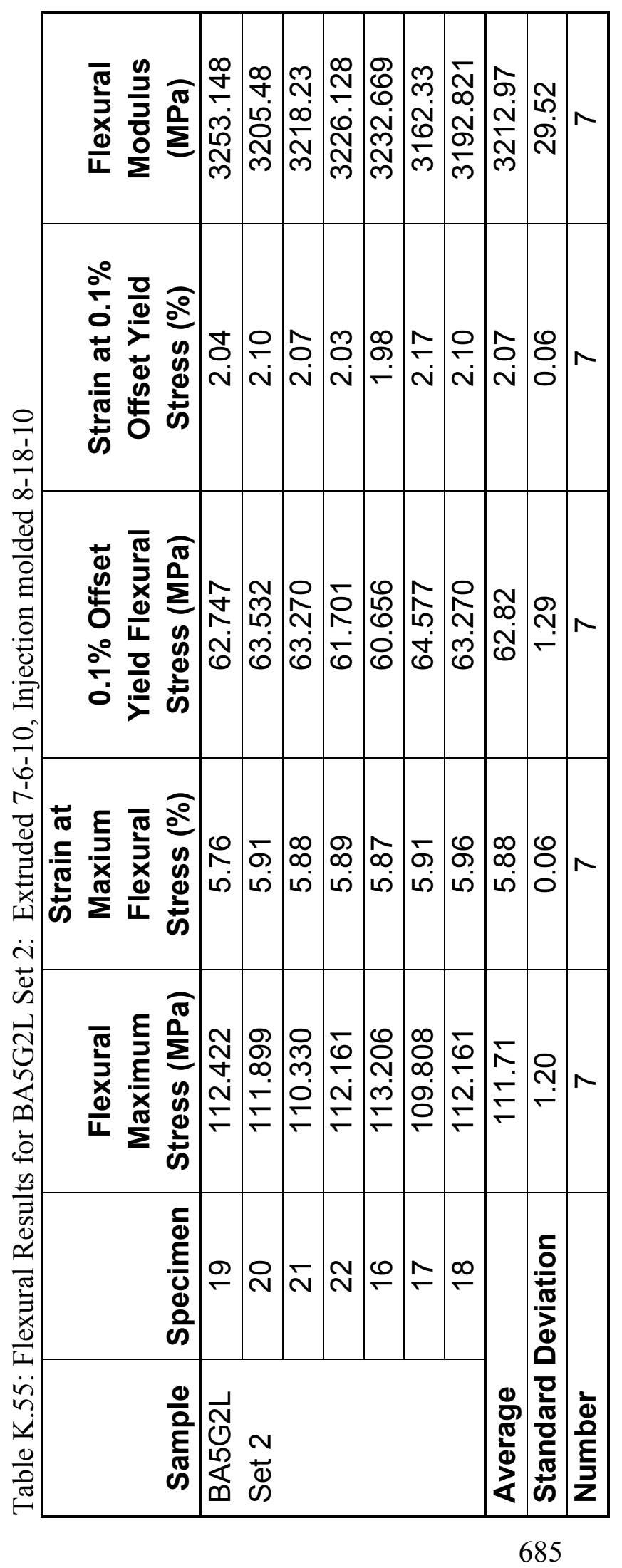




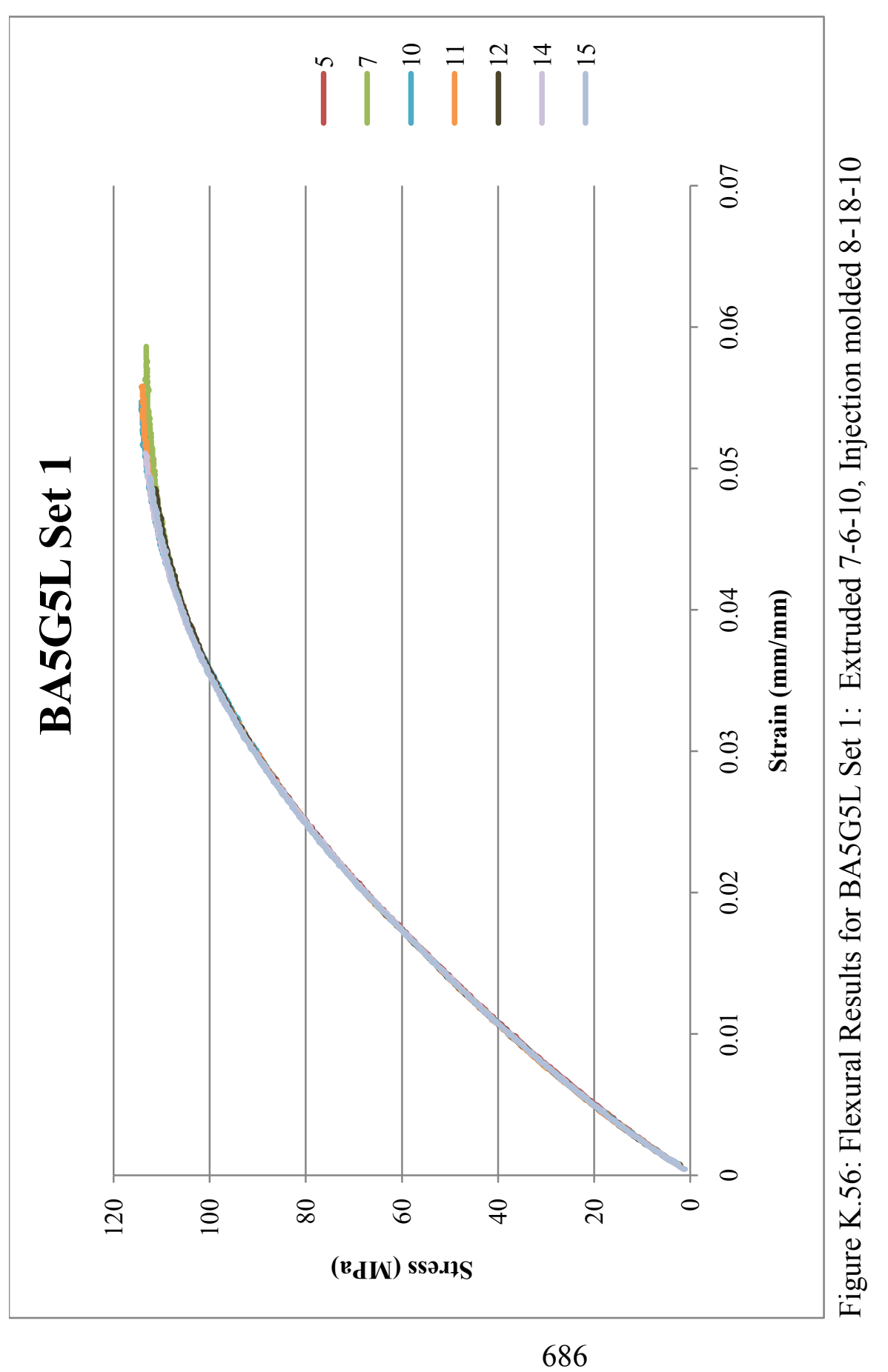




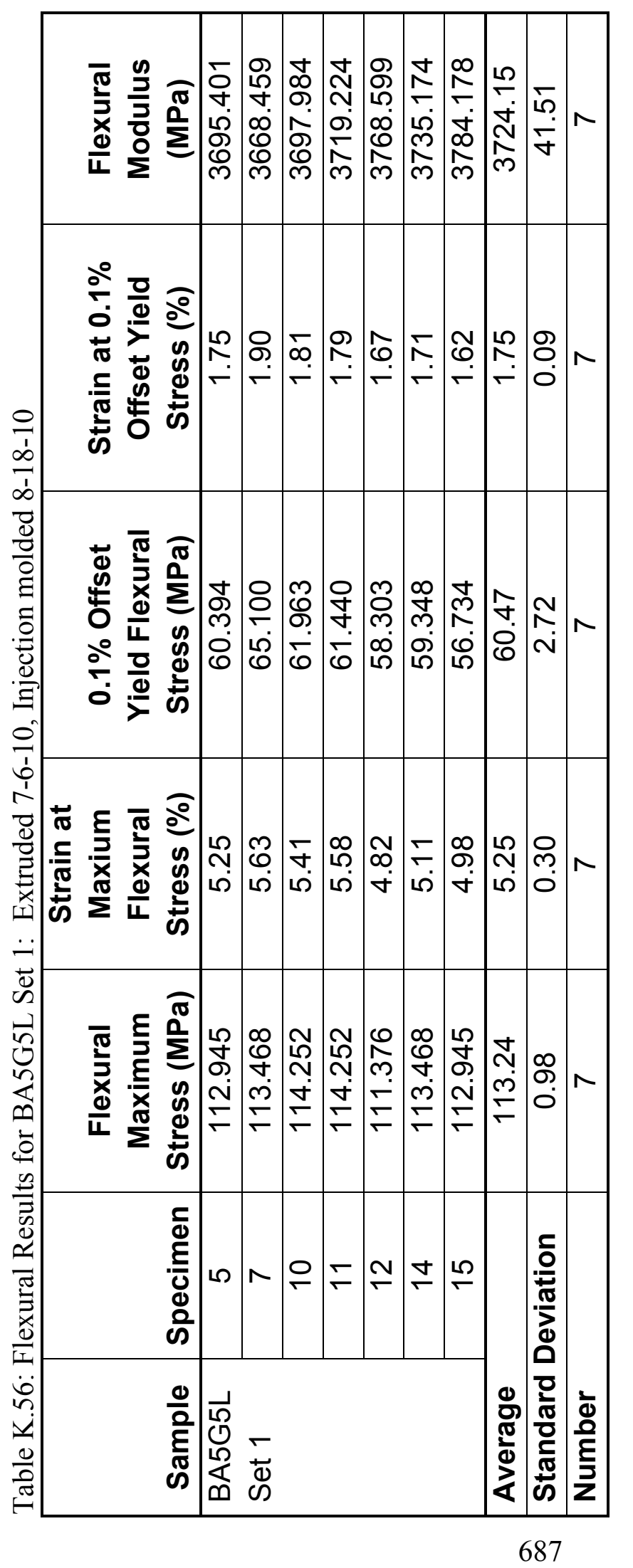




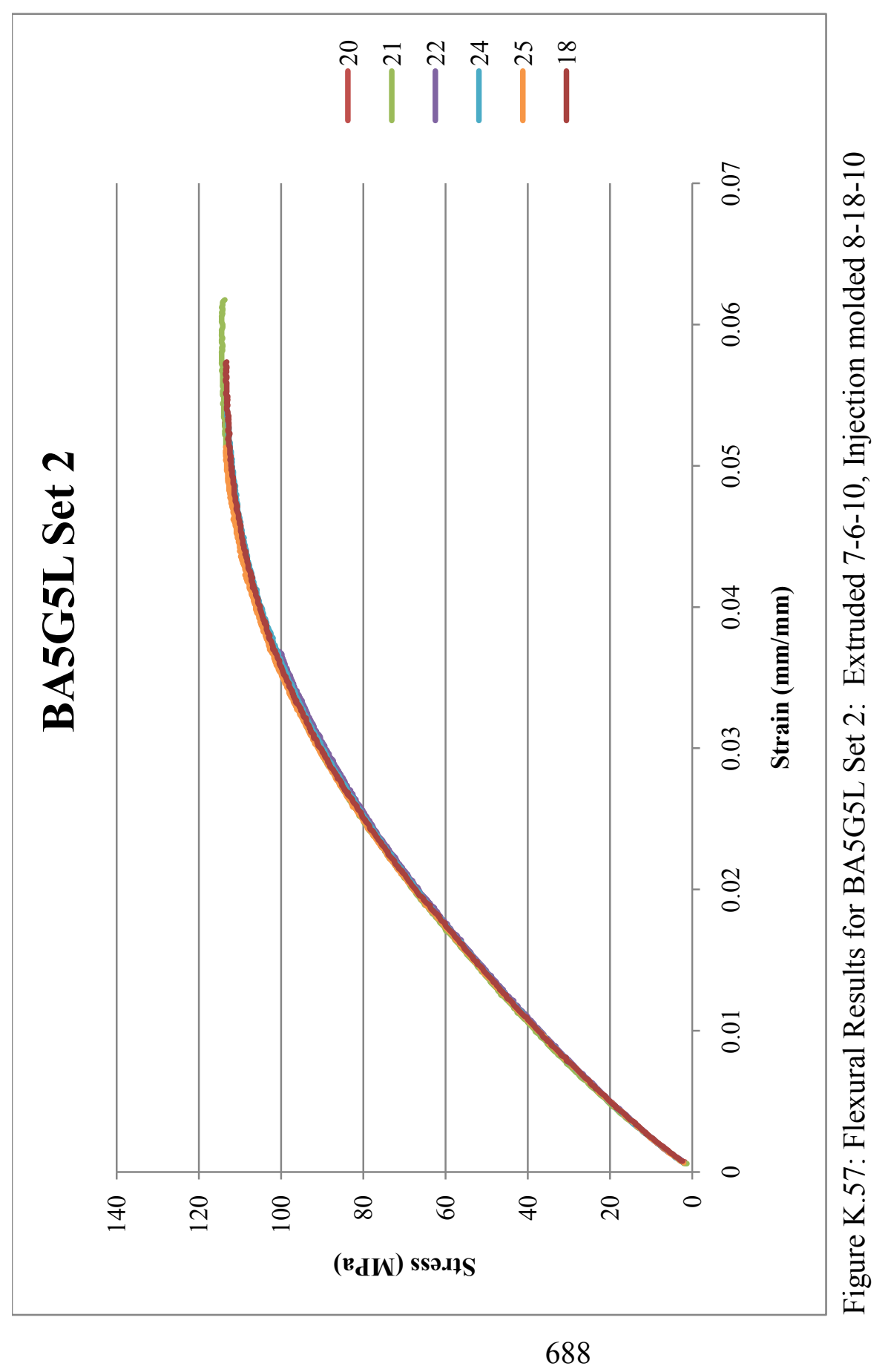




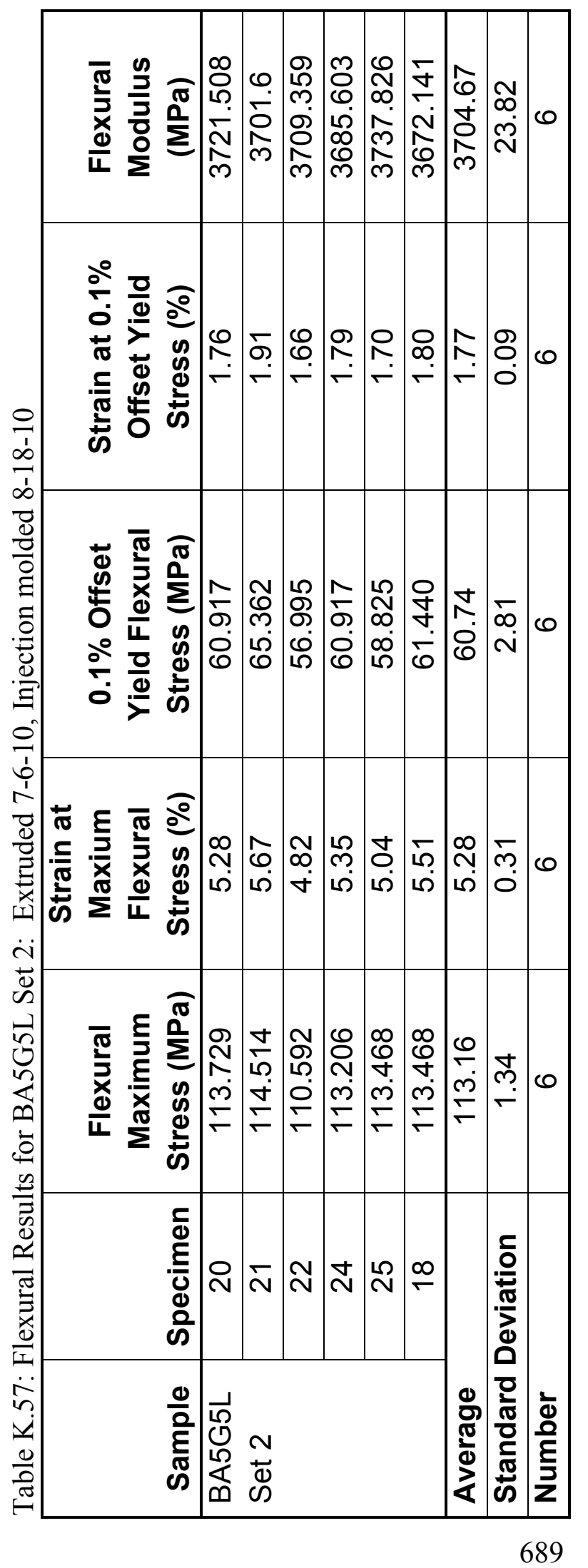




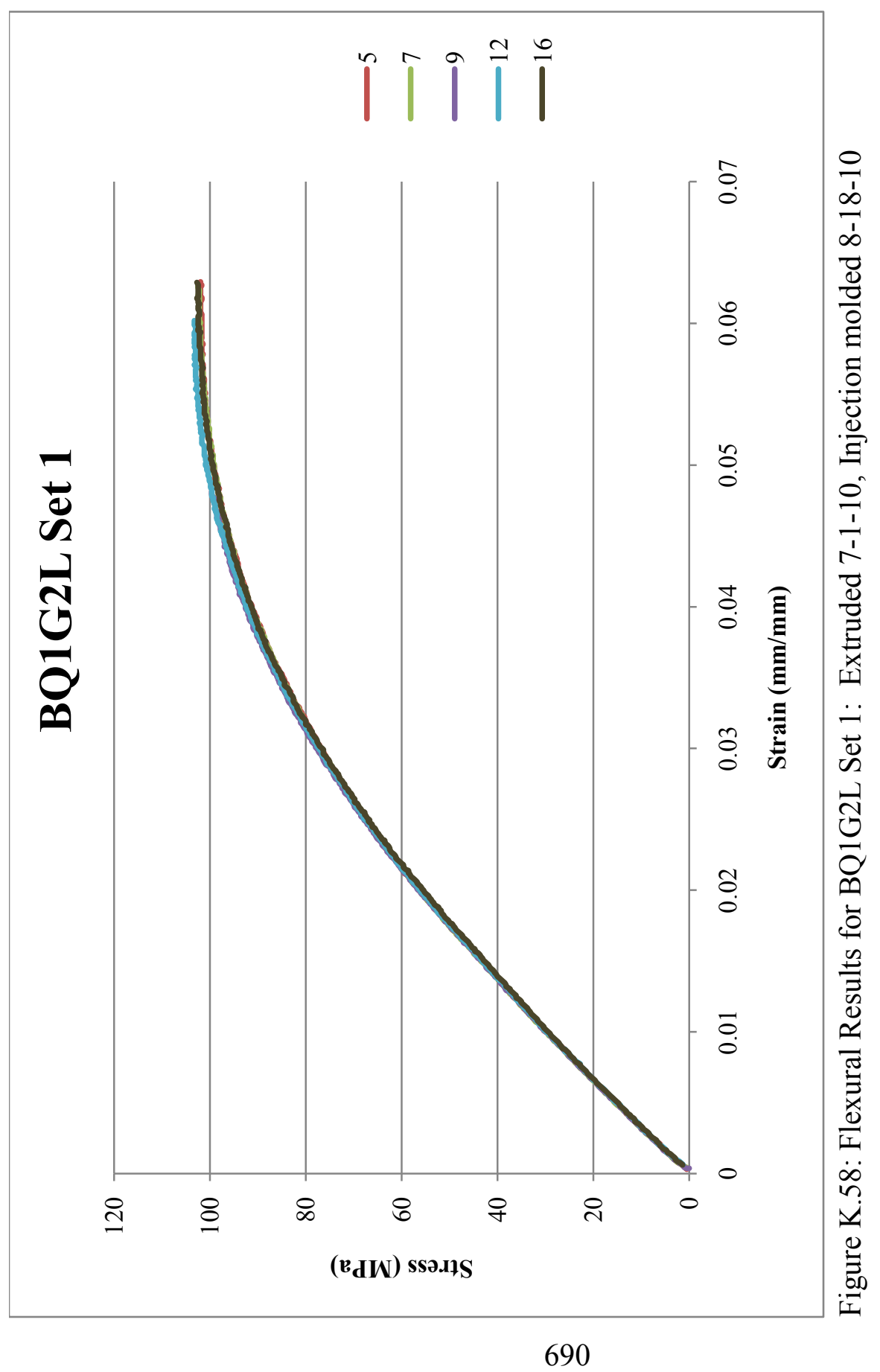




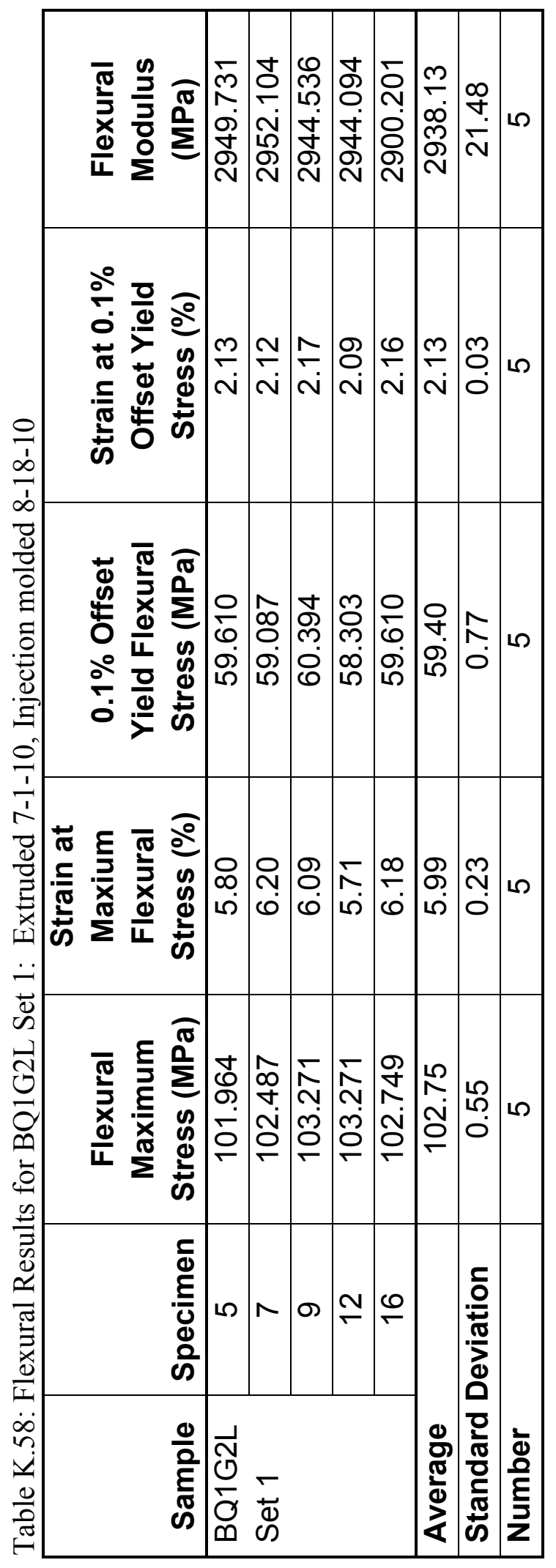




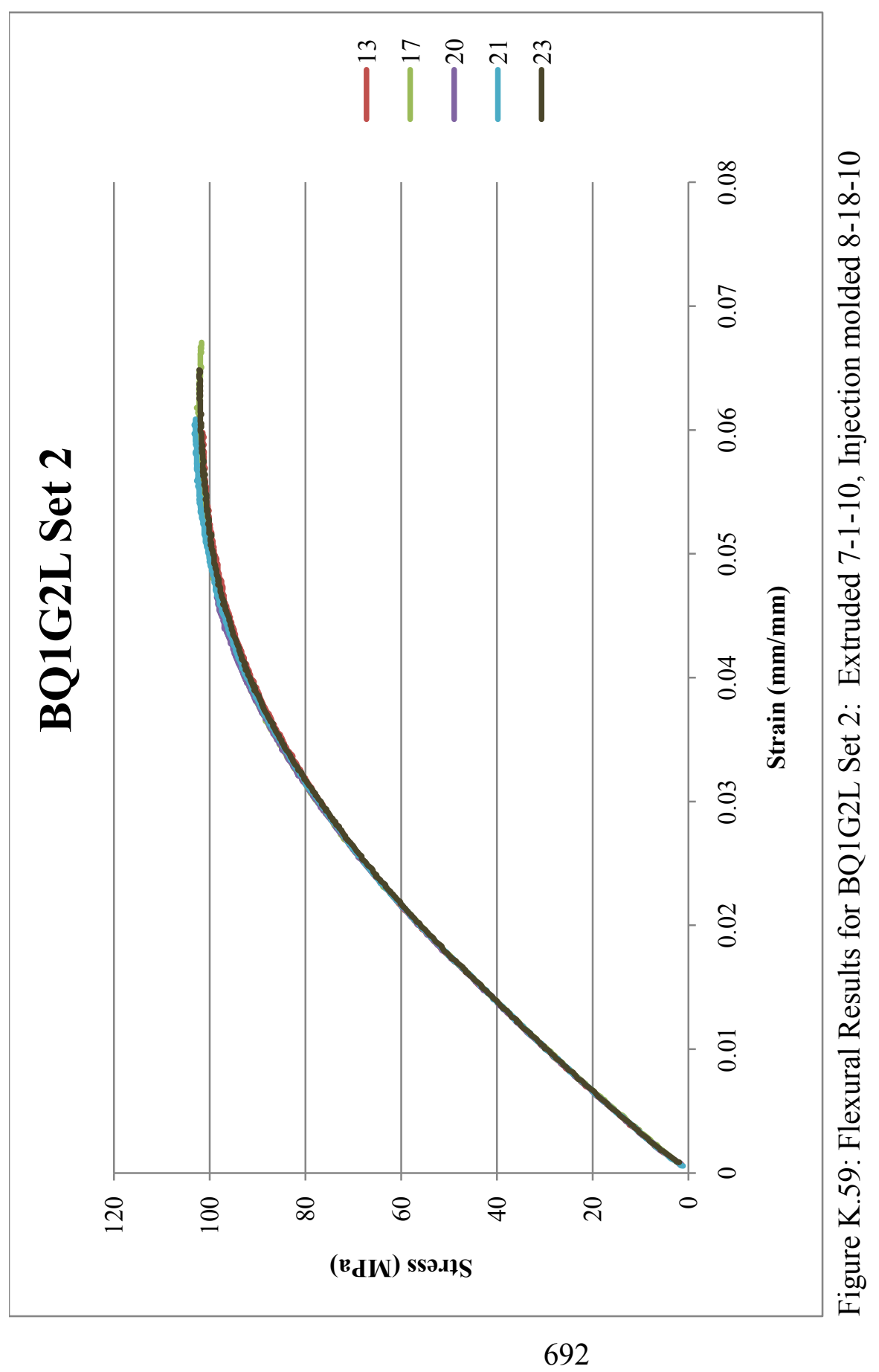




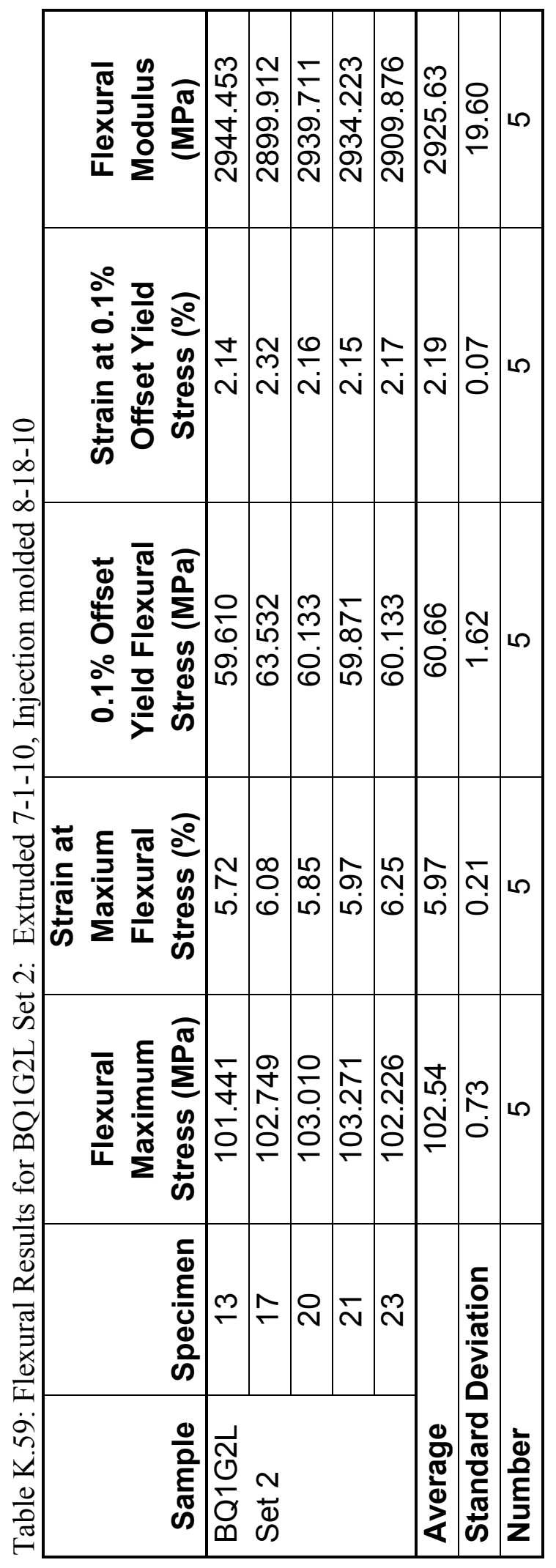




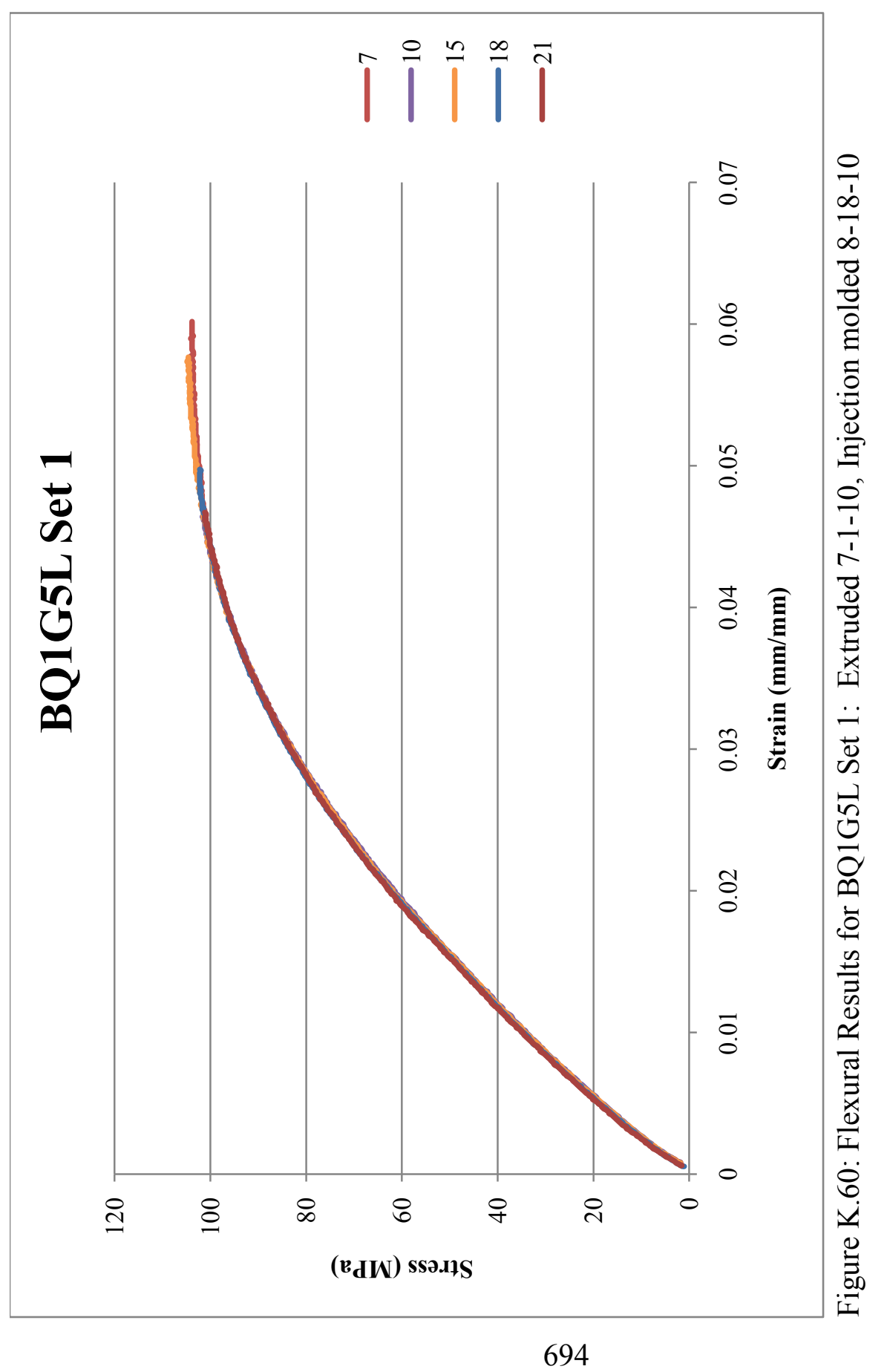




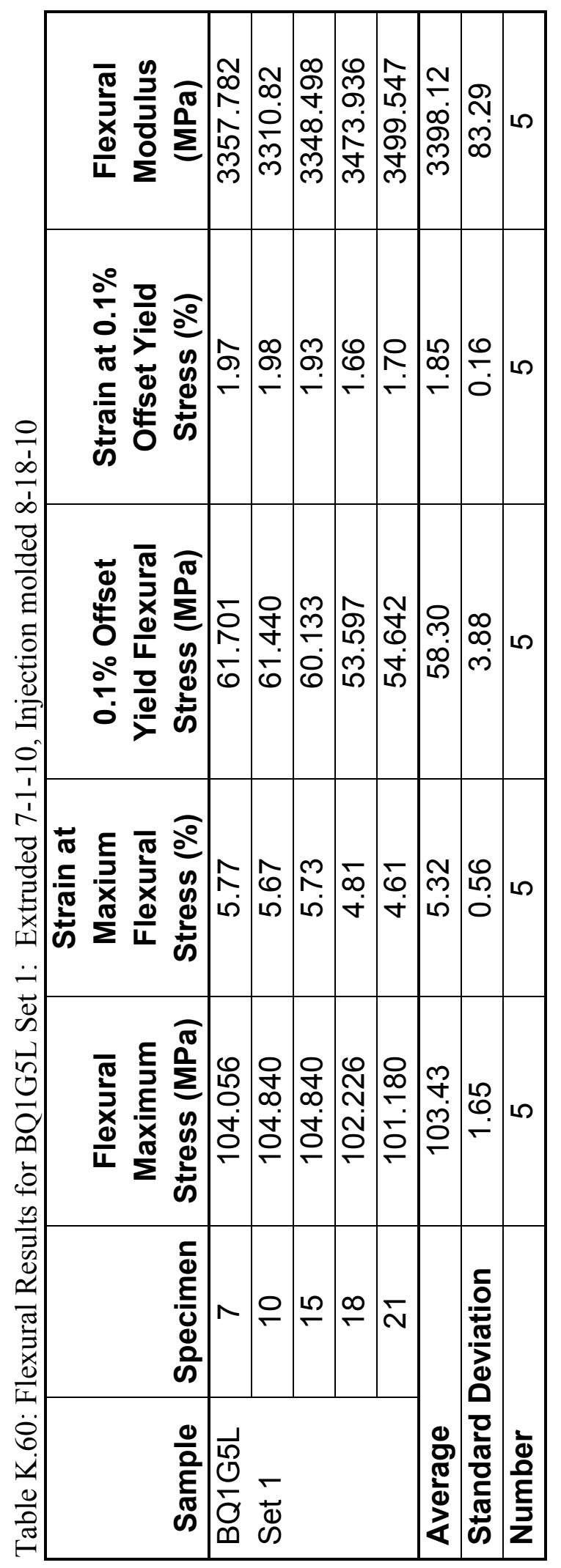




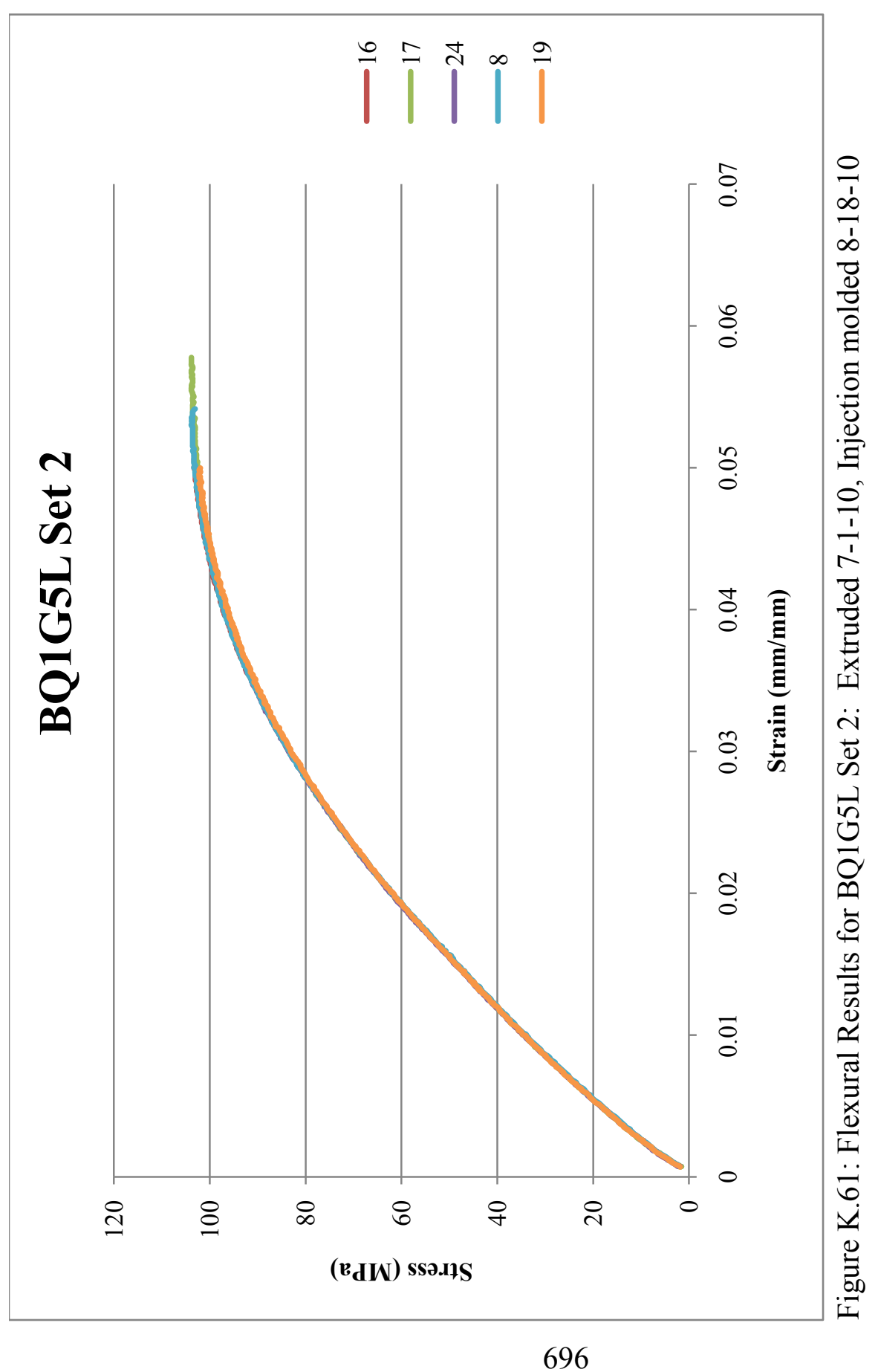




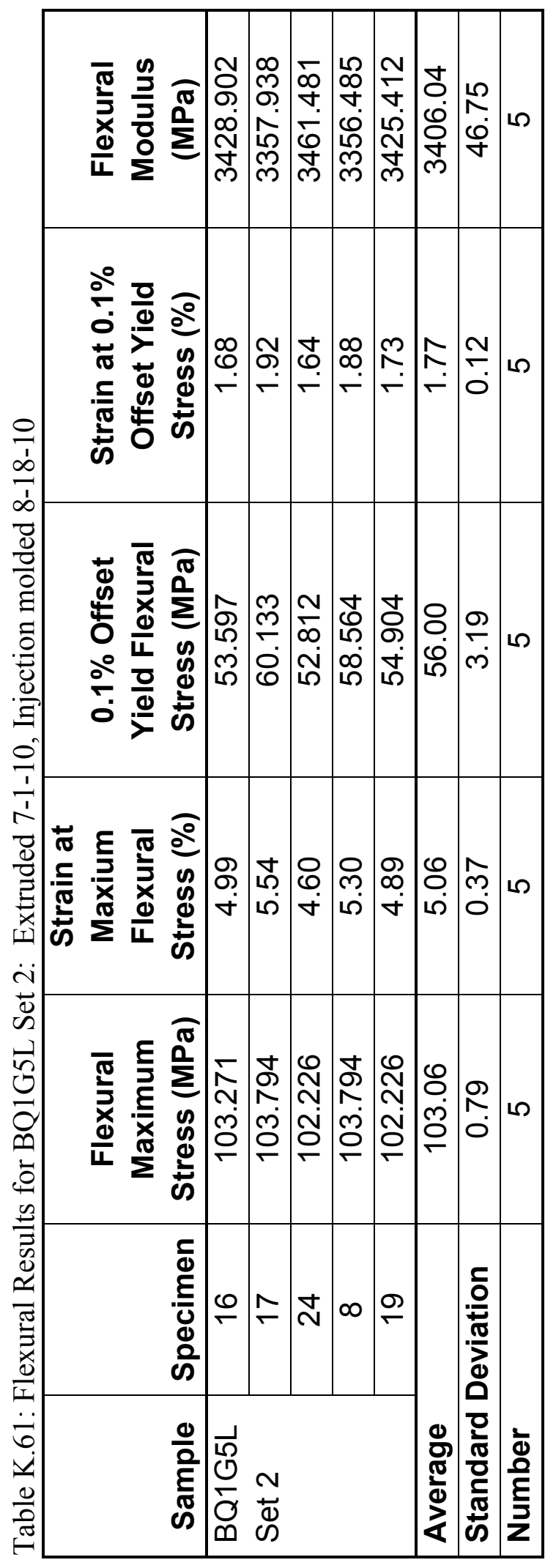




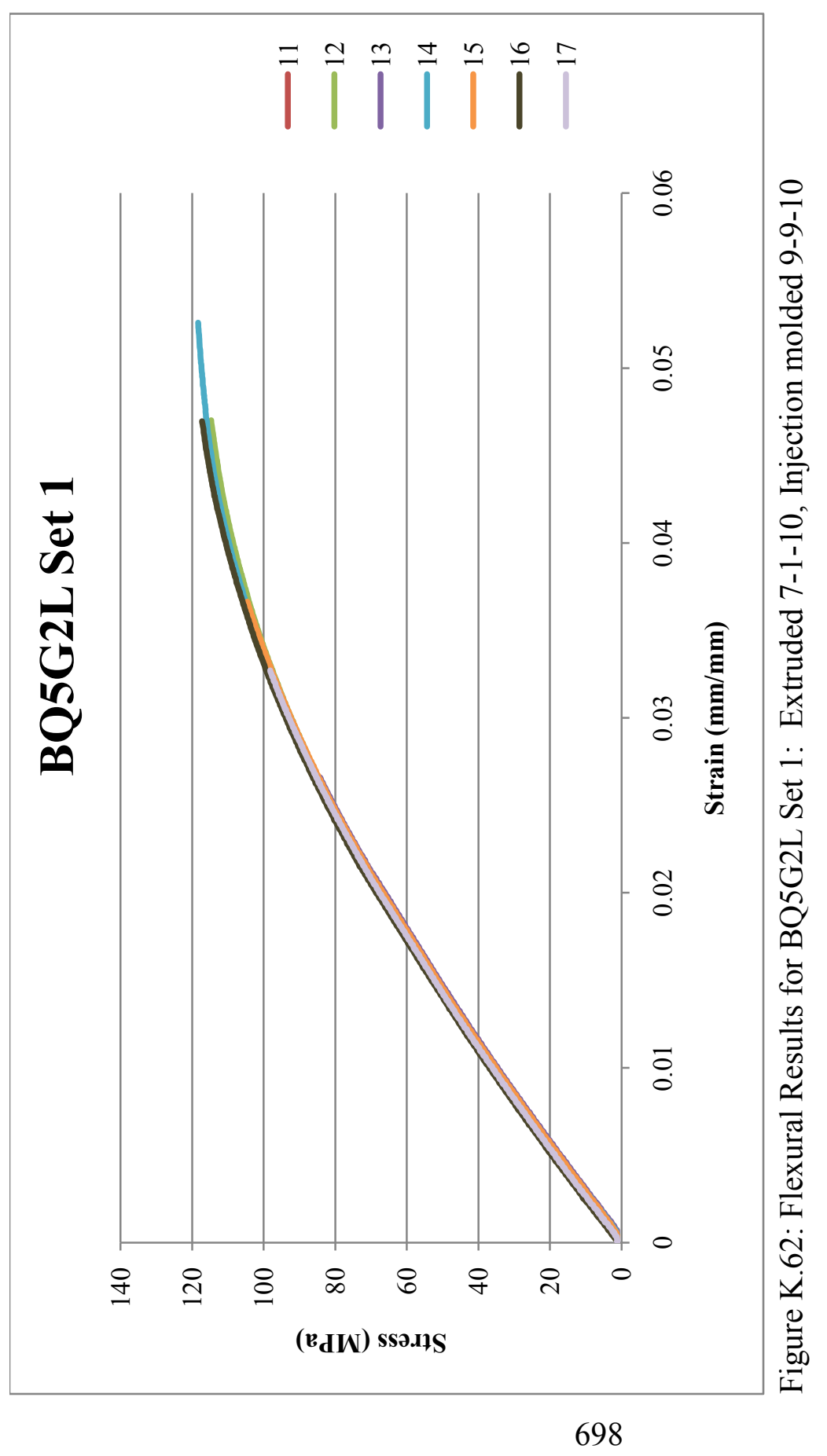




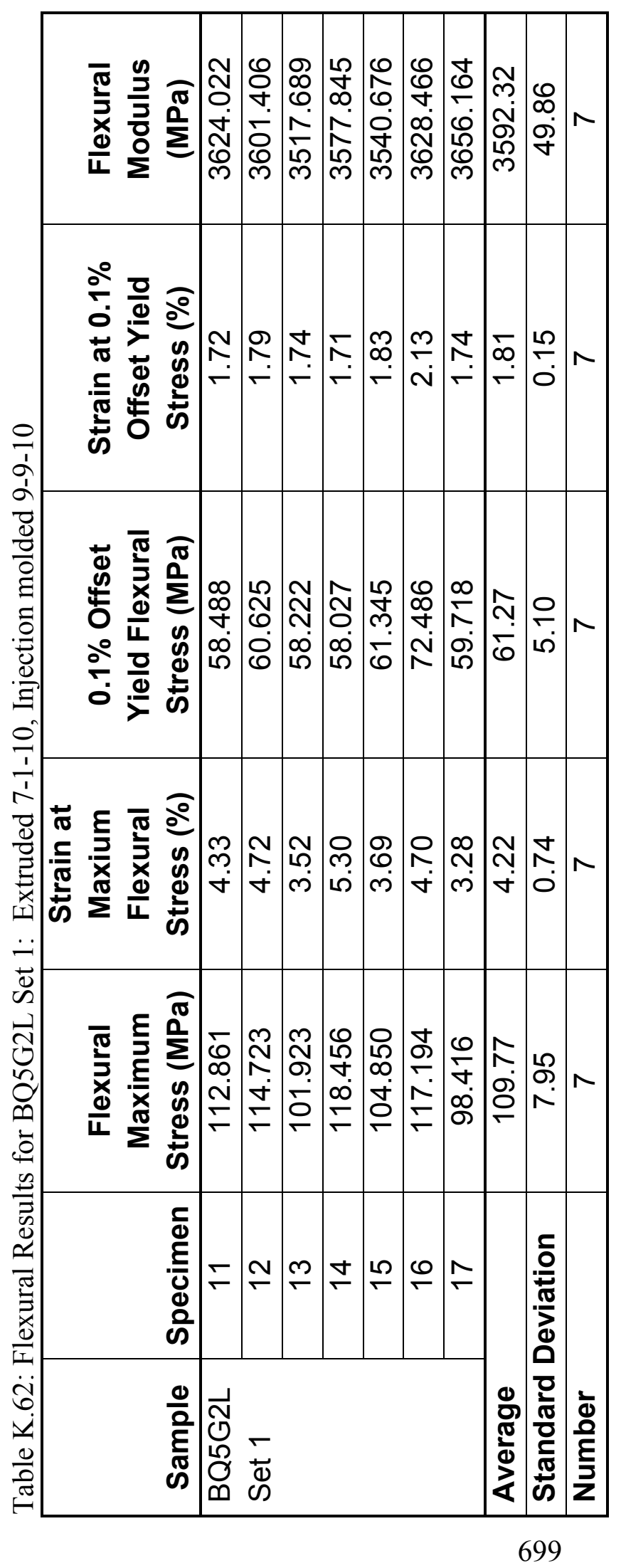




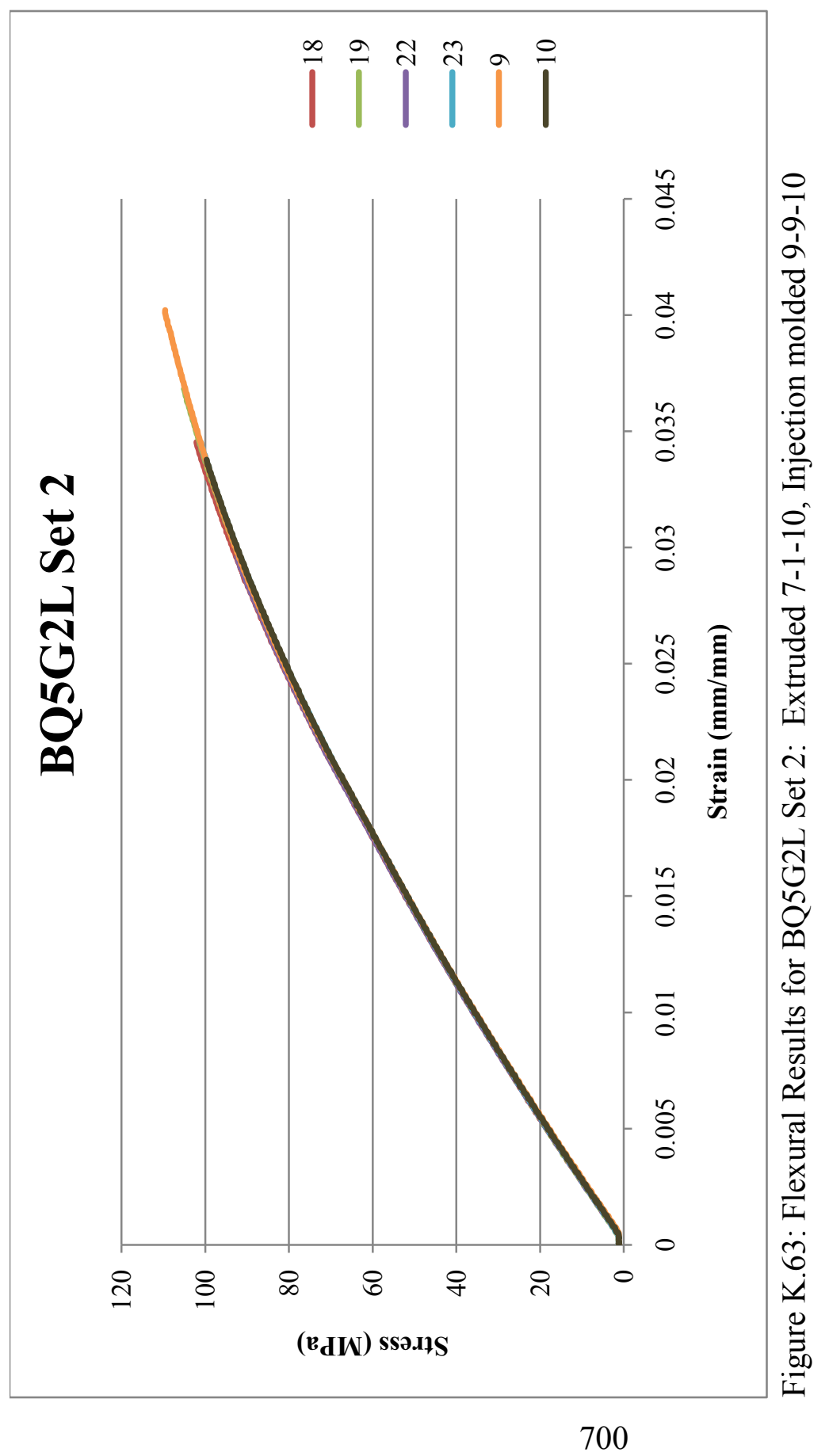




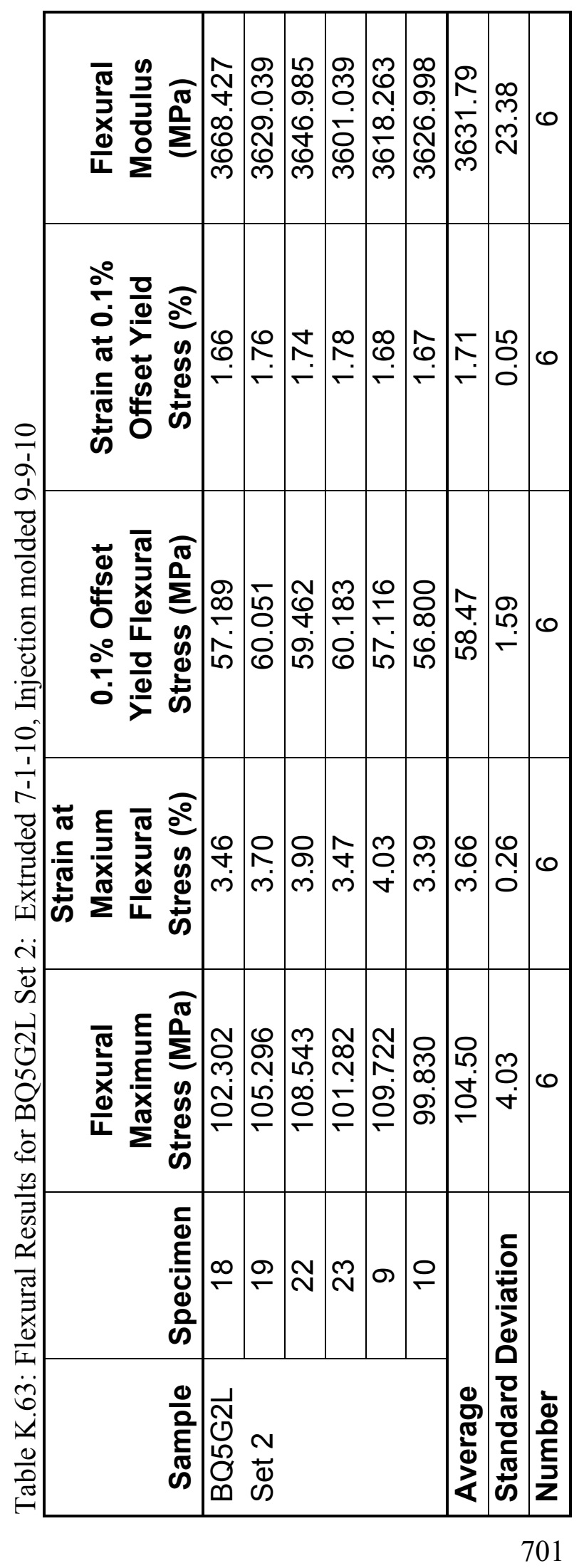




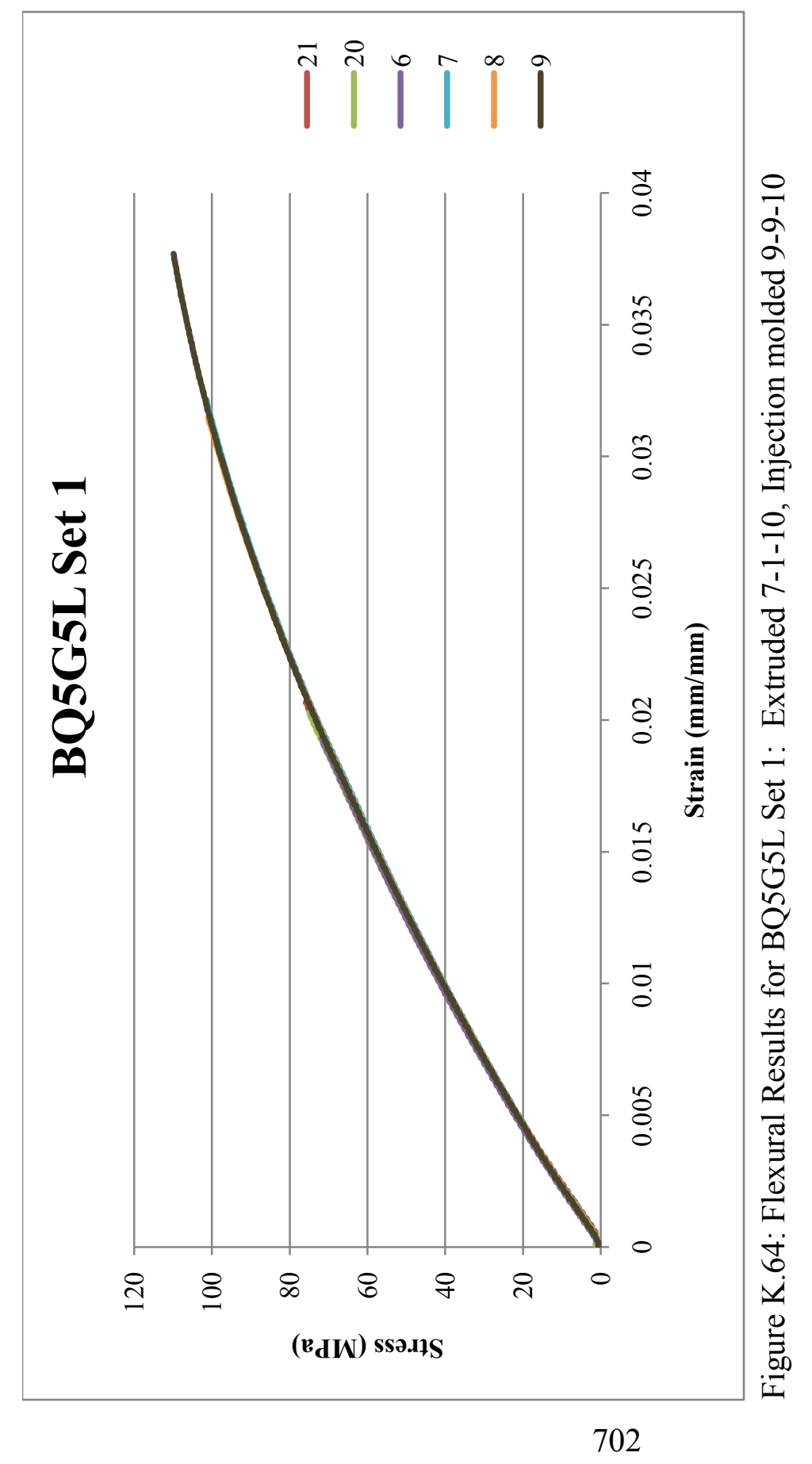




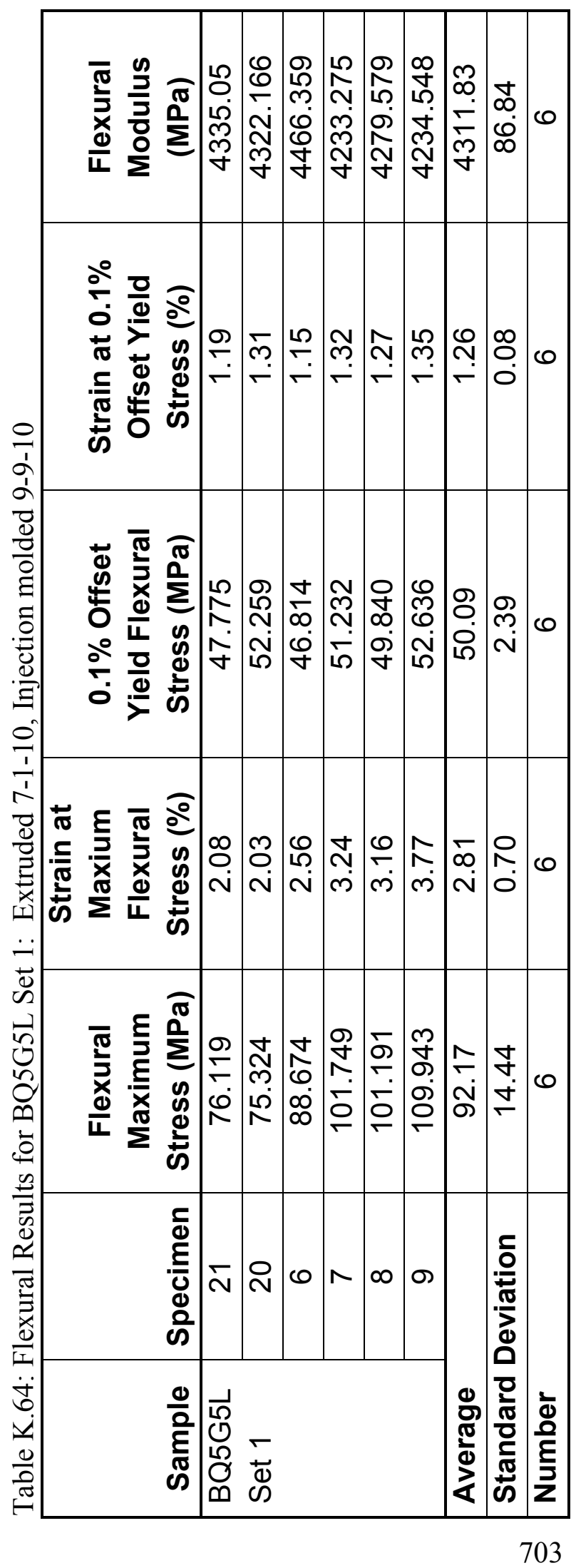




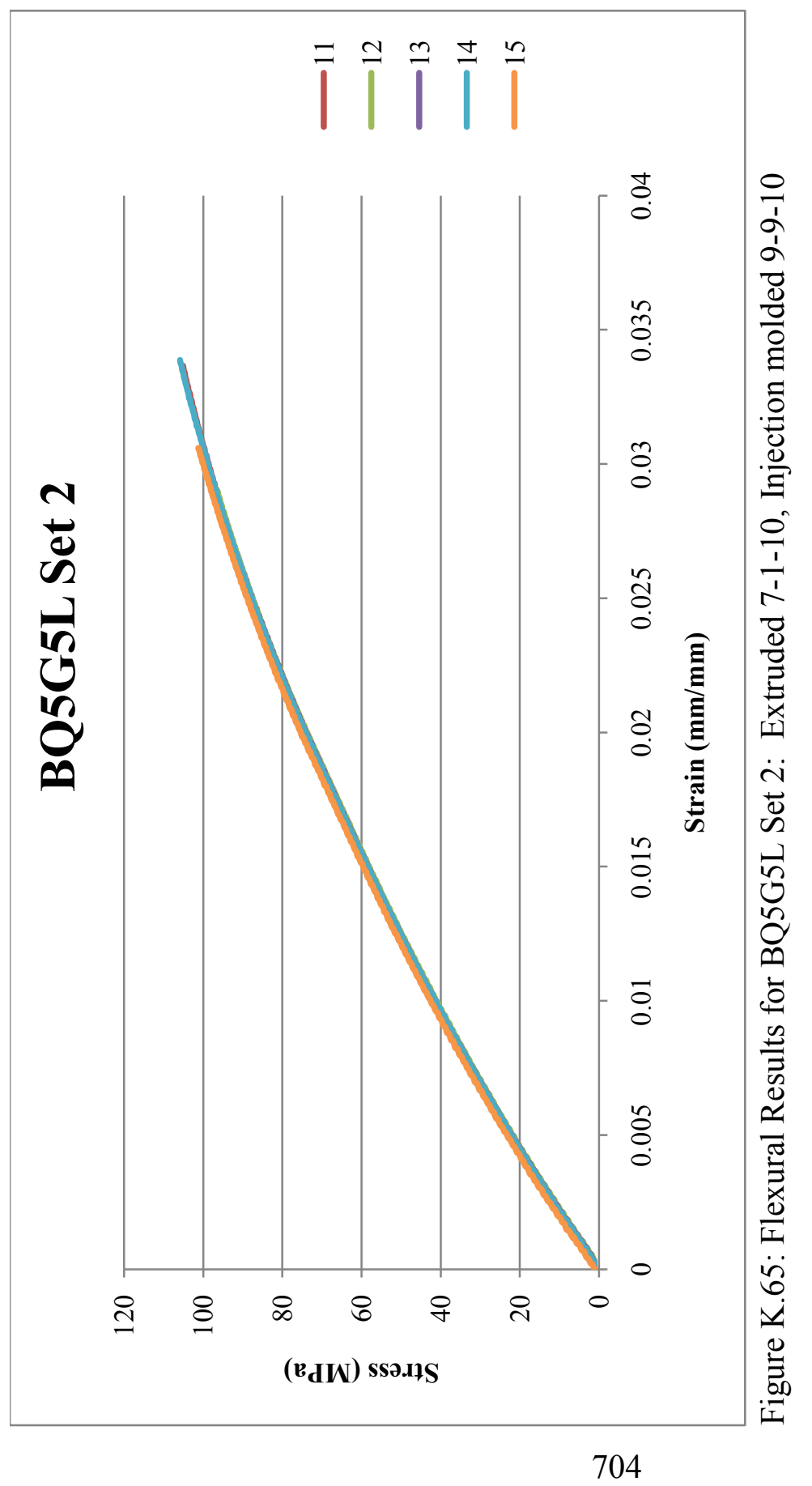




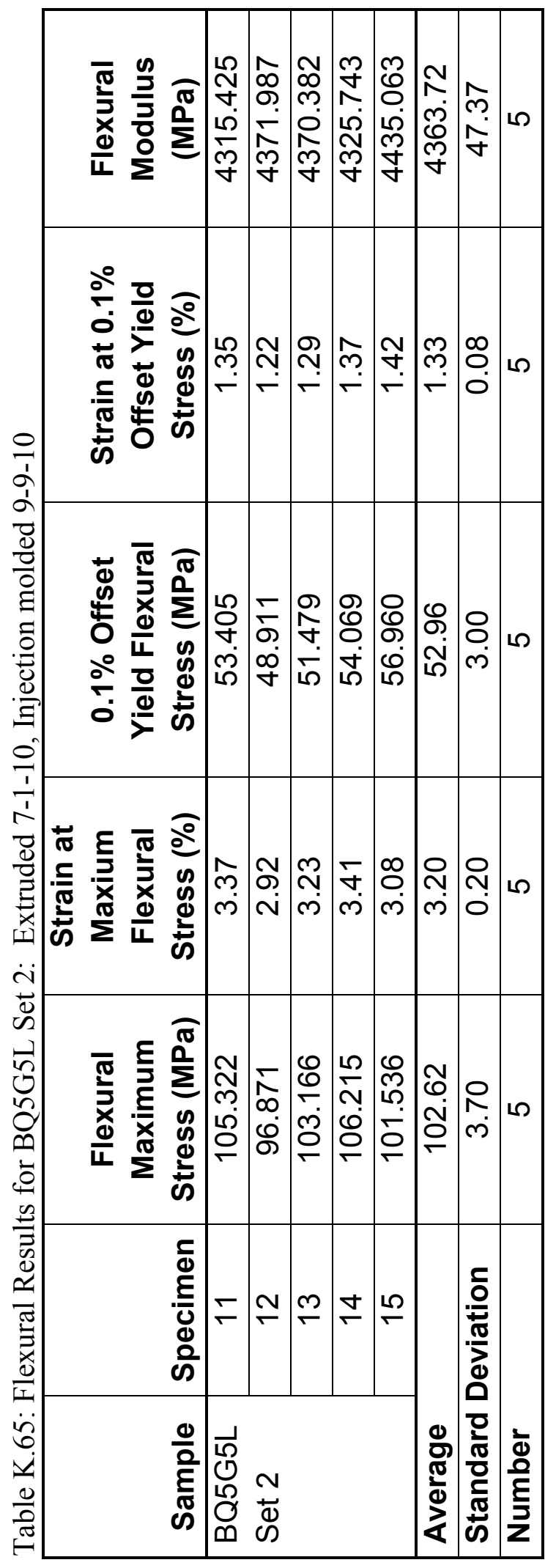




\section{Appendix L: Small Amplitude Oscillatory Shear Rheology Results}

\section{Appendix L.1: Polycarbonate with Carbon Nanotubes}

Table L.1: SAOS Results for BL: Lexan HF1130-111 at various temperatures

Formulation: $\mathrm{BL}$

\begin{tabular}{|c|c|c|}
\hline Temperature ${ }^{\circ} \mathrm{C}$ & Frequency (rad/s) & Complex Viscosity (Pa.s) \\
\hline \multirow[t]{10}{*}{210} & $1.00 \mathrm{E}-01$ & $5.15 \mathrm{E}+03$ \\
\hline & $2.15 \mathrm{E}-01$ & $5.08 \mathrm{E}+03$ \\
\hline & 4.64E-01 & $5.03 E+03$ \\
\hline & $1.00 \mathrm{E}+00$ & $4.98 \mathrm{E}+03$ \\
\hline & $2.15 \mathrm{E}+00$ & $4.89 \mathrm{E}+03$ \\
\hline & $4.64 \mathrm{E}+00$ & $4.69 \mathrm{E}+03$ \\
\hline & $1.00 \mathrm{E}+01$ & $4.25 \mathrm{E}+03$ \\
\hline & $2.15 \mathrm{E}+01$ & $3.71 \mathrm{E}+03$ \\
\hline & $4.64 \mathrm{E}+01$ & $2.98 \mathrm{E}+03$ \\
\hline & $1.00 \mathrm{E}+02$ & $2.19 \mathrm{E}+03$ \\
\hline \multirow[t]{10}{*}{270} & $1.00 \mathrm{E}-01$ & $3.66 \mathrm{E}+02$ \\
\hline & 2.15E-01 & $3.20 \mathrm{E}+02$ \\
\hline & 4.64E-01 & $2.84 \mathrm{E}+02$ \\
\hline & $1.00 \mathrm{E}+00$ & $2.68 \mathrm{E}+02$ \\
\hline & $2.15 \mathrm{E}+00$ & $2.62 \mathrm{E}+02$ \\
\hline & $4.64 \mathrm{E}+00$ & $2.61 \mathrm{E}+02$ \\
\hline & $1.00 \mathrm{E}+01$ & $2.59 \mathrm{E}+02$ \\
\hline & $2.15 \mathrm{E}+01$ & $2.57 \mathrm{E}+02$ \\
\hline & $4.64 \mathrm{E}+01$ & $2.52 \mathrm{E}+02$ \\
\hline & $1.00 \mathrm{E}+02$ & $2.36 \mathrm{E}+02$ \\
\hline \multirow[t]{10}{*}{230} & 1.00E-01 & $1.52 E+03$ \\
\hline & $2.15 \mathrm{E}-01$ & $1.45 \mathrm{E}+03$ \\
\hline & $4.64 \mathrm{E}-01$ & $1.43 \mathrm{E}+03$ \\
\hline & $1.00 \mathrm{E}+00$ & $1.43 \mathrm{E}+03$ \\
\hline & $2.15 \mathrm{E}+00$ & $1.42 \mathrm{E}+03$ \\
\hline & $4.64 \mathrm{E}+00$ & $1.41 \mathrm{E}+03$ \\
\hline & $1.00 \mathrm{E}+01$ & $1.37 \mathrm{E}+03$ \\
\hline & $2.15 \mathrm{E}+01$ & $1.30 \mathrm{E}+03$ \\
\hline & $4.64 \mathrm{E}+01$ & 1.17E+03 \\
\hline & $1.00 \mathrm{E}+02$ & $9.87 \mathrm{E}+02$ \\
\hline
\end{tabular}


Table L.2: SAOS Results for BQ2L: 2 wt\% Hyperion Fibrils (CNT) in Lexan HF1130111 at various temperatures

Formulation: BQ2L

\begin{tabular}{|c|c|c|}
\hline Temperature ${ }^{\circ} \mathrm{C}$ & Frequency (rad/s) & Complex Viscosity (Pa.s) \\
\hline \multirow[t]{10}{*}{210} & 1.00E-01 & 1.99E+04 \\
\hline & $2.15 \mathrm{E}-01$ & $1.18 \mathrm{E}+04$ \\
\hline & 4.64E-01 & $7.56 \mathrm{E}+03$ \\
\hline & $1.00 \mathrm{E}+00$ & $5.29 E+03$ \\
\hline & $2.15 \mathrm{E}+00$ & $3.96 \mathrm{E}+03$ \\
\hline & $4.64 \mathrm{E}+00$ & $3.13 E+03$ \\
\hline & $1.00 \mathrm{E}+01$ & $2.71 \mathrm{E}+03$ \\
\hline & $2.15 \mathrm{E}+01$ & $2.25 \mathrm{E}+03$ \\
\hline & $4.64 \mathrm{E}+01$ & $1.80 \mathrm{E}+03$ \\
\hline & $1.00 \mathrm{E}+02$ & $1.37 E+03$ \\
\hline \multirow[t]{10}{*}{270} & 1.00E-01 & $1.80 \mathrm{E}+04$ \\
\hline & $2.15 E-01$ & $8.83 E+03$ \\
\hline & 4.64E-01 & $4.32 E+03$ \\
\hline & $1.00 \mathrm{E}+00$ & $2.15 E+03$ \\
\hline & $2.15 \mathrm{E}+00$ & $1.12 \mathrm{E}+03$ \\
\hline & $4.64 \mathrm{E}+00$ & $6.20 \mathrm{E}+02$ \\
\hline & $1.00 \mathrm{E}+01$ & $3.86 \mathrm{E}+02$ \\
\hline & $2.15 E+01$ & $2.63 E+02$ \\
\hline & $4.64 \mathrm{E}+01$ & 1.93E+02 \\
\hline & $1.00 \mathrm{E}+02$ & $1.46 \mathrm{E}+02$ \\
\hline \multirow[t]{10}{*}{250} & 1.00E-01 & $1.28 \mathrm{E}+04$ \\
\hline & $2.15 \mathrm{E}-01$ & $6.59 \mathrm{E}+03$ \\
\hline & 4.64E-01 & $3.43 E+03$ \\
\hline & $1.00 \mathrm{E}+00$ & 1.87E+03 \\
\hline & $2.15 E+00$ & 1.11E+03 \\
\hline & $4.64 \mathrm{E}+00$ & $7.13 E+02$ \\
\hline & $1.00 \mathrm{E}+01$ & $5.20 \mathrm{E}+02$ \\
\hline & $2.15 E+01$ & $4.02 E+02$ \\
\hline & 4.64E+01 & $3.22 \mathrm{E}+02$ \\
\hline & $1.00 \mathrm{E}+02$ & $2.61 \mathrm{E}+02$ \\
\hline
\end{tabular}


Table L.2: SAOS Results for BQ2L: 2 wt\% Hyperion Fibrils (CNT) in Lexan HF1130111 at various temperatures continued

Formulation: BQ2L

\begin{tabular}{|c|c|c|}
\hline Temperature ${ }^{\circ} \mathrm{C}$ & Frequency (rad/s) & Complex Viscosity (Pa·s) \\
\hline $\mathbf{3 1 0}$ & $\mathbf{1 . 0 0 E}-01$ & $\mathbf{4 . 2 6 E + 0 4}$ \\
\hline & $2.15 \mathrm{E}-01$ & $2.11 \mathrm{E}+04$ \\
\hline & $4.64 \mathrm{E}-01$ & $1.02 \mathrm{E}+04$ \\
\hline & $1.00 \mathrm{E}+00$ & $4.94 \mathrm{E}+03$ \\
\hline & $2.15 \mathrm{E}+00$ & $2.40 \mathrm{E}+03$ \\
\hline & $4.64 \mathrm{E}+00$ & $1.18 \mathrm{E}+03$ \\
\hline & $1.00 \mathrm{E}+01$ & $5.96 \mathrm{E}+02$ \\
\hline & $2.15 \mathrm{E}+01$ & $3.14 \mathrm{E}+02$ \\
\hline & $4.64 \mathrm{E}+01$ & $1.76 \mathrm{E}+02$ \\
\hline & $1.00 \mathrm{E}+02$ & $1.04 \mathrm{E}+02$ \\
\hline
\end{tabular}


Table L.3: SAOS Results for BQ3L: 3 wt\% Hyperion Fibrils (CNT) in Lexan HF1130111 at various temperatures

Formulation: BQ3L

\begin{tabular}{|c|c|c|}
\hline Temperature ${ }^{\circ} \mathrm{C}$ & Frequency (rad/s) & Complex Viscosity (Pa.s) \\
\hline \multirow[t]{10}{*}{210} & 1.00E-01 & $2.00 \mathrm{E}+05$ \\
\hline & $2.15 \mathrm{E}-01$ & $1.03 E+05$ \\
\hline & 4.64E-01 & $7.86 \mathrm{E}+04$ \\
\hline & $1.00 \mathrm{E}+00$ & $3.07 E+04$ \\
\hline & $2.15 \mathrm{E}+00$ & $1.85 E+04$ \\
\hline & $4.64 \mathrm{E}+00$ & $1.19 \mathrm{E}+04$ \\
\hline & $1.00 \mathrm{E}+01$ & $8.88 \mathrm{E}+03$ \\
\hline & $2.15 \mathrm{E}+01$ & $6.07 E+03$ \\
\hline & $4.64 \mathrm{E}+01$ & $4.52 \mathrm{E}+03$ \\
\hline & $1.00 \mathrm{E}+02$ & $3.26 \mathrm{E}+03$ \\
\hline \multirow[t]{10}{*}{270} & 1.00E-01 & 1.47E+05 \\
\hline & $2.15 E-01$ & $7.19 \mathrm{E}+04$ \\
\hline & 4.64E-01 & $3.50 \mathrm{E}+04$ \\
\hline & $1.00 \mathrm{E}+00$ & $1.72 \mathrm{E}+04$ \\
\hline & $2.15 \mathrm{E}+00$ & $8.48 \mathrm{E}+03$ \\
\hline & $4.64 \mathrm{E}+00$ & $4.37 \mathrm{E}+03$ \\
\hline & $1.00 \mathrm{E}+01$ & $2.24 \mathrm{E}+03$ \\
\hline & $2.15 E+01$ & $1.29 \mathrm{E}+03$ \\
\hline & $4.64 \mathrm{E}+01$ & $7.93 E+02$ \\
\hline & $1.00 \mathrm{E}+02$ & $5.26 \mathrm{E}+02$ \\
\hline \multirow[t]{10}{*}{290} & 1.00E-01 & 1.16E+05 \\
\hline & $2.15 \mathrm{E}-01$ & $5.64 \mathrm{E}+04$ \\
\hline & 4.64E-01 & $2.75 \mathrm{E}+04$ \\
\hline & $1.00 \mathrm{E}+00$ & $1.35 \mathrm{E}+04$ \\
\hline & $2.15 \mathrm{E}+00$ & $6.76 \mathrm{E}+03$ \\
\hline & $4.64 \mathrm{E}+00$ & $3.42 \mathrm{E}+03$ \\
\hline & $1.00 \mathrm{E}+01$ & 1.67E+03 \\
\hline & $2.15 E+01$ & $9.09 \mathrm{E}+02$ \\
\hline & 4.64E+01 & $4.99 \mathrm{E}+02$ \\
\hline & $1.00 \mathrm{E}+02$ & $2.96 \mathrm{E}+02$ \\
\hline
\end{tabular}


Table L.3: SAOS Results for BQ3L: 3 wt\% Hyperion Fibrils (CNT) in Lexan HF1130111 at various temperatures continued

Formulation: BQ3L

\begin{tabular}{|c|c|c|}
\hline Temperature ${ }^{\circ} \mathrm{C}$ & Frequency (rad/s) & Complex Viscosity (Pa's) \\
\hline \multirow[t]{10}{*}{310} & $1.00 \mathrm{E}-01$ & $1.20 E+05$ \\
\hline & $2.15 \mathrm{E}-01$ & $5.86 \mathrm{E}+04$ \\
\hline & 4.64E-01 & $2.84 \mathrm{E}+04$ \\
\hline & $1.00 \mathrm{E}+00$ & $1.38 \mathrm{E}+04$ \\
\hline & $2.15 \mathrm{E}+00$ & $6.83 E+03$ \\
\hline & $4.64 \mathrm{E}+00$ & $3.41 \mathrm{E}+03$ \\
\hline & $1.00 \mathrm{E}+01$ & $1.65 \mathrm{E}+03$ \\
\hline & $2.15 \mathrm{E}+01$ & $8.74 \mathrm{E}+02$ \\
\hline & $4.64 \mathrm{E}+01$ & $4.59 \mathrm{E}+02$ \\
\hline & $1.00 \mathrm{E}+02$ & $2.57 \mathrm{E}+02$ \\
\hline \multirow[t]{10}{*}{330} & $1.00 \mathrm{E}-01$ & $1.14 \mathrm{E}+05$ \\
\hline & $2.15 \mathrm{E}-01$ & $5.65 \mathrm{E}+04$ \\
\hline & $4.64 \mathrm{E}-01$ & $2.77 \mathrm{E}+04$ \\
\hline & $1.00 \mathrm{E}+00$ & $1.35 \mathrm{E}+04$ \\
\hline & $2.15 \mathrm{E}+00$ & $6.66 \mathrm{E}+03$ \\
\hline & $4.64 \mathrm{E}+00$ & $3.33 \mathrm{E}+03$ \\
\hline & $1.00 \mathrm{E}+01$ & $1.63 \mathrm{E}+03$ \\
\hline & $2.15 \mathrm{E}+01$ & $8.64 \mathrm{E}+02$ \\
\hline & $4.64 \mathrm{E}+01$ & $4.46 \mathrm{E}+02$ \\
\hline & $1.00 \mathrm{E}+02$ & $2.39 \mathrm{E}+02$ \\
\hline \multirow[t]{10}{*}{350} & 1.00E-01 & $1.06 \mathrm{E}+05$ \\
\hline & $2.15 \mathrm{E}-01$ & $5.40 \mathrm{E}+04$ \\
\hline & 4.64E-01 & $2.76 \mathrm{E}+04$ \\
\hline & $1.00 \mathrm{E}+00$ & $1.37 \mathrm{E}+04$ \\
\hline & $2.15 \mathrm{E}+00$ & $6.78 \mathrm{E}+03$ \\
\hline & $4.64 \mathrm{E}+00$ & $3.39 \mathrm{E}+03$ \\
\hline & $1.00 \mathrm{E}+01$ & $1.65 \mathrm{E}+03$ \\
\hline & $2.15 E+01$ & $8.65 E+02$ \\
\hline & $4.64 \mathrm{E}+01$ & $4.49 \mathrm{E}+02$ \\
\hline & $1.00 \mathrm{E}+02$ & $2.36 \mathrm{E}+02$ \\
\hline
\end{tabular}


Table L.4: SAOS Results for BQ4L: 4 wt\% Hyperion Fibrils (CNT) in Lexan HF1130111 at various temperatures

Formulation: BQ4L

\begin{tabular}{|c|c|c|}
\hline Temperature ${ }^{\circ} \mathrm{C}$ & Frequency (rad/s) & Complex Viscosity (Pa.s) \\
\hline \multirow[t]{10}{*}{210} & 1.00E-01 & $4.65 E+05$ \\
\hline & $2.15 \mathrm{E}-01$ & $2.39 \mathrm{E}+05$ \\
\hline & 4.64E-01 & $1.80 \mathrm{E}+05$ \\
\hline & $1.00 \mathrm{E}+00$ & $6.59 \mathrm{E}+04$ \\
\hline & $2.15 \mathrm{E}+00$ & $3.71 \mathrm{E}+04$ \\
\hline & $4.64 \mathrm{E}+00$ & $1.95 \mathrm{E}+04$ \\
\hline & $1.00 \mathrm{E}+01$ & $1.59 E+04$ \\
\hline & $2.15 \mathrm{E}+01$ & $9.12 \mathrm{E}+03$ \\
\hline & $4.64 \mathrm{E}+01$ & $6.01 \mathrm{E}+03$ \\
\hline & $1.00 \mathrm{E}+02$ & $4.14 \mathrm{E}+03$ \\
\hline \multirow[t]{10}{*}{230} & 1.00E-01 & $3.94 \mathrm{E}+05$ \\
\hline & $2.15 E-01$ & $1.96 \mathrm{E}+05$ \\
\hline & 4.64E-01 & $9.70 \mathrm{E}+04$ \\
\hline & $1.00 \mathrm{E}+00$ & $4.92 \mathrm{E}+04$ \\
\hline & $2.15 \mathrm{E}+00$ & $2.53 E+04$ \\
\hline & $4.64 \mathrm{E}+00$ & 1.37E+04 \\
\hline & $1.00 \mathrm{E}+01$ & $7.75 \mathrm{E}+03$ \\
\hline & $2.15 E+01$ & $4.66 \mathrm{E}+03$ \\
\hline & $4.64 \mathrm{E}+01$ & $3.01 \mathrm{E}+03$ \\
\hline & $1.00 \mathrm{E}+02$ & $2.04 \mathrm{E}+03$ \\
\hline \multirow[t]{10}{*}{270} & 1.00E-01 & $3.14 \mathrm{E}+05$ \\
\hline & $2.15 \mathrm{E}-01$ & $1.57 \mathrm{E}+05$ \\
\hline & 4.64E-01 & $7.65 \mathrm{E}+04$ \\
\hline & $1.00 \mathrm{E}+00$ & $3.76 \mathrm{E}+04$ \\
\hline & $2.15 E+00$ & $1.86 \mathrm{E}+04$ \\
\hline & $4.64 \mathrm{E}+00$ & $9.15 \mathrm{E}+03$ \\
\hline & $1.00 \mathrm{E}+01$ & $4.42 \mathrm{E}+03$ \\
\hline & $2.15 E+01$ & $2.46 \mathrm{E}+03$ \\
\hline & $4.64 \mathrm{E}+01$ & $1.38 \mathrm{E}+03$ \\
\hline & $1.00 \mathrm{E}+02$ & $8.30 \mathrm{E}+02$ \\
\hline
\end{tabular}


Table L.4: SAOS Results for BQ4L: 4 wt\% Hyperion Fibrils (CNT) in Lexan HF1130111 at various temperatures continued

Formulation: BQ4L

\begin{tabular}{|c|c|c|}
\hline Temperature ${ }^{\circ} \mathrm{C}$ & Frequency (rad/s) & Complex Viscosity (Pa·s) \\
\hline $\mathbf{2 9 0}$ & $\mathbf{1 . 0 0 E}-\mathbf{0 1}$ & $\mathbf{2 . 8 5 E + 0 5}$ \\
\hline & $2.15 \mathrm{E}-01$ & $1.39 \mathrm{E}+05$ \\
\hline & $4.64 \mathrm{E}-01$ & $6.76 \mathrm{E}+04$ \\
\hline & $1.00 \mathrm{E}+00$ & $3.30 \mathrm{E}+04$ \\
\hline & $2.15 \mathrm{E}+00$ & $1.58 \mathrm{E}+04$ \\
\hline & $4.64 \mathrm{E}+00$ & $7.89 \mathrm{E}+03$ \\
\hline & $1.00 \mathrm{E}+01$ & $3.66 \mathrm{E}+03$ \\
\hline & $2.15 \mathrm{E}+01$ & $1.94 \mathrm{E}+03$ \\
\hline & $4.64 \mathrm{E}+01$ & $1.01 \mathrm{E}+03$ \\
\hline & $1.00 \mathrm{E}+02$ & $5.61 \mathrm{E}+02$ \\
\hline & $\mathbf{1 . 0 0 E}-01$ & $3.38 \mathrm{E}+05$ \\
\hline & $2.15 \mathrm{E}-01$ & $1.64 \mathrm{E}+05$ \\
\hline & $4.64 \mathrm{E}-01$ & $7.88 \mathrm{E}+04$ \\
\hline & $1.00 \mathrm{E}+00$ & $3.81 \mathrm{E}+04$ \\
\hline & $2.15 \mathrm{E}+00$ & $1.81 \mathrm{E}+04$ \\
\hline & $4.64 \mathrm{E}+00$ & $8.38 \mathrm{E}+03$ \\
\hline & $1.00 \mathrm{E}+01$ & $4.07 \mathrm{E}+03$ \\
\hline & $2.15 \mathrm{E}+01$ & $2.06 \mathrm{E}+03$ \\
\hline & $4.64 \mathrm{E}+01$ & $1.03 \mathrm{E}+03$ \\
\hline & $1.00 \mathrm{E}+02$ & $5.35 \mathrm{E}+02$ \\
\hline
\end{tabular}


Table L.5: SAOS Results for BQ5L: 5 wt\% Hyperion Fibrils (CNT) in Lexan HF1130111 at various temperatures

Formulation: BQ5L

\begin{tabular}{|c|c|c|}
\hline Temperature ${ }^{\circ} \mathrm{C}$ & Frequency (rad/s) & Complex Viscosity (Pa.s) \\
\hline \multirow[t]{10}{*}{210} & 1.00E-01 & $7.26 \mathrm{E}+05$ \\
\hline & $2.15 \mathrm{E}-01$ & $3.70 \mathrm{E}+05$ \\
\hline & 4.64E-01 & $2.73 E+05$ \\
\hline & $1.00 \mathrm{E}+00$ & $1.02 \mathrm{E}+05$ \\
\hline & $2.15 E+00$ & $5.52 E+04$ \\
\hline & $4.64 \mathrm{E}+00$ & $2.90 \mathrm{E}+04$ \\
\hline & $1.00 \mathrm{E}+01$ & $1.76 \mathrm{E}+04$ \\
\hline & $2.15 E+01$ & $1.09 \mathrm{E}+04$ \\
\hline & $4.64 \mathrm{E}+01$ & $6.84 \mathrm{E}+03$ \\
\hline & $1.00 \mathrm{E}+02$ & $4.57 \mathrm{E}+03$ \\
\hline \multirow[t]{10}{*}{270} & 1.00E-01 & $5.91 \mathrm{E}+05$ \\
\hline & $2.15 E-01$ & $3.04 \mathrm{E}+05$ \\
\hline & 4.64E-01 & $1.49 \mathrm{E}+05$ \\
\hline & $1.00 \mathrm{E}+00$ & $7.50 \mathrm{E}+04$ \\
\hline & $2.15 E+00$ & $3.59 \mathrm{E}+04$ \\
\hline & $4.64 \mathrm{E}+00$ & $1.68 \mathrm{E}+04$ \\
\hline & $1.00 \mathrm{E}+01$ & $8.43 E+03$ \\
\hline & $2.15 E+01$ & $4.39 E+03$ \\
\hline & $4.64 \mathrm{E}+01$ & $2.32 \mathrm{E}+03$ \\
\hline & $1.00 \mathrm{E}+02$ & $1.30 \mathrm{E}+03$ \\
\hline \multirow[t]{10}{*}{290} & 1.00E-01 & $5.41 \mathrm{E}+05$ \\
\hline & $2.15 \mathrm{E}-01$ & $2.66 \mathrm{E}+05$ \\
\hline & 4.64E-01 & $1.28 \mathrm{E}+05$ \\
\hline & $1.00 \mathrm{E}+00$ & $6.31 \mathrm{E}+04$ \\
\hline & $2.15 E+00$ & $3.05 E+04$ \\
\hline & $4.64 \mathrm{E}+00$ & $1.34 \mathrm{E}+04$ \\
\hline & $1.00 \mathrm{E}+01$ & $7.01 \mathrm{E}+03$ \\
\hline & $2.15 E+01$ & $3.49 \mathrm{E}+03$ \\
\hline & $4.64 \mathrm{E}+01$ & 1.77E+03 \\
\hline & $1.00 \mathrm{E}+02$ & $9.39 \mathrm{E}+02$ \\
\hline
\end{tabular}


Table L.5: SAOS Results for BQ5L: 5 wt\% Hyperion Fibrils (CNT) in Lexan HF1130111 at various temperatures continued

Formulation: BQ5L

\begin{tabular}{|c|c|c|}
\hline Temperature ${ }^{\circ} \mathrm{C}$ & Frequency (rad/s) & Complex Viscosity (Pa's) \\
\hline \multirow[t]{10}{*}{310} & $1.00 \mathrm{E}-01$ & $5.99 E+05$ \\
\hline & $2.15 \mathrm{E}-01$ & $2.94 \mathrm{E}+05$ \\
\hline & 4.64E-01 & $1.42 \mathrm{E}+05$ \\
\hline & $1.00 \mathrm{E}+00$ & $6.94 \mathrm{E}+04$ \\
\hline & $2.15 \mathrm{E}+00$ & $3.37 \mathrm{E}+04$ \\
\hline & $4.64 \mathrm{E}+00$ & $1.44 \mathrm{E}+04$ \\
\hline & $1.00 \mathrm{E}+01$ & $7.47 \mathrm{E}+03$ \\
\hline & $2.15 \mathrm{E}+01$ & $3.67 \mathrm{E}+03$ \\
\hline & $4.64 \mathrm{E}+01$ & $1.81 \mathrm{E}+03$ \\
\hline & $1.00 \mathrm{E}+02$ & $9.19 E+02$ \\
\hline \multirow{10}{*}{330} & $1.00 \mathrm{E}-01$ & $4.89 \mathrm{E}+05$ \\
\hline & 2.15E-01 & $2.43 E+05$ \\
\hline & $4.64 \mathrm{E}-01$ & $1.18 \mathrm{E}+05$ \\
\hline & $1.00 \mathrm{E}+00$ & $5.74 \mathrm{E}+04$ \\
\hline & $2.15 \mathrm{E}+00$ & $2.82 \mathrm{E}+04$ \\
\hline & $4.64 \mathrm{E}+00$ & $1.25 \mathrm{E}+04$ \\
\hline & $1.00 \mathrm{E}+01$ & $6.14 \mathrm{E}+03$ \\
\hline & $2.15 \mathrm{E}+01$ & $2.97 \mathrm{E}+03$ \\
\hline & $4.64 \mathrm{E}+01$ & $1.45 \mathrm{E}+03$ \\
\hline & $1.00 \mathrm{E}+02$ & $7.19 \mathrm{E}+02$ \\
\hline \multirow[t]{10}{*}{350} & 1.00E-01 & $5.49 \mathrm{E}+05$ \\
\hline & $2.15 \mathrm{E}-01$ & $2.75 \mathrm{E}+05$ \\
\hline & 4.64E-01 & $1.35 \mathrm{E}+05$ \\
\hline & $1.00 \mathrm{E}+00$ & $6.66 \mathrm{E}+04$ \\
\hline & $2.15 \mathrm{E}+00$ & $3.17 \mathrm{E}+04$ \\
\hline & $4.64 \mathrm{E}+00$ & $1.40 \mathrm{E}+04$ \\
\hline & $1.00 \mathrm{E}+01$ & $7.34 \mathrm{E}+03$ \\
\hline & $2.15 E+01$ & $3.52 \mathrm{E}+03$ \\
\hline & $4.64 \mathrm{E}+01$ & $1.67 \mathrm{E}+03$ \\
\hline & $1.00 \mathrm{E}+02$ & $8.21 \mathrm{E}+02$ \\
\hline
\end{tabular}


Table L.6: SAOS Results for BQ6L: 6 wt\% Hyperion Fibrils (CNT) in Lexan HF1130111 at various temperatures

Formulation: BQ6L

\begin{tabular}{|c|c|c|}
\hline Temperature ${ }^{\circ} \mathrm{C}$ & Frequency (rad/s) & Complex Viscosity (Pa.s) \\
\hline \multirow[t]{10}{*}{210} & 1.00E-01 & $1.24 \mathrm{E}+06$ \\
\hline & $2.15 \mathrm{E}-01$ & $6.17 \mathrm{E}+05$ \\
\hline & 4.64E-01 & $4.62 E+05$ \\
\hline & $1.00 \mathrm{E}+00$ & $1.58 \mathrm{E}+05$ \\
\hline & $2.15 \mathrm{E}+00$ & $9.83 E+04$ \\
\hline & $4.64 \mathrm{E}+00$ & $3.44 \mathrm{E}+04$ \\
\hline & $1.00 \mathrm{E}+01$ & $2.47 E+04$ \\
\hline & $2.15 \mathrm{E}+01$ & $1.35 \mathrm{E}+04$ \\
\hline & $4.64 \mathrm{E}+01$ & $8.18 \mathrm{E}+03$ \\
\hline & $1.00 \mathrm{E}+02$ & $5.05 E+03$ \\
\hline \multirow[t]{10}{*}{270} & 1.00E-01 & $8.67 E+05$ \\
\hline & $2.15 E-01$ & $4.27 \mathrm{E}+05$ \\
\hline & 4.64E-01 & $2.08 \mathrm{E}+05$ \\
\hline & $1.00 \mathrm{E}+00$ & $1.02 \mathrm{E}+05$ \\
\hline & $2.15 \mathrm{E}+00$ & $4.94 \mathrm{E}+04$ \\
\hline & $4.64 \mathrm{E}+00$ & $2.26 \mathrm{E}+04$ \\
\hline & $1.00 \mathrm{E}+01$ & $1.15 E+04$ \\
\hline & $2.15 E+01$ & $5.82 E+03$ \\
\hline & $4.64 \mathrm{E}+01$ & $2.98 \mathrm{E}+03$ \\
\hline & $1.00 \mathrm{E}+02$ & $1.60 \mathrm{E}+03$ \\
\hline \multirow[t]{10}{*}{290} & 1.00E-01 & $6.40 \mathrm{E}+05$ \\
\hline & $2.15 \mathrm{E}-01$ & $3.16 \mathrm{E}+05$ \\
\hline & 4.64E-01 & $1.53 \mathrm{E}+05$ \\
\hline & $1.00 \mathrm{E}+00$ & $7.61 \mathrm{E}+04$ \\
\hline & $2.15 E+00$ & $3.69 \mathrm{E}+04$ \\
\hline & $4.64 \mathrm{E}+00$ & $1.58 \mathrm{E}+04$ \\
\hline & $1.00 \mathrm{E}+01$ & $8.47 E+03$ \\
\hline & $2.15 \mathrm{E}+01$ & $4.15 \mathrm{E}+03$ \\
\hline & 4.64E+01 & $2.04 \mathrm{E}+03$ \\
\hline & $1.00 \mathrm{E}+02$ & $1.06 \mathrm{E}+03$ \\
\hline
\end{tabular}


Table L.6: SAOS Results for BQ6L: 6 wt\% Hyperion Fibrils (CNT) in Lexan HF1130111 at various temperatures continued

Formulation: BQ6L

\begin{tabular}{|c|c|c|}
\hline Temperature ${ }^{\circ} \mathrm{C}$ & Frequency (rad/s) & Complex Viscosity (Pa·s) \\
\hline $\mathbf{3 1 0}$ & $\mathbf{1 . 0 0 E}-01$ & $\mathbf{7 . 3 2 E + 0 5}$ \\
\hline & $2.15 \mathrm{E}-01$ & $3.57 \mathrm{E}+05$ \\
\hline & $4.64 \mathrm{E}-01$ & $1.73 \mathrm{E}+05$ \\
\hline & $1.00 \mathrm{E}+00$ & $8.51 \mathrm{E}+04$ \\
\hline & $2.15 \mathrm{E}+00$ & $4.09 \mathrm{E}+04$ \\
\hline & $4.64 \mathrm{E}+00$ & $1.71 \mathrm{E}+04$ \\
\hline & $1.00 \mathrm{E}+01$ & $9.12 \mathrm{E}+03$ \\
\hline & $2.15 \mathrm{E}+01$ & $4.42 \mathrm{E}+03$ \\
\hline & $4.64 \mathrm{E}+01$ & $2.14 \mathrm{E}+03$ \\
\hline & $1.00 \mathrm{E}+02$ & $1.08 \mathrm{E}+03$ \\
\hline
\end{tabular}


Table L.7: SAOS Results for BQ8L: 8 wt\% Hyperion Fibrils (CNT) in Lexan HF1130111 at various temperatures

Formulation: BQ8L

\begin{tabular}{|c|c|c|}
\hline Temperature ${ }^{\circ} \mathrm{C}$ & Frequency (rad/s) & Complex Viscosity (Pa.s) \\
\hline \multirow[t]{10}{*}{210} & 1.00E-01 & $3.08 E+06$ \\
\hline & $2.15 \mathrm{E}-01$ & $1.53 \mathrm{E}+06$ \\
\hline & 4.64E-01 & $1.13 E+06$ \\
\hline & $1.00 \mathrm{E}+00$ & $4.03 E+05$ \\
\hline & $2.15 \mathrm{E}+00$ & $2.92 \mathrm{E}+05$ \\
\hline & $4.64 \mathrm{E}+00$ & $7.69 \mathrm{E}+04$ \\
\hline & $1.00 \mathrm{E}+01$ & $5.49 E+04$ \\
\hline & $2.15 \mathrm{E}+01$ & $2.79 \mathrm{E}+04$ \\
\hline & $4.64 \mathrm{E}+01$ & $1.59 \mathrm{E}+04$ \\
\hline & $1.00 \mathrm{E}+02$ & $9.39 \mathrm{E}+03$ \\
\hline \multirow[t]{10}{*}{270} & 1.00E-01 & $2.04 E+06$ \\
\hline & $2.15 E-01$ & $1.02 \mathrm{E}+06$ \\
\hline & 4.64E-01 & $5.26 \mathrm{E}+05$ \\
\hline & $1.00 \mathrm{E}+00$ & $2.59 \mathrm{E}+05$ \\
\hline & $2.15 \mathrm{E}+00$ & $1.21 \mathrm{E}+05$ \\
\hline & $4.64 \mathrm{E}+00$ & $5.31 \mathrm{E}+04$ \\
\hline & $1.00 \mathrm{E}+01$ & $2.69 \mathrm{E}+04$ \\
\hline & $2.15 E+01$ & $1.35 E+04$ \\
\hline & $4.64 \mathrm{E}+01$ & $6.80 \mathrm{E}+03$ \\
\hline & $1.00 \mathrm{E}+02$ & $3.64 \mathrm{E}+03$ \\
\hline \multirow[t]{10}{*}{290} & 1.00E-01 & $1.85 E+06$ \\
\hline & $2.15 \mathrm{E}-01$ & $9.14 \mathrm{E}+05$ \\
\hline & 4.64E-01 & $4.66 \mathrm{E}+05$ \\
\hline & $1.00 \mathrm{E}+00$ & $2.33 E+05$ \\
\hline & $2.15 E+00$ & 1.19E+05 \\
\hline & $4.64 \mathrm{E}+00$ & $4.05 \mathrm{E}+04$ \\
\hline & $1.00 \mathrm{E}+01$ & $2.18 \mathrm{E}+04$ \\
\hline & $2.15 \mathrm{E}+01$ & $1.12 \mathrm{E}+04$ \\
\hline & 4.64E+01 & $5.31 \mathrm{E}+03$ \\
\hline & $1.00 \mathrm{E}+02$ & $2.64 \mathrm{E}+03$ \\
\hline
\end{tabular}


Table L.7: SAOS Results for BQ8L: 8 wt\% Hyperion Fibrils (CNT) in Lexan HF1130111 at various temperatures continued

Formulation: BQ8L

\begin{tabular}{|c|c|c|}
\hline Temperature ${ }^{\circ} \mathrm{C}$ & Frequency (rad/s) & Complex Viscosity (Pa's) \\
\hline \multirow[t]{10}{*}{310} & $1.00 \mathrm{E}-01$ & $1.78 \mathrm{E}+06$ \\
\hline & $2.15 \mathrm{E}-01$ & $8.72 \mathrm{E}+05$ \\
\hline & 4.64E-01 & $4.42 \mathrm{E}+05$ \\
\hline & $1.00 \mathrm{E}+00$ & $2.29 \mathrm{E}+05$ \\
\hline & $2.15 \mathrm{E}+00$ & $1.11 \mathrm{E}+05$ \\
\hline & $4.64 \mathrm{E}+00$ & $3.90 \mathrm{E}+04$ \\
\hline & $1.00 \mathrm{E}+01$ & $2.13 E+04$ \\
\hline & $2.15 \mathrm{E}+01$ & $1.08 \mathrm{E}+04$ \\
\hline & $4.64 \mathrm{E}+01$ & $5.15 \mathrm{E}+03$ \\
\hline & $1.00 \mathrm{E}+02$ & $2.58 \mathrm{E}+03$ \\
\hline \multirow{10}{*}{330} & $1.00 \mathrm{E}-01$ & $1.65 \mathrm{E}+06$ \\
\hline & $2.15 \mathrm{E}-01$ & $8.06 \mathrm{E}+05$ \\
\hline & $4.64 \mathrm{E}-01$ & $4.13 \mathrm{E}+05$ \\
\hline & $1.00 \mathrm{E}+00$ & $2.18 \mathrm{E}+05$ \\
\hline & $2.15 \mathrm{E}+00$ & $1.03 E+05$ \\
\hline & $4.64 \mathrm{E}+00$ & $3.72 \mathrm{E}+04$ \\
\hline & $1.00 \mathrm{E}+01$ & $2.01 \mathrm{E}+04$ \\
\hline & $2.15 \mathrm{E}+01$ & $1.03 E+04$ \\
\hline & $4.64 \mathrm{E}+01$ & $5.04 \mathrm{E}+03$ \\
\hline & $1.00 \mathrm{E}+02$ & $2.57 \mathrm{E}+03$ \\
\hline \multirow[t]{10}{*}{350} & 1.00E-01 & $1.61 \mathrm{E}+06$ \\
\hline & $2.15 \mathrm{E}-01$ & $7.86 \mathrm{E}+05$ \\
\hline & 4.64E-01 & $4.29 \mathrm{E}+05$ \\
\hline & $1.00 \mathrm{E}+00$ & $2.24 \mathrm{E}+05$ \\
\hline & $2.15 \mathrm{E}+00$ & $1.09 \mathrm{E}+05$ \\
\hline & $4.64 \mathrm{E}+00$ & $3.88 \mathrm{E}+04$ \\
\hline & $1.00 \mathrm{E}+01$ & $2.09 \mathrm{E}+04$ \\
\hline & $2.15 \mathrm{E}+01$ & $1.05 \mathrm{E}+04$ \\
\hline & $4.64 \mathrm{E}+01$ & $4.99 \mathrm{E}+03$ \\
\hline & $1.00 \mathrm{E}+02$ & $2.55 \mathrm{E}+03$ \\
\hline
\end{tabular}




\section{Appendix F.2: Polycarbonate with Carbon Black}

Table L.8: SAOS Results for BA2L: 2 wt\% Carbon Black (CB) in Lexan HF1130-111 at various temperatures

Formulation BA2L

\begin{tabular}{|c|c|c|}
\hline Temperature ${ }^{\circ} \mathrm{C}$ & Frequency (rad/s) & Complex Viscosity (Pa.s) \\
\hline \multirow[t]{10}{*}{250} & 1.00E-01 & $3.08 E+03$ \\
\hline & $2.15 \mathrm{E}-01$ & $2.23 E+03$ \\
\hline & 4.64E-01 & $1.63 E+03$ \\
\hline & $1.00 \mathrm{E}+00$ & $1.26 \mathrm{E}+03$ \\
\hline & $2.15 \mathrm{E}+00$ & $1.02 \mathrm{E}+03$ \\
\hline & $4.64 \mathrm{E}+00$ & $8.59 \mathrm{E}+02$ \\
\hline & $1.00 \mathrm{E}+01$ & $7.44 \mathrm{E}+02$ \\
\hline & $2.15 \mathrm{E}+01$ & $6.59 \mathrm{E}+02$ \\
\hline & $4.64 \mathrm{E}+01$ & $5.84 \mathrm{E}+02$ \\
\hline & $1.00 \mathrm{E}+02$ & $5.09 E+02$ \\
\hline \multirow[t]{10}{*}{270} & 1.00E-01 & $3.74 E+03$ \\
\hline & $2.15 \mathrm{E}-01$ & $2.26 \mathrm{E}+03$ \\
\hline & 4.64E-01 & $1.43 \mathrm{E}+03$ \\
\hline & $1.00 \mathrm{E}+00$ & $9.71 \mathrm{E}+02$ \\
\hline & $2.15 \mathrm{E}+00$ & $7.05 \mathrm{E}+02$ \\
\hline & $4.64 \mathrm{E}+00$ & $5.47 \mathrm{E}+02$ \\
\hline & $1.00 \mathrm{E}+01$ & $4.46 \mathrm{E}+02$ \\
\hline & $2.15 \mathrm{E}+01$ & $3.78 \mathrm{E}+02$ \\
\hline & $4.64 \mathrm{E}+01$ & $3.29 \mathrm{E}+02$ \\
\hline & $1.00 \mathrm{E}+02$ & $2.84 \mathrm{E}+02$ \\
\hline \multirow[t]{10}{*}{290} & 1.00E-01 & $4.57 E+03$ \\
\hline & $2.15 E-01$ & $2.49 \mathrm{E}+03$ \\
\hline & 4.64E-01 & $1.41 \mathrm{E}+03$ \\
\hline & $1.00 \mathrm{E}+00$ & $8.51 \mathrm{E}+02$ \\
\hline & $2.15 E+00$ & $5.51 \mathrm{E}+02$ \\
\hline & $4.64 \mathrm{E}+00$ & $3.83 E+02$ \\
\hline & $1.00 \mathrm{E}+01$ & $2.85 \mathrm{E}+02$ \\
\hline & $2.15 \mathrm{E}+01$ & $2.25 \mathrm{E}+02$ \\
\hline & $4.64 \mathrm{E}+01$ & $1.86 \mathrm{E}+02$ \\
\hline & $1.00 \mathrm{E}+02$ & $1.55 \mathrm{E}+02$ \\
\hline
\end{tabular}


Table L.8: SAOS Results for BA2L: 2 wt\% Carbon Black (CB) in Lexan HF1130-111 at various temperatures continued

Formulation BA2L

\begin{tabular}{|c|c|c|}
\hline Temperature ${ }^{\circ} \mathrm{C}$ & Frequency (rad/s) & Complex Viscosity (Pa·s) \\
\hline $\mathbf{3 1 0}$ & $\mathbf{1 . 0 0 E}-\mathbf{0 1}$ & $\mathbf{5 . 8 3 E + 0 3}$ \\
\hline & $2.15 \mathrm{E}-01$ & $3.03 \mathrm{E}+03$ \\
\hline & $4.64 \mathrm{E}-01$ & $1.57 \mathrm{E}+03$ \\
\hline & $1.00 \mathrm{E}+00$ & $8.57 \mathrm{E}+02$ \\
\hline & $2.15 \mathrm{E}+00$ & $4.95 \mathrm{E}+02$ \\
\hline & $4.64 \mathrm{E}+00$ & $3.08 \mathrm{E}+02$ \\
\hline & $1.00 \mathrm{E}+01$ & $2.05 \mathrm{E}+02$ \\
\hline & $2.15 \mathrm{E}+01$ & $1.47 \mathrm{E}+02$ \\
\hline & $4.64 \mathrm{E}+01$ & $1.12 \mathrm{E}+02$ \\
\hline & $1.00 \mathrm{E}+02$ & $8.67 \mathrm{E}+01$ \\
\hline & $\mathbf{1 . 0 0 E}-01$ & $\mathbf{7 . 3 0 E}+03$ \\
\hline & $2.15 \mathrm{E}-01$ & $3.68 \mathrm{E}+03$ \\
\hline & $4.64 \mathrm{E}-01$ & $1.82 \mathrm{E}+03$ \\
\hline & $1.00 \mathrm{E}+00$ & $9.43 \mathrm{E}+02$ \\
\hline & $2.15 \mathrm{E}+00$ & $5.05 \mathrm{E}+02$ \\
\hline & $4.64 \mathrm{E}+00$ & $2.89 \mathrm{E}+02$ \\
\hline & $1.00 \mathrm{E}+01$ & $1.79 \mathrm{E}+02$ \\
\hline & $2.15 \mathrm{E}+01$ & $1.20 \mathrm{E}+02$ \\
\hline & $4.64 \mathrm{E}+01$ & $8.70 \mathrm{E}+01$ \\
\hline & $1.00 \mathrm{E}+02$ & $6.41 \mathrm{E}+01$ \\
\hline
\end{tabular}


Table L.9: SAOS Results for BA3L: 3 wt\% Carbon Black (CB) in Lexan HF1130-111 at various temperatures

Formulation BA3L

\begin{tabular}{|c|c|c|}
\hline Temperature ${ }^{\circ} \mathrm{C}$ & Frequency (rad/s) & Complex Viscosity (Pa.s) \\
\hline \multirow[t]{10}{*}{250} & $1.00 \mathrm{E}-01$ & $3.91 \mathrm{E}+04$ \\
\hline & $2.15 \mathrm{E}-01$ & $1.96 \mathrm{E}+04$ \\
\hline & $4.64 \mathrm{E}-01$ & $1.02 \mathrm{E}+04$ \\
\hline & $1.00 \mathrm{E}+00$ & $5.57 \mathrm{E}+03$ \\
\hline & $2.15 \mathrm{E}+00$ & $3.29 \mathrm{E}+03$ \\
\hline & $4.64 \mathrm{E}+00$ & $2.11 \mathrm{E}+03$ \\
\hline & $1.00 \mathrm{E}+01$ & $1.47 \mathrm{E}+03$ \\
\hline & $2.15 \mathrm{E}+01$ & $1.09 \mathrm{E}+03$ \\
\hline & $4.64 \mathrm{E}+01$ & $8.47 \mathrm{E}+02$ \\
\hline & $1.00 \mathrm{E}+02$ & $6.69 \mathrm{E}+02$ \\
\hline \multirow[t]{10}{*}{270} & 1.00E-01 & $3.78 \mathrm{E}+04$ \\
\hline & $2.15 \mathrm{E}-01$ & $1.85 \mathrm{E}+04$ \\
\hline & 4.64E-01 & $9.17 E+03$ \\
\hline & $1.00 \mathrm{E}+00$ & $4.70 \mathrm{E}+03$ \\
\hline & $2.15 E+00$ & $2.55 E+03$ \\
\hline & $4.64 \mathrm{E}+00$ & $1.49 \mathrm{E}+03$ \\
\hline & $1.00 \mathrm{E}+01$ & $9.49 \mathrm{E}+02$ \\
\hline & $2.15 \mathrm{E}+01$ & $6.58 \mathrm{E}+02$ \\
\hline & $4.64 \mathrm{E}+01$ & $4.87 E+02$ \\
\hline & $1.00 \mathrm{E}+02$ & $3.74 \mathrm{E}+02$ \\
\hline \multirow[t]{10}{*}{290} & $1.00 \mathrm{E}-01$ & $3.86 \mathrm{E}+04$ \\
\hline & $2.15 \mathrm{E}-01$ & $1.88 \mathrm{E}+04$ \\
\hline & 4.64E-01 & $9.13 \mathrm{E}+03$ \\
\hline & $1.00 \mathrm{E}+00$ & $4.52 \mathrm{E}+03$ \\
\hline & $2.15 E+00$ & $2.32 \mathrm{E}+03$ \\
\hline & $4.64 \mathrm{E}+00$ & $1.27 \mathrm{E}+03$ \\
\hline & $1.00 \mathrm{E}+01$ & $7.46 \mathrm{E}+02$ \\
\hline & $2.15 E+01$ & $4.80 \mathrm{E}+02$ \\
\hline & $4.64 \mathrm{E}+01$ & $3.35 E+02$ \\
\hline & $1.00 \mathrm{E}+02$ & $2.46 \mathrm{E}+02$ \\
\hline
\end{tabular}


Table L.9: SAOS Results for BA3L: 3 wt\% Carbon Black (CB) in Lexan HF1130-111 at various temperatures continued

Formulation BA3L

\begin{tabular}{|c|c|c|}
\hline Temperature ${ }^{\circ} \mathrm{C}$ & Frequency (rad/s) & Complex Viscosity (Pa·s) \\
\hline $\mathbf{3 1 0}$ & $\mathbf{1 . 0 0 E}-\mathbf{0 1}$ & $\mathbf{4 . 1 0 E + 0 4}$ \\
\hline & $2.15 \mathrm{E}-01$ & $1.99 \mathrm{E}+04$ \\
\hline & $4.64 \mathrm{E}-01$ & $9.65 \mathrm{E}+03$ \\
\hline & $1.00 \mathrm{E}+00$ & $4.70 \mathrm{E}+03$ \\
\hline & $2.15 \mathrm{E}+00$ & $2.33 \mathrm{E}+03$ \\
\hline & $4.64 \mathrm{E}+00$ & $1.20 \mathrm{E}+03$ \\
\hline & $1.00 \mathrm{E}+01$ & $6.62 \mathrm{E}+02$ \\
\hline & $2.15 \mathrm{E}+01$ & $3.96 \mathrm{E}+02$ \\
\hline & $4.64 \mathrm{E}+01$ & $2.58 \mathrm{E}+02$ \\
\hline & $1.00 \mathrm{E}+02$ & $1.79 \mathrm{E}+02$ \\
\hline & $\mathbf{1 . 0 0 E}-01$ & $\mathbf{4 . 4 0 E}+04$ \\
\hline & $2.15 \mathrm{E}-01$ & $2.15 \mathrm{E}+04$ \\
\hline & $4.64 \mathrm{E}-01$ & $1.04 \mathrm{E}+04$ \\
\hline & $1.00 \mathrm{E}+00$ & $5.08 \mathrm{E}+03$ \\
\hline & $2.15 \mathrm{E}+00$ & $2.48 \mathrm{E}+03$ \\
\hline & $4.64 \mathrm{E}+00$ & $1.24 \mathrm{E}+03$ \\
\hline & $1.00 \mathrm{E}+01$ & $6.47 \mathrm{E}+02$ \\
\hline & $2.15 \mathrm{E}+01$ & $3.62 \mathrm{E}+02$ \\
\hline & $4.64 \mathrm{E}+01$ & $2.20 \mathrm{E}+02$ \\
\hline & $1.00 \mathrm{E}+02$ & $1.43 \mathrm{E}+02$ \\
\hline & & \\
\hline & & \\
\hline & & \\
\hline & & \\
\hline & &
\end{tabular}


Table L.10: SAOS Results for BA4L: 4 wt\% Carbon Black (CB) in Lexan HF1130-111 at various temperatures

Formulation BA4L

\begin{tabular}{|c|c|c|}
\hline Temperature ${ }^{\circ} \mathrm{C}$ & Frequency (rad/s) & Complex Viscosity (Pa.s) \\
\hline \multirow[t]{10}{*}{250} & $1.00 \mathrm{E}-01$ & $9.68 \mathrm{E}+04$ \\
\hline & $2.15 \mathrm{E}-01$ & $4.79 \mathrm{E}+04$ \\
\hline & $4.64 \mathrm{E}-01$ & $2.42 \mathrm{E}+04$ \\
\hline & $1.00 \mathrm{E}+00$ & $1.29 \mathrm{E}+04$ \\
\hline & $2.15 \mathrm{E}+00$ & $7.34 \mathrm{E}+03$ \\
\hline & $4.64 \mathrm{E}+00$ & $4.52 \mathrm{E}+03$ \\
\hline & $1.00 \mathrm{E}+01$ & $3.01 \mathrm{E}+03$ \\
\hline & $2.15 \mathrm{E}+01$ & $2.20 \mathrm{E}+03$ \\
\hline & $4.64 \mathrm{E}+01$ & $1.66 \mathrm{E}+03$ \\
\hline & $1.00 \mathrm{E}+02$ & $1.25 E+03$ \\
\hline \multirow[t]{10}{*}{270} & $1.00 \mathrm{E}-01$ & $9.16 \mathrm{E}+04$ \\
\hline & $2.15 \mathrm{E}-01$ & $4.45 E+04$ \\
\hline & 4.64E-01 & $2.19 E+04$ \\
\hline & $1.00 \mathrm{E}+00$ & $1.10 \mathrm{E}+04$ \\
\hline & $2.15 \mathrm{E}+00$ & $5.80 \mathrm{E}+03$ \\
\hline & $4.64 \mathrm{E}+00$ & $3.26 \mathrm{E}+03$ \\
\hline & $1.00 \mathrm{E}+01$ & $2.01 \mathrm{E}+03$ \\
\hline & $2.15 \mathrm{E}+01$ & $1.36 \mathrm{E}+03$ \\
\hline & $4.64 \mathrm{E}+01$ & $9.82 \mathrm{E}+02$ \\
\hline & $1.00 \mathrm{E}+02$ & $7.32 \mathrm{E}+02$ \\
\hline \multirow[t]{10}{*}{290} & $1.00 \mathrm{E}-01$ & $9.18 \mathrm{E}+04$ \\
\hline & $2.15 \mathrm{E}-01$ & $4.43 \mathrm{E}+04$ \\
\hline & 4.64E-01 & $2.14 \mathrm{E}+04$ \\
\hline & $1.00 \mathrm{E}+00$ & $1.05 \mathrm{E}+04$ \\
\hline & $2.15 \mathrm{E}+00$ & $5.26 \mathrm{E}+03$ \\
\hline & $4.64 \mathrm{E}+00$ & $2.76 \mathrm{E}+03$ \\
\hline & $1.00 \mathrm{E}+01$ & $1.57 \mathrm{E}+03$ \\
\hline & $2.15 E+01$ & $9.80 \mathrm{E}+02$ \\
\hline & $4.64 \mathrm{E}+01$ & $6.62 \mathrm{E}+02$ \\
\hline & $1.00 \mathrm{E}+02$ & $4.75 \mathrm{E}+02$ \\
\hline
\end{tabular}


Table L.10: SAOS Results for BA4L: 4 wt\% Carbon Black (CB) in Lexan HF1130-111 at various temperatures continued

Formulation BA4L

\begin{tabular}{|c|c|c|}
\hline Temperature ${ }^{\circ} \mathrm{C}$ & Frequency (rad/s) & Complex Viscosity (Pa·s) \\
\hline $\mathbf{3 1 0}$ & $\mathbf{1 . 0 0 E}-\mathbf{0 1}$ & $\mathbf{9 . 4 6 E + 0 4}$ \\
\hline & $2.15 \mathrm{E}-01$ & $4.56 \mathrm{E}+04$ \\
\hline & $4.64 \mathrm{E}-01$ & $2.19 \mathrm{E}+04$ \\
\hline & $1.00 \mathrm{E}+00$ & $1.05 \mathrm{E}+04$ \\
\hline & $2.15 \mathrm{E}+00$ & $5.15 \mathrm{E}+03$ \\
\hline & $4.64 \mathrm{E}+00$ & $2.59 \mathrm{E}+03$ \\
\hline & $1.00 \mathrm{E}+01$ & $1.37 \mathrm{E}+03$ \\
\hline & $2.15 \mathrm{E}+01$ & $7.91 \mathrm{E}+02$ \\
\hline & $4.64 \mathrm{E}+01$ & $4.99 \mathrm{E}+02$ \\
\hline & $1.00 \mathrm{E}+02$ & $3.40 \mathrm{E}+02$ \\
\hline & $\mathbf{1 . 0 0 E}-01$ & $\mathbf{9 . 8 1 E + 0 4}$ \\
\hline & $2.15 \mathrm{E}-01$ & $4.71 \mathrm{E}+04$ \\
\hline & $4.64 \mathrm{E}-01$ & $2.26 \mathrm{E}+04$ \\
\hline & $1.00 \mathrm{E}+00$ & $1.08 \mathrm{E}+04$ \\
\hline & $2.15 \mathrm{E}+00$ & $5.21 \mathrm{E}+03$ \\
\hline & $4.64 \mathrm{E}+00$ & $2.53 \mathrm{E}+03$ \\
\hline & $1.00 \mathrm{E}+01$ & $1.29 \mathrm{E}+03$ \\
\hline & $2.15 \mathrm{E}+01$ & $6.99 \mathrm{E}+02$ \\
\hline & $4.64 \mathrm{E}+01$ & $4.11 \mathrm{E}+02$ \\
\hline & $1.00 \mathrm{E}+02$ & $2.63 \mathrm{E}+02$ \\
\hline & &
\end{tabular}


Table L.11: SAOS Results for BA5L: 5 wt\% Carbon Black (CB) in Lexan HF1130-111 at various temperatures

Formulation BA5L

\begin{tabular}{|c|c|c|}
\hline Temperature ${ }^{\circ} \mathrm{C}$ & Frequency (rad/s) & Complex Viscosity (Pa.s) \\
\hline \multirow[t]{10}{*}{250} & $1.00 \mathrm{E}-01$ & $1.69 \mathrm{E}+05$ \\
\hline & $2.15 \mathrm{E}-01$ & $8.44 \mathrm{E}+04$ \\
\hline & $4.64 \mathrm{E}-01$ & $4.23 \mathrm{E}+04$ \\
\hline & $1.00 \mathrm{E}+00$ & $2.19 E+04$ \\
\hline & $2.15 \mathrm{E}+00$ & $1.20 \mathrm{E}+04$ \\
\hline & $4.64 \mathrm{E}+00$ & $6.95 \mathrm{E}+03$ \\
\hline & $1.00 \mathrm{E}+01$ & $4.31 \mathrm{E}+03$ \\
\hline & $2.15 \mathrm{E}+01$ & $3.04 \mathrm{E}+03$ \\
\hline & $4.64 \mathrm{E}+01$ & $2.18 \mathrm{E}+03$ \\
\hline & $1.00 \mathrm{E}+02$ & $1.59 \mathrm{E}+03$ \\
\hline \multirow[t]{10}{*}{270} & $1.00 \mathrm{E}-01$ & $1.77 \mathrm{E}+05$ \\
\hline & $2.15 \mathrm{E}-01$ & $8.68 \mathrm{E}+04$ \\
\hline & 4.64E-01 & $4.23 E+04$ \\
\hline & $1.00 \mathrm{E}+00$ & $2.09 E+04$ \\
\hline & $2.15 \mathrm{E}+00$ & $1.06 \mathrm{E}+04$ \\
\hline & $4.64 \mathrm{E}+00$ & $5.61 \mathrm{E}+03$ \\
\hline & $1.00 \mathrm{E}+01$ & $3.17 \mathrm{E}+03$ \\
\hline & $2.15 \mathrm{E}+01$ & $2.04 \mathrm{E}+03$ \\
\hline & $4.64 \mathrm{E}+01$ & $1.37 \mathrm{E}+03$ \\
\hline & $1.00 \mathrm{E}+02$ & $9.68 \mathrm{E}+02$ \\
\hline \multirow[t]{10}{*}{290} & $1.00 \mathrm{E}-01$ & $1.85 E+05$ \\
\hline & $2.15 \mathrm{E}-01$ & $8.95 E+04$ \\
\hline & 4.64E-01 & $4.31 E+04$ \\
\hline & $1.00 \mathrm{E}+00$ & $2.09 \mathrm{E}+04$ \\
\hline & $2.15 \mathrm{E}+00$ & $1.03 E+04$ \\
\hline & $4.64 \mathrm{E}+00$ & $5.11 \mathrm{E}+03$ \\
\hline & $1.00 \mathrm{E}+01$ & $2.69 \mathrm{E}+03$ \\
\hline & $2.15 E+01$ & $1.59 \mathrm{E}+03$ \\
\hline & $4.64 \mathrm{E}+01$ & $9.97 E+02$ \\
\hline & $1.00 \mathrm{E}+02$ & $6.70 \mathrm{E}+02$ \\
\hline
\end{tabular}


Table L.11: SAOS Results for BA5L: 5 wt\% Carbon Black (CB) in Lexan HF1130-111 at various temperatures continued

Formulation BA5L

\begin{tabular}{|c|c|c|}
\hline Temperature ${ }^{\circ} \mathrm{C}$ & Frequency (rad/s) & Complex Viscosity (Pa·s) \\
\hline $\mathbf{3 1 0}$ & $\mathbf{1 . 0 0 E}-\mathbf{0 1}$ & $\mathbf{1 . 9 3 E + 0 5}$ \\
\hline & $2.15 \mathrm{E}-01$ & $9.35 \mathrm{E}+04$ \\
\hline & $4.64 \mathrm{E}-01$ & $4.48 \mathrm{E}+04$ \\
\hline & $1.00 \mathrm{E}+00$ & $2.15 \mathrm{E}+04$ \\
\hline & $2.15 \mathrm{E}+00$ & $1.03 \mathrm{E}+04$ \\
\hline & $4.64 \mathrm{E}+00$ & $5.03 \mathrm{E}+03$ \\
\hline & $1.00 \mathrm{E}+01$ & $2.51 \mathrm{E}+03$ \\
\hline & $2.15 \mathrm{E}+01$ & $1.38 \mathrm{E}+03$ \\
\hline & $4.64 \mathrm{E}+01$ & $8.01 \mathrm{E}+02$ \\
\hline & $1.00 \mathrm{E}+02$ & $5.03 \mathrm{E}+02$ \\
\hline & $\mathbf{1 . 0 0 E}-01$ & $\mathbf{2 . 0 1 E}+05$ \\
\hline & $2.15 \mathrm{E}-01$ & $9.72 \mathrm{E}+04$ \\
\hline & $4.64 \mathrm{E}-01$ & $4.65 \mathrm{E}+04$ \\
\hline & $1.00 \mathrm{E}+00$ & $2.22 \mathrm{E}+04$ \\
\hline & $2.15 \mathrm{E}+00$ & $1.06 \mathrm{E}+04$ \\
\hline & $4.64 \mathrm{E}+00$ & $5.02 \mathrm{E}+03$ \\
\hline & $1.00 \mathrm{E}+01$ & $2.45 \mathrm{E}+03$ \\
\hline & $2.15 \mathrm{E}+01$ & $1.29 \mathrm{E}+03$ \\
\hline & $4.64 \mathrm{E}+01$ & $6.99 \mathrm{E}+02$ \\
\hline & $1.00 \mathrm{E}+02$ & $4.08 \mathrm{E}+02$ \\
\hline
\end{tabular}


Table L.12: SAOS Results for BA6L: 6 wt\% Carbon Black (CB) in Lexan HF1130-111 at various temperatures

Formulation BA6L

\begin{tabular}{|c|c|c|}
\hline Temperature ${ }^{\circ} \mathrm{C}$ & Frequency (rad/s) & Complex Viscosity (Pa.s) \\
\hline \multirow[t]{10}{*}{250} & $1.00 \mathrm{E}-01$ & $3.62 E+05$ \\
\hline & $2.15 \mathrm{E}-01$ & $1.75 \mathrm{E}+05$ \\
\hline & $4.64 \mathrm{E}-01$ & $8.45 E+04$ \\
\hline & $1.00 \mathrm{E}+00$ & $4.16 \mathrm{E}+04$ \\
\hline & $2.15 \mathrm{E}+00$ & $2.09 \mathrm{E}+04$ \\
\hline & $4.64 \mathrm{E}+00$ & $1.05 \mathrm{E}+04$ \\
\hline & $1.00 \mathrm{E}+01$ & $5.82 \mathrm{E}+03$ \\
\hline & $2.15 \mathrm{E}+01$ & $3.54 \mathrm{E}+03$ \\
\hline & $4.64 \mathrm{E}+01$ & $2.29 \mathrm{E}+03$ \\
\hline & $1.00 \mathrm{E}+02$ & $1.55 \mathrm{E}+03$ \\
\hline \multirow[t]{10}{*}{270} & $1.00 \mathrm{E}-01$ & $3.45 E+05$ \\
\hline & $2.15 \mathrm{E}-01$ & $1.67 \mathrm{E}+05$ \\
\hline & 4.64E-01 & $8.03 E+04$ \\
\hline & $1.00 \mathrm{E}+00$ & $3.89 E+04$ \\
\hline & $2.15 \mathrm{E}+00$ & $1.86 \mathrm{E}+04$ \\
\hline & $4.64 \mathrm{E}+00$ & $9.13 E+03$ \\
\hline & $1.00 \mathrm{E}+01$ & $4.67 \mathrm{E}+03$ \\
\hline & $2.15 \mathrm{E}+01$ & $2.65 \mathrm{E}+03$ \\
\hline & $4.64 \mathrm{E}+01$ & $1.58 \mathrm{E}+03$ \\
\hline & $1.00 \mathrm{E}+02$ & $1.01 \mathrm{E}+03$ \\
\hline \multirow[t]{10}{*}{290} & $1.00 \mathrm{E}-01$ & $3.44 E+05$ \\
\hline & $2.15 \mathrm{E}-01$ & $1.67 \mathrm{E}+05$ \\
\hline & 4.64E-01 & $7.99 \mathrm{E}+04$ \\
\hline & $1.00 \mathrm{E}+00$ & $3.85 E+04$ \\
\hline & $2.15 \mathrm{E}+00$ & $1.85 E+04$ \\
\hline & $4.64 \mathrm{E}+00$ & $8.86 \mathrm{E}+03$ \\
\hline & $1.00 \mathrm{E}+01$ & $4.33 \mathrm{E}+03$ \\
\hline & $2.15 E+01$ & $2.29 \mathrm{E}+03$ \\
\hline & $4.64 \mathrm{E}+01$ & $1.28 \mathrm{E}+03$ \\
\hline & $1.00 \mathrm{E}+02$ & $7.65 \mathrm{E}+02$ \\
\hline
\end{tabular}


Table L.12: SAOS Results for BA6L: 6 wt\% Carbon Black (CB) in Lexan HF1130-111 at various temperatures continued

Formulation BA6L

\begin{tabular}{|c|c|c|}
\hline Temperature ${ }^{\circ} \mathrm{C}$ & Frequency (rad/s) & Complex Viscosity (Pa·s) \\
\hline $\mathbf{3 1 0}$ & $\mathbf{1 . 0 0 E}-\mathbf{0 1}$ & $\mathbf{3 . 5 0 E + 0 5}$ \\
\hline & $2.15 \mathrm{E}-01$ & $1.69 \mathrm{E}+05$ \\
\hline & $4.64 \mathrm{E}-01$ & $8.07 \mathrm{E}+04$ \\
\hline & $1.00 \mathrm{E}+00$ & $3.89 \mathrm{E}+04$ \\
\hline & $2.15 \mathrm{E}+00$ & $1.86 \mathrm{E}+04$ \\
\hline & $4.64 \mathrm{E}+00$ & $8.56 \mathrm{E}+03$ \\
\hline & $1.00 \mathrm{E}+01$ & $4.15 \mathrm{E}+03$ \\
\hline & $2.15 \mathrm{E}+01$ & $2.15 \mathrm{E}+03$ \\
\hline & $4.64 \mathrm{E}+01$ & $1.12 \mathrm{E}+03$ \\
\hline & $1.00 \mathrm{E}+02$ & $6.30 \mathrm{E}+02$ \\
\hline & $\mathbf{1 . 0 0 E}-01$ & $3.51 \mathrm{E}+05$ \\
\hline & $2.15 \mathrm{E}-01$ & $1.72 \mathrm{E}+05$ \\
\hline & $4.64 \mathrm{E}-01$ & $8.24 \mathrm{E}+04$ \\
\hline & $1.00 \mathrm{E}+00$ & $3.96 \mathrm{E}+04$ \\
\hline & $2.15 \mathrm{E}+00$ & $1.89 \mathrm{E}+04$ \\
\hline & $4.64 \mathrm{E}+00$ & $8.75 \mathrm{E}+03$ \\
\hline & $1.00 \mathrm{E}+01$ & $4.16 \mathrm{E}+03$ \\
\hline & $2.15 \mathrm{E}+01$ & $2.10 \mathrm{E}+03$ \\
\hline & $4.64 \mathrm{E}+01$ & $1.06 \mathrm{E}+03$ \\
\hline & $1.00 \mathrm{E}+02$ & $5.61 \mathrm{E}+02$ \\
\hline
\end{tabular}


Table L.13: SAOS Results for BA8L: 8 wt\% Carbon Black (CB) in Lexan HF1130-111 at various temperatures

Formulation BA8L

\begin{tabular}{|c|c|c|}
\hline Temperature ${ }^{\circ} \mathrm{C}$ & Frequency (rad/s) & Complex Viscosity (Pa.s) \\
\hline \multirow[t]{10}{*}{250} & $1.00 \mathrm{E}-01$ & $1.12 \mathrm{E}+06$ \\
\hline & $2.15 \mathrm{E}-01$ & $5.49 \mathrm{E}+05$ \\
\hline & $4.64 \mathrm{E}-01$ & $2.68 \mathrm{E}+05$ \\
\hline & $1.00 \mathrm{E}+00$ & $1.29 \mathrm{E}+05$ \\
\hline & $2.15 \mathrm{E}+00$ & $6.40 \mathrm{E}+04$ \\
\hline & $4.64 \mathrm{E}+00$ & $2.92 \mathrm{E}+04$ \\
\hline & $1.00 \mathrm{E}+01$ & $1.66 \mathrm{E}+04$ \\
\hline & $2.15 \mathrm{E}+01$ & $8.64 \mathrm{E}+03$ \\
\hline & $4.64 \mathrm{E}+01$ & $5.06 \mathrm{E}+03$ \\
\hline & $1.00 \mathrm{E}+02$ & $3.04 \mathrm{E}+03$ \\
\hline \multirow[t]{10}{*}{270} & $1.00 \mathrm{E}-01$ & $1.08 \mathrm{E}+06$ \\
\hline & $2.15 \mathrm{E}-01$ & $5.22 \mathrm{E}+05$ \\
\hline & 4.64E-01 & $2.55 \mathrm{E}+05$ \\
\hline & $1.00 \mathrm{E}+00$ & $1.22 \mathrm{E}+05$ \\
\hline & $2.15 \mathrm{E}+00$ & $6.05 \mathrm{E}+04$ \\
\hline & $4.64 \mathrm{E}+00$ & $2.64 \mathrm{E}+04$ \\
\hline & $1.00 \mathrm{E}+01$ & $1.40 \mathrm{E}+04$ \\
\hline & $2.15 \mathrm{E}+01$ & $7.05 \mathrm{E}+03$ \\
\hline & $4.64 \mathrm{E}+01$ & $3.83 \mathrm{E}+03$ \\
\hline & $1.00 \mathrm{E}+02$ & $2.19 \mathrm{E}+03$ \\
\hline \multirow[t]{10}{*}{290} & $1.00 \mathrm{E}-01$ & $1.06 E+06$ \\
\hline & $2.15 \mathrm{E}-01$ & $5.13 \mathrm{E}+05$ \\
\hline & 4.64E-01 & $2.49 \mathrm{E}+05$ \\
\hline & $1.00 \mathrm{E}+00$ & $1.21 \mathrm{E}+05$ \\
\hline & $2.15 \mathrm{E}+00$ & $5.85 E+04$ \\
\hline & $4.64 \mathrm{E}+00$ & $2.54 \mathrm{E}+04$ \\
\hline & $1.00 \mathrm{E}+01$ & $1.31 \mathrm{E}+04$ \\
\hline & $2.15 E+01$ & $6.45 E+03$ \\
\hline & $4.64 \mathrm{E}+01$ & $3.33 E+03$ \\
\hline & $1.00 \mathrm{E}+02$ & $1.81 \mathrm{E}+03$ \\
\hline
\end{tabular}


Table L.13: SAOS Results for BA8L: 8 wt\% Carbon Black (CB) in Lexan HF1130-111 at various temperatures continued

Formulation BA8L

\begin{tabular}{|c|c|c|}
\hline Temperature ${ }^{\circ} \mathrm{C}$ & Frequency (rad/s) & Complex Viscosity (Pa·s) \\
\hline $\mathbf{3 1 0}$ & $\mathbf{1 . 0 0 E}-\mathbf{0 1}$ & $\mathbf{1 . 0 5 E}+\mathbf{0 6}$ \\
\hline & $2.15 \mathrm{E}-01$ & $5.09 \mathrm{E}+05$ \\
\hline & $4.64 \mathrm{E}-01$ & $2.46 \mathrm{E}+05$ \\
\hline & $1.00 \mathrm{E}+00$ & $1.17 \mathrm{E}+05$ \\
\hline & $2.15 \mathrm{E}+00$ & $5.74 \mathrm{E}+04$ \\
\hline & $4.64 \mathrm{E}+00$ & $2.46 \mathrm{E}+04$ \\
\hline & $1.00 \mathrm{E}+01$ & $1.26 \mathrm{E}+04$ \\
\hline & $2.15 \mathrm{E}+01$ & $6.13 \mathrm{E}+03$ \\
\hline & $4.64 \mathrm{E}+01$ & $3.07 \mathrm{E}+03$ \\
\hline & $1.00 \mathrm{E}+02$ & $1.61 \mathrm{E}+03$ \\
\hline & $\mathbf{1 . 0 0 E}-01$ & $\mathbf{1 . 0 2 E}+06$ \\
\hline & $2.15 \mathrm{E}-01$ & $4.98 \mathrm{E}+05$ \\
\hline & $4.64 \mathrm{E}-01$ & $2.39 \mathrm{E}+05$ \\
\hline & $1.00 \mathrm{E}+00$ & $1.16 \mathrm{E}+05$ \\
\hline & $2.15 \mathrm{E}+00$ & $5.39 \mathrm{E}+04$ \\
\hline & $4.64 \mathrm{E}+00$ & $2.42 \mathrm{E}+04$ \\
\hline & $1.00 \mathrm{E}+01$ & $1.23 \mathrm{E}+04$ \\
\hline & $2.15 \mathrm{E}+01$ & $5.94 \mathrm{E}+03$ \\
\hline & $4.64 \mathrm{E}+01$ & $2.91 \mathrm{E}+03$ \\
\hline & $1.00 \mathrm{E}+02$ & $1.48 \mathrm{E}+03$ \\
\hline
\end{tabular}




\section{Appendix L.3: Polycarbonate with Graphene Nanoplatelets}

Table L.14: SAOS Results for BG2L: $2 \mathrm{wt} \%$ Graphene Nanoplatelets (GNP) in Lexan HF1130-111 at various temperatures

Formulation BG2L

\begin{tabular}{|c|c|c|}
\hline Temperature ${ }^{\circ} \mathrm{C}$ & Frequency (rad/s) & Complex Viscosity (Pa.s) \\
\hline \multirow[t]{10}{*}{250} & 1.00E-01 & $8.41 E+02$ \\
\hline & $2.15 \mathrm{E}-01$ & $7.25 \mathrm{E}+02$ \\
\hline & 4.64E-01 & $6.53 \mathrm{E}+02$ \\
\hline & $1.00 \mathrm{E}+00$ & $6.22 \mathrm{E}+02$ \\
\hline & $2.15 \mathrm{E}+00$ & $6.10 \mathrm{E}+02$ \\
\hline & $4.64 \mathrm{E}+00$ & $5.97 \mathrm{E}+02$ \\
\hline & $1.00 \mathrm{E}+01$ & $5.86 \mathrm{E}+02$ \\
\hline & $2.15 \mathrm{E}+01$ & $5.71 \mathrm{E}+02$ \\
\hline & $4.64 \mathrm{E}+01$ & $5.41 \mathrm{E}+02$ \\
\hline & $1.00 \mathrm{E}+02$ & $4.87 \mathrm{E}+02$ \\
\hline \multirow[t]{10}{*}{270} & 1.00E-01 & 4.04E+02 \\
\hline & $2.15 \mathrm{E}-01$ & $3.36 \mathrm{E}+02$ \\
\hline & 4.64E-01 & $2.79 \mathrm{E}+02$ \\
\hline & $1.00 \mathrm{E}+00$ & $2.38 \mathrm{E}+02$ \\
\hline & $2.15 \mathrm{E}+00$ & $2.16 \mathrm{E}+02$ \\
\hline & $4.64 \mathrm{E}+00$ & $2.07 E+02$ \\
\hline & $1.00 \mathrm{E}+01$ & $2.03 E+02$ \\
\hline & $2.15 \mathrm{E}+01$ & $1.98 \mathrm{E}+02$ \\
\hline & $4.64 \mathrm{E}+01$ & $1.92 \mathrm{E}+02$ \\
\hline & $1.00 \mathrm{E}+02$ & $1.80 \mathrm{E}+02$ \\
\hline \multirow{10}{*}{290} & 1.00E-01 & $2.68 \mathrm{E}+02$ \\
\hline & $2.15 \mathrm{E}-01$ & $2.22 \mathrm{E}+02$ \\
\hline & $4.64 \mathrm{E}-01$ & $1.83 \mathrm{E}+02$ \\
\hline & $1.00 \mathrm{E}+00$ & $1.51 \mathrm{E}+02$ \\
\hline & $2.15 \mathrm{E}+00$ & $1.30 \mathrm{E}+02$ \\
\hline & $4.64 \mathrm{E}+00$ & $1.20 \mathrm{E}+02$ \\
\hline & $1.00 \mathrm{E}+01$ & $1.15 \mathrm{E}+02$ \\
\hline & $2.15 \mathrm{E}+01$ & $1.11 \mathrm{E}+02$ \\
\hline & $4.64 \mathrm{E}+01$ & $1.07 \mathrm{E}+02$ \\
\hline & $1.00 \mathrm{E}+02$ & $9.89 \mathrm{E}+01$ \\
\hline
\end{tabular}


Table L.14: SAOS Results for BG2L: 2 wt\% Graphene Nanoplatelets (GNP) in Lexan HF1130-111 at various temperatures continued

Formulation BG2L

\begin{tabular}{|c|c|c|}
\hline Temperature ${ }^{\circ} \mathrm{C}$ & Frequency (rad/s) & Complex Viscosity (Pa·s) \\
\hline $\mathbf{3 1 0}$ & $\mathbf{1 . 0 0 E}-\mathbf{0 1}$ & $\mathbf{2 . 0 0 E + 0 2}$ \\
\hline & $2.15 \mathrm{E}-01$ & $1.61 \mathrm{E}+02$ \\
\hline & $4.64 \mathrm{E}-01$ & $1.28 \mathrm{E}+02$ \\
\hline & $1.00 \mathrm{E}+00$ & $1.03 \mathrm{E}+02$ \\
\hline & $2.15 \mathrm{E}+00$ & $8.36 \mathrm{E}+01$ \\
\hline & $4.64 \mathrm{E}+00$ & $7.31 \mathrm{E}+01$ \\
\hline & $1.00 \mathrm{E}+01$ & $6.78 \mathrm{E}+01$ \\
\hline & $2.15 \mathrm{E}+01$ & $6.44 \mathrm{E}+01$ \\
\hline & $4.64 \mathrm{E}+01$ & $6.12 \mathrm{E}+01$ \\
\hline & $1.00 \mathrm{E}+02$ & $5.42 \mathrm{E}+01$ \\
\hline & $\mathbf{1 . 0 0 E}-01$ & $\mathbf{2 . 5 4 E + 0 2}$ \\
\hline & $2.15 \mathrm{E}-01$ & $1.52 \mathrm{E}+02$ \\
\hline & $4.64 \mathrm{E}-01$ & $1.07 \mathrm{E}+02$ \\
\hline & $1.00 \mathrm{E}+00$ & $8.10 \mathrm{E}+01$ \\
\hline & $2.15 \mathrm{E}+00$ & $6.51 \mathrm{E}+01$ \\
\hline & $4.64 \mathrm{E}+00$ & $5.55 \mathrm{E}+01$ \\
\hline & $1.00 \mathrm{E}+01$ & $4.92 \mathrm{E}+01$ \\
\hline & $2.15 \mathrm{E}+01$ & $4.54 \mathrm{E}+01$ \\
\hline & $4.64 \mathrm{E}+01$ & $4.21 \mathrm{E}+01$ \\
\hline & $1.00 \mathrm{E}+02$ & $3.52 \mathrm{E}+01$ \\
\hline
\end{tabular}


Table L.15: SAOS Results for BG3L: 3 wt $\%$ Graphene Nanoplatelets (GNP) in Lexan HF1130-111 at various temperatures

Formulation BG3L

\begin{tabular}{|c|c|c|}
\hline Temperature ${ }^{\circ} \mathrm{C}$ & Frequency (rad/s) & Complex Viscosity (Pa.s) \\
\hline \multirow[t]{10}{*}{250} & 1.00E-01 & $1.38 \mathrm{E}+03$ \\
\hline & $2.15 \mathrm{E}-01$ & $1.13 E+03$ \\
\hline & 4.64E-01 & $1.00 \mathrm{E}+03$ \\
\hline & $1.00 \mathrm{E}+00$ & $9.30 \mathrm{E}+02$ \\
\hline & $2.15 \mathrm{E}+00$ & $8.77 \mathrm{E}+02$ \\
\hline & $4.64 \mathrm{E}+00$ & $8.47 \mathrm{E}+02$ \\
\hline & $1.00 \mathrm{E}+01$ & $8.10 \mathrm{E}+02$ \\
\hline & $2.15 \mathrm{E}+01$ & $7.71 \mathrm{E}+02$ \\
\hline & $4.64 \mathrm{E}+01$ & $7.22 \mathrm{E}+02$ \\
\hline & $1.00 \mathrm{E}+02$ & $6.48 \mathrm{E}+02$ \\
\hline \multirow[t]{10}{*}{270} & $1.00 \mathrm{E}-01$ & $8.58 \mathrm{E}+02$ \\
\hline & $2.15 \mathrm{E}-01$ & $6.79 \mathrm{E}+02$ \\
\hline & 4.64E-01 & $5.67 \mathrm{E}+02$ \\
\hline & $1.00 \mathrm{E}+00$ & $4.96 \mathrm{E}+02$ \\
\hline & $2.15 \mathrm{E}+00$ & $4.55 \mathrm{E}+02$ \\
\hline & $4.64 \mathrm{E}+00$ & $4.26 \mathrm{E}+02$ \\
\hline & $1.00 \mathrm{E}+01$ & $4.08 \mathrm{E}+02$ \\
\hline & $2.15 \mathrm{E}+01$ & $3.87 \mathrm{E}+02$ \\
\hline & $4.64 \mathrm{E}+01$ & $3.69 \mathrm{E}+02$ \\
\hline & $1.00 \mathrm{E}+02$ & $3.41 \mathrm{E}+02$ \\
\hline \multirow[t]{10}{*}{290} & $1.00 \mathrm{E}-01$ & $6.61 \mathrm{E}+02$ \\
\hline & $2.15 \mathrm{E}-01$ & $4.99 \mathrm{E}+02$ \\
\hline & $4.64 \mathrm{E}-01$ & $3.88 \mathrm{E}+02$ \\
\hline & $1.00 \mathrm{E}+00$ & $3.17 \mathrm{E}+02$ \\
\hline & $2.15 \mathrm{E}+00$ & $2.75 \mathrm{E}+02$ \\
\hline & $4.64 \mathrm{E}+00$ & $2.50 \mathrm{E}+02$ \\
\hline & $1.00 \mathrm{E}+01$ & $2.33 E+02$ \\
\hline & $2.15 \mathrm{E}+01$ & $2.18 \mathrm{E}+02$ \\
\hline & $4.64 \mathrm{E}+01$ & $2.04 \mathrm{E}+02$ \\
\hline & $1.00 \mathrm{E}+02$ & $1.88 \mathrm{E}+02$ \\
\hline
\end{tabular}


Table L.15: SAOS Results for BG3L: 3 wt\% Graphene Nanoplatelets (GNP) in Lexan HF1130-111 at various temperatures continued

Formulation BG3L

\begin{tabular}{|c|c|c|}
\hline Temperature ${ }^{\circ} \mathrm{C}$ & Frequency (rad/s) & Complex Viscosity (Pa·s) \\
\hline $\mathbf{3 1 0}$ & $\mathbf{1 . 0 0 E}-\mathbf{0 1}$ & $\mathbf{8 . 4 9 E + 0 2}$ \\
\hline & $2.15 \mathrm{E}-01$ & $5.42 \mathrm{E}+02$ \\
\hline & $4.64 \mathrm{E}-01$ & $3.70 \mathrm{E}+02$ \\
\hline & $1.00 \mathrm{E}+00$ & $2.68 \mathrm{E}+02$ \\
\hline & $2.15 \mathrm{E}+00$ & $2.10 \mathrm{E}+02$ \\
\hline & $4.64 \mathrm{E}+00$ & $1.74 \mathrm{E}+02$ \\
\hline & $1.00 \mathrm{E}+01$ & $1.52 \mathrm{E}+02$ \\
\hline & $2.15 \mathrm{E}+01$ & $1.37 \mathrm{E}+02$ \\
\hline & $4.64 \mathrm{E}+01$ & $1.24 \mathrm{E}+02$ \\
\hline & $1.00 \mathrm{E}+02$ & $1.12 \mathrm{E}+02$ \\
\hline & $\mathbf{1 . 0 0 E}-01$ & $1.55 \mathrm{E}+03$ \\
\hline & $2.15 \mathrm{E}-01$ & $8.66 \mathrm{E}+02$ \\
\hline & $4.64 \mathrm{E}-01$ & $5.00 \mathrm{E}+02$ \\
\hline & $1.00 \mathrm{E}+00$ & $3.05 \mathrm{E}+02$ \\
\hline & $2.15 \mathrm{E}+00$ & $2.01 \mathrm{E}+02$ \\
\hline & $4.64 \mathrm{E}+00$ & $1.44 \mathrm{E}+02$ \\
\hline & $1.00 \mathrm{E}+01$ & $1.11 \mathrm{E}+02$ \\
\hline & $2.15 \mathrm{E}+01$ & $9.20 \mathrm{E}+01$ \\
\hline & $4.64 \mathrm{E}+01$ & $7.83 \mathrm{E}+01$ \\
\hline & $1.00 \mathrm{E}+02$ & $6.64 \mathrm{E}+01$ \\
\hline
\end{tabular}


Table L.16: SAOS Results for BG4L: 4 wt\% Graphene Nanoplatelets (GNP) in Lexan HF1130-111 at various temperatures

Formulation BG4L

\begin{tabular}{|c|c|c|}
\hline Temperature ${ }^{\circ} \mathrm{C}$ & Frequency (rad/s) & Complex Viscosity (Pa.s) \\
\hline \multirow[t]{10}{*}{250} & $1.00 \mathrm{E}-01$ & $1.77 \mathrm{E}+03$ \\
\hline & $2.15 \mathrm{E}-01$ & $1.29 \mathrm{E}+03$ \\
\hline & 4.64E-01 & $9.76 \mathrm{E}+02$ \\
\hline & $1.00 \mathrm{E}+00$ & $7.94 \mathrm{E}+02$ \\
\hline & $2.15 \mathrm{E}+00$ & $6.79 \mathrm{E}+02$ \\
\hline & $4.64 \mathrm{E}+00$ & $6.00 \mathrm{E}+02$ \\
\hline & $1.00 \mathrm{E}+01$ & $5.48 \mathrm{E}+02$ \\
\hline & $2.15 \mathrm{E}+01$ & $5.13 E+02$ \\
\hline & $4.64 \mathrm{E}+01$ & $4.75 \mathrm{E}+02$ \\
\hline & $1.00 \mathrm{E}+02$ & $4.30 \mathrm{E}+02$ \\
\hline \multirow[t]{10}{*}{270} & 1.00E-01 & $2.11 \mathrm{E}+03$ \\
\hline & $2.15 \mathrm{E}-01$ & $1.33 \mathrm{E}+03$ \\
\hline & 4.64E-01 & 8.97E+02 \\
\hline & $1.00 \mathrm{E}+00$ & $6.54 \mathrm{E}+02$ \\
\hline & $2.15 \mathrm{E}+00$ & $5.12 \mathrm{E}+02$ \\
\hline & $4.64 \mathrm{E}+00$ & $4.21 \mathrm{E}+02$ \\
\hline & $1.00 \mathrm{E}+01$ & $3.61 \mathrm{E}+02$ \\
\hline & $2.15 \mathrm{E}+01$ & $3.26 \mathrm{E}+02$ \\
\hline & $4.64 \mathrm{E}+01$ & $2.93 E+02$ \\
\hline & $1.00 \mathrm{E}+02$ & $2.62 \mathrm{E}+02$ \\
\hline \multirow[t]{10}{*}{290} & $1.00 \mathrm{E}-01$ & $3.52 \mathrm{E}+03$ \\
\hline & $2.15 \mathrm{E}-01$ & $1.97 \mathrm{E}+03$ \\
\hline & 4.64E-01 & $1.13 \mathrm{E}+03$ \\
\hline & $1.00 \mathrm{E}+00$ & $7.02 \mathrm{E}+02$ \\
\hline & $2.15 \mathrm{E}+00$ & $4.72 \mathrm{E}+02$ \\
\hline & $4.64 \mathrm{E}+00$ & $3.42 \mathrm{E}+02$ \\
\hline & $1.00 \mathrm{E}+01$ & $2.66 \mathrm{E}+02$ \\
\hline & $2.15 \mathrm{E}+01$ & $2.22 \mathrm{E}+02$ \\
\hline & $4.64 \mathrm{E}+01$ & $1.88 \mathrm{E}+02$ \\
\hline & $1.00 \mathrm{E}+02$ & $1.61 \mathrm{E}+02$ \\
\hline
\end{tabular}


Table L.16: SAOS Results for BG4L: 4 wt\% Graphene Nanoplatelets (GNP) in Lexan HF1130-111 at various temperatures continued

Formulation BG4L

\begin{tabular}{|c|c|c|}
\hline Temperature ${ }^{\circ} \mathrm{C}$ & Frequency (rad/s) & Complex Viscosity (Pa·s) \\
\hline $\mathbf{3 1 0}$ & $\mathbf{1 . 0 0 E}-\mathbf{0 1}$ & $\mathbf{6 . 4 9 E}+\mathbf{0 3}$ \\
\hline & $2.15 \mathrm{E}-01$ & $3.37 \mathrm{E}+03$ \\
\hline & $4.64 \mathrm{E}-01$ & $1.73 \mathrm{E}+03$ \\
\hline & $1.00 \mathrm{E}+00$ & $9.28 \mathrm{E}+02$ \\
\hline & $2.15 \mathrm{E}+00$ & $5.34 \mathrm{E}+02$ \\
\hline & $4.64 \mathrm{E}+00$ & $3.32 \mathrm{E}+02$ \\
\hline & $1.00 \mathrm{E}+01$ & $2.28 \mathrm{E}+02$ \\
\hline & $2.15 \mathrm{E}+01$ & $1.68 \mathrm{E}+02$ \\
\hline & $4.64 \mathrm{E}+01$ & $1.31 \mathrm{E}+02$ \\
\hline & $1.00 \mathrm{E}+02$ & $1.04 \mathrm{E}+02$ \\
\hline & $\mathbf{1 . 0 0 E}-01$ & $\mathbf{8 . 6 8 E}+03$ \\
\hline & $2.15 \mathrm{E}-01$ & $4.40 \mathrm{E}+03$ \\
\hline & $4.64 \mathrm{E}-01$ & $2.15 \mathrm{E}+03$ \\
\hline & $1.00 \mathrm{E}+00$ & $1.07 \mathrm{E}+03$ \\
\hline & $2.15 \mathrm{E}+00$ & $5.58 \mathrm{E}+02$ \\
\hline & $4.64 \mathrm{E}+00$ & $3.09 \mathrm{E}+02$ \\
\hline & $1.00 \mathrm{E}+01$ & $1.86 \mathrm{E}+02$ \\
\hline & $2.15 \mathrm{E}+01$ & $1.21 \mathrm{E}+02$ \\
\hline & $4.64 \mathrm{E}+01$ & $8.48 \mathrm{E}+01$ \\
\hline & $1.00 \mathrm{E}+02$ & $6.05 \mathrm{E}+01$ \\
\hline
\end{tabular}


Table L.17: SAOS Results for BG5L: 5 wt\% Graphene Nanoplatelets (GNP) in Lexan HF1130-111 at various temperatures

Formulation BG5L

\begin{tabular}{|c|c|c|}
\hline Temperature ${ }^{\circ} \mathrm{C}$ & Frequency (rad/s) & Complex Viscosity (Pa.s) \\
\hline \multirow[t]{10}{*}{250} & $1.00 \mathrm{E}-01$ & $5.09 \mathrm{E}+03$ \\
\hline & $2.15 \mathrm{E}-01$ & $3.66 \mathrm{E}+03$ \\
\hline & $4.64 \mathrm{E}-01$ & $2.82 \mathrm{E}+03$ \\
\hline & $1.00 \mathrm{E}+00$ & $2.28 \mathrm{E}+03$ \\
\hline & $2.15 \mathrm{E}+00$ & $1.92 \mathrm{E}+03$ \\
\hline & $4.64 \mathrm{E}+00$ & $1.65 \mathrm{E}+03$ \\
\hline & $1.00 \mathrm{E}+01$ & $1.49 \mathrm{E}+03$ \\
\hline & $2.15 \mathrm{E}+01$ & $1.35 \mathrm{E}+03$ \\
\hline & $4.64 \mathrm{E}+01$ & $1.20 \mathrm{E}+03$ \\
\hline & $1.00 \mathrm{E}+02$ & $1.05 \mathrm{E}+03$ \\
\hline \multirow[t]{10}{*}{270} & $1.00 \mathrm{E}-01$ & $3.90 \mathrm{E}+03$ \\
\hline & $2.15 \mathrm{E}-01$ & $2.58 \mathrm{E}+03$ \\
\hline & 4.64E-01 & $1.81 \mathrm{E}+03$ \\
\hline & $1.00 \mathrm{E}+00$ & $1.36 \mathrm{E}+03$ \\
\hline & $2.15 \mathrm{E}+00$ & $1.09 \mathrm{E}+03$ \\
\hline & $4.64 \mathrm{E}+00$ & $8.98 \mathrm{E}+02$ \\
\hline & $1.00 \mathrm{E}+01$ & $7.89 \mathrm{E}+02$ \\
\hline & $2.15 \mathrm{E}+01$ & $7.02 \mathrm{E}+02$ \\
\hline & $4.64 \mathrm{E}+01$ & $6.28 \mathrm{E}+02$ \\
\hline & $1.00 \mathrm{E}+02$ & $5.57 \mathrm{E}+02$ \\
\hline \multirow[t]{10}{*}{290} & 1.00E-01 & $6.08 \mathrm{E}+03$ \\
\hline & $2.15 \mathrm{E}-01$ & $3.47 E+03$ \\
\hline & $4.64 \mathrm{E}-01$ & $2.07 \mathrm{E}+03$ \\
\hline & $1.00 \mathrm{E}+00$ & $1.32 \mathrm{E}+03$ \\
\hline & $2.15 \mathrm{E}+00$ & $9.12 \mathrm{E}+02$ \\
\hline & $4.64 \mathrm{E}+00$ & $6.70 \mathrm{E}+02$ \\
\hline & $1.00 \mathrm{E}+01$ & $5.32 \mathrm{E}+02$ \\
\hline & $2.15 \mathrm{E}+01$ & $4.42 \mathrm{E}+02$ \\
\hline & $4.64 \mathrm{E}+01$ & $3.76 \mathrm{E}+02$ \\
\hline & $1.00 \mathrm{E}+02$ & $3.24 \mathrm{E}+02$ \\
\hline
\end{tabular}


Table L.17: SAOS Results for BG5L: $5 \mathrm{wt} \%$ Graphene Nanoplatelets (GNP) in Lexan HF1130-111 at various temperatures continued

Formulation BG5L

\begin{tabular}{|c|c|c|}
\hline Temperature ${ }^{\circ} \mathrm{C}$ & Frequency (rad/s) & Complex Viscosity (Pa·s) \\
\hline $\mathbf{3 1 0}$ & $\mathbf{1 . 0 0 E}-\mathbf{0 1}$ & $\mathbf{1 . 2 6 E + 0 4}$ \\
\hline & $2.15 \mathrm{E}-01$ & $6.57 \mathrm{E}+03$ \\
\hline & $4.64 \mathrm{E}-01$ & $3.43 \mathrm{E}+03$ \\
\hline & $1.00 \mathrm{E}+00$ & $1.87 \mathrm{E}+03$ \\
\hline & $2.15 \mathrm{E}+00$ & $1.09 \mathrm{E}+03$ \\
\hline & $4.64 \mathrm{E}+00$ & $6.81 \mathrm{E}+02$ \\
\hline & $1.00 \mathrm{E}+01$ & $4.69 \mathrm{E}+02$ \\
\hline & $2.15 \mathrm{E}+01$ & $3.43 \mathrm{E}+02$ \\
\hline & $4.64 \mathrm{E}+01$ & $2.65 \mathrm{E}+02$ \\
\hline & $1.00 \mathrm{E}+02$ & $2.12 \mathrm{E}+02$ \\
\hline & $\mathbf{1 . 0 0 E}-01$ & $1.79 \mathrm{E}+04$ \\
\hline & $2.15 \mathrm{E}-01$ & $9.10 \mathrm{E}+03$ \\
\hline & $4.64 \mathrm{E}-01$ & $4.50 \mathrm{E}+03$ \\
\hline & $1.00 \mathrm{E}+00$ & $2.27 \mathrm{E}+03$ \\
\hline & $2.15 \mathrm{E}+00$ & $1.19 \mathrm{E}+03$ \\
\hline & $4.64 \mathrm{E}+00$ & $6.70 \mathrm{E}+02$ \\
\hline & $1.00 \mathrm{E}+01$ & $4.06 \mathrm{E}+02$ \\
\hline & $2.15 \mathrm{E}+01$ & $2.66 \mathrm{E}+02$ \\
\hline & $4.64 \mathrm{E}+01$ & $1.86 \mathrm{E}+02$ \\
\hline & $1.00 \mathrm{E}+02$ & $1.37 \mathrm{E}+02$ \\
\hline
\end{tabular}


Table L.18: SAOS Results for BG6L: $6 \mathrm{wt} \%$ Graphene Nanoplatelets (GNP) in Lexan HF1130-111 at various temperatures

Formulation BG6L

\begin{tabular}{|c|c|c|}
\hline Temperature ${ }^{\circ} \mathrm{C}$ & Frequency (rad/s) & Complex Viscosity (Pa.s) \\
\hline \multirow[t]{10}{*}{250} & $1.00 \mathrm{E}-01$ & $5.48 \mathrm{E}+03$ \\
\hline & $2.15 \mathrm{E}-01$ & $3.96 \mathrm{E}+03$ \\
\hline & $4.64 \mathrm{E}-01$ & $3.02 E+03$ \\
\hline & $1.00 \mathrm{E}+00$ & $2.42 \mathrm{E}+03$ \\
\hline & $2.15 \mathrm{E}+00$ & $2.02 E+03$ \\
\hline & $4.64 \mathrm{E}+00$ & $1.72 \mathrm{E}+03$ \\
\hline & $1.00 \mathrm{E}+01$ & $1.58 \mathrm{E}+03$ \\
\hline & $2.15 \mathrm{E}+01$ & $1.42 \mathrm{E}+03$ \\
\hline & $4.64 \mathrm{E}+01$ & $1.26 \mathrm{E}+03$ \\
\hline & $1.00 \mathrm{E}+02$ & $1.09 \mathrm{E}+03$ \\
\hline \multirow[t]{10}{*}{270} & 1.00E-01 & $7.03 E+03$ \\
\hline & $2.15 \mathrm{E}-01$ & $4.31 \mathrm{E}+03$ \\
\hline & 4.64E-01 & $2.78 \mathrm{E}+03$ \\
\hline & $1.00 \mathrm{E}+00$ & $1.93 \mathrm{E}+03$ \\
\hline & $2.15 \mathrm{E}+00$ & $1.42 \mathrm{E}+03$ \\
\hline & $4.64 \mathrm{E}+00$ & $1.11 \mathrm{E}+03$ \\
\hline & $1.00 \mathrm{E}+01$ & $9.29 \mathrm{E}+02$ \\
\hline & $2.15 \mathrm{E}+01$ & $7.92 E+02$ \\
\hline & $4.64 \mathrm{E}+01$ & $6.82 \mathrm{E}+02$ \\
\hline & $1.00 \mathrm{E}+02$ & $5.91 \mathrm{E}+02$ \\
\hline \multirow[t]{10}{*}{290} & $1.00 \mathrm{E}-01$ & $1.38 \mathrm{E}+04$ \\
\hline & $2.15 \mathrm{E}-01$ & $7.38 \mathrm{E}+03$ \\
\hline & 4.64E-01 & $4.01 \mathrm{E}+03$ \\
\hline & $1.00 \mathrm{E}+00$ & $2.32 \mathrm{E}+03$ \\
\hline & $2.15 \mathrm{E}+00$ & $1.43 \mathrm{E}+03$ \\
\hline & $4.64 \mathrm{E}+00$ & $9.52 \mathrm{E}+02$ \\
\hline & $1.00 \mathrm{E}+01$ & $6.97 \mathrm{E}+02$ \\
\hline & $2.15 \mathrm{E}+01$ & $5.34 \mathrm{E}+02$ \\
\hline & $4.64 \mathrm{E}+01$ & $4.27 \mathrm{E}+02$ \\
\hline & $1.00 \mathrm{E}+02$ & $3.52 \mathrm{E}+02$ \\
\hline
\end{tabular}


Table L.18: SAOS Results for BG6L: $6 \mathrm{wt} \%$ Graphene Nanoplatelets (GNP) in Lexan HF1130-111 at various temperatures continued

Formulation BG6L

\begin{tabular}{|c|c|c|}
\hline Temperature ${ }^{\circ} \mathrm{C}$ & Frequency $(\mathrm{rad} / \mathrm{s})$ & Complex Viscosity (Pa·s) \\
\hline $\mathbf{3 1 0}$ & $\mathbf{1 . 0 0 E}-\mathbf{0 1}$ & $\mathbf{2 . 4 1 E + 0 4}$ \\
\hline & $2.15 \mathrm{E}-01$ & $1.24 \mathrm{E}+04$ \\
\hline & $4.64 \mathrm{E}-01$ & $6.17 \mathrm{E}+03$ \\
\hline & $1.00 \mathrm{E}+00$ & $3.17 \mathrm{E}+03$ \\
\hline & $2.15 \mathrm{E}+00$ & $1.71 \mathrm{E}+03$ \\
\hline & $4.64 \mathrm{E}+00$ & $9.92 \mathrm{E}+02$ \\
\hline & $1.00 \mathrm{E}+01$ & $6.25 \mathrm{E}+02$ \\
\hline & $2.15 \mathrm{E}+01$ & $4.22 \mathrm{E}+02$ \\
\hline & $4.64 \mathrm{E}+01$ & $3.02 \mathrm{E}+02$ \\
\hline & $1.00 \mathrm{E}+02$ & $2.29 \mathrm{E}+02$ \\
\hline & $\mathbf{1 . 0 0 E}-01$ & $\mathbf{2 . 8 9 E}+04$ \\
\hline & $2.15 \mathrm{E}-01$ & $1.42 \mathrm{E}+04$ \\
\hline & $4.64 \mathrm{E}-01$ & $6.84 \mathrm{E}+03$ \\
\hline & $1.00 \mathrm{E}+00$ & $3.36 \mathrm{E}+03$ \\
\hline & $2.15 \mathrm{E}+00$ & $1.71 \mathrm{E}+03$ \\
\hline & $4.64 \mathrm{E}+00$ & $9.11 \mathrm{E}+02$ \\
\hline & $1.00 \mathrm{E}+01$ & $5.21 \mathrm{E}+02$ \\
\hline & $2.15 \mathrm{E}+01$ & $3.20 \mathrm{E}+02$ \\
\hline & $4.64 \mathrm{E}+01$ & $2.10 \mathrm{E}+02$ \\
\hline & $1.00 \mathrm{E}+02$ & $1.47 \mathrm{E}+02$ \\
\hline
\end{tabular}


Table L.19: SAOS Results for BG8L: 8 wt\% Graphene Nanoplatelets (GNP) in Lexan HF1130-111 at various temperatures

Formulation BG8L

\begin{tabular}{|c|c|c|}
\hline Temperature ${ }^{\circ} \mathrm{C}$ & Frequency (rad/s) & Complex Viscosity (Pa.s) \\
\hline \multirow[t]{10}{*}{250} & $1.00 \mathrm{E}-01$ & $2.41 \mathrm{E}+04$ \\
\hline & $2.15 \mathrm{E}-01$ & $1.30 \mathrm{E}+04$ \\
\hline & $4.64 \mathrm{E}-01$ & $6.71 \mathrm{E}+03$ \\
\hline & $1.00 \mathrm{E}+00$ & $3.64 \mathrm{E}+03$ \\
\hline & $2.15 \mathrm{E}+00$ & $2.08 \mathrm{E}+03$ \\
\hline & $4.64 \mathrm{E}+00$ & $1.29 \mathrm{E}+03$ \\
\hline & $1.00 \mathrm{E}+01$ & $8.89 \mathrm{E}+02$ \\
\hline & $2.15 \mathrm{E}+01$ & $6.50 \mathrm{E}+02$ \\
\hline & $4.64 \mathrm{E}+01$ & $4.97 \mathrm{E}+02$ \\
\hline & $1.00 \mathrm{E}+02$ & $3.98 \mathrm{E}+02$ \\
\hline \multirow[t]{10}{*}{270} & 1.00E-01 & $3.40 \mathrm{E}+04$ \\
\hline & $2.15 \mathrm{E}-01$ & $1.65 \mathrm{E}+04$ \\
\hline & 4.64E-01 & $8.13 E+03$ \\
\hline & $1.00 \mathrm{E}+00$ & $4.15 E+03$ \\
\hline & $2.15 \mathrm{E}+00$ & $2.23 \mathrm{E}+03$ \\
\hline & $4.64 \mathrm{E}+00$ & $1.28 \mathrm{E}+03$ \\
\hline & $1.00 \mathrm{E}+01$ & $8.02 \mathrm{E}+02$ \\
\hline & $2.15 \mathrm{E}+01$ & $5.40 \mathrm{E}+02$ \\
\hline & $4.64 \mathrm{E}+01$ & $3.85 \mathrm{E}+02$ \\
\hline & $1.00 \mathrm{E}+02$ & $2.93 \mathrm{E}+02$ \\
\hline \multirow[t]{10}{*}{290} & 1.00E-01 & $4.04 E+04$ \\
\hline & $2.15 \mathrm{E}-01$ & $1.94 \mathrm{E}+04$ \\
\hline & 4.64E-01 & $9.23 E+03$ \\
\hline & $1.00 \mathrm{E}+00$ & $4.52 \mathrm{E}+03$ \\
\hline & $2.15 E+00$ & $2.29 E+03$ \\
\hline & $4.64 \mathrm{E}+00$ & $1.23 \mathrm{E}+03$ \\
\hline & $1.00 \mathrm{E}+01$ & $7.08 \mathrm{E}+02$ \\
\hline & $2.15 E+01$ & $4.40 \mathrm{E}+02$ \\
\hline & $4.64 \mathrm{E}+01$ & $2.93 \mathrm{E}+02$ \\
\hline & $1.00 \mathrm{E}+02$ & $2.10 \mathrm{E}+02$ \\
\hline
\end{tabular}


Table L.19: SAOS Results for BG8L: $8 \mathrm{wt} \%$ Graphene Nanoplatelets (GNP) in Lexan HF1130-111 at various temperatures continued

Formulation BG8L

\begin{tabular}{|c|c|c|}
\hline Temperature ${ }^{\circ} \mathrm{C}$ & Frequency $(\mathrm{rad} / \mathrm{s})$ & Complex Viscosity (Pa·s) \\
\hline $\mathbf{3 1 0}$ & $\mathbf{1 . 0 0 E}-\mathbf{0 1}$ & $\mathbf{4 . 6 6 E + 0 4}$ \\
\hline & $2.15 \mathrm{E}-01$ & $2.27 \mathrm{E}+04$ \\
\hline & $4.64 \mathrm{E}-01$ & $1.09 \mathrm{E}+04$ \\
\hline & $1.00 \mathrm{E}+00$ & $5.20 \mathrm{E}+03$ \\
\hline & $2.15 \mathrm{E}+00$ & $2.54 \mathrm{E}+03$ \\
\hline & $4.64 \mathrm{E}+00$ & $1.29 \mathrm{E}+03$ \\
\hline & $1.00 \mathrm{E}+01$ & $6.86 \mathrm{E}+02$ \\
\hline & $2.15 \mathrm{E}+01$ & $3.91 \mathrm{E}+02$ \\
\hline & $4.64 \mathrm{E}+01$ & $2.38 \mathrm{E}+02$ \\
\hline & $1.00 \mathrm{E}+02$ & $1.57 \mathrm{E}+02$ \\
\hline & $1.00 \mathrm{E}-01$ & $\mathbf{5 . 1 1 E}+04$ \\
\hline & $2.15 \mathrm{E}-01$ & $2.47 \mathrm{E}+04$ \\
\hline & $4.64 \mathrm{E}-01$ & $1.17 \mathrm{E}+04$ \\
\hline & $1.00 \mathrm{E}+00$ & $5.58 \mathrm{E}+03$ \\
\hline & $2.15 \mathrm{E}+00$ & $2.68 \mathrm{E}+03$ \\
\hline & $4.64 \mathrm{E}+00$ & $1.31 \mathrm{E}+03$ \\
\hline & $1.00 \mathrm{E}+01$ & $6.71 \mathrm{E}+02$ \\
\hline & $2.15 \mathrm{E}+01$ & $3.59 \mathrm{E}+02$ \\
\hline & $4.64 \mathrm{E}+01$ & $2.04 \mathrm{E}+02$ \\
\hline & $1.00 \mathrm{E}+02$ & $1.24 \mathrm{E}+02$ \\
\hline
\end{tabular}


Table L.20: SAOS Results for BG10L: $10 \mathrm{wt} \%$ Graphene Nanoplatelets (GNP) in Lexan HF1130-111 at various temperatures

Formulation BG10L

\begin{tabular}{|c|c|c|}
\hline Temperature ${ }^{\circ} \mathrm{C}$ & Frequency (rad/s) & Complex Viscosity (Pa.s) \\
\hline \multirow[t]{10}{*}{250} & $1.00 \mathrm{E}-01$ & $7.58 \mathrm{E}+04$ \\
\hline & $2.15 \mathrm{E}-01$ & $4.13 E+04$ \\
\hline & $4.64 \mathrm{E}-01$ & $2.27 \mathrm{E}+04$ \\
\hline & $1.00 \mathrm{E}+00$ & $1.30 \mathrm{E}+04$ \\
\hline & $2.15 \mathrm{E}+00$ & $7.82 \mathrm{E}+03$ \\
\hline & $4.64 \mathrm{E}+00$ & $5.42 \mathrm{E}+03$ \\
\hline & $1.00 \mathrm{E}+01$ & $3.75 E+03$ \\
\hline & $2.15 \mathrm{E}+01$ & $2.75 \mathrm{E}+03$ \\
\hline & $4.64 \mathrm{E}+01$ & $2.09 \mathrm{E}+03$ \\
\hline & $1.00 \mathrm{E}+02$ & $1.59 \mathrm{E}+03$ \\
\hline \multirow[t]{10}{*}{270} & 1.00E-01 & $8.94 \mathrm{E}+04$ \\
\hline & $2.15 \mathrm{E}-01$ & $4.63 \mathrm{E}+04$ \\
\hline & 4.64E-01 & $2.38 \mathrm{E}+04$ \\
\hline & $1.00 \mathrm{E}+00$ & $1.27 E+04$ \\
\hline & $2.15 \mathrm{E}+00$ & $7.13 \mathrm{E}+03$ \\
\hline & $4.64 \mathrm{E}+00$ & $4.35 \mathrm{E}+03$ \\
\hline & $1.00 \mathrm{E}+01$ & $2.68 \mathrm{E}+03$ \\
\hline & $2.15 \mathrm{E}+01$ & $1.85 \mathrm{E}+03$ \\
\hline & $4.64 \mathrm{E}+01$ & $1.31 \mathrm{E}+03$ \\
\hline & $1.00 \mathrm{E}+02$ & $9.69 \mathrm{E}+02$ \\
\hline \multirow[t]{10}{*}{290} & 1.00E-01 & $1.09 E+05$ \\
\hline & $2.15 E-01$ & $5.62 \mathrm{E}+04$ \\
\hline & 4.64E-01 & $2.78 \mathrm{E}+04$ \\
\hline & $1.00 \mathrm{E}+00$ & $1.41 \mathrm{E}+04$ \\
\hline & $2.15 \mathrm{E}+00$ & $7.53 \mathrm{E}+03$ \\
\hline & $4.64 \mathrm{E}+00$ & $4.14 \mathrm{E}+03$ \\
\hline & $1.00 \mathrm{E}+01$ & $2.34 \mathrm{E}+03$ \\
\hline & $2.15 E+01$ & $1.47 \mathrm{E}+03$ \\
\hline & $4.64 \mathrm{E}+01$ & $9.54 \mathrm{E}+02$ \\
\hline & $1.00 \mathrm{E}+02$ & $6.51 \mathrm{E}+02$ \\
\hline
\end{tabular}


Table L.20: SAOS Results for BG10L: $10 \mathrm{wt} \%$ Graphene Nanoplatelets (GNP) in Lexan HF1130-111 at various temperatures continued

Formulation BG10L

\begin{tabular}{|c|c|c|}
\hline Temperature ${ }^{\circ} \mathrm{C}$ & Frequency (rad/s) & Complex Viscosity (Pa·s) \\
\hline & $\mathbf{1 . 0 0 E}-\mathbf{0 1}$ & $\mathbf{1 . 3 1 E + 0 5}$ \\
\hline & $2.15 \mathrm{E}-01$ & $6.64 \mathrm{E}+04$ \\
\hline & $4.64 \mathrm{E}-01$ & $3.28 \mathrm{E}+04$ \\
\hline & $1.00 \mathrm{E}+00$ & $1.62 \mathrm{E}+04$ \\
\hline & $2.15 \mathrm{E}+00$ & $8.20 \mathrm{E}+03$ \\
\hline & $4.64 \mathrm{E}+00$ & $4.23 \mathrm{E}+03$ \\
\hline & $1.00 \mathrm{E}+01$ & $2.24 \mathrm{E}+03$ \\
\hline & $2.15 \mathrm{E}+01$ & $1.31 \mathrm{E}+03$ \\
\hline & $4.64 \mathrm{E}+01$ & $7.95 \mathrm{E}+02$ \\
\hline & $1.00 \mathrm{E}+02$ & $5.15 \mathrm{E}+02$ \\
\hline & $\mathbf{1 . 0 0 E}-01$ & $1.44 \mathrm{E}+05$ \\
\hline & $2.15 \mathrm{E}-01$ & $7.35 \mathrm{E}+04$ \\
\hline & $4.64 \mathrm{E}-01$ & $3.64 \mathrm{E}+04$ \\
\hline & $1.00 \mathrm{E}+00$ & $1.78 \mathrm{E}+04$ \\
\hline & $2.15 \mathrm{E}+00$ & $8.71 \mathrm{E}+03$ \\
\hline & $4.64 \mathrm{E}+00$ & $4.39 \mathrm{E}+03$ \\
\hline & $1.00 \mathrm{E}+01$ & $2.21 \mathrm{E}+03$ \\
\hline & $2.15 \mathrm{E}+01$ & $1.21 \mathrm{E}+03$ \\
\hline & $4.64 \mathrm{E}+01$ & $6.90 \mathrm{E}+02$ \\
\hline & $1.00 \mathrm{E}+02$ & $4.11 \mathrm{E}+02$ \\
\hline
\end{tabular}


Table L.21: SAOS Results for BG12L: $12 \mathrm{wt} \%$ Graphene Nanoplatelets (GNP) in Lexan HF1130-111 at various temperatures

Formulation BG12L

\begin{tabular}{|c|c|c|}
\hline Temperature ${ }^{\circ} \mathrm{C}$ & Frequency (rad/s) & Complex Viscosity (Pa.s) \\
\hline \multirow[t]{10}{*}{250} & $1.00 \mathrm{E}-01$ & $5.06 \mathrm{E}+05$ \\
\hline & $2.15 \mathrm{E}-01$ & $2.51 \mathrm{E}+05$ \\
\hline & $4.64 \mathrm{E}-01$ & $1.25 \mathrm{E}+05$ \\
\hline & $1.00 \mathrm{E}+00$ & $6.54 \mathrm{E}+04$ \\
\hline & $2.15 \mathrm{E}+00$ & $3.47 \mathrm{E}+04$ \\
\hline & $4.64 \mathrm{E}+00$ & $1.78 \mathrm{E}+04$ \\
\hline & $1.00 \mathrm{E}+01$ & $1.05 \mathrm{E}+04$ \\
\hline & $2.15 \mathrm{E}+01$ & $6.07 \mathrm{E}+03$ \\
\hline & $4.64 \mathrm{E}+01$ & $3.77 \mathrm{E}+03$ \\
\hline & $1.00 \mathrm{E}+02$ & $2.44 \mathrm{E}+03$ \\
\hline \multirow[t]{10}{*}{270} & 1.00E-01 & $4.00 \mathrm{E}+05$ \\
\hline & $2.15 \mathrm{E}-01$ & $1.98 \mathrm{E}+05$ \\
\hline & 4.64E-01 & $9.84 \mathrm{E}+04$ \\
\hline & $1.00 \mathrm{E}+00$ & $4.96 \mathrm{E}+04$ \\
\hline & $2.15 \mathrm{E}+00$ & $2.49 \mathrm{E}+04$ \\
\hline & $4.64 \mathrm{E}+00$ & $1.35 \mathrm{E}+04$ \\
\hline & $1.00 \mathrm{E}+01$ & $7.23 \mathrm{E}+03$ \\
\hline & $2.15 \mathrm{E}+01$ & $4.04 \mathrm{E}+03$ \\
\hline & $4.64 \mathrm{E}+01$ & $2.40 \mathrm{E}+03$ \\
\hline & $1.00 \mathrm{E}+02$ & $1.52 \mathrm{E}+03$ \\
\hline \multirow[t]{10}{*}{290} & 1.00E-01 & $3.62 E+05$ \\
\hline & $2.15 \mathrm{E}-01$ & $1.79 \mathrm{E}+05$ \\
\hline & 4.64E-01 & $8.78 E+04$ \\
\hline & $1.00 \mathrm{E}+00$ & $4.34 \mathrm{E}+04$ \\
\hline & $2.15 \mathrm{E}+00$ & $2.14 \mathrm{E}+04$ \\
\hline & $4.64 \mathrm{E}+00$ & $1.12 \mathrm{E}+04$ \\
\hline & $1.00 \mathrm{E}+01$ & $5.76 \mathrm{E}+03$ \\
\hline & $2.15 E+01$ & $3.15 E+03$ \\
\hline & $4.64 \mathrm{E}+01$ & $1.80 \mathrm{E}+03$ \\
\hline & $1.00 \mathrm{E}+02$ & $1.08 \mathrm{E}+03$ \\
\hline
\end{tabular}


Table L.21: SAOS Results for BG12L: $12 \mathrm{wt} \%$ Graphene Nanoplatelets (GNP) in Lexan HF1130-111 at various temperatures continued

Formulation BG12L

\begin{tabular}{|c|c|c|}
\hline Temperature ${ }^{\circ} \mathrm{C}$ & Frequency (rad/s) & Complex Viscosity (Pa·s) \\
\hline $\mathbf{3 1 0}$ & $\mathbf{1 . 0 0 E}-\mathbf{0 1}$ & $\mathbf{3 . 4 2 E}+\mathbf{0 5}$ \\
\hline & $2.15 \mathrm{E}-01$ & $1.73 \mathrm{E}+05$ \\
\hline & $4.64 \mathrm{E}-01$ & $8.52 \mathrm{E}+04$ \\
\hline & $1.00 \mathrm{E}+00$ & $4.19 \mathrm{E}+04$ \\
\hline & $2.15 \mathrm{E}+00$ & $2.06 \mathrm{E}+04$ \\
\hline & $4.64 \mathrm{E}+00$ & $1.02 \mathrm{E}+04$ \\
\hline & $1.00 \mathrm{E}+01$ & $5.17 \mathrm{E}+03$ \\
\hline & $2.15 \mathrm{E}+01$ & $2.76 \mathrm{E}+03$ \\
\hline & $4.64 \mathrm{E}+01$ & $1.51 \mathrm{E}+03$ \\
\hline & $1.00 \mathrm{E}+02$ & $8.67 \mathrm{E}+02$ \\
\hline & $\mathbf{1 . 0 0 E}-01$ & $\mathbf{2 . 9 1 E}+05$ \\
\hline & $2.15 \mathrm{E}-01$ & $1.50 \mathrm{E}+05$ \\
\hline & $4.64 \mathrm{E}-01$ & $7.61 \mathrm{E}+04$ \\
\hline & $1.00 \mathrm{E}+00$ & $3.73 \mathrm{E}+04$ \\
\hline & $2.15 \mathrm{E}+00$ & $1.87 \mathrm{E}+04$ \\
\hline & $4.64 \mathrm{E}+00$ & $9.59 \mathrm{E}+03$ \\
\hline & $1.00 \mathrm{E}+01$ & $4.73 \mathrm{E}+03$ \\
\hline & $2.15 \mathrm{E}+01$ & $2.43 \mathrm{E}+03$ \\
\hline & $4.64 \mathrm{E}+01$ & $1.30 \mathrm{E}+03$ \\
\hline & $1.00 \mathrm{E}+02$ & $7.26 \mathrm{E}+02$ \\
\hline
\end{tabular}


Table L.22: SAOS Results for BG15L: 15 wt\% Graphene Nanoplatelets (GNP) in Lexan HF1130-111 at various temperatures

Formulation BG15L

\begin{tabular}{|c|c|c|}
\hline Temperature ${ }^{\circ} \mathrm{C}$ & Frequency (rad/s) & Complex Viscosity (Pa.s) \\
\hline \multirow[t]{10}{*}{250} & 1.00E-01 & $8.37 \mathrm{E}+05$ \\
\hline & 2.15E-01 & $4.29 \mathrm{E}+05$ \\
\hline & $4.64 \mathrm{E}-01$ & $2.31 \mathrm{E}+05$ \\
\hline & $1.00 \mathrm{E}+00$ & $1.22 \mathrm{E}+05$ \\
\hline & $2.15 \mathrm{E}+00$ & $6.52 \mathrm{E}+04$ \\
\hline & $4.64 \mathrm{E}+00$ & $3.25 \mathrm{E}+04$ \\
\hline & $1.00 \mathrm{E}+01$ & $1.83 \mathrm{E}+04$ \\
\hline & $2.15 E+01$ & $1.02 E+04$ \\
\hline & $4.64 \mathrm{E}+01$ & $5.89 \mathrm{E}+03$ \\
\hline & $1.00 \mathrm{E}+02$ & $3.65 \mathrm{E}+03$ \\
\hline \multirow[t]{10}{*}{270} & 1.00E-01 & $5.78 \mathrm{E}+05$ \\
\hline & $2.15 \mathrm{E}-01$ & $3.15 \mathrm{E}+05$ \\
\hline & 4.64E-01 & $1.70 \mathrm{E}+05$ \\
\hline & $1.00 \mathrm{E}+00$ & $9.20 \mathrm{E}+04$ \\
\hline & $2.15 \mathrm{E}+00$ & $4.84 \mathrm{E}+04$ \\
\hline & $4.64 \mathrm{E}+00$ & $2.32 \mathrm{E}+04$ \\
\hline & $1.00 \mathrm{E}+01$ & $1.32 \mathrm{E}+04$ \\
\hline & $2.15 \mathrm{E}+01$ & $7.01 \mathrm{E}+03$ \\
\hline & $4.64 \mathrm{E}+01$ & $3.97 \mathrm{E}+03$ \\
\hline & $1.00 \mathrm{E}+02$ & $2.39 \mathrm{E}+03$ \\
\hline \multirow[t]{10}{*}{290} & 1.00E-01 & $5.61 \mathrm{E}+05$ \\
\hline & $2.15 \mathrm{E}-01$ & $3.11 \mathrm{E}+05$ \\
\hline & 4.64E-01 & $1.62 \mathrm{E}+05$ \\
\hline & $1.00 \mathrm{E}+00$ & $8.49 \mathrm{E}+04$ \\
\hline & $2.15 \mathrm{E}+00$ & $4.20 \mathrm{E}+04$ \\
\hline & $4.64 \mathrm{E}+00$ & $2.02 E+04$ \\
\hline & $1.00 \mathrm{E}+01$ & $1.13 E+04$ \\
\hline & $2.15 E+01$ & $5.84 \mathrm{E}+03$ \\
\hline & $4.64 \mathrm{E}+01$ & $3.17 \mathrm{E}+03$ \\
\hline & $1.00 \mathrm{E}+02$ & $1.82 \mathrm{E}+03$ \\
\hline
\end{tabular}


Table L.22: SAOS Results for BG15L: 15 wt\% Graphene Nanoplatelets (GNP) in Lexan HF1130-111 at various temperatures continued

Formulation BG15L

\begin{tabular}{|c|c|c|}
\hline Temperature ${ }^{\circ} \mathrm{C}$ & Frequency (rad/s) & Complex Viscosity (Pa·s) \\
\hline & $\mathbf{1 . 0 0 E}-\mathbf{0 1}$ & $\mathbf{5 . 7 1 E + 0 5}$ \\
\hline & $2.15 \mathrm{E}-01$ & $3.07 \mathrm{E}+05$ \\
\hline & $4.64 \mathrm{E}-01$ & $1.57 \mathrm{E}+05$ \\
\hline & $1.00 \mathrm{E}+00$ & $8.25 \mathrm{E}+04$ \\
\hline & $2.15 \mathrm{E}+00$ & $4.16 \mathrm{E}+04$ \\
\hline & $4.64 \mathrm{E}+00$ & $2.03 \mathrm{E}+04$ \\
\hline & $1.00 \mathrm{E}+01$ & $1.08 \mathrm{E}+04$ \\
\hline & $2.15 \mathrm{E}+01$ & $5.40 \mathrm{E}+03$ \\
\hline & $4.64 \mathrm{E}+01$ & $2.85 \mathrm{E}+03$ \\
\hline & $1.00 \mathrm{E}+02$ & $1.58 \mathrm{E}+03$ \\
\hline & $\mathbf{1 . 0 0 E}-01$ & $\mathbf{5 . 3 7 E}+05$ \\
\hline & $2.15 \mathrm{E}-01$ & $2.88 \mathrm{E}+05$ \\
\hline & $4.64 \mathrm{E}-01$ & $1.50 \mathrm{E}+05$ \\
\hline & $1.00 \mathrm{E}+00$ & $7.78 \mathrm{E}+04$ \\
\hline & $2.15 \mathrm{E}+00$ & $4.00 \mathrm{E}+04$ \\
\hline & $4.64 \mathrm{E}+00$ & $1.85 \mathrm{E}+04$ \\
\hline & $1.00 \mathrm{E}+01$ & $9.82 \mathrm{E}+03$ \\
\hline & $2.15 \mathrm{E}+01$ & $4.96 \mathrm{E}+03$ \\
\hline & $4.64 \mathrm{E}+01$ & $2.59 \mathrm{E}+03$ \\
\hline & $1.00 \mathrm{E}+02$ & $1.37 \mathrm{E}+03$ \\
\hline
\end{tabular}




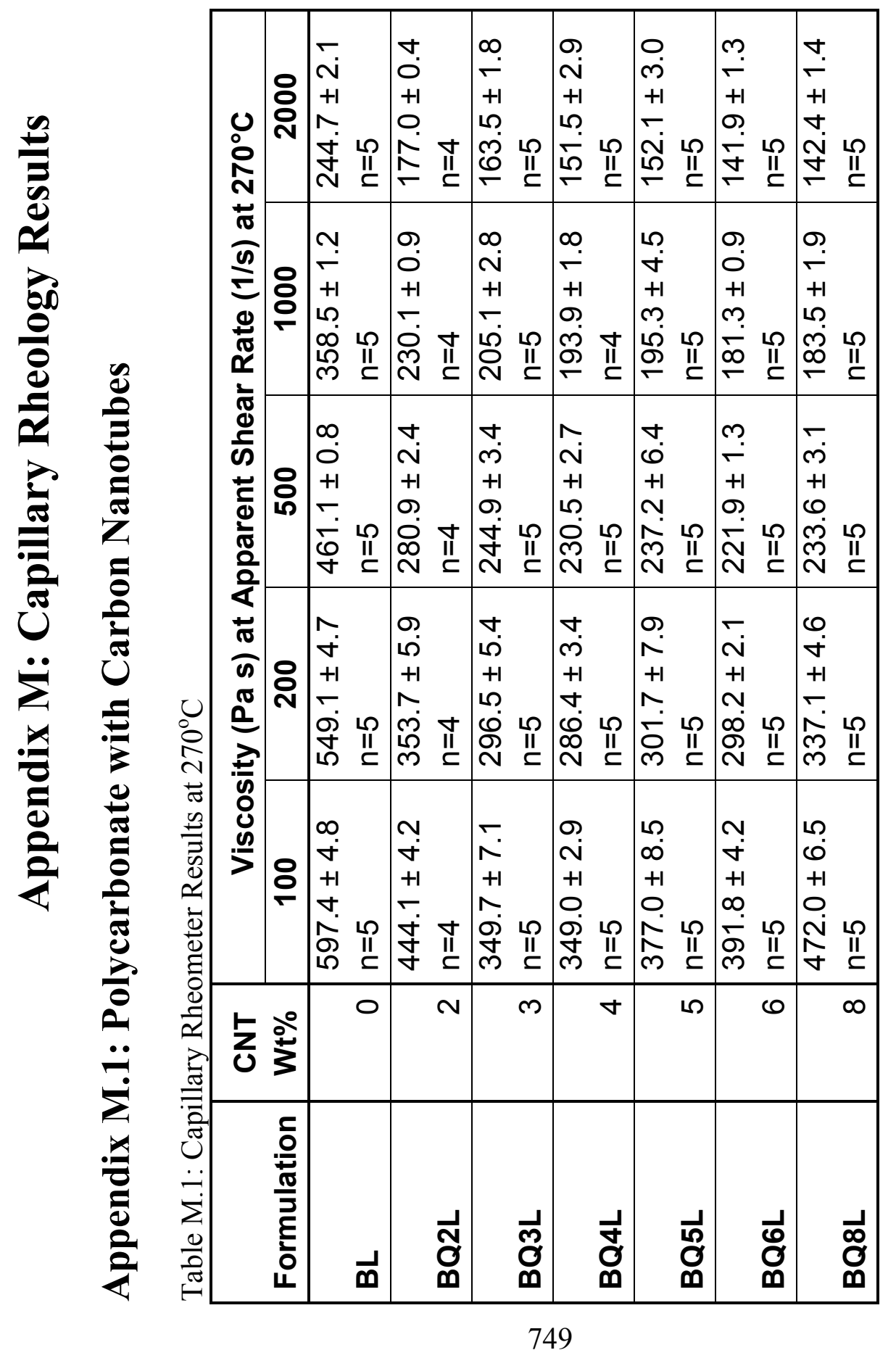




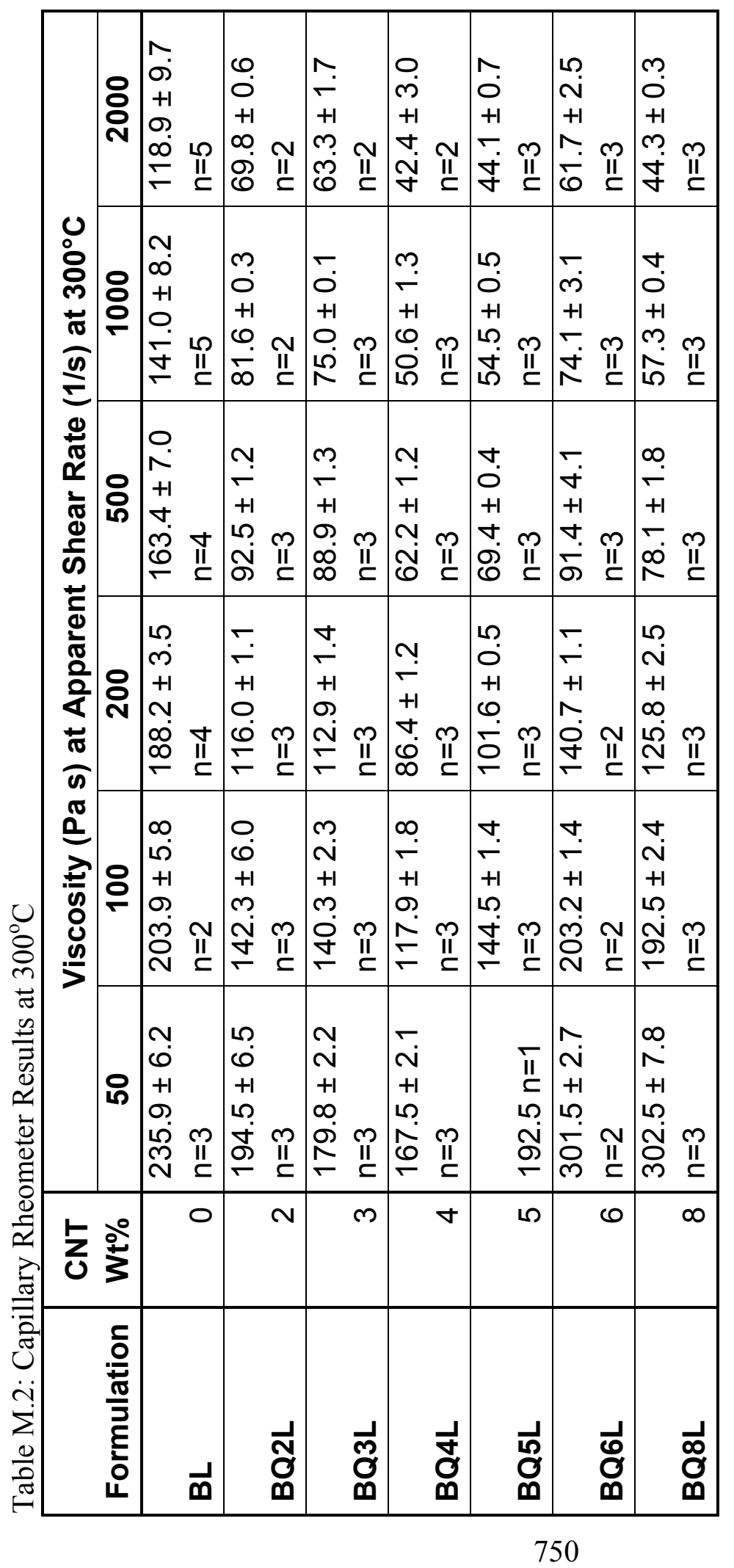




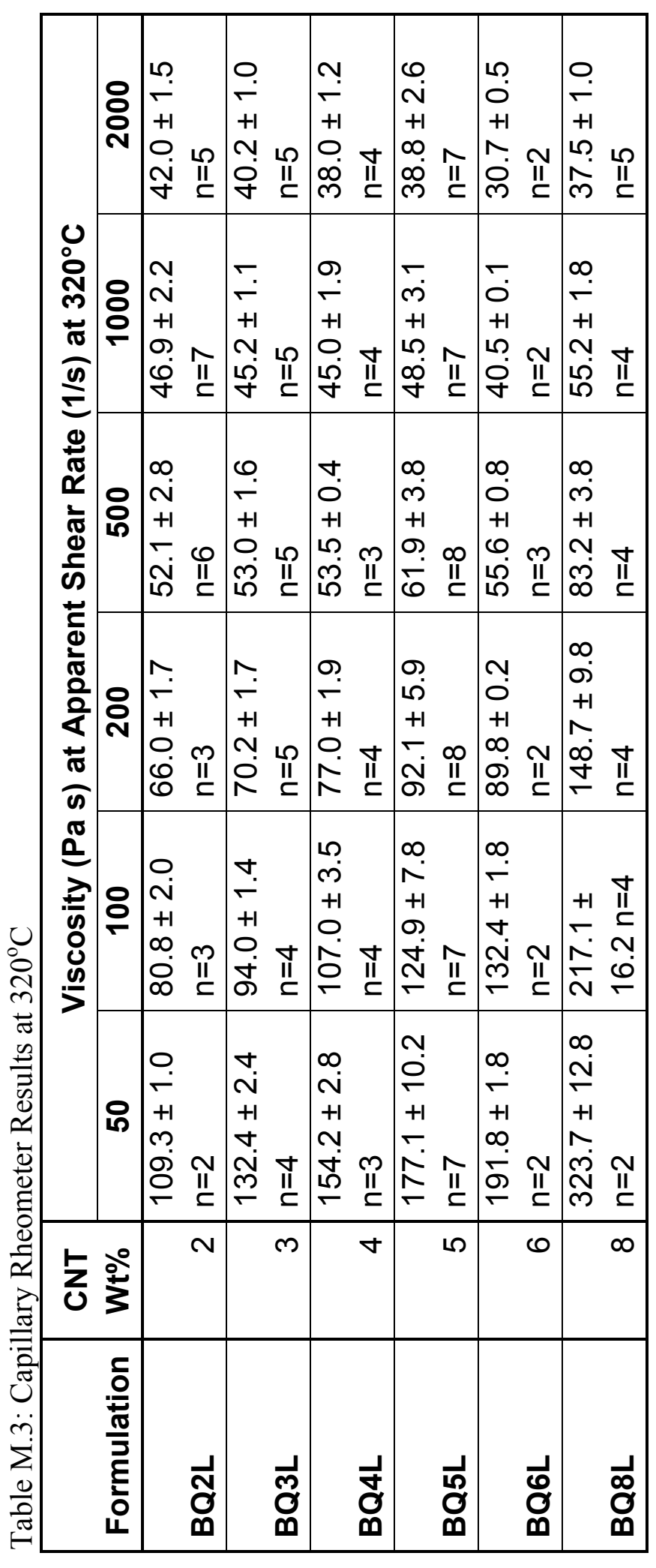




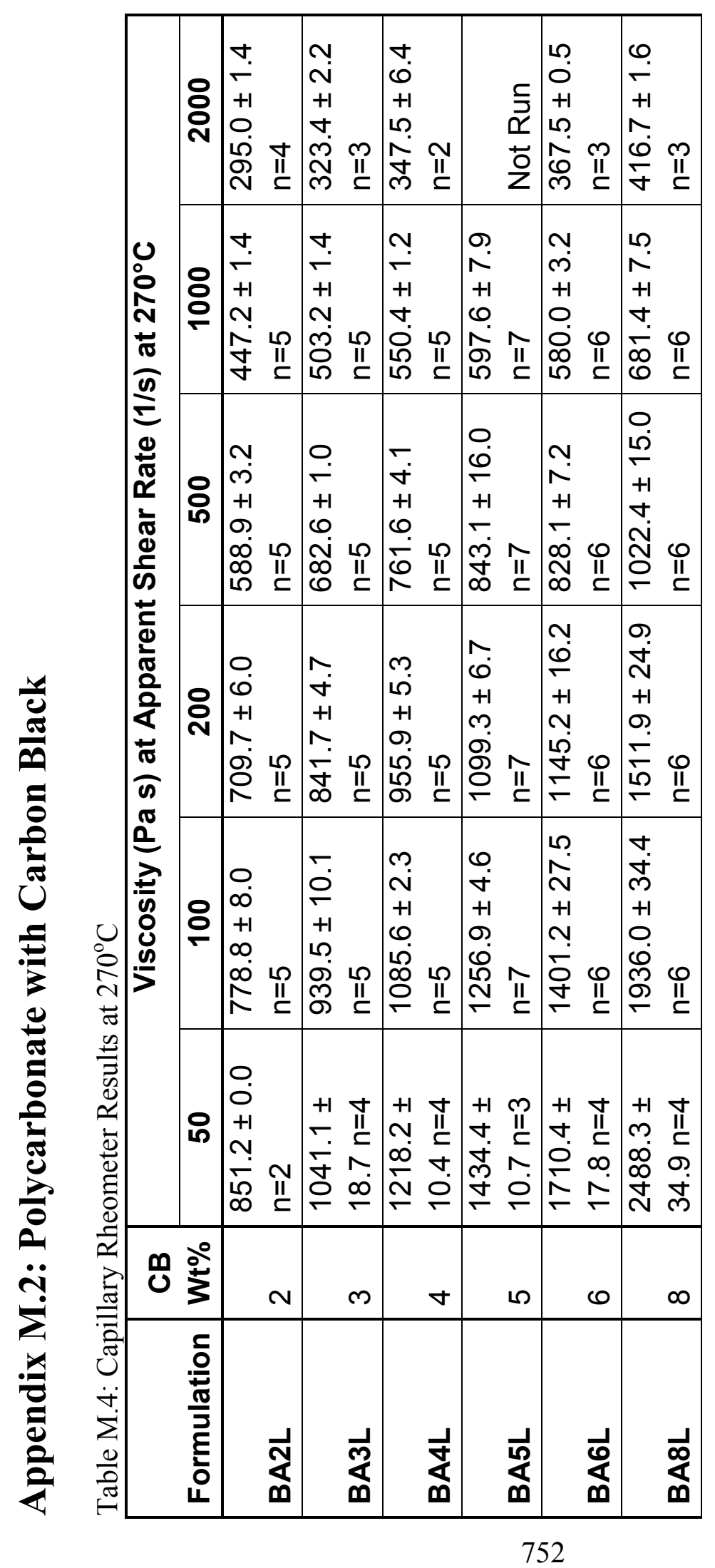




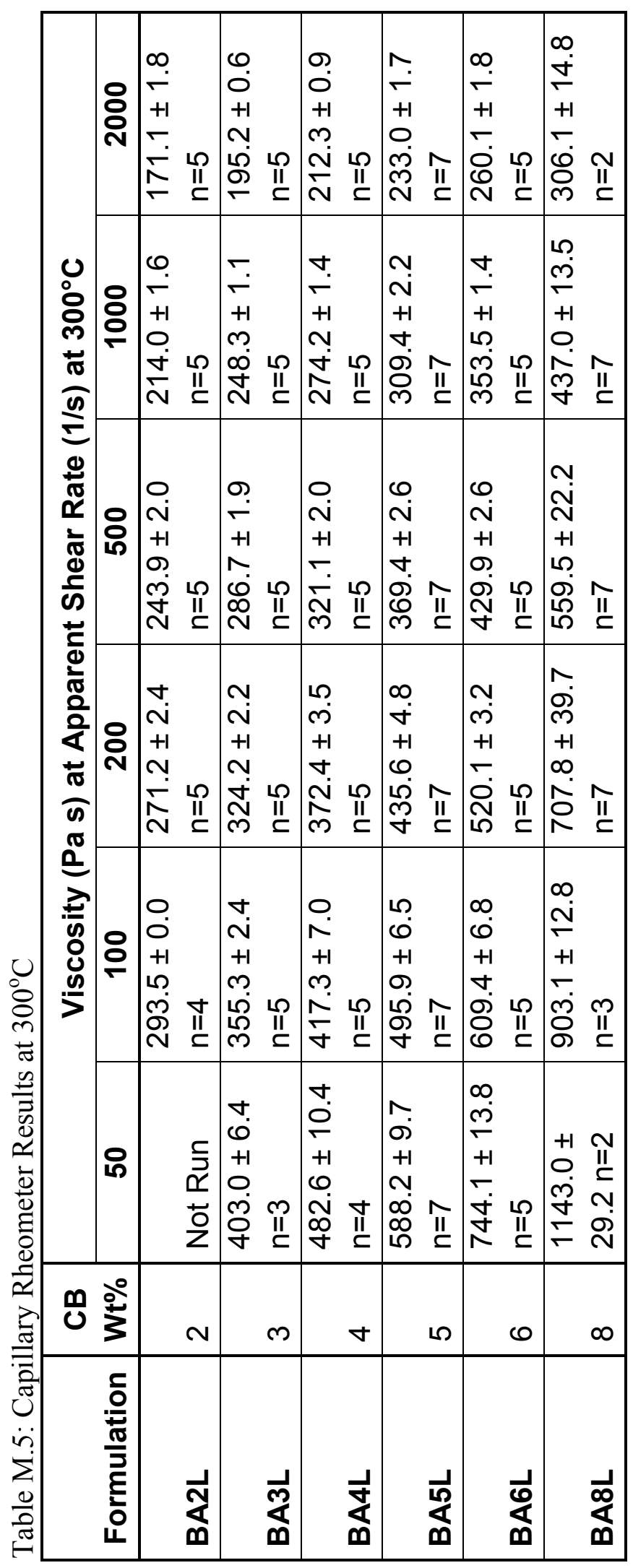




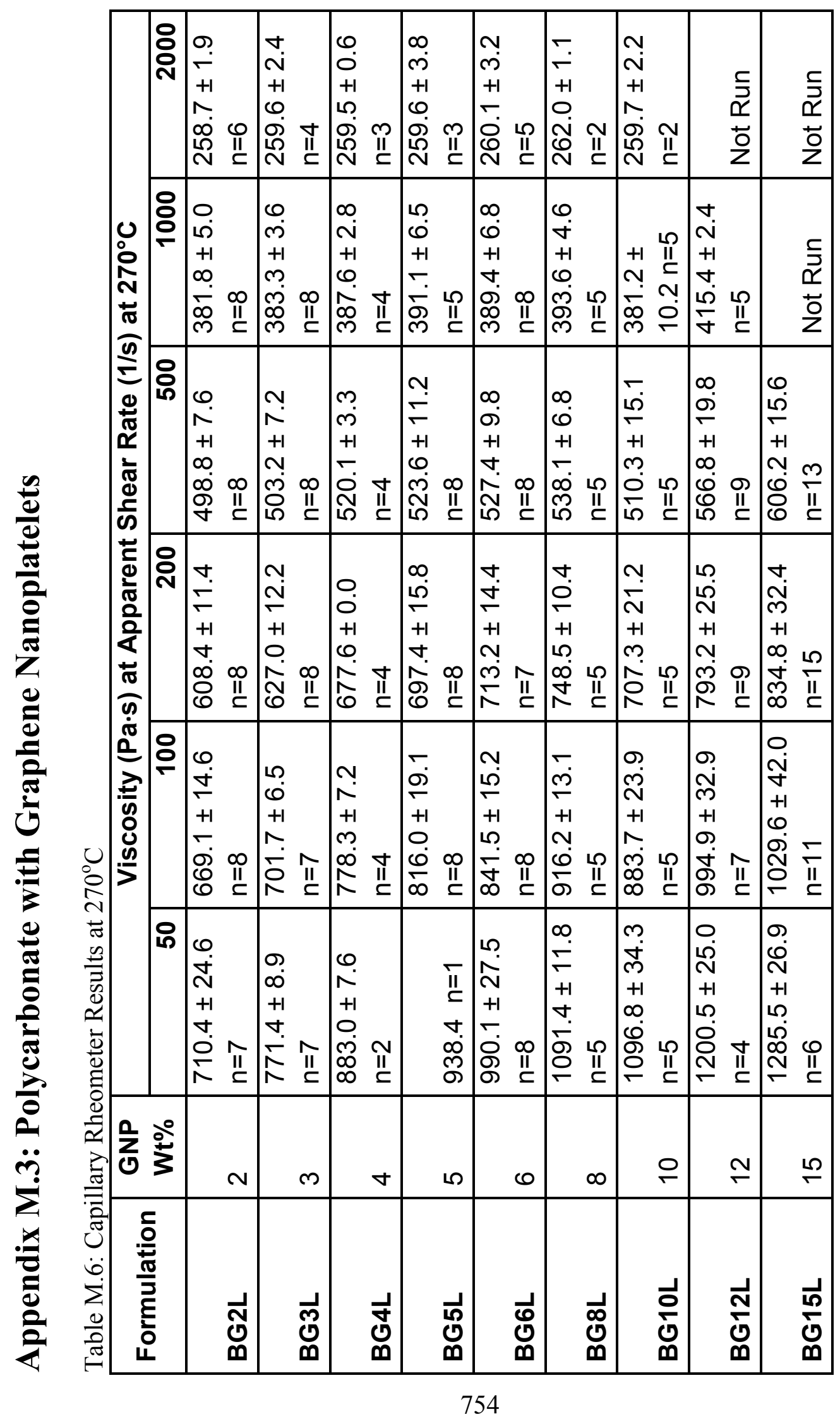




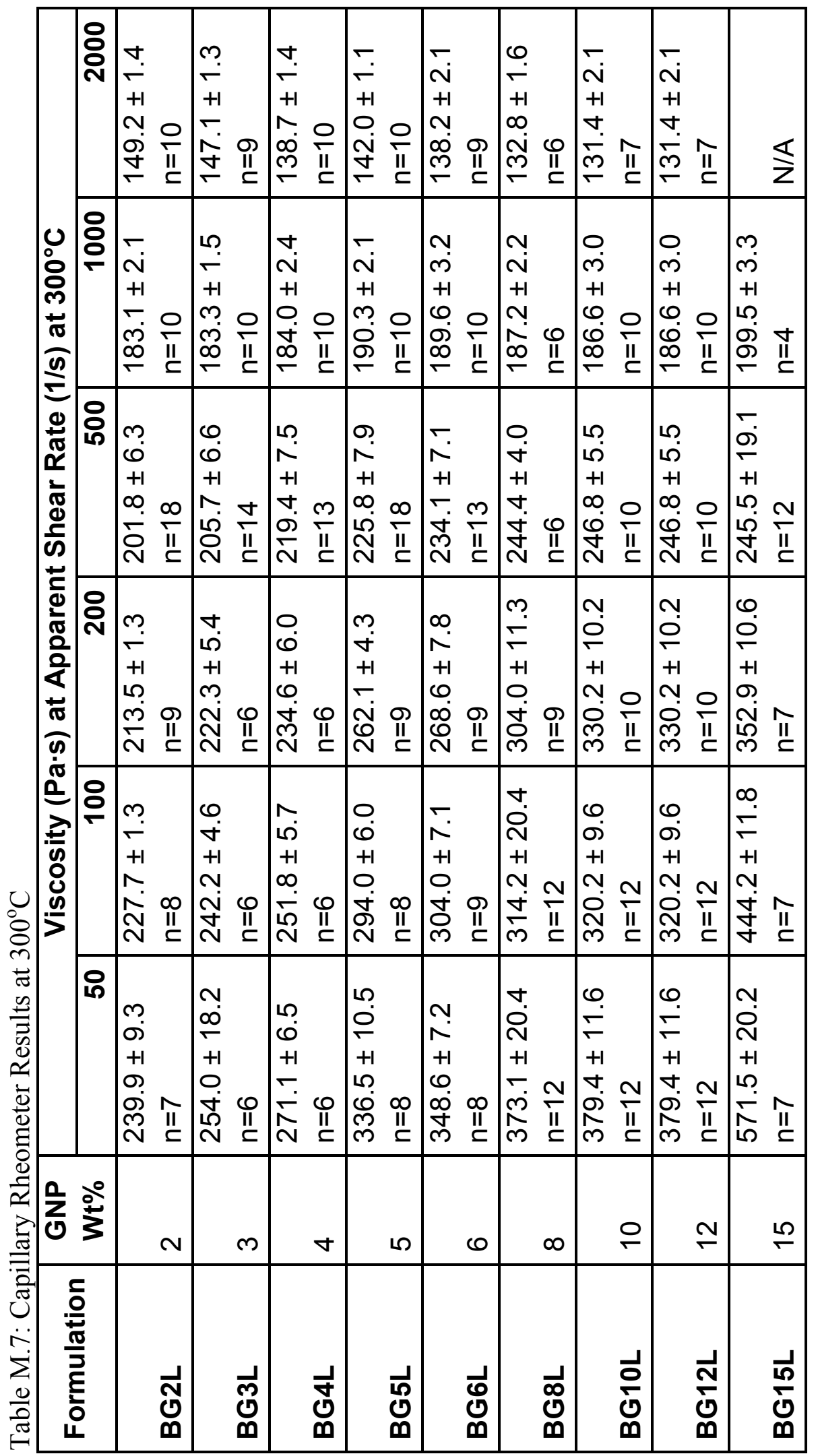




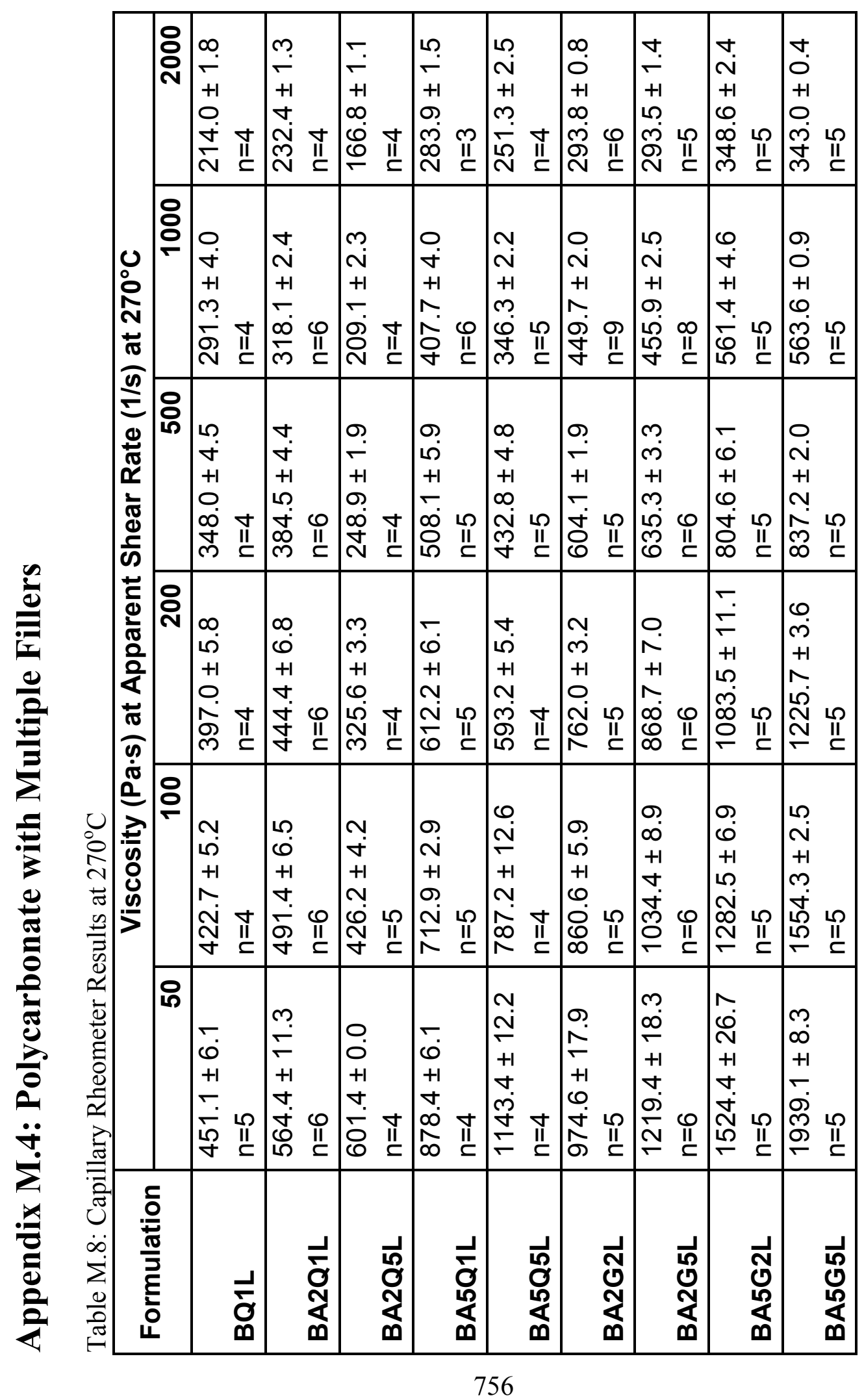




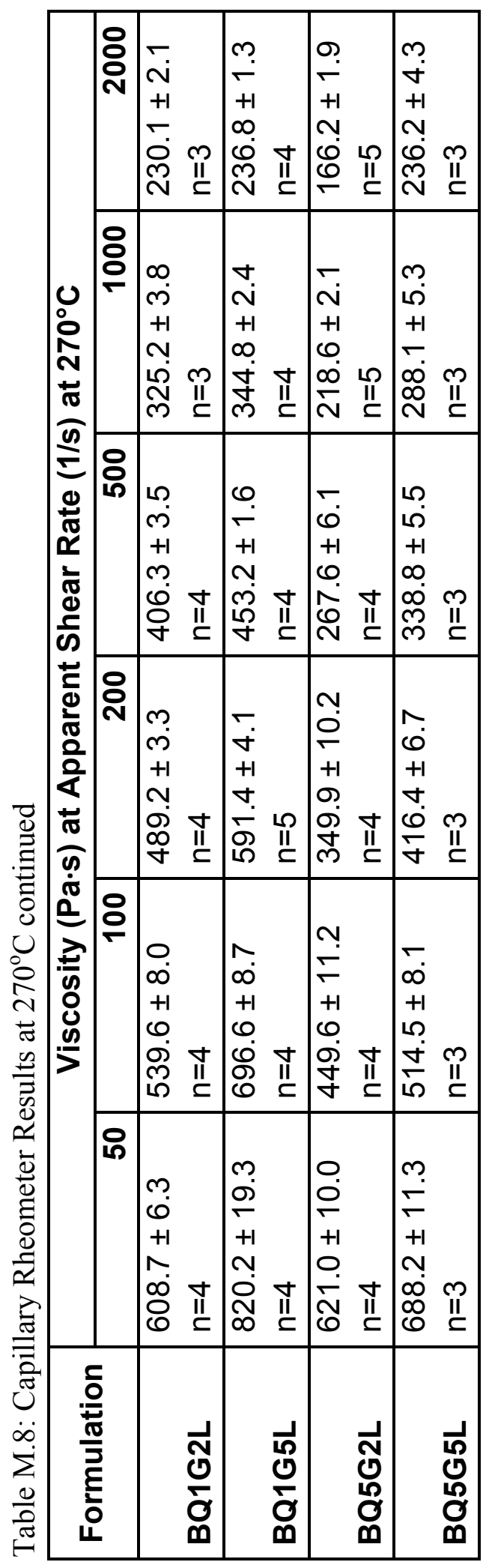

